\title{
Characterization of Gonadal Transcriptomes from the Turbot (Scophthalmus maximus)
}

\begin{tabular}{|c|c|}
\hline Journal: & Genome \\
\hline Manuscript ID & gen-2014-0190.R3 \\
\hline Manuscript Type: & Article \\
\hline Date Submitted by the Author: & 02-Oct-2015 \\
\hline Complete List of Authors: & $\begin{array}{l}\text { Hu, Yulong; Yellow Sea Fisheries Research Institute (YSFRI), } \\
\text { Huang, Meng; cornell university, } \\
\text { Wang, Weiji; Yellow Sea Fisheries Research Institute (YSFRI), } \\
\text { Guan, Jiantao; Yellow Sea Fisheries Research Institute (YSFRI), } \\
\text { Kong, Jie; Yellow Sea Fisheries Research Institute (YSFRI), }\end{array}$ \\
\hline Keyword: & Turbot, Transcriptome, sex, SNP \\
\hline \multicolumn{2}{|c|}{$\begin{array}{l}\text { Note: The following files were submitted by the author for peer review, but cannot be converted to } \\
\text { PDF. You must view these files (e.g. movies) online. }\end{array}$} \\
\hline $\begin{array}{l}\text { gen-2014-0190.R3 Supplement1 } \\
\text { gen-2014-0190.R3 Supplement2 }\end{array}$ & $\begin{array}{l}\text { 1-Dataset S1.rar } \\
\text { 2-Dataset S2.zip }\end{array}$ \\
\hline
\end{tabular}

\section{SCHOLARONE $^{\text {'x }}$}

Manuscripts 


\section{Characterization of Gonadal Transcriptomes from the Turbot (Scophthalmus maximus)}

Yulong Hu \#, Meng Huang \#, Weiji Wang, Jiantao Guan, Jie Kong*

Yellow Sea Fisheries Research Institute (YSFRI), Chinese Academy of Fishery

Sciences, Qingdao, China

Corresponding author:

Jie Kong, $\mathrm{PhD}$

Yellow Sea Fisheries Research Institute (YSFRI)

Chinese Academy of Fishery Sciences

Qingdao, China

Phone: 0086-532-85821650

Fax: 0086053285811514

Email: kongjie@sina.com 


\section{Abstract}

The mechanisms underlying sexual reproduction and sex ratio determination remains unclear in turbot, a flatfish of great commercial value. And there is limited information in the turbot database regarding genes related to the reproductive system. Here, we conducted high-throughput transcriptome profiling of turbot gonad tissues to better understand their reproductive functions and to supply essential gene sequence information for marker-assisted selection programs in the turbot industry. In this study, two gonad libraries representing sex differences in S.maximus yielded 453,818 high-quality reads that were assembled into 24,611 contigs and 33,713 singletons by using 454 pyrosequencing, 13,936 CS (contigs and singletons) of which were annotated using BLASTx. GO (Gene ontology) and KEGG (Kyoto Encyclopedia of Genes and Genomes) pathway analyses, revealed that various biological functions and processes were associated with many of the annotated CS (contigs and singletons). Expression analyses showed that 510 genes were differentially expressed in males versus females; $80 \%$ of these genes were annotated. In addition, 6,484 and 6,036 single nucleotide polymorphisms (SNPs) were identified in males and females libraries, respectively. This transcriptome resource will serve as the foundation for cDNA or SNP microarray construction, gene expression characterization, and sex-specific linkage mapping in turbot.

Key words: Turbot; Transcriptome; sex; SNP 


\section{Introduction}

The turbot (Scophthalmus maximus), also known as Psetta maxima, is an important economic flatfish species belonging to the family Scophthalmidae (order Pleuronectiformes). It is a marine flatfish species naturally distributed all along the European coasts, from Norway to Morocco in the Atlantic area, and across the northern coasts of the Mediterranean up to the Black Sea (Blanquer et al. 1992). Following, it was introduced to China as well for farming purposes in 1992 (Lei 2003). In 2012, turbot aquaculture production was 12,676 tons in Europe, while 64,000 tons in China, which represents a world leader in adult turbot production (FAO 2014).

During intensive farming, turbot females can exhibit over two-fold increase in their growth rate as compared to males (Husebye et al. 1994). This is among the highest sex-related differential growth rates in cultured marine fish (Piferrer et al. 1995). The difference in the growth rate could be observed as early as 8 months after hatching (Imsland et al. 1997), and it is maintained throughout the production cycle, even following sexual maturation (Piferrer et al. 2004). Under culture conditions, the males also mature earlier than females, which can reduce somatic growth and increase susceptibility to diseases and mortality (Imsland et al. 1997; Piferrer et al. 2003). Altogether, these factors result in loss of production during the grow-out phase and, therefore, all-female populations should be established to optimize turbot production.

The sex ratio depends on processes related to both genetic and environmental factors, which are directly related to the reproductive potential of the population (Penman et al. 2008). Flatfish have been reported to possess genetic systems ranging from polygenic to dominant sex-determining factors that are influenced by autosomal controls in male (XX/XY) or female heterogametic systems (ZZ/ZW) (Purdom 1972; Lincoln 1981; Tabata 1991; Howell et al. 1995; Aida and Arai 1998; Goto et al. 1999; 
Tvedt et al. 2006). The most common mode of GSD is determined by the inherited combination of sex-determining genes or polygenic systems (Oldfield 2005). Recently, the number of identified expressed sequence tags (ESTs) has increased very rapidly in turbot, it remains insufficient when compared with some other teleost (e.g. 15,598 vs 301,380; Salmon, GenBank/NCBI). Although several sex-associated markers have been discovered (Casas et al. 2005) and some linkage maps constructed or enriched (Bouza et al. 2007; Bouza et al. 2008; Bouza et al. 2012; Martínez et al. 2008), the sex-determining gene remains not defined and precisely sex-associated linkage maps are not available. However, development of a database of genes sequences has led to the identification of the major sex-determining region (Martínez et al. 2009) and mapping of DNA sex-specific markers and genes related to sex differentiation in turbot (Viñas et al. 2012).

RNA sequencing (RNA-seq) was used for transcriptome profiling, allowing us to rapidly identify the vast majority of transcriptomes and new splicing variants in a cost-effective manner. The Genome Sequencer FLX (GS FLX) System, powered by 454 sequencing technology, was selected as the sequencing platform because this technology is more appropriate than other next-generation sequencing platforms (such as the Illumina Solexa and ABI SOLiD) for de novo transcriptome sequencing in non-model organisms (Vera et al. 2008; Meyer et al. 2009; O’Neil et al. 2010). In particular, many transcriptome profiles of some marine species have been successfully mapped by using 454 sequencing (Kawahara-Miki et al. 2011; Milan et al. 2011; Hou et al. 2011; Du et al. 2012; Pereiro et al. 2012).

Recent studies have reported large-scale transcriptome profiling of S.maximus using 454 sequencing to identify candidate genes involved in immunity and sex differentiation (Pereiro et al. 2012; Rabis et al. 2013). Although the data (more than 
120,876 contigs and 237,580 singletons) obtained from those tissues (brain, hypophysis, gonad, gill, liver, spleen and head kidney) had found some genes involved in sex differentiation and maturation, more sequencing information is still needed to identify novel, key genes involved in gonad-specific or sex-associated genes. To supplement the genomic resources of the turbot, especially with respect to the sex transcriptome, we used a 454 pyrosequencing method to identify differentially expressed genes and potential genetic markers in gonad tissues. The data generated in this study will facilitate further research examining sex- and reproductive-related genes in the turbot, which will be useful for linkage map construction.

\section{Materials and methods}

\section{Ethics Statement}

The Institutional Animal Care and Use Committee (IACUC) of the Yellow Sea Fisheries Research Institute (YSFRI) approved this animal study. All of the fishes were sacrificed by an overdose of anesthesia to minimize suffering, and the experimental procedures were performed according to the institutional guidelines of the State Council of the People's Republic of China.

\section{Turbot sampling}

Gonad tissues from 12 male and 12 female turbot (aged 600 days) were collected for 454 sequencing. Artificial fertilization and maintenance of the larval cultures were performed by the Huanghai Aquatic Product Group Co., Ltd. (Haiyang, Shandong Province, China). Twenty juvenile fishes (aged 150 days) were provided by the Laboratory of Genetic Resources and Breeding for independent SNP validation at the Yellow Sea Fisheries Research Institute (Qingdao, Shandong Province, China). The analyzed turbot represented a mixture of unrelated families, and the number, age and 
mean value of morphometric data (standard length, width and weight) for each animal group were as follows: adult males $(n=12 ; 600$ days post-hatch; $29.5 \pm 1.6 \mathrm{~cm}, 24.0 \pm 1.0$ $\mathrm{cm}, 1398.7 \pm 88.4 \mathrm{~g})$; adult females $(n=12 ; 600$ days post-hatch; $30.7 \pm 0.9 \mathrm{~cm}, 25.0 \pm 0.6$ $\mathrm{cm}, 1519.8 \pm 165.7 \mathrm{~g})$; juvenile males $(n=10 ; 150$ days post-hatch; $8.8 \pm 1.1 \mathrm{~cm}, 6.7 \pm 1.0$ $\mathrm{cm}, 28.2 \pm 11.0 \mathrm{~g}$ ); juvenile females $(n=10 ; 150$ days post-hatch; $9.3 \pm 0.6 \mathrm{~cm}, 7.3 \pm 0.4$ $\mathrm{cm}, 36.2 \pm 7.8 \mathrm{~g}$ ). Gonads were removed from all turbot individuals for 454 sequencing and sex identification, and pterygiophore tissues were removed from juvenile turbot for SNP validation. For sex identification, the tissues were stained with hematoxylinand eosin, embedded in paraffin and sliced into $6-\mu \mathrm{m}$ sections. Phenotypic sex identification was conducted by microscopic observation. All of the samples subjected to 454 sequencing and SNP validation were flash frozen in liquid nitrogen and stored at $-80{ }^{\circ} \mathrm{C}$ until RNA extraction.

\section{Total RNA extraction, cDNA library construction and 454 GS-FLX sequencing}

Total RNA was extracted and purified from each tissue using the RNA prep pure Tissue Kit (Tiangen Biotech (Beijing) Co., Ltd., China) according to the manufacturer's protocol. The quantity and quality of the total RNA was assessed using an Ultrospec ${ }^{\mathrm{TM}} 2100$ pro UV/Visible Spectrophotometer (Amersham Biosciences, Uppsala, Sweden) and by gel electrophoresis. The final concentration of RNA was adjusted to $1 \mathrm{mg} / \mathrm{mL}$, and $18 \mathrm{~S}$ and $28 \mathrm{~S}$ ribosomal RNA bands were visualized by electrophoresis using denaturing agarose gel (1X) with MOPS buffer. Full-length enriched cDNA was synthesized from $1.5 \mu \mathrm{g}$ of two pooled (male and female) total RNA samples, and two independent transcriptome sequencing libraries were constructed as described previously (Meyer et al. 2009; Hou et al. 2011). Approximately $5 \mu \mathrm{g}$ of normalized cDNA from each library was used to generate a 
454 library, which was sequenced using the Roche Genome Sequencer FLX System (Roche, Basel, Switzerland). Male and female libraries were sequenced separately within $3 / 8$ of a 454 sequencing run. All of the sequencing reads obtained in this high-throughput sequence analysis were deposited in the Short Read Archive (SRA) database (http://www.ncbi.nlm.nih.gov/sra/) under the accession number SRP021171.

\section{Sequence assembly and functional annotation}

Seq-clean software (http://compbio.dfci.harvard.edu/tgi/software/) was used to process all of the pyrosequencing data prior to assembly. Short sequences $(<50 \mathrm{bp})$ and reads with contaminating vectors were discarded, and subsequently the adapters were trimmed. The pre-processed sequences were assembled using the CAP3 program, which has been used to compute overlaps among reads, construct multiple sequence alignments of reads, and to generate consensus sequences (Huang and Madan, 1999). To eliminate potential biases introduced by the RNA normalization process, only clean reads presenting a minimum length of $50 \mathrm{bp}$ and a minimum sequence identity of $90 \%$ were assembled. Similar contigs from the same gene ( $\geq 60 \%$ of the sequence with $>95 \%$ identity) were grouped into clusters using the BLAST Clust program (Altschul et al. 1997). Contigs or singletons $<100$ bp were removed, and then 58,324 CS (contigs and singletons) were selected from the two libraries for the annotation step.

For the gene annotation of S. maximus, all consensus contigs or singletons from each cluster were compared to the Swissprot/Metazoan Refseq/nr and uniprot/TrEMBLstats databases using BLASTx with an E-value threshold of $1 \mathrm{e}^{-5}$. Blast2GO software was used for the GO analysis (levels 2 and 3), and query sequences were assigned to the following GO terms: biological processes, molecular 
functions and cellular components (Götz et al. 2008). KEGG analysis was performed by submitting the data to the online KEGG Automatic Annotation Server (KAAS) to obtain an overview of the gene pathways networks (http://www.genome.jp/kegg/kaas/).

\section{Transcriptome comparison between male and female libraries}

To detect genes that might be involved in gonad differentiation, the relative expression of each unigene (calculated based on the total number of reads from the male or the female library) was compared between the libraries. The longest contig fragments or singletons were selected as the reference inputs for each realignment analysis (i.e., the unigene reference). BLASTN was used to align the reads from both libraries to the reference unigene set. After the raw reads were mapped to the reference unigene set the program IDEG6 (http://telethon.bio.unipd.it/bioinfo/IDEG6) selecting the Fisher's exact test option (Romualdi et al. 2003) was used to detect significant differences in differential expression between the sexes. To account for multiple testing, the false discovery rate (FDR) was calculated using the QVALUE program (Storey, 2002). In our study, to ensure consistent results, a strict algorithm was used to identify these genes: a gene expression level with a false discovery rate (FDR) $\leq 0.01$ and a $P$-value (Fisher's exact test) $\leq 0.05$ was selected to compare the two samples.

\section{SNP detection}

Potential SNPs, including insertion and deletion mutations, were identified using ssaha SNP software (Ning et al. 2005). SNP identification was limited to a minor allele frequency $>10 \%$.

\section{SNP validation}

Fifty predicted SNPs were selected for low-flux experimental validation. 
Pterygiophore tissue samples from 20 juvenile turbot were collected for SNP validation. Genomic DNA was extracted from pterygiophore tissues using the TIANamp marine animals DNA Kit (Tiangen Biotech Co. Ltd., Beijing, China). A matrix-assisted laser desorption/ionization-time of flight mass spectrometry (Sequenom Mass ARRAY, Bioyong Technologies Inc., Hong Kong) assay was used for individual genotyping.

\section{Results and discussion}

\section{Sequencing and assembly}

To maximize sex differences in transcript representation, male and female gonad cDNA libraries were constructed using 454 sequencing with 12 males and 12 females, respectively; all 24 samples were pooled for SNP or SSR identification. After a 3/8 single-run of 454 sequencing, 453,818 raw reads with an average length of 283 bases were obtained. The size distribution of raw reads from the two cDNA libraries is shown in Figure S1. Reads obtained in this high throughput sequence analysis have been submitted to the NCBI Sequence Read Archive. All of the reads were assembled into 24,611 contigs and 33,713 singletons, with an average contig length of 476bp. The statistical methods used for reads assembled, using Roche 454-pyrosequencing, are summarized in Table 1. The assembled contigs and singleton sequences are shown in dataset S1. Singletons with a length of $>100 \mathrm{bp}$ were selected for further analysis. Of 58,324 CS (singletons and contigs), 13,936(23.89\%) had a significant BLASTx result with proteins present in the Swissprot/Metazoan Refseq/nr and uniprot/TrEMBLstats databases, with $>30 \%$ protein similarity and an E-value $<1 \mathrm{e}^{-5}$.

Compared with the 454 sequencing data reported by Pereiro et al. (Pereiro et al. 2012), we found fewer high-quality reads and singletons. However, the tissues used in 
the current study differed from those used by Pereiro et al. In a recent report, a large-scale transcriptome profiling of gonad tissues was described (Ribas et al. 2013). Many reads from gonad tissues were supplied to the turbot transcriptome database; however, the gonad tissues were pooled with brain and hypophsis. Thus, some novel genes and sex-specific markers of the gonad may have been overlooked. Prior to the use of next-generation sequencing (NGS) technologies, there were only 15,598 ESTs and approximately 56,223 nucleotide sequences available for S. maximus in the NCBI GenBank database. An EST database constructed using cDNA libraries from turbot immune tissues of the turbot was previously enriched using strategies based on Sanger sequencing. A total of 1,073 contigs and 2,409 singletons were partially sequenced from three different cDNA libraries containing liver, spleen and head kidney tissues: turbot infected with Aeromonas salmonicida, Philasterides dicentrarchi and healthy fish (Pardo et al. 2008). The liver, kidney and gill libraries of nodavirus infected turbot provided 3,173 high-quality sequences and 768 unique genes (Park et al. 2009). Forty-nine ESTs were obtained from the kidney and spleen of turbot that were artificially infected with Vibrio harveyi (Wang et al. 2008). Many of the contigs identified in the present investigation may be used for further genomic and transcriptome studies in the turbot.

\section{Gene annotation analysis}

The Gene Ontology (GO) project is a major bioinformatics initiative that is commonly used to standardize the representation of genes and to categorize gene products across species and databases. To mitigate redundancy and avoid the inclusion of contigs from the same cluster with the same annotation, the longest contig from each cluster was submitted to the Blast2 GO suite for classification into

three functional groups (Cellular Component, Biological Process or Molecular 
Function) based on GO terminology (Conesa et al. 2005). In total, 7,710 (22.87\%) and 6,226 (25.30\%) CS (contigs and singletons) from males and females, respectively, were assigned to a GO category. The distribution of contigs in different GO categories (level 3) is shown in Figure 1. The Biological Process group comprised contigs involved in cellular metabolic processes (males, 17.48\%; females, $14.08 \%$ ), primary metabolic processes (males, 16.33\%; females, 14.62\%) and macromolecular metabolic processes (males, 15.14\%; females, 13.46\%), suggesting that there was a higher degree of metabolic activity in the males' sampled tissues. Some proteins related to reproduction in males were much higher than females, included three categories, regulation of biological processes, metabolic processes related to nitrogen compounds, and organization of cellular components. The results revealed similar representations between males and females in the following categories: cell part, intracellular, intracellular part and organelles. Finally, in the Molecular Function group, a transcriptome comparison revealed differences in the representation of GO terms between males and females. There were significant differences in structural constituents of the ribosome (males, 19.97\%; females, 12.06\%), oxidoreductase activity (males, 12.19\%; females, 5.06\%) and nucleoside binding (males, 3.18\%; females, 5.34\%). Gene annotation information obtained by comparing contigs or singletons against the Swiss-Prot database is shown in dataset S2.

\section{KEGG pathway analysis}

KEGG (Kyoto Encyclopedia of Genes and Genomes, KEGG) pathway analysis was conducted for the annotated contigs. The KEGG pathway, consisting mainly of graphical diagrams of biochemical pathways, is a knowledge base for the systematic analysis of gene function in terms of networks of genes and molecules (Hiroyuki et al. 1999). Enzyme commission (EC) numbers were assigned to 7,710 CS (contigs and 
singletons) for males and 6,226 CS for females, which were classified into 238 and 247 different pathways, respectively. A summary of the CS mapping in the primary pathway is shown in Table 2. Approximately $28 \%$ of the CS from both males and females were involved in metabolism, in particular, carbohydrate metabolism, nucleotide metabolism, and amino acid metabolism. Among these annotated CS in males and females, $18 \%$ and $28 \%$, respectively, were classified into the category of genetic information processing. Moreover, there was a preponderance of terms in the sub-categories of translation and of replication and repair. Cellular processes were represented by approximately $19 \%$ of the CS in both males and females. In addition, $13 \%$ and $10 \%$ of the annotated CS from males and females, respectively, were classified in the immune system and endocrine system categories, which are typically closely related to reproductive processes.

\section{Differential expression analysis comparing male and female turbot}

Compared with male turbot, female turbot grow faster and achieve a larger size; therefore, an all-female population would be of significant benefit for turbot aquaculture. Sex determination is important for turbot breeding with respect to the control of the proportions of each sex. Most of the previous studies examining turbot sex determination have focused on temperature-related, hormonal and genetic factors; however, genetic factors are under-reported. Molecular biology provides a powerful approach for research examining the genetic mechanism of sex determination. Furthermore, gene expression analyses provide indispensable information.

To identify differentially expressed genes, the sequencing coverage distribution was compared between the male and female libraries. There were 4,197 common genes in males and females, 28,138 exclusive genes in males and 12,017 exclusive genes in females. In these common genes, expression of 580 common expressed 
genes were detected above 10 , while there were 510 significant differentially expressed genes by Fisher test $(P \leqslant 0.05)$, of which 263 and 247 were down-regulated and up-regulated in males, respectively. All 4,197 common expressed genes, 580 common genes with expression above 10 and 510 differentially expressed genes $(P \leqslant 0.05)$ identified by transcriptome comparison between male and female turbots are presented in Table S1. Of these differentially expressed genes, 465 have been annotated previously; moreover, 277 clustered into 27 GO categories (biological processes, 11; cellular components, 6; molecular functions, 10) and 41 were mapped on to 25 terms in the KEGG pathway. For example, the geminin gene was expressed 56 times more in the female library compared with the male library; the nucleoprotein geminin plays an important role in cell proliferation, cell differentiation, and embryonic development and tumorigenesis, among others (Wohlschlegel et al. 2002; Hansel et al. 2003; Xouri et al. 2004). The pituitary tumor transforming (PTTG) gene, which demonstrated the highest differential expression level that was 71 times greater in females compared with males, is a key molecular component of the mitotic spindle checkpoint pathway, ensuring orderly progression of the cell cycle, and the cell division as well as genetic stability ( $\mathrm{Yu}$ et al. 2000). In addition, the expression of $P T T G$ increases substantially in response to estrogen stimulation, promoting the expression of fibroblast growth factor $\beta(b F G F)$ (Heaney et al. 1999). Meanwhile, the expression level of the protein ubiquitin $(\mathrm{Ub})$ was found to be 8 times greater in male gonad than female, the roles of which are mediated by its covalent attachment to target protein, a process known as ubiquitylation, may be involved in human reproductive process, like gametogenesis, modulation of steroid receptor concentration at the beginning of pregnancy (Bebington et al. 2001). Recently, there is increasing evidence that both ubiquitination and its reversal, deubiquitination, play 
crucial roles not only during the development of the immune system but also in the orchestration of an immune response by ensuring the proper functioning of the different cell types that constitute the immune system(Zinngrebe et al. 2014). In marine organism, Ub was found to be involved in the gonad development of Scylla paramamosain, an economically important breeding crab found along the southeast coast of China (Dai et al 2012). Other unannotated genes with significant changes in expression level are also worthy of further research. However, many genes that were not included in the comparison of the two libraries due to a low level of expression should also be noted. Furthermore, future studies should focus on more detailed transcriptome sequencing.

\section{SNP detection}

The 454 RNA-seq datas were also used to compare individual or pooled samples to detect molecular markers. The molecular markers (e.g., SNP) obtained from the transcribed regions have great potential for the identification of functional genes related to sex determination and sex-linked inherited economical traits.

Among the single base and indel mutations, 6,484 and 6,035 SNPs were identified in males and females, respectively, including transition and trans-version proportions of $72 \%$ and $28 \%, 71 \%$ and $29 \%$ in males and females, respectively (Table 3 ). Information of all SNPs detected in males and females are presented in Table S2. In addition, 7,799 and 8,698 insertion/deletion mutations were detected in males and females, respectively, with single nucleotide indels representing proportions of $97 \%$ in both samples. Comparison of these mutations in common genes that were expressed similarly in both males and females indicated that many genes contained unique or sex-linked mutations. For example, the gene glutathione peroxidase $4 \beta(G p x 4 b)$ had an insertion mutation that was detected in most of the female reads but was not 
detected in the male group. The same phenomenon was observed for the Ran gene encoding the GTP-binding nuclear protein; however, SNP screening revealed a repeated region (GTACT) inserted in this gene in the male group only. The frequency of the nucleophosmin gene was much higher in females than in males, and there were a large number of genes or contigs with opposite frequencies when comparing the different libraries. Fifty SNPs were subjected to experimental validation in twenty juvenile fishes (ten male and ten female) using the Mass ARRAY ${ }^{\circledR}$ method. Thirty-one of these SNPs could be validated, suggesting that approximately $60 \%$ of the predicted SNPs were indeed true SNPs. The results indicated that the gene frequencies of most true SNPs matched between the two methods with different flux (Table 4). Due to the limited number of samples and SNPs used in validation, we could not confirm all of the markers separately.

We anticipate that among the SNPs obtained using RNA-seq, there are many sex-specific markers that would be strong candidates for the identification of sex determination markers. In a previous study of sex determination in flatfish, many approaches were developed and several molecular markers were identified in the marine fish (Chen et al. 2008; Liao et al. 2009; Martínez et al. 2009; Kanako et al. 2010). In the tongue sole, a female-specific AFLP marker (CseF305) was isolated to effectively identify the sex of the half-smooth tongue sole (Chen et al. 2008). In the same study, a primary genetic linkage map, including 103 AFLP markers, 33 microsatellite markers, and one female-specific DNA marker, was constructed for the tongue sole, with an average spacing of $8.4 \mathrm{cM}$ and spanning $64.4 \%$ of the estimated genome size (Liao et al. 2009). The sex identification method used for the tongue sole provided an applied approach for sex-associated and for the possibility of an 
all-female population in the turbot. In further studies, validation using a low-flux method will be used in a large-scale population, and an effective sex identification system based on molecular markers will be established in the turbot.

In conclusion, using the 454 GS FLX platform to analyze a genital gland library, we obtained a large number of contigs for the turbot genomic database. These contigs supported gene digging and molecule characterization of gonad determination mechanisms at the whole genome level. In addition, the SSR and SNP data generated here in turbot were intended for sex identification and marker accumulation using genome-wide association analyses.

\section{Acknowledgments}

This research was supported financially by the sub-project "Elite Turbot Cultivation (2012AA10AA408-7)" of the National High-tech Research Develop Project (863), the special fund project "The Genetic Background and Mechanism of Important Traits in Turbot (2010-cb-04)" and "The Compare of Genome-wide Association Study Models in Turbot (20603022013026)" of the Basic Scientific Research Expenses of the Yellow Sea Fisheries Research Institute. 


\section{References}

Aida, S., and Arai, K., 1998. Sex ratio in the progeny of gynogenetic diploid marbled sole Limanda yokohamae males. Fish Sci. 64: 989-990.

Altschul, S.F., Madden, T.L., Schäffer, A.A., Zhang, J., Zhang, Z., et al, 1997. Gapped BLAST and PSI-BLAST, a new generation of protein database search programs. Nucleic Acids Res. 25: 3389-3402.

Bebington, C., Doherty, F.J., Fleming, S.D., 2001.The possible biological and reproductive functions of ubiquitin. Human Reproduction Update, 7(1): 102-111.

Blanquer, A., Alayse, J.P., Berrada-Rkhami, O., Berrebi, S., 1992. Allozyme variation in turbot (Psetta maxima) and brill (Scophthalmus rhombus) (Osteichthyes, Pleuronectiformes, Scophthalmidae) throughout their range in Europe. J. Fish Biol. 41: $725-36$

Bouza, C., Hermida, M., Millán, A., Vilas, R., Vera, M., et al, 2008. Characterization of EST-derived microsatellites for gene mapping and evolutionary genomics in turbot. Anim. Genet. 39: 666-670.

Bouza, C., Hermida, M., Pardo, B.G., Fernández, C., Fortes, G.G., et al, 2007. A microsatellite genetic map of the turbot (Scophthalmus maximus). Genetics, 177: $2457-2467$.

Bouza, C., Hermida, M., Pardo, B.G., Vera, M., Fernández, C., et al, 2012. An Expressed Sequence Tag (EST)-enriched genetic map of turbot (Scophthalmus maximus), a useful framework for comparative genomics across model and farmed teleosts. BMC Genet. 13: 54. 
Casas, L., Sánchez, L., Orban, L., 2005. Sex-associated DNA markers from turbot. Mar. Biol. Res. 7: 378-387.

Chen, S.L., Deng, S.P., Ma, H.Y., Tian, Y.S., Xu, J.Y., et a1, 2008. Molecular marker-assisted sex control in half-smooth tongue sole (Cynoglossus semilaevis). Aquaculture, 283: 7-12.

Conesa, A., Go“tz, S., Garc1'a-Go'mez, J.M., Terol, J., Talon, M., et al, 2005. Blast2GO, a universal tool for annotation, visualization and analysis in functional genomics research. Bioinformatics, 21: 3674-3676.

Dai, Y., Han K., Yan S., Zou, Z., Zhang Z., Wang, Y., 2012. Molecular characterization and expression profiles of $\mathrm{Sp}-\mathrm{Ub}$ during gonad development in Scylla paramamosain. J.F.S.C, 19(6): 946-955.

Du, H., Bao, Z., Hou, R., Wang, S., Su, H., et al, 2012. Transcriptome Sequencing and Characterization for the Sea Cucumber Apostichopus japonicus (Selenka,1867). PLoS One, 7: e33311.

Food and Agriculture Organization of the United Nations (FAO), 2014. The state of world fisheries and aquaculture. Rome, FAO.

Goto, R., Mori, T., Kawamata, K., Matsubara, T., Mizuno, S., Adachi, S., Yamauchi, K., 1999. Effects of temperature on gonad sex determination in barfin flounder Verasper moseri. Fish Sci. 65: 884-887.

Götz, S., García-Gómez, J.M., Terol, J., Williams, T.D., Nagaraj, S.H., et al, 2008. High-throughput functional annotation and data mining with the Blast2GO suite. Nucleic Acids Res. 36: 3420-3435. 
Hansel, D.E., Rahman, A., Hidalgo, M., Thuluvath, P.J., Lillemoe, K.D., et al, 2003. Identification of novel cellular targets in biliary tract cancers using global gene expression technology. Am. J. Pathol. 163: 217-229.

Heaney, A.P., Horwitz, G.A., Wang, Z., Singson, R., Melmed, S., 1999. Early involvement of estrogen-induced pituitary tumor transforming gene and fibroblast growth factor expression in prolactinoma pathogenesis. Nat Med. 5(11): 1317-1321. Hiroyuki, O., Susumu, G., Kazushige, S., Wataru, F., Hidemasa, B., et al, 1999. KEGG, Kyoto Encyclopedia of Genes and Genomes. Nucleic Acids Res. 27:29-34.

Hou, R., Bao, Z.M., Wang, S., Su, H.L., Li, Y., et al, 2011. Transcriptome sequencing and de novo analysis for Yesso scallop (Patinopecten yessoensis) using 454 GS FLX. PLoS One, 6: e21560.

Howell, B.R., Baynes, S.M., Thompson, D., 1995. Progress toward the identification of the sex determining mechanism of the sole, Solea solea (L.), by the induction of diploid gynogenesis. Aquacult Res. 26: 135-140.

Huang, X., Madan, A., 1999. CAP3, A DNA Sequence Assembly Program. Genome Res. 9: 868-877.

Husebye, H., Lund, S., Moller, M., Sunde, A., Krokan, H.E., 1994. A Bkm-related DNA sequence gives individual DNA finger-prints in turbot (Scophthalmus maximus), but neither Bkm-related, human SRYor human ZFY probes detect genetic sex-differences. Comp. Biochem. Physiol. B. 107: 69-73.

Imsland, A.K., Folkvord, A., Grung, G.L., Stefansson, S.O., Taranger, G.L., 1997. Sexual dimorphism in growth and maturation of turbot, Scophthalmus maximus 
(Rafinesque, 1810). Aquacult. Res. 28: 101-14.

Kanako, F., Kazunori, Y., Keita, H., Akiyuki, O., Kazuo, A., Masanori, O., 2010. Identification of the sex-linked locus in yellowtail, Seriola quinqueradiata. Aquaculture, 308: S51-S55.

Kawahara-Miki, R., Wada, K., Azuma, N., Chiba, S., 2011. Expression profiling without genome sequence information in a non-model species, Pandalid shrimp (Pandalus latirostris), by next-generation sequencing. PLoS One, 6: e26043.

Liao, X., Ma, H., Xu, G., Shao, C., Tian, Y., et al, 2009. Construction of a Genetic Linkage Map and Mapping of a Female-Specific DNA Marker in Half-Smooth Tongue Sole (Cynoglossus semilaevis). Mar Biotechnol. 11: 699-709.

Lincoln, R.F., 1981. Sexual maturation in female triploid plaice, Pleuronectes platessa, and plaice $\times$ flounder, Platichthys flesus, hybrids. J. Fish Biol. 19: 499-507.

Lei J.L. 2003. The development direction of turbot farming industry in China (in Chinese). Scientific Fish Farming, 7: 26-28.

Martínez, P., Bouza, C., Hermida, M., Fernández, J., Toro, M.A., et al, 2009. Identification of the Major Sex-Determining Region of Turbot (Scophthalmus maximus). Genetics, 183:1443-1452.

Martínez, P., Hermida, M., Pardo, B.G., Fernández, C., Castro, J., et al, 2008. Centromere-linkage in the turbot (Scophthalmus maximus) through half-tetrad analysis in diploid meiogynogenetics. Aquaculture, 280: 81-88.

Meyer, E., Aglyamova, G.V., Wang, S., Buchanan-Carter, J., Abrego, D., et al, 2009. Sequencing and de novo analysis of a coral larval transcriptome using 454 GSFlx. 
BMC Genomics, 10: 219.

Milan, M., Coppe, A., Reinhardt, R., Cancela, L.M., Leite, R.B., et al, 2011. Transcriptome sequencing and microarray development for the Manila clam, Ruditapes philippinarum, genomic tools for environmental monitoring. BMC Genomics, 12: 234.

Ning, Z., Caccamo, M., and Mullikin, J.C., 2005. ssahaSNP- a Polymorphism Detection Tool on a Whole Genome Scale. In proceedings of Computational Systems Bioinformatics Conference, IEEE, 8-12 Aug 2005. Computational Systems Bioinformatics Conference, IEEE. pp. 251-252.

O’Neil, S.T., Dzurisin, J.D., Czrmichael, R.D., Lobo, N.F., Emrich, S.J., et al, 2010. Population-level transcriptome sequencing of nonmodel organisms Erynnis propertius and Papilio zelicaon. BMC Genomics, 11: 310.

Oldfield, R.G, 2005. Genetic, abiotic and social influences on sex differentiation in cichlid fishes and the evolution of sequential hermaphroditism. Fish Fish. 6:93-110.

Pardo, B.G., Fernández, C., Millán, A., Bouza, C., Vázquez-López, A., et al, 2008. Expressed sequence tags (ESTs) from immune tissues of turbot (Scophthalmus maximus) challenged with pathogens. BMC Vet Res. 4: 37.

Park, K.C., Osborne, J.A., Montes, A., Dios, S., Nerland, A.H., et al, 2009. Immunological responses of turbot (Psetta maxima) to nodavirus infection or polyriboinosinic polyribocytidylic acid (pIC) stimulation, using expressed sequence tags (ESTs) analysis and cDNA microarrays. Fish Shellfish Immunol. 26: 91-108.

Penman, D.J., Piferrer, F., 2008. Fish gonadogenesis. Part I, genetic and 
environmental mechanisms of sex determination. Rev. Fish Sci. 16: 14-32.

Pereiro, P., Balseiro, P., Romero, A., Dios, S., Forn-Cuni, G., et al, 2012. High-Throughput Sequence Analysis of Turbot (Scophthalmus maximus) Transcriptome Using 454-Pyrosequencing for the Discovery of Antiviral Immune Genes. PLoS One, 7: e35369.

Piferrer, F., Blazquez, M., Felip, A., Ramos, J., Carrillo, M., Zanuy, S. 1995. Manipulación de la proporción de sexos en teleósteos y su aplicación en acuicultura. In Actas del V ${ }^{\mathrm{a}}$ Congreso Nacional de Acuicultura. Edited by Castelló, F, Calderer A. Barcelona: Publ. Univ. Barcelona. pp. 18-23.

Piferrer, F., Cal, R.M., Gomez, C., Bouza, C., Martinez, P., 2003. Induction of triploidy in the turbot (Scophthalmus maximus)-II.Effects of cold shock timing and induction of triploidy in a large volume of eggs. Aquaculture, 220: 821-31.

Piferrer, F., Cal, R.M., Gomez, C., Alvarez-Blazquez, B., Castro, J., Martinez, P., 2004. Induction of gynogenesis in the turbot (Scophthalmus maximus): Effects of UV irradiation on sperm motility, the Hertwig effect and viability during the first 6 months of age. Aquaculture, 238: 403-19.

Purdom, C.E., 1972. Induced polyploidy in plaice (Pleuronectes platessa) and its hybrid with the flounder (Platichthys flesus). Heredity, 29: 11-24.

Ribas, L., Pardo B.G., Fernández, C., Álvarez-Diós, J.A., Gómez-Tato, A., et al, 2013. A combined strategy involving Sanger and 454 pyrosequencing increases genomic resources to aid in the management of reproduction, disease control and genetic selection in the turbot (Scophthalmus maximus). BMC genomics, 14: 180. 
Romualdi, C., Bortoluzzi, S., D’Alessi, F., Danieli, G.A., 2003. IDEG6, a web tool for detection of differentially expressed genes in multiple tag sampling experiments. Physiol. Genomics, 12: 159-162.

Storey, J.D., 2002. A direct approach to false discovery rates. J. R. Stat. Soc. B. 64: 479-498.

Tabata, K., 1991. Induction of gynogenetic diploid males and presumption of sex determination in the hirame Paralichthys olivaceus. Nippon Suisan Gakkaishi, 57: 845-850.

Tvedt, H.B., Benfey, T.J., Martin-Robichaud, D.J., McGovan, C., Reith, M., 2006. Gynogenesis and sex determination in Atlantic halibut (Hippoglossus hippoglossus). Aquaculture, 252: 573-583.

Vera, J.C., Wheat, C.W., Fescemyer, H.W., Frilander, M.J., Crawford, D.L., et al, 2008. Rapid transcriptome characterization for a nonmodel organism using 454 pyrosequencing. Mol. Ecol. 17: 1636-1647.

Viñas, A., Taboada, X., Vale, L., Robledo, D., Hermida, M.,et al, 2012. Mapping of DNA sex-specific markers and genes related to sex differentiation in turbot (Scophthalmus maximus). Mar. Biotechnol. 14:655-663.

Wang, C., Zhang, X.H., Jia, A., Chen, J., Austin, B., 2008. Identification of immune related genes from kidney and spleen of turbot, Psetta maxima (L.), by suppression subtractive hybridization following challenge with Vibrio harveyi. J. Fish Dis. 31: $505-514$

Wohlschlegel, J.A., Kutok, J.L., Weng, A.P., Dutta, A., 2002. Expression of geminin 
as a marker of cell proliferation in normal tissues and malignancies. Am. J. Pathol. 161: 267-273.

Xouri, G., Lygerou, Z., Nishitani, H., Pachnis, V., Nurse, P., et al, 2004. Cdt1 and geminin are down-regulated upon cell cycle exit and are over-expressed in cancer-derived cell lines. Eur. J. Biochem. 271: 3368-3378.

Yu, R., Ren, S.G., Horwitz, G.A., Wang, Z., Melmed, S., 2000. Pituitary tumor transforming gene (PTTG) regulates placental JEG-3 cell division and survival, evidence from live cell imaging. Mol. Endocrinol. 14: 1137-1146.

Zinngrebe, J., Montinaro, A., Peltzer, N., Walczak, H., 2014. Ubiquitin in the immune system. EMBO reports, 15(1): 28-45. 


\section{Tables}

Table 1. Summary of male and female Scophthalmus maximus 454 pyrosequencing

\begin{tabular}{lll}
\hline & Male & Female \\
\hline Data generation & & \\
Number of Reads & 224,993 & 228,825 \\
Minimal length (bp) & 22 & 20 \\
Maximum length (bp) & 716 & 627 \\
Average length (bp) & 267.78 & 298.67 \\
Standard error of length & 169.73 & 161.57 \\
Total megabases & 68.34 & 60.25 \\
Assembly statistics & & \\
Number of reads assembled & $186,401(81.46 \%)$ & $163,726(72.77 \%)$ \\
Number of contigs & 15,795 & 8,816 \\
Average contig depth & 19.85 & 8.95 \\
Average contig length & 513 & 455 \\
N50 contig length & 609 & 497 \\
Number of singletons & 22,282 & 11,431 \\
Number of singletons $>100 b p$ & 17,953 & 9,546 \\
Number of annotated contigs & 4,371 & 3,686 \\
Number of annotated singletons & 3,339 & 2,540 \\
Total number of annotated CS & 7,710 & 6,226 \\
(contigs and singletons) & & \\
\hline
\end{tabular}


Table 2. KEGG pathway mapping for male and female turbot

\begin{tabular}{|c|c|c|c|}
\hline \multirow{2}{*}{ KEGG Pathways } & \multirow{2}{*}{ Sub-pathways } & \multicolumn{2}{|c|}{ No. of mapped KO /CS } \\
\hline & & Male & Female \\
\hline \multirow{11}{*}{ Metabolism } & Carbohydrate metabolism & $140 / 291$ & $122 / 207$ \\
\hline & Energy metabolism & $74 / 124$ & $72 / 118$ \\
\hline & Lipid metabolism & $89 / 150$ & $66 / 123$ \\
\hline & Nucleotide metabolism & $101 / 170$ & $91 / 149$ \\
\hline & Amino acid metabolism & $103 / 182$ & $94 / 197$ \\
\hline & Metabolism of other amino acids & $32 / 52$ & $35 / 68$ \\
\hline & Glycan biosynthesis and metabolism & $60 / 94$ & $45 / 70$ \\
\hline & Metabolism of cofactors and vitamins & $29 / 41$ & $34 / 54$ \\
\hline & Metabolism of terpenoids and polyketides & $8 / 17$ & $7 / 10$ \\
\hline & Biosynthesis of other secondary metabolites & $12 / 39$ & $9 / 16$ \\
\hline & Xenobiotic biodegradation and metabolism & $41 / 62$ & $33 / 76$ \\
\hline \multirow{4}{*}{$\begin{array}{l}\text { Genetic Information } \\
\text { Processing }\end{array}$} & Transcription & 98/161 & $99 / 160$ \\
\hline & Translation & $138 / 169$ & $123 / 263$ \\
\hline & Folding, sorting and degradation & $197 / 337$ & $189 / 303$ \\
\hline & Replication and repair & $69 / 114$ & $80 / 263$ \\
\hline \multirow{4}{*}{$\begin{array}{l}\text { Environmental } \\
\text { Information } \\
\text { Processing }\end{array}$} & & & \\
\hline & Membrane transport & $12 / 15$ & $5 / 6$ \\
\hline & Signal transduction & $201 / 374$ & $146 / 254$ \\
\hline & Signaling molecules and interaction & $40 / 63$ & $22 / 29$ \\
\hline \multirow{4}{*}{ Cellular Processes } & Transport and catabolism & $141 / 258$ & $125 / 194$ \\
\hline & Cell motility & $50 / 79$ & $31 / 52$ \\
\hline & Cell growth and death & $151 / 247$ & $161 / 308$ \\
\hline & Cell communication & $104 / 206$ & $82 / 156$ \\
\hline \multirow[t]{9}{*}{ Organismal Systems } & Immune system & $203 / 346$ & $141 / 226$ \\
\hline & Endocrine system & $107 / 216$ & $83 / 155$ \\
\hline & Circulatory system & $42 / 77$ & $28 / 49$ \\
\hline & Digestive system & $58 / 109$ & $34 / 59$ \\
\hline & Excretory system & $25 / 48$ & $25 / 34$ \\
\hline & Nervous system & $61 / 120$ & $37 / 75$ \\
\hline & Sensory system & $13 / 37$ & $13 / 29$ \\
\hline & Development & $24 / 37$ & $21 / 34$ \\
\hline & Environmental adaptation & $13 / 33$ & $9 / 24$ \\
\hline
\end{tabular}

Note: KO means KEGG Ortholog; CS means contigs and singletons. 
Table 3. Summary of SNPs/indels identified in the male and female transcriptome

\begin{tabular}{lll}
\hline Type & Male & Female \\
\hline Transition & & \\
$\mathrm{A} / \mathrm{G}$ & 2,334 & 2,176 \\
$\mathrm{C} / \mathrm{T}$ & 2,363 & 2,091 \\
Transversion & & \\
$\mathrm{A} / \mathrm{C}$ & 448 & 494 \\
$\mathrm{~A} / \mathrm{T}$ & 583 & 518 \\
$\mathrm{C} / \mathrm{G}$ & 271 & 299 \\
$\mathrm{G} / \mathrm{T}$ & 485 & 457 \\
Indel & 7,799 & 8,698 \\
Total & 14,283 & 14,734 \\
\hline
\end{tabular}


Table 4. Representation of SNPs scanning in 454 sequencing and MassARRAY

\begin{tabular}{|c|c|c|c|c|c|c|c|}
\hline \multirow[b]{2}{*}{ SNP index } & \multicolumn{3}{|c|}{454 sequencing result } & \multicolumn{4}{|c|}{ MassARRAY result } \\
\hline & Allele & $\begin{array}{c}\text { Reads number } \\
\text { of A allele }\end{array}$ & $\begin{array}{c}\text { Reads number of } \\
\text { B allele }\end{array}$ & Allele & $\begin{array}{l}\text { A allele } \\
\text { number }\end{array}$ & $\begin{array}{l}\text { B allele } \\
\text { number }\end{array}$ & $\begin{array}{c}\text { Ungenotyped } \\
\text { individuals }\end{array}$ \\
\hline SNP3806 & $\mathrm{A} / \mathrm{G}$ & 123 & 66 & $\mathrm{~A} / \mathrm{G}$ & 28 & 10 & 1 \\
\hline SNP1262 & $\mathrm{T} / \mathrm{C}$ & 59 & 49 & $\mathrm{~T} / \mathrm{C}$ & 20 & 20 & 0 \\
\hline SNP3801 & $\mathrm{C} / \mathrm{T}$ & 78 & 49 & $\mathrm{C} / \mathrm{T}$ & 10 & 30 & 0 \\
\hline SNP5435 & $\mathrm{C} / \mathrm{T}$ & 70 & 33 & $\mathrm{C} / \mathrm{T}$ & 26 & 14 & 0 \\
\hline SNP376 & $\mathrm{A} / \mathrm{G}$ & 298 & 25 & $\mathrm{~A} / \mathrm{G}$ & 22 & 18 & 0 \\
\hline SNP1488 & $\mathrm{G} / \mathrm{A}$ & 82 & 25 & $\mathrm{G} / \mathrm{A}$ & 31 & 3 & 3 \\
\hline SNP2777 & $\mathrm{G} / \mathrm{C}$ & 17 & 19 & $\mathrm{G} / \mathrm{C}$ & 4 & 32 & 2 \\
\hline SNP3321 & $\mathrm{G} / \mathrm{A}$ & 44 & 19 & $\mathrm{G} / \mathrm{A}$ & 14 & 26 & 0 \\
\hline SNP5045 & $\mathrm{A} / \mathrm{G}$ & 19 & 19 & $\mathrm{~A} / \mathrm{G}$ & 31 & 9 & 0 \\
\hline SNP1033 & $\mathrm{A} / \mathrm{G}$ & 78 & 18 & $\mathrm{~A} / \mathrm{G}$ & 25 & 15 & 0 \\
\hline SNP1715 & $\mathrm{A} / \mathrm{G}$ & 53 & 18 & $\mathrm{~A} / \mathrm{G}$ & 37 & 3 & 0 \\
\hline SNP578 & $\mathrm{C} / \mathrm{T}$ & 15 & 16 & $\mathrm{C} / \mathrm{T}$ & 4 & 36 & 0 \\
\hline SNP817 & $\mathrm{C} / \mathrm{T}$ & 47 & 15 & $\mathrm{C} / \mathrm{T}$ & 37 & 3 & 0 \\
\hline SNP1546 & $\mathrm{A} / \mathrm{C}$ & 16 & 14 & $\mathrm{~A} / \mathrm{C}$ & 14 & 8 & 9 \\
\hline SNP3006 & $\mathrm{C} / \mathrm{T}$ & 21 & 14 & $\mathrm{C} / \mathrm{T}$ & 21 & 19 & 0 \\
\hline SNP3053 & $\mathrm{C} / \mathrm{T}$ & 31 & 14 & $\mathrm{C} / \mathrm{T}$ & 29 & 11 & 0 \\
\hline SNP4353 & $\mathrm{A} / \mathrm{G}$ & 50 & 14 & $\mathrm{~A} / \mathrm{G}$ & 35 & 5 & 0 \\
\hline SNP2413 & $\mathrm{G} / \mathrm{T}$ & 38 & 13 & $\mathrm{G} / \mathrm{T}$ & 34 & 6 & 0 \\
\hline SNP5508 & $\mathrm{A} / \mathrm{G}$ & 18 & 13 & $\mathrm{~A} / \mathrm{G}$ & 34 & 6 & 0 \\
\hline SNP813 & $\mathrm{C} / \mathrm{A}$ & 26 & 12 & $\mathrm{C} / \mathrm{A}$ & 15 & 17 & 4 \\
\hline SNP1993 & $\mathrm{G} / \mathrm{A}$ & 13 & 12 & $\mathrm{G} / \mathrm{A}$ & 12 & 24 & 2 \\
\hline SNP2956 & $\mathrm{C} / \mathrm{A}$ & 195 & 12 & $\mathrm{C} / \mathrm{A}$ & 26 & 12 & 1 \\
\hline SNP524 & $\mathrm{A} / \mathrm{G}$ & 10 & 11 & $\mathrm{~A} / \mathrm{G}$ & 5 & 33 & 1 \\
\hline SNP1182 & $\mathrm{A} / \mathrm{G}$ & 16 & 11 & $\mathrm{~A} / \mathrm{G}$ & 38 & 2 & 0 \\
\hline SNP3268 & $\mathrm{G} / \mathrm{C}$ & 14 & 11 & $\mathrm{G} / \mathrm{C}$ & 16 & 20 & 2 \\
\hline SNP5681 & $\mathrm{A} / \mathrm{G}$ & 35 & 11 & $\mathrm{~A} / \mathrm{G}$ & 25 & 15 & 0 \\
\hline SNP665 & $\mathrm{T} / \mathrm{C}$ & 14 & 10 & $\mathrm{~T} / \mathrm{C}$ & 22 & 18 & 0 \\
\hline SNP1941 & $\mathrm{A} / \mathrm{C}$ & 12 & 10 & $\mathrm{~A} / \mathrm{C}$ & 30 & 10 & 0 \\
\hline SNP2226 & $\mathrm{G} / \mathrm{A}$ & 23 & 10 & $\mathrm{G} / \mathrm{A}$ & 26 & 14 & 0 \\
\hline SNP4007 & $\mathrm{C} / \mathrm{T}$ & 13 & 10 & $\mathrm{C} / \mathrm{T}$ & 12 & 28 & 0 \\
\hline SNP4709 & $\mathrm{G} / \mathrm{T}$ & 10 & 25 & $\mathrm{G} / \mathrm{T}$ & 36 & 4 & 0 \\
\hline
\end{tabular}




\section{Figures}

Figure 1. Gene ontology (GO) comparison (level 3) between females and males. (A) The proportion of contigs annotated for biological process, (B) The proportion of contigs annotated for cellular component, (C) The proportion of contigs annotated for molecular function 


\section{Supplementary material}

Dataset S1 Assembled contigs and singleton sequences. (ZIP)

Dataset S2 Gene annotation information obtained by comparing contigs or singletons against the Swiss-Prot database. (XLS)

Table S1 All common expressed genes, common genes with expression above 10 and differentially expressed genes identified by transcriptome comparison between male and female turbots. (XLS)

Table S2 Information of all SNPs detected in male and female. (XLS)

Figure S1 Overview of the raw reads obtained from the Scophthalmus maximus transcriptome. (A) Size distribution of the reads from male turbots. (B) Size distribution of the reads from female turbots. 

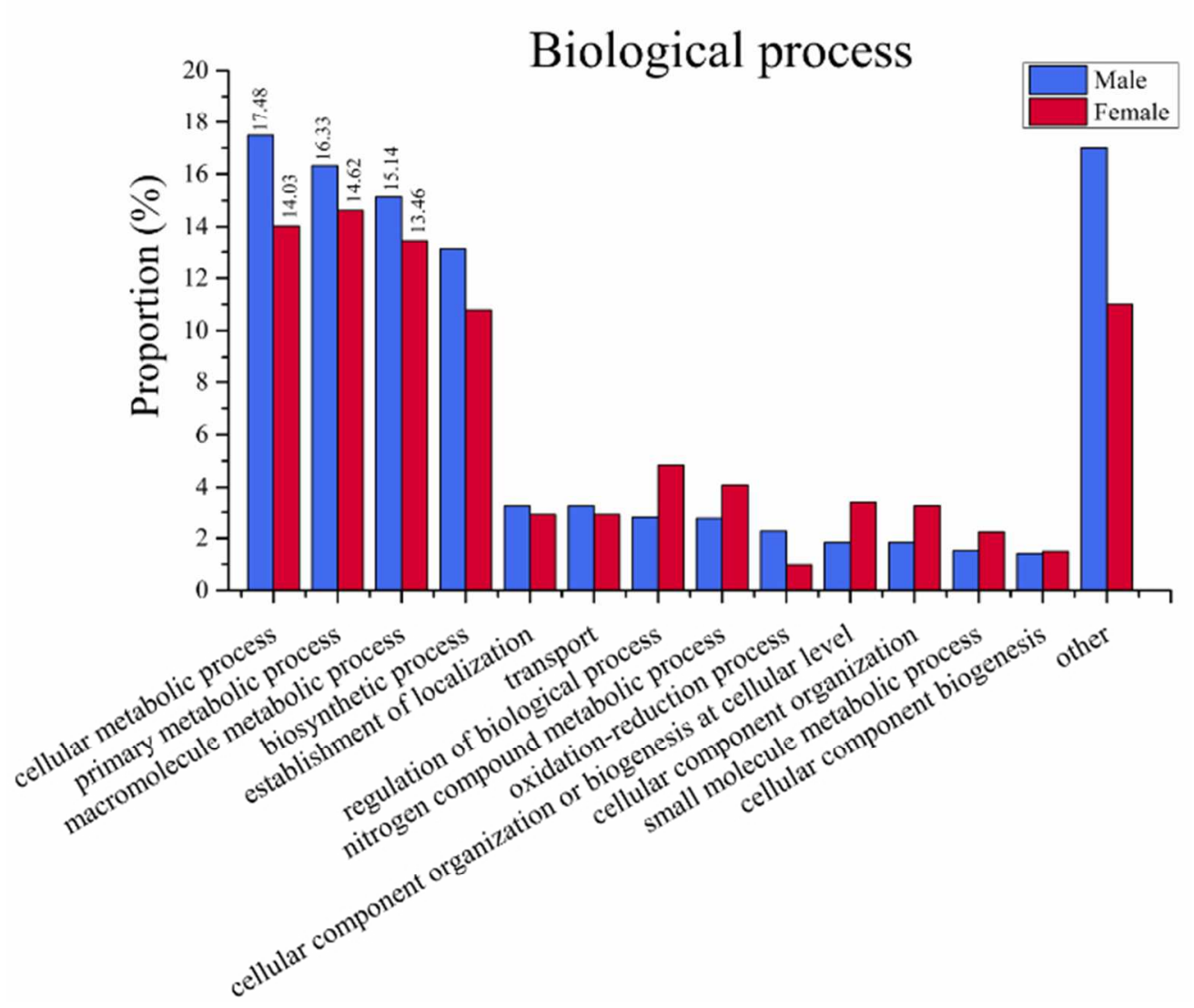

Figure 1. Gene ontology (GO) comparison (level 3) between females and males. (A) The proportion of contigs annotated for biological process, (B) The proportion of contigs annotated for cellular component, (C) The proportion of contigs annotated for molecular function 


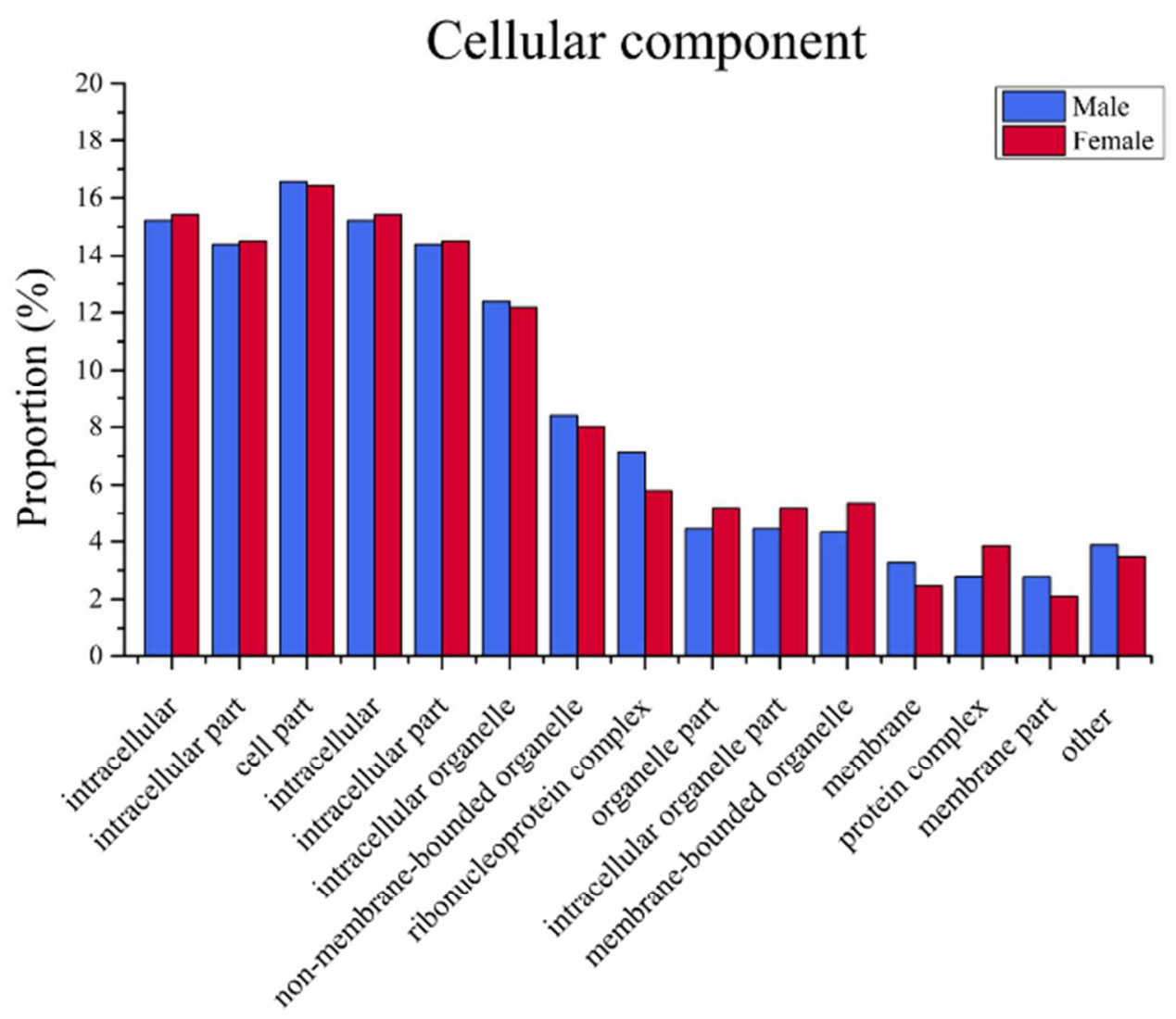

Figure 1. Gene ontology (GO) comparison (level 3) between females and males. (A) The proportion of contigs annotated for biological process, (B) The proportion of contigs annotated for cellular component, (C) The proportion of contigs annotated for molecular function 


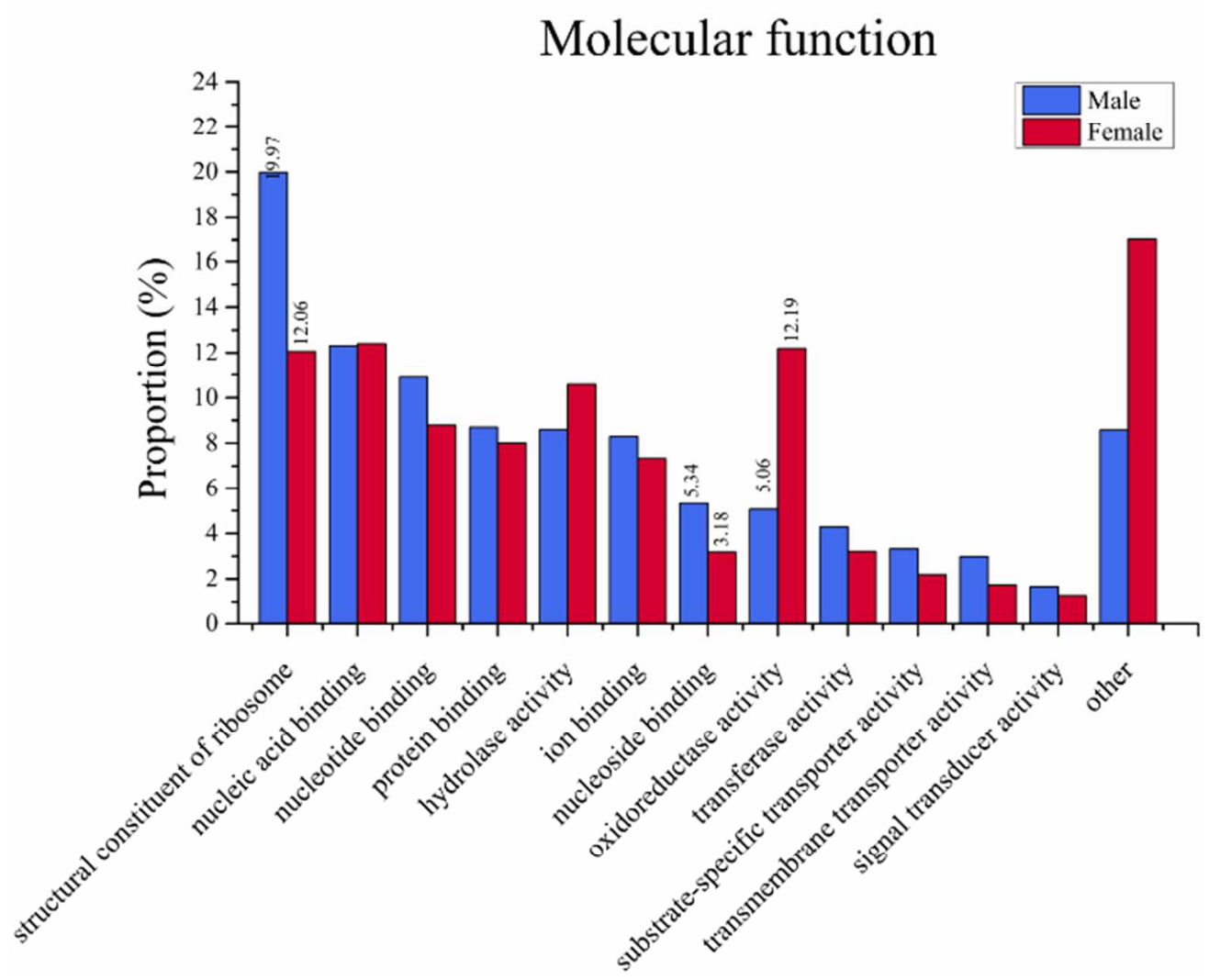

Figure 1. Gene ontology (GO) comparison (level 3) between females and males. (A) The proportion of contigs annotated for biological process, (B) The proportion of contigs annotated for cellular component, (C) The proportion of contigs annotated for molecular function 


\begin{tabular}{|c|c|c|}
\hline Unigene_ref_ID & MALE_expression & FEMALE_expressiMALE_unigene \\
\hline Unigene_GR6TTJD01AE1KJ_xiong & 26 & 69 GR6TTJD01AE1KJ_xion \\
\hline Unigene_UN_xiong_8786 & 184 & 26 UN_xiong_8786 \\
\hline Unigene_UN_xiong_1403 & 195 & 62 UN_xiong_1403 \\
\hline Unigene_UN_xiong_13350 & 1252 & 709 UN_xiong_13350 \\
\hline Unigene_UN_xiong_11583 & 127 & 214 UN_xiong_11583 \\
\hline Unigene_UN_xiong_7896 & 148 & 26 UN_xiong_7896 \\
\hline Unigene_GR6TTJD01A1S5H_xiong & 59 & 30 GR6TTJD01A1S5H_xion \\
\hline Unigene_UN_xiong_15270 & 114 & 62 UN_xiong_15270 \\
\hline Unigene_UN_xiong_14703 & 436 & 65 UN_xiong_14703 \\
\hline Unigene_GPGRCDD02HWK15_xiong & 54 & 90 GPGRCDD02HWK15_xion \\
\hline Unigene_UN_xiong_7949 & 149 & 399 UN_xiong_7949 \\
\hline Unigene_UN_xiong_10617 & 71 & 82 UN_xiong_10617 \\
\hline Unigene_UN_xiong_6592 & 44 & 25 UN_xiong_6592 \\
\hline Unigene_UN_xiong_8627 & 63 & 193 UN_xiong_8627 \\
\hline Unigene_UN_xiong_4949 & 84 & 469 UN_xiong_4949 \\
\hline Unigene_GP8I43W03FWRC5_xiong & 138 & 101 GP8I43W03FWRC5_xion \\
\hline Unigene_UN_xiong_4885 & 178 & 84 UN_xiong_4885 \\
\hline Unigene_UN_xiong_5778 & 148 & 71 UN_xiong_5778 \\
\hline Unigene_UN_xiong_709 & 43 & 25 UN_xiong_709 \\
\hline Unigene_UN_xiong_10542 & 163 & 239 UN_xiong_10542 \\
\hline Unigene_UN_xiong_5229 & 111 & 117 UN_xiong_5229 \\
\hline Unigene_UN_xiong_12937 & 60 & 30 UN_xiong_12937 \\
\hline Unigene_UN_xiong_9222 & 41 & 29 UN_xiong_9222 \\
\hline Unigene_UN_xiong_12107 & 47 & 24 UN_xiong_12107 \\
\hline Unigene_UN_xiong_496 & 132 & 175 UN_xiong_496 \\
\hline Unigene_GR6TTJD01BJWE7_xiong & 47 & 40 GR6TTJD01BJWE7_xion \\
\hline Unigene_UN_xiong_11556 & 60 & 57 UN_xiong_11556 \\
\hline Unigene_UN_xiong_15684 & 160 & 25 UN_xiong_15684 \\
\hline Unigene_UN_xiong_7193 & 67 & 51 UN_xiong_7193 \\
\hline Unigene_UN_xiong_1546 & 35 & 26 UN_xiong_1546 \\
\hline Unigene_UN_xiong_8441 & 67 & 33 UN_xiong_8441 \\
\hline Unigene_GPGRCDD02HSWPY_xiong & 30 & 36 GPGRCDD02HSWPY_xion \\
\hline Unigene_UN_xiong_11683 & 124 & 58 UN_xiong_11683 \\
\hline Unigene_UN_xiong_3634 & 293 & 1630 UN_xiong_3634 \\
\hline Unigene_GR6TTJD01BXBZ0_xiong & 42 & 40 GR6TTJD01BXBZ0_xion \\
\hline Unigene_UN_xiong_10796 & 62 & 214 UN_xiong_10796 \\
\hline Unigene_UN_xiong_5142 & 79 & 22 UN_xiong_5142 \\
\hline Unigene_UN_xiong_4985 & 106 & 84 UN_xiong_4985 \\
\hline Unigene_UN_xiong_5949 & 78 & 23 UN_xiong_5949 \\
\hline Unigene_GP8I43W03GWEPN_xiong & 35 & 302 GP8I43W03GWEPN_xion \\
\hline Unigene_UN_xiong_14639 & 98 & 1225 UN_xiong_14639 \\
\hline Unigene_UN_xiong_4223 & 35 & 161 UN_xiong_4223 \\
\hline Unigene_UN_xiong_12006 & 68 & 42 UN_xiong_12006 \\
\hline Unigene_UN_xiong_2777 & 98 & 43 UN_xiong_2777 \\
\hline Unigene_GR6TTJD01B9R70_xiong & 26 & 38 GR6TTJD01B9R70_xion \\
\hline Unigene_GPGRCDD02F8DJK_xiong & 81 & 144 GPGRCDD02F8DJK_xion \\
\hline Unigene UN xiong 9547 & 43 & 28 UN xiong 9547 \\
\hline Unigene_UN_xiong_14619 & 2290 & 4415 UN_xiong_14619 \\
\hline
\end{tabular}




\begin{tabular}{|c|c|c|}
\hline Unigene_UN_xiong_5805 & 44 & 225 UN_xiong_5805 \\
\hline Unigene_GPGRCDD02IR0T9_xiong & 41 & 44 GPGRCDD02IR0T9_xion \\
\hline Unigene_UN_xiong_7387 & 44 & 99 UN_xiong_7387 \\
\hline Unigene_GP8I43W03F10BK_xiong & 35 & 100 GP8I43W03F10BK_xion \\
\hline Unigene_GR6TTJD01A2A4F_xiong & 37 & 68 GR6TTJD01A2A4F_xion \\
\hline Unigene_GP8I43W03HECQT_xiong & 36 & 54 GP8I43W03HECQT_xion \\
\hline Unigene_GR6TTJD01BS7XY_xiong & 28 & 29 GR6TTJD01BS7XY_xion \\
\hline Unigene UN xiong 14606 & 114 & 144 UN_xiong_ 14606 \\
\hline Unigene_GPGRCDD02J1GTJ_xiong & 28 & 49 GPGRCDD02J1GTJ_xion \\
\hline Unigene_UN_xiong_10941 & 405 & 533 UN_xiong_10941 \\
\hline Unigene_GR6TTJD01AK5EF_xiong & 44 & 110 GR6TTJD01AK5EF_xion \\
\hline Unigene_UN_xiong_15716 & 116 & 25 UN_xiong_15716 \\
\hline Unigene_UN_xiong_14529 & 3996 & 257 UN_xiong_14529 \\
\hline Unigene_UN_xiong_15362 & 75 & 105 UN_xiong_15362 \\
\hline Unigene_UN_xiong_790 & 821 & 461 UN_xiong_790 \\
\hline Unigene_GR6TTJD01BIUJL_xiong & 78 & 76 GR6TTJD01BIUJL_xion \\
\hline Unigene_UN_xiong_9095 & 57 & 100 UN_xiong_9095 \\
\hline Unigene_UN_xiong_1428 & 226 & 954 UN_xiong_1428 \\
\hline Unigene_GR6TTJD01AVR2K_xiong & 19 & 78 GR6TTJD01AVR2K_xion \\
\hline Unigene_UN_xiong_5031 & 91 & 48 UN_xiong_5031 \\
\hline Unigene_UN_xiong_11863 & 135 & 55 UN_xiong_11863 \\
\hline Unigene_GP8I43W03HELZ0_xiong & 36 & 139 GP8I43W03HELZ0_xion \\
\hline Unigene_UN_xiong_3916 & 41 & 263 UN_xiong_3916 \\
\hline Unigene_UN_xiong_12140 & 171 & 44 UN_xiong_12140 \\
\hline Unigene UN xiong 15099 & 155 & 51 UN xiong 15099 \\
\hline Unigene_GR6TTJD01BYA27_xiong & 27 & 23 GR6TTJD01BYA27_xion \\
\hline Unigene_GR6TTJD01BNZ3M_xiong & 21 & 33 GR6TTJD01BNZ3M_xion \\
\hline Unigene_UN_xiong_7284 & 171 & 124 UN_xiong_7284 \\
\hline Unigene_UN_xiong_13697 & 142 & 204 UN_xiong_13697 \\
\hline Unigene_UN_xiong_5329 & 480 & 284 UN_xiong_5329 \\
\hline Unigene_UN_xiong_9429 & 440 & 928 UN_xiong_9429 \\
\hline Unigene_UN_xiong_14121 & 469 & 76 UN_xiong_14121 \\
\hline Unigene_UN_xiong_9664 & 99 & 60 UN_xiong_9664 \\
\hline Unigene_UN_xiong_7341 & 224 & 42 UN_xiong_7341 \\
\hline Unigene_GPGRCDD02GXAGU_xiong & 34 & 70 GPGRCDD02GXAGU_xion \\
\hline Unigene_UN_xiong_4290 & 137 & 21 UN_xiong_4290 \\
\hline Unigene_UN_xiong_1315 & 78 & 95 UN_xiong_1315 \\
\hline Unigene_UN_xiong_3054 & 75 & 175 UN_xiong_3054 \\
\hline Unigene_UN_xiong_847 & 163 & 90 UN_xiong_847 \\
\hline Unigene_UN_xiong_8540 & 176 & 24 UN_xiong_8540 \\
\hline Unigene_UN_xiong_4067 & 143 & 147 UN_xiong_4067 \\
\hline Unigene_UN_xiong_3396 & 55 & 551 UN_xiong_3396 \\
\hline Unigene_UN_xiong_1572 & 236 & 64 UN_xiong_1572 \\
\hline Unigene_UN_xiong_446 & 104 & 177 UN_xiong_446 \\
\hline Unigene_GR6TTJD01A0Q3B_xiong & 26 & 54 GR6TTJD01A0Q3B_xion \\
\hline Unigene UN xiong 12076 & 118 & 45 UN_xiong_ 12076 \\
\hline Unigene_UN_xiong_15292 & 117 & 48 UN_xiong_15292 \\
\hline Unigene_GR6TTJD01AHFY0_xiong & 26 & 41 GR6TTJD01AHFY0_xion \\
\hline Unigene_UN_xiong_3848 & 116 & 203 UN_xiong_3848 \\
\hline Unigene_UN_xiong_553 & 121 & 343 UN_xiong_553 \\
\hline
\end{tabular}




\begin{tabular}{|c|c|c|}
\hline Unigene_UN_xiong_7545 & 124 & 47 UN_xiong_7545 \\
\hline Unigene_GR6TTJD01A0GNE_xiong & 27 & 62 GR6TTJD01A0GNE_xion \\
\hline Unigene_UN_xiong_11094 & 312 & 22 UN_xiong_11094 \\
\hline Unigene_UN_xiong_14437 & 1702 & 739 UN_xiong_14437 \\
\hline Unigene_UN_xiong_14781 & 229 & 432 UN_xiong_14781 \\
\hline Unigene_UN_xiong_7419 & 63 & 668 UN_xiong_7419 \\
\hline Unigene_GPGRCDD02I4IYG_xiong & 21 & 36 GPGRCDD02I4IYG_xion \\
\hline Unigene_GR6TTJD01AMALN_xiong & 28 & 64 GR6TTJD01AMALN_xion \\
\hline Unigene_UN_xiong_3008 & 96 & 99 UN_xiong_3008 \\
\hline Unigene_UN_xiong_6386 & 202 & 494 UN_xiong_6386 \\
\hline Unigene_GR6TTJD01CAJU0_xiong & 28 & 22 GR6TTJD01CAJU0_xion \\
\hline Unigene_UN_xiong_14493 & 283 & 59 UN_xiong_14493 \\
\hline Unigene_GPGRCDD02HCYCH_xiong & 47 & 37 GPGRCDD02HCYCH_xion \\
\hline Unigene_UN_xiong_4351 & 295 & 166 UN_xiong_4351 \\
\hline Unigene_UN_xiong_14231 & 255 & 138 UN_xiong_14231 \\
\hline Unigene_UN_xiong_6787 & 126 & 229 UN_xiong_6787 \\
\hline Unigene_GP8I43W03HGEPH_xiong & 58 & 121 GP8I43W03HGEPH_xion \\
\hline Unigene_UN_xiong_14258 & 682 & 844 UN_xiong_14258 \\
\hline Unigene_UN_xiong_11001 & 161 & 148 UN_xiong_11001 \\
\hline Unigene_GR6TTJD01A810M_xiong & 36 & 22 GR6TTJD01A810M_xion \\
\hline Unigene_GPGRCDD02IGC0J_xiong & 56 & 520 GPGRCDD02IGC0J_xion \\
\hline Unigene_UN_xiong_4932 & 681 & 2295 UN_xiong_4932 \\
\hline Unigene_UN_xiong_3786 & 108 & 58 UN_xiong_3786 \\
\hline Unigene_UN_xiong_8913 & 199 & 73 UN_xiong_8913 \\
\hline Unigene_GP8I43W03F0QC0_xiong & 25 & 30 GP8I43W03F0QC0_xion \\
\hline Unigene_UN_xiong_11821 & 129 & 318 UN_xiong_11821 \\
\hline Unigene_UN_xiong_7764 & 94 & 92 UN_xiong_7764 \\
\hline Unigene_GPGRCDD02G08JC_xiong & 56 & 90 GPGRCDD02G08JC_xion \\
\hline Unigene_UN_xiong_10057 & 151 & 76 UN_xiong_10057 \\
\hline Unigene_UN_xiong_6458 & 174 & 137 UN_xiong_6458 \\
\hline Unigene_UN_xiong_13587 & 159 & 81 UN_xiong_13587 \\
\hline Unigene_UN_xiong_2017 & 55 & 64 UN_xiong_2017 \\
\hline Unigene_UN_xiong_14648 & 896 & 304 UN_xiong_14648 \\
\hline Unigene_UN_xiong_14885 & 855 & 992 UN_xiong_14885 \\
\hline Unigene_GPGRCDD02GLRS5_xiong & 28 & 211 GPGRCDD02GLRS5_xion \\
\hline Unigene_UN_xiong_896 & 233 & 253 UN_xiong_896 \\
\hline Unigene_GR6TTJD01B3W2T_xiong & 34 & 68 GR6TTJD01B3W2T_xion \\
\hline Unigene_UN_xiong_6468 & 76 & 44 UN_xiong_6468 \\
\hline Unigene_UN_xiong_748 & 109 & 183 UN_xiong_748 \\
\hline Unigene_UN_xiong_2721 & 72 & 58 UN_xiong_2721 \\
\hline Unigene_UN_xiong_326 & 117 & 37 UN_xiong_326 \\
\hline Unigene_UN_xiong_750 & 192 & 41 UN_xiong_750 \\
\hline Unigene_UN_xiong_2256 & 118 & 89 UN_xiong_2256 \\
\hline Unigene_UN_xiong_2413 & 302 & 190 UN_xiong_2413 \\
\hline Unigene_UN_xiong_8126 & 58 & 24 UN_xiong_8126 \\
\hline Unigene_UN_xiong_14444 & 1570 & 3640 UN_xiong_14444 \\
\hline Unigene_UN_xiong_8647 & 122 & 322 UN_xiong_8647 \\
\hline Unigene_UN_xiong_7701 & 142 & 20 UN_xiong_7701 \\
\hline Unigene_GR6TTJD01BMS3H_xiong & 32 & 41 GR6TTJD01BMS3H_xion \\
\hline Unigene_UN_xiong_12206 & 41 & 22 UN_xiong_12206 \\
\hline
\end{tabular}




\begin{tabular}{|c|c|c|}
\hline Unigene_UN_xiong_7517 & 131 & 35 UN_xiong_7517 \\
\hline Unigene_UN_xiong_11127 & 188 & 57 UN_xiong_11127 \\
\hline Unigene_UN_xiong_6700 & 102 & 51 UN_xiong_6700 \\
\hline Unigene_GP8I43W03GD053_xiong & 27 & 80 GP8I43W03GD053_xion \\
\hline Unigene_GPGRCDD02GUKZ8_xiong & 32 & 24 GPGRCDD02GUKZ8_xion \\
\hline Unigene_UN_xiong_555 & 91 & 44 UN_xiong_555 \\
\hline Unigene_GR6TTJD01BBNDM_xiong & 22 & 22 GR6TTJD01BBNDM_xion \\
\hline Unigene_GPGRCDD02F7TLR_xiong & 26 & 1712 GPGRCDD02F7TLR_xion \\
\hline Unigene_UN_xiong_13429 & 71 & 92 UN_xiong_13429 \\
\hline Unigene_UN_xiong_15563 & 433 & 76 UN_xiong_15563 \\
\hline Unigene_UN_xiong_3546 & 111 & 45 UN_xiong_3546 \\
\hline Unigene_UN_xiong_1132 & 80 & 23 UN_xiong_1132 \\
\hline Unigene_UN_xiong_14997 & 182 & 181 UN_xiong_14997 \\
\hline Unigene_UN_xiong_13319 & 74 & 25 UN_xiong_13319 \\
\hline Unigene_GR6TTJD01AWJNL_xiong & 30 & 24 GR6TTJD01AWJNL_xion \\
\hline Unigene_UN_xiong_14747 & 500 & 2772 UN_xiong_14747 \\
\hline Unigene_UN_xiong_701 & 249 & 117 UN_xiong_701 \\
\hline Unigene_GR6TTJD01BI0N9_xiong & 71 & 56 GR6TTJD01BI0N9_xion \\
\hline Unigene_UN_xiong_3347 & 71 & 40 UN_xiong_3347 \\
\hline Unigene_UN_xiong_2550 & 263 & 2564 UN_xiong_2550 \\
\hline Unigene_UN_xiong_7358 & 144 & 125 UN_xiong_7358 \\
\hline Unigene_GPGRCDD02FPC62_xiong & 67 & 55 GPGRCDD02FPC62_xion \\
\hline Unigene_UN_xiong_4687 & 90 & 270 UN_xiong_4687 \\
\hline Unigene_GP8I43W03HA05C_xiong & 23 & 22 GP8I43W03HA05C_xion \\
\hline Unigene UN xiong 8749 & 40 & 26 UN xiong 8749 \\
\hline Unigene_GR6TTJD01AR20V_xiong & 40 & 34 GR6TTJD01AR20V_xion \\
\hline Unigene_UN_xiong_5075 & 73 & 142 UN_xiong_5075 \\
\hline Unigene_UN_xiong_12801 & 43 & 485 UN_xiong_12801 \\
\hline Unigene_GR6TTJD01B8GDD_xiong & 41 & 51 GR6TTJD01B8GDD_xion \\
\hline Unigene_UN_xiong_10446 & 209 & 319 UN_xiong_10446 \\
\hline Unigene_UN_xiong_1390 & 123 & 123 UN_xiong_1390 \\
\hline Unigene_UN_xiong_5406 & 131 & 301 UN_xiong_5406 \\
\hline Unigene_GR6TTJD01CAI1I_xiong & 24 & 21 GR6TTJD01CAI1I_xion \\
\hline Unigene_GPGRCDD02F5LZF_xiong & 39 & 112 GPGRCDD02F5LZF_xion \\
\hline Unigene_GP8I43W03G8LMB_xiong & 39 & 27 GP8I43W03G8LMB_xion \\
\hline Unigene_UN_xiong_9369 & 75 & 42 UN_xiong_9369 \\
\hline Unigene_UN_xiong_12612 & 80 & 20 UN_xiong_12612 \\
\hline Unigene_UN_xiong_2566 & 233 & 69 UN_xiong_2566 \\
\hline Unigene_UN_xiong_7244 & 105 & 259 UN_xiong_7244 \\
\hline Unigene_UN_xiong_392 & 270 & 214 UN_xiong_392 \\
\hline Unigene_UN_xiong_4147 & 138 & 75 UN_xiong_4147 \\
\hline Unigene GPGRCDD02F50Z0 xiong & 65 & 47 GPGRCDD02F50Z0 xion \\
\hline Unigene_GPGRCDD02I000W_xiong & 27 & 29 GPGRCDD02I000W_xion \\
\hline Unigene_GP8I43W03GMA6A_xiong & 26 & 209 GP8I43W03GMA6A_xion \\
\hline Unigene_GR6TTJD01A8WBT_xiong & 30 & 470 GR6TTJD01A8WBT_xion \\
\hline Unigene UN xiong 11608 & 62 & 29 UN_xiong_11608 \\
\hline Unigene_UN_xiong_3417 & 126 & 622 UN_xiong_3417 \\
\hline Unigene_UN_xiong_6239 & 39 & 21 UN_xiong_6239 \\
\hline Unigene_UN_xiong_6546 & 34 & 26 UN_xiong_6546 \\
\hline Unigene_GR6TTJD01B7MKC_xiong & 24 & 80 GR6TTJD01B7MKC_xion \\
\hline
\end{tabular}




\begin{tabular}{|c|c|c|}
\hline Unigene_UN_xiong_11909 & 59 & 228 UN_xiong_11909 \\
\hline Unigene_UN_xiong_3585 & 174 & 66 UN_xiong_3585 \\
\hline Unigene_UN_xiong_14945 & 182 & 47 UN_xiong_14945 \\
\hline Unigene_UN_xiong_3834 & 47 & 93 UN_xiong_3834 \\
\hline Unigene_GR6TTJD01A0B5L_xiong & 99 & 58 GR6TTJD01A0B5L_xion \\
\hline Unigene_UN_xiong_3222 & 119 & 180 UN_xiong_3222 \\
\hline Unigene_UN_xiong_9063 & 192 & 581 UN_xiong_9063 \\
\hline Unigene_UN_xiong_13418 & 126 & 29 UN_xiong_13418 \\
\hline Unigene_UN_xiong_12490 & 59 & 132 UN_xiong_12490 \\
\hline Unigene_UN_xiong_3633 & 107 & 70 UN_xiong_3633 \\
\hline Unigene_UN_xiong_13451 & 119 & 1203 UN_xiong_13451 \\
\hline Unigene_UN_xiong_10662 & 93 & 22 UN_xiong_10662 \\
\hline Unigene_UN_xiong_15519 & 193 & 210 UN_xiong_15519 \\
\hline Unigene_UN_xiong_273 & 248 & 284 UN_xiong_273 \\
\hline Unigene_UN_xiong_14070 & 165 & 7554 UN_xiong_14070 \\
\hline Unigene_UN_xiong_14953 & 231 & 286 UN_xiong_14953 \\
\hline Unigene_UN_xiong_1494 & 1096 & 1338 UN_xiong_1494 \\
\hline Unigene_GP8I43W03F15MY_xiong & 37 & 135 GP8I43W03F15MY_xion \\
\hline Unigene_UN_xiong_399 & 2091 & 717 UN_xiong_399 \\
\hline Unigene_UN_xiong_2030 & 161 & 93 UN_xiong_2030 \\
\hline Unigene_UN_xiong_15422 & 104 & 262 UN_xiong_15422 \\
\hline Unigene_UN_xiong_215 & 294 & 91 UN_xiong_215 \\
\hline Unigene_GPGRCDD02IFYP4_xiong & 24 & 395 GPGRCDD02IFYP4_xion \\
\hline Unigene_UN_xiong_6419 & 251 & 339 UN_xiong_6419 \\
\hline Unigene_UN_xiong_4204 & 247 & 515 UN_xiong_4204 \\
\hline Unigene_UN_xiong_11435 & 270 & 104 UN_xiong_11435 \\
\hline Unigene_UN_xiong_5417 & 66 & 27 UN_xiong_5417 \\
\hline Unigene_UN_xiong_15690 & 181 & 375 UN_xiong_15690 \\
\hline Unigene_GR6TTJD01B003V_xiong & 24 & 78 GR6TTJD01B003V_xion \\
\hline Unigene_GR6TTJD01AFPS3_xiong & 95 & 88 GR6TTJD01AFPS3_xion \\
\hline Unigene_GPGRCDD02H7401_xiong & 46 & 61 GPGRCDD02H7401_xion \\
\hline Unigene_UN_xiong_2978 & 267 & 187 UN_xiong_2978 \\
\hline Unigene_UN_xiong_238 & 57 & 237 UN_xiong_238 \\
\hline Unigene_UN_xiong_312 & 29 & 71 UN_xiong_312 \\
\hline Unigene_UN_xiong_11224 & 63 & 336 UN_xiong_11224 \\
\hline Unigene_UN_xiong_13359 & 142 & 32 UN_xiong_13359 \\
\hline Unigene_UN_xiong_1111 & 212 & 253 UN_xiong_1111 \\
\hline Unigene_UN_xiong_2223 & 81 & 54 UN_xiong_2223 \\
\hline Unigene_UN_xiong_9264 & 123 & 62 UN_xiong_9264 \\
\hline Unigene_GR6TTJD01AFB3R_xiong & 30 & 22 GR6TTJD01AFB3R_xion \\
\hline Unigene_UN_xiong_2448 & 89 & 61 UN_xiong_2448 \\
\hline Unigene_UN_xiong_2972 & 323 & 29 UN_xiong_2972 \\
\hline Unigene_UN_xiong_7030 & 137 & 38 UN_xiong_7030 \\
\hline Unigene_UN_xiong_2530 & 212 & 46 UN_xiong_2530 \\
\hline Unigene_UN_xiong_996 & 77 & 42 UN_xiong_996 \\
\hline Unigene_UN_xiong_14354 & 529 & 435 UN_xiong_14354 \\
\hline Unigene_GR6TTJD01AHJ1A_xiong & 21 & 92 GR6TTJD01AHJ1A_xion \\
\hline Unigene_GR6TTJD01CD09W_xiong & 33 & 68 GR6TTJD01CD09W_xion \\
\hline Unigene_UN_xiong_14414 & 2462 & 7151 UN_xiong_14414 \\
\hline Unigene_GPGRCDD02IR375_xiong & 55 & 108 GPGRCDD02IR375_xion \\
\hline
\end{tabular}




\begin{tabular}{|c|c|c|}
\hline Unigene_UN_xiong_7224 & 67 & 32 UN_xiong_7224 \\
\hline Unigene_GPGRCDD02GNUZ9_xiong & 41 & 24 GPGRCDD02GNUZ9_xion \\
\hline Unigene_UN_xiong_9570 & 1245 & 1254 UN_xiong_9570 \\
\hline Unigene_UN_xiong_1675 & 823 & 69 UN_xiong_1675 \\
\hline Unigene_GR6TTJD01AKJKM_xiong & 58 & 88 GR6TTJD01AKJKM_xion \\
\hline Unigene_UN_xiong_3253 & 577 & 208 UN_xiong_3253 \\
\hline Unigene_GR6TTJD01B928W_xiong & 28 & 37 GR6TTJD01B928W_xion \\
\hline Unigene_GR6TTJD01AL8YA_xiong & 24 & 22 GR6TTJD01AL8YA_xion \\
\hline Unigene_UN_xiong_9068 & 53 & 115 UN_xiong_9068 \\
\hline Unigene_UN_xiong_3737 & 152 & 28 UN_xiong_3737 \\
\hline Unigene_UN_xiong_7045 & 221 & 29 UN_xiong_7045 \\
\hline Unigene_UN_xiong_14542 & 6371 & 1993 UN_xiong_14542 \\
\hline Unigene_UN_xiong_4428 & 145 & 30 UN_xiong_4428 \\
\hline Unigene_UN_xiong_7803 & 111 & 32 UN_xiong_7803 \\
\hline Unigene_UN_xiong_346 & 96 & 63 UN_xiong_346 \\
\hline Unigene_UN_xiong_12181 & 33 & 47 UN_xiong_12181 \\
\hline Unigene_UN_xiong_14367 & 308 & 39 UN_xiong_14367 \\
\hline Unigene_UN_xiong_2099 & 665 & 751 UN_xiong_2099 \\
\hline Unigene_UN_xiong_11060 & 49 & 99 UN_xiong_11060 \\
\hline Unigene_UN_xiong_6639 & 50 & 24 UN_xiong_6639 \\
\hline Unigene_GP8I43W03GX98S_xiong & 40 & 34 GP8I43W03GX98S_xion \\
\hline Unigene_UN_xiong_8069 & 178 & 79 UN_xiong_8069 \\
\hline Unigene_UN_xiong_2273 & 100 & 67 UN_xiong_2273 \\
\hline Unigene_UN_xiong_6515 & 86 & 73 UN_xiong_6515 \\
\hline Unigene_UN_xiong_13712 & 138 & 343 UN_xiong_13712 \\
\hline Unigene_GR6TTJD01AMMP5_xiong & 41 & 40 GR6TTJD01AMMP5_xion \\
\hline Unigene_UN_xiong_974 & 166 & 326 UN_xiong_974 \\
\hline Unigene_UN_xiong_692 & 245 & 210 UN_xiong_692 \\
\hline Unigene_UN_xiong_11225 & 556 & 278 UN_xiong_11225 \\
\hline Unigene_GPGRCDD02GTY95_xiong & 28 & 68 GPGRCDD02GTY95_xion \\
\hline Unigene_UN_xiong_6015 & 115 & 157 UN_xiong_6015 \\
\hline Unigene_UN_xiong_14683 & 315 & 223 UN_xiong_14683 \\
\hline Unigene_UN_xiong_2113 & 107 & 65 UN_xiong_2113 \\
\hline Unigene_GPGRCDD02JENGV_xiong & 42 & 32 GPGRCDD02JENGV_xion \\
\hline Unigene_UN_xiong_13030 & 149 & 183 UN_xiong_13030 \\
\hline Unigene_UN_xiong_10726 & 230 & 63 UN_xiong_10726 \\
\hline Unigene_GR6TTJD01A5DDA_xiong & 26 & 25 GR6TTJD01A5DDA_xion \\
\hline Unigene_UN_xiong_13318 & 232 & 648 UN_xiong_13318 \\
\hline Unigene_UN_xiong_6711 & 144 & 149 UN_xiong_6711 \\
\hline Unigene_UN_xiong_14474 & 150 & 171 UN_xiong_14474 \\
\hline Unigene_GPGRCDD02HLFB4_xiong & 29 & 140 GPGRCDD02HLFB4_xion \\
\hline Unigene_UN_xiong_109 & 119 & 119 UN_xiong_109 \\
\hline Unigene_GR6TTJD01AESHN_xiong & 87 & 29 GR6TTJD01AESHN_xion \\
\hline Unigene_UN_xiong_14912 & 481 & 144 UN_xiong_14912 \\
\hline Unigene_UN_xiong_8341 & 31 & 136 UN_xiong_8341 \\
\hline Unigene_UN_xiong_12969 & 182 & 123 UN_xiong_12969 \\
\hline Unigene_UN_xiong_222 & 63 & 62 UN_xiong_222 \\
\hline Unigene_UN_xiong_5410 & 227 & 242 UN_xiong_5410 \\
\hline Unigene_UN_xiong_5526 & 68 & 118 UN_xiong_5526 \\
\hline Unigene_UN_xiong_7692 & 66 & 44 UN_xiong_7692 \\
\hline
\end{tabular}




\begin{tabular}{|c|c|c|}
\hline Unigene_GPGRCDD02HSZC1_xiong & 25 & 166 GPGRCDD02HSZC1_xion \\
\hline Unigene_GR6TTJD01AFR3D_xiong & 23 & 181 GR6TTJD01AFR3D_xion \\
\hline Unigene_UN_xiong_15372 & 649 & 516 UN_xiong_15372 \\
\hline Unigene_GR6TTJD01A0UE2_xiong & 31 & 119 GR6TTJD01A0UE2_xion \\
\hline Unigene_UN_xiong_8738 & 97 & 43 UN_xiong_8738 \\
\hline Unigene_UN_xiong_10668 & 123 & 44 UN_xiong_10668 \\
\hline Unigene_UN_xiong_4807 & 70 & 81 UN_xiong_4807 \\
\hline Unigene_UN_xiong_13708 & 74 & 271 UN_xiong_13708 \\
\hline Unigene_GPGRCDD02JX0UP_xiong & 76 & 148 GPGRCDD02JX0UP_xion \\
\hline Unigene_GPGRCDD02GK4DZ_xiong & 22 & 237 GPGRCDD02GK4DZ_xion \\
\hline Unigene_UN_xiong_10818 & 76 & 25 UN_xiong_10818 \\
\hline Unigene_UN_xiong_9969 & 122 & 74 UN_xiong_9969 \\
\hline Unigene_GR6TTJD01BNLLF_xiong & 39 & 69 GR6TTJD01BNLLF_xion \\
\hline Unigene_UN_xiong_15449 & 350 & 176 UN_xiong_15449 \\
\hline Unigene_GR6TTJD01BIBAY_xiong & 39 & 80 GR6TTJD01BIBAY_xion \\
\hline Unigene_GR6TTJD01B37K5_xiong & 22 & 26 GR6TTJD01B37K5_xion \\
\hline Unigene_UN_xiong_7068 & 35 & 105 UN_xiong_7068 \\
\hline Unigene_UN_xiong_469 & 265 & 33 UN_xiong_469 \\
\hline Unigene_UN_xiong_11627 & 228 & 52 UN_xiong_11627 \\
\hline Unigene_UN_xiong_10652 & 1141 & 46 UN_xiong_10652 \\
\hline Unigene_UN_xiong_10151 & 49 & 144 UN_xiong_10151 \\
\hline Unigene_UN_xiong_5357 & 233 & 89 UN_xiong_5357 \\
\hline Unigene_UN_xiong_13314 & 97 & 47 UN_xiong_13314 \\
\hline Unigene_UN_xiong_148 & 173 & 895 UN_xiong_148 \\
\hline Unigene_GR6TTJD01AYS64_xiong & 45 & 82 GR6TTJD01AYS64_xion \\
\hline Unigene_UN_xiong_14182 & 84 & 187 UN_xiong_14182 \\
\hline Unigene_UN_xiong_14548 & 199 & 208 UN_xiong_14548 \\
\hline Unigene_UN_xiong_3481 & 286 & 224 UN_xiong_3481 \\
\hline Unigene_UN_xiong_1517 & 133 & 212 UN_xiong_1517 \\
\hline Unigene_GR6TTJD01B036H_xiong & 56 & 239 GR6TTJD01B036H_xion \\
\hline Unigene_UN_xiong_4647 & 41 & 56 UN_xiong_4647 \\
\hline Unigene_GR6TTJD01A9N3I_xiong & 41 & 45 GR6TTJD01A9N3I_xion \\
\hline Unigene_UN_xiong_8429 & 42 & 60 UN_xiong_8429 \\
\hline Unigene_UN_xiong_240 & 63 & 97 UN_xiong_240 \\
\hline Unigene_GPGRCDD02GNYZC_xiong & 83 & 263 GPGRCDD02GNYZC_xion \\
\hline Unigene_UN_xiong_14066 & 52 & 769 UN_xiong_14066 \\
\hline Unigene_UN_xiong_4544 & 732 & 130 UN_xiong_4544 \\
\hline Unigene_UN_xiong_14459 & 185 & 209 UN_xiong_14459 \\
\hline Unigene_UN_xiong_7231 & 463 & 274 UN_xiong_7231 \\
\hline Unigene_UN_xiong_14975 & 194 & 175 UN_xiong_14975 \\
\hline Unigene_UN_xiong_9544 & 310 & 121 UN_xiong_9544 \\
\hline Unigene_UN_xiong_3111 & 148 & 40 UN_xiong_3111 \\
\hline Unigene_UN_xiong_14012 & 128 & 116 UN_xiong_14012 \\
\hline Unigene_UN_xiong_4095 & 60 & 38 UN_xiong_4095 \\
\hline Unigene_GR6TTJD01BNK1X_xiong & 76 & 100 GR6TTJD01BNK1X_xion \\
\hline Unigene_UN_xiong_11786 & 107 & 48 UN_xiong_11786 \\
\hline Unigene_UN_xiong_481 & 169 & 84 UN_xiong_481 \\
\hline Unigene_UN_xiong_8413 & 165 & 290 UN_xiong_8413 \\
\hline Unigene_GR6TTJD01AZK39_xiong & 35 & 55 GR6TTJD01AZK39_xion \\
\hline Unigene_GPGRCDD02HVQAJ_xiong & 33 & 26 GPGRCDD02HVQAJ_xion \\
\hline
\end{tabular}




\begin{tabular}{|c|c|c|}
\hline Unigene_GP8I43W03GC643_xiong & 25 & 94 GP8I43W03GC643_xion \\
\hline Unigene_UN_xiong_2470 & 68 & 64 UN_xiong_2470 \\
\hline Unigene_GP8I43W03G1IU1_xiong & 38 & 32 GP8I43W03G1IU1_xion \\
\hline Unigene_GR6TTJD01A2I6Z_xiong & 35 & 112 GR6TTJD01A2I6Z_xion \\
\hline Unigene_UN_xiong_11975 & 51 & 40 UN_xiong_11975 \\
\hline Unigene_UN_xiong_6297 & 183 & 103 UN_xiong_6297 \\
\hline Unigene_UN_xiong_14306 & 160 & 68 UN_xiong_14306 \\
\hline Unigene_UN_xiong_11636 & 337 & 37 UN_xiong_11636 \\
\hline Unigene_UN_xiong_14524 & 837 & 294 UN_xiong_14524 \\
\hline Unigene_UN_xiong_14863 & 208 & 59 UN_xiong_14863 \\
\hline Unigene_GR6TTJD01BLZZA_xiong & 26 & 22 GR6TTJD01BLZZA_xion \\
\hline Unigene_GP8I43W03FVBYW_xiong & 38 & 120 GP8I43W03FVBYW_xion \\
\hline Unigene_UN_xiong_4859 & 100 & 154 UN_xiong_4859 \\
\hline Unigene_UN_xiong_7054 & 129 & 84 UN_xiong_7054 \\
\hline Unigene_UN_xiong_2239 & 291 & 160 UN_xiong_2239 \\
\hline Unigene_UN_xiong_3590 & 256 & 613 UN_xiong_3590 \\
\hline Unigene_UN_xiong_6926 & 163 & 81 UN_xiong_6926 \\
\hline Unigene_UN_xiong_2573 & 148 & 66 UN_xiong_2573 \\
\hline Unigene_UN_xiong_9227 & 342 & 79 UN_xiong_9227 \\
\hline Unigene_UN_xiong_13607 & 360 & 5649 UN_xiong_13607 \\
\hline Unigene_GP8I43W03F8RXP_xiong & 29 & 41 GP8I43W03F8RXP_xion \\
\hline Unigene_UN_xiong_10370 & 132 & 65 UN_xiong_10370 \\
\hline Unigene_UN_xiong_2521 & 326 & 474 UN_xiong_2521 \\
\hline Unigene_UN_xiong_6771 & 84 & 100 UN_xiong_6771 \\
\hline Unigene_UN_xiong_949 & 10881 & 2441 UN_xiong_949 \\
\hline Unigene_UN_xiong_11730 & 44 & 22 UN_xiong_11730 \\
\hline Unigene_UN_xiong_4614 & 234 & 30 UN_xiong_4614 \\
\hline Unigene_UN_xiong_14677 & 496 & 3268 UN_xiong_14677 \\
\hline Unigene_UN_xiong_6402 & 127 & 249 UN_xiong_6402 \\
\hline Unigene_UN_xiong_88 & 89 & 63 UN_xiong_88 \\
\hline Unigene_UN_xiong_10308 & 52 & 80 UN_xiong_10308 \\
\hline Unigene_UN_xiong_1027 & 227 & 764 UN_xiong_1027 \\
\hline Unigene_GR6TTJD01A0I55_xiong & 28 & 193 GR6TTJD01A0I55_xion \\
\hline Unigene_UN_xiong_13643 & 82 & 40 UN_xiong_13643 \\
\hline Unigene_UN_xiong_12574 & 64 & 21 UN_xiong_12574 \\
\hline Unigene_UN_xiong_13273 & 78 & 89 UN_xiong_13273 \\
\hline Unigene_UN_xiong_12975 & 369 & 221 UN_xiong_12975 \\
\hline Unigene_GPGRCDD02GAAMK_xiong & 31 & 60 GPGRCDD02GAAMK_xion \\
\hline Unigene_UN_xiong_3976 & 176 & 45 UN_xiong_3976 \\
\hline Unigene_UN_xiong_8113 & 316 & 42 UN_xiong_8113 \\
\hline Unigene_UN_xiong_15062 & 179 & 79 UN_xiong_15062 \\
\hline Unigene_GP8I43W03HBPNX_xiong & 20 & 136 GP8I43W03HBPNX_xion \\
\hline Unigene_UN_xiong_7707 & 338 & 1511 UN_xiong_7707 \\
\hline Unigene_UN_xiong_427 & 50 & 61 UN_xiong_427 \\
\hline Unigene_UN_xiong_3105 & 984 & 3163 UN_xiong_3105 \\
\hline Unigene_UN_xiong_11996 & 62 & 145 UN_xiong_11996 \\
\hline Unigene_UN_xiong_3628 & 241 & 366 UN_xiong_3628 \\
\hline Unigene_UN_xiong_3793 & 240 & 125 UN_xiong_3793 \\
\hline Unigene_UN_xiong_12588 & 48 & 477 UN_xiong_12588 \\
\hline Unigene_GR6TTJD01A6DJ0_xiong & 26 & 47 GR6TTJD01A6DJ0_xion \\
\hline
\end{tabular}




\begin{tabular}{|c|c|c|}
\hline Unigene_UN_xiong_3181 & 232 & 126 UN_xiong_3181 \\
\hline Unigene_UN_xiong_6981 & 73 & 60 UN_xiong_6981 \\
\hline Unigene_UN_xiong_2600 & 36 & 60 UN_xiong_2600 \\
\hline Unigene_UN_xiong_7819 & 54 & 73 UN_xiong_7819 \\
\hline Unigene_UN_xiong_4493 & 278 & 80 UN_xiong_4493 \\
\hline Unigene_UN_xiong_6837 & 98 & 63 UN_xiong_6837 \\
\hline Unigene_GR6TTJD01AEPHX_xiong & 37 & 61 GR6TTJD01AEPHX_xion \\
\hline Unigene_UN_xiong_13984 & 38 & 104 UN_xiong_13984 \\
\hline Unigene_UN_xiong_6509 & 61 & 42 UN_xiong_6509 \\
\hline Unigene_UN_xiong_6650 & 85 & 61 UN_xiong_6650 \\
\hline Unigene_GR6TTJD01ASCEC_xiong & 26 & 44 GR6TTJD01ASCEC_xion \\
\hline Unigene_UN_xiong_1154 & 163 & 39 UN_xiong_1154 \\
\hline Unigene_GP8I43W03HDLX9_xiong & 44 & 26 GP8I43W03HDLX9_xion \\
\hline Unigene_UN_xiong_2697 & 253 & 109 UN_xiong_2697 \\
\hline Unigene_UN_xiong_2755 & 192 & 571 UN_xiong_2755 \\
\hline Unigene_UN_xiong_10411 & 47 & 48 UN_xiong_10411 \\
\hline Unigene_UN_xiong_2167 & 152 & 40 UN_xiong_2167 \\
\hline Unigene_UN_xiong_1885 & 100 & 81 UN_xiong_1885 \\
\hline Unigene_UN_xiong_5260 & 93 & 173 UN_xiong_5260 \\
\hline Unigene_UN_xiong_10862 & 110 & 58 UN_xiong_10862 \\
\hline Unigene_GR6TTJD01BNEYA_xiong & 77 & 177 GR6TTJD01BNEYA_xion \\
\hline Unigene_UN_xiong_2938 & 47 & 48 UN_xiong_2938 \\
\hline Unigene_UN_xiong_5040 & 73 & 134 UN_xiong_5040 \\
\hline Unigene_UN_xiong_3356 & 165 & 192 UN_xiong_3356 \\
\hline Unigene_UN_xiong_13660 & 56 & 92 UN_xiong_13660 \\
\hline Unigene_UN_xiong_14363 & 278 & 63 UN_xiong_14363 \\
\hline Unigene_UN_xiong_7295 & 143 & 329 UN_xiong_7295 \\
\hline Unigene_UN_xiong_158 & 299 & 78 UN_xiong_158 \\
\hline Unigene_UN_xiong_6447 & 106 & 93 UN_xiong_6447 \\
\hline Unigene_UN_xiong_3935 & 264 & 322 UN_xiong_3935 \\
\hline Unigene_UN_xiong_14038 & 241 & 393 UN_xiong_14038 \\
\hline Unigene_UN_xiong_10484 & 117 & 56 UN_xiong_10484 \\
\hline Unigene_UN_xiong_15009 & 71 & 4703 UN_xiong_15009 \\
\hline Unigene_GPGRCDD02GQ41R_xiong & 37 & 1608 GPGRCDD02GQ41R_xion \\
\hline Unigene_UN_xiong_4153 & 70 & 47 UN_xiong_4153 \\
\hline Unigene_GR6TTJD01BYSCR_xiong & 21 & 32 GR6TTJD01BYSCR_xion \\
\hline Unigene_UN_xiong_10301 & 267 & 142 UN_xiong_10301 \\
\hline Unigene_UN_xiong_9007 & 132 & 28 UN_xiong_9007 \\
\hline Unigene_UN_xiong_759 & 172 & 232 UN_xiong_759 \\
\hline Unigene_UN_xiong_1628 & 557 & 240 UN_xiong_1628 \\
\hline Unigene_UN_xiong_1118 & 84 & 59 UN_xiong_1118 \\
\hline Unigene_UN_xiong_13034 & 82 & 232 UN_xiong_13034 \\
\hline Unigene_GPGRCDD02JXM8R_xiong & 35 & 571 GPGRCDD02JXM8R_xion \\
\hline Unigene_UN_xiong_15136 & 269 & 447 UN_xiong_15136 \\
\hline Unigene_UN_xiong_6033 & 65 & 159 UN_xiong_6033 \\
\hline Unigene_UN_xiong_879 & 214 & 183 UN_xiong_879 \\
\hline Unigene_UN_xiong_5167 & 106 & 225 UN_xiong_5167 \\
\hline Unigene_UN_xiong_14965 & 157 & 377 UN_xiong_14965 \\
\hline Unigene_UN_xiong_15453 & 144 & 89 UN_xiong_15453 \\
\hline Unigene_GR6TTJD01A20N0_xiong & 78 & 50 GR6TTJD01A20N0_xion \\
\hline
\end{tabular}




\begin{tabular}{|c|c|c|}
\hline Unigene_UN_xiong_7871 & 101 & 821 UN_xiong_7871 \\
\hline Unigene_UN_xiong_11899 & 72 & 271 UN_xiong_11899 \\
\hline Unigene_GR6TTJD01AHE0X_xiong & 53 & 29 GR6TTJD01AHE0X_xion \\
\hline Unigene_GR6TTJD01A1EHD_xiong & 44 & 44 GR6TTJD01A1EHD_xion \\
\hline Unigene_UN_xiong_11425 & 75 & 182 UN_xiong_11425 \\
\hline Unigene_UN_xiong_15102 & 170 & 72 UN_xiong_15102 \\
\hline Unigene_UN_xiong_2706 & 117 & 235 UN_xiong_2706 \\
\hline Unigene_UN_xiong_2637 & 4241 & 1501 UN_xiong_2637 \\
\hline Unigene_UN_xiong_14734 & 69 & 29 UN_xiong_14734 \\
\hline Unigene_UN_xiong_10584 & 3141 & 1811 UN_xiong_10584 \\
\hline Unigene_UN_xiong_1408 & 53 & 24 UN_xiong_1408 \\
\hline Unigene_UN_xiong_12751 & 71 & 57 UN_xiong_12751 \\
\hline Unigene_UN_xiong_3902 & 147 & 428 UN_xiong_3902 \\
\hline Unigene_UN_xiong_5346 & 139 & 92 UN_xiong_5346 \\
\hline Unigene_GR6TTJD01A0MB1_xiong & 66 & 40 GR6TTJD01A0MB1_xion \\
\hline Unigene_UN_xiong_11376 & 286 & 297 UN_xiong_11376 \\
\hline Unigene_UN_xiong_1720 & 60 & 63 UN_xiong_1720 \\
\hline Unigene_UN_xiong_674 & 42 & 120 UN_xiong_674 \\
\hline Unigene_GP8I43W03FZ7Z0_xiong & 34 & 133 GP8I43W03FZ7Z0_xion \\
\hline Unigene_UN_xiong_9324 & 505 & 76 UN_xiong_9324 \\
\hline Unigene_UN_xiong_10903 & 142 & 111 UN_xiong_10903 \\
\hline Unigene_UN_xiong_5786 & 85 & 35 UN_xiong_5786 \\
\hline Unigene_UN_xiong_335 & 839 & 3951 UN_xiong_335 \\
\hline Unigene_GR6TTJD01B3K3L_xiong & 31 & 29 GR6TTJD01B3K3L_xion \\
\hline Unigene_GR6TTJD01CDNOW_xiong & 27 & 26 GR6TTJD01CDNOW_xion \\
\hline Unigene_UN_xiong_10360 & 220 & 592 UN_xiong_10360 \\
\hline Unigene_UN_xiong_5083 & 92 & 63 UN_xiong_5083 \\
\hline Unigene_UN_xiong_1735 & 469 & 301 UN_xiong_1735 \\
\hline Unigene_UN_xiong_10859 & 93 & 381 UN_xiong_10859 \\
\hline Unigene_UN_xiong_3290 & 49 & 117 UN_xiong_3290 \\
\hline Unigene_GPGRCDD02HDPWR_xiong & 26 & 127 GPGRCDD02HDPWR_xion \\
\hline Unigene_UN_xiong_9456 & 338 & 613 UN_xiong_9456 \\
\hline Unigene_UN_xiong_765 & 174 & 26 UN_xiong_765 \\
\hline Unigene_UN_xiong_823 & 396 & 493 UN_xiong_823 \\
\hline Unigene_UN_xiong_13657 & 129 & 55 UN_xiong_13657 \\
\hline Unigene_UN_xiong_3882 & 115 & 41 UN_xiong_3882 \\
\hline Unigene_UN_xiong_5837 & 148 & 25 UN_xiong_5837 \\
\hline Unigene_UN_xiong_9110 & 109 & 169 UN_xiong_9110 \\
\hline Unigene_UN_xiong_12561 & 133 & 100 UN_xiong_12561 \\
\hline Unigene_UN_xiong_7492 & 50 & 49 UN_xiong_7492 \\
\hline Unigene_GP8I43W03GMVTL_xiong & 61 & 316 GP8I43W03GMVTL_xion \\
\hline Unigene_UN_xiong_9125 & 104 & 133 UN_xiong_9125 \\
\hline Unigene_GR6TTJD01A83XQ_xiong & 23 & 132 GR6TTJD01A83XQ_xion \\
\hline Unigene_UN_xiong_2216 & 106 & 104 UN_xiong_2216 \\
\hline Unigene_GP8I43W03GLZN4_xiong & 29 & 22 GP8I43W03GLZN4_xion \\
\hline Unigene_GP8I43W03FTABU_xiong & 53 & 67 GP8I43W03FTABU_xion \\
\hline Unigene_UN_xiong_2561 & 46 & 49 UN_xiong_2561 \\
\hline Unigene_UN_xiong_11196 & 273 & 19628 UN_xiong_11196 \\
\hline Unigene_UN_xiong_5478 & 65 & 70 UN_xiong_5478 \\
\hline Unigene_GPGRCDD02IDOGW_xiong & 24 & 108 GPGRCDD02IDOGW_xion \\
\hline
\end{tabular}




\begin{tabular}{|c|c|c|}
\hline Unigene_UN_xiong_8753 & 69 & 29 UN_xiong_8753 \\
\hline Unigene_GP8I43W03GHUUC_xiong & 27 & 26 GP8I43W03GHUUC_xion \\
\hline Unigene_UN_xiong_14438 & 2105 & 2102 UN_xiong_14438 \\
\hline Unigene_GR6TTJD01BHQZE_xiong & 22 & 45 GR6TTJD01BHQZE_xion \\
\hline Unigene_GPGRCDD02F1Q6Z_xiong & 32 & 32 GPGRCDD02F1Q6Z_xion \\
\hline Unigene_UN_xiong_7675 & 90 & 408 UN_xiong_7675 \\
\hline Unigene_UN_xiong_6270 & 165 & 457 UN_xiong_6270 \\
\hline Unigene_GR6TTJD01AQ3AN_xiong & 79 & 28 GR6TTJD01AQ3AN_xion \\
\hline Unigene_UN_xiong_1056 & 81 & 451 UN_xiong_1056 \\
\hline Unigene_UN_xiong_13752 & 84 & 54 UN_xiong_13752 \\
\hline Unigene_UN_xiong_4601 & 51 & 63 UN_xiong_4601 \\
\hline Unigene_UN_xiong_5823 & 277 & 28 UN_xiong_5823 \\
\hline Unigene_UN_xiong_1801 & 254 & 196 UN_xiong_1801 \\
\hline Unigene_GPGRCDD02IKOJZ_xiong & 26 & 27 GPGRCDD02IK0JZ_xion \\
\hline Unigene_UN_xiong_389 & 308 & 51 UN_xiong_389 \\
\hline Unigene_GR6TTJD01AEA0J_xiong & 78 & 54 GR6TTJD01AEA0J_xion \\
\hline Unigene_UN_xiong_2461 & 98 & 82 UN_xiong_2461 \\
\hline Unigene_UN_xiong_9942 & 43 & 24 UN_xiong_9942 \\
\hline Unigene_UN_xiong_726 & 188 & 100 UN_xiong_726 \\
\hline Unigene_GP8I43W03FYL6W_xiong & 45 & 45 GP8I43W03FYL6W_xion \\
\hline Unigene_UN_xiong_7757 & 98 & 89 UN_xiong_7757 \\
\hline Unigene_UN_xiong_8308 & 79 & 102 UN_xiong_8308 \\
\hline Unigene_UN_xiong_3627 & 79 & 176 UN_xiong_3627 \\
\hline Unigene_GPGRCDD02F0FU5_xiong & 27 & 31 GPGRCDD02F0FU5_xion \\
\hline Unigene_UN_xiong_5333 & 135 & 124 UN_xiong_5333 \\
\hline Unigene_UN_xiong_942 & 246 & 115 UN_xiong_942 \\
\hline Unigene_UN_xiong_4355 & 133 & 53 UN_xiong_4355 \\
\hline Unigene_UN_xiong_13465 & 323 & 140 UN_xiong_13465 \\
\hline Unigene_GR6TTJD01BTNQA_xiong & 29 & 46 GR6TTJD01BTNQA_xion \\
\hline Unigene_UN_xiong_3826 & 101 & 372 UN_xiong_3826 \\
\hline Unigene_UN_xiong_183 & 111 & 59 UN_xiong_183 \\
\hline Unigene_GPGRCDD02GDHPW_xiong & 23 & 41 GPGRCDD02GDHPW_xion \\
\hline Unigene_UN_xiong_8081 & 111 & 90 UN_xiong_8081 \\
\hline Unigene_UN_xiong_5109 & 377 & 391 UN_xiong_5109 \\
\hline Unigene_UN_xiong_14932 & 1037 & 1185 UN_xiong_14932 \\
\hline Unigene_GP8I43W03F7YB6_xiong & 52 & 92 GP8I43W03F7YB6_xion \\
\hline Unigene_UN_xiong_4704 & 87 & 40 UN_xiong_4704 \\
\hline Unigene_UN_xiong_7164 & 203 & 10946 UN_xiong_7164 \\
\hline Unigene_UN_xiong_848 & 200 & 104 UN_xiong_848 \\
\hline Unigene_UN_xiong_4628 & 272 & 243 UN_xiong_4628 \\
\hline Unigene_UN_xiong_7051 & 77 & 397 UN_xiong_7051 \\
\hline Unigene_GR6TTJD01A8196_xiong & 29 & 186 GR6TTJD01A8196_xion \\
\hline Unigene_UN_xiong_11550 & 206 & 47 UN_xiong_11550 \\
\hline Unigene_GP8I43W03GCZHI_xiong & 27 & 30 GP8I43W03GCZHI_xion \\
\hline Unigene_UN_xiong_6118 & 52 & 48 UN_xiong_6118 \\
\hline Unigene_UN_xiong_3420 & 490 & 599 UN_xiong_3420 \\
\hline Unigene_UN_xiong_4695 & 40 & 57 UN_xiong_4695 \\
\hline Unigene_UN_xiong_7337 & 55 & 21 UN_xiong_7337 \\
\hline Unigene_UN_xiong_12962 & 59 & 113 UN_xiong_12962 \\
\hline Unigene_UN_xiong_4291 & 340 & 445 UN_xiong_4291 \\
\hline
\end{tabular}




\begin{tabular}{|c|c|c|}
\hline Unigene_UN_xiong_14286 & 799 & 401 UN_xiong_14286 \\
\hline Unigene_GPGRCDD02HZ5X4_xiong & 25 & 182 GPGRCDD02HZ5X4_xion \\
\hline Unigene_UN_xiong_10981 & 83 & 208 UN_xiong_10981 \\
\hline Unigene_UN_xiong_537 & 175 & 463 UN_xiong_537 \\
\hline Unigene_UN_xiong_11987 & 43 & 42 UN_xiong_11987 \\
\hline Unigene_UN_xiong_4436 & 925 & 275 UN_xiong_4436 \\
\hline Unigene_UN_xiong_5198 & 62 & 90 UN_xiong_5198 \\
\hline Unigene_UN_xiong_914 & 227 & 54 UN_xiong_914 \\
\hline Unigene_GPGRCDD02H4FA0_xiong & 31 & 32 GPGRCDD02H4FA0_xion \\
\hline Unigene_UN_xiong_2137 & 63 & 46 UN_xiong_2137 \\
\hline Unigene_UN_xiong_734 & 175 & 72 UN_xiong_734 \\
\hline Unigene_UN_xiong_889 & 220 & 177 UN_xiong_889 \\
\hline Unigene_UN_xiong_3114 & 309 & 1994 UN_xiong_3114 \\
\hline Unigene_UN_xiong_13271 & 67 & 33 UN_xiong_13271 \\
\hline Unigene_GR6TTJD01AWGKE_xiong & 40 & 44 GR6TTJD01AWGKE_xion \\
\hline Unigene_UN_xiong_11119 & 60 & 89 UN_xiong_11119 \\
\hline Unigene_UN_xiong_714 & 167 & 99 UN_xiong_714 \\
\hline Unigene_UN_xiong_11932 & 109 & 377 UN_xiong_11932 \\
\hline Unigene_UN_xiong_1206 & 158 & 204 UN_xiong_1206 \\
\hline Unigene_UN_xiong_4186 & 351 & 154 UN_xiong_4186 \\
\hline Unigene_UN_xiong_2542 & 71 & 41 UN_xiong_2542 \\
\hline Unigene_UN_xiong_2606 & 153 & 233 UN_xiong_2606 \\
\hline Unigene_UN_xiong_6062 & 78 & 64 UN_xiong_6062 \\
\hline Unigene_UN_xiong_2049 & 108 & 363 UN_xiong_2049 \\
\hline Unigene_UN_xiong_13344 & 188 & 257 UN_xiong_13344 \\
\hline Unigene_UN_xiong_2475 & 219 & 301 UN_xiong_2475 \\
\hline Unigene_UN_xiong_1121 & 58 & 73 UN_xiong_1121 \\
\hline Unigene_UN_xiong_15293 & 184 & 54 UN_xiong_15293 \\
\hline Unigene_GR6TTJD01ARJGV_xiong & 41 & 97 GR6TTJD01ARJGV_xion \\
\hline Unigene_UN_xiong_14357 & 134 & 159 UN_xiong_14357 \\
\hline Unigene_GR6TTJD01A0WZM_xiong & 32 & 23 GR6TTJD01A0WZM_xion \\
\hline Unigene_GR6TTJD01ARKCM_xiong & 28 & 175 GR6TTJD01ARKCM_xion \\
\hline Unigene_UN_xiong_9158 & 103 & 167 UN_xiong_9158 \\
\hline Unigene_UN_xiong_9527 & 86 & 44 UN_xiong_9527 \\
\hline Unigene_UN_xiong_5903 & 344 & 58 UN_xiong_5903 \\
\hline Unigene_UN_xiong_12462 & 207 & 75 UN_xiong_12462 \\
\hline Unigene_UN_xiong_85 & 93 & 33 UN_xiong_85 \\
\hline Unigene_UN_xiong_5243 & 68 & 170 UN_xiong_5243 \\
\hline Unigene_GP8I43W03HE0C3_xiong & 44 & 274 GP8I43W03HEOC3_xion \\
\hline Unigene_UN_xiong_5253 & 78 & 258 UN_xiong_5253 \\
\hline Unigene_UN_xiong_3440 & 152 & 176 UN_xiong_3440 \\
\hline Unigene_UN_xiong_6978 & 972 & 700 UN_xiong_6978 \\
\hline Unigene_UN_xiong_14170 & 163 & 59 UN_xiong_14170 \\
\hline Unigene_UN_xiong_13883 & 582 & 137 UN_xiong_13883 \\
\hline Unigene_GR6TTJD01A6BF0_xiong & 29 & 55 GR6TTJD01A6BF0_xion \\
\hline Unigene_GR6TTJD01AMMV1_xiong & 25 & 154 GR6TTJD01AMMV1_xion \\
\hline Unigene_UN_xiong_12066 & 117 & 93 UN_xiong_12066 \\
\hline Unigene_UN_xiong_401 & 168 & 272 UN_xiong_401 \\
\hline Unigene_UN_xiong_11757 & 117 & 25 UN_xiong_11757 \\
\hline Unigene_UN_xiong_7652 & 147 & 50 UN_xiong_7652 \\
\hline
\end{tabular}




\begin{tabular}{|c|c|c|}
\hline Unigene_GPGRCDD02J0BJ2_xiong & 41 & 39 GPGRCDD02J0BJ2_xion \\
\hline Unigene_UN_xiong_9501 & 90 & 108 UN_xiong_9501 \\
\hline Unigene_UN_xiong_14895 & 377 & 109 UN_xiong_14895 \\
\hline Unigene_UN_xiong_5436 & 48 & 20 UN_xiong_5436 \\
\hline Unigene_UN_xiong_9088 & 88 & 273 UN_xiong_9088 \\
\hline Unigene_UN_xiong_14924 & 1396 & 559 UN_xiong_14924 \\
\hline Unigene_UN_xiong_9997 & 161 & 122 UN_xiong_9997 \\
\hline Unigene_GR6TTJD01B27VG xiong & 28 & 61 GR6TTJD01B27VG xion \\
\hline Unigene_UN_xiong_14686 & 97 & 28 UN_xiong_14686 \\
\hline Unigene_UN_xiong_14405 & 192 & 45 UN_xiong_14405 \\
\hline Unigene_UN_xiong_4469 & 47 & 104 UN_xiong_4469 \\
\hline Unigene_UN_xiong_14638 & 135 & 254 UN_xiong_14638 \\
\hline Unigene_UN_xiong_12998 & 217 & 34 UN_xiong_12998 \\
\hline Unigene_UN_xiong_1173 & 87 & 20 UN_xiong_1173 \\
\hline Unigene_UN_xiong_990 & 138 & 63 UN_xiong_990 \\
\hline Unigene_UN_xiong_10412 & 74 & 105 UN_xiong_10412 \\
\hline Unigene_UN_xiong_2248 & 463 & 1949 UN_xiong_2248 \\
\hline Unigene_GPGRCDD02HPKJ0_xiong & 25 & 24 GPGRCDD02HPKJ0_xion \\
\hline Unigene_UN_xiong_9876 & 160 & 68 UN_xiong_9876 \\
\hline Unigene_UN_xiong_11723 & 105 & 306 UN_xiong_11723 \\
\hline Unigene_UN_xiong_5444 & 131 & 135 UN_xiong_5444 \\
\hline Unigene_GR6TTJD01AYBT3_xiong & 23 & 44 GR6TTJD01AYBT3_xion \\
\hline Unigene_UN_xiong_2105 & 276 & 612 UN_xiong_2105 \\
\hline Unigene_UN_xiong_7740 & 96 & 30 UN_xiong_7740 \\
\hline Unigene_UN_xiong_1994 & 142 & 56 UN_xiong_1994 \\
\hline Unigene_UN_xiong_1436 & 681 & 545 UN_xiong_1436 \\
\hline Unigene_UN_xiong_10111 & 155 & 154 UN_xiong_10111 \\
\hline Unigene_UN_xiong_5777 & 814 & 366 UN_xiong_5777 \\
\hline Unigene_UN_xiong_3047 & 594 & 187 UN_xiong_3047 \\
\hline Unigene_UN_xiong_2430 & 51 & 25 UN_xiong_2430 \\
\hline Unigene_UN_xiong_14320 & 260 & 172 UN_xiong_14320 \\
\hline Unigene_UN_xiong_4423 & 108 & 109 UN_xiong_4423 \\
\hline Unigene_GP8I43W03GVNF2_xiong & 34 & 34 GP8I43W03GVNF2_xion \\
\hline Unigene_UN_xiong_1358 & 191 & 68 UN_xiong_1358 \\
\hline Unigene_UN_xiong_8531 & 58 & 103 UN_xiong_8531 \\
\hline Unigene_UN_xiong_12936 & 168 & 233 UN_xiong_12936 \\
\hline Unigene_GPGRCDD02H62GC_xiong & 31 & 2315 GPGRCDD02H62GC_xion \\
\hline Unigene_UN_xiong_14326 & 950 & 1315 UN_xiong_14326 \\
\hline Unigene_GR6TTJD01AY58D_xiong & 22 & 45 GR6TTJD01AY58D_xion \\
\hline Unigene_UN_xiong_5545 & 451 & 1844 UN_xiong_5545 \\
\hline Unigene_UN_xiong_2788 & 248 & 262 UN_xiong_2788 \\
\hline Unigene_UN xiong 13417 & 138 & 281 UN_xiong_13417 \\
\hline Unigene_GP8I43W03F2GCU_xiong & 34 & 123 GP8I43W03F2GCU_xion \\
\hline Unigene_GR6TTJD01BY0CP_xiong & 32 & 26 GR6TTJD01BY0CP_xion \\
\hline Unigene_UN_xiong_12005 & 53 & 90 UN_xiong_12005 \\
\hline Unigene GPGRCDD02GX3FC xiong & 39 & 29 GPGRCDD02GX3FC xion \\
\hline Unigene_UN_xiong_5588 & 49 & 70 UN_xiong_5588 \\
\hline Unigene_UN_xiong_9970 & 89 & 98 UN_xiong_9970 \\
\hline Unigene_UN_xiong_10438 & 136 & 51 UN_xiong_10438 \\
\hline Unigene_GR6TTJD01B9E2N_xiong & 33 & 49 GR6TTJD01B9E2N_xion \\
\hline
\end{tabular}




\begin{tabular}{|c|c|c|}
\hline Unigene_UN_xiong_12728 & 110 & 59 UN_xiong_12728 \\
\hline Unigene_GPGRCDD02I3IPZ_xiong & 93 & 38 GPGRCDD02I3IPZ_xion \\
\hline Unigene_UN_xiong_3000 & 147 & 54 UN_xiong_3000 \\
\hline Unigene_UN_xiong_11751 & 44 & 104 UN_xiong_11751 \\
\hline Unigene_UN_xiong_1942 & 282 & 826 UN_xiong_1942 \\
\hline Unigene_UN_xiong_1565 & 179 & 305 UN_xiong_1565 \\
\hline Unigene_UN_xiong_4523 & 70 & 62 UN_xiong_4523 \\
\hline Unigene_UN_xiong_15715 & 366 & 152 UN_xiong_15715 \\
\hline Unigene_UN_xiong_14274 & 616 & 164 UN_xiong_14274 \\
\hline Unigene_UN_xiong_1873 & 100 & 50 UN_xiong_1873 \\
\hline Unigene_UN_xiong_14213 & 201 & 55 UN_xiong_14213 \\
\hline Unigene_UN_xiong_6861 & 55 & 495 UN_xiong_6861 \\
\hline Unigene_UN_xiong_10831 & 42 & 27 UN_xiong_10831 \\
\hline Unigene_GR6TTJD01BH8GB_xiong & 27 & 50 GR6TTJD01BH8GB_xion \\
\hline Unigene_UN_xiong_13804 & 54 & 47 UN_xiong_13804 \\
\hline Unigene_UN_xiong_1131 & 59 & 37 UN_xiong_1131 \\
\hline Unigene_GR6TTJD01B74MY_xiong & 35 & 35 GR6TTJD01B74MY_xion \\
\hline Unigene_UN_xiong_4159 & 47 & 117 UN_xiong_4159 \\
\hline Unigene_UN_xiong_6027 & 319 & 84 UN_xiong_6027 \\
\hline Unigene_UN_xiong_10069 & 399 & 357 UN_xiong_10069 \\
\hline Unigene_UN_xiong_13709 & 122 & 79 UN_xiong_13709 \\
\hline Unigene_UN_xiong_3941 & 304 & 23 UN_xiong_3941 \\
\hline Unigene_UN_xiong_6229 & 62 & 31 UN_xiong_6229 \\
\hline Unigene_GR6TTJD01AF600_xiong & 21 & 21 GR6TTJD01AF600_xion \\
\hline Unigene_UN_xiong_13056 & 54 & 38 UN_xiong_13056 \\
\hline Unigene_UN_xiong_12706 & 174 & 1269 UN_xiong_12706 \\
\hline Unigene_UN_xiong_6842 & 88 & 44 UN_xiong_6842 \\
\hline Unigene_UN_xiong_13450 & 172 & 197 UN_xiong_13450 \\
\hline Unigene_UN_xiong_4166 & 51 & 25 UN_xiong_4166 \\
\hline Unigene_UN_xiong_256 & 1100 & 476 UN_xiong_256 \\
\hline Unigene_UN_xiong_14208 & 1351 & 2697 UN_xiong_14208 \\
\hline Unigene_UN_xiong_6513 & 645 & 191 UN_xiong_6513 \\
\hline Unigene_GR6TTJD01A21CW_xiong & 37 & 32 GR6TTJD01A21CW_xion \\
\hline Unigene_GPGRCDD02FWLOS_xiong & 46 & 41 GPGRCDD02FWLOS_xion \\
\hline Unigene_GPGRCDD02IHCYL_xiong & 44 & 74 GPGRCDD02IHCYL_xion \\
\hline Unigene_GR6TTJD01BT215_xiong & 24 & 33 GR6TTJD01BT215_xion \\
\hline Unigene_UN_xiong_2803 & 118 & 690 UN_xiong_2803 \\
\hline Unigene_UN_xiong_9698 & 287 & 381 UN_xiong_9698 \\
\hline Unigene_GP8I43W03GY9FI_xiong & 53 & 28 GP8I43W03GY9FI_xion \\
\hline Unigene_GR6TTJD01AEHAH_xiong & 30 & 30 GR6TTJD01AEHAH_xion \\
\hline Unigene_GR6TTJD01BUUPV_xiong & 42 & 40 GR6TTJD01BUUPV_xion \\
\hline Unigene_GPGRCDD02HBR57_xiong & 26 & 66 GPGRCDD02HBR57_xion \\
\hline Unigene_GPGRCDD02IRM8G_xiong & 30 & 26 GPGRCDD02IRM8G_xion \\
\hline Unigene_GPGRCDD02GV2HV_xiong & 53 & 53 GPGRCDD02GV2HV_xion \\
\hline Unigene_GPGRCDD02GBMIE_xiong & 23 & 28 GPGRCDD02GBMIE_xion \\
\hline Unigene_GPGRCDD02G4EE7_xiong & 110 & 117 GPGRCDD02G4EE7_xion \\
\hline Unigene_GPGRCDD02JTFEW_xiong & 66 & 154 GPGRCDD02JTFEW_xion \\
\hline Unigene_UN_xiong_14797 & 435 & 147 UN_xiong_14797 \\
\hline Unigene_UN_xiong_651 & 57 & 53 UN_xiong_651 \\
\hline Unigene_UN_xiong_14687 & 385 & 454 UN_xiong_14687 \\
\hline
\end{tabular}




\begin{tabular}{|c|c|c|}
\hline Unigene_UN_xiong_11569 & 241 & 110 UN_xiong_11569 \\
\hline Unigene_UN_xiong_437 & 536 & 268 UN_xiong_437 \\
\hline Unigene_GP8I43W03HFPH8_xiong & 31 & 69 GP8I43W03HFPH8_xion \\
\hline Unigene_UN_xiong_1475 & 40 & 65 UN_xiong_1475 \\
\hline Unigene_UN_xiong_12637 & 69 & 78 UN_xiong_12637 \\
\hline Unigene_UN_xiong_11178 & 120 & 193 UN_xiong_11178 \\
\hline Unigene_GP8I43W03HDCAG_xiong & 44 & 41 GP8I43W03HDCAG_xion \\
\hline Unigene UN xiong $14780^{-}$ & 354 & 384 UN_xiong_14780 \\
\hline Unigene_GR6TTJD01BXF3R_xiong & 38 & 26 GR6TTJD01BXF3R_xion \\
\hline Unigene_UN_xiong_14383 & 447 & 175 UN_xiong_14383 \\
\hline Unigene_UN_xiong_13957 & 78 & 76 UN_xiong_13957 \\
\hline Unigene_UN_xiong_1308 & 125 & 102 UN_xiong_1308 \\
\hline Unigene_UN_xiong_4136 & 92 & 320 UN_xiong_4136 \\
\hline Unigene_UN_xiong_2996 & 274 & 337 UN_xiong_2996 \\
\hline Unigene_UN_xiong_14835 & 328 & 1170 UN_xiong_14835 \\
\hline Unigene_UN_xiong_9276 & 67 & 35 UN_xiong_9276 \\
\hline Unigene_GR6TTJD01AKA50_xiong & 43 & 29 GR6TTJD01AKA50_xion \\
\hline Unigene_UN_xiong_8735 & 69 & 74 UN_xiong_8735 \\
\hline Unigene_UN_xiong_1926 & 134 & 104 UN_xiong_1926 \\
\hline Unigene_UN_xiong_11877 & 80 & 36 UN_xiong_11877 \\
\hline Unigene_UN_xiong_10808 & 108 & 122 UN_xiong_10808 \\
\hline Unigene_UN_xiong_14521 & 222 & 116 UN_xiong_14521 \\
\hline Unigene_UN_xiong_3132 & 59 & 79 UN_xiong_3132 \\
\hline Unigene_UN_xiong_1507 & 89 & 66 UN_xiong_1507 \\
\hline Unigene_UN_xiong_14395 & 1243 & 95 UN_xiong_14395 \\
\hline Unigene_UN_xiong_14988 & 137 & 192 UN_xiong_14988 \\
\hline Unigene_UN_xiong_6353 & 83 & 27 UN_xiong_6353 \\
\hline Unigene_UN_xiong_8834 & 47 & 223 UN_xiong_8834 \\
\hline Unigene_GR6TTJD01B00VV_xiong & 47 & 137 GR6TTJD01B00VV_xion \\
\hline Unigene_UN_xiong_10353 & 276 & 82 UN_xiong_10353 \\
\hline Unigene_UN_xiong_5976 & 38 & 34 UN_xiong_5976 \\
\hline Unigene_UN_xiong_1987 & 283 & 5118 UN_xiong_1987 \\
\hline Unigene_UN_xiong_9761 & 781 & 990 UN_xiong_9761 \\
\hline Unigene_UN_xiong_6096 & 85 & 21 UN_xiong_6096 \\
\hline Unigene_UN_xiong_8596 & 168 & 182 UN_xiong_8596 \\
\hline Unigene_UN_xiong_758 & 385 & 68 UN_xiong_758 \\
\hline Unigene_UN_xiong_14737 & 367 & 504 UN_xiong_14737 \\
\hline Unigene_UN_xiong_5225 & 65 & 47 UN_xiong_5225 \\
\hline Unigene_UN_xiong_4837 & 88 & 22 UN_xiong_4837 \\
\hline Unigene_GPGRCDD02I9SVP_xiong & 33 & 61 GPGRCDD02I9SVP_xion \\
\hline Unigene UN xiong 4039 & 192 & 886 UN xiong 4039 \\
\hline Unigene UN xiong 10901 & 194 & 105 UN_xiong_10901 \\
\hline Unigene_GPGRCDD02GMUC4_xiong & 28 & 24 GPGRCDD02GMUC4_xion \\
\hline Unigene_UN_xiong_6938 & 810 & 66 UN_xiong_6938 \\
\hline Unigene_UN_xiong_5548 & 46 & 58 UN_xiong_5548 \\
\hline Unigene_UN_xiong_11488 & 68 & 78 UN_xiong_11488 \\
\hline Unigene_UN_xiong_5591 & 184 & 46 UN_xiong_5591 \\
\hline Unigene_UN_xiong_2634 & 59 & 23 UN_xiong_2634 \\
\hline Unigene_UN_xiong_8448 & 58 & 28 UN_xiong_8448 \\
\hline Unigene GP8I43W03GY816 xiong & 21 & 27 GP8I43W03GY816 xion \\
\hline
\end{tabular}




\begin{tabular}{|c|c|c|}
\hline Unigene_UN_xiong_5498 & 102 & 29 UN_xiong_5498 \\
\hline Unigene_UN_xiong_1652 & 103 & 141 UN_xiong_1652 \\
\hline Unigene_UN_xiong_12425 & 136 & 70 UN_xiong_12425 \\
\hline Unigene_UN_xiong_12626 & 93 & 63 UN_xiong_12626 \\
\hline Unigene_GP8I43W03F0NU9_xiong & 29 & 145 GP8I43W03F0NU9_xion \\
\hline Unigene_GR6TTJD01A8Y25_xiong & 39 & 74 GR6TTJD01A8Y25_xion \\
\hline Unigene_UN_xiong_6037 & 155 & 65 UN_xiong_6037 \\
\hline Unigene_GR6TTJD01AU2SM_xiong & 40 & 41 GR6TTJD01AU2SM_xion \\
\hline Unigene_UN_xiong_12115 & 243 & 1345 UN_xiong_12115 \\
\hline Unigene_GR6TTJD01B77WA_xiong & 23 & 180 GR6TTJD01B77WA_xion \\
\hline Unigene_UN_xiong_4244 & 236 & 245 UN_xiong_4244 \\
\hline Unigene_UN_xiong_12942 & 71 & 189 UN_xiong_12942 \\
\hline Unigene_UN_xiong_2038 & 104 & 27 UN_xiong_2038 \\
\hline Unigene_UN_xiong_2317 & 2928 & 2498 UN_xiong_2317 \\
\hline Unigene_UN_xiong_7071 & 73 & 24 UN_xiong_7071 \\
\hline Unigene_UN_xiong_2503 & 110 & 33 UN_xiong_2503 \\
\hline Unigene_UN_xiong_9563 & 44 & 76 UN_xiong_9563 \\
\hline Unigene_UN_xiong_5179 & 51 & 426 UN_xiong_5179 \\
\hline Unigene_UN_xiong_2226 & 216 & 186 UN_xiong_2226 \\
\hline Unigene_UN_xiong_2186 & 418 & 838 UN_xiong_2186 \\
\hline Unigene_UN_xiong_8554 & 53 & 36 UN_xiong_8554 \\
\hline Unigene_UN_xiong_15511 & 719 & 1473 UN_xiong_15511 \\
\hline Unigene_GR6TTJD01A1SH0_xiong & 33 & 44 GR6TTJD01A1SH0_xion \\
\hline Unigene_UN_xiong_15361 & 290 & 205 UN_xiong_15361 \\
\hline Unigene_UN_xiong_5808 & 170 & 89 UN_xiong_5808 \\
\hline Unigene_GP8I43W03GB1M2_xiong & 57 & 51 GP8I43W03GB1M2_xion \\
\hline Unigene_UN_xiong_4032 & 153 & 372 UN_xiong_4032 \\
\hline Unigene_GPGRCDD02IE2WY_xiong & 26 & 26 GPGRCDD02IE2WY_xion \\
\hline Unigene_GR6TTJD01AFXT9_xiong & 61 & 55 GR6TTJD01AFXT9_xion \\
\hline Unigene_UN_xiong_194 & 2025 & 549 UN_xiong_194 \\
\hline Unigene_GR6TTJD01CB0MT_xiong & 52 & 100 GR6TTJD01CB0MT_xion \\
\hline Unigene_UN_xiong_9229 & 238 & 366 UN_xiong_9229 \\
\hline Unigene_UN_xiong_12865 & 45 & 53 UN_xiong_12865 \\
\hline Unigene_UN_xiong_14666 & 185 & 169 UN_xiong_14666 \\
\hline Unigene_UN_xiong_1283 & 63 & 70 UN_xiong_1283 \\
\hline Unigene_UN_xiong_1077 & 106 & 34 UN_xiong_1077 \\
\hline Unigene_GR6TTJD01BMHT8_xiong & 41 & 66 GR6TTJD01BMHT8_xion \\
\hline Unigene_UN_xiong_9137 & 112 & 26 UN_xiong_9137 \\
\hline Unigene_UN_xiong_11123 & 63 & 32 UN_xiong_11123 \\
\hline Unigene_UN_xiong_14332 & 128 & 304 UN_xiong_14332 \\
\hline Unigene_UN_xiong_5718 & 133 & 62 UN_xiong_5718 \\
\hline Unigene_UN_xiong_2199 & 165 & 807 UN_xiong_2199 \\
\hline Unigene_UN_xiong_11463 & 49 & 79 UN_xiong_11463 \\
\hline Unigene_UN_xiong_10815 & 687 & 108 UN_xiong_10815 \\
\hline Unigene_UN_xiong_5059 & 72 & 195 UN_xiong_5059 \\
\hline Unigene_GR6TTJD01BXLEP_xiong & 42 & 26 GR6TTJD01BXLEP_xion \\
\hline Unigene_GR6TTJD01CBAEC_xiong & 21 & 42 GR6TTJD01CBAEC_xion \\
\hline Unigene_UN_xiong_9483 & 85 & 653 UN_xiong_9483 \\
\hline Unigene_UN_xiong_5761 & 140 & 446 UN_xiong_5761 \\
\hline Unigene_UN_xiong_14658 & 392 & 1433 UN_xiong_14658 \\
\hline
\end{tabular}




\begin{tabular}{|c|c|c|}
\hline Unigene_UN_xiong_14883 & 304 & 172 UN_xiong_14883 \\
\hline Unigene_UN_xiong_1415 & 67 & 62 UN_xiong_1415 \\
\hline Unigene_UN_xiong_10747 & 139 & 96 UN_xiong_10747 \\
\hline Unigene_UN_xiong_14812 & 1115 & 852 UN_xiong_14812 \\
\hline Unigene_UN_xiong_3968 & 433 & 344 UN_xiong_3968 \\
\hline Unigene_UN_xiong_4865 & 259 & 188 UN_xiong_4865 \\
\hline Unigene_UN_xiong_2485 & 52 & 1222 UN_xiong_2485 \\
\hline Unigene_GR6TTJD01B9N04_xiong & 29 & 67 GR6TTJD01B9N04_xion \\
\hline Unigene_UN_xiong_11934 & 61 & 80 UN_xiong_11934 \\
\hline Unigene_GR6TTJD01B9ZM5_xiong & 43 & 63 GR6TTJD01B9ZM5_xion \\
\hline Unigene_UN_xiong_3507 & 155 & 40 UN_xiong_3507 \\
\hline Unigene_UN_xiong_14844 & 172 & 302 UN_xiong_14844 \\
\hline Unigene_UN_xiong_10717 & 51 & 186 UN_xiong_10717 \\
\hline Unigene_UN_xiong_3033 & 1765 & 1111 UN_xiong_3033 \\
\hline Unigene_UN_xiong_6298 & 133 & 48 UN_xiong_6298 \\
\hline Unigene_UN_xiong_3128 & 320 & 2409 UN_xiong_3128 \\
\hline Unigene_UN_xiong_387 & 426 & 617 UN_xiong_387 \\
\hline Unigene_UN_xiong_5977 & 312 & 513 UN_xiong_5977 \\
\hline Unigene_UN_xiong_14080 & 75 & 436 UN_xiong_14080 \\
\hline Unigene_GR6TTJD01AWIMR_xiong & 26 & 57 GR6TTJD01AWIMR_xion \\
\hline Unigene_GPGRCDD02I83Z3_xiong & 23 & 28 GPGRCDD02I83Z3_xion \\
\hline Unigene_GPGRCDD02HMYJS_xiong & 37 & 39 GPGRCDD02HMYJS_xion \\
\hline Unigene_UN_xiong_5097 & 43 & 27 UN_xiong_5097 \\
\hline Unigene_GR6TTJD01AT98W_xiong & 30 & 38 GR6TTJD01AT98W_xion \\
\hline Unigene_UN_xiong_14307 & 839 & 2621 UN_xiong_14307 \\
\hline Unigene_UN_xiong_1826 & 171 & 258 UN_xiong_1826 \\
\hline Unigene_UN_xiong_10983 & 77 & 43 UN_xiong_10983 \\
\hline Unigene_UN_xiong_7138 & 441 & 398 UN_xiong_7138 \\
\hline Unigene_UN_xiong_5575 & 205 & 128 UN_xiong_5575 \\
\hline Unigene_UN_xiong_14678 & 65 & 41 UN_xiong_14678 \\
\hline Unigene_UN_xiong_9694 & 89 & 389 UN_xiong_9694 \\
\hline Unigene_UN_xiong_12351 & 152 & 95 UN_xiong_12351 \\
\hline Unigene_GPGRCDD02IL1VA_xiong & 75 & 367 GPGRCDD02IL1VA_xion \\
\hline Unigene_UN_xiong_9571 & 184 & 77 UN_xiong_9571 \\
\hline Unigene_UN_xiong_2905 & 690 & 984 UN_xiong_2905 \\
\hline Unigene_GPGRCDD02ITM02_xiong & 41 & 164 GPGRCDD02ITM02_xion \\
\hline Unigene_GPGRCDD02I0LU3_xiong & 20 & 26 GPGRCDD02I0LU3_xion \\
\hline Unigene_GPGRCDD02FLZQG_xiong & 36 & 135 GPGRCDD02FLZQG_xion \\
\hline Unigene_GR6TTJD01BZR9D_xiong & 45 & 42 GR6TTJD01BZR9D_xion \\
\hline Unigene_UN_xiong_4589 & 55 & 25 UN_xiong_4589 \\
\hline Unigene_GP8I43W03GULZR_xiong & 30 & 138 GP8I43W03GULZR_xion \\
\hline Unigene_UN_xiong_14837 & 298 & 70 UN_xiong_14837 \\
\hline Unigene_UN_xiong_4600 & 88 & 218 UN_xiong_4600 \\
\hline Unigene_UN_xiong_5959 & 234 & 21 UN_xiong_5959 \\
\hline Unigene_GP8I43W03GY7GB_xiong & 29 & 86 GP8I43W03GY7GB_xion \\
\hline Unigene_UN_xiong_13626 & 62 & 305 UN_xiong_13626 \\
\hline Unigene_UN_xiong_2021 & 182 & 58 UN_xiong_2021 \\
\hline Unigene_UN_xiong_2520 & 1151 & 1044 UN_xiong_2520 \\
\hline Unigene_UN_xiong_11842 & 102 & 115 UN_xiong_11842 \\
\hline Unigene_UN_xiong_14872 & 90 & 76 UN_xiong_14872 \\
\hline
\end{tabular}




\begin{tabular}{|c|c|c|}
\hline Unigene_UN_xiong_3158 & 151 & 36 UN_xiong_3158 \\
\hline Unigene_UN_xiong_14366 & 214 & 375 UN_xiong_14366 \\
\hline Unigene_UN_xiong_15789 & 182 & 279 UN_xiong_15789 \\
\hline Unigene_UN_xiong_14665 & 1757 & 1058 UN_xiong_14665 \\
\hline Unigene_UN_xiong_5740 & 88 & 209 UN_xiong_5740 \\
\hline Unigene_GR6TTJD01AFI5D_xiong & 29 & 85 GR6TTJD01AFI5D_xion \\
\hline Unigene_UN_xiong_9232 & 192 & 465 UN_xiong_9232 \\
\hline Unigene_UN_xiong_4649 & 131 & 66 UN_xiong_4649 \\
\hline Unigene_GP8I43W03G5DI9_xiong & 51 & 542 GP8I43W03G5DI9_xion \\
\hline Unigene_GP8I43W03GR0S9_xiong & 38 & 36 GP8I43W03GR0S9_xion \\
\hline Unigene_UN_xiong_3265 & 318 & 1130 UN_xiong_3265 \\
\hline Unigene_UN_xiong_11408 & 143 & 76 UN_xiong_11408 \\
\hline Unigene_UN_xiong_4115 & 107 & 145 UN_xiong_4115 \\
\hline Unigene_UN_xiong_7019 & 154 & 78 UN_xiong_7019 \\
\hline Unigene_UN_xiong_14255 & 205 & 93 UN_xiong_14255 \\
\hline Unigene_UN_xiong_12012 & 95 & 222 UN_xiong_12012 \\
\hline Unigene_UN_xiong_223 & 195 & 105 UN_xiong_223 \\
\hline Unigene_GPGRCDD02HODBB_xiong & 68 & 60 GPGRCDD02HODBB_xion \\
\hline Unigene_UN_xiong_15045 & 3603 & 576 UN_xiong_15045 \\
\hline Unigene_UN_xiong_7476 & 194 & 525 UN_xiong_7476 \\
\hline Unigene_UN_xiong_327 & 143 & 91 UN_xiong_327 \\
\hline Unigene_UN_xiong_5287 & 108 & 5966 UN_xiong_5287 \\
\hline Unigene_UN_xiong_15334 & 419 & 629 UN_xiong_15334 \\
\hline Unigene_UN_xiong_539 & 115 & 23 UN_xiong_539 \\
\hline Unigene_UN_xiong_2596 & 90 & 30 UN_xiong_2596 \\
\hline Unigene_UN_xiong_11475 & 226 & 496 UN_xiong_11475 \\
\hline Unigene_UN_xiong_3778 & 145 & 67 UN_xiong_3778 \\
\hline Unigene_GR6TTJD01A94E7_xiong & 71 & 46 GR6TTJD01A94E7_xion \\
\hline Unigene_UN_xiong_15243 & 3729 & 595 UN_xiong_15243 \\
\hline Unigene_GR6TTJD01BD06K_xiong & 41 & 265 GR6TTJD01BD06K_xion \\
\hline Unigene_GR6TTJD01AES4K_xiong & 45 & 47 GR6TTJD01AES4K_xion \\
\hline Unigene_GR6TTJD01BTKNE_xiong & 22 & 82 GR6TTJD01BTKNE_xion \\
\hline Unigene_UN_xiong_14834 & 215 & 321 UN_xiong_14834 \\
\hline Unigene_UN_xiong_7125 & 91 & 38 UN_xiong_7125 \\
\hline Unigene_UN_xiong_11449 & 219 & 84 UN_xiong_11449 \\
\hline Unigene_GR6TTJD01AW00K_xiong & 26 & 25 GR6TTJD01AW00K_xion \\
\hline Unigene_UN_xiong_6441 & 176 & 536 UN_xiong_6441 \\
\hline Unigene_UN_xiong_11041 & 172 & 145 UN_xiong_11041 \\
\hline Unigene_UN_xiong_973 & 154 & 57 UN_xiong_973 \\
\hline Unigene_UN_xiong_15 & 66 & 214 UN_xiong_15 \\
\hline Unigene_UN_xiong_3053 & 63 & 167 UN_xiong_3053 \\
\hline Unigene_UN_xiong_10608 & 183 & 62 UN_xiong_10608 \\
\hline Unigene_UN_xiong_2422 & 46 & 200 UN_xiong_2422 \\
\hline Unigene_UN_xiong_15778 & 70 & 51 UN_xiong_15778 \\
\hline Unigene_UN_xiong_7681 & 40 & 71 UN_xiong_7681 \\
\hline Unigene_UN_xiong_12491 & 366 & 189 UN_xiong_12491 \\
\hline Unigene_UN_xiong_9366 & 38 & 121 UN_xiong_9366 \\
\hline Unigene_UN_xiong_11245 & 189 & 37 UN_xiong_11245 \\
\hline Unigene_UN_xiong_10595 & 131 & 144 UN_xiong_10595 \\
\hline Unigene_UN_xiong_13671 & 64 & 25 UN_xiong_13671 \\
\hline
\end{tabular}




\begin{tabular}{|c|c|c|}
\hline Unigene_GPGRCDD02IBL8U_xiong & 34 & 104 GPGRCDD02IBL8U_xion \\
\hline Unigene_UN_xiong_4390 & 75 & 47 UN_xiong_4390 \\
\hline Unigene_UN_xiong_3557 & 62 & 1066 UN_xiong_3557 \\
\hline Unigene_GP8I43W03GYMPF_xiong & 26 & 98 GP8I43W03GYMPF_xion \\
\hline Unigene_UN_xiong_14516 & 412 & 314 UN_xiong_14516 \\
\hline Unigene_UN_xiong_5331 & 32 & 62 UN_xiong_5331 \\
\hline Unigene_GR6TTJD01AKDCK_xiong & 45 & 98 GR6TTJD01AKDCK_xion \\
\hline Unigene_GR6TTJD01AH67A_xiong & 26 & 69 GR6TTJD01AH67A_xion \\
\hline Unigene_UN_xiong_2576 & 77 & 574 UN_xiong_2576 \\
\hline Unigene_GPGRCDD02JUJYD_xiong & 33 & 50 GPGRCDD02JUJYD_xion \\
\hline Unigene_UN_xiong_4257 & 93 & 66 UN_xiong_4257 \\
\hline Unigene_UN_xiong_3155 & 207 & 406 UN_xiong_3155 \\
\hline Unigene_UN_xiong_2484 & 556 & 286 UN_xiong_2484 \\
\hline Unigene_GP8I43W03FS6NH_xiong & 76 & 242 GP8I43W03FS6NH_xion \\
\hline Unigene_UN_xiong_14727 & 1181 & 649 UN_xiong_14727 \\
\hline Unigene_GPGRCDD02H5HFJ_xiong & 29 & 101 GPGRCDD02H5HFJ_xion \\
\hline Unigene_UN_xiong_7586 & 212 & 543 UN_xiong_7586 \\
\hline Unigene_UN_xiong_14509 & 3681 & 754 UN_xiong_14509 \\
\hline Unigene_UN_xiong_1736 & 41 & 333 UN_xiong_1736 \\
\hline Unigene_UN_xiong_7527 & 637 & 312 UN_xiong_7527 \\
\hline Unigene_UN_xiong_10833 & 201 & 400 UN_xiong_10833 \\
\hline Unigene_UN_xiong_11095 & 33 & 446 UN_xiong_11095 \\
\hline Unigene_GPGRCDD02JWP03_xiong & 35 & 77 GPGRCDD02JWP03_xion \\
\hline Unigene_GP8I43W03GEAYN_xiong & 27 & 78 GP8I43W03GEAYN_xion \\
\hline Unigene_UN_xiong_6273 & 299 & 1811 UN_xiong_6273 \\
\hline Unigene_UN_xiong_398 & 83 & 39 UN_xiong_398 \\
\hline Unigene_UN_xiong_14060 & 361 & 37 UN_xiong_14060 \\
\hline Unigene_UN_xiong_9784 & 92 & 25 UN_xiong_9784 \\
\hline Unigene_GPGRCDD02JZYSI_xiong & 98 & 83 GPGRCDD02JZYSI_xion \\
\hline Unigene_UN_xiong_10677 & 90 & 29 UN_xiong_10677 \\
\hline Unigene_GR6TTJD01AJ51R_xiong & 39 & 92 GR6TTJD01AJ51R_xion \\
\hline Unigene_UN_xiong_8056 & 185 & 631 UN_xiong_8056 \\
\hline Unigene_GR6TTJD01BJV0X_xiong & 57 & 62 GR6TTJD01BJV0X_xion \\
\hline Unigene_UN_xiong_55 & 236 & 313 UN_xiong_55 \\
\hline Unigene_UN_xiong_3860 & 157 & 44 UN_xiong_3860 \\
\hline Unigene_UN_xiong_12775 & 52 & 106 UN_xiong_12775 \\
\hline Unigene_UN_xiong_5248 & 245 & 175 UN_xiong_5248 \\
\hline Unigene_UN_xiong_2780 & 104 & 180 UN_xiong_2780 \\
\hline Unigene_UN_xiong_1215 & 229 & 89 UN_xiong_1215 \\
\hline Unigene_UN_xiong_12184 & 211 & 42 UN_xiong_12184 \\
\hline Unigene_UN_xiong_7836 & 65 & 31 UN_xiong_7836 \\
\hline Unigene_UN_xiong_2765 & 346 & 43 UN_xiong_2765 \\
\hline Unigene_GR6TTJD01A9TCM_xiong & 24 & 34 GR6TTJD01A9TCM_xion \\
\hline Unigene_UN_xiong_4161 & 205 & 72 UN_xiong_4161 \\
\hline Unigene_GP8I43W03F94VR_xiong & 32 & 63 GP8I43W03F94VR_xion \\
\hline Unigene_UN_xiong_15554 & 1435 & 1495 UN_xiong_15554 \\
\hline Unigene_UN_xiong_13747 & 145 & 61 UN_xiong_13747 \\
\hline Unigene_UN_xiong_8562 & 50 & 26 UN_xiong_8562 \\
\hline Unigene_GPGRCDD02HSHYZ_xiong & 24 & 40 GPGRCDD02HSHYZ_xion \\
\hline Unigene_UN_xiong_11976 & 44 & 157 UN_xiong_11976 \\
\hline
\end{tabular}




\begin{tabular}{|c|c|c|}
\hline Unigene_UN_xiong_6435 & 167 & 67 UN_xiong_6435 \\
\hline Unigene_UN_xiong_5268 & 238 & 68 UN_xiong_5268 \\
\hline Unigene_GPGRCDD02HPVA7_xiong & 48 & 25 GPGRCDD02HPVA7_xion \\
\hline Unigene_GP8I43W03F9I3L_xiong & 59 & 41 GP8I43W03F9I3L_xion \\
\hline Unigene_UN_xiong_8361 & 59 & 332 UN_xiong_8361 \\
\hline Unigene_GP8I43W03GCZ8U_xiong & 58 & 56 GP8I43W03GCZ8U_xion \\
\hline Unigene_GR6TTJD01BKTLD_xiong & 33 & 34 GR6TTJD01BKTLD_xion \\
\hline Unigene_GPGRCDD02FV490_xiong & 31 & 30 GPGRCDD02FV490_xion \\
\hline Unigene_GR6TTJD01B0FFM_xiong & 41 & 28 GR6TTJD01B0FFM_xion \\
\hline Unigene_UN_xiong_2698 & 273 & 235 UN_xiong_2698 \\
\hline Unigene_UN_xiong_8484 & 190 & 95 UN_xiong_8484 \\
\hline Unigene_UN_xiong_1057 & 122 & 153 UN_xiong_1057 \\
\hline Unigene_UN_xiong_3629 & 52 & 64 UN_xiong_3629 \\
\hline Unigene_UN_xiong_3977 & 43 & 51 UN_xiong_3977 \\
\hline Unigene_UN_xiong_8601 & 45 & 65 UN_xiong_8601 \\
\hline Unigene_GR6TTJD01CDZ4E_xiong & 105 & 226 GR6TTJD01CDZ4E_xion \\
\hline Unigene_UN_xiong_2856 & 138 & 57 UN_xiong_2856 \\
\hline Unigene_UN_xiong_9555 & 145 & 282 UN_xiong_9555 \\
\hline Unigene_UN_xiong_1813 & 42 & 68 UN_xiong_1813 \\
\hline Unigene_UN_xiong_7097 & 522 & 57 UN_xiong_7097 \\
\hline Unigene_UN_xiong_3309 & 104 & 69 UN_xiong_3309 \\
\hline Unigene_UN_xiong_13950 & 76 & 30 UN_xiong_13950 \\
\hline Unigene_UN_xiong_3911 & 82 & 306 UN_xiong_3911 \\
\hline Unigene_UN_xiong_4247 & 156 & 481 UN_xiong_4247 \\
\hline Unigene_UN_xiong_8884 & 165 & 356 UN_xiong_8884 \\
\hline Unigene_UN_xiong_4755 & 70 & 27 UN_xiong_4755 \\
\hline Unigene_UN_xiong_6172 & 84 & 78 UN_xiong_6172 \\
\hline Unigene_GR6TTJD01A00EL_xiong & 31 & 123 GR6TTJD01A00EL_xion \\
\hline Unigene_UN_xiong_3999 & 287 & 132 UN_xiong_3999 \\
\hline Unigene_UN_xiong_7079 & 271 & 130 UN_xiong_7079 \\
\hline Unigene_GPGRCDD02GVALW_xiong & 52 & 28 GPGRCDD02GVALW_xion \\
\hline Unigene_UN_xiong_5314 & 133 & 141 UN_xiong_5314 \\
\hline Unigene_GPGRCDD02J2X54_xiong & 31 & 28 GPGRCDD02J2X54_xion \\
\hline Unigene_UN_xiong_1141 & 193 & 91 UN_xiong_1141 \\
\hline Unigene_UN_xiong_2168 & 430 & 173 UN_xiong_2168 \\
\hline Unigene_UN_xiong_13985 & 53 & 48 UN_xiong_13985 \\
\hline Unigene_UN_xiong_14247 & 405 & 102 UN_xiong_14247 \\
\hline Unigene_UN_xiong_4992 & 130 & 91 UN_xiong_4992 \\
\hline Unigene_UN_xiong_11988 & 134 & 23 UN_xiong_11988 \\
\hline Unigene_UN_xiong_8021 & 132 & 93 UN_xiong_8021 \\
\hline Unigene_UN_xiong_14279 & 1022 & 149 UN_xiong_14279 \\
\hline Unigene_UN_xiong_14783 & 2055 & 1154 UN_xiong_14783 \\
\hline Unigene_UN_xiong_4263 & 125 & 44 UN_xiong_4263 \\
\hline Unigene_UN_xiong_14362 & 266 & 251 UN_xiong_14362 \\
\hline Unigene_UN_xiong_11794 & 81 & 47 UN_xiong_11794 \\
\hline Unigene_UN_xiong_4494 & 692 & 447 UN_xiong_4494 \\
\hline Unigene_GPGRCDD02GCG9Y_xiong & 164 & 19 GPGRCDD02GCG9Y_xion \\
\hline Unigene_UN_xiong_15130 & 1065 & 4408 UN_xiong_15130 \\
\hline Unigene_UN_xiong_500 & 75 & 22 UN_xiong_500 \\
\hline Unigene_UN_xiong_5912 & 518 & 169 UN_xiong_5912 \\
\hline
\end{tabular}




\begin{tabular}{|c|c|c|}
\hline Unigene_UN_xiong_12470 & 98 & 235 UN_xiong_12470 \\
\hline Unigene_UN_xiong_8841 & 61 & 94 UN_xiong_8841 \\
\hline Unigene_UN_xiong_13661 & 147 & 437 UN_xiong_13661 \\
\hline Unigene_UN_xiong_5589 & 164 & 682 UN_xiong_5589 \\
\hline Unigene_UN_xiong_4767 & 107 & 163 UN_xiong_4767 \\
\hline Unigene_GR6TTJD01A2994_xiong & 32 & 69 GR6TTJD01A2994_xion \\
\hline Unigene_UN_xiong_4926 & 126 & 199 UN_xiong_4926 \\
\hline Unigene_UN_xiong_3490 & 51 & 24 UN_xiong_3490 \\
\hline Unigene_UN_xiong_13392 & 64 & 87 UN_xiong_13392 \\
\hline Unigene_GPGRCDD02I78TS_xiong & 55 & 204 GPGRCDD02I78TS_xion \\
\hline Unigene_UN_xiong_3161 & 187 & 255 UN_xiong_3161 \\
\hline Unigene_UN_xiong_14540 & 351 & 38 UN_xiong_14540 \\
\hline Unigene_GR6TTJD01B4N7E_xiong & 43 & 84 GR6TTJD01B4N7E_xion \\
\hline Unigene_UN_xiong_8690 & 86 & 62 UN_xiong_8690 \\
\hline Unigene_GR6TTJD01BNHPE_xiong & 46 & 36 GR6TTJD01BNHPE_xion \\
\hline Unigene_GP8I43W03GGJVD_xiong & 48 & 74 GP8I43W03GGJVD_xion \\
\hline Unigene_UN_xiong_9679 & 180 & 60 UN_xiong_9679 \\
\hline Unigene_UN_xiong_140 & 144 & 156 UN_xiong_140 \\
\hline Unigene_UN_xiong_3232 & 70 & 53 UN_xiong_3232 \\
\hline Unigene_UN_xiong_6798 & 71 & 561 UN_xiong_6798 \\
\hline Unigene_UN_xiong_2320 & 171 & 35 UN_xiong_2320 \\
\hline Unigene_GR6TTJD01ALTT4_xiong & 37 & 91 GR6TTJD01ALTT4_xion \\
\hline Unigene_UN_xiong_11991 & 72 & 41 UN_xiong_11991 \\
\hline Unigene_UN_xiong_11572 & 61 & 39 UN_xiong_11572 \\
\hline Unigene_GPGRCDD02IAGT0_xiong & 32 & 30 GPGRCDD02IAGT0_xion \\
\hline Unigene_UN_xiong_9887 & 101 & 26 UN_xiong_9887 \\
\hline Unigene_UN_xiong_12191 & 33 & 24 UN_xiong_12191 \\
\hline Unigene_UN_xiong_5387 & 361 & 536 UN_xiong_5387 \\
\hline Unigene_UN_xiong_11288 & 121 & 197 UN_xiong_11288 \\
\hline Unigene_UN_xiong_4513 & 79 & 57 UN_xiong_4513 \\
\hline Unigene_UN_xiong_11823 & 85 & 1807 UN_xiong_11823 \\
\hline Unigene_UN_xiong_4280 & 40 & 70 UN_xiong_4280 \\
\hline Unigene_UN_xiong_933 & 47 & 28 UN_xiong_933 \\
\hline Unigene_UN_xiong_3438 & 142 & 62 UN_xiong_3438 \\
\hline Unigene_GPGRCDD02F0NF6_xiong & 45 & 30 GPGRCDD02FONF6_xion \\
\hline Unigene_UN_xiong_12088 & 74 & 334 UN_xiong_12088 \\
\hline Unigene_GR6TTJD01AT2GK_xiong & 38 & 39 GR6TTJD01AT2GK_xion \\
\hline Unigene_UN_xiong_5549 & 262 & 2153 UN_xiong_5549 \\
\hline Unigene_UN_xiong_4819 & 120 & 133 UN_xiong_4819 \\
\hline Unigene_GR6TTJD01B0UC9_xiong & 24 & 21 GR6TTJD01B0UC9_xion \\
\hline Unigene_UN_xiong_8803 & 129 & 476 UN_xiong_8803 \\
\hline Unigene_UN_xiong_9662 & 84 & 35 UN_xiong_9662 \\
\hline Unigene_UN_xiong_2548 & 311 & 53 UN_xiong_2548 \\
\hline Unigene_UN_xiong_5790 & 31 & 58 UN_xiong_5790 \\
\hline Unigene_UN_xiong_2411 & 206 & 397 UN_xiong_2411 \\
\hline Unigene_UN_xiong_6097 & 62 & 50 UN_xiong_6097 \\
\hline Unigene_GPGRCDD02IM7JN_xiong & 22 & 780 GPGRCDD02IM7JN_xion \\
\hline Unigene_UN_xiong_4711 & 202 & 435 UN_xiong_4711 \\
\hline Unigene_GR6TTJD01AH9KM_xiong & 22 & 22 GR6TTJD01AH9KM_xion \\
\hline Unigene_UN_xiong_3681 & 100 & 498 UN_xiong_3681 \\
\hline
\end{tabular}




\begin{tabular}{|c|c|c|}
\hline Unigene_GP8I43W03GSTN5_xiong & 24 & 45 GP8I43W03GSTN5_xion \\
\hline Unigene_GP8I43W03GT0L9_xiong & 25 & 21 GP8I43W03GT0L9_xion \\
\hline Unigene_UN_xiong_4289 & 280 & 392 UN_xiong_4289 \\
\hline Unigene_UN_xiong_2278 & 85 & 90 UN_xiong_2278 \\
\hline Unigene_UN_xiong_3469 & 81 & 137 UN_xiong_3469 \\
\hline Unigene_UN_xiong_369 & 302 & 62 UN_xiong_369 \\
\hline Unigene_UN_xiong_14020 & 136 & 47 UN_xiong_14020 \\
\hline Unigene_UN_xiong_4038 & 344 & 818 UN_xiong_4038 \\
\hline Unigene_UN_xiong_10241 & 39 & 183 UN_xiong_10241 \\
\hline Unigene_UN_xiong_3035 & 77 & 88 UN_xiong_3035 \\
\hline Unigene_UN_xiong_6694 & 713 & 538 UN_xiong_6694 \\
\hline Unigene_UN_xiong_12242 & 78 & 3422 UN_xiong_12242 \\
\hline Unigene_GR6TTJD01AF7CM_xiong & 42 & 40 GR6TTJD01AF7CM_xion \\
\hline Unigene_GPGRCDD02G1ZD3_xiong & 41 & 40 GPGRCDD02G1ZD3_xion \\
\hline Unigene_UN_xiong_4876 & 43 & 100 UN_xiong_4876 \\
\hline Unigene_UN_xiong_11438 & 43 & 62 UN_xiong_11438 \\
\hline Unigene_UN_xiong_6572 & 55 & 87 UN_xiong_6572 \\
\hline Unigene_UN_xiong_1492 & 152 & 214 UN_xiong_1492 \\
\hline Unigene_GPGRCDD02JJCK5_xiong & 22 & 82 GPGRCDD02JJCK5_xion \\
\hline Unigene_UN_xiong_11907 & 122 & 112 UN_xiong_11907 \\
\hline Unigene_UN_xiong_9266 & 122 & 228 UN_xiong_9266 \\
\hline Unigene_UN_xiong_13065 & 105 & 181 UN_xiong_13065 \\
\hline Unigene_UN_xiong_4348 & 183 & 161 UN_xiong_4348 \\
\hline Unigene_UN_xiong_5148 & 194 & 271 UN_xiong_5148 \\
\hline Unigene UN xiong 1822 & 141 & 230 UN xiong 1822 \\
\hline Unigene_UN_xiong_14271 & 852 & 737 UN_xiong_14271 \\
\hline Unigene_GR6TTJD01A0FGW_xiong & 29 & 87 GR6TTJD01A0FGW_xion \\
\hline Unigene_UN_xiong_2509 & 270 & 43 UN_xiong_2509 \\
\hline Unigene UN xiong 8922 & 296 & 548 UN xiong 8922 \\
\hline Unigene_UN_xiong_15512 & 41 & 45 UN_xiong_15512 \\
\hline Unigene_UN_xiong_14422 & 546 & 66 UN_xiong_14422 \\
\hline Unigene_GR6TTJD01BK65C_xiong & 33 & 42 GR6TTJD01BK65C_xion \\
\hline Unigene_GR6TTJD01AKT8Q_xiong & 24 & 39 GR6TTJD01AKT8Q_xion \\
\hline Unigene_UN_xiong_7900 & 192 & 52 UN_xiong_7900 \\
\hline Unigene_UN_xiong_8994 & 592 & 346 UN_xiong_8994 \\
\hline Unigene_GR6TTJD01BV050_xiong & 30 & 32 GR6TTJD01BV050_xion \\
\hline Unigene_GR6TTJD01AWNSS_xiong & 47 & 34 GR6TTJD01AWNSS_xion \\
\hline Unigene_UN_xiong_6790 & 64 & 240 UN_xiong_6790 \\
\hline Unigene_UN_xiong_15513 & 41 & 23 UN_xiong_15513 \\
\hline Unigene_UN_xiong_12945 & 62 & 172 UN_xiong_12945 \\
\hline Unigene_UN_xiong_14299 & 305 & 166 UN_xiong_14299 \\
\hline Unigene UN xiong 14520 & 779 & 1509 UN xiong 14520 \\
\hline Unigene_GPGRCDD02FG340_xiong & 34 & 163 GPGRCDD02FG340_xion \\
\hline Unigene_UN_xiong_3382 & 312 & 222 UN_xiong_3382 \\
\hline Unigene_UN_xiong_6673 & 145 & 29 UN_xiong_6673 \\
\hline Unigene GPGRCDD02FKSTM xiong & 58 & 63 GPGRCDD02FKSTM xion \\
\hline Unigene_GR6TTJD01AHEW1_xiong & 22 & 67 GR6TTJD01AHEW1_xion \\
\hline Unigene_UN_xiong_14513 & 314 & 162 UN_xiong_14513 \\
\hline Unigene_GP8I43W03FQYRR_xiong & 47 & 55 GP8I43W03FQYRR_xion \\
\hline Unigene_GPGRCDD02HQSOZ_xiong & 22 & 29 GPGRCDD02HQSOZ_xion \\
\hline
\end{tabular}




\begin{tabular}{|c|c|c|}
\hline Unigene_UN_xiong_7163 & 143 & 1148 UN_xiong_7163 \\
\hline Unigene_UN_xiong_2079 & 59 & 74 UN_xiong_2079 \\
\hline Unigene_UN_xiong_6145 & 103 & 108 UN_xiong_6145 \\
\hline Unigene_UN_xiong_13267 & 133 & 120 UN_xiong_13267 \\
\hline Unigene_UN_xiong_10532 & 90 & 111 UN_xiong_10532 \\
\hline Unigene_UN_xiong_70 & 81 & 62 UN_xiong_70 \\
\hline Unigene_GR6TTJD01AQNEQ_xiong & 36 & 35 GR6TTJD01AQNEQ_xion \\
\hline Unigene_GPGRCDD02IEJOA_xiong & 72 & 92 GPGRCDD02IEJ0A_xion \\
\hline Unigene_UN_xiong_1113 & 182 & 378 UN_xiong_1113 \\
\hline Unigene_UN_xiong_1846 & 81 & 104 UN_xiong_1846 \\
\hline Unigene_GR6TTJD01BZKMJ_xiong & 22 & 115 GR6TTJD01BZKMJ_xion \\
\hline Unigene_UN_xiong_4503 & 88 & 28 UN_xiong_4503 \\
\hline Unigene_GPGRCDD02G56A3_xiong & 32 & 55 GPGRCDD02G56A3_xion \\
\hline Unigene_UN_xiong_641 & 330 & 22 UN_xiong_641 \\
\hline Unigene_UN_xiong_3419 & 244 & 93 UN_xiong_3419 \\
\hline Unigene_UN_xiong_2261 & 69 & 39 UN_xiong_2261 \\
\hline Unigene_GP8I43W03F0KZR_xiong & 33 & 111 GP8I43W03F0KZR_xion \\
\hline Unigene_UN_xiong_8485 & 81 & 35 UN_xiong_8485 \\
\hline Unigene_UN_xiong_9489 & 74 & 73 UN_xiong_9489 \\
\hline Unigene_UN_xiong_11125 & 259 & 117 UN_xiong_11125 \\
\hline Unigene_UN_xiong_9691 & 353 & 28 UN_xiong_9691 \\
\hline Unigene_UN_xiong_1392 & 352 & 766 UN_xiong_1392 \\
\hline Unigene_UN_xiong_8320 & 59 & 34 UN_xiong_8320 \\
\hline Unigene_UN_xiong_2808 & 570 & 423 UN_xiong_2808 \\
\hline Unigene_GR6TTJD01AYDC2_xiong & 24 & 27 GR6TTJD01AYDC2_xion \\
\hline Unigene_UN_xiong_13147 & 114 & 25 UN_xiong_13147 \\
\hline Unigene_UN_xiong_12134 & 46 & 197 UN_xiong_12134 \\
\hline Unigene_UN_xiong_11837 & 223 & 99 UN_xiong_11837 \\
\hline Unigene_UN_xiong_13044 & 119 & 118 UN_xiong_13044 \\
\hline Unigene_UN_xiong_11211 & 99 & 127 UN_xiong_11211 \\
\hline Unigene_UN_xiong_1088 & 60 & 21 UN_xiong_1088 \\
\hline Unigene_GR6TTJD01AQIA9_xiong & 68 & 72 GR6TTJD01AQIA9_xion \\
\hline Unigene_UN_xiong_6117 & 46 & 36 UN_xiong_6117 \\
\hline Unigene_GR6TTJD01BK35G_xiong & 30 & 102 GR6TTJD01BK35G_xion \\
\hline Unigene_UN_xiong_1914 & 156 & 53 UN_xiong_1914 \\
\hline Unigene_UN_xiong_4135 & 71 & 24 UN_xiong_4135 \\
\hline Unigene_UN_xiong_11487 & 82 & 153 UN_xiong_11487 \\
\hline Unigene_UN_xiong_11605 & 89 & 175 UN_xiong_11605 \\
\hline Unigene_UN_xiong_6113 & 125 & 183 UN_xiong_6113 \\
\hline Unigene_GR6TTJD01AGQ05_xiong & 32 & 23 GR6TTJD01AGQ05_xion \\
\hline Unigene_UN_xiong_11735 & 60 & 23 UN_xiong_11735 \\
\hline Unigene_GR6TTJD01A3LMS_xiong & 35 & 100 GR6TTJD01A3LMS_xion \\
\hline Unigene_GR6TTJD01BZCX1_xiong & 31 & 29 GR6TTJD01BZCX1_xion \\
\hline Unigene_GR6TTJD01ANVTP_xiong & 61 & 65 GR6TTJD01ANVTP_xion \\
\hline Unigene_UN_xiong_3986 & 70 & 864 UN_xiong_3986 \\
\hline Unigene_UN_xiong_711 & 433 & 156 UN_xiong_711 \\
\hline Unigene_GPGRCDD02FS9XI_xiong & 29 & 26 GPGRCDD02FS9XI_xion \\
\hline Unigene_UN_xiong_8609 & 92 & 23 UN_xiong_8609 \\
\hline Unigene_UN_xiong_6463 & 284 & 150 UN_xiong_6463 \\
\hline Unigene_UN_xiong_5290 & 252 & 105 UN_xiong_5290 \\
\hline
\end{tabular}




\begin{tabular}{|c|c|c|}
\hline Unigene_GPGRCDD02H304C_xiong & 56 & 80 GPGRCDD02H304C_xion \\
\hline Unigene_UN_xiong_15430 & 78 & 634 UN_xiong_15430 \\
\hline Unigene_UN_xiong_7507 & 105 & 42 UN_xiong_7507 \\
\hline Unigene_UN_xiong_12897 & 99 & 114 UN_xiong_12897 \\
\hline Unigene_UN_xiong_12316 & 120 & 139 UN_xiong_12316 \\
\hline Unigene_UN_xiong_15197 & 93 & 26 UN_xiong_15197 \\
\hline Unigene_UN_xiong_11967 & 671 & 856 UN_xiong_11967 \\
\hline Unigene_UN_xiong_8614 & 88 & 43 UN_xiong_8614 \\
\hline Unigene_UN_xiong_15660 & 553 & 2562 UN_xiong_15660 \\
\hline Unigene_UN_xiong_5512 & 153 & 61 UN_xiong_5512 \\
\hline Unigene_UN_xiong_14327 & 969 & 814 UN_xiong_14327 \\
\hline Unigene_UN_xiong_15008 & 95 & 46 UN_xiong_15008 \\
\hline Unigene_UN_xiong_12967 & 133 & 132 UN_xiong_12967 \\
\hline Unigene_UN_xiong_3363 & 94 & 38 UN_xiong_3363 \\
\hline Unigene_GR6TTJD01BSG50_xiong & 27 & 101 GR6TTJD01BSG50_xion \\
\hline Unigene_GR6TTJD01B0H8H_xiong & 77 & 68 GR6TTJD01B0H8H_xion \\
\hline Unigene_GP8I43W03HCPRU_xiong & 27 & 151 GP8I43W03HCPRU_xion \\
\hline Unigene_UN_xiong_8269 & 150 & 22 UN_xiong_8269 \\
\hline Unigene_UN_xiong_11113 & 218 & 7116 UN_xiong_11113 \\
\hline Unigene_UN_xiong_6557 & 149 & 67 UN_xiong_6557 \\
\hline Unigene_UN_xiong_11144 & 52 & 170 UN_xiong_11144 \\
\hline Unigene_UN_xiong_14042 & 202 & 482 UN_xiong_14042 \\
\hline Unigene_UN_xiong_11492 & 122 & 585 UN_xiong_11492 \\
\hline Unigene_UN_xiong_6002 & 340 & 292 UN_xiong_6002 \\
\hline Unigene_GR6TTJD01BA8KZ_xiong & 26 & 31 GR6TTJD01BA8KZ_xion \\
\hline Unigene_UN_xiong_12339 & 85 & 211 UN_xiong_12339 \\
\hline Unigene_UN_xiong_13509 & 67 & 41 UN_xiong_13509 \\
\hline Unigene_UN_xiong_15687 & 309 & 1692 UN_xiong_15687 \\
\hline Unigene_UN_xiong_5437 & 205 & 486 UN_xiong_5437 \\
\hline Unigene_GPGRCDD02HGZOL_xiong & 34 & 31 GPGRCDD02HGZ0L_xion \\
\hline Unigene_UN_xiong_14050 & 42 & 137 UN_xiong_14050 \\
\hline Unigene_UN_xiong_11910 & 93 & 211 UN_xiong_11910 \\
\hline Unigene_UN_xiong_2880 & 590 & 3689 UN_xiong_2880 \\
\hline Unigene_UN_xiong_12386 & 150 & 60 UN_xiong_12386 \\
\hline Unigene_UN_xiong_2185 & 70 & 68 UN_xiong_2185 \\
\hline Unigene_UN_xiong_14685 & 287 & 375 UN_xiong_14685 \\
\hline Unigene_GR6TTJD01BDS1G_xiong & 76 & 58 GR6TTJD01BDS1G_xion \\
\hline Unigene_UN_xiong_8625 & 73 & 49 UN_xiong_8625 \\
\hline Unigene_GR6TTJD01BVK0L_xiong & 75 & 68 GR6TTJD01BVK0L_xion \\
\hline Unigene_UN_xiong_1959 & 117 & 120 UN_xiong_1959 \\
\hline Unigene_UN_xiong_3766 & 86 & 121 UN_xiong_3766 \\
\hline Unigene_UN_xiong_11535 & 150 & 21 UN_xiong_11535 \\
\hline Unigene_GR6TTJD01AXFSL_xiong & 42 & 28 GR6TTJD01AXFSL_xion \\
\hline Unigene_UN_xiong_5771 & 176 & 47 UN_xiong_5771 \\
\hline Unigene_UN_xiong_620 & 88 & 36 UN_xiong_620 \\
\hline Unigene_UN_xiong_15219 & 1857 & 2117 UN_xiong_15219 \\
\hline Unigene_UN_xiong_7363 & 54 & 100 UN_xiong_7363 \\
\hline Unigene_UN_xiong_10619 & 46 & 546 UN_xiong_10619 \\
\hline Unigene_UN_xiong_8536 & 365 & 55 UN_xiong_8536 \\
\hline Unigene_UN_xiong_300 & 115 & 62 UN_xiong_300 \\
\hline
\end{tabular}




\begin{tabular}{|c|c|c|}
\hline Unigene_UN_xiong_1364 & 713 & 983 UN_xiong_1364 \\
\hline Unigene_UN_xiong_9352 & 70 & 32 UN_xiong_9352 \\
\hline Unigene_GR6TTJD01A29NN_xiong & 47 & 27 GR6TTJD01A29NN_xion \\
\hline Unigene_UN_xiong_6026 & 309 & 25 UN_xiong_6026 \\
\hline Unigene_GP8I43W03G67ZL_xiong & 38 & 34 GP8I43W03G67ZL_xion \\
\hline Unigene_GP8I43W03G4063_xiong & 27 & 73 GP8I43W03G4063_xion \\
\hline Unigene_GPGRCDD02J0HCX_xiong & 62 & 366 GPGRCDD02J0HCX_xion \\
\hline Unigene_GR6TTJD01BHBRB_xiong & 34 & 26 GR6TTJD01BHBRB_xion \\
\hline Unigene_UN_xiong_7793 & 48 & 184 UN_xiong_7793 \\
\hline Unigene_UN_xiong_14663 & 260 & 648 UN_xiong_14663 \\
\hline Unigene_UN_xiong_13635 & 153 & 281 UN_xiong_13635 \\
\hline Unigene_GR6TTJD01AH9NB_xiong & 62 & 58 GR6TTJD01AH9NB_xion \\
\hline Unigene_UN_xiong_14805 & 716 & 725 UN_xiong_14805 \\
\hline Unigene_UN_xiong_12414 & 40 & 110 UN_xiong_12414 \\
\hline Unigene_UN_xiong_8573 & 628 & 96 UN_xiong_8573 \\
\hline Unigene_UN_xiong_14675 & 251 & 82 UN_xiong_14675 \\
\hline Unigene_UN_xiong_8505 & 99 & 224 UN_xiong_8505 \\
\hline Unigene_UN_xiong_12927 & 68 & 214 UN_xiong_12927 \\
\hline Unigene_GR6TTJD01B27UQ_xiong & 26 & 54 GR6TTJD01B27UQ_xion \\
\hline Unigene_UN_xiong_14139 & 68 & 87 UN_xiong_14139 \\
\hline Unigene_UN_xiong_2816 & 108 & 23 UN_xiong_2816 \\
\hline Unigene_GP8I43W03FY3VP_xiong & 28 & 45 GP8I43W03FY3VP_xion \\
\hline Unigene_UN_xiong_5490 & 48 & 108 UN_xiong_5490 \\
\hline Unigene_UN_xiong_14899 & 242 & 192 UN_xiong_14899 \\
\hline Unigene_UN_xiong_12650 & 43 & 41 UN_xiong_12650 \\
\hline Unigene_UN_xiong_5108 & 174 & 58 UN_xiong_5108 \\
\hline Unigene_UN_xiong_9829 & 65 & 50 UN_xiong_9829 \\
\hline Unigene_UN_xiong_11585 & 96 & 46 UN_xiong_11585 \\
\hline Unigene_UN_xiong_8544 & 36 & 160 UN_xiong_8544 \\
\hline Unigene_UN_xiong_8136 & 407 & 188 UN_xiong_8136 \\
\hline Unigene_GR6TTJD01BDXAW_xiong & 27 & 20 GR6TTJD01BDXAW_xion \\
\hline Unigene_GPGRCDD02G2Y56_xiong & 30 & 48 GPGRCDD02G2Y56_xion \\
\hline Unigene_UN_xiong_3939 & 95 & 32 UN_xiong_3939 \\
\hline Unigene_GP8I43W03GB3R9_xiong & 24 & 352 GP8I43W03GB3R9_xion \\
\hline Unigene_UN_xiong_9526 & 126 & 47 UN_xiong_9526 \\
\hline Unigene_GPGRCDD02HHCSB_xiong & 23 & 109 GPGRCDD02HHCSB_xion \\
\hline Unigene_UN_xiong_15375 & 1763 & 572 UN_xiong_15375 \\
\hline Unigene_UN_xiong_11690 & 57 & 84 UN_xiong_11690 \\
\hline Unigene_UN_xiong_2288 & 244 & 208 UN_xiong_2288 \\
\hline Unigene_UN_xiong_14207 & 1023 & 88 UN_xiong_14207 \\
\hline Unigene_UN_xiong_6747 & 80 & 90 UN_xiong_6747 \\
\hline Unigene_UN_xiong_6514 & 132 & 158 UN_xiong_6514 \\
\hline Unigene_UN_xiong_876 & 509 & 277 UN_xiong_876 \\
\hline Unigene_UN_xiong_4270 & 272 & 44 UN_xiong_4270 \\
\hline Unigene_UN_xiong_6304 & 584 & 341 UN_xiong_6304 \\
\hline Unigene_UN_xiong_14384 & 486 & 226 UN_xiong_14384 \\
\hline Unigene_UN_xiong_8721 & 87 & 78 UN_xiong_8721 \\
\hline Unigene_GPGRCDD02HA1BC_xiong & 50 & 53 GPGRCDD02HA1BC_xion \\
\hline Unigene_UN_xiong_8033 & 53 & 44 UN_xiong_8033 \\
\hline Unigene_GR6TTJD01AW9R0_xiong & 30 & 25 GR6TTJD01AW9R0_xion \\
\hline
\end{tabular}




\begin{tabular}{|c|c|c|}
\hline Unigene_UN_xiong_1579 & 165 & 211 UN_xiong_1579 \\
\hline Unigene_UN_xiong_14469 & 776 & 121 UN_xiong_14469 \\
\hline Unigene_UN_xiong_3287 & 81 & 34 UN_xiong_3287 \\
\hline Unigene_UN_xiong_1666 & 128 & 41 UN_xiong_1666 \\
\hline Unigene_UN_xiong_12033 & 196 & 58 UN_xiong_12033 \\
\hline Unigene_UN_xiong_6740 & 310 & 211 UN_xiong_6740 \\
\hline Unigene_UN_xiong_15671 & 99 & 100 UN_xiong_15671 \\
\hline Unigene_GP8I43W03HFVNM_xiong & 37 & 52 GP8I43W03HFVNM_xion \\
\hline Unigene_UN_xiong_1596 & 289 & 328 UN_xiong_1596 \\
\hline Unigene_GPGRCDD02IYL41_xiong & 65 & 98 GPGRCDD02IYL41_xion \\
\hline Unigene_UN_xiong_5033 & 67 & 56 UN_xiong_5033 \\
\hline Unigene_UN_xiong_2220 & 377 & 94 UN_xiong_2220 \\
\hline Unigene_UN_xiong_6516 & 147 & 33 UN_xiong_6516 \\
\hline Unigene_GP8I43W03G8SZ7_xiong & 25 & 110 GP8I43W03G8SZ7_xion \\
\hline Unigene_UN_xiong_1313 & 105 & 46 UN_xiong_1313 \\
\hline Unigene_GPGRCDD02IZBCM_xiong & 23 & 53 GPGRCDD02IZBCM_xion \\
\hline Unigene_UN_xiong_4703 & 237 & 33 UN_xiong_4703 \\
\hline Unigene_UN_xiong_10982 & 61 & 130 UN_xiong_10982 \\
\hline Unigene_UN_xiong_14256 & 110 & 60 UN_xiong_14256 \\
\hline Unigene_UN_xiong_4341 & 98 & 22 UN_xiong_4341 \\
\hline Unigene_UN_xiong_6384 & 675 & 413 UN_xiong_6384 \\
\hline Unigene_UN_xiong_3249 & 126 & 175 UN_xiong_3249 \\
\hline Unigene_GPGRCDD02GPU6P_xiong & 93 & 53 GPGRCDD02GPU6P_xion \\
\hline Unigene_UN_xiong_7599 & 270 & 55 UN_xiong_7599 \\
\hline Unigene_UN_xiong_15593 & 42 & 39 UN_xiong_15593 \\
\hline Unigene_UN_xiong_1340 & 62 & 245 UN_xiong_1340 \\
\hline Unigene_UN_xiong_1034 & 69 & 487 UN_xiong_1034 \\
\hline Unigene_UN_xiong_4612 & 181 & 271 UN_xiong_4612 \\
\hline Unigene_UN_xiong_14752 & 242 & 119 UN_xiong_14752 \\
\hline Unigene_GP8I43W03GX40W_xiong & 44 & 60 GP8I43W03GX40W_xion \\
\hline Unigene_UN_xiong_14495 & 128 & 23 UN_xiong_14495 \\
\hline Unigene_UN_xiong_15294 & 198 & 61 UN_xiong_15294 \\
\hline Unigene_UN_xiong_7923 & 54 & 30 UN_xiong_7923 \\
\hline Unigene_GP8I43W03GCOYP_xiong & 27 & 26 GP8I43W03GC0YP_xion \\
\hline Unigene_UN_xiong_4474 & 211 & 34 UN_xiong_4474 \\
\hline Unigene_UN_xiong_14315 & 126 & 328 UN_xiong_14315 \\
\hline Unigene_GR6TTJD01BTJBD_xiong & 47 & 58 GR6TTJD01BTJBD_xion \\
\hline Unigene_UN_xiong_4442 & 104 & 101 UN_xiong_4442 \\
\hline Unigene_UN_xiong_9582 & 162 & 66 UN_xiong_9582 \\
\hline Unigene_UN_xiong_9759 & 48 & 30 UN_xiong_9759 \\
\hline Unigene_GR6TTJD01A0LVD_xiong & 32 & 75 GR6TTJD01A0LVD_xion \\
\hline Unigene_UN_xiong_10199 & 40 & 31 UN_xiong_10199 \\
\hline Unigene_GPGRCDD02HKP93_xiong & 26 & 74 GPGRCDD02HKP93_xion \\
\hline Unigene_UN_xiong_2853 & 65 & 176 UN_xiong_2853 \\
\hline Unigene_UN_xiong_798 & 52 & 25 UN_xiong_798 \\
\hline Unigene_GPGRCDD02GLM7K_xiong & 23 & 43 GPGRCDD02GLM7K_xion \\
\hline Unigene_UN_xiong_13667 & 1185 & 881 UN_xiong_13667 \\
\hline Unigene_GR6TTJD01AYT26_xiong & 32 & 82 GR6TTJD01AYT26_xion \\
\hline Unigene_UN_xiong_8742 & 41 & 20 UN_xiong_8742 \\
\hline Unigene_UN_xiong_8790 & 149 & 1048 UN_xiong_8790 \\
\hline
\end{tabular}




\begin{tabular}{|c|c|c|}
\hline Unigene_UN_xiong_9353 & 55 & 71 UN_xiong_9353 \\
\hline Unigene_UN_xiong_12586 & 93 & 23 UN_xiong_12586 \\
\hline Unigene_UN_xiong_13681 & 114 & 222 UN_xiong_13681 \\
\hline Unigene_UN_xiong_15790 & 332 & 318 UN_xiong_15790 \\
\hline Unigene_UN_xiong_1883 & 30 & 21 UN_xiong_1883 \\
\hline Unigene_UN_xiong_14574 & 552 & 44 UN_xiong_14574 \\
\hline Unigene_UN_xiong_4512 & 57 & 162 UN_xiong_4512 \\
\hline Unigene_UN_xiong_8203 & 321 & 286 UN_xiong_8203 \\
\hline Unigene_GR6TTJD01CBCZZ_xiong & 34 & 173 GR6TTJD01CBCZZ_xion \\
\hline Unigene_GR6TTJD01B2KC7_xiong & 30 & 356 GR6TTJD01B2KC7_xion \\
\hline Unigene_UN_xiong_2820 & 67 & 236 UN_xiong_2820 \\
\hline Unigene_GR6TTJD01CBMCA_xiong & 52 & 57 GR6TTJD01CBMCA_xion \\
\hline Unigene GP8I43W03HAM8A xiong & 28 & 27 GP8I43W03HAM8A xion \\
\hline Unigene_UN_xiong_11696 & 102 & 175 UN_xiong_11696 \\
\hline Unigene_UN_xiong_92 & 90 & 49 UN_xiong_92 \\
\hline Unigene_GPGRCDD02G0V24_xiong & 24 & 31 GPGRCDD02G0V24_xion \\
\hline Unigene_UN_xiong_13716 & 122 & 182 UN_xiong_13716 \\
\hline Unigene_GPGRCDD02HUNA1_xiong & 23 & 26 GPGRCDD02HUNA1_xion \\
\hline Unigene_UN_xiong_12835 & 104 & 439 UN_xiong_12835 \\
\hline Unigene_GR6TTJD01BDVB9_xiong & 39 & 138 GR6TTJD01BDVB9_xion \\
\hline Unigene UN xiong 8985 & 137 & 100 UN xiong 8985 \\
\hline Unigene_GP8I43W03F7IZX_xiong & 72 & 76 GP8I43W03F7IZX_xion \\
\hline Unigene_UN_xiong_13545 & 146 & 160 UN_xiong_13545 \\
\hline Unigene_UN_xiong_14385 & 1219 & 515 UN_xiong_14385 \\
\hline Unigene_UN_xiong_15160 & 1367 & 76 UN_xiong_15160 \\
\hline Unigene_UN_xiong_5020 & 1176 & $741 \mathrm{UN}$ xiong 5020 \\
\hline Unigene_UN_xiong_443 & 397 & 68 UN_xiong_443 \\
\hline Unigene_UN_xiong_7308 & 109 & 39 UN_xiong_7308 \\
\hline Unigene_UN_xiong_7592 & 121 & 60 UN_xiong_7592 \\
\hline Unigene_UN_xiong_14313 & 831 & 762 UN_xiong_14313 \\
\hline Unigene_UN_xiong_11841 & 143 & 60 UN_xiong_11841 \\
\hline Unigene_UN_xiong_4534 & 52 & 79 UN_xiong_4534 \\
\hline Unigene_GPGRCDD02GW54J_xiong & 39 & 74 GPGRCDD02GW54J_xion \\
\hline Unigene_UN_xiong_3859 & 77 & 27 UN_xiong_3859 \\
\hline Unigene_UN_xiong_11384 & 153 & 72 UN_xiong_11384 \\
\hline Unigene_UN_xiong_3750 & 80 & 30 UN_xiong_3750 \\
\hline Unigene_UN_xiong_3070 & 75 & 1316 UN_xiong_3070 \\
\hline Unigene_GR6TTJD01AFT1C_xiong & 20 & 267 GR6TTJD01AFT1C_xion \\
\hline Unigene_GR6TTJD01BNBH6_xiong & 41 & 41 GR6TTJD01BNBH6_xion \\
\hline Unigene_GR6TTJD01B4768_xiong & 30 & 23 GR6TTJD01B4768_xion \\
\hline Unigene_GR6TTJD01APBPF_xiong & 19 & 119 GR6TTJD01APBPF_xion \\
\hline Unigene_UN_xiong_3904 & 1200 & 635 UN_xiong_3904 \\
\hline Unigene_UN_xiong_11233 & 98 & 224 UN_xiong_11233 \\
\hline Unigene_UN_xiong_8272 & 49 & 24 UN_xiong_8272 \\
\hline Unigene_GR6TTJD01B57TB_xiong & 24 & 45 GR6TTJD01B57TB_xion \\
\hline Unigene_UN_xiong_12091 & 427 & 41 UN_xiong_12091 \\
\hline Unigene_GP8I43W03FZ6G2_xiong & 54 & 192 GP8I43W03FZ6G2_xion \\
\hline Unigene_UN_xiong_772 & 239 & 117 UN_xiong_772 \\
\hline Unigene_GR6TTJD01ATUS3_xiong & 23 & 45 GR6TTJD01ATUS3_xion \\
\hline Unigene_UN_xiong_7633 & 331 & 166 UN_xiong_7633 \\
\hline
\end{tabular}




\begin{tabular}{|c|c|c|}
\hline Unigene_GR6TTJD01A7GRT_xiong & 27 & 29 GR6TTJD01A7GRT_xion \\
\hline Unigene_UN_xiong_11668 & 80 & 68 UN_xiong_11668 \\
\hline Unigene_UN_xiong_1143 & 58 & 45 UN_xiong_1143 \\
\hline Unigene_UN_xiong_14479 & 309 & 62 UN_xiong_14479 \\
\hline Unigene_UN_xiong_2463 & 108 & 117 UN_xiong_2463 \\
\hline Unigene_UN_xiong_8318 & 166 & 102 UN_xiong_8318 \\
\hline Unigene_GP8I43W03F1K0T_xiong & 69 & 42 GP8I43W03F1K0T_xion \\
\hline Unigene_GP8I43W03GAGUY_xiong & 32 & 213 GP8I43W03GAGUY_xion \\
\hline Unigene_UN_xiong_7816 & 219 & 73 UN_xiong_7816 \\
\hline Unigene_UN_xiong_15036 & 381 & 50 UN_xiong_15036 \\
\hline Unigene_UN_xiong_2202 & 206 & 172 UN_xiong_2202 \\
\hline Unigene_UN_xiong_7498 & 248 & 1303 UN_xiong_7498 \\
\hline Unigene_UN_xiong_15237 & 320 & 23 UN_xiong_15237 \\
\hline Unigene_UN_xiong_11238 & 142 & 111 UN_xiong_11238 \\
\hline Unigene_UN_xiong_11026 & 62 & 77 UN_xiong_11026 \\
\hline Unigene_UN_xiong_4922 & 100 & 58 UN_xiong_4922 \\
\hline Unigene_UN_xiong_12190 & 117 & 97 UN_xiong_12190 \\
\hline Unigene_UN_xiong_1149 & 91 & 37 UN_xiong_1149 \\
\hline Unigene_UN_xiong_9077 & 43 & 46 UN_xiong_9077 \\
\hline Unigene_UN_xiong_3157 & 214 & 1194 UN_xiong_3157 \\
\hline Unigene_UN_xiong_1334 & 52 & 29 UN_xiong_1334 \\
\hline Unigene_GR6TTJD01AVEC1_xiong & 29 & 104 GR6TTJD01AVEC1_xion \\
\hline Unigene_UN_xiong_15100 & 112 & 40 UN_xiong_15100 \\
\hline Unigene_GPGRCDD02IP43Z_xiong & 31 & 140 GPGRCDD02IP43Z_xion \\
\hline Unigene_UN_xiong_14981 & 73 & 21 UN_xiong_14981 \\
\hline Unigene UN xiong_1054 & 184 & 46 UN xiong 1054 \\
\hline Unigene_GPGRCDD02JHI10_xiong & 33 & 99 GPGRCDD02JHI10_xion \\
\hline Unigene_GP8I43W03GIN4V_xiong & 23 & 402 GP8I43W03GIN4V_xion \\
\hline Unigene_UN_xiong_333 & 1190 & 366 UN_xiong_333 \\
\hline Unigene_UN_xiong_14373 & 206 & 74 UN_xiong_14373 \\
\hline Unigene_UN_xiong_2214 & 62 & 98 UN_xiong_2214 \\
\hline Unigene_UN_xiong_13655 & 78 & 113 UN_xiong_13655 \\
\hline Unigene_UN_xiong_4561 & 272 & 31 UN_xiong_4561 \\
\hline Unigene_GP8I43W03HB86U_xiong & 47 & 65 GP8I43W03HB86U_xion \\
\hline Unigene_UN_xiong_3616 & 205 & 41 UN_xiong_3616 \\
\hline Unigene_UN_xiong_7172 & 68 & 284 UN_xiong_7172 \\
\hline Unigene_UN_xiong_3861 & 130 & 126 UN_xiong_3861 \\
\hline Unigene_GR6TTJD01BQ42Z_xiong & 31 & 41 GR6TTJD01BQ42Z_xion \\
\hline Unigene_UN_xiong_14706 & 179 & 99 UN_xiong_14706 \\
\hline Unigene_UN_xiong_1632 & 140 & 24 UN_xiong_1632 \\
\hline Unigene_UN_xiong_1733 & 48 & 23 UN_xiong_1733 \\
\hline Unigene_UN_xiong_5042 & 63 & 36 UN_xiong_5042 \\
\hline Unigene_UN_xiong_3845 & 880 & 187 UN_xiong_3845 \\
\hline Unigene_UN_xiong_5933 & 52 & 64 UN_xiong_5933 \\
\hline Unigene_UN_xiong_5431 & 94 & 127 UN_xiong_5431 \\
\hline Unigene_UN_xiong_8055 & 50 & 27 UN_xiong_8055 \\
\hline Unigene_UN_xiong_11885 & 69 & 57 UN_xiong_11885 \\
\hline Unigene_GPGRCDD02I8WD8_xiong & 23 & 143 GPGRCDD02I8WD8_xion \\
\hline Unigene_GR6TTJD01BYCSL_xiong & 31 & 24 GR6TTJD01BYCSL_xion \\
\hline Unigene_UN_xiong_362 & 323 & 194 UN_xiong_362 \\
\hline
\end{tabular}




\begin{tabular}{|c|c|c|}
\hline Unigene_GPGRCDD02JNHVE_xiong & 43 & 145 GPGRCDD02JNHVE_xion \\
\hline Unigene_GPGRCDD02GDXLM_xiong & 43 & 123 GPGRCDD02GDXLM_xion \\
\hline Unigene_UN_xiong_10770 & 190 & 143 UN_xiong_10770 \\
\hline Unigene_UN_xiong_14294 & 596 & 22 UN_xiong_14294 \\
\hline Unigene_UN_xiong_6171 & 267 & 232 UN_xiong_6171 \\
\hline Unigene_UN_xiong_12188 & 63 & 71 UN_xiong_12188 \\
\hline Unigene_UN_xiong_13283 & 255 & 154 UN_xiong_13283 \\
\hline Unigene_UN_xiong_8810 & 85 & 29 UN_xiong_8810 \\
\hline Unigene_UN_xiong_12364 & 101 & 40 UN_xiong_12364 \\
\hline Unigene_UN_xiong_791 & 217 & 66 UN_xiong_791 \\
\hline Unigene_UN_xiong_14145 & 365 & 21 UN_xiong_14145 \\
\hline Unigene_UN_xiong_13887 & 42 & 90 UN_xiong_13887 \\
\hline Unigene_UN_xiong_14228 & 673 & 1356 UN_xiong_14228 \\
\hline Unigene_UN_xiong_10262 & 102 & 21 UN_xiong_10262 \\
\hline Unigene_UN_xiong_10416 & 284 & 76 UN_xiong_10416 \\
\hline Unigene_UN_xiong_607 & 458 & 436 UN_xiong_607 \\
\hline Unigene_UN_xiong_1256 & 108 & 30 UN_xiong_1256 \\
\hline Unigene_UN_xiong_7588 & 64 & 120 UN_xiong_7588 \\
\hline Unigene_UN_xiong_1763 & 121 & 508 UN_xiong_1763 \\
\hline Unigene_UN_xiong_12413 & 312 & 102 UN_xiong_12413 \\
\hline Unigene_UN_xiong_4268 & 2602 & 4653 UN_xiong_4268 \\
\hline Unigene_UN_xiong_10908 & 62 & 62 UN_xiong_10908 \\
\hline Unigene_GPGRCDD02GYG6Q_xiong & 37 & 66 GPGRCDD02GYG6Q_xion \\
\hline Unigene_UN_xiong_9534 & 125 & 50 UN_xiong_9534 \\
\hline Unigene_UN_xiong_11724 & 40 & 41 UN_xiong_11724 \\
\hline Unigene_UN_xiong_15378 & 63 & 280 UN_xiong_15378 \\
\hline Unigene_UN_xiong_2845 & 48 & 130 UN_xiong_2845 \\
\hline Unigene_UN_xiong_7366 & 39 & 118 UN_xiong_7366 \\
\hline Unigene_UN_xiong_983 & 232 & 748 UN_xiong_983 \\
\hline Unigene_UN_xiong_15424 & 526 & 1250 UN_xiong_15424 \\
\hline Unigene_UN_xiong_3442 & 68 & 29 UN_xiong_3442 \\
\hline Unigene_GR6TTJD01BBL7C_xiong & 37 & 36 GR6TTJD01BBL7C_xion \\
\hline Unigene_UN_xiong_8116 & 260 & 126 UN_xiong_8116 \\
\hline Unigene_UN_xiong_6233 & 130 & 37 UN_xiong_6233 \\
\hline Unigene_UN_xiong_3508 & 160 & 45 UN_xiong_3508 \\
\hline Unigene_UN_xiong_7776 & 92 & 224 UN_xiong_7776 \\
\hline Unigene_UN_xiong_431 & 141 & 49 UN_xiong_431 \\
\hline Unigene_UN_xiong_3209 & 89 & 26 UN_xiong_3209 \\
\hline Unigene_UN_xiong_9415 & 218 & 24 UN_xiong_9415 \\
\hline Unigene_UN_xiong_4334 & 48 & 25 UN_xiong_4334 \\
\hline Unigene_UN_xiong_9647 & 96 & 23 UN_xiong_9647 \\
\hline Unigene_UN_xiong_14097 & 398 & 78 UN_xiong_14097 \\
\hline Unigene_UN_xiong_8852 & 186 & 130 UN_xiong_8852 \\
\hline Unigene_UN_xiong_7678 & 64 & 33 UN_xiong_7678 \\
\hline Unigene_UN_xiong_14569 & 569 & 197 UN_xiong_14569 \\
\hline Unigene_GPGRCDD02FM109_xiong & 48 & 198 GPGRCDD02FM109_xion \\
\hline Unigene_GR6TTJD01BK44I_xiong & 28 & 150 GR6TTJD01BK44I_xion \\
\hline Unigene_UN_xiong_182 & 88 & 222 UN_xiong_182 \\
\hline Unigene_GPGRCDD02HMAKQ_xiong & 40 & 75 GPGRCDD02HMAKQ_xion \\
\hline Unigene_UN_xiong_9770 & 79 & 44 UN_xiong_9770 \\
\hline
\end{tabular}




\begin{tabular}{|c|c|c|}
\hline Unigene_GPGRCDD02IRUW8_xiong & 41 & 167 GPGRCDD02IRUW8_xion \\
\hline Unigene_UN_xiong_13767 & 111 & 243 UN_xiong_13767 \\
\hline Unigene_GR6TTJD01B83UZ_xiong & 40 & 46 GR6TTJD01B83UZ_xion \\
\hline Unigene_GR6TTJD01BLHYQ_xiong & 26 & 72 GR6TTJD01BLHYQ_xion \\
\hline Unigene_UN_xiong_1061 & 131 & 214 UN_xiong_1061 \\
\hline Unigene_UN_xiong_15308 & 342 & 1419 UN_xiong_15308 \\
\hline Unigene_UN_xiong_9100 & 66 & 62 UN_xiong_9100 \\
\hline Unigene_UN_xiong_972 & 573 & 39 UN_xiong_972 \\
\hline Unigene_UN_xiong_4479 & 68 & 39 UN_xiong_4479 \\
\hline Unigene_UN_xiong_12520 & 101 & 79 UN_xiong_12520 \\
\hline Unigene_GR6TTJD01CFFHF_xiong & 29 & 28 GR6TTJD01CFFHF_xion \\
\hline Unigene_UN_xiong_14369 & 299 & 155 UN_xiong_14369 \\
\hline Unigene_UN_xiong_1732 & 135 & 57 UN_xiong_1732 \\
\hline Unigene_UN_xiong_13100 & 51 & 51 UN_xiong_13100 \\
\hline Unigene_UN_xiong_14347 & 5749 & 12920 UN_xiong_14347 \\
\hline Unigene_GR6TTJD01AMMVS_xiong & 95 & 60 GR6TTJD01AMMVS_xion \\
\hline Unigene_UN_xiong_13737 & 119 & 78 UN_xiong_13737 \\
\hline Unigene_UN_xiong_1683 & 256 & 273 UN_xiong_1683 \\
\hline Unigene_UN_xiong_13323 & 205 & 31 UN_xiong_13323 \\
\hline Unigene_UN_xiong_11953 & 87 & 144 UN_xiong_11953 \\
\hline Unigene_UN_xiong_3196 & 135 & 321 UN_xiong_3196 \\
\hline Unigene_UN_xiong_14616 & 554 & 154 UN_xiong_14616 \\
\hline Unigene_UN_xiong_2741 & 412 & 309 UN_xiong_2741 \\
\hline Unigene_GP8I43W03G0AIN_xiong & 28 & 138 GP8I43W03G0AIN_xion \\
\hline Unigene_UN_xiong_11715 & 48 & 85 UN_xiong_11715 \\
\hline Unigene_UN_xiong_15345 & 220 & 346 UN_xiong_15345 \\
\hline Unigene_UN_xiong_712 & 198 & 55 UN_xiong_712 \\
\hline Unigene_UN_xiong_10898 & 134 & 30 UN_xiong_10898 \\
\hline Unigene_UN_xiong_12729 & 159 & 318 UN_xiong_12729 \\
\hline Unigene_UN_xiong_5032 & 48 & 124 UN_xiong_5032 \\
\hline Unigene_GPGRCDD02FKN71_xiong & 51 & 47 GPGRCDD02FKN71_xion \\
\hline Unigene_UN_xiong_6548 & 164 & 34 UN_xiong_6548 \\
\hline Unigene_UN_xiong_4650 & 302 & 24 UN_xiong_4650 \\
\hline Unigene_UN_xiong_15587 & 554 & 1672 UN_xiong_15587 \\
\hline Unigene_UN_xiong_12354 & 66 & 45 UN_xiong_12354 \\
\hline Unigene_UN_xiong_8883 & 160 & 145 UN_xiong_8883 \\
\hline Unigene_UN_xiong_2453 & 91 & 61 UN_xiong_2453 \\
\hline Unigene_UN_xiong_417 & 171 & 189 UN_xiong_417 \\
\hline Unigene_GPGRCDD02G3Y6C_xiong & 24 & 58 GPGRCDD02G3Y6C_xion \\
\hline Unigene_UN_xiong_1796 & 75 & 29 UN_xiong_1796 \\
\hline Unigene_UN_xiong_15653 & 107 & 31 UN_xiong_15653 \\
\hline Unigene_UN_xiong_838 & 194 & 187 UN_xiong_838 \\
\hline Unigene_UN_xiong_3129 & 92 & 94 UN_xiong_3129 \\
\hline Unigene_UN_xiong_1758 & 115 & 105 UN_xiong_1758 \\
\hline Unigene_UN_xiong_1623 & 100 & 28 UN_xiong_1623 \\
\hline Unigene_UN_xiong_4783 & 890 & 85 UN_xiong_4783 \\
\hline Unigene_UN_xiong_7260 & 95 & 75 UN_xiong_7260 \\
\hline Unigene_GPGRCDD02JCSBE_xiong & 23 & 54 GPGRCDD02JCSBE_xion \\
\hline Unigene_UN_xiong_618 & 217 & 24 UN_xiong_618 \\
\hline Unigene_UN_xiong_9191 & 72 & 345 UN_xiong_9191 \\
\hline
\end{tabular}




\begin{tabular}{|c|c|c|}
\hline Unigene_GR6TTJD01BN60I_xiong & 28 & 41 GR6TTJD01BN60I_xion \\
\hline Unigene_UN_xiong_11971 & 77 & 117 UN_xiong_11971 \\
\hline Unigene_UN_xiong_14014 & 238 & 26 UN_xiong_14014 \\
\hline Unigene_UN_xiong_14951 & 96 & 234 UN_xiong_14951 \\
\hline Unigene_GPGRCDD02JD5KE_xiong & 30 & 26 GPGRCDD02JD5KE_xion \\
\hline Unigene_UN_xiong_451 & 157 & 273 UN_xiong_451 \\
\hline Unigene_UN_xiong_2277 & 253 & 44 UN_xiong_2277 \\
\hline Unigene_UN_xiong_4829 & 95 & 220 UN_xiong_4829 \\
\hline Unigene_UN_xiong_5502 & 59 & 53 UN_xiong_5502 \\
\hline Unigene_UN_xiong_9738 & 296 & 281 UN_xiong_9738 \\
\hline Unigene_GR6TTJD01A5EGT_xiong & 22 & 35 GR6TTJD01A5EGT_xion \\
\hline Unigene_UN_xiong_8467 & 118 & 32 UN_xiong_8467 \\
\hline Unigene_UN_xiong_14970 & 305 & 184 UN_xiong_14970 \\
\hline Unigene_GP8I43W03FSLKB_xiong & 34 & 26 GP8I43W03FSLKB_xion \\
\hline Unigene_UN_xiong_1642 & 84 & 122 UN_xiong_1642 \\
\hline Unigene_GR6TTJD01B24XD_xiong & 29 & 29 GR6TTJD01B24XD_xion \\
\hline Unigene_UN_xiong_3483 & 306 & 152 UN_xiong_3483 \\
\hline Unigene_GR6TTJD01B5I09_xiong & 21 & 95 GR6TTJD01B5I09_xion \\
\hline Unigene_UN_xiong_9546 & 1221 & 908 UN_xiong_9546 \\
\hline Unigene_UN_xiong_11308 & 370 & 969 UN_xiong_11308 \\
\hline Unigene_UN_xiong_4470 & 73 & 86 UN_xiong_4470 \\
\hline Unigene_GPGRCDD02FSYWW_xiong & 29 & 34 GPGRCDD02FSYWW_xion \\
\hline Unigene_UN_xiong_1606 & 52 & 27 UN_xiong_1606 \\
\hline Unigene_UN_xiong_1393 & 76 & 80 UN_xiong_1393 \\
\hline Unigene_UN_xiong_15533 & 152 & 792 UN_xiong_15533 \\
\hline Unigene_UN_xiong_15467 & 93 & 102 UN_xiong_15467 \\
\hline Unigene_UN_xiong_4744 & 201 & 25 UN_xiong_4744 \\
\hline Unigene_GR6TTJD01APL5S_xiong & 19 & 77 GR6TTJD01APL5S_xion \\
\hline Unigene_UN_xiong_6149 & 85 & 126 UN_xiong_6149 \\
\hline Unigene_UN_xiong_2523 & 667 & 189 UN_xiong_2523 \\
\hline Unigene_GR6TTJD01AYJF4_xiong & 40 & 28 GR6TTJD01AYJF4_xion \\
\hline Unigene_UN_xiong_4645 & 91 & 217 UN_xiong_4645 \\
\hline Unigene_UN_xiong_11673 & 361 & 53 UN_xiong_11673 \\
\hline Unigene_UN_xiong_1612 & 67 & 31 UN_xiong_1612 \\
\hline Unigene_UN_xiong_15490 & 336 & 455 UN_xiong_15490 \\
\hline Unigene_GR6TTJD01AR7AL_xiong & 22 & 169 GR6TTJD01AR7AL_xion \\
\hline Unigene_UN_xiong_10563 & 598 & 410 UN_xiong_10563 \\
\hline Unigene_UN_xiong_4279 & 134 & 33 UN_xiong_4279 \\
\hline Unigene_GPGRCDD02GIYZZ_xiong & 20 & 70 GPGRCDD02GIYZZ_xion \\
\hline Unigene_UN_xiong_14818 & 385 & 28 UN_xiong_14818 \\
\hline Unigene_GR6TTJD01BWA7V_xiong & 34 & 33 GR6TTJD01BWA7V_xion \\
\hline Unigene_UN_xiong_15111 & 97 & 63 UN_xiong_15111 \\
\hline Unigene_UN_xiong_5987 & 36 & 108 UN_xiong_5987 \\
\hline Unigene_UN_xiong_3219 & 97 & 77 UN_xiong_3219 \\
\hline Unigene_UN_xiong_1345 & 131 & 65 UN_xiong_1345 \\
\hline Unigene_UN_xiong_15780 & 133 & 51 UN_xiong_15780 \\
\hline Unigene_GPGRCDD02I2AIL_xiong & 62 & 182 GPGRCDD02I2AIL_xion \\
\hline Unigene_UN_xiong_5280 & 134 & 438 UN_xiong_5280 \\
\hline Unigene_GR6TTJD01BNN3K_xiong & 21 & 39 GR6TTJD01BNN3K_xion \\
\hline Unigene_UN_xiong_4473 & 317 & 77 UN_xiong_4473 \\
\hline
\end{tabular}




\begin{tabular}{|c|c|c|}
\hline Unigene_GR6TTJD01BL1SZ_xiong & 24 & 86 GR6TTJD01BL1SZ_xion \\
\hline Unigene_UN_xiong_4259 & 38 & 87 UN_xiong_4259 \\
\hline Unigene_GR6TTJD01B5FDX_xiong & 76 & 67 GR6TTJD01B5FDX_xion \\
\hline Unigene_GR6TTJD01BN7AF_xiong & 67 & 41 GR6TTJD01BN7AF_xion \\
\hline Unigene_UN_xiong_8350 & 76 & 33 UN_xiong_8350 \\
\hline Unigene_UN_xiong_11672 & 202 & 306 UN_xiong_11672 \\
\hline Unigene_UN_xiong_10934 & 130 & 113 UN_xiong_10934 \\
\hline Unigene_UN_xiong_1587 & 157 & 51 UN_xiong_1587 \\
\hline Unigene_GP8I43W03F4756_xiong & 43 & 52 GP8I43W03F4756_xion \\
\hline Unigene_UN_xiong_14689 & 537 & 168 UN_xiong_14689 \\
\hline Unigene_UN_xiong_5724 & 91 & 43 UN_xiong_5724 \\
\hline Unigene_UN_xiong_8380 & 121 & 194 UN_xiong_8380 \\
\hline Unigene_UN_xiong_7971 & 111 & 42 UN_xiong_7971 \\
\hline Unigene_UN_xiong_15284 & 70 & 102 UN_xiong_15284 \\
\hline Unigene_UN_xiong_11942 & 81 & 702 UN_xiong_11942 \\
\hline Unigene_UN_xiong_2196 & 52 & 34 UN_xiong_2196 \\
\hline Unigene_UN_xiong_14795 & 585 & 1784 UN_xiong_14795 \\
\hline Unigene_GPGRCDD02GKEGT_xiong & 79 & 98 GPGRCDD02GKEGT_xion \\
\hline Unigene_GPGRCDD02HG2XQ_xiong & 28 & 30 GPGRCDD02HG2XQ_xion \\
\hline Unigene_UN_xiong_1044 & 86 & 85 UN_xiong_1044 \\
\hline Unigene_UN_xiong_3056 & 569 & 98 UN_xiong_3056 \\
\hline Unigene_UN_xiong_10730 & 109 & 37 UN_xiong_10730 \\
\hline Unigene_UN_xiong_6228 & 1070 & 328 UN_xiong_6228 \\
\hline Unigene_UN_xiong_13 & 222 & 289 UN_xiong_13 \\
\hline Unigene_UN_xiong_6354 & 64 & 131 UN_xiong_6354 \\
\hline Unigene_UN_xiong_2414 & 114 & 184 UN_xiong_2414 \\
\hline Unigene_UN_xiong_5064 & 62 & 184 UN_xiong_5064 \\
\hline Unigene_GR6TTJD01BJ5EP_xiong & 32 & 46 GR6TTJD01BJ5EP_xion \\
\hline Unigene_UN_xiong_10700 & 139 & 110 UN_xiong_10700 \\
\hline Unigene_UN_xiong_8934 & 427 & 40 UN_xiong_8934 \\
\hline Unigene_GR6TTJD01CD8EU_xiong & 33 & 23 GR6TTJD01CD8EU_xion \\
\hline Unigene_UN_xiong_2965 & 129 & 75 UN_xiong_2965 \\
\hline Unigene_GP8I43W03F59B8_xiong & 40 & 267 GP8I43W03F59B8_xion \\
\hline Unigene_GR6TTJD01BALZE_xiong & 29 & 23 GR6TTJD01BALZE_xion \\
\hline Unigene_UN_xiong_214 & 50 & 41 UN_xiong_214 \\
\hline Unigene_UN_xiong_2881 & 149 & 28 UN_xiong_2881 \\
\hline Unigene_GR6TTJD01ASQNP_xiong & 21 & 60 GR6TTJD01ASQNP_xion \\
\hline Unigene_GR6TTJD01BLM2T_xiong & 45 & 27 GR6TTJD01BLM2T_xion \\
\hline Unigene_GPGRCDD02FPV81_xiong & 48 & 30 GPGRCDD02FPV81_xion \\
\hline Unigene_UN_xiong_10037 & 44 & 64 UN_xiong_10037 \\
\hline Unigene_UN_xiong_9494 & 964 & 367 UN_xiong_9494 \\
\hline Unigene_UN_xiong_7713 & 84 & 43 UN_xiong_7713 \\
\hline Unigene_UN_xiong_4146 & 123 & 222 UN_xiong_4146 \\
\hline Unigene_UN_xiong_12325 & 239 & 237 UN_xiong_12325 \\
\hline Unigene_UN_xiong_10341 & 141 & 30 UN_xiong_10341 \\
\hline Unigene_UN_xiong_12798 & 159 & 90 UN_xiong_12798 \\
\hline Unigene_UN_xiong_13936 & 169 & 68 UN_xiong_13936 \\
\hline Unigene_UN_xiong_5370 & 479 & 180 UN_xiong_5370 \\
\hline Unigene_GR6TTJD01BK6NR_xiong & 63 & 64 GR6TTJD01BK6NR_xion \\
\hline Unigene_UN_xiong_3169 & 373 & 635 UN_xiong_3169 \\
\hline
\end{tabular}




\begin{tabular}{|c|c|c|}
\hline Unigene_UN_xiong_4019 & 114 & 37 UN_xiong_4019 \\
\hline Unigene_UN_xiong_13280 & 156 & 37 UN_xiong_13280 \\
\hline Unigene_GPGRCDD02IVX64_xiong & 50 & 54 GPGRCDD02IVX64_xion \\
\hline Unigene_UN_xiong_10444 & 110 & 60 UN_xiong_10444 \\
\hline Unigene_GR6TTJD01AMJH7_xiong & 25 & 28 GR6TTJD01AMJH7_xion \\
\hline Unigene_UN_xiong_12628 & 747 & 69 UN_xiong_12628 \\
\hline Unigene_GP8I43W03G0RUQ_xiong & 21 & 25 GP8I43W03G0RUQ_xion \\
\hline Unigene_UN_xiong_4109 & 884 & 535 UN_xiong_4109 \\
\hline Unigene_UN_xiong_1302 & 104 & 66 UN_xiong_1302 \\
\hline Unigene_UN_xiong_1547 & 47 & 247 UN_xiong_1547 \\
\hline Unigene_UN_xiong_11779 & 30 & 25 UN_xiong_11779 \\
\hline Unigene_UN_xiong_8986 & 91 & 24 UN_xiong_8986 \\
\hline Unigene_GP8I43W03GLAF8_xiong & 31 & 40 GP8I43W03GLAF8_xion \\
\hline Unigene_UN_xiong_10850 & 53 & 176 UN_xiong_10850 \\
\hline Unigene_GP8I43W03GQD39_xiong & 59 & 117 GP8I43W03GQD39_xion \\
\hline Unigene_UN_xiong_13461 & 80 & 45 UN_xiong_13461 \\
\hline Unigene_UN_xiong_11820 & 78 & 21 UN_xiong_11820 \\
\hline Unigene_UN_xiong_4693 & 66 & 209 UN_xiong_4693 \\
\hline Unigene_UN_xiong_3569 & 73 & 35 UN_xiong_3569 \\
\hline Unigene_UN_xiong_4712 & 632 & 2997 UN_xiong_4712 \\
\hline Unigene_UN_xiong_15162 & 500 & 364 UN_xiong_15162 \\
\hline Unigene_UN_xiong_2213 & 449 & 110 UN_xiong_2213 \\
\hline Unigene_UN_xiong_2985 & 56 & 208 UN_xiong_2985 \\
\hline Unigene_UN_xiong_2558 & 140 & 24 UN_xiong_2558 \\
\hline Unigene_UN_xiong_2209 & 518 & 525 UN_xiong_2209 \\
\hline Unigene_UN_xiong_3541 & 142 & 31 UN xiong 3541 \\
\hline Unigene_GR6TTJD01BTPZ1_xiong & 50 & 34 GR6TTJD01BTPZ1_xion \\
\hline Unigene_UN_xiong_8892 & 130 & 112 UN_xiong_8892 \\
\hline Unigene_UN_xiong_9889 & 76 & 89 UN_xiong_9889 \\
\hline Unigene_UN_xiong_10073 & 58 & 96 UN_xiong_10073 \\
\hline Unigene_GP8I43W03F0K0J_xiong & 46 & 27 GP8I43W03F0K0J_xion \\
\hline Unigene_GR6TTJD01B3EW8_xiong & 21 & 59 GR6TTJD01B3EW8_xion \\
\hline Unigene_UN_xiong_9051 & 66 & 63 UN_xiong_9051 \\
\hline Unigene_UN_xiong_7355 & 120 & 54 UN_xiong_7355 \\
\hline Unigene_UN_xiong_3741 & 2134 & 3893 UN_xiong_3741 \\
\hline Unigene_UN_xiong_10681 & 164 & 73 UN_xiong_10681 \\
\hline Unigene_UN_xiong_1605 & 50 & 46 UN_xiong_1605 \\
\hline Unigene_GPGRCDD02F4ZA0_xiong & 30 & 36 GPGRCDD02F4ZA0_xion \\
\hline Unigene_UN_xiong_10826 & 172 & 23 UN_xiong_10826 \\
\hline Unigene_GP8I43W03GMNH6_xiong & 70 & 645 GP8I43W03GMNH6_xion \\
\hline Unigene_UN_xiong_1363 & 80 & 29 UN_xiong_1363 \\
\hline Unigene_GR6TTJD01BUX8M_xiong & 20 & 38 GR6TTJD01BUX8M_xion \\
\hline Unigene_UN_xiong_3230 & 181 & 221 UN_xiong_3230 \\
\hline Unigene_UN_xiong_1298 & 246 & 51 UN_xiong_1298 \\
\hline Unigene_UN_xiong_12124 & 51 & 45 UN_xiong_12124 \\
\hline Unigene_GPGRCDD02G6HMN_xiong & 27 & 25 GPGRCDD02G6HMN_xion \\
\hline Unigene_GR6TTJD01B9G93_xiong & 45 & 56 GR6TTJD01B9G93_xion \\
\hline Unigene_UN_xiong_4091 & 1332 & 68944 UN_xiong_4091 \\
\hline Unigene_GPGRCDD02JTMIX_xiong & 35 & 2434 GPGRCDD02JTMIX_xion \\
\hline Unigene_UN_xiong_4030 & 184 & 326 UN_xiong_4030 \\
\hline
\end{tabular}




\begin{tabular}{|c|c|c|}
\hline Unigene_UN_xiong_11880 & 320 & 84 UN_xiong_11880 \\
\hline Unigene_GR6TTJD01CAR27_xiong & 44 & 33 GR6TTJD01CAR27_xion \\
\hline Unigene_UN_xiong_4083 & 157 & 66 UN_xiong_4083 \\
\hline Unigene_UN_xiong_5147 & 312 & 169 UN_xiong_5147 \\
\hline Unigene_GPGRCDD02JERW6_xiong & 95 & 91 GPGRCDD02JERW6_xion \\
\hline Unigene_UN_xiong_2184 & 56 & 32 UN_xiong_2184 \\
\hline Unigene_UN_xiong_9430 & 122 & 96 UN_xiong_9430 \\
\hline Unigene_GP8I43W03FSSYJ_xiong & 26 & 243 GP8I43W03FSSYJ_xion \\
\hline Unigene_UN_xiong_8777 & 40 & 127 UN_xiong_8777 \\
\hline Unigene_UN_xiong_14773 & 105 & 50 UN_xiong_14773 \\
\hline Unigene_GPGRCDD02GTTTE_xiong & 31 & 34 GPGRCDD02GTTTE_xion \\
\hline Unigene_UN_xiong_13067 & 113 & 56 UN_xiong_13067 \\
\hline Unigene_GR6TTJD01BKTUV_xiong & 23 & 29 GR6TTJD01BKTUV_xion \\
\hline Unigene_UN_xiong_5810 & 127 & 31 UN_xiong_5810 \\
\hline Unigene_GR6TTJD01ATW7H_xiong & 53 & 29 GR6TTJD01ATW7H_xion \\
\hline Unigene_UN_xiong_4222 & 70 & 24 UN_xiong_4222 \\
\hline Unigene_UN_xiong_10169 & 154 & 113 UN_xiong_10169 \\
\hline Unigene_UN_xiong_15018 & 86 & 38 UN_xiong_15018 \\
\hline Unigene_UN_xiong_3523 & 4254 & 1236 UN_xiong_3523 \\
\hline Unigene_GP8I43W03HF5BV_xiong & 39 & 27 GP8I43W03HF5BV_xion \\
\hline Unigene_UN_xiong_14998 & 274 & 85 UN_xiong_14998 \\
\hline Unigene_UN_xiong_7290 & 61 & 26 UN_xiong_7290 \\
\hline Unigene_UN_xiong_14735 & 76 & 233 UN_xiong_14735 \\
\hline Unigene_UN_xiong_8866 & 80 & 45 UN_xiong_8866 \\
\hline Unigene_GPGRCDD02IM547_xiong & 35 & 71 GPGRCDD02IM547_xion \\
\hline Unigene_UN_xiong_9503 & 115 & 275 UN_xiong_9503 \\
\hline Unigene_GR6TTJD01CCR6M_xiong & 31 & 26 GR6TTJD01CCR6M_xion \\
\hline Unigene_UN_xiong_14705 & 422 & 124 UN_xiong_14705 \\
\hline Unigene_UN_xiong_2084 & 72 & 28 UN_xiong_2084 \\
\hline Unigene_UN_xiong_3872 & 194 & 52 UN_xiong_3872 \\
\hline Unigene_UN_xiong_11639 & 130 & 189 UN_xiong_11639 \\
\hline Unigene_UN_xiong_14106 & 234 & 149 UN_xiong_14106 \\
\hline Unigene_UN_xiong_5614 & 226 & 383 UN_xiong_5614 \\
\hline Unigene_UN_xiong_14942 & 111 & 97 UN_xiong_14942 \\
\hline Unigene_UN_xiong_4230 & 97 & 548 UN_xiong_4230 \\
\hline Unigene_GPGRCDD02F5TSW_xiong & 25 & 26 GPGRCDD02F5TSW_xion \\
\hline Unigene_UN_xiong_531 & 94 & 963 UN_xiong_531 \\
\hline Unigene_UN_xiong_1411 & 131 & 62 UN_xiong_1411 \\
\hline Unigene_UN_xiong_5539 & 107 & 22 UN_xiong_5539 \\
\hline Unigene_UN_xiong_6529 & 816 & 159 UN_xiong_6529 \\
\hline Unigene_UN_xiong_15196 & 44 & 459 UN_xiong_15196 \\
\hline Unigene_UN_xiong_441 & 140 & 53 UN_xiong_441 \\
\hline Unigene_UN_xiong_9851 & 199 & 47 UN_xiong_9851 \\
\hline Unigene_UN_xiong_14868 & 155 & 3621 UN_xiong_14868 \\
\hline Unigene_UN_xiong_9692 & 80 & 78 UN_xiong_9692 \\
\hline Unigene_UN_xiong_3390 & 3494 & 3184 UN_xiong_3390 \\
\hline Unigene_UN_xiong_9629 & 128 & 81 UN_xiong_9629 \\
\hline Unigene_UN_xiong_3436 & 467 & 243 UN_xiong_3436 \\
\hline Unigene_UN_xiong_12460 & 58 & 56 UN_xiong_12460 \\
\hline Unigene_UN_xiong_3701 & 36 & 287 UN_xiong_3701 \\
\hline
\end{tabular}




\begin{tabular}{|c|c|c|}
\hline Unigene_UN_xiong_9243 & 787 & 764 UN_xiong_9243 \\
\hline Unigene_UN_xiong_12305 & 85 & 97 UN_xiong_12305 \\
\hline Unigene_GR6TTJD01ASJGP_xiong & 53 & 64 GR6TTJD01ASJGP_xion \\
\hline Unigene_UN_xiong_9316 & 679 & 433 UN_xiong_9316 \\
\hline Unigene_UN_xiong_11520 & 46 & 92 UN_xiong_11520 \\
\hline Unigene_GP8I43W03F1BHI_xiong & 39 & 41 GP8I43W03F1BHI_xion \\
\hline Unigene_UN_xiong_8310 & 53 & 46 UN_xiong_8310 \\
\hline Unigene_GR6TTJD01A90AP_xiong & 90 & 57 GR6TTJD01A90AP_xion \\
\hline Unigene_UN_xiong_1260 & 92 & 25 UN_xiong_1260 \\
\hline Unigene_UN_xiong_12656 & 173 & 94 UN_xiong_12656 \\
\hline Unigene_UN_xiong_14490 & 689 & 418 UN_xiong_14490 \\
\hline Unigene_GR6TTJD01AQ1L1_xiong & 74 & 62 GR6TTJD01AQ1L1_xion \\
\hline Unigene_UN_xiong_12048 & 91 & 43 UN_xiong_12048 \\
\hline Unigene_UN_xiong_3725 & 231 & 21 UN_xiong_3725 \\
\hline Unigene_UN_xiong_15326 & 113 & 80 UN_xiong_15326 \\
\hline Unigene_UN_xiong_10638 & 91 & 160 UN_xiong_10638 \\
\hline Unigene_UN_xiong_1314 & 280 & 33 UN_xiong_1314 \\
\hline Unigene_UN_xiong_15278 & 208 & 205 UN_xiong_15278 \\
\hline Unigene_UN_xiong_4453 & 166 & 808 UN_xiong_4453 \\
\hline Unigene_UN_xiong_6503 & 61 & 128 UN_xiong_6503 \\
\hline Unigene_GP8I43W03GBUEQ_xiong & 53 & 44 GP8I43W03GBUEQ_xion \\
\hline Unigene_UN_xiong_15358 & 206 & 78 UN_xiong_15358 \\
\hline Unigene_GR6TTJD01BL5W4_xiong & 25 & 83 GR6TTJD01BL5W4_xion \\
\hline Unigene_UN_xiong_793 & 830 & 42 UN_xiong_793 \\
\hline Unigene_UN_xiong_5305 & 96 & 417 UN_xiong_5305 \\
\hline Unigene_GR6TTJD01BMP0H_xiong & 40 & 78 GR6TTJD01BMP0H_xion \\
\hline Unigene_UN_xiong_3600 & 176 & 778 UN_xiong_3600 \\
\hline Unigene_UN_xiong_4555 & 50 & 119 UN_xiong_4555 \\
\hline Unigene_UN_xiong_3369 & 1784 & 2997 UN_xiong_3369 \\
\hline Unigene_UN_xiong_15039 & 373 & 256 UN_xiong_15039 \\
\hline Unigene_UN_xiong_14751 & 157 & 42 UN_xiong_14751 \\
\hline Unigene_GPGRCDD02G9MGD_xiong & 35 & 40 GPGRCDD02G9MGD_xion \\
\hline Unigene_GP8I43W03F5P80_xiong & 37 & 61 GP8I43W03F5P80_xion \\
\hline Unigene_UN_xiong_6806 & 144 & 95 UN_xiong_6806 \\
\hline Unigene_GP8I43W03GDCJH_xiong & 33 & 36 GP8I43W03GDCJH_xion \\
\hline Unigene_GR6TTJD01CBUIF_xiong & 31 & 64 GR6TTJD01CBUIF_xion \\
\hline Unigene_UN_xiong_2047 & 77 & 239 UN_xiong_2047 \\
\hline Unigene_GPGRCDD02JR211_xiong & 54 & 54 GPGRCDD02JR211_xion \\
\hline Unigene_UN_xiong_5107 & 175 & 187 UN_xiong_5107 \\
\hline Unigene_UN_xiong_11755 & 277 & 589 UN_xiong_11755 \\
\hline Unigene_UN_xiong_1279 & 315 & 160 UN_xiong_1279 \\
\hline Unigene_UN_xiong_5675 & 41 & 230 UN_xiong_5675 \\
\hline Unigene_UN_xiong_13077 & 64 & 317 UN_xiong_13077 \\
\hline Unigene_UN_xiong_11629 & 67 & 56 UN_xiong_11629 \\
\hline Unigene_UN_xiong_7381 & 87 & 167 UN_xiong_7381 \\
\hline Unigene_UN_xiong_9972 & 109 & 269 UN_xiong_9972 \\
\hline Unigene_UN_xiong_7929 & 53 & 200 UN_xiong_7929 \\
\hline Unigene_GP8I43W03FYJLN_xiong & 66 & 130 GP8I43W03FYJLN_xion \\
\hline Unigene_UN_xiong_9905 & 77 & 120 UN_xiong_9905 \\
\hline Unigene_GR6TTJD01BY31P_xiong & 23 & 37 GR6TTJD01BY31P_xion \\
\hline
\end{tabular}




\begin{tabular}{|c|c|c|}
\hline Unigene_UN_xiong_1685 & 741 & 399 UN_xiong_1685 \\
\hline Unigene_UN_xiong_8061 & 58 & 39 UN_xiong_8061 \\
\hline Unigene_UN_xiong_14884 & 242 & 89 UN_xiong_14884 \\
\hline Unigene_UN_xiong_4863 & 60 & 51 UN_xiong_4863 \\
\hline Unigene_UN_xiong_290 & 2121 & 317 UN_xiong_290 \\
\hline Unigene_UN_xiong_13126 & 59 & 75 UN_xiong_13126 \\
\hline Unigene_UN_xiong_11968 & 51 & 207 UN_xiong_11968 \\
\hline Unigene_UN_xiong_4639 & 99 & 338 UN_xiong_4639 \\
\hline Unigene_UN_xiong_7384 & 57 & 159 UN_xiong_7384 \\
\hline Unigene_GR6TTJD01CFI69_xiong & 92 & 87 GR6TTJD01CFI69_xion \\
\hline Unigene_UN_xiong_2681 & 91 & 80 UN_xiong_2681 \\
\hline Unigene_UN_xiong_13598 & 602 & 153 UN_xiong_13598 \\
\hline Unigene_UN_xiong_11102 & 55 & 52 UN_xiong_11102 \\
\hline Unigene_GR6TTJD01BCSL5_xiong & 23 & 28 GR6TTJD01BCSL5_xion \\
\hline Unigene_UN_xiong_12157 & 140 & 35 UN_xiong_12157 \\
\hline Unigene_UN_xiong_6626 & 101 & 34 UN_xiong_6626 \\
\hline Unigene_UN_xiong_9661 & 63 & 99 UN_xiong_9661 \\
\hline Unigene_UN_xiong_3250 & 71 & 36 UN_xiong_3250 \\
\hline Unigene_UN_xiong_4812 & 78 & 321 UN_xiong_4812 \\
\hline Unigene_UN_xiong_3940 & 229 & 46 UN_xiong_3940 \\
\hline Unigene_UN_xiong_4987 & 126 & 81 UN_xiong_4987 \\
\hline Unigene_GR6TTJD01AW090_xiong & 21 & 63 GR6TTJD01AW090_xion \\
\hline Unigene_GR6TTJD01BDQZ4_xiong & 30 & 19 GR6TTJD01BDQZ4_xion \\
\hline Unigene_UN_xiong_13806 & 107 & 105 UN_xiong_13806 \\
\hline Unigene_UN_xiong_10618 & 62 & 22 UN_xiong_10618 \\
\hline Unigene_UN_xiong_8729 & 86 & 25 UN_xiong_8729 \\
\hline Unigene_GPGRCDD02JQL07_xiong & 40 & 197 GPGRCDD02JQL07_xion \\
\hline Unigene_UN_xiong_3517 & 75 & 23 UN_xiong_3517 \\
\hline Unigene_GR6TTJD01BZIOR_xiong & 27 & 109 GR6TTJD01BZI0R_xion \\
\hline Unigene_UN_xiong_8049 & 761 & 83 UN_xiong_8049 \\
\hline Unigene_UN_xiong_12449 & 59 & 47 UN_xiong_12449 \\
\hline Unigene_GPGRCDD02IODPP_xiong & 88 & 50 GPGRCDD02IODPP_xion \\
\hline Unigene_GR6TTJD01AWLNN_xiong & 100 & 67 GR6TTJD01AWLNN_xion \\
\hline Unigene_UN_xiong_10524 & 94 & 29 UN_xiong_10524 \\
\hline Unigene_GR6TTJD01AHDSQ_xiong & 27 & 58 GR6TTJD01AHDSQ_xion \\
\hline Unigene_UN_xiong_9781 & 230 & 30 UN_xiong_9781 \\
\hline Unigene_UN_xiong_8376 & 224 & 381 UN_xiong_8376 \\
\hline Unigene_GPGRCDD02JJRMX_xiong & 21 & 62 GPGRCDD02JJRMX_xion \\
\hline Unigene_GPGRCDD02F7EAG_xiong & 27 & 31 GPGRCDD02F7EAG_xion \\
\hline Unigene_UN_xiong_9775 & 308 & 42 UN_xiong_9775 \\
\hline Unigene_GR6TTJD01AYSN3_xiong & 59 & 163 GR6TTJD01AYSN3_xion \\
\hline Unigene_UN_xiong_10541 & 117 & 175 UN_xiong_10541 \\
\hline Unigene_GR6TTJD01B713X_xiong & 48 & 48 GR6TTJD01B713X_xion \\
\hline Unigene_UN_xiong_10103 & 296 & 364 UN_xiong_10103 \\
\hline Unigene_UN_xiong_14508 & 1374 & 155 UN_xiong_14508 \\
\hline Unigene_UN_xiong_3947 & 122 & 80 UN_xiong_3947 \\
\hline Unigene_UN_xiong_14051 & 60 & 28 UN_xiong_14051 \\
\hline Unigene_UN_xiong_11645 & 524 & 165 UN_xiong_11645 \\
\hline Unigene_UN_xiong_3763 & 169 & 148 UN_xiong_3763 \\
\hline Unigene_UN_xiong_15787 & 559 & 1291 UN_xiong_15787 \\
\hline
\end{tabular}




\begin{tabular}{|c|c|c|}
\hline Unigene_UN_xiong_5440 & 36 & 23 UN_xiong_5440 \\
\hline Unigene_GR6TTJD01BGZ4A_xiong & 22 & 102 GR6TTJD01BGZ4A_xion \\
\hline Unigene_UN_xiong_10202 & 55 & 35 UN_xiong_10202 \\
\hline Unigene_GP8I43W03G2HFS_xiong & 32 & 34 GP8I43W03G2HFS_xion \\
\hline Unigene_UN_xiong_3756 & 43 & 23 UN_xiong_3756 \\
\hline Unigene_UN_xiong_5787 & 82 & 99 UN_xiong_5787 \\
\hline Unigene_UN_xiong_4726 & 941 & 1647 UN_xiong_4726 \\
\hline Unigene_UN_xiong_14272 & 233 & 4341 UN_xiong_14272 \\
\hline Unigene_UN_xiong_2828 & 84 & 111 UN_xiong_2828 \\
\hline Unigene_UN_xiong_5653 & 197 & 100 UN_xiong_5653 \\
\hline Unigene_UN_xiong_6466 & 192 & 52 UN_xiong_6466 \\
\hline Unigene_UN_xiong_7670 & 612 & 118 UN_xiong_7670 \\
\hline Unigene_UN_xiong_14618 & 1356 & 4284 UN_xiong_14618 \\
\hline Unigene_UN_xiong_12552 & 110 & 587 UN_xiong_12552 \\
\hline Unigene_GPGRCDD02I032M_xiong & 50 & 46 GPGRCDD02I032M_xion \\
\hline Unigene_GP8I43W03F49KU_xiong & 54 & 111 GP8I43W03F49KU_xion \\
\hline Unigene_UN_xiong_5161 & 205 & 289 UN_xiong_5161 \\
\hline Unigene_UN_xiong_10858 & 75 & 25 UN_xiong_10858 \\
\hline Unigene_UN_xiong_9674 & 114 & 58 UN_xiong_9674 \\
\hline Unigene_UN_xiong_9031 & 122 & 20 UN_xiong_9031 \\
\hline Unigene_GPGRCDD02I7E28_xiong & 55 & 56 GPGRCDD02I7E28_xion \\
\hline Unigene_UN_xiong_10337 & 92 & 227 UN_xiong_10337 \\
\hline Unigene_GR6TTJD01A00Z2_xiong & 28 & 43 GR6TTJD01A00Z2_xion \\
\hline Unigene_UN_xiong_13466 & 324 & 32 UN_xiong_13466 \\
\hline Unigene_UN_xiong_14964 & 256 & 537 UN_xiong_14964 \\
\hline Unigene_UN_xiong_4660 & 75 & 186 UN_xiong_4660 \\
\hline Unigene_GPGRCDD02H46VH_xiong & 26 & 35 GPGRCDD02H46VH_xion \\
\hline Unigene_GP8I43W03FUD0H_xiong & 27 & 21 GP8I43W03FUD0H_xion \\
\hline Unigene_GR6TTJD01BF4WE_xiong & 20 & 74 GR6TTJD01BF4WE_xion \\
\hline Unigene_GPGRCDD02GNNK3_xiong & 47 & 76 GPGRCDD02GNNK3_xion \\
\hline Unigene_GR6TTJD01AJP8S_xiong & 30 & 66 GR6TTJD01AJP8S_xion \\
\hline Unigene_UN_xiong_7560 & 186 & 36 UN_xiong_7560 \\
\hline Unigene_UN_xiong_10824 & 106 & 24 UN_xiong_10824 \\
\hline Unigene_UN_xiong_4919 & 53 & 76 UN_xiong_4919 \\
\hline Unigene_UN_xiong_5946 & 80 & 29 UN_xiong_5946 \\
\hline Unigene_UN_xiong_10417 & 105 & 198 UN_xiong_10417 \\
\hline Unigene_GPGRCDD02ID5XW_xiong & 28 & 34 GPGRCDD02ID5XW_xion \\
\hline Unigene_GP8I43W03HE54E_xiong & 24 & 42 GP8I43W03HE54E_xion \\
\hline Unigene_GPGRCDD02IYB9I_xiong & 89 & 211 GPGRCDD02IYB9I_xion \\
\hline Unigene_UN_xiong_13364 & 86 & 26 UN_xiong_13364 \\
\hline Unigene_UN_xiong_14889 & 714 & 135 UN_xiong_14889 \\
\hline Unigene_UN_xiong_631 & 91 & 417 UN_xiong_631 \\
\hline Unigene_GPGRCDD02F8AFJ_xiong & 56 & 53 GPGRCDD02F8AFJ_xion \\
\hline Unigene_UN_xiong_8687 & 302 & 327 UN_xiong_8687 \\
\hline Unigene_UN_xiong_13831 & 103 & 52 UN_xiong_13831 \\
\hline Unigene_UN_xiong_242 & 185 & 48 UN_xiong_242 \\
\hline Unigene_UN_xiong_12602 & 57 & 20 UN_xiong_12602 \\
\hline Unigene_UN_xiong_10293 & 60 & 298 UN_xiong_10293 \\
\hline Unigene_UN_xiong_14372 & 102 & 37 UN_xiong_14372 \\
\hline Unigene_GPGRCDD02I2HQW_xiong & 30 & 33 GPGRCDD02I2HQW_xion \\
\hline
\end{tabular}




\begin{tabular}{|c|c|c|}
\hline Unigene_GR6TTJD01A1QLE_xiong & 24 & 130 GR6TTJD01A1QLE_xion \\
\hline Unigene_UN_xiong_4643 & 279 & 1417 UN_xiong_4643 \\
\hline Unigene_UN_xiong_13886 & 79 & 25 UN_xiong_13886 \\
\hline Unigene_GP8I43W03GWLVR_xiong & 72 & 67 GP8I43W03GWLVR_xion \\
\hline Unigene_UN_xiong_14647 & 110 & 80 UN_xiong_14647 \\
\hline Unigene_UN_xiong_1840 & 158 & 22 UN_xiong_1840 \\
\hline Unigene_UN_xiong_11165 & 59 & 26 UN_xiong_11165 \\
\hline Unigene_GR6TTJD01BLA3X_xiong & 26 & 24 GR6TTJD01BLA3X_xion \\
\hline Unigene_UN_xiong_2344 & 54 & 414 UN_xiong_2344 \\
\hline Unigene_UN_xiong_11660 & 117 & 293 UN_xiong_11660 \\
\hline Unigene_UN_xiong_15556 & 2968 & 1647 UN_xiong_15556 \\
\hline Unigene_GPGRCDD02HGKBO_xiong & 28 & 898 GPGRCDD02HGKBO_xion \\
\hline Unigene_GR6TTJD01CDXM4_xiong & 26 & 23 GR6TTJD01CDXM4_xion \\
\hline Unigene_UN_xiong_10954 & 248 & 31 UN_xiong_10954 \\
\hline Unigene_UN_xiong_9659 & 53 & 61 UN_xiong_9659 \\
\hline Unigene_GR6TTJD01A21DM_xiong & 22 & 45 GR6TTJD01A21DM_xion \\
\hline Unigene_UN_xiong_14952 & 139 & 100 UN_xiong_14952 \\
\hline Unigene_UN_xiong_10309 & 157 & 354 UN_xiong_10309 \\
\hline Unigene_UN_xiong_4237 & 6655 & 326 UN_xiong_4237 \\
\hline Unigene_UN_xiong_6056 & 120 & 23 UN_xiong_6056 \\
\hline Unigene_UN_xiong_11796 & 83 & 67 UN_xiong_11796 \\
\hline Unigene_UN_xiong_2290 & 158 & 58 UN_xiong_2290 \\
\hline Unigene_UN_xiong_7639 & 75 & 112 UN_xiong_7639 \\
\hline Unigene_UN_xiong_2696 & 95 & 30 UN_xiong_2696 \\
\hline Unigene_UN_xiong_7589 & 299 & 194 UN_xiong_7589 \\
\hline Unigene_UN_xiong_8012 & 58 & 27 UN_xiong_8012 \\
\hline Unigene_UN_xiong_984 & 156 & 318 UN_xiong_984 \\
\hline Unigene_UN_xiong_14595 & 133 & 65 UN_xiong_14595 \\
\hline Unigene_UN_xiong_15382 & 6739 & 793 UN_xiong_15382 \\
\hline Unigene_UN_xiong_5378 & 320 & 155 UN_xiong_5378 \\
\hline Unigene_UN_xiong_11886 & 87 & 78 UN_xiong_11886 \\
\hline Unigene_UN_xiong_14725 & 255 & 70 UN_xiong_14725 \\
\hline Unigene_GR6TTJD01BE04C_xiong & 24 & 39 GR6TTJD01BE04C_xion \\
\hline Unigene_GR6TTJD01ARQBG_xiong & 30 & 31 GR6TTJD01ARQBG_xion \\
\hline Unigene_GPGRCDD02IX7YD_xiong & 23 & 102 GPGRCDD02IX7YD_xion \\
\hline Unigene_GP8I43W03HBULJ_xiong & 43 & 76 GP8I43W03HBULJ_xion \\
\hline Unigene_UN_xiong_14905 & 12337 & 11775 UN_xiong_14905 \\
\hline Unigene_UN_xiong_13604 & 86 & 129 UN_xiong_13604 \\
\hline Unigene_UN_xiong_13016 & 535 & 766 UN_xiong_13016 \\
\hline Unigene_UN_xiong_13813 & 66 & 65 UN_xiong_13813 \\
\hline Unigene_UN_xiong_1155 & 200 & 411 UN_xiong_1155 \\
\hline Unigene_UN_xiong_2973 & 823 & 746 UN_xiong_2973 \\
\hline Unigene_GP8I43W03HCG81_xiong & 38 & 40 GP8I43W03HCG81_xion \\
\hline Unigene_UN_xiong_316 & 79 & 212 UN_xiong_316 \\
\hline Unigene_UN_xiong_4833 & 91 & 177 UN_xiong_4833 \\
\hline Unigene_UN_xiong_9043 & 55 & 28 UN_xiong_9043 \\
\hline Unigene_UN_xiong_9328 & 77 & 108 UN_xiong_9328 \\
\hline Unigene_UN_xiong_4496 & 165 & 281 UN_xiong_4496 \\
\hline Unigene_UN_xiong_1690 & 1483 & 227 UN_xiong_1690 \\
\hline Unigene_UN_xiong_13311 & 117 & 204 UN_xiong_13311 \\
\hline
\end{tabular}




\begin{tabular}{|c|c|c|}
\hline Unigene_GPGRCDD02F43H4_xiong & 66 & 91 GPGRCDD02F43H4_xion \\
\hline Unigene_UN_xiong_234 & 66 & 167 UN_xiong_234 \\
\hline Unigene_UN_xiong_12521 & 142 & 118 UN_xiong_12521 \\
\hline Unigene_UN_xiong_6377 & 74 & 66 UN_xiong_6377 \\
\hline Unigene_UN_xiong_2274 & 204 & 221 UN_xiong_2274 \\
\hline Unigene_GR6TTJD01A3DK7_xiong & 39 & 46 GR6TTJD01A3DK7_xion \\
\hline Unigene_UN_xiong_7733 & 53 & 126 UN_xiong_7733 \\
\hline Unigene_UN_xiong_3801 & 60 & 19 UN_xiong_3801 \\
\hline Unigene_GR6TTJD01AFQ86_xiong & 24 & 21 GR6TTJD01AFQ86_xion \\
\hline Unigene_UN_xiong_3071 & 58 & 67 UN_xiong_3071 \\
\hline Unigene_UN_xiong_7147 & 107 & 138 UN_xiong_7147 \\
\hline Unigene_GR6TTJD01BT6VN_xiong & 23 & 26 GR6TTJD01BT6VN_xion \\
\hline Unigene_UN_xiong_3286 & 144 & 396 UN_xiong_3286 \\
\hline Unigene_UN_xiong_1233 & 42 & 136 UN_xiong_1233 \\
\hline Unigene_UN_xiong_9306 & 60 & 104 UN_xiong_9306 \\
\hline Unigene_GR6TTJD01BI2PU_xiong & 26 & 27 GR6TTJD01BI2PU_xion \\
\hline Unigene_UN_xiong_4514 & 408 & 277 UN_xiong_4514 \\
\hline Unigene_UN_xiong_2878 & 64 & 108 UN_xiong_2878 \\
\hline Unigene_UN_xiong_138 & 290 & 74 UN_xiong_138 \\
\hline Unigene_UN_xiong_544 & 222 & 259 UN_xiong_544 \\
\hline Unigene_UN_xiong_6225 & 81 & 29 UN_xiong_6225 \\
\hline Unigene_GPGRCDD02FY507_xiong & 27 & 37 GPGRCDD02FY507_xion \\
\hline Unigene_UN_xiong_5707 & 142 & 358 UN_xiong_5707 \\
\hline Unigene_GPGRCDD02I5ZUC_xiong & 85 & 1288 GPGRCDD02I5ZUC_xion \\
\hline Unigene_GPGRCDD02IMEMR_xiong & 20 & 49 GPGRCDD02IMEMR_xion \\
\hline Unigene_UN_xiong_14497 & 568 & 341 UN_xiong_14497 \\
\hline Unigene_UN_xiong_11612 & 543 & 30 UN_xiong_11612 \\
\hline Unigene_UN_xiong_10847 & 697 & 398 UN_xiong_10847 \\
\hline Unigene_UN_xiong_1280 & 2641 & 765 UN_xiong_1280 \\
\hline Unigene_GR6TTJD01BGS1G_xiong & 37 & 32 GR6TTJD01BGS1G_xion \\
\hline Unigene_UN_xiong_3802 & 224 & 534 UN_xiong_3802 \\
\hline Unigene_UN_xiong_14877 & 1204 & 383 UN_xiong_14877 \\
\hline Unigene_UN_xiong_10186 & 82 & 253 UN_xiong_10186 \\
\hline Unigene_UN_xiong_4533 & 470 & 1243 UN_xiong_4533 \\
\hline Unigene_UN_xiong_1187 & 235 & 100 UN_xiong_1187 \\
\hline Unigene_GR6TTJD01BKMQ6_xiong & 28 & 23 GR6TTJD01BKMQ6_xion \\
\hline Unigene_UN_xiong_8138 & 64 & 53 UN_xiong_8138 \\
\hline Unigene_UN_xiong_5023 & 86 & 25 UN_xiong_5023 \\
\hline Unigene_UN_xiong_1894 & 62 & 359 UN_xiong_1894 \\
\hline Unigene_UN_xiong_15624 & 462 & 460 UN_xiong_15624 \\
\hline Unigene_UN_xiong_11993 & 61 & 26 UN_xiong_11993 \\
\hline Unigene_GP8I43W03GLRHZ_xiong & 73 & 224 GP8I43W03GLRHZ_xion \\
\hline Unigene_GR6TTJD01B599R_xiong & 32 & 124 GR6TTJD01B599R_xion \\
\hline Unigene_UN_xiong_3622 & 51 & 92 UN_xiong_3622 \\
\hline Unigene_GP8I43W03F0AKI_xiong & 27 & 24 GP8I43W03F0AKI_xion \\
\hline Unigene_GR6TTJD01A452U_xiong & 30 & 31 GR6TTJD01A452U_xion \\
\hline Unigene_UN_xiong_14915 & 161 & 272 UN_xiong_14915 \\
\hline Unigene_GR6TTJD01AJJKG_xiong & 31 & 89 GR6TTJD01AJJKG_xion \\
\hline Unigene_UN_xiong_5445 & 42 & 43 UN_xiong_5445 \\
\hline Unigene_GP8I43W03F4Z5R_xiong & 51 & 46 GP8I43W03F4Z5R_xion \\
\hline
\end{tabular}




\begin{tabular}{|c|c|c|}
\hline Unigene_UN_xiong_13087 & 42 & 93 UN_xiong_13087 \\
\hline Unigene_UN_xiong_270 & 320 & 87 UN_xiong_270 \\
\hline Unigene_UN_xiong_6275 & 50 & 91 UN_xiong_6275 \\
\hline Unigene_GR6TTJD01CD9GC_xiong & 62 & 80 GR6TTJD01CD9GC_xion \\
\hline Unigene_UN_xiong_7881 & 150 & 224 UN_xiong_7881 \\
\hline Unigene_UN_xiong_15139 & 920 & 469 UN_xiong_15139 \\
\hline Unigene_UN_xiong_1287 & 145 & 109 UN_xiong_1287 \\
\hline Unigene GP8I43W03GMLSQ xiong & 29 & 20 GP8I43W03GMLSQ xion \\
\hline Unigene_UN_xiong_1015 & 345 & 986 UN_xiong_1015 \\
\hline Unigene_UN_xiong_3631 & 36 & 32 UN_xiong_3631 \\
\hline Unigene_UN_xiong_11811 & 246 & 163 UN_xiong_11811 \\
\hline Unigene_GP8I43W03F5H7X_xiong & 36 & 50 GP8I43W03F5H7X_xion \\
\hline Unigene_UN_xiong_14478 & 236 & 357 UN_xiong_14478 \\
\hline Unigene_GPGRCDD02FJH27_xiong & 94 & 238 GPGRCDD02FJH27_xion \\
\hline Unigene_UN_xiong_4548 & 89 & 406 UN_xiong_4548 \\
\hline Unigene_UN_xiong_4784 & 75 & 331 UN_xiong_4784 \\
\hline Unigene_GPGRCDD02IHRLP_xiong & 22 & 115 GPGRCDD02IHRLP_xion \\
\hline Unigene_UN_xiong_6922 & 31 & 154 UN_xiong_6922 \\
\hline Unigene_GP8I43W03G1BBF_xiong & 27 & 123 GP8I43W03G1BBF_xion \\
\hline Unigene_UN_xiong_3495 & 426 & 397 UN_xiong_3495 \\
\hline Unigene_UN_xiong_4615 & 161 & 1079 UN_xiong_4615 \\
\hline Unigene_UN_xiong_15043 & 353 & 561 UN_xiong_15043 \\
\hline Unigene_GPGRCDD02GFWWN_xiong & 24 & 85 GPGRCDD02GFWWN_xion \\
\hline Unigene_UN_xiong_9962 & 145 & 166 UN_xiong_9962 \\
\hline Unigene UN xiong 15692 & 387 & 215 UN xiong 15692 \\
\hline Unigene_GPGRCDD02F3323_xiong & 21 & 25 GPGRCDD02F3323_xion \\
\hline Unigene_UN_xiong_14849 & 272 & 111 UN_xiong_14849 \\
\hline Unigene_GPGRCDD02JVJ83_xiong & 20 & 456 GPGRCDD02JVJ83_xion \\
\hline Unigene_GPGRCDD02G5SQA_xiong & 28 & 107 GPGRCDD02G5SQA_xion \\
\hline Unigene_GR6TTJD01CDZ53_xiong & 38 & 32 GR6TTJD01CDZ53_xion \\
\hline Unigene_UN_xiong_13566 & 211 & 71 UN_xiong_13566 \\
\hline Unigene_UN_xiong_1462 & 131 & 1214 UN_xiong_1462 \\
\hline Unigene_GPGRCDD02HRJOH_xiong & 23 & 30 GPGRCDD02HRJOH_xion \\
\hline Unigene_UN_xiong_14262 & 179 & 71 UN_xiong_14262 \\
\hline Unigene_UN_xiong_589 & 1575 & 312 UN_xiong_589 \\
\hline Unigene_UN_xiong_10440 & 78 & 29 UN_xiong_10440 \\
\hline Unigene_UN_xiong_14842 & 502 & 4128 UN_xiong_14842 \\
\hline Unigene_UN_xiong_1333 & 398 & 749 UN_xiong_1333 \\
\hline Unigene_GPGRCDD02IDS4N_xiong & 62 & 72 GPGRCDD02IDS4N_xion \\
\hline Unigene_UN_xiong_7823 & 154 & 85 UN_xiong_7823 \\
\hline Unigene_GPGRCDD02JHC7A_xiong & 56 & 153 GPGRCDD02JHC7A_xion \\
\hline Unigene GR6TTJD01BTIXF xiong & 30 & 31 GR6TTJD01BTIXF xion \\
\hline Unigene_UN_xiong_6146 & 57 & 324 UN_xiong_6146 \\
\hline Unigene_UN_xiong_464 & 92 & 59 UN_xiong_464 \\
\hline Unigene_UN_xiong_10562 & 206 & 522 UN_xiong_10562 \\
\hline Unigene_UN xiong 14821 & 61 & 179 UN_xiong_14821 \\
\hline Unigene_UN_xiong_4276 & 1091 & 364 UN_xiong_4276 \\
\hline Unigene_GR6TTJD01B7720_xiong & 31 & 26 GR6TTJD01B7720_xion \\
\hline Unigene_UN_xiong_12884 & 1457 & 107 UN_xiong_12884 \\
\hline Unigene GPGRCDD02G536C xiong & 26 & 33 GPGRCDD02G536C xion \\
\hline
\end{tabular}




\begin{tabular}{|c|c|c|}
\hline Unigene_UN_xiong_14067 & 243 & 70 UN_xiong_14067 \\
\hline Unigene_GPGRCDD02HBSMH_xiong & 25 & 42 GPGRCDD02HBSMH_xion \\
\hline Unigene_UN_xiong_6263 & 105 & 125 UN_xiong_6263 \\
\hline Unigene_UN_xiong_112 & 102 & 1687 UN_xiong_112 \\
\hline Unigene_UN_xiong_6167 & 116 & 33 UN_xiong_6167 \\
\hline Unigene_UN_xiong_5457 & 234 & 82 UN_xiong_5457 \\
\hline Unigene_UN_xiong_662 & 455 & 386 UN_xiong_662 \\
\hline Unigene_UN_xiong_6570 & 73 & 73 UN_xiong_6570 \\
\hline Unigene_UN_xiong_15567 & 121 & 51 UN_xiong_15567 \\
\hline Unigene_UN_xiong_5322 & 50 & 83 UN_xiong_5322 \\
\hline Unigene_UN_xiong_1099 & 545 & 117 UN_xiong_1099 \\
\hline Unigene_UN_xiong_7136 & 91 & 35 UN_xiong_7136 \\
\hline Unigene_GR6TTJD01BFR1T_xiong & 56 & 34 GR6TTJD01BFR1T_xion \\
\hline Unigene_GR6TTJD01B4J83_xiong & 85 & 76 GR6TTJD01B4J83_xion \\
\hline Unigene_UN_xiong_11461 & 104 & 113 UN_xiong_11461 \\
\hline Unigene_GR6TTJD01BL2I7_xiong & 77 & 325 GR6TTJD01BL2I7_xion \\
\hline Unigene_UN_xiong_6878 & 343 & 300 UN_xiong_6878 \\
\hline Unigene_UN_xiong_3218 & 155 & 71 UN_xiong_3218 \\
\hline Unigene_UN_xiong_6992 & 120 & 66 UN_xiong_6992 \\
\hline Unigene_UN_xiong_2711 & 189 & 27 UN_xiong_2711 \\
\hline Unigene_GR6TTJD01BNYOU_xiong & 33 & 21 GR6TTJD01BNYOU_xion \\
\hline Unigene_UN_xiong_14330 & 551 & 207 UN_xiong_14330 \\
\hline Unigene_GR6TTJD01A3IGY_xiong & 23 & 21 GR6TTJD01A3IGY_xion \\
\hline Unigene_UN_xiong_12244 & 60 & 100 UN_xiong_12244 \\
\hline Unigene_UN_xiong_15702 & 235 & 297 UN_xiong_15702 \\
\hline Unigene_UN_xiong_12422 & 30 & 258 UN_xiong_12422 \\
\hline Unigene_UN_xiong_7323 & 58 & 249 UN_xiong_7323 \\
\hline Unigene_UN_xiong_2562 & 83 & 274 UN_xiong_2562 \\
\hline Unigene_UN_xiong_13515 & 619 & 2300 UN_xiong_13515 \\
\hline Unigene_GPGRCDD02JTMV4_xiong & 20 & 72 GPGRCDD02JTMV4_xion \\
\hline Unigene_GR6TTJD01CAEG4_xiong & 43 & 44 GR6TTJD01CAEG4_xion \\
\hline Unigene_GPGRCDD02H6BZ0_xiong & 24 & 80 GPGRCDD02H6BZ0_xion \\
\hline Unigene_UN_xiong_1728 & 74 & 122 UN_xiong_1728 \\
\hline Unigene_GR6TTJD01BQULS_xiong & 25 & 160 GR6TTJD01BQULS_xion \\
\hline Unigene_GP8I43W03F5CLI_xiong & 27 & 275 GP8I43W03F5CLI_xion \\
\hline Unigene_GPGRCDD02FQPCL_xiong & 26 & 23 GPGRCDD02FQPCL_xion \\
\hline Unigene_GR6TTJD01BM15A_xiong & 35 & 83 GR6TTJD01BM15A_xion \\
\hline Unigene_UN_xiong_4208 & 81 & 95 UN_xiong_4208 \\
\hline Unigene_UN_xiong_8115 & 265 & 144 UN_xiong_8115 \\
\hline Unigene_GR6TTJD01AWPMW_xiong & 39 & 40 GR6TTJD01AWPMW_xion \\
\hline Unigene_UN_xiong_1383 & 142 & 30 UN_xiong_1383 \\
\hline Unigene_UN_xiong_591 & 49 & 95 UN_xiong_591 \\
\hline Unigene_UN_xiong_3642 & 63 & 41 UN_xiong_3642 \\
\hline Unigene_GP8I43W03FPNF8_xiong & 50 & 45 GP8I43W03FPNF8_xion \\
\hline Unigene_GP8I43W03GYYGE_xiong & 47 & 45 GP8I43W03GYYGE_xion \\
\hline Unigene_GR6TTJD01BJDEK_xiong & 28 & 63 GR6TTJD01BJDEK_xion \\
\hline Unigene_UN_xiong_7775 & 113 & 154 UN_xiong_7775 \\
\hline Unigene_UN_xiong_1247 & 898 & 1921 UN_xiong_1247 \\
\hline Unigene_UN_xiong_13097 & 898 & 848 UN_xiong_13097 \\
\hline Unigene_UN_xiong_14978 & 2492 & 699 UN_xiong_14978 \\
\hline
\end{tabular}




\begin{tabular}{|c|c|c|}
\hline Unigene_UN_xiong_971 & 294 & 1869 UN_xiong_971 \\
\hline Unigene_UN_xiong_12920 & 69 & 21 UN_xiong_12920 \\
\hline Unigene_UN_xiong_1199 & 412 & 97 UN_xiong_1199 \\
\hline Unigene_UN_xiong_9634 & 72 & 94 UN_xiong_9634 \\
\hline Unigene_UN_xiong_14541 & 397 & 251 UN_xiong_14541 \\
\hline Unigene_UN_xiong_1893 & 32 & 81 UN_xiong_1893 \\
\hline Unigene_UN_xiong_12517 & 55 & 33 UN_xiong_12517 \\
\hline Unigene_GPGRCDD02H8B8D_xiong & 20 & 194 GPGRCDD02H8B8D_xion \\
\hline Unigene_UN_xiong_1089 & 100 & 68 UN_xiong_1089 \\
\hline Unigene_UN_xiong_12956 & 78 & 219 UN_xiong_12956 \\
\hline Unigene_UN_xiong_13553 & 98 & 19 UN_xiong_13553 \\
\hline Unigene_UN_xiong_2574 & 147 & 107 UN_xiong_2574 \\
\hline Unigene_GR6TTJD01BY511_xiong & 46 & 65 GR6TTJD01BY511_xion \\
\hline Unigene_UN_xiong_9163 & 619 & 392 UN_xiong_9163 \\
\hline Unigene_UN_xiong_8838 & 146 & 345 UN_xiong_8838 \\
\hline Unigene_UN_xiong_8825 & 73 & 32 UN_xiong_8825 \\
\hline Unigene_UN_xiong_14409 & 766 & 929 UN_xiong_14409 \\
\hline Unigene_UN_xiong_3263 & 79 & 26 UN_xiong_3263 \\
\hline Unigene_UN_xiong_2659 & 82 & 22 UN_xiong_2659 \\
\hline Unigene_UN_xiong_13969 & 99 & 30 UN_xiong_13969 \\
\hline Unigene_UN_xiong_11018 & 35 & 67 UN_xiong_11018 \\
\hline Unigene_UN_xiong_1654 & 251 & 72 UN_xiong_1654 \\
\hline Unigene_UN_xiong_6142 & 75 & 76 UN_xiong_6142 \\
\hline Unigene_UN_xiong_247 & 39 & 61 UN_xiong_247 \\
\hline Unigene_GP8I43W03FSFCD_xiong & 56 & 242 GP8I43W03FSFCD_xion \\
\hline Unigene_GPGRCDD02IUZ01_xiong & 47 & 46 GPGRCDD02IUZ01_xion \\
\hline Unigene_UN_xiong_3040 & 253 & 270 UN_xiong_3040 \\
\hline Unigene_UN_xiong_9221 & 112 & 74 UN_xiong_9221 \\
\hline Unigene_UN_xiong_3687 & 65 & 43 UN_xiong_3687 \\
\hline Unigene_UN_xiong_145 & 459 & 135 UN_xiong_145 \\
\hline Unigene_GPGRCDD02IAIMZ_xiong & 32 & 53 GPGRCDD02IAIMZ_xion \\
\hline Unigene_UN_xiong_6111 & 91 & 90 UN_xiong_6111 \\
\hline Unigene_UN_xiong_14810 & 189 & 152 UN_xiong_14810 \\
\hline Unigene_GR6TTJD01A6LNA_xiong & 30 & 60 GR6TTJD01A6LNA_xion \\
\hline Unigene_UN_xiong_15021 & 343 & 26 UN_xiong_15021 \\
\hline Unigene_GR6TTJD01AQP3E_xiong & 26 & 72 GR6TTJD01AQP3E_xion \\
\hline Unigene_UN_xiong_3696 & 70 & 60 UN_xiong_3696 \\
\hline Unigene_UN_xiong_4121 & 53 & 42 UN_xiong_4121 \\
\hline Unigene_UN_xiong_8660 & 94 & 41 UN_xiong_8660 \\
\hline Unigene_UN_xiong_5480 & 223 & 103 UN_xiong_5480 \\
\hline Unigene_UN_xiong_11477 & 278 & 37 UN_xiong_11477 \\
\hline Unigene_UN_xiong_8917 & 51 & 41 UN_xiong_8917 \\
\hline Unigene_GP8I43W03GDAJL_xiong & 63 & 84 GP8I43W03GDAJL_xion \\
\hline Unigene_UN_xiong_4674 & 40 & 270 UN_xiong_4674 \\
\hline Unigene_UN_xiong_13871 & 56 & 78 UN_xiong_13871 \\
\hline Unigene_UN_xiong_5714 & 196 & 102 UN_xiong_5714 \\
\hline Unigene_UN_xiong_14435 & 528 & 44 UN_xiong_14435 \\
\hline Unigene_UN_xiong_9071 & 73 & 335 UN_xiong_9071 \\
\hline Unigene_UN_xiong_3203 & 1131 & 1187 UN_xiong_3203 \\
\hline Unigene_UN_xiong_1529 & 29 & 138 UN_xiong_1529 \\
\hline
\end{tabular}




\begin{tabular}{|c|c|c|}
\hline Unigene_UN_xiong_13915 & 73 & 52 UN_xiong_13915 \\
\hline Unigene_GR6TTJD01AFKY9_xiong & 37 & 66 GR6TTJD01AFKY9_xion \\
\hline Unigene_UN_xiong_9239 & 50 & 111 UN_xiong_9239 \\
\hline Unigene_UN_xiong_7202 & 192 & 25 UN_xiong_7202 \\
\hline Unigene_UN_xiong_3091 & 88 & 120 UN_xiong_3091 \\
\hline Unigene_UN_xiong_15647 & 193 & 160 UN_xiong_15647 \\
\hline Unigene_GPGRCDD02JIUUR_xiong & 26 & 26 GPGRCDD02JIUUR_xion \\
\hline Unigene_UN_xiong_6173 & 210 & 237 UN_xiong_6173 \\
\hline Unigene_UN_xiong_7858 & 50 & 20 UN_xiong_7858 \\
\hline Unigene_GPGRCDD02JX3JI_xiong & 36 & 40 GPGRCDD02JX3JI_xion \\
\hline Unigene_UN_xiong_13647 & 106 & 29 UN_xiong_13647 \\
\hline Unigene_GR6TTJD01B715J_xiong & 61 & 53 GR6TTJD01B715J_xion \\
\hline Unigene_GR6TTJD01B7CPE_xiong & 21 & 65 GR6TTJD01B7CPE_xion \\
\hline Unigene_GR6TTJD01CCB3U_xiong & 21 & 200 GR6TTJD01CCB3U_xion \\
\hline Unigene_GR6TTJD01BTR90_xiong & 24 & 93 GR6TTJD01BTR90_xion \\
\hline Unigene_GR6TTJD01ANEBV_xiong & 22 & 425 GR6TTJD01ANEBV_xion \\
\hline Unigene_GR6TTJD01B1KFF_xiong & 29 & 39 GR6TTJD01B1KFF_xion \\
\hline Unigene_UN_xiong_4580 & 562 & 114 UN_xiong_4580 \\
\hline Unigene_UN_xiong_14265 & 251 & 396 UN_xiong_14265 \\
\hline Unigene_UN_xiong_5994 & 95 & 166 UN_xiong_5994 \\
\hline Unigene_UN_xiong_169 & 143 & 956 UN_xiong_169 \\
\hline Unigene_UN_xiong_10079 & 80 & 272 UN_xiong_10079 \\
\hline Unigene_GR6TTJD01B07KL_xiong & 26 & 89 GR6TTJD01B07KL_xion \\
\hline Unigene_UN_xiong_15023 & 449 & 79 UN_xiong_15023 \\
\hline Unigene_GPGRCDD02FUDGA_xiong & 31 & 37 GPGRCDD02FUDGA_xion \\
\hline Unigene_UN_xiong_4108 & 98 & 1931 UN_xiong_4108 \\
\hline Unigene_UN_xiong_3595 & 117 & 167 UN_xiong_3595 \\
\hline Unigene_GPGRCDD02H2UOR_xiong & 37 & 35 GPGRCDD02H2U0R_xion \\
\hline Unigene_GPGRCDD02H5RWL_xiong & 43 & 359 GPGRCDD02H5RWL_xion \\
\hline Unigene_GPGRCDD02G9UBT_xiong & 25 & 32 GPGRCDD02G9UBT_xion \\
\hline Unigene_UN_xiong_2525 & 188 & 28 UN_xiong_2525 \\
\hline Unigene_UN_xiong_14260 & 124 & 417 UN_xiong_14260 \\
\hline Unigene_UN_xiong_14840 & 162 & 823 UN_xiong_14840 \\
\hline Unigene_UN_xiong_13595 & 109 & 142 UN_xiong_13595 \\
\hline Unigene_UN_xiong_9965 & 50 & 39 UN_xiong_9965 \\
\hline Unigene_GP8I43W03FRBZW_xiong & 34 & 65 GP8I43W03FRBZW_xion \\
\hline Unigene_GPGRCDD02JT300_xiong & 63 & 49 GPGRCDD02JT300_xion \\
\hline Unigene_UN_xiong_5587 & 47 & 23 UN_xiong_5587 \\
\hline Unigene_GR6TTJD01B5ZYT_xiong & 38 & 33 GR6TTJD01B5ZYT_xion \\
\hline Unigene_UN_xiong_13013 & 281 & 584 UN_xiong_13013 \\
\hline Unigene_UN_xiong_6534 & 246 & 27 UN_xiong_6534 \\
\hline Unigene_GPGRCDD02JJP9E_xiong & 32 & 35 GPGRCDD02JJP9E_xion \\
\hline Unigene_GR6TTJD01AIQ25_xiong & 26 & 32 GR6TTJD01AIQ25_xion \\
\hline Unigene_UN_xiong_1513 & 363 & 23 UN_xiong_1513 \\
\hline Unigene_UN_xiong_14749 & 448 & 726 UN_xiong_14749 \\
\hline Unigene_UN_xiong_15786 & 398 & 324 UN_xiong_15786 \\
\hline Unigene_UN_xiong_6511 & 206 & 107 UN_xiong_6511 \\
\hline Unigene_UN_xiong_8630 & 284 & 595 UN_xiong_8630 \\
\hline Unigene_UN_xiong_11680 & 139 & 5026 UN_xiong_11680 \\
\hline Unigene_UN_xiong_15527 & 141 & 78 UN_xiong_15527 \\
\hline
\end{tabular}




\begin{tabular}{|c|c|c|}
\hline Unigene_GPGRCDD02GF51M_xiong & 51 & 96 GPGRCDD02GF51M_xion \\
\hline Unigene_GP8I43W03GA0HX_xiong & 27 & 45 GP8I43W03GAOHX_xion \\
\hline Unigene_UN_xiong_9524 & 111 & 80 UN_xiong_9524 \\
\hline Unigene_UN_xiong_10179 & 1109 & 64 UN_xiong_10179 \\
\hline Unigene_UN_xiong_4641 & 54 & 208 UN_xiong_4641 \\
\hline Unigene_GP8I43W03GH0PX_xiong & 25 & 95 GP8I43W03GHOPX_xion \\
\hline Unigene_UN_xiong_10987 & 55 & 108 UN_xiong_10987 \\
\hline Unigene_UN_xiong_14527 & 273 & 176 UN_xiong_14527 \\
\hline Unigene_UN_xiong_5214 & 122 & 25 UN_xiong_5214 \\
\hline Unigene_GR6TTJD01B70IV_xiong & 24 & 90 GR6TTJD01B70IV_xion \\
\hline Unigene_UN_xiong_15774 & 299 & 233 UN_xiong_15774 \\
\hline Unigene_UN_xiong_11159 & 55 & 93 UN_xiong_11159 \\
\hline Unigene_GR6TTJD01BU8EY_xiong & 21 & 29 GR6TTJD01BU8EY_xion \\
\hline Unigene_GR6TTJD01AJ3LK_xiong & 50 & 116 GR6TTJD01AJ3LK_xion \\
\hline Unigene_GPGRCDD02JB6SN_xiong & 57 & 71 GPGRCDD02JB6SN_xion \\
\hline Unigene_GPGRCDD02J1Z6N_xiong & 59 & 90 GPGRCDD02J1Z6N_xion \\
\hline Unigene_UN_xiong_10580 & 347 & 70 UN_xiong_10580 \\
\hline Unigene_GP8I43W03GYJWH_xiong & 41 & 39 GP8I43W03GYJWH_xion \\
\hline Unigene_GR6TTJD01BFPRC_xiong & 30 & 23 GR6TTJD01BFPRC_xion \\
\hline Unigene_UN_xiong_2611 & 1976 & 1719 UN_xiong_2611 \\
\hline Unigene_GP8I43W03G55FQ_xiong & 28 & 26 GP8I43W03G55FQ_xion \\
\hline Unigene_UN_xiong_13966 & 69 & 98 UN_xiong_13966 \\
\hline Unigene_UN_xiong_8417 & 140 & 75 UN_xiong_8417 \\
\hline Unigene_UN_xiong_14563 & 1317 & 1304 UN_xiong_14563 \\
\hline Unigene_GR6TTJD01CB6LM_xiong & 27 & 50 GR6TTJD01CB6LM_xion \\
\hline Unigene_GR6TTJD01BDRIT_xiong & 28 & 41 GR6TTJD01BDRIT_xion \\
\hline Unigene_UN_xiong_1971 & 78 & 83 UN_xiong_1971 \\
\hline Unigene_UN_xiong_14770 & 132 & 45 UN_xiong_14770 \\
\hline Unigene_UN_xiong_8920 & 541 & 265 UN_xiong_8920 \\
\hline Unigene_UN_xiong_15542 & 87 & 29 UN_xiong_15542 \\
\hline Unigene_UN_xiong_38 & 112 & 42 UN_xiong_38 \\
\hline Unigene_UN_xiong_14717 & 477 & 381 UN_xiong_14717 \\
\hline Unigene_UN_xiong_4277 & 29 & 38 UN_xiong_4277 \\
\hline Unigene_UN_xiong_13488 & 168 & 141 UN_xiong_13488 \\
\hline Unigene_UN_xiong_9168 & 80 & 265 UN_xiong_9168 \\
\hline Unigene_UN_xiong_9688 & 97 & 45 UN_xiong_9688 \\
\hline Unigene_UN_xiong_14990 & 174 & 134 UN_xiong_14990 \\
\hline Unigene_UN_xiong_9178 & 118 & 28 UN_xiong_9178 \\
\hline Unigene_UN_xiong_10756 & 91 & 35 UN_xiong_10756 \\
\hline Unigene_UN_xiong_10374 & 119 & 68 UN_xiong_10374 \\
\hline Unigene_GR6TTJD01BZ4KD_xiong & 30 & 92 GR6TTJD01BZ4KD_xion \\
\hline Unigene_UN_xiong_5818 & 129 & 95 UN_xiong_5818 \\
\hline Unigene_UN_xiong_8120 & 169 & 535 UN_xiong_8120 \\
\hline Unigene_UN_xiong_4780 & 496 & 47 UN_xiong_4780 \\
\hline Unigene_UN_xiong_601 & 56 & 36 UN_xiong_601 \\
\hline Unigene_UN_xiong_10122 & 245 & 51 UN_xiong_10122 \\
\hline Unigene_UN_xiong_12473 & 61 & 152 UN_xiong_12473 \\
\hline Unigene_UN_xiong_1588 & 397 & 1639 UN_xiong_1588 \\
\hline Unigene_UN_xiong_15503 & 1081 & 2346 UN_xiong_15503 \\
\hline Unigene_UN_xiong_7884 & 125 & 28 UN_xiong_7884 \\
\hline
\end{tabular}




\begin{tabular}{|c|c|c|}
\hline Unigene_UN_xiong_13720 & 160 & 37 UN_xiong_13720 \\
\hline Unigene_UN_xiong_5353 & 168 & 316 UN_xiong_5353 \\
\hline Unigene_UN_xiong_11496 & 91 & 26 UN_xiong_11496 \\
\hline Unigene_UN_xiong_629 & 653 & 104 UN_xiong_629 \\
\hline Unigene_UN_xiong_5483 & 112 & 64 UN_xiong_5483 \\
\hline Unigene_UN_xiong_7854 & 661 & 278 UN_xiong_7854 \\
\hline Unigene_UN_xiong_1766 & 59 & 96 UN_xiong_1766 \\
\hline Unigene_UN_xiong_15586 & 88 & 69 UN_xiong_15586 \\
\hline Unigene_UN_xiong_1200 & 335 & 234 UN_xiong_1200 \\
\hline Unigene_UN_xiong_6761 & 124 & 170 UN_xiong_6761 \\
\hline Unigene_UN_xiong_10251 & 152 & 102 UN_xiong_10251 \\
\hline Unigene_GR6TTJD01BDQNT_xiong & 29 & 49 GR6TTJD01BDQNT_xion \\
\hline Unigene_UN_xiong_7984 & 46 & 44 UN_xiong_7984 \\
\hline Unigene_UN_xiong_15570 & 32 & 50 UN_xiong_15570 \\
\hline Unigene_UN_xiong_353 & 405 & 146 UN_xiong_353 \\
\hline Unigene_UN_xiong_15049 & 283 & 198 UN_xiong_15049 \\
\hline Unigene_GR6TTJD01B3LBH_xiong & 34 & 42 GR6TTJD01B3LBH_xion \\
\hline Unigene_UN_xiong_1964 & 57 & 27 UN_xiong_1964 \\
\hline Unigene_UN_xiong_4867 & 623 & 75 UN_xiong_4867 \\
\hline Unigene_UN_xiong_13896 & 191 & 172 UN_xiong_13896 \\
\hline Unigene_UN_xiong_12227 & 123 & 278 UN_xiong_12227 \\
\hline Unigene_UN_xiong_1807 & 2282 & 437 UN_xiong_1807 \\
\hline Unigene_GPGRCDD02GIPK2_xiong & 35 & 110 GPGRCDD02GIPK2_xion \\
\hline Unigene_UN_xiong_4846 & 170 & 39 UN_xiong_4846 \\
\hline Unigene_UN_xiong_14470 & 380 & 846 UN_xiong_14470 \\
\hline Unigene_UN_xiong_12570 & 66 & 102 UN_xiong_12570 \\
\hline Unigene_UN_xiong_12198 & 70 & 53 UN_xiong_12198 \\
\hline Unigene_UN_xiong_14297 & 131 & 58 UN_xiong_14297 \\
\hline Unigene UN xiong 12139 & 244 & 4019 UN xiong 12139 \\
\hline Unigene_UN_xiong_6760 & 104 & 83 UN_xiong_6760 \\
\hline Unigene_UN_xiong_14427 & 194 & 802 UN_xiong_14427 \\
\hline Unigene_UN_xiong_12675 & 452 & 197 UN_xiong_12675 \\
\hline Unigene_UN_xiong_12487 & 59 & 65 UN_xiong_12487 \\
\hline Unigene_UN_xiong_632 & 130 & 147 UN_xiong_632 \\
\hline Unigene_UN_xiong_10604 & 184 & 33 UN_xiong_10604 \\
\hline Unigene_UN_xiong_5755 & 90 & 75 UN_xiong_5755 \\
\hline Unigene_UN_xiong_3281 & 39 & 30 UN_xiong_3281 \\
\hline Unigene_UN_xiong_14460 & 258 & 39 UN_xiong_14460 \\
\hline Unigene_UN_xiong_5130 & 202 & 10038 UN_xiong_5130 \\
\hline Unigene_UN_xiong_15175 & 339 & 377 UN_xiong_15175 \\
\hline Unigene_UN_xiong_1005 & 156 & 404 UN_xiong_1005 \\
\hline Unigene GR6TTJD01AXVBV xiong & 49 & 64 GR6TTJD01AXVBV xion \\
\hline Unigene_UN_xiong_3997 & 615 & 135 UN_xiong_3997 \\
\hline Unigene_GR6TTJD01A8PQD_xiong & 34 & 35 GR6TTJD01A8PQD_xion \\
\hline Unigene_UN_xiong_6541 & 129 & 519 UN_xiong_6541 \\
\hline Unigene_UN_xiong_13569 & 4533 & 53675 UN_xiong_13569 \\
\hline Unigene_UN_xiong_13217 & 122 & 2691 UN_xiong_13217 \\
\hline Unigene_UN_xiong_10591 & 364 & 40 UN_xiong_10591 \\
\hline Unigene_GR6TTJD01BRMAG_xiong & 28 & 37 GR6TTJD01BRMAG_xion \\
\hline Unigene_UN_xiong_2347 & 45 & 57 UN_xiong_2347 \\
\hline
\end{tabular}




\begin{tabular}{|c|c|c|}
\hline Unigene_UN_xiong_419 & 405 & 74 UN_xiong_419 \\
\hline Unigene_UN_xiong_8732 & 186 & 387 UN_xiong_8732 \\
\hline Unigene_UN_xiong_5425 & 97 & 76 UN_xiong_5425 \\
\hline Unigene_UN_xiong_12684 & 44 & 99 UN_xiong_12684 \\
\hline Unigene_GP8I43W03GN4UY_xiong & 40 & 51 GP8I43W03GN4UY_xion \\
\hline Unigene_UN_xiong_2768 & 36 & 126 UN_xiong_2768 \\
\hline Unigene_UN_xiong_7815 & 42 & 278 UN_xiong_7815 \\
\hline Unigene_UN_xiong_14199 & 66 & 125 UN_xiong_14199 \\
\hline Unigene_UN_xiong_610 & 116 & 41 UN_xiong_610 \\
\hline Unigene_UN_xiong_1379 & 78 & 1132 UN_xiong_1379 \\
\hline Unigene_UN_xiong_6577 & 100 & 69 UN_xiong_6577 \\
\hline Unigene_UN_xiong_9042 & 43 & 116 UN_xiong_9042 \\
\hline Unigene_UN_xiong_14176 & 128 & 77 UN_xiong_14176 \\
\hline Unigene_UN_xiong_10682 & 120 & 63 UN_xiong_10682 \\
\hline Unigene_UN_xiong_11809 & 65 & 43 UN_xiong_11809 \\
\hline Unigene_GPGRCDD02IOEE3_xiong & 76 & 64 GPGRCDD02I0EE3_xion \\
\hline Unigene_UN_xiong_762 & 103 & 98 UN_xiong_762 \\
\hline Unigene_UN_xiong_1182 & 42 & 25 UN_xiong_1182 \\
\hline Unigene_UN_xiong_13614 & 53 & 60 UN_xiong_13614 \\
\hline Unigene_UN_xiong_13640 & 37 & 86 UN_xiong_13640 \\
\hline Unigene_UN_xiong_4266 & 61 & 600 UN_xiong_4266 \\
\hline Unigene_GPGRCDD02GREDH_xiong & 38 & 77 GPGRCDD02GREDH_xion \\
\hline Unigene_UN_xiong_6123 & 32 & 56 UN_xiong_6123 \\
\hline Unigene_UN_xiong_13743 & 55 & 82 UN_xiong_13743 \\
\hline Unigene_UN_xiong_8041 & 45 & 41 UN_xiong_8041 \\
\hline Unigene_UN_xiong_4059 & 327 & 136 UN_xiong_4059 \\
\hline Unigene_UN_xiong_2340 & 767 & 122 UN_xiong_2340 \\
\hline Unigene_UN_xiong_3822 & 72 & 449 UN_xiong_3822 \\
\hline Unigene_GP8I43W03GDUGS_xiong & 37 & 50 GP8I43W03GDUGS_xion \\
\hline Unigene_UN_xiong_5508 & 117 & 291 UN_xiong_5508 \\
\hline Unigene_UN_xiong_12557 & 88 & 23 UN_xiong_12557 \\
\hline Unigene_UN_xiong_6795 & 33 & 116 UN_xiong_6795 \\
\hline Unigene_UN_xiong_14962 & 2668 & 1944 UN_xiong_14962 \\
\hline Unigene_UN_xiong_11383 & 58 & 33 UN_xiong_11383 \\
\hline Unigene_UN_xiong_2751 & 53 & 137 UN_xiong_2751 \\
\hline Unigene_UN_xiong_13181 & 70 & 29 UN_xiong_13181 \\
\hline Unigene_GR6TTJD01BJHDB_xiong & 21 & 53 GR6TTJD01BJHDB_xion \\
\hline Unigene_UN_xiong_1170 & 437 & 37 UN_xiong_1170 \\
\hline Unigene_UN_xiong_13845 & 417 & 508 UN_xiong_13845 \\
\hline Unigene_UN_xiong_1842 & 782 & 80 UN_xiong_1842 \\
\hline Unigene_UN_xiong_12555 & 165 & 52 UN_xiong_12555 \\
\hline Unigene_UN_xiong_14859 & 645 & 226 UN_xiong_14859 \\
\hline Unigene_UN_xiong_7090 & 47 & 144 UN_xiong_7090 \\
\hline Unigene_UN_xiong_13109 & 105 & 148 UN_xiong_13109 \\
\hline Unigene_UN_xiong_8471 & 150 & 216 UN_xiong_8471 \\
\hline Unigene_GP8I43W03FPFMZ_xiong & 38 & 40 GP8I43W03FPFMZ_xion \\
\hline Unigene_UN_xiong_2183 & 60 & 50 UN_xiong_2183 \\
\hline Unigene_UN_xiong_6836 & 141 & 31 UN_xiong_6836 \\
\hline Unigene_UN_xiong_9565 & 31 & 39 UN_xiong_9565 \\
\hline Unigene_UN_xiong_13959 & 131 & 34 UN_xiong_13959 \\
\hline
\end{tabular}




\begin{tabular}{|c|c|c|}
\hline Unigene_UN_xiong_11771 & 136 & 59 UN_xiong_11771 \\
\hline Unigene_UN_xiong_11465 & 57 & 33 UN_xiong_11465 \\
\hline Unigene_UN_xiong_11271 & 73 & 25 UN_xiong_11271 \\
\hline Unigene_UN_xiong_2821 & 38 & 224 UN_xiong_2821 \\
\hline Unigene_UN_xiong_14511 & 147 & 46 UN_xiong_14511 \\
\hline Unigene_UN_xiong_7876 & 236 & 35 UN_xiong_7876 \\
\hline Unigene_GR6TTJD01A0AJL_xiong & 38 & 24 GR6TTJD01A0AJL_xion \\
\hline Unigene_UN_xiong_922 & 99 & 69 UN_xiong_922 \\
\hline Unigene_UN_xiong_1012 & 59 & 29 UN_xiong_1012 \\
\hline Unigene_GPGRCDD02F5NMB_xiong & 35 & 66 GPGRCDD02F5NMB_xion \\
\hline Unigene_UN_xiong_4359 & 1567 & 1585 UN_xiong_4359 \\
\hline Unigene_GR6TTJD01BLKML_xiong & 29 & 63 GR6TTJD01BLKML_xion \\
\hline Unigene_UN_xiong_3349 & 90 & 115 UN_xiong_3349 \\
\hline Unigene_UN_xiong_13713 & 742 & 1384 UN_xiong_13713 \\
\hline Unigene_UN_xiong_4497 & 326 & 163 UN_xiong_4497 \\
\hline Unigene_UN_xiong_14566 & 1884 & 690 UN_xiong_14566 \\
\hline Unigene_UN_xiong_2270 & 408 & 55 UN_xiong_2270 \\
\hline Unigene_GR6TTJD01B00JE_xiong & 27 & 67 GR6TTJD01B00JE_xion \\
\hline Unigene_UN_xiong_11327 & 305 & 49 UN_xiong_11327 \\
\hline Unigene_UN_xiong_15747 & 280 & 110 UN_xiong_15747 \\
\hline Unigene_GR6TTJD01B80XL_xiong & 85 & 85 GR6TTJD01B80XL_xion \\
\hline Unigene_GR6TTJD01BG9KN_xiong & 31 & 41 GR6TTJD01BG9KN_xion \\
\hline Unigene_UN_xiong_3659 & 776 & 36 UN_xiong_3659 \\
\hline Unigene_UN_xiong_4172 & 420 & 955 UN_xiong_4172 \\
\hline Unigene_UN_xiong_13476 & 303 & 83 UN_xiong_13476 \\
\hline Unigene_UN_xiong_13925 & 1128 & 12535 UN_xiong_13925 \\
\hline Unigene_GR6TTJD01B9MYG_xiong & 21 & 103 GR6TTJD01B9MYG_xion \\
\hline Unigene_GR6TTJD01BXQVR_xiong & 38 & 34 GR6TTJD01BXQVR_xion \\
\hline Unigene_UN_xiong_6371 & 146 & 118 UN_xiong_6371 \\
\hline Unigene_UN_xiong_9788 & 113 & 45 UN_xiong_9788 \\
\hline Unigene_UN_xiong_4305 & 81 & 48 UN_xiong_4305 \\
\hline Unigene_GP8I43W03GBC5G_xiong & 25 & 190 GP8I43W03GBC5G_xion \\
\hline Unigene_UN_xiong_13135 & 94 & 154 UN_xiong_13135 \\
\hline Unigene_UN_xiong_1129 & 193 & 27 UN_xiong_1129 \\
\hline Unigene_UN_xiong_6316 & 86 & 97 UN_xiong_6316 \\
\hline Unigene_UN_xiong_15740 & 80 & 125 UN_xiong_15740 \\
\hline Unigene_GR6TTJD01A9NES_xiong & 21 & 56 GR6TTJD01A9NES_xion \\
\hline Unigene_UN_xiong_6279 & 155 & 38 UN_xiong_6279 \\
\hline Unigene_UN_xiong_5827 & 206 & 94 UN_xiong_5827 \\
\hline Unigene_GR6TTJD01B48BI_xiong & 33 & 37 GR6TTJD01B48BI_xion \\
\hline Unigene_UN_xiong_8880 & 158 & 28 UN_xiong_8880 \\
\hline Unigene_UN_xiong_7632 & 54 & 22 UN_xiong_7632 \\
\hline Unigene_GR6TTJD01BZWHH_xiong & 35 & 38 GR6TTJD01BZWHH_xion \\
\hline Unigene_UN_xiong_8118 & 98 & 56 UN_xiong_8118 \\
\hline Unigene_UN_xiong_5320 & 44 & 65 UN_xiong_5320 \\
\hline Unigene_UN_xiong_1775 & 237 & 39 UN_xiong_1775 \\
\hline Unigene_UN_xiong_10855 & 200 & 32 UN_xiong_10855 \\
\hline Unigene_UN_xiong_14283 & 178 & 640 UN_xiong_14283 \\
\hline Unigene_GR6TTJD01CC035_xiong & 31 & 29 GR6TTJD01CC035_xion \\
\hline Unigene_UN_xiong_8462 & 121 & 64 UN_xiong_8462 \\
\hline
\end{tabular}




\begin{tabular}{|c|c|c|}
\hline Unigene_GR6TTJD01A65D7_xiong & 27 & 30 GR6TTJD01A65D7_xion \\
\hline Unigene_GR6TTJD01BK1K6_xiong & 24 & 104 GR6TTJD01BK1K6_xion \\
\hline Unigene_GPGRCDD02G0F2I_xiong & 22 & 23 GPGRCDD02G0F2I_xion \\
\hline Unigene_GR6TTJD01BTI68_xiong & 27 & 82 GR6TTJD01BTI68_xion \\
\hline Unigene_UN_xiong_7810 & 137 & 259 UN_xiong_7810 \\
\hline Unigene_UN_xiong_594 & 221 & 159 UN_xiong_594 \\
\hline Unigene_GPGRCDD02H5V6F_xiong & 43 & 51 GPGRCDD02H5V6F_xion \\
\hline Unigene_GR6TTJD01ATK9Z_xiong & 27 & 275 GR6TTJD01ATK9Z_xion \\
\hline Unigene_UN_xiong_15520 & 921 & 719 UN_xiong_15520 \\
\hline Unigene_UN_xiong_5313 & 53 & 25 UN_xiong_5313 \\
\hline Unigene_UN_xiong_4619 & 239 & 502 UN_xiong_4619 \\
\hline Unigene_GR6TTJD01BHLC2_xiong & 32 & 21 GR6TTJD01BHLC2_xion \\
\hline Unigene_UN_xiong_10869 & 87 & 62 UN_xiong_10869 \\
\hline Unigene_UN_xiong_929 & 40 & 113 UN_xiong_929 \\
\hline Unigene_GPGRCDD02GRWYQ_xiong & 30 & 68 GPGRCDD02GRWYQ_xion \\
\hline Unigene_GR6TTJD01A2LL8_xiong & 20 & 62 GR6TTJD01A2LL8_xion \\
\hline Unigene_UN_xiong_4394 & 169 & 26 UN_xiong_4394 \\
\hline Unigene_UN_xiong_10977 & 170 & 44 UN_xiong_10977 \\
\hline Unigene_UN_xiong_510 & 113 & 24 UN_xiong_510 \\
\hline Unigene_UN_xiong_4882 & 82 & 42 UN_xiong_4882 \\
\hline Unigene_UN_xiong_4175 & 114 & 67 UN_xiong_4175 \\
\hline Unigene_UN_xiong_200 & 112 & 58 UN_xiong_200 \\
\hline Unigene_UN_xiong_2117 & 131 & 52 UN_xiong_2117 \\
\hline Unigene_UN_xiong_14236 & 338 & 118 UN_xiong_14236 \\
\hline Unigene_GPGRCDD02IVZYL_xiong & 76 & 76 GPGRCDD02IVZYL_xion \\
\hline Unigene_UN_xiong_13395 & 63 & 45 UN_xiong_13395 \\
\hline Unigene_UN_xiong_5135 & 357 & 120 UN_xiong_5135 \\
\hline Unigene_GR6TTJD01A04RP_xiong & 23 & 33 GR6TTJD01A04RP_xion \\
\hline Unigene_UN_xiong_1472 & 147 & 64 UN_xiong_1472 \\
\hline Unigene_UN_xiong_1719 & 220 & 686 UN_xiong_1719 \\
\hline Unigene_UN_xiong_3672 & 39 & 132 UN_xiong_3672 \\
\hline Unigene_UN_xiong_2739 & 54 & 86 UN_xiong_2739 \\
\hline Unigene_GPGRCDD02HHWUB_xiong & 23 & 25 GPGRCDD02HHWUB_xion \\
\hline Unigene_UN_xiong_7441 & 58 & 175 UN_xiong_7441 \\
\hline Unigene_UN_xiong_12363 & 420 & 157 UN_xiong_12363 \\
\hline Unigene_UN_xiong_11239 & 216 & 29 UN_xiong_11239 \\
\hline Unigene_GR6TTJD01B9XQ1_xiong & 19 & 57 GR6TTJD01B9XQ1_xion \\
\hline Unigene_UN_xiong_3005 & 150 & 269 UN_xiong_3005 \\
\hline Unigene_UN_xiong_8071 & 255 & 618 UN_xiong_8071 \\
\hline Unigene_GPGRCDD02G0CGB_xiong & 53 & 79 GPGRCDD02G0CGB_xion \\
\hline Unigene_UN_xiong_8703 & 66 & 32 UN_xiong_8703 \\
\hline Unigene_UN_xiong_2479 & 227 & 105 UN_xiong_2479 \\
\hline Unigene_UN_xiong_6615 & 87 & 58 UN_xiong_6615 \\
\hline Unigene_GP8I43W03GAT2R_xiong & 33 & 31 GP8I43W03GAT2R_xion \\
\hline Unigene_GR6TTJD01BBW2S_xiong & 32 & 120 GR6TTJD01BBW2S_xion \\
\hline Unigene_UN_xiong_8871 & 80 & 33 UN_xiong_8871 \\
\hline Unigene_UN_xiong_8684 & 260 & 336 UN_xiong_8684 \\
\hline Unigene_UN_xiong_1407 & 75 & 145 UN_xiong_1407 \\
\hline Unigene_UN_xiong_7020 & 231 & 79 UN_xiong_7020 \\
\hline Unigene_UN_xiong_15557 & 512 & 519 UN_xiong_15557 \\
\hline
\end{tabular}




\begin{tabular}{|c|c|c|}
\hline Unigene_GPGRCDD02FYYL7_xiong & 23 & 91 GPGRCDD02FYYL7_xion \\
\hline Unigene_UN_xiong_14253 & 184 & 196 UN_xiong_14253 \\
\hline Unigene_GP8I43W03G9H1L_xiong & 32 & 35 GP8I43W03G9H1L_xion \\
\hline Unigene_GP8I43W03G22RZ_xiong & 29 & 91 GP8I43W03G22RZ_xion \\
\hline Unigene_UN_xiong_8535 & 107 & 210 UN_xiong_8535 \\
\hline Unigene_UN_xiong_3558 & 282 & 52 UN_xiong_3558 \\
\hline Unigene_UN_xiong_10526 & 136 & 43 UN_xiong_10526 \\
\hline Unigene_UN_xiong_14937 & 583 & 180 UN_xiong_14937 \\
\hline Unigene_GR6TTJD01BSSCM_xiong & 47 & 38 GR6TTJD01BSSCM_xion \\
\hline Unigene_UN_xiong_8950 & 75 & 294 UN_xiong_8950 \\
\hline Unigene_GPGRCDD02JNNZB_xiong & 50 & 28 GPGRCDD02JNNZB_xion \\
\hline Unigene_UN_xiong_13538 & 80 & 25 UN_xiong_13538 \\
\hline Unigene_GR6TTJD01AUNWU_xiong & 38 & 78 GR6TTJD01AUNWU_xion \\
\hline Unigene_GR6TTJD01AX6WR_xiong & 36 & 28 GR6TTJD01AX6WR_xion \\
\hline Unigene_UN_xiong_4164 & 103 & 51 UN_xiong_4164 \\
\hline Unigene_GPGRCDD02GSC5F_xiong & 25 & 99 GPGRCDD02GSC5F_xion \\
\hline Unigene_UN_xiong_9300 & 194 & 160 UN_xiong_9300 \\
\hline Unigene_UN_xiong_9226 & 136 & 259 UN_xiong_9226 \\
\hline Unigene_UN_xiong_13964 & 501 & 45 UN_xiong_13964 \\
\hline Unigene_UN_xiong_6000 & 134 & 113 UN_xiong_6000 \\
\hline Unigene_UN_xiong_14206 & 3667 & 1136 UN_xiong_14206 \\
\hline Unigene_GR6TTJD01A5D3B_xiong & 25 & 40 GR6TTJD01A5D3B_xion \\
\hline Unigene_UN_xiong_3698 & 43 & 22 UN_xiong_3698 \\
\hline Unigene_GR6TTJD01ANBVC_xiong & 21 & 134 GR6TTJD01ANBVC_xion \\
\hline Unigene UN xiong 10421 & 62 & 159 UN xiong 10421 \\
\hline Unigene_UN_xiong_9908 & 355 & 111 UN_xiong_9908 \\
\hline Unigene_UN_xiong_11150 & 119 & 78 UN_xiong_11150 \\
\hline Unigene_GPGRCDD02GVGK5_xiong & 32 & 52 GPGRCDD02GVGK5_xion \\
\hline Unigene_GR6TTJD01AQMYP_xiong & 44 & 37 GR6TTJD01AQMYP_xion \\
\hline Unigene_UN_xiong_8517 & 687 & 4441 UN_xiong_8517 \\
\hline Unigene_UN_xiong_15543 & 781 & 439 UN_xiong_15543 \\
\hline Unigene_GPGRCDD02GL6VP_xiong & 37 & 33 GPGRCDD02GL6VP_xion \\
\hline Unigene_GPGRCDD02JP79T_xiong & 60 & 41 GPGRCDD02JP79T_xion \\
\hline Unigene_UN_xiong_706 & 465 & 56 UN_xiong_706 \\
\hline Unigene_GR6TTJD01AWTIP_xiong & 33 & 79 GR6TTJD01AWTIP_xion \\
\hline Unigene_UN_xiong_10204 & 192 & 37 UN_xiong_10204 \\
\hline Unigene_UN_xiong_4821 & 52 & 23 UN_xiong_4821 \\
\hline Unigene_UN_xiong_7500 & 181 & 40 UN_xiong_7500 \\
\hline Unigene_GR6TTJD01A1DC9_xiong & 43 & 27 GR6TTJD01A1DC9_xion \\
\hline Unigene_UN_xiong_12548 & 90 & 42 UN_xiong_12548 \\
\hline Unigene_UN_xiong_5103 & 56 & 137 UN_xiong_5103 \\
\hline Unigene GP8I43W03FY0S2 xiong & 25 & 161 GP8I43W03FY0S2 xion \\
\hline Unigene_UN_xiong_8456 & 114 & 123 UN_xiong_8456 \\
\hline Unigene_GPGRCDD02I8W7W_xiong & 44 & 43 GPGRCDD02I8W7W_xion \\
\hline Unigene_UN_xiong_3578 & 39 & 63 UN_xiong_3578 \\
\hline Unigene_UN xiong 12766 & 95 & 163 UN_xiong_12766 \\
\hline Unigene_UN_xiong_4889 & 98 & 57 UN_xiong_4889 \\
\hline Unigene_UN_xiong_6563 & 77 & 125 UN_xiong_6563 \\
\hline Unigene_GPGRCDD02FXNP3_xiong & 65 & 64 GPGRCDD02FXNP3_xion \\
\hline Unigene_GP8I43W03GSA1E_xiong & 25 & 19 GP8I43W03GSA1E_xion \\
\hline
\end{tabular}




\begin{tabular}{|c|c|c|}
\hline Unigene_GP8I43W03F0VHR_xiong & 27 & 395 GP8I43W03F0VHR_xion \\
\hline Unigene_GPGRCDD02HJ3A1_xiong & 33 & 29 GPGRCDD02HJ3A1_xion \\
\hline Unigene_GR6TTJD01AQK7H_xiong & 41 & 47 GR6TTJD01AQK7H_xion \\
\hline Unigene_GR6TTJD01BS57G_xiong & 25 & 23 GR6TTJD01BS57G_xion \\
\hline Unigene_UN_xiong_1243 & 47 & 27 UN_xiong_1243 \\
\hline Unigene_UN_xiong_3764 & 69 & 61 UN_xiong_3764 \\
\hline Unigene_UN_xiong_10156 & 33 & 163 UN_xiong_10156 \\
\hline Unigene_UN_xiong_13766 & 115 & 99 UN_xiong_13766 \\
\hline Unigene_UN_xiong_3342 & 165 & 56 UN_xiong_3342 \\
\hline Unigene_UN_xiong_12045 & 55 & 391 UN_xiong_12045 \\
\hline Unigene_UN_xiong_13503 & 110 & 36 UN_xiong_13503 \\
\hline Unigene_UN_xiong_12090 & 65 & 38 UN_xiong_12090 \\
\hline Unigene_GPGRCDD02G3E59_xiong & 38 & 56 GPGRCDD02G3E59_xion \\
\hline Unigene_UN_xiong_12616 & 120 & 263 UN_xiong_12616 \\
\hline Unigene_UN_xiong_14270 & 220 & 1442 UN_xiong_14270 \\
\hline Unigene_UN_xiong_15561 & 65 & 158 UN_xiong_15561 \\
\hline Unigene_UN_xiong_6451 & 124 & 93 UN_xiong_6451 \\
\hline Unigene_UN_xiong_2677 & 215 & 124 UN_xiong_2677 \\
\hline Unigene_UN_xiong_11244 & 141 & 259 UN_xiong_11244 \\
\hline Unigene_UN_xiong_14881 & 569 & 966 UN_xiong_14881 \\
\hline Unigene_UN_xiong_15310 & 93 & 33 UN_xiong_15310 \\
\hline Unigene_GPGRCDD02G8RZM_xiong & 46 & 650 GPGRCDD02G8RZM_xion \\
\hline Unigene_UN_xiong_3084 & 154 & 48 UN_xiong_3084 \\
\hline Unigene_UN_xiong_4168 & 1238 & 2348 UN_xiong_4168 \\
\hline Unigene_GR6TTJD01AHJJC_xiong & 24 & 313 GR6TTJD01AHJJC_xion \\
\hline Unigene_GR6TTJD01BQTHU_xiong & 22 & 168 GR6TTJD01BQTHU_xion \\
\hline Unigene_UN_xiong_14960 & 171 & 228 UN_xiong_14960 \\
\hline Unigene_UN_xiong_7087 & 41 & 40 UN_xiong_7087 \\
\hline Unigene_UN_xiong_3092 & 60 & 24 UN_xiong_3092 \\
\hline Unigene_UN_xiong_14334 & 1216 & 828 UN_xiong_14334 \\
\hline Unigene_GR6TTJD01A0W15_xiong & 27 & 78 GR6TTJD01A0W15_xion \\
\hline Unigene_UN_xiong_1416 & 206 & 412 UN_xiong_1416 \\
\hline Unigene_UN_xiong_1756 & 303 & 371 UN_xiong_1756 \\
\hline Unigene_UN_xiong_12155 & 68 & 52 UN_xiong_12155 \\
\hline Unigene_UN_xiong_8488 & 56 & 88 UN_xiong_8488 \\
\hline Unigene_UN_xiong_13320 & 95 & 55 UN_xiong_13320 \\
\hline Unigene_UN_xiong_7280 & 154 & 20 UN_xiong_7280 \\
\hline Unigene_GPGRCDD02FMEWN_xiong & 36 & 28 GPGRCDD02FMEWN_xion \\
\hline Unigene_UN_xiong_1982 & 269 & 100 UN_xiong_1982 \\
\hline Unigene_UN_xiong_7648 & 209 & 28 UN_xiong_7648 \\
\hline Unigene_GR6TTJD01CDEKI_xiong & 26 & 60 GR6TTJD01CDEKI_xion \\
\hline Unigene_UN_xiong_4724 & 380 & 282 UN_xiong_4724 \\
\hline Unigene_UN_xiong_9903 & 51 & 99 UN_xiong_9903 \\
\hline Unigene_UN_xiong_4440 & 42 & 38 UN_xiong_4440 \\
\hline Unigene_UN_xiong_307 & 238 & 1059 UN_xiong_307 \\
\hline Unigene_UN_xiong_2275 & 45 & 25 UN_xiong_2275 \\
\hline Unigene_UN_xiong_801 & 67 & 69 UN_xiong_801 \\
\hline Unigene_UN_xiong_3922 & 208 & 106 UN_xiong_3922 \\
\hline Unigene_UN_xiong_1119 & 76 & 25 UN_xiong_1119 \\
\hline Unigene_UN_xiong_11784 & 105 & 66 UN_xiong_11784 \\
\hline
\end{tabular}




\begin{tabular}{|c|c|c|}
\hline Unigene_GR6TTJD01BHS8B_xiong & 44 & 43 GR6TTJD01BHS8B_xion \\
\hline Unigene_UN_xiong_12272 & 51 & 27 UN_xiong_12272 \\
\hline Unigene_UN_xiong_4504 & 102 & 40 UN_xiong_4504 \\
\hline Unigene_GP8I43W03F3A82_xiong & 27 & 43 GP8I43W03F3A82_xion \\
\hline Unigene_UN_xiong_14308 & 2896 & 3434 UN_xiong_14308 \\
\hline Unigene_UN_xiong_14826 & 121 & 231 UN_xiong_14826 \\
\hline Unigene_GP8I43W03HE1E5_xiong & 21 & 360 GP8I43W03HE1E5_xion \\
\hline Unigene_UN_xiong_13015 & 204 & 258 UN_xiong_13015 \\
\hline Unigene_UN_xiong_9466 & 89 & 216 UN_xiong_9466 \\
\hline Unigene_GR6TTJD01AXQJ2_xiong & 62 & 38 GR6TTJD01AXQJ2_xion \\
\hline Unigene_UN_xiong_11687 & 151 & 116 UN_xiong_11687 \\
\hline Unigene_GR6TTJD01A1T9D_xiong & 29 & 42 GR6TTJD01A1T9D_xion \\
\hline Unigene_UN_xiong_4430 & 143 & 1933 UN_xiong_4430 \\
\hline Unigene_UN_xiong_10210 & 107 & 76 UN_xiong_10210 \\
\hline Unigene_UN_xiong_14804 & 165 & 252 UN_xiong_14804 \\
\hline Unigene_UN_xiong_562 & 129 & 294 UN_xiong_562 \\
\hline Unigene_UN_xiong_2087 & 66 & 123 UN_xiong_2087 \\
\hline Unigene_UN_xiong_7469 & 109 & 186 UN_xiong_7469 \\
\hline Unigene_UN_xiong_14083 & 157 & 29 UN_xiong_14083 \\
\hline Unigene_UN_xiong_9482 & 161 & 20 UN_xiong_9482 \\
\hline Unigene_GPGRCDD02FTRWR_xiong & 21 & 44 GPGRCDD02FTRWR_xion \\
\hline Unigene_UN_xiong_5674 & 216 & 31 UN_xiong_5674 \\
\hline Unigene_UN_xiong_15785 & 542 & 2215 UN_xiong_15785 \\
\hline Unigene_GPGRCDD02FNNXJ_xiong & 40 & 223 GPGRCDD02FNNXJ_xion \\
\hline Unigene_UN_xiong_1465 & 77 & 45 UN_xiong_1465 \\
\hline Unigene_UN_xiong_6739 & 395 & 45 UN_xiong_6739 \\
\hline Unigene_UN_xiong_8023 & 192 & 44 UN_xiong_8023 \\
\hline Unigene_UN_xiong_2554 & 42 & 965 UN_xiong_2554 \\
\hline Unigene_UN_xiong_13241 & 84 & 578 UN_xiong_13241 \\
\hline Unigene_UN_xiong_8936 & 25 & 186 UN_xiong_8936 \\
\hline Unigene_UN_xiong_10528 & 173 & 301 UN_xiong_10528 \\
\hline Unigene_UN_xiong_13198 & 78 & 111 UN_xiong_13198 \\
\hline Unigene_GPGRCDD02FV32C_xiong & 25 & 233 GPGRCDD02FV32C_xion \\
\hline Unigene_UN_xiong_9830 & 94 & 1135 UN_xiong_9830 \\
\hline Unigene_UN_xiong_8657 & 97 & 110 UN_xiong_8657 \\
\hline Unigene_UN_xiong_1326 & 105 & 541 UN_xiong_1326 \\
\hline Unigene_GP8I43W03GRU2L_xiong & 48 & 31 GP8I43W03GRU2L_xion \\
\hline Unigene_UN_xiong_8886 & 136 & 227 UN_xiong_8886 \\
\hline Unigene_GPGRCDD02GUVY2_xiong & 22 & 52 GPGRCDD02GUVY2_xion \\
\hline Unigene_UN_xiong_717 & 100 & 151 UN_xiong_717 \\
\hline Unigene_GR6TTJD01A3M52_xiong & 20 & 123 GR6TTJD01A3M52_xion \\
\hline Unigene_UN_xiong_8653 & 45 & 103 UN_xiong_8653 \\
\hline Unigene_GR6TTJD01BG2VL_xiong & 21 & 23 GR6TTJD01BG2VL_xion \\
\hline Unigene_UN_xiong_5815 & 87 & 22 UN_xiong_5815 \\
\hline Unigene_UN_xiong_3811 & 72 & 478 UN_xiong_3811 \\
\hline Unigene_UN_xiong_13437 & 138 & 75 UN_xiong_13437 \\
\hline Unigene_UN_xiong_5177 & 42 & 25 UN_xiong_5177 \\
\hline Unigene_GR6TTJD01AP7HD_xiong & 22 & 28 GR6TTJD01AP7HD_xion \\
\hline Unigene_UN_xiong_6414 & 256 & 50 UN_xiong_6414 \\
\hline Unigene_UN_xiong_4621 & 174 & 73 UN_xiong_4621 \\
\hline
\end{tabular}




\begin{tabular}{|c|c|c|}
\hline Unigene_UN_xiong_4084 & 319 & 955 UN_xiong_4084 \\
\hline Unigene_UN_xiong_3857 & 150 & 61 UN_xiong_3857 \\
\hline Unigene_UN_xiong_2497 & 68 & 71 UN_xiong_2497 \\
\hline Unigene_UN_xiong_12315 & 160 & 60 UN_xiong_12315 \\
\hline Unigene_UN_xiong_4791 & 91 & 374 UN_xiong_4791 \\
\hline Unigene_UN_xiong_4658 & 118 & 89 UN_xiong_4658 \\
\hline Unigene_UN_xiong_6286 & 88 & 43 UN_xiong_6286 \\
\hline Unigene GPGRCDD02JD3FA xiong & 95 & 69 GPGRCDD02JD3FA xion \\
\hline Unigene_UN_xiong_6704 & 247 & 41 UN_xiong_6704 \\
\hline Unigene_GPGRCDD02F77T2_xiong & 21 & 33 GPGRCDD02F77T2_xion \\
\hline Unigene_UN_xiong_683 & 94 & 75 UN_xiong_683 \\
\hline Unigene_UN_xiong_13063 & 376 & 34 UN_xiong_13063 \\
\hline Unigene_UN_xiong_2265 & 292 & 500 UN_xiong_2265 \\
\hline Unigene_UN_xiong_13269 & 162 & 133 UN_xiong_13269 \\
\hline Unigene_GPGRCDD02GRF6P_xiong & 45 & 43 GPGRCDD02GRF6P_xion \\
\hline Unigene_GPGRCDD02ISOIA_xiong & 28 & 68 GPGRCDD02IS0IA_xion \\
\hline Unigene_UN_xiong_549 & 142 & 74 UN_xiong_549 \\
\hline Unigene_UN_xiong_2958 & 266 & 1616 UN_xiong_2958 \\
\hline Unigene_UN_xiong_3988 & 104 & 43 UN_xiong_3988 \\
\hline Unigene_UN_xiong_8096 & 99 & 51 UN_xiong_8096 \\
\hline Unigene_UN_xiong_7955 & 98 & 113 UN_xiong_7955 \\
\hline Unigene_UN_xiong_4898 & 112 & 28 UN_xiong_4898 \\
\hline Unigene_UN_xiong_5424 & 391 & 53 UN_xiong_5424 \\
\hline Unigene_GR6TTJD01ANNRN_xiong & 29 & 117 GR6TTJD01ANNRN_xion \\
\hline Unigene UN xiong 9425 & 149 & 24 UN xiong 9425 \\
\hline Unigene_UN_xiong_5472 & 50 & 1641 UN_xiong_5472 \\
\hline Unigene_UN_xiong_246 & 230 & 374 UN_xiong_246 \\
\hline Unigene_GP8I43W03G0AN2_xiong & 23 & 135 GP8I43W03G0AN2_xion \\
\hline Unigene_GR6TTJD01BV4KK_xiong & 29 & 45 GR6TTJD01BV4KK_xion \\
\hline Unigene_UN_xiong_488 & 250 & 446 UN_xiong_488 \\
\hline Unigene_UN_xiong_1043 & 409 & 396 UN_xiong_1043 \\
\hline Unigene_UN_xiong_10741 & 202 & 614 UN_xiong_10741 \\
\hline Unigene_UN_xiong_1001 & 180 & 165 UN_xiong_1001 \\
\hline Unigene_UN_xiong_696 & 118 & 41 UN_xiong_696 \\
\hline Unigene_UN_xiong_821 & 80 & 25 UN_xiong_821 \\
\hline Unigene_UN_xiong_5692 & 215 & 32 UN_xiong_5692 \\
\hline Unigene_UN_xiong_12856 & 224 & 181 UN_xiong_12856 \\
\hline Unigene_UN_xiong_828 & 72 & 101 UN_xiong_828 \\
\hline Unigene_UN_xiong_2689 & 364 & 137 UN_xiong_2689 \\
\hline Unigene_UN_xiong_5120 & 81 & 69 UN_xiong_5120 \\
\hline Unigene_UN_xiong_1542 & 185 & 55 UN_xiong_1542 \\
\hline Unigene UN xiong 216 & 838 & $125 \mathrm{UN}$ xiong 216 \\
\hline Unigene_GR6TTJD01BR3BP_xiong & 28 & 59 GR6TTJD01BR3BP_xion \\
\hline Unigene_UN_xiong_4288 & 193 & 42 UN_xiong_4288 \\
\hline Unigene_UN_xiong_13634 & 60 & 714 UN_xiong_13634 \\
\hline Unigene GPGRCDD02H03IC xiong & 36 & 65 GPGRCDD02H03IC xion \\
\hline Unigene_GPGRCDD02J0CD5_xiong & 32 & 59 GPGRCDD02J0CD5_xion \\
\hline Unigene_UN_xiong_1145 & 90 & 156 UN_xiong_1145 \\
\hline Unigene_UN_xiong_4995 & 355 & 241 UN_xiong_4995 \\
\hline Unigene_UN_xiong_9365 & 145 & 82 UN_xiong_9365 \\
\hline
\end{tabular}




\begin{tabular}{|c|c|c|}
\hline Unigene_UN_xiong_14662 & 127 & 39 UN_xiong_14662 \\
\hline Unigene_UN_xiong_15445 & 95 & 43 UN_xiong_15445 \\
\hline Unigene_GR6TTJD01B3UH9_xiong & 46 & 55 GR6TTJD01B3UH9_xion \\
\hline Unigene_UN_xiong_928 & 105 & 113 UN_xiong_928 \\
\hline Unigene_UN_xiong_5650 & 237 & 29 UN_xiong_5650 \\
\hline Unigene_GPGRCDD02I3RW9_xiong & 30 & 47 GPGRCDD02I3RW9_xion \\
\hline Unigene_UN_xiong_9861 & 166 & 35 UN_xiong_9861 \\
\hline Unigene_UN_xiong_3118 & 397 & 127 UN_xiong_3118 \\
\hline Unigene_UN_xiong_12838 & 36 & 53 UN_xiong_12838 \\
\hline Unigene_UN_xiong_1868 & 59 & 140 UN_xiong_1868 \\
\hline Unigene_UN_xiong_5630 & 110 & 98 UN_xiong_5630 \\
\hline Unigene_UN_xiong_210 & 153 & 253 UN_xiong_210 \\
\hline Unigene_GP8I43W03HC1GP_xiong & 38 & 148 GP8I43W03HC1GP_xion \\
\hline Unigene_UN_xiong_3294 & 100 & 22 UN_xiong_3294 \\
\hline Unigene_UN_xiong_486 & 149 & 165 UN_xiong_486 \\
\hline Unigene_GPGRCDD02INVGD_xiong & 31 & 24 GPGRCDD02INVGD_xion \\
\hline Unigene_GP8I43W03G05KS_xiong & 23 & 23 GP8I43W03G05KS_xion \\
\hline Unigene_UN_xiong_42 & 55 & 85 UN_xiong_42 \\
\hline Unigene_UN_xiong_3502 & 40 & 130 UN_xiong_3502 \\
\hline Unigene_UN_xiong_1512 & 278 & 349 UN_xiong_1512 \\
\hline Unigene_GR6TTJD01B0PK3_xiong & 29 & 34 GR6TTJD01BOPK3_xion \\
\hline Unigene_UN_xiong_10441 & 206 & 68 UN_xiong_10441 \\
\hline Unigene_UN_xiong_2313 & 222 & 254 UN_xiong_2313 \\
\hline Unigene_UN_xiong_5560 & 52 & 87 UN_xiong_5560 \\
\hline Unigene_UN_xiong_13349 & 138 & 70 UN_xiong_13349 \\
\hline Unigene_UN_xiong_8346 & 71 & 52 UN_xiong_8346 \\
\hline Unigene_GR6TTJD01B063R_xiong & 36 & 106 GR6TTJD01B063R_xion \\
\hline Unigene_UN_xiong_5668 & 127 & 272 UN_xiong_5668 \\
\hline Unigene_UN_xiong_4066 & 57 & 29 UN_xiong_4066 \\
\hline Unigene_UN_xiong_12892 & 83 & 23 UN_xiong_12892 \\
\hline Unigene_UN_xiong_14325 & 537 & 627 UN_xiong_14325 \\
\hline Unigene_UN_xiong_5809 & 281 & 37 UN_xiong_5809 \\
\hline Unigene_UN_xiong_12097 & 63 & 25 UN_xiong_12097 \\
\hline Unigene_UN_xiong_10564 & 54 & 26 UN_xiong_10564 \\
\hline Unigene_GR6TTJD01APCMA_xiong & 30 & 53 GR6TTJD01APCMA_xion \\
\hline Unigene_UN_xiong_11431 & 216 & 28 UN_xiong_11431 \\
\hline Unigene_UN_xiong_6469 & 63 & 35 UN_xiong_6469 \\
\hline Unigene_UN_xiong_8223 & 123 & 22 UN_xiong_8223 \\
\hline Unigene_UN_xiong_15386 & 174 & 92 UN_xiong_15386 \\
\hline Unigene_UN_xiong_9609 & 119 & 228 UN_xiong_9609 \\
\hline Unigene_GP8I43W03G8MC2_xiong & 65 & 48 GP8I43W03G8MC2_xion \\
\hline Unigene_UN_xiong_14303 & 169 & 87 UN_xiong_14303 \\
\hline Unigene_UN_xiong_14314 & 355 & 108 UN_xiong_14314 \\
\hline Unigene_UN_xiong_4710 & 189 & 172 UN_xiong_4710 \\
\hline Unigene_UN_xiong_352 & 83 & 126 UN_xiong_352 \\
\hline Unigene_UN_xiong_1167 & 331 & 373 UN_xiong_1167 \\
\hline Unigene_GPGRCDD02HF8KY_xiong & 43 & 43 GPGRCDD02HF8KY_xion \\
\hline Unigene_UN_xiong_14117 & 59 & 22 UN_xiong_14117 \\
\hline Unigene_UN_xiong_9409 & 69 & 38 UN_xiong_9409 \\
\hline Unigene_GR6TTJD01AER55_xiong & 27 & 26 GR6TTJD01AER55_xion \\
\hline
\end{tabular}




\begin{tabular}{|c|c|c|}
\hline Unigene_GPGRCDD02GZ14A_xiong & 27 & 85 GPGRCDD02GZ14A_xion \\
\hline Unigene_UN_xiong_15044 & 102 & 138 UN_xiong_15044 \\
\hline Unigene_UN_xiong_1792 & 448 & 55 UN_xiong_1792 \\
\hline Unigene_UN_xiong_14084 & 55 & 125 UN_xiong_14084 \\
\hline Unigene_UN_xiong_13218 & 50 & 90 UN_xiong_13218 \\
\hline Unigene_GR6TTJD01BV7FD_xiong & 70 & 160 GR6TTJD01BV7FD_xion \\
\hline Unigene_GR6TTJD01B41WQ_xiong & 47 & 187 GR6TTJD01B41WQ_xion \\
\hline Unigene UN xiong 10084 & 107 & 139 UN_xiong_10084 \\
\hline Unigene_GPGRCDD02IKPL6_xiong & 28 & 34 GPGRCDD02IKPL6_xion \\
\hline Unigene_UN_xiong_9715 & 233 & 83 UN_xiong_9715 \\
\hline Unigene_GPGRCDD02GTQ68_xiong & 28 & 43 GPGRCDD02GTQ68_xion \\
\hline Unigene_GPGRCDD02F2WQE_xiong & 114 & 287 GPGRCDD02F2WQE_xion \\
\hline Unigene_UN_xiong_3274 & 38 & 223 UN_xiong_3274 \\
\hline Unigene_UN_xiong_12240 & 126 & 400 UN_xiong_12240 \\
\hline Unigene_UN_xiong_14454 & 289 & 410 UN_xiong_14454 \\
\hline Unigene_GP8I43W03G02Q8_xiong & 47 & 96 GP8I43W03G02Q8_xion \\
\hline Unigene_UN_xiong_14816 & 141 & 85 UN_xiong_14816 \\
\hline Unigene_UN_xiong_2324 & 210 & 359 UN_xiong_2324 \\
\hline Unigene_UN_xiong_14674 & 614 & 207 UN_xiong_14674 \\
\hline Unigene_GR6TTJD01AQRP8_xiong & 24 & 148 GR6TTJD01AQRP8_xion \\
\hline Unigene_UN_xiong_9644 & 129 & 75 UN_xiong_9644 \\
\hline Unigene_UN_xiong_8411 & 310 & 291 UN_xiong_8411 \\
\hline Unigene_UN_xiong_15604 & 103 & 30 UN_xiong_15604 \\
\hline Unigene_UN_xiong_1211 & 67 & 91 UN_xiong_1211 \\
\hline Unigene UN xiong 8117 & 315 & 1002 UN xiong 8117 \\
\hline Unigene_GPGRCDD02HVHR0_xiong & 60 & 1033 GPGRCDD02HVHR0_xion \\
\hline Unigene_UN_xiong_7743 & 172 & 380 UN_xiong_7743 \\
\hline Unigene_UN_xiong_78 & 202 & 142 UN_xiong_78 \\
\hline Unigene_UN_xiong_2119 & 455 & 202 UN_xiong_2119 \\
\hline Unigene_UN_xiong_3844 & 5482 & 4555 UN_xiong_3844 \\
\hline Unigene_UN_xiong_14873 & 571 & 207 UN_xiong_14873 \\
\hline Unigene_GPGRCDD02IVNNV_xiong & 68 & 207 GPGRCDD02IVNNV_xion \\
\hline Unigene_UN_xiong_14344 & 594 & 480 UN_xiong_14344 \\
\hline Unigene_GR6TTJD01BFI0A_xiong & 32 & 62 GR6TTJD01BFI0A_xion \\
\hline Unigene_UN_xiong_13116 & 68 & 41 UN_xiong_13116 \\
\hline Unigene_UN_xiong_13990 & 91 & 46 UN_xiong_13990 \\
\hline Unigene_UN_xiong_2956 & 2083 & 725 UN_xiong_2956 \\
\hline Unigene_UN_xiong_3503 & 343 & 241 UN_xiong_3503 \\
\hline Unigene_UN_xiong_4252 & 93 & 295 UN_xiong_4252 \\
\hline Unigene_GR6TTJD01B7QHD_xiong & 44 & 160 GR6TTJD01B7QHD_xion \\
\hline Unigene_UN_xiong_12894 & 161 & 39 UN_xiong_12894 \\
\hline Unigene_UN_xiong_7259 & 51 & 33 UN_xiong_7259 \\
\hline Unigene_UN_xiong_1087 & 63 & 763 UN_xiong_1087 \\
\hline Unigene_GR6TTJD01CDE5L_xiong & 42 & 77 GR6TTJD01CDE5L_xion \\
\hline Unigene_UN_xiong_4826 & 160 & 122 UN_xiong_4826 \\
\hline Unigene_UN xiong 11505 & 58 & 25 UN xiong 11505 \\
\hline Unigene_UN_xiong_100 & 155 & 340 UN_xiong_100 \\
\hline Unigene_UN_xiong_653 & 231 & 30 UN_xiong_653 \\
\hline Unigene_GR6TTJD01B47FG_xiong & 46 & 96 GR6TTJD01B47FG_xion \\
\hline Unigene_UN_xiong_11734 & 69 & 144 UN_xiong_11734 \\
\hline
\end{tabular}




\begin{tabular}{|c|c|c|}
\hline Unigene_UN_xiong_14906 & 169 & 177 UN_xiong_14906 \\
\hline Unigene_UN_xiong_13322 & 108 & 43 UN_xiong_13322 \\
\hline Unigene_UN_xiong_15700 & 126 & 83 UN_xiong_15700 \\
\hline Unigene_UN_xiong_1374 & 38 & 423 UN_xiong_1374 \\
\hline Unigene_GR6TTJD01BVPG5_xiong & 42 & 24 GR6TTJD01BVPG5_xion \\
\hline Unigene_UN_xiong_3124 & 112 & 54 UN_xiong_3124 \\
\hline Unigene_GP8I43W03FZRA3_xiong & 61 & 42 GP8I43W03FZRA3_xion \\
\hline Unigene_UN_xiong_1060 & 279 & 1544 UN_xiong_1060 \\
\hline Unigene_UN_xiong_2459 & 147 & 79 UN_xiong_2459 \\
\hline Unigene_UN_xiong_3090 & 116 & 55 UN_xiong_3090 \\
\hline Unigene_GPGRCDD02FYVI9_xiong & 32 & 57 GPGRCDD02FYVI9_xion \\
\hline Unigene_UN_xiong_3494 & 119 & 27 UN_xiong_3494 \\
\hline Unigene_UN_xiong_1781 & 45 & 55 UN xiong 1781 \\
\hline Unigene_UN_xiong_3028 & 53 & 28 UN_xiong_3028 \\
\hline Unigene_GPGRCDD02G6XJT_xiong & 61 & 74 GPGRCDD02G6XJT_xion \\
\hline Unigene_UN_xiong_11180 & 61 & 86 UN_xiong_11180 \\
\hline Unigene_UN_xiong_8731 & 185 & 21 UN_xiong_8731 \\
\hline Unigene_UN_xiong_14016 & 69 & 62 UN_xiong_14016 \\
\hline Unigene_GPGRCDD02G3X8H_xiong & 57 & 610 GPGRCDD02G3X8H_xion \\
\hline Unigene_GR6TTJD01BH57B_xiong & 37 & 68 GR6TTJD01BH57B_xion \\
\hline Unigene_GR6TTJD01A1Q8I_xiong & 38 & 122 GR6TTJD01A1Q8I_xion \\
\hline Unigene_UN_xiong_5954 & 88 & 81 UN_xiong_5954 \\
\hline Unigene_UN_xiong_13255 & 285 & 1070 UN_xiong_13255 \\
\hline Unigene_GPGRCDD02J22CA_xiong & 34 & 55 GPGRCDD02J22CA_xion \\
\hline Unigene_UN_xiong_465 & 1218 & 182 UN_xiong_465 \\
\hline Unigene_UN_xiong_2495 & 198 & 445 UN xiong 2495 \\
\hline Unigene_UN_xiong_8501 & 56 & 176 UN_xiong_8501 \\
\hline Unigene_UN_xiong_1804 & 91 & 35 UN_xiong_1804 \\
\hline Unigene_GR6TTJD01AHYFF_xiong & 27 & 54 GR6TTJD01AHYFF_xion \\
\hline Unigene_UN_xiong_226 & 85 & 151 UN_xiong_226 \\
\hline Unigene_UN_xiong_6054 & 59 & 175 UN_xiong_6054 \\
\hline Unigene_UN_xiong_5283 & 74 & 87 UN_xiong_5283 \\
\hline Unigene_GR6TTJD01ARMC0_xiong & 38 & 52 GR6TTJD01ARMC0_xion \\
\hline Unigene_GR6TTJD01AL4G7_xiong & 25 & 44 GR6TTJD01AL4G7_xion \\
\hline Unigene_UN_xiong_5845 & 80 & 20 UN_xiong_5845 \\
\hline Unigene_UN_xiong_40 & 167 & 235 UN_xiong_40 \\
\hline Unigene_GR6TTJD01BHJ2E_xiong & 22 & 69 GR6TTJD01BHJ2E_xion \\
\hline Unigene_UN_xiong_10048 & 53 & 131 UN_xiong_10048 \\
\hline Unigene_UN_xiong_9964 & 239 & 116 UN_xiong_9964 \\
\hline Unigene_UN_xiong_4599 & 198 & 571 UN_xiong_4599 \\
\hline Unigene_UN_xiong_12432 & 91 & 35 UN_xiong_12432 \\
\hline Unigene_GR6TTJD01CFBLW_xiong & 60 & 130 GR6TTJD01CFBLW_xion \\
\hline Unigene_UN_xiong_7807 & 101 & 25 UN_xiong_7807 \\
\hline Unigene_GPGRCDD02HS7AY_xiong & 28 & 31 GPGRCDD02HS7AY_xion \\
\hline Unigene_UN_xiong_3855 & 99 & 28 UN_xiong_3855 \\
\hline Unigene_GPGRCDD02HYMG2_xiong & 96 & 62 GPGRCDD02HYMG2_xion \\
\hline Unigene_UN_xiong_2662 & 1246 & 174 UN_xiong_2662 \\
\hline Unigene_UN_xiong_8042 & 74 & 28 UN_xiong_8042 \\
\hline Unigene_GP8I43W03GBEIK_xiong & 27 & 58 GP8I43W03GBEIK_xion \\
\hline Unigene_UN_xiong_570 & 98 & 26 UN_xiong_570 \\
\hline
\end{tabular}




\begin{tabular}{|c|c|c|}
\hline Unigene_GP8I43W03G8WZN_xiong & 24 & 20 GP8I43W03G8WZN_xion \\
\hline Unigene_UN_xiong_12519 & 187 & 187 UN_xiong_12519 \\
\hline Unigene_UN_xiong_8858 & 206 & 103 UN_xiong_8858 \\
\hline Unigene_UN_xiong_13174 & 508 & 113 UN_xiong_13174 \\
\hline Unigene_UN_xiong_5361 & 58 & 25 UN_xiong_5361 \\
\hline Unigene_UN_xiong_13870 & 412 & 58 UN_xiong_13870 \\
\hline Unigene_UN_xiong_11320 & 132 & 175 UN_xiong_11320 \\
\hline Unigene_UN_xiong_7574 & 223 & 222 UN_xiong_7574 \\
\hline Unigene_UN_xiong_12428 & 157 & 224 UN_xiong_12428 \\
\hline Unigene_GR6TTJD01APUWQ_xiong & 22 & 132 GR6TTJD01APUWQ_xion \\
\hline Unigene_UN_xiong_7266 & 78 & 121 UN_xiong_7266 \\
\hline Unigene_UN_xiong_3321 & 166 & 29 UN_xiong_3321 \\
\hline Unigene_UN_xiong_4517 & 70 & 188 UN_xiong_4517 \\
\hline Unigene_UN_xiong_98 & 2222 & 107 UN_xiong_98 \\
\hline Unigene_UN_xiong_15541 & 138 & 435 UN_xiong_15541 \\
\hline Unigene_UN_xiong_5828 & 50 & 27 UN_xiong_5828 \\
\hline Unigene_UN_xiong_843 & 82 & 21 UN_xiong_843 \\
\hline Unigene_GR6TTJD01AF6ED_xiong & 32 & 37 GR6TTJD01AF6ED_xion \\
\hline Unigene_UN_xiong_9928 & 79 & 40 UN_xiong_9928 \\
\hline Unigene_GR6TTJD01AK64Z_xiong & 24 & 391 GR6TTJD01AK64Z_xion \\
\hline Unigene_UN_xiong_12604 & 78 & 52 UN_xiong_12604 \\
\hline Unigene_GPGRCDD02I4R8J_xiong & 63 & 702 GPGRCDD02I4R8J_xion \\
\hline Unigene_UN_xiong_8638 & 59 & 22 UN_xiong_8638 \\
\hline Unigene_UN_xiong_4545 & 82 & 32 UN_xiong_4545 \\
\hline Unigene_GPGRCDD02I5V34_xiong & 51 & 99 GPGRCDD02I5V34_xion \\
\hline Unigene_UN_xiong_13095 & 381 & 55 UN_xiong_13095 \\
\hline Unigene_GP8I43W03HEKB7_xiong & 57 & 131 GP8I43W03HEKB7_xion \\
\hline Unigene_UN_xiong_4304 & 285 & 142 UN_xiong_4304 \\
\hline Unigene_UN_xiong_10123 & 97 & 147 UN_xiong_10123 \\
\hline Unigene_UN_xiong_4618 & 232 & 841 UN_xiong_4618 \\
\hline Unigene_UN_xiong_3678 & 88 & 406 UN_xiong_3678 \\
\hline Unigene_UN_xiong_3864 & 288 & 293 UN_xiong_3864 \\
\hline Unigene_GPGRCDD02IIHLA_xiong & 35 & 29 GPGRCDD02IIHLA_xion \\
\hline Unigene_UN_xiong_7329 & 44 & 43 UN_xiong_7329 \\
\hline Unigene_UN_xiong_15078 & 340 & 291 UN_xiong_15078 \\
\hline Unigene_UN_xiong_15731 & 126 & 78 UN_xiong_15731 \\
\hline Unigene_GR6TTJD01CD3A4_xiong & 27 & 44 GR6TTJD01CD3A4_xion \\
\hline Unigene_UN_xiong_8722 & 130 & 270 UN_xiong_8722 \\
\hline Unigene_UN_xiong_13798 & 72 & 23 UN_xiong_13798 \\
\hline Unigene_UN_xiong_15354 & 65 & 101 UN_xiong_15354 \\
\hline Unigene_UN_xiong_11615 & 110 & 74 UN_xiong_11615 \\
\hline Unigene_UN_xiong_12496 & 99 & 678 UN_xiong_12496 \\
\hline Unigene_UN_xiong_13023 & 75 & 30 UN_xiong_13023 \\
\hline Unigene_UN_xiong_1190 & 79 & 68 UN_xiong_1190 \\
\hline Unigene_UN_xiong_10844 & 134 & 162 UN_xiong_10844 \\
\hline Unigene_UN_xiong_5336 & 58 & 23 UN_xiong_5336 \\
\hline Unigene_UN_xiong_4976 & 316 & 111 UN_xiong_4976 \\
\hline Unigene_GR6TTJD01AY4G5_xiong & 32 & 53 GR6TTJD01AY4G5_xion \\
\hline Unigene_UN_xiong_14590 & 529 & 820 UN_xiong_14590 \\
\hline Unigene_UN_xiong_12152 & 63 & 35 UN_xiong_12152 \\
\hline
\end{tabular}




\begin{tabular}{|c|c|c|}
\hline Unigene_UN_xiong_14699 & 215 & 332 UN_xiong_14699 \\
\hline Unigene_GPGRCDD02H0BEK_xiong & 65 & 74 GPGRCDD02H0BEK_xion \\
\hline Unigene_UN_xiong_14203 & 584 & 224 UN_xiong_14203 \\
\hline Unigene_UN_xiong_8354 & 104 & 128 UN_xiong_8354 \\
\hline Unigene_GP8I43W03GBFJQ_xiong & 40 & 45 GP8I43W03GBFJQ_xion \\
\hline Unigene_UN_xiong_1881 & 267 & 205 UN_xiong_1881 \\
\hline Unigene_GR6TTJD01BW9D2_xiong & 23 & 93 GR6TTJD01BW9D2_xion \\
\hline Unigene_UN_xiong_13555 & 48 & 36 UN_xiong_13555 \\
\hline Unigene_UN_xiong_7006 & 52 & 67 UN_xiong_7006 \\
\hline Unigene_UN_xiong_7098 & 73 & 109 UN_xiong_7098 \\
\hline Unigene_UN_xiong_330 & 64 & 586 UN_xiong_330 \\
\hline Unigene_GPGRCDD02IU94D_xiong & 41 & 39 GPGRCDD02IU94D_xion \\
\hline Unigene_GR6TTJD01A0RMS_xiong & 40 & 160 GR6TTJD01A0RMS_xion \\
\hline Unigene_UN_xiong_4393 & 393 & 21 UN_xiong_4393 \\
\hline Unigene_UN_xiong_13278 & 105 & 224 UN_xiong_13278 \\
\hline Unigene_GR6TTJD01B1FX7_xiong & 87 & 175 GR6TTJD01B1FX7_xion \\
\hline Unigene_UN_xiong_9496 & 165 & 32 UN_xiong_9496 \\
\hline Unigene_UN_xiong_4215 & 123 & 77 UN_xiong_4215 \\
\hline Unigene_UN_xiong_29 & 251 & 629 UN_xiong_29 \\
\hline Unigene_UN_xiong_2445 & 51 & 41 UN_xiong_2445 \\
\hline Unigene_UN_xiong_3789 & 80 & 354 UN_xiong_3789 \\
\hline Unigene_UN_xiong_452 & 158 & 2923 UN_xiong_452 \\
\hline Unigene_UN_xiong_8693 & 60 & 28 UN_xiong_8693 \\
\hline Unigene_UN_xiong_3662 & 158 & 253 UN_xiong_3662 \\
\hline Unigene_GP8I43W03F55S9_xiong & 36 & 40 GP8I43W03F55S9_xion \\
\hline Unigene_GR6TTJD01A6DJK_xiong & 28 & 26 GR6TTJD01A6DJK_xion \\
\hline Unigene_UN_xiong_5136 & 64 & 53 UN_xiong_5136 \\
\hline Unigene_UN_xiong_3229 & 43 & 208 UN_xiong_3229 \\
\hline Unigene_UN_xiong_1703 & 279 & 83 UN_xiong_1703 \\
\hline Unigene_UN_xiong_11034 & 64 & 66 UN_xiong_11034 \\
\hline Unigene_UN_xiong_14472 & 500 & 674 UN_xiong_14472 \\
\hline Unigene_UN_xiong_11016 & 175 & 30 UN_xiong_11016 \\
\hline Unigene_UN_xiong_9265 & 58 & 32 UN_xiong_9265 \\
\hline Unigene_UN_xiong_15211 & 63 & 42 UN_xiong_15211 \\
\hline Unigene_UN_xiong_3996 & 153 & 141 UN_xiong_3996 \\
\hline Unigene_UN_xiong_15721 & 79 & 50 UN_xiong_15721 \\
\hline Unigene_UN_xiong_596 & 108 & 40 UN_xiong_596 \\
\hline Unigene_UN_xiong_9394 & 108 & 348 UN_xiong_9394 \\
\hline Unigene_UN_xiong_609 & 250 & 43 UN_xiong_609 \\
\hline Unigene_UN_xiong_2189 & 82 & 20 UN_xiong_2189 \\
\hline Unigene_UN_xiong_15686 & 51 & 140 UN_xiong_15686 \\
\hline Unigene_UN_xiong_15622 & 193 & 44 UN_xiong_15622 \\
\hline Unigene_UN_xiong_15034 & 395 & 274 UN_xiong_15034 \\
\hline Unigene_GR6TTJD01AW00J_xiong & 68 & 61 GR6TTJD01AW00J_xion \\
\hline Unigene_UN_xiong_1296 & 127 & 48 UN_xiong_1296 \\
\hline Unigene_UN_xiong_13033 & 748 & 822 UN_xiong_13033 \\
\hline Unigene_UN_xiong_7406 & 114 & 115 UN_xiong_7406 \\
\hline Unigene_GPGRCDD02GXYD5_xiong & 21 & 26 GPGRCDD02GXYD5_xion \\
\hline Unigene_UN_xiong_12237 & 198 & 303 UN_xiong_12237 \\
\hline Unigene_UN_xiong_3991 & 113 & 101 UN_xiong_3991 \\
\hline
\end{tabular}




\begin{tabular}{|c|c|c|}
\hline Unigene_GP8I43W03G3GR2_xiong & 41 & 32 GP8I43W03G3GR2_xion \\
\hline Unigene_UN_xiong_12683 & 43 & 42 UN_xiong_12683 \\
\hline Unigene_UN_xiong_2891 & 86 & 26 UN_xiong_2891 \\
\hline Unigene_UN_xiong_2106 & 129 & 84 UN_xiong_2106 \\
\hline Unigene_GR6TTJD01A2MFZ_xiong & 24 & 58 GR6TTJD01A2MFZ_xion \\
\hline Unigene_UN_xiong_11382 & 44 & 24 UN_xiong_11382 \\
\hline Unigene_UN_xiong_2571 & 46 & 217 UN_xiong_2571 \\
\hline Unigene_UN_xiong_542 & 100 & 456 UN_xiong_542 \\
\hline Unigene_UN_xiong_7832 & 158 & 522 UN_xiong_7832 \\
\hline Unigene_GR6TTJD01AWZAY_xiong & 32 & 46 GR6TTJD01AWZAY_xion \\
\hline Unigene_GR6TTJD01B5FNS_xiong & 63 & 66 GR6TTJD01B5FNS_xion \\
\hline Unigene_UN_xiong_4372 & 75 & 256 UN_xiong_4372 \\
\hline Unigene_UN_xiong_7386 & 179 & 375 UN_xiong_7386 \\
\hline Unigene_UN_xiong_1324 & 136 & 224 UN_xiong_1324 \\
\hline Unigene_GPGRCDD02JM3Z8_xiong & 29 & 23 GPGRCDD02JM3Z8_xion \\
\hline Unigene_UN_xiong_8566 & 78 & 101 UN_xiong_8566 \\
\hline Unigene_UN_xiong_13249 & 92 & 23 UN_xiong_13249 \\
\hline Unigene_UN_xiong_12556 & 282 & 48 UN_xiong_12556 \\
\hline Unigene_UN_xiong_10723 & 316 & 96 UN_xiong_10723 \\
\hline Unigene_GPGRCDD02HEFCY_xiong & 51 & 34 GPGRCDD02HEFCY_xion \\
\hline Unigene_UN_xiong_5705 & 360 & 53 UN_xiong_5705 \\
\hline Unigene_UN_xiong_15492 & 213 & 47 UN_xiong_15492 \\
\hline Unigene_UN_xiong_10710 & 111 & 192 UN_xiong_10710 \\
\hline Unigene_UN_xiong_14034 & 109 & 197 UN_xiong_14034 \\
\hline Unigene_UN_xiong_13612 & 122 & 123 UN_xiong_13612 \\
\hline Unigene_UN_xiong_4752 & 296 & 84 UN_xiong_4752 \\
\hline Unigene_GR6TTJD01BLNS9_xiong & 26 & 71 GR6TTJD01BLNS9_xion \\
\hline Unigene_UN_xiong_8091 & 160 & 38 UN_xiong_8091 \\
\hline Unigene_UN_xiong_4232 & 42 & 120 UN_xiong_4232 \\
\hline Unigene_UN_xiong_12232 & 67 & 777 UN_xiong_12232 \\
\hline Unigene_UN_xiong_2943 & 83 & 23 UN_xiong_2943 \\
\hline Unigene_UN_xiong_3658 & 306 & 78 UN_xiong_3658 \\
\hline Unigene_UN_xiong_13735 & 140 & 35 UN_xiong_13735 \\
\hline Unigene_GR6TTJD01CEZ6B_xiong & 24 & 45 GR6TTJD01CEZ6B_xion \\
\hline Unigene_UN_xiong_13180 & 93 & 86 UN_xiong_13180 \\
\hline Unigene_UN_xiong_3689 & 46 & 377 UN_xiong_3689 \\
\hline Unigene_UN_xiong_7171 & 41 & 24 UN_xiong_7171 \\
\hline Unigene_GR6TTJD01BSVN1_xiong & 24 & 22 GR6TTJD01BSVN1_xion \\
\hline Unigene_UN_xiong_13205 & 197 & 175 UN_xiong_13205 \\
\hline Unigene_GR6TTJD01B500L_xiong & 56 & 20 GR6TTJD01B500L_xion \\
\hline Unigene_UN_xiong_6984 & 195 & 31 UN_xiong_6984 \\
\hline Unigene_UN_xiong_2889 & 367 & 601 UN_xiong_2889 \\
\hline Unigene_GR6TTJD01AIFV6_xiong & 66 & 79 GR6TTJD01AIFV6_xion \\
\hline Unigene_UN_xiong_5113 & 529 & 319 UN_xiong_5113 \\
\hline Unigene_UN_xiong_14374 & 198 & 516 UN_xiong_14374 \\
\hline Unigene_UN_xiong_4403 & 148 & 25 UN_xiong_4403 \\
\hline Unigene_UN_xiong_8037 & 806 & 49 UN_xiong_8037 \\
\hline Unigene_UN_xiong_3878 & 744 & 517 UN_xiong_3878 \\
\hline Unigene_UN_xiong_9960 & 72 & 45 UN_xiong_9960 \\
\hline Unigene_UN_xiong_8143 & 251 & 492 UN_xiong_8143 \\
\hline
\end{tabular}




\begin{tabular}{|c|c|c|}
\hline Unigene_UN_xiong_3649 & 108 & 39 UN_xiong_3649 \\
\hline Unigene_UN_xiong_12554 & 48 & 22 UN_xiong_12554 \\
\hline Unigene_UN_xiong_5403 & 150 & 55 UN_xiong_5403 \\
\hline Unigene_UN_xiong_10799 & 155 & 1701 UN_xiong_10799 \\
\hline Unigene_UN_xiong_11219 & 220 & 32 UN_xiong_11219 \\
\hline Unigene_GPGRCDD02FPBBK_xiong & 64 & 322 GPGRCDD02FPBBK_xion \\
\hline Unigene_GPGRCDD02H08K8_xiong & 96 & 85 GPGRCDD02H08K8_xion \\
\hline Unigene_UN_xiong_15562 & 1158 & 109 UN_xiong_15562 \\
\hline Unigene_GR6TTJD01A8NLQ_xiong & 28 & 42 GR6TTJD01A8NLQ_xion \\
\hline Unigene_UN_xiong_14707 & 50 & 194 UN_xiong_14707 \\
\hline Unigene_UN_xiong_15366 & 60 & 55 UN_xiong_15366 \\
\hline Unigene_UN_xiong_8905 & 67 & 309 UN_xiong_8905 \\
\hline Unigene_UN_xiong_1262 & 65 & 27 UN_xiong_1262 \\
\hline Unigene_UN_xiong_11251 & 198 & 1028 UN_xiong_11251 \\
\hline Unigene_UN_xiong_12824 & 53 & 55 UN_xiong_12824 \\
\hline Unigene_UN_xiong_2829 & 258 & 158 UN_xiong_2829 \\
\hline Unigene_GP8I43W03GLPRY_xiong & 26 & 165 GP8I43W03GLPRY_xion \\
\hline Unigene_UN_xiong_2713 & 388 & 34 UN_xiong_2713 \\
\hline Unigene_UN_xiong_15065 & 199 & 240 UN_xiong_15065 \\
\hline Unigene_UN_xiong_3434 & 92 & 39 UN_xiong_3434 \\
\hline Unigene_UN_xiong_12333 & 63 & 40 UN_xiong_12333 \\
\hline Unigene_UN_xiong_8812 & 298 & 132 UN_xiong_8812 \\
\hline Unigene_GP8I43W03F9YMW_xiong & 28 & 43 GP8I43W03F9YMW_xion \\
\hline Unigene_UN_xiong_11921 & 97 & 28 UN_xiong_11921 \\
\hline Unigene_UN_xiong_9338 & 110 & 39 UN_xiong_9338 \\
\hline Unigene_UN_xiong_3780 & 134 & 162 UN_xiong_3780 \\
\hline Unigene_UN_xiong_7831 & 51 & 25 UN_xiong_7831 \\
\hline Unigene_GPGRCDD02FWPLM_xiong & 24 & 24 GPGRCDD02FWPLM_xion \\
\hline Unigene_UN_xiong_6327 & 42 & 25 UN_xiong_6327 \\
\hline Unigene_UN_xiong_1457 & 847 & 702 UN_xiong_1457 \\
\hline Unigene_UN_xiong_13275 & 76 & 308 UN_xiong_13275 \\
\hline Unigene_UN_xiong_12050 & 561 & 405 UN_xiong_12050 \\
\hline Unigene_UN_xiong_13433 & 300 & 240 UN_xiong_13433 \\
\hline Unigene_UN_xiong_3085 & 342 & 261 UN_xiong_3085 \\
\hline Unigene_UN_xiong_3397 & 107 & 246 UN_xiong_3397 \\
\hline Unigene_UN_xiong_4908 & 301 & 129 UN_xiong_4908 \\
\hline Unigene_UN_xiong_14512 & 964 & 1537 UN_xiong_14512 \\
\hline Unigene_UN_xiong_14312 & 7251 & 4154 UN_xiong_14312 \\
\hline Unigene_GP8I43W03HCAZG_xiong & 27 & 29 GP8I43W03HCAZG_xion \\
\hline Unigene_UN_xiong_13115 & 30 & 78 UN_xiong_13115 \\
\hline Unigene_UN_xiong_2396 & 83 & 243 UN_xiong_2396 \\
\hline Unigene_UN_xiong_1591 & 67 & 53 UN_xiong_1591 \\
\hline Unigene_UN_xiong_12200 & 105 & 105 UN_xiong_12200 \\
\hline Unigene_UN_xiong_3703 & 329 & 245 UN_xiong_3703 \\
\hline Unigene_UN_xiong_15516 & 127 & 206 UN_xiong_15516 \\
\hline Unigene_UN_xiong_15517 & 76 & 48 UN_xiong_15517 \\
\hline Unigene_UN_xiong_12871 & 45 & 34 UN_xiong_12871 \\
\hline Unigene_UN_xiong_3757 & 121 & 224 UN_xiong_3757 \\
\hline Unigene_GPGRCDD02HGHSX_xiong & 54 & 40 GPGRCDD02HGHSX_xion \\
\hline Unigene_UN_xiong_12384 & 197 & 366 UN_xiong_12384 \\
\hline
\end{tabular}




\begin{tabular}{|c|c|c|}
\hline Unigene_UN_xiong_12525 & 87 & 33 UN_xiong_12525 \\
\hline Unigene_UN_xiong_8617 & 99 & 34 UN_xiong_8617 \\
\hline Unigene_GR6TTJD01AW8T1_xiong & 29 & 27 GR6TTJD01AW8T1_xion \\
\hline Unigene_GP8I43W03GVIPC_xiong & 30 & 29 GP8I43W03GVIPC_xion \\
\hline Unigene_UN_xiong_13446 & 161 & 85 UN_xiong_13446 \\
\hline Unigene_GR6TTJD01BUSLS_xiong & 36 & 83 GR6TTJD01BUSLS_xion \\
\hline Unigene_UN_xiong_6051 & 128 & 30 UN_xiong_6051 \\
\hline Unigene_UN xiong 14802 & 142 & 225 UN_xiong_14802 \\
\hline Unigene_UN_xiong_4124 & 183 & 51 UN_xiong_4124 \\
\hline Unigene_UN_xiong_14035 & 392 & 268 UN_xiong_14035 \\
\hline Unigene_UN_xiong_959 & 124 & 26 UN_xiong_959 \\
\hline Unigene_GP8I43W03GHB42_xiong & 26 & 179 GP8I43W03GHB42_xion \\
\hline Unigene_GPGRCDD02HIVNH_xiong & 50 & 47 GPGRCDD02HIVNH_xion \\
\hline Unigene_GR6TTJD01ASWWK_xiong & 39 & 373 GR6TTJD01ASWWK_xion \\
\hline Unigene_UN_xiong_13125 & 102 & 43 UN_xiong_13125 \\
\hline Unigene_UN_xiong_4382 & 41 & 52 UN_xiong_4382 \\
\hline Unigene_UN_xiong_10774 & 50 & 65 UN_xiong_10774 \\
\hline Unigene_UN_xiong_3039 & 68 & 62 UN_xiong_3039 \\
\hline Unigene_UN_xiong_14024 & 195 & 66 UN_xiong_14024 \\
\hline Unigene_UN_xiong_9477 & 46 & 80 UN_xiong_9477 \\
\hline Unigene_GR6TTJD01AQPV0_xiong & 21 & 114 GR6TTJD01AQPV0_xion \\
\hline Unigene_UN_xiong_11658 & 39 & 67 UN_xiong_11658 \\
\hline Unigene_GP8I43W03F3H9P_xiong & 23 & 327 GP8I43W03F3H9P_xion \\
\hline Unigene_UN_xiong_4387 & 162 & 25 UN_xiong_4387 \\
\hline Unigene UN xiong 11151 & 81 & $48 \mathrm{UN}$ xiong 11151 \\
\hline Unigene_GR6TTJD01AGKC2_xiong & 24 & 57 GR6TTJD01AGKC2_xion \\
\hline Unigene_GR6TTJD01BHJ0N_xiong & 21 & 34 GR6TTJD01BHJ0N_xion \\
\hline Unigene_UN_xiong_9899 & 124 & 198 UN_xiong_9899 \\
\hline Unigene_GR6TTJD01BJS1Y_xiong & 41 & 22 GR6TTJD01BJS1Y_xion \\
\hline Unigene_UN_xiong_14620 & 929 & 57 UN_xiong_14620 \\
\hline Unigene_UN_xiong_14968 & 323 & 323 UN_xiong_14968 \\
\hline Unigene_GPGRCDD02FUHMZ_xiong & 55 & 86 GPGRCDD02FUHMZ_xion \\
\hline Unigene_GR6TTJD01A37VV_xiong & 24 & 55 GR6TTJD01A37VV_xion \\
\hline Unigene_UN_xiong_5553 & 80 & 70 UN_xiong_5553 \\
\hline Unigene_GP8I43W03HC664_xiong & 25 & 71 GP8I43W03HC664_xion \\
\hline Unigene_UN_xiong_4975 & 426 & 208 UN_xiong_4975 \\
\hline Unigene_GR6TTJD01A8KF9_xiong & 28 & 119 GR6TTJD01A8KF9_xion \\
\hline Unigene_UN_xiong_11983 & 92 & 76 UN_xiong_11983 \\
\hline Unigene_UN_xiong_14278 & 189 & 288 UN_xiong_14278 \\
\hline Unigene_UN_xiong_4583 & 296 & 208 UN_xiong_4583 \\
\hline Unigene UN xiong 11385 & 35 & 44 UN xiong 11385 \\
\hline Unigene_UN_xiong_9902 & 170 & 21 UN_xiong_9902 \\
\hline Unigene_UN_xiong_1456 & 55 & 32 UN_xiong_1456 \\
\hline Unigene_UN_xiong_2121 & 60 & 113 UN_xiong_2121 \\
\hline Unigene_GPGRCDD02IJ023_xiong & 95 & 522 GPGRCDD02IJ023_xion \\
\hline Unigene UN xiong 13965 & 59 & 67 UN_xiong_ 13965 \\
\hline Unigene_GP8I43W03GQ3AG_xiong & 35 & 67 GP8I43W03GQ3AG_xion \\
\hline Unigene_UN_xiong_4321 & 47 & 20 UN_xiong_4321 \\
\hline Unigene_UN_xiong_3137 & 141 & 74 UN_xiong_3137 \\
\hline Unigene_UN_xiong_4317 & 68 & 68 UN_xiong_4317 \\
\hline
\end{tabular}




\begin{tabular}{|c|c|c|}
\hline Unigene_UN_xiong_14352 & 242 & 236 UN_xiong_14352 \\
\hline Unigene_GR6TTJD01A91I7_xiong & 32 & 37 GR6TTJD01A91I7_xion \\
\hline Unigene_UN_xiong_7501 & 43 & 35 UN_xiong_7501 \\
\hline Unigene_UN_xiong_3403 & 134 & 167 UN_xiong_3403 \\
\hline Unigene_UN_xiong_14708 & 136 & 100 UN_xiong_14708 \\
\hline Unigene_UN_xiong_6847 & 42 & 65 UN_xiong_6847 \\
\hline Unigene_GPGRCDD02JJNIU_xiong & 33 & 118 GPGRCDD02JJNIU_xion \\
\hline Unigene_UN_xiong_11167 & 140 & 246 UN_xiong_11167 \\
\hline Unigene_GR6TTJD01B76KT_xiong & 26 & 115 GR6TTJD01B76KT_xion \\
\hline Unigene_GPGRCDD02I5F7G_xiong & 95 & 52 GPGRCDD02I5F7G_xion \\
\hline Unigene_GP8I43W03GRV65_xiong & 93 & 294 GP8I43W03GRV65_xion \\
\hline Unigene_UN_xiong_5750 & 47 & 141 UN_xiong_5750 \\
\hline Unigene_UN_xiong_13377 & 67 & 105 UN_xiong_13377 \\
\hline Unigene_UN_xiong_1931 & 588 & 606 UN_xiong_1931 \\
\hline Unigene_GPGRCDD02IRGXT_xiong & 22 & 46 GPGRCDD02IRGXT_xion \\
\hline Unigene_GP8I43W03FNS13_xiong & 38 & 27 GP8I43W03FNS13_xion \\
\hline Unigene_GPGRCDD02G1RK8_xiong & 24 & 29 GPGRCDD02G1RK8_xion \\
\hline Unigene_UN_xiong_14065 & 67 & 30 UN_xiong_14065 \\
\hline Unigene_GP8I43W03G8UNV_xiong & 22 & 59 GP8I43W03G8UNV_xion \\
\hline Unigene_UN_xiong_14239 & 1466 & 362 UN_xiong_14239 \\
\hline Unigene_UN_xiong_3042 & 72 & 60 UN_xiong_3042 \\
\hline Unigene_GPGRCDD02GZ9XD_xiong & 23 & 197 GPGRCDD02GZ9XD_xion \\
\hline Unigene_UN_xiong_12530 & 56 & 21 UN_xiong_12530 \\
\hline Unigene_UN_xiong_3352 & 263 & 1453 UN_xiong_3352 \\
\hline Unigene_GR6TTJD01AIFX7_xiong & 29 & 27 GR6TTJD01AIFX7_xion \\
\hline Unigene_GR6TTJD01BXIPS_xiong & 49 & 26 GR6TTJD01BXIPS_xion \\
\hline Unigene_GPGRCDD02H2XWD_xiong & 83 & 92 GPGRCDD02H2XWD_xion \\
\hline Unigene_UN_xiong_14441 & 325 & 1144 UN_xiong_14441 \\
\hline Unigene_GR6TTJD01A77CH_xiong & 72 & 72 GR6TTJD01A77CH_xion \\
\hline Unigene_GPGRCDD02HFM97_xiong & 74 & 80 GPGRCDD02HFM97_xion \\
\hline Unigene_GR6TTJD01AX8I1_xiong & 22 & 60 GR6TTJD01AX8I1_xion \\
\hline Unigene_GR6TTJD01BHZPP_xiong & 22 & 70 GR6TTJD01BHZPP_xion \\
\hline Unigene_UN_xiong_14550 & 84 & 45 UN_xiong_14550 \\
\hline Unigene_UN_xiong_10036 & 75 & 104 UN_xiong_10036 \\
\hline Unigene_UN_xiong_4239 & 129 & 33 UN_xiong_4239 \\
\hline Unigene_GR6TTJD01AUK9B_xiong & 43 & 27 GR6TTJD01AUK9B_xion \\
\hline Unigene_UN_xiong_10740 & 69 & 32 UN_xiong_10740 \\
\hline Unigene_UN_xiong_1557 & 205 & 71 UN_xiong_1557 \\
\hline Unigene_UN_xiong_14002 & 259 & 271 UN_xiong_14002 \\
\hline Unigene_UN_xiong_8937 & 187 & 64 UN_xiong_8937 \\
\hline Unigene_UN_xiong_7007 & 78 & 21 UN_xiong_7007 \\
\hline Unigene_UN_xiong_3293 & 197 & 334 UN_xiong_3293 \\
\hline Unigene_UN_xiong_9449 & 243 & 208 UN_xiong_9449 \\
\hline Unigene_UN_xiong_577 & 263 & 112 UN_xiong_577 \\
\hline Unigene_GPGRCDD02H91BW_xiong & 37 & 36 GPGRCDD02H91BW_xion \\
\hline Unigene_GR6TTJD01AY1VN_xiong & 35 & 72 GR6TTJD01AY1VN_xion \\
\hline Unigene_GR6TTJD01B6T8D_xiong & 27 & 24 GR6TTJD01B6T8D_xion \\
\hline Unigene_GR6TTJD01B44VV_xiong & 28 & 212 GR6TTJD01B44VV_xion \\
\hline Unigene_UN_xiong_2093 & 133 & 117 UN_xiong_2093 \\
\hline Unigene_UN_xiong_5686 & 99 & 85 UN_xiong_5686 \\
\hline
\end{tabular}


Unigene_UN_xiong_870

Unigene_GR6TTJD01BES4D_xiong

Unigene_UN_xiong_14887

Unigene_GR6TTJD01B8ZJR_xiong

Unigene_UN_xiong_15471

Unigene_GR6TTJD01A4LSA_xiong

Unigene_GR6TTJD01BL9BB_xiong

Unigene_UN_xiong_12016

Unigene_UN_xiong_12096

Unigene_UN_xiong_6934

Unigene_UN_xiong_8591

Unigene_UN_xiong_4607

Unigene_UN_xiong_1877

Unigene_UN_xiong_14690

Unigene_UN_xiong_3117

Unigene_UN_xiong_3637

Unigene_UN_xiong_14091

Unigene_GPGRCDD02H54YP_xiong

Unigene_UN_xiong_14593

Unigene_UN_xiong_5257

Unigene_UN_xiong_14891

Unigene_UN_xiong_4138

Unigene_GP8I43W03GWOL9_xiong

Unigene_GR6TTJD01BIMLX_xiong

Unigene_UN_xiong_1050

Unigene_UN_xiong_5460

Unigene_UN_xiong_14525

Unigene_UN_xiong_2299

Unigene_UN_xiong_2673

Unigene_UN_xiong_4877

Unigene_UN_xiong_9206

Unigene_UN_xiong_4471

Unigene_UN_xiong_10883

Unigene_UN_xiong_5944

Unigene_UN_xiong_7649

Unigene_UN_xiong_2088

Unigene_UN_xiong_1042

Unigene_UN_xiong_9010

Unigene_UN_xiong_12618

Unigene_UN_xiong_6083

Unigene_GPGRCDD02H0A92_xiong

Unigene_UN_xiong_343

Unigene_UN_xiong_14907

Unigene_UN_xiong_9129

Unigene_UN_xiong_899

Unigene_UN_xiong_7330

Unigene_UN_xiong_1511

Unigene_UN_xiong_14710

Unigene_GR6TTJD01AEDYK_xiong

Unigene_GR6TTJD01AJ8K6_xiong
35

23

243

37

125

23

27

112

80

190

543

69

92

335

96

629

191

87

686

103

820

90

25

31

54

89

1055

47

78

62

62

87

135

66

68

102

52

119

58

132

89

435

2105

72

70

154

152

283

50

37
47 UN_xiong_870

53 GR6TTJD01BES4D_xion

386 UN_xiong_14887

22 GR6TTJD01B8ZJR_xion

173 UN_xiong_15471

21 GR6TTJD01A4LSA_xion

22 GR6TTJD01BL9BB_xion

29 UN_xiong_12016

45 UN_xiong_12096

174 UN_xiong_6934

542 UN_xiong_8591

34 UN_xiong_4607

54 UN_xiong_1877

72 UN_xiong_14690

146 UN_xiong_3117

168 UN_xiong_3637

96 UN_xiong_14091

268 GPGRCDD02H54YP_xion

707 UN_xiong_14593

48 UN_xiong_5257

199 UN_xiong_14891

19 UN_xiong_4138

51 GP8I43W03GWOL9_xion

84 GR6TTJD01BIMLX_xion

57 UN_xiong_1050

93 UN_xiong_5460

1044 UN_xiong_14525

1316 UN_xiong_2299

7523 UN_xiong_2673

516 UN_xiong_4877

95 UN_xiong_9206

93 UN_xiong_4471

438 UN_xiong_10883

25 UN_xiong_5944

61 UN_xiong_7649

27 UN_xiong_2088

35 UN_xiong_1042

64 UN_xiong_9010

47 UN_xiong_12618

216 UN_xiong_6083

104 GPGRCDD02HOA92_xion

1771 UN_xiong_343

2812 UN_xiong_14907

27 UN_xiong_9129

275 UN_xiong_899

120 UN_xiong_7330

256 UN_xiong_1511

1040 UN_xiong_14710

184 GR6TTJD01AEDYK_xion

82 GR6TTJD01AJ8K6_xion 


\begin{tabular}{|c|c|c|}
\hline Unigene_UN_xiong_485 & 98 & 103 UN_xiong_485 \\
\hline Unigene_UN_xiong_7559 & 596 & 184 UN_xiong_7559 \\
\hline Unigene_GP8I43W03FSY2I_xiong & 21 & 85 GP8I43W03FSY2I_xion \\
\hline Unigene_UN_xiong_2601 & 120 & 96 UN_xiong_2601 \\
\hline Unigene_UN_xiong_5494 & 29 & 25 UN_xiong_5494 \\
\hline Unigene_UN_xiong_4036 & 403 & 136 UN_xiong_4036 \\
\hline Unigene_GR6TTJD01B9IX7_xiong & 51 & 28 GR6TTJD01B9IX7_xion \\
\hline Unigene_UN_xiong_9548 & 100 & 169 UN_xiong_9548 \\
\hline Unigene_GP8I43W03F7GGI_xiong & 54 & 96 GP8I43W03F7GGI_xion \\
\hline Unigene_UN_xiong_15676 & 196 & 102 UN_xiong_15676 \\
\hline Unigene_UN_xiong_11439 & 243 & 25 UN_xiong_11439 \\
\hline Unigene_UN_xiong_4982 & 109 & 53 UN_xiong_4982 \\
\hline Unigene_UN_xiong_6356 & 108 & 208 UN_xiong_6356 \\
\hline Unigene_UN_xiong_1072 & 73 & 1257 UN_xiong_1072 \\
\hline Unigene_UN_xiong_14784 & 551 & 642 UN_xiong_14784 \\
\hline Unigene_GPGRCDD02IFID5_xiong & 98 & 171 GPGRCDD02IFID5_xion \\
\hline Unigene_UN_xiong_1191 & 170 & 86 UN_xiong_1191 \\
\hline Unigene_GR6TTJD01AXY1Q_xiong & 206 & 21 GR6TTJD01AXY1Q_xion \\
\hline Unigene_GR6TTJD01CBSS5_xiong & 26 & 23 GR6TTJD01CBSS5_xion \\
\hline Unigene_UN_xiong_8084 & 81 & 52 UN_xiong_8084 \\
\hline Unigene_UN_xiong_13970 & 379 & 452 UN_xiong_13970 \\
\hline Unigene_GR6TTJD01CC2T2_xiong & 25 & 222 GR6TTJD01CC2T2_xion \\
\hline Unigene_GR6TTJD01BEEXC_xiong & 28 & 37 GR6TTJD01BEEXC_xion \\
\hline Unigene_UN_xiong_3372 & 669 & 347 UN_xiong_3372 \\
\hline Unigene_GPGRCDD02H8GJX_xiong & 71 & 85 GPGRCDD02H8GJX_xion \\
\hline Unigene_GR6TTJD01BPEK4_xiong & 27 & 30 GR6TTJD01BPEK4_xion \\
\hline Unigene_UN_xiong_9026 & 115 & 32 UN_xiong_9026 \\
\hline Unigene_UN_xiong_13694 & 96 & 41 UN_xiong_13694 \\
\hline Unigene_GPGRCDD02I04WR_xiong & 38 & 27 GPGRCDD02I04WR_xion \\
\hline Unigene_GR6TTJD01BBK2T_xiong & 40 & 108 GR6TTJD01BBK2T_xion \\
\hline Unigene_UN_xiong_7115 & 67 & 87 UN_xiong_7115 \\
\hline Unigene_GPGRCDD02HPMIJ_xiong & 29 & 27 GPGRCDD02HPMIJ_xion \\
\hline Unigene_UN_xiong_5175 & 76 & 135 UN_xiong_5175 \\
\hline Unigene_UN_xiong_9981 & 89 & 112 UN_xiong_9981 \\
\hline Unigene_GR6TTJD01A2ZJA_xiong & 34 & 48 GR6TTTD01A2ZJA_xion \\
\hline Unigene_UN_xiong_3175 & 59 & 113 UN_xiong_3175 \\
\hline Unigene_UN_xiong_14388 & 109 & 79 UN_xiong_14388 \\
\hline Unigene_GR6TTJD01B2TSB_xiong & 65 & 78 GR6TTJD01B2TSB_xion \\
\hline Unigene_UN_xiong_13810 & 127 & 43 UN_xiong_13810 \\
\hline Unigene_UN_xiong_11708 & 273 & 213 UN_xiong_11708 \\
\hline Unigene_UN_xiong_1785 & 114 & 477 UN_xiong_1785 \\
\hline Unigene_UN_xiong_10733 & 917 & 707 UN_xiong_10733 \\
\hline Unigene_UN_xiong_125 & 52 & 29 UN_xiong_125 \\
\hline Unigene_UN_xiong_11947 & 105 & 66 UN_xiong_11947 \\
\hline Unigene_UN_xiong_74 & 33 & 329 UN_xiong_74 \\
\hline Unigene_UN_xiong_6254 & 89 & 41 UN_xiong_6254 \\
\hline Unigene_UN_xiong_4081 & 176 & 458 UN_xiong_4081 \\
\hline Unigene_UN_xiong_2029 & 192 & 97 UN_xiong_2029 \\
\hline Unigene_UN_xiong_9067 & 87 & 33 UN_xiong_9067 \\
\hline Unigene_UN_xiong_14851 & 179 & 232 UN_xiong_14851 \\
\hline
\end{tabular}




\begin{tabular}{|c|c|c|}
\hline Unigene_GR6TTJD01BNSBS_xiong & 31 & 24 GR6TTJD01BNSBS_xion \\
\hline Unigene_UN_xiong_487 & 119 & 61 UN_xiong_487 \\
\hline Unigene_GP8I43W03G3R9E_xiong & 72 & 88 GP8I43W03G3R9E_xion \\
\hline Unigene_UN_xiong_18 & 47 & 143 UN_xiong_18 \\
\hline Unigene_GPGRCDD02GGXFH_xiong & 23 & 25 GPGRCDD02GGXFH_xion \\
\hline Unigene_UN_xiong_4343 & 248 & 62 UN_xiong_4343 \\
\hline Unigene_UN_xiong_3121 & 122 & 44 UN_xiong_3121 \\
\hline Unigene_UN_xiong_155 & 1556 & 788 UN_xiong_155 \\
\hline Unigene_UN_xiong_5520 & 72 & 174 UN_xiong_5520 \\
\hline Unigene_UN_xiong_8979 & 141 & 473 UN_xiong_8979 \\
\hline Unigene_GR6TTJD01BKF5H_xiong & 27 & 24 GR6TTJD01BKF5H_xion \\
\hline Unigene_UN_xiong_14398 & 328 & 245 UN_xiong_14398 \\
\hline Unigene_UN_xiong_6724 & 38 & 52 UN_xiong_6724 \\
\hline Unigene_GR6TTJD01A3DGJ_xiong & 33 & 65 GR6TTJD01A3DGJ_xion \\
\hline Unigene_UN_xiong_10266 & 63 & 45 UN_xiong_10266 \\
\hline Unigene_GR6TTJD01AZUQG_xiong & 21 & 21 GR6TTJD01AZUQG_xion \\
\hline Unigene_UN_xiong_12599 & 52 & 42 UN_xiong_12599 \\
\hline Unigene_GPGRCDD02ISB80_xiong & 24 & 29 GPGRCDD02ISB80_xion \\
\hline Unigene_GP8I43W03HEH9A_xiong & 31 & 37 GP8I43W03HEH9A_xion \\
\hline Unigene_UN_xiong_3153 & 236 & 86 UN_xiong_3153 \\
\hline Unigene_UN_xiong_3596 & 44 & 25 UN_xiong_3596 \\
\hline Unigene_UN_xiong_4991 & 122 & 35 UN_xiong_4991 \\
\hline Unigene_UN_xiong_2570 & 196 & 172 UN_xiong_2570 \\
\hline Unigene_GR6TTJD01BNFNX_xiong & 23 & 31 GR6TTJD01BNFNX_xion \\
\hline Unigene_GP8I43W03GRKV0_xiong & 62 & 51 GP8I43W03GRKV0_xion \\
\hline Unigene_UN_xiong_2925 & 74 & 84 UN_xiong_2925 \\
\hline Unigene_UN_xiong_14736 & 501 & 1210 UN_xiong_14736 \\
\hline Unigene_GR6TTJD01BRER0_xiong & 21 & 28 GR6TTJD01BRER0_xion \\
\hline Unigene_UN_xiong_396 & 548 & 91 UN_xiong_396 \\
\hline Unigene_GR6TTJD01ALHKP_xiong & 41 & 71 GR6TTJD01ALHKP_xion \\
\hline Unigene_UN_xiong_13685 & 58 & 67 UN_xiong_13685 \\
\hline Unigene_UN_xiong_6049 & 76 & 22 UN_xiong_6049 \\
\hline Unigene_GP8I43W03GL706_xiong & 36 & 66 GP8I43W03GL706_xion \\
\hline Unigene_UN_xiong_3245 & 155 & 78 UN_xiong_3245 \\
\hline Unigene_UN_xiong_766 & 226 & 119 UN_xiong_766 \\
\hline Unigene_GR6TTJD01BF809_xiong & 28 & 71 GR6TTJD01BF809_xion \\
\hline Unigene_GR6TTJD01BC7WF_xiong & 20 & 28 GR6TTJD01BC7WF_xion \\
\hline Unigene_UN_xiong_3277 & 52 & 30 UN_xiong_3277 \\
\hline Unigene_UN_xiong_1070 & 269 & 256 UN_xiong_1070 \\
\hline Unigene_UN_xiong_14246 & 650 & 78 UN_xiong_14246 \\
\hline Unigene_UN_xiong_3924 & 201 & 75 UN_xiong_3924 \\
\hline Unigene_GR6TTJD01B0CCT_xiong & 21 & 62 GR6TTJD01B0CCT_xion \\
\hline Unigene_UN_xiong_2306 & 46 & 60 UN_xiong_2306 \\
\hline Unigene_UN_xiong_5459 & 155 & 53 UN_xiong_5459 \\
\hline Unigene_UN_xiong_4179 & 778 & 662 UN_xiong_4179 \\
\hline Unigene_GPGRCDD02HZXFE_xiong & 29 & 59 GPGRCDD02HZXFE_xion \\
\hline Unigene_UN_xiong_4111 & 112 & 43 UN_xiong_4111 \\
\hline Unigene_UN_xiong_5638 & 439 & 48 UN_xiong_5638 \\
\hline Unigene_GR6TTJD01BRMW2_xiong & 38 & 37 GR6TTJD01BRMW2_xion \\
\hline Unigene_UN_xiong_14178 & 94 & 141 UN_xiong_14178 \\
\hline
\end{tabular}




\begin{tabular}{|c|c|c|}
\hline Unigene_UN_xiong_4401 & 602 & 204 UN_xiong_4401 \\
\hline Unigene_UN_xiong_2181 & 1021 & 564 UN_xiong_2181 \\
\hline Unigene_UN_xiong_10518 & 151 & 106 UN_xiong_10518 \\
\hline Unigene_UN_xiong_57 & 83 & 96 UN_xiong_57 \\
\hline Unigene_GR6TTJD01AJAFC_xiong & 25 & 28 GR6TTJD01AJAFC_xion \\
\hline Unigene_GR6TTJD01AZ7TY_xiong & 42 & 37 GR6TTJD01AZ7TY_xion \\
\hline Unigene_GP8I43W03GVW7F_xiong & 35 & 26 GP8I43W03GVW7F_xion \\
\hline Unigene_GP8I43W03GPV6E_xiong & 26 & 69 GP8I43W03GPV6E_xion \\
\hline Unigene_GPGRCDD02GSH9V_xiong & 31 & 61 GPGRCDD02GSH9V_xion \\
\hline Unigene_GR6TTJD01B0EAL_xiong & 35 & 45 GR6TTJD01B0EAL_xion \\
\hline Unigene_GR6TTJD01BHZC6 xiong & 30 & 41 GR6TTJD01BHZC6 xion \\
\hline Unigene_GR6TTJD01CDHL4_xiong & 40 & 42 GR6TTJD01CDHL4_xion \\
\hline Unigene_UN_xiong_7726 & 51 & 400 UN_xiong_7726 \\
\hline Unigene_GP8I43W03F591A_xiong & 62 & 131 GP8I43W03F591A_xion \\
\hline Unigene_GR6TTJD01AGU3X_xiong & 41 & 62 GR6TTJD01AGU3X_xion \\
\hline Unigene_UN_xiong_3455 & 48 & 627 UN_xiong_3455 \\
\hline Unigene_UN_xiong_11021 & 336 & 102 UN_xiong_11021 \\
\hline Unigene_UN_xiong_14488 & 184 & 1073 UN_xiong_14488 \\
\hline Unigene_UN_xiong_11973 & 47 & 74 UN_xiong_11973 \\
\hline Unigene_GR6TTJD01A0WH5_xiong & 27 & 23 GR6TTJD01A0WH5_xion \\
\hline Unigene_UN_xiong_5784 & 278 & 975 UN_xiong_5784 \\
\hline Unigene_GPGRCDD02JAU04_xiong & 75 & 286 GPGRCDD02JAU04_xion \\
\hline Unigene_UN_xiong_9552 & 233 & 3108 UN_xiong_9552 \\
\hline Unigene UN xiong 4546 & 335 & 425 UN xiong 4546 \\
\hline Unigene_UN_xiong_5580 & 175 & 167 UN_xiong_5580 \\
\hline Unigene_UN_xiong_13986 & 120 & 205 UN_xiong_13986 \\
\hline Unigene_UN_xiong_14075 & 123 & 113 UN_xiong_14075 \\
\hline Unigene UN xiong 8846 & 74 & 79 UN xiong 8846 \\
\hline Unigene_UN_xiong_14608 & 533 & 638 UN_xiong_14608 \\
\hline Unigene_GP8I43W03F0XE7_xiong & 53 & 81 GP8I43W03F0XE7_xion \\
\hline Unigene_UN_xiong_10153 & 90 & 38 UN_xiong_10153 \\
\hline Unigene_UN_xiong_1771 & 130 & 127 UN_xiong_1771 \\
\hline Unigene_UN_xiong_9106 & 48 & 126 UN_xiong_9106 \\
\hline Unigene_GR6TTJD01B08VN_xiong & 28 & 43 GR6TTJD01B08VN_xion \\
\hline Unigene_UN_xiong_13057 & 81 & 96 UN_xiong_13057 \\
\hline Unigene_UN_xiong_6009 & 66 & 63 UN_xiong_6009 \\
\hline Unigene_UN_xiong_7540 & 69 & 21 UN_xiong_7540 \\
\hline Unigene_UN_xiong_14761 & 396 & 371 UN_xiong_14761 \\
\hline Unigene_UN_xiong_6698 & 472 & 314 UN_xiong_6698 \\
\hline Unigene_GPGRCDD02ISK5G_xiong & 44 & 29 GPGRCDD02ISK5G_xion \\
\hline Unigene_UN_xiong_7806 & 153 & 26 UN_xiong_7806 \\
\hline Unigene_UN_xiong_14377 & 1386 & 46636 UN_xiong_14377 \\
\hline Unigene_GR6TTJD01BUIJ7_xiong & 22 & 59 GR6TTJD01BUIJ7_xion \\
\hline Unigene_UN_xiong_285 & 106 & 70 UN_xiong_285 \\
\hline Unigene UN xiong 12762 & 368 & 280 UN xiong_12762 \\
\hline Unigene_UN_xiong_9213 & 242 & 50 UN_xiong_9213 \\
\hline Unigene_UN_xiong_1976 & 108 & 28 UN_xiong_1976 \\
\hline Unigene_UN_xiong_15754 & 200 & 323 UN_xiong_15754 \\
\hline Unigene_UN_xiong_864 & 836 & 56 UN_xiong_864 \\
\hline Unigene_UN_xiong_3839 & 322 & 29 UN_xiong_3839 \\
\hline
\end{tabular}




\begin{tabular}{|c|c|c|}
\hline Unigene_UN_xiong_8692 & 197 & 26 UN_xiong_8692 \\
\hline Unigene_UN_xiong_7565 & 701 & 336 UN_xiong_7565 \\
\hline Unigene_UN_xiong_2646 & 164 & 30 UN_xiong_2646 \\
\hline Unigene_GP8I43W03HCOF5_xiong & 49 & 52 GP8I43W03HCOF5_xion \\
\hline Unigene_GPGRCDD02H0IA0_xiong & 23 & 21 GPGRCDD02H0IA0_xion \\
\hline Unigene_UN_xiong_10802 & 135 & 367 UN_xiong_10802 \\
\hline Unigene_GR6TTJD01B06ZH_xiong & 33 & 73 GR6TTJD01B06ZH_xion \\
\hline Unigene_UN_xiong_9575 & 78 & 45 UN_xiong_9575 \\
\hline Unigene_UN_xiong_3934 & 78 & 48 UN_xiong_3934 \\
\hline Unigene_UN_xiong_2063 & 155 & 47 UN_xiong_2063 \\
\hline Unigene_UN_xiong_13608 & 95 & 205 UN_xiong_13608 \\
\hline Unigene_GR6TTJD01A51SY_xiong & 54 & 58 GR6TTJD01A51SY_xion \\
\hline Unigene_UN_xiong_8683 & 244 & 268 UN_xiong_8683 \\
\hline Unigene_GPGRCDD02HWQFE_xiong & 29 & 22 GPGRCDD02HWQFE_xion \\
\hline Unigene_UN_xiong_2056 & 110 & 37 UN_xiong_2056 \\
\hline Unigene_UN_xiong_14158 & 110 & 51 UN_xiong_14158 \\
\hline Unigene_UN_xiong_380 & 188 & 128 UN_xiong_380 \\
\hline Unigene_UN_xiong_2039 & 61 & 55 UN_xiong_2039 \\
\hline Unigene_UN_xiong_11262 & 120 & 65 UN_xiong_11262 \\
\hline Unigene_UN_xiong_9596 & 125 & 70 UN_xiong_9596 \\
\hline Unigene_UN_xiong_13562 & 179 & 84 UN_xiong_13562 \\
\hline Unigene_UN_xiong_5252 & 64 & 65 UN_xiong_5252 \\
\hline Unigene_UN_xiong_7399 & 38 & 1045 UN_xiong_7399 \\
\hline Unigene_UN_xiong_6191 & 34 & 43 UN_xiong_6191 \\
\hline Unigene_GR6TTJD01AF5I0_xiong & 48 & 343 GR6TTJD01AF5I0_xion \\
\hline Unigene_UN_xiong_5737 & 336 & 1417 UN_xiong_5737 \\
\hline Unigene_UN_xiong_11304 & 166 & 230 UN_xiong_11304 \\
\hline Unigene_UN_xiong_212 & 75 & 41 UN_xiong_212 \\
\hline Unigene_GP8I43W03F5KHZ_xiong & 48 & 146 GP8I43W03F5KHZ_xion \\
\hline Unigene_UN_xiong_7538 & 68 & 119 UN_xiong_7538 \\
\hline Unigene_UN_xiong_5754 & 44 & 162 UN_xiong_5754 \\
\hline Unigene_UN_xiong_2109 & 209 & 52 UN_xiong_2109 \\
\hline Unigene_UN_xiong_14896 & 367 & 118 UN_xiong_14896 \\
\hline Unigene_UN_xiong_12177 & 215 & 74 UN_xiong_12177 \\
\hline Unigene_GP8I43W03GCU21_xiong & 88 & 93 GP8I43W03GCU21_xion \\
\hline Unigene_UN_xiong_434 & 99 & 50 UN_xiong_434 \\
\hline Unigene_UN_xiong_3735 & 73 & 4296 UN_xiong_3735 \\
\hline Unigene_UN_xiong_11710 & 146 & 170 UN_xiong_11710 \\
\hline Unigene_UN_xiong_15025 & 68 & 53 UN_xiong_15025 \\
\hline Unigene_UN_xiong_12102 & 56 & 169 UN_xiong_12102 \\
\hline Unigene_GR6TTJD01A40XM_xiong & 34 & 50 GR6TTJD01A40XM_xion \\
\hline Unigene_GR6TTJD01BZJJ0_xiong & 59 & 60 GR6TTJD01BZJJ0_xion \\
\hline Unigene_GR6TTJD01A087W_xiong & 74 & 58 GR6TTJD01A087W_xion \\
\hline Unigene_UN_xiong_13405 & 112 & 67 UN_xiong_13405 \\
\hline Unigene_UN_xiong_1952 & 111 & 40 UN_xiong_1952 \\
\hline Unigene_UN_xiong_2616 & 147 & 4536 UN_xiong_2616 \\
\hline Unigene_GR6TTJD01BXYY7_xiong & 20 & 35 GR6TTJD01BXYY7_xion \\
\hline Unigene_UN_xiong_11849 & 112 & 34 UN_xiong_11849 \\
\hline Unigene_GR6TTJD01BYOAF_xiong & 27 & 56 GR6TTJD01BYOAF_xion \\
\hline Unigene_GR6TTJD01CA3MQ_xiong & 31 & 32 GR6TTJD01CA3MQ_xion \\
\hline
\end{tabular}




\begin{tabular}{|c|c|c|}
\hline Unigene_GR6TTJD01B4YUZ_xiong & 25 & 35 GR6TTJD01B4YUZ_xion \\
\hline Unigene_GP8I43W03GVME5_xiong & 43 & 39 GP8I43W03GVME5_xion \\
\hline Unigene_UN_xiong_15447 & 75 & 81 UN_xiong_15447 \\
\hline Unigene_UN_xiong_3504 & 150 & 199 UN_xiong_3504 \\
\hline Unigene_GR6TTJD01BY9YW_xiong & 24 & 20 GR6TTJD01BY9YW_xion \\
\hline Unigene_UN_xiong_10706 & 278 & 63 UN_xiong_10706 \\
\hline Unigene_UN_xiong_13705 & 108 & 83 UN_xiong_13705 \\
\hline Unigene_UN_xiong_5441 & 218 & 124 UN_xiong_5441 \\
\hline Unigene_UN_xiong_14545 & 2739 & 77 UN_xiong_14545 \\
\hline Unigene_UN_xiong_14637 & 300 & 176 UN_xiong_14637 \\
\hline Unigene_UN_xiong_8267 & 52 & 28 UN_xiong_8267 \\
\hline Unigene_UN_xiong_2538 & 111 & 124 UN_xiong_2538 \\
\hline Unigene_GR6TTJD01APSAV_xiong & 23 & 23 GR6TTJD01APSAV_xion \\
\hline Unigene_UN_xiong_15383 & 2533 & 586 UN_xiong_15383 \\
\hline Unigene_UN_xiong_586 & 237 & 108 UN_xiong_586 \\
\hline Unigene_UN_xiong_8419 & 240 & 1634 UN_xiong_8419 \\
\hline Unigene_UN_xiong_5489 & 220 & 411 UN_xiong_5489 \\
\hline Unigene_UN_xiong_3215 & 121 & 76 UN_xiong_3215 \\
\hline Unigene_UN_xiong_4481 & 174 & 377 UN_xiong_4481 \\
\hline Unigene_UN_xiong_8334 & 118 & 29 UN_xiong_8334 \\
\hline Unigene_UN_xiong_7637 & 51 & 27 UN_xiong_7637 \\
\hline Unigene_UN_xiong_1201 & 520 & 303 UN_xiong_1201 \\
\hline Unigene_GR6TTJD01AD710_xiong & 28 & 43 GR6TTJD01AD710_xion \\
\hline Unigene_UN_xiong_14365 & 333 & 79 UN_xiong_14365 \\
\hline Unigene_GR6TTJD01A7UAX_xiong & 24 & 106 GR6TTJD01A7UAX_xion \\
\hline Unigene_UN_xiong_2094 & 120 & 214 UN_xiong_2094 \\
\hline Unigene_UN_xiong_9643 & 266 & 681 UN_xiong_9643 \\
\hline Unigene_GR6TTJD01BYMRA_xiong & 33 & 51 GR6TTJD01BYMRA_xion \\
\hline Unigene_UN_xiong_8960 & 117 & 295 UN_xiong_8960 \\
\hline Unigene_UN_xiong_4606 & 91 & 256 UN_xiong_4606 \\
\hline Unigene_GR6TTJD01BWYVN_xiong & 22 & 30 GR6TTJD01BWYVN_xion \\
\hline Unigene_UN_xiong_7960 & 39 & 20 UN_xiong_7960 \\
\hline Unigene_UN_xiong_15487 & 313 & 78 UN_xiong_15487 \\
\hline Unigene_UN_xiong_11502 & 179 & 187 UN_xiong_11502 \\
\hline Unigene_GP8I43W03GZM50_xiong & 31 & 69 GP8I443W03GZM50_xion \\
\hline Unigene_GR6TTJD01AT9NQ_xiong & 57 & 30 GR6TTJD01AT9NQ_xion \\
\hline Unigene_UN_xiong_1321 & 70 & 249 UN_xiong_1321 \\
\hline Unigene_UN_xiong_1162 & 103 & 120 UN_xiong_1162 \\
\hline Unigene_UN_xiong_1742 & 92 & 154 UN_xiong_1742 \\
\hline Unigene_UN_xiong_11649 & 88 & 27 UN_xiong_11649 \\
\hline Unigene_GR6TTJD01A1DAH_xiong & 23 & 295 GR6TTJD01A1DAH_xion \\
\hline Unigene_GP8I43W03GNKJZ_xiong & 51 & 29 GP8I43W03GNKJZ_xion \\
\hline Unigene_UN_xiong_13173 & 83 & 40 UN_xiong_13173 \\
\hline Unigene_UN_xiong_15374 & 794 & 349 UN_xiong_15374 \\
\hline Unigene_UN_xiong_5990 & 92 & 46 UN_xiong_5990 \\
\hline Unigene_UN_xiong_13601 & 224 & 49 UN_xiong_13601 \\
\hline Unigene_UN_xiong_14318 & 157 & 123 UN_xiong_14318 \\
\hline Unigene_UN_xiong_5723 & 338 & 23 UN_xiong_5723 \\
\hline Unigene_UN_xiong_4912 & 237 & 232 UN_xiong_4912 \\
\hline Unigene_UN_xiong_3879 & 45 & 82 UN_xiong_3879 \\
\hline
\end{tabular}




\begin{tabular}{|c|c|c|}
\hline Unigene_UN_xiong_595 & 120 & 246 UN_xiong_595 \\
\hline Unigene_GR6TTJD01A3BJG_xiong & 39 & 74 GR6TTJD01A3BJG_xion \\
\hline Unigene_UN_xiong_5859 & 112 & 56 UN_xiong_5859 \\
\hline Unigene_UN_xiong_10587 & 194 & 126 UN_xiong_10587 \\
\hline Unigene_UN_xiong_1670 & 84 & 21 UN_xiong_1670 \\
\hline Unigene_UN_xiong_12439 & 72 & 23 UN_xiong_12439 \\
\hline Unigene_UN_xiong_8216 & 71 & 45 UN_xiong_8216 \\
\hline Unigene_UN_xiong_10185 & 336 & 68 UN_xiong_10185 \\
\hline Unigene_UN_xiong_3537 & 248 & 373 UN_xiong_3537 \\
\hline Unigene_GP8I43W03F5614_xiong & 98 & 272 GP8I43W03F5614_xion \\
\hline Unigene_GR6TTJD01A21FM_xiong & 28 & 72 GR6TTJD01A21FM_xion \\
\hline Unigene_UN_xiong_12485 & 283 & 137 UN_xiong_12485 \\
\hline Unigene_UN_xiong_2863 & 138 & 33 UN_xiong_2863 \\
\hline Unigene_UN_xiong_15793 & 147 & 57 UN_xiong_15793 \\
\hline Unigene_UN_xiong_1657 & 1790 & 278 UN_xiong_1657 \\
\hline Unigene_UN_xiong_8410 & 68 & 2169 UN_xiong_8410 \\
\hline Unigene_GPGRCDD02IQ8NX_xiong & 26 & 29 GPGRCDD02IQ8NX_xion \\
\hline Unigene_UN_xiong_5536 & 65 & 73 UN_xiong_5536 \\
\hline Unigene_UN_xiong_9359 & 433 & 1569 UN_xiong_9359 \\
\hline Unigene_UN_xiong_7852 & 234 & 950 UN_xiong_7852 \\
\hline Unigene_GR6TTJD01AY8IS_xiong & 49 & 64 GR6TTJD01AY8IS_xion \\
\hline Unigene_UN_xiong_2708 & 180 & 81 UN_xiong_2708 \\
\hline Unigene_UN_xiong_10894 & 120 & 123 UN_xiong_10894 \\
\hline Unigene_GR6TTJD01BS4V3_xiong & 26 & 45 GR6TTJD01BS4V3_xion \\
\hline Unigene UN xiong 11719 & 126 & 167 UN xiong 11719 \\
\hline Unigene_UN_xiong_14295 & 90 & 25 UN_xiong_14295 \\
\hline Unigene_UN_xiong_988 & 1029 & 631 UN_xiong_988 \\
\hline Unigene_UN_xiong_12764 & 197 & 620 UN_xiong_12764 \\
\hline Unigene UN xiong 10876 & 48 & 155 UN xiong 10876 \\
\hline Unigene_UN_xiong_2210 & 155 & 82 UN_xiong_2210 \\
\hline Unigene_UN_xiong_11998 & 64 & 139 UN_xiong_11998 \\
\hline Unigene_UN_xiong_348 & 365 & 1642 UN_xiong_348 \\
\hline Unigene_UN_xiong_1214 & 256 & 24 UN_xiong_1214 \\
\hline Unigene_GR6TTJD01CCF87_xiong & 46 & 94 GR6TTJD01CCF87_xion \\
\hline Unigene_UN_xiong_12229 & 113 & 339 UN_xiong_12229 \\
\hline Unigene_UN_xiong_167 & 112 & 235 UN_xiong_167 \\
\hline Unigene_UN_xiong_12977 & 198 & 136 UN_xiong_12977 \\
\hline Unigene_GR6TTJD01AKL7B_xiong & 21 & 38 GR6TTJD01AKL7B_xion \\
\hline Unigene_UN_xiong_603 & 125 & 44 UN_xiong_603 \\
\hline Unigene_UN_xiong_9092 & 39 & 24 UN_xiong_9092 \\
\hline Unigene_UN_xiong_1692 & 191 & 47 UN_xiong_1692 \\
\hline Unigene GR6TTJD01AN5CR xiong & 59 & 82 GR6TTJD01AN5CR xion \\
\hline Unigene_GR6TTJD01BKMTD_xiong & 75 & 160 GR6TTJD01BKMTD_xion \\
\hline Unigene_GR6TTJD01B23TX_xiong & 25 & 39 GR6TTJD01B23TX_xion \\
\hline Unigene_UN_xiong_6996 & 239 & 87 UN_xiong_6996 \\
\hline Unigene UN xiong 4129 & 102 & 84 UN xiong 4129 \\
\hline Unigene_UN_xiong_12757 & 90 & 434 UN_xiong_12757 \\
\hline Unigene_GPGRCDD02F8XIS_xiong & 49 & 44 GPGRCDD02F8XIS_xion \\
\hline Unigene_UN_xiong_13017 & 341 & 59 UN_xiong_13017 \\
\hline Unigene_UN_xiong_455 & 155 & 306 UN_xiong_455 \\
\hline
\end{tabular}




\begin{tabular}{|c|c|c|}
\hline Unigene_UN_xiong_11571 & 126 & 94 UN_xiong_11571 \\
\hline Unigene_UN_xiong_355 & 136 & 245 UN_xiong_355 \\
\hline Unigene_UN_xiong_13542 & 66 & 63 UN_xiong_13542 \\
\hline Unigene_UN_xiong_13462 & 273 & 111 UN_xiong_13462 \\
\hline Unigene_UN_xiong_7974 & 169 & 165 UN_xiong_7974 \\
\hline Unigene_UN_xiong_6295 & 136 & 105 UN_xiong_6295 \\
\hline Unigene_UN_xiong_8025 & 206 & 49 UN_xiong_8025 \\
\hline Unigene_UN_xiong_6482 & 88 & 66 UN_xiong_6482 \\
\hline Unigene_UN_xiong_14263 & 814 & 341 UN_xiong_14263 \\
\hline Unigene_UN_xiong_12619 & 125 & 170 UN_xiong_12619 \\
\hline Unigene_UN_xiong_10930 & 91 & 87 UN_xiong_10930 \\
\hline Unigene_UN_xiong_4656 & 196 & 43 UN_xiong_4656 \\
\hline Unigene_GR6TTJD01A4K2E_xiong & 22 & 142 GR6TTJD01A4K2E_xion \\
\hline Unigene_GP8I43W03FR5R5_xiong & 30 & 107 GP8I43W03FR5R5_xion \\
\hline Unigene_GP8I43W03GBAIQ_xiong & 78 & 57 GP8I43W03GBAIQ_xion \\
\hline Unigene_UN_xiong_14913 & 179 & 86 UN_xiong_14913 \\
\hline Unigene_UN_xiong_9760 & 123 & 983 UN_xiong_9760 \\
\hline Unigene_UN_xiong_15592 & 126 & 118 UN_xiong_15592 \\
\hline Unigene_UN_xiong_12342 & 371 & 138 UN_xiong_12342 \\
\hline Unigene_UN_xiong_6452 & 449 & 519 UN_xiong_6452 \\
\hline Unigene_UN_xiong_10253 & 173 & 87 UN_xiong_10253 \\
\hline Unigene_UN_xiong_10537 & 84 & 43 UN_xiong_10537 \\
\hline Unigene_UN_xiong_14229 & 270 & 180 UN_xiong_14229 \\
\hline Unigene_UN_xiong_891 & 152 & 147 UN_xiong_891 \\
\hline Unigene_UN_xiong_8393 & 72 & 147 UN_xiong_8393 \\
\hline Unigene_UN_xiong_8651 & 340 & 125 UN_xiong_8651 \\
\hline Unigene_UN_xiong_1888 & 352 & 60 UN_xiong_1888 \\
\hline Unigene_GPGRCDD02F158F_xiong & 71 & 69 GPGRCDD02F158F_xion \\
\hline Unigene_UN_xiong_11562 & 121 & 184 UN_xiong_11562 \\
\hline Unigene_UN_xiong_9901 & 195 & 132 UN_xiong_9901 \\
\hline Unigene_GP8I43W03GD5UV_xiong & 29 & 149 GP8I43W03GD5UV_xion \\
\hline Unigene_UN_xiong_3960 & 130 & 37 UN_xiong_3960 \\
\hline Unigene_UN_xiong_4035 & 117 & 64 UN_xiong_4035 \\
\hline Unigene_UN_xiong_13498 & 144 & 140 UN_xiong_13498 \\
\hline Unigene_UN_xiong_752 & 89 & 139 UN_xiong_752 \\
\hline Unigene_GR6TTJD01B6750_xiong & 31 & 67 GR6TTJD01B6750_xion \\
\hline Unigene_GPGRCDD02HR3QN_xiong & 56 & 27 GPGRCDD02HR3QN_xion \\
\hline Unigene_UN_xiong_12982 & 93 & 136 UN_xiong_12982 \\
\hline Unigene_GR6TTJD01BUFBC_xiong & 35 & 88 GR6TTJD01BUFBC_xion \\
\hline Unigene_GR6TTJD01AV4RU_xiong & 55 & 42 GR6TTJD01AV4RU_xion \\
\hline Unigene_UN_xiong_2983 & 304 & 71 UN_xiong_2983 \\
\hline Unigene_GP8I43W03GIG19_xiong & 93 & 28 GP8I43W03GIG19_xion \\
\hline Unigene_UN_xiong_3983 & 116 & 86 UN_xiong_3983 \\
\hline Unigene_UN_xiong_9856 & 48 & 183 UN_xiong_9856 \\
\hline Unigene_UN_xiong_15656 & 54 & 135 UN_xiong_15656 \\
\hline Unigene_GPGRCDD02GMZLI_xiong & 68 & 75 GPGRCDD02GMZLI_xion \\
\hline Unigene_UN_xiong_319 & 191 & 184 UN_xiong_319 \\
\hline Unigene_UN_xiong_11175 & 94 & 63 UN_xiong_11175 \\
\hline Unigene_GPGRCDD02GKHGA_xiong & 73 & 71 GPGRCDD02GKHGA_xion \\
\hline Unigene_UN_xiong_14568 & 389 & 183 UN_xiong_14568 \\
\hline
\end{tabular}




\begin{tabular}{|c|c|c|}
\hline Unigene_GR6TTJD01B51C7_xiong & 36 & 32 GR6TTJD01B51C7_xion \\
\hline Unigene_GR6TTJD01B97SD_xiong & 29 & 49 GR6TTJD01B97SD_xion \\
\hline Unigene_UN_xiong_9146 & 226 & 234 UN_xiong_9146 \\
\hline Unigene_UN_xiong_6936 & 290 & 130 UN_xiong_6936 \\
\hline Unigene_GP8I43W03FLBZF_xiong & 38 & 54 GP8I43W03FLBZF_xion \\
\hline Unigene_UN_xiong_12654 & 86 & 153 UN_xiong_12654 \\
\hline Unigene_UN_xiong_3051 & 71 & 859 UN_xiong_3051 \\
\hline Unigene_UN_xiong_5690 & 66 & 48 UN_xiong_5690 \\
\hline Unigene_UN_xiong_5927 & 60 & 45 UN_xiong_5927 \\
\hline Unigene_GR6TTJD01CAEEM_xiong & 27 & 105 GR6TTJD01CAEEM_xion \\
\hline Unigene_GP8I43W03GTXKQ_xiong & 32 & 41 GP8I43W03GTXKQ_xion \\
\hline Unigene_UN_xiong_657 & 318 & 58 UN_xiong_657 \\
\hline Unigene_UN_xiong_1232 & 104 & 684 UN_xiong_1232 \\
\hline Unigene_UN_xiong_15055 & 120 & 324 UN_xiong_15055 \\
\hline Unigene_GR6TTJD01BVW85_xiong & 38 & 52 GR6TTJD01BVW85_xion \\
\hline Unigene_GP8I43W03FUAAY_xiong & 29 & 25 GP8I43W03FUAAY_xion \\
\hline Unigene_UN_xiong_2987 & 683 & 290 UN_xiong_2987 \\
\hline Unigene_GPGRCDD02JZNN6_xiong & 37 & 665 GPGRCDD02JZNN6_xion \\
\hline Unigene_UN_xiong_2360 & 412 & 452 UN_xiong_2360 \\
\hline Unigene_UN_xiong_14340 & 112 & 64 UN_xiong_14340 \\
\hline Unigene_UN_xiong_12679 & 420 & 946 UN_xiong_12679 \\
\hline Unigene_UN_xiong_13873 & 75 & 50 UN_xiong_13873 \\
\hline Unigene_GPGRCDD02JLCPW_xiong & 41 & 25 GPGRCDD02JLCPW_xion \\
\hline Unigene_UN_xiong_13384 & 175 & 59 UN_xiong_13384 \\
\hline Unigene_UN_xiong_13695 & 51 & 22 UN_xiong_13695 \\
\hline Unigene_UN_xiong_3829 & 72 & 86 UN_xiong_3829 \\
\hline Unigene_UN_xiong_12794 & 72 & 25 UN_xiong_12794 \\
\hline Unigene_UN_xiong_2609 & 61 & 61 UN_xiong_2609 \\
\hline Unigene_UN_xiong_13725 & 137 & 181 UN_xiong_13725 \\
\hline Unigene_UN_xiong_10735 & 116 & 56 UN_xiong_10735 \\
\hline Unigene_GPGRCDD02HHQRY_xiong & 22 & 50 GPGRCDD02HHQRY_xion \\
\hline Unigene_UN_xiong_5474 & 163 & 45 UN_xiong_5474 \\
\hline Unigene_UN_xiong_11352 & 68 & 166 UN_xiong_11352 \\
\hline Unigene_UN_xiong_2687 & 125 & 246 UN_xiong_2687 \\
\hline Unigene_UN_xiong_4803 & 202 & 19 UN_xiong_4803 \\
\hline Unigene_GR6TTJD01B0HU2_xiong & 32 & 51 GR6TTJD01B0HU2_xion \\
\hline Unigene_UN_xiong_6210 & 443 & 171 UN_xiong_6210 \\
\hline Unigene_UN_xiong_5028 & 126 & 81 UN_xiong_5028 \\
\hline Unigene_GPGRCDD02HE7WY_xiong & 31 & 40 GPGRCDD02HE7WY_xion \\
\hline Unigene_UN_xiong_1305 & 94 & 57 UN_xiong_1305 \\
\hline Unigene_GPGRCDD02HML6C_xiong & 37 & 94 GPGRCDD02HML6C_xion \\
\hline Unigene_UN_xiong_7587 & 37 & 90 UN_xiong_7587 \\
\hline Unigene_UN_xiong_5310 & 206 & 196 UN_xiong_5310 \\
\hline Unigene_GR6TTJD01AIX9U_xiong & 42 & 64 GR6TTJD01AIX9U_xion \\
\hline Unigene_UN_xiong_14632 & 222 & 188 UN_xiong_14632 \\
\hline Unigene_UN_xiong_6552 & 49 & 21 UN_xiong_6552 \\
\hline Unigene_UN_xiong_14399 & 246 & 190 UN_xiong_14399 \\
\hline Unigene_UN_xiong_7451 & 78 & 388 UN_xiong_7451 \\
\hline Unigene_UN_xiong_1049 & 214 & 71 UN_xiong_1049 \\
\hline Unigene_UN_xiong_13971 & 107 & 96 UN_xiong_13971 \\
\hline
\end{tabular}




\begin{tabular}{|c|c|c|}
\hline Unigene_UN_xiong_8451 & 110 & 131 UN_xiong_8451 \\
\hline Unigene_GPGRCDD02GSVDQ_xiong & 26 & 284 GPGRCDD02GSVDQ_xion \\
\hline Unigene_UN_xiong_7746 & 170 & 25 UN_xiong_7746 \\
\hline Unigene_UN_xiong_8530 & 1935 & 1396 UN_xiong_8530 \\
\hline Unigene_UN_xiong_397 & 103 & 66 UN_xiong_397 \\
\hline Unigene_UN_xiong_3186 & 103 & 127 UN_xiong_3186 \\
\hline Unigene_UN_xiong_3886 & 149 & 915 UN_xiong_3886 \\
\hline Unigene_GP8I43W03GQWMR_xiong & 34 & 110 GP8I43W03GQWMR_xion \\
\hline Unigene_UN_xiong_15434 & 204 & 1984 UN_xiong_15434 \\
\hline Unigene_UN_xiong_491 & 141 & 400 UN_xiong_491 \\
\hline Unigene_GR6TTJD01B8MML_xiong & 53 & 54 GR6TTJD01B8MML_xion \\
\hline Unigene_GP8I43W03GSCIX_xiong & 52 & 78 GP8I43W03GSCIX_xion \\
\hline Unigene_UN_xiong_2319 & 87 & 101 UN_xiong_2319 \\
\hline Unigene_UN_xiong_14546 & 1672 & 495 UN_xiong_14546 \\
\hline Unigene_UN_xiong_12390 & 637 & 1276 UN_xiong_12390 \\
\hline Unigene_UN_xiong_1304 & 125 & 84 UN_xiong_1304 \\
\hline Unigene_UN_xiong_14660 & 1300 & 319 UN_xiong_14660 \\
\hline Unigene_UN_xiong_9332 & 82 & 24 UN_xiong_9332 \\
\hline Unigene_GR6TTJD01A0AE7_xiong & 25 & 45 GR6TTJD01A0AE7_xion \\
\hline Unigene_UN_xiong_1478 & 1338 & 775 UN_xiong_1478 \\
\hline Unigene_GR6TTJD01BHVEI_xiong & 27 & 282 GR6TTJD01BHVEI_xion \\
\hline Unigene_UN_xiong_12872 & 135 & 29 UN_xiong_12872 \\
\hline Unigene_UN_xiong_9833 & 164 & 25 UN_xiong_9833 \\
\hline Unigene_UN_xiong_557 & 393 & 246 UN_xiong_557 \\
\hline Unigene_UN_xiong_3575 & 78 & 62 UN_xiong_3575 \\
\hline Unigene_UN_xiong_7246 & 71 & 31 UN_xiong_7246 \\
\hline Unigene_GR6TTJD01APU9L_xiong & 99 & 112 GR6TTJD01APU9L_xion \\
\hline Unigene_GR6TTJD01AJY40_xiong & 26 & 25 GR6TTJD01AJY40_xion \\
\hline Unigene_UN_xiong_9138 & 62 & 131 UN_xiong_9138 \\
\hline Unigene_UN_xiong_1488 & 118 & 130 UN_xiong_1488 \\
\hline Unigene_UN_xiong_15235 & 328 & 2209 UN_xiong_15235 \\
\hline Unigene_UN_xiong_3234 & 72 & 25 UN_xiong_3234 \\
\hline Unigene_GPGRCDD02IY69Y_xiong & 49 & 105 GPGRCDD02IY69Y_xion \\
\hline Unigene_GP8I43W03F0DBU_xiong & 25 & 70 GP8I43W03F0DBU_xion \\
\hline Unigene_UN_xiong_14230 & 78 & 350 UN_xiong_14230 \\
\hline Unigene_GR6TTJD01AF6W7_xiong & 34 & 30 GR6TTJD01AF6W7_xion \\
\hline Unigene_UN_xiong_2622 & 351 & 442 UN_xiong_2622 \\
\hline Unigene_UN_xiong_508 & 60 & 31 UN_xiong_508 \\
\hline Unigene_UN_xiong_6084 & 241 & 102 UN_xiong_6084 \\
\hline Unigene_UN_xiong_3776 & 339 & 108 UN_xiong_3776 \\
\hline Unigene_UN_xiong_585 & 69 & 21 UN_xiong_585 \\
\hline Unigene_UN_xiong_7069 & 265 & 77 UN_xiong_7069 \\
\hline Unigene_GPGRCDD02G3Q7R_xiong & 25 & 62 GPGRCDD02G3Q7R_xion \\
\hline Unigene_UN_xiong_5776 & 72 & 48 UN_xiong_5776 \\
\hline Unigene_UN_xiong_14731 & 266 & 234 UN_xiong_14731 \\
\hline Unigene_UN_xiong_1704 & 1053 & 44 UN_xiong_1704 \\
\hline Unigene_GP8I43W03F20ZR_xiong & 28 & 86 GP8I43W03F20ZR_xion \\
\hline Unigene_UN_xiong_2887 & 432 & 55 UN_xiong_2887 \\
\hline Unigene_UN_xiong_14791 & 606 & 1279 UN_xiong_14791 \\
\hline Unigene_UN_xiong_9279 & 94 & 89 UN_xiong_9279 \\
\hline
\end{tabular}




\begin{tabular}{|c|c|c|}
\hline Unigene_GR6TTJD01ANB23_xiong & 23 & 21 GR6TTJD01ANB23_xion \\
\hline Unigene_GR6TTJD01A1KYR_xiong & 31 & 59 GR6TTJD01A1KYR_xion \\
\hline Unigene_GR6TTJD01BUU57_xiong & 87 & 120 GR6TTJD01BUU57_xion \\
\hline Unigene_UN_xiong_6041 & 99 & 43 UN_xiong_6041 \\
\hline Unigene_GPGRCDD02FZ756_xiong & 28 & 85 GPGRCDD02FZ756_xion \\
\hline Unigene_GR6TTJD01AFKL6_xiong & 26 & 30 GR6TTJD01AFKL6_xion \\
\hline Unigene_UN_xiong_1246 & 217 & 156 UN_xiong_1246 \\
\hline Unigene_UN_xiong_11895 & 55 & 106 UN_xiong_11895 \\
\hline Unigene_UN_xiong_7717 & 190 & 42 UN_xiong_7717 \\
\hline Unigene_UN_xiong_7934 & 33 & 165 UN_xiong_7934 \\
\hline Unigene_GR6TTJD01AFU20_xiong & 34 & 49 GR6TTJD01AFU20_xion \\
\hline Unigene_UN_xiong_628 & 106 & 72 UN_xiong_628 \\
\hline Unigene_UN_xiong_15178 & 164 & 70 UN_xiong_15178 \\
\hline Unigene_UN_xiong_10777 & 63 & 40 UN_xiong_10777 \\
\hline Unigene_UN_xiong_10029 & 159 & 62 UN_xiong_10029 \\
\hline Unigene_UN_xiong_1570 & 777 & 2591 UN_xiong_1570 \\
\hline Unigene_UN_xiong_6646 & 92 & 48 UN_xiong_6646 \\
\hline Unigene_GPGRCDD02G800S_xiong & 49 & 139 GPGRCDD02G800S_xion \\
\hline Unigene_GR6TTJD01A9FQJ_xiong & 93 & 81 GR6TTJD01A9FQJ_xion \\
\hline Unigene_UN_xiong_1812 & 529 & 1527 UN_xiong_1812 \\
\hline Unigene_GP8I43W03GDTVW_xiong & 39 & 25 GP8I43W03GDTVW_xion \\
\hline Unigene_UN_xiong_15041 & 360 & 53 UN_xiong_15041 \\
\hline Unigene_UN_xiong_11986 & 192 & 95 UN_xiong_11986 \\
\hline Unigene_GR6TTJD01BW76I_xiong & 44 & 43 GR6TTJD01BW76I_xion \\
\hline Unigene_UN_xiong_8067 & 167 & 41 UN_xiong_8067 \\
\hline Unigene_GR6TTJD01B77WJ_xiong & 23 & 23 GR6TTJD01B77WJ_xion \\
\hline Unigene_GPGRCDD02HKYJ3_xiong & 32 & 41 GPGRCDD02HKYJ3_xion \\
\hline Unigene_GP8I43W03F29L4_xiong & 28 & 71 GP8I43W03F29L4_xion \\
\hline Unigene_UN_xiong_7443 & 122 & 1581 UN_xiong_7443 \\
\hline Unigene_GR6TTJD01BRVRQ_xiong & 22 & 24 GR6TTJD01BRVRQ_xion \\
\hline Unigene_GPGRCDD02JLIQT_xiong & 35 & 61 GPGRCDD02JLIQT_xion \\
\hline Unigene_GPGRCDD02JOKQ1_xiong & 70 & 224 GPGRCDD02J0KQ1_xion \\
\hline Unigene_UN_xiong_4398 & 133 & 185 UN_xiong_4398 \\
\hline Unigene_UN_xiong_3944 & 65 & 117 UN_xiong_3944 \\
\hline Unigene_UN_xiong_5326 & 75 & 120 UN_xiong_5326 \\
\hline Unigene_UN_xiong_4707 & 265 & 286 UN_xiong_4707 \\
\hline Unigene_UN_xiong_9379 & 92 & 68 UN_xiong_9379 \\
\hline Unigene_GP8I43W03GWRCN_xiong & 38 & 145 GP8I43W03GWRCN_xion \\
\hline Unigene_UN_xiong_8292 & 56 & 58 UN_xiong_8292 \\
\hline Unigene_UN_xiong_4148 & 80 & 47 UN_xiong_4148 \\
\hline Unigene_UN_xiong_5551 & 51 & 22 UN_xiong_5551 \\
\hline Unigene_UN_xiong_9949 & 112 & 227 UN_xiong_9949 \\
\hline Unigene_GPGRCDD02HLSC6_xiong & 43 & 29 GPGRCDD02HLSC6_xion \\
\hline Unigene_UN_xiong_15193 & 674 & 39 UN_xiong_15193 \\
\hline Unigene_GPGRCDD02HJUSG_xiong & 35 & 42 GPGRCDD02HJUSG_xion \\
\hline Unigene_GPGRCDD02I8SK5_xiong & 19 & 51 GPGRCDD02I8SK5_xion \\
\hline Unigene_UN_xiong_13171 & 56 & 27 UN_xiong_13171 \\
\hline Unigene_GPGRCDD02IJAHI_xiong & 49 & 26 GPGRCDD02IJAHI_xion \\
\hline Unigene_GR6TTJD01BX4U3_xiong & 26 & 135 GR6TTJD01BX4U3_xion \\
\hline Unigene_UN_xiong_15667 & 200 & 285 UN_xiong_15667 \\
\hline
\end{tabular}




\begin{tabular}{|c|c|c|}
\hline Unigene_GPGRCDD02F81FJ_xiong & 30 & 121 GPGRCDD02F81FJ_xion \\
\hline Unigene_GPGRCDD02F92SX_xiong & 32 & 63 GPGRCDD02F92SX_xion \\
\hline Unigene_UN_xiong_5132 & 260 & 305 UN_xiong_5132 \\
\hline Unigene_GR6TTJD01AF1VJ_xiong & 29 & 46 GR6TTJD01AF1VJ_xion \\
\hline Unigene_UN_xiong_812 & 207 & 96 UN_xiong_812 \\
\hline Unigene_GR6TTJD01ASSVX_xiong & 36 & 70 GR6TTJD01ASSVX_xion \\
\hline Unigene_UN_xiong_1786 & 223 & 49 UN_xiong_1786 \\
\hline Unigene_GPGRCDD02GV1NZ_xiong & 27 & 314 GPGRCDD02GV1NZ_xion \\
\hline Unigene_UN_xiong_15403 & 270 & 341 UN_xiong_15403 \\
\hline Unigene_UN_xiong_4952 & 181 & 350 UN_xiong_4952 \\
\hline Unigene_GPGRCDD02HP1YF_xiong & 98 & 215 GPGRCDD02HP1YF_xion \\
\hline Unigene_UN_xiong_379 & 208 & 84 UN_xiong_379 \\
\hline Unigene_UN_xiong_15020 & 303 & 131 UN_xiong_15020 \\
\hline Unigene_UN_xiong_14237 & 112 & 84 UN_xiong_14237 \\
\hline Unigene_UN_xiong_12492 & 78 & 51 UN_xiong_12492 \\
\hline Unigene_UN_xiong_1920 & 316 & 270 UN_xiong_1920 \\
\hline Unigene_UN_xiong_15664 & 209 & 2414 UN_xiong_15664 \\
\hline Unigene_UN_xiong_14251 & 865 & 1054 UN_xiong_14251 \\
\hline Unigene_UN_xiong_11663 & 100 & 50 UN_xiong_11663 \\
\hline Unigene_GPGRCDD02F2AJ5_xiong & 27 & 77 GPGRCDD02F2AJ5_xion \\
\hline Unigene_UN_xiong_6525 & 233 & 101 UN_xiong_6525 \\
\hline Unigene_GPGRCDD02GC6F7_xiong & 30 & 33 GPGRCDD02GC6F7_xion \\
\hline Unigene_UN_xiong_6618 & 53 & 65 UN_xiong_6618 \\
\hline Unigene_UN_xiong_6908 & 65 & 41 UN_xiong_6908 \\
\hline Unigene_UN_xiong_1386 & 113 & 21 UN_xiong_1386 \\
\hline Unigene_UN_xiong_4219 & 312 & 33 UN_xiong_4219 \\
\hline Unigene_UN_xiong_11339 & 64 & 70 UN_xiong_11339 \\
\hline Unigene_GR6TTJD01ASAS8_xiong & 74 & 65 GR6TTJD01ASAS8_xion \\
\hline Unigene_GR6TTJD01ASN4A_xiong & 30 & 139 GR6TTJD01ASN4A_xion \\
\hline Unigene_UN_xiong_1241 & 255 & 549 UN_xiong_1241 \\
\hline Unigene_UN_xiong_3466 & 38 & 314 UN_xiong_3466 \\
\hline Unigene_UN_xiong_7289 & 172 & 50 UN_xiong_7289 \\
\hline Unigene_UN_xiong_14224 & 409 & 365 UN_xiong_14224 \\
\hline Unigene_UN_xiong_4183 & 157 & 112 UN_xiong_4183 \\
\hline Unigene_UN_xiong_3521 & 859 & 2102 UN_xiong_3521 \\
\hline Unigene_GR6TTJD01A2MAF_xiong & 36 & 52 GR6TTJD01A2MAF_xion \\
\hline Unigene_GPGRCDD02ICRIK_xiong & 26 & 216 GPGRCDD02ICRIK_xion \\
\hline Unigene_GP8I43W03GZDIW_xiong & 28 & 95 GP8I43W03GZDIW_xion \\
\hline Unigene_UN_xiong_9586 & 220 & 121 UN_xiong_9586 \\
\hline Unigene_GR6TTJD01AYKG0_xiong & 22 & 26 GR6TTJD01AYKG0_xion \\
\hline Unigene_UN_xiong_9218 & 60 & 349 UN_xiong_9218 \\
\hline Unigene_UN_xiong_14285 & 2489 & 1939 UN_xiong_14285 \\
\hline Unigene_UN_xiong_4314 & 79 & 22 UN_xiong_4314 \\
\hline Unigene_UN_xiong_3544 & 1234 & 77 UN_xiong_3544 \\
\hline Unigene_GP8I43W03FI1R0_xiong & 30 & 30 GP8I43W03FI1R0_xion \\
\hline Unigene_UN_xiong_14215 & 446 & 64 UN_xiong_14215 \\
\hline Unigene_UN_xiong_9652 & 636 & 1141 UN_xiong_9652 \\
\hline Unigene_GP8I43W03G07QB_xiong & 61 & 78 GP8I43W03G07QB_xion \\
\hline Unigene_UN_xiong_11580 & 330 & 303 UN_xiong_11580 \\
\hline Unigene_GP8I43W03G52Z6_xiong & 50 & 43 GP8I43W03G52Z6_xion \\
\hline
\end{tabular}




\begin{tabular}{|c|c|c|}
\hline Unigene_UN_xiong_9881 & 82 & 330 UN_xiong_9881 \\
\hline Unigene_UN_xiong_5449 & 46 & 91 UN_xiong_5449 \\
\hline Unigene_UN_xiong_2191 & 189 & 288 UN_xiong_2191 \\
\hline Unigene_UN_xiong_13092 & 274 & 202 UN_xiong_13092 \\
\hline Unigene_GPGRCDD02I70LB_xiong & 30 & 27 GPGRCDD02I70LB_xion \\
\hline Unigene_GR6TTJD01BNA4S_xiong & 21 & 62 GR6TTJD01BNA4S_xion \\
\hline Unigene_UN_xiong_2188 & 66 & 45 UN_xiong_2188 \\
\hline Unigene_UN_xiong_14801 & 681 & 437 UN_xiong_14801 \\
\hline Unigene_GR6TTJD01AQ5ZW_xiong & 61 & 25 GR6TTJD01AQ5ZW_xion \\
\hline Unigene_GPGRCDD02JCNPC_xiong & 28 & 157 GPGRCDD02JCNPC_xion \\
\hline Unigene_GR6TTJD01BAEUX_xiong & 26 & 87 GR6TTJD01BAEUX_xion \\
\hline Unigene_UN_xiong_10905 & 247 & 357 UN_xiong_10905 \\
\hline Unigene_UN_xiong_7905 & 199 & 84 UN_xiong_7905 \\
\hline Unigene_UN_xiong_7361 & 90 & 60 UN_xiong_7361 \\
\hline Unigene_UN_xiong_4162 & 79 & 195 UN_xiong_4162 \\
\hline Unigene_UN_xiong_1378 & 132 & 42 UN_xiong_1378 \\
\hline Unigene_UN_xiong_248 & 88 & 179 UN_xiong_248 \\
\hline Unigene_GPGRCDD02JL05R_xiong & 52 & 49 GPGRCDD02JL05R_xion \\
\hline Unigene_UN_xiong_1850 & 1314 & 67 UN_xiong_1850 \\
\hline Unigene_UN_xiong_804 & 248 & 21 UN_xiong_804 \\
\hline Unigene_UN_xiong_1124 & 73 & 39 UN_xiong_1124 \\
\hline Unigene_UN_xiong_1259 & 256 & 119 UN_xiong_1259 \\
\hline Unigene_UN_xiong_9214 & 95 & 100 UN_xiong_9214 \\
\hline Unigene_UN_xiong_13142 & 261 & 47 UN_xiong_13142 \\
\hline Unigene_UN_xiong_3823 & 593 & 43 UN_xiong_3823 \\
\hline Unigene_GR6TTJD01BL9ST_xiong & 27 & 45 GR6TTJD01BL9ST_xion \\
\hline Unigene_UN_xiong_13906 & 74 & 26 UN_xiong_13906 \\
\hline Unigene_UN_xiong_8286 & 265 & 131 UN_xiong_8286 \\
\hline Unigene_UN_xiong_3384 & 606 & 475 UN_xiong_3384 \\
\hline Unigene_UN_xiong_261 & 231 & 54 UN_xiong_261 \\
\hline Unigene_UN_xiong_2716 & 33 & 21 UN_xiong_2716 \\
\hline Unigene_GPGRCDD02GGCUC_xiong & 66 & 74 GPGRCDD02GGCUC_xion \\
\hline Unigene_UN_xiong_14838 & 325 & 227 UN_xiong_14838 \\
\hline Unigene_GR6TTJD01AFCUA_xiong & 27 & 80 GR6TTJD01AFCUA_xion \\
\hline Unigene_UN_xiong_8123 & 42 & 31 UN_xiong_8123 \\
\hline Unigene_GR6TTJD01A03J6_xiong & 24 & 39 GR6TTJD01A03J6_xion \\
\hline Unigene_UN_xiong_9886 & 93 & 26 UN_xiong_9886 \\
\hline Unigene_UN_xiong_2825 & 163 & 168 UN_xiong_2825 \\
\hline Unigene_UN_xiong_15755 & 962 & 1446 UN_xiong_15755 \\
\hline Unigene_GP8I43W03G8QY2_xiong & 30 & 149 GP8I43W03G8QY2_xion \\
\hline Unigene_UN_xiong_10445 & 61 & 144 UN_xiong_10445 \\
\hline Unigene_UN_xiong_3675 & 494 & 221 UN_xiong_3675 \\
\hline Unigene_GR6TTJD01BJ42K_xiong & 28 & 27 GR6TTJD01BJ42K_xion \\
\hline Unigene_UN_xiong_9105 & 4634 & 3671 UN_xiong_9105 \\
\hline Unigene_GR6TTJD01A1UKL_xiong & 29 & 169 GR6TTJD01A1UKL_xion \\
\hline Unigene_UN_xiong_10838 & 136 & 202 UN_xiong_10838 \\
\hline Unigene_UN_xiong_4413 & 73 & 141 UN_xiong_4413 \\
\hline Unigene_UN_xiong_14992 & 104 & 3118 UN_xiong_14992 \\
\hline Unigene_UN_xiong_10854 & 118 & 51 UN_xiong_10854 \\
\hline Unigene_GR6TTJD01A4BD8_xiong & 22 & 40 GR6TTJD01A4BD8_xion \\
\hline
\end{tabular}




\begin{tabular}{|c|c|c|}
\hline Unigene_UN_xiong_9553 & 2174 & 91 UN_xiong_9553 \\
\hline Unigene_GR6TTJD01B70A0_xiong & 105 & 55 GR6TTJD01B70A0_xion \\
\hline Unigene_UN_xiong_5938 & 73 & 70 UN_xiong_5938 \\
\hline Unigene_UN_xiong_11417 & 110 & 114 UN_xiong_11417 \\
\hline Unigene_GPGRCDD02H9T8Q_xiong & 32 & 116 GPGRCDD02H9T8Q_xion \\
\hline Unigene_UN_xiong_6851 & 56 & 44 UN_xiong_6851 \\
\hline Unigene_UN_xiong_13557 & 538 & 59 UN_xiong_13557 \\
\hline Unigene_UN_xiong_3334 & 90 & 23 UN_xiong_3334 \\
\hline Unigene_UN_xiong_14376 & 826 & 94 UN_xiong_14376 \\
\hline Unigene_UN_xiong_6815 & 28 & 118 UN_xiong_6815 \\
\hline Unigene_UN_xiong_4642 & 265 & 264 UN_xiong_4642 \\
\hline Unigene_GR6TTJD01BKE87_xiong & 41 & 25 GR6TTJD01BKE87_xion \\
\hline Unigene_UN xiong 1011 & 97 & 501 UN_xiong 1011 \\
\hline Unigene_GP8I43W03GL1VD_xiong & 28 & 125 GP8I43W03GL1VD_xion \\
\hline Unigene_UN_xiong_15191 & 510 & 623 UN_xiong_15191 \\
\hline Unigene_UN_xiong_825 & 355 & 131 UN_xiong_825 \\
\hline Unigene_UN_xiong_12916 & 104 & 676 UN_xiong_12916 \\
\hline Unigene_GR6TTJD01A6VM5_xiong & 41 & 51 GR6TTJD01A6VM5_xion \\
\hline Unigene_GR6TTJD01AXJ1X_xiong & 62 & 62 GR6TTJD01AXJ1X_xion \\
\hline Unigene_UN_xiong_8066 & 46 & 23 UN_xiong_8066 \\
\hline Unigene_GR6TTJD01A80YI_xiong & 21 & 21 GR6TTJD01A80YI_xion \\
\hline Unigene_GR6TTJD01BQEHV_xiong & 58 & 138 GR6TTJD01BQEHV_xion \\
\hline Unigene_GP8I43W03GSFMW_xiong & 58 & 48 GP8I43W03GSFMW_xion \\
\hline Unigene_UN_xiong_6672 & 448 & 308 UN_xiong_6672 \\
\hline Unigene_UN_xiong_3383 & 85 & 337 UN_xiong_3383 \\
\hline Unigene_GR6TTJD01A0SZV_xiong & 38 & 85 GR6TTJD01A0SZV_xion \\
\hline Unigene_GPGRCDD02G9L30_xiong & 79 & 79 GPGRCDD02G9L30_xion \\
\hline Unigene_UN_xiong_3885 & 366 & 50 UN_xiong_3885 \\
\hline Unigene_UN_xiong_15479 & 61 & 179 UN_xiong_15479 \\
\hline Unigene_UN_xiong_2440 & 65 & 131 UN_xiong_2440 \\
\hline Unigene_UN_xiong_11303 & 125 & 47 UN_xiong_11303 \\
\hline Unigene_UN_xiong_5889 & 92 & 53 UN_xiong_5889 \\
\hline Unigene_GP8I43W03GL46Q_xiong & 33 & 28 GP8I43W03GL46Q_xion \\
\hline Unigene_GPGRCDD02HQLDD_xiong & 26 & 209 GPGRCDD02HQLDD_xion \\
\hline Unigene_UN_xiong_2057 & 246 & 175 UN_xiong_2057 \\
\hline Unigene_UN_xiong_4518 & 318 & 90 UN_xiong_4518 \\
\hline Unigene_UN_xiong_13224 & 165 & 84 UN_xiong_13224 \\
\hline Unigene_UN_xiong_3510 & 31 & 167 UN_xiong_3510 \\
\hline Unigene_GP8I43W03G17JX_xiong & 24 & 46 GP8I43W03G17JX_xion \\
\hline Unigene_UN_xiong_2314 & 52 & 54 UN_xiong_2314 \\
\hline Unigene_UN_xiong_13526 & 199 & 65 UN_xiong_13526 \\
\hline Unigene_UN_xiong_7089 & 92 & 172 UN_xiong_7089 \\
\hline Unigene_UN_xiong_6196 & 84 & 69 UN_xiong_6196 \\
\hline Unigene_UN_xiong_12059 & 78 & 59 UN_xiong_12059 \\
\hline Unigene_UN_xiong_13394 & 223 & 153 UN_xiong_13394 \\
\hline Unigene_UN_xiong_13144 & 185 & 366 UN_xiong_13144 \\
\hline Unigene_GPGRCDD02F2B90_xiong & 56 & 77 GPGRCDD02F2B90_xion \\
\hline Unigene_UN_xiong_1837 & 45 & 87 UN_xiong_1837 \\
\hline Unigene_GR6TTJD01A7JEM_xiong & 28 & 121 GR6TTJD01A7JEM_xion \\
\hline Unigene_UN_xiong_1317 & 70 & 192 UN_xiong_1317 \\
\hline
\end{tabular}


Unigene_UN_xiong_24 54

Unigene_GR6TTJD01BWCLX_xiong 28

Unigene_UN_xiong_2419 48

Unigene_UN_xiong_8445 80

Unigene_UN_xiong_2797 258

Unigene_UN_xiong_5164 61

Unigene_UN_xiong_1586 927

Unigene_UN_xiong_2103 312

Unigene_GPGRCDD02F4VFP_xiong 23

Unigene_UN_xiong_13396 143

Unigene_UN_xiong_12881 46

Unigene_UN_xiong_5385 132

Unigene_GPGRCDD02F1EGQ_xiong 42

Unigene_UN_xiong_12024 274

Unigene_UN_xiong_5138 42

Unigene_UN_xiong_1706 81

Unigene_UN_xiong_853 238

Unigene_GP8I43W03FUQV0_xiong 28

Unigene_UN_xiong_5592 174

Unigene UN xiong_15105 4711

Unigene_UN_xiong_11516 209

Unigene_UN_xiong_3244 258

Unigene_UN_xiong_7456 121

Unigene_UN_xiong_2837 99

Unigene_UN_xiong_14141 104

Unigene_UN_xiong_5662

Unigene_GR6TTJD01B00KN_xiong

Unigene_UN_xiong_14225

Unigene_UN_xiong_8793

Unigene_GR6TTJD01AGBVM_xiong 69

Unigene_UN_xiong_2218 213

Unigene_UN_xiong_12279 130

Unigene_UN_xiong_12248 82

Unigene_UN_xiong_122 661

Unigene_GP8I43W03FR5MS_xiong 55

Unigene_GR6TTJD01ATWM0_xiong 20

Unigene_UN_xiong_6917 82

Unigene_UN_xiong_4652 37

Unigene_GP8I43W03GEJSL_xiong 28

Unigene_UN_xiong_7616 81

Unigene_UN_xiong_2165 216

Unigene_UN_xiong_7790 167

Unigene_GR6TTJD01AJ4SS_xiong 21

Unigene_GPGRCDD02HJA5Z_xiong 37

Unigene_UN_xiong_8140 441

Unigene_UN_xiong_3787 195

Unigene_UN_xiong_8082 302

Unigene_GP8I43W03GTP00_xiong 28

Unigene_UN_xiong_15071 207

Unigene_GR6TTJD01A1VVT_xiong 39
87 UN_xiong_24

208 GR6TTJD01BWCLX_xion

91 UN_xiong_2419

1408 UN_xiong_8445

135 UN_xiong_2797

21 UN_xiong_5164

807 UN_xiong_1586

50 UN_xiong_2103

26 GPGRCDD02F4VFP_xion

240 UN_xiong_13396

28 UN_xiong_12881

24 UN_xiong_5385

66 GPGRCDD02F1EGQ_xion

86 UN_xiong_12024

119 UN_xiong_5138

536 UN_xiong_1706

547 UN_xiong_853

48 GP8I43W03FUQV0_xion

72 UN_xiong_5592

2446 UN_xiong_15105

103 UN_xiong_11516

92 UN_xiong_3244

34 UN_xiong_7456

76 UN_xiong_2837

126 UN_xiong_14141

149 UN xiong 5662

84 GR6TTJD01B00KN_xion

24 UN_xiong_14225

196 UN_xiong_8793

49 GR6TTJD01AGBVM_xion

1139 UN xiong 2218

71 UN_xiong_12279

73 UN_xiong_12248

2915 UN_xiong_122

61 GP8I43W03FR5MS_xion

130 GR6TTJD01ATWM0_xion

93 UN_xiong_6917

280 UN_xiong_4652

29 GP8I43W03GEJSL_xion

108 UN_xiong_7616

244 UN_xiong_2165

19 UN_xiong_7790

63 GR6TTJD01AJ4SS_xion

178 GPGRCDD02HJA5Z_xion

507 UN_xiong_8140

1564 UN_xiong_3787

103 UN_xiong_8082

101 GP8I43W03GTP00_xion

46 UN_xiong_15071

39 GR6TTJD01A1VVT_xion 


\begin{tabular}{|c|c|c|}
\hline Unigene_UN_xiong_6623 & 108 & 23 UN_xiong_6623 \\
\hline Unigene_UN_xiong_4795 & 253 & 2864 UN_xiong_4795 \\
\hline Unigene_UN_xiong_7727 & 109 & 36 UN_xiong_7727 \\
\hline Unigene_UN_xiong_5964 & 52 & 60 UN_xiong_5964 \\
\hline Unigene_UN_xiong_11693 & 45 & 95 UN_xiong_11693 \\
\hline Unigene_GPGRCDD02GGX3A_xiong & 58 & 100 GPGRCDD02GGX3A_xion \\
\hline Unigene_UN_xiong_10610 & 205 & 196 UN_xiong_10610 \\
\hline Unigene_UN_xiong_2305 & 88 & 219 UN_xiong_2305 \\
\hline Unigene_UN_xiong_11592 & 143 & 89 UN_xiong_11592 \\
\hline Unigene_UN_xiong_10971 & 108 & 42 UN_xiong_10971 \\
\hline Unigene_UN_xiong_150 & 312 & 2165 UN_xiong_150 \\
\hline Unigene_UN_xiong_14440 & 1775 & 1689 UN_xiong_14440 \\
\hline Unigene_UN_xiong_110 & 112 & 265 UN_xiong_110 \\
\hline Unigene_UN_xiong_2730 & 88 & 192 UN_xiong_2730 \\
\hline Unigene_UN_xiong_4683 & 79 & 50 UN_xiong_4683 \\
\hline Unigene_UN_xiong_10154 & 214 & 22 UN_xiong_10154 \\
\hline Unigene_UN_xiong_6282 & 108 & 42 UN_xiong_6282 \\
\hline Unigene_UN_xiong_14588 & 99 & 182 UN_xiong_14588 \\
\hline Unigene_UN_xiong_5217 & 70 & 26 UN_xiong_5217 \\
\hline Unigene_GPGRCDD02IURKV_xiong & 28 & 104 GPGRCDD02IURKV_xion \\
\hline Unigene_UN_xiong_947 & 191 & 245 UN_xiong_947 \\
\hline Unigene_GR6TTJD01ATSDD_xiong & 51 & 56 GR6TTJD01ATSDD_xion \\
\hline Unigene_UN_xiong_10027 & 2497 & 1155 UN_xiong_10027 \\
\hline Unigene_UN_xiong_14011 & 95 & 32 UN_xiong_14011 \\
\hline Unigene_UN_xiong_8577 & 47 & 27 UN_xiong_8577 \\
\hline Unigene_UN_xiong_2858 & 79 & 44 UN_xiong_2858 \\
\hline Unigene_UN_xiong_6480 & 121 & 57 UN_xiong_6480 \\
\hline Unigene_UN_xiong_3597 & 353 & 24 UN_xiong_3597 \\
\hline Unigene_UN_xiong_5941 & 535 & 225 UN_xiong_5941 \\
\hline Unigene_GPGRCDD02F470T_xiong & 64 & 91 GPGRCDD02F470T_xion \\
\hline Unigene_UN_xiong_9309 & 97 & 180 UN_xiong_9309 \\
\hline Unigene_UN_xiong_10942 & 295 & 220 UN_xiong_10942 \\
\hline Unigene_UN_xiong_978 & 50 & 56 UN_xiong_978 \\
\hline Unigene_UN_xiong_8426 & 65 & 39 UN_xiong_8426 \\
\hline Unigene_UN_xiong_1329 & 400 & 404 UN_xiong_1329 \\
\hline Unigene_UN_xiong_6788 & 77 & 322 UN_xiong_6788 \\
\hline Unigene_UN_xiong_6686 & 85 & 46 UN_xiong_6686 \\
\hline Unigene_UN_xiong_3399 & 35 & 24 UN_xiong_3399 \\
\hline Unigene_UN_xiong_11914 & 88 & 389 UN_xiong_11914 \\
\hline Unigene_GPGRCDD02HUQY9_xiong & 42 & 39 GPGRCDD02HUQY9_xion \\
\hline Unigene_UN_xiong_6187 & 40 & 104 UN_xiong_6187 \\
\hline Unigene_UN_xiong_10607 & 57 & 27 UN_xiong_10607 \\
\hline Unigene_UN_xiong_7697 & 62 & 37 UN_xiong_7697 \\
\hline Unigene_UN_xiong_141 & 150 & 1702 UN_xiong_141 \\
\hline Unigene_UN_xiong_1085 & 87 & 1622 UN_xiong_1085 \\
\hline Unigene_GP8I43W03GR792_xiong & 59 & 58 GP8I43W03GR792_xion \\
\hline Unigene_UN_xiong_14846 & 121 & 332 UN_xiong_14846 \\
\hline Unigene_UN_xiong_5956 & 88 & 115 UN_xiong_5956 \\
\hline Unigene_UN_xiong_1528 & 215 & 129 UN_xiong_1528 \\
\hline Unigene_GR6TTJD01A92NG_xiong & 50 & 36 GR6TTJD01A92NG_xion \\
\hline
\end{tabular}




\begin{tabular}{|c|c|c|}
\hline Unigene_UN_xiong_12852 & 102 & 362 UN_xiong_12852 \\
\hline Unigene_UN_xiong_14918 & 266 & 93 UN_xiong_14918 \\
\hline Unigene_UN_xiong_499 & 113 & 356 UN_xiong_499 \\
\hline Unigene_UN_xiong_2849 & 999 & 62 UN_xiong_2849 \\
\hline Unigene_GPGRCDD02FWTUC_xiong & 36 & 48 GPGRCDD02FWTUC_xion \\
\hline Unigene_UN_xiong_999 & 107 & 153 UN_xiong_999 \\
\hline Unigene_UN_xiong_2747 & 132 & 227 UN_xiong_2747 \\
\hline Unigene_GP8I43W03FNJEG_xiong & 42 & 41 GP8I43W03FNJEG_xion \\
\hline Unigene_UN_xiong_61 & 84 & 78 UN_xiong_61 \\
\hline Unigene_UN_xiong_14848 & 493 & 1041 UN_xiong_14848 \\
\hline Unigene_GPGRCDD02IH19S_xiong & 75 & 68 GPGRCDD02IH19S_xion \\
\hline Unigene_GPGRCDD02H57IS_xiong & 27 & 26 GPGRCDD02H57IS_xion \\
\hline Unigene_UN_xiong_5507 & 214 & 47 UN_xiong_5507 \\
\hline Unigene_UN_xiong_6296 & 77 & 83 UN_xiong_6296 \\
\hline Unigene_UN_xiong_15395 & 63 & 125 UN_xiong_15395 \\
\hline Unigene_UN_xiong_15681 & 237 & 27 UN_xiong_15681 \\
\hline Unigene_GR6TTJD01B7IIM_xiong & 22 & 28 GR6TTJD01B7IIM_xion \\
\hline Unigene_UN_xiong_11332 & 269 & 366 UN_xiong_11332 \\
\hline Unigene_UN_xiong_6997 & 67 & 47 UN_xiong_6997 \\
\hline Unigene_UN_xiong_6076 & 79 & 43 UN_xiong_6076 \\
\hline Unigene_UN_xiong_10010 & 339 & 120 UN_xiong_10010 \\
\hline Unigene_UN_xiong_9093 & 42 & 178 UN_xiong_9093 \\
\hline Unigene_UN_xiong_7918 & 100 & 115 UN_xiong_7918 \\
\hline Unigene_GPGRCDD02HRLLW_xiong & 24 & 23 GPGRCDD02HRLLW_xion \\
\hline Unigene_UN_xiong_1857 & 109 & 468 UN_xiong_1857 \\
\hline Unigene_GP8I43W03FMQ1T_xiong & 40 & 203 GP8I43W03FMQ1T_xion \\
\hline Unigene_UN_xiong_15032 & 466 & 4948 UN_xiong_15032 \\
\hline Unigene_UN_xiong_7053 & 57 & 74 UN_xiong_7053 \\
\hline Unigene_UN_xiong_9132 & 49 & 65 UN_xiong_9132 \\
\hline Unigene_UN_xiong_9624 & 90 & 25 UN_xiong_9624 \\
\hline Unigene_GR6TTJD01B5LQD_xiong & 43 & 111 GR6TTJD01B5LQD_xion \\
\hline Unigene_GR6TTJD01BD0QX_xiong & 49 & 172 GR6TTJD01BD0QX_xion \\
\hline Unigene_GR6TTJD01B0ETR_xiong & 28 & 78 GR6TTJD01B0ETR_xion \\
\hline Unigene_UN_xiong_10654 & 87 & 25 UN_xiong_10654 \\
\hline Unigene_UN_xiong_4464 & 50 & 288 UN_xiong_4464 \\
\hline Unigene_UN_xiong_1197 & 120 & 25 UN_xiong_1197 \\
\hline Unigene_UN_xiong_2991 & 97 & 2899 UN_xiong_2991 \\
\hline Unigene_UN_xiong_8198 & 91 & 22 UN_xiong_8198 \\
\hline Unigene_UN_xiong_14718 & 246 & 12389 UN_xiong 14718 \\
\hline Unigene_GR6TTJD01BZHF4_xiong & 45 & 38 GR6TTJD01BZHF4_xion \\
\hline Unigene_UN_xiong_10082 & 53 & 95 UN_xiong_10082 \\
\hline Unigene_UN_xiong_5186 & 201 & 124 UN_xiong_5186 \\
\hline Unigene_GP8I43W03HB8X3_xiong & 100 & 56 GP8I43W03HB8X3_xion \\
\hline Unigene_UN_xiong_7772 & 99 & 88 UN_xiong_7772 \\
\hline Unigene_UN_xiong_10414 & 369 & 336 UN_xiong_10414 \\
\hline Unigene_UN_xiong_4029 & 45 & 68 UN_xiong_4029 \\
\hline Unigene_UN_xiong_8829 & 34 & 85 UN_xiong_8829 \\
\hline Unigene_UN_xiong_12443 & 100 & 72 UN_xiong_12443 \\
\hline Unigene_GPGRCDD02GCOCL_xiong & 26 & 80 GPGRCDD02GCOCL_xion \\
\hline Unigene_UN_xiong_1765 & 151 & 45 UN_xiong_1765 \\
\hline
\end{tabular}




\begin{tabular}{|c|c|c|}
\hline Unigene_UN_xiong_14748 & 55 & 73 UN_xiong_14748 \\
\hline Unigene_UN_xiong_9292 & 53 & 136 UN_xiong_9292 \\
\hline Unigene_UN_xiong_10886 & 172 & 439 UN_xiong_10886 \\
\hline Unigene_UN_xiong_13684 & 77 & 37 UN_xiong_13684 \\
\hline Unigene_GR6TTJD01BQB1P_xiong & 33 & 109 GR6TTJD01BQB1P_xion \\
\hline Unigene_UN_xiong_14814 & 128 & 220 UN_xiong_14814 \\
\hline Unigene_UN_xiong_10058 & 125 & 23 UN_xiong_10058 \\
\hline Unigene_GR6TTJD01BDGRA_xiong & 25 & 71 GR6TTJD01BDGRA_xion \\
\hline Unigene_UN_xiong_14486 & 560 & 629 UN_xiong_14486 \\
\hline Unigene_UN_xiong_7465 & 426 & 121 UN_xiong_7465 \\
\hline Unigene_UN_xiong_4265 & 61 & 71 UN_xiong_4265 \\
\hline Unigene_UN_xiong_13480 & 5608 & 8578 UN_xiong_13480 \\
\hline Unigene_UN_xiong_11858 & 81 & 238 UN_xiong_11858 \\
\hline Unigene_UN_xiong_1348 & 126 & 325 UN_xiong_1348 \\
\hline Unigene_UN_xiong_2615 & 130 & 77 UN_xiong_2615 \\
\hline Unigene_UN_xiong_11182 & 119 & 86 UN_xiong_11182 \\
\hline Unigene_UN_xiong_7320 & 37 & 99 UN_xiong_7320 \\
\hline Unigene_UN_xiong_13257 & 116 & 55 UN_xiong_13257 \\
\hline Unigene_UN_xiong_15337 & 569 & 156 UN_xiong_15337 \\
\hline Unigene_UN_xiong_2832 & 304 & 91 UN_xiong_2832 \\
\hline Unigene_UN_xiong_14059 & 209 & 161 UN_xiong_14059 \\
\hline Unigene_UN_xiong_1743 & 402 & 500 UN_xiong_1743 \\
\hline Unigene_UN_xiong_4004 & 124 & 46 UN_xiong_4004 \\
\hline Unigene_UN_xiong_386 & 4364 & 2000 UN_xiong_386 \\
\hline Unigene_GR6TTJD01CCLGV_xiong & 47 & 187 GR6TTTJD01CCLGV_xion \\
\hline Unigene_UN_xiong_3900 & 274 & 60 UN_xiong_3900 \\
\hline Unigene_GP8I43W03F80ZJ_xiong & 29 & 54 GP8I43W03F80ZJ_xion \\
\hline Unigene_UN_xiong_10019 & 108 & 105 UN_xiong_10019 \\
\hline Unigene_UN_xiong_14567 & 2813 & 1157 UN_xiong_14567 \\
\hline Unigene_GPGRCDD02GBX8H_xiong & 21 & 34 GPGRCDD02GBX8H_xion \\
\hline Unigene_UN_xiong_2251 & 299 & 933 UN_xiong_2251 \\
\hline Unigene_GPGRCDD02GI27W_xiong & 20 & 28 GPGRCDD02GI27W_xion \\
\hline Unigene_UN_xiong_15705 & 88 & 112 UN_xiong_15705 \\
\hline Unigene_UN_xiong_3142 & 588 & 960 UN_xiong_3142 \\
\hline Unigene_UN_xiong_12646 & 56 & 228 UN_xiong_12646 \\
\hline Unigene_UN_xiong_6714 & 70 & 68 UN_xiong_6714 \\
\hline Unigene_UN_xiong_2091 & 161 & 27 UN_xiong_2091 \\
\hline Unigene_UN_xiong_10344 & 198 & 37 UN_xiong_10344 \\
\hline Unigene_UN_xiong_7062 & 131 & 38 UN_xiong_7062 \\
\hline Unigene_UN_xiong_14684 & 271 & 258 UN_xiong_14684 \\
\hline Unigene_GPGRCDD02JSZRM_xiong & 42 & 103 GPGRCDD02JSZRM_xion \\
\hline Unigene_UN_xiong_12159 & 74 & 96 UN_xiong_12159 \\
\hline Unigene_GP8I43W03GFS34_xiong & 44 & 218 GP8I43W03GFS34_xion \\
\hline Unigene_GP8I43W03GT2DX_xiong & 27 & 31 GP8I43W03GT2DX_xion \\
\hline Unigene_GP8I43W03F0ITH_xiong & 39 & 238 GP8I43W03F0ITH_xion \\
\hline Unigene_UN_xiong_3730 & 114 & 111 UN_xiong_3730 \\
\hline Unigene_UN_xiong_5997 & 364 & 106 UN_xiong_5997 \\
\hline Unigene_UN_xiong_13600 & 240 & 67 UN_xiong_13600 \\
\hline Unigene_GP8I43W03FYBL9_xiong & 21 & 395 GP8I43W03FYBL9_xion \\
\hline Unigene_UN_xiong_10479 & 48 & 71 UN_xiong_10479 \\
\hline
\end{tabular}




\begin{tabular}{|c|c|c|}
\hline Unigene_GR6TTJD01BVUBF_xiong & 22 & 23 GR6TTJD01BVUBF_xion \\
\hline Unigene_UN_xiong_13924 & 865 & 988 UN_xiong_13924 \\
\hline Unigene_GR6TTJD01AH8WC_xiong & 27 & 35 GR6TTJD01AH8WC_xion \\
\hline Unigene_UN_xiong_2778 & 66 & 40 UN_xiong_2778 \\
\hline Unigene_GR6TTJD01BGSEZ_xiong & 38 & 28 GR6TTJD01BGSEZ_xion \\
\hline Unigene_GPGRCDD02I7CSD_xiong & 31 & 21 GPGRCDD02I7CSD_xion \\
\hline Unigene_UN_xiong_992 & 79 & 61 UN_xiong_992 \\
\hline Unigene_GPGRCDD02F7PPP_xiong & 65 & 38 GPGRCDD02F7PPP_xion \\
\hline Unigene_UN_xiong_6332 & 68 & 110 UN_xiong_6332 \\
\hline Unigene_UN_xiong_1555 & 56 & 205 UN_xiong_1555 \\
\hline Unigene_UN_xiong_6676 & 55 & 70 UN_xiong_6676 \\
\hline Unigene_GPGRCDD02F0UEU_xiong & 22 & 489 GPGRCDD02F0UEU_xion \\
\hline Unigene_GR6TTJD01AV4KI_xiong & 26 & 41 GR6TTJD01AV4KI_xion \\
\hline Unigene_UN_xiong_134 & 144 & 299 UN_xiong_134 \\
\hline Unigene_UN_xiong_1858 & 75 & 58 UN_xiong_1858 \\
\hline Unigene_UN_xiong_4578 & 128 & 79 UN_xiong_4578 \\
\hline Unigene_GR6TTJD01A0CHX_xiong & 30 & 81 GR6TTJD01A0CHX_xion \\
\hline Unigene_GR6TTJD01BLJHC_xiong & 64 & 66 GR6TTJD01BLJHC_xion \\
\hline Unigene_UN_xiong_3341 & 134 & 28 UN_xiong_3341 \\
\hline Unigene_GPGRCDD02G0VCV_xiong & 24 & 50 GPGRCDD02G0VCV_xion \\
\hline Unigene_UN_xiong_10473 & 236 & 391 UN_xiong_10473 \\
\hline Unigene_UN_xiong_1377 & 72 & 76 UN_xiong_1377 \\
\hline Unigene_GP8I43W03HBHQ2_xiong & 56 & 41 GP8I43W03HBHQ2_xion \\
\hline Unigene_UN_xiong_930 & 50 & 48 UN_xiong_930 \\
\hline Unigene_GR6TTJD01BUIMU_xiong & 29 & 29 GR6TTJD01BUIMU_xion \\
\hline Unigene_UN_xiong_10183 & 192 & 48 UN_xiong_10183 \\
\hline Unigene_UN_xiong_1438 & 854 & 271 UN_xiong_1438 \\
\hline Unigene_UN_xiong_10090 & 247 & 506 UN_xiong_10090 \\
\hline Unigene_UN_xiong_14056 & 31 & 51 UN_xiong_14056 \\
\hline Unigene_UN_xiong_7151 & 194 & 406 UN_xiong_7151 \\
\hline Unigene_GR6TTJD01BW6TK_xiong & 25 & 24 GR6TTJD01BW6TK_xion \\
\hline Unigene_UN_xiong_10494 & 57 & 187 UN_xiong_10494 \\
\hline Unigene_GPGRCDD02JFC6Q_xiong & 37 & 59 GPGRCDD02JFC6Q_xion \\
\hline Unigene_GR6TTJD01BBJRH_xiong & 29 & 63 GR6TTJD01BBJRH_xion \\
\hline Unigene_UN_xiong_7428 & 126 & 177 UN_xiong_7428 \\
\hline Unigene_GP8I43W03GIHL4_xiong & 93 & 158 GP8I43W03GIHL4_xion \\
\hline Unigene_GPGRCDD02HS50A_xiong & 53 & 51 GPGRCDD02HS50A_xion \\
\hline Unigene_UN_xiong_3682 & 31 & 191 UN_xiong_3682 \\
\hline Unigene_UN_xiong_7563 & 108 & 31 UN_xiong_7563 \\
\hline Unigene_UN_xiong_14856 & 207 & 228 UN_xiong_14856 \\
\hline Unigene_UN_xiong_4098 & 208 & 174 UN_xiong_4098 \\
\hline Unigene_UN_xiong_14788 & 196 & 40 UN_xiong_14788 \\
\hline Unigene_UN_xiong_8765 & 304 & 20 UN_xiong_8765 \\
\hline Unigene_UN_xiong_7800 & 193 & 66 UN_xiong_7800 \\
\hline Unigene_GR6TTJD01B1CRX_xiong & 41 & 27 GR6TTJD01B1CRX_xion \\
\hline Unigene_GR6TTJD01BXS0F_xiong & 39 & 141 GR6TTJD01BXS0F_xion \\
\hline Unigene_UN_xiong_2672 & 71 & 74 UN_xiong_2672 \\
\hline Unigene_UN_xiong_7935 & 87 & 117 UN_xiong_7935 \\
\hline Unigene_UN_xiong_14323 & 329 & 50 UN_xiong_14323 \\
\hline Unigene_GR6TTJD01BUE26_xiong & 35 & 28 GR6TTJD01BUE26_xion \\
\hline
\end{tabular}




\begin{tabular}{|c|c|c|}
\hline Unigene_UN_xiong_2205 & 54 & 158 UN_xiong_2205 \\
\hline Unigene_UN_xiong_10467 & 67 & 45 UN_xiong_10467 \\
\hline Unigene_UN_xiong_11054 & 431 & 159 UN_xiong_11054 \\
\hline Unigene_UN_xiong_3841 & 65 & 26 UN_xiong_3841 \\
\hline Unigene_UN_xiong_6224 & 227 & 31 UN_xiong_6224 \\
\hline Unigene_UN_xiong_139 & 292 & 357 UN_xiong_139 \\
\hline Unigene_GR6TTJD01AR9TY_xiong & 111 & 34 GR6TTJD01AR9TY_xion \\
\hline Unigene_UN_xiong_4346 & 117 & 72 UN_xiong_4346 \\
\hline Unigene_UN_xiong_5202 & 62 & 34 UN_xiong_5202 \\
\hline Unigene_UN_xiong_12671 & 848 & 810 UN_xiong_12671 \\
\hline Unigene_GP8I43W03GFE6C_xiong & 29 & 156 GP8I43W03GFE6C_xion \\
\hline Unigene_UN_xiong_142 & 607 & 404 UN_xiong_142 \\
\hline Unigene_UN_xiong_7269 & 82 & 147 UN_xiong_7269 \\
\hline Unigene_UN_xiong_15349 & 225 & 177 UN_xiong_15349 \\
\hline Unigene_UN_xiong_14681 & 209 & 229 UN_xiong_14681 \\
\hline Unigene_GP8I43W03GNJ1Q_xiong & 93 & 577 GP8I43W03GNJ1Q_xion \\
\hline Unigene_UN_xiong_7761 & 176 & 82 UN_xiong_7761 \\
\hline Unigene_GPGRCDD02I4DZ3_xiong & 26 & 47 GPGRCDD02I4DZ3_xion \\
\hline Unigene_GR6TTJD01BMMLZ_xiong & 24 & 25 GR6TTJD01BMMLZ_xion \\
\hline Unigene_UN_xiong_11817 & 101 & 67 UN_xiong_11817 \\
\hline Unigene_UN_xiong_274 & 336 & 87 UN_xiong_274 \\
\hline Unigene_UN_xiong_14453 & 1023 & 514 UN_xiong_14453 \\
\hline Unigene_UN_xiong_6390 & 46 & 182 UN_xiong_6390 \\
\hline Unigene_UN_xiong_15042 & 159 & 26 UN_xiong_15042 \\
\hline Unigene UN xiong 6359 & 104 & 49 UN xiong 6359 \\
\hline Unigene_GR6TTJD01BGNM9_xiong & 37 & 191 GR6TTJD01BGNM9_xion \\
\hline Unigene_GR6TTJD01ATT4J_xiong & 27 & 98 GR6TTJD01ATT4J_xion \\
\hline Unigene_GR6TTJD01A3QI5_xiong & 51 & 99 GR6TTJD01A3QI5_xion \\
\hline Unigene_GR6TTJD01AW09R_xiong & 23 & 37 GR6TTJD01AW09R_xion \\
\hline Unigene_UN_xiong_14774 & 383 & 594 UN_xiong_14774 \\
\hline Unigene_GR6TTJD01AH8FD_xiong & 51 & 39 GR6TTJD01AH8FD_xion \\
\hline Unigene_UN_xiong_5623 & 216 & 472 UN_xiong_5623 \\
\hline Unigene_GPGRCDD02I4SHA_xiong & 78 & 66 GPGRCDD02I4SHA_xion \\
\hline Unigene_GP8I43W03FK234_xiong & 26 & 23 GP8I43W03FK234_xion \\
\hline Unigene_GPGRCDD02I1J9W_xiong & 86 & 87 GPGRCDD02I1J9W_xion \\
\hline Unigene_UN_xiong_9271 & 94 & 22 UN_xiong_9271 \\
\hline Unigene_UN_xiong_11602 & 337 & 91 UN_xiong_11602 \\
\hline Unigene_UN_xiong_8108 & 182 & 30 UN_xiong_8108 \\
\hline Unigene_UN_xiong_6779 & 163 & 97 UN_xiong_6779 \\
\hline Unigene_UN_xiong_1117 & 47 & 20 UN_xiong_1117 \\
\hline Unigene_GR6TTJD01A7NP0_xiong & 34 & 112 GR6TTJD01A7NP0_xion \\
\hline Unigene UN xiong 4892 & 45 & 32 UN xiong 4892 \\
\hline Unigene_GR6TTJD01BSZ50_xiong & 51 & 49 GR6TTJD01BSZ50_xion \\
\hline Unigene_GR6TTJD01AMF6Z_xiong & 22 & 340 GR6TTJD01AMF6Z_xion \\
\hline Unigene_UN_xiong_16 & 313 & 275 UN_xiong_16 \\
\hline Unigene_UN xiong 11149 & 217 & 224 UN_xiong_11149 \\
\hline Unigene_GR6TTJD01AGPJA_xiong & 29 & 21 GR6TTJD01AGPJA_xion \\
\hline Unigene_UN_xiong_3098 & 339 & 317 UN_xiong_3098 \\
\hline Unigene_UN_xiong_3465 & 44 & 31 UN_xiong_3465 \\
\hline Unigene_GR6TTJD01BITGD_xiong & 62 & 112 GR6TTJD01BITGD_xion \\
\hline
\end{tabular}




\begin{tabular}{|c|c|c|}
\hline Unigene_GR6TTJD01CD85H_xiong & 24 & 31 GR6TTJD01CD85H_xion \\
\hline Unigene_UN_xiong_1600 & 77 & 235 UN_xiong_1600 \\
\hline Unigene_UN_xiong_11868 & 57 & 580 UN_xiong_11868 \\
\hline Unigene_UN_xiong_13763 & 241 & 71 UN_xiong_13763 \\
\hline Unigene_UN_xiong_13085 & 63 & 99 UN_xiong_13085 \\
\hline Unigene_UN_xiong_426 & 122 & 24 UN_xiong_426 \\
\hline Unigene_UN_xiong_13696 & 51 & 61 UN_xiong_13696 \\
\hline Unigene_UN_xiong_912 & 142 & 26 UN_xiong_912 \\
\hline Unigene_GR6TTJD01ALXZU_xiong & 25 & 104 GR6TTJD01ALXZU_xion \\
\hline Unigene_UN_xiong_10749 & 1333 & 89 UN_xiong_10749 \\
\hline Unigene_UN_xiong_2555 & 1189 & 634 UN_xiong_2555 \\
\hline Unigene_UN_xiong_10077 & 85 & 82 UN_xiong_10077 \\
\hline Unigene_UN_xiong_3468 & 131 & 678 UN_xiong_3468 \\
\hline Unigene_GPGRCDD02G89IR_xiong & 25 & 92 GPGRCDD02G89IR_xion \\
\hline Unigene_UN_xiong_14607 & 135 & 109 UN_xiong_14607 \\
\hline Unigene_UN_xiong_14926 & 378 & 109 UN_xiong_14926 \\
\hline Unigene_UN_xiong_7495 & 224 & 259 UN_xiong_7495 \\
\hline Unigene_UN_xiong_7162 & 42 & 24 UN_xiong_7162 \\
\hline Unigene_UN_xiong_15256 & 97 & 78 UN_xiong_15256 \\
\hline Unigene_UN_xiong_1532 & 81 & 33 UN_xiong_1532 \\
\hline Unigene_UN_xiong_5453 & 122 & 841 UN_xiong_5453 \\
\hline Unigene_GP8I43W03FKMVL_xiong & 34 & 107 GP8I43W03FKMVL_xion \\
\hline Unigene_UN_xiong_4182 & 127 & 301 UN_xiong_4182 \\
\hline Unigene_GP8I43W03HGHUJ_xiong & 67 & 262 GP8I43W03HGHUJ_xion \\
\hline Unigene UN xiong 6381 & 92 & 49 UN xiong 6381 \\
\hline Unigene_UN_xiong_4692 & 170 & 74 UN_xiong_4692 \\
\hline Unigene_UN_xiong_14716 & 3412 & 1562 UN_xiong_14716 \\
\hline Unigene_UN_xiong_14119 & 186 & 115 UN_xiong_14119 \\
\hline Unigene_GPGRCDD02JUV6Q_xiong & 23 & 21 GPGRCDD02JUV6Q_xion \\
\hline Unigene_UN_xiong_13753 & 66 & 74 UN_xiong_13753 \\
\hline Unigene_GPGRCDD02HN6MB_xiong & 24 & 35 GPGRCDD02HN6MB_xion \\
\hline Unigene_GPGRCDD02IY2A5_xiong & 24 & 55 GPGRCDD02IY2A5_xion \\
\hline Unigene_UN_xiong_3917 & 50 & 26 UN_xiong_3917 \\
\hline Unigene_UN_xiong_4212 & 69 & 98 UN_xiong_4212 \\
\hline Unigene_GP8I43W03FWAQJ_xiong & 68 & 59 GP8I43W03FWAQJ_xion \\
\hline Unigene_UN_xiong_13859 & 43 & 52 UN_xiong_13859 \\
\hline Unigene_UN_xiong_8569 & 1036 & 2445 UN_xiong_8569 \\
\hline Unigene_UN_xiong_9462 & 83 & 27 UN_xiong_9462 \\
\hline Unigene_UN_xiong_3429 & 106 & 152 UN_xiong_3429 \\
\hline Unigene_UN_xiong_9506 & 33 & 41 UN_xiong_9506 \\
\hline Unigene_UN_xiong_9880 & 28 & 101 UN_xiong_9880 \\
\hline Unigene_UN_xiong_938 & 121 & 289 UN_xiong_938 \\
\hline Unigene_UN_xiong_15569 & 77 & 34 UN_xiong_15569 \\
\hline Unigene_GR6TTJD01A3XSJ_xiong & 56 & 32 GR6TTJD01A3XSJ_xion \\
\hline Unigene_UN_xiong_8001 & 178 & 81 UN_xiong_8001 \\
\hline Unigene_UN xiong 14219 & 174 & 45 UN xiong 14219 \\
\hline Unigene_GPGRCDD02FY58S_xiong & 27 & 52 GPGRCDD02FY58S_xion \\
\hline Unigene_GP8I43W03GXT9M_xiong & 53 & 64 GP8I43W03GXT9M_xion \\
\hline Unigene_UN_xiong_12070 & 204 & 996 UN_xiong_12070 \\
\hline Unigene UN xiong 8726 & 297 & 542 UN xiong 8726 \\
\hline
\end{tabular}




\begin{tabular}{|c|c|c|}
\hline Unigene_UN_xiong_5779 & 169 & 76 UN_xiong_5779 \\
\hline Unigene_GR6TTJD01BD75M_xiong & 20 & 22 GR6TTJD01BD75M_xion \\
\hline Unigene_UN_xiong_11142 & 99 & 112 UN_xiong_11142 \\
\hline Unigene_UN_xiong_13073 & 65 & 63 UN_xiong_13073 \\
\hline Unigene_GPGRCDD02II9WZ_xiong & 66 & 52 GPGRCDD02II9WZ_xion \\
\hline Unigene_UN_xiong_2824 & 143 & 41 UN_xiong_2824 \\
\hline Unigene_UN_xiong_3079 & 256 & 2269 UN_xiong_3079 \\
\hline Unigene_UN_xiong_7992 & 58 & 44 UN_xiong_7992 \\
\hline Unigene_UN_xiong_14214 & 110 & 62 UN_xiong_14214 \\
\hline Unigene_UN_xiong_3719 & 64 & 24 UN_xiong_3719 \\
\hline Unigene_UN_xiong_2224 & 80 & 461 UN_xiong_2224 \\
\hline Unigene_GP8I43W03F1UP2_xiong & 50 & 121 GP8I43W03F1UP2_xion \\
\hline Unigene_GR6TTJD01AP7VG_xiong & 21 & 43 GR6TTJD01AP7VG_xion \\
\hline Unigene_UN_xiong_6450 & 53 & 195 UN_xiong_6450 \\
\hline Unigene_UN_xiong_9816 & 245 & 72 UN_xiong_9816 \\
\hline Unigene_UN_xiong_9217 & 64 & 27 UN_xiong_9217 \\
\hline Unigene_UN_xiong_6496 & 144 & 140 UN_xiong_6496 \\
\hline Unigene_UN_xiong_14037 & 139 & 156 UN_xiong_14037 \\
\hline Unigene_UN_xiong_13907 & 34 & 84 UN_xiong_13907 \\
\hline Unigene_UN_xiong_5874 & 40 & 105 UN_xiong_5874 \\
\hline Unigene_UN_xiong_5193 & 34 & 54 UN_xiong_5193 \\
\hline Unigene_UN_xiong_535 & 138 & 145 UN_xiong_535 \\
\hline Unigene_UN_xiong_7315 & 63 & 298 UN_xiong_7315 \\
\hline Unigene_UN_xiong_9900 & 57 & 40 UN_xiong_9900 \\
\hline Unigene_UN_xiong_1961 & 48 & 264 UN_xiong_1961 \\
\hline Unigene_GP8I43W03FR4I5_xiong & 24 & 21 GP8I43W03FR4I5_xion \\
\hline Unigene_UN_xiong_4828 & 128 & 85 UN_xiong_4828 \\
\hline Unigene_UN_xiong_340 & 296 & 135 UN_xiong_340 \\
\hline Unigene_UN_xiong_9082 & 188 & 26 UN_xiong_9082 \\
\hline Unigene_UN_xiong_13143 & 203 & 57 UN_xiong_13143 \\
\hline Unigene_UN_xiong_10788 & 87 & 42 UN_xiong_10788 \\
\hline Unigene_UN_xiong_4983 & 78 & 288 UN_xiong_4983 \\
\hline Unigene_GR6TTJD01BR417_xiong & 31 & 43 GR6TTJD01BR417_xion \\
\hline Unigene_UN_xiong_260 & 556 & 70 UN_xiong_260 \\
\hline Unigene_UN_xiong_751 & 779 & 376 UN_xiong_751 \\
\hline Unigene_UN_xiong_10957 & 89 & 44 UN_xiong_10957 \\
\hline Unigene_UN_xiong_8079 & 73 & 206 UN_xiong_8079 \\
\hline Unigene_UN_xiong_8002 & 40 & 38 UN_xiong_8002 \\
\hline Unigene_GR6TTJD01BAEVC_xiong & 39 & 92 GR6TTJD01BAEVC_xion \\
\hline Unigene_UN_xiong_14828 & 215 & 119 UN_xiong_14828 \\
\hline Unigene_UN_xiong_6035 & 39 & 24 UN_xiong_6035 \\
\hline Unigene_UN_xiong_2515 & 453 & 73 UN_xiong_2515 \\
\hline Unigene_UN_xiong_14082 & 194 & 134 UN_xiong_14082 \\
\hline Unigene_GR6TTJD01B6GN9_xiong & 40 & 476 GR6TTJD01B6GN9_xion \\
\hline Unigene_UN_xiong_6972 & 112 & 44 UN_xiong_6972 \\
\hline Unigene_GR6TTJD01ATF7W_xiong & 41 & 196 GR6TTJD01ATF7W_xion \\
\hline Unigene_UN_xiong_8496 & 105 & 102 UN_xiong_8496 \\
\hline Unigene_UN_xiong_8850 & 951 & 655 UN_xiong_8850 \\
\hline Unigene_UN_xiong_10425 & 120 & 175 UN_xiong_10425 \\
\hline Unigene_GR6TTJD01BDRHB_xiong & 24 & 28 GR6TTJD01BDRHB_xion \\
\hline
\end{tabular}




\begin{tabular}{|c|c|}
\hline Unigene_UN_xiong_15205 & 48 \\
\hline Unigene_UN_xiong_9911 & 158 \\
\hline Unigene_UN_xiong_14995 & 338 \\
\hline Unigene_UN_xiong_6909 & 759 \\
\hline Unigene_GPGRCDD02I302F_xiong & 45 \\
\hline Unigene_GR6TTJD01ATJWS_xiong & 41 \\
\hline Unigene_UN_xiong_5301 & 171 \\
\hline Unigene_UN_xiong_14276 & 839 \\
\hline Unigene_UN_xiong_8944 & 521 \\
\hline Unigene_UN_xiong_9666 & 59 \\
\hline Unigene_UN_xiong_15322 & 30 \\
\hline Unigene_UN_xiong_4178 & 392 \\
\hline Unigene_UN_xiong_10220 & 282 \\
\hline Unigene_UN_xiong_14273 & 7734 \\
\hline Unigene_UN_xiong_14623 & 4746 \\
\hline Unigene_UN_xiong_12860 & 189 \\
\hline Unigene_UN_xiong_11553 & 116 \\
\hline Unigene_UN_xiong_10207 & 231 \\
\hline Unigene_UN_xiong_329 & 146 \\
\hline Unigene_UN_xiong_2081 & 108 \\
\hline Unigene_UN_xiong_10060 & 249 \\
\hline Unigene_GR6TTJD01B1PBW_xiong & 55 \\
\hline Unigene_UN_xiong_12118 & 213 \\
\hline Unigene_UN_xiong_241 & 1 \\
\hline Unigene_GP8I43W03F1R03_xiong & \\
\hline Unigene_GPGRCDD02H7N3Y_xiong & 66 \\
\hline Unigene_GR6TTJD01BAVA9_xiong & 22 \\
\hline Unigene_UN_xiong_5820 & 44 \\
\hline Unigene_UN_xiong_9827 & 88 \\
\hline Unigene_UN_xiong_9628 & 59 \\
\hline Unigene_UN_xiong_5559 & 188 \\
\hline Unigene_GP8I43W03GNTKR_xiong & 32 \\
\hline Unigene_UN_xiong_8839 & 139 \\
\hline Unigene_UN_xiong_9046 & 103 \\
\hline Unigene_UN_xiong_1991 & 83 \\
\hline Unigene_GR6TTJD01B4SPI_xiong & 28 \\
\hline Unigene_GP8I43W03HDRKG_xiong & 43 \\
\hline Unigene_UN_xiong_8628 & 275 \\
\hline Unigene_UN_xiong_14880 & 364 \\
\hline Unigene_UN_xiong_7967 & 85 \\
\hline Unigene_UN_xiong_12826 & 211 \\
\hline Unigene_UN_xiong_15775 & 64 \\
\hline Unigene_GR6TTJD01AMKAM_xiong & 34 \\
\hline Unigene_GPGRCDD02H93KS_xiong & 54 \\
\hline Unigene_GR6TTJD01A39W0_xiong & 55 \\
\hline Unigene_UN_xiong_22 & 85 \\
\hline Unigene_UN_xiong_3087 & 88 \\
\hline Unigene_GR6TTJD01B9L0I_xiong & 57 \\
\hline Unigene_UN_xiong_2134 & 408 \\
\hline
\end{tabular}

141 UN_xiong_15205
166 UN_xiong_9911
1040 UN_xiong_14995
271 UN_xiong_6909
34 GPGRCDD02I302F_xion
84 GR6TTJD01ATJWS_xion

109 UN_xiong_5301

23 UN_xiong_14276

149 UN_xiong_8944

127 UN_xiong_9666

212 UN_xiong_15322

50 UN_xiong_4178

97 UN_xiong_10220

2707 UN_xiong_14273

2395 UN_xiong_14623

168 UN_xiong_12860

103 UN_xiong_11553

61 UN_xiong_10207

323 UN_xiong_329

158 UN_xiong_2081

108 UN_xiong_10060

76 GR6TTJD01B1PBW_xion

44 UN_xiong_12118

734 UN_xiong_241

52 GP8I43W03F1R03_xion

68 GPGRCDD02H7N3Y_xion

41 GR6TTJD01BAVA9_xion

46 UN_xiong_5820

85 UN_xiong_9827

21 UN_xiong_9628

33 UN_xiong_5559

308 GP8I43W03GNTKR_xion

61 UN_xiong_8839

169 UN_xiong_9046

28 UN_xiong_1991

95 GR6TTJD01B4SPI_xion

56 GP8I43W03HDRKG_xion

426 UN_xiong_8628

84 UN_xiong_14880

39 UN_xiong_7967

119 UN_xiong_12826

46 UN_xiong_15775

23 GR6TTJD01AMKAM_xion

108 GPGRCDD02H93KS_xion

29 GR6TTJD01A39W0_xion

208 UN_xiong_22

161 UN_xiong_3087

54 GR6TTJD01B9L0I_xion

198 UN_xiong_2134 


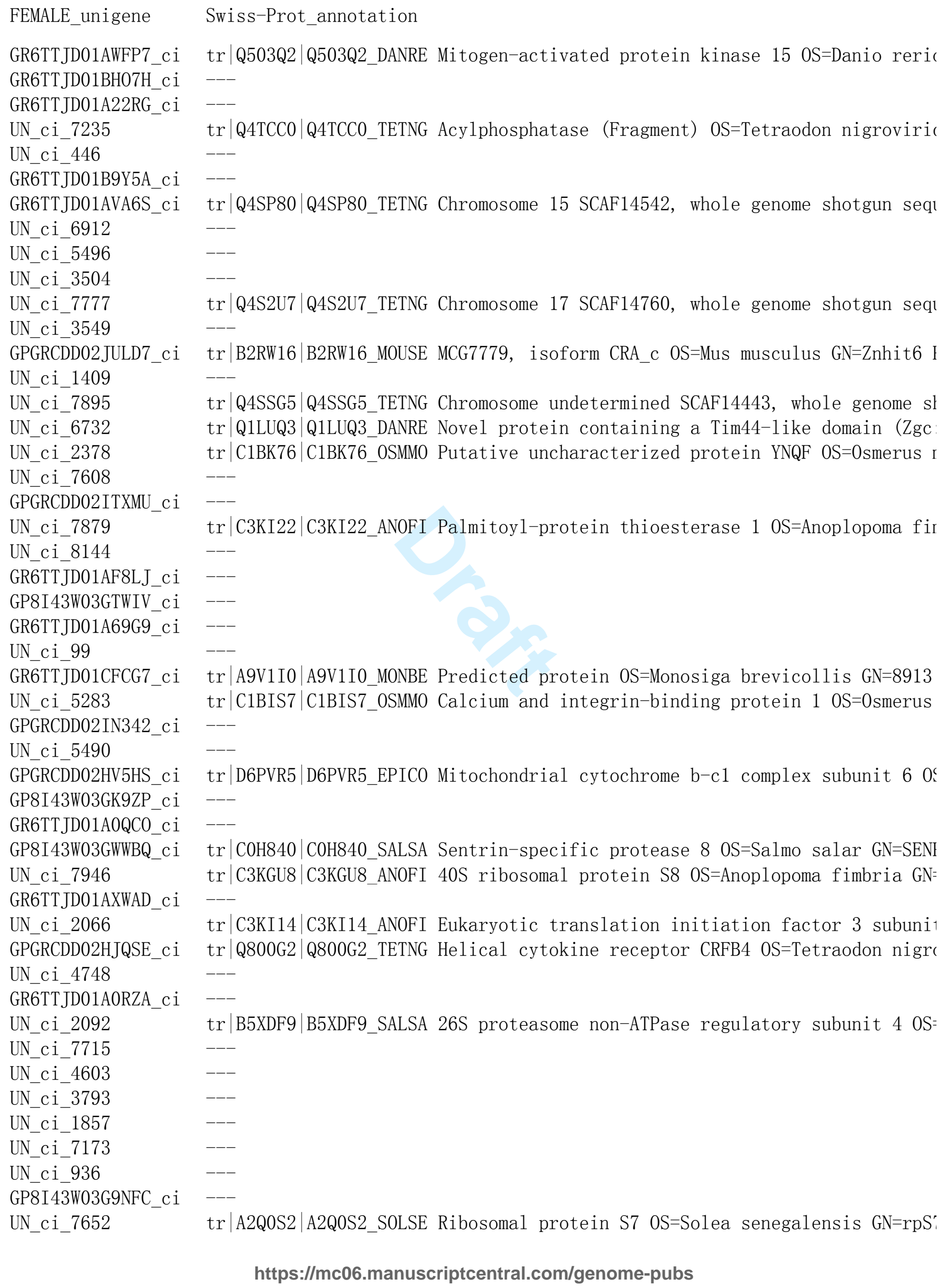




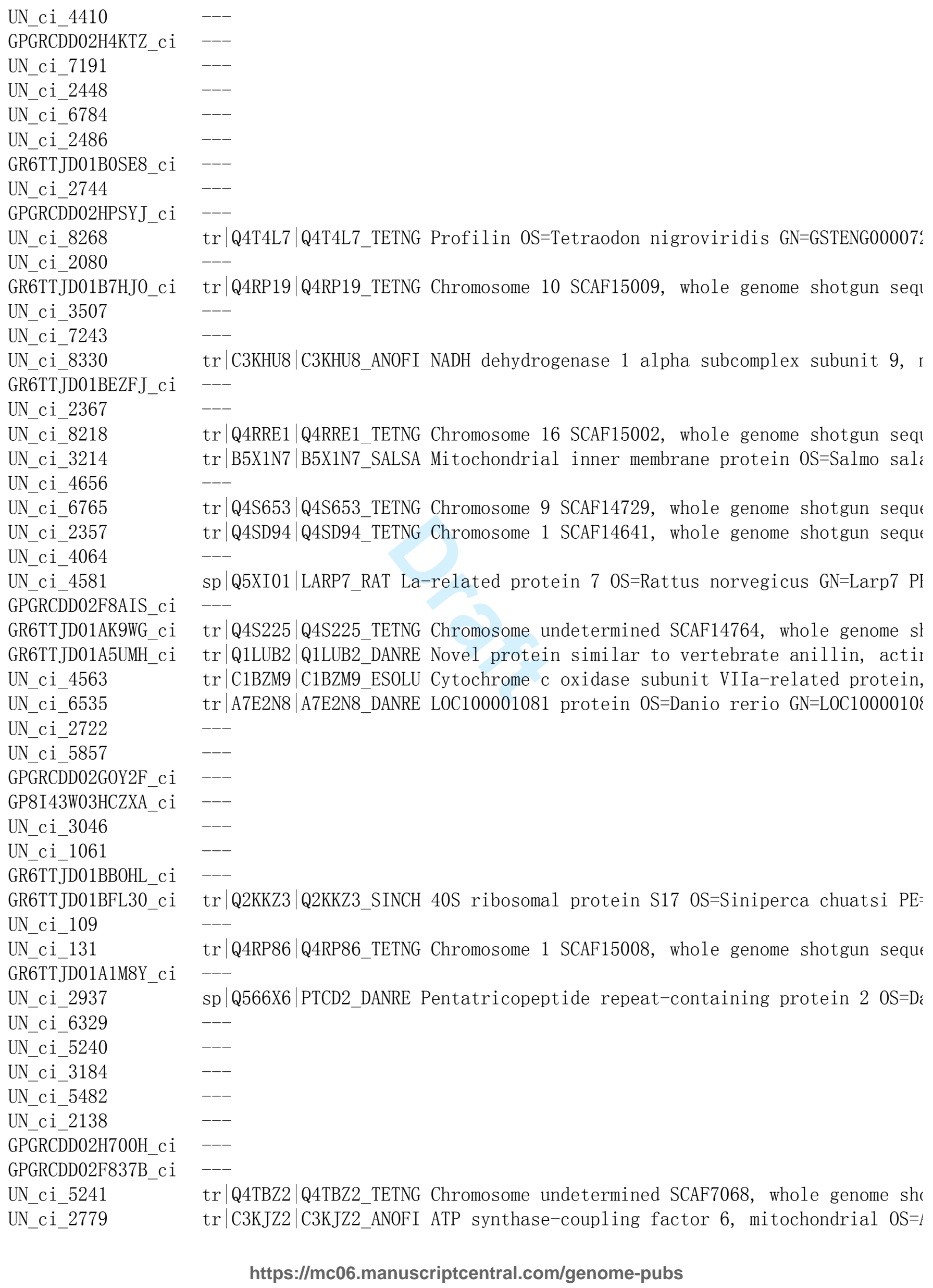




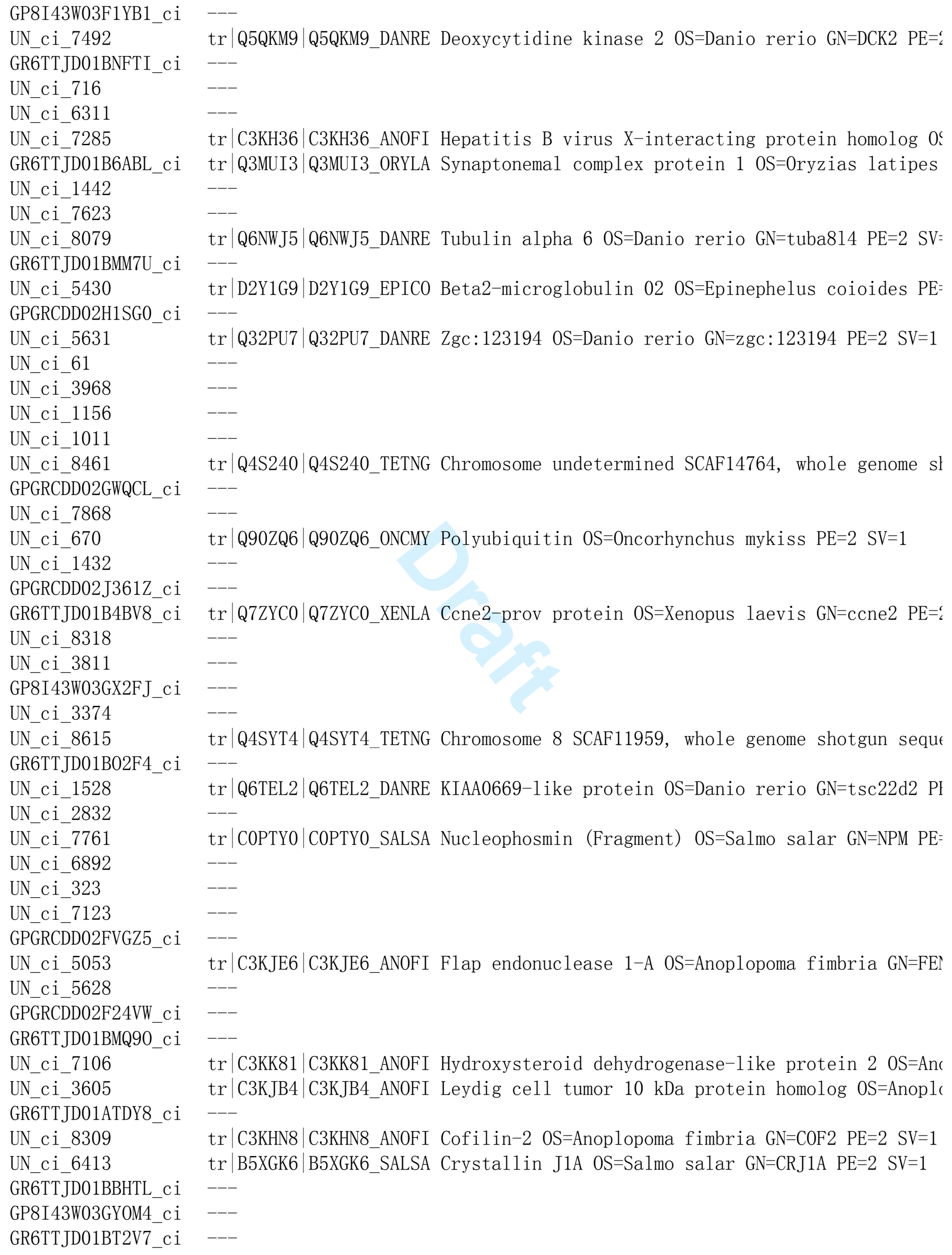




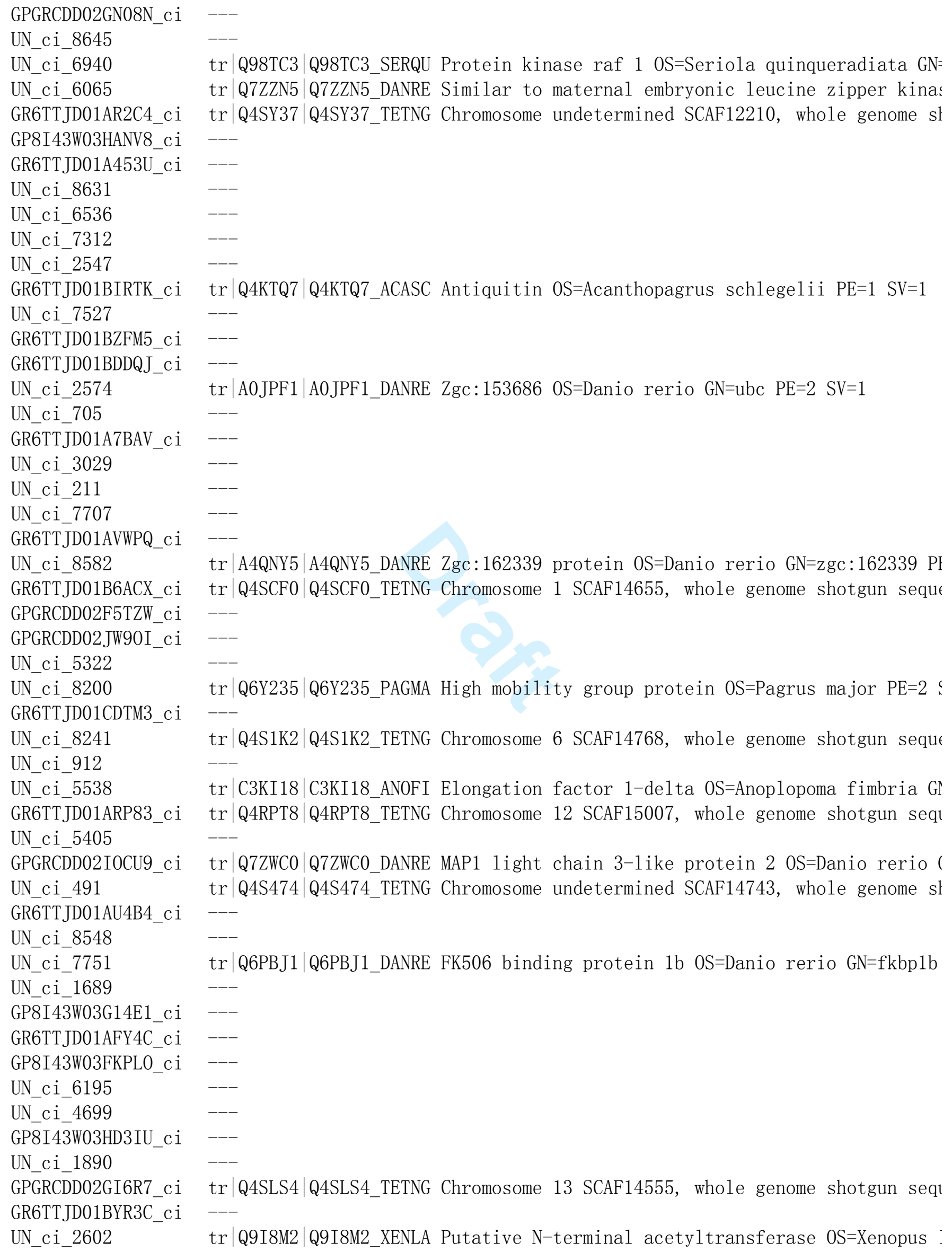

UN_ci_2574 tr $\mid$ A0JPF1 $\mid$ A0JPF1_DANRE Zgc:153686 OS=Danio rerio GN=ubc PE=2 SV=1

tr $\mid$ Q98TC3 $\mid$ Q98TC3_SERQU Protein kinase raf 1 OS=Seriola quinqueradiata GN= tr|Q7ZZN5|Q7ZZN5_DANRE Similar to maternal embryonic leucine zipper kinas tr $\mid$ Q4SY37|Q4SY37_TETNG Chromosome undetermined SCAF12210, whole genome sl

$-$

$\operatorname{tr} \mid$ Q4KTQ7 |Q4KTQ7_ACASC Antiquitin OS=Acanthopagrus schlegelii PE=1 SV=1

tr $\mid$ A4QNY5|A4QNY5_DANRE Zgc: 162339 protein OS=Danio rerio GN=zgc: $162339 \mathrm{PI}$ tr $\mid$ Q4SCF0|Q4SCF0_TETNG Chromosome 1 SCAF14655, whole genome shotgun sequt

$\operatorname{tr} \mid$ Q6Y235 $\mid$ Q6Y235_PAGMA High mobility group protein OS=Pagrus major PE=2 ؛ tr $\mid$ Q4S1K2 $\mid$ Q4S1K2_TETNG Chromosome 6 SCAF14768, whole genome shotgun sequt $\operatorname{tr} \mid$ C3KI18 $\mid$ C3KI18_ANOFI Elongation factor 1-de1ta 0S=Anoplopoma fimbria Gl tr $\mid$ Q4RPT8|Q4RPT8_TETNG Chromosome 12 SCAF15007, whole genome shotgun seq1 tr $\mid$ Q7ZWC0 $\mid$ Q7ZWC0_DANRE MAP1 light chain 3-1ike protein 2 OS=Danio rerio ( $\operatorname{tr} \mid$ Q4S474|Q4S474_TETNG Chromosome undetermined SCAF14743, whole genome sl $\operatorname{tr} \mid$ Q6PBJ1 $\mid$ Q6PBJ1_DANRE FK506 binding protein 1b 0S=Danio rerio GN=fkbp1b

tr $\mid$ Q4SLS4 $\mid$ Q4SLS4_TETNG Chromosome 13 SCAF14555, whole genome shotgun seq1 tr $\mid$ Q9I8M2 |Q9I8M2_XENLA Putative N-terminal acety1transferase 0S=Xenopus 


\begin{tabular}{|c|c|}
\hline UN_ci_3101 & tr $\mid$ C0H9B9 $\mid$ C0H9B9_SALSA RMD5 homolog B OS=Salmo salar GN=RMD5B PE=4 SV=1 \\
\hline UN_ci_674 & --- \\
\hline UN_ci_2915 & -- \\
\hline GPGRCDD02I5K55_ci & --- \\
\hline UN_ci_6301 & sp $\mid$ Q6AZW1 $\mid$ KISHB_DANRE Protein kish-B OS=Danio rerio GN=tmem167b PE=2 SV=: \\
\hline UN_ci_5769 & -- \\
\hline GP8I43W03HBKQM_ci & --- \\
\hline UN_ci_6796 & $\operatorname{tr} \mid$ D6WA77|D6WA77_TRICA Putative uncharacterized protein 0S=Tribolium cas \\
\hline UN_ci_4303 & tr $\mid$ Q4S2Y0|Q4S2Y0_TETNG Chromosome 8 SCAF14759, whole genome shotgun sequt \\
\hline UN_ci_5483 & $\operatorname{tr} \mid$ C3KIW9 $\mid$ C3KIW9_ANOFI Nuclear nucleic acid-binding protein C1D OS=Anoplc \\
\hline $\begin{array}{l}\text { GPGRCDD02FQNW9_ci } \\
\text { UN ci } 6589\end{array}$ & tr|A4IIR2 $\mid$ A4IIR2_XENTR LOC100125043 protein OS=Xenopus tropicalis GN=LOC: \\
\hline UN_ci_4159 & $\operatorname{tr} \mid$ Q4S8P8|Q4S8P8_TETNG Chromosome 7 SCAF14703, whole genome shotgun seque \\
\hline UN_ci_8324 & tr $\mid$ Q4RBH1|Q4RBH1_TETNG Chromosome undetermined SCAF21483, whole genome sl \\
\hline UN_ci_76-7659 & --- \\
\hline UN_ci_4613 & $\operatorname{tr} \mid$ C1BL65|C1BL65_0SMM0 Dolichy1-diphosphooligosaccharide--protein glycos! \\
\hline UN_ci_4441 & --- \\
\hline UN_ci_2022 & --- \\
\hline UN_ci_4197 & --- \\
\hline UN_ci_878 & --- \\
\hline UN_ci_1658 & --- \\
\hline UN_ci_8110 & --- \\
\hline UN_ci_4293 & --- \\
\hline UN_ci_8137 & $\operatorname{tr} \mid$ B5X7F1 $\mid$ B5X7F1_SALSA Lin-52 homolog 0S=Salmo salar GN=LIN52 PE=4 SV=1 \\
\hline UN_ci_1394 & sp $\mid$ Q8N531|FBXL6_HUMAN F-box/LRR-repeat protein 6 OS=Homo sapiens GN=FBXLt \\
\hline GR6TTJD01ANH3G_ci & $\operatorname{tr} \mid$ Q5RG21|Q5RG21_DANRE Integrin beta 1 binding protein 1 OS=Danio rerio ( \\
\hline UN_ci_7860 & --- \\
\hline UN_ci_7242 & --- \\
\hline GR6TTJD01B06E3_ci & --- \\
\hline $\begin{array}{l}\text { GP8I43W03FKJ09_ci } \\
\text { UN_ci_1154 }\end{array}$ & $\begin{array}{l}\text { tr } \mid \text { Q4RS02 } \mid \text { Q4RS02_TETNG Chromosome } 7 \text { SCAF15001, whole genome shotgun sequt } \\
---\end{array}$ \\
\hline UN_ci_1661 & $\operatorname{tr} \mid$ C3KIV7|C3KIV7_ANOFI C12orf62 homolog 0S=Anoplopoma fimbria GN=CL062 PI \\
\hline UN_ci_6273 & sp $\mid$ A2RV29|ZN346_DANRE Zinc finger protein 346 OS=Danio rerio GN=znf346 PI \\
\hline UN_ci_8203 & --- \\
\hline GP8I43W03FZUCX_ci & $\operatorname{tr} \mid$ B5X3J0|B5X3J0_SALSA Ras-related protein Rab-7a OS=Salmo salar GN=RAB7 \\
\hline UN_ci_51 & $\operatorname{tr} \mid$ C1BZE3 $\mid$ C1BZE3_ESOLU Rab-1ike protein 3 OS=Esox lucius GN=RABL3 PE=2 ST \\
\hline UN_ci_1586 & --- \\
\hline GPGRCDD02HVIPA_ci & $\operatorname{tr} \mid$ C3KJC1 $\mid$ C3KJC1_ANOFI Fructose-1,6-bisphosphatase 1 OS=Anoplopoma fimbr: \\
\hline GR6TTJD01AJ9KU_ci & --- \\
\hline UN_ci_5588 & --- \\
\hline GR6TTJJD01BCVV0_ci & --- \\
\hline UN_ci_2580 & tr|Q4RU26|Q4RU26_TETNG Chromosome 1 SCAF14995, whole genome shotgun sequt \\
\hline UN_ci_3254 & $\operatorname{tr} \mid$ Q4SSD9|Q4SSD9_TETNG ATP synthase subunit alpha OS=Tetraodon nigroviric \\
\hline GR6TTJD01BSDKP_ci & --- \\
\hline UN_ci_8068 & tr|B3DLM7|B3DLM7_XENTR L0C100170503 protein (Fragment) OS=Xenopus tropice \\
\hline UN_ci_8783 & $\operatorname{tr} \mid$ Q2HYMO $\mid$ Q2HYM0_ICTPU Putative uncharacterized protein (Fragment) OS=Ic1 \\
\hline UN_ci_3088 & --- \\
\hline UN_ci_239 & $\operatorname{tr} \mid$ C3KINO $\mid$ C3KIN0_ANOFI GTP-binding nuclear protein Ran OS=Anoplopoma fiml \\
\hline UN_ci_8621 & \\
\hline
\end{tabular}




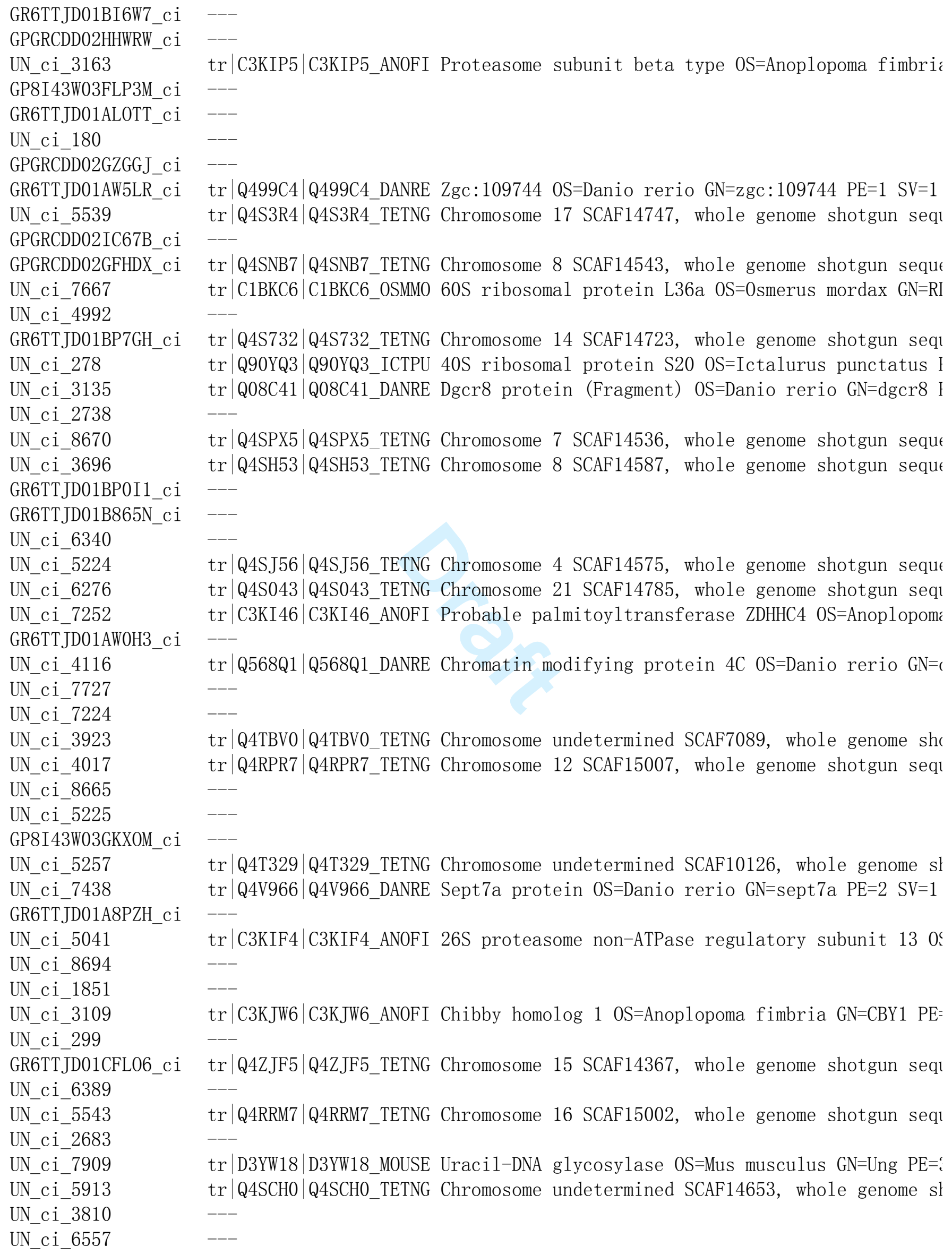




\begin{abstract}
UN_ci_2714
UN_ci_4694

UN_ci_854

UN_ci_2264

UN_ci_5206

UN_ci_4976

UN_ci_4080

UN_ci_8027

UN_ci_4830

UN_ci_2662

GPGRCDD02H843H_ci

UN_ci_4751

UN_ci_5170

UN_ci_1239

UN_ci_6257

GP8I43W03G2V77_ci

UN_ci_7981

GPGRCDD02G2ZPT_ci

UN_ci_820

UN_ci_5428

UN_ci_3554

GR6TTJD01AUCXF_ci

UN_ci_6480

UN_ci_1994

UN_ci_535

UN_ci_6462

UN_ci_3355

UN_ci_5872

UN_ci_6043

UN ci 3271

UN_ci_7332

GR6TTJD01BPF0I_ci

UN_ci_6794

UN_ci_6662

UN_ci_7769

UN_ci_1767

UN_ci_4891

UN_ci_332

UN_ci_4430

UN_ci_2560

UN_ci_3206

GP8I43W03FR3VX_ci

UN_ci_2047

GPGRCDD02HFK7S_ci

GP8I43W03HEUDC_ci

GR6TTJD01AYUKQ_ci

GR6TTJD01CD6HW_ci

UN_ci_3906

UN_ci 6608

GPGRCDD02JM96G_ci

tr $\mid$ B5XE35|B5XE35_SALSA Nucleoside diphosphate kinase OS=Salmo salar GN=NI $\operatorname{tr} \mid$ Q4SVP1|Q4SVP1_TETNG Chromosome undetermined SCAF13749, whole genome sl

$\operatorname{tr} \mid$ Q4JG04 $\mid$ Q4JG04_DANRE Atypical protein kinase C zeta OS=Danio rerio GN=1

tr |Q8CGQ6|Q8CGQ6_RAT Zinc finger protein 161 OS=Rattus norvegicus GN=Zfp

sp|P0C7V7|SC11B_HUMAN Putative signal peptidase complex catalytic subuni1 $\operatorname{tr} \mid$ B6ZIW1 $\mid$ B6ZIW1_ORYLA Inner centromere protein OS=0ryzias latipes GN=inc

sp $\mid$ Q7ZWW7 |TS31B_XENLA Tetraspanin-31-B OS=Xenopus laevis GN=tspan31-b PE=

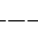

tr |Q4SXC6 |Q4SXC6_TETNG Chromosome undetermined SCAF12505, whole genome sl tr $\mid$ C1BLE0|C1BLE0_0SMM0 Mitotic spindle assembly checkpoint protein MAD2B

$\operatorname{tr} \mid$ Q4SQ68|Q4SQ68_TETNG Chromosome 19 SCAF14535, whole genome shotgun seq1

$\operatorname{tr} \mid$ C3KIG8|C3KIG8_ANOFI DCN1-1ike protein 5 OS=Anoplopoma fimbria GN=DCNL!

tr|C1BM92|C1BM92_0SMM0 SH3 domain-binding glutamic acid-rich-like proteir tr $\mid$ Q4RGQ3 $\mid$ Q4RGQ3_TETNG Chromosome 4 SCAF15094, whole genome shotgun seque

$-$
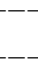

$\operatorname{tr} \mid$ Q45KR0 $\mid$ Q45KR0_LARCR Heat shock protein 60 OS=Larimichthys crocea GN=Hs tr $\mid$ A0EZW3 $\mid$ A0EZW3_PSEMX Receptor for activated protein kinase C (Fragment, tr|C1BYS8|C1BYS8_ES0LU 3-oxoacy1-acy1-carrier-protein reductase 0S=Esox tr $\mid$ Q6GPN8|Q6GPN8_XENLA LOC443622 protein (Fragment) OS=Xenopus laevis GN= $\operatorname{tr} \mid$ Q501Z8|Q501Z8_DANRE Zgc:112384 OS=Danio rerio GN=zgc:112384 PE=2 SV=1

sp $\mid$ Q5M8G4|LRC40_XENTR Leucine-rich repeat-containing protein 40 OS=Xenop $\operatorname{tr} \mid$ Q6DC52 |Q6DC52_DANRE Zgc:101107 OS=Danio rerio GN=zgc:101107 PE=2 SV=1 $\operatorname{tr} \mid$ Q4T9X6|Q4T9X6_TETNG Chromosome undetermined SCAF7485, whole genome she sp |Q7ZW86|CWC27_DANRE Peptidy1-prolyl cis-trans isomerase CWC27 homolog ( $--$

tr $\mid$ C1BJK8|C1BJK8_0SMM0 Transcription initiation factor IIE subunit beta ( $\operatorname{tr} \mid$ D2Y3F1|D2Y3F1_RIVMA 0-6-methylguanine DNA-methyltransferase OS=Rivulus
\end{abstract}




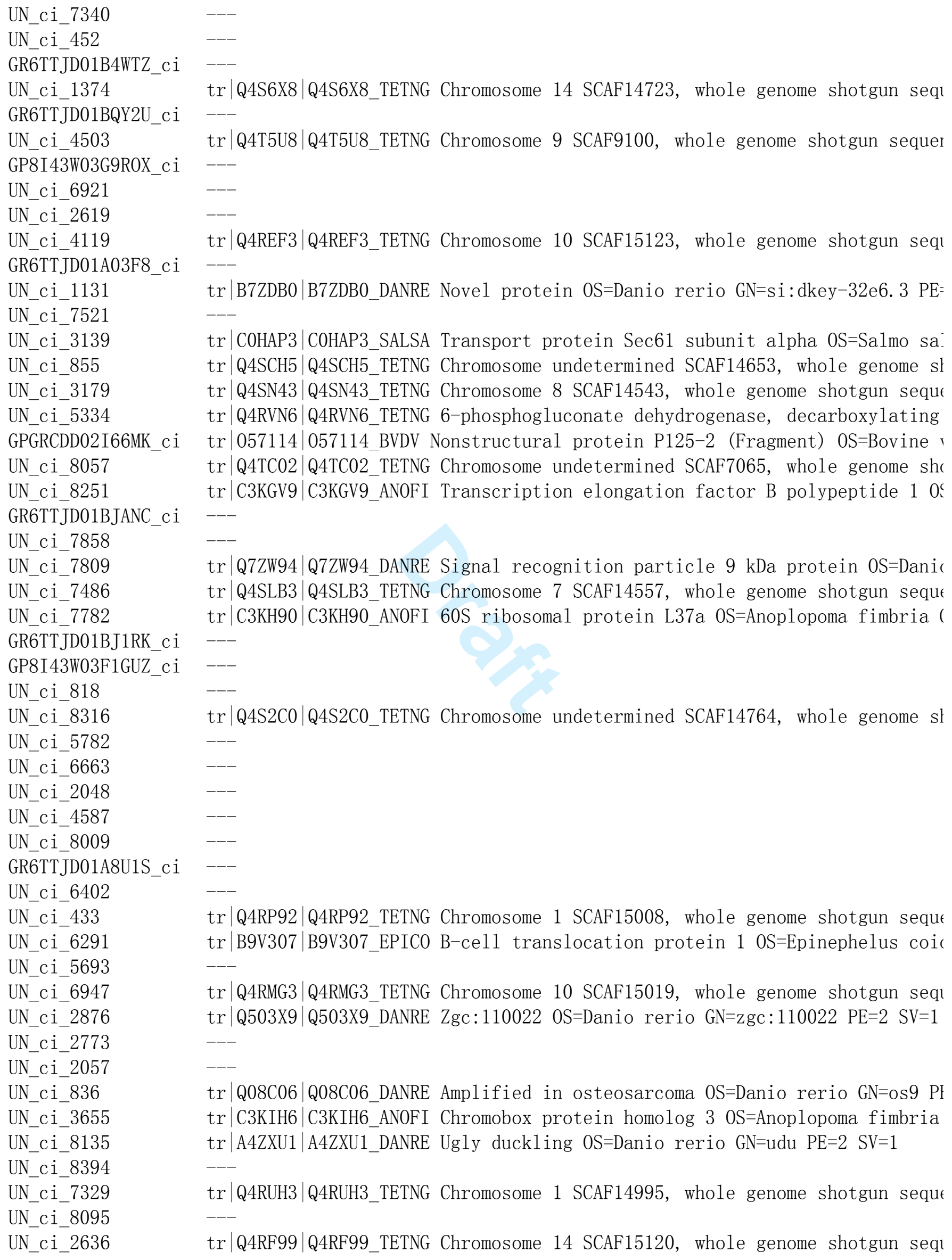

tr $\mid$ Q4S6X8|Q4S6X8_TETNG Chromosome 14 SCAF14723, whole genome shotgun seq1 $\operatorname{tr} \mid$ Q4T5U8 $\mid$ Q4T5U8_TETNG Chromosome 9 SCAF9100, whole genome shotgun sequer $--$

tr $\mid$ Q4REF3 $\mid$ Q4REF3_TETNG Chromosome 10 SCAF15123, whole genome shotgun seq $\operatorname{tr} \mid$ B7ZDB0 |B7ZDB0_DANRE Novel protein OS=Danio rerio GN=si:dkey-32e6. 3 PE= $-$ tr $\mid$ COHAP3 $\mid$ COHAP3_SALSA Transport protein Sec61 subunit alpha OS=Salmo sa: tr $\mid$ Q4SCH5 $\mid$ Q4SCH5_TETNG Chromosome undetermined SCAF14653, whole genome sl tr $\mid$ Q4SN43|Q4SN43_TETNG Chromosome 8 SCAF14543, whole genome shotgun seque tr |Q4RVN6|Q4RVN6_TETNG 6-phosphogluconate dehydrogenase, decarboxylating $\operatorname{tr}|057114| 057114$ BVDV Nonstructural protein P125-2 (Fragment) 0S=Bovine r $\operatorname{tr} \mid$ Q4TC02 $\mid$ Q4TC02_TETNG Chromosome undetermined SCAF7065, whole genome shc tr|C3KGV9|C3KGV9_ANOFI Transcription elongation factor B polypeptide 10 亿

tr $\mid$ Q7ZW94|Q7ZW94_DANRE Signal recognition particle $9 \mathrm{kDa}$ protein OS=Danic tr $\mid$ Q4SLB3|Q4SLB3_TETNG Chromosome 7 SCAF14557, whole genome shotgun sequt tr $\mid$ C3KH90|C3KH90_ANOFI 60S ribosomal protein L37a OS=Anoplopoma fimbria (

$\operatorname{tr} \mid$ Q4S2C0 |Q4S2C0_TETNG Chromosome undetermined SCAF14764, whole genome sl

tr|Q4RP92|Q4RP92_TETNG Chromosome 1 SCAF15008, whole genome shotgun sequt $\operatorname{tr} \mid$ B9V307|B9V307_EPIC0 B-ce11 translocation protein 1 OS=Epinephelus coic

tr $\mid$ Q4RMG3 |Q4RMG3_TETNG Chromosome 10 SCAF15019, whole genome shotgun seq1 $\operatorname{tr} \mid$ Q503X9|Q503X9_DANRE Zgc:110022 OS=Danio rerio GN=zgc:110022 PE=2 SV=1

$\operatorname{tr} \mid$ Q08C06 |Q08C06_DANRE Amplified in osteosarcoma 0S=Danio rerio GN=os9 PI tr|C3KIH6|C3KIH6_ANOFI Chromobox protein homolog 3 OS=Anoplopoma fimbria $\operatorname{tr} \mid$ A4ZXU1 $\mid$ A4ZXU1_DANRE Ugly duckling OS=Danio rerio GN=udu PE=2 SV=1

tr $\mid$ Q4RUH3 |Q4RUH3_TETNG Chromosome 1 SCAF14995, whole genome shotgun sequt $\operatorname{tr} \mid$ Q4RF99|Q4RF99_TETNG Chromosome 14 SCAF15120, whole genome shotgun seq1 


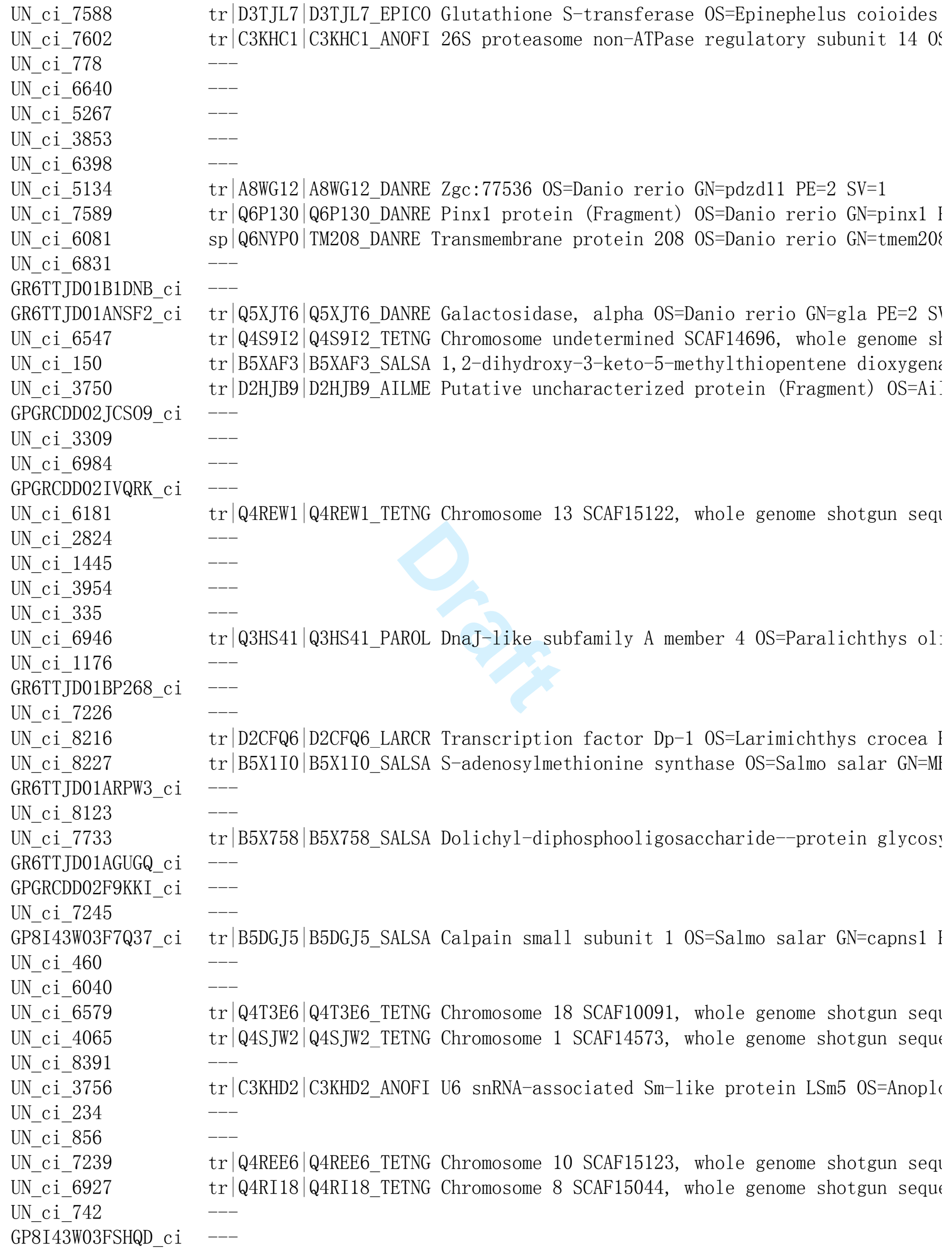

tr|Q4REW1 | Q4REW1_TETNG Chromosome 13 SCAF15122, whole genome shotgun seq1

$--+$

$\operatorname{tr} \mid$ Q3HS41|Q3HS41_PAROL DnaJ-1ike subfamily A member 4 OS=Paralichthys o1:

\section{$---$}

tr|D2CFQ6|D2CFQ6_LARCR Transcription factor Dp-1 0S=Larimichthys crocea I $\operatorname{tr} \mid$ B5X1I0|B5X1I0_SALSA S-adenosylmethionine synthase OS=Salmo salar GN=MI

tr|B5X758|B5X758_SALSA Dolichyl-diphosphooligosaccharide--protein glycos! 


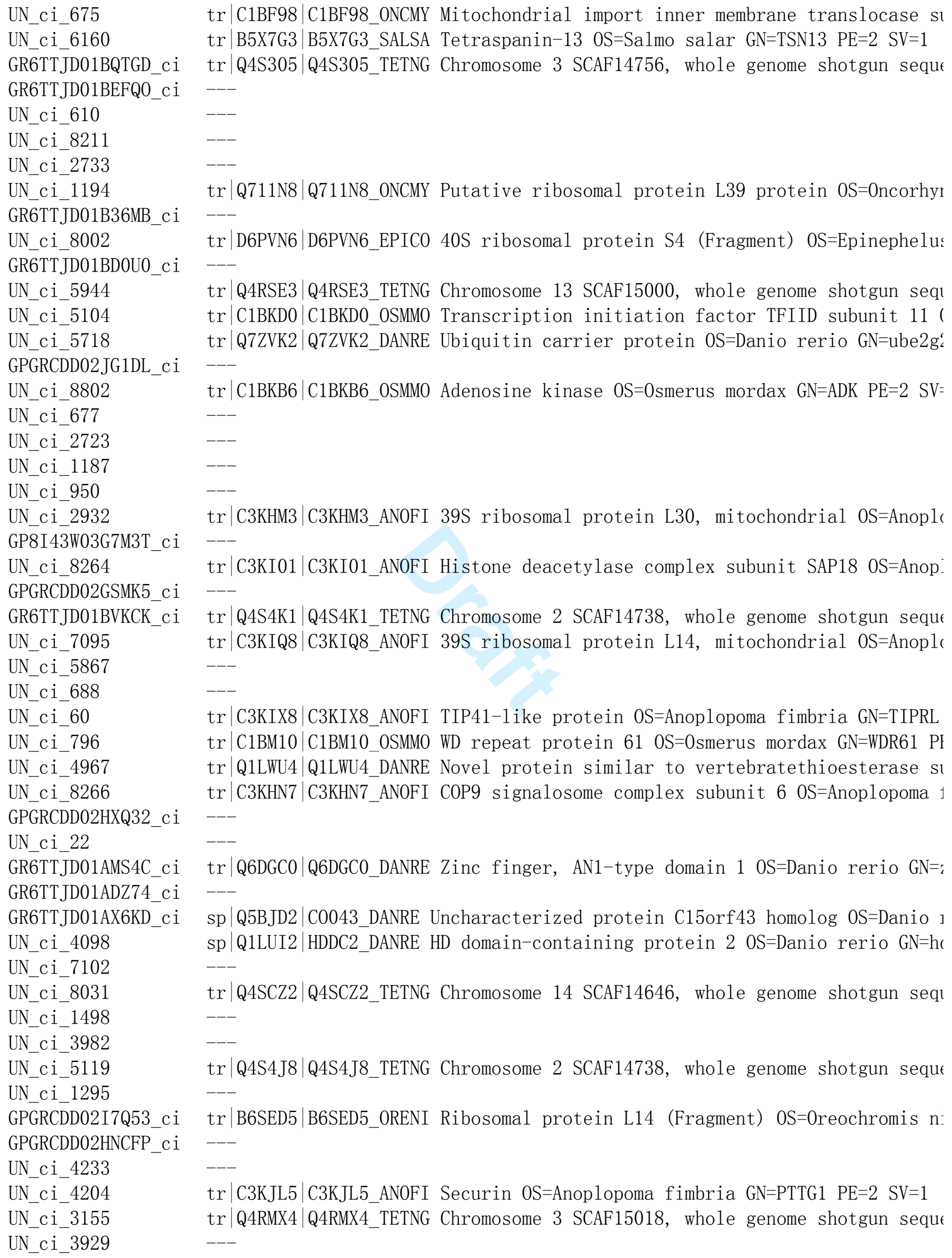

tr $\mid$ C1BF98|C1BF98_ONCMY Mitochondrial import inner membrane translocase si $\operatorname{tr} \mid$ B5X7G3 |B5X7G3_SALSA Tetraspanin-13 OS=Salmo salar GN=TSN13 PE=2 SV=1 tr $\mid$ Q4S305|Q4S305_TETNG Chromosome 3 SCAF14756, whole genome shotgun sequt $\operatorname{tr} \mid$ Q711N8 $\mid$ Q711N8_ONCMY Putative ribosomal protein L39 protein OS=0ncorhyr tr|D6PVN6|D6PVN6_EPIC0 40S ribosomal protein S4 (Fragment) OS=Epinephelus tr $\mid$ Q4RSE3 |Q4RSE3_TETNG Chromosome 13 SCAF15000, whole genome shotgun seq1 tr $\mid$ C1BKD0|C1BKD0_0SMM0 Transcription initiation factor TFIID subunit 11 ( $\operatorname{tr} \mid$ Q7ZVK2 $\mid$ Q7ZVK2_DANRE Ubiquitin carrier protein OS=Danio rerio GN=ube2g، $\operatorname{tr} \mid$ C1BKB6 $\mid$ C1BKB6_0SMM0 Adenosine kinase OS=0smerus mordax GN=ADK PE=2 SV= $---$

$\operatorname{tr} \mid$ C3KHM3 $\mid$ C3KHM3_ANOFI 39S ribosomal protein L30, mitochondrial OS=Anop1c tr $\mid$ C3KI01|C3KI01_ANOFI Histone deacetylase complex subunit SAP18 OS=Anop tr $\mid$ Q4S4K1 $\mid$ Q4S4K1_TETNG Chromosome 2 SCAF14738, whole genome shotgun sequt $\operatorname{tr} \mid$ C3KIQ8 $\mid$ C3KIQ8_ANOFI 39S ribosomal protein L14, mitochondrial OS=Anoplc $---$

$\operatorname{tr} \mid$ C3KIX8 $\mid$ C3KIX8_ANOFI TIP41-1ike protein OS=Anoplopoma fimbria GN=TIPRL $\operatorname{tr} \mid$ C1BM10 $\mid$ C1BM10_0SMM0 WD repeat protein 61 OS=0smerus mordax GN=WDR61 PI tr $\mid$ Q1LWU4 $\mid$ Q1LWU4_DANRE Novel protein similar to vertebratethioesterase si $\operatorname{tr} \mid$ C3KHN7 |C3KHN7_ANOFI COP9 signalosome complex subunit 6 OS=Anoplopoma 1

$\operatorname{tr} \mid$ Q6DGC0 $\mid$ Q6DGC0_DANRE Zinc finger, AN1-type domain 1 OS=Danio rerio GN=: sp |Q5BJD2 |C0043_DANRE Uncharacterized protein C15orf43 homolog OS=Danio ] sp $\mid$ Q1LUI2 $\mid$ HDDC2_DANRE HD domain-containing protein 2 OS=Danio rerio GN=hr $\operatorname{tr} \mid$ Q4SCZ2 $\mid$ Q4SCZ2_TETNG Chromosome 14 SCAF14646, whole genome shotgun seq1 $---$

tr $\mid$ Q4S4J8|Q4S4J8_TETNG Chromosome 2 SCAF14738, whole genome shotgun sequt tr $\mid$ B6SED5 $\mid$ B6SED5_0RENI Ribosomal protein L14 (Fragment) 0S=0reochromis n: $\operatorname{tr} \mid$ C3KJL5 $\mid$ C3KJL5_ANOFI Securin OS=Anoplopoma fimbria GN=PTTG1 PE=2 SV=1 tr $\mid$ Q4RMX4|Q4RMX4_TETNG Chromosome 3 SCAF15018, whole genome shotgun sequt 


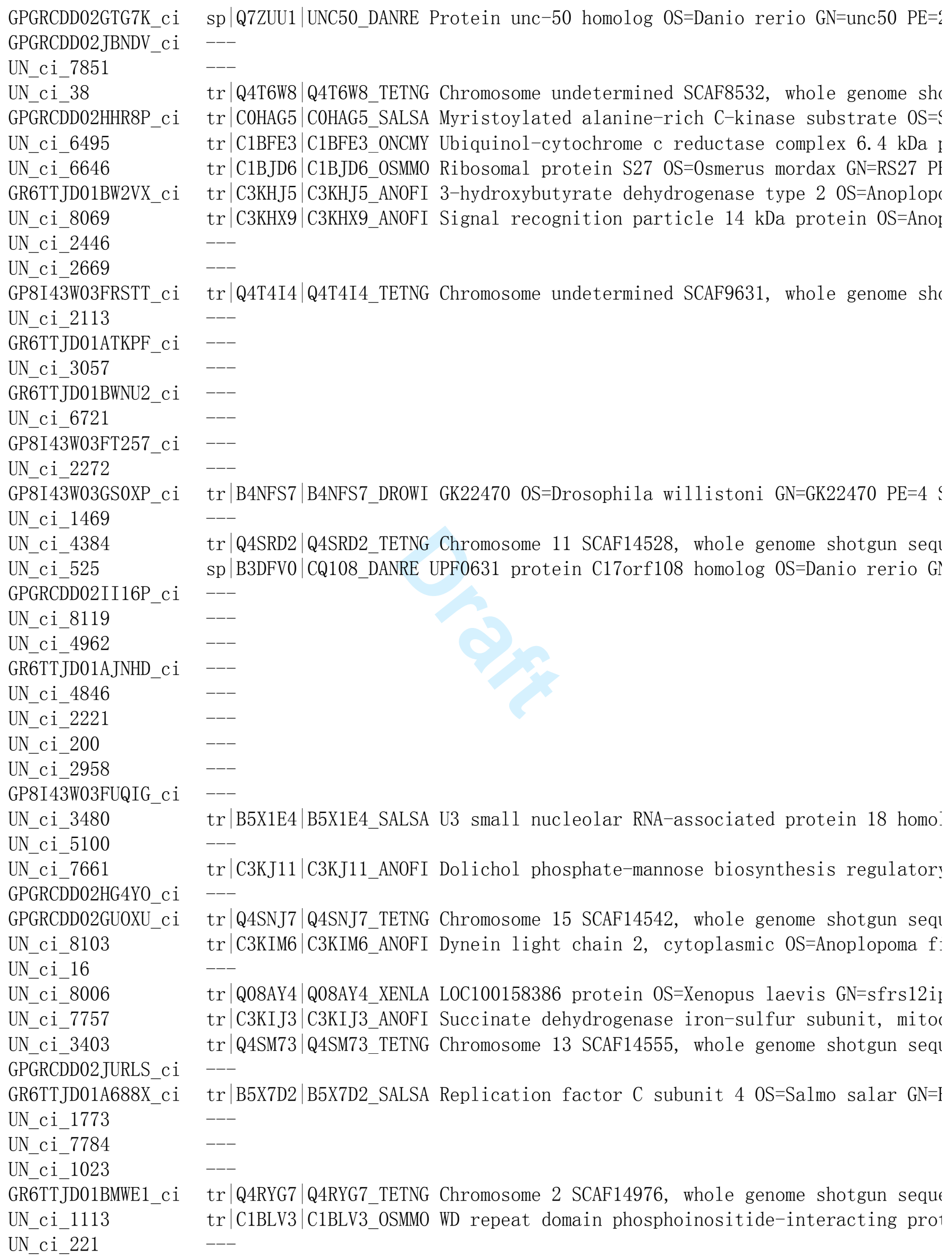




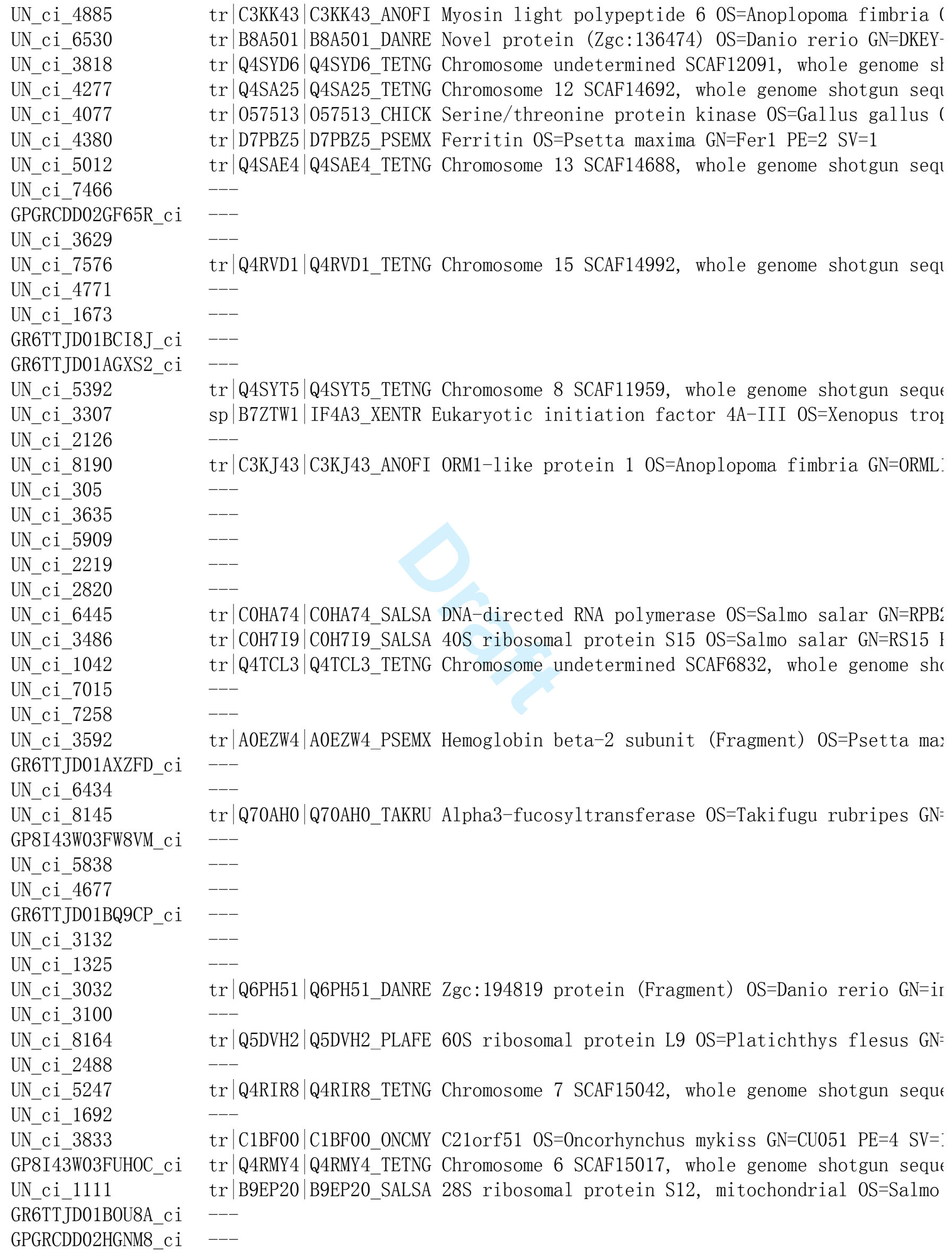

tr $\mid$ C3KK43|C3KK43_ANOFI Myosin light polypeptide 6 OS=Anoplopoma fimbria ( $\operatorname{tr} \mid$ B8A501|B8A501_DANRE Novel protein (Zgc:136474) OS=Danio rerio GN=DKEYtr |Q4SYD6|Q4SYD6_TETNG Chromosome undetermined SCAF12091, whole genome sl tr|Q4SA25|Q4SA25_TETNG Chromosome 12 SCAF14692, whole genome shotgun seqr $\operatorname{tr}|057513| 057513$ CHICK Serine/threonine protein kinase 0S=Gallus gallus ( tr $\mid$ D7PBZ5|D7PBZ5_PSEMX Ferritin OS=Psetta maxima GN=Fer1 PE=2 SV=1 tr $\mid$ Q4SAE4|Q4SAE4_TETNG Chromosome 13 SCAF14688, whole genome shotgun seqr tr |Q4RVD1|Q4RVD1_TETNG Chromosome 15 SCAF14992, whole genome shotgun seq1 tr $\mid$ Q4SYT5 $\mid$ Q4SYT5_TETNG Chromosome 8 SCAF11959, whole genome shotgun seque sp $\mid$ B7ZTW1|IF4A3_XENTR Eukaryotic initiation factor 4A-III OS=Xenopus tror $\operatorname{tr} \mid$ C3KJ43|C3KJ43_ANOFI ORM1-1ike protein 1 OS=Anoplopoma fimbria GN=0RML

tr $\mid$ COHA74 $\mid$ COHA74_SALSA DNA-directed RNA polymerase OS=Salmo salar GN=RPB، tr $\mid$ COH7I9|COH7I9_SALSA 40S ribosomal protein S15 0S=Salmo salar GN=RS15 I tr $\mid$ Q4TCL3 $\mid$ Q4TCL3_TETNG Chromosome undetermined SCAF6832, whole genome shi

$\operatorname{tr} \mid$ A0EZW4 $\mid$ A0EZW4_PSEMX Hemoglobin beta-2 subunit (Fragment) OS=Psetta ma:

$\operatorname{tr} \mid$ Q70AHO $\mid$ Q70AH0_TAKRU Alpha3-fucosyltransferase OS=Takifugu rubripes GN=

$\operatorname{tr} \mid$ Q6PH51 |Q6PH51_DANRE Zgc:194819 protein (Fragment) OS=Danio rerio GN=ir $\operatorname{tr} \mid$ Q5DVH2 $\mid$ Q5DVH2_PLAFE 60S ribosomal protein L9 0S=Platichthys flesus GN= tr $\mid$ Q4RIR8|Q4RIR8_TETNG Chromosome 7 SCAF15042, whole genome shotgun sequt $\operatorname{tr} \mid$ C1BF00 $\mid$ C1BF00_ONCMY C21orf51 OS=0ncorhynchus mykiss GN=CU051 PE=4 SV=: tr $\mid$ Q4RMY4|Q4RMY4_TETNG Chromosome 6 SCAF15017, whole genome shotgun sequt tr|B9EP20|B9EP20_SALSA 28S ribosomal protein S12, mitochondrial OS=Salmo 


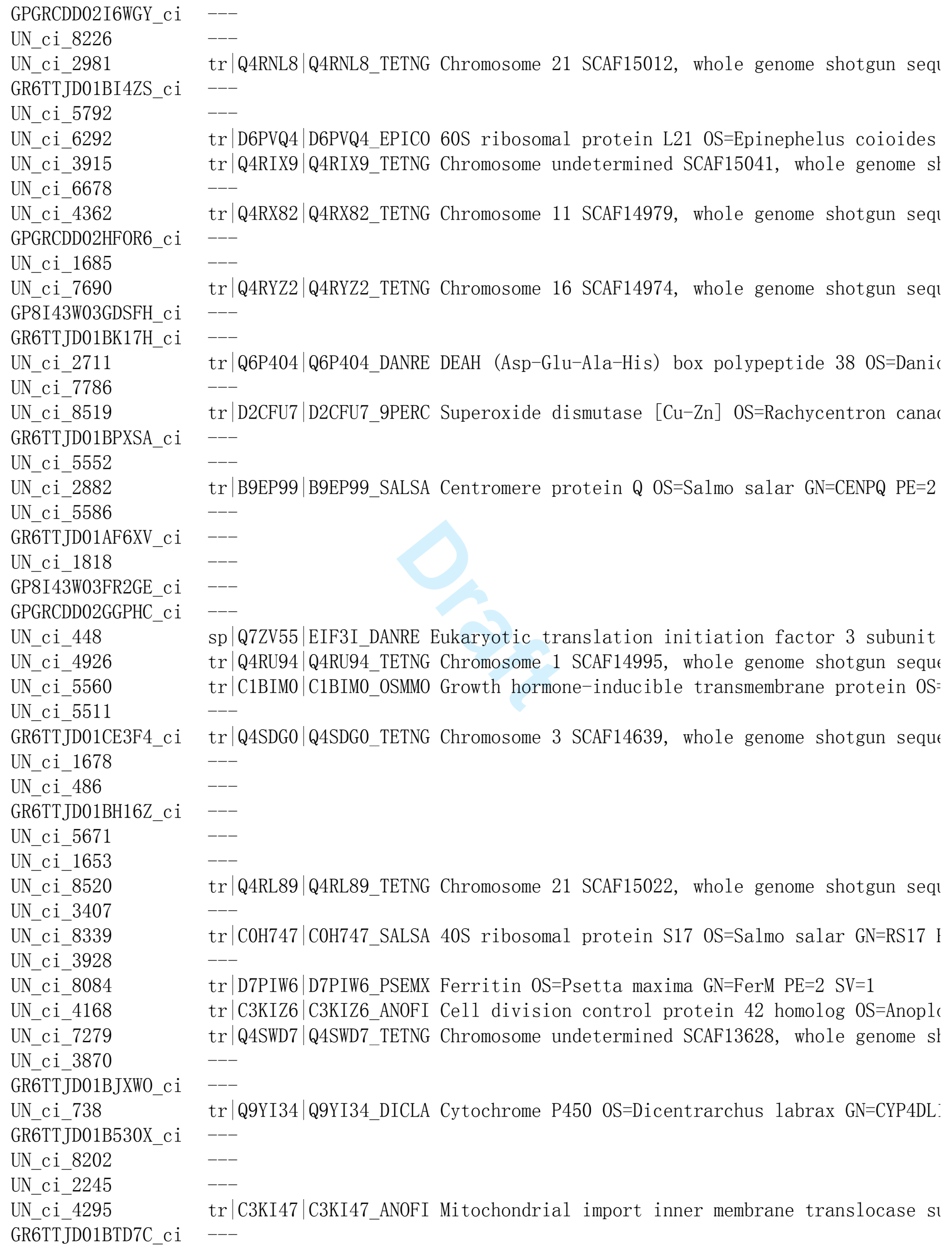




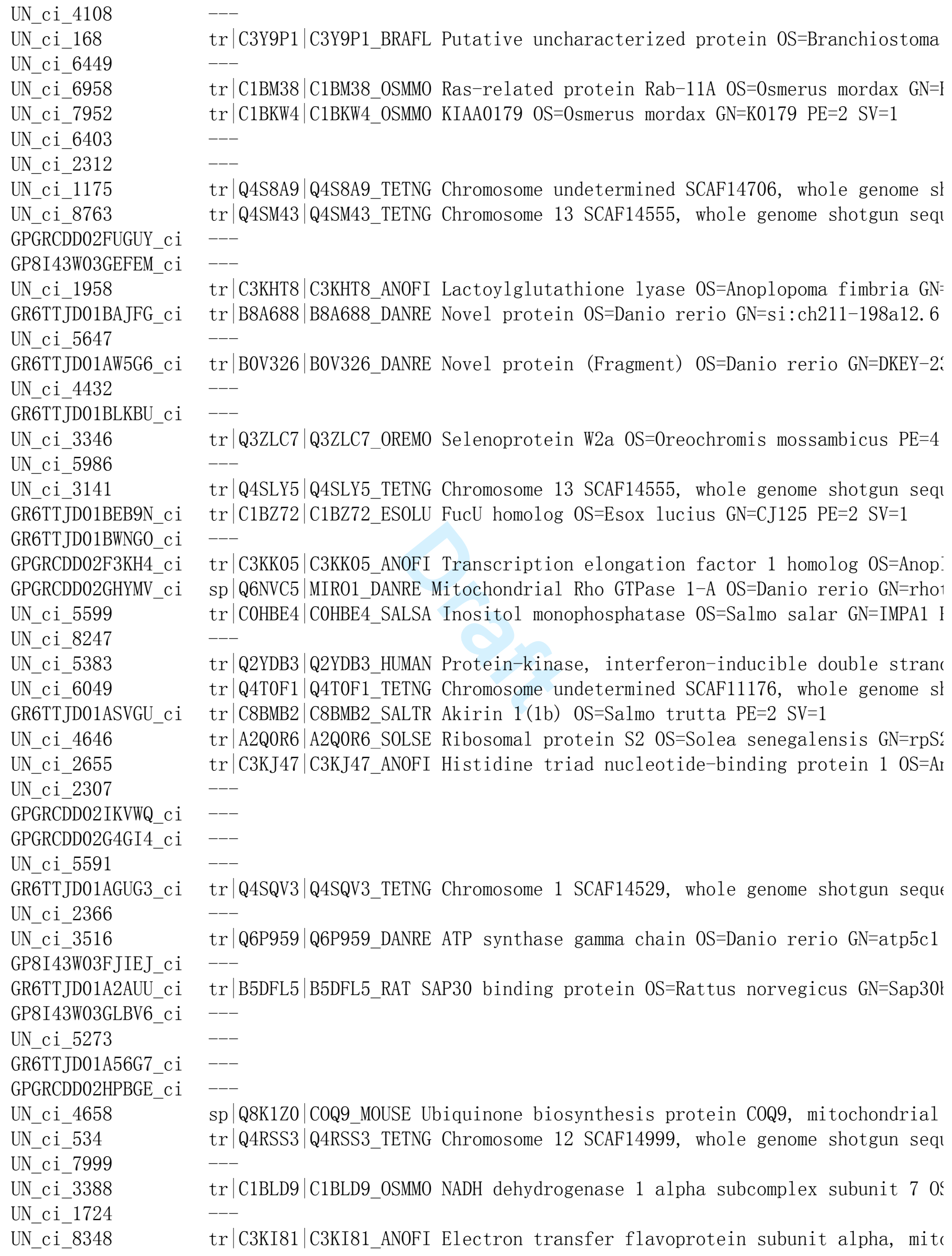

$\operatorname{tr} \mid$ C3Y9P1 $\mid$ C3Y9P1_BRAFL Putative uncharacterized protein OS=Branchiostoma

$\operatorname{tr} \mid$ C1BM38 $\mid$ C1BM38_0SMM0 Ras-related protein Rab-11A OS=0smerus mordax GN=I $\operatorname{tr} \mid$ C1BKW4 $\mid$ C1BKW4_0SMM0 KIAA0179 0S=0smerus mordax GN=K0179 PE=2 SV=1

tr $\mid$ Q4S8A9|Q4S8A9_TETNG Chromosome undetermined SCAF14706, whole genome sl tr $\mid$ Q4SM43|Q4SM43_TETNG Chromosome 13 SCAF14555, whole genome shotgun seq1

$\operatorname{tr} \mid$ C3KHT8 $\mid$ C3KHT8_ANOFI Lactoylglutathione 1yase OS=Anoplopoma fimbria GN= $\operatorname{tr} \mid$ B8A688|B8A688_DANRE Novel protein OS=Danio rerio GN=si:ch211-198a12. 6

tr $\mid$ B0V326|B0V326_DANRE Novel protein (Fragment) OS=Danio rerio GN=DKEY-2: $\operatorname{tr} \mid$ Q3ZLC7 $\mid$ Q3ZLC7_OREMO Selenoprotein W2a OS=0reochromis mossambicus PE=4 tr $\mid$ Q4SLY5|Q4SLY5_TETNG Chromosome 13 SCAF14555, whole genome shotgun seq1 $\operatorname{tr} \mid$ C1BZ72 $\mid$ C1BZ72_ESOLU FucU homolog OS=Esox lucius GN=CJ125 PE=2 SV=1

tr|C3KK05|C3KK05_ANOFI Transcription elongation factor 1 homolog OS=Anop: sp |Q6NVC5 |MIR01_DANRE Mitochondrial Rho GTPase 1-A OS=Danio rerio GN=rho1 $\operatorname{tr} \mid$ COHBE4 $\mid$ COHBE4_SALSA Inositol monophosphatase OS=Salmo salar GN=IMPA1 I

$\operatorname{tr} \mid$ Q2YDB3 $\mid$ Q2YDB3_HUMAN Protein-kinase, interferon-inducible double stranc tr $\mid$ Q4T0F1 $\mid$ Q4T0F1_TETNG Chromosome undetermined SCAF11176, whole genome sl tr $\mid$ C8BMB2 $\mid$ C8BMB2_SALTR Akirin 1(1b) OS=Salmo trutta PE=2 SV=1

tr $\mid$ A2Q0R6 $\mid$ A2Q0R6_SOLSE Ribosomal protein S2 OS=Solea senegalensis GN=rpS؛ $\operatorname{tr} \mid$ C3KJ47 $\mid$ C3KJ47_ANOFI Histidine triad nucleotide-binding protein 1 OS=Ar

tr $\mid$ Q4SQV3 $\mid$ Q4SQV3_TETNG Chromosome 1 SCAF14529, whole genome shotgun sequt tr $\mid$ Q6P959|Q6P959_DANRE ATP synthase gamma chain OS=Danio rerio GN=atp5c1 tr $\mid$ B5DFL5 $\mid$ B5DFL5_RAT SAP30 binding protein OS=Rattus norvegicus GN=Sap30k sp |Q8K1Z0|C0Q9_MOUSE Ubiquinone biosynthesis protein C0Q9, mitochondrial tr $\mid$ Q4RSS3|Q4RSS3_TETNG Chromosome 12 SCAF14999, whole genome shotgun seq1 tr $\mid$ C1BLD9 $\mid$ C1BLD9_0SMMO NADH dehydrogenase 1 alpha subcomplex subunit 7 0 $\operatorname{tr} \mid$ C3KI81|C3KI81_ANOFI Electron transfer flavoprotein subunit alpha, mit 


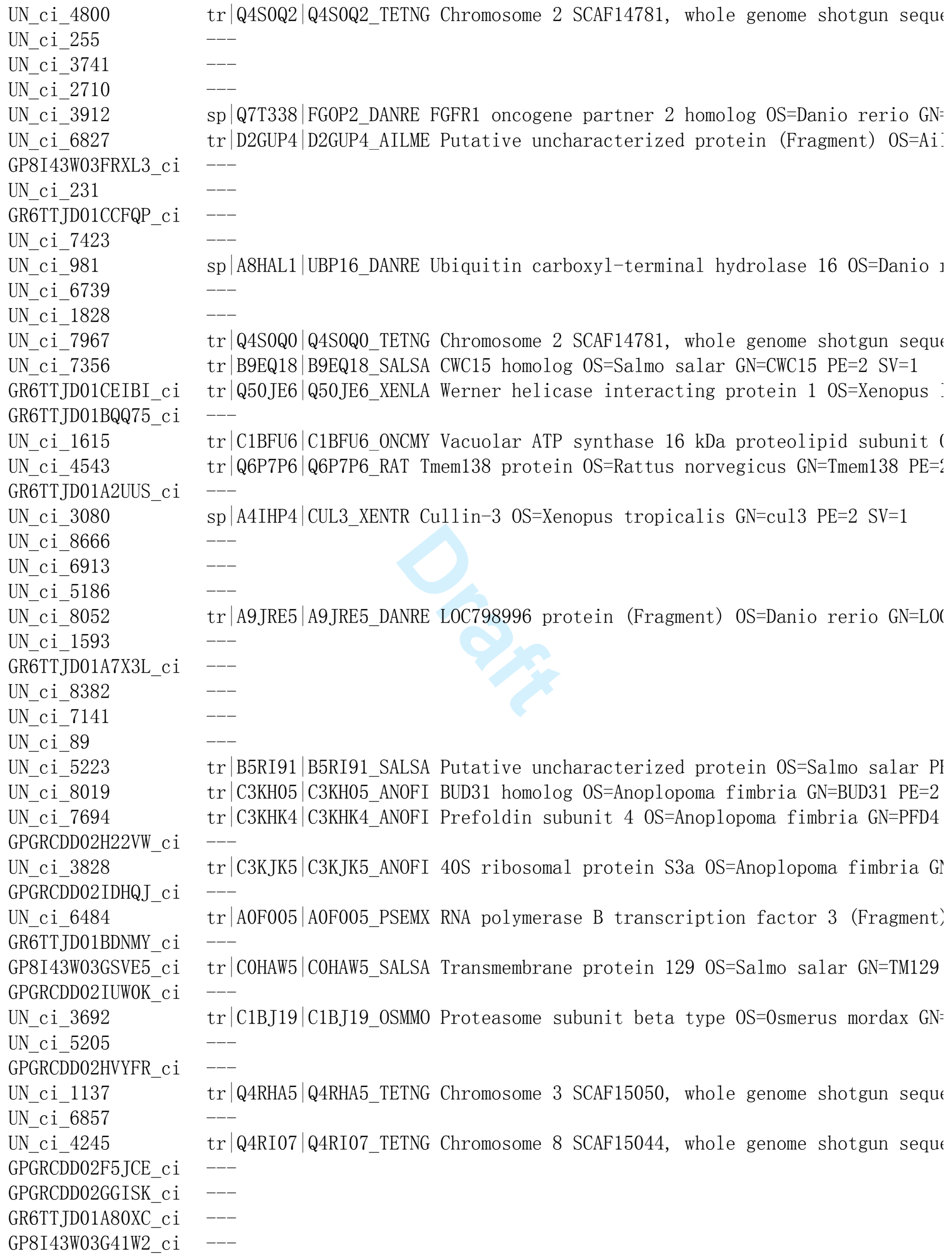

tr $\mid$ Q4S0Q2 |Q4S0Q2_TETNG Chromosome 2 SCAF14781, whole genome shotgun sequt

sp $\mid$ Q7T338|FG0P2_DANRE FGFR1 oncogene partner 2 homolog OS=Danio rerio GN= $\operatorname{tr} \mid$ D2GUP4|D2GUP4_AILME Putative uncharacterized protein (Fragment) OS=Ai:

sp $\mid$ A8HAL1|UBP16_DANRE Ubiquitin carboxy1-terminal hydrolase 16 0S=Danio ]

tr $\mid$ Q4S0Q0 $\mid$ Q4S0Q0_TETNG Chromosome 2 SCAF14781, whole genome shotgun sequt $\operatorname{tr} \mid$ B9EQ18 $\mid$ B9EQ18_SALSA CWC15 homolog OS=Salmo salar GN=CWC15 PE=2 SV=1 $\operatorname{tr} \mid$ Q50JE6|Q50JE6_XENLA Werner helicase interacting protein 1 OS=Xenopus

tr|C1BFU6|C1BFU6_0NCMY Vacuolar ATP synthase $16 \mathrm{kDa}$ proteolipid subunit ( $\operatorname{tr} \mid$ Q6P7P6 $\mid$ Q6P7P6_RAT Tmem138 protein 0S=Rattus norvegicus GN=Tmem138 PE=‘ sp $\mid$ A4IHP4 $\mid$ CUL3_XENTR Cullin-3 OS=Xenopus tropicalis GN=cul3 PE=2 SV=1

$---$

tr $\mid$ A9JRE5 $\mid$ A9JRE5_DANRE LOC798996 protein (Fragment) OS=Danio rerio GN=LOC

tr|B5RI91|B5RI91_SALSA Putative uncharacterized protein OS=Salmo salar PI $\operatorname{tr} \mid$ C3KH05|C3KH05_ANOFI BUD31 homolog OS=Anoplopoma fimbria GN=BUD31 PE=2 $\operatorname{tr} \mid$ C3KHK4 $\mid$ C3KHK4_ANOFI Prefoldin subunit 4 OS=Anoplopoma fimbria GN=PFD4 $\operatorname{tr} \mid$ C3KJK5 $\mid$ C3KJK5_ANOFI 40S ribosomal protein S3a 0S=Anoplopoma fimbria G! tr $\mid$ A0F005 $\mid$ A0F005_PSEMX RNA polymerase B transcription factor 3 (Fragment) tr $\mid$ COHAW5 $\mid$ COHAW5_SALSA Transmembrane protein 129 OS=Salmo salar GN=TM129 $\operatorname{tr} \mid$ C1BJ19 $\mid$ C1BJ19_0SMM0 Proteasome subunit beta type OS=0smerus mordax GN= tr $\mid$ Q4RHA5 |Q4RHA5_TETNG Chromosome 3 SCAF15050, whole genome shotgun sequt tr $\mid$ Q4RI07|Q4RI07_TETNG Chromosome 8 SCAF15044, whole genome shotgun seque https://mc06.manuscriptcentral.com/genome-pubs 


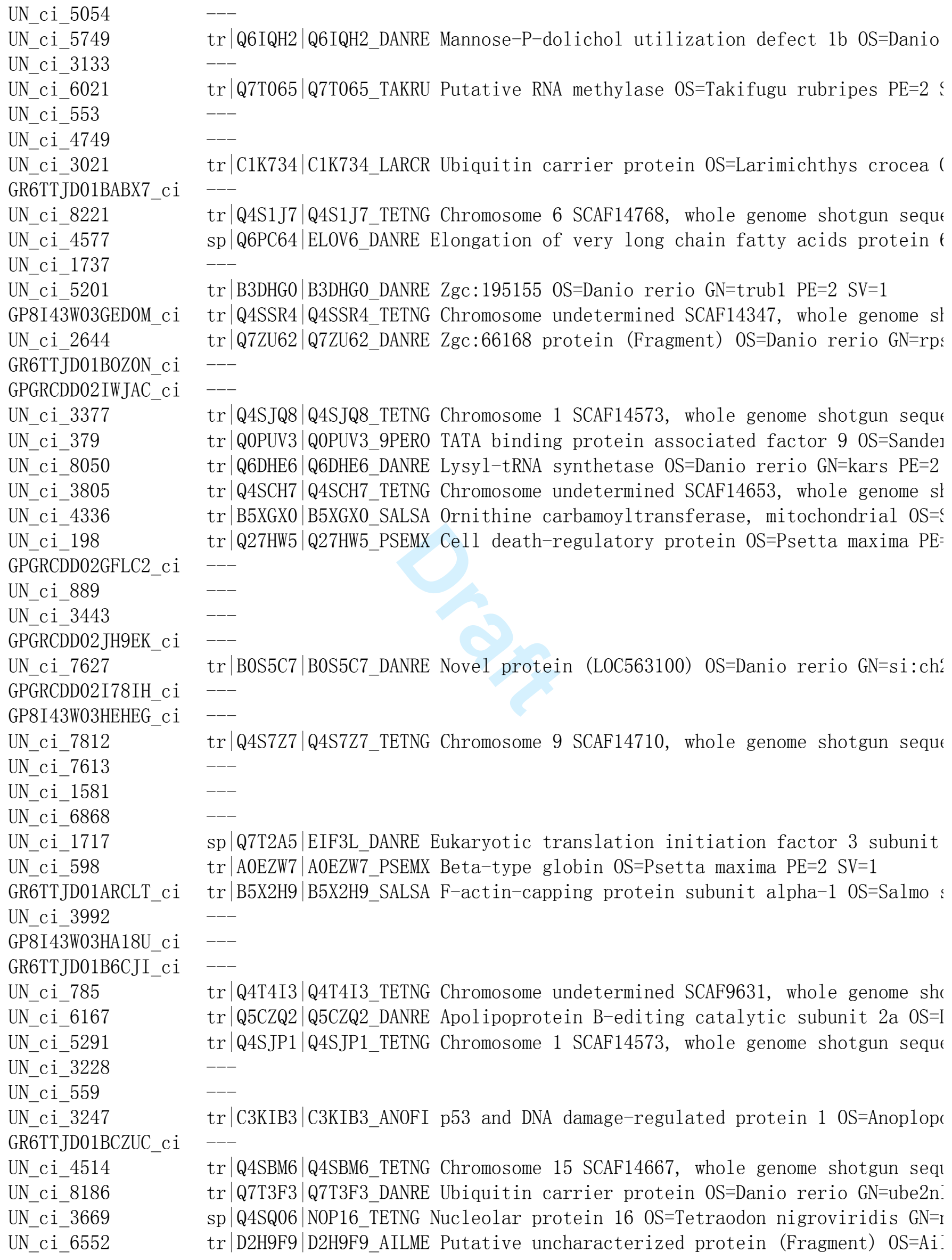

$\operatorname{tr} \mid$ Q6IQH2 $\mid$ Q6IQH2_DANRE Mannose-P-dolichol utilization defect 1b OS=Danio $\operatorname{tr} \mid$ Q7T065 $\mid$ Q7T065_TAKRU Putative RNA methylase OS=Takifugu rubripes PE=2 ؛

$\operatorname{tr} \mid$ C1K734 $\mid$ C1K734_LARCR Ubiquitin carrier protein 0S=Larimichthys crocea ( $\operatorname{tr} \mid$ Q4S1J7|Q4S1J7_TETNG Chromosome 6 SCAF14768, whole genome shotgun sequt sp|Q6PC64|EL0V6_DANRE Elongation of very long chain fatty acids protein $t$

$\operatorname{tr} \mid$ B3DHG0|B3DHG0_DANRE Zgc:195155 OS=Danio rerio GN=trub1 PE=2 SV=1 tr $\mid$ Q4SSR4|Q4SSR4_TETNG Chromosome undetermined SCAF14347, whole genome sl $\operatorname{tr} \mid$ Q7ZU62 $\mid$ Q7ZU62_DANRE Zgc:66168 protein (Fragment) OS=Danio rerio GN=rp؛

tr $\mid$ Q4SJQ8 |Q4SJQ8_TETNG Chromosome 1 SCAF14573, whole genome shotgun sequt $\operatorname{tr} \mid$ Q0PUV3 $\mid$ Q0PUV3_9PER0 TATA binding protein associated factor 9 OS=Sande] $\operatorname{tr} \mid$ Q6DHE6 $\mid$ Q6DHE6_DANRE Lysy1-tRNA synthetase OS=Danio rerio GN=kars PE=2 tr $\mid$ Q4SCH7 |Q4SCH7_TETNG Chromosome undetermined SCAF14653, whole genome sl $\operatorname{tr} \mid$ B5XGX0|B5XGX0_SALSA Ornithine carbamoyltransferase, mitochondrial OS=؟ $\operatorname{tr} \mid$ Q27HW5 $\mid$ Q27HW5_PSEMX Ce11 death-regulatory protein OS=Psetta maxima PE=

$\operatorname{tr} \mid$ B0S5C7 |BOS5C7_DANRE Novel protein (L0C563100) OS=Danio rerio GN=si:ch؛ tr $\mid$ Q4S7Z7|Q4S7Z7_TETNG Chromosome 9 SCAF14710, whole genome shotgun sequt $---$

sp $\mid$ Q7T2A5|EIF3L_DANRE Eukaryotic translation initiation factor 3 subunit tr $\mid$ A0EZW7 $\mid$ A0EZW7_PSEMX Beta-type globin OS=Psetta maxima PE=2 SV=1 $\operatorname{tr} \mid$ B5X2H9|B5X2H9_SALSA F-actin-capping protein subunit alpha-1 OS=Salmo : $---$

tr $\mid$ Q4T4I3|Q4T4I3_TETNG Chromosome undetermined SCAF9631, whole genome sh $\operatorname{tr} \mid$ Q5CZQ2 |Q5CZQ2_DANRE Apolipoprotein B-editing catalytic subunit 2a OS=I tr |Q4SJP1|Q4SJP1_TETNG Chromosome 1 SCAF14573, whole genome shotgun seque

tr $\mid$ C3KIB3 $\mid$ C3KIB3_ANOFI p53 and DNA damage-regulated protein 1 OS=Anoplop tr $\mid$ Q4SBM6 |Q4SBM6_TETNG Chromosome 15 SCAF14667, whole genome shotgun seq1 $\operatorname{tr} \mid$ Q7T3F3 $\mid$ Q7T3F3_DANRE Ubiquitin carrier protein OS=Danio rerio GN=ube2n. sp $\mid$ Q4SQ06 |NOP16_TETNG Nucleolar protein 16 OS=Tetraodon nigroviridis $\mathrm{GN}=\mathrm{r}$ tr $\mid$ D2H9F9 $\mid$ D2H9F9_AILME Putative uncharacterized protein (Fragment) OS=Ai: 


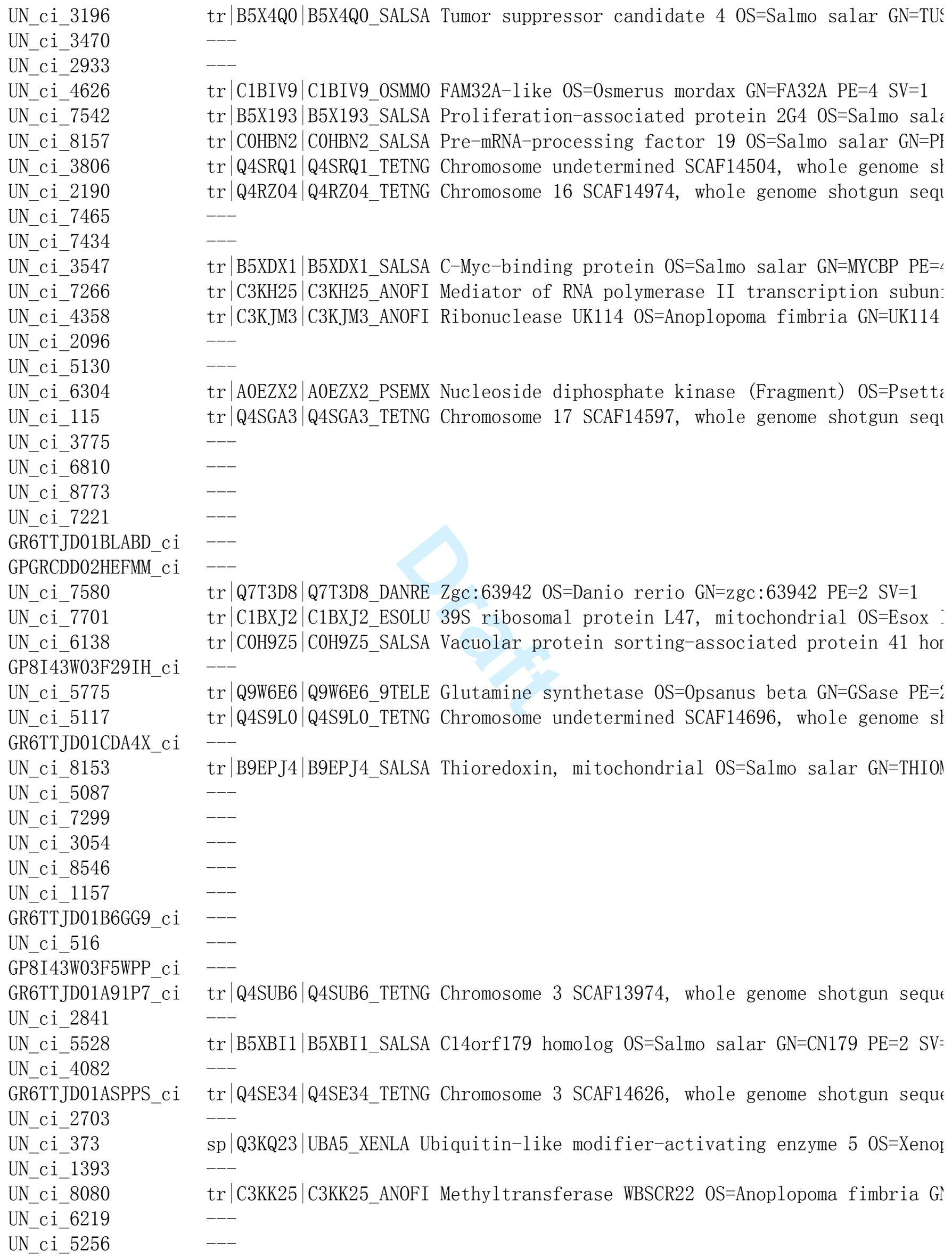

$\operatorname{tr} \mid$ B5X4Q0|B5X4Q0_SALSA Tumor suppressor candidate 4 OS=Salmo salar GN=TU؛

tr $\mid$ C1BIV9|C1BIV9_0SMM0 FAM32A-1ike OS=0smerus mordax GN=FA32A PE=4 SV=1

$\operatorname{tr} \mid$ B5X193|B5X193_SALSA Proliferation-associated protein 2G4 0S=Salmo sali $\operatorname{tr} \mid$ COHBN2 $\mid$ COHBN2_SALSA Pre-mRNA-processing factor 19 OS=Salmo salar GN=PI tr $\mid$ Q4SRQ1|Q4SRQ1_TETNG Chromosome undetermined SCAF14504, whole genome sl tr|Q4RZ04|Q4RZ04_TETNG Chromosome 16 SCAF14974, whole genome shotgun seqr

$\operatorname{tr} \mid$ B5XDX1 $\mid$ B5XDX1_SALSA C-Myc-binding protein 0S=Salmo salar GN=MYCBP PE= $\operatorname{tr} \mid$ C3KH25|C3KH25_ANOFI Mediator of RNA polymerase II transcription subun $\operatorname{tr} \mid$ C3KJM3 $\mid$ C3KJM3_ANOFI Ribonuclease UK114 OS=Anoplopoma fimbria GN=UK114

tr $\mid$ A0EZX2|A0EZX2_PSEMX Nucleoside diphosphate kinase (Fragment) OS=Psettc tr $\mid$ Q4SGA3|Q4SGA3_TETNG Chromosome 17 SCAF14597, whole genome shotgun seq1

tr|Q7T3D8|Q7T3D8_DANRE Zgc:63942 OS=Danio rerio GN=zgc:63942 PE=2 SV=1 $\operatorname{tr} \mid$ C1BXJ2|C1BXJ2_ESOLU 39S ribosomal protein L47, mitochondrial 0S=Esox tr $\mid$ COH9Z5|COH9Z5_SALSA Vacuolar protein sorting-associated protein 41 hor

tr $\mid$ Q9W6E6 $\mid$ Q9W6E6_9TELE Glutamine synthetase OS=0psanus beta GN=GSase PE=؛ $\operatorname{tr} \mid$ Q4S9L0|Q4S9L0_TETNG Chromosome undetermined SCAF14696, whole genome sl

tr|B9EPJ4|B9EPJ4_SALSA Thioredoxin, mitochondrial OS=Salmo salar GN=THIO!

tr $\mid$ Q4SUB6 |Q4SUB6_TETNG Chromosome 3 SCAF13974, whole genome shotgun sequt $\operatorname{tr} \mid$ B5XBI1|B5XBI1_SALSA C14orf179 homolog 0S=Salmo salar GN=CN179 PE=2 SV= tr $\mid$ Q4SE34|Q4SE34_TETNG Chromosome 3 SCAF14626, whole genome shotgun sequt sp|Q3KQ23|UBA5_XENLA Ubiquitin-1ike modifier-activating enzyme 5 OS=Xenor tr|C3KK25|C3KK25_ANOFI Methyltransferase WBSCR22 OS=Anoplopoma fimbria GI $---$ 


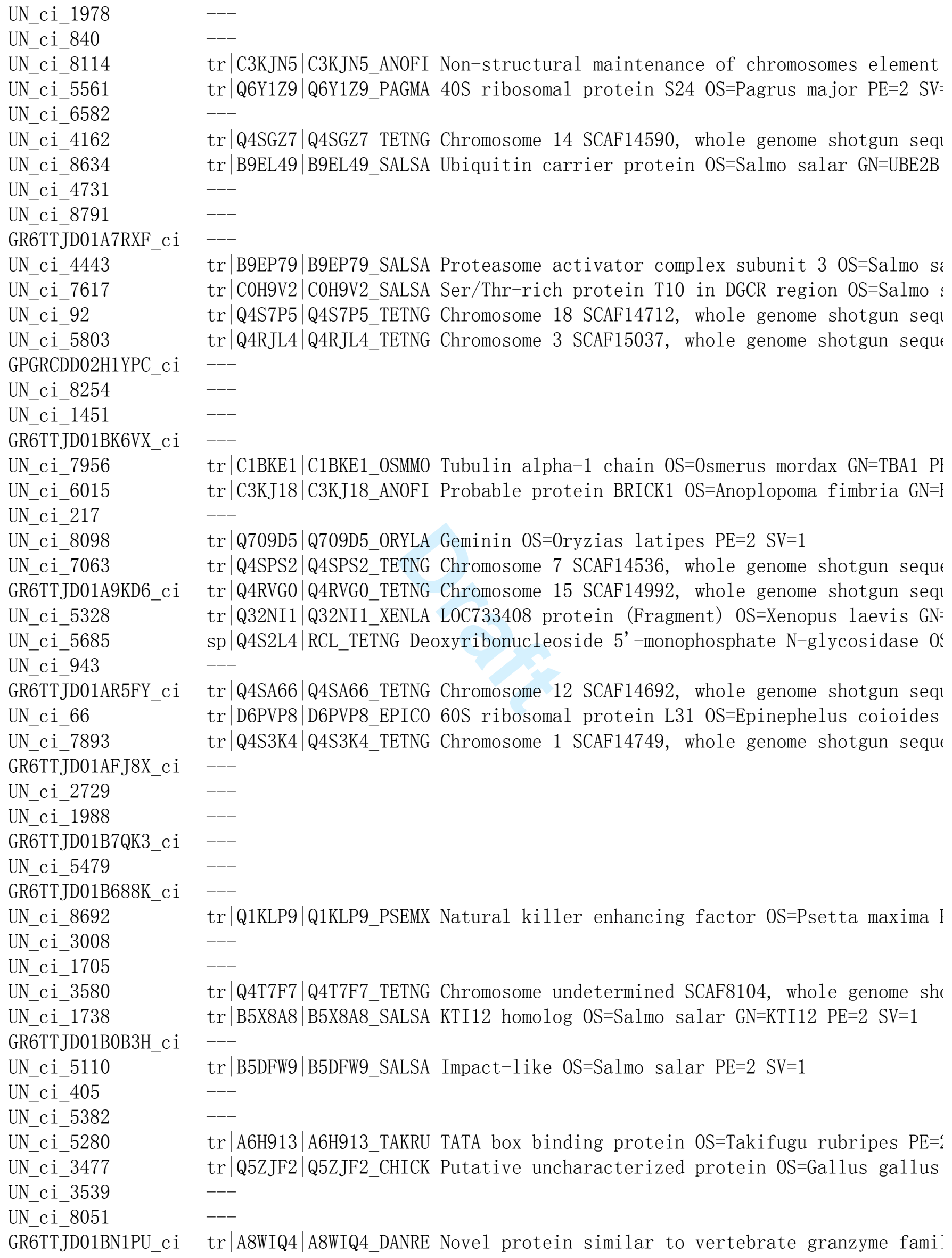




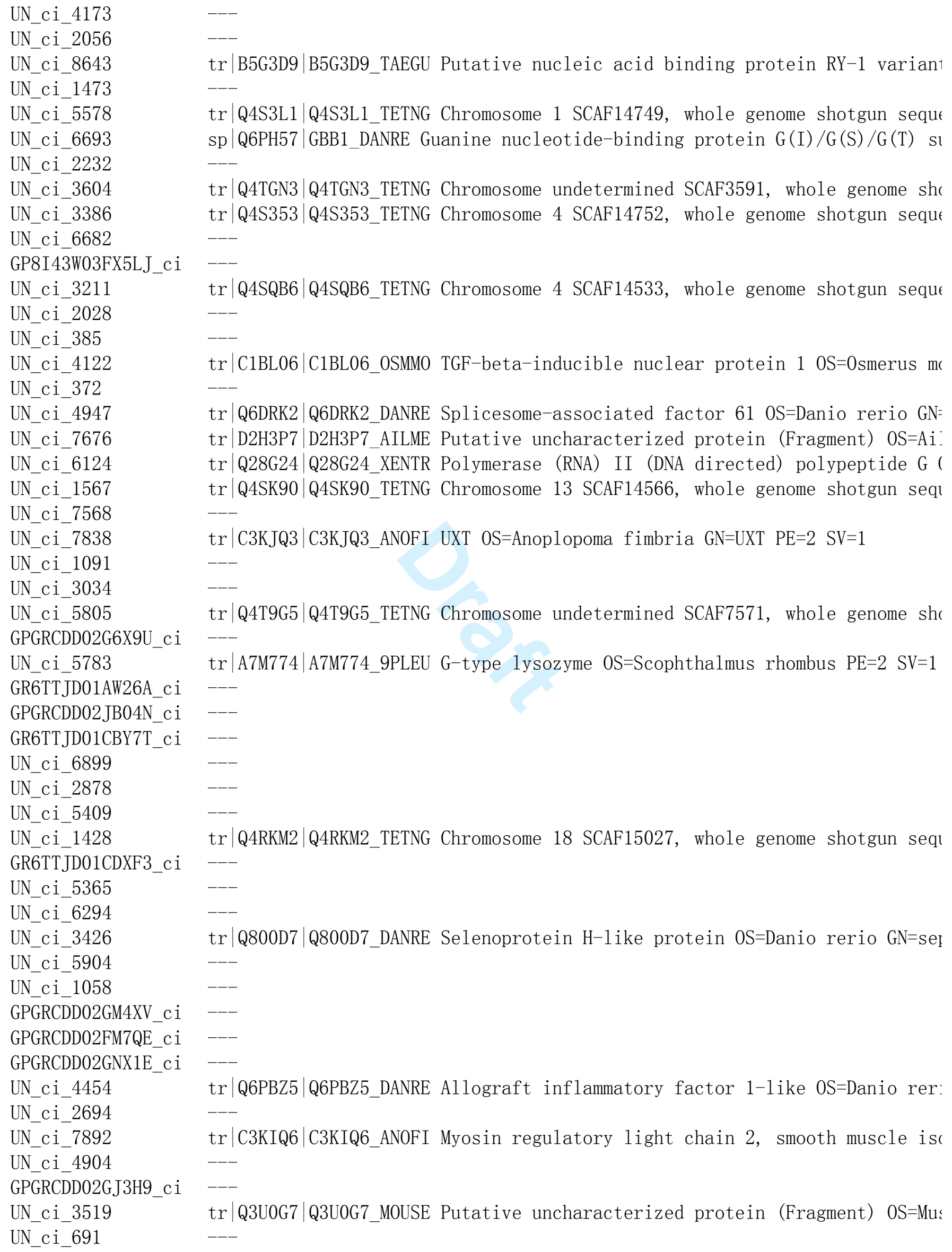




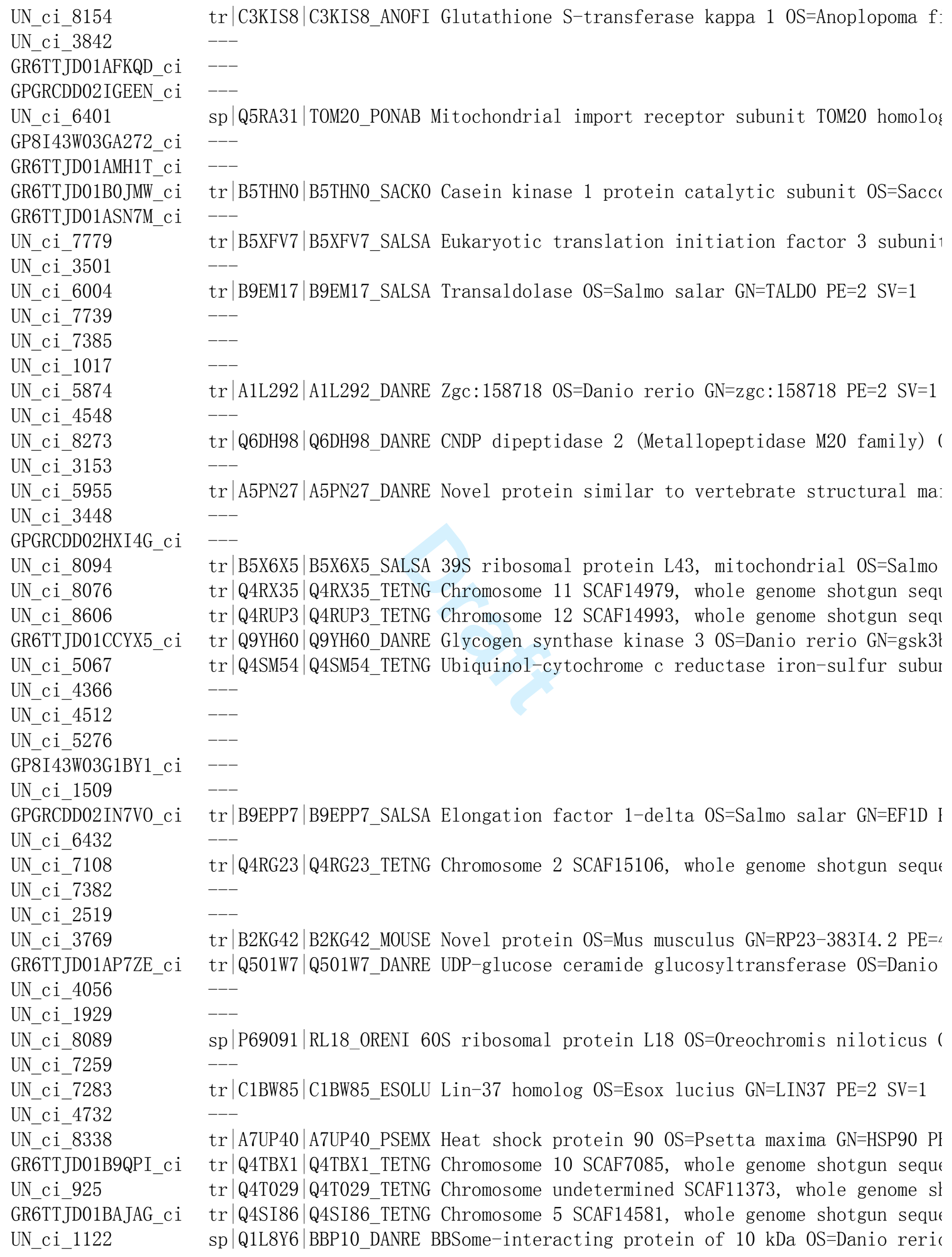

GR6TTJD01B0JMW_ci tr|B5THN0|B5THNO_SACK0 Casein kinase 1 protein catalytic subunit 0S=Sacce

$\operatorname{tr} \mid$ C3KIS8 $\mid$ C3KIS8_ANOFI Glutathione S-transferase kappa 1 OS=Anoplopoma $f$ :

sp |Q5RA31|T0M20_PONAB Mitochondrial import receptor subunit T0M20 homolok tr|B5XFV7|B5XFV7_SALSA Eukaryotic translation initiation factor 3 subuni1 $\operatorname{tr} \mid$ B9EM17|B9EM17_SALSA Transaldolase OS=Salmo salar GN=TALD0 PE=2 SV=1

$---$

$\operatorname{tr} \mid$ A1L292 $\mid$ A1L292_DANRE Zgc:158718 OS=Danio rerio GN=zgc:158718 PE=2 SV=1 tr $\mid$ Q6DH98|Q6DH98_DANRE CNDP dipeptidase 2 (Metallopeptidase M20 family) ( tr $\mid$ A5PN27|A5PN27_DANRE Novel protein similar to vertebrate structural ma: tr|B5X6X5|B5X6X5_SALSA 39S ribosomal protein L43, mitochondrial 0S=Salmo tr $\mid$ Q4RX35|Q4RX35_TETNG Chromosome 11 SCAF14979, whole genome shotgun sequ tr $\mid$ Q4RUP3 $\mid$ Q4RUP3_TETNG Chromosome 12 SCAF14993, whole genome shotgun sequ $\operatorname{tr} \mid$ Q9YH60|Q9YH60_DANRE Glycogen synthase kinase 3 OS=Danio rerio GN=gsk31 tr $\mid$ Q4SM54|Q4SM54_TETNG Ubiquinol-cytochrome c reductase iron-sulfur subur

tr|B9EPP7|B9EPP7_SALSA Elongation factor 1-delta 0S=Salmo salar GN=EF1D I tr $\mid$ Q4RG23|Q4RG23_TETNG Chromosome 2 SCAF15106, whole genome shotgun sequt $---$

$\operatorname{tr} \mid$ B2KG42 $\mid$ B2KG42_MOUSE Novel protein OS=Mus musculus GN=RP23-383I4. 2 PE=^ $\operatorname{tr} \mid$ Q501W7 $\mid$ Q501W7_DANRE UDP-glucose ceramide glucosyltransferase 0S=Danio $---$

sp|P69091|RL18_ORENI 60S ribosomal protein L18 0S=0reochromis niloticus ( $\operatorname{tr} \mid$ C1BW85 $\mid$ C1BW85_ESOLU Lin-37 homolog OS=Esox lucius GN=LIN37 PE=2 SV=1 tr $\mid$ A7UP40 $\mid$ A7UP40_PSEMX Heat shock protein 90 OS=Psetta maxima GN=HSP90 PI $\operatorname{tr} \mid$ Q4TBX1|Q4TBX1_TETNG Chromosome 10 SCAF7085, whole genome shotgun sequt $\operatorname{tr} \mid$ Q4T029|Q4T029_TETNG Chromosome undetermined SCAF11373, whole genome sl tr $\mid$ Q4SI86|Q4SI86_TETNG Chromosome 5 SCAF14581, whole genome shotgun sequt sp |Q1L8Y6|BBP10_DANRE BBSome-interacting protein of $10 \mathrm{kDa}$ OS=Danio reric 


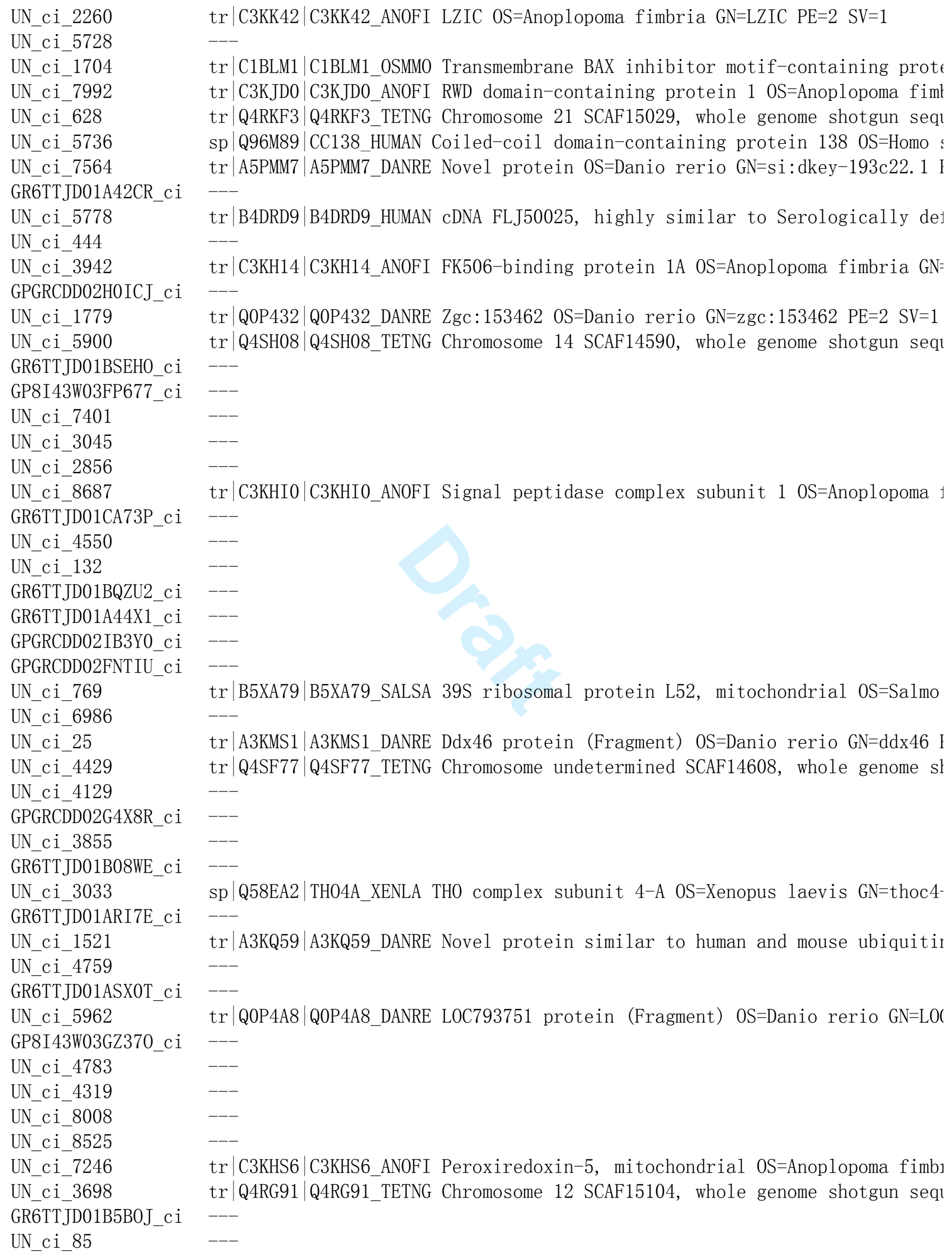

$\operatorname{tr} \mid$ C3KK42 $\mid$ C3KK42_ANOFI LZIC OS=Anoplopoma fimbria GN=LZIC PE=2 SV=1

tr $\mid$ C1BLM1 $\mid$ C1BLM1_0SMM0 Transmembrane BAX inhibitor motif-containing prot $\operatorname{tr} \mid$ C3KJDO $\mid$ C3KJD0_ANOFI RWD domain-containing protein 1 OS=Anoplopoma fiml tr $\mid$ Q4RKF3|Q4RKF3_TETNG Chromosome 21 SCAF15029, whole genome shotgun sequ sp |Q96M89|CC138_HUMAN Coiled-coil domain-containing protein 138 OS=Homo : $\operatorname{tr} \mid$ A5PMM7 $\mid$ A5PMM7_DANRE Nove1 protein OS=Danio rerio GN=si:dkey-193c22. 1 I tr|B4DRD9|B4DRD9_HUMAN cDNA FLJ50025, highly similar to Serologically dej $\operatorname{tr} \mid$ C3KH14|C3KH14_ANOFI FK506-binding protein 1A 0S=Anoplopoma fimbria GN= $\operatorname{tr} \mid$ Q0P432|Q0P432_DANRE Zgc:153462 OS=Danio rerio GN=zgc:153462 PE=2 SV=1 tr $\mid$ Q4SH08|Q4SH08_TETNG Chromosome 14 SCAF14590, whole genome shotgun seq1

$\operatorname{tr} \mid$ C3KHIO $\mid$ C3KHI0_ANOFI Signal peptidase complex subunit 1 OS=Anoplopoma 1 tr $\mid$ B5XA79|B5XA79_SALSA 39S ribosomal protein L52, mitochondrial 0S=Salmo $\operatorname{tr} \mid$ A3KMS1 $\mid$ A3KMS1_DANRE Ddx46 protein (Fragment) OS=Danio rerio GN=ddx46 I $\operatorname{tr} \mid$ Q4SF77 $\mid$ Q4SF77_TETNG Chromosome undetermined SCAF14608, whole genome sl sp $\mid$ Q58EA2 $\mid$ TH04A_XENLA TH0 complex subunit 4-A OS=Xenopus laevis GN=thoc4$\operatorname{tr} \mid$ A3KQ59|A3KQ59_DANRE Novel protein similar to human and mouse ubiquitir $\operatorname{tr} \mid$ Q0P4A8 $\mid$ Q0P4A8_DANRE LOC793751 protein (Fragment) OS=Danio rerio GN=LOC $\operatorname{tr} \mid$ C3KHS6 |C3KHS6_ANOFI Peroxiredoxin-5, mitochondrial OS=Anoplopoma fimb] tr $\mid$ Q4RG91|Q4RG91_TETNG Chromosome 12 SCAF15104, whole genome shotgun seqr 


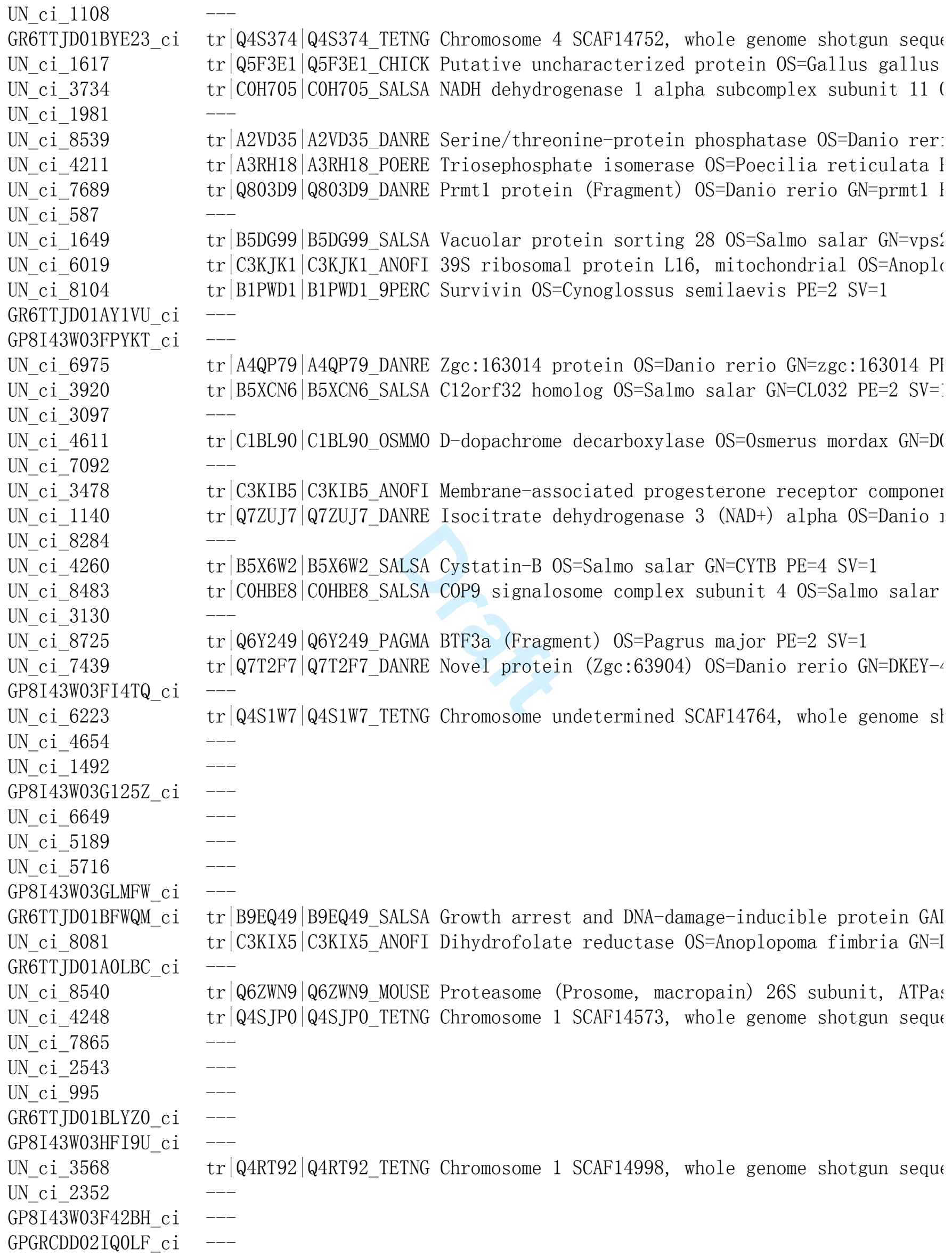

tr $\mid$ Q4S374|Q4S374_TETNG Chromosome 4 SCAF14752, whole genome shotgun sequt tr $\mid$ Q5F3E1|Q5F3E1_CHICK Putative uncharacterized protein OS=Gallus gallus tr $\mid$ COH705|COH705_SALSA NADH dehydrogenase 1 alpha subcomplex subunit 11 (

tr $\mid$ A2VD35 $\mid$ A2VD35_DANRE Serine/threonine-protein phosphatase OS=Danio rer $\operatorname{tr} \mid$ A3RH18|A3RH18_POERE Triosephosphate isomerase OS=Poecilia reticulata I $\operatorname{tr} \mid$ Q803D9 $\mid$ Q803D9_DANRE Prmt1 protein (Fragment) OS=Danio rerio GN=prmt1 I

tr|B5DG99|B5DG99_SALSA Vacuolar protein sorting 28 OS=Salmo salar GN=vps‘ $\operatorname{tr} \mid$ C3KJK1 $\mid$ C3KJK1_ANOFI 39S ribosomal protein L16, mitochondrial OS=Anop1c $\operatorname{tr} \mid$ B1PWD1|B1PWD1_9PERC Survivin OS=Cynoglossus semilaevis PE=2 SV=1

$\operatorname{tr} \mid$ A4QP79|A4QP79_DANRE Zgc: 163014 protein OS=Danio rerio GN=zgc:163014 PI tr $\mid$ B5XCN6 |B5XCN6_SALSA C12orf32 homolog OS=Salmo salar GN=CL032 PE=2 SV=:

$\operatorname{tr} \mid$ C1BL90 $\mid$ C1BL90_0SMM0 D-dopachrome decarboxylase 0S=0smerus mordax GN=D( tr|C3KIB5|C3KIB5_ANOFI Membrane-associated progesterone receptor componer $\operatorname{tr} \mid$ Q7ZUJ7|Q7ZUJ7_DANRE Isocitrate dehydrogenase $3(\mathrm{NAD+})$ alpha 0S=Danio $]$

$\operatorname{tr} \mid$ B5X6W2 $\mid$ B5X6W2_SALSA Cystatin-B 0S=Salmo salar GN=CYTB PE=4 SV=1

tr $\mid$ COHBE8 $\mid$ COHBE8_SALSA COP9 signalosome complex subunit 4 OS=Salmo salar

tr $\mid$ Q6Y249 $\mid$ Q6Y249_PAGMA BTF3a (Fragment) OS=Pagrus major PE=2 SV=1

tr $\mid$ Q7T2F7 $\mid$ Q7T2F7_DANRE Novel protein (Zgc:63904) OS=Danio rerio GN=DKEY-ょ tr $\mid$ Q4S1W7|Q4S1W7_TETNG Chromosome undetermined SCAF14764, whole genome sl

tr|B9EQ49|B9EQ49_SALSA Growth arrest and DNA-damage-inducible protein GAI $\operatorname{tr} \mid$ C3KIX5|C3KIX5_ANOFI Dihydrofolate reductase OS=Anoplopoma fimbria GN=I tr $\mid$ Q6ZWN9 $\mid$ Q6ZWN9_MOUSE Proteasome (Prosome, macropain) 26S subunit, ATPa؛ tr|Q4SJP0|Q4SJP0_TETNG Chromosome 1 SCAF14573, whole genome shotgun seque

tr $\mid$ Q4RT92|Q4RT92_TETNG Chromosome 1 SCAF14998, whole genome shotgun sequt 


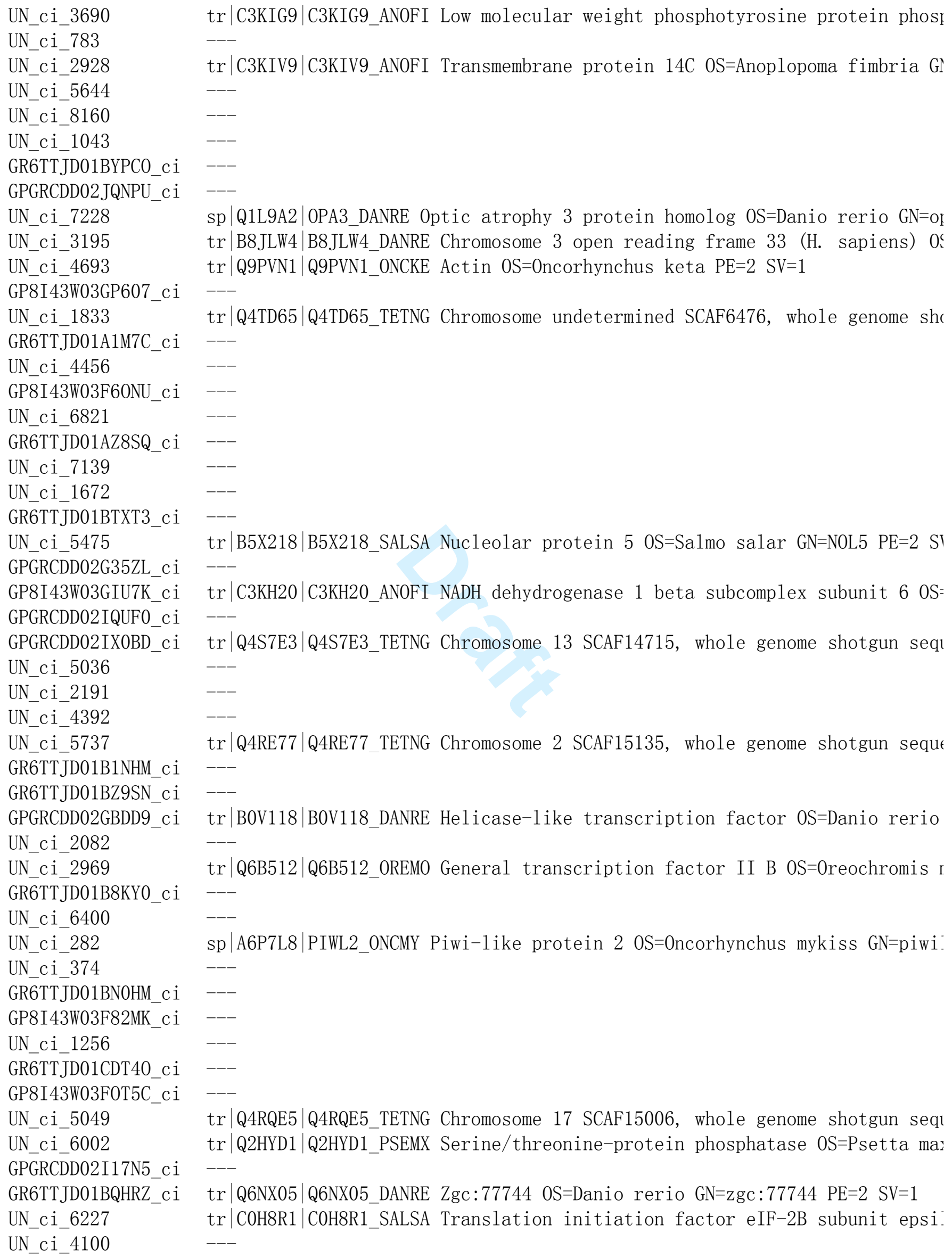


UN_ci_3567

UN_ci_8261

UN_ci_3940

UN_ci_7217

UN_ci_3883

GPGRCDD02JE7BJ_ci

UN_ci_8768 GP8I43W03GKGCQ ci

UN_ci_3921

UN_ci_6882

UN_ci_6832

GPGRCDD02G5L3D_ci

UN_ci_5212

GR6TTJD01BN00T_ci GPGRCDD02IN88V_ci GR6TTJD01BKDV7_ci

UN_ci_583

GR6TTJD01AR0FA_ci

UN_ci_7696

UN_ci_4665

UN_ci_6421

UN_ci_8070

UN_ci 7405

UN_ci 4669

GPGRCDD02ILKYV_ci

UN_ci_4161

UN_ci_2458

UN_ci_3682

UN_ci_5388

GP8I43W03FV6TN_ci

UN_ci_1847

UN ci 6007

GPGRCDD02H7PEA_ci

UN_ci_7487

UN_ci_475

UN ci 8270

GR6TTJD01AS351_ci

UN_ci_5272

GR6TTJD01AECT9_ci

UN_ci_7758

UN_ci_970

GR6TTJD01BMRUB ci GR6TTJD01B5QIT_ci GP8I43W03GKI31_ci

GP8I43W03FSX10_ci

UN_ci_257

UN_ci_5274

UN_ci_3666

UN_ci_2804

UN_ci_7940 tr $\mid$ Q4S2C7 $\mid$ Q4S2C7_TETNG Chromosome undetermined SCAF14764, whole genome sl tr $\mid$ Q9PUG4 $\mid$ Q9PUG4_GADM0 Beta-2 tubulin OS=Gadus morhua PE=2 SV=1

$\operatorname{tr} \mid$ Q6K0C3 $\mid$ Q6K0C3_SPAAU Cytosolic alanine aminotransferase 0S=Sparus aura1

tr $\mid$ C9E6G0|C9E6G0_0RYLA Smal1 ubiquitin-related modifier 1 OS=0ryzias lat

tr|B3DFW5|B3DFW5_DANRE Si:dkey-13i19.8 OS=Danio rerio GN=si:dkey-13i19. 8

tr $\mid$ C3KJ13|C3KJ13_ANOFI SRA stem-loop-interacting RNA-binding protein, mi1 tr|Q4RQ24|Q4RQ24_TETNG Chromosome 17 SCAF15006, whole genome shotgun seq1 $\operatorname{tr} \mid$ Q5RKM2 $\mid$ Q5RKM2_DANRE NADH-ubiquinone oxidoreductase $75 \mathrm{kDa}$ subunit OS=I

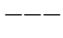

$\operatorname{tr} \mid$ C3KJI9|C3KJI9_ANOFI C17orf89 OS=Anoplopoma fimbria GN=CQ089 PE=4 SV=1 $---$

tr|B7X8Y8|B7X8Y8_PAGMA Elongation factor 1-alpha 0S=Pagrus major GN=42Sp? tr|Q4RT54|Q4RT54_TETNG Chromosome 12 SCAF14999, whole genome shotgun sequ

tr $\mid$ C1BIP5|C1BIP5_0SMM0 U3 sma11 nucleolar RNA-interacting protein 2 OS=0 tr|Q4RLD5|Q4RLD5_TETNG Chromosome 21 SCAF15022, whole genome shotgun sequ $\operatorname{tr} \mid$ Q8AYL1 $\mid$ Q8AYL1_ORYLA ZPC2 (Fragment) OS=0ryzias latipes PE=4 SV=1

$\operatorname{tr} \mid$ C3KK71 $\mid$ C3KK71_ANOFI RNA-binding protein 8A OS=Anoplopoma fimbria GN=RI $\operatorname{tr} \mid$ Q4S836 $\mid$ Q4S836_TETNG Chromosome 9 SCAF14710, whole genome shotgun seque $\operatorname{tr} \mid$ C3KJT2 $\mid$ C3KJT2_ANOFI NADH dehydrogenase 1 beta subcomplex subunit 5 , m:

tr $\mid$ B5X9N4 $\mid$ B5X9N4_SALSA DNA-binding protein RFXANK OS=Salmo salar GN=RFXK $\operatorname{tr} \mid$ COPUA0|COPUA0_SALSA Serine/threonine-protein phosphatase 2A $65 \mathrm{kDa}$ re६ $\operatorname{tr} \mid$ C1BJN5 $\mid$ C1BJN5_0SMM0 Cathepsin Z OS=0smerus mordax GN=CATZ PE=2 SV=1 tr $\mid$ Q4RL02|Q4RL02_TETNG Chromosome 1 SCAF15025, whole genome shotgun sequt tr $\mid$ Q6PGV9|Q6PGV9_DANRE Peptidylprolyl isomerase (Cyclophilin)-1ike 4 OS=I $\operatorname{tr} \mid$ Q6GQN0 $\mid$ Q6GQN0_DANRE Testis specific, 14 OS=Danio rerio GN=tsga14 PE=2 tr $\mid$ Q4RRF1|Q4RRF1_TETNG Chromosome 16 SCAF15002, whole genome shotgun seq1 $\operatorname{tr} \mid$ C1BLM2 $\mid$ C1BLM2_0SMMO PKHD-type hydroxylase YER049W OS=0smerus mordax G! $\operatorname{tr} \mid$ Q8UVG1 $\mid$ Q8UVG1_EPIC0 Ribosomal protein L32 OS=Epinephelus coioides PE=‘ sp $\mid$ P79896|ADHX_SPAAU Alcohol dehydrogenase class-3 0S=Sparus aurata PE=2

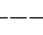

tr $\mid$ Q4TAA9 $\mid$ Q4TAA9_TETNG Chromosome 12 SCAF7391, whole genome shotgun seque 


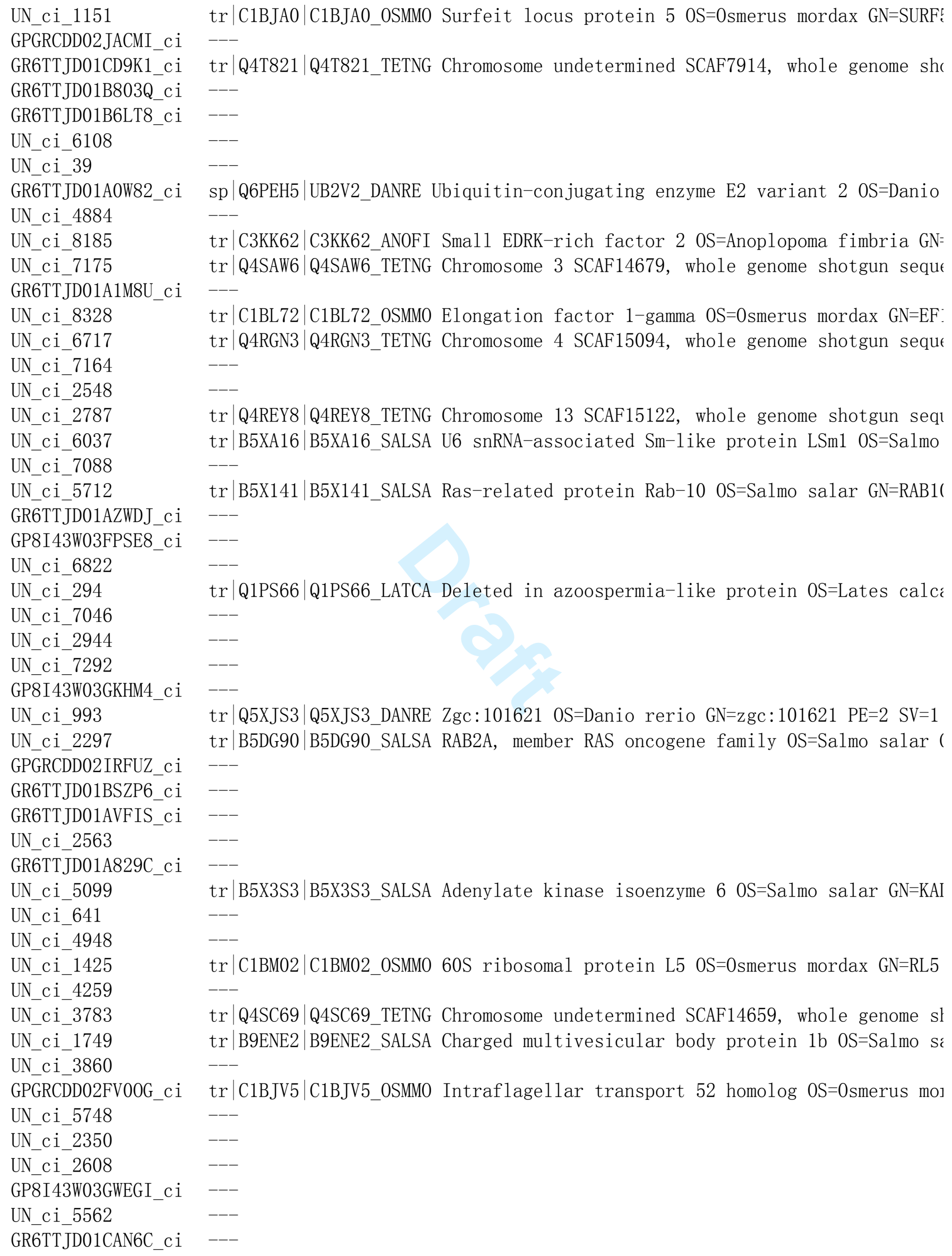

$\operatorname{tr} \mid$ C1BJA0|C1BJA0_0SMM0 Surfeit locus protein 5 OS=0smerus mordax GN=SURF? $\operatorname{tr} \mid$ Q4T821|Q4T821_TETNG Chromosome undetermined SCAF7914, whole genome sh sp |Q6PEH5|UB2V2_DANRE Ubiquitin-conjugating enzyme E2 variant 2 OS=Danio tr $\mid$ C3KK62 $\mid$ C3KK62_ANOFI Smal1 EDRK-rich factor 2 OS=Anoplopoma fimbria GN= tr|Q4SAW6|Q4SAW6_TETNG Chromosome 3 SCAF14679, whole genome shotgun sequt $\operatorname{tr} \mid$ C1BL72 $\mid$ C1BL72_0SMM0 Elongation factor 1-gamma OS=0smerus mordax GN=EF: tr $\mid$ Q4RGN3|Q4RGN3_TETNG Chromosome 4 SCAF15094, whole genome shotgun sequt $---$

tr $\mid$ Q4REY8|Q4REY8_TETNG Chromosome 13 SCAF15122, whole genome shotgun seq1 tr $\mid$ B5XA16|B5XA16_SALSA U6 snRNA-associated Sm-1ike protein LSm1 OS=Salmo

$\operatorname{tr} \mid$ B5X141|B5X141_SALSA Ras-related protein Rab-10 0S=Salmo salar GN=RAB1( tr $\mid$ Q1PS66 |Q1PS66_LATCA Deleted in azoospermia-1ike protein OS=Lates calcé tr $\mid$ Q5XJS3 |Q5XJS3_DANRE Zgc:101621 OS=Danio rerio GN=zgc:101621 PE=2 SV=1 tr|B5DG90|B5DG90_SALSA RAB2A, member RAS oncogene family OS=Salmo salar (

$\operatorname{tr} \mid$ B5X3S3|B5X3S3_SALSA Adenylate kinase isoenzyme 6 OS=Salmo salar GN=KAI $\operatorname{tr} \mid$ C1BM02 $\mid$ C1BM02_0SMM0 60S ribosomal protein L5 0S=0smerus mordax GN=RL5 tr $\mid$ Q4SC69|Q4SC69_TETNG Chromosome undetermined SCAF14659, whole genome sl tr|B9ENE2|B9ENE2_SALSA Charged multivesicular body protein 1b 0S=Salmo sć tr $\mid$ C1BJV5|C1BJV5_0SMM0 Intraflagellar transport 52 homolog 0S=0smerus mo] 


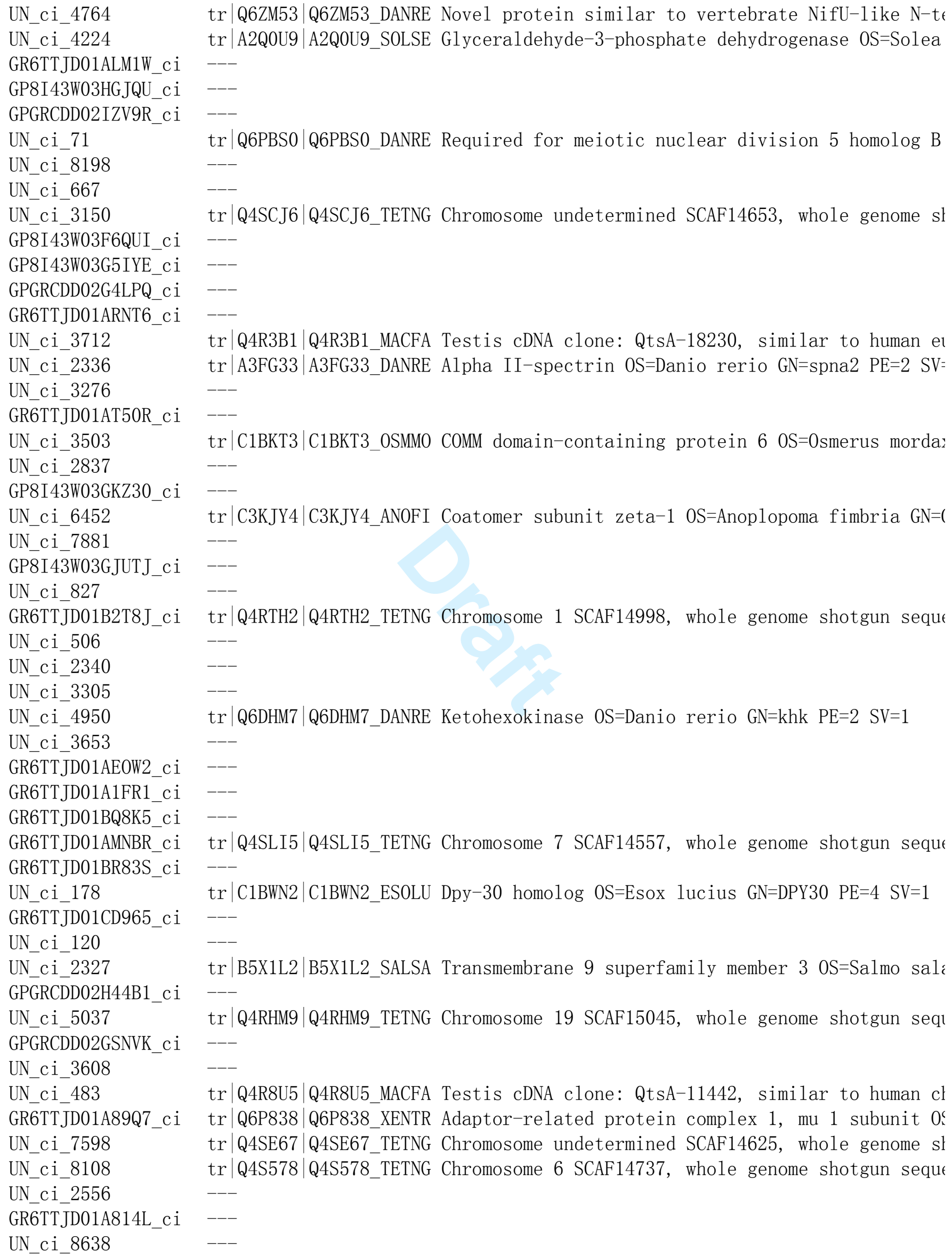




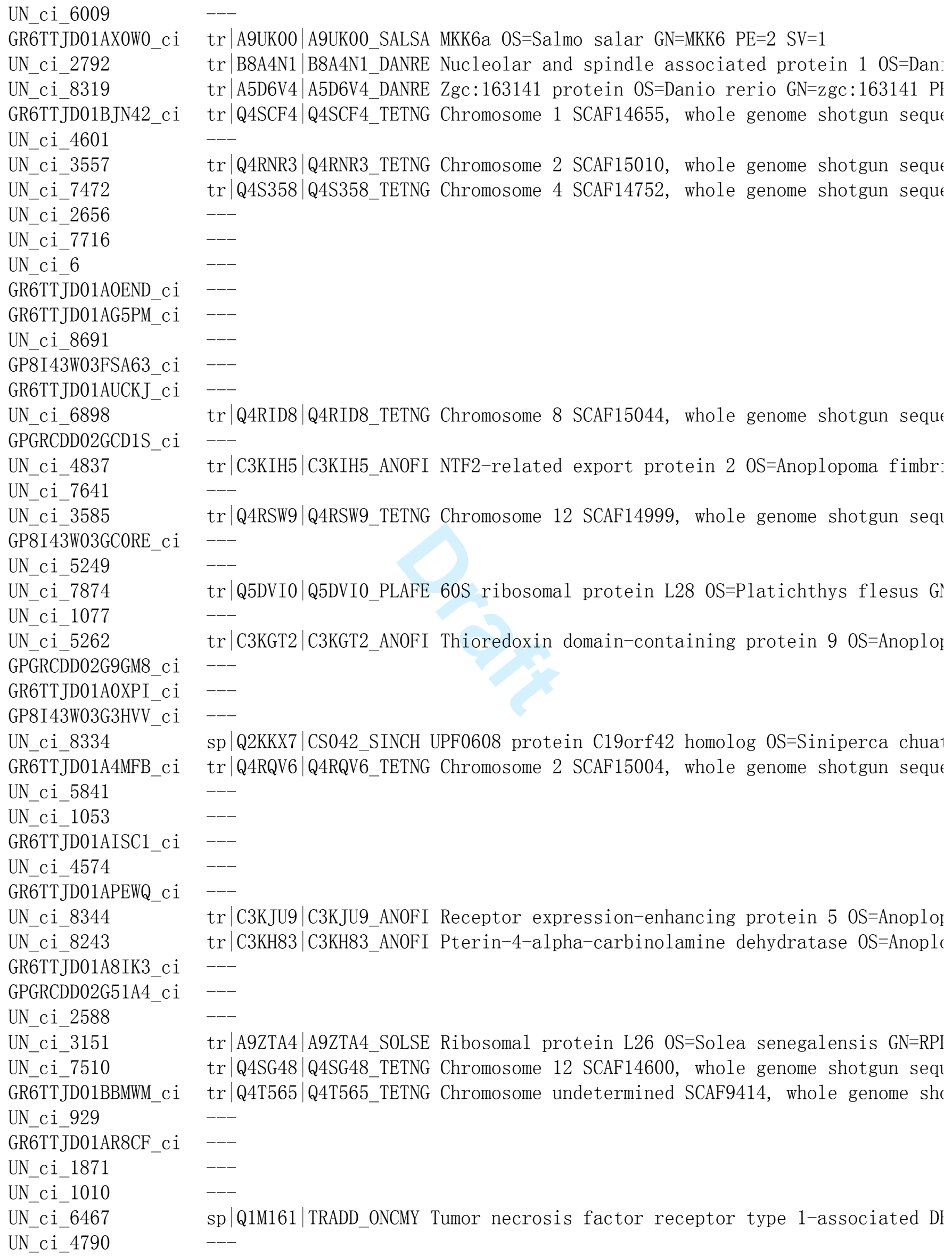

sp |Q1M161|TRADD_0NCMY Tumor necrosis factor receptor type 1-associated DI 


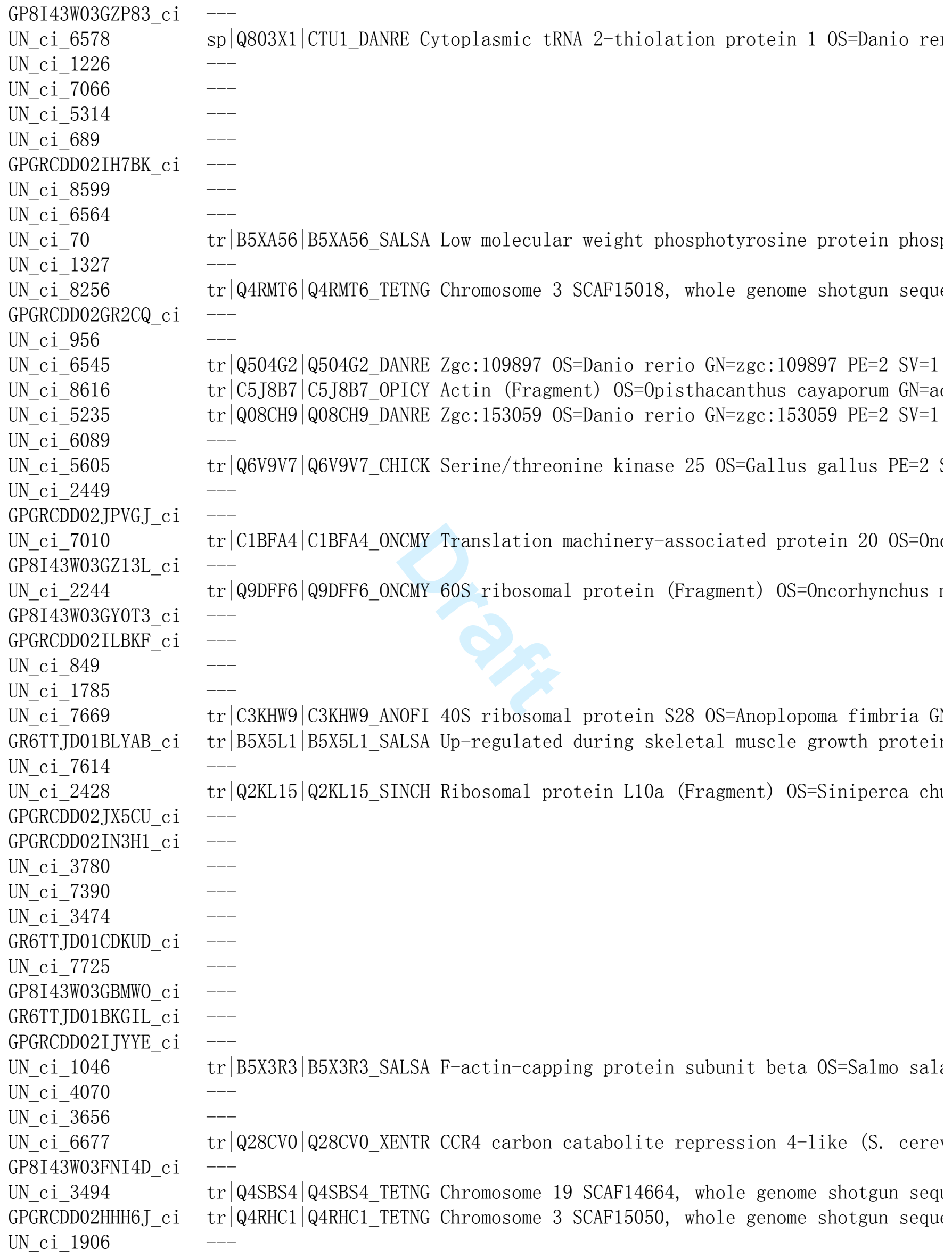




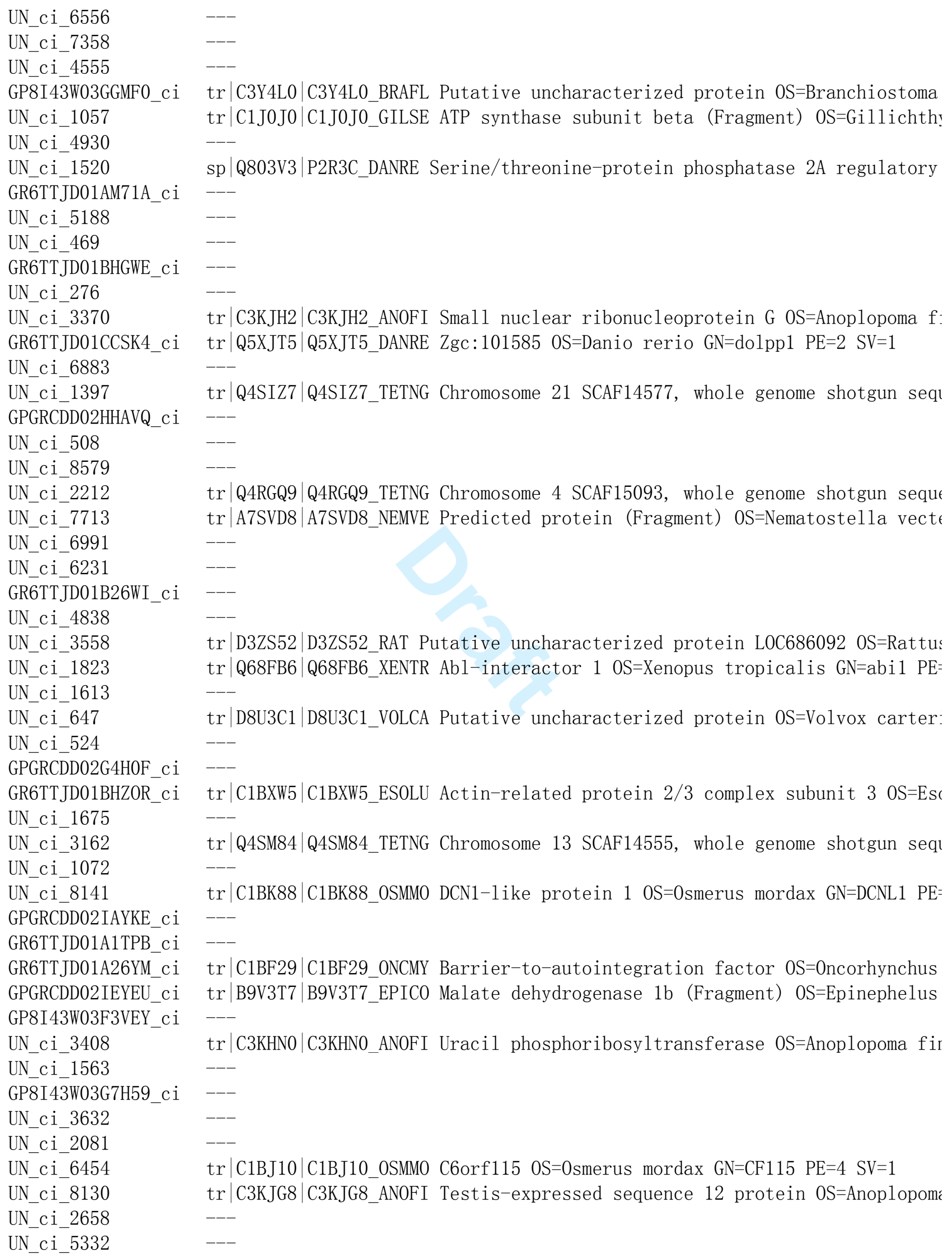




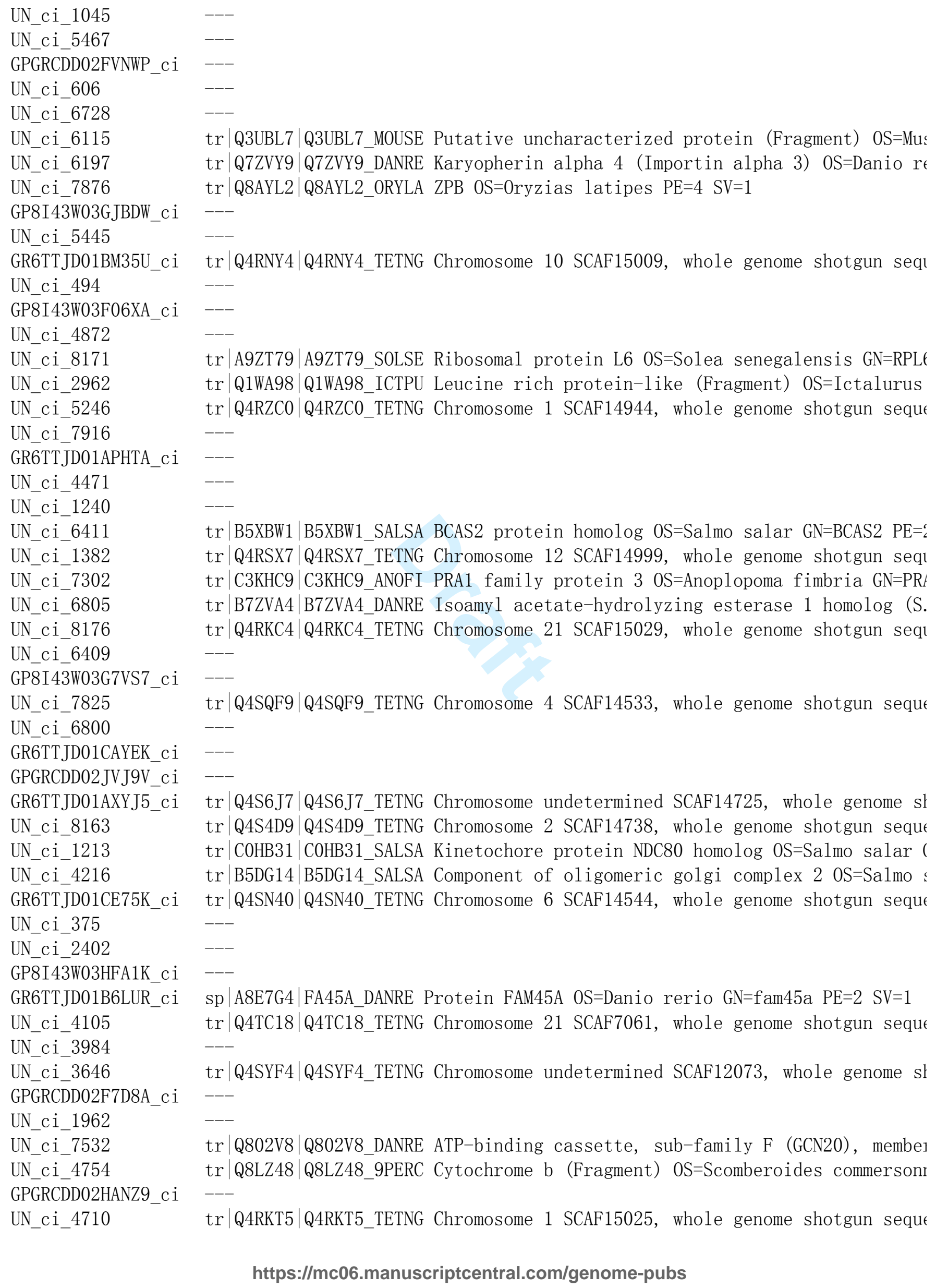

$\operatorname{tr} \mid$ Q3UBL7 $\mid$ Q3UBL7_MOUSE Putative uncharacterized protein (Fragment) OS=Mus $\operatorname{tr} \mid$ Q7ZVY9 $\mid$ Q7ZVY9_DANRE Karyopherin alpha 4 (Importin alpha 3) OS=Danio rt $\operatorname{tr} \mid$ Q8AYL2 $\mid$ Q8AYL2_ORYLA ZPB OS=0ryzias latipes PE=4 SV=1

tr $\mid$ Q4RNY4 |Q4RNY4_TETNG Chromosome 10 SCAF15009, whole genome shotgun seqı $---$

$\operatorname{tr} \mid$ A9ZT79|A9ZT79_SOLSE Ribosomal protein L6 OS=Solea senegalensis GN=RPLt $\operatorname{tr} \mid$ Q1WA98|Q1WA98_ICTPU Leucine rich protein-1ike (Fragment) OS=Ictalurus tr $\mid$ Q4RZC0|Q4RZC0_TETNG Chromosome 1 SCAF14944, whole genome shotgun sequt $---$

$\operatorname{tr} \mid$ B5XBW1 $\mid$ B5XBW1_SALSA BCAS2 protein homolog OS=Salmo salar GN=BCAS2 PE=‘ tr $\mid$ Q4RSX7|Q4RSX7_TETNG Chromosome 12 SCAF14999, whole genome shotgun seq1 tr $\mid$ C3KHC9 $\mid$ C3KHC9_ANOFI PRA1 family protein 3 OS=Anoplopoma fimbria GN=PR $f$ tr|B7ZVA4|B7ZVA4_DANRE Isoamyl acetate-hydrolyzing esterase 1 homolog (S. tr $\mid$ Q4RKC4|Q4RKC4_TETNG Chromosome 21 SCAF15029, whole genome shotgun seqi

tr $\mid$ Q4SQF9 $\mid$ Q4SQF9_TETNG Chromosome 4 SCAF14533, whole genome shotgun sequt $---$

tr $\mid$ Q4S6J7|Q4S6J7_TETNG Chromosome undetermined SCAF14725, whole genome s1 tr $\mid$ Q4S4D9|Q4S4D9_TETNG Chromosome 2 SCAF14738, whole genome shotgun sequt $\operatorname{tr} \mid$ C0HB31 $\mid$ COHB31_SALSA Kinetochore protein NDC80 homolog 0S=Salmo salar ( tr|B5DG14|B5DG14_SALSA Component of oligomeric golgi complex 2 0S=Salmo : tr $\mid$ Q4SN40|Q4SN40_TETNG Chromosome 6 SCAF14544, whole genome shotgun sequt $---$

sp $\mid$ A8E7G4 $\mid$ FA45A_DANRE Protein FAM45A OS=Danio rerio GN=fam45a PE=2 SV=1 $\operatorname{tr} \mid$ Q4TC18|Q4TC18_TETNG Chromosome 21 SCAF7061, whole genome shotgun sequt tr $\mid$ Q4SYF4|Q4SYF4_TETNG Chromosome undetermined SCAF12073, whole genome sl $\operatorname{tr} \mid$ Q802V8|Q802V8_DANRE ATP-binding cassette, sub-family F (GCN20), membe] $\operatorname{tr} \mid$ Q8LZ48|Q8LZ48_9PERC Cytochrome b (Fragment) OS=Scomberoides commersonr tr $\mid$ Q4RKT5|Q4RKT5_TETNG Chromosome 1 SCAF15025, whole genome shotgun sequt 


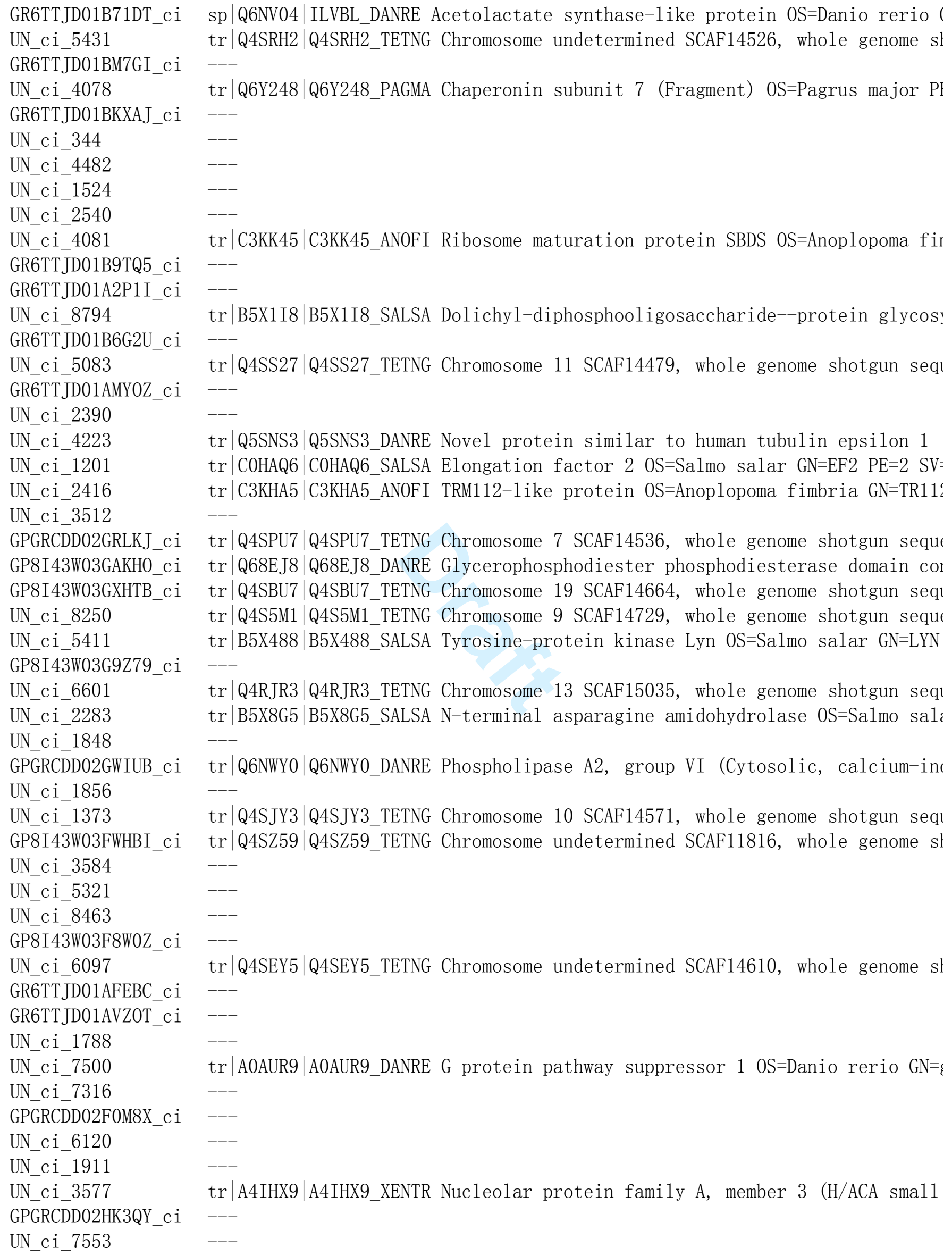

sp $\mid$ Q6NV04|ILVBL_DANRE Acetolactate synthase-1ike protein OS=Danio rerio ( tr $\mid$ Q4SRH2 $\mid$ Q4SRH2_TETNG Chromosome undetermined SCAF14526, whole genome sl tr $\mid$ Q6Y248|Q6Y248_PAGMA Chaperonin subunit 7 (Fragment) OS=Pagrus major PI $\operatorname{tr} \mid$ C3KK45 $\mid$ C3KK45_ANOFI Ribosome maturation protein SBDS OS=Anoplopoma fir tr|B5X1I8|B5X1I8_SALSA Dolichyl-diphosphooligosaccharide--protein glycos! tr $\mid$ Q4SS27|Q4SS27_TETNG Chromosome 11 SCAF14479, whole genome shotgun seq1 $\operatorname{tr} \mid$ Q5SNS3 $\mid$ Q5SNS3_DANRE Novel protein similar to human tubulin epsilon 1 tr $\mid$ COHAQ6 $\mid$ COHAQ6_SALSA Elongation factor 2 OS=Salmo salar GN=EF2 PE=2 SV= $\operatorname{tr} \mid$ C3KHA5 $\mid$ C3KHA5_ANOFI TRM112-1ike protein OS=Anoplopoma fimbria GN=TR11: tr $\mid$ Q4SPU7|Q4SPU7_TETNG Chromosome 7 SCAF14536, whole genome shotgun seque tr |Q68EJ8|Q68EJ8_DANRE Glycerophosphodiester phosphodiesterase domain cor tr $\mid$ Q4SBU7|Q4SBU7_TETNG Chromosome 19 SCAF14664, whole genome shotgun seq1 tr $\mid$ Q4S5M1 |Q4S5M1_TETNG Chromosome 9 SCAF14729, whole genome shotgun sequt tr $\mid$ B5X488|B5X488_SALSA Tyrosine-protein kinase Lyn OS=Salmo salar GN=LYN tr $\mid$ Q4RJR3 |Q4RJR3_TETNG Chromosome 13 SCAF15035, whole genome shotgun sequ tr|B5X8G5|B5X8G5_SALSA N-terminal asparagine amidohydrolase 0S=Salmo sali tr $\mid$ Q6NWY0|Q6NWY0_DANRE Phospholipase A2, group VI (Cytosolic, calcium-inc tr $\mid$ Q4SJY3|Q4SJY3_TETNG Chromosome 10 SCAF14571, whole genome shotgun seq1 tr $\mid$ Q4SZ59|Q4SZ59_TETNG Chromosome undetermined SCAF11816, whole genome s1

$\operatorname{tr} \mid$ Q4SEY5 $\mid$ Q4SEY5_TETNG Chromosome undetermined SCAF14610, whole genome sl $---$

$\operatorname{tr} \mid$ AOAUR9 $\mid$ A0AUR9_DANRE G protein pathway suppressor 1 OS=Danio rerio $G N=\xi$ $---$ 


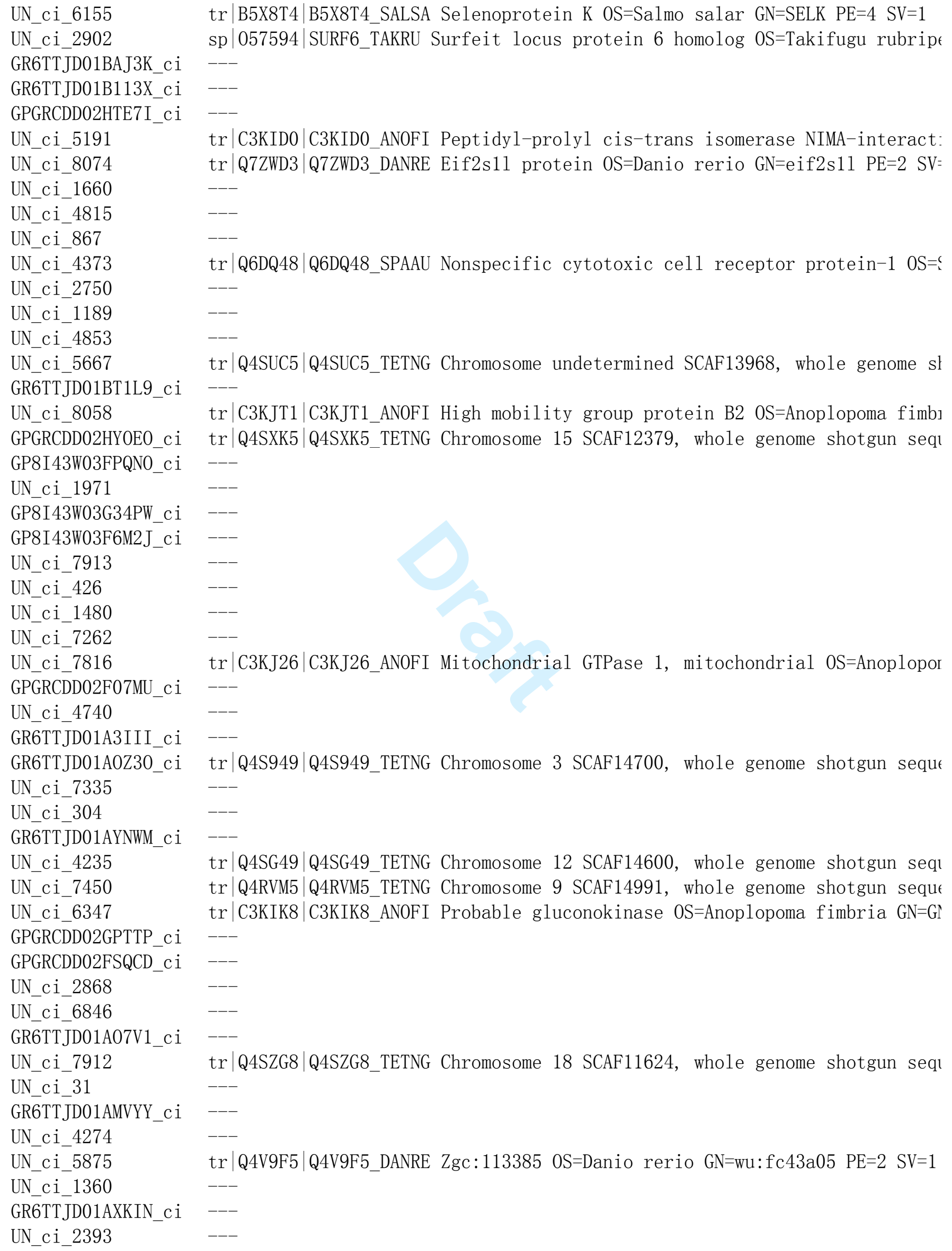




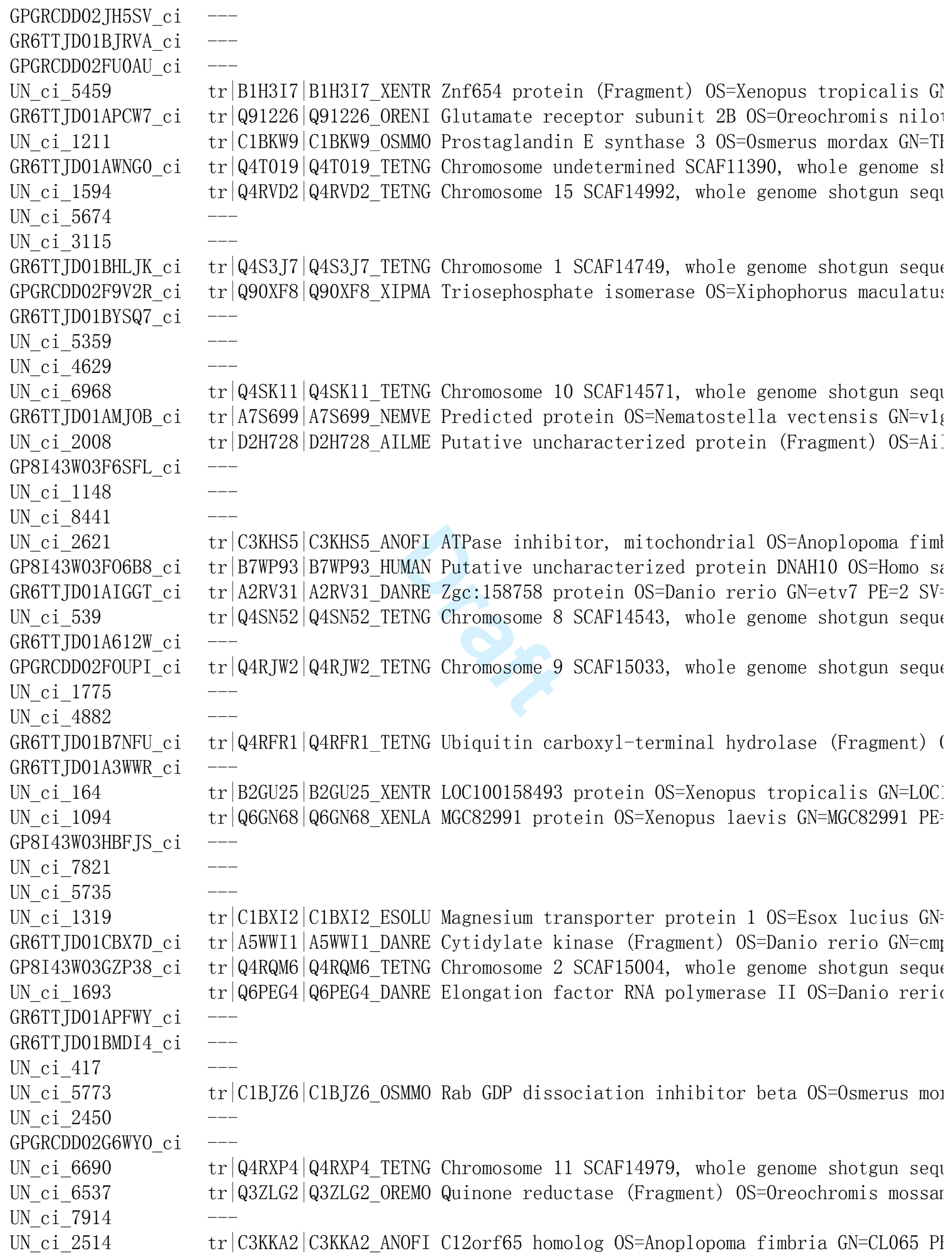

tr $\mid$ B1H3I7|B1H3I7_XENTR Znf654 protein (Fragment) OS=Xenopus tropicalis G! $\operatorname{tr} \mid$ Q91226|Q91226_ORENI Glutamate receptor subunit 2B OS=0reochromis nilo1 tr $\mid$ C1BKW9|C1BKW9_0SMM0 Prostaglandin E synthase 3 OS=0smerus mordax GN=TI tr $\mid$ Q4T019|Q4T019_TETNG Chromosome undetermined SCAF11390, whole genome sl tr|Q4RVD2|Q4RVD2_TETNG Chromosome 15 SCAF14992, whole genome shotgun seq1 $---$

tr $\mid$ Q4S3J7 $\mid$ Q4S3J7_TETNG Chromosome 1 SCAF14749, whole genome shotgun sequt $\operatorname{tr} \mid$ Q90XF8|Q90XF8_XIPMA Triosephosphate isomerase OS=Xiphophorus maculatus

$\operatorname{tr} \mid$ Q4SK11|Q4SK11_TETNG Chromosome 10 SCAF14571, whole genome shotgun seq1 $\operatorname{tr} \mid$ A7S699|A7S699_NEMVE Predicted protein 0S=Nematostella vectensis GN=v1 $\operatorname{tr} \mid$ D2H728|D2H728_AILME Putative uncharacterized protein (Fragment) OS=Ai:

tr $\mid$ C3KHS5 $\mid$ C3KHS5_ANOFI ATPase inhibitor, mitochondrial OS=Anoplopoma fiml tr|B7WP93|B7WP93_HUMAN Putative uncharacterized protein DNAH10 0S=Homo si $\operatorname{tr} \mid$ A2RV31 $\mid$ A2RV31_DANRE Zgc: 158758 protein 0S=Danio rerio GN=etv7 PE=2 SV= tr $\mid$ Q4SN52|Q4SN52_TETNG Chromosome 8 SCAF14543, whole genome shotgun sequt tr $\mid$ Q4RJW2 $\mid$ Q4RJW2_TETNG Chromosome 9 SCAF15033, whole genome shotgun sequt $---$

$\operatorname{tr} \mid$ Q4RFR1|Q4RFR1_TETNG Ubiquitin carboxy1-terminal hydrolase (Fragment) ( tr $\mid$ B2GU25 |B2GU25_XENTR LOC100158493 protein OS=Xenopus tropicalis GN=LOC: $\operatorname{tr} \mid$ Q6GN68 $\mid$ Q6GN68_XENLA MGC82991 protein 0S=Xenopus laevis GN=MGC82991 PE= $---$

$\operatorname{tr} \mid$ C1BXI2|C1BXI2_ESOLU Magnesium transporter protein 1 OS=Esox lucius GN= $\operatorname{tr} \mid$ A5WWI1 $\mid$ A5WWI1_DANRE Cytidylate kinase (Fragment) OS=Danio rerio GN=cmr tr |Q4RQM6|Q4RQM6_TETNG Chromosome 2 SCAF15004, whole genome shotgun sequt tr|Q6PEG4|Q6PEG4_DANRE Elongation factor RNA polymerase II OS=Danio reric $---$

$\operatorname{tr} \mid$ C1BJZ6 $\mid$ C1BJZ6_0SMM0 Rab GDP dissociation inhibitor beta 0S=0smerus mo] $---$

tr|Q4RXP4|Q4RXP4_TETNG Chromosome 11 SCAF14979, whole genome shotgun seq1 tr $\mid$ Q3ZLG2 $\mid$ Q3ZLG2_OREM0 Quinone reductase (Fragment) OS=0reochromis mossar $\operatorname{tr} \mid$ C3KKA2 $\mid$ C3KKA2_ANOFI C12orf65 homolog OS=Anoplopoma fimbria GN=CL065 PI 


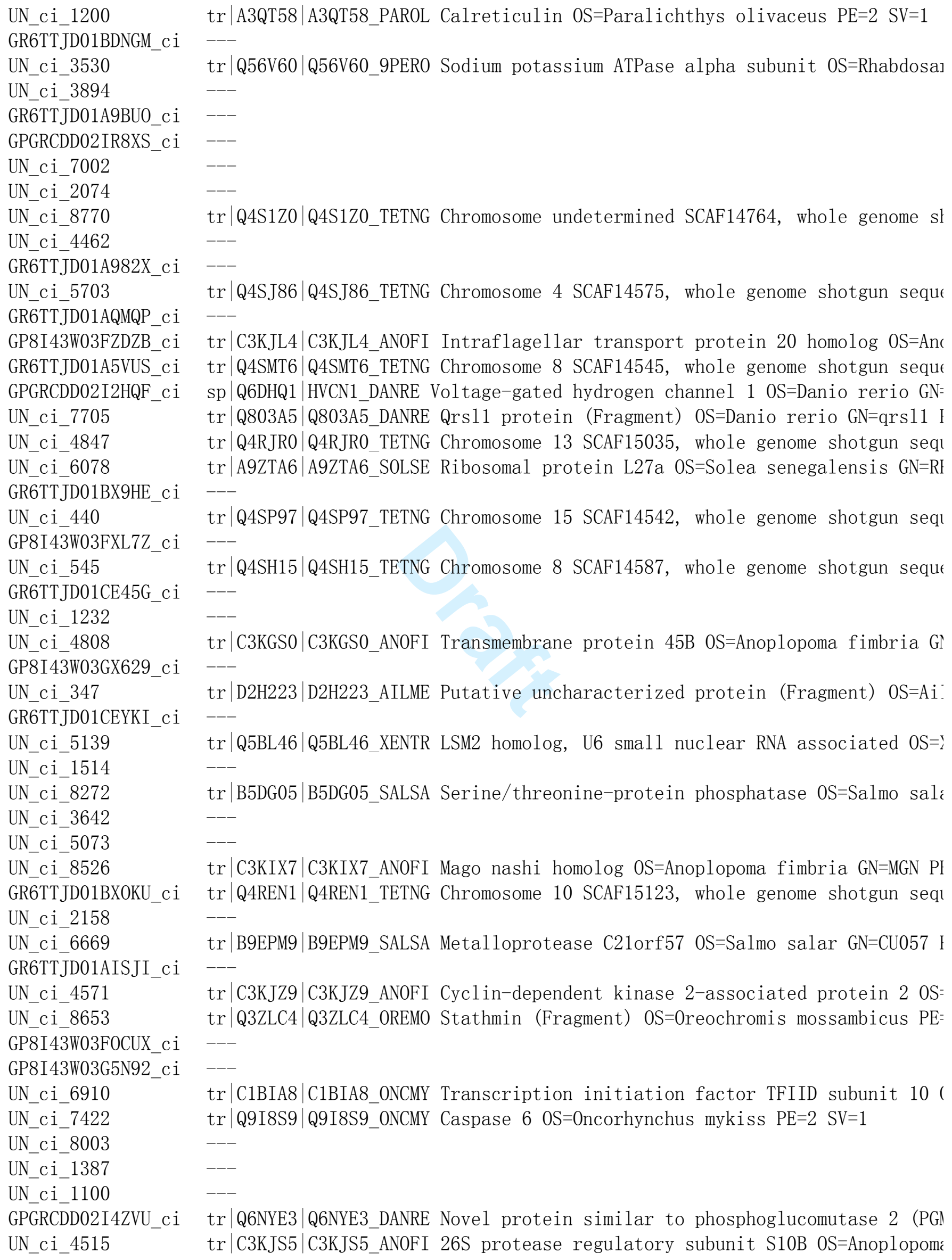

$\operatorname{tr} \mid$ A3QT58 $\mid$ A3QT58_PAR0L Calreticulin OS=Paralichthys olivaceus PE=2 SV=1

$\operatorname{tr} \mid$ Q56V60|Q56V60_9PER0 Sodium potassium ATPase alpha subunit OS=Rhabdosa]

$\operatorname{tr} \mid$ Q4S1Z0|Q4S1Z0_TETNG Chromosome undetermined SCAF14764, whole genome sl tr $\mid$ Q4SJ86|Q4SJ86_TETNG Chromosome 4 SCAF14575, whole genome shotgun seque tr $\mid$ C3KJL4|C3KJL4_ANOFI Intraflagellar transport protein 20 homolog OS=Anc tr $\mid$ Q4SMT6 |Q4SMT6_TETNG Chromosome 8 SCAF14545, whole genome shotgun sequt sp |Q6DHQ1 |HVCN1_DANRE Voltage-gated hydrogen channe1 1 OS=Danio rerio GN= $\operatorname{tr} \mid$ Q803A5 $\mid$ Q803A5_DANRE Qrs11 protein (Fragment) OS=Danio rerio GN=qrs11 I tr $\mid$ Q4RJR0|Q4RJR0_TETNG Chromosome 13 SCAF15035, whole genome shotgun seq1 $\operatorname{tr} \mid$ A9ZTA6 $\mid$ A9ZTA6_SOLSE Ribosomal protein L27a OS=Solea senegalensis GN=RI tr $\mid$ Q4SP97|Q4SP97_TETNG Chromosome 15 SCAF14542, whole genome shotgun seq1 tr $\mid$ Q4SH15|Q4SH15_TETNG Chromosome 8 SCAF14587, whole genome shotgun sequt $\operatorname{tr} \mid$ C3KGS0 $\mid$ C3KGS0_ANOFI Transmembrane protein 45B 0S=Anoplopoma fimbria G! tr $\mid$ D2H223 |D2H223_AILME Putative uncharacterized protein (Fragment) OS=Ai: tr $\mid$ Q5BL46|Q5BL46_XENTR LSM2 homolog, U6 small nuclear RNA associated OS=` tr $\mid$ B5DG05|B5DG05_SALSA Serine/threonine-protein phosphatase OS=Salmo sali

$\operatorname{tr} \mid$ C3KIX7 $\mid$ C3KIX7_ANOFI Mago nashi homolog 0S=Anoplopoma fimbria GN=MGN PI tr $\mid$ Q4REN1|Q4REN1_TETNG Chromosome 10 SCAF15123, whole genome shotgun seq1 tr | B9EPM9|B9EPM9_SALSA Metalloprotease C21orf57 0S=Salmo salar GN=CU057 I $\operatorname{tr} \mid$ C3KJZ9|C3KJZ9_ANOFI Cyclin-dependent kinase 2-associated protein 2 OS= $\operatorname{tr} \mid$ Q3ZLC4 $\mid$ Q3ZLC4_OREMO Stathmin (Fragment) OS=0reochromis mossambicus PE=

$\operatorname{tr} \mid$ C1BIA8|C1BIA8_0NCMY Transcription initiation factor TFIID subunit 10 ( $\operatorname{tr} \mid$ Q9I8S9 |Q9I8S9_ONCMY Caspase 6 OS=0ncorhynchus mykiss PE=2 SV=1

$---$

tr|Q6NYE3 |Q6NYE3_DANRE Nove1 protein similar to phosphoglucomutase 2 (PG) tr $\mid$ C3KJS5 $\mid$ C3KJS5_ANOFI 26S protease regulatory subunit S10B OS=Anoplopomi 


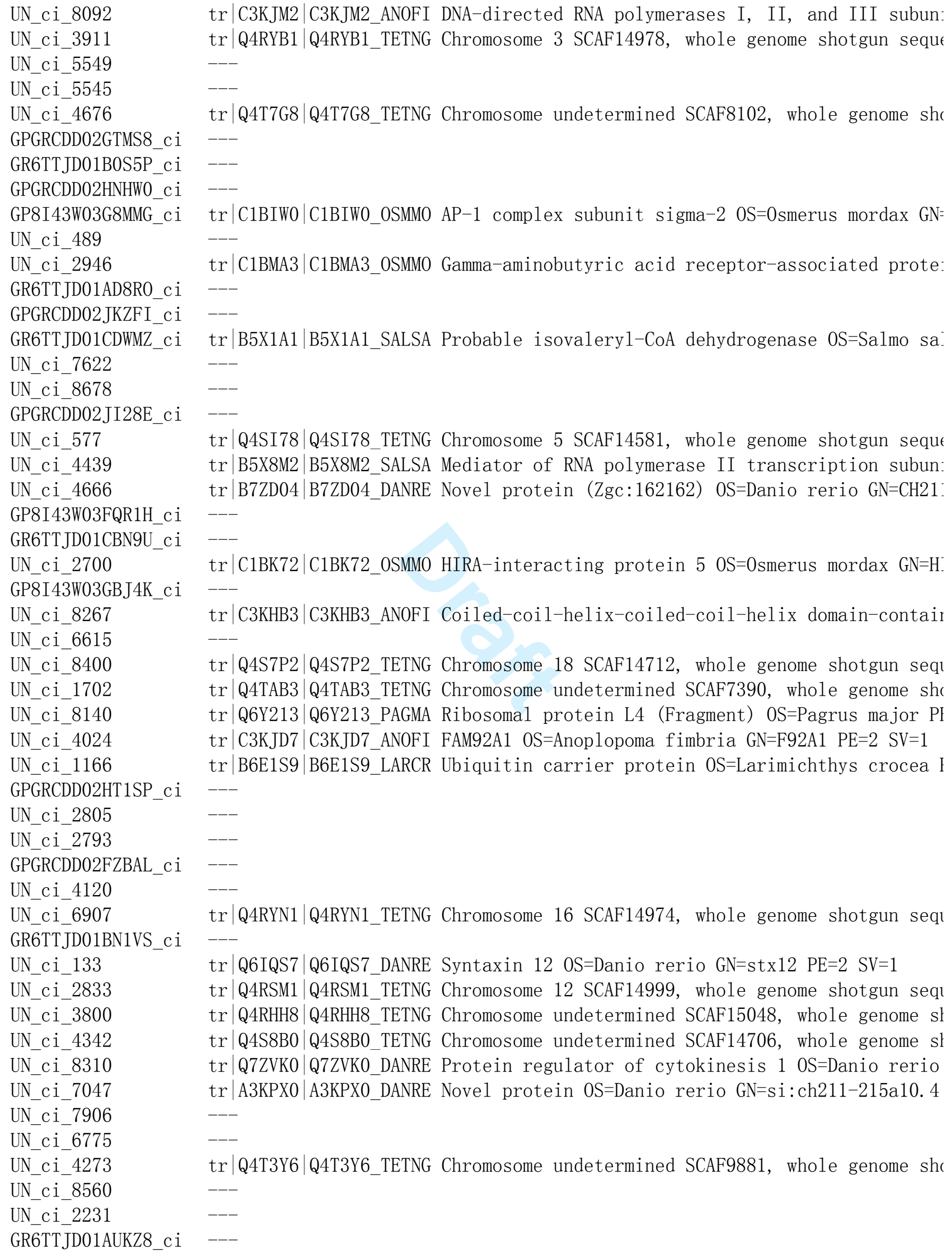

tr $\mid$ Q4SI78 $\mid$ Q4SI78_TETNG Chromosome 5 SCAF14581, whole genome shotgun sequt $\operatorname{tr|B5X8M2|B5X8M2\_ SALSA~Mediator~of~RNA~polymerase~II~transcription~subun:~}$ $\operatorname{tr} \mid$ B7ZD04|B7ZD04_DANRE Novel protein (Zgc:162162) OS=Danio rerio GN=CH21.

$\operatorname{tr} \mid$ C1BK72 $\mid$ C1BK72_OSMM0 HIRA-interacting protein 5 OS=0smerus mordax GN=H. $\operatorname{tr} \mid$ C3KHB3 $\mid$ C3KHB3_ANOFI Coiled-coil-helix-coiled-coil-helix domain-contair $---$

tr $\mid$ Q4S7P2 $\mid$ Q4S7P2_TETNG Chromosome 18 SCAF14712, whole genome shotgun seq1 tr $\mid$ Q4TAB3 $\mid$ Q4TAB3_TETNG Chromosome undetermined SCAF7390, whole genome she $\operatorname{tr} \mid$ Q6Y213 $\mid$ Q6Y213_PAGMA Ribosomal protein L4 (Fragment) OS=Pagrus major PI $\operatorname{tr} \mid$ C3KJD7|C3KJD7_ANOFI FAM92A1 OS=Anoplopoma fimbria GN=F92A1 PE=2 SV=1 tr $\mid$ B6E1S9|B6E1S9_LARCR Ubiquitin carrier protein OS=Larimichthys crocea I

tr|Q4RYN1|Q4RYN1_TETNG Chromosome 16 SCAF14974, whole genome shotgun seq1 $\operatorname{tr} \mid$ Q6IQS7 |Q6IQS7_DANRE Syntaxin 12 OS=Danio rerio GN=stx12 PE=2 SV=1 tr $\mid$ Q4RSM1|Q4RSM1_TETNG Chromosome 12 SCAF14999, whole genome shotgun sequ tr $\mid$ Q4RHH8|Q4RHH8_TETNG Chromosome undetermined SCAF15048, whole genome sl $\operatorname{tr} \mid$ Q4S8B0|Q4S8B0_TETNG Chromosome undetermined SCAF14706, whole genome sl $\operatorname{tr} \mid$ Q7ZVK0|Q7ZVK0_DANRE Protein regulator of cytokinesis 1 OS=Danio rerio $\operatorname{tr} \mid$ A3KPX0 $\mid$ A3KPXO_DANRE Novel protein OS=Danio rerio GN=si:ch211-215a10. 4

tr $\mid$ Q4T3Y6|Q4T3Y6_TETNG Chromosome undetermined SCAF9881, whole genome sh 


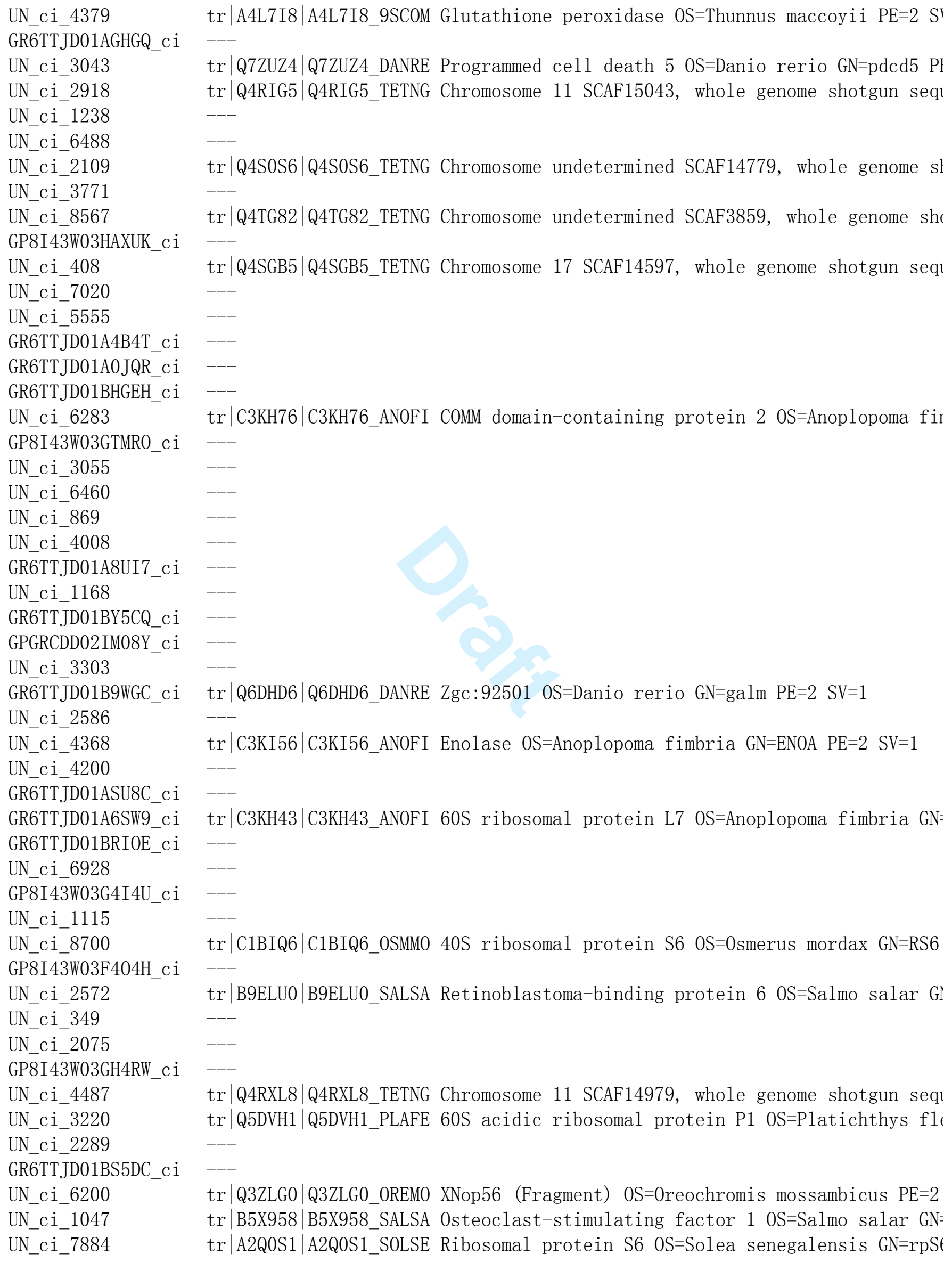

$\operatorname{tr} \mid$ A4L7I8|A4L7I8_9SCOM Glutathione peroxidase 0S=Thunnus maccoyii PE=2 SI tr $\mid$ Q7ZUZ4|Q7ZUZ4_DANRE Programmed cel1 death 5 OS=Danio rerio GN=pdcd5 PI tr $\mid$ Q4RIG5|Q4RIG5_TETNG Chromosome 11 SCAF15043, whole genome shotgun seqi

tr $\mid$ Q4S0S6 |Q4S0S6_TETNG Chromosome undetermined SCAF14779, whole genome sl $\operatorname{tr} \mid$ Q4TG82|Q4TG82_TETNG Chromosome undetermined SCAF3859, whole genome shi tr $\mid$ Q4SGB5|Q4SGB5_TETNG Chromosome 17 SCAF14597, whole genome shotgun seq1 $\operatorname{tr} \mid$ C3KH76|C3KH76_ANOFI COMM domain-containing protein 2 OS=Anoplopoma fir $\operatorname{tr} \mid$ Q6DHD6 | Q6DHD6_DANRE Zgc:92501 OS=Danio rerio GN=galm PE=2 SV=1 $\operatorname{tr} \mid$ C3KI56 $\mid$ C3KI56_ANOFI Enolase OS=Anoplopoma fimbria GN=ENOA PE=2 SV=1

$\operatorname{tr} \mid$ C3KH43|C3KH43_ANOFI 60S ribosomal protein L7 OS=Anoplopoma fimbria GN= $--$

tr $\mid$ C1BIQ6 $\mid$ C1BIQ6_0SMM0 40S ribosomal protein S6 0S=0smerus mordax GN=RS6 tr $\mid$ B9ELU0|B9ELU0_SALSA Retinoblastoma-binding protein 6 OS=Salmo salar GI $---$

tr $\mid$ Q4RXL8|Q4RXL8_TETNG Chromosome 11 SCAF14979, whole genome shotgun seq1 tr |Q5DVH1 |Q5DVH1_PLAFE 60S acidic ribosomal protein P1 0S=Platichthys f1

tr $\mid$ Q3ZLG0 $\mid$ Q3ZLG0_OREM0 XNop56 (Fragment) OS=0reochromis mossambicus PE=2 $\operatorname{tr} \mid$ B5X958|B5X958_SALSA 0steoclast-stimulating factor 1 OS=Salmo salar GN= $\operatorname{tr} \mid$ A2Q0S1 $\mid$ A2Q0S1_SOLSE Ribosomal protein S6 OS=Solea senegalensis GN=rpSt 


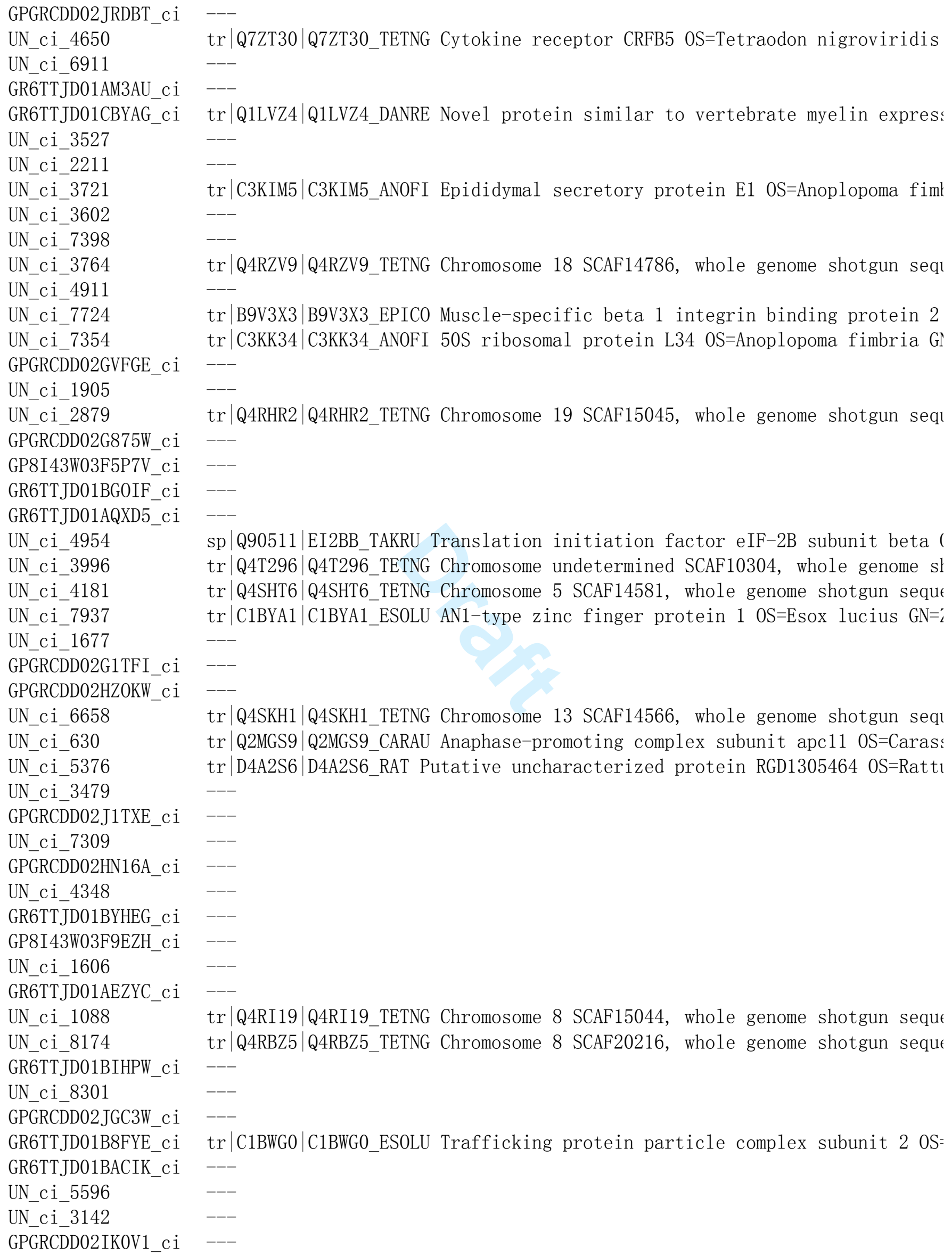

tr $\mid$ Q7ZT30|Q7ZT30 TETNG Cytokine receptor CRFB5 OS=Tetraodon nigroviridis

tr|C3KIM5 $\mid$ C3KIM5_ANOFI Epididymal secretory protein E1 OS=Anoplopoma fiml $---$

tr $\mid$ Q4RZV9|Q4RZV9_TETNG Chromosome 18 SCAF14786, whole genome shotgun seqı $\operatorname{tr} \mid$ B9V3X3|B9V3X3_EPIC0 Muscle-specific beta 1 integrin binding protein 2 tr $\mid$ C3KK34|C3KK34_ANOFI 50S ribosomal protein L34 0S=Anoplopoma fimbria Gl

tr $\mid$ Q4RHR2 |Q4RHR2_TETNG Chromosome 19 SCAF15045, whole genome shotgun seq1 sp |Q90511|EI2BB_TAKRU Translation initiation factor eIF-2B subunit beta ( tr|Q4T296|Q4T296_TETNG Chromosome undetermined SCAF10304, whole genome sl tr $\mid$ Q4SHT6|Q4SHT6_TETNG Chromosome 5 SCAF14581, whole genome shotgun sequt $\operatorname{tr} \mid$ C1BYA1 $\mid$ C1BYA1_ESOLU AN1-type zinc finger protein 1 OS=Esox lucius GN='

tr $\mid$ Q4SKH1|Q4SKH1_TETNG Chromosome 13 SCAF14566, whole genome shotgun seq1 tr $\mid$ Q2MGS9|Q2MGS9_CARAU Anaphase-promoting complex subunit apc11 0S=Caras: tr|D4A2S6|D4A2S6_RAT Putative uncharacterized protein RGD1305464 OS=Rattı

tr $\mid$ Q4RI19|Q4RI19_TETNG Chromosome 8 SCAF15044, whole genome shotgun sequt tr $\mid$ Q4RBZ5|Q4RBZ5_TETNG Chromosome 8 SCAF20216, whole genome shotgun sequt

tr $\mid$ C1BWG0 $\mid$ C1BWG0_ESOLU Trafficking protein particle complex subunit 2 OS= 


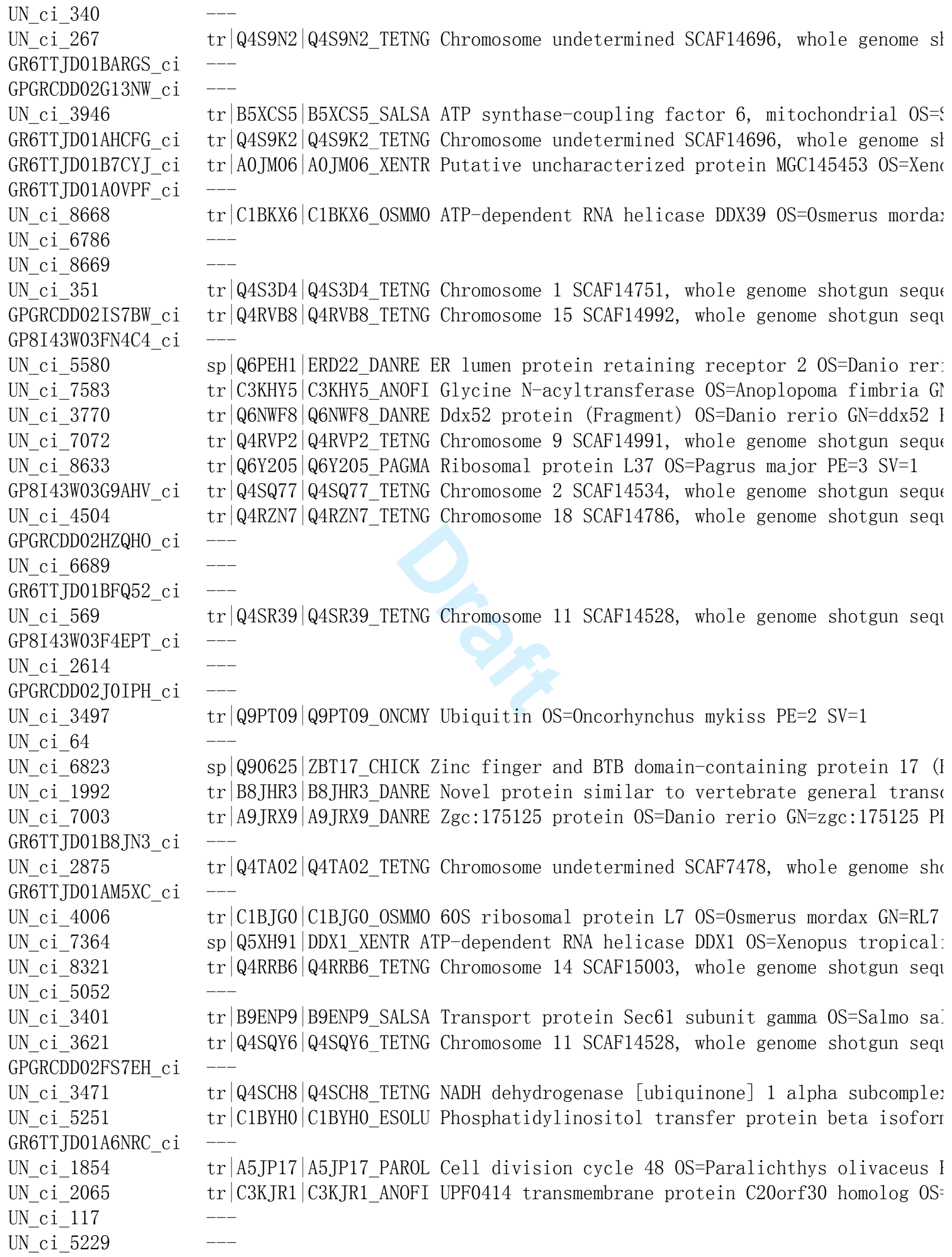

tr |Q4S9N2 |Q4S9N2_TETNG Chromosome undetermined SCAF14696, whole genome sl

$\operatorname{tr} \mid$ B5XCS5|B5XCS5_SALSA ATP synthase-coupling factor 6, mitochondrial 0S=: $\operatorname{tr} \mid$ Q4S9K2 $\mid$ Q4S9K2_TETNG Chromosome undetermined SCAF14696, whole genome sl tr|A0JM06|A0JM06_XENTR Putative uncharacterized protein MGC145453 OS=Xenc $\operatorname{tr} \mid$ C1BKX6 $\mid$ C1BKX6_0SMM0 ATP-dependent RNA helicase DDX39 0S=0smerus morda: tr |Q4S3D4|Q4S3D4_TETNG Chromosome 1 SCAF14751, whole genome shotgun sequt tr|Q4RVB8|Q4RVB8_TETNG Chromosome 15 SCAF14992, whole genome shotgun seqr sp |Q6PEH1 |ERD22_DANRE ER lumen protein retaining receptor 2 OS=Danio rer: tr $\mid$ C3KHY5|C3KHY5_ANOFI Glycine N-acyltransferase OS=Anoplopoma fimbria G! tr $\mid$ Q6NWF8 $\mid$ Q6NWF8_DANRE Ddx52 protein (Fragment) OS=Danio rerio GN=ddx52 I tr|Q4RVP2|Q4RVP2_TETNG Chromosome 9 SCAF14991, whole genome shotgun sequt $\operatorname{tr} \mid$ Q6Y205|Q6Y205_PAGMA Ribosomal protein L37 OS=Pagrus major PE=3 SV=1 tr $\mid$ Q4SQ77|Q4SQ77_TETNG Chromosome 2 SCAF14534, whole genome shotgun sequt tr|Q4RZN7|Q4RZN7_TETNG Chromosome 18 SCAF14786, whole genome shotgun seq1

tr $\mid$ Q4SR39|Q4SR39_TETNG Chromosome 11 SCAF14528, whole genome shotgun seq1 $\operatorname{tr} \mid$ Q9PT09 $\mid$ Q9PT09_ONCMY Ubiquitin OS=0ncorhynchus mykiss PE=2 SV=1

sp|Q90625|ZBT17_CHICK Zinc finger and BTB domain-containing protein 17 (I tr|B8JHR3|B8JHR3_DANRE Novel protein similar to vertebrate general transi tr|A9JRX9|A9JRX9_DANRE Zgc:175125 protein 0S=Danio rerio GN=zgc:175125 PI $\operatorname{tr} \mid$ Q4TA02 |Q4TA02_TETNG Chromosome undetermined SCAF7478, whole genome sh tr $\mid$ C1BJG0|C1BJG0_0SMM0 60S ribosomal protein L7 0S=0smerus mordax GN=RL7 sp $\mid$ Q5XH91|DDX1_XENTR ATP-dependent RNA helicase DDX1 OS=Xenopus tropical tr $\mid$ Q4RRB6|Q4RRB6_TETNG Chromosome 14 SCAF15003, whole genome shotgun seqr tr $\mid$ B9ENP9|B9ENP9_SALSA Transport protein Sec61 subunit gamma 0S=Salmo sa: tr $\mid$ Q4SQY6|Q4SQY6_TETNG Chromosome 11 SCAF14528, whole genome shotgun seq1 $\operatorname{tr} \mid$ Q4SCH8 $\mid$ Q4SCH8_TETNG NADH dehydrogenase [ubiquinone] 1 alpha subcomple: tr $\mid$ C1BYHO|C1BYH0_ESOLU Phosphatidylinositol transfer protein beta isoforr tr|A5JP17|A5JP17_PAR0L Ce11 division cycle 48 0S=Paralichthys olivaceus I $\operatorname{tr} \mid$ C3KJR1|C3KJR1_ANOFI UPF0414 transmembrane protein C20orf30 homolog OS= 


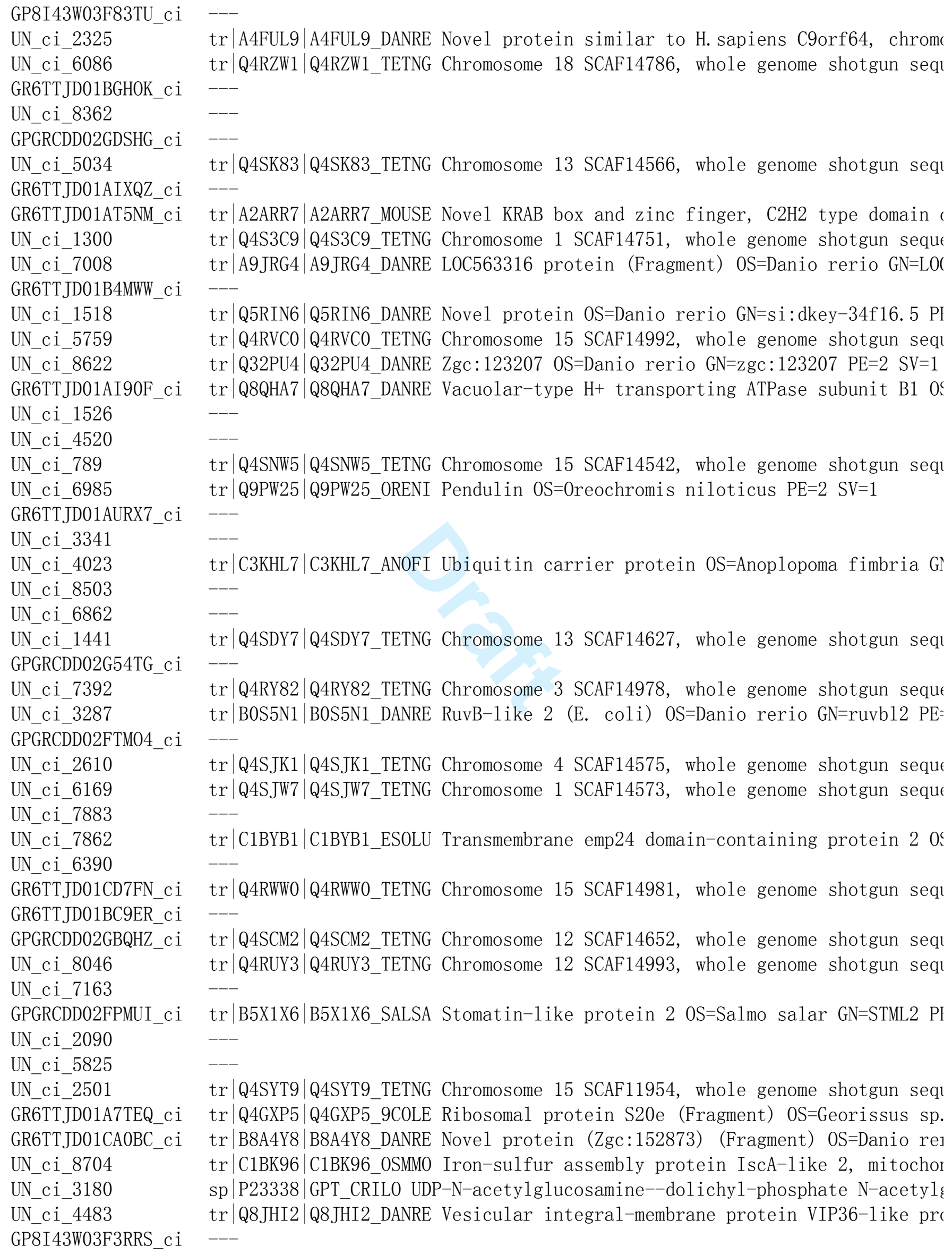

tr $\mid$ A4FUL9 $\mid$ A4FUL9_DANRE Novel protein similar to H. sapiens C9orf64, chrom tr $\mid$ Q4RZW1|Q4RZW1_TETNG Chromosome 18 SCAF14786, whole genome shotgun seq1

tr $\mid$ Q4SK83|Q4SK83_TETNG Chromosome 13 SCAF14566, whole genome shotgun seqr

$\operatorname{tr} \mid$ A2ARR7 $\mid$ A2ARR7_MOUSE Nove1 KRAB box and zinc finger, C2H2 type domain ( tr $\mid$ Q4S3C9|Q4S3C9_TETNG Chromosome 1 SCAF14751, whole genome shotgun sequt tr $\mid$ A9JRG4 $\mid$ A9JRG4_DANRE LOC563316 protein (Fragment) OS=Danio rerio GN=LOC

tr $\mid$ Q5RIN6 $\mid$ Q5RIN6_DANRE Novel protein OS=Danio rerio GN=si:dkey-34f16. 5 PI tr $\mid$ Q4RVC0|Q4RVC0_TETNG Chromosome 15 SCAF14992, whole genome shotgun seq1 $\operatorname{tr} \mid$ Q32PU4 $\mid$ Q32PU4_DANRE Zgc:123207 OS=Danio rerio GN=zgc:123207 PE=2 SV=1 tr $\mid$ Q8QHA7|Q8QHA7_DANRE Vacuolar-type H+ transporting ATPase subunit B1 0؛

$---$

tr $\mid$ Q4SNW5|Q4SNW5_TETNG Chromosome 15 SCAF14542, whole genome shotgun seqr tr $\mid$ Q9PW25 |Q9PW25_ORENI Pendulin OS=0reochromis niloticus PE=2 SV=1

$\operatorname{tr} \mid$ C3KHL7 $\mid$ C3KHL7_ANOFI Ubiquitin carrier protein OS=Anoplopoma fimbria G! $---$

tr $\mid$ Q4SDY7|Q4SDY7_TETNG Chromosome 13 SCAF14627, whole genome shotgun seq1 tr $\mid$ Q4RY82|Q4RY82_TETNG Chromosome 3 SCAF14978, whole genome shotgun sequt $\operatorname{tr} \mid$ B0S5N1 |BOS5N1_DANRE RuvB-1ike 2 (E. coli) OS=Danio rerio GN=ruvb12 PE=

tr $\mid$ Q4SJK1 $\mid$ Q4SJK1_TETNG Chromosome 4 SCAF14575, whole genome shotgun sequt tr $\mid$ Q4SJW7|Q4SJW7_TETNG Chromosome 1 SCAF14573, whole genome shotgun sequt $\operatorname{tr} \mid$ C1BYB1|C1BYB1_ES0LU Transmembrane emp24 domain-containing protein 2 0؛ tr $\mid$ Q4RWW0|Q4RWW0_TETNG Chromosome 15 SCAF14981, whole genome shotgun seq1 tr $\mid$ Q4SCM2 $\mid$ Q4SCM2_TETNG Chromosome 12 SCAF14652, whole genome shotgun seq1 tr |Q4RUY3|Q4RUY3_TETNG Chromosome 12 SCAF14993, whole genome shotgun seq1 $\operatorname{tr} \mid$ B5X1X6|B5X1X6_SALSA Stomatin-1ike protein 2 OS=Salmo salar GN=STML2 PI $---$

tr $\mid$ Q4SYT9 $\mid$ Q4SYT9_TETNG Chromosome 15 SCAF11954, whole genome shotgun seq1 tr $\mid$ Q4GXP5|Q4GXP5_9COLE Ribosomal protein S20e (Fragment) 0S=Georissus sp. tr $\mid$ B8A4Y8|B8A4Y8_DANRE Novel protein (Zgc:152873) (Fragment) OS=Danio re] tr|C1BK96|C1BK96_0SMM0 Iron-sulfur assembly protein IscA-1ike 2, mitochor sp |P23338|GPT_CRIL0 UDP-N-acetylglucosamine--dolichyl-phosphate N-acetylई tr $\mid$ Q8JHI2|Q8JHI2_DANRE Vesicular integral-membrane protein VIP36-1ike prc https://mc06.manuscriptcentral.com/genome-pubs 


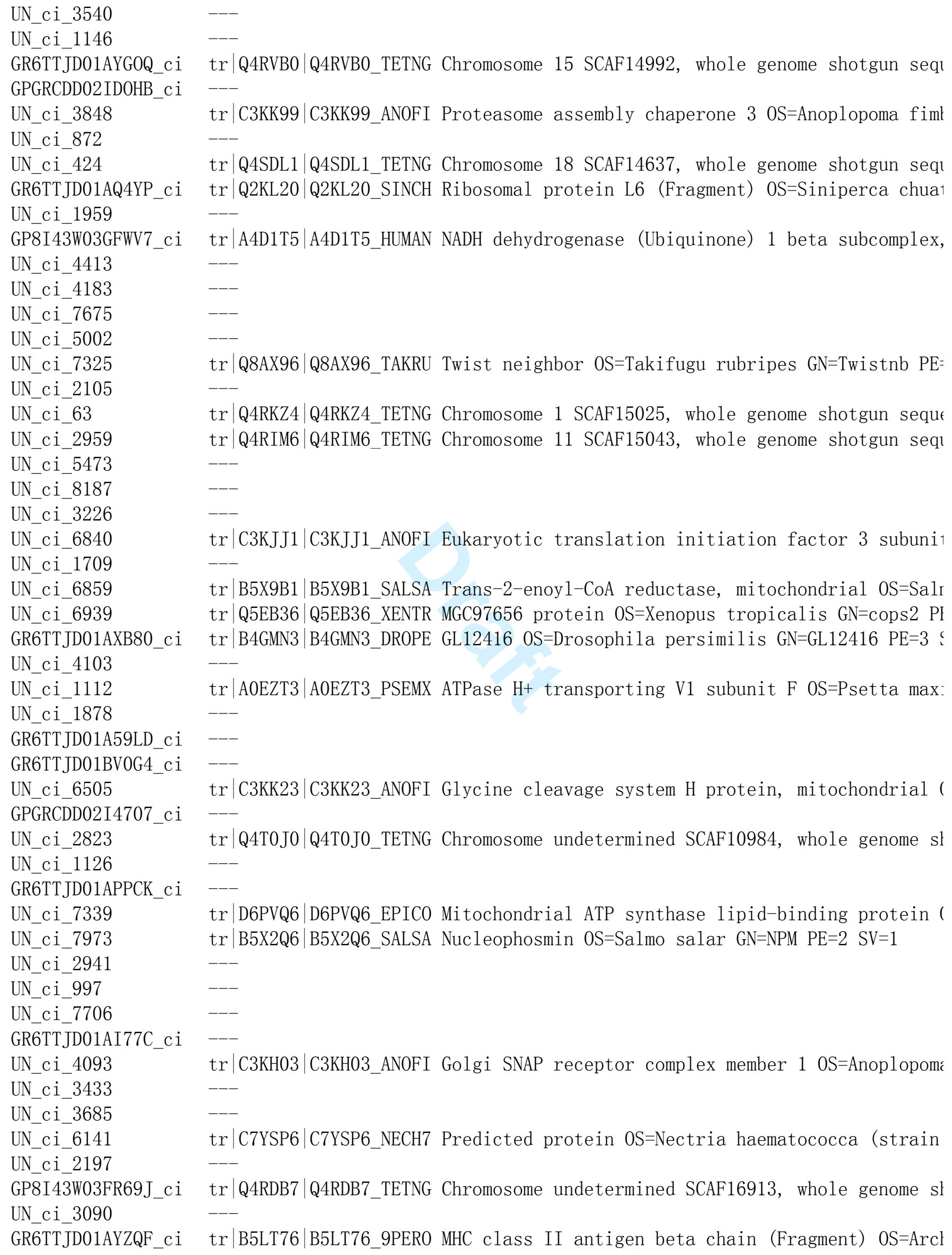




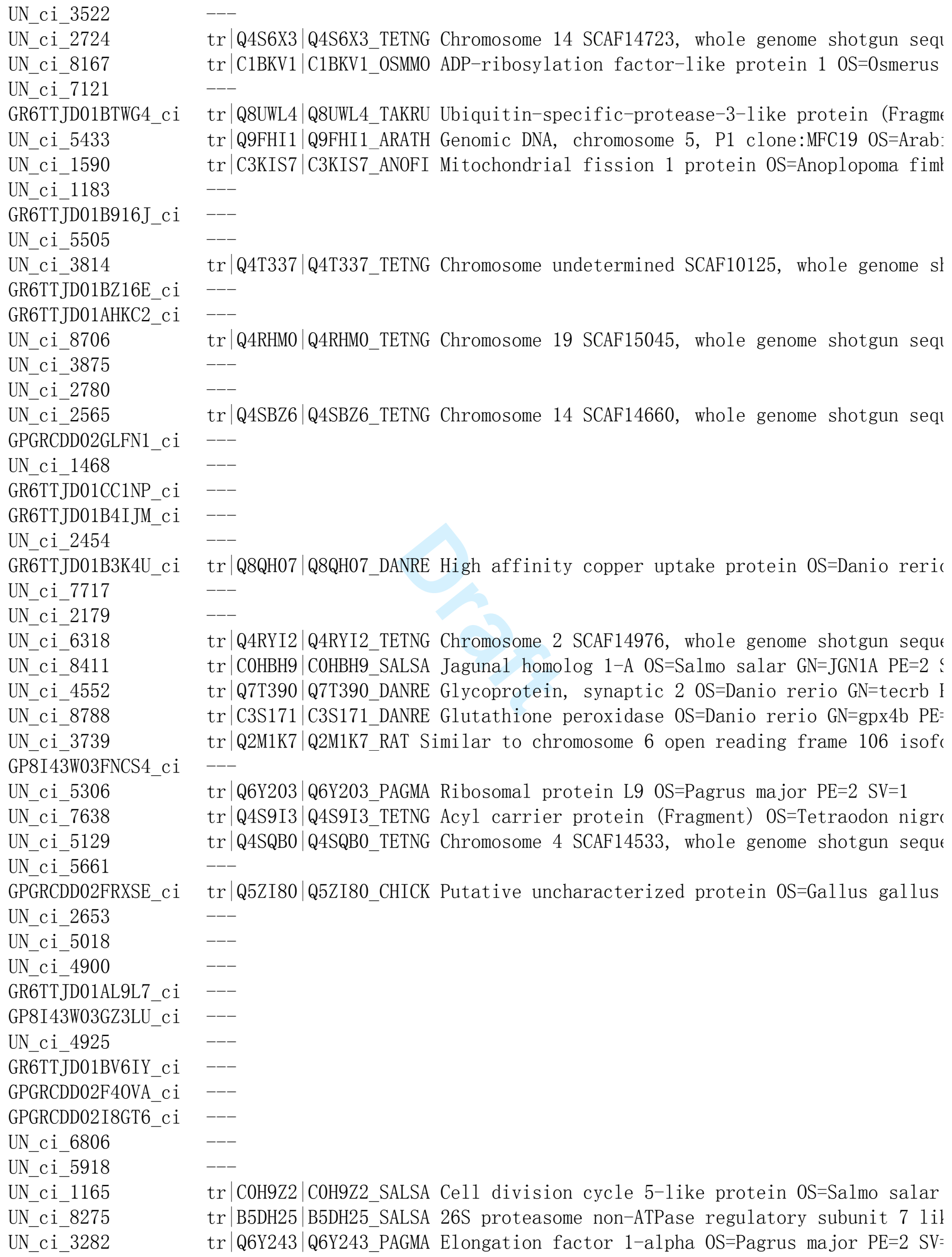

tr $\mid$ Q4S6X3|Q4S6X3_TETNG Chromosome 14 SCAF14723, whole genome shotgun seq1 $\operatorname{tr} \mid$ C1BKV1 $\mid$ C1BKV1_0SMM0 ADP-ribosylation factor-1ike protein 1 OS=0smerus

$\operatorname{tr} \mid$ Q8UWL4 $\mid$ Q8UWL4_TAKRU Ubiquitin-specific-protease-3-1ike protein (Fragm tr|Q9FHI1|Q9FHI1_ARATH Genomic DNA, chromosome 5, P1 clone:MFC19 OS=Arab $\operatorname{tr} \mid$ C3KIS7 $\mid$ C3KIS7_ANOFI Mitochondrial fission 1 protein OS=Anoplopoma fiml tr $\mid$ Q4T337|Q4T337_TETNG Chromosome undetermined SCAF10125, whole genome sh tr |Q4RHM0 |Q4RHM0_TETNG Chromosome 19 SCAF15045, whole genome shotgun seqr $---$

tr $\mid$ Q4SBZ6 $\mid$ Q4SBZ6_TETNG Chromosome 14 SCAF14660, whole genome shotgun seq1 tr $\mid$ Q8QH07 $\mid$ Q8QH07_DANRE High affinity copper uptake protein OS=Danio reric tr $\mid$ Q4RYI2|Q4RYI2_TETNG Chromosome 2 SCAF14976, whole genome shotgun sequt $\operatorname{tr} \mid$ COHBH9|COHBH9_SALSA Jagunal homolog 1-A OS=Salmo salar GN=JGN1A PE=2 ؛ $\operatorname{tr} \mid$ Q7T390|Q7T390_DANRE Glycoprotein, synaptic 2 OS=Danio rerio GN=tecrb I $\operatorname{tr} \mid$ C3S171 $\mid$ C3S171_DANRE Glutathione peroxidase OS=Danio rerio GN=gpx4b PE= tr $\mid$ Q2M1K7|Q2M1K7_RAT Similar to chromosome 6 open reading frame 106 isof،

tr $\mid$ Q6Y203 |Q6Y203_PAGMA Ribosomal protein L9 OS=Pagrus major PE=2 SV=1 tr $\mid$ Q4S9I3 Q4S9I3_TETNG Acyl carrier protein (Fragment) OS=Tetraodon nigrc tr $\mid$ Q4SQB0|Q4SQB0_TETNG Chromosome 4 SCAF14533, whole genome shotgun sequt $\operatorname{tr} \mid$ Q5ZI80 $\mid$ Q5ZI80_CHICK Putative uncharacterized protein OS=Gallus gallus tr $\mid$ COH9Z2 $\mid$ COH9Z2_SALSA Cell division cycle 5-like protein OS=Salmo salar tr|B5DH25|B5DH25_SALSA 26S proteasome non-ATPase regulatory subunit 7 lil $\operatorname{tr} \mid$ Q6Y243 $\mid$ Q6Y243_PAGMA Elongation factor 1-alpha OS=Pagrus major PE=2 SV= 


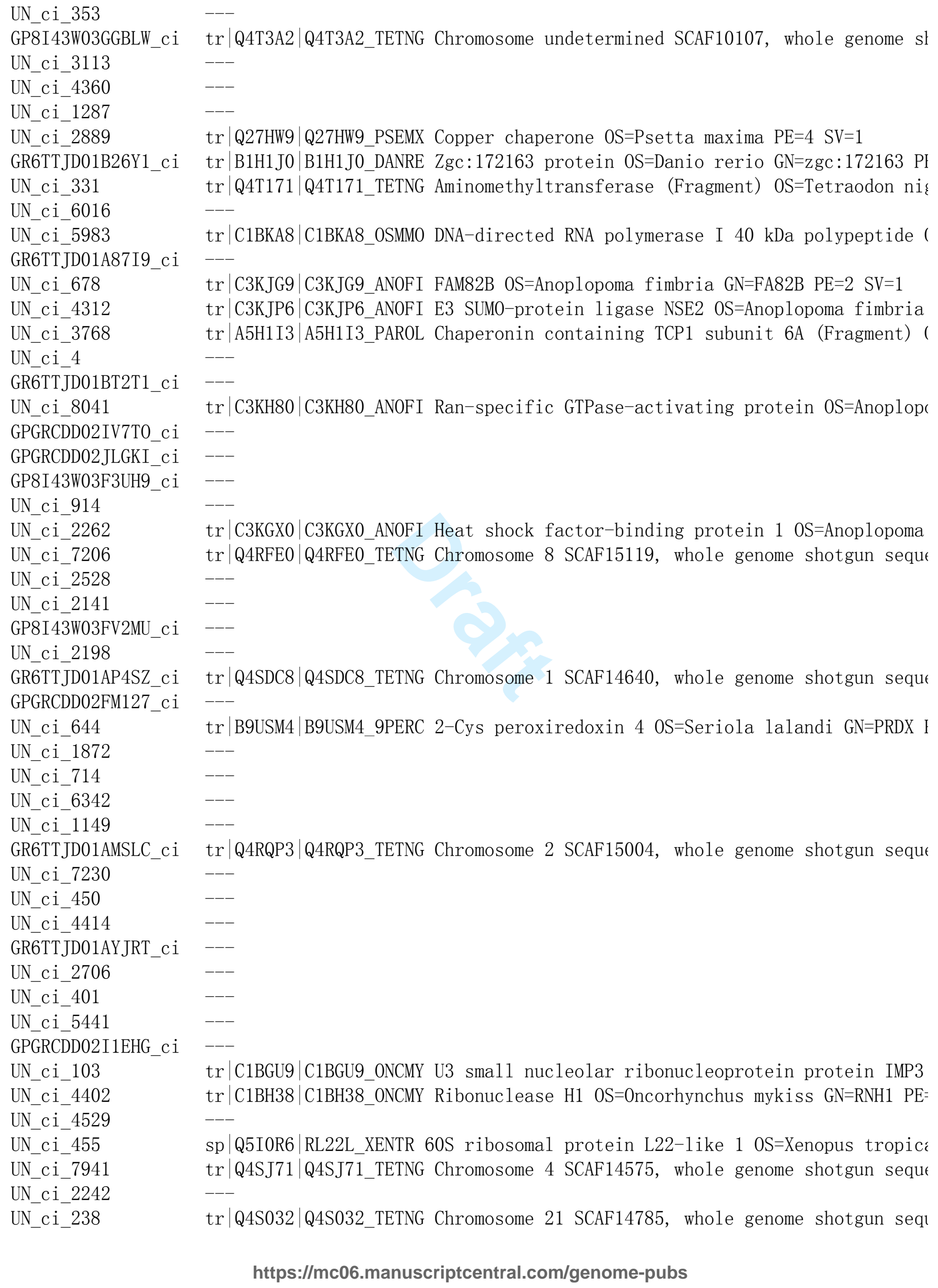




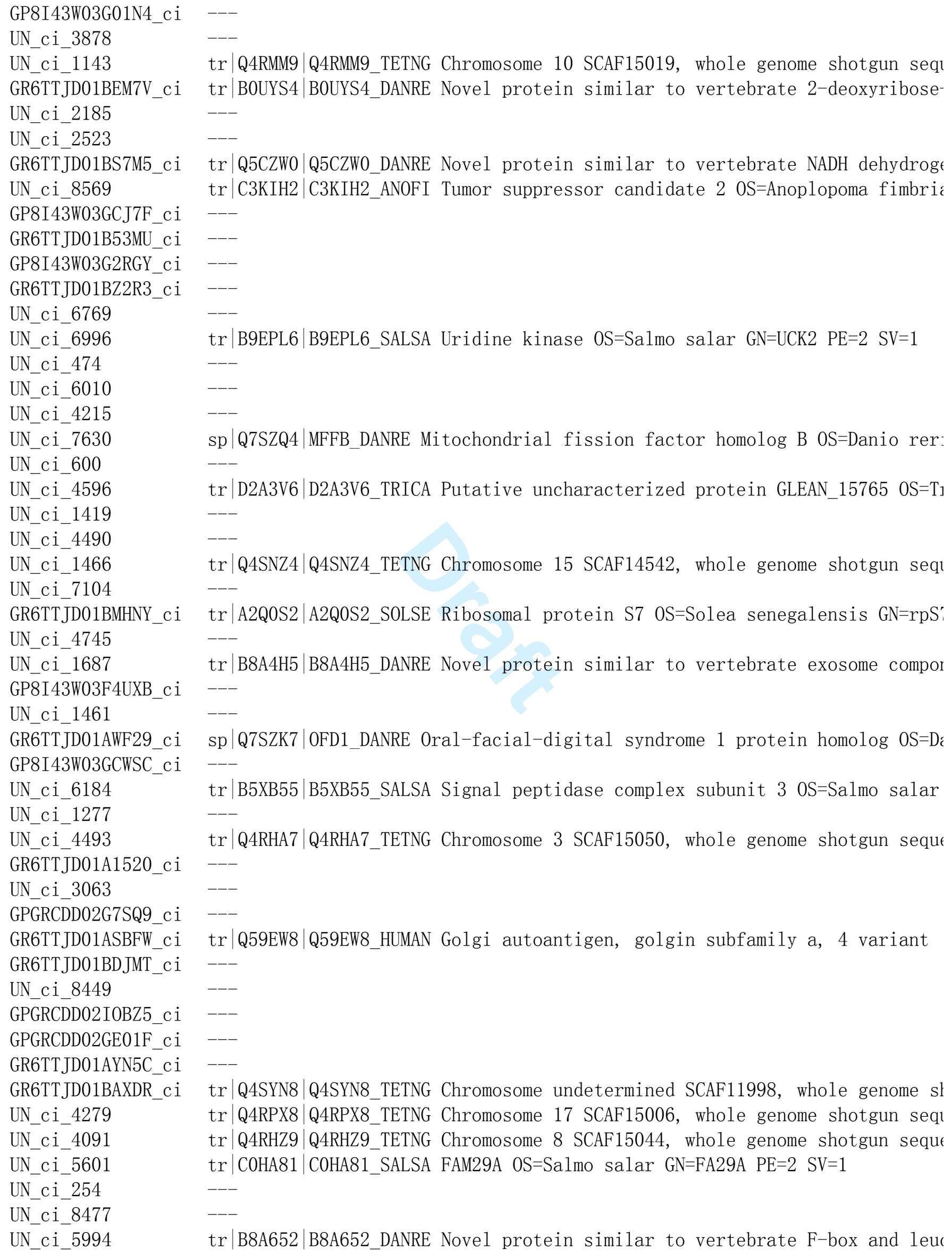




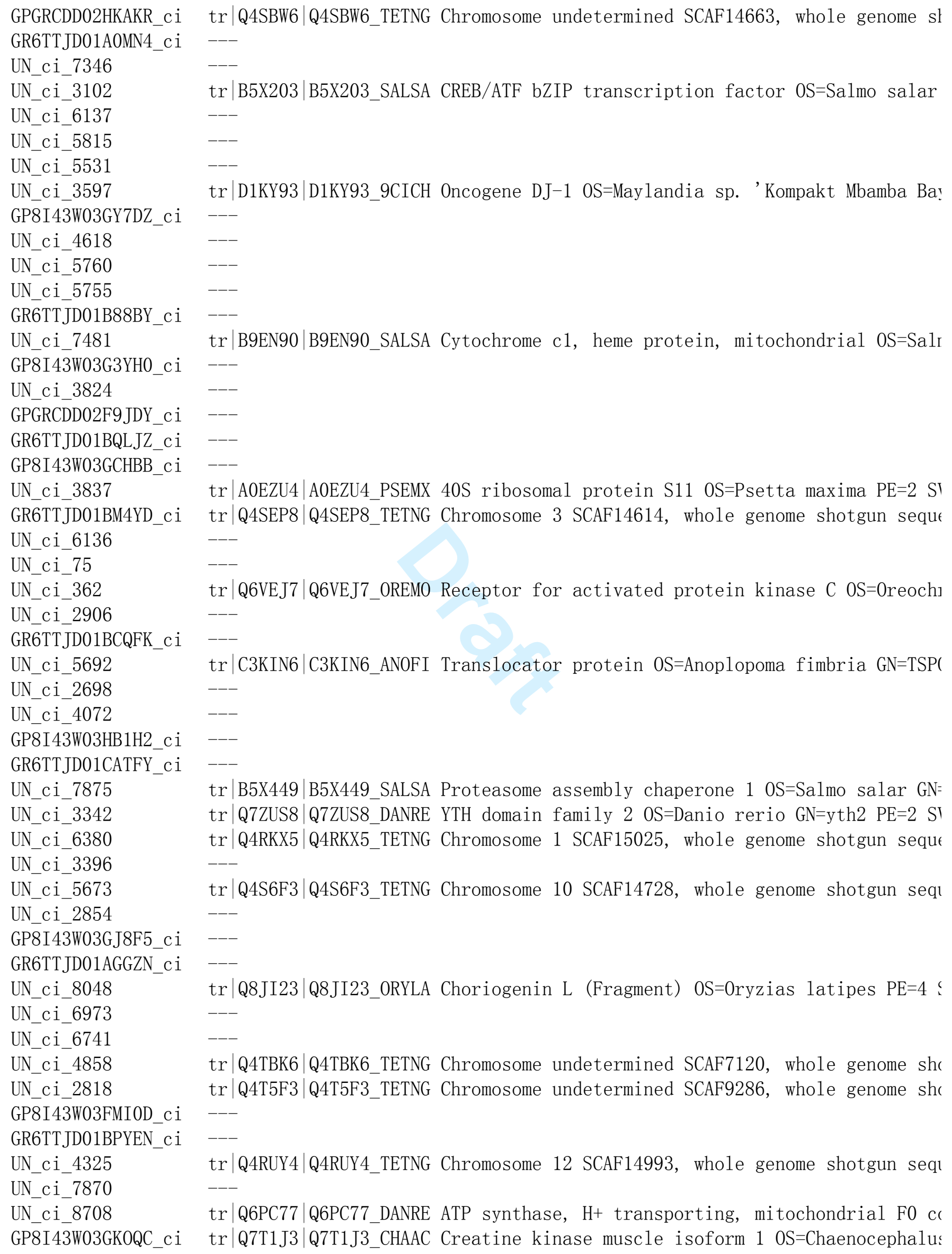




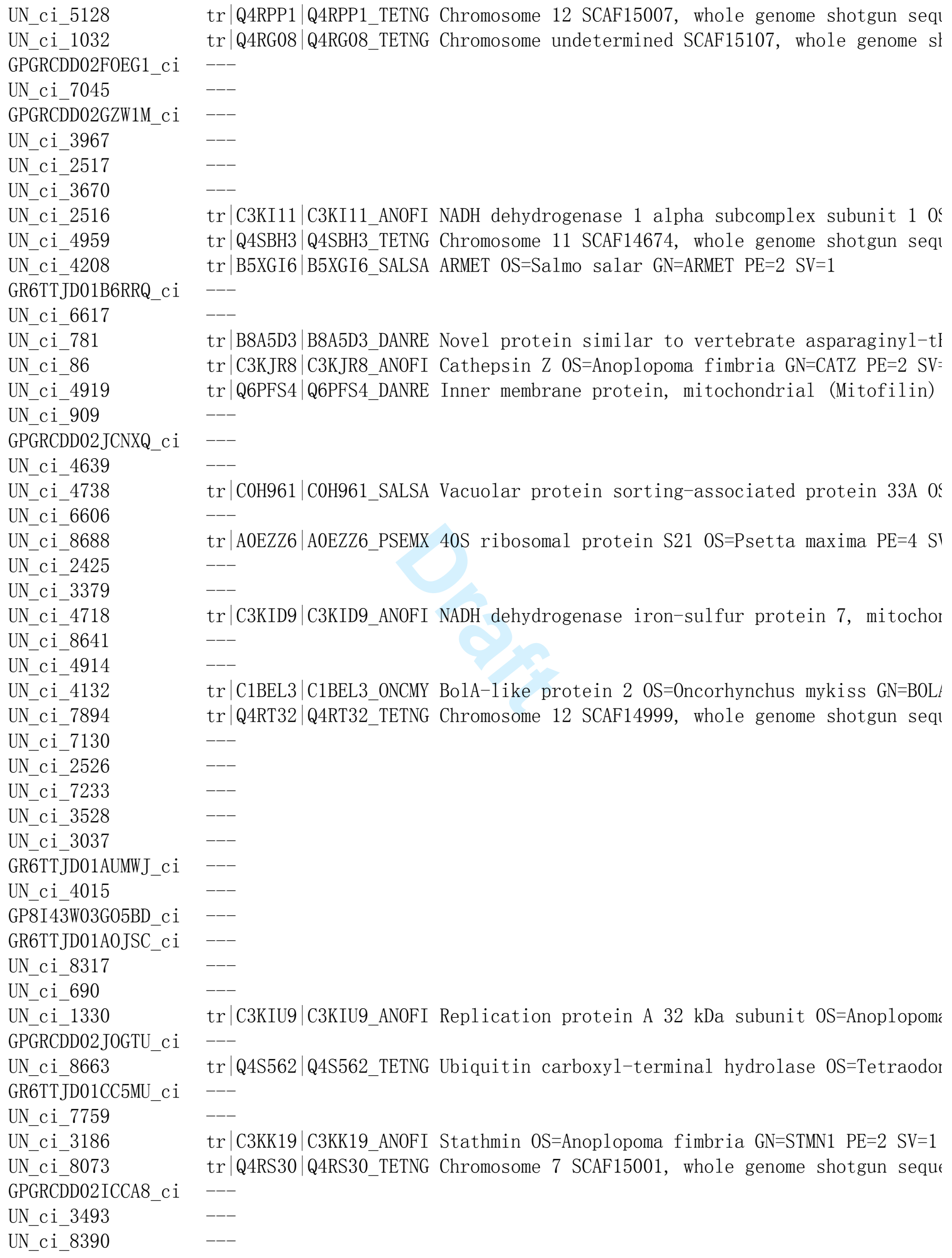




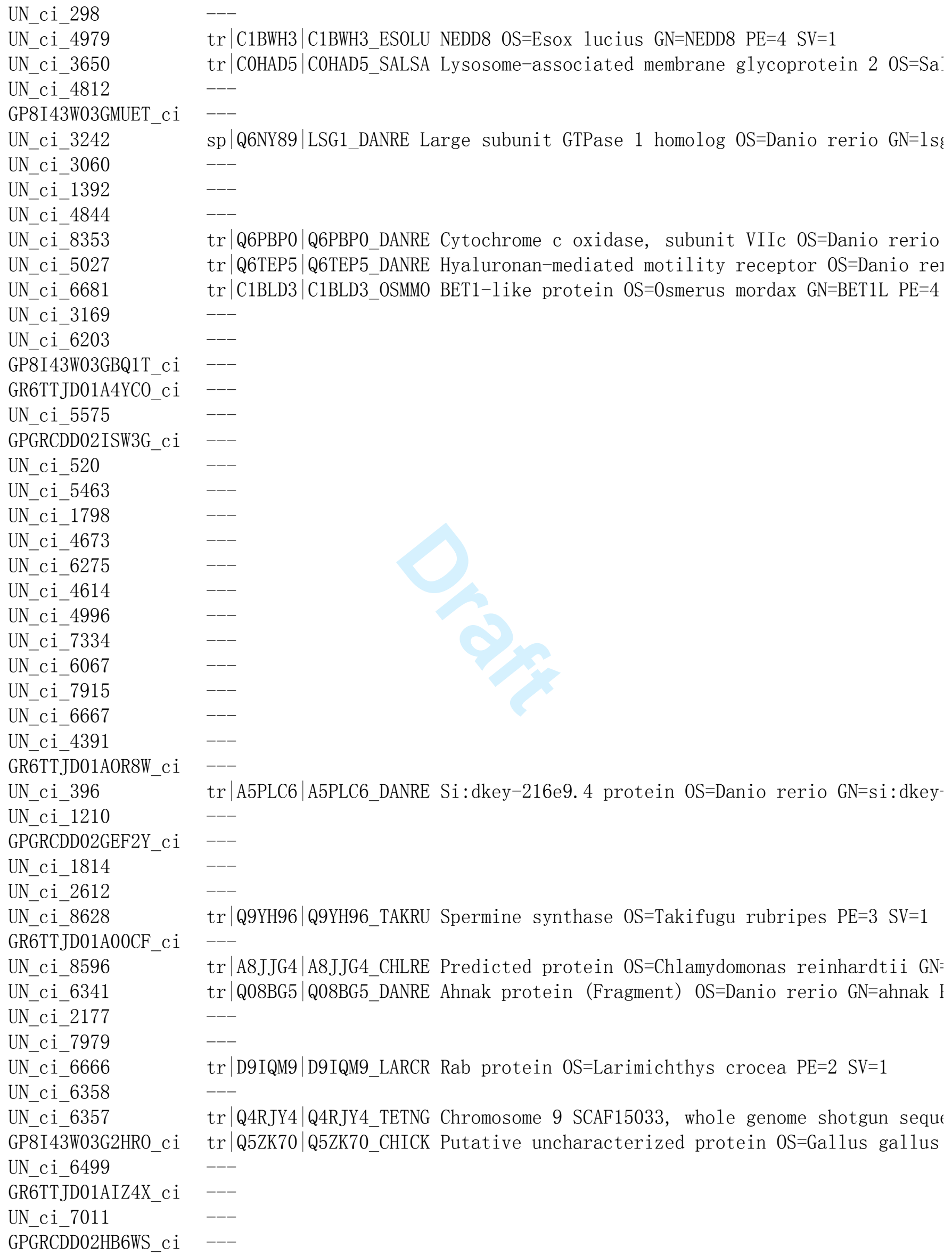

$\operatorname{tr} \mid$ C1BWH3 $\mid$ C1BWH3 ESOLU NEDD8 OS=Esox lucius GN=NEDD8 PE=4 SV=1

$\operatorname{tr} \mid$ COHAD5 $\mid$ COHAD5_SALSA Lysosome-associated membrane glycoprotein 2 OS=Sa

sp $\mid$ Q6NY89|LSG1_DANRE Large subunit GTPase 1 homolog OS=Danio rerio GN=1s

$\operatorname{tr} \mid$ Q6PBP0 $\mid$ Q6PBP0_DANRE Cytochrome c oxidase, subunit VIIc OS=Danio rerio $\operatorname{tr} \mid$ Q6TEP5 $\mid$ Q6TEP5_DANRE Hyaluronan-mediated motility receptor OS=Danio re] $\operatorname{tr} \mid$ C1BLD3 $\mid$ C1BLD3_0SMM0 BET1-1ike protein 0S=0smerus mordax GN=BET1L PE=4

$-$

$\operatorname{tr} \mid$ A5PLC6 |A5PLC6_DANRE Si:dkey-216e9. 4 protein OS=Danio rerio GN=si:dkey-

$\operatorname{tr} \mid$ Q9YH96 $\mid$ Q9YH96_TAKRU Spermine synthase OS=Takifugu rubripes PE=3 SV=1 tr $\mid$ A8JJG4 $\mid$ A8JJG4_CHLRE Predicted protein OS=Chlamydomonas reinhardtii GN= tr $\mid$ Q08BG5 $\mid$ Q08BG5_DANRE Ahnak protein (Fragment) OS=Danio rerio GN=ahnak I

tr|D9IQM9|D9IQM9_LARCR Rab protein OS=Larimichthys crocea PE=2 SV=1

tr $\mid$ Q4RJY4|Q4RJY4_TETNG Chromosome 9 SCAF15033, whole genome shotgun sequt tr $\mid$ Q5ZK70 $\mid$ Q5ZK70_CHICK Putative uncharacterized protein OS=Gallus gallus

\section{https://mc06.manuscriptcentral.com/genome-pubs}




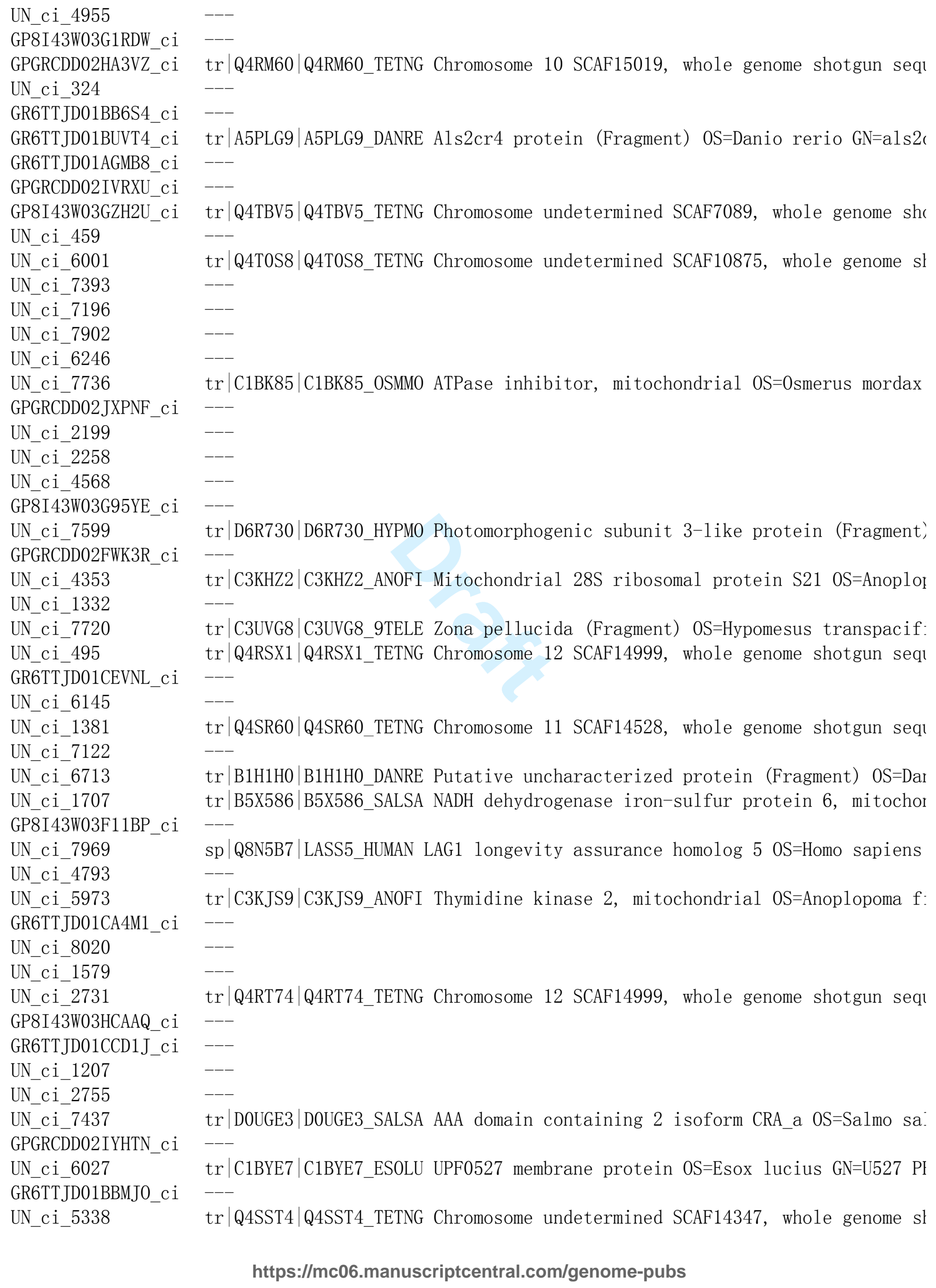




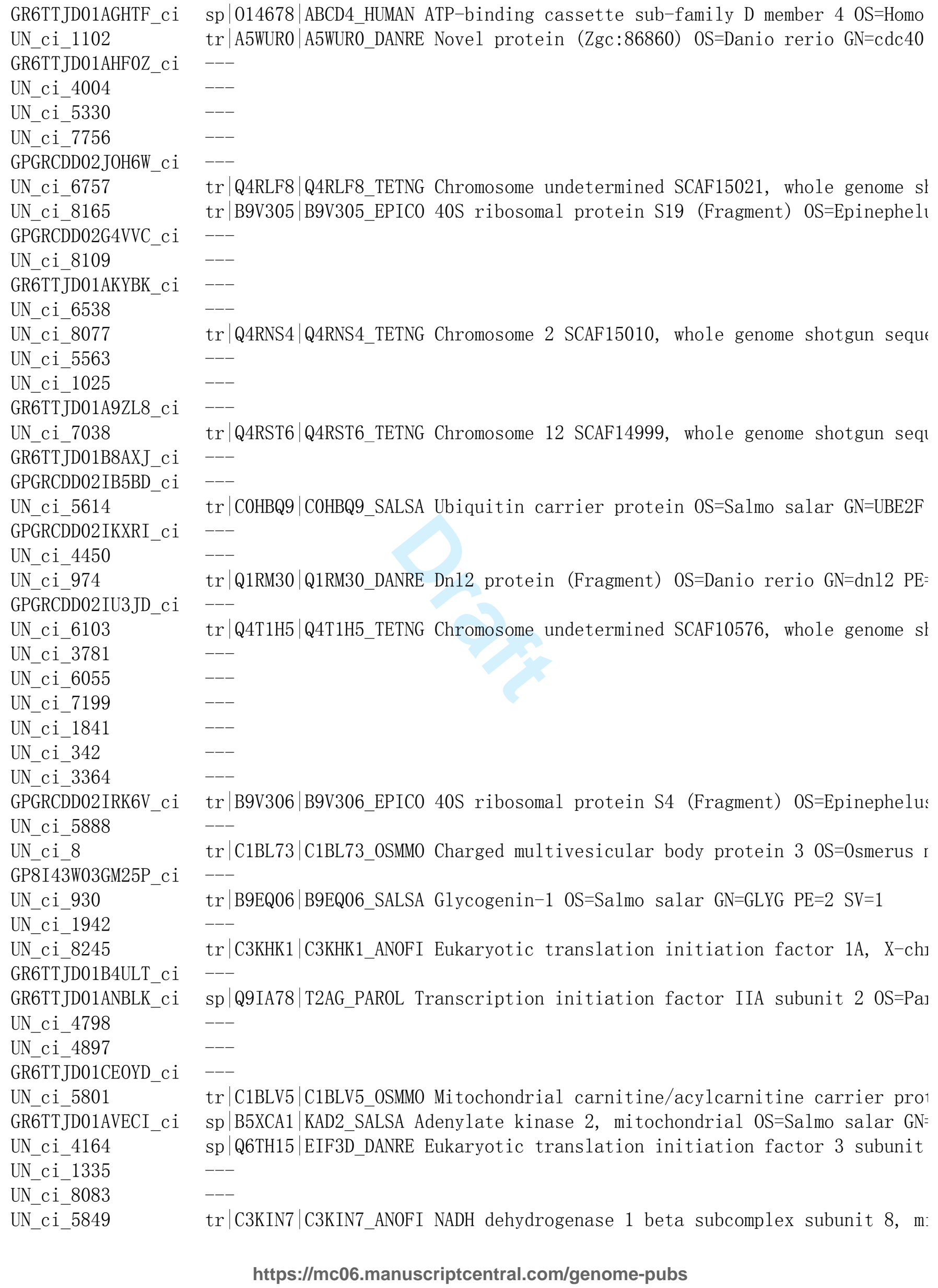




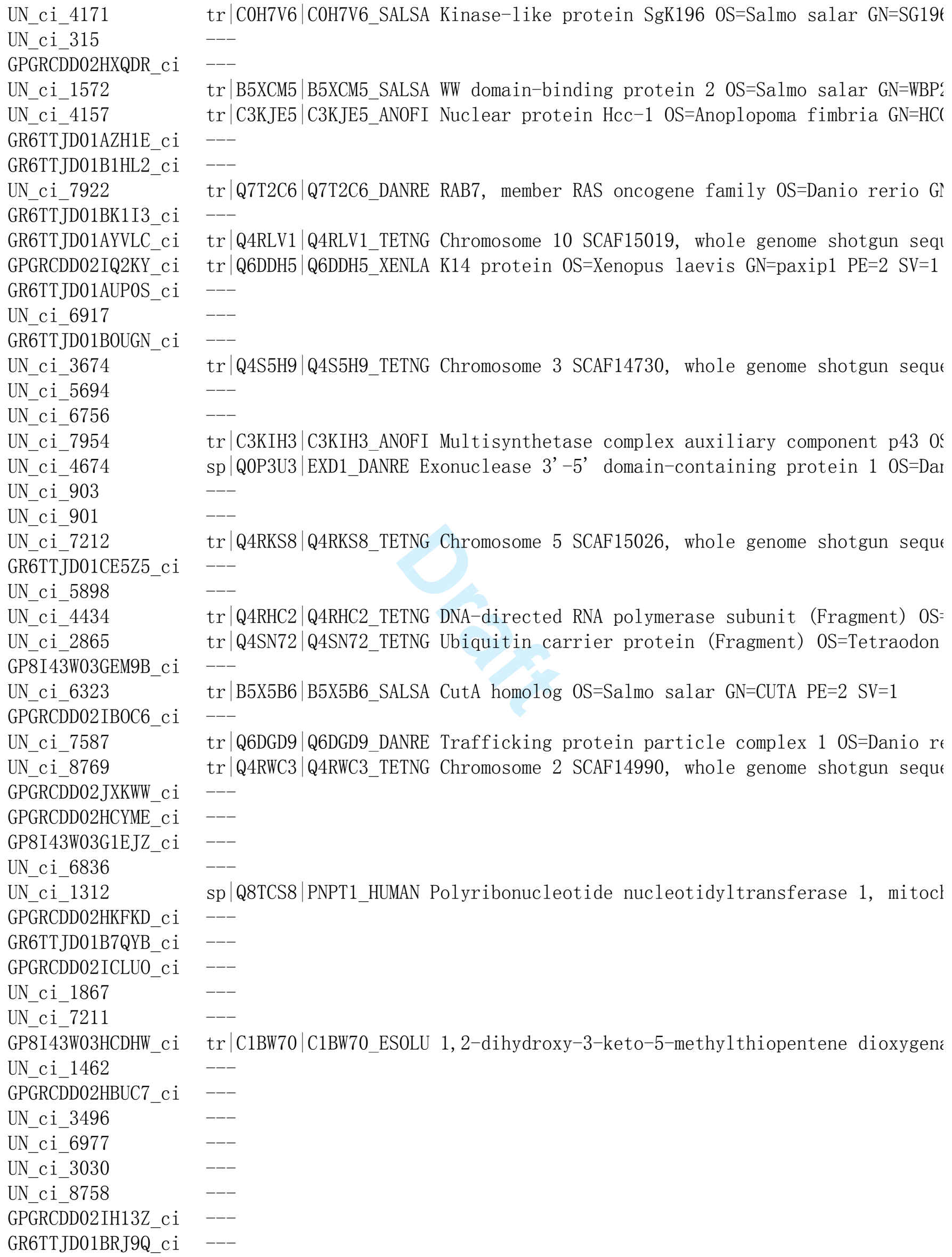




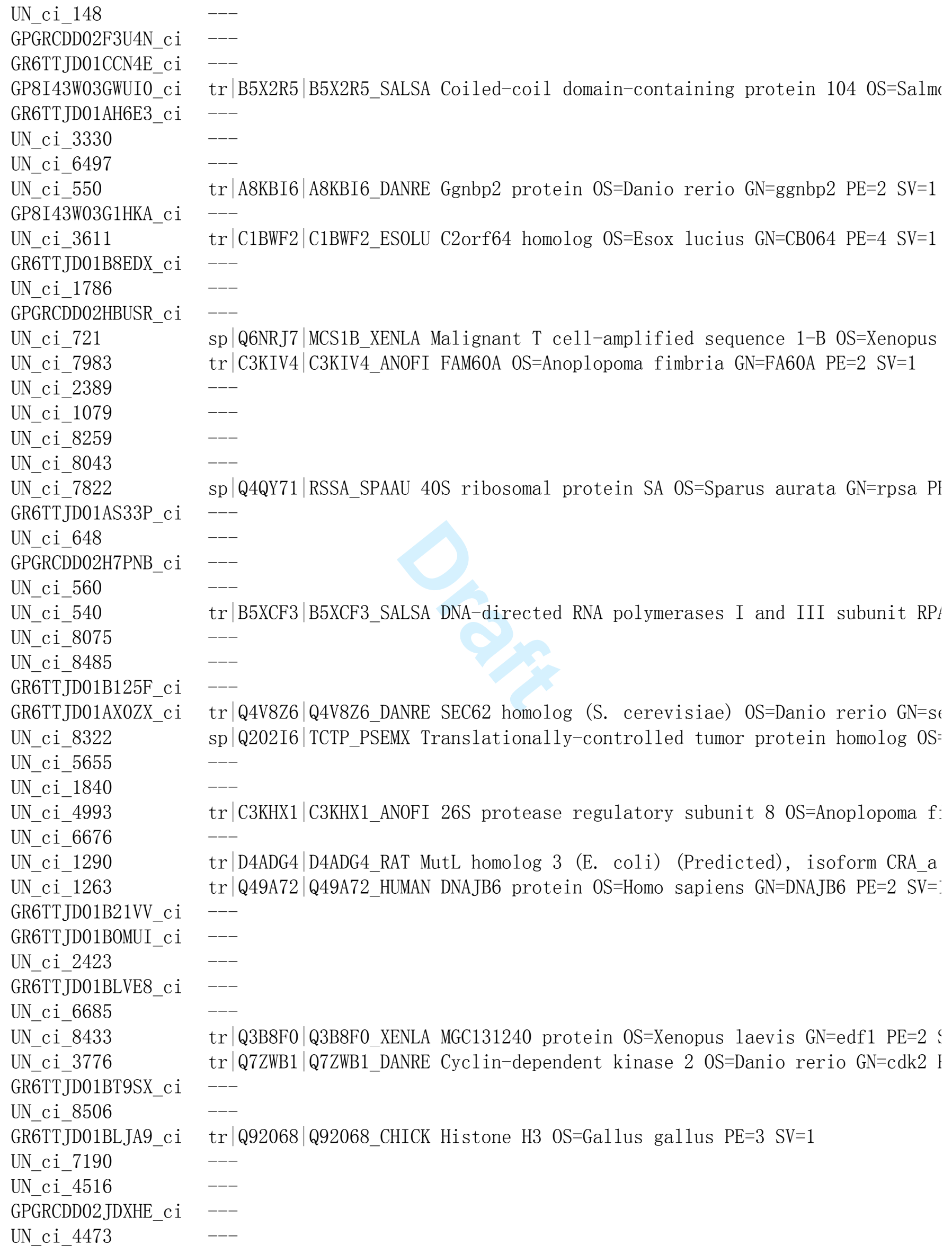




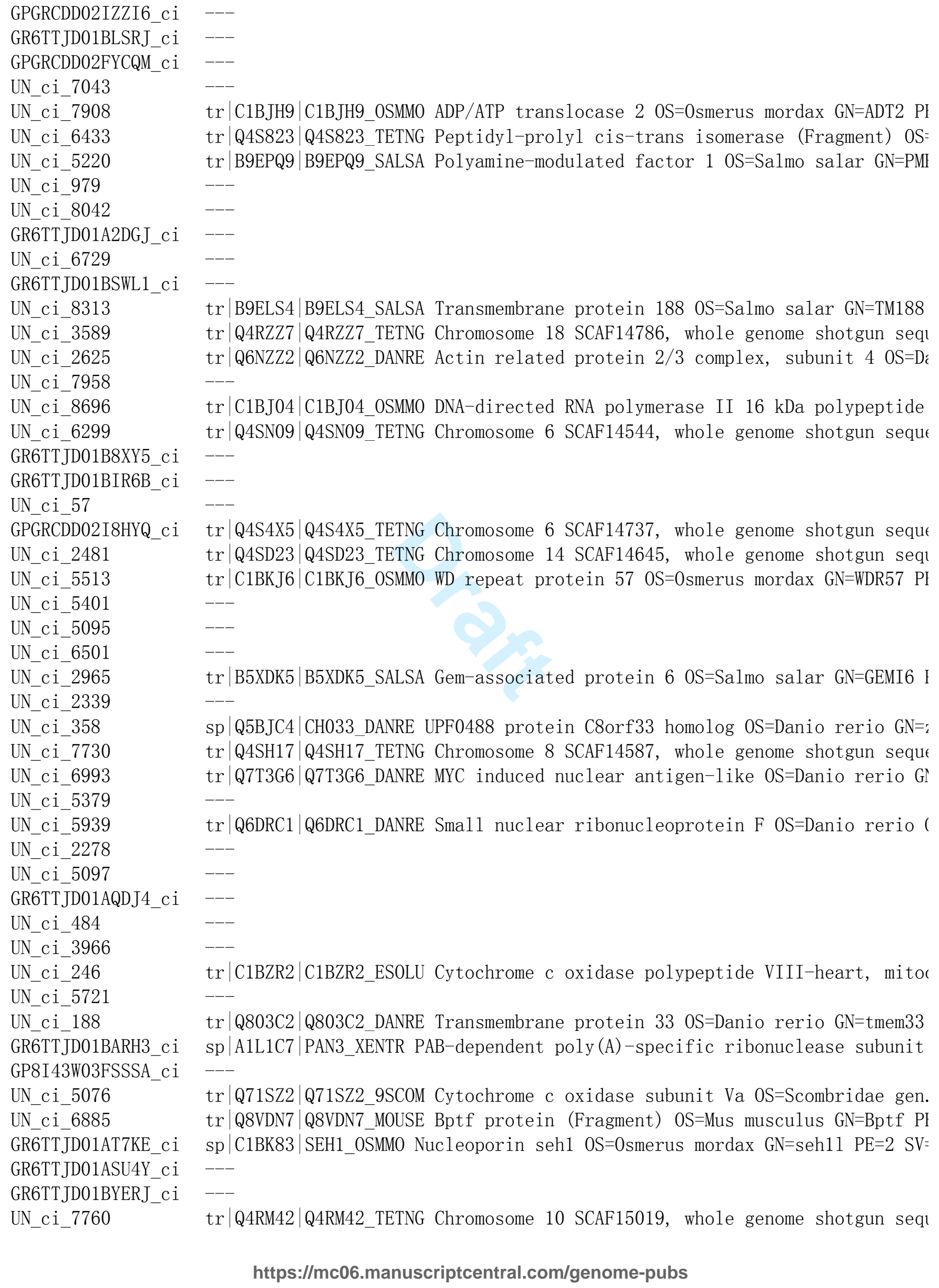




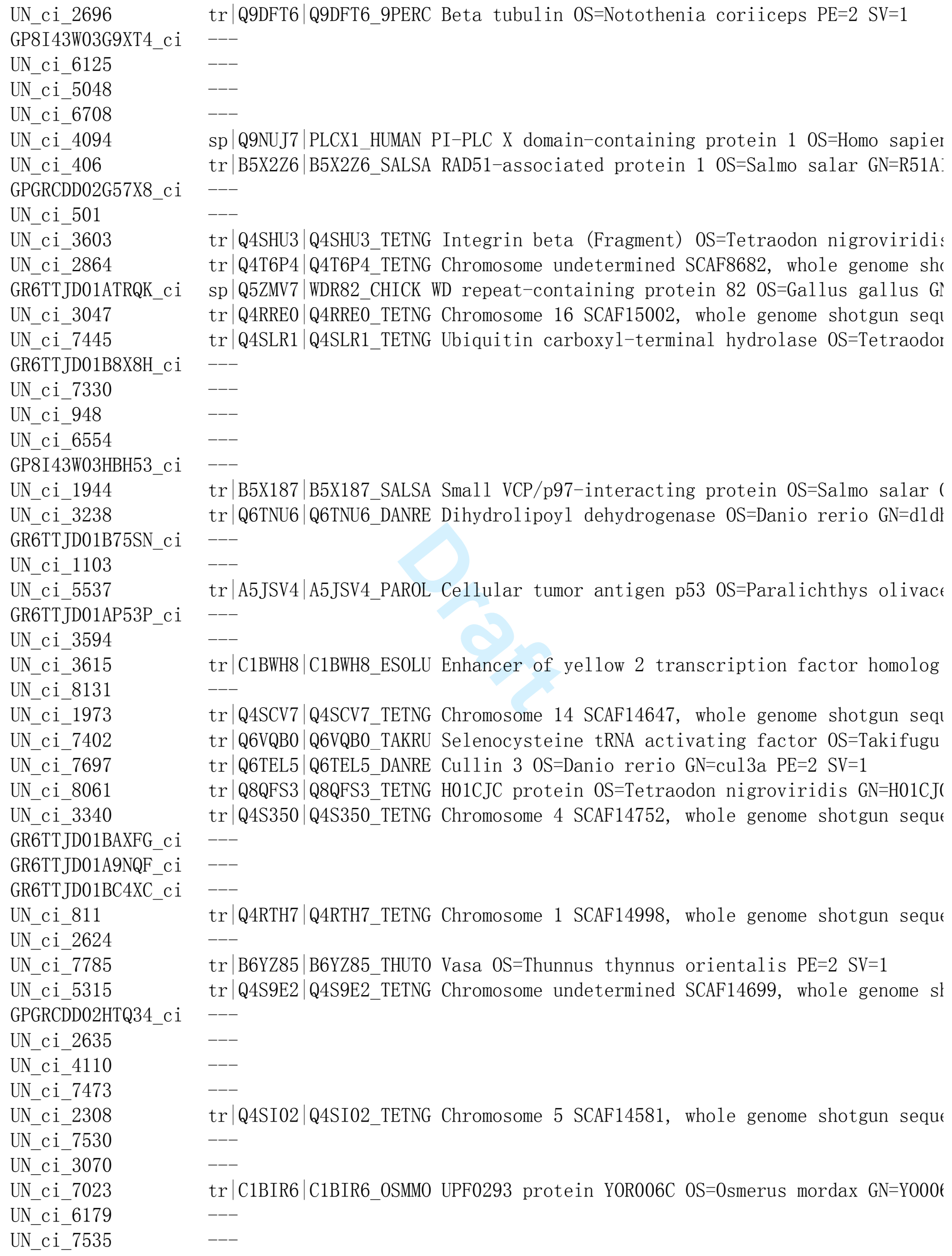

tr $\mid$ Q9DFT6 $\mid$ Q9DFT6_9PERC Beta tubulin OS=Notothenia coriiceps PE=2 SV=1

sp |Q9NUJ7|PLCX1_HUMAN PI-PLC X domain-containing protein 1 OS=Homo sapier tr|B5X2Z6|B5X2Z6_SALSA RAD51-associated protein 1 OS=Salmo salar GN=R51A:

tr $\mid$ Q4SHU3 $\mid$ Q4SHU3_TETNG Integrin beta (Fragment) OS=Tetraodon nigroviridis tr $\mid$ Q4T6P4|Q4T6P4_TETNG Chromosome undetermined SCAF8682, whole genome shc sp |Q5ZMV7|WDR82_CHICK WD repeat-containing protein 82 OS=Gallus gallus G! tr $\mid$ Q4RRE0|Q4RRE0_TETNG Chromosome 16 SCAF15002, whole genome shotgun seq1 $\operatorname{tr} \mid$ Q4SLR1 $\mid$ Q4SLR1_TETNG Ubiquitin carboxy1-terminal hydrolase 0S=Tetraodor

tr|B5X187|B5X187_SALSA Sma11 VCP/p97-interacting protein 0S=Salmo salar ( tr $\mid$ Q6TNU6 |Q6TNU6_DANRE Dihydrolipoyl dehydrogenase OS=Danio rerio GN=dldl

tr|A5JSV4|A5JSV4_PAR0L Cellular tumor antigen p53 0S=Paralichthys olivace $\operatorname{tr} \mid$ C1BWH8|C1BWH8_ESOLU Enhancer of yellow 2 transcription factor homolog tr $\mid$ Q4SCV7|Q4SCV7_TETNG Chromosome 14 SCAF14647, whole genome shotgun seq1 tr $\mid$ Q6VQB0|Q6VQB0_TAKRU Selenocysteine tRNA activating factor OS=Takifugu tr $\mid$ Q6TEL5 $\mid$ Q6TEL5_DANRE Cullin 3 OS=Danio rerio GN=cu13a PE=2 SV=1 $\operatorname{tr} \mid$ Q8QFS3 $\mid$ Q8QFS3_TETNG H01CJC protein OS=Tetraodon nigroviridis GN=H01CJ( tr $\mid$ Q4S350|Q4S350_TETNG Chromosome 4 SCAF14752, whole genome shotgun sequt

$\operatorname{tr} \mid$ Q4RTH7 |Q4RTH7_TETNG Chromosome 1 SCAF14998, whole genome shotgun sequt $\operatorname{tr} \mid$ B6YZ85|B6YZ85_THUT0 Vasa OS=Thunnus thynnus orientalis PE=2 SV=1 tr $\mid$ Q4S9E2 $\mid$ Q4S9E2_TETNG Chromosome undetermined SCAF14699, whole genome sl $---$ 


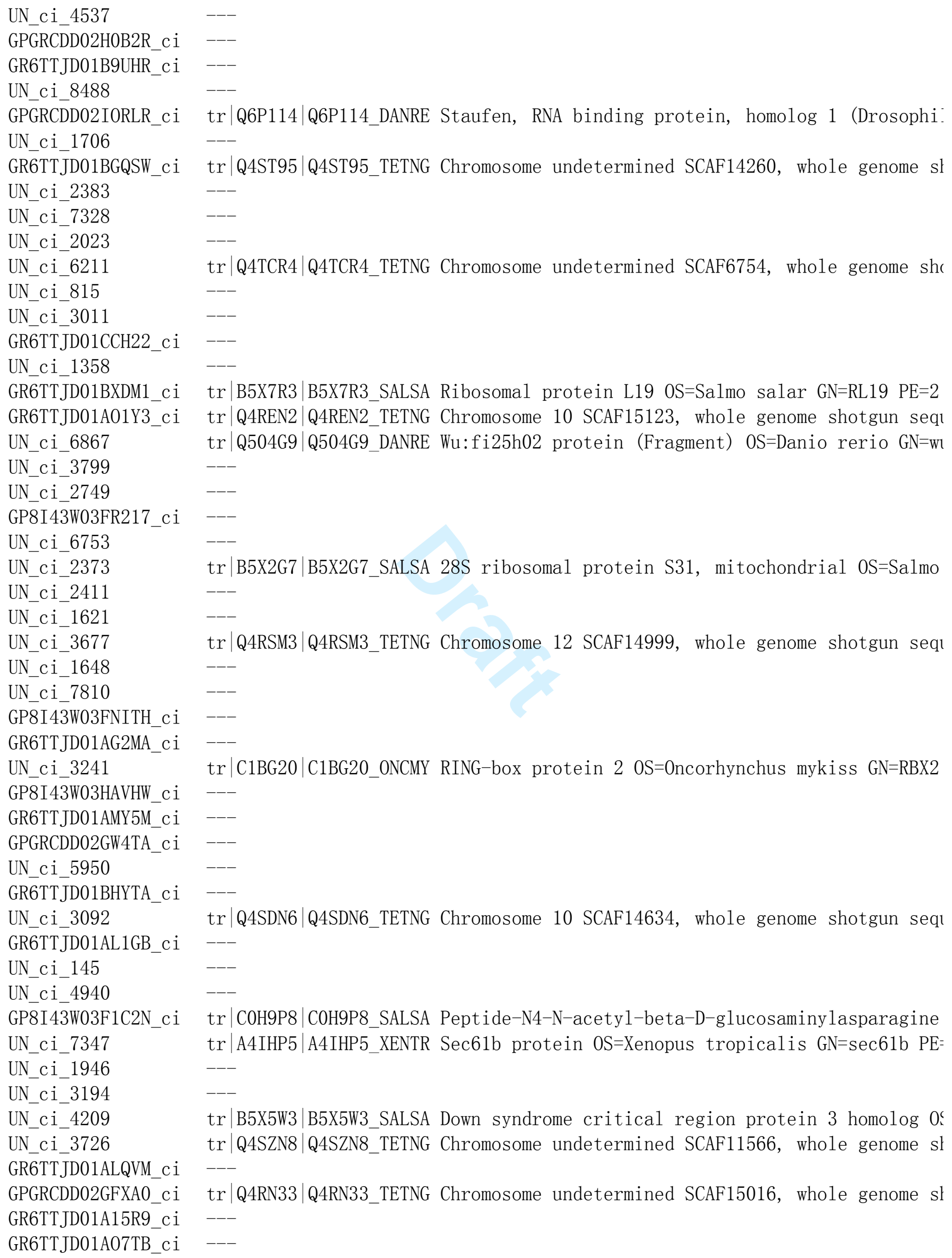




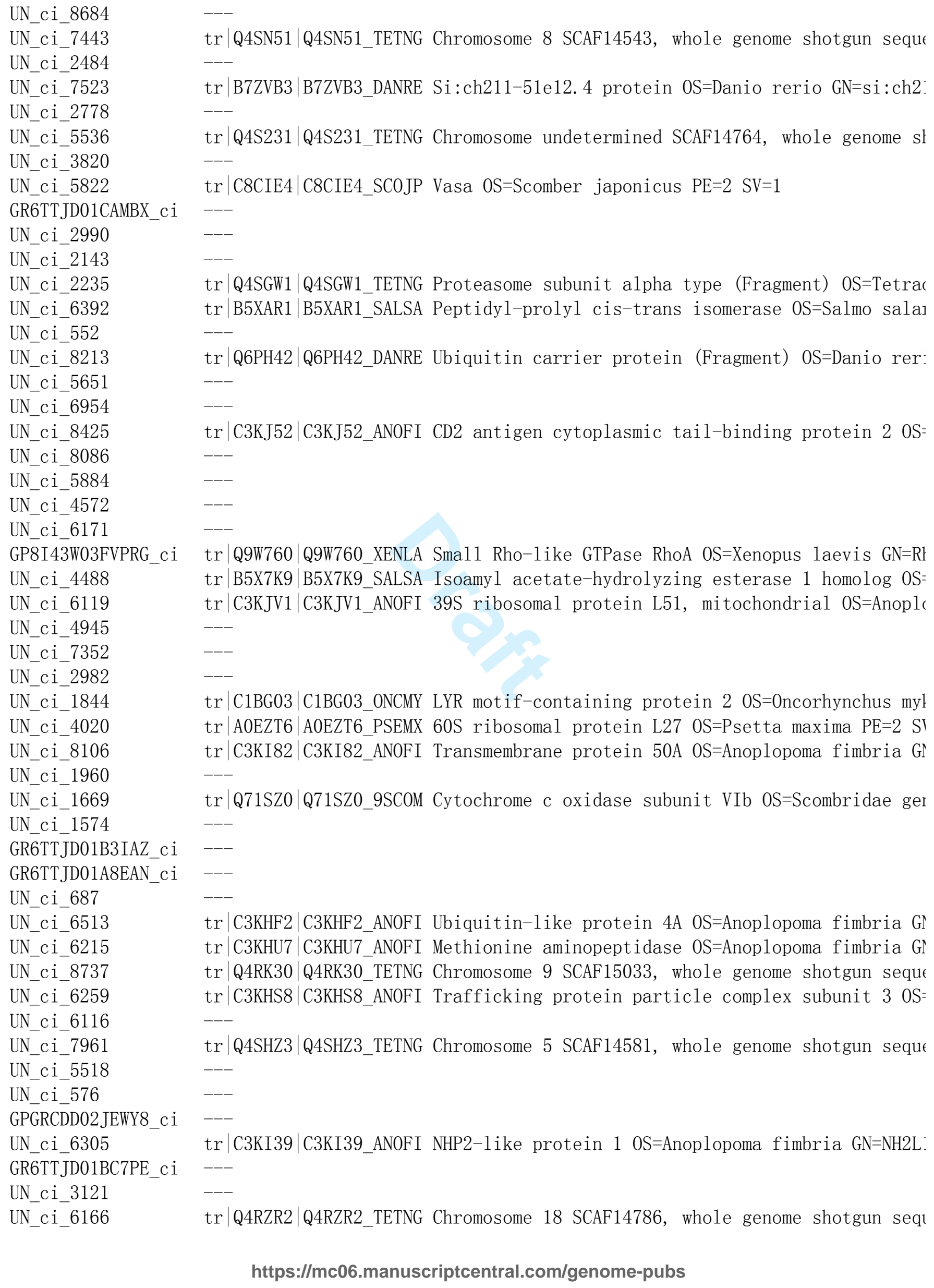

tr $\mid$ Q4SN51 |Q4SN51_TETNG Chromosome 8 SCAF14543, whole genome shotgun sequt tr $\mid$ B7ZVB3 |B7ZVB3_DANRE Si:ch211-51e12. 4 protein OS=Danio rerio GN=si:ch2: $\operatorname{tr} \mid$ Q4S231|Q4S231_TETNG Chromosome undetermined SCAF14764, whole genome sl $\operatorname{tr} \mid$ C8CIE4 $\mid$ C8CIE4_SC0JP Vasa OS=Scomber japonicus PE=2 SV=1

tr $\mid$ Q4SGW1 $\mid$ Q4SGW1_TETNG Proteasome subunit alpha type (Fragment) OS=Tetrar tr|B5XAR1|B5XAR1_SALSA Peptidy1-prolyl cis-trans isomerase 0S=Salmo sala] $\operatorname{tr} \mid$ Q6PH42 $\mid$ Q6PH42_DANRE Ubiquitin carrier protein (Fragment) OS=Danio rer: tr $\mid$ C3KJ52 $\mid$ C3KJ52_ANOFI CD2 antigen cytoplasmic tail-binding protein 2 OS= $---$

$\operatorname{tr} \mid$ Q9W760 $\mid$ Q9W760_XENLA Smal1 Rho-1ike GTPase RhoA OS=Xenopus laevis GN=Rl tr $\mid$ B5X7K9|B5X7K9_SALSA Isoamy1 acetate-hydrolyzing esterase 1 homolog 0S= tr $\mid$ C3KJV1|C3KJV1_ANOFI 39S ribosomal protein L51, mitochondrial OS=Anoplc

$\operatorname{tr} \mid$ C1BG03 $\mid$ C1BG03_ONCMY LYR motif-containing protein 2 OS=0ncorhynchus myl tr|A0EZT6|A0EZT6_PSEMX 60S ribosomal protein L27 0S=Psetta maxima PE=2 ST $\operatorname{tr} \mid$ C3KI82 $\mid$ C3KI82_ANOFI Transmembrane protein 50A 0S=Anoplopoma fimbria GI $\operatorname{tr} \mid$ Q71SZ0 $\mid$ Q71SZ0_9SCOM Cytochrome c oxidase subunit VIb OS=Scombridae ger

$\operatorname{tr} \mid$ C3KHF2 $\mid$ C3KHF2_ANOFI Ubiquitin-1ike protein 4A OS=Anoplopoma fimbria G! $\operatorname{tr} \mid$ C3KHU7 |C3KHU7_ANOFI Methionine aminopeptidase OS=Anoplopoma fimbria G! tr $\mid$ Q4RK30|Q4RK30_TETNG Chromosome 9 SCAF15033, whole genome shotgun sequt tr|C3KHS8|C3KHS8_ANOFI Trafficking protein particle complex subunit 3 OS= tr $\mid$ Q4SHZ3|Q4SHZ3_TETNG Chromosome 5 SCAF14581, whole genome shotgun sequt $---$ $\operatorname{tr} \mid$ C3KI39 $\mid$ C3KI39_ANOFI NHP2-1ike protein 1 OS=Anoplopoma fimbria GN=NH2L:

tr $\mid$ Q4RZR2 |Q4RZR2_TETNG Chromosome 18 SCAF14786, whole genome shotgun seq1 


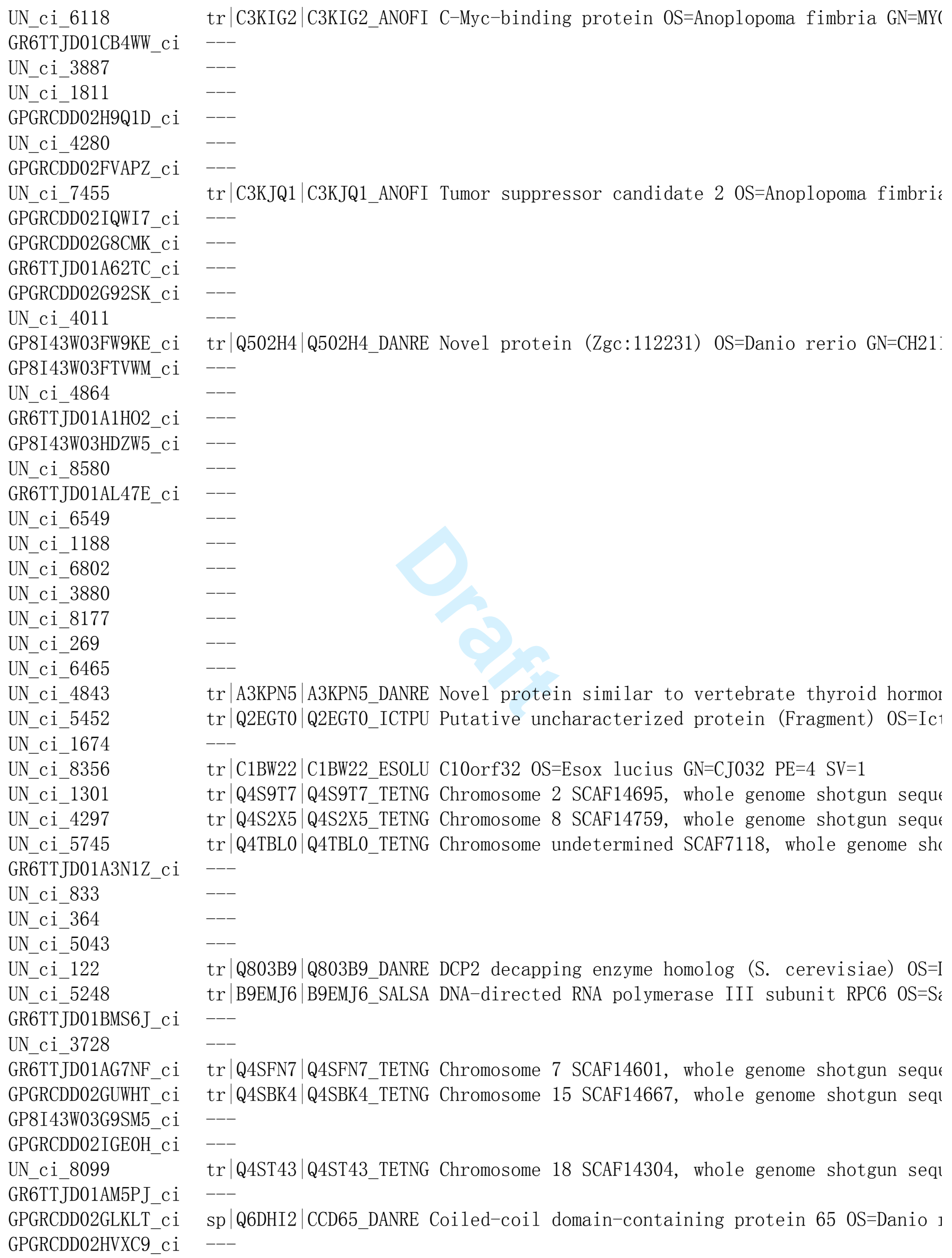




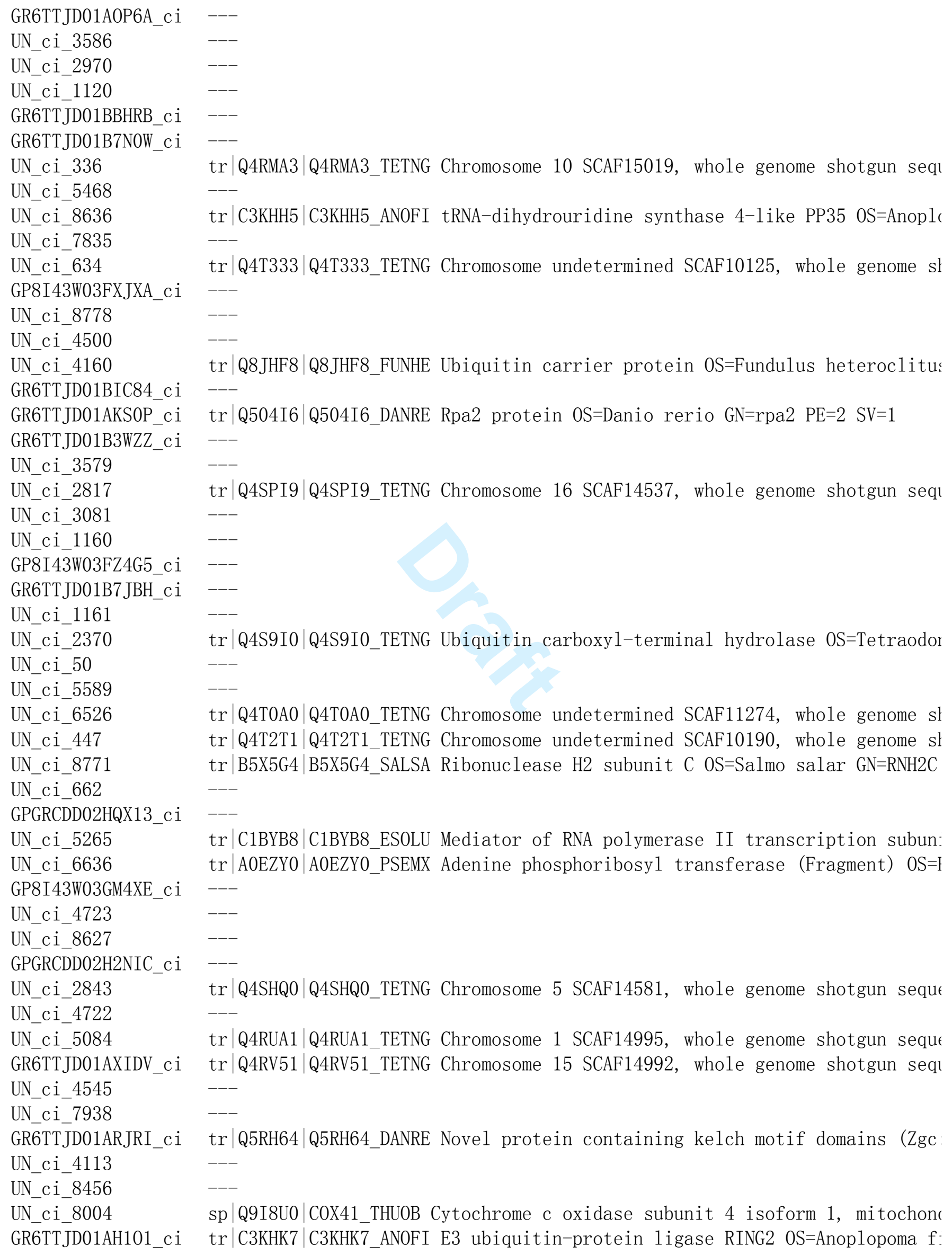




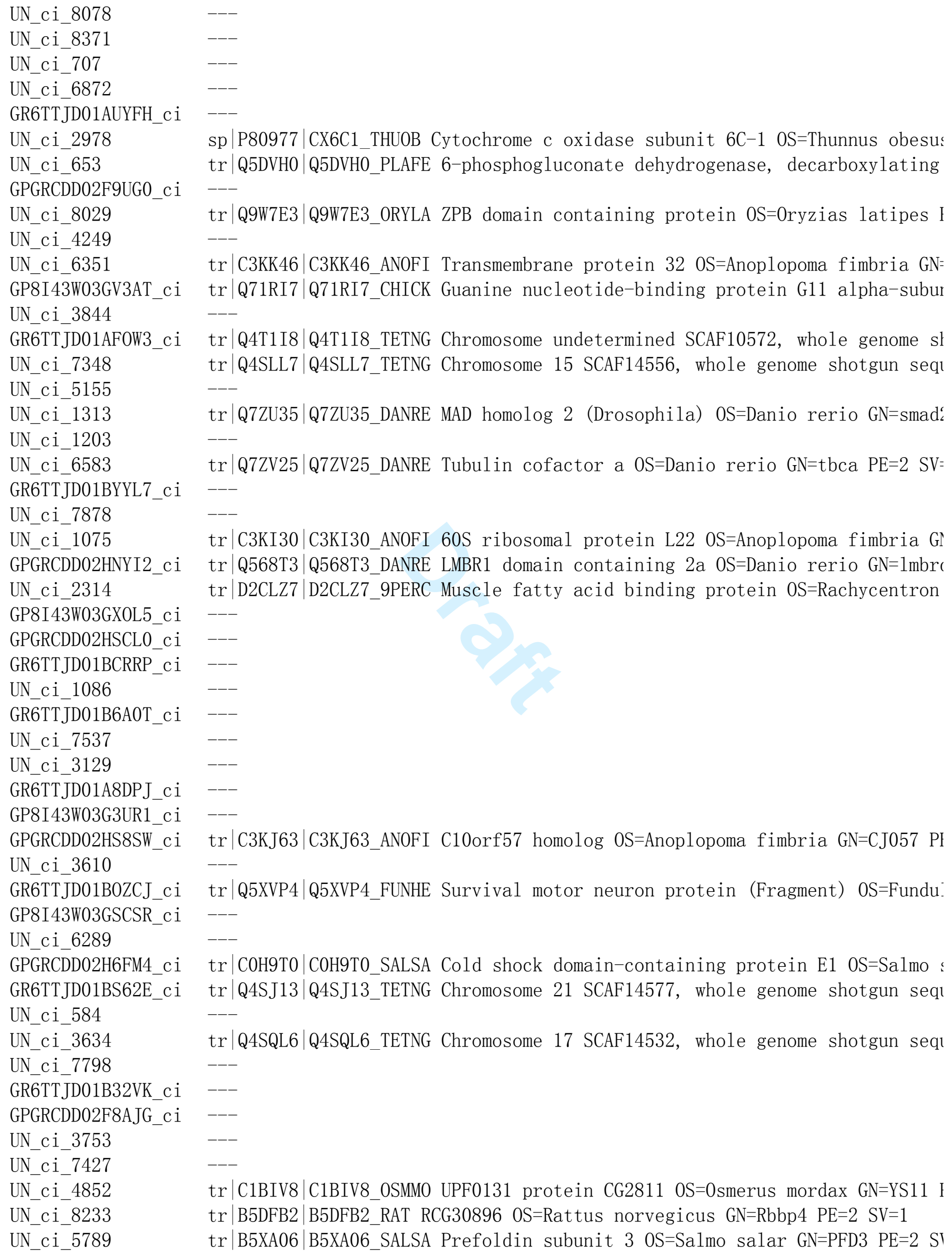

sp $\mid$ P80977|CX6C1_THUOB Cytochrome c oxidase subunit 6C-1 0S=Thunnus obesus tr |Q5DVH0|Q5DVH0_PLAFE 6-phosphogluconate dehydrogenase, decarboxylating tr $\mid$ Q9W7E3 $\mid$ Q9W7E3_0RYLA ZPB domain containing protein OS=0ryzias latipes I tr|C3KK46|C3KK46_ANOFI Transmembrane protein 32 OS=Anoplopoma fimbria GN= $\operatorname{tr} \mid$ Q71RI7|Q71RI7_CHICK Guanine nucleotide-binding protein G11 alpha-subur $\operatorname{tr}$ Q4T1I8|Q4T1I8_TETNG Chromosome undetermined SCAF10572, whole genome sl tr $\mid$ Q4SLL7|Q4SLL7_TETNG Chromosome 15 SCAF14556, whole genome shotgun seq1 tr $\mid$ Q7ZU35 $\mid$ Q7ZU35_DANRE MAD homolog 2 (Drosophila) OS=Danio rerio GN=smad؛ $\operatorname{tr} \mid$ Q7ZV25 $\mid$ Q7ZV25_DANRE Tubulin cofactor a OS=Danio rerio GN=tbca PE=2 SV=

$\operatorname{tr} \mid$ C3KI30|C3KI30_ANOFI 60S ribosomal protein L22 0S=Anoplopoma fimbria G! $\operatorname{tr} \mid$ Q568T3 $\mid$ Q568T3_DANRE LMBR1 domain containing 2a OS=Danio rerio GN=1mbrc tr|D2CLZ7|D2CLZ7_9PERC Muscle fatty acid binding protein 0S=Rachycentron

tr $\mid$ C3KJ63|C3KJ63_ANOFI C10orf57 homolog OS=Anoplopoma fimbria GN=CJ057 PI $\operatorname{tr} \mid$ Q5XVP4 $\mid$ Q5XVP4_FUNHE Survival motor neuron protein (Fragment) OS=Fundu: $---$

tr $\mid$ COH9T0 $\mid$ COH9T0_SALSA Cold shock domain-containing protein E1 OS=Salmo : tr $\mid$ Q4SJ13|Q4SJ13_TETNG Chromosome 21 SCAF14577, whole genome shotgun seq1 $---$ tr $\mid$ Q4SQL6|Q4SQL6_TETNG Chromosome 17 SCAF14532, whole genome shotgun seqı 


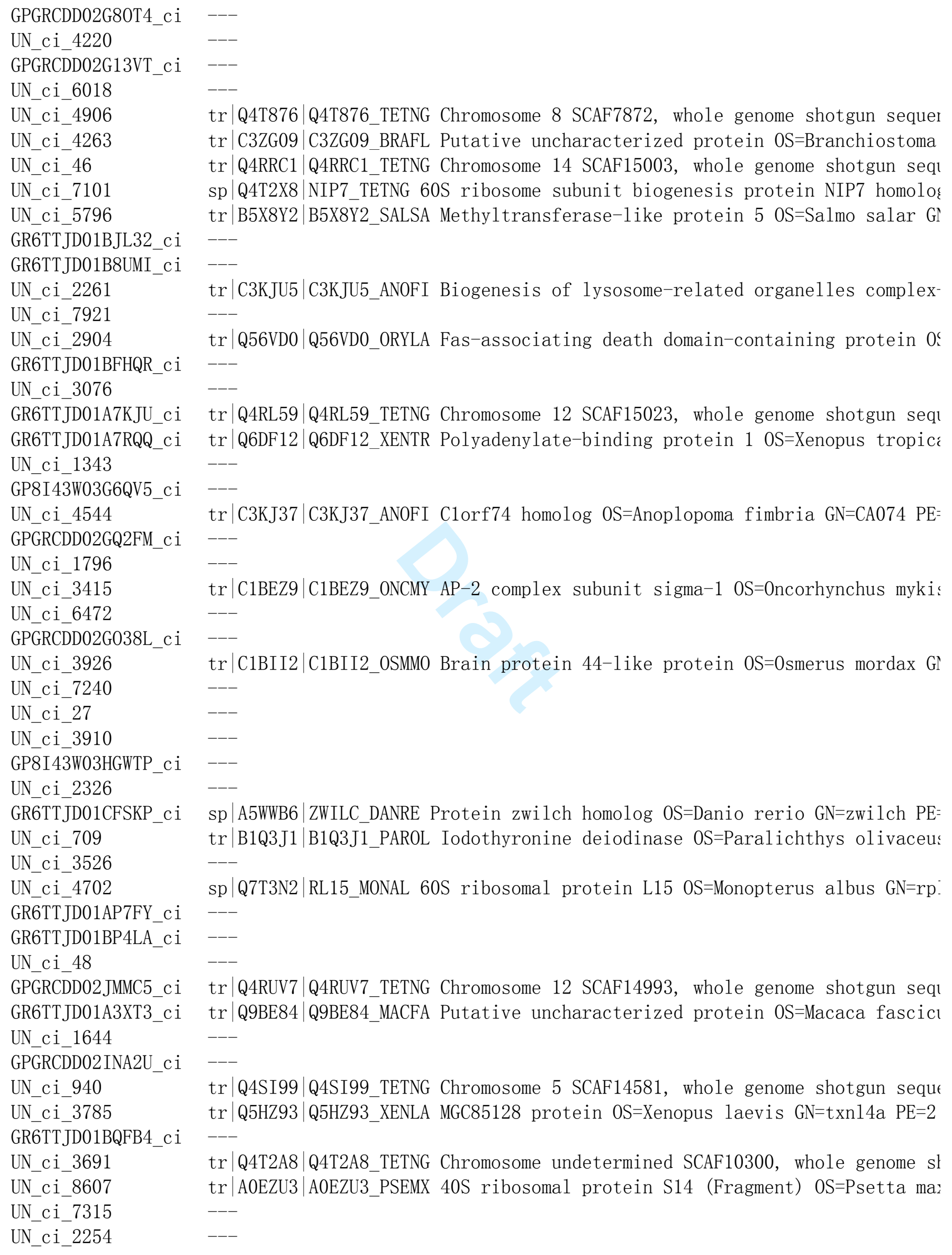




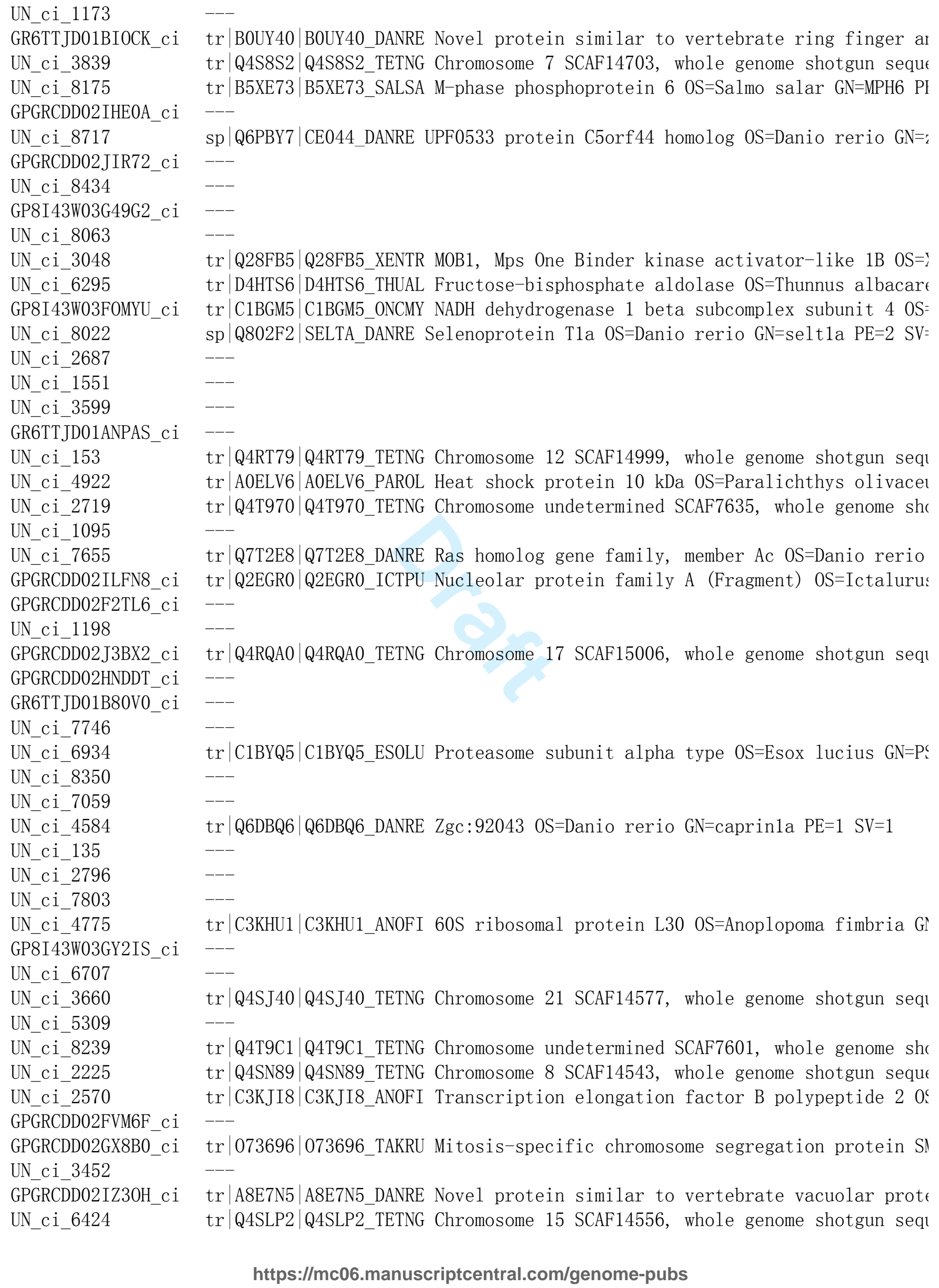




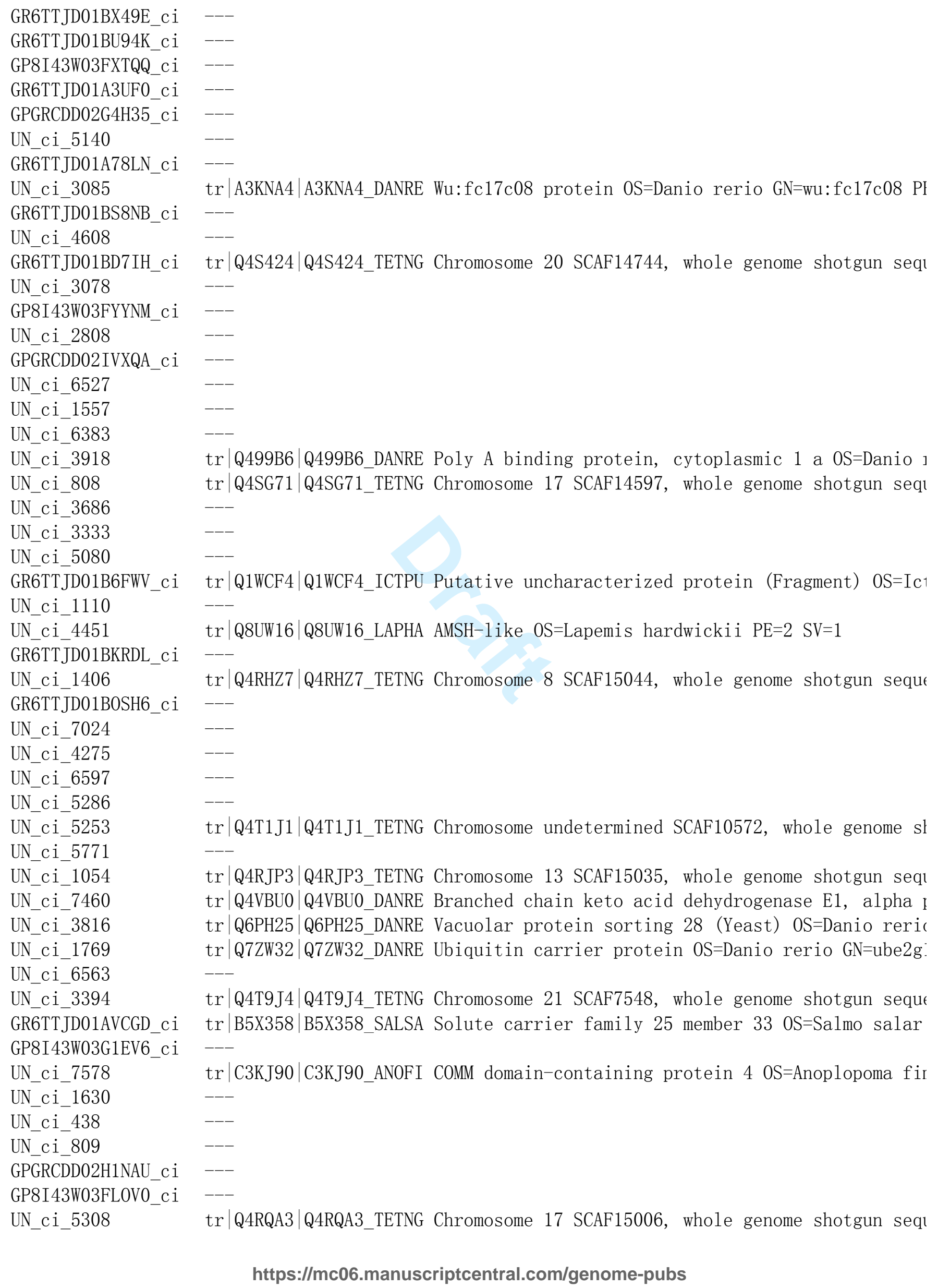




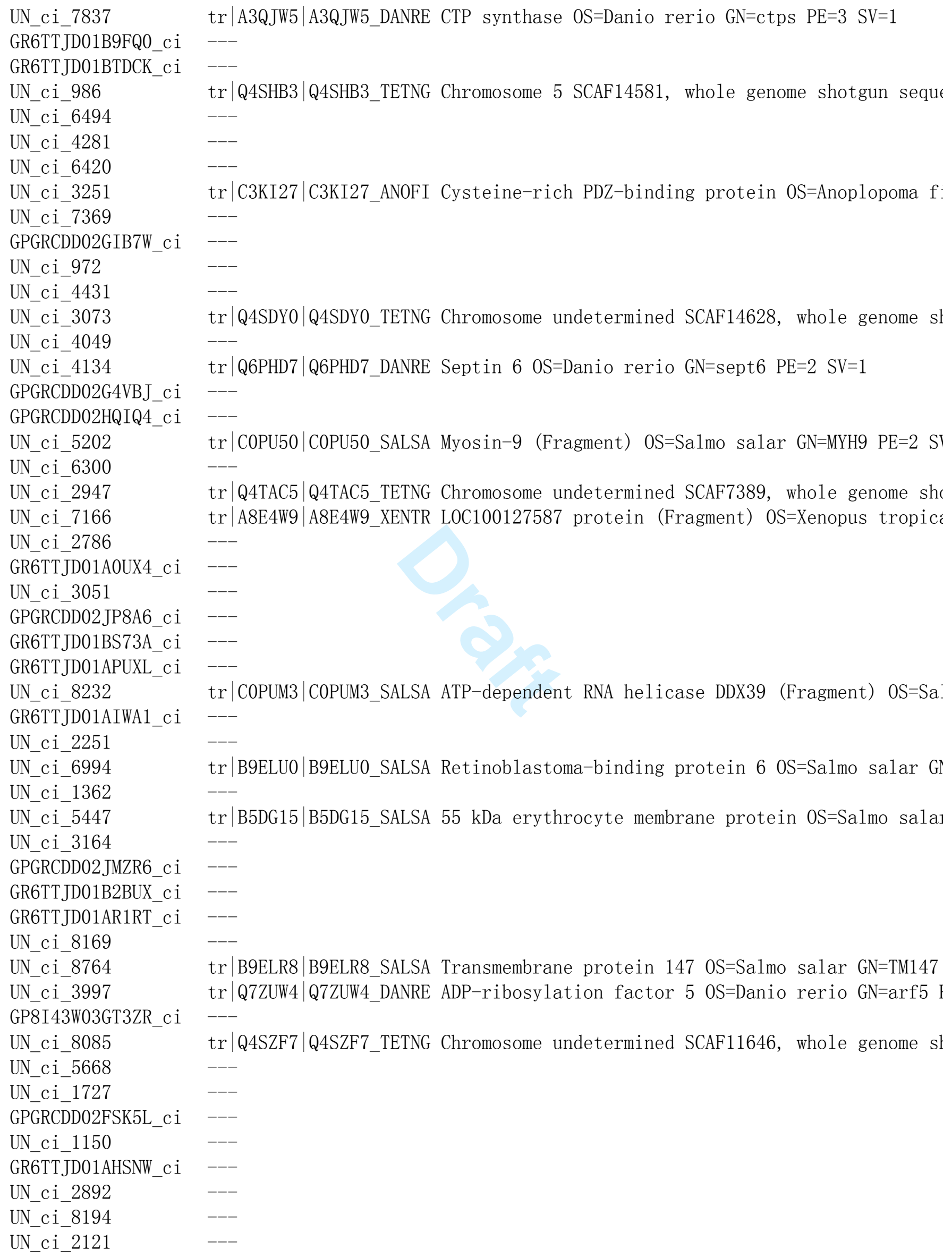




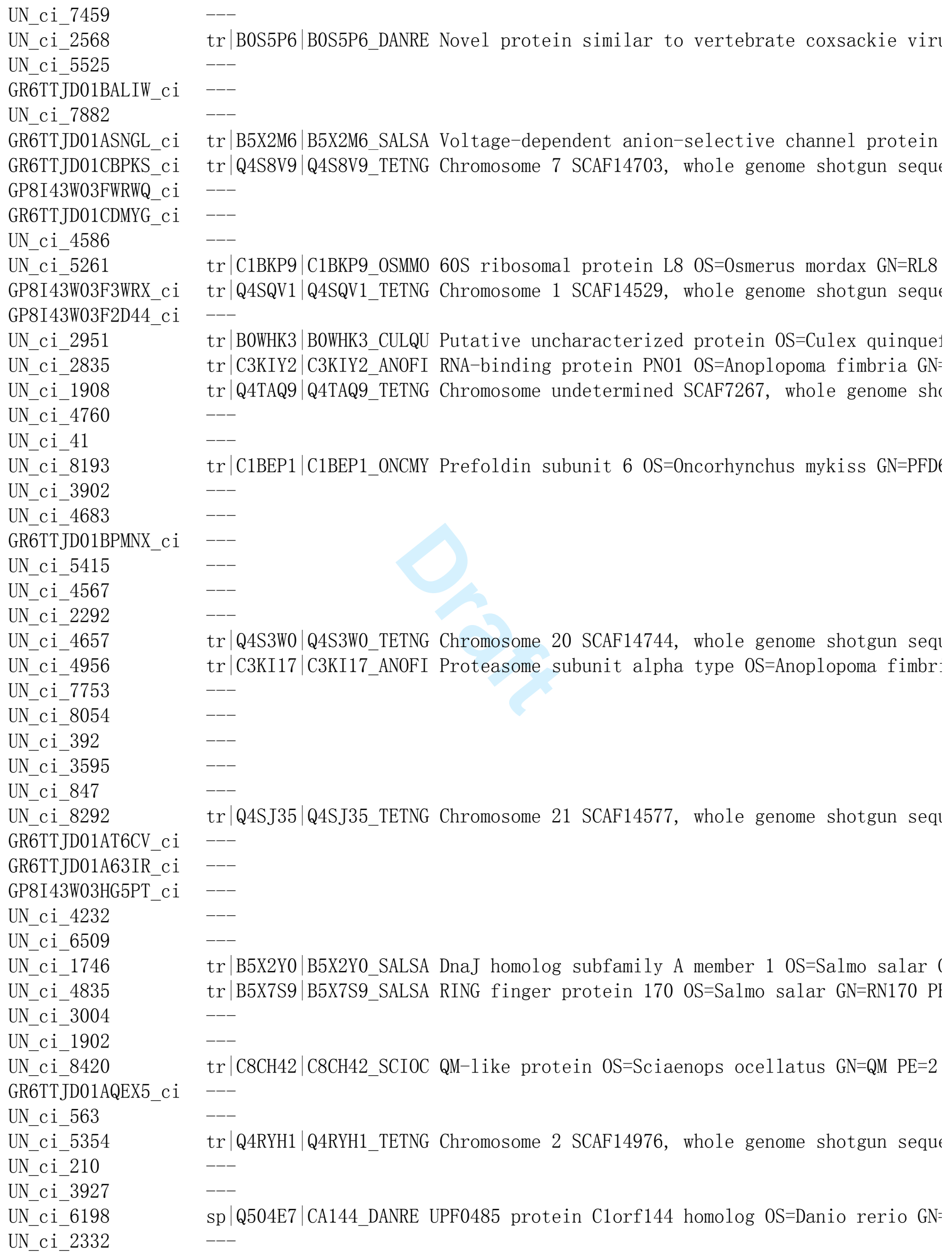

UN_ci_7459

UN_ci 2568

Ci 5525

UN_ci 7882

GR6TTJD01ASNGL ci

GR6TTJD01CBPKS c

GP8I43W03FWRWQ_ci

GR6TTJD01CDMYG_ci

UN_ci_4586

GP8I43W03F3WRX ci

GP8I43W03F2D44 c

UN_ci_2951

UN_ci_2835

UN_ci_1908

UN ci 4760

UN ci 4

UN ci 8193

UN_ci_3902

UN ci 4683

UN ci 5415

UN ci 4567

UN ci 2292

UN ci 4657

UN ci 4956

UN ci 7753

UN ci 8054

UN ci 392

UN ci 3595

UN ci 847

UN ci 8292

GR6TTJD01AT6CV C

GR6TTJD01A63IR_c

GP8I43W03HG5PT c

UN ci 4232

UN c1 6509

ci 1746

UN ci 4835

UN ci 3004

UN ci 1902

UN ci 8420

GR6TTJD01AQEX5 ci

UN ci 563

UN ci 535

UN ci 210

UN ci 6198

UN_ci_2332 $\operatorname{tr} \mid$ B0S5P6|B0S5P6 DANRE Novel protein similar to vertebrate coxsackie virı

tr B5X2M6 B5X2M6 SALSA Voltage-dependent anion-selective channel protein Q4S8V9 Q4S8V9 TETNG Chromosome 7 SCAF14703, whole genome shotgun sequt

tr $\mid$ C1BKP9|C1BKP9_0SMM0 60S ribosomal protein L8 0S=0smerus mordax GN=RL8

$\operatorname{tr} \mid$ BOWHK3 BOWHK3 CULQU Putative uncharacterized protein OS=Culex quinque $\operatorname{tr} \mid$ C3KIY2 $\mid$ C3KIY2_ANOFI RNA-binding protein PNO1 OS=Anoplopoma fimbria GN= $\operatorname{tr} \mid$ Q4TAQ9|Q4TAQ9_TETNG Chromosome undetermined SCAF7267, whole genome she

$\operatorname{tr} \mid$ C1BEP1 $\mid$ C1BEP1 0NCMY Prefoldin subunit 6 OS=0ncorhynchus mykiss GN=PFDt

tr |Q4S3W0|Q4S3W0_TETNG Chromosome 20 SCAF14744, whole genome shotgun seqr $\operatorname{tr} \mid$ C3KI17|C3KI17_ANOFI Proteasome subunit alpha type 0S=Anoplopoma fimbr

tr|Q4SJ35|Q4SJ35_TETNG Chromosome 21 SCAF14577, whole genome shotgun seq

tr |B5X2Y0|B5X2Y0_SALSA DnaJ homolog subfamily A member 1 0S=Salmo salar ( tr B5X7S9|B5X7S9_SALSA RING finger protein 170 OS=Salmo salar GN=RN170 PI

$\operatorname{tr}|\mathrm{C} 8 \mathrm{CH} 42| \mathrm{C} 8 \mathrm{CH} 42$ SCIOC QM-1ike protein OS=Sciaenops ocellatus GN=QM PE=2

$\operatorname{tr} \mid$ Q4RYH1 |Q4RYH1_TETNG Chromosome 2 SCAF14976, whole genome shotgun sequt

sp |Q504E7|CA144_DANRE UPF0485 protein C1orf144 homolog OS=Danio rerio GN= 


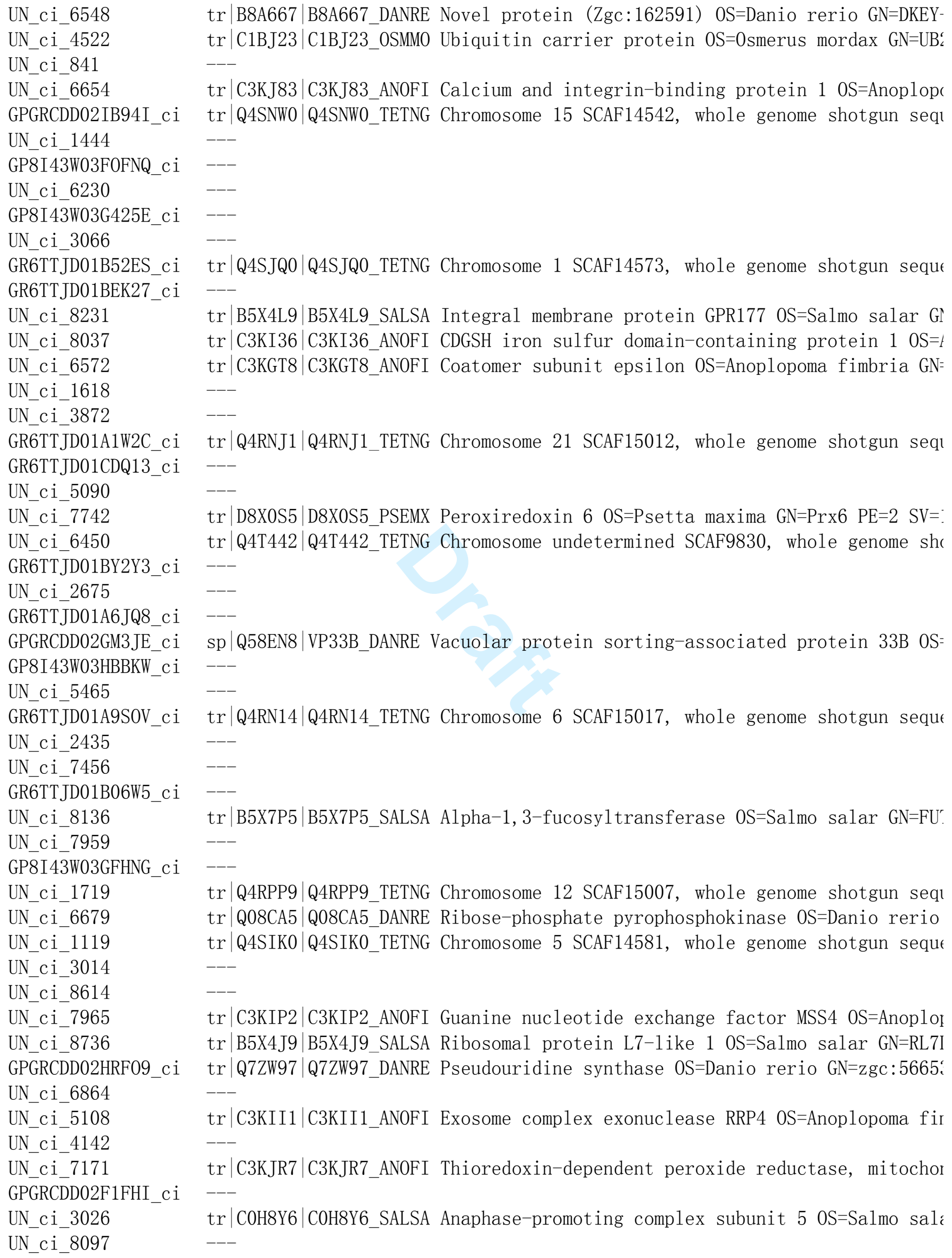

$\operatorname{tr} \mid$ B8A667|B8A667_DANRE Novel protein (Zgc:162591) OS=Danio rerio GN=DKEY$\operatorname{tr} \mid$ C1BJ23 $\mid$ C1BJ23_0SMM0 Ubiquitin carrier protein OS=0smerus mordax GN=UB؛

tr $\mid$ C3KJ83|C3KJ83_ANOFI Calcium and integrin-binding protein 1 OS=Anoplop $\operatorname{tr} \mid$ Q4SNW0|Q4SNW0_TETNG Chromosome 15 SCAF14542, whole genome shotgun seq

tr|Q4SJQ0|Q4SJQ0_TETNG Chromosome 1 SCAF14573, whole genome shotgun seque tr|B5X4L9|B5X4L9_SALSA Integral membrane protein GPR177 0S=Salmo salar G! tr|C3KI36|C3KI36_ANOFI CDGSH iron sulfur domain-containing protein 1 OS=r $\operatorname{tr} \mid$ C3KGT8 $\mid$ C3KGT8_ANOFI Coatomer subunit epsilon OS=Anoplopoma fimbria GN=

$\operatorname{tr} \mid$ Q4RNJ1|Q4RNJ1_TETNG Chromosome 21 SCAF15012, whole genome shotgun seq1 $\operatorname{tr} \mid$ D8X0S5 $\mid$ D8X0S5_PSEMX Peroxiredoxin 6 OS=Psetta maxima GN=Prx6 PE=2 SV=: $\operatorname{tr} \mid$ Q4T442|Q4T442_TETNG Chromosome undetermined SCAF9830, whole genome shi sp |Q58EN8|VP33B_DANRE Vacuolar protein sorting-associated protein 33B OS= tr $\mid$ Q4RN14|Q4RN14_TETNG Chromosome 6 SCAF15017, whole genome shotgun sequt $---$ tr $\mid$ B5X7P5|B5X7P5_SALSA Alpha-1, 3-fucosyltransferase OS=Salmo salar GN=FU'. tr |Q4RPP9|Q4RPP9_TETNG Chromosome 12 SCAF15007, whole genome shotgun seq1 tr $\mid$ Q08CA5 |Q08CA5_DANRE Ribose-phosphate pyrophosphokinase OS=Danio rerio tr $\mid$ Q4SIK0|Q4SIK0_TETNG Chromosome 5 SCAF14581, whole genome shotgun sequt

$\operatorname{tr} \mid$ C3KIP2 $\mid$ C3KIP2_ANOFI Guanine nucleotide exchange factor MSS4 0S=Anoplor $\operatorname{tr} \mid$ B5X4J9|B5X4J9_SALSA Ribosomal protein L7-1ike 1 OS=Salmo salar GN=RL7I $\operatorname{tr} \mid$ Q7ZW97|Q7ZW97_DANRE Pseudouridine synthase OS=Danio rerio GN=zgc:5665: $\operatorname{tr} \mid$ C3KII1 $\mid$ C3KII1_ANOFI Exosome complex exonuclease RRP4 OS=Anoplopoma fir tr|C3KJR7|C3KJR7_ANOFI Thioredoxin-dependent peroxide reductase, mitochor tr $\mid$ COH8Y6 $\mid$ COH8Y6_SALSA Anaphase-promoting complex subunit 5 OS=Salmo sali 


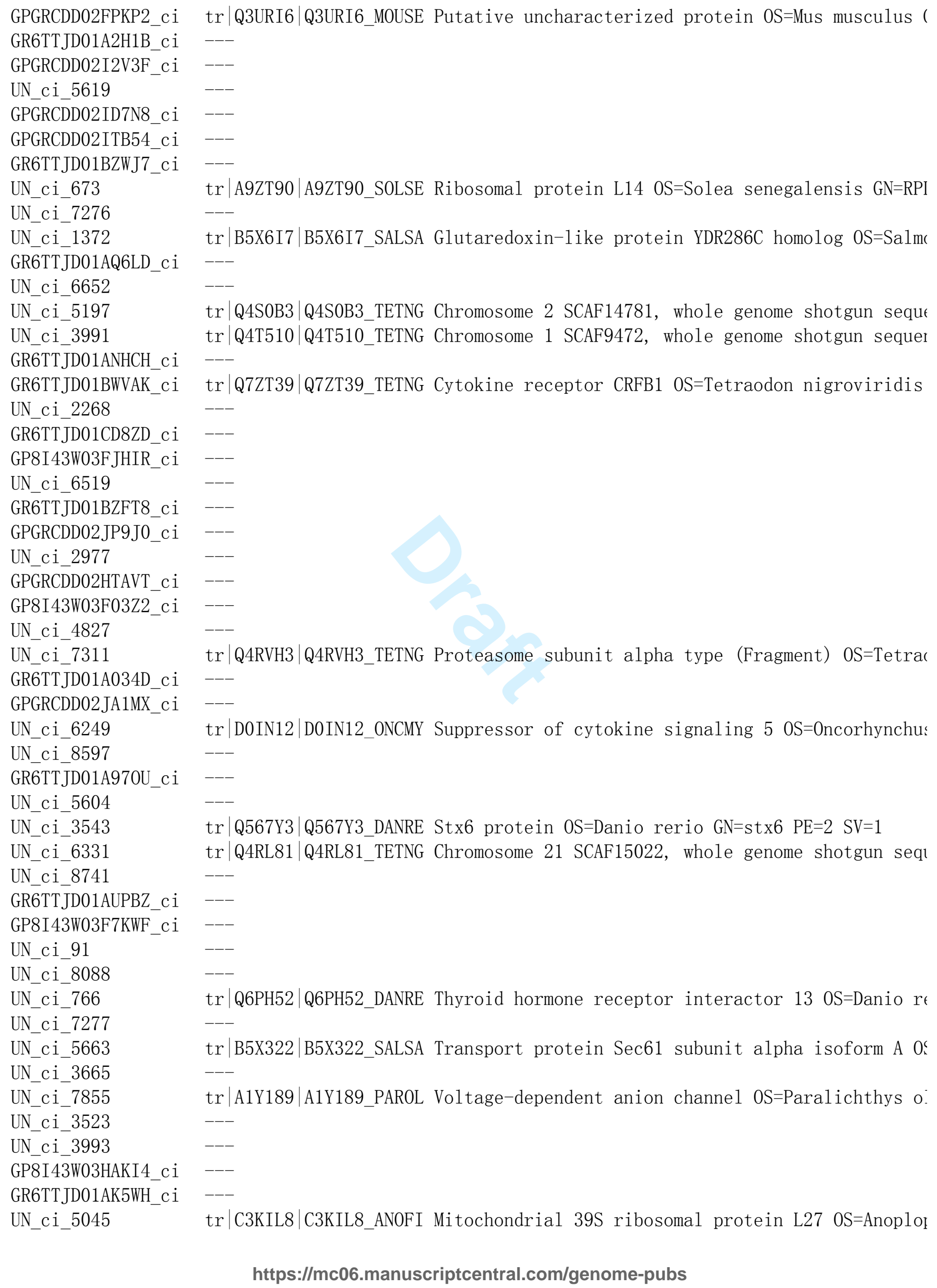




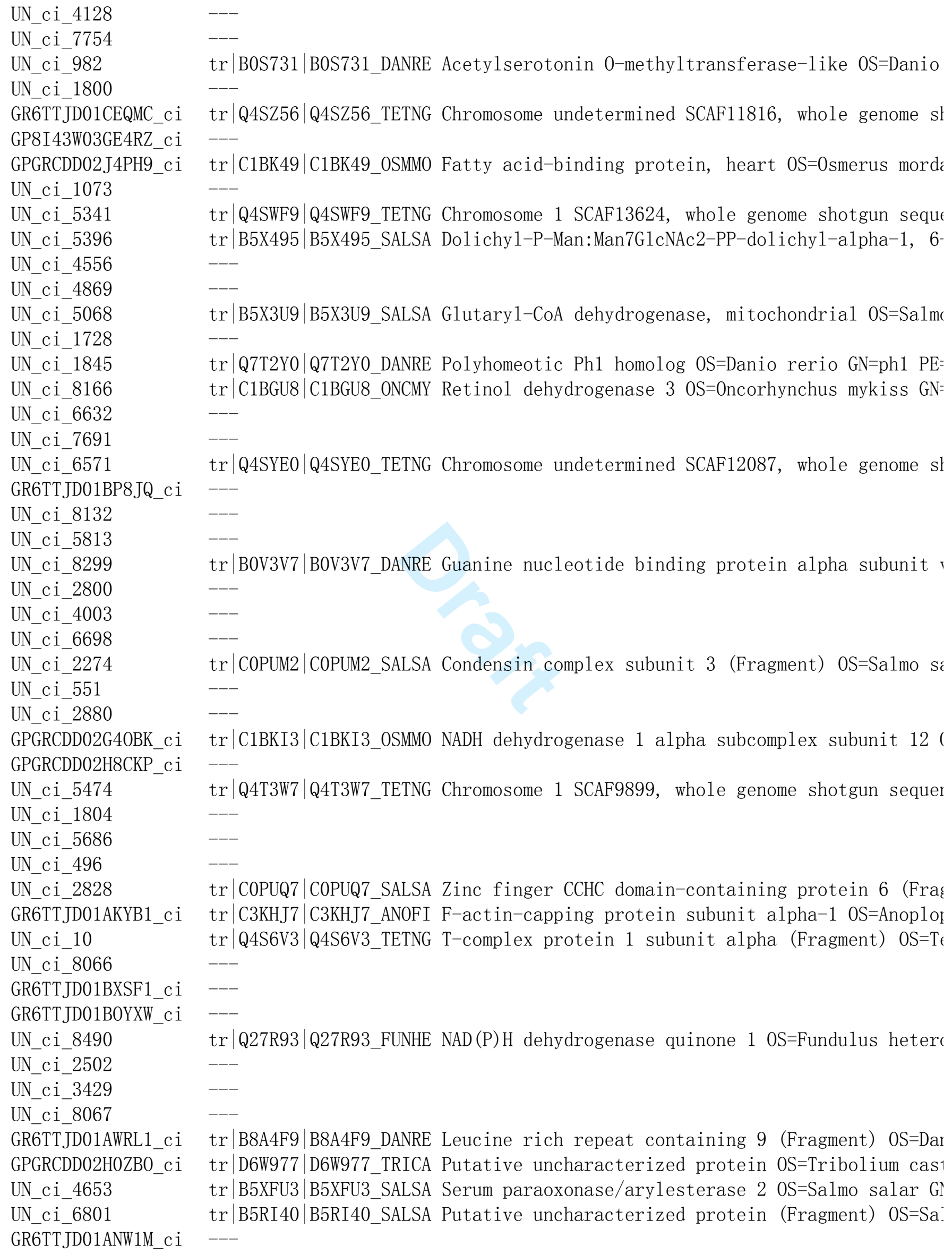

$\operatorname{tr} \mid$ B0S731|B0S731_DANRE Acetylserotonin 0-methyltransferase-1ike OS=Danio

tr $\mid$ Q4SZ56 |Q4SZ56_TETNG Chromosome undetermined SCAF11816, whole genome sl $---$

$\operatorname{tr} \mid$ C1BK49 $\mid$ C1BK49_0SMM0 Fatty acid-binding protein, heart 0S=0smerus mordi tr $\mid$ Q4SWF9 $\mid$ Q4SWF9_TETNG Chromosome 1 SCAF13624, whole genome shotgun sequt tr $\mid$ B5X495|B5X495_SALSA Dolichy1-P-Man:Man7G1cNAc2-PP-dolichy1-alpha-1, 6-

$---$

tr|B5X3U9|B5X3U9_SALSA Glutary1-CoA dehydrogenase, mitochondrial OS=Salm

$\operatorname{tr} \mid$ Q7T2Y0 $\mid$ Q7T2Y0_DANRE Polyhomeotic Ph1 homolog OS=Danio rerio GN=ph1 PE= $\operatorname{tr} \mid$ C1BGU8 $\mid$ C1BGU8_0NCMY Retinol dehydrogenase 3 OS=0ncorhynchus mykiss GN=

$\operatorname{tr} \mid$ Q4SYE0|Q4SYE0_TETNG Chromosome undetermined SCAF12087, whole genome sl $--$

$\operatorname{tr} \mid$ B0V3V7|B0V3V7_DANRE Guanine nucleotide binding protein alpha subunit

$--$

$\operatorname{tr} \mid$ COPUM2 $\mid$ COPUM2_SALSA Condensin complex subunit 3 (Fragment) OS=Salmo š́

tr|C1BKI3|C1BKI3_0SMM0 NADH dehydrogenase 1 alpha subcomplex subunit 12 ( tr $\mid$ Q4T3W7|Q4T3W7_TETNG Chromosome 1 SCAF9899, whole genome shotgun sequer $---$

tr $\mid$ COPUQ7|COPUQ7_SALSA Zinc finger CCHC domain-containing protein 6 (Fra $\operatorname{tr} \mid$ C3KHJ7 $\mid$ C3KHJ7_ANOFI F-actin-capping protein subunit alpha-1 0S=Anoplor $\operatorname{tr} \mid$ Q4S6V3 $\mid$ Q4S6V3_TETNG T-complex protein 1 subunit alpha (Fragment) OS=Tt

$\operatorname{tr} \mid$ Q27R93|Q27R93_FUNHE NAD (P)H dehydrogenase quinone 1 OS=Fundulus heter

$\operatorname{tr} \mid$ B8A4F9 $\mid$ B8A4F9_DANRE Leucine rich repeat containing 9 (Fragment) OS=Dar tr $\mid$ D6W977|D6W977_TRICA Putative uncharacterized protein 0S=Tribolium cas1 tr|B5XFU3|B5XFU3_SALSA Serum paraoxonase/arylesterase 2 OS=Salmo salar G! $\operatorname{tr} \mid$ B5RI40|B5RI40_SALSA Putative uncharacterized protein (Fragment) OS=Sa: 


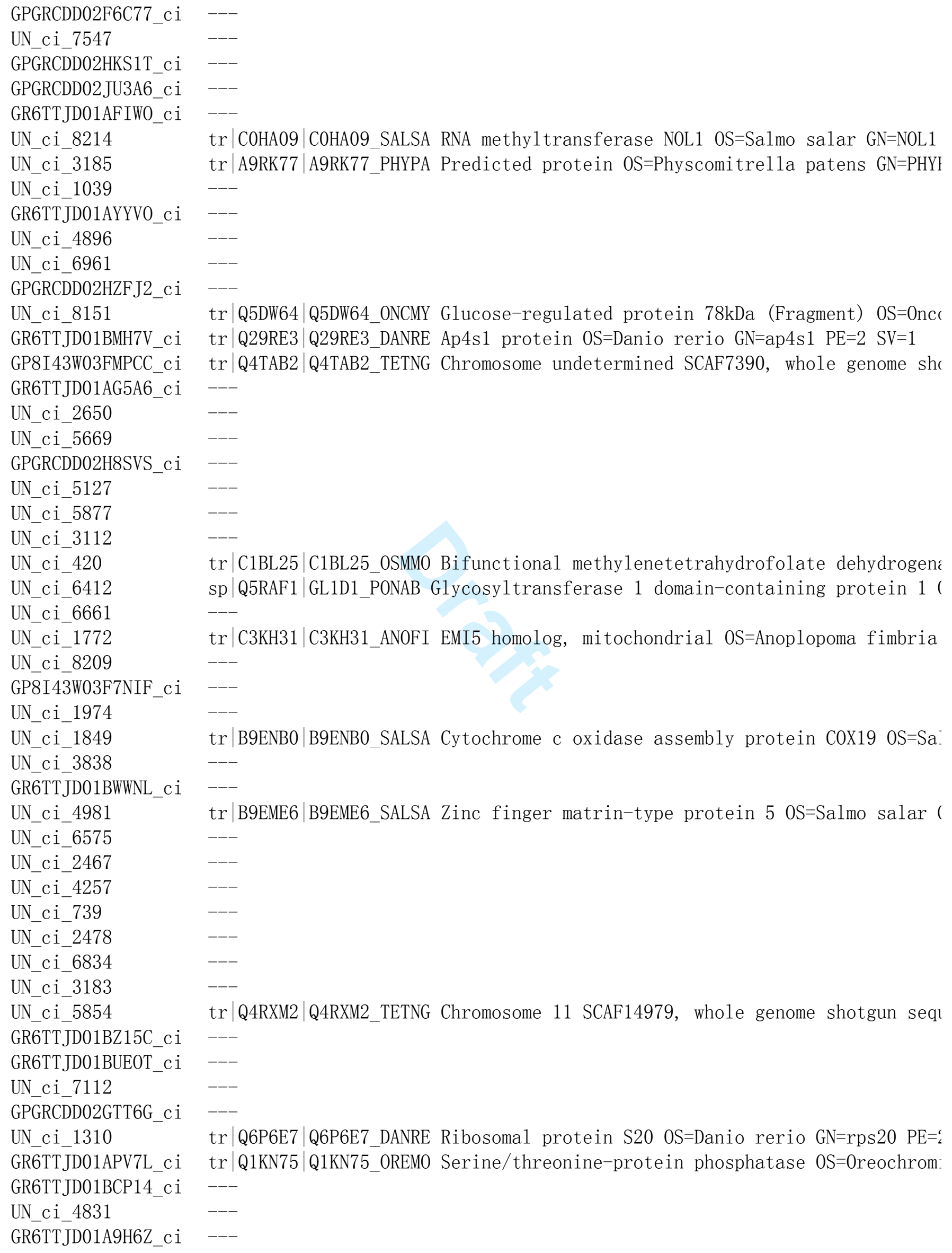




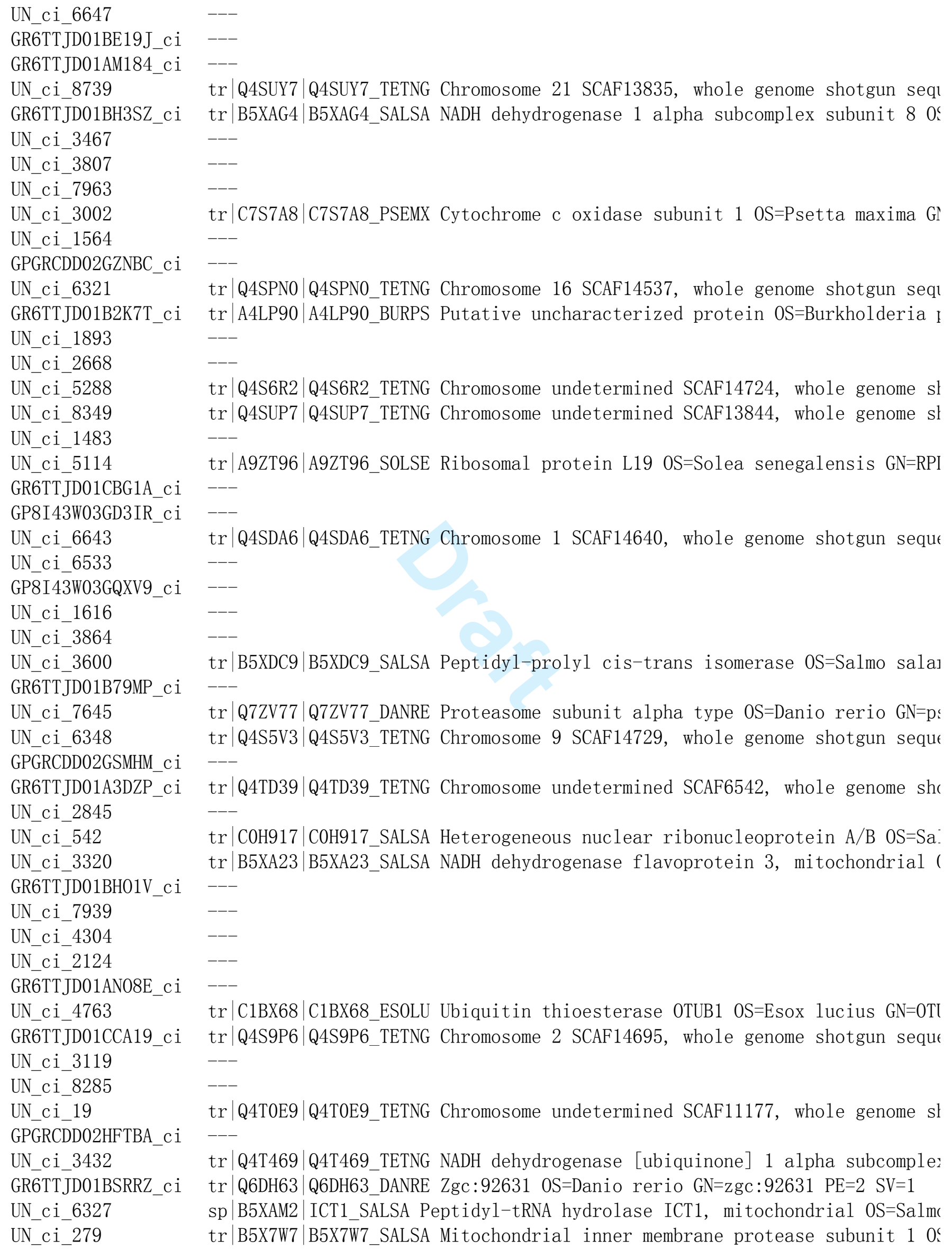




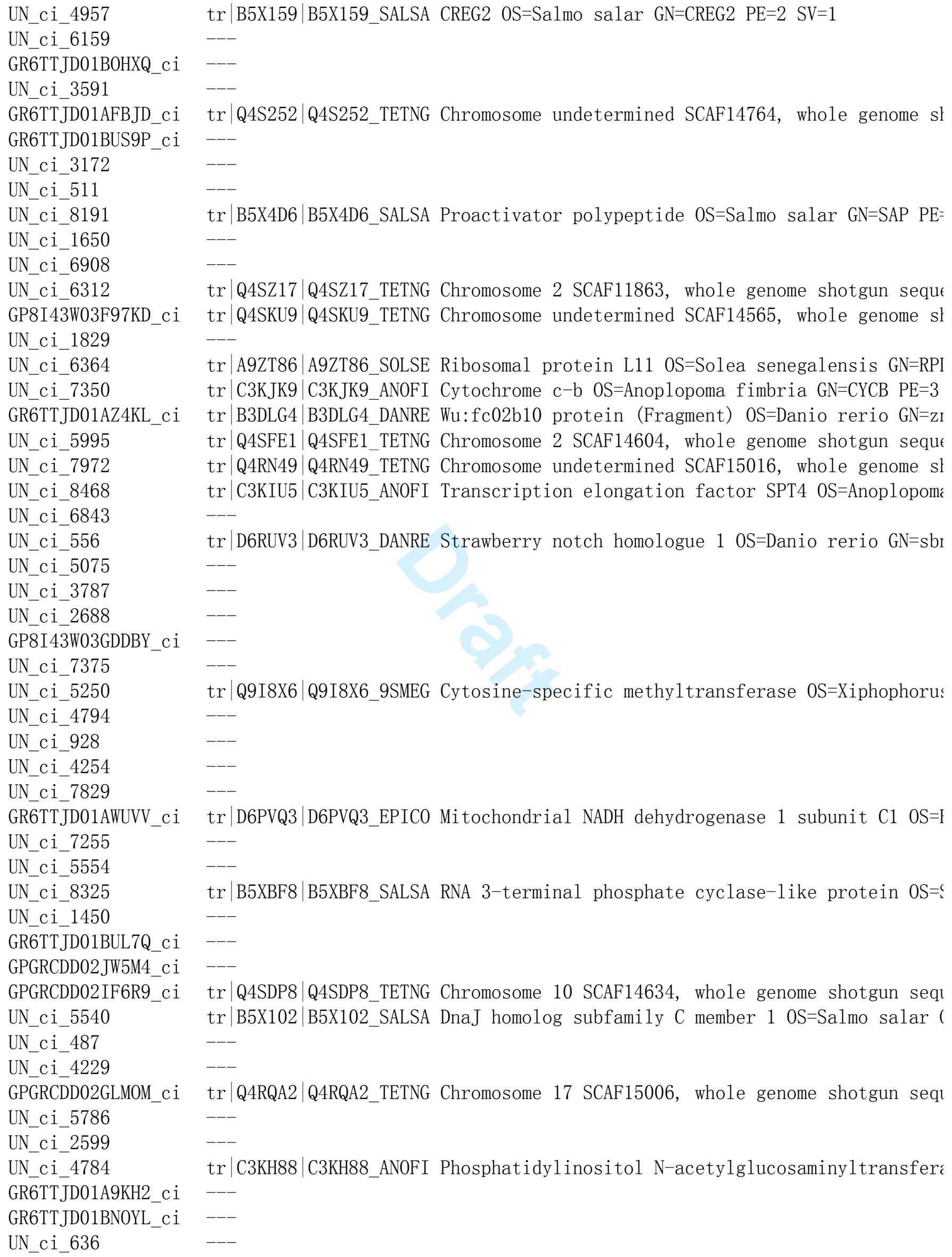




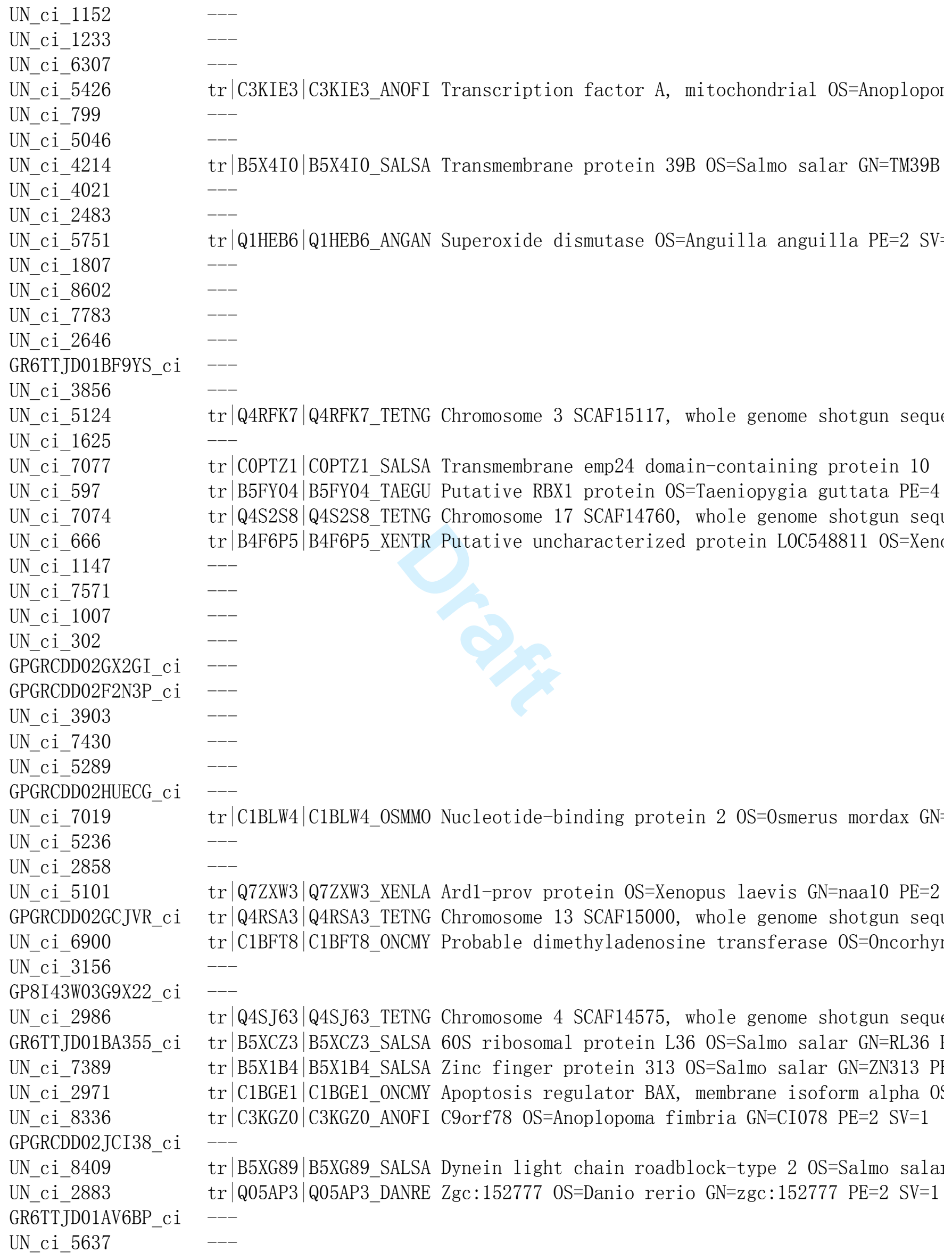




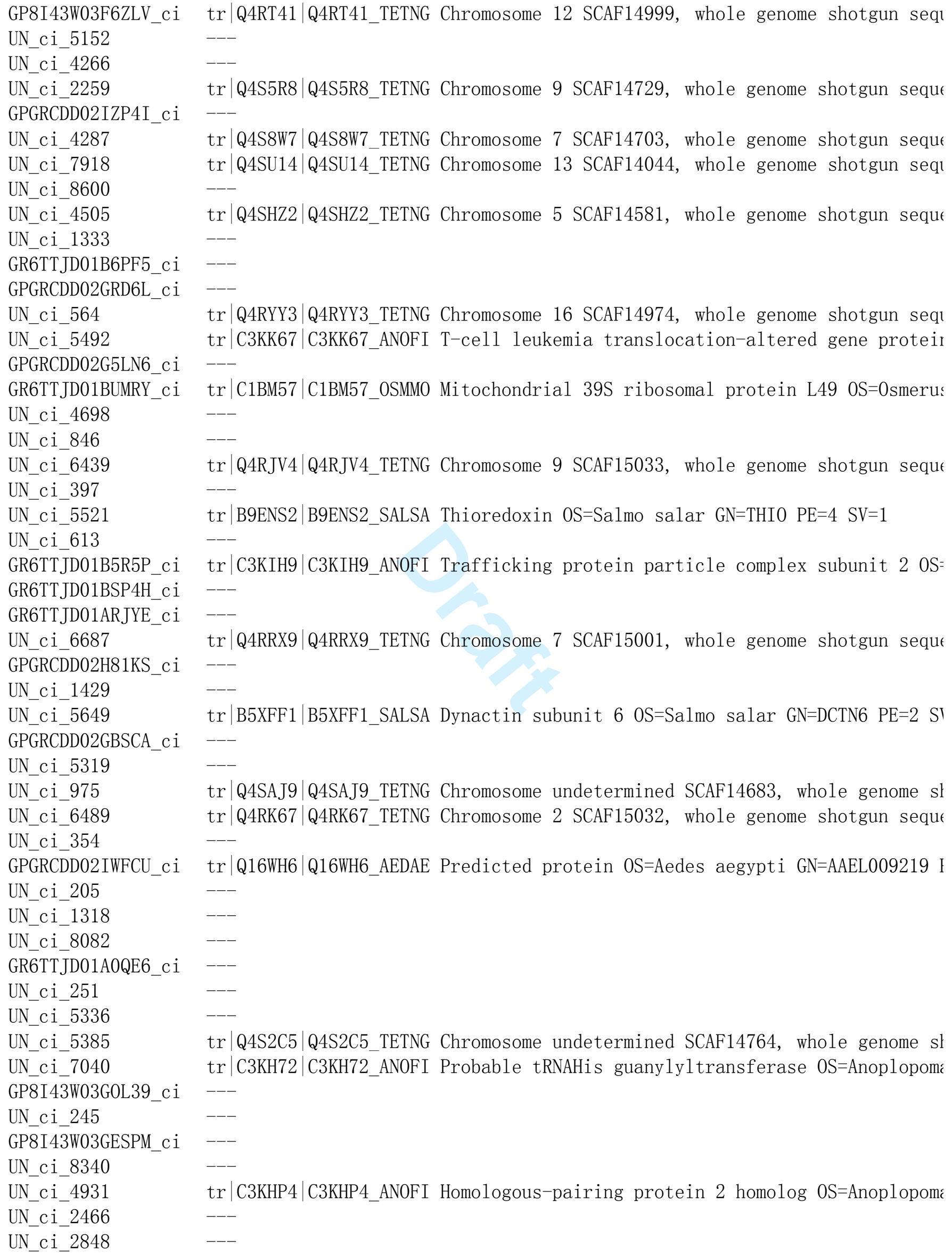

tr $\mid$ Q4RYY3|Q4RYY3_TETNG Chromosome 16 SCAF14974, whole genome shotgun seq1 tr|C3KK67|C3KK67_ANOFI T-cel1 leukemia translocation-altered gene proteir tr|C1BM57|C1BM57_0SMM0 Mitochondrial 39S ribosomal protein L49 0S=0smeru؛

$---$

tr|Q4RJV4|Q4RJV4_TETNG Chromosome 9 SCAF15033, whole genome shotgun sequt $\operatorname{tr} \mid$ B9ENS2 $\mid$ B9ENS2_SALSA Thioredoxin OS=Salmo salar GN=THI0 PE=4 SV=1 tr|C3KIH9|C3KIH9_ANOFI Trafficking protein particle complex subunit 2 OS= tr $\mid$ Q4RRX9|Q4RRX9_TETNG Chromosome 7 SCAF15001, whole genome shotgun sequt $\operatorname{tr} \mid$ B5XFF1|B5XFF1_SALSA Dynactin subunit 6 OS=Salmo salar GN=DCTN6 PE=2 SI $\operatorname{tr}$ |Q4SAJ9 |Q4SAJ9_TETNG Chromosome undetermined SCAF14683, whole genome sl tr $\mid$ Q4RK67|Q4RK67_TETNG Chromosome 2 SCAF15032, whole genome shotgun sequt tr $\mid$ Q16WH6 $\mid$ Q16WH6_AEDAE Predicted protein OS=Aedes aegypti GN=AAEL009219 I

tr $\mid$ Q4S2C5 $\mid$ Q4S2C5_TETNG Chromosome undetermined SCAF14764, whole genome sl tr $\mid$ C3KH72 $\mid$ C3KH72_ANOFI Probable tRNAHis guanylyltransferase OS=Anoplopomi $\operatorname{tr} \mid$ C3KHP4 $\mid$ C3KHP4_ANOFI Homologous-pairing protein 2 homolog OS=Anoplopomc 


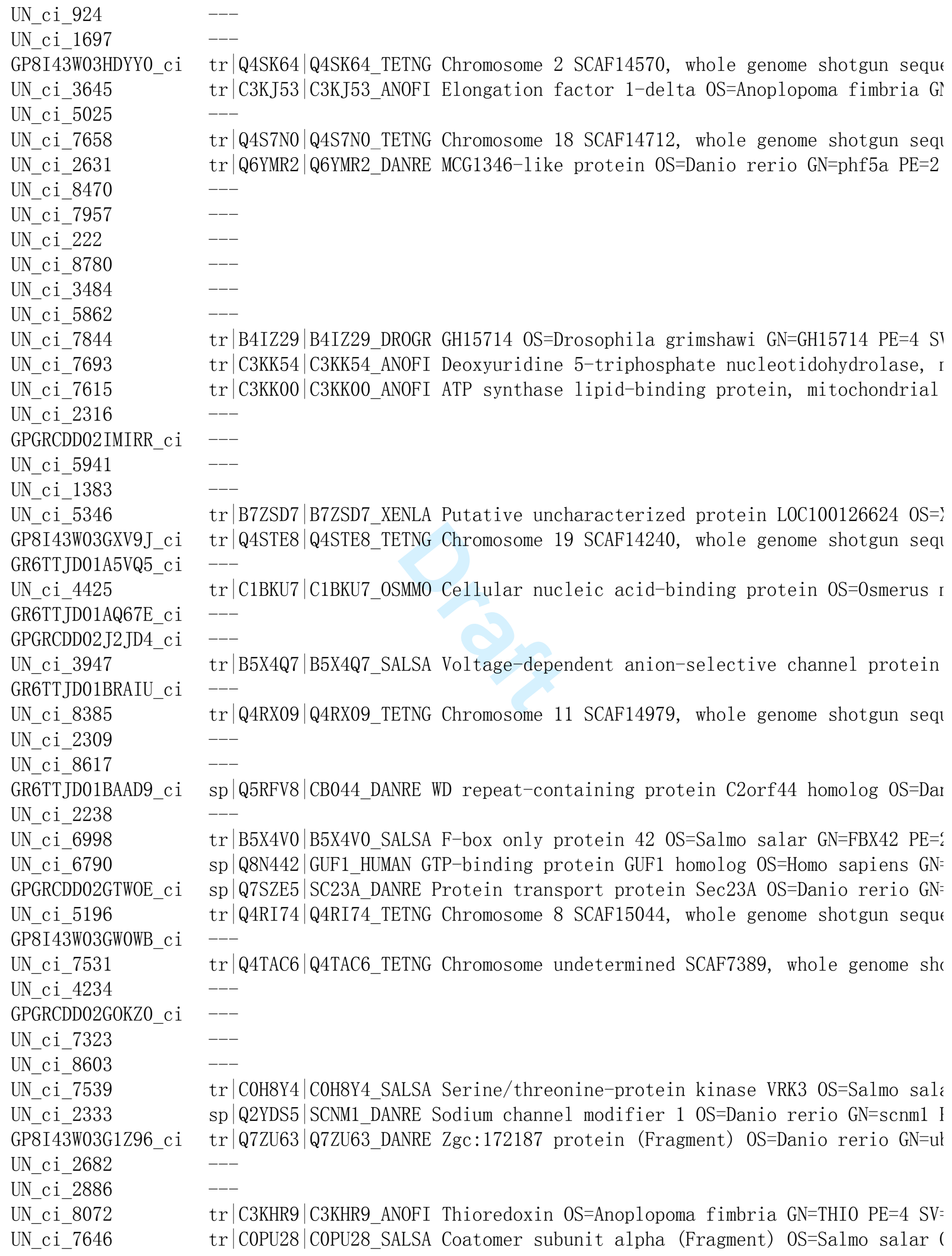

tr $\mid$ Q4SK64|Q4SK64_TETNG Chromosome 2 SCAF14570, whole genome shotgun sequt $\operatorname{tr} \mid$ C3KJ53 $\mid$ C3KJ53_ANOFI Elongation factor 1-delta OS=Anoplopoma fimbria G! tr $\mid$ Q4S7N0 |Q4S7N0_TETNG Chromosome 18 SCAF14712, whole genome shotgun seq1 tr $\mid$ Q6YMR2 $\mid$ Q6YMR2_DANRE MCG1346-1ike protein OS=Danio rerio GN=phf5a PE=2

tr|B4IZ29|B4IZ29_DR0GR GH15714 OS=Drosophila grimshawi GN=GH15714 PE=4 SI tr|C3KK54|C3KK54_ANOFI Deoxyuridine 5-triphosphate nucleotidohydrolase, I tr $\mid$ C3KK00|C3KK00_ANOFI ATP synthase lipid-binding protein, mitochondrial

tr $\mid$ B7ZSD7|B7ZSD7_XENLA Putative uncharacterized protein LOC100126624 OS=: tr|Q4STE8|Q4STE8_TETNG Chromosome 19 SCAF14240, whole genome shotgun seqi $\operatorname{tr} \mid$ C1BKU7 $\mid$ C1BKU7_0SMM0 Cellular nucleic acid-binding protein 0S=0smerus r $\operatorname{tr} \mid$ B5X4Q7|B5X4Q7_SALSA Voltage-dependent anion-selective channel protein tr $\mid$ Q4RX09|Q4RX09_TETNG Chromosome 11 SCAF14979, whole genome shotgun seq1 $---$

sp $\mid$ Q5RFV8|CB044_DANRE WD repeat-containing protein C2orf44 homolog OS=Dar $\operatorname{tr} \mid$ B5X4V0 $\mid$ B5X4V0_SALSA F-box only protein 42 OS=Salmo salar GN=FBX42 PE=‘ sp $\mid$ Q8N442|GUF1_HUMAN GTP-binding protein GUF1 homolog OS=Homo sapiens GN= sp|Q7SZE5|SC23A_DANRE Protein transport protein Sec23A OS=Danio rerio GN= tr|Q4RI74|Q4RI74_TETNG Chromosome 8 SCAF15044, whole genome shotgun sequt tr |Q4TAC6|Q4TAC6_TETNG Chromosome undetermined SCAF7389, whole genome shi $---$

$\operatorname{tr} \mid$ COH8Y4 $\mid$ COH8Y4_SALSA Serine/threonine-protein kinase VRK3 OS=Salmo sali sp |Q2YDS5|SCNM1_DANRE Sodium channe1 modifier 1 OS=Danio rerio GN=scnm1 I tr $\mid$ Q7ZU63|Q7ZU63_DANRE Zgc:172187 protein (Fragment) OS=Danio rerio GN=ul $---$

$\operatorname{tr} \mid$ C3KHR9 $\mid$ C3KHR9_ANOFI Thioredoxin OS=Anoplopoma fimbria GN=THI0 PE=4 SV= tr $\mid$ COPU28 $\mid$ COPU28_SALSA Coatomer subunit alpha (Fragment) 0S=Salmo salar ( 


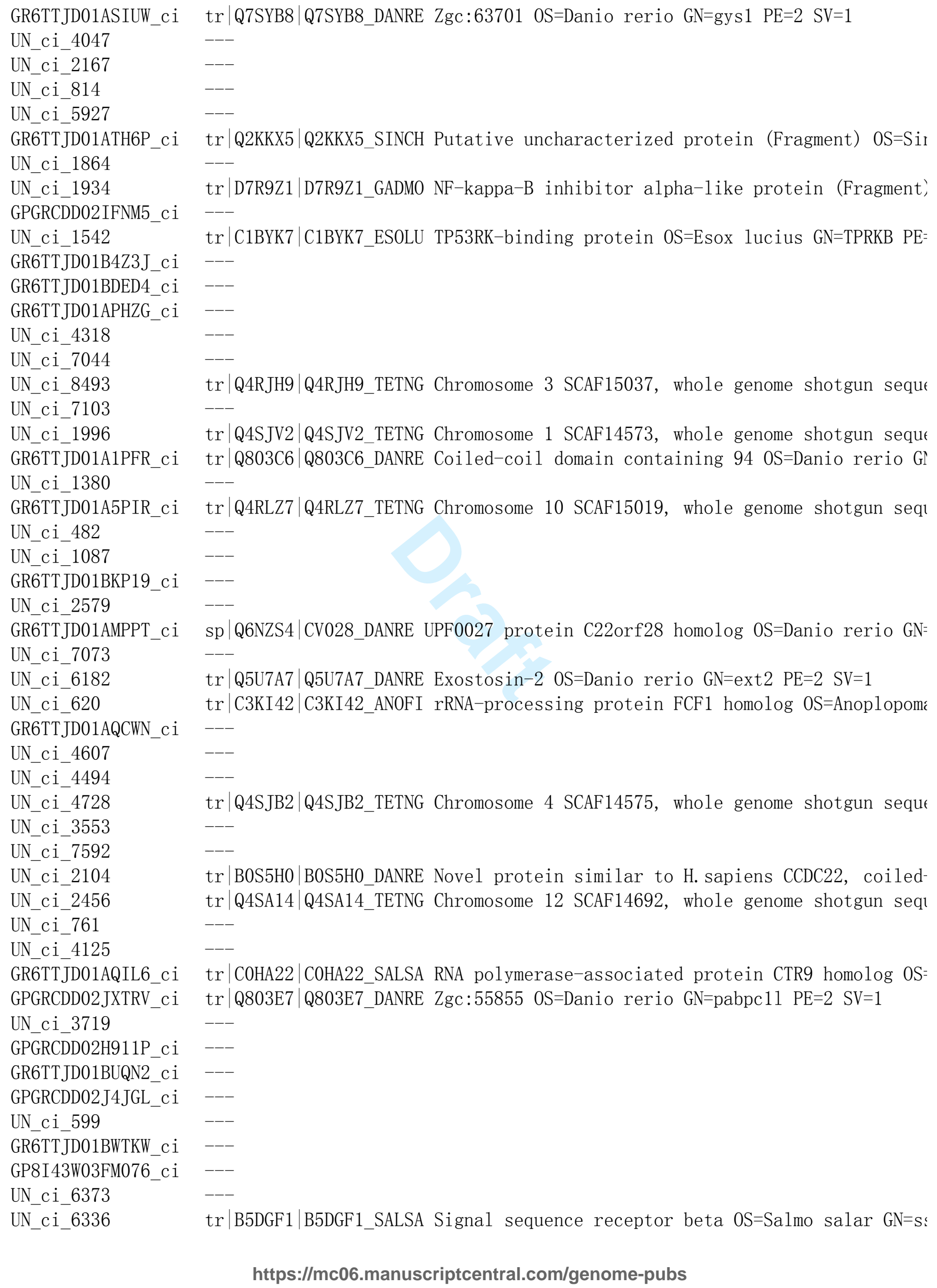




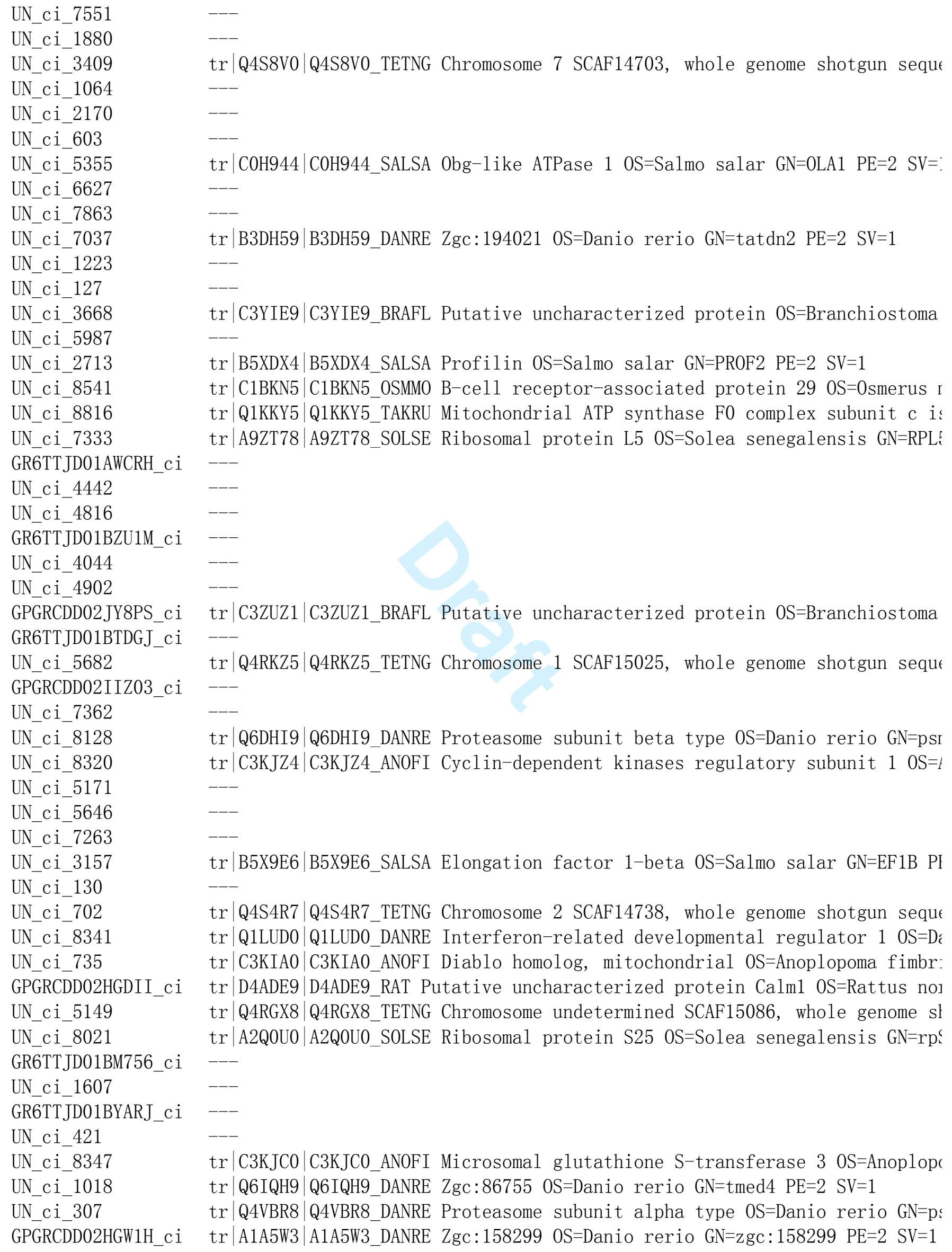




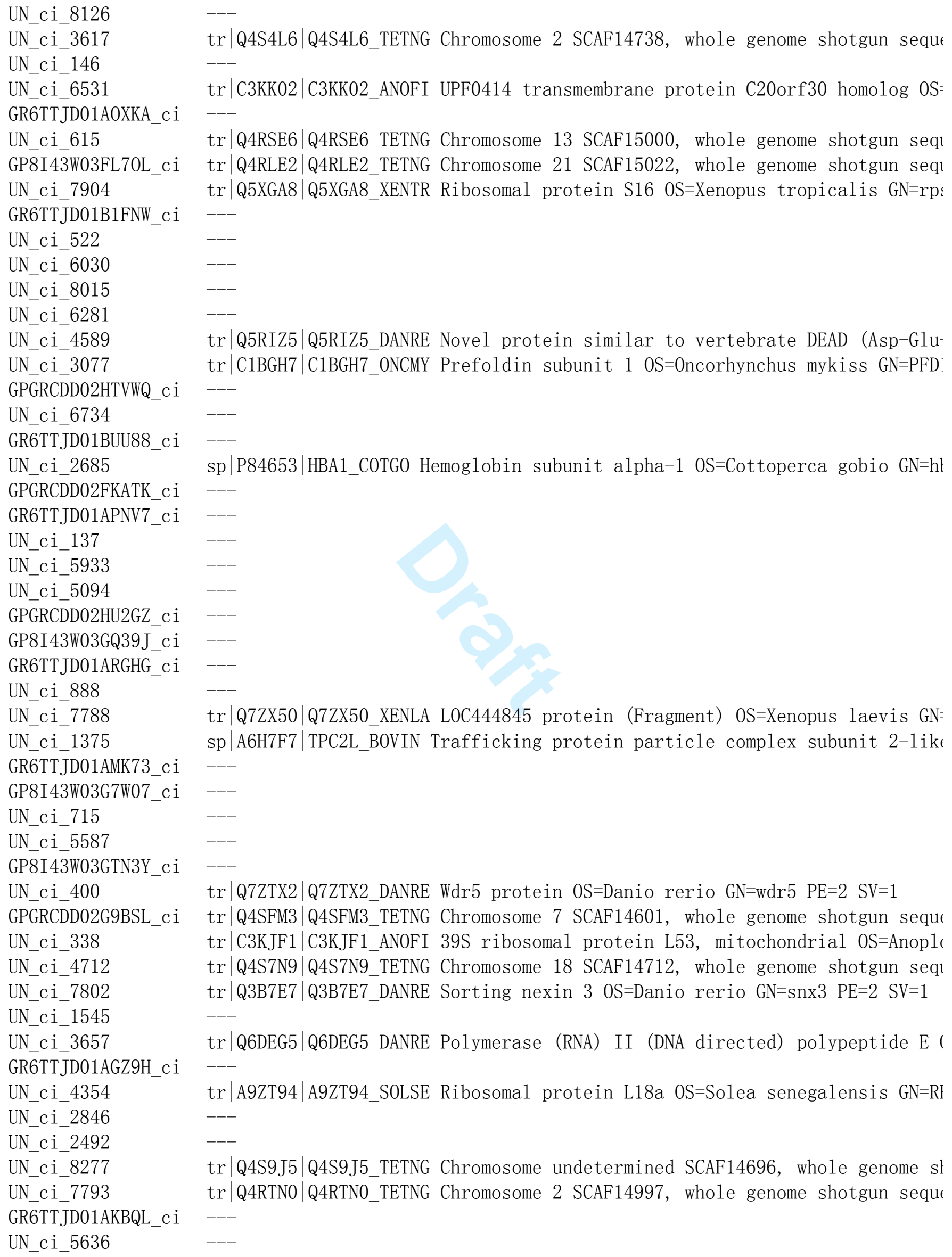

tr $\mid$ Q4S4L6 |Q4S4L6_TETNG Chromosome 2 SCAF14738, whole genome shotgun sequt $\operatorname{tr} \mid$ C3KK02|C3KK02_ANOFI UPF0414 transmembrane protein C20orf30 homolog OS= tr $\mid$ Q4RSE6 |Q4RSE6_TETNG Chromosome 13 SCAF15000, whole genome shotgun sequ tr|Q4RLE2|Q4RLE2_TETNG Chromosome 21 SCAF15022, whole genome shotgun seqr $\operatorname{tr} \mid$ Q5XGA8 $\mid$ Q5XGA8_XENTR Ribosomal protein S16 OS=Xenopus tropicalis GN=rps

$\operatorname{tr} \mid$ Q5RIZ5 |Q5RIZ5_DANRE Novel protein similar to vertebrate DEAD (Asp-Glu$\operatorname{tr} \mid$ C1BGH7 $\mid$ C1BGH7_ONCMY Prefoldin subunit 1 OS=0ncorhynchus mykiss GN=PFD:

sp $\mid$ P84653|HBA1_COTG0 Hemoglobin subunit alpha-1 OS=Cottoperca gobio GN=hl

$\operatorname{tr} \mid$ Q7ZX50|Q7ZX50_XENLA LOC444845 protein (Fragment) OS=Xenopus laevis GN= sp|A6H7F7|TPC2L_B0VIN Trafficking protein particle complex subunit 2-likt

$\operatorname{tr} \mid$ Q7ZTX2 $\mid$ Q7ZTX2_DANRE Wdr5 protein OS=Danio rerio GN=wdr5 PE=2 SV=1 tr $\mid$ Q4SFM3 $\mid$ Q4SFM3_TETNG Chromosome 7 SCAF14601, whole genome shotgun sequt $\operatorname{tr} \mid$ C3KJF1|C3KJF1_ANOFI 39S ribosomal protein L53, mitochondrial OS=Anoplc tr $\mid$ Q4S7N9|Q4S7N9_TETNG Chromosome 18 SCAF14712, whole genome shotgun seqr $\operatorname{tr} \mid$ Q3B7E7 $\mid$ Q3B7E7_DANRE Sorting nexin 3 OS=Danio rerio GN=snx3 PE=2 SV=1

tr $\mid$ Q6DEG5 $\mid$ Q6DEG5_DANRE Polymerase (RNA) II (DNA directed) polypeptide E ( tr $\mid$ A9ZT94|A9ZT94_SOLSE Ribosomal protein L18a OS=Solea senegalensis GN=RI

tr $\mid$ Q4S9J5|Q4S9J5_TETNG Chromosome undetermined SCAF14696, whole genome sl tr $\mid$ Q4RTN0|Q4RTN0_TETNG Chromosome 2 SCAF14997, whole genome shotgun seque 


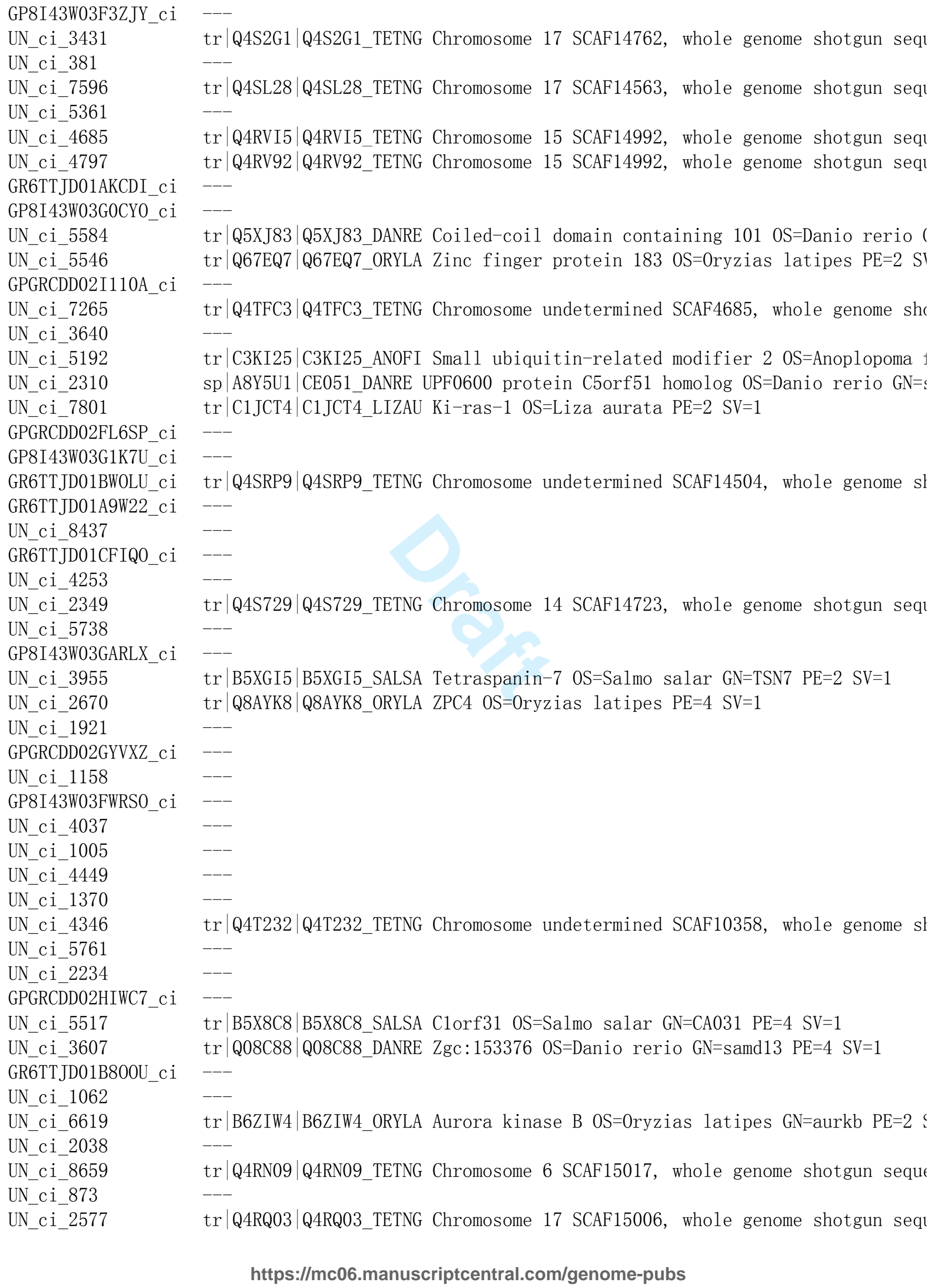

tr |Q4T232|Q4T232_TETNG Chromosome undetermined SCAF10358, whole genome st

tr $\mid$ B5X8C8 $\mid$ B5X8C8_SALSA C1orf31 OS=Salmo salar GN=CA031 PE=4 SV=1 $\operatorname{tr} \mid$ Q08C88 $\mid$ Q08C88_DANRE Zgc:153376 OS=Danio rerio GN=samd13 PE=4 SV=1

tr $\mid$ B6ZIW4 $\mid$ B6ZIW4_0RYLA Aurora kinase B OS=0ryzias latipes GN=aurkb PE=2 ؛ $---$

tr|Q4RN09|Q4RN09_TETNG Chromosome 6 SCAF15017, whole genome shotgun sequt tr $\mid$ Q4RQ03 $\mid$ Q4RQ03_TETNG Chromosome 17 SCAF15006, whole genome shotgun sequ 


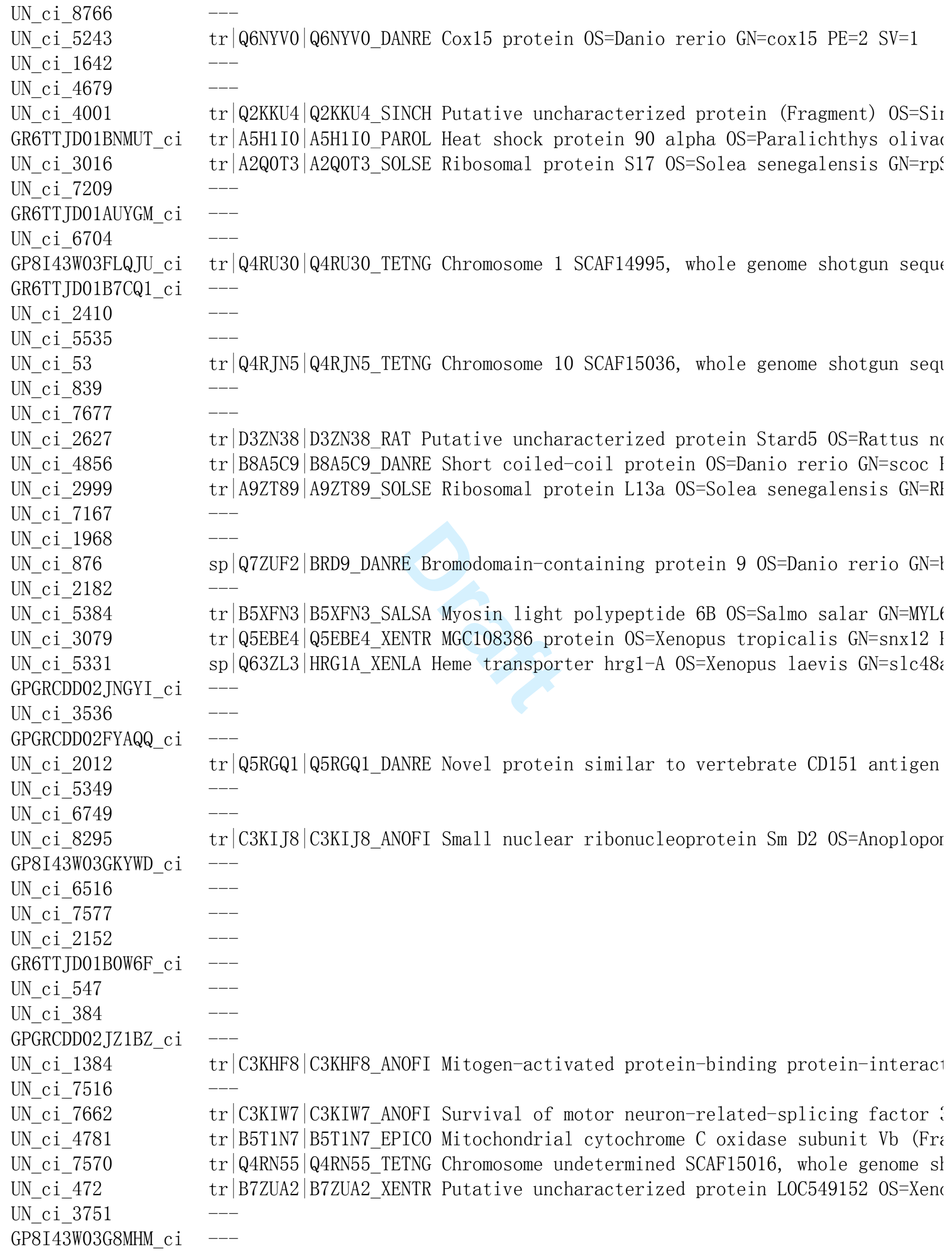

$\operatorname{tr} \mid$ Q6NYV0 $\mid$ Q6NYV0_DANRE Cox15 protein OS=Danio rerio GN=cox15 PE=2 SV=1

tr $\mid$ Q2KKU4 $\mid$ Q2KKU4_SINCH Putative uncharacterized protein (Fragment) OS=Sir tr|A5H1I0|A5H1I0_PAR0L Heat shock protein 90 alpha OS=Paralichthys olivar $\operatorname{tr} \mid$ A2Q0T3 $\mid$ A2Q0T3_SOLSE Ribosomal protein S17 OS=Solea senegalensis GN=rp؛

tr $\mid$ Q4RU30|Q4RU30_TETNG Chromosome 1 SCAF14995, whole genome shotgun sequt $---$

tr $\mid$ Q4RJN5|Q4RJN5_TETNG Chromosome 10 SCAF15036, whole genome shotgun seqi

tr|D3ZN38|D3ZN38_RAT Putative uncharacterized protein Stard5 0S=Rattus nc $\operatorname{tr} \mid$ B8A5C9|B8A5C9_DANRE Short coiled-coil protein OS=Danio rerio GN=scoc I $\operatorname{tr} \mid$ A9ZT89|A9ZT89_SOLSE Ribosomal protein L13a OS=Solea senegalensis GN=RI $---$

sp |Q7ZUF2 |BRD9_DANRE Bromodomain-containing protein 9 OS=Danio rerio GN=l tr $\mid$ B5XFN3 $\mid$ B5XFN3_SALSA Myosin light polypeptide 6B OS=Salmo salar GN=MYLt $\operatorname{tr} \mid$ Q5EBE4 $\mid$ Q5EBE4_XENTR MGC108386 protein 0S=Xenopus tropicalis GN=snx12 I sp $\mid$ Q63ZL3|HRG1A_XENLA Heme transporter hrg1-A OS=Xenopus laevis GN=slc48i

$\operatorname{tr} \mid$ Q5RGQ1|Q5RGQ1_DANRE Nove1 protein similar to vertebrate CD151 antigen

$\operatorname{tr} \mid$ C3KIJ8|C3KIJ8_ANOFI Smal1 nuclear ribonucleoprotein Sm D2 0S=Anoplopor $---$

tr $\mid$ C3KHF8|C3KHF8_ANOFI Mitogen-activated protein-binding protein-interac1 tr $\mid$ C3KIW7 |C3KIW7_ANOFI Survival of motor neuron-related-splicing factor : tr|B5T1N7|B5T1N7_EPIC0 Mitochondrial cytochrome C oxidase subunit Vb (Frí tr|Q4RN55|Q4RN55_TETNG Chromosome undetermined SCAF15016, whole genome sl $\operatorname{tr} \mid$ B7ZUA2|B7ZUA2_XENTR Putative uncharacterized protein L0C549152 OS=Xenc 


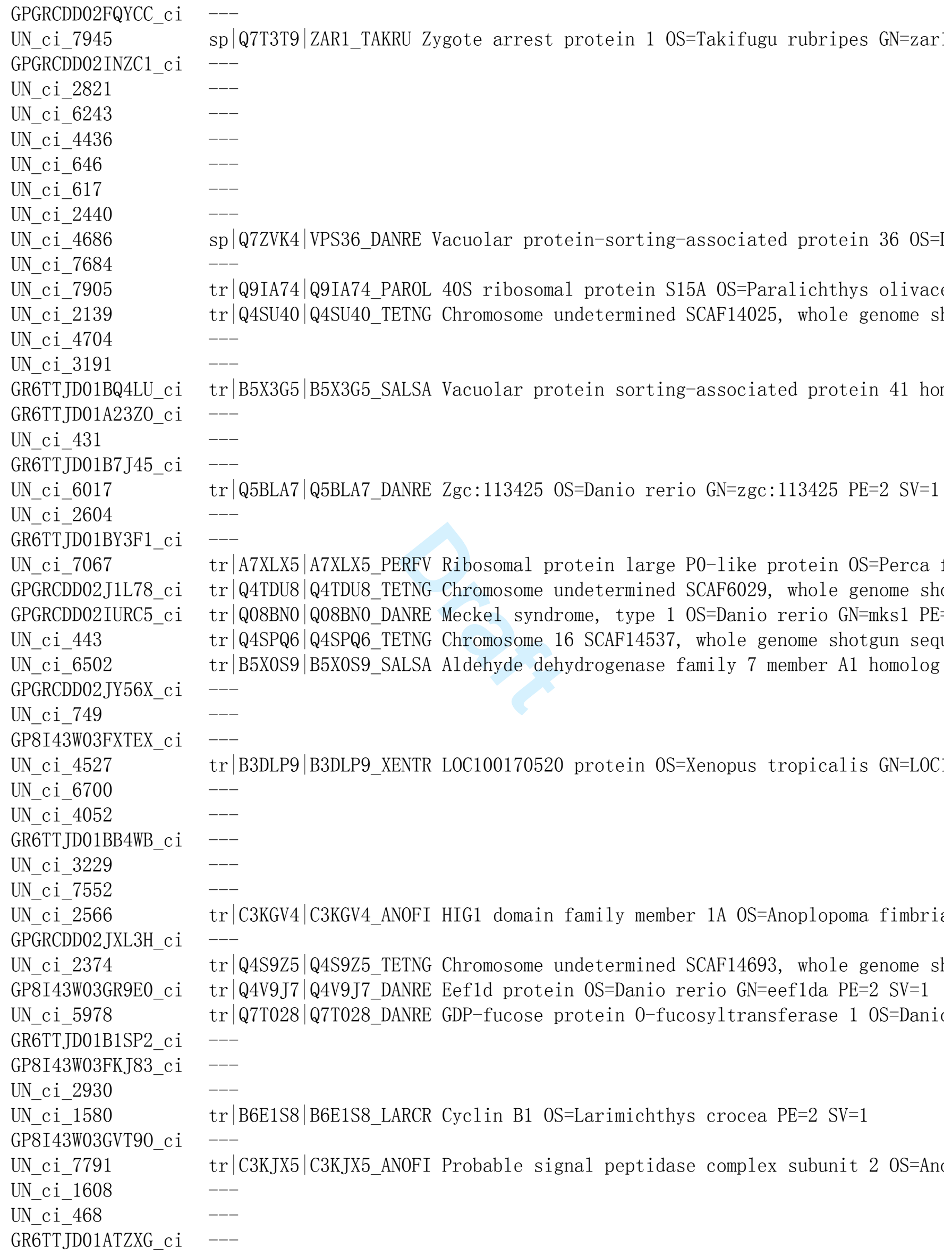




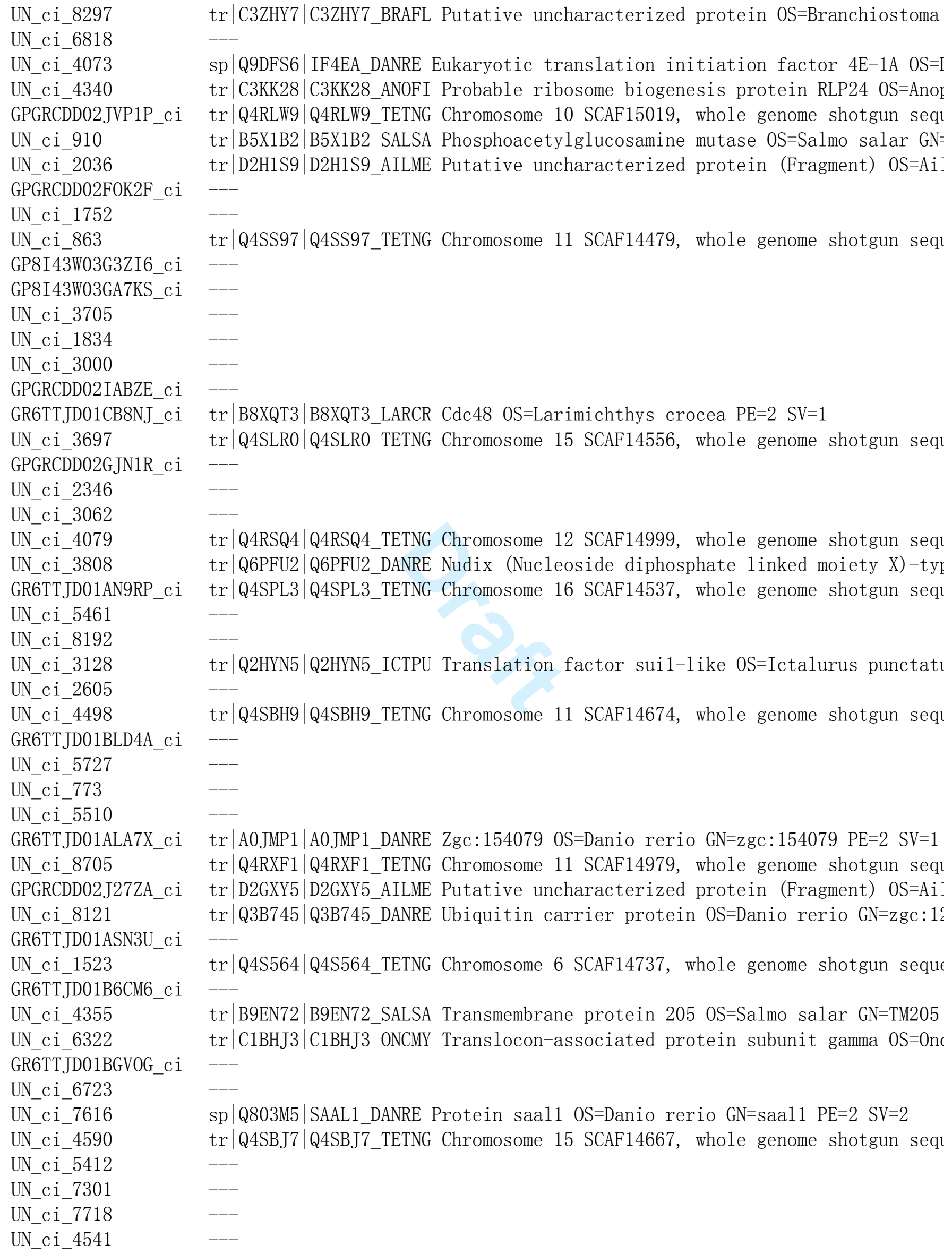

tr $\mid$ C3ZHY7 $\mid$ C3ZHY7_BRAFL Putative uncharacterized protein 0S=Branchiostoma

sp |Q9DFS6 |IF4EA_DANRE Eukaryotic translation initiation factor 4E-1A OS=I tr $\mid$ C3KK28|C3KK28_ANOFI Probable ribosome biogenesis protein RLP24 OS=Anor tr $\mid$ Q4RLW9|Q4RLW9_TETNG Chromosome 10 SCAF15019, whole genome shotgun seq1 $\operatorname{tr} \mid$ B5X1B2 |B5X1B2_SALSA Phosphoacetylglucosamine mutase OS=Salmo salar GN= $\operatorname{tr} \mid$ D2H1S9|D2H1S9_AILME Putative uncharacterized protein (Fragment) OS=Ai:

tr $\mid$ Q4SS97|Q4SS97_TETNG Chromosome 11 SCAF14479, whole genome shotgun sequ $\operatorname{tr} \mid$ B8XQT3 $\mid$ B8XQT3_LARCR Cdc48 OS=Larimichthys crocea PE=2 SV=1 tr $\mid$ Q4SLR0 $\mid$ Q4SLR0_TETNG Chromosome 15 SCAF14556, whole genome shotgun seq

tr |Q4RSQ4|Q4RSQ4_TETNG Chromosome 12 SCAF14999, whole genome shotgun seq1 tr $\mid$ Q6PFU2 $\mid$ Q6PFU2_DANRE Nudix (Nucleoside diphosphate linked moiety X)-tyr tr $\mid$ Q4SPL3|Q4SPL3_TETNG Chromosome 16 SCAF14537, whole genome shotgun seq1

$\operatorname{tr} \mid$ Q2HYN5 |Q2HYN5_ICTPU Translation factor sui1-1ike OS=Ictalurus punctatı tr $\mid$ Q4SBH9 |Q4SBH9_TETNG Chromosome 11 SCAF14674, whole genome shotgun seq1 $\operatorname{tr} \mid$ A0JMP1 $\mid$ A0JMP1_DANRE Zgc:154079 OS=Danio rerio GN=zgc:154079 PE=2 SV=1 $\operatorname{tr} \mid$ Q4RXF1|Q4RXF1_TETNG Chromosome 11 SCAF14979, whole genome shotgun seqr tr $\mid$ D2GXY5|D2GXY5_AILME Putative uncharacterized protein (Fragment) OS=Ai: $\operatorname{tr} \mid$ Q3B745|Q3B745_DANRE Ubiquitin carrier protein OS=Danio rerio GN=zgc: 1؛ tr $\mid$ Q4S564|Q4S564_TETNG Chromosome 6 SCAF14737, whole genome shotgun sequt $\operatorname{tr} \mid$ B9EN72 $\mid$ B9EN72_SALSA Transmembrane protein 205 OS=Salmo salar GN=TM205 $\operatorname{tr} \mid$ C1BHJ3 $\mid$ C1BHJ3_ONCMY Translocon-associated protein subunit gamma OS=0nc sp $\mid$ Q803M5 |SAAL1_DANRE Protein saal1 OS=Danio rerio GN=saal1 PE=2 SV=2 tr $\mid$ Q4SBJ7|Q4SBJ7_TETNG Chromosome 15 SCAF14667, whole genome shotgun seq 


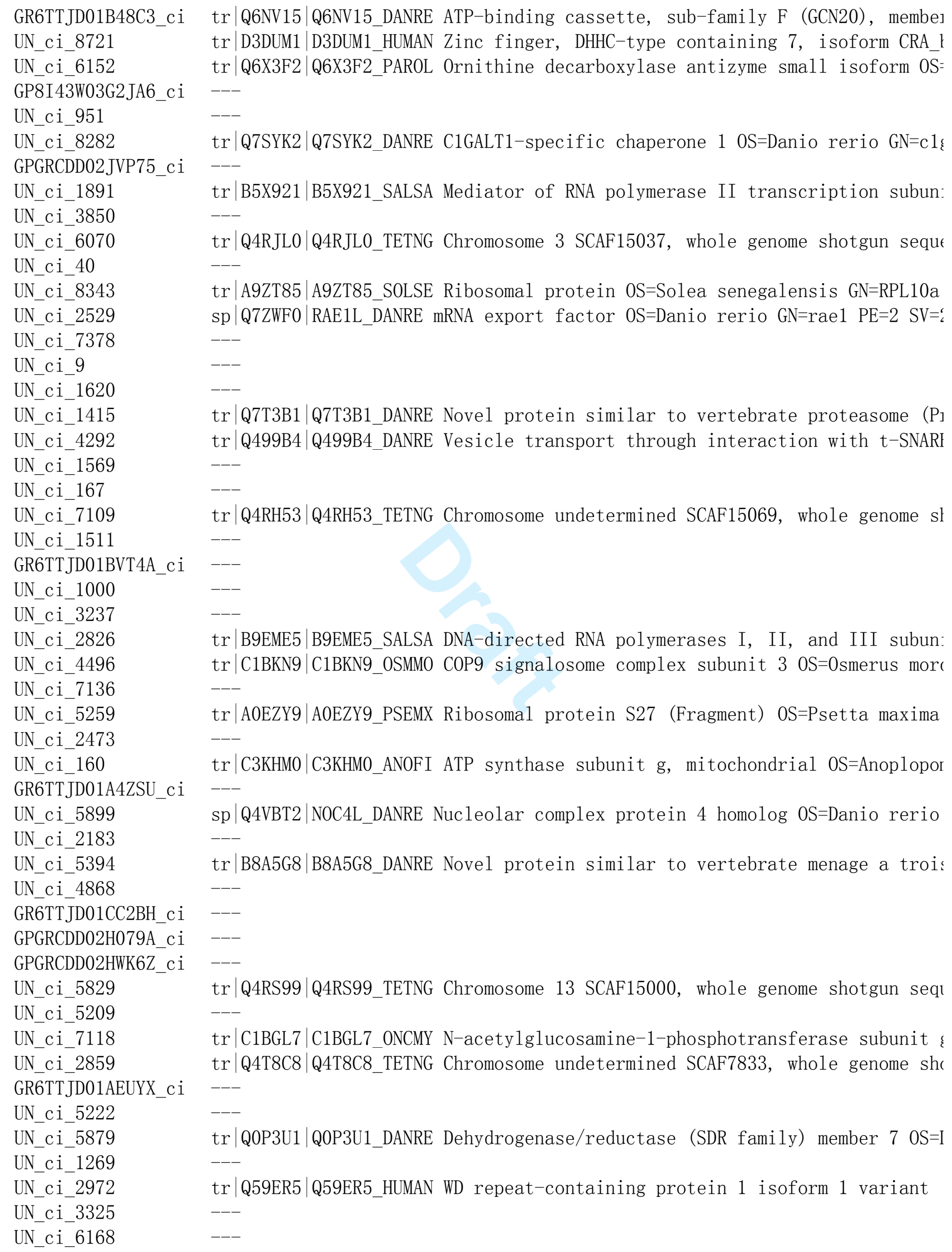




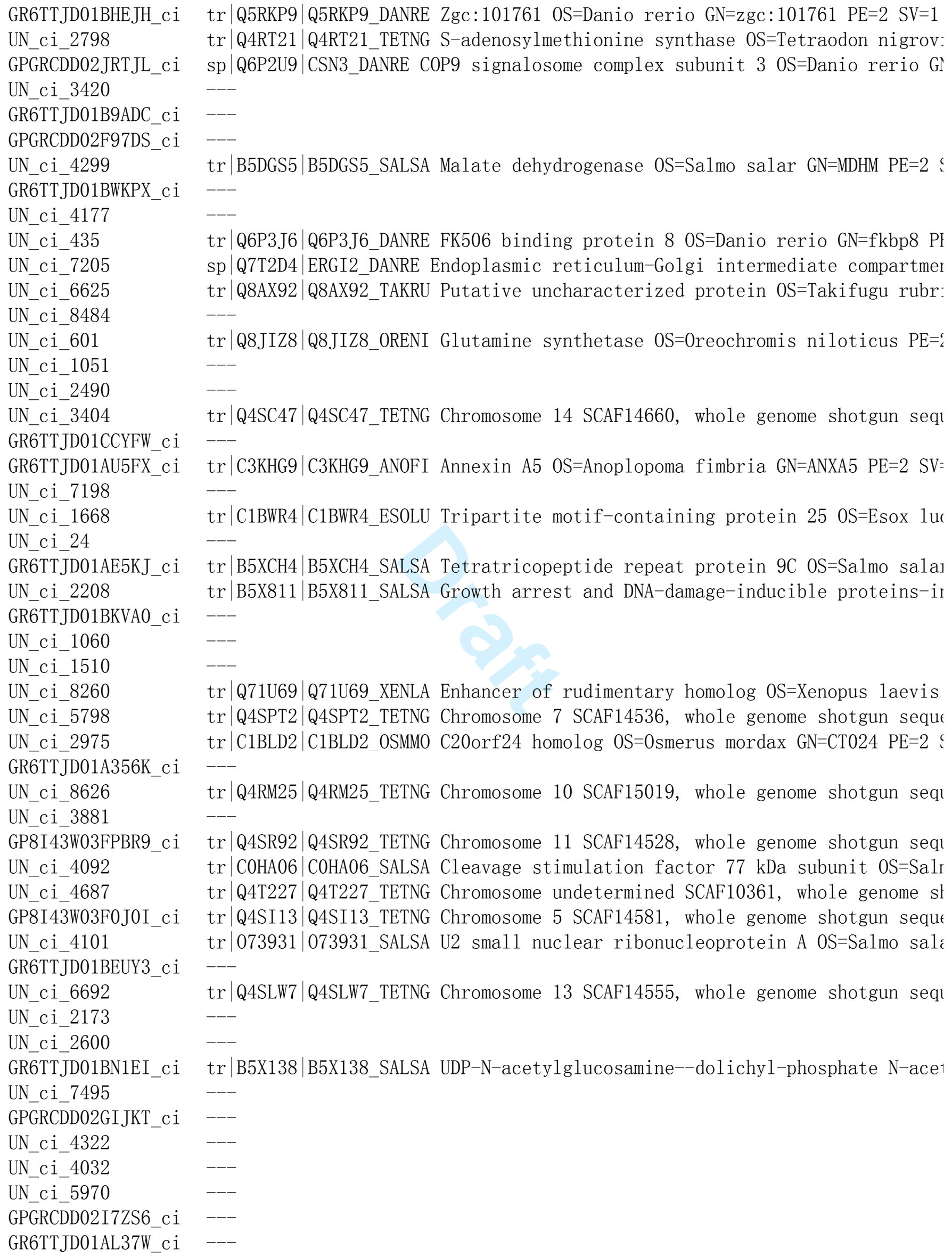

$\operatorname{tr} \mid$ Q5RKP9|Q5RKP9 DANRE Zgc:101761 OS=Danio rerio GN=zgc:101761 PE=2 SV=1 $\operatorname{tr} \mid$ Q4RT21|Q4RT21_TETNG S-adenosylmethionine synthase OS=Tetraodon nigrov sp|Q6P2U9|CSN3_DANRE COP9 signalosome complex subunit 3 OS=Danio rerio G!

$\operatorname{tr} \mid$ B5DGS5 $\mid$ B5DGS5_SALSA Malate dehydrogenase OS=Salmo salar GN=MDHM PE=2 ؛

tr $\mid$ Q6P3J6 $\mid$ Q6P3J6_DANRE FK506 binding protein 8 OS=Danio rerio GN=fkbp8 PI sp |Q7T2D4|ERGI2_DANRE Endoplasmic reticulum-Golgi intermediate compartmer $\operatorname{tr} \mid$ Q8AX92 $\mid$ Q8AX92_TAKRU Putative uncharacterized protein OS=Takifugu rubr:

$\operatorname{tr} \mid$ Q8JIZ8 $\mid$ Q8JIZ8_ORENI Glutamine synthetase OS=0reochromis niloticus PE=‘ $---$

tr $\mid$ Q4SC47|Q4SC47_TETNG Chromosome 14 SCAF14660, whole genome shotgun seq $\operatorname{tr} \mid$ C3KHG9|C3KHG9_ANOFI Annexin A5 OS=Anoplopoma fimbria GN=ANXA5 PE=2 SV= $\operatorname{tr} \mid$ C1BWR $\mid$ C1BWR4_ESOLU Tripartite motif-containing protein 25 OS=Esox luc $\operatorname{tr} \mid$ B5XCH4 |B5XCH4_SALSA Tetratricopeptide repeat protein 9C 0S=Salmo sala] $\operatorname{tr} \mid$ B5X811|B5X811_SALSA Growth arrest and DNA-damage-inducible proteins-ir

$\operatorname{tr} \mid$ Q71U69 $\mid$ Q71U69_XENLA Enhancer of rudimentary homolog OS=Xenopus laevis tr $\mid$ Q4SPT2 $\mid$ Q4SPT2_TETNG Chromosome 7 SCAF14536, whole genome shotgun sequt $\operatorname{tr} \mid$ C1BLD2 $\mid$ C1BLD2_0SMM0 C20orf24 homolog OS=0smerus mordax GN=CT024 PE=2 ؛ tr|Q4RM25|Q4RM25_TETNG Chromosome 10 SCAF15019, whole genome shotgun seq $--$

tr $\mid$ Q4SR92 $\mid$ Q4SR92_TETNG Chromosome 11 SCAF14528, whole genome shotgun seq1 tr|COHA06|COHA06_SALSA Cleavage stimulation factor $77 \mathrm{kDa}$ subunit OS=Salr tr $\mid$ Q4T227|Q4T227_TETNG Chromosome undetermined SCAF10361, whole genome sl tr|Q4SI13|Q4SI13_TETNG Chromosome 5 SCAF14581, whole genome shotgun sequt $\operatorname{tr}|073931| 073931 \_S A L S A$ U2 small nuclear ribonucleoprotein A OS=Salmo sali tr $\mid$ Q4SLW7 |Q4SLW7_TETNG Chromosome 13 SCAF14555, whole genome shotgun seq1 $---$ tr $\mid$ B5X138|B5X138_SALSA UDP-N-acetylglucosamine--dolichyl-phosphate N-ace1 


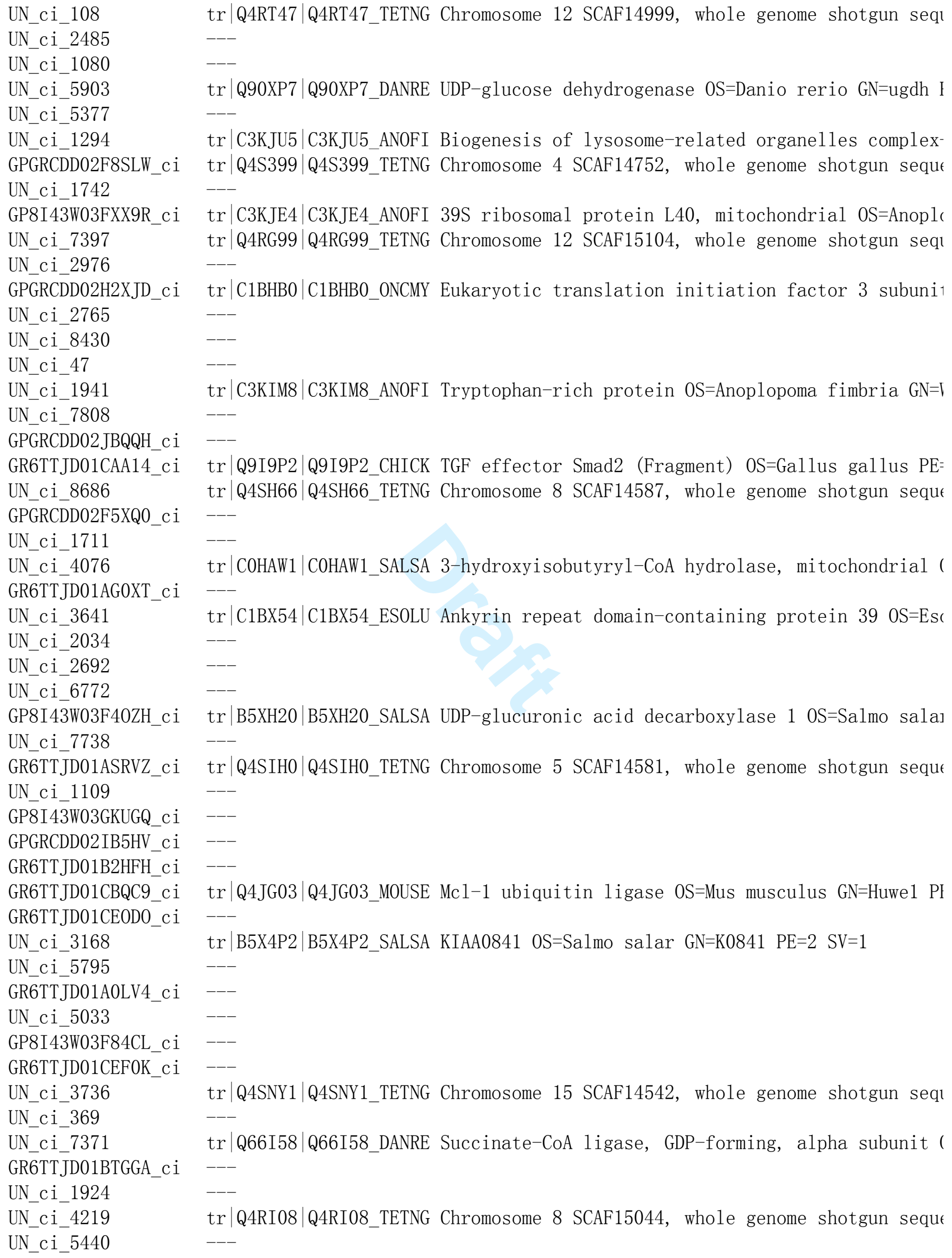




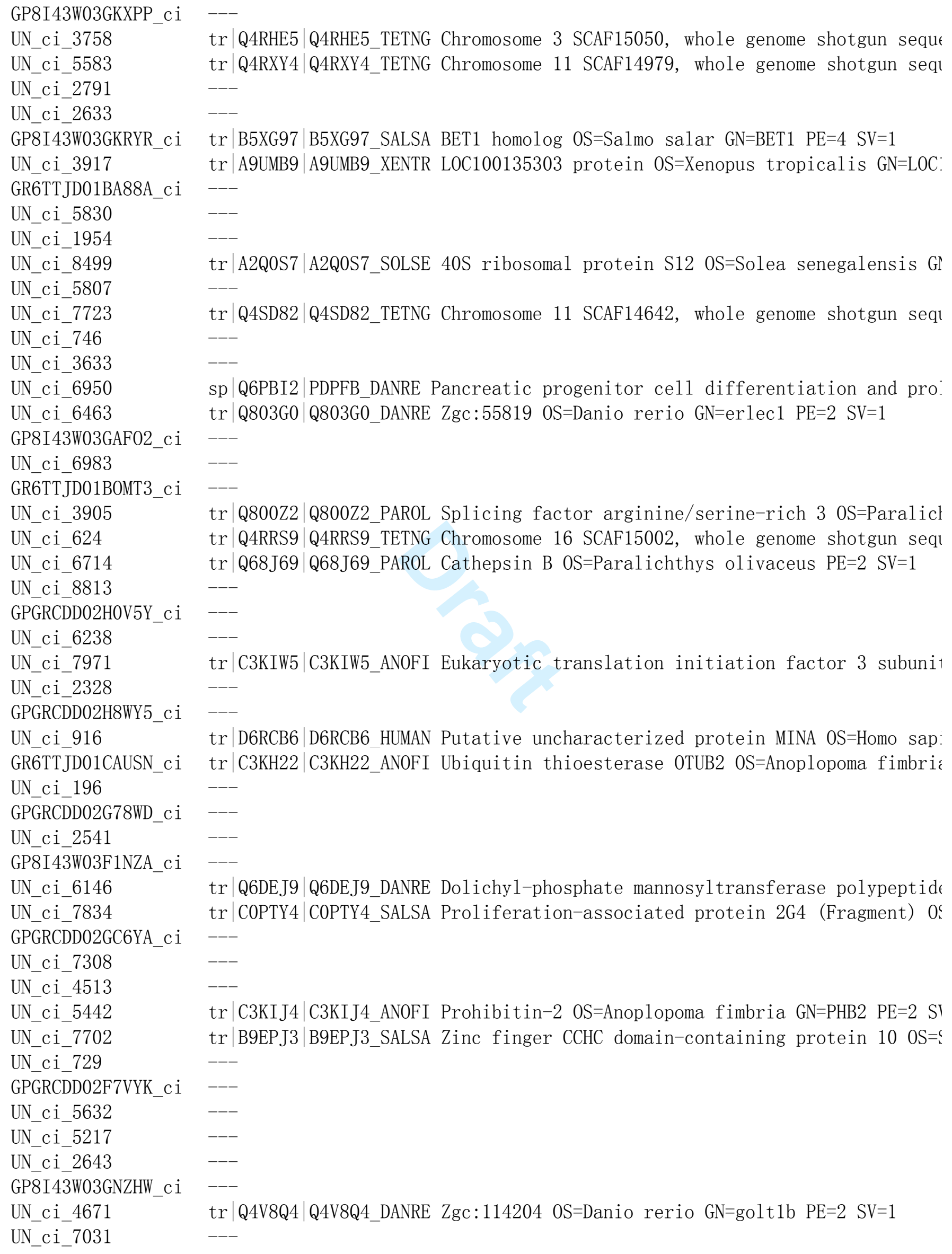

tr|Q4RHE5|Q4RHE5_TETNG Chromosome 3 SCAF15050, whole genome shotgun sequt tr $\mid$ Q4RXY4|Q4RXY4_TETNG Chromosome 11 SCAF14979, whole genome shotgun seq1

tr $\mid$ B5XG97|B5XG97_SALSA BET1 homolog 0S=Salmo salar GN=BET1 PE=4 SV=1 tr $\mid$ A9UMB9 $\mid$ A9UMB9_XENTR LOC100135303 protein OS=Xenopus tropicalis GN=LOC:

$\operatorname{tr} \mid$ A2Q0S7 $\mid$ A2Q0S7_SOLSE 40S ribosomal protein S12 0S=Solea senegalensis GI $\operatorname{tr} \mid$ Q4SD82|Q4SD82_TETNG Chromosome 11 SCAF14642, whole genome shotgun seq1 $---$

sp $\mid$ Q6PBI2 $\mid$ PDPFB_DANRE Pancreatic progenitor cell differentiation and pro: $\operatorname{tr} \mid$ Q803G0 |Q803G0_DANRE Zgc:55819 0S=Danio rerio GN=erlec1 PE=2 SV=1

$\operatorname{tr} \mid$ Q800Z2 $\mid$ Q800Z2_PAROL Splicing factor arginine/serine-rich 3 OS=Paralicl tr $\mid$ Q4RRS9|Q4RRS9_TETNG Chromosome 16 SCAF15002, whole genome shotgun seq1 $\operatorname{tr} \mid$ Q68J69|Q68J69_PAR0L Cathepsin B OS=Paralichthys olivaceus PE=2 SV=1

tr|C3KIW5|C3KIW5_ANOFI Eukaryotic translation initiation factor 3 subuni1

tr $\mid$ D6RCB6 $\mid$ D6RCB6_HUMAN Putative uncharacterized protein MINA 0S=Homo sap: $\operatorname{tr} \mid$ C3KH22|C3KH22_ANOFI Ubiquitin thioesterase OTUB2 OS=Anoplopoma fimbric

tr $\mid$ Q6DEJ9|Q6DEJ9_DANRE Dolichy1-phosphate mannosyltransferase polypeptidt tr|COPTY4|COPTY4_SALSA Proliferation-associated protein 2G4 (Fragment) 0 s

tr $\mid$ C3KIJ4|C3KIJ4_ANOFI Prohibitin-2 OS=Anoplopoma fimbria GN=PHB2 PE=2 SI $\operatorname{tr} \mid$ B9EPJ3|B9EPJ3_SALSA Zinc finger CCHC domain-containing protein 10 OS=؛

$\operatorname{tr} \mid$ Q4V8Q4 |Q4V8Q4_DANRE Zgc:114204 OS=Danio rerio GN=golt1b PE=2 SV=1 


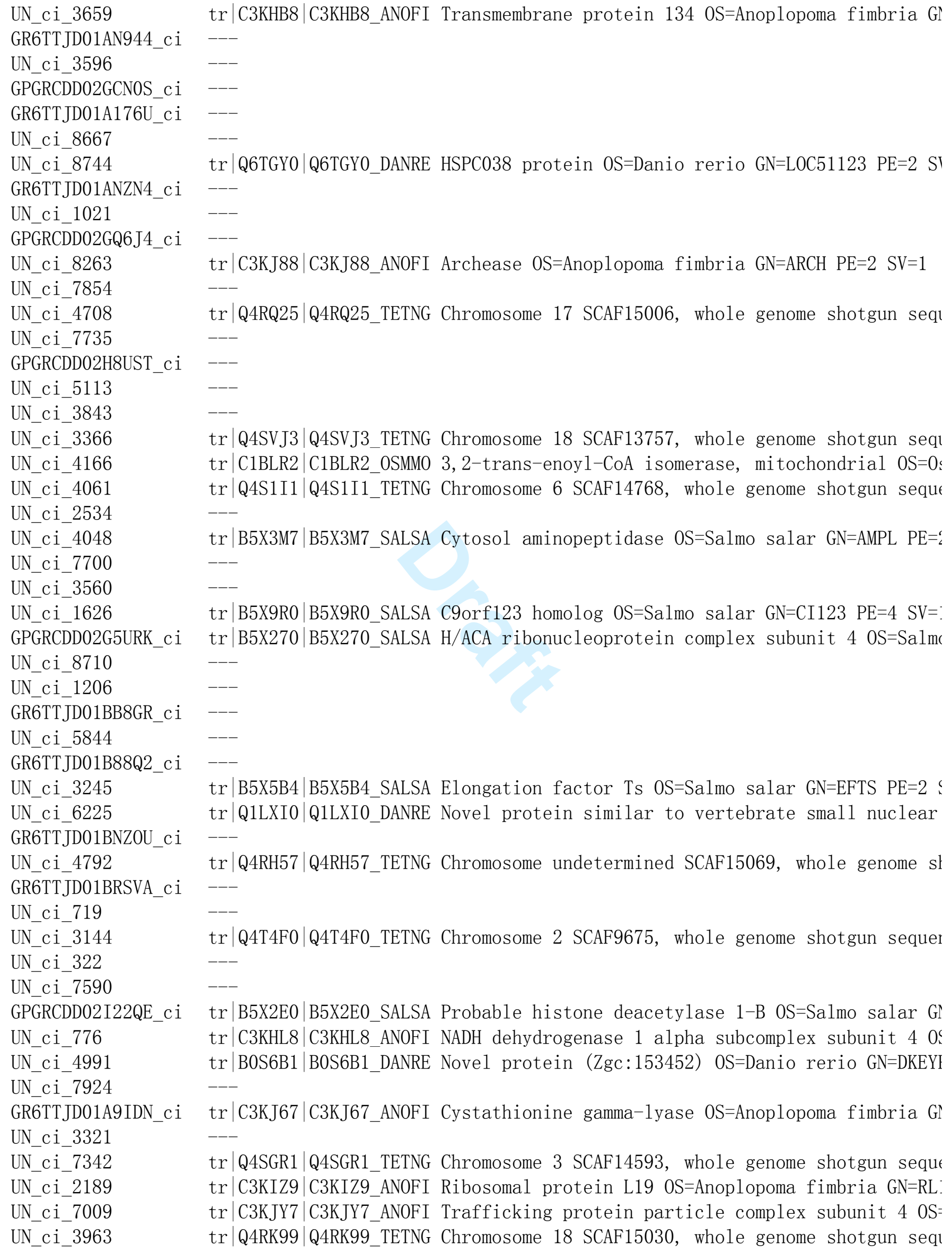




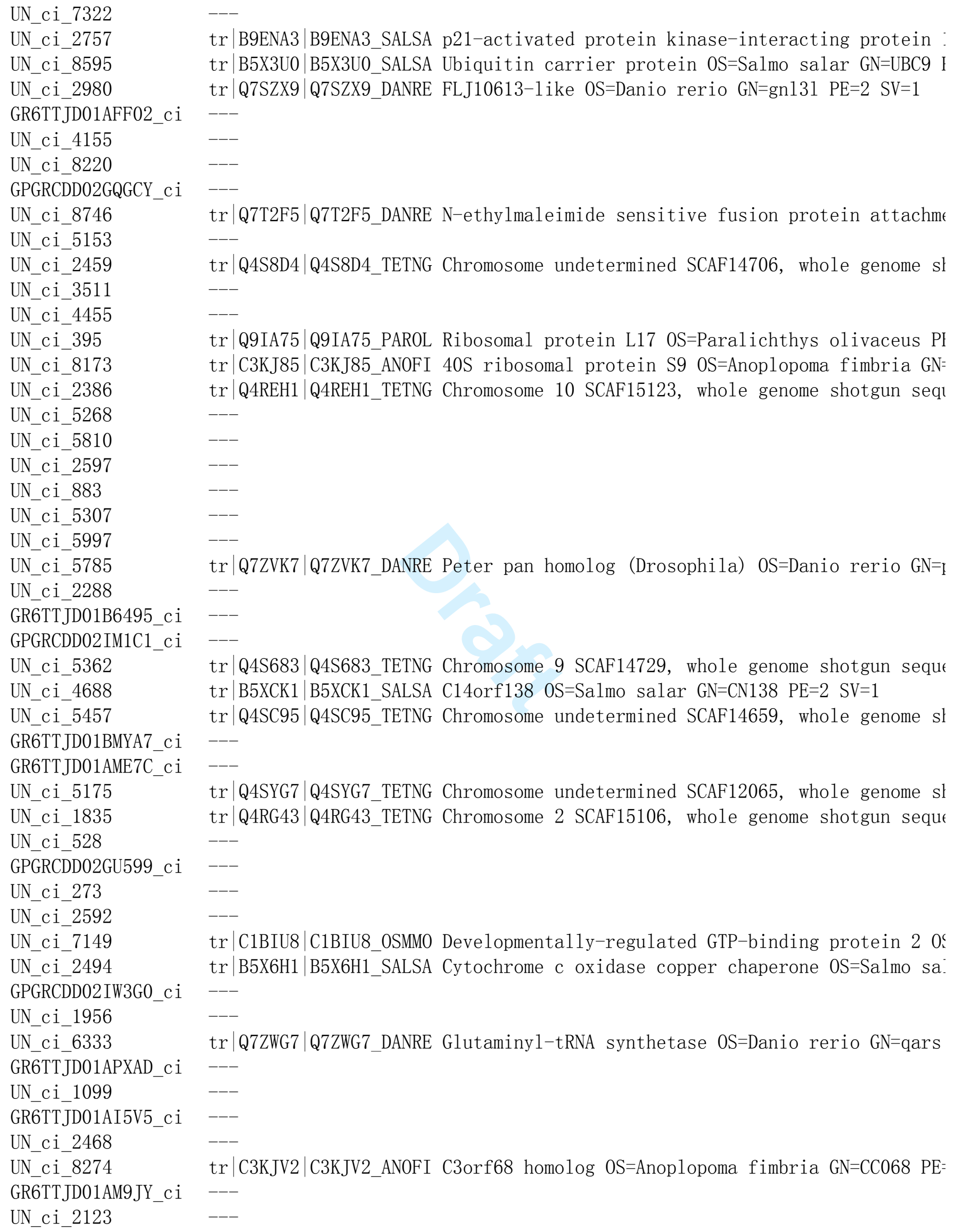

UN_ci_8274 $\operatorname{tr} \mid$ C3KJV2 $\mid$ C3KJV2_ANOFI C3orf68 homolog OS=Anoplopoma fimbria GN=CC068 PE=

tr $\mid$ Q7T2F5|Q7T2F5_DANRE N-ethylmaleimide sensitive fusion protein attachm tr $\mid$ Q4S8D4|Q4S8D4_TETNG Chromosome undetermined SCAF14706, whole genome sl

tr $\mid$ Q9IA75|Q9IA75_PAR0L Ribosomal protein L17 0S=Paralichthys olivaceus PI $\operatorname{tr} \mid$ C3KJ85|C3KJ85_ANOFI 40S ribosomal protein S9 0S=Anoplopoma fimbria GN= tr $\mid$ Q4REH1 |Q4REH1_TETNG Chromosome 10 SCAF15123, whole genome shotgun seq1

$\operatorname{tr} \mid$ Q7ZVK7 |Q7ZVK7_DANRE Peter pan homolog (Drosophila) OS=Danio rerio GN=1

tr $\mid$ Q4S683|Q4S683_TETNG Chromosome 9 SCAF14729, whole genome shotgun sequt $\operatorname{tr} \mid$ B5XCK1 $\mid$ B5XCK1_SALSA C14orf138 OS=Salmo salar GN=CN138 PE=2 SV=1 tr $\mid$ Q4SC95|Q4SC95_TETNG Chromosome undetermined SCAF14659, whole genome sl

$\operatorname{tr} \mid$ Q4SYG7|Q4SYG7_TETNG Chromosome undetermined SCAF12065, whole genome sl tr $\mid$ Q4RG43|Q4RG43_TETNG Chromosome 2 SCAF15106, whole genome shotgun sequt

tr $\mid$ C1BIU8 $\mid$ C1BIU8_0SMM0 Developmentally-regulated GTP-binding protein 2 0s tr|B5X6H1|B5X6H1_SALSA Cytochrome c oxidase copper chaperone OS=Salmo sa:

$\operatorname{tr} \mid$ Q7ZWG7 |Q7ZWG7_DANRE Glutaminy1-tRNA synthetase OS=Danio rerio GN=qars 
ว $\mathrm{GN}=\operatorname{mapk} 15 \mathrm{PE}=2 \mathrm{SV}=1$

dis GN=GSTENG00003377001 PE=3 SV=1

lence. (Fragment) OS=Tetraodon nigroviridis GN=GSTENG00014966001 PE=4 SV=1

lence. (Fragment) OS=Tetraodon nigroviridis GN=GSTENG00024925001 PE=4 SV=1

IE $=2 \quad \mathrm{SV}=1$

1otgun sequence OS=Tetraodon nigroviridis GN=GSTENG00013440001 PE=4 SV=1 :110764) OS=Danio rerio GN=timm44 $\mathrm{PE}=2 \mathrm{SV}=1$ nordax $\quad \mathrm{GN}=\mathrm{YNQF} \quad \mathrm{PE}=2 \mathrm{SV}=1$

nbria GN=PPT1 PE=2 SV=1

$\mathrm{PE}=4 \mathrm{SV}=1$

$\operatorname{mordax}$ GN=KIP1 PE=2 SV=1

j=Epinephelus coioides $\mathrm{PE}=4 \mathrm{SV}=1$

ग8 $\mathrm{PE}=2 \mathrm{SV}=1$

$=\mathrm{RS} 8 \mathrm{PE}=2 \quad \mathrm{SV}=1$

t I OS=Anoplopoma fimbria GN=EIF3I $\mathrm{PE}=2 \mathrm{SV}=1$

गviridis $\mathrm{GN}=\mathrm{crfb} 4 \mathrm{PE}=2 \mathrm{SV}=1$

$=$ Salmo salar GN=PSD4 PE=2 SV=1

$7 \quad \mathrm{PE}=2 \quad \mathrm{SV}=1$ 
$244001 \mathrm{PE}=3 \mathrm{SV}=1$

lence. (Fragment) OS=Tetraodon nigroviridis GN=GSTENG00031315001 PE=4 SV=1

nitochondrial OS=Anoplopoma fimbria GN=NDUA9 PE=2 SV=1

lence. (Fragment) OS=Tetraodon nigroviridis GN=GSTENG00030206001 PE=4 SV=1 ar GN=IMMT PE=2 SV=1

ənce OS=Tetraodon nigroviridis GN=GSTENG00023443001 PE=4 SV=1

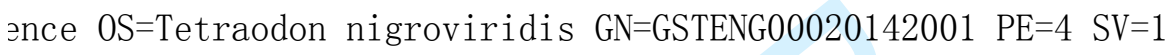

$\mathrm{Z}=1 \quad \mathrm{SV}=2$

lotgun sequence OS=Tetraodon nigroviridis GN=GSTENG00025283001 PE=4 SV=1

a binding protein (Scraps homolog, Drosophila) (ANLN) OS=Danio rerio GN=si:ch211-197n10.2 F mitochondrial OS=Esox lucius $\mathrm{GN}=\mathrm{COX} 7 \mathrm{R} \quad \mathrm{PE}=4 \mathrm{SV}=1$

$31 \mathrm{PE}=2 \mathrm{SV}=1$

$=2 \mathrm{SV}=1$

ənce. (Fragment) OS=Tetraodon nigroviridis GN=GSTENG00031223001 PE=3 SV=1

ænio rerio $\mathrm{GN}=$ ptcd2 $\mathrm{PE}=2 \mathrm{SV}=1$

stgun sequence. (Fragment) OS=Tetraodon nigroviridis GN=GSTENG00003540001 PE=4 SV=1 Anoplopoma fimbria GN=ATP5J PE=3 SV=1 
$2 \mathrm{SV}=1$

j=Anoplopoma fimbria GN=XIP PE=4 SV=1

$\mathrm{GN}=\mathrm{SYCP} 1 \quad \mathrm{PE}=2 \quad \mathrm{SV}=1$

$=1$

$=3 \mathrm{SV}=1$

lotgun sequence. (Fragment) OS=Tetraodon nigroviridis GN=GSTENG00025267001 PE=4 SV=1

$2 \mathrm{SV}=1$

ənce. (Fragment) OS=Tetraodon nigroviridis GN=GSTENG00010178001 PE=4 SV=1

$\Xi=2 \quad S V=1$

$=2 \mathrm{SV}=1$

$\mathrm{V} 1 \mathrm{~A} \quad \mathrm{PE}=2 \mathrm{SV}=1$

splopoma fimbria GN=HSDL2 PE=2 SV=1

spoma fimbria $\mathrm{GN}=\mathrm{L} 10 \mathrm{~K} \quad \mathrm{PE}=4 \mathrm{SV}=1$ 
$=\mathrm{cRAF} \quad \mathrm{PE}=2 \mathrm{SV}=1$

se $0 \mathrm{~S}=$ Danio rerio $\mathrm{GN}=$ melk $\mathrm{PE}=2 \mathrm{SV}=1$

rotgun sequence. (Fragment) OS=Tetraodon nigroviridis GN=GSTENG00010523001 PE=4 SV=1

$\mathrm{z}=2 \mathrm{SV}=1$

ənce. (Fragment) OS=Tetraodon nigroviridis GN=GSTENG00020526001 PE=4 SV=1

jV=1

ənce. (Fragment) OS=Tetraodon nigroviridis GN=GSTENG00025511001 PE=4 SV=1

$\mathrm{V}=\mathrm{EF} 1 \mathrm{D} \quad \mathrm{PE}=2 \quad \mathrm{SV}=1$

lence OS=Tetraodon nigroviridis GN=GSTENG00030938001 PE=4 SV=1

$3 \mathrm{~N}=\mathrm{MAP} 1-\mathrm{LC} 3 \mathrm{C} \quad \mathrm{PE}=2 \quad \mathrm{SV}=1$

rotgun sequence. (Fragment) OS=Tetraodon nigroviridis GN=GSTENG00024315001 PE=4 SV=1

$\mathrm{PE}=2 \mathrm{SV}=1$

lence OS=Tetraodon nigroviridis GN=GSTENG00016104001 PE=4 SV=1

laevis $\mathrm{GN}=$ naa16 $\mathrm{PE}=2 \mathrm{SV}=1$

https://mc06.manuscriptcentral.com/genome-pubs 
taneum GN=TcasGA2_TC000449 PE=4 SV=1

ənce. (Fragment) OS=Tetraodon nigroviridis GN=GSTENG00024879001 PE=4 SV=1 spoma fimbria $\mathrm{GN}=\mathrm{C} 1 \mathrm{D} \quad \mathrm{PE}=2 \mathrm{SV}=1$

$100125043 \mathrm{PE}=4 \mathrm{SV}=1$

ənce. (Fragment) OS=Tetraodon nigroviridis GN=GSTENG00022244001 PE=4 SV=1 rotgun sequence $0 \mathrm{~S}=$ Tetraodon nigroviridis $\mathrm{GN}=\mathrm{GSTENG} 00038513001 \mathrm{PE}=4 \mathrm{SV}=1$

yltransferase subunit DAD1 OS=0smerus mordax GN=DAD1 PE=4 SV=1

j $\mathrm{PE}=2 \mathrm{SV}=1$

IN=i tgb1bp1 PE=2 SV=1

ənce. (Fragment) 0S=Tetraodon nigroviridis GN=GSTENG00029930001 PE=3 SV=1

$\mathrm{Z}=4 \mathrm{SV}=1$

$\mathrm{\Xi}=2 \mathrm{SV}=1$

^ $\mathrm{PE}=2 \mathrm{SV}=1$

$\mathrm{V}=1$

ia $\mathrm{GN}=\mathrm{F} 16 \mathrm{P} 1 \quad \mathrm{PE}=2 \mathrm{SV}=1$

ənce OS=Tetraodon nigroviridis GN=GSTENG00028981001 PE=4 SV=1

dis GN=GSTENG00013477001 PE=3 SV=1

¥lis GN=LOC100170503 PE=2 SV=1

talurus punctatus $\mathrm{PE}=2 \mathrm{SV}=1$

oria $\mathrm{GN}=\mathrm{RAN} \mathrm{PE}=2 \mathrm{SV}=1$ 
ə $\mathrm{GN}=\mathrm{PSB} 1 \mathrm{~A} \quad \mathrm{PE}=2 \mathrm{SV}=1$

ıence. (Fragment) OS=Tetraodon nigroviridis GN=GSTENG00024542001 PE=4 SV=1

ənce. (Fragment) OS=Tetraodon nigroviridis GN=GSTENG00015362001 PE=4 SV=1 $36 \mathrm{~A} \quad \mathrm{PE}=3 \mathrm{SV}=1$

ıence. (Fragment) OS=Tetraodon nigroviridis GN=GSTENG00023013001 PE=4 SV=1

? $=4 \quad \mathrm{SV}=1$

IE $=2 \quad \mathrm{SV}=1$

ənce OS=Tetraodon nigroviridis GN=GSTENG00014630001 PE=4 SV=1

ənce. (Fragment) OS=Tetraodon nigroviridis GN=GSTENG00018324001 PE=4 SV=1

ənce OS=Tetraodon nigroviridis GN=GSTENG00017354001 PE=4 SV=1

lence. (Fragment) OS=Tetraodon nigroviridis GN=GSTENG00026200001 PE=4 SV=1

ə fimbria $\mathrm{GN}=\mathrm{ZDHC} 4 \mathrm{PE}=2 \mathrm{SV}=1$

shmp4c PE=2 SV=1

Jtgun sequence. (Fragment) OS=Tetraodon nigroviridis GN=GSTENG00003601001 PE=4 SV=1

lence OS=Tetraodon nigroviridis GN=GSTENG00030961001 PE=4 SV=1

ıotgun sequence OS=Tetraodon nigroviridis GN=GSTENG00008032001 PE=4 SV=1

j=Anoplopoma fimbria GN=PSD13 PE=2 SV=1

$=2 \quad \mathrm{SV}=1$

lence. $0 \mathrm{~S}=$ Tetraodon nigroviridis $\mathrm{GN}=\mathrm{NKEF} \mathrm{PE}=2 \mathrm{SV}=1$

lence. (Fragment) OS=Tetraodon nigroviridis GN=GSTENG00030100001 PE=4 SV=1

$3 \mathrm{SV}=1$

ıotgun sequence. (Fragment) OS=Tetraodon nigroviridis GN=GSTENG00020493001 PE=4 SV=1 
JK3 $\mathrm{PE}=2 \mathrm{SV}=1$

rotgun sequence OS=Tetraodon nigroviridis GN=GSTENG00011891001 PE=4 SV=1

orkcz PE=2 SV=1

$161 \quad \mathrm{PE}=2 \mathrm{SV}=1$

t $\mathrm{SEC} 11 \mathrm{~B}$ 0S=Homo sapiens $\mathrm{GN}=\mathrm{SEC} 11 \mathrm{~B}$ PE=5 SV=1

zenp $\mathrm{PE}=2 \mathrm{SV}=1$

$=2 \mathrm{SV}=1$

ıotgun sequence. (Fragment) OS=Tetraodon nigroviridis GN=GSTENG00010923001 PE=4 SV=1 OS=0smerus mordax GN=MD2L2 $\mathrm{PE}=2 \mathrm{SV}=1$

lence OS=Tetraodon nigroviridis GN=GSTENG00014497001 PE=4 SV=1

¡ $\mathrm{PE}=2 \mathrm{SV}=1$

1 $0 \mathrm{~S}=0$ smerus mordax $\mathrm{GN}=\mathrm{SH} 3 \mathrm{~L} 1 \mathrm{PE}=4 \mathrm{SV}=1$

ence. (Fragment) OS=Tetraodon nigroviridis GN=GSTENG00034706001 PE=4 SV=1

sp60 $\mathrm{PE}=4 \mathrm{SV}=1$

) $\mathrm{OS}=\mathrm{Psetta}$ maxima $\mathrm{PE}=2 \mathrm{SV}=1$

lucius $\mathrm{GN}=\mathrm{FABG} \mathrm{PE}=2 \mathrm{SV}=1$

$=\mathrm{LOC} 443622$ PE=2 SV=1

is tropicalis GN=1rrc40 $\mathrm{PE}=2 \mathrm{SV}=1$

Jtgun sequence OS=Tetraodon nigroviridis GN=GSTENG00004538001 PE=4 SV=1

JS=Danio rerio $\mathrm{GN}=\mathrm{cwc} 27 \mathrm{PE}=2 \mathrm{SV}=1$

JS=0smerus mordax GN=T2EB PE=2 SV=1

s marmoratus $\mathrm{GN}=06-\mathrm{MGMT} \mathrm{PE}=2 \mathrm{SV}=1$ 
ıence. (Fragment) OS=Tetraodon nigroviridis GN=GSTENG00023080001 PE=4 SV=1 ıce. (Fragment) OS=Tetraodon nigroviridis GN=GSTENG00006630001 PE=4 SV=1

lence OS=Tetraodon nigroviridis GN=GSTENG00035787001 PE=4 SV=1

$=4 \mathrm{SV}=1$

lar $\mathrm{GN}=\mathrm{SC} 61 \mathrm{~A} \quad \mathrm{PE}=2 \mathrm{SV}=1$

lotgun sequence OS=Tetraodon nigroviridis GN=GSTENG00020488001 PE=4 SV=1

ənce. (Fragment) OS=Tetraodon nigroviridis GN=GSTENG00015448001 PE=4 SV=1 OS=Tetraodon nigroviridis GN=GSTENG00028241001 PE=3 SV=1

viral diarrhea virus GN=p125 PE=2 SV=1

stgun sequence OS=Tetraodon nigroviridis GN=GSTENG00003525001 PE=4 SV=1

$\mathrm{j}=$ Anoplopoma fimbria GN=ELOC PE=4 SV=1

ว rerio $\mathrm{GN}=\mathrm{srp} 9 \mathrm{PE}=2 \mathrm{SV}=1$

ənce. (Fragment) OS=Tetraodon nigroviridis GN=GSTENG00016316001 PE=4 SV=1

IN=RL37A PE=4 SV=1

ıotgun sequence. (Fragment) OS=Tetraodon nigroviridis GN=GSTENG00025158001 PE=3 SV=1

ənce. (Fragment) OS=Tetraodon nigroviridis GN=GSTENG00031216001 PE=4 SV=1 jides $\mathrm{PE}=2 \mathrm{SV}=1$

lence. (Fragment) OS=Tetraodon nigroviridis GN=GSTENG00032048001 PE=4 SV=1

$\mathrm{z}=2 \mathrm{SV}=1$

$\mathrm{GN}=\mathrm{CBX} 3 \quad \mathrm{PE}=2 \quad \mathrm{SV}=1$

ənce OS=Tetraodon nigroviridis GN=GSTENG00028795001 PE=4 SV=1

lence. (Fragment) OS=Tetraodon nigroviridis GN=GSTENG00035419001 PE=4 SV=1 
$\mathrm{PE}=2 \quad \mathrm{SV}=1$

j=Anoplopoma fimbria GN=PSDE PE=2 SV=1

PE $=2 \quad \mathrm{SV}=1$

$3 \mathrm{PE}=2 \mathrm{SV}=1$

$\mathrm{J}=1$

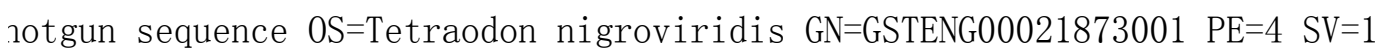

zse $0 \mathrm{~S}=$ Salmo salar GN=MTND $\mathrm{PE}=2 \mathrm{SV}=1$

luropoda melanoleuca GN=PANDA_011387 PE=4 SV=1

lence OS=Tetraodon nigroviridis GN=GSTENG00035588001 PE=3 SV=1

ivaceus $\mathrm{GN}=\mathrm{H} \operatorname{sp} 40 \mathrm{~A} 4 \mathrm{PE}=2 \mathrm{SV}=1$

गE $=2 \quad \mathrm{SV}=1$

ITK2 $\mathrm{PE}=2 \mathrm{SV}=1$

yltransferase subunit 4 OS=Salmo salar $\mathrm{GN}=0 \mathrm{ST} 4 \mathrm{PE}=4 \mathrm{SV}=1$

PE $=2 \quad S V=1$

lence OS=Tetraodon nigroviridis GN=GSTENG00007887001 PE=4 SV=1

znce OS=Tetraodon nigroviridis GN=GSTENG00017023001 PE=3 SV=1

spoma fimbria GN=LSM5 PE=4 SV=1

lence OS=Tetraodon nigroviridis GN=GSTENG00035794001 PE=4 SV=1

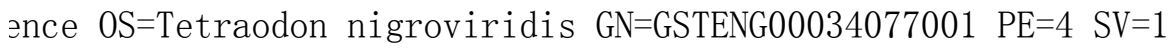


dbunit Tim8 A OS=0ncorhynchus mykiss GN=TIM8A PE=4 SV=1

ənce. (Fragment) OS=Tetraodon nigroviridis GN=GSTENG00024850001 PE=4 SV=1

ichus mykiss $\mathrm{PE}=4 \mathrm{SV}=1$

s coioides $\mathrm{PE}=2 \mathrm{SV}=1$

lence OS=Tetraodon nigroviridis GN=GSTENG00029735001 PE=4 SV=1

JS=0smerus mordax GN=TAF11 PE=2 SV=1

$2 \mathrm{PE}=2 \mathrm{SV}=1$

$=1$

spoma fimbria GN=RM30 PE=2 SV=1

lopoma fimbria GN=SAP18 PE=2 SV=1

ənce OS=Tetraodon nigroviridis GN=GSTENG00024147001 PE=4 SV=1

spoma fimbria GN=RM14 PE=2 SV=1

$\mathrm{PE}=2 \quad \mathrm{SV}=1$

$\mathrm{Z}=2 \mathrm{SV}=1$

uperfamily member 2 (THEM2) OS=Danio rerio $\mathrm{GN}=\operatorname{acot} 13 \mathrm{PE}=4 \mathrm{SV}=1$

Eimbria GN=CSN6 PE=2 SV=1

zfand1 $\mathrm{PE}=2 \mathrm{SV}=1$

rerio GN=zgc: $111992 \mathrm{PE}=2 \mathrm{SV}=1$

$\operatorname{tdc} 2 \mathrm{PE}=2 \mathrm{SV}=1$

ıence. (Fragment) OS=Tetraodon nigroviridis GN=GSTENG00020274001 PE=4 SV=1

ənce. (Fragment) OS=Tetraodon nigroviridis GN=GSTENG00024150001 PE=3 SV=1

iloticus $\mathrm{PE}=2 \mathrm{SV}=1$

ənce. (Fragment) OS=Tetraodon nigroviridis GN=GSTENG00031835001 PE=4 SV=1 
$2 \mathrm{SV}=1$

Jtgun sequence OS=Tetraodon nigroviridis GN=GSTENG00006076001 PE=3 SV=1

Jalmo salar GN=MARCS $P E=2$ SV=1

orotein $0 \mathrm{~S}=0$ ncorhynchus mykiss $\mathrm{GN}=\mathrm{UCR} 11 \mathrm{PE}=4 \mathrm{SV}=1$

$\mathrm{z}=3 \mathrm{SV}=1$

sma fimbria $\mathrm{GN}=\mathrm{BDH} 2 \mathrm{PE}=2 \mathrm{SV}=1$

Jlopoma fimbria GN=SRP14 PE=4 SV=1

Jtgun sequence. (Fragment) OS=Tetraodon nigroviridis GN=GSTENG00007300001 PE=4 SV=1

$3 \mathrm{~V}=1$

lence. (Fragment) OS=Tetraodon nigroviridis GN=GSTENG00013964001 PE=4 SV=1

$\mathrm{V}=\mathrm{Zgc}: 193694 \mathrm{PE}=3 \mathrm{SV}=1$

$\log$ 0S=Salmo salar $\mathrm{GN}=\mathrm{UTP} 18 \mathrm{PE}=2 \mathrm{SV}=1$

y protein $0 \mathrm{~S}=$ Anoplopoma fimbria $\mathrm{GN}=\mathrm{DPM} 2 \mathrm{PE}=4 \mathrm{SV}=1$

lence OS=Tetraodon nigroviridis GN=GSTENG00015265001 PE=4 SV=1

imbria GN=DYL2 PE=4 SV=1

ว1 $\mathrm{PE}=2 \mathrm{SV}=1$

shondrial OS=Anoplopoma fimbria GN=DHSB PE=2 SV=1

lence OS=Tetraodon nigroviridis GN=GSTENG00015908001 PE=3 SV=1

RFC4 PE=2 SV=1

ance. (Fragment) OS=Tetraodon nigroviridis GN=GSTENG00026970001 PE=4 SV=1

tein 4 OS=0smerus mordax GN=WIPI4 PE=2 SV=1 
IN $=$ MYL6 $\quad$ PE $=2 \quad \mathrm{SV}=1$

$-9 \mathrm{G} 4.3 \mathrm{PE}=4 \mathrm{SV}=1$

1otgun sequence. (Fragment) OS=Tetraodon nigroviridis GN=GSTENG00010382001 PE=4 SV=1

lence. (Fragment) OS=Tetraodon nigroviridis GN=GSTENG00021635001 PE=3 SV=1

IN $=$ akt1 $\quad \mathrm{PE}=2 \quad \mathrm{SV}=1$

lence. (Fragment) OS=Tetraodon nigroviridis GN=GSTENG00021492001 PE=4 SV=1

ıence. (Fragment) OS=Tetraodon nigroviridis GN=GSTENG00028389001 PE=4 SV=1

ənce. (Fragment) OS=Tetraodon nigroviridis GN=GSTENG00010176001 PE=4 SV=1

गicalis $\mathrm{GN}=\mathrm{e}$ if4a3 $\mathrm{PE}=2 \mathrm{SV}=1$

$1 \mathrm{PE}=2 \mathrm{SV}=1$

2 $\mathrm{PE}=2 \mathrm{SV}=1$

गE $=2 \quad \mathrm{SV}=1$

stgun sequence. (Fragment) OS=Tetraodon nigroviridis GN=GSTENG00003252001 PE=4 SV=1

xima $\mathrm{PE}=2 \mathrm{SV}=1$

$=$ fut $9 \mathrm{~b} \quad \mathrm{PE}=2 \mathrm{SV}=1$

n:7148063 PE=2 SV=1

$=r p 19 \quad \mathrm{PE}=2 \mathrm{SV}=1$

ənce. (Fragment) OS=Tetraodon nigroviridis GN=GSTENG00033770001 PE=4 SV=1

1

ənce. (Fragment) OS=Tetraodon nigroviridis GN=GSTENG00031822001 PE=4 SV=1 salar $\mathrm{GN}=\mathrm{RT} 12 \mathrm{PE}=2 \mathrm{SV}=1$ 
lence. (Fragment) OS=Tetraodon nigroviridis GN=GSTENG00031498001 PE=4 SV=1

$\mathrm{PE}=2 \mathrm{SV}=1$

lotgun sequence. (Fragment) OS=Tetraodon nigroviridis GN=GSTENG00033686001 PE=4 SV=1

lence. (Fragment) OS=Tetraodon nigroviridis GN=GSTENG00027526001 PE=4 SV=1

lence OS=Tetraodon nigroviridis GN=GSTENG00026727001 PE=3 SV=1

) rerio $\mathrm{GN}=\mathrm{dh} \times 38 \mathrm{PE}=2 \mathrm{SV}=1$

Jum GN=SOD1 PE=2 SV=1

$\mathrm{SV}=1$

I OS=Danio rerio GN=eif3i $\mathrm{PE}=2 \mathrm{SV}=1$

ənce OS=Tetraodon nigroviridis GN=GSTENG00028899001 PE=4 SV=1

$=0$ smerus mordax GN=GHITM PE=2 SV=1

ənce OS=Tetraodon nigroviridis GN=GSTENG00020063001 PE=4 SV=1

lence. (Fragment) OS=Tetraodon nigroviridis GN=GSTENG00032595001 PE=4 SV=1

?E=2 SV=1

spoma fimbria GN=CDC42 $\mathrm{PE}=2 \mathrm{SV}=1$

rotgun sequence. (Fragment) OS=Tetraodon nigroviridis GN=GSTENG00011542001 PE=4 SV=1

$1 \mathrm{PE}=2 \mathrm{SV}=1$

dbunit Tim13 0S=Anoplopoma fimbria GN=TIM13 PE=4 SV=1 
floridae GN=BRAFLDRAFT_125176 PE=4 SV=1

RB11A PE=2 SV=1

rotgun sequence. (Fragment) OS=Tetraodon nigroviridis GN=GSTENG00022436001 PE=3 SV=1

lence OS=Tetraodon nigroviridis GN=GSTENG00015941001 PE=4 SV=1

$=$ LGUL PE $=2 \quad \mathrm{SV}=1$

$\mathrm{PE}=4 \mathrm{SV}=1$

3C22. 5-001 PE=4 SV=1

$\mathrm{SV}=2$

lence. (Fragment) OS=Tetraodon nigroviridis GN=GSTENG00016015001 PE=4 SV=1

lopoma fimbria GN=ELOF1 PE=4 SV=1

t1a $P E=2 \quad S V=1$

?E $=2 \quad \mathrm{SV}=1$

ted RNA dependent inhibitor, repressor of (P58 repressor) OS=Homo sapiens GN=PRKRIR PE=2 SI rotgun sequence OS=Tetraodon nigroviridis GN=GSTENG00009376001 PE=3 SV=1

2 $\mathrm{PE}=2 \mathrm{SV}=1$

.oplopoma fimbria GN=HINT1 PE=2 SV=1

ənce. (Fragment) OS=Tetraodon nigroviridis GN=GSTENG00014208001 PE=4 SV=1

$\mathrm{PE}=2 \quad \mathrm{SV}=1$

sp $\mathrm{PE}=2 \mathrm{SV}=1$

OS=Mus musculus GN=Coq9 PE=1 SV=1

lence OS=Tetraodon nigroviridis GN=GSTENG00029567001 PE=4 SV=1

$\zeta=0$ smerus $\operatorname{mordax}$ GN=NDUA7 $\mathrm{PE}=4 \mathrm{SV}=1$

chondrial OS=Anoplopoma fimbria GN=ETFA PE=2 SV=1 
ənce. (Fragment) OS=Tetraodon nigroviridis GN=GSTENG00025920001 PE=4 SV=1

=fgfr $1 \mathrm{op} 2 \mathrm{PE}=2 \mathrm{SV}=1$

luropoda melanoleuca GN=PANDA_000373 PE=4 SV=1

rerio $\mathrm{GN}=\mathrm{usp} 16 \mathrm{PE}=3 \mathrm{SV}=1$

ənce OS=Tetraodon nigroviridis GN=GSTENG00025926001 PE=4 SV=1

laevis GN=wrnip1 PE=2 SV=1

JS=0ncorhynchus mykiss GN=VATL PE=2 SV=1

$2 \mathrm{SV}=1$

$3798996 \quad \mathrm{PE}=2 \mathrm{SV}=1$

$\mathrm{Z}=2 \mathrm{SV}=1$

$\mathrm{SV}=1$

$\mathrm{PE}=2 \mathrm{SV}=1$

$\mathrm{V}=\mathrm{RS} 3 \mathrm{~A} \quad \mathrm{PE}=2 \quad \mathrm{SV}=1$

) $\mathrm{OS}=$ Psetta maxima $\mathrm{PE}=2 \mathrm{SV}=1$

$\mathrm{PE}=2 \quad \mathrm{SV}=1$

$=\mathrm{PSB} 3 \quad \mathrm{PE}=2 \quad \mathrm{SV}=1$

ənce. (Fragment) OS=Tetraodon nigroviridis GN=GSTENG00034434001 PE=4 SV=1

ənce. (Fragment) OS=Tetraodon nigroviridis GN=GSTENG00034091001 PE=4 SV=1 
rerio $\mathrm{GN}=$ mpdulb $\mathrm{PE}=2 \mathrm{SV}=1$

SV=1

IN=UB2D4 PE=2 SV=1

ənce OS=Tetraodon nigroviridis GN=GSTENG00025517001 PE=4 SV=1

j OS=Danio rerio GN=elov16 $\mathrm{PE}=2 \mathrm{SV}=1$

rotgun sequence. (Fragment) OS=Tetraodon nigroviridis GN=GSTENG00013298001 PE=4 SV=1 s27a $\mathrm{PE}=2 \mathrm{SV}=1$

ənce. (Fragment) OS=Tetraodon nigroviridis GN=GSTENG00017093001 PE=4 SV=1

$r$ vitreus $\mathrm{PE}=2 \mathrm{SV}=1$

$\mathrm{SV}=1$

ıotgun sequence. (Fragment) OS=Tetraodon nigroviridis GN=GSTENG00020486001 PE=4 SV=1

Jalmo salar GN=0TC $\mathrm{PE}=2 \mathrm{SV}=1$

$=2 \quad \mathrm{SV}=2$

211-51h9. $6 \mathrm{PE}=4 \mathrm{SV}=1$

ənce OS=Tetraodon nigroviridis GN=GSTENG00022568001 PE=4 SV=1

L OS=Danio rerio GN=eif31 $\mathrm{PE}=2 \mathrm{SV}=1$

salar $\mathrm{GN}=\mathrm{CAZA1} \mathrm{PE}=2 \mathrm{SV}=1$

stgun sequence OS=Tetraodon nigroviridis GN=GSTENG00007301001 PE=4 SV=1

Janio rerio $\mathrm{GN}=$ apobec2a $\mathrm{PE}=2 \mathrm{SV}=1$

ənce. (Fragment) 0S=Tetraodon nigroviridis GN=GSTENG00017122001 PE=4 SV=1

วma fimbria GN=PDRG1 PE=2 SV=1

lence. (Fragment) OS=Tetraodon nigroviridis GN=GSTENG00020903001 PE=4 SV=1

1 $\mathrm{PE}=2 \mathrm{SV}=1$

.op16 $\mathrm{PE}=3 \mathrm{SV}=1$

luropoda melanoleuca GN=PANDA_006953 PE=4 SV=1 
SC4 PE=2 SV=1

ar $\mathrm{GN}=\mathrm{PA} 2 \mathrm{G} 4 \mathrm{PE}=2 \mathrm{SV}=1$

रP19 $\mathrm{PE}=2 \mathrm{SV}=1$

rotgun sequence OS=Tetraodon nigroviridis GN=GSTENG00013803001 PE=4 SV=1

lence. (Fragment) OS=Tetraodon nigroviridis GN=GSTENG00026711001 PE=4 SV=1

$4 \mathrm{SV}=1$

it 10 OS=Anoplopoma fimbria GN=MED10 PE=2 SV=1

$\mathrm{PE}=2 \mathrm{SV}=1$

ə maxima $\mathrm{PE}=2 \mathrm{SV}=1$

lence. (Fragment) OS=Tetraodon nigroviridis GN=GSTENG00018743001 PE=4 SV=1

lucius $\mathrm{GN}=\mathrm{RM} 47 \mathrm{PE}=2 \mathrm{SV}=1$

nolog $0 \mathrm{~S}=$ Salmo salar GN=VPS41 PE=2 SV=1

$2 \mathrm{SV}=1$

rotgun sequence OS=Tetraodon nigroviridis GN=GSTENG00021829001 PE=4 SV=1

4 $\mathrm{PE}=2 \quad \mathrm{SV}=1$

ənce. (Fragment) OS=Tetraodon nigroviridis GN=GSTENG00012540001 PE=4 SV=1

$=1$

ənce. (Fragment) OS=Tetraodon nigroviridis GN=GSTENG00019736001 PE=4 SV=1

Jus laevis GN=uba5 PE=2 SV=1

V=WBS22 PE=2 SV=1 
1 homolog 0S=Anoplopoma fimbria GN=NSE1 PE=2 SV=1

$=1$

lence OS=Tetraodon nigroviridis GN=GSTENG00018392001 PE=4 SV=1

$\mathrm{PE}=2 \mathrm{SV}=1$

эlar GN=PSME3 PE=2 SV=1

salar $\mathrm{GN}=\mathrm{T} 10 \mathrm{PE}=2 \mathrm{SV}=1$

lence OS=Tetraodon nigroviridis GN=GSTENG00022689001 PE=4 SV=1

ance. (Fragment) OS=Tetraodon nigroviridis GN=GSTENG00033383001 PE=3 SV=1

$\mathrm{z}=2 \mathrm{SV}=1$

3RK1 PE=4 SV=1

ənce OS=Tetraodon nigroviridis GN=GSTENG00014695001 PE=4 SV=1

lence. (Fragment) OS=Tetraodon nigroviridis GN=GSTENG00028349001 PE=3 SV=1

$=\mathrm{L} 0 \mathrm{C} 733408$ PE=2 SV=1

$\mathrm{j}=$ Tetraodon nigroviridis $\mathrm{GN}=\mathrm{rc} 1 \mathrm{PE}=3 \quad \mathrm{SV}=2$

lence. (Fragment) OS=Tetraodon nigroviridis GN=GSTENG00021590001 PE=4 SV=1 $\mathrm{PE}=2 \mathrm{SV}=1$

ənce OS=Tetraodon nigroviridis GN=GSTENG00024610001 PE $=4$ SV=1

?E $=2 \quad \mathrm{SV}=1$

Jtgun sequence. (Fragment) OS=Tetraodon nigroviridis GN=GSTENG00005742001 PE=3 SV=1

$2 \mathrm{SV}=1$

GN=RCJMB04_18k24 PE=4 SV=1

ly OS=Danio rerio GN=CH211-165B19. 8-001 PE=3 SV=1 
t 5 OS=Taeniopygia guttata $\mathrm{PE}=2 \mathrm{SV}=1$

ənce OS=Tetraodon nigroviridis GN=GSTENG00024602001 PE=4 SV=1

dbunit beta -1 OS=Danio rerio $\mathrm{GN}=$ gnb1 $\mathrm{PE}=2 \mathrm{SV}=1$

Jtgun sequence. (Fragment) OS=Tetraodon nigroviridis GN=GSTENG00001016001 PE=4 SV=1

ənce. (Fragment) OS=Tetraodon nigroviridis GN=GSTENG00024788001 PE=4 SV=1

ənce OS=Tetraodon nigroviridis GN=GSTENG00014438001 PE=4 SV=1

$\operatorname{rdax}$ GN=TIP1 PE=2 SV=1

=sf3a3 PE=2 SV=1

luropoda melanoleuca GN=PANDA_004337 PE=3 SV=1

JS=Xenopus tropicalis GN=polr2g PE=2 SV=1

sence. (Fragment) OS=Tetraodon nigroviridis GN=GSTENG00016829001 PE=4 SV=1

Jtgun sequence. (Fragment) OS=Tetraodon nigroviridis GN=GSTENG00004757001 PE=4 SV=1

lence OS=Tetraodon nigroviridis GN=GSTENG00032873001 PE=4 SV=1

गh $\mathrm{PE}=2 \mathrm{SV}=1$

io $\mathrm{GN}=$ aif11 $\mathrm{PE}=2 \mathrm{SV}=1$

गform 0S=Anoplopoma fimbria GN=MLRN PE=2 SV=1

s musculus GN=Epb4. 1 PE=2 SV=1 
imbria $\mathrm{GN}=\mathrm{GSTK} 1 \quad \mathrm{PE}=2 \mathrm{SV}=1$

3. OS=Pongo abelii GN=TOMM20 PE=2 SV=1

jglossus kowalevskii $\mathrm{PE}=2 \mathrm{SV}=1$

t G OS=Salmo salar GN=EIF3G PE=2 SV=1

JS=Danio rerio $\mathrm{GN}=\mathrm{cndp} 2 \mathrm{PE}=2 \mathrm{SV}=1$

intenance of chromosomes 6 (SMC6) OS=Danio rerio GN=si:dkey-119f1. 1 PE=4 SV=1

salar GN=RM43 PE=2 SV=1

lence. (Fragment) OS=Tetraodon nigroviridis GN=GSTENG00027582001 PE=3 SV=1

uence. (Fragment) OS=Tetraodon nigroviridis GN=GSTENG00028701001 PE $=4 \mathrm{SV}=1$

ว $\mathrm{PE}=2 \mathrm{SV}=1$

ait (Fragment) OS=Tetraodon nigroviridis GN=GSTENG00015928001 PE=3 SV=1

?E $=2 \quad \mathrm{SV}=1$

ənce. (Fragment) OS=Tetraodon nigroviridis GN=GSTENG00035051001 PE=4 SV=1

$1 \mathrm{SV}=1$

rerio $\mathrm{GN}=$ ugcg $\mathrm{PE}=2 \mathrm{SV}=1$

$3 \mathrm{~N}=\mathrm{rp} 118 \quad \mathrm{PE}=2 \quad \mathrm{SV}=2$

$\mathrm{z}=2 \mathrm{SV}=1$

ənce. (Fragment) OS=Tetraodon nigroviridis GN=GSTENG00003570001 PE=4 SV=1

rotgun sequence. (Fragment) OS=Tetraodon nigroviridis GN=GSTENG00009546001 PE=4 SV=1

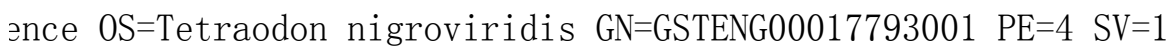

ว $\mathrm{GN}=$ bbip10 $\mathrm{PE}=2 \mathrm{SV}=1$ 
əin 4 OS=0smerus mordax GN=TMBI4 PE=2 SV=1

oria GN=RWDD1 $\mathrm{PE}=2 \mathrm{SV}=1$

lence OS=Tetraodon nigroviridis GN=GSTENG00032982001 PE=4 SV=1

sapiens $\mathrm{GN}=\mathrm{CCDC} 138 \mathrm{PE}=1 \mathrm{SV}=1$

?E $=4 \quad \mathrm{SV}=1$

fined colon cancer antigen 8 OS=Homo sapiens $\mathrm{PE}=2 \mathrm{SV}=1$

$=$ FKB1A PE=3 SV=1

lence OS=Tetraodon nigroviridis GN=GSTENG00018378001 PE=3 SV=1

Eimbria $\mathrm{GN}=\mathrm{SPCS} 1 \mathrm{PE}=4 \mathrm{SV}=1$

salar GN=RM52 PE=2 SV=1

?E=2 SV=1

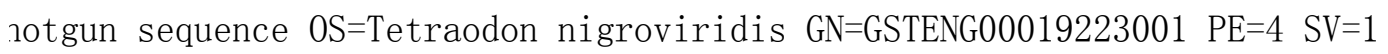

-a $\mathrm{PE}=2 \mathrm{SV}=1$

a specific peptidase 39 (USP39) $0 \mathrm{~S}=$ Danio rerio $\mathrm{GN}=\mathrm{usp} 39 \mathrm{PE}=4 \mathrm{SV}=1$

$7793751 \quad \mathrm{PE}=2$ SV=1

ria GN=PRDX5 PE=2 SV=1

lence OS=Tetraodon nigroviridis GN=GSTENG00034911001 PE=4 SV=1 
ənce OS=Tetraodon nigroviridis GN=GSTENG00024766001 PE=3 SV=1

GN=RCJMB04_19p6 PE=2 SV=1

JS=Salmo salar GN=NDUAB $\mathrm{PE}=2 \quad \mathrm{SV}=1$

io $\mathrm{GN}=\mathrm{zgc}: 110801 \mathrm{PE}=2 \mathrm{SV}=1$

गE $=2 \quad \mathrm{SV}=1$

?E $=2 \quad \mathrm{SV}=1$

$28 \mathrm{PE}=2 \mathrm{SV}=1$

spoma fimbria $\mathrm{GN}=\mathrm{RM} 16 \quad \mathrm{PE}=2 \mathrm{SV}=1$

$\mathrm{z}=2 \mathrm{SV}=1$

1

JPD $\quad \mathrm{PE}=4 \quad \mathrm{SV}=1$

it 1 OS=Anoplopoma fimbria GN=PGRC1 PE=2 SV=1

rerio $\mathrm{GN}=$ idh3a $\mathrm{PE}=2 \mathrm{SV}=1$

$\mathrm{GN}=\mathrm{CSN} 4 \quad \mathrm{PE}=2 \quad \mathrm{SV}=1$

41N20. 1 PE=2 SV=1

ıotgun sequence. (Fragment) OS=Tetraodon nigroviridis GN=GSTENG00025358001 PE=4 SV=1

JD45 alpha 0S=Salmo salar GN=GA45A PE=2 SV=1

JYR $\mathrm{PE}=2 \mathrm{SV}=1$

se, 4 OS=Mus musculus GN=Psmc4 PE=2 SV=1

ənce. (Fragment) OS=Tetraodon nigroviridis GN=GSTENG00017123001 PE=4 SV=1

ənce. (Fragment) OS=Tetraodon nigroviridis GN=GSTENG00029357001 PE=4 SV=1 
shatase $0 \mathrm{~S}=$ Anoplopoma fimbria GN=PPAC PE=2 SV=1

$\mathrm{V}=\mathrm{TM} 14 \mathrm{C} \quad \mathrm{PE}=4 \quad \mathrm{SV}=1$

गa3 $\mathrm{PE}=2 \mathrm{SV}=1$

j=Danio rerio $\mathrm{GN}=\mathrm{c} 3 \mathrm{orf} 33 \mathrm{PE}=4 \mathrm{SV}=1$

tgun sequence OS=Tetraodon nigroviridis GN=GSTENG00002963001 PE=4 SV=1

$V=1$

=Anoplopoma fimbria GN=NDUB6 PE=2 SV=1

lence. (Fragment) OS=Tetraodon nigroviridis GN=GSTENG00022824001 PE=3 SV=1

ənce OS=Tetraodon nigroviridis GN=GSTENG00035891001 PE=4 SV=1

$\mathrm{GN}=\mathrm{h} 1 \mathrm{tf} \quad \mathrm{PE}=4 \mathrm{SV}=1$

nossambicus $\mathrm{PE}=2 \mathrm{SV}=1$

$12 \quad \mathrm{PE}=2 \mathrm{SV}=1$

lence. (Fragment) OS=Tetraodon nigroviridis GN=GSTENG00030642001 PE=4 SV=1 xima $\mathrm{GN}=\mathrm{PP} 1 \mathrm{cb} \mathrm{PE}=2 \mathrm{SV}=1$

lon $0 \mathrm{~S}=$ Salmo salar $\mathrm{GN}=\mathrm{EI} 2 \mathrm{BE} \quad \mathrm{PE}=2 \mathrm{SV}=1$ 
lotgun sequence. (Fragment) OS=Tetraodon nigroviridis GN=GSTENG00025145001 PE=4 SV=1

ta $\mathrm{PE}=2 \mathrm{SV}=1$

ipes GN=SUM01 PE=4 SV=1

$\mathrm{PE}=4 \mathrm{SV}=1$

tochondrial OS=Anoplopoma fimbria GN=SLIRP PE=4 SV=1

lence OS=Tetraodon nigroviridis GN=GSTENG00030807001 PE=4 SV=1

Janio rerio $\mathrm{GN}=$ ndufs $1 \mathrm{PE}=2 \mathrm{SV}=1$

j0 $\mathrm{PE}=2 \mathrm{SV}=1$

lence. (Fragment) OS=Tetraodon nigroviridis GN=GSTENG00029413001 PE=3 SV=1

smerus mordax GN=U3IP2 PE=2 SV=1

ıence. (Fragment) OS=Tetraodon nigroviridis GN=GSTENG00032537001 PE=3 SV=1

3M8A $\quad \mathrm{PE}=2 \quad \mathrm{SV}=1$

ənce OS=Tetraodon nigroviridis GN=GSTENG00022528001 PE=3 SV=1

itochondrial 0S=Anoplopoma fimbria GN=NDUB5 PE=2 SV=1

$\mathrm{PE}=2 \mathrm{SV}=1$

zulatory subunit A beta isoform (Fragment) $0 \mathrm{~S}=\mathrm{Salmo}$ salar $\mathrm{GN}=2 \mathrm{AAB} \quad \mathrm{PE}=2 \mathrm{SV}=1$

ənce. (Fragment) OS=Tetraodon nigroviridis GN=GSTENG00032711001 PE=4 SV=1

Janio rerio $\mathrm{GN}=$ ppil $\mathrm{PE}=2 \mathrm{SV}=1$

$\mathrm{SV}=1$

lence. (Fragment) OS=Tetraodon nigroviridis GN=GSTENG00030193001 PE=4 SV=1

$\mathrm{V}=\mathrm{YEN} 9 \quad \mathrm{PE}=2 \quad \mathrm{SV}=1$

$2 \mathrm{SV}=1$

$\mathrm{SV}=1$

ənce OS=Tetraodon nigroviridis GN=GSTENG00004294001 PE=4 SV=1 
j $\mathrm{PE}=2 \mathrm{SV}=1$

Jtgun sequence. (Fragment) OS=Tetraodon nigroviridis GN=GSTENG00005444001 PE=4 SV=1

rerio $\mathrm{GN}=\mathrm{ube} 2 \mathrm{v} 2 \mathrm{PE}=2 \mathrm{SV}=1$

$=\mathrm{SERF} 2 \mathrm{PE}=4 \mathrm{SV}=1$

ənce OS=Tetraodon nigroviridis GN=GSTENG00021231001 PE=4 SV=1

$1 \mathrm{G} \quad \mathrm{PE}=2 \mathrm{SV}=1$

ənce OS=Tetraodon nigroviridis GN=GSTENG00034729001 PE=4 SV=1

lence OS=Tetraodon nigroviridis GN=GSTENG00035558001 PE=4 SV=1

salar GN=LSM1 $\mathrm{PE}=2 \mathrm{SV}=1$

) $\mathrm{PE}=2 \mathrm{SV}=1$

arifer $\mathrm{GN}=$ dazl $\mathrm{PE}=2 \mathrm{SV}=1$

$\mathrm{J} \mathrm{N}=\mathrm{rab2a} \quad \mathrm{PE}=2 \quad \mathrm{SV}=1$

)6 $\mathrm{PE}=2 \mathrm{SV}=1$

$\mathrm{PE}=2 \mathrm{SV}=1$

1otgun sequence. (Fragment) OS=Tetraodon nigroviridis GN=GSTENG00020646001 PE=4 SV=1

Ælar GN=CHM1B PE=2 SV=1

rdax GN=IFT52 PE=2 SV=1 
ərminal domain containing (NIFUN) OS=Danio rerio GN=si:ch211-191d15. 2 PE=4 SV=1 senegalensis GN=GAPD2 $\mathrm{PE}=2 \mathrm{SV}=1$

(S. cerevisiae) $0 \mathrm{~S}=$ Danio rerio $\mathrm{GN}=\mathrm{rmnd} 5 \mathrm{~b} \mathrm{PE}=2 \mathrm{SV}=1$

rotgun sequence OS=Tetraodon nigroviridis GN=GSTENG00020465001 PE=4 SV=1

dkaryotic translation initiation factor 3, subunit 10theta, 150/170kDa (EIF3S10), 0S=Macace $=1$

$\times \mathrm{GN}=\mathrm{COMD} 6 \mathrm{PE}=2 \mathrm{SV}=1$

20PZ1 PE=2 SV=1

ənce OS=Tetraodon nigroviridis GN=GSTENG00029250001 PE=4 SV=1

ənce. (Fragment) OS=Tetraodon nigroviridis GN=GSTENG00016226001 PE=4 SV=1

ar $\mathrm{GN}=\mathrm{TM} 9 \mathrm{~S} 3 \mathrm{PE}=2 \mathrm{SV}=1$

lence OS=Tetraodon nigroviridis GN=GSTENG00034262001 PE=4 SV=1

Iitobiase, di-N-acetyl- (CTBS), OS=Macaca fascicularis $\mathrm{PE}=2 \mathrm{SV}=1$

$\zeta=X e n o p u s$ tropical is GN=ap1m1 PE=2 SV=1

rotgun sequence OS=Tetraodon nigroviridis GN=GSTENG00019694001 PE=4 SV=1

ance OS=Tetraodon nigroviridis GN=GSTENG00023858001 PE=4 SV=1 
io rerio $\mathrm{GN}=$ nusap1 $\mathrm{PE}=4 \mathrm{SV}=1$

$\mathrm{z}=2 \mathrm{SV}=1$

ənce. (Fragment) OS=Tetraodon nigroviridis GN=GSTENG00020521001 PE=4 SV=1

ənce OS=Tetraodon nigroviridis GN=GSTENG00031446001 PE $=4$ SV=1

ənce $0 \mathrm{~S}=$ Tetraodon nigroviridis GN=GSTENG00024782001 PE=4 SV=1

ənce. (Fragment) OS=Tetraodon nigroviridis GN=GSTENG00033927001 PE=4 SV=1

ia $\mathrm{GN}=\mathrm{NXT} 2 \mathrm{PE}=2 \quad \mathrm{SV}=1$

ıence. (Fragment) OS=Tetraodon nigroviridis GN=GSTENG00029514001 PE=4 SV=1

$\mathrm{V}=\mathrm{rp} 128 \quad \mathrm{PE}=2 \quad \mathrm{SV}=1$

गoma fimbria GN=TXND9 PE=2 SV=1

tsi $\mathrm{PE}=3 \mathrm{SV}=1$

ənce. (Fragment) OS=Tetraodon nigroviridis GN=GSTENG00030437001 PE=4 SV=1

गoma fimbria GN=REEP5 PE=2 SV=1

spoma fimbria GN=PHS PE=3 SV=1

$26 \mathrm{PE}=2 \mathrm{SV}=1$

lence OS=Tetraodon nigroviridis GN=GSTENG00018811001 PE=4 SV=1

Jtgun sequence $0 \mathrm{~S}=$ Tetraodon nigroviridis GN=GSTENG00006968001 PE=4 SV=1

¿ATH domain protein OS=0ncorhynchus mykiss GN=tradd PE=2 SV=1 
rio $\mathrm{GN}=$ ctu1 $\mathrm{PE}=2 \mathrm{SV}=1$

shatase $0 \mathrm{~S}=$ Salmo salar $\mathrm{GN}=\mathrm{PPAC} \mathrm{PE}=2 \mathrm{SV}=1$

ənce OS=Tetraodon nigroviridis GN=GSTENG00031888001 PE=4 SV=1

it $\mathrm{PE}=2 \mathrm{SV}=1$

jV=1

zorhynchus mykiss GN=TMA20 PE=2 SV=1

nykiss $\mathrm{PE}=2 \mathrm{SV}=1$

$\mathrm{V}=\mathrm{RS} 28 \quad \mathrm{PE}=4 \quad \mathrm{SV}=1$

1 5 OS=Salmo salar GN=USMG5 $\mathrm{PE}=4 \mathrm{SV}=1$

latsi $\mathrm{PE}=2 \mathrm{SV}=1$

ar $\mathrm{GN}=\mathrm{CAPZB} \mathrm{PE}=2 \mathrm{SV}=1$

visiae) OS=Xenopus tropicalis $\mathrm{GN}=\mathrm{ccrn} 41 \mathrm{PE}=2 \mathrm{SV}=1$

lence. (Fragment) OS=Tetraodon nigroviridis GN=GSTENG00020840001 PE=4 SV=1

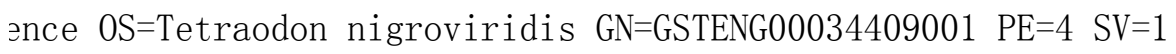


floridae GN=BRAFLDRAFT_211971 PE=4 SV=1

ys seta $\mathrm{PE}=2 \mathrm{SV}=1$

subunit B' subunit gamma OS=Danio rerio GN=ppp2r3c PE=2 SV=1

imbria GN=RUXG PE=4 SV=1

lence OS=Tetraodon nigroviridis GN=GSTENG00017418001 PE=4 SV=1

ənce OS=Tetraodon nigroviridis GN=GSTENG00034700001 PE=4 SV=1

əns is $\mathrm{GN}=\mathrm{v} 1 \mathrm{~g} 134024 \mathrm{PE}=4 \mathrm{SV}=1$

s norvegicus $\mathrm{GN}=\mathrm{L} 0 \mathrm{C6} 66092 \mathrm{PE}=4 \mathrm{SV}=1$

$=2 \mathrm{SV}=1$

i f. nagariensis GN=VOLCADRAFT_93889 PE=4 SV=1

गx lucius GN=ARPC3 PE=2 SV=1

lence. (Fragment) OS=Tetraodon nigroviridis GN=GSTENG00015895001 PE=4 SV=1

$=2 \mathrm{SV}=1$

mykiss $\mathrm{GN}=\mathrm{BAF} \mathrm{PE}=4 \mathrm{SV}=1$

coioides $\mathrm{PE}=2 \mathrm{SV}=1$

nbria $\mathrm{GN}=\mathrm{UPP} \quad \mathrm{PE}=2 \quad \mathrm{SV}=1$

ə fimbria GN=TEX12 PE=2 SV=1 
s musculus $\mathrm{GN}=2410042 \mathrm{D} 21 \mathrm{Rik} \mathrm{PE}=2 \mathrm{SV}=1$

ərio $\mathrm{GN}=\mathrm{kpna} 4 \mathrm{PE}=2 \mathrm{SV}=1$

lence OS=Tetraodon nigroviridis GN=GSTENG00031357001 PE=3 SV=1

j $\mathrm{PE}=2 \mathrm{SV}=1$

punctatus $\mathrm{PE}=2 \mathrm{SV}=1$

ənce OS=Tetraodon nigroviridis GN=GSTENG00026555001 PE=3 SV=1

$2 \mathrm{SV}=1$

lence. (Fragment) OS=Tetraodon nigroviridis GN=GSTENG00029506001 PE=4 SV=1

$4 F 3 \quad P E=2 \quad S V=1$

cerevisiae) $0 \mathrm{~S}=$ Danio rerio $\mathrm{GN}=$ iah1 $\mathrm{PE}=2 \mathrm{SV}=1$

lence. (Fragment) OS=Tetraodon nigroviridis GN=GSTENG00033020001 PE=4 SV=1

ənce OS=Tetraodon nigroviridis GN=GSTENG00014386001 PE=4 SV=1

rotgun sequence. (Fragment) OS=Tetraodon nigroviridis GN=GSTENG00023248001 PE=4 SV=1

ənce. (Fragment) OS=Tetraodon nigroviridis GN=GSTENG00024220001 PE=4 SV=1

$3 \mathrm{~N}=\mathrm{NDC} 80 \quad \mathrm{PE}=2 \mathrm{SV}=1$

salar $\mathrm{GN}=\operatorname{cog} 2 \mathrm{PE}=2 \mathrm{SV}=1$

ənce OS=Tetraodon nigroviridis GN=GSTENG00015451001 PE=4 SV=1

ənce OS=Tetraodon nigroviridis GN=GSTENG00003506001 PE=4 SV=1

ıotgun sequence. (Fragment) OS=Tetraodon nigroviridis GN=GSTENG00010358001 PE=4 SV=1

r 2 OS=Danio rerio $\mathrm{GN}=$ abcf $2 \mathrm{PE}=2 \mathrm{SV}=1$

aianus $\mathrm{GN}=$ cytb $\mathrm{PE}=3 \mathrm{SV}=1$

ənce. (Fragment) OS=Tetraodon nigroviridis GN=GSTENG00032795001 PE=4 SV=1 
IN=ilvb1 PE=2 SV=1

ıotgun sequence. (Fragment) OS=Tetraodon nigroviridis GN=GSTENG00013913001 PE=4 SV=1

$\mathrm{I}=2 \mathrm{SV}=1$

nbria GN=SBDS PE=2 SV=1

yltransferase subunit STT3A OS=Salmo salar GN=STT3A PE=2 SV=1

lence. (Fragment) OS=Tetraodon nigroviridis GN=GSTENG00013639001 PE=4 SV=1

(TUBE1) OS=Danio rerio GN=tube1 $\mathrm{PE}=3 \mathrm{SV}=1$

$=1$

$2 \mathrm{PE}=2 \mathrm{SV}=1$

ənce. (Fragment) OS=Tetraodon nigroviridis GN=GSTENG00014662001 PE=4 SV=1 ataining 1 OS=Danio rerio $\mathrm{GN}=\mathrm{gdpd} 1 \mathrm{PE}=2 \mathrm{SV}=1$

lence. (Fragment) OS=Tetraodon nigroviridis GN=GSTENG00020808001 PE=4 SV=1

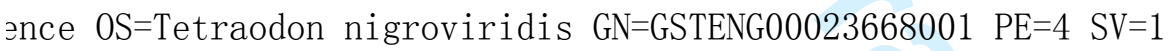

$\mathrm{PE}=2 \mathrm{SV}=1$

lence. (Fragment) OS=Tetraodon nigroviridis GN=GSTENG00033317001 PE=4 SV=1 ar GN=NTAN1 PE=2 SV=1

dependent) $0 \mathrm{~S}=$ Danio rerio $\mathrm{GN}=\mathrm{pla} 2 \mathrm{~g} 6 \mathrm{PE}=2 \mathrm{SV}=1$

lence. (Fragment) OS=Tetraodon nigroviridis GN=GSTENG00016999001 PE=4 SV=1

rotgun sequence. (Fragment) OS=Tetraodon nigroviridis GN=GSTENG00009999001 PE=3 SV=1

ıotgun sequence. (Fragment) OS=Tetraodon nigroviridis GN=GSTENG00019333001 PE=4 SV=1

эps $1 \quad \mathrm{PE}=2 \mathrm{SV}=1$

nucleolar RNPs) 0S=Xenopus tropicalis $\mathrm{GN}=$ nop10 PE=4 SV=1 
2s $\mathrm{GN}=\operatorname{surf6} \mathrm{PE}=3 \mathrm{SV}=1$

ing 4 OS=Anoplopoma fimbria GN=PIN4 PE=2 SV=1

$=1$

sparus aurata GN=NCCRP-1 PE=2 SV=1

lotgun sequence. (Fragment) OS=Tetraodon nigroviridis GN=GSTENG00012530001 PE=3 SV=1

ria $\mathrm{GN}=\mathrm{HMGB} 2 \mathrm{PE}=2 \mathrm{SV}=1$

ıence. (Fragment) OS=Tetraodon nigroviridis GN=GSTENG00010805001 PE=4 SV=1

na fimbria GN=MTG1 PE=2 SV=1

ənce. (Fragment) OS=Tetraodon nigroviridis GN=GSTENG00022057001 PE=4 SV=1

lence OS=Tetraodon nigroviridis GN=GSTENG00018810001 PE=4 SV=1

ənce OS=Tetraodon nigroviridis GN=GSTENG00028264001 PE=3 SV=1

VTK $\mathrm{PE}=2 \mathrm{SV}=1$

lence OS=Tetraodon nigroviridis GN=GSTENG00009848001 PE=4 SV=1 
$\mathrm{V}=\mathrm{znf} 654 \mathrm{PE}=2 \mathrm{SV}=1$

ticus $\mathrm{PE}=2 \mathrm{SV}=1$

IBP $\mathrm{PE}=2 \mathrm{SV}=1$

rotgun sequence OS=Tetraodon nigroviridis GN=GSTENG00009556001 PE=4 SV=1

lence. (Fragment) OS=Tetraodon nigroviridis GN=GSTENG00028388001 PE=4 SV=1

ənce. (Fragment) OS=Tetraodon nigroviridis GN=GSTENG00024620001 PE=4 SV=1 s $\mathrm{PE}=2 \mathrm{SV}=1$

lence OS=Tetraodon nigroviridis GN=GSTENG00016951001 PE=4 SV=1 э235000 PE=3 SV=1

luropoda melanoleuca GN=PANDA_005916 PE=4 SV=1

oria GN=ATIF1 $\mathrm{PE}=4 \mathrm{SV}=1$

əpiens GN=DNAH10 PE=4 SV=2

$=1$

ənce. (Fragment) OS=Tetraodon nigroviridis GN=GSTENG00015434001 PE=4 SV=1

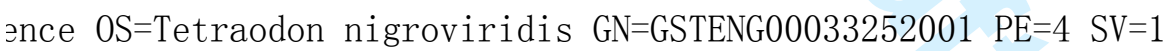

JS=Tetraodon nigroviridis GN=GSTENG00035209001 PE=3 SV=1

$100158493 \mathrm{PE}=2 \mathrm{SV}=1$

$=2 \mathrm{SV}=1$

$=$ MAGT $1 \quad \mathrm{PE}=2 \quad \mathrm{SV}=1$

गk $\mathrm{PE}=3 \mathrm{SV}=1$

ance OS=Tetraodon nigroviridis GN=GSTENG00030538001 PE $=4 \mathrm{SV}=1$

) $\mathrm{GN}=\mathrm{e} 11 \mathrm{PE}=2 \mathrm{SV}=1$

$\operatorname{rdax} \quad \mathrm{GN}=\mathrm{GDIB} \quad \mathrm{PE}=2 \quad \mathrm{SV}=1$

lence. (Fragment) OS=Tetraodon nigroviridis GN=GSTENG00027336001 PE=4 SV=1 nbicus $\mathrm{PE}=2 \mathrm{SV}=1$

$\mathrm{Z}=2 \mathrm{SV}=1$ 
rgus sarba $\mathrm{PE}=2 \mathrm{SV}=1$

lotgun sequence. (Fragment) OS=Tetraodon nigroviridis GN=GSTENG00025323001 PE=4 SV=1

ənce. (Fragment) OS=Tetraodon nigroviridis GN=GSTENG00017319001 PE=4 SV=1

splopoma fimbria GN=IFT20 PE=2 SV=1

ənce. (Fragment) OS=Tetraodon nigroviridis GN=GSTENG00015602001 PE=4 SV=1

$=h v c n 1 \quad \mathrm{PE}=2 \mathrm{SV}=1$

?E=2 SV=1

lence. (Fragment) OS=Tetraodon nigroviridis GN=GSTENG00033323001 PE=4 SV=1

ग27a $\mathrm{PE}=2$ SV=1

lence. (Fragment) OS=Tetraodon nigroviridis GN=GSTENG00014947001 PE=4 SV=1

ənce. (Fragment) OS=Tetraodon nigroviridis GN=GSTENG00018370001 PE=4 SV=1

$\mathrm{V}=\mathrm{TM} 45 \mathrm{~B} \quad \mathrm{PE}=2 \quad \mathrm{SV}=1$

luropoda melanoleuca GN=PANDA_003630 PE=4 SV=1

Xenopus tropicalis $\mathrm{GN}=1 \mathrm{sm} 2 \mathrm{PE}=4 \mathrm{SV}=1$

ar $\mathrm{GN}=\mathrm{ppp} 1 \mathrm{cc}$ PE=2 SV=1

$\mathrm{z}=2 \mathrm{SV}=1$

lence OS=Tetraodon nigroviridis GN=GSTENG00035697001 PE=4 SV=1

PE=2 SV=1

=Anoplopoma fimbria GN=CDKA2 $\mathrm{PE}=4 \mathrm{SV}=1$

$=2 \mathrm{SV}=1$

JS=0ncorhynchus mykiss GN=TAF10 PE=2 SV=1

12, zgc:77113) OS=Danio rerio GN=pgm2 PE=2 SV=1

a fimbria GN=PRS10 PE=2 SV=1 
it RPABC5 OS=Anoplopoma fimbria GN=RPAB5 PE=3 SV=1

ənce. (Fragment) 0S=Tetraodon nigroviridis GN=GSTENG00027061001 PE=4 SV=1

stgun sequence. (Fragment) OS=Tetraodon nigroviridis GN=GSTENG00005730001 PE=4 SV=1

$=A P 1 S 2 \quad P E=2 \quad S V=1$

in-like 1 OS=0smerus mordax GN=GRL1 PE=3 SV=1

$\operatorname{lar}$ GN=IVD PE=2 SV=1

ənce. (Fragment) 0S=Tetraodon nigroviridis GN=GSTENG00017805001 PE=4 SV=1

it 20 OS=Salmo salar GN=MED20 PE=2 SV=1

1-223P8. 4 PE=4 SV=1

IRP5 PE=2 SV=1

ling protein 7 OS=Anoplopoma fimbria GN=CHCH7 $\mathrm{PE}=4$ SV=1

lence OS=Tetraodon nigroviridis GN=GSTENG00022692001 PE=4 SV=1

tgun sequence $0 \mathrm{~S}=$ Tetraodon nigroviridis GN=GSTENG00004290001 PE=4 SV=1

$\mathrm{z}=2 \mathrm{SV}=1$

PE $=2 \quad \mathrm{SV}=1$

lence OS=Tetraodon nigroviridis GN=GSTENG00026867001 PE=4 SV=1

lence OS=Tetraodon nigroviridis GN=GSTENG00029628001 PE=4 SV=1

notgun sequence. (Fragment) OS=Tetraodon nigroviridis GN=GSTENG00034342001 PE=4 SV=1

rotgun sequence. (Fragment) OS=Tetraodon nigroviridis GN=GSTENG00022435001 PE=4 SV=1

$\mathrm{GN}=$ prcla $\mathrm{PE}=2 \mathrm{SV}=1$

$\mathrm{PE}=4 \mathrm{SV}=1$

Jtgun sequence. (Fragment) OS=Tetraodon nigroviridis GN=GSTENG00007581001 PE=4 SV=1 
$\mathrm{V}=2$

$\mathrm{z}=2 \mathrm{SV}=1$

lence. (Fragment) OS=Tetraodon nigroviridis GN=GSTENG00033898001 PE=4 SV=1

rotgun sequence OS=Tetraodon nigroviridis GN=GSTENG00025888001 PE=4 SV=1

Jtgun sequence. (Fragment) OS=Tetraodon nigroviridis GN=GSTENG00001229001 PE=4 SV=1

lence. (Fragment) OS=Tetraodon nigroviridis GN=GSTENG00018716001 PE=4 SV=1

nbria $\mathrm{GN}=\mathrm{COMD} 2 \mathrm{PE}=2 \quad \mathrm{SV}=1$

$=$ RL7 PE=2 SV=1

$\mathrm{PE}=2 \quad \mathrm{SV}=1$

V=RBBP $6 \quad$ PE=2 SV=1

lence OS=Tetraodon nigroviridis GN=GSTENG00027365001 PE=4 SV=1

əsus GN=rplp1 PE=4 SV=1

$\mathrm{SV}=1$

$=0 \mathrm{STF} 1 \quad \mathrm{PE}=2 \quad \mathrm{SV}=1$

j $\mathrm{PE}=2 \mathrm{SV}=1$ 
$\mathrm{GN}=\mathrm{crfb} 5 \mathrm{PE}=2 \mathrm{SV}=1$

sion factor 2 (MYEF2) (Fragment) OS=Danio rerio GN=hnrnpm PE=1 SV=1

oria $\mathrm{GN}=\mathrm{NPC} 2 \mathrm{PE}=2 \mathrm{SV}=1$

lence. (Fragment) OS=Tetraodon nigroviridis GN=GSTENG00026307001 PE=4 SV=1

OS=Epinephelus coioides $\mathrm{PE}=2 \mathrm{SV}=1$

$\mathrm{V}=\mathrm{RM} 34 \quad \mathrm{PE}=2 \quad \mathrm{SV}=1$

ıence. (Fragment) OS=Tetraodon nigroviridis GN=GSTENG00034212001 PE=4 SV=1

JS=Takifugu rubripes GN=eif2b2 $\mathrm{PE}=3 \mathrm{SV}=1$

rotgun sequence. (Fragment) OS=Tetraodon nigroviridis GN=GSTENG00008434001 PE=4 SV=1

ənce. (Fragment) 0S=Tetraodon nigroviridis GN=GSTENG00018007001 PE=4 SV=1

ZFAN1 PE=2 SV=1

lence. (Fragment) OS=Tetraodon nigroviridis GN=GSTENG00016728001 PE=4 SV=1 sius auratus $\mathrm{GN}=\operatorname{apc} 11 \mathrm{PE}=4 \mathrm{SV}=1$

is norvegicus GN=RGD1305464 $\mathrm{PE}=4 \mathrm{SV}=1$

ənce. (Fragment) OS=Tetraodon nigroviridis GN=GSTENG00034076001 PE=4 SV=1

ənce. (Fragment) 0S=Tetraodon nigroviridis GN=GSTENG00036631001 PE=4 SV=1

=Esox lucius GN=TPPC2 PE=2 SV=1 
rotgun sequence. (Fragment) OS=Tetraodon nigroviridis GN=GSTENG00021806001 PE=4 SV=1

Jalmo salar GN=ATP5J PE=2 SV=1

notgun sequence. (Fragment) OS=Tetraodon nigroviridis GN=GSTENG00021842001 PE=4 SV=1

spus tropicalis $\mathrm{GN}=$ recq14 $\mathrm{PE}=2 \mathrm{SV}=1$

x GN=DDX39 PE=2 SV=1

ənce OS=Tetraodon nigroviridis GN=GSTENG00024696001 PE=3 SV=1

lence. (Fragment) OS=Tetraodon nigroviridis GN=GSTENG00028405001 PE=4 SV=1

io $\mathrm{GN}=\mathrm{kde} \operatorname{lr} 2 \mathrm{PE}=2 \mathrm{SV}=1$

$\mathrm{V}=\mathrm{GLYAT} \quad \mathrm{PE}=2 \quad \mathrm{SV}=1$

गE $=2 \quad \mathrm{SV}=1$

ənce. (Fragment) OS=Tetraodon nigroviridis GN=GSTENG00028235001 PE=4 SV=1

ənce. (Fragment) OS=Tetraodon nigroviridis GN=GSTENG00014485001 PE=4 SV=1

lence. (Fragment) OS=Tetraodon nigroviridis GN=GSTENG00026395001 PE=4 SV=1

lence OS=Tetraodon nigroviridis GN=GSTENG00014087001 PE=4 SV=1

Fragment) $0 \mathrm{~S}=\mathrm{Gallus}$ gallus $\mathrm{GN}=\mathrm{ZBTB} 17 \mathrm{PE}=2 \mathrm{SV}=1$

zription factor IIF, polypeptide 2, 30kDa (GTF2F2, zgc:86775) OS=Danio rerio GN=DKEY-151M2 $\leftarrow$

$\mathrm{z}=2 \mathrm{SV}=1$

Jtgun sequence OS=Tetraodon nigroviridis GN=GSTENG00004506001 PE=4 SV=1

$\mathrm{PE}=2 \mathrm{SV}=1$

is $\mathrm{GN}=\mathrm{ddx} 1 \mathrm{PE}=2 \mathrm{SV}=1$

lence. (Fragment) OS=Tetraodon nigroviridis GN=GSTENG00030239001 PE=4 SV=1

lar $\mathrm{GN}=\mathrm{SC} 61 \mathrm{G} \quad \mathrm{PE}=4 \mathrm{SV}=1$

uence. (Fragment) OS=Tetraodon nigroviridis GN=GSTENG00014161001 PE=4 SV=1

$\times$ subunit 6 OS=Tetraodon nigroviridis GN=GSTENG00020485001 PE=3 SV=1

n $0 \mathrm{~S}=$ Esox lucius GN=PIPNB PE=2 SV=1

PE $=2 \quad \mathrm{SV}=1$

=Anoplopoma fimbria GN=CT030 PE=2 SV=1 
गsome 9 open reading frame 64 (C9orf64, zgc:136564) OS=Danio rerio GN=CH211-37P17. 6 PE=2 SI lence. (Fragment) OS=Tetraodon nigroviridis GN=GSTENG00026305001 PE=4 SV=1

lence. (Fragment) OS=Tetraodon nigroviridis GN=GSTENG00016836001 PE=4 SV=1

zontaining protein OS=Mus musculus GN=Gm14412 PE=4 SV=1

ance OS=Tetraodon nigroviridis GN=GSTENG00024701001 PE $=4$ SV=1

$5563316 \mathrm{PE}=2 \mathrm{SV}=1$

$\mathrm{z}=2 \mathrm{SV}=1$

lence. (Fragment) OS=Tetraodon nigroviridis GN=GSTENG00028403001 PE=4 SV=1

j=Danio rerio GN=atp6v1ba $\mathrm{PE}=2 \mathrm{SV}=1$

lence OS=Tetraodon nigroviridis GN=GSTENG00015100001 PE=4 SV=1

$\mathrm{J}=\mathrm{UBE} 2 \mathrm{~A} \quad \mathrm{PE}=2 \quad \mathrm{SV}=1$

lence. (Fragment) OS=Tetraodon nigroviridis GN=GSTENG00019795001 PE=3 SV=1

ənce. (Fragment) OS=Tetraodon nigroviridis GN=GSTENG00027102001 PE=4 SV=1 $=4 \mathrm{SV}=1$

ənce. (Fragment) OS=Tetraodon nigroviridis GN=GSTENG00017172001 PE=4 SV=1

ance. (Fragment) OS=Tetraodon nigroviridis GN=GSTENG00017018001 PE=3 SV=1

j=Esox lucius GN=TMED2 PE=2 SV=1

lence. (Fragment) OS=Tetraodon nigroviridis GN=GSTENG00027669001 PE=4 SV=1

lence. (Fragment) OS=Tetraodon nigroviridis GN=GSTENG00020435001 PE=4 SV=1

lence OS=Tetraodon nigroviridis GN=GSTENG00028592001 PE=4 SV=1

$\Xi=2 \quad S V=1$

lence. (Fragment) OS=Tetraodon nigroviridis GN=GSTENG00010170001 PE=4 SV=1

$\mathrm{APV}-2005 \mathrm{GN}=\mathrm{rpS} 20$ e $\mathrm{PE}=2 \mathrm{SV}=1$

rio GN=DKEY-9503. 3 PE=4 SV=1

adrial 0S=0smerus mordax GN=ISAM2 PE=2 SV=1

slucosaminephosphotransferase 0S=Cricetulus longicaudatus GN=DPAGT1 PE=2 SV=1

stein $0 \mathrm{~S}=$ Danio rerio $\mathrm{GN}=1 \mathrm{man} 21 \mathrm{a} \mathrm{PE}=2 \mathrm{SV}=1$ 
lence. (Fragment) OS=Tetraodon nigroviridis GN=GSTENG00028415001 PE=4 SV=1 Jria GN=PSMG3 PE=2 SV=1

lence. (Fragment) OS=Tetraodon nigroviridis GN=GSTENG00019989001 PE=4 SV=1 tsi $\mathrm{PE}=2 \mathrm{SV}=1$

2, 8kDa OS=Homo sapiens GN=NDUFB2 PE=4 SV=1

$=4 \mathrm{SV}=1$

ənce OS=Tetraodon nigroviridis GN=GSTENG00032719001 PE $=4$ SV=1

lence OS=Tetraodon nigroviridis GN=GSTENG00033822001 PE=4 SV=1

t M OS=Anoplopoma fimbria GN=EIF3M PE=2 SV=1

no salar GN=MECR $\mathrm{PE}=2 \mathrm{SV}=1$

$\mathrm{z}=2 \mathrm{SV}=1$

$\mathrm{sV}=1$

ima $\mathrm{PE}=4 \mathrm{SV}=1$

JS=Anoplopoma fimbria GN=GCSH PE=2 SV=1

ıotgun sequence. (Fragment) OS=Tetraodon nigroviridis GN=GSTENG00009306001 PE=4 SV=1

JS=Epinephelus coioides PE=2 SV=1

a fimbria GN=GOSR1 PE=2 SV=1

77-13-4 / FGSC 9596 / MPVI) GN=NECHADRAFT_80808 PE=4 SV=1

ıotgun sequence. (Fragment) OS=Tetraodon nigroviridis GN=GSTENG00036259001 PE=4 SV=1

loplites interruptus GN=Arin-DAB PE=2 SV=1 
lence. (Fragment) OS=Tetraodon nigroviridis GN=GSTENG00023085001 PE=4 SV=1 mordax GN=ARL1 PE=2 SV=1

ənt) $0 \mathrm{~S}=$ Takifugu rubripes $\mathrm{GN}=\mathrm{USP} 3 \mathrm{~L} \quad \mathrm{PE}=4 \mathrm{SV}=1$

idopsis thaliana GN=At5g $45550 \mathrm{PE}=2 \mathrm{SV}=1$

oria $\mathrm{GN}=\mathrm{FIS} 1 \mathrm{PE}=2 \mathrm{SV}=1$

lotgun sequence. (Fragment) OS=Tetraodon nigroviridis GN=GSTENG00008021001 PE=3 SV=1

lence. (Fragment) OS=Tetraodon nigroviridis GN=GSTENG00034273001 PE=4 SV=1

lence OS=Tetraodon nigroviridis GN=GSTENG00020739001 PE=3 SV=1

) $\mathrm{GN}=\mathrm{slc} 31 \mathrm{a} 1 \mathrm{PE}=2 \mathrm{SV}=1$

ənce. (Fragment) OS=Tetraodon nigroviridis GN=GSTENG00026953001 PE=4 SV=1

$\mathrm{jV}=1$

$\mathrm{E}=2 \mathrm{SV}=1$

$=2 \mathrm{SV}=1$

orm a OS=Rattus norvegicus GN=LOC294154 PE=2 SV=1

गviridis GN=GSTENG00021872001 PE=3 SV=1

ənce. (Fragment) OS=Tetraodon nigroviridis GN=GSTENG00014445001 PE=4 SV=1

GN=RCJMB04_29h3 PE=2 SV=1

$\mathrm{GN}=\mathrm{CDC} 5 \mathrm{~L} \quad \mathrm{PE}=2 \mathrm{SV}=1$

ке 2 OS=Salmo salar $\mathrm{PE}=2 \mathrm{SV}=1$

$=1$ 
ıotgun sequence OS=Tetraodon nigroviridis GN=GSTENG00007939001 PE=4 SV=1

$\mathrm{Z}=2 \quad \mathrm{SV}=1$

๖roviridis GN=GSTENG00008972001 PE=3 SV=1

JS=0smerus mordax GN=RPA5 PE=2 SV=1

GN=NSE2 $\quad \mathrm{PE}=2 \quad \mathrm{SV}=1$

JS=Paralichthys olivaceus GN=Cctz PE=2 SV=1

गma fimbria GN=RANG PE=2 SV=1

fimbria $\mathrm{GN}=\mathrm{HSBP} 1 \mathrm{PE}=4 \mathrm{SV}=1$

ənce. (Fragment) OS=Tetraodon nigroviridis GN=GSTENG00035363001 PE=4 SV=1

ənce. (Fragment) OS=Tetraodon nigroviridis GN=GSTENG00020104001 PE=4 SV=1

E $=2 \quad \mathrm{SV}=1$

ənce OS=Tetraodon nigroviridis GN=GSTENG00030520001 PE=4 SV=1

OS=0ncorhynchus mykiss GN=IMP3 PE=2 SV=1

$=2 \mathrm{SV}=1$

¥1 is $\mathrm{GN}=\operatorname{rp} 12211 \mathrm{PE}=2 \mathrm{SV}=1$

ance. (Fragment) OS=Tetraodon nigroviridis GN=GSTENG00017334001 PE=4 SV=1

lence OS=Tetraodon nigroviridis GN=GSTENG00026214001 PE=4 SV=1 
lence. (Fragment) OS=Tetraodon nigroviridis GN=GSTENG00031959001 PE=4 SV=1

-5-phosphate aldolase homolog (C. elegans) (DERA, zgc:101610) OS=Danio rerio GN=DKEY-150I1?

ənase (Ubiquinone) 1 alpha subcomplex, assembly factor 1 (NDUFAF1, zgc:113197) 0S=Danio rer ə $\mathrm{GN}=\mathrm{TUSC} 2 \mathrm{PE}=4 \mathrm{SV}=1$

io $\mathrm{GN}=z g c: 66022 \mathrm{PE}=1 \mathrm{SV}=1$

ribolium castaneum GN=GLEAN_15765 PE=4 SV=1

lence. (Fragment) OS=Tetraodon nigroviridis GN=GSTENG00015064001 PE=3 SV=1

$7 \mathrm{PE}=2 \mathrm{SV}=1$

lent 1 (EXOSC1) OS=Danio rerio $\mathrm{GN}=\operatorname{exosc} 1 \mathrm{PE}=4 \mathrm{SV}=1$

ænio rerio GN=ofd1 $\mathrm{PE}=2 \mathrm{SV}=1$

GN=SPCS3 PE=2 SV=1

ənce OS=Tetraodon nigroviridis GN=GSTENG00034432001 PE=4 SV=1

(Fragment) OS=Homo sapiens $\mathrm{PE}=2 \quad \mathrm{SV}=1$

rotgun sequence OS=Tetraodon nigroviridis GN=GSTENG00010234001 PE=4 SV=1

lence. (Fragment) OS=Tetraodon nigroviridis GN=GSTENG00030881001 PE=4 SV=1

ənce. (Fragment) OS=Tetraodon nigroviridis GN=GSTENG00034101001 PE=4 SV=1

sine-rich repeat protein family OS=Danio rerio GN=DKEY-9I23. 3 PE=4 SV=1 
rotgun sequence. (Fragment) OS=Tetraodon nigroviridis GN=GSTENG00020783001 PE=4 SV=1

$\mathrm{GN}=\mathrm{ZHANG} \quad \mathrm{PE}=2 \quad \mathrm{SV}=1$

y' GN=Dj1 PE=2 SV=1

no salar GN=CY1 PE=2 SV=1

$\mathrm{V}=1$

ənce. (Fragment) OS=Tetraodon nigroviridis GN=GSTENG00019443001 PE=4 SV=1

romis mossambicus $\mathrm{PE}=2 \mathrm{SV}=1$

) $\mathrm{PE}=2 \mathrm{SV}=1$

$=$ PSMG1 PE=2 SV=1

$\mathrm{V}=1$

ənce. (Fragment) OS=Tetraodon nigroviridis GN=GSTENG00032745001 PE=4 SV=1

lence. (Fragment) OS=Tetraodon nigroviridis GN=GSTENG00023316001 PE=4 SV=1

$j \mathrm{~V}=1$

Jtgun sequence OS=Tetraodon nigroviridis GN=GSTENG00003719001 PE=4 SV=1

stgun sequence. (Fragment) OS=Tetraodon nigroviridis GN=GSTENG00006838001 PE=4 SV=1

lence OS=Tetraodon nigroviridis GN=GSTENG00028591001 PE=4 SV=1

mmplex, subunit d OS=Danio rerio $\mathrm{GN}=$ atp5h $\mathrm{PE}=2 \mathrm{SV}=1$

s aceratus $\mathrm{PE}=2 \mathrm{SV}=1$ 
lence. (Fragment) OS=Tetraodon nigroviridis GN=GSTENG00030996001 PE=3 SV=1 rotgun sequence. (Fragment) OS=Tetraodon nigroviridis GN=GSTENG00035068001 PE=4 SV=1

J=Anoplopoma fimbria GN=NDUA1 PE=4 SV=1

lence. (Fragment) OS=Tetraodon nigroviridis GN=GSTENG00020966001 PE=4 SV=1

2NA synthetase (NARS) OS=Danio rerio GN=si:dkey-274m14. 2 PE=3 SV=1 $=1$ OS=Danio rerio $\mathrm{GN}=$ immt $\mathrm{PE}=2 \mathrm{SV}=1$

j=Salmo salar GN=VP33A PE=2 SV=1

$V=1$

adrial OS=Anoplopoma fimbria GN=NDUS7 PE=2 SV=1

$42 \quad \mathrm{PE}=3 \mathrm{SV}=1$

lence. (Fragment) OS=Tetraodon nigroviridis GN=GSTENG00029438001 PE=4 SV=1

ə fimbria GN=RFA2 $\mathrm{PE}=2 \mathrm{SV}=1$

1. nigroviridis GN=GSTENG00023876001 PE=3 SV=1

ənce. (Fragment) OS=Tetraodon nigroviridis GN=GSTENG00029894001 PE=4 SV=1 
lmo salar GN=LAMP2 $\mathrm{PE}=2$ SV=1

э1 PE=2 SV=1

$\mathrm{GN}=\operatorname{cox} 7 \mathrm{c} \quad \mathrm{PE}=4 \mathrm{SV}=1$

rio $\mathrm{GN}=\mathrm{hmm}$ PE=2 $\mathrm{SV}=1$

$\mathrm{SV}=1$

$-216 \mathrm{e} 9.4 \mathrm{PE}=2 \mathrm{SV}=1$

=CHLREDRAFT_155068 PE=4 SV=1

?E $=1 \quad \mathrm{SV}=1$

ənce OS=Tetraodon nigroviridis GN=GSTENG00033224001 PE=4 SV=1 $\mathrm{GN}=\mathrm{RCJMB} 04 \_12 \mathrm{~m} 17 \quad \mathrm{PE}=2 \quad \mathrm{SV}=1$ 
lence. (Fragment) OS=Tetraodon nigroviridis GN=GSTENG00032199001 PE=4 SV=1

$\approx r 4 \mathrm{a} \quad \mathrm{PE}=2 \mathrm{SV}=1$

Jtgun sequence OS=Tetraodon nigroviridis GN=GSTENG00003596001 PE=4 SV=1

lotgun sequence. (Fragment) OS=Tetraodon nigroviridis GN=GSTENG00009171001 PE=4 SV=1

$\mathrm{GN}=\mathrm{ATIF} 1 \quad \mathrm{PE}=4 \quad \mathrm{SV}=1$

) $0 \mathrm{~S}=$ Hypophthalmichthys molitrix $\mathrm{PE}=2 \mathrm{SV}=1$

soma fimbria GN=RT21 PE=4 SV=1

icus $\mathrm{PE}=2 \mathrm{SV}=1$

lence. (Fragment) OS=Tetraodon nigroviridis GN=GSTENG00029512001 PE=4 SV=1

lence. (Fragment) OS=Tetraodon nigroviridis GN=GSTENG00014058001 PE=4 SV=1

aio rerio $\mathrm{GN}=\mathrm{si}: \mathrm{ch} 211-153 \mathrm{j} 24.6 \mathrm{PE}=2 \mathrm{SV}=1$

adrial OS=Salmo salar GN=NDUS6 $\mathrm{PE}=2 \mathrm{SV}=1$

GN=LASS5 PE=1 SV=1

imbria $\mathrm{GN}=\mathrm{KITM} \quad \mathrm{PE}=2 \mathrm{SV}=1$

lence. (Fragment) OS=Tetraodon nigroviridis GN=GSTENG00029380001 PE=4 SV=1

lar $\mathrm{GN}=\mathrm{ATAD} 2 \mathrm{PE}=3 \mathrm{SV}=1$

$\Xi=2 \quad S V=1$

rotgun sequence OS=Tetraodon nigroviridis GN=GSTENG00013272001 PE=4 SV=1 
sapiens $\mathrm{GN}=\mathrm{ABCD} 4 \mathrm{PE}=2 \mathrm{SV}=1$

$\mathrm{PE}=4 \mathrm{SV}=1$

lotgun sequence. (Fragment) OS=Tetraodon nigroviridis GN=GSTENG00032508001 PE=4 SV=1 is coioides $\mathrm{PE}=2 \mathrm{SV}=1$

ənce. (Fragment) OS=Tetraodon nigroviridis GN=GSTENG00031434001 PE=4 SV=1

lence. (Fragment) OS=Tetraodon nigroviridis GN=GSTENG00029551001 PE=4 SV=1

\section{$\mathrm{PE}=2 \mathrm{SV}=1$}

$=2 \quad \mathrm{SV}=1$

1otgun sequence. (Fragment) OS=Tetraodon nigroviridis GN=GSTENG00008805001 PE=4 SV=1

s coioides $\mathrm{PE}=2 \mathrm{SV}=1$

nordax GN=CHMP3 PE=2 SV=1

romosomal OS=Anoplopoma fimbria GN=IF1AX PE=2 SV=1

ralichthys olivaceus GN=gtf2a $2 \quad \mathrm{PE}=3 \quad \mathrm{SV}=1$

tein $0 \mathrm{~S}=0$ smerus mordax $\mathrm{GN}=\mathrm{MCAT} \mathrm{PE}=2 \mathrm{SV}=1$

$=$ ak2 $\mathrm{PE}=2 \mathrm{SV}=1$

D OS=Danio rerio $\mathrm{GN}=$ eif3d $\mathrm{PE}=2 \mathrm{SV}=1$

itochondrial 0S=Anoplopoma fimbria GN=NDUB8 PE=2 SV=1 
j $\mathrm{PE}=2 \mathrm{SV}=1$

$2 \mathrm{PE}=2 \mathrm{SV}=1$

$\frown 1 \quad \mathrm{PE}=2 \mathrm{SV}=1$

$\mathrm{V}=\mathrm{rab} 7 \mathrm{PE}=2 \quad \mathrm{SV}=1$

lence OS=Tetraodon nigroviridis GN=GSTENG00032331001 PE=3 SV=1

ənce. (Fragment) OS=Tetraodon nigroviridis GN=GSTENG00023730001 PE=4 SV=1

j=Anoplopoma fimbria GN=MCA1 PE=2 SV=1

aio rerio $\mathrm{GN}=\operatorname{exd} 1 \mathrm{PE}=2 \mathrm{SV}=1$

ənce OS=Tetraodon nigroviridis GN=GSTENG00032803001 PE=4 SV=1

$=$ Tetraodon nigroviridis GN=GSTENG00034408001 PE=3 SV=1

nigroviridis GN=GSTENG00015412001 PE=3 SV=1

ərio GN=trappc1 $\mathrm{PE}=2 \mathrm{SV}=1$

ənce. (Fragment) OS=Tetraodon nigroviridis GN=GSTENG00027932001 PE=4 SV=1

londrial 0S=Homo sapiens GN=PNPT1 PE=1 SV=2

əse OS=Esox lucius GN=MTND PE=2 SV=1 
) salar $\mathrm{GN}=\mathrm{CC} 104 \mathrm{PE}=2 \mathrm{SV}=1$

laevis GN=mcts $1-b \quad P E=2 \quad S V=1$

$\mathrm{z}=2 \quad \mathrm{SV}=2$

AC2 OS=Salmo salar GN=RPAC2 $\mathrm{PE}=4 \mathrm{SV}=1$

əc62 $\mathrm{PE}=2 \mathrm{SV}=1$

=Psetta maxima GN=tpt $1 \quad \mathrm{PE}=2 \mathrm{SV}=1$

imbria GN=PRS8 PE=2 SV=1

OS=Rattus norvegicus GN=M1h3 PE=4 SV=1

1

$\mathrm{SV}=1$

गE $=1 \quad \mathrm{SV}=1$ 
$\mathrm{z}=2 \mathrm{SV}=1$

=Tetraodon nigroviridis GN=GSTENG00022542001 PE=3 SV=1

$\because 1 \mathrm{PE}=2 \mathrm{SV}=1$

$\mathrm{PE}=2 \mathrm{SV}=1$

lence. (Fragment) 0S=Tetraodon nigroviridis GN=GSTENG00026260001 PE=4 SV=1 ənio rerio $\mathrm{GN}=\operatorname{arpc} 41 \mathrm{PE}=2 \mathrm{SV}=1$

OS=0smerus mordax GN=RPB4 PE=2 SV=1

snce OS=Tetraodon nigroviridis GN=GSTENG00015494001 PE=3 SV=1

ənce. (Fragment) 0S=Tetraodon nigroviridis GN=GSTENG00023993001 PE=4 SV=1

sence. (Fragment) OS=Tetraodon nigroviridis GN=GSTENG00020229001 PE=4 SV=1 $\mathrm{z}=2 \mathrm{SV}=1$

?E=2 SV=1

zgc: $112185 \mathrm{PE}=2 \mathrm{SV}=1$

ence OS=Tetraodon nigroviridis GN=GSTENG00018368001 PE $=4$ SV=1

$\mathrm{v}=$ minal $\mathrm{PE}=2 \quad \mathrm{SV}=1$

$3 \mathrm{~N}=\operatorname{snrpf} 1 \quad \mathrm{PE}=4 \mathrm{SV}=1$

shondrial 0S=Esox lucius GN=COX81 PE=4 SV=1

$\mathrm{PE}=2 \mathrm{SV}=1$

3 OS=Xenopus tropicalis $\mathrm{GN}=$ pan $3 \mathrm{PE}=2 \mathrm{SV}=1$

sp. $\quad \mathrm{PE}=2 \mathrm{SV}=1$

$\mathrm{z}=2 \mathrm{SV}=1$

$=1$

lence. (Fragment) OS=Tetraodon nigroviridis GN=GSTENG00032218001 PE=4 SV=1 
is $\mathrm{GN}=\mathrm{PLCXD} 1 \mathrm{PE}=2 \mathrm{SV}=1$

$1 \mathrm{PE}=2 \mathrm{SV}=1$

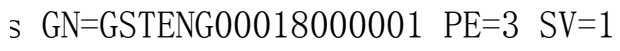

Jtgun sequence. (Fragment) OS=Tetraodon nigroviridis GN=GSTENG00006203001 PE=4 SV=1 $\mathrm{V}=$ WDR82 $\mathrm{PE}=2 \quad \mathrm{SV}=1$

lence OS=Tetraodon nigroviridis GN=GSTENG00030207001 PE $=4$ SV=1

a nigroviridis GN=GSTENG00016122001 PE=3 SV=1

IN $=$ SVIP $\quad \mathrm{PE}=4 \quad \mathrm{SV}=1$

. $\mathrm{PE}=2 \mathrm{SV}=1$

əus $\mathrm{GN}=\mathrm{p} 53 \quad \mathrm{PE}=2 \mathrm{SV}=1$

OS=Esox lucius GN=ENY2 PE=4 SV=1

lence. (Fragment) OS=Tetraodon nigroviridis GN=GSTENG00020318001 PE=4 SV=1 rubripes $\mathrm{PE}=2 \mathrm{SV}=1$

¿ $\mathrm{PE}=2 \mathrm{SV}=1$

ənce. (Fragment) OS=Tetraodon nigroviridis GN=GSTENG00024792001 PE=4 SV=1

ənce. (Fragment) OS=Tetraodon nigroviridis GN=GSTENG00029244001 PE=4 SV=1

lotgun sequence OS=Tetraodon nigroviridis GN=GSTENG00021930001 PE=4 SV=1

ənce. (Fragment) OS=Tetraodon nigroviridis GN=GSTENG00017907001 PE=4 SV=1

j $\mathrm{PE}=2 \mathrm{SV}=1$ 
la) $\mathrm{OS}=$ Danio rerio $\mathrm{GN}=$ stau $1 \mathrm{PE}=2 \mathrm{SV}=1$

rotgun sequence. (Fragment) OS=Tetraodon nigroviridis GN=GSTENG00013054001 PE=4 SV=1

stgun sequence. (Fragment) OS=Tetraodon nigroviridis GN=GSTENG00003182001 PE=4 SV=1

$\mathrm{SV}=1$

lence. (Fragment) OS=Tetraodon nigroviridis GN=GSTENG00035695001 PE=4 SV=1 u:fi25h02 PE=2 SV=1

salar GN=RT31 PE=2 SV=1

lence. (Fragment) OS=Tetraodon nigroviridis GN=GSTENG00029626001 PE=4 SV=1

$\mathrm{PE}=4 \mathrm{SV}=1$

lence OS=Tetraodon nigroviridis GN=GSTENG00019949001 PE=4 SV=1

amidase F OS=Salmo salar GN=PNGF PE=2 SV=1

$=4 \mathrm{SV}=1$

j=Salmo salar GN=DSCR3 PE=2 SV=1

rotgun sequence. (Fragment) OS=Tetraodon nigroviridis GN=GSTENG00009758001 PE=4 SV=1

. 
ənce. (Fragment) OS=Tetraodon nigroviridis GN=GSTENG00015435001 PE=4 SV=1

11-222h17. 5 PE=2 SV=1

rotgun sequence OS=Tetraodon nigroviridis GN=GSTENG00025277001 PE=3 SV=1

Jdon nigroviridis GN=GSTENG00018444001 PE=3 SV=1

r $\mathrm{GN}=\mathrm{PPIB}$ PE $=2 \mathrm{SV}=1$

io $\mathrm{GN}=\mathrm{ube} 2131 \mathrm{PE}=2 \mathrm{SV}=1$

=Anoplopoma fimbria GN=CD2B2 PE=2 SV=1

1०A $\mathrm{PE}=2 \mathrm{SV}=1$

$=$ Salmo salar GN=IAH1 PE=2 SV=1

spoma fimbria $\mathrm{GN}=\mathrm{RM} 51 \mathrm{PE}=2 \mathrm{SV}=1$

«iss GN=LYRM2 PE=4 SV=1

$\mathrm{V}=1$

$\mathrm{V}=\mathrm{TM} 50 \mathrm{~A} \quad \mathrm{PE}=2 \quad \mathrm{SV}=1$

า. $\mathrm{sp} . \mathrm{PE}=4 \mathrm{SV}=1$

$\mathrm{J}=\mathrm{UBL} 4 \mathrm{~A} \quad \mathrm{PE}=2 \quad \mathrm{SV}=1$

V=AMPM1 PE=2 SV=1

ance. (Fragment) OS=Tetraodon nigroviridis GN=GSTENG00033161001 PE=4 SV=1

=Anoplopoma fimbria GN=TPPC3 PE=2 SV=1

ənce. (Fragment) OS=Tetraodon nigroviridis GN=GSTENG00017921001 PE=4 SV=1

$1 \mathrm{PE}=2 \mathrm{SV}=1$

lence. (Fragment) OS=Tetraodon nigroviridis GN=GSTENG00026366001 PE=4 SV=1 
JBP $\mathrm{PE}=4 \mathrm{SV}=1$

ə $\mathrm{GN}=\mathrm{TUSC} 2 \mathrm{PE}=4 \mathrm{SV}=1$

$1-147 \mathrm{G} 19.2 \mathrm{PE}=4 \mathrm{SV}=1$

le receptor interactor 12 (TRIP12) (Fragment) OS=Danio rerio GN=CH211-272F3.4-001 PE=4 SV=1 talurus punctatus $\mathrm{PE}=2 \mathrm{SV}=1$

ənce OS=Tetraodon nigroviridis GN=GSTENG00021745001 PE=4 SV=1

ənce. (Fragment) OS=Tetraodon nigroviridis GN=GSTENG00024884001 PE=4 SV=1

stgun sequence. (Fragment) OS=Tetraodon nigroviridis GN=GSTENG00003714001 PE=3 SV=1

Janio rerio $\mathrm{GN}=\mathrm{dcp} 2 \mathrm{PE}=2 \mathrm{SV}=1$

almo salar GN=RPC6 $\mathrm{PE}=2 \mathrm{SV}=1$

ənce OS=Tetraodon nigroviridis GN=GSTENG00019014001 PE=4 SV=1

lence OS=Tetraodon nigroviridis GN=GSTENG00020931001 PE=3 SV=1

lence. (Fragment) OS=Tetraodon nigroviridis GN=GSTENG00013131001 PE=4 SV=1

rerio $\mathrm{GN}=\operatorname{ccdc} 65 \mathrm{PE}=2 \mathrm{SV}=2$ 
lence OS=Tetraodon nigroviridis GN=GSTENG00032135001 PE=4 SV=1

spoma fimbria GN=DUS4L PE=2 SV=1

lotgun sequence. (Fragment) OS=Tetraodon nigroviridis GN=GSTENG00008026001 PE=4 SV=1

s $\mathrm{PE}=2 \mathrm{SV}=1$

ıence. (Fragment) OS=Tetraodon nigroviridis GN=GSTENG00014804001 PE=4 SV=1

a nigroviridis GN=GSTENG00021876001 PE=3 SV=1

rotgun sequence OS=Tetraodon nigroviridis GN=GSTENG00009444001 PE=4 SV=1

rotgun sequence. (Fragment) OS=Tetraodon nigroviridis GN=GSTENG00008193001 PE=4 SV=1

$\mathrm{PE}=2 \mathrm{SV}=1$

it 29 OS=Esox lucius GN=MED29 PE=2 SV=1

?setta maxima $\mathrm{PE}=2 \mathrm{SV}=1$

ənce. (Fragment) OS=Tetraodon nigroviridis GN=GSTENG00018053001 PE=4 SV=1

ənce. (Fragment) OS=Tetraodon nigroviridis GN=GSTENG00028891001 PE=4 SV=1

lence. (Fragment) OS=Tetraodon nigroviridis GN=GSTENG00028508001 PE=4 SV=1

:55663) (Fragment) OS=Danio rerio GN=k1hdc10 PE=2 SV=1

trial OS=Thunnus obesus $\mathrm{PE}=2 \mathrm{SV}=1$

imbria GN=RING2 PE=2 SV=1 
s $\mathrm{PE}=1 \mathrm{SV}=2$

(Fragment) 0S=Platichthys flesus $\mathrm{GN}=$ pgd $\mathrm{PE}=2 \mathrm{SV}=1$

PE=2 SV=1

$=$ TMM $32 \mathrm{PE}=2 \mathrm{SV}=1$

iit $0 \mathrm{~S}=$ Gallus gallus $\mathrm{PE}=2 \mathrm{SV}=1$

lotgun sequence. (Fragment) OS=Tetraodon nigroviridis GN=GSTENG00008790001 PE=4 SV=1

lence. (Fragment) OS=Tetraodon nigroviridis GN=GSTENG00016183001 PE=3 SV=1

$2 \mathrm{PE}=2 \mathrm{SV}=1$

$=1$

$\mathrm{V}=\mathrm{RL} 22 \quad \mathrm{PE}=2 \quad \mathrm{SV}=1$

$12 \mathrm{a} \quad \mathrm{PE}=2 \mathrm{SV}=1$

canadum $\mathrm{PE}=2 \mathrm{SV}=1$

$\exists=2 \quad S V=1$

lus heteroclitus $\mathrm{PE}=2 \mathrm{SV}=1$

salar $\mathrm{GN}=\mathrm{CSDE} 1 \mathrm{PE}=2 \mathrm{SV}=1$

lence. (Fragment) OS=Tetraodon nigroviridis GN=GSTENG00017399001 PE=4 SV=1

lence. (Fragment) OS=Tetraodon nigroviridis GN=GSTENG00014317001 PE=4 SV=1

PE $=2 \quad \mathrm{SV}=1$

$V=1$ 
ice. (Fragment) OS=Tetraodon nigroviridis GN=GSTENG00005366001 PE=4 SV=1 floridae GN=BRAFLDRAFT_113832 PE=4 SV=1

lence. (Fragment) OS=Tetraodon nigroviridis GN=GSTENG00030230001 PE=4 SV=1 3. OS=Tetraodon nigroviridis GN=nip7 $\mathrm{PE}=3 \mathrm{SV}=1$

$\mathrm{V}=\mathrm{METL} 5 \mathrm{PE}=2 \quad \mathrm{SV}=1$

-1 subunit 1 OS=Anoplopoma fimbria GN=BL1S1 PE=2 SV=1

j=0ryzias latipes $\mathrm{PE}=2 \mathrm{SV}=1$

lence. (Fragment) OS=Tetraodon nigroviridis GN=GSTENG00032635001 PE=4 SV=1 al is GN=pabpc1 $\mathrm{PE}=2 \mathrm{SV}=1$

$=2 \mathrm{SV}=1$

is $\mathrm{GN}=\mathrm{AP} 2 \mathrm{~S} 1 \mathrm{PE}=2 \mathrm{SV}=1$

$\mathrm{J}=\mathrm{BR} 44 \mathrm{~L} \quad \mathrm{PE}=4 \quad \mathrm{SV}=1$

$=2 \mathrm{SV}=1$

s $\mathrm{GN}=\mathrm{dio} 1 \mathrm{PE}=2 \mathrm{SV}=1$

$115 \mathrm{PE}=2 \mathrm{SV}=3$

lence. (Fragment) OS=Tetraodon nigroviridis GN=GSTENG00028622001 PE=4 SV=1 dlaris $\mathrm{PE}=2 \mathrm{SV}=1$

ənce. (Fragment) OS=Tetraodon nigroviridis GN=GSTENG00017779001 PE=4 SV=1 $\mathrm{SV}=1$

rotgun sequence OS=Tetraodon nigroviridis GN=GSTENG00008419001 PE=4 SV=1 xima $\mathrm{PE}=2 \mathrm{SV}=1$ 
ad WD repeat domain 2 (RFWD2, zgc:163067) OS=Danio rerio GN=DKEY-39F8. 2 PE=4 SV=1 ənce. (Fragment) OS=Tetraodon nigroviridis GN=GSTENG00022210001 PE=4 SV=1 $\mathrm{z}=2 \mathrm{SV}=1$

zgc:73187 PE=2 SV=2

Xenopus tropicalis $\mathrm{GN}=$ mobkl1b PE $=2 \mathrm{SV}=1$

es $\mathrm{GN}=\mathrm{aldA} \mathrm{PE}=2 \mathrm{SV}=1$

$=0$ corhynchus mykiss GN=NDUB4 PE=2 SV=1

$=3$

lence OS=Tetraodon nigroviridis GN=GSTENG00029374001 PE=4 SV=1

is $\mathrm{GN}=\mathrm{hsp} 10 \mathrm{PE}=3 \mathrm{SV}=1$

stgun sequence. (Fragment) OS=Tetraodon nigroviridis GN=GSTENG00004894001 PE=4 SV=1

$\mathrm{GN}=$ rhoac $\mathrm{PE}=2 \mathrm{SV}=1$

s punctatus $\mathrm{PE}=2 \mathrm{SV}=1$

lence. (Fragment) OS=Tetraodon nigroviridis GN=GSTENG00030695001 PE=3 SV=1

¡A4 PE=2 SV=1

$\mathrm{V}=\mathrm{RL} 30 \quad \mathrm{PE}=4 \quad \mathrm{SV}=1$

lence. (Fragment) OS=Tetraodon nigroviridis GN=GSTENG00017370001 PE=4 SV=1

Jtgun sequence OS=Tetraodon nigroviridis GN=GSTENG00004818001 PE=4 SV=1

ənce. (Fragment) OS=Tetraodon nigroviridis GN=GSTENG00015394001 PE=4 SV=1

$\zeta=$ Anoplopoma fimbria GN=ELOB PE=4 SV=1

IC1 homolog OS=Takifugu rubripes GN=SMC1 PE=4 SV=1

əin sorting 45A (Yeast) (VPS45A) OS=Danio rerio GN=DKEY-200H23. 3-001 PE=4 SV=1

lence OS=Tetraodon nigroviridis GN=GSTENG00016149001 PE=4 SV=1 
$\Xi=2 \quad S V=1$

lence. (Fragment) OS=Tetraodon nigroviridis GN=GSTENG00024382001 PE=4 SV=1

rerio $\mathrm{GN}=$ pabpcla $\mathrm{PE}=2 \mathrm{SV}=1$

lence. (Fragment) OS=Tetraodon nigroviridis GN=GSTENG00018785001 PE=4 SV=1

talurus punctatus $\mathrm{PE}=2 \mathrm{SV}=1$

ənce. (Fragment) OS=Tetraodon nigroviridis GN=GSTENG00034106001 PE=4 SV=1

rotgun sequence OS=Tetraodon nigroviridis GN=GSTENG00008786001 PE=4 SV=1

lence OS=Tetraodon nigroviridis GN=GSTENG00033341001 PE=4 SV=1

วolypeptide $0 \mathrm{~S}=$ Danio rerio $\mathrm{GN}=$ bckdha $\mathrm{PE}=2 \mathrm{SV}=1$

ว $\mathrm{GN}=$ vps $28 \mathrm{PE}=2 \mathrm{SV}=1$

$1 \mathrm{PE}=2 \mathrm{SV}=1$

ənce. (Fragment) 0S=Tetraodon nigroviridis GN=GSTENG00004715001 PE=4 SV=1 $\mathrm{GN}=\mathrm{S} 2533 \quad \mathrm{PE}=2 \quad \mathrm{SV}=1$

nbria $\mathrm{GN}=\mathrm{COMD} 4 \mathrm{PE}=2 \quad \mathrm{SV}=1$

lence. (Fragment) OS=Tetraodon nigroviridis GN=GSTENG00030692001 PE=4 SV=1 
ənce. (Fragment) OS=Tetraodon nigroviridis GN=GSTENG00018243001 PE=4 SV=1

imbria GN=CRIPT PE=4 SV=1

.

$\mathrm{V}=1$

Jtgun sequence OS=Tetraodon nigroviridis GN=GSTENG00004276001 PE=4 SV=1 ælis GN=LOC100127587 PE=2 SV=1

lmo salar GN=DDX39 PE=2 SV=1

$\mathrm{V}=\mathrm{RBBP} 6 \quad \mathrm{PE}=2 \quad \mathrm{SV}=1$

r $\quad$ GN=mpp1 $\mathrm{PE}=2 \quad \mathrm{SV}=1$

$\mathrm{PE}=2 \mathrm{SV}=1$

PE $=2 \quad \mathrm{SV}=1$

rotgun sequence. (Fragment) OS=Tetraodon nigroviridis GN=GSTENG00009864001 PE=4 SV=1 
Is and adenovirus receptor (CXADR) OS=Danio rerio GN=si:ch211-145m1. 1 PE=4 SV=1

2 OS=Salmo salar GN=VDAC2 PE=2 SV=1

ənce. (Fragment) OS=Tetraodon nigroviridis GN=GSTENG00022166001 PE=4 SV=1

$\mathrm{PE}=2 \quad \mathrm{SV}=1$

ənce. (Fragment) OS=Tetraodon nigroviridis GN=GSTENG00014210001 PE=4 SV=1

fasciatus GN=CpipJ_CPIJ006503 PE=4 SV=1

$=\mathrm{PN} 01 \mathrm{PE}=2 \mathrm{SV}=1$

stgun sequence. (Fragment) OS=Tetraodon nigroviridis GN=GSTENG00004090001 PE=4 SV=1

j $\mathrm{PE}=2 \mathrm{SV}=1$

lence OS=Tetraodon nigroviridis GN=GSTENG00024467001 PE=4 SV=1

ia $\mathrm{GN}=\mathrm{PSA} 3 \mathrm{PE}=2 \mathrm{SV}=1$

lence OS=Tetraodon nigroviridis GN=GSTENG00017376001 PE=4 SV=1

IN=DNJA1 $\mathrm{PE}=2 \quad \mathrm{SV}=1$

$z=2 \quad$ SV=1

$\mathrm{SV}=1$

ənce. (Fragment) OS=Tetraodon nigroviridis GN=GSTENG00026966001 PE=4 SV=1

=zgc: $109926 \mathrm{PE}=1 \mathrm{SV}=1$ 
-121B10. $3 \mathrm{PE}=4 \mathrm{SV}=1$

$2 \mathrm{G} 1 \quad \mathrm{PE}=2 \mathrm{SV}=1$

sma fimbria GN=CIB1 $\mathrm{PE}=2 \mathrm{SV}=1$

lence. (Fragment) OS=Tetraodon nigroviridis GN=GSTENG00015106001 PE=4 SV=1

ənce. (Fragment) OS=Tetraodon nigroviridis GN=GSTENG00017104001 PE=4 SV=1

$\mathrm{V}=\mathrm{GP} 177 \quad \mathrm{PE}=2 \quad \mathrm{SV}=1$

łnoplopoma fimbria GN=CISD1 $\mathrm{PE}=4 \mathrm{SV}=1$

$=\mathrm{COPE} \quad \mathrm{PE}=2 \mathrm{SV}=1$

1ence. (Fragment) OS=Tetraodon nigroviridis GN=GSTENG00031530001 PE=4 SV=1

1

tgun sequence $0 \mathrm{~S}=$ Tetraodon nigroviridis GN=GSTENG00007500001 PE=4 SV=1

=Danio rerio GN=vps33b $\mathrm{PE}=2 \mathrm{SV}=1$

ənce OS=Tetraodon nigroviridis GN=GSTENG00031784001 PE=4 SV=1

Г9 $\mathrm{PE}=2 \mathrm{SV}=1$

lence OS=Tetraodon nigroviridis GN=GSTENG00030988001 PE=4 SV=1

$\mathrm{GN}=\operatorname{prps} 1 \mathrm{~b} \quad \mathrm{PE}=2 \quad \mathrm{SV}=1$

ənce. (Fragment) OS=Tetraodon nigroviridis GN=GSTENG00017656001 PE=4 SV=1

गoma fimbria GN=MSS4 PE=2 SV=1

, $\mathrm{PE}=2 \mathrm{SV}=1$

$3 \mathrm{PE}=2 \mathrm{SV}=1$

nbria GN=EXOS2 PE=2 SV=1

adrial OS=Anoplopoma fimbria GN=PRDX3 PE=2 SV=1

ar GN=ANC5 PE=2 SV=1 
$3 \mathrm{~N}=\mathrm{Zfp} 560 \quad \mathrm{PE}=2 \quad \mathrm{SV}=1$

$14 \mathrm{PE}=2 \mathrm{SV}=1$

ว salar GN=YD286 PE=2 SV=1

ənce. (Fragment) 0S=Tetraodon nigroviridis GN=GSTENG00026104001 PE=4 SV=1 nce $0 \mathrm{~S}=$ Tetraodon nigroviridis $\mathrm{GN}=\mathrm{GSTENG00007051001} \mathrm{PE=4} \mathrm{SV=1}$

$\mathrm{GN}=\mathrm{crfb} 1 \quad \mathrm{PE}=2 \quad \mathrm{SV}=1$

Jdon nigroviridis GN=GSTENG00028335001 PE=3 SV=1

s mykiss $\mathrm{GN}=\operatorname{socs} 5 \mathrm{PE}=2 \mathrm{SV}=1$

lence OS=Tetraodon nigroviridis GN=GSTENG00032604001 PE=4 SV=1

ərio $\mathrm{GN}=\operatorname{trip} 13 \mathrm{PE}=2 \mathrm{SV}=1$

j=Salmo salar GN=S61A1 PE=2 SV=1

livaceus $\mathrm{GN}=\mathrm{VDAC} \quad \mathrm{PE}=2 \mathrm{SV}=1$

गoma fimbria GN=RM27 PE=2 SV=1 
rerio $\mathrm{GN}=$ asmt $1 \mathrm{PE}=3 \mathrm{SV}=1$

lotgun sequence. (Fragment) OS=Tetraodon nigroviridis GN=GSTENG00010002001 PE=4 SV=1

चx $\mathrm{GN}=\mathrm{FABPH} \mathrm{PE}=3 \mathrm{SV}=1$

ənce. (Fragment) OS=Tetraodon nigroviridis GN=GSTENG00011516001 PE=4 SV=1

-mannosyltransferase OS=Salmo salar GN=ALG12 PE=2 SV=1

) salar $\mathrm{GN}=\mathrm{GCDH} \mathrm{PE}=2 \mathrm{SV}=1$

$=2 \mathrm{SV}=1$

$=\mathrm{RDH} 3 \quad \mathrm{PE}=2 \quad \mathrm{SV}=1$

ıotgun sequence. (Fragment) OS=Tetraodon nigroviridis GN=GSTENG00010378001 PE=4 SV=1

$\checkmark 1$ subtype (Fragment) OS=Danio rerio GN=gnav1 PE=2 SV=1

əlar GN=CND3 PE=2 SV=1

JS=0smerus mordax GN=NDUAC PE=2 SV=1

xce. (Fragment) OS=Tetraodon nigroviridis GN=GSTENG00007605001 PE=3 SV=1

эment) $\mathrm{OS}=\mathrm{Sa}$ lmo salar $\mathrm{GN}=\mathrm{ZCHC} 6 \mathrm{PE}=2 \mathrm{SV}=1$

गoma fimbria $\mathrm{GN}=\mathrm{CAZA} 1 \mathrm{PE}=2 \mathrm{SV}=1$

atraodon nigroviridis GN=GSTENG00023111001 PE=3 SV=1

xlitus $\mathrm{PE}=2 \mathrm{SV}=1$

aio rerio $\mathrm{GN}=1$ rrc9 $\mathrm{PE}=4 \mathrm{SV}=1$

taneum GN=TcasGA2_TC000634 PE=4 SV=1

$\mathrm{V}=\mathrm{P} 0 \mathrm{~N} 2 \quad \mathrm{PE}=2 \quad \mathrm{SV}=1$

lmo salar $\mathrm{PE}=2 \mathrm{SV}=1$ 
$\mathrm{PE}=2 \quad \mathrm{SV}=1$

?ADRAFT_67260 PE=4 SV=1

Jrhynchus mykiss $\mathrm{GN}=\mathrm{GRP} 78 \quad \mathrm{PE}=2 \mathrm{SV}=1$

stgun sequence. (Fragment) OS=Tetraodon nigroviridis GN=GSTENG00004291001 PE=4 SV=1

zse/cyclohydrolase, mitochondrial $0 \mathrm{~S}=0$ smerus mordax GN=MTDC PE=2 SV=1

JS=Pongo abeli i GN=GLT1D1 PE=2 SV=1

$\mathrm{GN}=\mathrm{EMI} 5 \quad \mathrm{PE}=2 \quad \mathrm{SV}=1$

lmo salar GN=COX19 PE=4 SV=1

3N=ZMAT5 PE=2 SV=1

lence. (Fragment) OS=Tetraodon nigroviridis GN=GSTENG00027361001 PE=3 SV=1

$2 \mathrm{SV}=1$

is mossambicus $\mathrm{PE}=2 \mathrm{SV}=1$ 
lence. (Fragment) OS=Tetraodon nigroviridis GN=GSTENG00012247001 PE=3 SV=1 $j=$ Salmo salar GN=NDUA8 $\mathrm{PE}=2 \mathrm{SV}=1$

$\mathrm{V}=\mathrm{COI} \quad \mathrm{PE}=3 \mathrm{SV}=1$

lence. (Fragment) OS=Tetraodon nigroviridis GN=GSTENG00014751001 PE=4 SV=1 गseudomallei 305 GN=BURPS305_2067 PE=4 SV=1

1otgun sequence OS=Tetraodon nigroviridis GN=GSTENG00023162001 PE=4 SV=1 rotgun sequence OS=Tetraodon nigroviridis GN=GSTENG00012362001 PE=4 SV=1

19-1ike2 PE=2 SV=1

ənce. (Fragment) OS=Tetraodon nigroviridis GN=GSTENG00020129001 PE=4 SV=1

r GN=PPIL3 PE=2 SV=1

sma4 $\mathrm{PE}=2 \mathrm{SV}=1$

ənce. (Fragment) OS=Tetraodon nigroviridis GN=GSTENG00023567001 PE=4 SV=1

Jtgun sequence. (Fragment) OS=Tetraodon nigroviridis GN=GSTENG00003007001 PE=4 SV=1

lmo salar GN=ROAA $\mathrm{PE}=2 \mathrm{SV}=1$

JS=Salmo salar GN=NDUV3 $\mathrm{PE}=4 \mathrm{SV}=1$

JB1 $\mathrm{PE}=2 \quad \mathrm{SV}=1$

ənce. (Fragment) 0S=Tetraodon nigroviridis GN=GSTENG00021791001 PE=4 SV=1

ıotgun sequence. (Fragment) OS=Tetraodon nigroviridis GN=GSTENG00009379001 PE=4 SV=1

x subunit 2 OS=Tetraodon nigroviridis GN=GSTENG00007462001 PE=3 SV=1

) salar $\mathrm{GN}=$ ict1 $\mathrm{PE}=2 \mathrm{SV}=1$

j=Salmo salar GN=IMP1L PE=2 SV=1 
lotgun sequence. (Fragment) OS=Tetraodon nigroviridis GN=GSTENG00025252001 PE=4 SV=1

$=2 \mathrm{SV}=1$

ənce OS=Tetraodon nigroviridis GN=GSTENG00010059001 PE=3 SV=1

rotgun sequence. (Fragment) OS=Tetraodon nigroviridis GN=GSTENG00016545001 PE=4 SV=1

$11 \mathrm{PE}=2 \mathrm{SV}=1$

$\mathrm{SV}=1$

ıf $280 d \mathrm{dE}=2 \mathrm{SV}=1$

ənce OS=Tetraodon nigroviridis GN=GSTENG00019140001 PE=4 SV=1

rotgun sequence OS=Tetraodon nigroviridis GN=GSTENG00031736001 PE=4 SV=1

a fimbria GN=SPT4H PE=4 SV=1

101 $\mathrm{PE}=2 \mathrm{SV}=1$

s maculatus x Xiphophorus helleri GN=dnmt-1 PE=2 SV=1

Epinephelus coioides $\mathrm{PE}=4 \mathrm{SV}=1$

Jalmo salar GN=RCL1 PE=2 SV=1

lence OS=Tetraodon nigroviridis GN=GSTENG00019934001 PE=4 SV=1

3N=DNJC1 PE=2 SV=1

lence. (Fragment) OS=Tetraodon nigroviridis GN=GSTENG00030693001 PE=4 SV=1

ase subunit P OS=Anoplopoma fimbria GN=PIGP PE=2 SV=1 
na fimbria GN=TFAM $P E=2 \quad S V=1$

$\mathrm{PE}=2 \quad \mathrm{SV}=1$

$=1$

ənce. (Fragment) OS=Tetraodon nigroviridis GN=GSTENG00035276001 PE=4 SV=1

(Fragment) 0S=Salmo salar GN=TMEDA $\mathrm{PE}=2 \mathrm{SV}=1$

$\mathrm{SV}=1$

lence. (Fragment) OS=Tetraodon nigroviridis GN=GSTENG00024949001 PE=4 SV=1

spus tropicalis $\mathrm{GN}=$ tmem $87 \mathrm{~b} \quad \mathrm{PE}=2 \mathrm{SV}=1$

$=\mathrm{NUBP} 2 \quad \mathrm{PE}=2 \quad \mathrm{SV}=1$

$\mathrm{SV}=1$

lence OS=Tetraodon nigroviridis GN=GSTENG00029794001 PE=4 SV=1

xchus mykiss GN=DIMT1 PE=2 SV=1

ənce OS=Tetraodon nigroviridis GN=GSTENG00017345001 PE=4 SV=1

?E $=2 \quad \mathrm{SV}=1$

$\mathrm{Z}=2 \mathrm{SV}=1$

$j=0$ corhynchus mykiss GN=BAXA PE=2 SV=1

r $\mathrm{GN}=\mathrm{DLRB} 2 \mathrm{PE}=4 \mathrm{SV}=1$ 
lence. (Fragment) OS=Tetraodon nigroviridis GN=GSTENG00029426001 PE=4 SV=1

ənce. (Fragment) OS=Tetraodon nigroviridis GN=GSTENG00023614001 PE=4 SV=1

ənce. (Fragment) OS=Tetraodon nigroviridis GN=GSTENG00022158001 PE=4 SV=1 lence. (Fragment) OS=Tetraodon nigroviridis GN=GSTENG00012678001 PE=4 SV=1

ənce. (Fragment) OS=Tetraodon nigroviridis GN=GSTENG00017922001 PE=4 SV=1

lence. (Fragment) OS=Tetraodon nigroviridis GN=GSTENG00026737001 PE=4 SV=1 a homolog OS=Anoplopoma fimbria GN=TCTA $\mathrm{PE}=4 \mathrm{SV}=1$

s $\operatorname{mordax} \mathrm{GN}=\mathrm{RM} 49 \mathrm{PE}=2 \mathrm{SV}=1$

ənce OS=Tetraodon nigroviridis GN=GSTENG00033260001 PE=4 SV=1

=Anoplopoma fimbria GN=TPPC2 PE=2 SV=1

ənce. (Fragment) OS=Tetraodon nigroviridis GN=GSTENG00029956001 PE=4 SV=1

$\mathrm{V}=1$

lotgun sequence. (Fragment) OS=Tetraodon nigroviridis GN=GSTENG00021420001 PE=4 SV=1 ənce OS=Tetraodon nigroviridis GN=GSTENG00033110001 PE=3 SV=1

PE $=4 \quad \mathrm{SV}=1$

lotgun sequence. (Fragment) OS=Tetraodon nigroviridis GN=GSTENG00025147001 PE=4 SV=1 ə fimbria $\mathrm{GN}=\mathrm{THG} 1 \mathrm{PE}=2 \mathrm{SV}=1$

ə fimbria GN=HOP2 $\mathrm{PE}=2 \quad \mathrm{SV}=1$ 
ənce. (Fragment) OS=Tetraodon nigroviridis GN=GSTENG00016860001 PE=4 SV=1

$\mathrm{V}=\mathrm{EF} 1 \mathrm{D} \quad \mathrm{PE}=2 \quad \mathrm{SV}=1$

lence OS=Tetraodon nigroviridis GN=GSTENG00022705001 PE=4 SV=1

$\mathrm{SV}=1$

$\mathrm{V}=1$

nitochondrial OS=Anoplopoma fimbria GN=DUT PE=2 SV=1

OS=Anoplopoma fimbria GN=AT5G3 PE=2 SV=1

Xenopus laevis GN=LOC100126624 PE=2 SV=1

lence OS=Tetraodon nigroviridis GN=GSTENG00012989001 PE=4 SV=1

nordax $\mathrm{GN}=\mathrm{CNBP} \mathrm{PE}=2 \mathrm{SV}=1$

1 OS=Salmo salar GN=VDAC1 PE=2 SV=1

lence. (Fragment) OS=Tetraodon nigroviridis GN=GSTENG00027613001 PE=3 SV=1

nio rerio $\mathrm{GN}=\mathrm{si}$ :dkeyp-115a10. $2 \mathrm{PE}=2 \mathrm{SV}=2$

$2 \mathrm{SV}=1$

$=$ GUF $1 \quad \mathrm{PE}=1 \quad \mathrm{SV}=1$

$=\sec 23 \mathrm{a} \quad \mathrm{PE}=2 \mathrm{SV}=1$

ənce. (Fragment) OS=Tetraodon nigroviridis GN=GSTENG00034003001 PE=4 SV=1

stgun sequence. (Fragment) OS=Tetraodon nigroviridis GN=GSTENG00004275001 PE=4 SV=1

ar $\mathrm{GN}=\mathrm{VRK} 3 \mathrm{PE}=2 \mathrm{SV}=1$

$\mathrm{E}=2 \mathrm{SV}=1$

गb $\mathrm{PE}=2 \mathrm{SV}=1$

$=1$

IN $=$ COPA PE=2 SV=1 
aiperca chuatsi $\mathrm{PE}=2 \mathrm{SV}=1$

) $\mathrm{OS}=$ Gadus morhua $\mathrm{PE}=2 \mathrm{SV}=1$

$=2 \mathrm{SV}=1$

ənce OS=Tetraodon nigroviridis GN=GSTENG00033425001 PE=4 SV=1

ənce. (Fragment) 0S=Tetraodon nigroviridis GN=GSTENG00017033001 PE=4 SV=1

$\mathrm{V}=\operatorname{ccdc} 94 \quad \mathrm{PE}=2 \quad \mathrm{SV}=1$

lence. (Fragment) OS=Tetraodon nigroviridis GN=GSTENG00032278001 PE=4 SV=1

=zgc: 76871 PE=2 SV=1

ə fimbria GN=FCF1 PE=2 SV=1

ənce. (Fragment) OS=Tetraodon nigroviridis GN=GSTENG00017291001 PE=4 SV=1

- coil domain containing 22 (CCDC22, zgc:114058) OS=Danio rerio GN=CH211-233C3. 4 PE=4 SV=1 lence. (Fragment) OS=Tetraodon nigroviridis GN=GSTENG00021646001 PE=4 SV=1

$=$ Salmo salar GN=CTR9 PE=2 SV=1

sr2 $\mathrm{PE}=2 \mathrm{SV}=1$ 
ənce. (Fragment) OS=Tetraodon nigroviridis GN=GSTENG00022178001 PE=4 SV=1

1

floridae GN=BRAFLDRAFT_261551 PE=4 SV=1

nordax GN=BAP29 PE=2 SV=1

soform 1 OS=Takifugu rubripes $\mathrm{GN}=\mathrm{Atp} 5 \mathrm{~g} 1 \mathrm{PE}=3 \mathrm{SV}=1$

¡ $\mathrm{PE}=2 \mathrm{SV}=1$

floridae GN=BRAFLDRAFT_96468 PE=4 SV=1

ənce. (Fragment) OS=Tetraodon nigroviridis GN=GSTENG00032718001 PE=4 SV=1

nb2 $\mathrm{PE}=2 \mathrm{SV}=1$

łnoplopoma fimbria GN=CKS1 PE=4 SV=1

$\exists=2 \quad S V=1$

ənce OS=Tetraodon nigroviridis GN=GSTENG00024068001 PE=4 SV=1

anio rerio $\mathrm{GN}=$ ifrd1 $\mathrm{PE}=2 \mathrm{SV}=1$

ia $\mathrm{GN}=\mathrm{DBLOH} \mathrm{PE}=2 \mathrm{SV}=1$

rvegicus $\mathrm{GN}=\mathrm{Calm} 1 \mathrm{PE}=4 \mathrm{SV}=1$

rotgun sequence. (Fragment) OS=Tetraodon nigroviridis GN=GSTENG00034610001 PE=4 SV=1

ऽ25 PE=2 SV=1

วma fimbria GN=MGST3 PE=2 SV=1

sma2 $\mathrm{PE}=2 \mathrm{SV}=1$ 
ənce. (Fragment) OS=Tetraodon nigroviridis GN=GSTENG00024126001 PE=4 SV=1

=Anoplopoma fimbria GN=CT030 PE=4 SV=1

lence. (Fragment) OS=Tetraodon nigroviridis GN=GSTENG00029731001 PE=4 SV=1

lence. (Fragment) OS=Tetraodon nigroviridis GN=GSTENG00032527001 PE=3 SV=1

s16 $\mathrm{PE}=2 \mathrm{SV}=1$

-Ala-Asp) box polypeptide 1 (DDX1) (Fragment) OS=Danio rerio GN=ddx1 PE=2 SV=1

$1 \mathrm{PE}=2 \mathrm{SV}=1$

Ja1 $\mathrm{PE}=1 \quad \mathrm{SV}=2$

$=\mathrm{L} 0 \mathrm{C} 444845 \mathrm{PE}=2$ SV=1

ə protein $0 \mathrm{~S}=\mathrm{Bos}$ taurus $\mathrm{GN}=\mathrm{TRAPPC} 2 \mathrm{~L}$ PE=2 SV=1

ənce OS=Tetraodon nigroviridis GN=GSTENG00019029001 PE=4 SV=1

spoma fimbria GN=RM53 PE=4 SV=1

lence. (Fragment) OS=Tetraodon nigroviridis GN=GSTENG00022695001 PE=4 SV=1

JS=Danio rerio GN=polr2eb $\mathrm{PE}=2 \mathrm{SV}=1$

गL18a PE=2 SV=1

lotgun sequence. (Fragment) OS=Tetraodon nigroviridis GN=GSTENG00021851001 PE=4 SV=1

ance OS=Tetraodon nigroviridis GN=GSTENG00029182001 PE=4 SV=1 
lence. (Fragment) OS=Tetraodon nigroviridis GN=GSTENG00025100001 PE=4 SV=1

lence. (Fragment) OS=Tetraodon nigroviridis GN=GSTENG00016445001 PE=4 SV=1

lence. (Fragment) OS=Tetraodon nigroviridis GN=GSTENG00028316001 PE=4 SV=1

sence OS=Tetraodon nigroviridis GN=GSTENG00028448001 PE=4 SV=1

$3 \mathrm{~N}=\operatorname{ccdc} 101 \quad \mathrm{PE}=2 \quad \mathrm{SV}=1$

$\mathrm{V}=1$

stgun sequence. (Fragment) OS=Tetraodon nigroviridis GN=GSTENG00001721001 PE=4 SV=1

fimbria $\mathrm{GN}=\mathrm{SUM} 02 \mathrm{PE}=4 \mathrm{SV}=1$

si:dkey-46a10. 3 PE=2 SV=1

rotgun sequence OS=Tetraodon nigroviridis GN=GSTENG00013805001 PE=4 SV=1

lence OS=Tetraodon nigroviridis GN=GSTENG00023016001 PE=4 SV=1

rotgun sequence OS=Tetraodon nigroviridis GN=GSTENG00008524001 PE=4 SV=1

$\mathrm{j}=1$

ənce. (Fragment) OS=Tetraodon nigroviridis GN=GSTENG00031789001 PE=4 SV=1

lence OS=Tetraodon nigroviridis GN=GSTENG00030849001 PE=4 SV=1 
niperca chuatsi $\mathrm{PE}=2 \mathrm{SV}=1$

zeus $\mathrm{GN}=H$ sp90alpha $\mathrm{PE}=2 \mathrm{SV}=1$

$\lesssim 17 \quad \mathrm{PE}=2 \mathrm{SV}=1$

ənce. (Fragment) OS=Tetraodon nigroviridis GN=GSTENG00028977001 PE=3 SV=1

lence OS=Tetraodon nigroviridis GN=GSTENG00033355001 PE=4 SV=1

Jrvegicus GN=Stard5 $\mathrm{PE}=4 \mathrm{SV}=1$

?E $=4 \quad \mathrm{SV}=1$

?L13a-1ike1 PE=2 SV=1

$\operatorname{rrd} 9 \mathrm{PE}=2 \mathrm{SV}=1$

jB $\mathrm{PE}=2 \mathrm{SV}=1$

?E=2 SV=1

¥1-a $\mathrm{PE}=2 \quad \mathrm{SV}=1$

(CD151) OS=Danio rerio GN=si:dkey-21h14. $7 \mathrm{PE}=4 \mathrm{SV}=1$

na fimbria GN=SMD2 $\mathrm{PE}=4 \mathrm{SV}=1$

ting protein $0 \mathrm{~S}=$ Anoplopoma fimbria $\mathrm{GN}=\mathrm{MAPIP} \mathrm{PE}=2 \mathrm{SV}=1$

30 OS=Anoplopoma fimbria GN=SPF30 PE=2 SV=1

agment) OS=Epinephelus coioides $\mathrm{PE}=2 \mathrm{SV}=1$

rotgun sequence. (Fragment) OS=Tetraodon nigroviridis GN=GSTENG00031728001 PE=4 SV=1

spus tropicalis GN=L0C549152 PE=2 SV=1 
$1 \mathrm{PE}=2 \mathrm{SV}=1$

Janio rerio GN=vps36 $\mathrm{PE}=2 \mathrm{SV}=1$

əus $\mathrm{PE}=2 \mathrm{SV}=1$

rotgun sequence OS=Tetraodon nigroviridis GN=GSTENG00012641001 PE=4 SV=1

nolog OS=Salmo salar GN=VPS41 PE=2 SV=1

flavescens PE=2 SV=1

stgun sequence. (Fragment) OS=Tetraodon nigroviridis GN=GSTENG00002618001 PE=4 SV=1 $=2 \mathrm{SV}=1$

lence. (Fragment) OS=Tetraodon nigroviridis GN=GSTENG00014716001 PE=4 SV=1

OS=Salmo salar GN=AL7A1 PE=2 SV=1

$100170520 \quad \mathrm{PE}=2 \mathrm{SV}=1$

ə $\mathrm{GN}=\mathrm{HIG} 1 \mathrm{~A} \quad \mathrm{PE}=4 \mathrm{SV}=1$

ıotgun sequence. (Fragment) OS=Tetraodon nigroviridis GN=GSTENG00021675001 PE=3 SV=1

ว rerio $\mathrm{GN}=$ pofut1 $\mathrm{PE}=2 \mathrm{SV}=1$

splopoma fimbria GN=SPCS2 PE=2 SV=1 
floridae GN=BRAFLDRAFT_59924 PE=4 SV=1

Janio rerio $\mathrm{GN}=\mathrm{eif4}$ ela $\mathrm{PE}=1 \mathrm{SV}=1$

गlopoma fimbria GN=RLP24 PE=2 SV=1

lence OS=Tetraodon nigroviridis GN=GSTENG00032312001 PE=4 SV=1

$=$ AGM1 PE=2 SV=1

luropoda melanoleuca GN=PANDA_003513 PE=4 SV=1

lence. (Fragment) OS=Tetraodon nigroviridis GN=GSTENG00013537001 PE=4 SV=1

lence. (Fragment) OS=Tetraodon nigroviridis GN=GSTENG00016123001 PE=4 SV=1

lence. (Fragment) OS=Tetraodon nigroviridis GN=GSTENG00029587001 PE=4 SV=1 se motif 9 OS=Danio rerio GN=nudt9 $\mathrm{PE}=2 \mathrm{SV}=1$

lence $0 \mathrm{~S}=$ Tetraodon nigroviridis GN=GSTENG00014775001 PE=4 SV=1

1S $\mathrm{PE}=4 \mathrm{SV}=1$

lence. (Fragment) OS=Tetraodon nigroviridis GN=GSTENG00020960001 PE=3 SV=1

lence. (Fragment) OS=Tetraodon nigroviridis GN=GSTENG00027439001 PE=4 SV=1 luropoda melanoleuca GN=PANDA_001804 PE=4 SV=1

$23190 \mathrm{PE}=2 \mathrm{SV}=1$

ənce. (Fragment) OS=Tetraodon nigroviridis GN=GSTENG00023873001 PE=4 SV=1

$\mathrm{PE}=2 \mathrm{SV}=1$

zorhynchus mykiss GN=SSRG PE=2 SV=1

lence. (Fragment) OS=Tetraodon nigroviridis GN=GSTENG00020941001 PE=4 SV=1 
r 1 OS=Danio rerio $\mathrm{GN}=$ abcf1 $\mathrm{PE}=2 \mathrm{SV}=1$

ว OS=Homo sapiens $\mathrm{GN}=\mathrm{ZDHHC7} \mathrm{PE}=4 \mathrm{SV}=1$

=Paralichthys olivaceus $\mathrm{PE}=2 \mathrm{SV}=1$

salt1c1 PE=2 SV=1

it 11 OS=Salmo salar GN=MED11 PE=4 SV=1

ənce. (Fragment) 0S=Tetraodon nigroviridis GN=GSTENG00033388001 PE=3 SV=1

$\mathrm{PE}=2 \quad \mathrm{SV}=1$

2

rosome, macropain) 26S subunit, ATPase, 2 (PSMC2) (Zgc:63995) OS=Danio rerio GN=psmc2 PE=2 Is homolog $1 \mathrm{~A}$ (Yeast) OS=Danio rerio GN=vtila $\mathrm{PE}=2 \mathrm{SV}=1$

lotgun sequence. (Fragment) OS=Tetraodon nigroviridis GN=GSTENG00034508001 PE=4 SV=1

it RPABC4 OS=Salmo salar GN=RPAB4 PE=4 SV=1

Jax GN=CSN3 PE=2 SV=1

$\mathrm{PE}=2 \quad \mathrm{SV}=1$

na fimbria $\mathrm{GN}=\mathrm{ATP} 5 \mathrm{~L} \quad \mathrm{PE}=4 \mathrm{SV}=1$

$\mathrm{GN}=$ noc $41 \quad \mathrm{PE}=2 \quad \mathrm{SV}=1$

s homolog 1, cyclin $\mathrm{H}$ assembly factor (Xenopus laevis) (MNAT1, zgc:101599) 0S=Danio rerio (

lence. (Fragment) OS=Tetraodon nigroviridis GN=GSTENG00029800001 PE=4 SV=1

samma $\mathrm{OS}=0$ ncorhynchus mykiss $\mathrm{GN}=\mathrm{GNPTG} \mathrm{PE}=2 \mathrm{SV}=1$

stgun sequence. (Fragment) OS=Tetraodon nigroviridis GN=GSTENG00005294001 PE=4 SV=1

Janio rerio $\mathrm{GN}=\mathrm{dhrs}$ $\mathrm{PE}=2 \mathrm{SV}=1$

(Fragment) 0S=Homo sapiens $\mathrm{PE}=2 \mathrm{SV}=1$ 
iridis GN=GSTENG00029452001 PE=3 SV=1

$\mathrm{V}=\operatorname{cops} 3 \mathrm{PE}=2 \mathrm{SV}=1$

$j \mathrm{~V}=1$

$\mathrm{z}=2 \mathrm{SV}=1$

at protein 2 OS=Danio rerio $\mathrm{GN}=$ ergic2 $\mathrm{PE}=2 \mathrm{SV}=1$

ipes $\mathrm{PE}=4 \mathrm{SV}=1$

$2 \mathrm{SV}=1$

lence OS=Tetraodon nigroviridis GN=GSTENG00020671001 PE=4 SV=1

$=1$

zius GN=TRI25 PE=2 SV=1

r $\mathrm{GN}=\mathrm{TTC} 9 \mathrm{C}$ PE=2 SV=1

teracting protein 1 OS=Salmo salar $\mathrm{GN}=\mathrm{G} 45 \mathrm{IP}$ PE=2 SV=1

$\mathrm{GN}=$ erh $\mathrm{PE}=3 \mathrm{SV}=1$

ənce. (Fragment) OS=Tetraodon nigroviridis GN=GSTENG00014685001 PE=4 SV=1

$\mathrm{s}=1$

lence. (Fragment) OS=Tetraodon nigroviridis GN=GSTENG00032237001 PE=4 SV=1

lence OS=Tetraodon nigroviridis GN=GSTENG00014021001 PE=4 SV=1

no salar GN=CSTF3 PE=2 SV=1

notgun sequence OS=Tetraodon nigroviridis GN=GSTENG00008530001 PE=4 SV=1

ənce. (Fragment) OS=Tetraodon nigroviridis GN=GSTENG00017893001 PE=4 SV=1

ar $\mathrm{GN}=\mathrm{U} 2 \mathrm{~A}^{\prime} \quad \mathrm{PE}=2 \mathrm{SV}=2$

lence. (Fragment) OS=Tetraodon nigroviridis GN=GSTENG00016036001 PE=4 SV=1

tylglucosaminephosphotransferase $0 \mathrm{~S}=$ Salmo salar GN=GPT PE=2 SV=1 
lence OS=Tetraodon nigroviridis GN=GSTENG00029420001 PE=4 SV=1

PE $=2 \quad$ SV=1

-1 subunit 1 OS=Anoplopoma fimbria GN=BL1S1 PE=2 SV=1

ənce. (Fragment) 0S=Tetraodon nigroviridis GN=GSTENG00024739001 PE=4 SV=1

spoma fimbria GN=RM40 PE=2 SV=1

lence OS=Tetraodon nigroviridis GN=GSTENG00034902001 PE=4 SV=1

t 12 OS=0ncorhynchus mykiss GN=IF3C PE=2 SV=1

NRB PE=2 SV=1

$=2 \quad \mathrm{SV}=1$

ənce. (Fragment) 0S=Tetraodon nigroviridis GN=GSTENG00018308001 PE=4 SV=1

JS=Salmo salar GN=HIBCH PE=2 SV=1

วx lucius GN=ANR39 PE=2 SV=1

r GN=UXS1 PE=2 SV=1

ənce. (Fragment) OS=Tetraodon nigroviridis GN=GSTENG00017691001 PE=4 SV=1

$\mathrm{\Xi}=2 \mathrm{SV}=1$

lence OS=Tetraodon nigroviridis GN=GSTENG00015081001 PE=4 SV=1

JS=Danio rerio $\mathrm{GN}=\operatorname{suclg} 1 \mathrm{PE}=2 \mathrm{SV}=1$

ənce. (Fragment) OS=Tetraodon nigroviridis GN=GSTENG00034089001 PE=3 SV=1 
ənce OS=Tetraodon nigroviridis GN=GSTENG00034383001 PE=4 SV=1

lence. (Fragment) OS=Tetraodon nigroviridis GN=GSTENG00027236001 PE=4 SV=1

$100135303 \mathrm{PE}=2 \mathrm{SV}=1$

$\mathrm{V}=\mathrm{rpS} 12 \quad \mathrm{PE}=2 \quad \mathrm{SV}=1$

lence OS=Tetraodon nigroviridis GN=GSTENG00020155001 PE=4 SV=1

liferation factor B OS=Danio rerio $\mathrm{GN}=$ ppdpfb $\mathrm{PE}=2 \mathrm{SV}=1$

thys olivaceus $\mathrm{PE}=2 \mathrm{SV}=1$

lence. (Fragment) OS=Tetraodon nigroviridis GN=GSTENG00030024001 PE=3 SV=1

t E OS=Anoplopoma fimbria GN=EIF3E PE=2 SV=1

iens GN=MINA $\mathrm{PE}=4 \mathrm{SV}=1$

ə $\mathrm{GN}=0 \mathrm{TUB} 2 \mathrm{PE}=2 \mathrm{SV}=1$

ว 1 , catalytic subunit $0 \mathrm{~S}=$ Danio rerio $\mathrm{GN}=\mathrm{dpm} 1 \mathrm{PE}=2 \mathrm{SV}=1$

j=Salmo salar GN=PA2G4 PE=2 SV=1

$\mathrm{V}=1$

jalmo salar GN=ZCH10 PE=2 SV=1 
$\mathrm{V}=\mathrm{TM} 134 \quad \mathrm{PE}=2 \quad \mathrm{SV}=1$

$\mathrm{V}=1$

lence OS=Tetraodon nigroviridis GN=GSTENG00030806001 PE=4 SV=1

lence. (Fragment) OS=Tetraodon nigroviridis GN=GSTENG00011945001 PE=4 SV=1 smerus mordax $\mathrm{GN}=\mathrm{D} 3 \mathrm{D} 2 \mathrm{PE}=2 \mathrm{SV}=1$

ənce. (Fragment) OS=Tetraodon nigroviridis GN=GSTENG00025551001 PE=4 SV=1

$2 \mathrm{SV}=1$

1

) salar GN=DKC1 $\mathrm{PE}=2 \mathrm{SV}=1$

$\mathrm{SV}=1$

RNA activating complex, polypeptide 5, 19kDa (SNAPC5) OS=Danio rerio GN=snapc5 PE=4 SV=1 rotgun sequence OS=Tetraodon nigroviridis GN=GSTENG00034503001 PE=3 SV=1

ıce OS=Tetraodon nigroviridis GN=GSTENG00007352001 PE=4 SV=1

V=HDA1B $\quad$ PE $=2 \quad$ SV=1

$\zeta=$ Anoplopoma fimbria $\mathrm{GN}=\mathrm{NDUA} 4 \mathrm{PE}=4 \mathrm{SV}=1$

?-104C8. 1 PE=4 SV=1

$\mathrm{V}=\mathrm{CGL} \quad \mathrm{PE}=2 \quad \mathrm{SV}=1$

ənce OS=Tetraodon nigroviridis GN=GSTENG00018520001 PE=3 SV=1

$19 \mathrm{PE}=2 \mathrm{SV}=1$

=Anoplopoma fimbria GN=TPPC4 PE=2 SV=1

lence. (Fragment) OS=Tetraodon nigroviridis GN=GSTENG00033052001 PE=4 SV=1 
$1-1$ ike $0 \mathrm{~S}=$ Salmo salar $\mathrm{GN}=\mathrm{PK} 1 \mathrm{IP} \quad \mathrm{PE}=2 \mathrm{SV}=1$

?E=2 SV=1

ənt protein alpha $0 \mathrm{~S}=$ Danio rerio $\mathrm{GN}=$ napa $\mathrm{PE}=2 \mathrm{SV}=1$

lotgun sequence. (Fragment) OS=Tetraodon nigroviridis GN=GSTENG00022380001 PE=3 SV=1

$\mathrm{I}=2 \mathrm{SV}=1$

=RS9 $\mathrm{PE}=2 \mathrm{SV}=1$

lence OS=Tetraodon nigroviridis GN=GSTENG00035765001 PE=4 SV=1

эpan $\mathrm{PE}=2 \mathrm{SV}=1$

ənce. (Fragment) OS=Tetraodon nigroviridis GN=GSTENG00023401001 PE=4 SV=1

rotgun sequence OS=Tetraodon nigroviridis GN=GSTENG00020604001 PE=4 SV=1

notgun sequence OS=Tetraodon nigroviridis GN=GSTENG00010340001 PE=4 SV=1

ənce OS=Tetraodon nigroviridis GN=GSTENG00035024001 PE=4 SV=1

j=0smerus mordax GN=DRG2 PE=2 SV=1

lar $\mathrm{GN}=\mathrm{COX} 17 \quad \mathrm{PE}=4 \quad \mathrm{SV}=1$

$\mathrm{PE}=2 \mathrm{SV}=1$

$=4 \mathrm{SV}=1$ 
${ }^{\mathrm{P}}=1 \quad \mathrm{SV}=1$ - 
a fascicularis $\mathrm{PE}=2 \mathrm{SV}=1$ 
3. $4 \mathrm{PE}=4 \mathrm{SV}=1$ 
$I=1$

https://mc06.manuscriptcentral.com/genome-pubs 
j. $4 \mathrm{PE}=4 \mathrm{SV}=1$

:io $\mathrm{GN}=$ ndufaf $1 \mathrm{PE}=2 \mathrm{SV}=1$ 
$\mathrm{SV}=1$

iN=mnat $1 \quad \mathrm{PE}=4 \quad \mathrm{SV}=1$ 


\begin{tabular}{|c|c|c|}
\hline Unigene_ref_ID & xiong_expression & ci_expression Fisher_p_value \\
\hline Unigene_UN_xiong_14529 & 3996 & 257 \\
\hline Unigene_UN_xiong_14070 & 165 & 7554 \\
\hline Unigene_UN_xiong_399 & 2091 & 717 \\
\hline Unigene_UN_xiong_14542 & 6371 & 1993 \\
\hline Unigene_UN_xiong_13607 & 360 & 5649 \\
\hline Unigene_UN_xiong_949 & 10881 & 2441 \\
\hline Unigene_UN_xiong_2637 & 4241 & 1501 \\
\hline Unigene_UN_xiong_11196 & 273 & 19628 \\
\hline Unigene_UN_xiong_7164 & 203 & 10946 \\
\hline Unigene_UN_xiong_1987 & 283 & 5118 \\
\hline Unigene_UN_xiong_194 & 2025 & 549 \\
\hline Unigene_UN_xiong_15045 & 3603 & 576 \\
\hline Unigene UN xiong 5287 & 108 & 5966 \\
\hline Unigene_UN_xiong_15243 & 3729 & 595 \\
\hline Unigene_UN_xiong_14509 & 3681 & 754 \\
\hline Unigene_UN_xiong_11113 & 218 & 7116 \\
\hline Unigene_UN_xiong_4091 & 1332 & 68944 \\
\hline Unigene_UN_xiong_3523 & 4254 & 1236 \\
\hline Unigene_UN_xiong_14868 & 155 & 3621 \\
\hline Unigene_UN_xiong_14272 & 233 & 4341 \\
\hline Unigene_UN_xiong_4237 & 6655 & 326 \\
\hline Unigene_UN_xiong_15382 & 6739 & 793 \\
\hline Unigene_UN_xiong_14905 & 12337 & 11775 \\
\hline Unigene_UN_xiong_1280 & 2641 & 765 \\
\hline Unigene_UN_xiong_14842 & 502 & 4128 \\
\hline Unigene_UN_xiong_14978 & 2492 & 699 \\
\hline Unigene_UN_xiong_11680 & 139 & 5026 \\
\hline Unigene_UN_xiong_1807 & 2282 & 437 \\
\hline Unigene_UN xiong 12139 & 244 & 4019 \\
\hline Unigene_UN_xiong_5130 & 202 & 10038 \\
\hline Unigene_UN_xiong_13569 & 4533 & 53675 \\
\hline Unigene_UN_xiong_13925 & 1128 & 12535 \\
\hline Unigene_UN_xiong_2662 & 1246 & 174 \\
\hline Unigene_UN_xiong_14312 & 7251 & 4154 \\
\hline Unigene_UN_xiong_9552 & 233 & 3108 \\
\hline Unigene_UN_xiong_14377 & 1386 & 46636 \\
\hline Unigene_UN_xiong_15383 & 2533 & 586 \\
\hline Unigene_UN xiong 1657 & 1790 & 278 \\
\hline Unigene_UN_xiong_14546 & 1672 & 495 \\
\hline Unigene_UN_xiong_15105 & 4711 & 2446 \\
\hline Unigene_UN_xiong_10027 & 2497 & 1155 \\
\hline Unigene_UN_xiong_15032 & 466 & 4948 \\
\hline Unigene_UN_xiong_14718 & 246 & 12389 \\
\hline Unigene_UN_xiong_386 & 4364 & 2000 \\
\hline Unigene_UN_xiong_14567 & 2813 & 1157 \\
\hline Unigene_UN_xiong_14716 & 3412 & 1562 \\
\hline Unigene_UN xiong_ 14273 & 7734 & 2707 \\
\hline Unigene_UN_xiong_14623 & 4746 & 2395 \\
\hline Unigene_UN_xiong_10584 & 3141 & $18111.03753785626662 \mathrm{e}^{-322}$ \\
\hline Unigene_UN_xiong_14566 & 1884 & 690 6. $26913325946737 \mathrm{e}^{-314}$ \\
\hline Unigene_UN xiong_15375 & 1763 & $5726.4228533959362 \mathrm{e}^{-323}$ \\
\hline Unigene UN xiong 15556 & 2968 & $16477.27758696324156 \mathrm{e}^{-321}$ \\
\hline
\end{tabular}


Unigene_UN_xiong_14239

Unigene_UN_xiong_8517

Unigene_UN_xiong_3844

Unigene_UN_xiong_4795

Unigene_UN_xiong_14660

Unigene_UN_xiong_9105

Unigene_UN_xiong_15664

Unigene_UN_xiong_14437

Unigene_UN_xiong_2880

Unigene_UN_xiong_216

Unigene_UN_xiong_333

Unigene_UN_xiong_14677

Unigene_UN_xiong_14877

Unigene_UN_xiong_14783

Unigene_UN_xiong_4430

Unigene_UN_xiong_14924

Unigene_UN_xiong_3845

Unigene_UN_xiong_6228

Unigene_UN_xiong_3079

Unigene_UN_xiong_14962

Unigene_UN_xiong_155

Unigene_UN_xiong_3128

Unigene_UN_xiong_14891

Unigene_UN_xiong_335

Unigene_UN_xiong_4436

Unigene_UN_xiong_5549

Unigene_UN_xiong_14385

Unigene_UN_xiong_10799

Unigene_UN_xiong_14665

Unigene_UN_xiong_15130

Unigene_UN_xiong_1438

Unigene_UN_xiong_14648

Unigene_UN_xiong_14747

Unigene_UN_xiong_256

Unigene_UN_xiong_14285

Unigene_UN_xiong_3390

Unigene_UN_xiong_2317

Unigene_UN_xiong_8530

Unigene_UN_xiong_4712

Unigene_UN_xiong_3904

Unigene_UN_xiong_1478

Unigene_UN_xiong_2555

Unigene_UN_xiong_13350

Unigene_UN_xiong_3637

Unigene_UN_xiong_14727

Unigene_UN_xiong_6909

Unigene_UN_xiong_14274

Unigene_UN_xiong_3787

Unigene_UN_xiong_14453

Unigene_UN_xiong_14263

Unigene_UN_xiong_122

Unigene_UN_xiong_13451

Unigene_UN_xiong_8419

Unigene_UN_xiong_15660
1466

687

5482

253

1300

4634

209

1702

590

838

1190

496

1204

2055

143

1396

880

1070

256

2668

1556

320

820

839

925

262

1219

155

1757

1065

854

896

500

1100

2489

3494

2928

1935

632

1200

1338

1189

1252

629

1181

759

616

195

1023

814

661

119

240

553
362 9. $45641646140146 \mathrm{e}^{-321}$

$4441 \quad 9.73 \mathrm{E}-299$

$4555 \quad 1.43 \mathrm{E}-297$

$2864 \quad 1.80 \mathrm{E}-294$

$319 \quad 6.22 \mathrm{E}-286$

$3671 \quad 2.91 \mathrm{E}-279$

$2414 \quad 2.01 \mathrm{E}-251$

$739 \quad 1.71 \mathrm{E}-243$

$36892.82 \mathrm{E}-240$

$125 \quad 1.15 \mathrm{E}-232$

$366 \quad 2.67 \mathrm{E}-227$

$3268 \quad 1.16 \mathrm{E}-224$

$383 \quad 1.59 \mathrm{E}-224$

$1154 \quad 1.24 \mathrm{E}-219$

1933 2. $36 \mathrm{E}-218$

$559 \quad 4.55 \mathrm{E}-216$

187 1. $10 \mathrm{E}-209$

$328 \quad 5.20 \mathrm{E}-205$

2269

1944

788

2409

199

3951

275

2153

515

1701

1058

4408

271

304

2772

476

1939

3184

2498

1396

2997

635

775

634

2. $22 \mathrm{E}-200$

1. $19 \mathrm{E}-190$

1. $80 \mathrm{E}-189$

1. $97 \mathrm{E}-187$

2. $60 \mathrm{E}-182$

2. $17 \mathrm{E}-181$

2. $93 \mathrm{E}-181$

6. $11 \mathrm{E}-180$

6. $99 \mathrm{E}-180$

9. $17 \mathrm{E}-173$

2. $49 \mathrm{E}-171$

3. $01 \mathrm{E}-164$

4. $67 \mathrm{E}-160$

9. $91 \mathrm{E}-160$

1. $10 \mathrm{E}-158$

1. $14 \mathrm{E}-158$

1. $13 \mathrm{E}-156$

4. $64 \mathrm{E}-153$

3. $04 \mathrm{E}-151$

1. $79 \mathrm{E}-141$

1. $33 \mathrm{E}-139$

3. $79 \mathrm{E}-139$

4. $45 \mathrm{E}-138$

1. $20 \mathrm{E}-136$

2. $83 \mathrm{E}-133$

1. $61 \mathrm{E}-132$

7. $45 \mathrm{E}-131$

2. $05 \mathrm{E}-130$

3. $10 \mathrm{E}-130$

8. $66 \mathrm{E}-129$

2. $11 \mathrm{E}-126$

8. $96 \mathrm{E}-122$

1. $22 \mathrm{E}-121$

6. $88 \mathrm{E}-117$

6. $92 \mathrm{E}-117$

1. $51 \mathrm{E}-115$ 
Unigene_UN_xiong_6273

Unigene_UN_xiong_14616

Unigene_UN_xiong_5777

Unigene_UN_xiong_15374

Unigene_UN_xiong_14859

Unigene_UN_xiong_3047

Unigene_UN_xiong_2181

Unigene_UN_xiong_14414

Unigene_UN_xiong_14937

Unigene_UN_xiong_14674

Unigene_UN_xiong_5020

Unigene_UN_xiong_4401

Unigene_UN_xiong_2958

Unigene_UN_xiong_14689

Unigene_UN_xiong_751

Unigene_UN_xiong_14569

Unigene_UN_xiong_14270

Unigene_UN_xiong_11645

Unigene_UN_xiong_14286

Unigene_UN_xiong_14334

Unigene_UN_xiong_7854

Unigene_UN_xiong_988

Unigene_UN_xiong_2611

Unigene_UN_xiong_14873

Unigene_UN_xiong_15687

Unigene_UN_xiong_5912

Unigene_UN_xiong_3634

Unigene_UN_xiong_145

Unigene_UN_xiong_790

Unigene_UN_xiong_1060

Unigene_UN_xiong_14618

Unigene_UN_xiong_7465

Unigene_UN_xiong_4109

Unigene_UN_xiong_9546

Unigene_UN_xiong_1685

Unigene_UN_xiong_15543

Unigene_UN_xiong_13667

Unigene_UN_xiong_1628

Unigene_UN_xiong_15785

Unigene_UN_xiong_7527

Unigene_UN_xiong_5941

Unigene_UN_xiong_8790

Unigene_UN_xiong_12115

Unigene_UN_xiong_8850

Unigene_UN_xiong_2248

Unigene_UN_xiong_10847

Unigene_UN_xiong_14812

Unigene_UN_xiong_4643

Unigene_UN_xiong_6978

Unigene_UN_xiong_14438

Unigene_UN_xiong_14383

Unigene_UN_xiong_7498

Unigene_UN_xiong_13515

Unigene_UN_xiong_12363

\begin{tabular}{|c|c|c|}
\hline 299 & 1811 & 3. $33 \mathrm{E}-115$ \\
\hline 554 & 154 & 4. $06 \mathrm{E}-114$ \\
\hline 814 & 366 & 1. $53 \mathrm{E}-113$ \\
\hline 794 & 349 & $2.50 \mathrm{E}-113$ \\
\hline 645 & 226 & 8. $11 \mathrm{E}-113$ \\
\hline 594 & 187 & 1. $23 \mathrm{E}-112$ \\
\hline 1021 & 564 & 1. $55 \mathrm{E}-112$ \\
\hline 2462 & 7151 & 2. $43 \mathrm{E}-112$ \\
\hline 583 & 180 & 4. $68 \mathrm{E}-112$ \\
\hline 614 & 207 & 1. $12 \mathrm{E}-110$ \\
\hline 1176 & 741 & 3. $99 \mathrm{E}-108$ \\
\hline 602 & 204 & 4. $25 \mathrm{E}-108$ \\
\hline 266 & 1616 & 2. $69 \mathrm{E}-103$ \\
\hline 537 & 168 & 1. $86 \mathrm{E}-102$ \\
\hline 779 & 376 & 3. $43 \mathrm{E}-101$ \\
\hline 569 & 197 & 1. $11 \mathrm{E}-100$ \\
\hline 220 & 1442 & 8. $25 \mathrm{E}-100$ \\
\hline 524 & 165 & 1. $46 \mathrm{E}-99$ \\
\hline 799 & 401 & 2. $40 \mathrm{E}-99$ \\
\hline 1216 & 828 & 6. $22 \mathrm{E}-99$ \\
\hline 661 & 278 & 1. $02 \mathrm{E}-98$ \\
\hline 1029 & 631 & 1. $30 \mathrm{E}-98$ \\
\hline 1976 & 1719 & 3. $16 \mathrm{E}-98$ \\
\hline 571 & 207 & 1. $92 \mathrm{E}-97$ \\
\hline 309 & 1692 & 3. $04 \mathrm{E}-96$ \\
\hline 518 & 169 & 6. $64 \mathrm{E}-96$ \\
\hline 293 & 1630 & 1. $88 \mathrm{E}-94$ \\
\hline 459 & 135 & 1. $94 \mathrm{E}-91$ \\
\hline 821 & 461 & 3. $89 \mathrm{E}-89$ \\
\hline 279 & 1544 & 5. $09 \mathrm{E}-89$ \\
\hline 1356 & 4284 & 4. $92 \mathrm{E}-88$ \\
\hline 426 & 121 & 4. $06 \mathrm{E}-87$ \\
\hline 884 & 535 & 1. $33 \mathrm{E}-86$ \\
\hline 1221 & 908 & 3. $46 \mathrm{E}-85$ \\
\hline 741 & 399 & $6.15 \mathrm{E}-85$ \\
\hline 781 & 439 & $9.24 \mathrm{E}-85$ \\
\hline 1185 & 881 & $8.96 \mathrm{E}-83$ \\
\hline 557 & 240 & 9. $23 \mathrm{E}-82$ \\
\hline 542 & 2215 & 9. $73 \mathrm{E}-82$ \\
\hline 637 & 312 & 1. $18 \mathrm{E}-81$ \\
\hline 535 & 225 & 2. $50 \mathrm{E}-80$ \\
\hline 149 & 1048 & 7. $50 \mathrm{E}-78$ \\
\hline 243 & 1345 & $9.99 \mathrm{E}-78$ \\
\hline 951 & 655 & 2. $63 \mathrm{E}-76$ \\
\hline 463 & 1949 & 5. $62 \mathrm{E}-76$ \\
\hline 697 & 398 & 3. $56 \mathrm{E}-74$ \\
\hline 1115 & 852 & 4. $45 \mathrm{E}-74$ \\
\hline 279 & 1417 & 1. $44 \mathrm{E}-73$ \\
\hline 972 & 700 & 2. $74 \mathrm{E}-72$ \\
\hline 2105 & 2102 & 3. $91 \mathrm{E}-72$ \\
\hline 447 & 175 & 7. $86 \mathrm{E}-72$ \\
\hline 248 & 1303 & $1.01 \mathrm{E}-70$ \\
\hline 619 & 2300 & 1. $14 \mathrm{E}-70$ \\
\hline 420 & 157 & 2. $06 \mathrm{E}-70$ \\
\hline
\end{tabular}

https://mc06.manuscriptcentral.com/genome-pubs 
Unigene_UN_xiong_14440

Unigene_UN_xiong_3675

Unigene_UN_xiong_8920

Unigene_UN_xiong_3105

Unigene_UN_xiong_5545

Unigene_UN_xiong_14490

Unigene_UN_xiong_2168

Unigene_UN_xiong_6384

Unigene_UN_xiong_7707

Unigene_UN_xiong_2119

Unigene_UN_xiong_2218

Unigene_UN_xiong_12342

Unigene_UN_xiong_9316

Unigene_UN_xiong_1570

Unigene_UN_xiong_4494

Unigene_UN_xiong_1588

Unigene_UN_xiong_5453

Unigene_UN_xiong_14801

Unigene_UN_xiong_8994

Unigene_UN_xiong_2689

Unigene_UN_xiong_6304

Unigene_UN_xiong_825

Unigene_UN_xiong_10733

Unigene_UN_xiong_3878

Unigene_UN_xiong_15520

Unigene_UN_xiong_12413

Unigene_UN_xiong_876

Unigene_UN_xiong_14347

Unigene_UN_xiong_9163

Unigene_UN_xiong_14497

Unigene_UN_xiong_4932

Unigene_UN_xiong_3436

Unigene_UN_xiong_8136

Unigene_UN_xiong_5737

Unigene_UN_xiong_11251

Unigene_UN_xiong_4975

Unigene_UN_xiong_15308

Unigene_UN_xiong_1201

Unigene_UN_xiong_14327

Unigene_UN_xiong_14307

Unigene_UN_xiong_5113

Unigene_UN_xiong_2520

Unigene_UN_xiong_142

Unigene_UN_xiong_4359

Unigene_UN_xiong_14308

Unigene_UN_xiong_4059

Unigene_UN_xiong_12070

Unigene_UN_xiong_6694

Unigene_UN_xiong_10563

Unigene_UN_xiong_1586

Unigene_UN_xiong_14563

Unigene_UN_xiong_9359

Unigene_UN_xiong_307

Unigene_UN_xiong_12491

\begin{tabular}{|c|c|c|}
\hline 1775 & 1689 & $2.51 \mathrm{E}-70$ \\
\hline 494 & 221 & 4. $04 \mathrm{E}-70$ \\
\hline 541 & 265 & 1. $03 \mathrm{E}-69$ \\
\hline 984 & 3163 & 1. $21 \mathrm{E}-68$ \\
\hline 451 & 1844 & 2. $44 \mathrm{E}-68$ \\
\hline 689 & 418 & $9.83 \mathrm{E}-68$ \\
\hline 430 & 173 & 1. $41 \mathrm{E}-67$ \\
\hline 675 & 413 & $1.88 \mathrm{E}-65$ \\
\hline 338 & 1511 & 2. $40 \mathrm{E}-65$ \\
\hline 455 & 202 & 3. $86 \mathrm{E}-65$ \\
\hline 213 & 1139 & 1. $55 \mathrm{E}-63$ \\
\hline 371 & 138 & 1. $17 \mathrm{E}-62$ \\
\hline 679 & 433 & 3. $66 \mathrm{E}-62$ \\
\hline 777 & 2591 & 3. $77 \mathrm{E}-62$ \\
\hline 692 & 447 & 4. $66 \mathrm{E}-62$ \\
\hline 397 & 1639 & 5. $61 \mathrm{E}-62$ \\
\hline 122 & 841 & 1. $03 \mathrm{E}-61$ \\
\hline 681 & 437 & 1. $27 \mathrm{E}-61$ \\
\hline 592 & 346 & 1. $57 \mathrm{E}-61$ \\
\hline 364 & 137 & 8. $65 \mathrm{E}-61$ \\
\hline 584 & 341 & 1. $16 \mathrm{E}-60$ \\
\hline 355 & 131 & 2. $93 \mathrm{E}-60$ \\
\hline 917 & 707 & 3. $43 \mathrm{E}-60$ \\
\hline 744 & 517 & 3. $15 \mathrm{E}-59$ \\
\hline 921 & 719 & 5. $15 \mathrm{E}-59$ \\
\hline 312 & 102 & 2. $59 \mathrm{E}-58$ \\
\hline 509 & 277 & 3. $31 \mathrm{E}-58$ \\
\hline 5749 & 12920 & 6. $20 \mathrm{E}-58$ \\
\hline 619 & 392 & 2. $58 \mathrm{E}-57$ \\
\hline 568 & 341 & 5. $47 \mathrm{E}-57$ \\
\hline 681 & 2295 & 9. $76 \mathrm{E}-57$ \\
\hline 467 & 243 & 1. $77 \mathrm{E}-56$ \\
\hline 407 & 188 & 4. $03 \mathrm{E}-56$ \\
\hline 336 & 1417 & 7. $28 \mathrm{E}-56$ \\
\hline 198 & 1028 & 1. $84 \mathrm{E}-55$ \\
\hline 426 & 208 & 2. $46 \mathrm{E}-55$ \\
\hline 342 & 1419 & 2. $20 \mathrm{E}-54$ \\
\hline 520 & 303 & 2. $47 \mathrm{E}-54$ \\
\hline 969 & 814 & 2. $68 \mathrm{E}-53$ \\
\hline 839 & 2621 & $6.78 \mathrm{E}-53$ \\
\hline 529 & 319 & 7. $69 \mathrm{E}-53$ \\
\hline 1151 & 1044 & 2. $07 \mathrm{E}-52$ \\
\hline 607 & 404 & 2. $93 \mathrm{E}-52$ \\
\hline 1567 & 1585 & 3. $70 \mathrm{E}-52$ \\
\hline 2896 & 3434 & 7. $06 \mathrm{E}-52$ \\
\hline 327 & 136 & $2.52 \mathrm{E}-50$ \\
\hline 204 & 996 & 1. $52 \mathrm{E}-49$ \\
\hline 713 & 538 & $3.26 \mathrm{E}-49$ \\
\hline 598 & 410 & 4. $72 \mathrm{E}-49$ \\
\hline 927 & 807 & 4. $62 \mathrm{E}-47$ \\
\hline 1317 & 1304 & 5. $05 \mathrm{E}-47$ \\
\hline 433 & 1569 & 2. $72 \mathrm{E}-46$ \\
\hline 238 & 1059 & 6. $11 \mathrm{E}-46$ \\
\hline 366 & 189 & 4. $79 \mathrm{E}-4$ \\
\hline
\end{tabular}


Unigene_UN_xiong_15449

Unigene_UN_xiong_14271

Unigene_UN_xiong_15554

Unigene_UN_xiong_15692

Unigene_UN_xiong_14840

Unigene_UN_xiong_14658

Unigene_UN_xiong_15533

Unigene_UN_xiong_13462

Unigene_UN_xiong_12050

Unigene_UN_xiong_9570

Unigene_UN_xiong_1436

Unigene_UN_xiong_14884

Unigene_UN_xiong_4179

Unigene_UN_xiong_6936

Unigene_UN_xiong_2808

Unigene_UN_xiong_4039

Unigene_UN_xiong_15372

Unigene_UN_xiong_2199

Unigene_UN_xiong_4453

Unigene_UN_xiong_15219

Unigene_UN_xiong_3384

Unigene_UN_xiong_1428

Unigene_UN_xiong_14525

Unigene_UN_xiong_2973

Unigene_UN_xiong_557

Unigene_UN_xiong_12975

Unigene_UN_xiong_14541

Unigene_UN_xiong_14313

Unigene_UN_xiong_13097

Unigene_UN_xiong_6672

Unigene_UN_xiong_4304

Unigene_UN_xiong_14344

Unigene_UN_xiong_7852

Unigene_UN_xiong_14299

Unigene_UN_xiong_12671

Unigene_UN_xiong_14795

Unigene_UN_xiong_3203

Unigene_UN_xiong_3600

Unigene_UN_xiong_14835

Unigene_UN_xiong_14883

Unigene_UN_xiong_2239

Unigene_UN_xiong_4351

Unigene_UN_xiong_3265

Unigene_UN_xiong_15034

Unigene_UN_xiong_14710

Unigene_UN_xiong_14441

Unigene_UN_xiong_9964

Unigene_UN_xiong_14637

Unigene_UN_xiong_14354

Unigene_UN_xiong_15039

Unigene_UN_xiong_15587

Unigene_UN_xiong_14970

Unigene_UN_xiong_9243

Unigene_UN_xiong_4995

\begin{tabular}{|c|c|c|}
\hline 350 & 176 & 2. $09 \mathrm{E}-44$ \\
\hline 852 & 737 & 4. $83 \mathrm{E}-44$ \\
\hline 1435 & 1495 & 1. $50 \mathrm{E}-43$ \\
\hline 387 & 215 & 1. $74 \mathrm{E}-43$ \\
\hline 162 & 823 & 1. $93 \mathrm{E}-43$ \\
\hline 392 & 1433 & 3. $20 \mathrm{E}-43$ \\
\hline 152 & 792 & 3. $26 \mathrm{E}-43$ \\
\hline 273 & 111 & 3. $37 \mathrm{E}-43$ \\
\hline 561 & 405 & 2. $32 \mathrm{E}-42$ \\
\hline 1245 & 1254 & 2. $57 \mathrm{E}-42$ \\
\hline 681 & 545 & $5.98 \mathrm{E}-42$ \\
\hline 242 & 89 & 7. 50E-42 \\
\hline 778 & 662 & 7. $97 \mathrm{E}-42$ \\
\hline 290 & 130 & 1. $02 \mathrm{E}-41$ \\
\hline 570 & 423 & 5. $69 \mathrm{E}-41$ \\
\hline 192 & 886 & $6.19 \mathrm{E}-41$ \\
\hline 649 & 516 & 1. $40 \mathrm{E}-40$ \\
\hline 165 & 807 & 1. $72 \mathrm{E}-40$ \\
\hline 166 & 808 & 2. $22 \mathrm{E}-40$ \\
\hline 1857 & 2117 & 3. $37 \mathrm{E}-40$ \\
\hline 606 & 475 & 4. $11 \mathrm{E}-39$ \\
\hline 226 & 954 & $2.98 \mathrm{E}-38$ \\
\hline 1055 & 1044 & $3.86 \mathrm{E}-38$ \\
\hline 823 & 746 & 4. $38 \mathrm{E}-38$ \\
\hline 393 & 246 & 1. $06 \mathrm{E}-37$ \\
\hline 369 & 221 & 1. $30 \mathrm{E}-37$ \\
\hline 397 & 251 & 1. $99 \mathrm{E}-37$ \\
\hline 831 & 762 & $2.31 \mathrm{E}-37$ \\
\hline 898 & 848 & $3.34 \mathrm{E}-37$ \\
\hline 448 & 308 & 4. $86 \mathrm{E}-37$ \\
\hline 285 & 142 & $9.85 \mathrm{E}-37$ \\
\hline 594 & 480 & 3. $46 \mathrm{E}-36$ \\
\hline 234 & 950 & 1. $12 \mathrm{E}-35$ \\
\hline 305 & 166 & 1. $62 \mathrm{E}-35$ \\
\hline 848 & 810 & 3. $20 \mathrm{E}-34$ \\
\hline 585 & 1784 & 3. $74 \mathrm{E}-34$ \\
\hline 1131 & 1187 & $6.41 \mathrm{E}-34$ \\
\hline 176 & 778 & 8. $87 \mathrm{E}-34$ \\
\hline 328 & 1170 & 8. $94 \mathrm{E}-34$ \\
\hline 304 & 172 & 1. $00 \mathrm{E}-33$ \\
\hline 291 & 160 & 1. $92 \mathrm{E}-33$ \\
\hline 295 & 166 & $6.11 \mathrm{E}-33$ \\
\hline 318 & 1130 & 2. $12 \mathrm{E}-32$ \\
\hline 395 & 274 & 2. $58 \mathrm{E}-32$ \\
\hline 283 & 1040 & 3. $86 \mathrm{E}-32$ \\
\hline 325 & 1144 & 4. $32 \mathrm{E}-32$ \\
\hline 239 & 116 & 4. $80 \mathrm{E}-32$ \\
\hline 300 & 176 & 8. 09E-32 \\
\hline 529 & 435 & 3. $07 \mathrm{E}-31$ \\
\hline 373 & 256 & 3. $39 \mathrm{E}-31$ \\
\hline 554 & 1672 & $3.53 \mathrm{E}-31$ \\
\hline 305 & 184 & 3. $60 \mathrm{E}-31$ \\
\hline 787 & 764 & 2. $02 \mathrm{E}-30$ \\
\hline 355 & 241 & 3. $29 \mathrm{E}-30$ \\
\hline
\end{tabular}


Unigene_UN_xiong_14717

Unigene_UN_xiong_3793

Unigene_UN_xiong_2413

Unigene_UN_xiong_14516

Unigene_UN_xiong_3968

Unigene_UN_xiong_3503

Unigene_UN_xiong_3181

Unigene_UN_xiong_1200

Unigene_UN_xiong_5589

Unigene_UN_xiong_6740

Unigene_UN_xiong_13283

Unigene_UN_xiong_12762

Unigene_UN_xiong_14527

Unigene_UN_xiong_662

Unigene_UN_xiong_14683

Unigene_UN_xiong_1812

Unigene_UN_xiong_14805

Unigene_UN_xiong_15786

Unigene_UN_xiong_14398

Unigene_UN_xiong_3085

Unigene_UN_xiong_2677

Unigene_UN_xiong_15049

Unigene_UN_xiong_14932

Unigene_UN_xiong_14106

Unigene_UN_xiong_14593

Unigene_UN_xiong_8117

Unigene_UN_xiong_14444

Unigene_UN_xiong_7138

Unigene_UN_xiong_14995

Unigene_UN_xiong_13092

Unigene_UN_xiong_14224

Unigene_UN_xiong_4865

Unigene_UN_xiong_14907

Unigene_UN_xiong_8591

Unigene_UN_xiong_10069

Unigene_UN_xiong_2251

Unigene_UN_xiong_13033

Unigene_UN_xiong_13924

Unigene_UN_xiong_14283

Unigene_UN_xiong_1931

Unigene_UN_xiong_607

Unigene_UN_xiong_3481

Unigene_UN_xiong_3495

Unigene_UN_xiong_15078

Unigene_UN_xiong_6002

Unigene_UN_xiong_11708

Unigene_UN_xiong_2209

Unigene_UN_xiong_6878

Unigene_UN_xiong_1494

Unigene_UN_xiong_14885

Unigene_UN_xiong_4084

Unigene_UN_xiong_15557

Unigene_UN_xiong_1920

Unigene_UN_xiong_10414

\begin{tabular}{|c|c|c|}
\hline 477 & 381 & 4. $36 \mathrm{E}-30$ \\
\hline 240 & 125 & 7. $99 \mathrm{E}-30$ \\
\hline 302 & 190 & 3. $56 \mathrm{E}-29$ \\
\hline 412 & 314 & 1. $51 \mathrm{E}-28$ \\
\hline 433 & 344 & 9. $32 \mathrm{E}-28$ \\
\hline 343 & 241 & 1. $07 \mathrm{E}-27$ \\
\hline 232 & 126 & 1. $68 \mathrm{E}-27$ \\
\hline 335 & 234 & 2. $46 \mathrm{E}-27$ \\
\hline 164 & 682 & 4. $31 \mathrm{E}-27$ \\
\hline 310 & 211 & 1. $37 \mathrm{E}-26$ \\
\hline 255 & 154 & 3. $26 \mathrm{E}-26$ \\
\hline 368 & 280 & 9. $79 \mathrm{E}-26$ \\
\hline 273 & 176 & 1. $83 \mathrm{E}-25$ \\
\hline 455 & 386 & 2. $39 \mathrm{E}-25$ \\
\hline 315 & 223 & 2. $55 \mathrm{E}-25$ \\
\hline 529 & 1527 & 5. $65 \mathrm{E}-25$ \\
\hline 716 & 725 & 1. $15 \mathrm{E}-24$ \\
\hline 398 & 324 & 2. $29 \mathrm{E}-24$ \\
\hline 328 & 245 & $5.58 \mathrm{E}-24$ \\
\hline 342 & 261 & 5. $75 \mathrm{E}-24$ \\
\hline 215 & 124 & 1. $07 \mathrm{E}-23$ \\
\hline 283 & 198 & 2. $69 \mathrm{E}-23$ \\
\hline 1037 & 1185 & $5.33 \mathrm{E}-23$ \\
\hline 234 & 149 & 1. $49 \mathrm{E}-22$ \\
\hline 686 & 707 & 1. $83 \mathrm{E}-22$ \\
\hline 315 & 1002 & 2. $63 \mathrm{E}-22$ \\
\hline 1570 & 3640 & 1. $68 \mathrm{E}-21$ \\
\hline 441 & 398 & 2. $29 \mathrm{E}-21$ \\
\hline 338 & 1040 & 3. $95 \mathrm{E}-21$ \\
\hline 274 & 202 & 9. $41 \mathrm{E}-21$ \\
\hline 409 & 365 & 1. $70 \mathrm{E}-20$ \\
\hline 259 & 188 & 3. $60 \mathrm{E}-20$ \\
\hline 2105 & 2812 & 3. $98 \mathrm{E}-20$ \\
\hline 543 & 542 & $5.88 \mathrm{E}-20$ \\
\hline 399 & 357 & 7. $32 \mathrm{E}-20$ \\
\hline 299 & 933 & 7. $78 \mathrm{E}-20$ \\
\hline 748 & 822 & 1. $22 \mathrm{E}-19$ \\
\hline 865 & 988 & 1. $89 \mathrm{E}-19$ \\
\hline 178 & 640 & $1.89 \mathrm{E}-19$ \\
\hline 588 & 606 & 1. $90 \mathrm{E}-19$ \\
\hline 458 & 436 & 2. $17 \mathrm{E}-19$ \\
\hline 286 & 224 & 2. $19 \mathrm{E}-19$ \\
\hline 426 & 397 & 3. $55 \mathrm{E}-19$ \\
\hline 340 & 291 & 5. $22 \mathrm{E}-19$ \\
\hline 340 & 292 & 7. $97 \mathrm{E}-19$ \\
\hline 273 & 213 & $9.80 \mathrm{E}-19$ \\
\hline 518 & 525 & 2. $99 \mathrm{E}-18$ \\
\hline 343 & 300 & 3. $40 \mathrm{E}-18$ \\
\hline 1096 & 1338 & 3. $71 \mathrm{E}-18$ \\
\hline 855 & 992 & 4. $42 \mathrm{E}-18$ \\
\hline 319 & 955 & 4. $54 \mathrm{E}-18$ \\
\hline 512 & 519 & 4. $57 \mathrm{E}-18$ \\
\hline 316 & 270 & 8. $34 \mathrm{E}-18$ \\
\hline 369 & 336 & 1. $01 \mathrm{E}-1$ \\
\hline
\end{tabular}




\begin{tabular}{|c|c|c|c|}
\hline Unigene_UN_xiong_14761 & 396 & 371 & 1. $20 \mathrm{E}-17$ \\
\hline Unigene_UN_xiong_983 & 232 & 748 & 1. $20 \mathrm{E}-17$ \\
\hline Unigene_UN_xiong_3521 & 859 & 2102 & 2. $09 \mathrm{E}-17$ \\
\hline Unigene_UN_xiong_9694 & 89 & 389 & 2. $32 \mathrm{E}-17$ \\
\hline Unigene_UN_xiong_15624 & 462 & 460 & 3. $07 \mathrm{E}-17$ \\
\hline Unigene_UN_xiong_1043 & 409 & 396 & 1. $01 \mathrm{E}-16$ \\
\hline Unigene_UN_xiong_16 & 313 & 275 & 1. $34 \mathrm{E}-16$ \\
\hline Unigene_UN_xiong_8569 & 1036 & 2445 & 1. $36 \mathrm{E}-16$ \\
\hline Unigene_UN_xiong_8203 & 321 & 286 & 2. $00 \mathrm{E}-16$ \\
\hline Unigene_UN_xiong_14899 & 242 & 192 & 3. $90 \mathrm{E}-16$ \\
\hline Unigene_UN_xiong_2099 & 665 & 751 & $5.53 \mathrm{E}-16$ \\
\hline Unigene_UN_xiong_11580 & 330 & 303 & 8. $97 \mathrm{E}-16$ \\
\hline Unigene_UN_xiong_4533 & 470 & 1243 & $3.74 \mathrm{E}-15$ \\
\hline Unigene_UN_xiong_1942 & 282 & 826 & 7. $29 \mathrm{E}-15$ \\
\hline Unigene_UN_xiong_14251 & 865 & 1054 & $8.57 \mathrm{E}-15$ \\
\hline Unigene_UN_xiong_6171 & 267 & 232 & $8.94 \mathrm{E}-15$ \\
\hline Unigene_UN_xiong_889 & 220 & 177 & 1. $83 \mathrm{E}-14$ \\
\hline Unigene_UN_xiong_13480 & 5608 & 8578 & 2. $75 \mathrm{E}-14$ \\
\hline Unigene_UN xiong 14731 & 266 & 234 & 2. $83 \mathrm{E}-14$ \\
\hline Unigene_UN_xiong_15790 & 332 & 318 & 2. $99 \mathrm{E}-14$ \\
\hline Unigene_UN_xiong_2288 & 244 & 208 & 3. $95 \mathrm{E}-14$ \\
\hline Unigene_UN_xiong_8411 & 310 & 291 & 3. $96 \mathrm{E}-14$ \\
\hline Unigene UN xiong 4628 & 272 & 243 & 4. $79 \mathrm{E}-14$ \\
\hline Unigene_UN_xiong_14486 & 560 & 629 & 6. $03 \mathrm{E}-14$ \\
\hline Unigene_UN_xiong_12764 & 197 & 620 & 6. $38 \mathrm{E}-14$ \\
\hline Unigene_UN_xiong_14409 & 766 & 929 & 1. $43 \mathrm{E}-13$ \\
\hline Unigene_UN xiong 7832 & 158 & 522 & 2. $05 \mathrm{E}-13$ \\
\hline Unigene_UN_xiong_9969 & 122 & 74 & $2.89 \mathrm{E}-13$ \\
\hline Unigene_UN_xiong_9738 & 296 & 281 & 3. $39 \mathrm{E}-13$ \\
\hline Unigene_UN_xiong_14990 & 174 & 134 & 9. $14 \mathrm{E}-13$ \\
\hline Unigene_UN xiong 10741 & 202 & 614 & 1. $28 \mathrm{E}-12$ \\
\hline Unigene_UN_xiong_14968 & 323 & 323 & $1.99 \mathrm{E}-12$ \\
\hline Unigene_UN_xiong_2226 & 216 & 186 & 2. $20 \mathrm{E}-12$ \\
\hline Unigene_UN_xiong_8120 & 169 & 535 & 2. $29 \mathrm{E}-12$ \\
\hline Unigene_UN_xiong_14362 & 266 & 251 & 3. $55 \mathrm{E}-12$ \\
\hline Unigene_UN_xiong_14684 & 271 & 258 & 4. $47 \mathrm{E}-12$ \\
\hline Unigene_UN_xiong_14784 & 551 & 642 & 5. $75 \mathrm{E}-12$ \\
\hline Unigene_UN_xiong_14325 & 537 & 627 & 1. $17 \mathrm{E}-11$ \\
\hline Unigene_UN_xiong_11308 & 370 & 969 & 1. $18 \mathrm{E}-11$ \\
\hline Unigene UN xiong 2360 & 412 & 452 & 1. $49 \mathrm{E}-11$ \\
\hline Unigene_UN_xiong_14260 & 124 & 417 & 1. $99 \mathrm{E}-11$ \\
\hline Unigene_UN_xiong_6441 & 176 & 536 & $2.82 \mathrm{E}-11$ \\
\hline Unigene_UN_xiong 8985 & 137 & 100 & 2. $84 \mathrm{E}-11$ \\
\hline Unigene_UN_xiong_14258 & 682 & 844 & 3. $69 \mathrm{E}-11$ \\
\hline Unigene_UN_xiong_9761 & 781 & 990 & 3. $82 \mathrm{E}-11$ \\
\hline Unigene_UN_xiong_13205 & 197 & 175 & 9. $63 \mathrm{E}-11$ \\
\hline Unigene_UN_xiong_3864 & 288 & 293 & 1. $04 \mathrm{E}-10$ \\
\hline Unigene_UN_xiong_4247 & 156 & 481 & 1. $39 \mathrm{E}-10$ \\
\hline Unigene_UN_xiong_4642 & 265 & 264 & 1. $46 \mathrm{E}-10$ \\
\hline Unigene_UN_xiong_14780 & 354 & 384 & 1. $75 \mathrm{E}-10$ \\
\hline Unigene_UN_xiong_14608 & 533 & 638 & 1. $93 \mathrm{E}-10$ \\
\hline Unigene UN xiong 10903 & 142 & 111 & 2. $11 \mathrm{E}-10$ \\
\hline Unigene_UN_xiong_8140 & 441 & 507 & 2. $35 \mathrm{E}-10$ \\
\hline Unigene_UN_xiong_6452 & 449 & 519 & 2. $36 \mathrm{E}-10$ \\
\hline
\end{tabular}


Unigene_UN_xiong_13318

Unigene_UN_xiong_4348

Unigene_UN_xiong_14352

Unigene_UN_xiong_4599

Unigene_UN_xiong_14975

Unigene_UN_xiong_13896

Unigene_UN_xiong_11376

Unigene_UN_xiong_14736

Unigene_UN_xiong_3763

Unigene_UN_xiong_14666

Unigene_UN_xiong_5310

Unigene_UN_xiong_11967

Unigene_UN_xiong_15503

Unigene_UN_xiong_15191

Unigene_UN_xiong_14002

Unigene_UN_xiong_13661

Unigene_UN_xiong_3420

Unigene_UN_xiong_1167

Unigene_UN_xiong_838

Unigene_UN_xiong_4244

Unigene_UN_xiong_14687

Unigene_UN_xiong_10360

Unigene_UN_xiong_2788

Unigene_UN_xiong_15787

Unigene_UN_xiong_9146

Unigene_UN_xiong_3902

Unigene_UN_xiong_13970

Unigene_UN_xiong_9643

Unigene_UN_xiong_14326

Unigene_UN_xiong_13845

Unigene_UN_xiong_12519

Unigene_UN_xiong_7476

Unigene_UN_xiong_14997

Unigene_UN_xiong_1596

Unigene_UN_xiong_8683

Unigene_UN_xiong_14548

Unigene_UN_xiong_891

Unigene_UN_xiong_1247

Unigene_UN_xiong_14663

Unigene_UN_xiong_29

Unigene_UN_xiong_4038

Unigene_UN_xiong_10111

Unigene_UN_xiong_14374

Unigene_UN_xiong_11502

Unigene_UN_xiong_14681

Unigene_UN_xiong_7586

Unigene_UN_xiong_273

Unigene_UN_xiong_14856

Unigene_UN_xiong_1364

Unigene_UN_xiong_14906

Unigene_UN_xiong_553

Unigene_UN_xiong_15519

Unigene_UN_xiong_5132

Unigene_UN_xiong_4081

\begin{tabular}{|c|c|c|}
\hline 232 & 648 & 2. $45 \mathrm{E}-10$ \\
\hline 183 & 161 & 2. $45 \mathrm{E}-10$ \\
\hline 242 & 236 & 2. $65 \mathrm{E}-10$ \\
\hline 198 & 571 & 3. $26 \mathrm{E}-10$ \\
\hline 194 & 175 & 3. $39 \mathrm{E}-10$ \\
\hline 191 & 172 & $3.57 \mathrm{E}-10$ \\
\hline 286 & 297 & 4. $71 \mathrm{E}-10$ \\
\hline 501 & 1210 & 4. $97 \mathrm{E}-10$ \\
\hline 169 & 148 & 1. $25 \mathrm{E}-09$ \\
\hline 185 & 169 & 1. $31 \mathrm{E}-09$ \\
\hline 206 & 196 & 1. $42 \mathrm{E}-09$ \\
\hline 671 & 856 & 1. $70 \mathrm{E}-09$ \\
\hline 1081 & 2346 & 2. $26 \mathrm{E}-09$ \\
\hline 510 & 623 & 3. $34 \mathrm{E}-09$ \\
\hline 259 & 271 & 4. $89 \mathrm{E}-09$ \\
\hline 147 & 437 & 7. $14 \mathrm{E}-09$ \\
\hline 490 & 599 & 7. $48 \mathrm{E}-09$ \\
\hline 331 & 373 & 9. $43 \mathrm{E}-09$ \\
\hline 194 & 187 & $9.51 \mathrm{E}-09$ \\
\hline 236 & 245 & 1. $87 \mathrm{E}-08$ \\
\hline 385 & 454 & 1. $98 \mathrm{E}-08$ \\
\hline 220 & 592 & 2. $15 \mathrm{E}-08$ \\
\hline 248 & 262 & 2. $17 \mathrm{E}-08$ \\
\hline 559 & 1291 & 2. $23 \mathrm{E}-08$ \\
\hline 226 & 234 & 2. $80 \mathrm{E}-08$ \\
\hline 147 & 428 & 3. $25 \mathrm{E}-08$ \\
\hline 379 & 452 & $5.87 \mathrm{E}-08$ \\
\hline 266 & 681 & 6. $08 \mathrm{E}-08$ \\
\hline 950 & 1315 & $6.56 \mathrm{E}-08$ \\
\hline 417 & 508 & 7. $45 \mathrm{E}-08$ \\
\hline 187 & 187 & 8. $77 \mathrm{E}-08$ \\
\hline 194 & 525 & $9.89 \mathrm{E}-08$ \\
\hline 182 & 181 & 1. $02 \mathrm{E}-07$ \\
\hline 289 & 328 & 1. $22 \mathrm{E}-07$ \\
\hline 244 & 268 & 2. $38 \mathrm{E}-07$ \\
\hline 199 & 208 & 2. $96 \mathrm{E}-07$ \\
\hline 152 & 147 & 3. $80 \mathrm{E}-07$ \\
\hline 898 & 1921 & 4. $21 \mathrm{E}-07$ \\
\hline 260 & 648 & 7. $71 \mathrm{E}-07$ \\
\hline 251 & 629 & 8. $78 \mathrm{E}-07$ \\
\hline 344 & 818 & 1. $03 \mathrm{E}-06$ \\
\hline 155 & 154 & 1. $06 \mathrm{E}-06$ \\
\hline 198 & 516 & 1. $08 \mathrm{E}-06$ \\
\hline 179 & 187 & 1. $18 \mathrm{E}-06$ \\
\hline 209 & 229 & 1. $34 \mathrm{E}-06$ \\
\hline 212 & 543 & 1. $46 \mathrm{E}-06$ \\
\hline 248 & 284 & 1. $64 \mathrm{E}-06$ \\
\hline 207 & 228 & 2. $01 \mathrm{E}-06$ \\
\hline 713 & 983 & 2. $02 \mathrm{E}-06$ \\
\hline 169 & 177 & 2. $43 \mathrm{E}-06$ \\
\hline 121 & 343 & 2. $62 \mathrm{E}-06$ \\
\hline 193 & 210 & 2. $86 \mathrm{E}-06$ \\
\hline 260 & 305 & 3. $33 \mathrm{E}-06$ \\
\hline 176 & 458 & 4. $36 \mathrm{E}-06$ \\
\hline
\end{tabular}

https://mc06.manuscriptcentral.com/genome-pubs 
Unigene_UN_xiong_4172

Unigene_UN_xiong_7949

Unigene_UN_xiong_2313

Unigene_UN_xiong_6173

Unigene_UN_xiong_1756

Unigene_UN_xiong_2622

Unigene_UN_xiong_4067

Unigene_UN_xiong_8071

Unigene_UN_xiong_139

Unigene_UN_xiong_10103

Unigene_UN_xiong_12679

Unigene_UN_xiong_14846

Unigene_UN_xiong_4693

Unigene_UN_xiong_1005

Unigene_UN_xiong_3935

Unigene_UN_xiong_2996

Unigene_UN_xiong_10941

Unigene_UN_xiong_15055

Unigene_UN_xiong_1512

Unigene_UN_xiong_14208

Unigene_UN_xiong_14470

Unigene_UN_xiong_8647

Unigene_UN_xiong_4291

Unigene_UN_xiong_9232

Unigene_UN_xiong_14791

Unigene_UN_xiong_14619

Unigene_UN_xiong_11417

Unigene_UN_xiong_14042

Unigene_UN_xiong_5707

Unigene_UN_xiong_5437

Unigene_UN_xiong_14685

Unigene_UN_xiong_8684

Unigene_UN_xiong_15755

Unigene_UN_xiong_13712

Unigene_UN_xiong_15511

Unigene_UN_xiong_14848

Unigene_UN_xiong_13016

Unigene_UN_xiong_9698

Unigene_UN_xiong_14737

Unigene_UN_xiong_1392

Unigene_UN_xiong_55

Unigene_UN_xiong_11332

Unigene_UN_xiong_14228

Unigene_UN_xiong_6419

Unigene_UN_xiong_7955

Unigene_UN_xiong_4182

Unigene_UN_xiong_11475

Unigene_UN_xiong_12390

Unigene_UN_xiong_6263

Unigene_UN_xiong_4289

Unigene_UN_xiong_1206

Unigene_UN_xiong_1241

Unigene_UN_xiong_14951

Unigene_UN_xiong_11755

\begin{tabular}{|c|c|c|}
\hline 420 & 955 & 5. $41 \mathrm{E}-06$ \\
\hline 149 & 399 & $5.55 \mathrm{E}-06$ \\
\hline 222 & 254 & $5.59 \mathrm{E}-06$ \\
\hline 210 & 237 & 5. $63 \mathrm{E}-06$ \\
\hline 303 & 371 & $5.78 \mathrm{E}-06$ \\
\hline 351 & 442 & 6. $40 \mathrm{E}-06$ \\
\hline 143 & 147 & 7. $62 \mathrm{E}-06$ \\
\hline 255 & 618 & 7. $62 \mathrm{E}-06$ \\
\hline 292 & 357 & $8.27 \mathrm{E}-06$ \\
\hline 296 & 364 & $9.85 \mathrm{E}-06$ \\
\hline 420 & 946 & 1. $12 \mathrm{E}-05$ \\
\hline 121 & 332 & 1. $30 \mathrm{E}-05$ \\
\hline 66 & 209 & 1. $37 \mathrm{E}-05$ \\
\hline 156 & 404 & 1. $96 \mathrm{E}-05$ \\
\hline 264 & 322 & 2. $03 \mathrm{E}-05$ \\
\hline 274 & 337 & 2. $08 \mathrm{E}-05$ \\
\hline 405 & 533 & 2. $16 \mathrm{E}-05$ \\
\hline 120 & 324 & 3. $24 \mathrm{E}-05$ \\
\hline 278 & 349 & 4. $63 \mathrm{E}-05$ \\
\hline 1351 & 2697 & 5. $68 \mathrm{E}-05$ \\
\hline 380 & 846 & $6.85 \mathrm{E}-05$ \\
\hline 122 & 322 & 7. $61 \mathrm{E}-05$ \\
\hline 340 & 445 & 8. $19 \mathrm{E}-05$ \\
\hline 192 & 465 & 0.000113842 \\
\hline 606 & 1279 & 0.000113925 \\
\hline 2290 & 4415 & 0.000115013 \\
\hline 110 & 114 & 0.000123248 \\
\hline 202 & 482 & 0.000156752 \\
\hline 142 & 358 & 0.000162563 \\
\hline 205 & 486 & 0.000198153 \\
\hline 287 & 375 & 0.000271018 \\
\hline 260 & 336 & 0.000340404 \\
\hline 962 & 1446 & 0.000367605 \\
\hline 138 & 343 & 0.000371784 \\
\hline 719 & 1473 & 0.000410546 \\
\hline 493 & 1041 & 0.000455935 \\
\hline 535 & 766 & 0.000483569 \\
\hline 287 & 381 & 0.000534225 \\
\hline 367 & 504 & 0.000549823 \\
\hline 352 & 766 & 0.000555159 \\
\hline 236 & 313 & 0.001615934 \\
\hline 269 & 366 & 0.002245386 \\
\hline 673 & 1356 & 0.002301629 \\
\hline 251 & 339 & 0.002354303 \\
\hline 98 & 113 & 0.003254974 \\
\hline 127 & 301 & 0.003544136 \\
\hline 226 & 496 & 0.004181513 \\
\hline 637 & 1276 & 0.004651094 \\
\hline 105 & 125 & 0.004844714 \\
\hline 280 & 392 & 0.004998584 \\
\hline 158 & 204 & 0.005264702 \\
\hline 255 & 549 & 0.005331294 \\
\hline 96 & 234 & 0.005945418 \\
\hline 277 & 589 & 0.006520803 \\
\hline
\end{tabular}

https://mc06.manuscriptcentral.com/genome-pubs 
Unigene_UN_xiong_14454

Unigene_UN_xiong_5406

Unigene_UN_xiong_2475

Unigene_UN_xiong_3161

Unigene_UN_xiong_13344

Unigene_UN_xiong_3504

Unigene_UN_xiong_2521

Unigene_UN_xiong_13725

Unigene_UN_xiong_14964

Unigene_UN_xiong_12227

Unigene_UN_xiong_15334

Unigene_UN_xiong_5387

Unigene_UN_xiong_14520

Unigene_UN_xiong_4481

Unigene_UN_xiong_4168

Unigene_UN_xiong_4619

Unigene_UN_xiong_4204

Unigene_UN_xiong_5161

Unigene_UN_xiong_2186

Unigene_UN_xiong_12936

Unigene_UN_xiong_14512

Unigene_UN_xiong_15667

Unigene_UN_xiong_14590

Unigene_UN_xiong_7151

Unigene_UN_xiong_10090

Unigene_UN_xiong_7386

Unigene_UN_xiong_8732

Unigene_UN_xiong_15471

Unigene_UN_xiong_12428

Unigene_UN_xiong_1155

Unigene_UN_xiong_1113

Unigene_UN_xiong_14774

Unigene_UN_xiong_3537

Unigene_UN_xiong_14834

Unigene_UN_xiong_10542

Unigene_UN_xiong_3741

Unigene_UN_xiong_8628

Unigene_UN_xiong_10425

Unigene_UN_xiong_10446

Unigene_UN_xiong_8143

Unigene_UN_xiong_13417

Unigene_UN_xiong_13713

Unigene_UN_xiong_12237

Unigene_UN_xiong_12729

Unigene_UN_xiong_14699

Unigene_UN_xiong_15043

Unigene_UN_xiong_13144

Unigene_UN_xiong_15789

Unigene_UN_xiong_14804

Unigene_UN_xiong_3369

Unigene_UN_xiong_14265

Unigene_UN_xiong_974

Unigene_UN_xiong_14749

Unigene_UN_xiong_1333

\begin{tabular}{|c|c|c|}
\hline 289 & 410 & 0.007478477 \\
\hline 131 & 301 & 0.008042897 \\
\hline 219 & 301 & 0.008141499 \\
\hline 187 & 255 & 0.011613861 \\
\hline 188 & 257 & 0.011913152 \\
\hline 150 & 199 & 0.012219281 \\
\hline 326 & 474 & 0.012405025 \\
\hline 137 & 181 & 0.014376075 \\
\hline 256 & 537 & 0.016371574 \\
\hline 123 & 278 & 0.016921315 \\
\hline 419 & 629 & 0.017471221 \\
\hline 361 & 536 & 0.018248326 \\
\hline 779 & 1509 & 0.018838957 \\
\hline 174 & 377 & 0.018875139 \\
\hline 1238 & 2348 & 0.018959565 \\
\hline 239 & 502 & 0.019779951 \\
\hline 247 & 515 & 0.021615442 \\
\hline 205 & 289 & 0.021844835 \\
\hline 418 & 838 & 0.022087708 \\
\hline 168 & 233 & 0.025519663 \\
\hline 964 & 1537 & 0.028951514 \\
\hline 200 & 285 & 0.029863689 \\
\hline 529 & 820 & 0.033762136 \\
\hline 194 & 406 & 0.037636371 \\
\hline 247 & 506 & 0.040760726 \\
\hline 179 & 375 & 0.046839504 \\
\hline 186 & 387 & 0.050683503 \\
\hline 125 & 173 & 0.053851126 \\
\hline 157 & 224 & 0.05522878 \\
\hline 200 & 411 & 0.058560121 \\
\hline 182 & 378 & 0.058840575 \\
\hline 383 & 594 & 0.072534796 \\
\hline 248 & 373 & 0.072841397 \\
\hline 215 & 321 & 0.079932141 \\
\hline 163 & 239 & 0.087305763 \\
\hline 2134 & 3893 & 0.104458217 \\
\hline 275 & 426 & 0.125784195 \\
\hline 120 & 175 & 0.130662854 \\
\hline 209 & 319 & 0.135562804 \\
\hline 251 & 492 & 0.137140144 \\
\hline 138 & 281 & 0.141207477 \\
\hline 742 & 1384 & 0.148882573 \\
\hline 198 & 303 & 0.150260609 \\
\hline 159 & 318 & 0.167713932 \\
\hline 215 & 332 & 0.168306626 \\
\hline 353 & 561 & 0.169107784 \\
\hline 185 & 366 & 0.170057092 \\
\hline 182 & 279 & 0.175470187 \\
\hline 165 & 252 & 0.185899391 \\
\hline 1784 & 2997 & 0.200604179 \\
\hline 251 & 396 & 0. 220222013 \\
\hline 166 & 326 & 0.223383838 \\
\hline 448 & 726 & 0.224869645 \\
\hline 398 & 749 & 0.23134604 \\
\hline
\end{tabular}


Unigene_UN_xiong_15345

Unigene_UN_xiong_2411

Unigene_UN_xiong_14887

Unigene_UN_xiong_6402

Unigene_UN_xiong_9555

Unigene_UN_xiong_2889

Unigene_UN_xiong_4268

Unigene_UN_xiong_8535

Unigene_UN_xiong_14781

Unigene_UN_xiong_14802

Unigene_UN_xiong_15754

Unigene_UN_xiong_246

Unigene_UN_xiong_14038

Unigene_UN_xiong_8922

Unigene_UN_xiong_5489

Unigene_UN_xiong_9226

Unigene_UN_xiong_5353

Unigene_UN_xiong_14638

Unigene_UN_xiong_12384

Unigene_UN_xiong_15516

Unigene_UN_xiong_10473

Unigene_UN_xiong_15136

Unigene_UN_xiong_9158

Unigene_UN_xiong_6083

Unigene_UN_xiong_9652

Unigene_UN_xiong_9456

Unigene_UN_xiong_14881

Unigene_UN_xiong_13635

Unigene_UN_xiong_5614

Unigene_UN_xiong_6787

Unigene_UN_xiong_3293

Unigene_UN_xiong_14915

Unigene_UN_xiong_8376

Unigene_UN_xiong_2265

Unigene_UN_xiong_4496

Unigene_UN_xiong_488

Unigene_UN_xiong_2324

Unigene_UN_xiong_1145

Unigene_UN_xiong_8413

Unigene_UN_xiong_4726

Unigene_UN_xiong_14844

Unigene_UN_xiong_11167

\begin{tabular}{|c|c|c|}
\hline 220 & 346 & 0.238153124 \\
\hline 206 & 397 & 0.253472577 \\
\hline 243 & 386 & 0.263141651 \\
\hline 127 & 249 & 0.308563201 \\
\hline 145 & 282 & 0.314463503 \\
\hline 367 & 601 & 0.332810409 \\
\hline 2602 & 4653 & 0.333706844 \\
\hline 107 & 210 & 0.350409201 \\
\hline 229 & 432 & 0.352676075 \\
\hline 142 & 225 & 0.385420513 \\
\hline 200 & 323 & 0.388156067 \\
\hline 230 & 374 & 0.39790181 \\
\hline 241 & 393 & 0.409201762 \\
\hline 296 & 548 & 0.431270204 \\
\hline 220 & 411 & 0.431938809 \\
\hline 136 & 259 & 0.433003363 \\
\hline 168 & 316 & 0.449992061 \\
\hline 135 & 254 & 0.493846498 \\
\hline 197 & 366 & 0.511188034 \\
\hline 127 & 206 & 0.531128936 \\
\hline 236 & 391 & 0.53363664 \\
\hline 269 & 447 & 0.534317246 \\
\hline 103 & 167 & 0.569412057 \\
\hline 132 & 216 & 0.577541069 \\
\hline 636 & 1141 & 0.587453667 \\
\hline 338 & 613 & 0.589745415 \\
\hline 569 & 966 & 0.59568812 \\
\hline 153 & 281 & 0.653488546 \\
\hline 226 & 383 & 0.736217166 \\
\hline 126 & 229 & 0.740905497 \\
\hline 197 & 334 & 0.75230802 \\
\hline 161 & 272 & 0.764467855 \\
\hline 224 & 381 & 0.767449092 \\
\hline 292 & 500 & 0.796058166 \\
\hline 165 & 281 & 0.805746206 \\
\hline 250 & 446 & 0.813163295 \\
\hline 210 & 359 & 0.827566696 \\
\hline 90 & 156 & 0.947282686 \\
\hline 165 & 290 & 0.961180014 \\
\hline 941 & 1647 & 0.967381223 \\
\hline 172 & 302 & \\
\hline 140 & 246 & \\
\hline
\end{tabular}

https://mc06.manuscriptcentral.com/genome-pubs 
1 UN_xiong_14529 UN_ci_3507

0 UN_xiong_14070 UN_ci_8324

1 UN_xiong_399 UN_ci_2022

1 UN_xiong_14542 UN_ci_7667

0 UN_xiong_13607 UN_ci_8251

1 UN_xiong_949 UN_ci_7782

1 UN_xiong_2637 UN_ci_1194

0 UN_xiong_11196 UN_ci_4204

0 UN_xiong_7164 UN_ci_8103

0 UN_xiong_1987 UN_ci_8019

1 UN_xiong_194 UN_ci_7812

1 UN_xiong_15045 UN_ci_7956

0 UN_xiong_5287 UN_ci_8098

1 UN_xiong_15243 UN_ci_66

1 UN_xiong_14509 UN_ci_7676

0 UN_xiong_11113 UN_ci_7696

0 UN_xiong_4091 UN_ci_6537

1 UN_xiong_3523 UN_ci_6078

0 UN_xiong_14868 UN_ci_6910

0 UN_xiong_14272 UN_ci_3721

1 UN_xiong_4237 UN_ci_8633

1 UN_xiong_15382 UN_ci_3497

1 UN_xiong_14905 UN_ci_4006

1 UN_xiong_1280 UN_ci_3287

0 UN_xiong_14842 UN_ci_7339

1 UN_xiong_14978 UN_ci_3282

0 UN_xiong_11680 UN_ci_8477

1 UN_xiong_1807 UN_ci_8688

0 UN_xiong_12139 UN_ci_7894

0 UN_xiong_5130 UN_ci_8317

0 UN_xiong_13569 UN_ci_3186

0 UN_xiong_13925 UN_ci_7720

1 UN_xiong_2662 UN_ci_8099

1 UN_xiong_14312 UN_ci_4775

0 UN xiong 9552 UN ci 8299

0 UN xiong 14377 UN ci 8490

1 UN_xiong_15383 UN_ci 1893

1 UN_xiong_1657 UN_ci_6364

1 UN_xiong_14546 UN_ci_7844

1 UN_xiong_15105 UN_ci_2999

1 UN_xiong_10027 UN_ci_7067

0 UN_xiong_15032 UN_ci_3128

0 UN_xiong_14718 UN_ci_1523

1 UN_xiong_386 UN_ci_1000

1 UN_xiong_14567 UN_ci_5259

1 UN_xiong_14716 UN_ci_7971

1 UN_xiong_14273 UN_ci_395

1 UN_xiong_14623 UN_ci_8173

1 UN_xiong_10584 UN_ci_8002

1 UN_xiong_14566 UN_ci_7736

1 UN_xiong_15375 UN_ci_641

1 UN_xiong_15556 UN_ci_8669

tr $\mid$ Q4RBH1 $\mid$ Q4RBH1_TETNG Chrc

tr $\mid$ C1BKC6 $\mid$ C1BKC6_0SMM0 60S

tr|C3KGV9|C3KGV9_ANOFI Trar

tr $\mid$ C3KH90|C3KH90_ANOFI 60S

tr $\mid$ Q711N8 $\mid$ Q711N8_ONCMY Pute

$\operatorname{tr} \mid$ C3KJL5 $\mid$ C3KJL5_ANOFI Sect

tr|C3KIM6|C3KIM6_ANOFI Dyne

tr $\mid$ C3KH05|C3KH05_ANOFI BUD:

tr $\mid$ Q4S7Z7 $\mid$ Q4S7Z7_TETNG Chrc

tr $\mid$ C1BKE1 $\mid$ C1BKE1_OSMM0 Tubr

tr $\mid$ Q709D5 $\mid$ Q709D5_0RYLA Gemi

tr $\mid$ D6PVP8 $\mid$ D6PVP8_EPIC0 60S

tr $\mid$ D2H3P7 $\mid$ D2H3P7_AILME Pute

tr|B7X8Y8|B7X8Y8_PAGMA Elor

tr $\mid$ Q3ZLG2 $\mid$ Q3ZLG2_OREMO Quir

tr $\mid$ A9ZTA6 $\mid$ A9ZTA6_SOLSE Ribc

tr $\mid$ C1BIA8 $\mid$ C1BIA8_ONCMY Trar

tr|C3KIM5|C3KIM5_ANOFI Epic

tr $\mid$ Q6Y205|Q6Y205_PAGMA Ribc

tr $\mid$ Q9PT09|Q9PT09_ONCMY Ubic tr $\mid$ C1BJG0|C1BJG0_OSMM0 60S tr|B0S5N1|B0S5N1_DANRE RuvE tr |D6PVQ6 |D6PVQ6_EPIC0 Mitc tr $\mid$ Q6Y243|Q6Y243_PAGMA Elor

tr $\mid$ A0EZZ6 $\mid$ A0EZZ6_PSEMX 40S tr $\mid$ Q4RT32|Q4RT32_TETNG Chrc

tr $\mid$ C3KK19 $\mid$ C3KK19_ANOFI Stat tr |C3UVG8|C3UVG8_9TELE Zone tr $\mid$ Q4ST43|Q4ST43_TETNG Chrc tr $\mid$ C3KHU1 $\mid$ C3KHU1_ANOFI 60S $\operatorname{tr} \mid$ B0V3V7|BOV3V7_DANRE Guar tr $\mid$ Q27R93|Q27R93_FUNHE NAD (

tr|A9ZT86|A9ZT86_SOLSE Ribc tr|B4IZ29|B4IZ29_DROGR GH1E tr $\mid$ A9ZT89|A9ZT89_SOLSE Ribc tr|A7XLX5 |A7XLX5_PERFV Ribc tr | Q2HYN5 |Q2HYN5_ICTPU Trar tr $\mid$ Q4S564|Q4S564_TETNG Chrc

tr $\mid$ A0EZY9 $\mid$ A0EZY9_PSEMX Ribc tr|C3KIW5|C3KIW5_ANOFI Euke tr|Q9IA75|Q9IA75_PAROL Ribc tr $\mid$ C3KJ85|C3KJ85_ANOFI 40S tr $\mid$ D6PVN6|D6PVN6_EPIC0 40S $\operatorname{tr} \mid$ C1BK85 $\mid$ C1BK85_0SMM0 ATPc 
1 UN_xiong_14239 UN_ci_2947

0 UN_xiong_8517 UN_ci_7587

1 UN_xiong_3844 UN_ci_4020

0 UN_xiong_4795 UN_ci_7945

1 UN_xiong_14660 UN_ci_2316

1 UN_xiong_9105 UN_ci_4354

0 UN_xiong_15664 UN_ci_8816

1 UN_xiong_14437 UN_ci_716

0 UN_xiong_2880 GPGRCDD02H7PEA_ci

1 UN_xiong_216 UN_ci_2635

1 UN_xiong_333 UN_ci_7669

0 UN_xiong_14677 UN_ci_818

1 UN_xiong_14877 UN_ci_6169

1 UN_xiong_14783 UN_ci_8089

0 UN_xiong_4430 UN_ci_8313

1 UN_xiong_14924 UN_ci_6292

1 UN_xiong_3845 UN_ci_1046

1 UN_xiong_6228 UN_ci_7913

0 UN_xiong_3079 UN_ci_8744

1 UN_xiong_14962 UN_ci_1210

1 UN_xiong_155 UN_ci_673

0 UN_xiong_3128 UN_ci_6304

1 UN_xiong_14891 UN_ci_4683

0 UN_xiong_335 UN_ci_8264

1 UN_xiong_4436 UN_ci_4380

0 UN_xiong_5549 UN_ci_1521

1 UN_xiong_14385 UN_ci_7874

0 UN_xiong_10799 UN_ci_8175

1 UN_xiong_14665 UN_ci_5561

0 UN_xiong_15130 UN_ci_925

1 UN_xiong_1438 UN_ci_1510

1 UN_xiong_14648 UN_ci_2832

0 UN_xiong_14747 UN_ci_2574

1 UN_xiong_256 UN_ci_4646

1 UN_xiong_14285 UN_ci_8021

1 UN_xiong_3390 UN_ci_8003

1 UN_xiong_2317 UN_ci_2644

1 UN_xiong_8530 UN_ci_3645

0 UN_xiong_4712 UN_ci_1148

1 UN_xiong_3904 UN_ci_3151

1 UN_xiong_1478 UN_ci_1383

1 UN_xiong_2555 UN_ci_8499

1 UN_xiong_13350 UN_ci_7235

1 UN_xiong_3637 UN_ci_1908

1 UN_xiong_14727 UN_ci_4122

1 UN_xiong_6909 UN_ci_2980

1 UN_xiong_14274 UN_ci_8763

0 UN_xiong_3787 UN_ci_4781

1 UN_xiong_14453 UN_ci_1711

1 UN_xiong_14263 UN_ci_2483

0 UN_xiong_122 UN_ci_8295

0 UN_xiong_13451 UN_ci_5483

0 UN_xiong_8419 UN_ci_5288

0 UN_xiong_15660 UN_ci_3921 tr $\mid$ Q4TAC5 $\mid$ Q4TAC5_TETNG Chrc tr $\mid$ Q6DGD9|Q6DGD9_DANRE Traf tr $\mid$ A0EZT6 |A0EZT6_PSEMX 60S sp |Q7T3T9|ZAR1_TAKRU Zygot $\epsilon$

tr|A9ZT94|A9ZT94_SOLSE Ribc tr $\mid$ Q1KKY5|Q1KKY5_TAKRU Mitc

tr $\mid$ C1BJN5 |C1BJN5_0SMM0 Catr tr|C3KHW9|C3KHW9_ANOFI 40S

tr $\mid$ Q4SJW7 $\mid$ Q4SJW7_TETNG Chrc sp |P69091|RL18_0RENI 60S ri tr|B9ELS4|B9ELS4_SALSA Trar tr $\mid$ D6PVQ4 |D6PVQ4_EPIC0 60S

$\operatorname{tr} \mid$ B5X3R3 |B5X3R3_SALSA F-ac

tr $\mid$ Q6TGY0 $\mid$ Q6TGY0_DANRE HSPC

tr $\mid$ A9ZT90|A9ZT90_SOLSE Ribc tr $\mid$ A0EZX2 |A0EZX2_PSEMX Nuc1

tr|C3KI01|C3KI01_ANOFI Hist tr|D7PBZ5|D7PBZ5_PSEMX Ferr tr|A3KQ59|A3KQ59_DANRE Nove tr $\mid$ Q5DVI0 $\mid$ Q5DVI0_PLAFE 60S

tr $\mid$ B5XE73|B5XE73_SALSA M-pr tr $\mid$ Q6Y1Z9|Q6Y1Z9_PAGMA 40S tr $\mid$ Q4T029|Q4T029_TETNG Chrc

tr $\mid$ A0JPF1 $\mid$ A0JPF1_DANRE Zgc: tr|A2Q0R6|A2Q0R6_SOLSE Ribc tr $\mid$ A2Q0U0 $\mid$ A2Q0U0_SOLSE Ribc

tr $\mid$ Q7ZU62 |Q7ZU62_DANRE Zgc: tr $\mid$ C3KJ53|C3KJ53_ANOFI Elor

tr $\mid$ A9ZTA4 $\mid$ A9ZTA4_SOLSE Ribc

tr $\mid$ A2Q0S7 $\mid$ A2Q0S7_SOLSE 40S tr $\mid$ Q4TCC0 $\mid$ Q4TCC0_TETNG Acy 1 tr $\mid$ Q4TAQ9 $\mid$ Q4TAQ9_TETNG Chrc tr $\mid$ C1BL06|C1BL06_0SMM0 TGFtr $\mid$ Q7SZX9 $\mid$ Q7SZX9_DANRE FLJ1 tr $\mid$ Q4SM43 $\mid$ Q4SM43 TETNG Chrc tr|B5T1N7|B5T1N7_EPIC0 Mitc

$\operatorname{tr} \mid$ C3KIJ8 $\mid$ C3KIJ8_ANOFI Smal tr $\mid$ C3KIW9 $\mid$ C3KIW9_ANOFI Nuc1 tr $\mid$ Q4S6R2 | Q4S6R2_TETNG Chrc $\operatorname{tr} \mid$ C3KJ13|C3KJ13_ANOFI SRA 
0 UN_xiong_6273 UN_ci_5805

1 UN_xiong_14616 UN_ci_6411

1 UN_xiong_5777 UN_ci_5560

1 UN_xiong_15374 UN_ci_8285

1 UN_xiong_14859 UN_ci_7979

1 UN_xiong_3047 UN_ci_5511

1 UN_xiong_2181 UN_ci_7754

0 UN_xiong_14414 UN_ci_239

1 UN_xiong_14937 UN_ci_7922

1 UN_xiong_14674 UN_ci_8086

1 UN_xiong_5020 UN_ci_5262

1 UN_xiong_4401 UN_ci_4128

0 UN_xiong_2958 UN_ci_6554

1 UN_xiong_14689 UN_ci_867

1 UN_xiong_751 UN_ci_4792

1 UN_xiong_14569 UN_ci_3632

0 UN_xiong_14270 UN_ci_7983

1 UN_xiong_11645 UN_ci_6200

1 UN_xiong_14286 UN_ci_4885

1 UN_xiong_14334 UN_ci_8322

1 UN_xiong_7854 UN_ci_3967

1 UN_xiong_988 UN_ci_7375

1 UN_xiong_2611 UN_ci_3837

1 UN_xiong_14873 UN_ci_8106

0 UN_xiong_15687 UN_ci_3682

1 UN_xiong_5912 UN_ci_1122

0 UN_xiong_3634 UN_ci_7946

1 UN_xiong_145 UN_ci_644

1 UN xiong 790 UN ci 8330

0 UN_xiong_1060 UN_ci_7455

0 UN_xiong_14618 UN_ci_7724

1 UN_xiong_7465 UN_ci_6070

1 UN_xiong_4109 UN_ci_1594

1 UN_xiong_9546 UN_ci_1201

1 UN_xiong_1685 UN_ci_4379

1 UN_xiong_15543 UN_ci_8769

1 UN_xiong_13667 UN_ci_8108

1 UN_xiong_1628 UN_ci_6040

0 UN_xiong_15785 UN_ci_2481

1 UN_xiong_7527 UN_ci_1567

1 UN_xiong_5941 UN_ci_749

0 UN_xiong_8790 UN_ci_8638

0 UN_xiong_12115 UN_ci_8221

1 UN_xiong_8850 UN_ci_2189

0 UN_xiong_2248 UN_ci_8519

1 UN_xiong_10847 UN_ci_7392

1 UN_xiong_14812 UN_ci_4626

0 UN_xiong_4643 UN_ci_267

1 UN_xiong_6978 UN_ci_8164

1 UN_xiong_14438 UN_ci_7851

1 UN_xiong_14383 UN_ci_7423

0 UN_xiong_7498 UN_ci_8256

0 UN_xiong_13515 UN_ci_8788

1 UN_xiong_12363 UN_ci_8 tr $\mid$ Q4T9G5 $\mid$ Q4T9G5_TETNG Chrc tr|B5XBW1|B5XBW1_SALSA BCA tr|C1BIM0|C1BIM0_0SMM0 Gron

tr $\mid$ C3KINO |C3KINO_ANOFI GTP$\operatorname{tr} \mid$ Q7T2C6 $\mid$ Q7T2C6_DANRE RAB7

tr $\mid$ C3KGT2 $\mid$ C3KGT2_ANOFI Thic

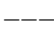

tr |Q4RH57|Q4RH57_TETNG Chrc

tr|C3KIV4|C3KIV4_ANOFI FAME tr $\mid$ Q3ZLGO $\mid$ Q3ZLGO_OREMO XNor tr $\mid$ C3KK43|C3KK43_ANOFI Myos sp |Q202I6|TCTP_PSEMX Trans1

$--$

tr $\mid$ AOEZU4 $\mid$ A0EZU4_PSEMX 40S

tr|C3KI82|C3KI82_ANOFI Trar tr $\mid$ C3KJT2 $\mid$ C3KJT2_ANOFI NADF sp |Q1L8Y6|BBP10_DANRE BBSon tr|C3KGU8|C3KGU8_ANOFI 40S tr $\mid$ B9USM4 | B9USM4_9PERC 2-Cy tr $\mid$ C3KHU8 $\mid$ C3KHU8_ANOFI NADF $\operatorname{tr} \mid$ C3KJQ1|C3KJQ1_ANOFI Tumc tr|B9V3X3|B9V3X3_EPIC0 Musc tr $\mid$ Q4RJL0 $\mid$ Q4RJL0_TETNG Chrc tr $\mid$ Q4RVD2 $\mid$ Q4RVD2_TETNG Chrc tr $\mid$ COHAQ6 $\mid$ COHAQ6_SALSA Elor tr|A4L7I8|A4L7I8_9SCOM Glut tr $\mid$ Q4RWC3 $\mid$ Q4RWC3_TETNG Chrc tr $\mid$ Q4S578|Q4S578_TETNG Chrc

tr $\mid$ Q4SD23 $\mid$ Q4SD23_TETNG Chrc tr $\mid$ Q4SK90 $\mid$ Q4SK90_TETNG Chrc

$\operatorname{tr} \mid$ Q4S1J7|Q4S1J7_TETNG Chrc tr $\mid$ C3KIZ9 $\mid$ C3KIZ9_ANOFI Ribc tr $\mid$ D2CFU7 |D2CFU7 9PERC Sup tr $\mid$ Q4RY82 $\mid$ Q4RY82_TETNG Chrc tr $\mid$ C1BIV9|C1BIV9 OSMM0 FAM: tr $\mid$ Q4S9N2 $\mid$ Q4S9N2_TETNG Chrc tr $\mid$ Q5DVH2 $\mid$ Q5DVH2_PLAFE 60S

tr | Q4RMT6 |Q4RMT6_TETNG Chrc tr $\mid$ C3S171 $\mid$ C3S171_DANRE Glut tr $\mid$ C1BL73|C1BL73_0SMM0 Char 
1 UN_xiong_14440 UN_ci_7905

1 UN_xiong_3675 UN_ci_3657

1 UN_xiong_8920 UN_ci_4072

0 UN_xiong_3105 UN_ci_3655

0 UN_xiong_5545 UN_ci_8084

1 UN_xiong_14490 UN_ci_2946

1 UN_xiong_2168 UN_ci_7108

1 UN_xiong_6384 UN_ci_6452

0 UN_xiong_7707 UN_ci_2057

1 UN_xiong_2119 UN_ci_1844

0 UN_xiong_2218 UN_ci_2012

1 UN_xiong_12342 UN_ci_7077

1 UN_xiong_9316 UN_ci_5545

0 UN_xiong_1570 UN_ci_8493

1 UN_xiong_4494 UN_ci_8338

0 UN_xiong_1588 UN_ci_7870

0 UN_xiong_5453 UN_ci_3905

1 UN_xiong_14801 UN_ci_7904

1 UN_xiong_8994 UN_ci_5716

1 UN_xiong_2689 UN_ci_7785

1 UN_xiong_6304 UN_ci_5748

1 UN_xiong_825 UN_ci_2310

1 UN_xiong_10733 UN_ci_8736

1 UN_xiong_3878 UN_ci_8607

1 UN_xiong_15520 UN_ci_8165

1 UN_xiong_12413 UN_ci_2212

1 UN_xiong_876 UN_ci_3860

0 UN_xiong_14347 UN_ci_8171

1 UN_xiong_9163 UN_ci_3768

1 UN_xiong_14497 UN_ci_1441

0 UN_xiong_4932 UN_ci_670

1 UN_xiong_3436 UN_ci_1100

1 UN_xiong_8136 UN_ci_2297

0 UN_xiong_5737 UN_ci_1772

0 UN_xiong_11251 UN_ci_8022

1 UN_xiong_4975 UN_ci_1054

0 UN_xiong_15308 UN_ci_6115

1 UN_xiong_1201 UN_ci_6643

1 UN_xiong_14327 UN_ci_6832

0 UN_xiong_14307 UN_ci_7701

1 UN_xiong_5113 UN_ci_940

1 UN_xiong_2520 UN_ci_8080

1 UN_xiong_142 GPGRCDD02H2XJD_ci

0 UN xiong 4359 UN ci 6001

0 UN_xiong_14308 UN_ci_7908

1 UN_xiong_4059 UN_ci_7334

0 UN_xiong_12070 UN_ci_4671

1 UN_xiong_6694 UN_ci_6019

1 UN_xiong_10563 UN_ci_8463

1 UN_xiong_1586 UN_ci_3016

1 UN_xiong_14563 UN_ci_362

0 UN_xiong_9359 UN_ci_7972

0 UN_xiong_307 UN_ci_8506

1 UN_xiong_12491 UN_ci_5280 tr $\mid$ Q9IA74 $\mid$ Q9IA74_PAR0L 40S

tr $\mid$ Q6DEG5 $\mid$ Q6DEG5_DANRE Poly

tr|C3KIH6|C3KIH6_ANOFI Chrc tr|D7PIW6 |D7PIW6_PSEMX Ferr tr $\mid$ C1BMA3 $\mid$ C1BMA3_0SMM0 Gamn tr $\mid$ Q4RG23|Q4RG23_TETNG Chrc tr|C3KJY4|C3KJY4_ANOFI Coat

tr $\mid$ C1BG03 $\mid$ C1BG03_ONCMY LYR tr $\mid$ Q5RGQ1 $\mid$ Q5RGQ1_DANRE Nov $\epsilon$ tr $\mid$ COPTZ1|COPTZ1_SALSA Trar

tr $\mid$ Q4RJH9 |Q4RJH9_TETNG Chrc tr $\mid$ A7UP40|A7UP40_PSEMX Heat

$\operatorname{tr} \mid$ Q800Z2 |Q800Z2_PAR0L Spli tr |Q5XGA8 |Q5XGA8_XENTR Ribc

tr|B6YZ85|B6YZ85_THUT0 Vase

sp |A8Y5U1 | CE051_DANRE UPF0€ tr|B5X4J9|B5X4J9_SALSA Ribc tr $\mid$ AOEZU3 $\mid$ A0EZU3_PSEMX 40S tr|B9V305|B9V305_EPIC0 40S tr $\mid$ Q4RGQ9|Q4RGQ9_TETNG Chrc tr $\mid$ A9ZT79|A9ZT79_SOLSE Ribc tr $\mid$ A5H1I3 $\mid$ A5H1I3_PAR0L Char tr $\mid$ Q4SDY7 $\mid$ Q4SDY7_TETNG Chrc tr $\mid$ Q90ZQ6 |Q90ZQ6_ONCMY Poly

tr|B5DG90|B5DG90_SALSA RAB2 tr|C3KH31|C3KH31_ANOFI EMIE sp |Q802F2 | SELTA_DANRE Seler tr $\mid$ Q4RJP3 $\mid$ Q4RJP3_TETNG Chrc tr $\mid$ Q3UBL7 $\mid$ Q3UBL7_MOUSE Pute tr $\mid$ Q4SDA6 $\mid$ Q4SDA6_TETNG Chrc tr $\mid$ Q5RKM2 $\mid$ Q5RKM2_DANRE NADF tr $\mid$ C1BXJ2 $\mid$ C1BXJ2_ESOLU 39S tr $\mid$ Q4SI99 $\mid$ Q4SI99_TETNG Chrc tr|C3KK25|C3KK25_ANOFI Metr tr $\mid$ C1BHBO $\mid$ C1BHB0_ONCMY Eukc tr $\mid$ Q4T0S8 $\mid$ Q4T0S8_TETNG Chrc tr $\mid$ C1BJH9 $\mid$ C1BJH9_OSMMO ADP/

tr $\mid$ Q4V8Q4 $\mid$ Q4V8Q4_DANRE Zgc: tr $\mid$ C3KJK1|C3KJK1_ANOFI 39S

tr $\mid$ A2Q0T3 $\mid$ A2Q0T3_SOLSE Ribc $\operatorname{tr} \mid$ Q6VEJ7 $\mid$ Q6VEJ7_OREM0 Rec tr $\mid$ Q4RN49|Q4RN49_TETNG Chrc

$\operatorname{tr} \mid$ A6H913 |A6H913_TAKRU TATA 
1 UN_xiong_15449 UN_ci_1239

1 UN_xiong_14271 UN_ci_8725

0 UN_xiong_15554 UN_ci_7892

1 UN_xiong_15692 UN_ci_6939

0 UN_xiong_14840 UN_ci_1277

0 UN_xiong_14658 UN_ci_6552

0 UN_xiong_15533 UN_ci_8250

1 UN_xiong_13462 UN_ci_5426

1 UN_xiong_12050 UN_ci_8350

0 UN_xiong_9570 UN_ci_3163

1 UN_xiong_1436 UN_ci_448

1 UN_xiong_14884 UN_ci_3043

1 UN_xiong_4179 UN_ci_7855

1 UN_xiong_6936 UN_ci_2259

1 UN_xiong_2808 GP8I43W03GIU7K_ci

0 UN_xiong_4039 UN_ci_3692

1 UN_xiong_15372 UN_ci_854

0 UN_xiong_2199 UN_ci_5291

0 UN_xiong_4453 UN_ci_4439

0 UN_xiong_15219 UN_ci_257

1 UN_xiong_3384 UN_ci_7788

0 UN_xiong_1428 UN_ci_8218

1 UN_xiong_14525 UN_ci_4956

1 UN_xiong_2973 UN_ci_3621

1 UN_xiong_557 UN_ci_4425

1 UN_xiong_12975 UN_ci_433

1 UN_xiong_14541 UN_ci_1287

1 UN_xiong_14313 UN_ci_8334

1 UN_xiong_13097 UN_ci_8275

1 UN_xiong_6672 UN_ci_4253

1 UN_xiong_4304 UN_ci_5589

1 UN_xiong_14344 UN_ci_1669

0 UN_xiong_7852 UN_ci_8468

1 UN_xiong_14299 UN_ci_4248

1 UN_xiong_12671 UN_ci_7397

0 UN_xiong_14795 UN_ci_8058

0 UN_xiong_3203 UN_ci_2242

0 UN_xiong_3600 UN_ci_8400

0 UN_xiong_14835 UN_ci_7356

1 UN_xiong_14883 UN_ci_3196

1 UN_xiong_2239 UN_ci_855

1 UN_xiong_4351 UN_ci_5631

0 UN_xiong_3265 UN_ci_4443

1 UN_xiong_15034 UN_ci_7798

0 UN_xiong_14710 UN_ci_3927

0 UN_xiong_14441 UN_ci_8232

1 UN_xiong_9964 UN_ci_122

1 UN_xiong_14637 UN_ci_1564

1 UN_xiong_14354 UN_ci_8068

1 UN_xiong_15039 UN_ci_4024

0 UN_xiong_15587 UN_ci_8163

1 UN_xiong_14970 UN_ci_8794

1 UN_xiong_9243 UN_ci_8092

1 UN_xiong_4995 UN_ci_6179 tr $\mid$ Q6Y249|Q6Y249_PAGMA BTFE

tr|C3KIQ6|C3KIQ6_ANOFI Myos

tr $\mid$ Q5EB36 $\mid$ Q5EB36_XENTR MGC

tr $\mid$ D2H9F9 $\mid$ D2H9F9_AILME Pute tr $\mid$ Q4S5M1 | Q4S5M1_TETNG Chrc tr|C3KIE3|C3KIE3_ANOFI Trar

tr $\mid$ C3KIP5 $\mid$ C3KIP5_ANOFI Prot sp $\mid$ Q7ZV55|EIF3I_DANRE Eukar tr $\mid$ Q7ZUZ4 $\mid$ Q7ZUZ4_DANRE Pro६ tr $\mid$ A1Y189|A1Y189_PAR0L Volt tr $\mid$ Q4S5R8 $\mid$ Q4S5R8_TETNG Chrc tr $\mid$ C3KH20 $\mid$ C3KH20_ANOFI NADF tr $\mid$ C1BJ19|C1BJ19_0SMM0 Prot tr $\mid$ Q4SVP1 $\mid$ Q4SVP1_TETNG Chrc tr $\mid$ Q4SJP1 $\mid$ Q4SJP1_TETNG Chrc tr $\mid$ B5X8M2|B5X8M2_SALSA Medi tr $\mid$ Q8UVG1 $\mid$ Q8UVG1_EPIC0 Ribc tr $\mid$ Q7ZX50 |Q7ZX50_XENLA LOCA tr $\mid$ Q4RRE1 $\mid$ Q4RRE1_TETNG Chrc tr $\mid$ C3KI17|C3KI17_ANOFI Prot tr $\mid$ Q4SQY6 |Q4SQY6_TETNG Chrc tr $\mid$ C1BKU7 $\mid$ C1BKU7_0SMM0 Ce11 tr $\mid$ Q4RP92 $\mid$ Q4RP92_TETNG Chrc

sp | Q2KKX7 | CS042_SINCH UPFOE tr|B5DH25|B5DH25_SALSA 26S

tr $\mid$ Q71SZ0 $\mid$ Q71SZ0_9SC0M Cytc tr|C3KIU5|C3KIU5_ANOFI Trar tr $\mid$ Q4SJP0 $\mid$ Q4SJP0_TETNG Chrc tr $\mid$ Q4RG99|Q4RG99_TETNG Chrc tr $\mid$ C3KJT1 $\mid$ C3KJT1_ANOFI Higr

tr $\mid$ Q4S7P2 $\mid$ Q4S7P2_TETNG Chrc $\operatorname{tr} \mid$ B9EQ18|B9EQ18_SALSA CWC1 $\operatorname{tr} \mid$ B5X4Q0 | B5X4Q0_SALSA Tumc tr $\mid$ Q4SCH5 | Q4SCH5_TETNG Chrc tr $\mid$ Q32PU7 | Q32PU7_DANRE Zgc: tr|B9EP79|B9EP79_SALSA Prot

tr $\mid$ COPUM3 $\mid$ COPUM3_SALSA ATPtr $\mid$ Q803B9 $\mid$ Q803B9_DANRE DCP 2

tr | B3DLM7 |B3DLM7_XENTR LOC1 tr $\mid$ C3KJD7 $\mid$ C3KJD7_ANOFI FAMC tr $\mid$ Q4S4D9|Q4S4D9_TETNG Chrc $\operatorname{tr} \mid$ B5X1I8|B5X1I8_SALSA Doli tr $\mid$ C3KJM2 $\mid$ C3KJM2_ANOFI DNA- 
1 UN_xiong_14717 UN_ci_7875

1 UN_xiong_3793 UN_ci_7329

1 UN_xiong_2413 UN_ci_3605

1 UN_xiong_14516 UN_ci_5578

1 UN_xiong_3968 UN_ci_7542

1 UN_xiong_3503 UN_ci_6513

1 UN_xiong_3181 UN_ci_7588

1 UN_xiong_1200 UN_ci_2516

0 UN_xiong_5589 UN_ci_7992

1 UN_xiong_6740 UN_ci_71

1 UN_xiong_13283 UN_ci_1520

1 UN_xiong_12762 UN_ci_8067

1 UN_xiong_14527 UN_ci_3597

1 UN_xiong_662 UN_ci_1590

1 UN_xiong_14683 UN_ci_8665

0 UN_xiong_1812 UN_ci_1380

0 UN_xiong_14805 UN_ci_8328

1 UN_xiong_15786 UN_ci_4091

1 UN_xiong_14398 UN_ci_6652

1 UN_xiong_3085 UN_ci_4584

1 UN_xiong_2677 UN_ci_8259

1 UN_xiong_15049 UN_ci_4919

0 UN_xiong_14932 UN_ci_7661

1 UN_xiong_14106 UN_ci_8272

0 UN_xiong_14593 UN_ci_8193

0 UN_xiong_8117 UN_ci_6119

0 UN_xiong_14444 UN_ci_8309

1 UN_xiong_7138 UN_ci_5775

0 UN_xiong_14995 UN_ci_8595

1 UN_xiong_13092 UN_ci_6531

1 UN_xiong_14224 UN_ci_5646

1 UN_xiong_4865 UN_ci_8157

0 UN_xiong_14907 UN_ci_8420

1 UN_xiong_8591 UN_ci_5261

1 UN_xiong_10069 UN_ci_3141

0 UN_xiong_2251 UN_ci_160

0 UN_xiong_13033 UN_ci_3753

0 UN_xiong_13924 UN_ci_2798

0 UN_xiong_14283 UN_ci_6027

0 UN_xiong_1931 UN_ci_4049

1 UN_xiong_607 UN_ci_1397

1 UN_xiong_3481 UN_ci_5872

1 UN_xiong_3495 UN_ci_8187

1 UN_xiong_15078 UN_ci_6636

1 UN_xiong_6002 UN_ci_4669

1 UN_xiong_11708 UN_ci_8614

0 UN_xiong_2209 UN_ci_539

1 UN_xiong_6878 UN_ci_2565

0 UN_xiong_1494 UN_ci_4613

0 UN_xiong_14885 UN_ci_7761

0 UN_xiong_4084 UN_ci_2696

0 UN_xiong_15557 UN_ci_5849

1 UN_xiong_1920 UN_ci_8541

1 UN_xiong_10414 UN_ci_7616 tr $\mid$ B5X449|B5X449_SALSA Prot tr $\mid$ Q4RUH3 $\mid$ Q4RUH3_TETNG Chrc tr $\mid$ C3KJB4|C3KJB4_ANOFI Leyc tr $\mid$ Q4S3L1|Q4S3L1_TETNG Chrc tr|B5X193|B5X193_SALSA Pro1 tr $\mid$ C3KHF2 $\mid$ C3KHF2_ANOFI Ubic tr|D3TJL7|D3TJL7_EPIC0 Glut tr $\mid$ C3KI11|C3KI11_ANOFI NADF tr $\mid$ C3KJDO $\mid$ C3KJDO_ANOFI RWD $\operatorname{tr} \mid$ Q6PBS0 $\mid$ Q6PBS0_DANRE Requ sp |Q803V3 |P2R3C_DANRE Serir

tr|D1KY93|D1KY93_9CICH Once tr|C3KIS7|C3KIS7_ANOFI Mitc

tr $\mid$ C1BL72 $\mid$ C1BL72_0SMM0 E1or tr $\mid$ Q4RHZ9|Q4RHZ9_TETNG Chrc

tr | Q6DBQ6 | Q6DBQ6_DANRE Zgc:

$\operatorname{tr} \mid$ Q6PFS4 $\mid$ Q6PFS4_DANRE Inne tr $\mid$ C3KJ11|C3KJ11_ANOFI Doli tr $\mid$ B5DG05|B5DG05_SALSA Seri tr $\mid$ C1BEP1 $\mid$ C1BEP1_0NCMY Pref tr $\mid$ C3KJV1|C3KJV1_ANOFI 39S tr $\mid$ C3KHN8|C3KHN8_ANOFI Cofi tr $\mid$ Q9W6E6 $\mid$ Q9W6E6_9TELE Glut $\operatorname{tr} \mid$ B5X3U0 |B5X3U0_SALSA Ubic $\operatorname{tr} \mid$ C3KK02|C3KK02_ANOFI UPFC

tr $\mid$ COHBN2 $\mid$ COHBN2_SALSA Pre$\operatorname{tr} \mid$ C8CH42 $\mid$ C8CH42_SCIOC QM-1 tr $\mid$ C1BKP9 $\mid$ C1BKP9_0SMM0 60S tr $\mid$ Q4SLY5 $\mid$ Q4SLY5_TETNG Chrc tr $\mid$ C3KHMO $\mid$ C3KHMO_ANOFI ATP

tr $\mid$ Q4RT21 |Q4RT21_TETNG S-ac tr $\mid$ C1BYE7 $\mid$ C1BYE7_ESOLU UPFC

tr $\mid$ Q4SIZ7 $\mid$ Q4SIZ7_TETNG Chrc tr $\mid$ A0EZW3 $\mid$ A0EZW3_PSEMX Rec $\epsilon$

tr $\mid$ A0EZYO $\mid$ A0EZYO_PSEMX Ader $\operatorname{tr} \mid$ Q8AYL1 $\mid$ Q8AYL1_ORYLA ZPČ

tr $\mid$ Q4SN52 |Q4SN52_TETNG Chrc tr $\mid$ Q4SBZ6 $\mid$ Q4SBZ6_TETNG Chrc tr $\mid$ C1BL65 $\mid$ C1BL65_0SMM0 Doli tr $\mid$ COPTYO $\mid$ COPTYO_SALSA Nuc1 tr $\mid$ Q9DFT6 $\mid$ Q9DFT6_9PERC Bete $\operatorname{tr} \mid$ C3KIN7 $\mid$ C3KIN7_ANOFI NADF tr $\mid$ C1BKN5 $\mid$ C1BKN5_OSMM0 B-ce sp $\mid$ Q803M5 |SAAL1_DANRE Prot $\epsilon$ 
1 UN_xiong_14761 UN_ci_10

0 UN_xiong_983 UN_ci_647

0 UN_xiong_3521 UN_ci_3157

0 UN_xiong_9694 UN_ci_8153

1 UN_xiong_15624 UN_ci_7163

1 UN_xiong_1043 UN_ci_7697

1 UN_xiong_16 UN_ci_369

0 UN_xiong_8569 UN_ci_7834

1 UN_xiong_8203 UN_ci_7472

1 UN_xiong_14899 UN_ci_294

0 UN_xiong_2099 UN_ci_8670

1 UN_xiong_11580 UN_ci_307

0 UN_xiong_4533 UN_ci_7862

0 UN_xiong_1942 UN_ci_7952

0 UN_xiong_14251 UN_ci_7333

1 UN_xiong_6171 UN_ci_1057

1 UN_xiong_889 UN_ci_4771

0 UN_xiong_13480 UN_ci_8343

1 UN_xiong_14731 UN_ci_2333

1 UN_xiong_15790 UN_ci_8319

1 UN_xiong_2288 UN_ci_1425

1 UN_xiong_8411 UN_ci_6171

1 UN_xiong_4628 UN_ci_8006

0 UN_xiong_14486 UN_ci_3850

0 UN_xiong_12764 UN_ci_5250

0 UN_xiong_14409 UN_ci_8041

0 UN_xiong_7832 UN_ci_5796

1 UN_xiong_9969 UN_ci_4751

1 UN xiong 9738 UN ci 4081

1 UN_xiong_14990 UN_ci_2854

0 UN_xiong_10741 UN_ci_8061

0 UN_xiong_14968 UN_ci_4275

1 UN_xiong_2226 UN_ci_8050

0 UN_xiong_8120 UN_ci_4858

1 UN_xiong_14362 UN_ci_7283

1 UN_xiong_14684 UN_ci_5829

0 UN_xiong_14784 UN_ci_6572

0 UN_xiong_14325 UN_ci_3241

0 UN_xiong_11308 UN_ci_2416

0 UN_xiong_2360 UN_ci_6439

0 UN_xiong_14260 UN_ci_6184

0 UN_xiong_6441 UN_ci_8692

1 UN_xiong_8985 UN_ci_3585

0 UN_xiong_14258 UN_ci_1011

0 UN_xiong_9761 UN_ci_7694

1 UN_xiong_13205 UN_ci_48

0 UN_xiong_3864 UN_ci 662

0 UN_xiong_4247 UN_ci_8076

1 UN_xiong_4642 UN_ci_5546

0 UN_xiong_14780 UN_ci_231

0 UN_xiong_14608 UN_ci_2880

1 UN_xiong_10903 UN_ci_2932

0 UN_xiong_8140 UN_ci_7662

0 UN_xiong_6452 UN_ci_597 tr $\mid$ Q4S6V3 $\mid$ Q4S6V3_TETNG T-cc tr $\mid$ D8U3C1 $\mid$ D8U3C1_VOLCA Pute tr|B5X9E6 |B5X9E6_SALSA E1or tr|B9EPJ4|B9EPJ4_SALSA Thic

tr $\mid$ Q6TEL5 $\mid$ Q6TEL5_DANRE Cu11

tr $\mid$ COPTY4 $\mid$ COPTY4_SALSA Pro1 tr $\mid$ Q4S358|Q4S358_TETNG Chrc tr |Q1PS66|Q1PS66_LATCA Del€ tr $\mid$ Q4SPX5 $\mid$ Q4SPX5_TETNG Chrc tr $\mid$ Q4VBR8 $\mid$ Q4VBR8_DANRE Prot tr $\mid$ C1BYB1 $\mid$ C1BYB1_ESOLU Trar $\operatorname{tr} \mid$ C1BKW4 $\mid$ C1BKW4_0SMM0 KIAA tr $\mid$ A9ZT78|A9ZT78_SOLSE Ribc tr $\mid$ C1J0J0|C1J0J0_GILSE ATP

tr|A9ZT85|A9ZT85_S0LSE Ribc sp |Q2YDS5 |SCNM1_DANRE Sodic $\operatorname{tr} \mid$ A5D6V4 $\mid$ A5D6V4_DANRE Zgc: tr $\mid$ C1BM02 $\mid$ C1BM02_OSMM0 60S

$\operatorname{tr} \mid$ Q08AY4 $\mid$ Q08AY4_XENLA LOC1

tr |Q9I8X6|Q9I8X6_9SMEG Cytc tr $\mid$ C3KH80|C3KH80_ANOFI Ran$\operatorname{tr} \mid$ B5X8Y2|B5X8Y2_SALSA Metr sp $\mid$ Q7ZWW7 |TS31B_XENLA Tetre tr $\mid$ C3KK45|C3KK45_ANOFI Ribc

tr $\mid$ Q8QFS3 $\mid$ Q8QFS3_TETNG H01C

tr $\mid$ Q6DHE6 |Q6DHE6_DANRE Lysy tr $\mid$ Q4TBK6 $\mid$ Q4TBK6_TETNG Chrc tr $\mid$ C1BW85|C1BW85_ES0LU Lintr $\mid$ Q4RS99|Q4RS99_TETNG Chrc tr $\mid$ C3KGT8 $\mid$ C3KGT8_ANOFI Coat $\operatorname{tr} \mid$ C1BG20 $\mid$ C1BG20_0NCMY RINC tr $\mid$ C3KHA5 $\mid$ C3KHA5_ANOFI TRM1 tr $\mid$ Q4RJV4 $\mid$ Q4RJV4_TETNG Chrc tr|B5XB55|B5XB55_SALSA Sigr tr $\mid$ Q1KLP9|Q1KLP9_PSEMX Nate tr $\mid$ Q4RSW9 $\mid$ Q4RSW9_TETNG Chrc

$\operatorname{tr} \mid$ C3KHK4 $\mid$ C3KHK4_ANOFI Pref

tr |Q4RX35|Q4RX35_TETNG Chrc tr |Q67EQ7|Q67EQ7_ORYLA Zinc

tr $\mid$ C3KHM3 $\mid$ C3KHM3_ANOFI 39S tr|C3KIW7 |C3KIW7_ANOFI Surv tr|B5FY04|B5FY04_TAEGU Pute 
0 UN_xiong_13318 UN_ci_5041

1 UN_xiong_4348 UN_ci_4260

1 UN_xiong_14352 UN_ci_7837

0 UN_xiong_4599 UN_ci_5248

1 UN_xiong_14975 UN_ci_2560

1 UN_xiong_13896 UN_ci_4738

0 UN_xiong_11376 UN_ci_8802

0 UN_xiong_14736 UN_ci_7311

1 UN_xiong_3763 UN_ci_1047

1 UN_xiong_14666 UN_ci_1717

1 UN_xiong_5310 UN_ci_7040

0 UN_xiong_11967 UN_ci_8768

0 UN_xiong_15503 UN_ci_8708

0 UN_xiong_15191 UN_ci_5192

0 UN_xiong_14002 UN_ci_8764

0 UN_xiong_13661 UN_ci_1704

0 UN_xiong_3420 UN_ci_7784

0 UN_xiong_1167 UN_ci_3726

1 UN_xiong_838 UN_ci_4105

0 UN_xiong_4244 UN_ci_1737

0 UN_xiong_14687 UN_ci_8348

0 UN_xiong_10360 UN_ci_7095

0 UN_xiong_2788 UN_ci_4168

0 UN_xiong_15787 UN_ci_7884

0 UN_xiong_9146 UN_ci_4266

0 UN_xiong_3902 UN_ci_5104

0 UN_xiong_13970 UN_ci_7742

0 UN_xiong_9643 UN_ci_3600

0 UN_xiong_14326 UN_ci_8339

0 UN_xiong_13845 UN_ci_8596

0 UN_xiong_12519 UN_ci_3586

0 UN_xiong_7476 UN_ci_6015

1 UN_xiong_14997 UN_ci_7527

0 UN_xiong_1596 UN_ci_3150

0 UN_xiong_8683 UN_ci_8151

0 UN_xiong_14548 UN_ci_3355

1 UN_xiong_891 UN_ci_7571

0 UN_xiong_1247 UN_ci_1165

0 UN_xiong_14663 UN_ci_8185

0 UN_xiong_29 UN_ci_6583

0 UN_xiong_4038 UN_ci_7689

1 UN xiong 10111 UN ci 4926

0 UN xiong 14374 UN ci 3785

0 UN xiong 11502 UN ci 542

0 UN_xiong_14681 UN_ci_47

0 UN_xiong 7586 UN_ci_4947

0 UN_xiong_273 UN_ci_4159

0 UN_xiong_14856 UN_ci_6692

0 UN_xiong_1364 UN_ci_1151

0 UN_xiong_14906 UN_ci_6118

0 UN_xiong_553 UN_ci_2779

0 UN_xiong_15519 UN_ci_6589

0 UN_xiong_5132 UN_ci_3409

0 UN_xiong_4081 UN_ci_7171 tr $\mid$ C3KIF4|C3KIF4_ANOFI 26S

tr|B5X6W2 |B5X6W2_SALSA Cyst tr $\mid$ A3QJW5 $\mid$ A3QJW5_DANRE CTP tr $\mid$ B9EMJ6|B9EMJ6_SALSA DNA$\operatorname{tr} \mid$ Q6DC52 $\mid$ Q6DC52_DANRE Zgc: tr $\mid$ COH961|COH961_SALSA Vact tr $\mid$ C1BKB6 $\mid$ C1BKB6_0SMM0 Ader tr $\mid$ Q4RVH3 $\mid$ Q4RVH3_TETNG Prot tr|B5X958|B5X958_SALSA 0st $\epsilon$ sp $\mid$ Q7T2A5|EIF3L_DANRE Eukar tr $\mid$ C3KH72 $\mid$ C3KH72_ANOFI Prok tr $\mid$ B3DFW5 |B3DFW5_DANRE Si:c tr $\mid$ Q6PC77 $\mid$ Q6PC77_DANRE ATP $\operatorname{tr} \mid$ C3KI25|C3KI25_ANOFI Smal tr |B9ELR8|B9ELR8_SALSA Trar tr $\mid$ C1BLM1 $\mid$ C1BLM1_0SMM0 Trar

tr $\mid$ Q4SZN8 $\mid$ Q4SZN8_TETNG Chrc tr $\mid$ Q4TC18|Q4TC18_TETNG Chrc

tr $\mid$ C3KI81 $\mid$ C3KI81_ANOFI Elec tr|C3KIQ8|C3KIQ8_ANOFI 39S

tr|C3KIZ6|C3KIZ6_ANOFI Ce11 tr $\mid$ A2Q0S1|A2Q0S1_SOLSE Ribc

tr $\mid$ C1BKD0 $\mid$ C1BKD0_0SMM0 Trar tr $\mid$ D8X0S5|D8X0S5_PSEMX Perc tr $\mid$ B5XDC9 $\mid$ B5XDC9_SALSA Pept tr $\mid$ COH747|COH747_SALSA 40S tr $\mid$ A8JJG4 $\mid$ A8JJG4_CHLRE Prec

tr $\mid$ C3KJ18|C3KJ18_ANOFI Prok

tr $\mid$ Q4SCJ6 $\mid$ Q4SCJ6_TETNG Chrc tr $\mid$ Q5DW64 $\mid$ Q5DW64_ONCMY Gluc $\operatorname{tr} \mid$ Q45KR0 $\mid$ Q45KR0_LARCR Heat

tr $\mid$ COH9Z2 $\mid$ COH9Z2_SALSA Cel1 $\operatorname{tr} \mid$ C3KK62 $\mid$ C3KK62_ANOFI Smal $\operatorname{tr} \mid$ Q7ZV25 $\mid$ Q7ZV25_DANRE Tubr tr $\mid$ Q803D9 $\mid$ Q803D9_DANRE Prmt tr $\mid$ Q4RU94 |Q4RU94_TETNG Chrc $\operatorname{tr} \mid$ Q5HZ93 $\mid$ Q5HZ93_XENLA MGCE tr $\mid$ COH917|COH917_SALSA Het $\epsilon$

tr $\mid$ Q6DRK2 $\mid$ Q6DRK2_DANRE Sp1i tr $\mid$ Q4S8P8 $\mid$ Q4S8P8_TETNG Chrc tr $\mid$ Q4SLW7 $\mid$ Q4SLW7_TETNG Chrc tr $\mid$ C1BJA0 $\mid$ C1BJA0_0SMM0 Surf tr $\mid$ C3KIG2 $\mid$ C3KIG2_ANOFI C-My tr $\mid$ C3KJZ2 $\mid$ C3KJZ2_ANOFI ATP

tr $\mid$ Q4S8V0 $\mid$ Q4S8V0_TETNG Chrc tr $\mid$ C3KJR7|C3KJR7_ANOFI Thic 
0 UN_xiong_4172 UN_ci_4353

0 UN_xiong_7949 UN_ci_7777

0 UN_xiong_2313 UN_ci_2373

0 UN_xiong_6173 UN_ci_8569

0 UN_xiong_1756 UN_ci_4993

0 UN_xiong_2622 UN_ci_5196

0 UN_xiong_4067 UN_ci_2937

0 UN_xiong_8071 UN_ci_8245

0 UN_xiong_139 UN_ci_1294

0 UN_xiong_10103 UN_ci_4487

0 UN_xiong_12679 UN_ci_5521

0 UN_xiong_14846 UN_ci_7791

0 UN_xiong_4693 UN_ci_2008

0 UN_xiong_1005 UN_ci_1330

0 UN_xiong_3935 UN_ci_8216

0 UN_xiong_2996 UN_ci_7967

0 UN_xiong_10941 UN_ci_8268

0 UN_xiong_15055 UN_ci_5492

0 UN_xiong_1512 UN_ci_2749

0 UN_xiong_14208 UN_ci_2655

0 UN_xiong_14470 UN_ci_4718

0 UN_xiong_8647 UN_ci_6413

0 UN_xiong_4291 UN_ci_221

0 UN_xiong_9232 UN_ci_8634

0 UN_xiong_14791 UN_ci_8072

0 UN_xiong_14619 UN_ci_7652

0 UN_xiong_11417 UN_ci_7596

0 UN_xiong_14042 UN_ci_8070

0 UN_xiong_5707 UN_ci_4023

0 UN_xiong_5437 UN_ci_5388

0 UN_xiong_14685 UN_ci_8270

0 UN_xiong_8684 UN_ci_4164

0 UN_xiong_15755 UN_ci_4712

0 UN_xiong_13712 UN_ci_7252

0 UN_xiong_15511 UN_ci_198

0 UN_xiong_14848 UN_ci_863

0 UN_xiong_13016 UN_ci_8321

0 UN_xiong_9698 UN_ci_3516

0 UN_xiong_14737 UN_ci_6484

0 UN_xiong_1392 UN_ci_5475

0 UN_xiong_55 UN_ci_1428

0 UN_xiong_11332 UN_ci_3697

0 UN_xiong_14228 UN_ci_3370

0 UN_xiong_6419 UN_ci_4293

0 UN_xiong_7955 UN_ci_3238

0 UN_xiong_4182 UN_ci_6714

0 UN_xiong_11475 UN_ci_5685

0 UN_xiong_12390 UN_ci_7693

0 UN_xiong_6263 UN_ci_8167

0 UN_xiong_4289 UN_ci_1617

0 UN_xiong_1206 UN_ci_8190

0 UN_xiong_1241 UN_ci_8128

0 UN_xiong_14951 UN_ci_4078

0 UN_xiong_11755 UN_ci_2833 tr $\mid$ C3KHZ2 $\mid$ C3KHZ2_ANOFI Mitc tr $\mid$ Q4S2U7 $\mid$ Q4S2U7_TETNG Chrc $\operatorname{tr} \mid$ B5X2G7|B5X2G7_SALSA 28S tr $\mid$ C3KIH2 $\mid$ C3KIH2_ANOFI Tumc tr $\mid$ C3KHX1|C3KHX1_ANOFI 26S tr $\mid$ Q4RI74|Q4RI74_TETNG Chrc sp |Q566X6|PTCD2_DANRE Pente tr $\mid$ C3KHK1|C3KHK1_ANOFI Euke tr $\mid$ C3KJU5|C3KJU5_ANOFI Biog tr $\mid$ Q4RXL8 $\mid$ Q4RXL8_TETNG Chrc tr|B9ENS2|B9ENS2_SALSA Thic tr $\mid$ C3KJX5 $\mid$ C3KJX5_ANOFI Prok tr|D2H728|D2H728_AILME Putc tr|C3KIU9|C3KIU9_ANOFI Rep1 tr|D2CFQ6 |D2CFQ6_LARCR Trar tr $\mid$ Q4SOQ0 $\mid$ Q4SOQ0_TETNG Chrc tr $\mid$ Q4T4L7|Q4T4L7_TETNG Prof $\operatorname{tr} \mid$ C3KK67|C3KK67_ANOFI T-c€

tr $\mid$ C3KJ47|C3KJ47_ANOFI Hist $\operatorname{tr} \mid$ C3KID9|C3KID9_ANOFI NADF tr|B5XGK6|B5XGK6_SALSA Crys

tr $\mid$ B9EL49|B9EL49_SALSA Ubic tr|C3KHR9|C3KHR9_ANOFI Thic tr $\mid$ A2Q0S2 |A2Q0S2_SOLSE Ribc tr $\mid$ Q4SL28|Q4SL28_TETNG Chrc tr $\mid$ C1BIP5 $\mid$ C1BIP5_0SMM0 U3 s $\operatorname{tr} \mid$ C3KHL7|C3KHL7_ANOFI Ubic

tr $\mid$ Q6PGV9 |Q6PGV9_DANRE Pept sp |Q6TH15|EIF3D_DANRE Eukar tr $\mid$ Q4S7N9 $\mid$ Q4S7N9_TETNG Chrc tr $\mid$ C3KI46|C3KI46_ANOFI Prok tr $\mid$ Q27HW5 $\mid$ Q27HW5_PSEMX Ce11 tr $\mid$ Q4SS97|Q4SS97_TETNG Chrc tr $\mid$ Q4RRB6 |Q4RRB6_TETNG Chrc tr $\mid$ Q6P959|Q6P959_DANRE ATP tr $\mid$ A0F005 $\mid$ A0F005_PSEMX RNA $\operatorname{tr} \mid$ B5X218|B5X218_SALSA Nuc1 tr $\mid$ Q4RKM2 |Q4RKM2_TETNG Chrc tr $\mid$ Q4SLRO $\mid$ Q4SLR0_TETNG Chrc tr $\mid$ C3KJH2 $\mid$ C3KJH2_ANOFI Smal

tr|Q6TNU6|Q6TNU6_DANRE Dihy tr $\mid$ Q68J69|Q68J69_PAR0L Catr sp |Q4S2L4|RCL_TETNG Deoxyri tr $\mid$ C3KK54|C3KK54_ANOFI Deox tr $\mid$ C1BKV1 $\mid$ C1BKV1_0SMM0 ADPtr $\mid$ Q5F3E1 $\mid$ Q5F3E1_CHICK Pute $\operatorname{tr} \mid$ C3KJ43|C3KJ43_ANOFI ORM1 tr $\mid$ Q6DHI9|Q6DHI9_DANRE Prot tr $\mid$ Q6Y248|Q6Y248_PAGMA Char tr $\mid$ Q4RSM1 | Q4RSM1_TETNG Chrc 
0 UN_xiong_14454 UN_ci_8213

0 UN_xiong_5406 UN_ci_5538

0 UN_xiong_2475 UN_ci_3486

0 UN_xiong_3161 UN_ci_3942

0 UN_xiong_13344 UN_ci_6445

0 UN_xiong_3504 UN_ci_8739

0 UN_xiong_2521 UN_ci_7809

0 UN_xiong_13725 UN_ci_5649

0 UN_xiong_14964 UN_ci_7937

0 UN_xiong_12227 UN_ci_6606

0 UN_xiong_15334 UN_ci_7063

0 UN_xiong_5387 UN_ci_769

0 UN_xiong_14520 UN_ci_7865

0 UN_xiong_4481 UN_ci_5114

0 UN_xiong_4168 UN_ci_560

0 UN_xiong_4619 UN_ci_8109

0 UN_xiong_4204 UN_ci_8137

0 UN_xiong_5161 UN_ci_2879

0 UN_xiong_2186 UN_ci_3805

0 UN_xiong_12936 UN_ci_8520

0 UN_xiong_14512 UN_ci_7803

0 UN_xiong_15667 UN_ci_6336

0 UN_xiong_14590 UN_ci_8004

0 UN_xiong_7151 UN_ci_2975

0 UN_xiong_10090 UN_ci_8260

0 UN_xiong_7386 UN_ci_7921

0 UN_xiong_8732 UN_ci_4979

0 UN_xiong_15471 UN_ci_7882

0 UN xiong_12428 UN ci 8636

0 UN_xiong_1155 UN_ci_3401

0 UN_xiong_1113 UN_ci_7228

0 UN_xiong_14774 UN_ci_7738

0 UN_xiong_3537 UN_ci_8191

0 UN_xiong_14834 UN_ci_1988

0 UN_xiong_10542 UN_ci_7879

0 UN_xiong_3741 UN_ci_7821

0 UN_xiong_8628 UN_ci_7149

0 UN_xiong_10425 UN_ci_7009

0 UN_xiong_10446 UN_ci_8241

0 UN_xiong_8143 UN_ci_2254

0 UN_xiong_13417 UN_ci_7279

0 UN_xiong_13713 UN_ci_7902

0 UN_xiong_12237 UN_ci_8233

0 UN_xiong_12729 UN_ci_7825

0 UN_xiong_14699 UN_ci_8078

0 UN_xiong_15043 UN_ci_6840

0 UN_xiong_13144 UN_ci_6619

0 UN_xiong_15789 UN_ci_8114

0 UN_xiong_14804 UN_ci_2625

0 UN_xiong_3369 UN_ci_8140

0 UN_xiong_14265 UN_ci_600

0 UN_xiong_974 UN_ci_4116

0 UN_xiong_14749 UN_ci_4279

0 UN_xiong_1333 UN_ci_7973 tr $\mid$ Q6PH42 | Q6PH42_DANRE Ubic tr $\mid$ C3KI18|C3KI18_ANOFI Elor tr|COH7I9|COH7I9_SALSA 40S tr $\mid$ C3KH14|C3KH14_ANOFI FK5C tr $\mid$ COHA74 $\mid$ COHA74_SALSA DNAtr $\mid$ Q4SUY7|Q4SUY7_TETNG Chrc tr $\mid$ Q7ZW94|Q7ZW94_DANRE Sigr $\operatorname{tr} \mid$ B5XFF1|B5XFF1_SALSA Dyne $\operatorname{tr} \mid$ C1BYA1 $\mid$ C1BYA1_ESOLU AN1-

tr $\mid$ Q4SPS2 $\mid$ Q4SPS2_TETNG Chrc tr|B5XA79|B5XA79_SALSA 39S

tr |A9ZT96|A9ZT96_SOLSE Ribc

\section{$---$}

tr $\mid$ B5X7F1 $\mid$ B5X7F1_SALSA Lintr $\mid$ Q4RHR2 | Q4RHR2_TETNG Chrc tr $\mid$ Q4SCH7 $\mid$ Q4SCH7_TETNG Chrc tr $\mid$ Q4RL89|Q4RL89_TETNG Chrc

tr $\mid$ B5DGF1|B5DGF1_SALSA Sigr sp |Q9I8U0|COX41_THUOB Cytoc tr $\mid$ C1BLD2 $\mid$ C1BLD2_OSMM0 C20c tr $\mid$ Q71U69|Q71U69_XENLA Enhe

tr $\mid$ C1BWH3 $\mid$ C1BWH3_ESOLU NEDC

tr|C3KHH5|C3KHH5_ANOFI tRNA tr|B9ENP9|B9ENP9_SALSA Trar sp |Q1L9A2 |OPA3_DANRE Optic

tr|B5X4D6|B5X4D6_SALSA Proc

tr|C3KI22|C3KI22_ANOFI Paln

tr $\mid$ C1BIU8 $\mid$ C1BIU8_0SMM0 Devє tr|C3KJY7|C3KJY7_ANOFI Traf tr $\mid$ Q4S1K2 | Q4S1K2_TETNG Chrc

tr $\mid$ Q4SWD7 $\mid$ Q4SWD7_TETNG Chrc

tr $\mid$ B5DFB2 $\mid$ B5DFB2_RAT RCG30E tr $\mid$ Q4SQF9 $\mid$ Q4SQF9_TETNG Chrc

$\operatorname{tr} \mid$ C3KJJ1 $\mid$ C3KJJ1_ANOFI Euke tr|B6ZIW4|B6ZIW4_ORYLA Aurc tr $\mid$ C3KJN5 $\mid$ C3KJN5_ANOFI Nontr $\mid$ Q6NZZ2 $\mid$ Q6NZZ2_DANRE Acti tr $\mid$ Q6Y213|Q6Y213_PAGMA Ribc

$\operatorname{tr} \mid$ Q568Q1 $\mid$ Q568Q1_DANRE Chrc tr $\mid$ Q4RPX8 $\mid$ Q4RPX8_TETNG Chrc $\operatorname{tr} \mid$ B5X2Q6 | B5X2Q6_SALSA Nuc1 
0 UN_xiong_15345 UN_ci_8176

0 UN_xiong_2411 UN_ci_8008

0 UN_xiong_14887 UN_ci_5525

0 UN_xiong_6402 UN_ci_8316

0 UN_xiong_9555 UN_ci_8273

0 UN_xiong_2889 UN_ci_1644

0 UN_xiong_4268 UN_ci_7713

0 UN_xiong_8535 UN_ci_4157

0 UN_xiong_14781 UN_ci_6311

0 UN_xiong_14802 UN_ci_3085

0 UN_xiong_15754 UN_ci_4653

0 UN_xiong_246 UN_ci_3615

0 UN_xiong_14038 UN_ci_8227

0 UN_xiong_8922 UN_ci_6223

0 UN_xiong_5489 UN_ci_8349

0 UN_xiong_9226 UN_ci_7954

0 UN_xiong_5353 UN_ci_1032

0 UN_xiong_14638 UN_ci_7690

0 UN_xiong_12384 UN_ci_6424

0 UN_xiong_15516 UN_ci_2570

0 UN_xiong_10473 UN_ci_1668

0 UN_xiong_15136 UN_ci_3756

0 UN_xiong_9158 UN_ci_8145

0 UN_xiong_6083 UN_ci_4835

0 UN_xiong_9652 UN_ci_8347

0 UN_xiong_9456 UN_ci_8266

0 UN_xiong_14881 UN_ci_7822

0 UN_xiong_13635 UN_ci_7175

0 UN xiong_5614 UN ci 3642

0 UN_xiong_6787 UN_ci_3968

0 UN_xiong_3293 UN_ci_8085

0 UN_xiong_14915 UN_ci_8704

0 UN_xiong_8376 UN_ci_1115

0 UN_xiong_2265 UN_ci_3047

0 UN_xiong_4496 UN_ci_2065

0 UN_xiong_488 UN_ci_7402

0 UN_xiong_2324 UN_ci_8425

0 UN_xiong_1145 UN_ci_7023

0 UN_xiong_8413 UN_ci_3906

0 UN_xiong_4726 UN_ci_2211

0 UN_xiong_14844 UN_ci_7266

0 UN_xiong_11167 UN_ci_3251 tr $\mid$ Q4RKC4 $\mid$ Q4RKC4_TETNG Chrc

tr $\mid$ Q4S2C0 $\mid$ Q4S2C0_TETNG Chrc tr $\mid$ Q6DH98|Q6DH98_DANRE CNDF

tr $\mid$ A7SVD8 $\mid$ A7SVD8_NEMVE Prec tr $\mid$ C3KJE5 $\mid$ C3KJE5_ANOFI Nuc1

tr $\mid$ A3KNA4 $\mid$ A3KNA4_DANRE Wu:f tr|B5XFU3|B5XFU3_SALSA Sert tr $\mid$ C1BWH8 $\mid$ C1BWH8_ESOLU Enhe tr|B5X1I0|B5X1I0_SALSA S-ac tr $\mid$ Q4S1W7 $\mid$ Q4S1W7_TETNG Chrc tr $\mid$ Q4SUP7 $\mid$ Q4SUP7_TETNG Chrc $\operatorname{tr} \mid$ C3KIH3 $\mid$ C3KIH3_ANOFI Mult tr $\mid$ Q4RG08|Q4RG08_TETNG Chrc tr $\mid$ Q4RYZ2 |Q4RYZ2_TETNG Chrc tr $\mid$ Q4SLP2 $\mid$ Q4SLP2_TETNG Chrc $\operatorname{tr} \mid$ C3KJI8|C3KJI8_ANOFI Trar tr $\mid$ C1BWR4 $\mid$ C1BWR4_ESOLU TriF tr $\mid$ C3KHD2 $\mid$ C3KHD2_ANOFI U6 s tr $\mid$ Q70AHO $\mid$ Q70AHO_TAKRU Alpr tr $\mid$ B5X7S9|B5X7S9_SALSA RINC tr $\mid$ C3KJCO $\mid$ C3KJCO_ANOFI Micr $\operatorname{tr} \mid$ C3KHN7 $\mid$ C3KHN7_ANOFI COPC sp $\mid$ Q4QY71 | RSSA_SPAAU 40S ri tr $\mid$ Q4SAW6 |Q4SAW6_TETNG Chrc

tr $\mid$ Q4SZF7 $\mid$ Q4SZF7_TETNG Chrc tr|C1BK96|C1BK96_0SMM0 Iror

tr $\mid$ Q4RRE0 $\mid$ Q4RRE0_TETNG Chrc tr $\mid$ C3KJR1|C3KJR1_ANOFI UPFC $\operatorname{tr} \mid$ Q6VQB0 $\mid$ Q6VQB0_TAKRU Se1 $\epsilon$ tr $\mid$ C3KJ52|C3KJ52_ANOFI CD2 tr $\mid$ C1BIR6|C1BIR6_OSMM0 UPFC tr $\mid$ C1BJK8|C1BJK8_0SMM0 Trar

tr $\mid$ C3KH25|C3KH25_ANOFI Medi $\operatorname{tr} \mid$ C3KI27|C3KI27_ANOFI Cyst 
mosome undetermined SCAF21483, whole genome shotgun sequence OS=Tetraodon nigrovi

ribosomal protein L36a OS=0smerus mordax GN=RL36A PE=3 SV=1

iscription elongation factor B polypeptide 1 OS=Anoplopoma fimbria GN=ELOC PE=4 SV ribosomal protein L37a OS=Anoplopoma fimbria GN=RL37A PE=4 SV=1

ttive ribosomal protein 239 protein $0 \mathrm{~S}=0$ ncorhynchus mykiss $\mathrm{PE}=4 \mathrm{SV}=1$

Irin 0S=Anoplopoma fimbria GN=PTTG1 PE=2 SV=1

in 1ight chain 2, cytoplasmic OS=Anoplopoma fimbria GN=DYL2 PE=4 SV=1

i1 homolog OS=Anoplopoma fimbria GN=BUD31 PE=2 SV=1

mosome 9 SCAF14710, whole genome shotgun sequence OS=Tetraodon nigroviridis GN=GS

11 in alpha-1 chain OS=0smerus mordax GN=TBA1 PE=2 SV=1

nin OS=0ryzias latipes $\mathrm{PE}=2 \mathrm{SV}=1$

ribosomal protein L31 OS=Epinephelus coioides $\mathrm{PE}=2 \mathrm{SV}=1$

itive uncharacterized protein (Fragment) OS=Ailuropoda melanoleuca GN=PANDA_004337

Igation factor 1-alpha 0S=Pagrus major GN=42Sp50 PE=2 SV=1

Ione reductase (Fragment) $0 \mathrm{~S}=0$ reochromis mossambicus $\mathrm{PE}=2 \mathrm{SV}=1$

somal protein L27a OS=Solea senegalensis GN=RPL27a PE=2 SV=1

iscription initiation factor TFIID subunit 10 OS=0ncorhynchus mykiss GN=TAF10 PE=C

lidymal secretory protein E1 OS=Anoplopoma fimbria GN=NPC2 PE=2 SV=1

somal protein L37 OS=Pagrus major $\mathrm{PE}=3 \mathrm{SV}=1$

luitin OS=0ncorhynchus mykiss $\mathrm{PE}=2 \mathrm{SV}=1$

ribosomal protein $\mathrm{L} 7$ OS=0smerus mordax GN=RL7 PE=2 SV=1

3-1ike 2 (E. coli) OS=Danio rerio GN=ruvb12 $\mathrm{PE}=4 \mathrm{SV}=1$

chondrial ATP synthase lipid-binding protein OS=Epinephelus coioides PE=2 SV=1

igation factor $1-$ alpha $0 \mathrm{~S}=$ Pagrus major $\mathrm{PE}=2 \mathrm{SV}=1$

ribosomal protein $\mathrm{S} 21$ OS=Psetta maxima $\mathrm{PE}=4 \mathrm{SV}=1$

smosome 12 SCAF14999, whole genome shotgun sequence. (Fragment) OS=Tetraodon nigrc

hmin OS=Anoplopoma fimbria GN=STMN1 PE=2 SV=1

ı pellucida (Fragment) OS=Hypomesus transpacificus $\mathrm{PE}=2 \mathrm{SV}=1$

mosome 18 SCAF14304, whole genome shotgun sequence. (Fragment) OS=Tetraodon nigrc ribosomal protein L30 OS=Anoplopoma fimbria GN=RL30 PE=4 SV=1

ıine nucleotide binding protein alpha subunit v1 subtype (Fragment) OS=Danio reric (P) H dehydrogenase quinone 1 OS=Fundulus heteroclitus $\mathrm{PE}=2 \mathrm{SV}=1$

somal protein L11 OS=Solea senegalensis GN=RPL11 PE=2 SV=1

¡714 OS=Drosophila grimshawi GN=GH15714 PE=4 SV=1

somal protein L13a OS=Solea senegalensis GN=RPL13a-1ike1 PE=2 SV=1

somal protein large $\mathrm{P} 0-1$ ike protein $0 \mathrm{~S}=$ Perca flavescens $\mathrm{PE}=2 \mathrm{SV}=1$

islation factor sui1-1ike $0 \mathrm{~S}=$ Ictalurus punctatus $\mathrm{PE}=4 \mathrm{SV}=1$

mosome 6 SCAF14737, whole genome shotgun sequence. (Fragment) OS=Tetraodon nigrov

somal protein S27 (Fragment) OS=Psetta maxima PE=2 SV=1

iryotic translation initiation factor 3 subunit E OS=Anoplopoma fimbria GN=EIF3E F somal protein L17 OS=Paralichthys olivaceus PE=2 SV=1

ribosomal protein S9 OS=Anoplopoma fimbria GN=RS9 PE=2 SV=1

ribosomal protein S4 (Fragment) OS=Epinephelus coioides PE=2 SV=1

ise inhibitor, mitochondrial OS=0smerus mordax GN=ATIF1 PE=4 SV=1 
mosome undetermined SCAF7389, whole genome shotgun sequence OS=Tetraodon nigrovir ficking protein particle complex 1 OS=Danio rerio $\mathrm{GN}=$ trappc1 $\mathrm{PE}=2 \mathrm{SV}=1$

ribosomal protein $\mathrm{L} 27$ OS=Psetta maxima $\mathrm{PE}=2 \mathrm{SV}=1$

: arrest protein 1 OS=Takifugu rubripes $\mathrm{GN}=\mathrm{zar} 1 \mathrm{PE}=2 \mathrm{SV}=1$

somal protein L18a OS=Solea senegalensis GN=RPL18a PE=2 SV=1

chondrial ATP synthase F0 complex subunit $\mathrm{c}$ isoform 1 OS=Takifugu rubripes GN=Atr

ıepsin Z OS=0smerus mordax GN=CATZ PE=2 SV=1

ribosomal protein $\mathrm{S} 28$ 0S=Anoplopoma fimbria GN=RS28 PE=4 SV=1

mosome 1 SCAF14573, whole genome shotgun sequence. (Fragment) OS=Tetraodon nigrov bosomal protein L18 0S=0reochromis niloticus $\mathrm{GN}=\mathrm{rp} 118 \mathrm{PE}=2 \mathrm{SV}=2$

Ismembrane protein 188 OS=Salmo salar GN=TM188 PE=2 SV=1

ribosomal protein L21 OS=Epinephelus coioides $\mathrm{PE}=2 \mathrm{SV}=1$

:tin-capping protein subunit beta $0 \mathrm{~S}=\mathrm{Salmo}$ salar $\mathrm{GN}=\mathrm{CAPZB} \mathrm{PE}=2 \mathrm{SV}=1$

:038 protein 0S=Danio rerio GN=L0C51123 PE=2 SV=1

somal protein L14 OS=Solea senegalensis GN=RPL14 PE=2 SV=1

eoside diphosphate kinase (Fragment) $0 \mathrm{~S}=$ Psetta maxima $\mathrm{PE}=2 \mathrm{SV}=1$

;one deacetylase complex subunit SAP18 OS=Anoplopoma fimbria GN=SAP18 PE=2 SV=1

-itin $0 \mathrm{~S}=$ Psetta maxima $\mathrm{GN}=\mathrm{Fer} 1 \mathrm{PE}=2 \mathrm{SV}=1$

$\$ 1$ protein similar to human and mouse ubiquitin specific peptidase 39 (USP39) OS=[

ribosomal protein L28 0S=Platichthys flesus GN=rp128 PE=2 SV=1

lase phosphoprotein 6 OS=Salmo salar GN=MPH6 PE=2 SV=1

ribosomal protein $\mathrm{S} 24$ OS=Pagrus major $\mathrm{PE}=2 \mathrm{SV}=1$

smosome undetermined SCAF11373, whole genome shotgun sequence. (Fragment) OS=Tetra

153686 OS=Danio rerio $\mathrm{GN}=\mathrm{ubc} \mathrm{PE}=2 \mathrm{SV}=1$

somal protein $\mathrm{S} 2$ OS=Solea senegalensis $\mathrm{GN}=\mathrm{rpS} 2 \mathrm{PE}=2 \mathrm{SV}=1$

somal protein $\mathrm{S} 25 \mathrm{OS}=\mathrm{Solea}$ senegalensis $\mathrm{GN}=\mathrm{rpS} 25 \mathrm{PE}=2 \mathrm{SV}=1$

66168 protein (Fragment) $0 \mathrm{~S}=$ Danio rerio $\mathrm{GN}=\mathrm{rps} 27 \mathrm{a} \mathrm{PE}=2 \mathrm{SV}=1$

Igation factor 1 -delta $0 \mathrm{~S}=$ Anoplopoma fimbria $\mathrm{GN}=\mathrm{EF} 1 \mathrm{D} \quad \mathrm{PE}=2 \mathrm{SV}=1$

ssomal protein L26 OS=Solea senegalensis GN=RPL26 PE=2 SV=1

ribosomal protein $\mathrm{S} 12$ 0S=Solea senegalensis $\mathrm{GN}=\mathrm{rpS} 12 \mathrm{PE}=2 \mathrm{SV}=1$

phosphatase (Fragment) OS=Tetraodon nigroviridis GN=GSTENG00003377001 PE=3 SV=1

mosome undetermined SCAF7267, whole genome shotgun sequence. (Fragment) OS=Tetrac -beta-inducible nuclear protein 1 OS=0smerus mordax GN=TIP1 PE=2 SV=1

0613-1ike 0S=Danio rerio GN=gn131 PE=2 SV=1

mosome 13 SCAF14555, whole genome shotgun sequence OS=Tetraodon nigroviridis GN=C. chondrial cytochrome $\mathrm{C}$ oxidase subunit $\mathrm{Vb}$ (Fragment) $0 \mathrm{~S}=$ Epinephelus coioides $\mathrm{PE}=\check{C}$

1 nuclear ribonucleoprotein Sm D2 OS=Anoplopoma fimbria GN=SMD2 PE=4 SV=1 ear nucleic acid-binding protein C1D OS=Anoplopoma fimbria GN=C1D PE=2 SV=1

mosome undetermined SCAF14724, whole genome shotgun sequence OS=Tetraodon nigrovi stem-1oop-interacting RNA-binding protein, mitochondrial OS=Anoplopoma fimbria GN 
mosome undetermined SCAF7571, whole genome shotgun sequence. (Fragment) 0S=Tetrac i2 protein homolog $0 \mathrm{~S}=$ Salmo salar GN=BCAS2 $\mathrm{PE}=2 \mathrm{SV}=1$

th hormone-inducible transmembrane protein $0 \mathrm{~S}=0$ smerus $\operatorname{mordax} \mathrm{GN}=\mathrm{GHITM} \mathrm{PE}=2 \mathrm{SV}=1$

-binding nuclear protein Ran OS=Anoplopoma fimbria GN=RAN PE=2 SV=1

', member RAS oncogene family OS=Danio rerio GN=rab7 $\mathrm{PE}=2 \mathrm{SV}=1$

redoxin domain-containing protein 9 OS=Anoplopoma fimbria GN=TXND9 PE=2 SV=1

mosome undetermined SCAF15069, whole genome shotgun sequence OS=Tetraodon nigrovi

j0A OS=Anoplopoma fimbria GN=FA60A PE=2 SV=1

,56 (Fragment) 0S=0reochromis mossambicus $\mathrm{PE}=2 \mathrm{SV}=1$

;in light polypeptide 6 OS=Anoplopoma fimbria GN=MYL6 PE=2 SV=1

ationally-controlled tumor protein homolog 0S=Psetta maxima GN=tpt1 PE=2 SV=1

ribosomal protein $\mathrm{S} 11$ OS=Psetta maxima $\mathrm{PE}=2 \mathrm{SV}=1$

ismembrane protein 50A OS=Anoplopoma fimbria GN=TM50A PE=2 SV=1

I dehydrogenase 1 beta subcomplex subunit 5, mitochondrial OS=Anoplopoma fimbria C. le-interacting protein of $10 \mathrm{kDa}$ OS=Danio rerio GN=bbip10 $\mathrm{PE}=2 \mathrm{SV}=1$

ribosomal protein $\mathrm{S} 8$ OS=Anoplopoma fimbria GN=RS8 PE=2 SV=1

${ }^{\prime}$ S peroxiredoxin 4 OS=Seriola lalandi GN=PRDX PE=2 SV=1

I dehydrogenase 1 alpha subcomplex subunit 9, mitochondrial OS=Anoplopoma fimbria or suppressor candidate 2 OS=Anoplopoma fimbria GN=TUSC2 PE=4 SV=1

:le-specific beta 1 integrin binding protein 2 OS=Epinephelus coioides $\mathrm{PE}=2 \mathrm{SV}=1$

mosome 3 SCAF15037, whole genome shotgun sequence. (Fragment) OS=Tetraodon nigrov mosome 15 SCAF14992, whole genome shotgun sequence. (Fragment) OS=Tetraodon nigrc igation factor 2 OS=Salmo salar $\mathrm{GN}=\mathrm{EF} 2 \mathrm{PE}=2 \mathrm{SV}=1$

athione peroxidase $0 \mathrm{~S}=$ Thunnus maccoyii $\mathrm{PE}=2 \mathrm{SV}=2$

mosome 2 SCAF14990, whole genome shotgun sequence. (Fragment) OS=Tetraodon nigrov mosome 6 SCAF14737, whole genome shotgun sequence OS=Tetraodon nigroviridis GN=G؟

mosome 14 SCAF14645, whole genome shotgun sequence. (Fragment) OS=Tetraodon nigrc mosome 13 SCAF14566, whole genome shotgun sequence. (Fragment) OS=Tetraodon nigrc

mosome 6 SCAF14768, whole genome shotgun sequence OS=Tetraodon nigroviridis GN=Gई somal protein L19 OS=Anoplopoma fimbria GN=RL19 PE=2 SV=1

roxide dismutase $[\mathrm{Cu}-\mathrm{Zn}]$ OS=Rachycentron canadum GN=S0D1 PE=2 SV=1

mosome 3 SCAF14978, whole genome shotgun sequence. (Fragment) OS=Tetraodon nigrov i2A-1ike 0S=0smerus mordax GN=FA32A PE=4 SV=1

mosome undetermined SCAF14696, whole genome shotgun sequence. (Fragment) OS=Tetra ribosomal protein L9 0S=Platichthys flesus GN=rp19 PE=2 SV=1

mosome 3 SCAF15018, whole genome shotgun sequence OS=Tetraodon nigroviridis GN=Gई athione peroxidase $0 \mathrm{~S}=$ Danio rerio $\mathrm{GN}=\mathrm{gpx} 4 \mathrm{~b} \mathrm{PE}=2 \mathrm{SV}=1$

ged multivesicular body protein 3 OS=0smerus mordax GN=CHMP3 PE=2 SV=1 
ribosomal protein $\mathrm{S} 15 \mathrm{~A}$ 0S=Paralichthys olivaceus $\mathrm{PE}=2 \mathrm{SV}=1$

rmerase (RNA) II (DNA directed) polypeptide E OS=Danio rerio GN=polr2eb PE=2 SV=1

mobox protein homolog 3 OS=Anoplopoma fimbria GN=CBX3 PE=2 SV=1

-itin $0 \mathrm{~S}=$ Psetta maxima $\mathrm{GN}=\mathrm{FerM} \mathrm{PE}=2 \mathrm{SV}=1$

la-aminobutyric acid receptor-associated protein-1ike 1 OS=0smerus mordax GN=GRL1

mosome 2 SCAF15106, whole genome shotgun sequence. (Fragment) OS=Tetraodon nigrov omer subunit zeta-1 OS=Anoplopoma fimbria GN=COPZ1 PE=2 SV=1

motif-containing protein 2 OS=0ncorhynchus mykiss GN=LYRM2 PE=4 SV=1

:1 protein similar to vertebrate CD151 antigen (CD151) OS=Danio rerio GN=si:dkey-2 Ismembrane emp24 domain-containing protein 10 (Fragment) OS=Salmo salar GN=TMEDA F

mosome 3 SCAF15037, whole genome shotgun sequence 0S=Tetraodon nigroviridis GN=G؟ shock protein 90 OS=Psetta maxima GN=HSP90 PE=2 SV=1

cing factor arginine/serine-rich 3 OS=Paralichthys olivaceus $\mathrm{PE}=2 \mathrm{SV}=1$ somal protein $\mathrm{S} 16$ OS=Xenopus tropicalis $\mathrm{GN}=\operatorname{rps} 16 \mathrm{PE}=2 \mathrm{SV}=1$

เ OS=Thunnus thynnus orientalis $\mathrm{PE}=2 \mathrm{SV}=1$

j00 protein C5orf51 homolog 0S=Danio rerio GN=si:dkey-46a10. 3 PE=2 SV=1

somal protein L7-1ike 1 OS=Salmo salar GN=RL7L PE=2 SV=1

ribosomal protein S14 (Fragment) $0 \mathrm{~S}=P$ setta maxima $\mathrm{PE}=2 \mathrm{SV}=1$

ribosomal protein $\mathrm{S} 19$ (Fragment) $0 \mathrm{~S}=$ Epinephelus coioides $\mathrm{PE}=2 \mathrm{SV}=1$

mosome 4 SCAF15093, whole genome shotgun sequence OS=Tetraodon nigroviridis GN=GS

ssomal protein L6 OS=Solea senegalensis GN=RPL6 PE=2 SV=1

seronin containing TCP1 subunit 6A (Fragment) $0 \mathrm{~S}=\mathrm{Paralichthys}$ olivaceus GN=Cctz PE mosome 13 SCAF14627, whole genome shotgun sequence. (Fragment) 0S=Tetraodon nigrc rubiquitin $0 \mathrm{~S}=0$ ncorhynchus mykiss $\mathrm{PE}=2 \mathrm{SV}=1$

:A, member RAS oncogene family OS=Salmo salar GN=rab2a PE=2 SV=1

; homolog, mitochondrial OS=Anoplopoma fimbria GN=EMI5 PE=2 SV=1

loprotein $\mathrm{T} 1 \mathrm{a}$ OS=Danio rerio $\mathrm{GN}=\mathrm{selt1a} \mathrm{PE}=2 \mathrm{SV}=3$

mosome 13 SCAF15035, whole genome shotgun sequence OS=Tetraodon nigroviridis GN=C. itive uncharacterized protein (Fragment) OS=Mus musculus GN=2410042D21Rik PE=2 SV= mosome 1 SCAF14640, whole genome shotgun sequence. (Fragment) OS=Tetraodon nigrov I-ubiquinone oxidoreductase $75 \mathrm{kDa}$ subunit $0 \mathrm{~S}=$ Danio rerio $\mathrm{GN}=$ ndufs1 $\mathrm{PE}=2 \mathrm{SV}=1$

ribosomal protein L47, mitochondrial OS=Esox lucius GN=RM47 PE=2 SV=1

mosome 5 SCAF14581, whole genome shotgun sequence. (Fragment) 0S=Tetraodon nigrov iyltransferase WBSCR22 OS=Anoplopoma fimbria GN=WBS22 PE=2 SV=1

Iryotic translation initiation factor 3 subunit 12 OS=0ncorhynchus mykiss GN=IF3C mosome undetermined SCAF10875, whole genome shotgun sequence. (Fragment) OS=Tetra 'ATP translocase 2 OS=0 smerus mordax GN=ADT2 $\mathrm{PE}=2 \mathrm{SV}=1$

114204 OS=Danio rerio $\mathrm{GN}=$ golt1b $\mathrm{PE}=2 \mathrm{SV}=1$

ribosomal protein L16, mitochondrial OS=Anoplopoma fimbria GN=RM16 PE=2 SV=1

somal protein $\mathrm{S} 17$ OS=Solea senegalensis $\mathrm{GN}=\mathrm{rpS17} \mathrm{PE}=2 \mathrm{SV}=1$

sptor for activated protein kinase $\mathrm{C}$ OS=0reochromis mossambicus $\mathrm{PE}=2 \mathrm{SV}=1$

mosome undetermined SCAF15016, whole genome shotgun sequence OS=Tetraodon nigrovi

I box binding protein $0 \mathrm{~S}=$ Takifugu rubripes $\mathrm{PE}=2 \mathrm{SV}=1$ 
ia (Fragment) $0 \mathrm{~S}=$ Pagrus major $\mathrm{PE}=2 \mathrm{SV}=1$

;in regulatory light chain 2, smooth muscle isoform $0 \mathrm{~S}=$ Anoplopoma fimbria GN=MLRN 17656 protein $0 \mathrm{~S}=$ Xenopus tropicalis $\mathrm{GN}=\operatorname{cops} 2 \mathrm{PE}=2 \mathrm{SV}=1$

itive uncharacterized protein (Fragment) OS=Ailuropoda melanoleuca GN=PANDA_00695? mosome 9 SCAF14729, whole genome shotgun sequence OS=Tetraodon nigroviridis GN=GS iscription factor A, mitochondrial OS=Anoplopoma fimbria GN=TFAM PE=2 SV=1

:easome subunit beta type OS=Anoplopoma fimbria GN=PSB1A PE=2 SV=1

yotic translation initiation factor 3 subunit I OS=Danio rerio GN=eif3i $\mathrm{PE}=2 \mathrm{SV}=1$ srammed cell death 5 OS=Danio rerio GN=pdcd5 PE=2 SV=1

age-dependent anion channel OS=Paralichthys olivaceus GN=VDAC PE=2 SV=1

mosome 9 SCAF14729, whole genome shotgun sequence. (Fragment) OS=Tetraodon nigrov I dehydrogenase 1 beta subcomplex subunit 6 OS=Anoplopoma fimbria GN=NDUB6 PE=2 SV easome subunit beta type 0S=0smerus mordax $\mathrm{GN}=\mathrm{PSB} 3 \mathrm{PE}=2 \mathrm{SV}=1$

mosome undetermined SCAF13749, whole genome shotgun sequence OS=Tetraodon nigrovi mosome 1 SCAF14573, whole genome shotgun sequence. (Fragment) 0S=Tetraodon nigrov ator of RNA polymerase II transcription subunit 20 OS=Salmo salar GN=MED20 PE=2 $s$ somal protein L32 OS=Epinephelus coioides $\mathrm{PE}=2 \mathrm{SV}=1$

144845 protein (Fragment) $0 \mathrm{~S}=$ Xenopus laevis GN=LOC444845 PE=2 SV=1

mosome 16 SCAF15002, whole genome shotgun sequence. (Fragment) 0S=Tetraodon nigrc easome subunit alpha type OS=Anoplopoma fimbria GN=PSA3 PE=2 SV=1

mosome 11 SCAF14528, whole genome shotgun sequence. (Fragment) 0S=Tetraodon nigrc ular nucleic acid-binding protein $0 \mathrm{~S}=0$ smerus mordax $\mathrm{GN}=\mathrm{CNBP} \mathrm{PE}=2 \mathrm{SV}=1$

mosome 1 SCAF15008, whole genome shotgun sequence. (Fragment) OS=Tetraodon nigrov

i08 protein C19orf42 homolog OS=Siniperca chuatsi PE=3 SV=1

proteasome non-ATPase regulatory subunit 7 like 2 OS=Salmo salar $\mathrm{PE}=2 \mathrm{SV}=1$

shrome c oxidase subunit VIb OS=Scombridae gen. sp. $\mathrm{PE}=4 \mathrm{SV}=1$

iscription elongation factor SPT4 $0 \mathrm{~S}=$ Anoplopoma fimbria GN=SPT4H PE=4 SV=1

mosome 1 SCAF14573, whole genome shotgun sequence. (Fragment) OS=Tetraodon nigrov mosome 12 SCAF15104, whole genome shotgun sequence OS=Tetraodon nigroviridis $\mathrm{GN}=C$. 1 mobility group protein B2 OS=Anoplopoma fimbria GN=HMGB2 PE=2 SV=1

mosome 18 SCAF14712, whole genome shotgun sequence OS=Tetraodon nigroviridis GN=C. 5 homolog OS=Salmo salar GN=CWC15 PE=2 SV=1

or suppressor candidate $4 \mathrm{OS}=\mathrm{Salmo}$ salar $\mathrm{GN}=\mathrm{TUSC} 4 \mathrm{PE}=2 \mathrm{SV}=1$

mosome undetermined SCAF14653, whole genome shotgun sequence OS=Tetraodon nigrovi 123194 OS=Danio rerio GN=zgc: $123194 \mathrm{PE}=2 \mathrm{SV}=1$

:easome activator complex subunit 3 OS=Salmo salar GN=PSME3 PE=2 SV=1

-dependent RNA helicase DDX39 (Fragment) OS=Salmo salar GN=DDX39 PE=2 SV=1

? decapping enzyme homolog (S. cerevisiae) OS=Danio rerio $\mathrm{GN}=\mathrm{dcp} 2 \mathrm{PE}=2 \mathrm{SV}=1$

00170503 protein (Fragment) OS=Xenopus tropicalis GN=LOC100170503 PE=2 SV=1

12A1 OS=Anoplopoma fimbria GN=F92A1 PE=2 SV=1

mosome 2 SCAF14738, whole genome shotgun sequence. (Fragment) OS=Tetraodon nigrov chyl-diphosphooligosaccharide--protein glycosyltransferase subunit STT3A OS=Salmc

-directed RNA polymerases I, II, and III subunit RPABC5 OS=Anoplopoma fimbria GN=F 
;easome assembly chaperone 1 OS=Salmo salar GN=PSMG1 PE=2 SV=1

mosome 1 SCAF14995, whole genome shotgun sequence OS=Tetraodon nigroviridis GN=GS lig cell tumor $10 \mathrm{kDa}$ protein homolog OS=Anoplopoma fimbria GN=L10K $\mathrm{PE}=4 \mathrm{SV}=1$

mosome 1 SCAF14749, whole genome shotgun sequence OS=Tetraodon nigroviridis GN=GS iferation-associated protein $2 \mathrm{G} 4$ OS=Salmo salar $\mathrm{GN}=\mathrm{PA} 2 \mathrm{G} 4 \mathrm{PE}=2 \mathrm{SV}=1$

luitin-like protein $4 \mathrm{~A}$ OS=Anoplopoma fimbria GN=UBL4A PE=2 SV=1

athione $\mathrm{S}$-transferase $\mathrm{OS}=$ Epinephelus coioides $\mathrm{PE}=2 \mathrm{SV}=1$

I dehydrogenase 1 alpha subcomplex subunit 1 OS=Anoplopoma fimbria GN=NDUA1 PE=4 $s$ domain-containing protein 1 OS=Anoplopoma fimbria GN=RWDD1 PE=2 SV=1

lired for meiotic nuclear division 5 homolog B (S. cerevisiae) OS=Danio rerio $\mathrm{GN}=\mathrm{r}$ e/threonine-protein phosphatase $2 \mathrm{~A}$ regulatory subunit B' subunit gamma OS=Danio

gene DJ-1 OS=Maylandia sp. 'Kompakt Mbamba Bay' GN=Dj1 PE=2 SV=1

chondrial fission 1 protein $0 \mathrm{~S}=$ Anoplopoma fimbria GN=FIS1 $\mathrm{PE}=2 \mathrm{SV}=1$

Igation factor 1-gamma $0 \mathrm{~S}=0$ smerus mordax $\mathrm{GN}=\mathrm{EF} 1 \mathrm{G} \quad \mathrm{PE}=2 \quad \mathrm{SV}=1$

mosome 8 SCAF15044, whole genome shotgun sequence. (Fragment) OS=Tetraodon nigrov

92043 OS=Danio rerio GN=caprin1a $\mathrm{PE}=1 \mathrm{SV}=1$

rr membrane protein, mitochondrial (Mitofilin) OS=Danio rerio $\mathrm{GN}=i m m t \quad \mathrm{PE}=2 \mathrm{SV}=1$

chol phosphate-mannose biosynthesis regulatory protein $0 \mathrm{~S}=$ Anoplopoma fimbria $\mathrm{GN}=[$ ne/threonine-protein phosphatase $0 \mathrm{~S}=\mathrm{Salmo}$ salar $\mathrm{GN}=$ ppp1cc $\mathrm{PE}=2 \mathrm{SV}=1$

oldin subunit 6 OS=0ncorhynchus mykiss GN=PFD6 PE=2 SV=1

ribosomal protein L51, mitochondrial OS=Anoplopoma fimbria GN=RM51 PE=2 SV=1

lin-2 OS=Anoplopoma fimbria GN=COF2 $\mathrm{PE}=2 \mathrm{SV}=1$

amine synthetase $0 \mathrm{~S}=0$ psanus beta $\mathrm{GN}=\mathrm{GSase} \mathrm{PE}=2 \mathrm{SV}=1$

juitin carrier protein $0 \mathrm{~S}=\mathrm{Salmo}$ salar $\mathrm{GN}=\mathrm{UBC} 9 \mathrm{PE}=2 \mathrm{SV}=1$

1414 transmembrane protein C20orf30 homolog OS=Anoplopoma fimbria GN=CT030 PE=4 SV

-mRNA-processing factor 19 OS=Salmo salar GN=PRP19 PE=2 SV=1

ike protein $0 \mathrm{~S}=\mathrm{Sc}$ iaenops ocellatus $\mathrm{GN}=\mathrm{QM} \quad \mathrm{PE}=2 \mathrm{SV}=1$

ribosomal protein L8 OS=0

mosome 13 SCAF14555, whole genome shotgun sequence. (Fragment) OS=Tetraodon nigrc synthase subunit $\mathrm{g}$, mitochondrial $0 \mathrm{~S}=$ Anoplopoma fimbria GN=ATP5L PE=4 SV=1

lenosylmethionine synthase OS=Tetraodon nigroviridis GN=GSTENG00029452001 PE=3 SV= 1527 membrane protein OS=Esox lucius GN=U527 PE=2 SV=1

mosome 21 SCAF14577, whole genome shotgun sequence OS=Tetraodon nigroviridis GN=C. :ptor for activated protein kinase C (Fragment) OS=Psetta maxima PE=2 SV=1

Iine phosphoribosyl transferase (Fragment) OS=Psetta maxima $\mathrm{PE}=2 \mathrm{SV}=1$

: (Fragment) 0S=0ryzias latipes $\mathrm{PE}=4 \mathrm{SV}=1$

mosome 8 SCAF14543, whole genome shotgun sequence. (Fragment) 0S=Tetraodon nigrov mosome 14 SCAF14660, whole genome shotgun sequence $0 S=$ Tetraodon nigroviridis $\mathrm{GN}=C$ chyl-diphosphooligosaccharide--protein glycosyltransferase subunit DAD1 OS=0smert eophosmin (Fragment) $0 \mathrm{~S}=\mathrm{Salmo}$ salar $\mathrm{GN}=\mathrm{NPM} \mathrm{PE}=2 \mathrm{SV}=1$

$\checkmark$ tubulin $0 \mathrm{~S}=$ Notothenia coriiceps $\mathrm{PE}=2 \mathrm{SV}=1$

I dehydrogenase 1 beta subcomplex subunit 8 , mitochondrial OS=Anoplopoma fimbria $C$ :11 receptor-associated protein 29 OS=0smerus mordax GN=BAP29 PE=2 SV=1

in saal1 $0 \mathrm{~S}=$ Danio rerio $\mathrm{GN}=$ saal1 $\mathrm{PE}=2 \mathrm{SV}=2$ 
mplex protein 1 subunit alpha (Fragment) OS=Tetraodon nigroviridis GN=GSTENG0002? itive uncharacterized protein $0 \mathrm{~S}=$ Volvox carteri $\mathrm{f}$. nagariensis GN=VOLCADRAFT_9388C Igation factor 1 -beta $0 \mathrm{~S}=\mathrm{Salmo}$ salar $\mathrm{GN}=\mathrm{EF} 1 \mathrm{~B} \mathrm{PE}=2 \mathrm{SV}=1$ redoxin, mitochondrial $0 \mathrm{~S}=\mathrm{Salmo}$ salar GN=THIOM $\mathrm{PE}=2 \mathrm{SV}=1$

in $3 \mathrm{OS}=$ Danio rerio $\mathrm{GN}=\mathrm{cul} 3 \mathrm{a} \mathrm{PE}=2 \mathrm{SV}=1$

iferation-associated protein 2G4 (Fragment) $0 \mathrm{~S}=\mathrm{Salmo}$ salar GN=PA2G4 PE=2 SV=1 mosome $4 \mathrm{SCAF} 14752$, whole genome shotgun sequence $0 \mathrm{~S}=$ Tetraodon nigroviridis $\mathrm{GN}=\mathrm{G} S$ :ted in azoospermia-1ike protein $0 \mathrm{~S}=$ Lates calcarifer $\mathrm{GN}=$ dazl $\mathrm{PE}=2 \mathrm{SV}=1$

mosome 7 SCAF14536, whole genome shotgun sequence OS=Tetraodon nigroviridis $\mathrm{GN}=G_{S}^{S}$ :easome subunit alpha type $0 \mathrm{~S}=$ Danio rerio $\mathrm{GN}=\mathrm{psma} 2 \mathrm{PE}=2 \mathrm{SV}=1$ Ismembrane emp24 domain-containing protein 2 OS=Esox lucius GN=TMED2 PE=2 SV=1 10179 OS=0smerus mordax GN=K0179 PE=2 SV=1 isomal protein L5 OS=Solea senegalensis GN=RPL5 PE=2 SV=1 synthase subunit beta (Fragment) $0 \mathrm{~S}=\mathrm{Gilli}$ ichthys seta $\mathrm{PE}=2 \mathrm{SV}=1$

somal protein $0 \mathrm{~S}=$ Solea senegalensis $\mathrm{GN}=\mathrm{RPL} 10 \mathrm{a} \mathrm{PE}=2 \mathrm{SV}=1$ Im channel modifier 1 OS=Danio rerio $\mathrm{GN}=\mathrm{Scnm} 1 \mathrm{PE}=2 \mathrm{SV}=1$ 163141 protein $0 \mathrm{~S}=$ Danio rerio $\mathrm{GN}=\mathrm{zgc}: 163141 \mathrm{PE}=2 \mathrm{SV}=1$ ribosomal protein L5 OS=0smerus mordax GN=RL5 PE=2 SV=1

00158386 protein $0 \mathrm{~S}=$ Xenopus laevis GN=sfrs12ip1 PE=2 SV=1

sine-specific methyltransferase 0S=Xiphophorus maculatus $x$ Xiphophorus helleri GN -specific GTPase-activating protein OS=Anoplopoma fimbria GN=RANG PE=2 SV=1 lyltransferase- 1 ike protein 5 OS=Salmo salar GN=METL5 PE=2 SV=1 ıspanin-31-B OS=Xenopus laevis GN=tspan31-b PE=2 SV=1 some maturation protein SBDS OS=Anoplopoma fimbria GN=SBDS PE=2 SV=1

; $\mathrm{JC}$ protein $0 \mathrm{~S}=$ Tetraodon nigroviridis $\mathrm{GN}=\mathrm{H} 01 \mathrm{CJC}$ PE $=2 \mathrm{SV}=1$

r1-tRNA synthetase OS=Danio rerio GN=kars $\mathrm{PE}=2 \mathrm{SV}=1$

mosome undetermined SCAF7120, whole genome shotgun sequence OS=Tetraodon nigrovir -37 homolog OS=Esox lucius GN=LIN37 PE=2 SV=1

mosome 13 SCAF15000, whole genome shotgun sequence. (Fragment) OS=Tetraodon nigrc :omer subunit epsilon $0 \mathrm{~S}=$ Anoplopoma fimbria $\mathrm{GN}=\mathrm{COPE} \mathrm{PE}=2 \mathrm{SV}=1$

i-box protein 2 OS=0ncorhynchus mykiss $\mathrm{GN}=\mathrm{RBX} 2 \mathrm{PE}=4 \mathrm{SV}=1$

.12-1ike protein $0 \mathrm{~S}=$ Anoplopoma fimbria GN=TR112 PE=2 SV=1

mosome 9 SCAF15033, whole genome shotgun sequence OS=Tetraodon nigroviridis GN=GS al peptidase complex subunit 3 OS=Salmo salar GN=SPCS3 PE=2 SV=1

Iral killer enhancing factor $0 \mathrm{~S}=$ Psetta maxima $\mathrm{PE}=2 \mathrm{SV}=1$

mosome 12 SCAF14999, whole genome shotgun sequence. (Fragment) OS=Tetraodon nigrc

oldin subunit 4 OS=Anoplopoma fimbria GN=PFD4 PE=2 SV=1

mosome 11 SCAF14979, whole genome shotgun sequence. (Fragment) 0S=Tetraodon nigrc : finger protein 183 OS=0ryzias latipes $\mathrm{PE}=2 \mathrm{SV}=1$

ribosomal protein L30, mitochondrial OS=Anoplopoma fimbria GN=RM30 PE=2 SV=1 rival of motor neuron-related-splicing factor 30 OS=Anoplopoma fimbria GN=SPF30 PE itive RBX1 protein $0 \mathrm{~S}=$ Taeniopygia guttata $\mathrm{PE}=4 \mathrm{SV}=1$ 
proteasome non-ATPase regulatory subunit 13 OS=Anoplopoma fimbria GN=PSD13 PE=2 $\varsigma$ atin-B $0 \mathrm{~S}=$ Salmo salar $\mathrm{GN}=\mathrm{CYTB} \mathrm{PE}=4 \mathrm{SV}=1$

synthase $0 \mathrm{~S}=$ Danio rerio $\mathrm{GN}=$ ctps $\mathrm{PE}=3 \mathrm{SV}=1$

-directed RNA polymerase III subunit RPC6 OS=Salmo salar GN=RPC6 PE=2 SV=1

101107 OS=Danio rerio GN=zgc: 101107 PE=2 SV=1

tolar protein sorting-associated protein 33A OS=Salmo salar GN=VP33A PE=2 SV=1

losine kinase $0 \mathrm{~S}=0$ smerus mordax $\mathrm{GN}=\mathrm{ADK} \mathrm{PE}=2 \mathrm{SV}=1$

easome subunit alpha type (Fragment) OS=Tetraodon nigroviridis GN=GSTENG00028335C soclast-stimulating factor 1 OS=Salmo salar GN=0STF1 PE=2 SV=1

yotic translation initiation factor 3 subunit $\mathrm{L}$ OS=Danio rerio $\mathrm{GN}=\mathrm{e}$ if31 $\mathrm{PE}=2 \mathrm{SV}=1$ able tRNAHis guanylyltransferase 0S=Anoplopoma fimbria GN=THG1 PE=2 SV=1

lkey-13i19. 8 OS=Danio rerio $\mathrm{GN}=\mathrm{si}: \mathrm{dkey}-13 \mathrm{i} 19.8 \mathrm{PE}=4 \mathrm{SV}=1$

synthase, $\mathrm{H}+$ transporting, mitochondrial F0 complex, subunit d OS=Danio rerio $\mathrm{GN}=$

1 ubiquitin-related modifier 2 OS=Anoplopoma fimbria GN=SUM02 PE=4 SV=1

ismembrane protein 147 OS=Salmo salar GN=TM147 PE=2 SV=1

Ismembrane BAX inhibitor motif-containing protein 4 OS=0smerus mordax GN=TMBI4 PE=

mosome undetermined SCAF11566, whole genome shotgun sequence. (Fragment) OS=Tetra mosome 21 SCAF7061, whole genome shotgun sequence OS=Tetraodon nigroviridis $\mathrm{GN}=\mathrm{G} S$

:tron transfer flavoprotein subunit alpha, mitochondrial OS=Anoplopoma fimbria GN= ribosomal protein L14, mitochondrial OS=Anoplopoma fimbria GN=RM14 PE=2 SV=1

division control protein 42 homolog OS=Anoplopoma fimbria GN=CDC42 PE=2 SV=1

ssomal protein $\mathrm{S} 6$ OS=Solea senegalensis $\mathrm{GN}=\mathrm{rpS} 6 \mathrm{PE}=2 \mathrm{SV}=1$

Iscription initiation factor TFIID subunit 11 OS=0smerus mordax GN=TAF11 PE=2 SV=1 xiredoxin 6 OS=Psetta maxima GN=Prx6 $\mathrm{PE}=2 \mathrm{SV}=1$

idyl-prolyl cis-trans isomerase OS=Salmo salar GN=PPIL3 PE=2 SV=1

ribosomal protein $\mathrm{S} 17$ OS=Salmo salar GN=RS17 $\mathrm{PE}=2 \mathrm{SV}=1$

licted protein 0S=Chlamydomonas reinhardtii GN=CHLREDRAFT_155068 PE=4 SV=1

able protein BRICK1 OS=Anoplopoma fimbria GN=BRK1 PE=4 SV=1

mosome undetermined SCAF14653, whole genome shotgun sequence OS=Tetraodon nigrovi :ose-regulated protein 78kDa (Fragment) OS=0ncorhynchus mykiss GN=GRP78 PE=2 SV=1

shock protein 60 OS=Larimichthys crocea $\mathrm{GN}=\mathrm{Hsp} 60 \mathrm{PE}=4 \mathrm{SV}=1$

division cycle 5 -1ike protein $0 \mathrm{~S}=\mathrm{Salmo}$ salar $\mathrm{GN}=\mathrm{CDC5L} \mathrm{PE}=2 \mathrm{SV}=1$

1 EDRK-rich factor 2 OS=Anoplopoma fimbria GN=SERF2 PE=4 SV=1

1 in cofactor a $0 \mathrm{~S}=$ Danio rerio $\mathrm{GN}=$ tbca $\mathrm{PE}=2 \mathrm{SV}=1$

:1 protein (Fragment) OS=Danio rerio GN=prmt1 $\mathrm{PE}=2 \mathrm{SV}=1$

mosome 1 SCAF14995, whole genome shotgun sequence 0S=Tetraodon nigroviridis GN=G؟ 35128 protein $0 \mathrm{~S}=$ Xenopus laevis $\mathrm{GN}=\mathrm{txn} 14$ a $\mathrm{PE}=2 \mathrm{SV}=1$

rogeneous nuclear ribonucleoprotein A/B OS=Salmo salar GN=ROAA PE=2 SV=1

cesome-associated factor 61 OS=Danio rerio $\mathrm{GN}=\mathrm{sf} 3 \mathrm{a} 3 \mathrm{PE}=2 \mathrm{SV}=1$

mosome 7 SCAF14703, whole genome shotgun sequence. (Fragment) 0S=Tetraodon nigrov mosome 13 SCAF14555, whole genome shotgun sequence. (Fragment) OS=Tetraodon nigrc eit locus protein 5 OS=0smerus mordax GN=SURF5 PE=2 SV=1

${ }^{r} \mathrm{C}$-binding protein $0 \mathrm{~S}=$ Anoplopoma fimbria $\mathrm{GN}=\mathrm{MYCBP} \mathrm{PE}=4 \mathrm{SV}=1$

synthase-coupling factor 6 , mitochondrial $0 \mathrm{~S}=$ Anoplopoma fimbria GN=ATP5J PE=3 SV=

mosome 7 SCAF14703, whole genome shotgun sequence. (Fragment) OS=Tetraodon nigrov rredoxin-dependent peroxide reductase, mitochondrial OS=Anoplopoma fimbria GN=PRDX 
chondrial 28S ribosomal protein S21 OS=Anoplopoma fimbria GN=RT21 PE=4 SV=1 mosome 17 SCAF14760, whole genome shotgun sequence. (Fragment) OS=Tetraodon nigrc ribosomal protein $\mathrm{S} 31$, mitochondrial $0 \mathrm{~S}=$ Salmo salar GN=RT31 $\mathrm{PE}=2 \mathrm{SV}=1$

Ir suppressor candidate 2 OS=Anoplopoma fimbria GN=TUSC2 PE=4 SV=1 protease regulatory subunit 8 OS=Anoplopoma fimbria GN=PRS 8 PE=2 SV=1 mosome 8 SCAF15044, whole genome shotgun sequence. (Fragment) 0S=Tetraodon nigrov itricopeptide repeat-containing protein 2 OS=Danio rerio GN=ptcd2 $\mathrm{PE}=2 \mathrm{SV}=1$ iryotic translation initiation factor $1 \mathrm{~A}, \mathrm{X}$-chromosomal $0 \mathrm{~S}=$ Anoplopoma fimbria GN=I ;enesis of 1 ysosome-related organelles complex-1 subunit 1 OS=Anoplopoma fimbria $C$ mosome 11 SCAF14979, whole genome shotgun sequence $0 \mathrm{~S}=$ Tetraodon nigroviridis $\mathrm{GN}=\mathrm{C}$ redoxin $0 \mathrm{~S}=\mathrm{Salmo}$ salar $\mathrm{GN}=\mathrm{THIO} \mathrm{PE}=4 \mathrm{SV}=1$

able signal peptidase complex subunit 2 OS=Anoplopoma fimbria GN=SPCS2 PE=2 SV=1

itive uncharacterized protein (Fragment) OS=Ailuropoda melanoleuca GN=PANDA_005916 ication protein A $32 \mathrm{kDa}$ subunit $0 \mathrm{~S}=$ Anoplopoma fimbria GN=RFA2 PE=2 SV=1

Iscription factor Dp-1 OS=Larimichthys crocea $\mathrm{PE}=2 \mathrm{SV}=1$

mosome 2 SCAF14781, whole genome shotgun sequence $0 \mathrm{~S}=$ Tetraodon nigroviridis $\mathrm{GN}=\mathrm{G} S$ :ilin OS=Tetraodon nigroviridis GN=GSTENG00007244001 PE=3 SV=1

:11 leukemia translocation-altered gene protein homolog OS=Anoplopoma fimbria GN=T

idine triad nucleotide-binding protein 1 OS=Anoplopoma fimbria GN=HINT1 PE=2 SV=1 I dehydrogenase iron-sulfur protein 7, mitochondrial OS=Anoplopoma fimbria GN=NDUS ;tallin J1A 0S=Salmo salar GN=CRJ1A PE=2 SV=1

luitin carrier protein $\mathrm{OS}=\mathrm{Salmo}$ salar $\mathrm{GN}=\mathrm{UBE} 2 \mathrm{~B} \quad \mathrm{PE}=2 \mathrm{SV}=1$

redoxin $0 \mathrm{~S}=$ Anoplopoma fimbria $\mathrm{GN}=\mathrm{THIO} \mathrm{PE}=4 \mathrm{SV}=1$

isomal protein $\mathrm{S} 7 \mathrm{OS}=\mathrm{Solea}$ senegalensis $\mathrm{GN}=\mathrm{rpS} 7 \mathrm{PE}=2 \mathrm{SV}=1$

mosome 17 SCAF14563, whole genome shotgun sequence. (Fragment) OS=Tetraodon nigrc ;mal1 nucleolar RNA-interacting protein 2 OS=0smerus mordax GN=U3IP2 PE=2 SV=1

juitin carrier protein $0 \mathrm{~S}=$ Anoplopoma fimbria $\mathrm{GN}=\mathrm{UBE} 2 \mathrm{~A} \quad \mathrm{PE}=2 \mathrm{SV}=1$

idylprolyl isomerase (Cyclophilin)-1ike 4 OS=Danio rerio GN=ppi14 $\mathrm{PE}=2 \mathrm{SV}=1$

yotic translation initiation factor 3 subunit D OS=Danio rerio GN=eif3d PE=2 SV=1 mosome 18 SCAF14712, whole genome shotgun sequence. (Fragment) OS=Tetraodon nigrc able palmitoyltransferase ZDHHC4 OS=Anoplopoma fimbria GN=ZDHC4 PE=2 SV=1

death-regulatory protein $0 \mathrm{~S}=P$ setta maxima $\mathrm{PE}=2 \mathrm{SV}=2$

mosome 11 SCAF14479, whole genome shotgun sequence. (Fragment) OS=Tetraodon nigrc mosome 14 SCAF15003, whole genome shotgun sequence. (Fragment) OS=Tetraodon nigrc synthase gamma chain $0 \mathrm{~S}=$ Danio rerio $\mathrm{GN}=\mathrm{atp} 5 \mathrm{c} 1 \mathrm{PE}=2 \mathrm{SV}=1$

polymerase B transcription factor 3 (Fragment) $0 \mathrm{~S}=$ Psetta maxima $\mathrm{PE}=2 \mathrm{SV}=1$

eolar protein 5 OS=Salmo salar GN=NOL5 $\mathrm{PE}=2 \mathrm{SV}=1$

mosome 18 SCAF15027, whole genome shotgun sequence OS=Tetraodon nigroviridis GN=C. mosome $15 \mathrm{SCAF} 14556$, whole genome shotgun sequence. (Fragment) 0S=Tetraodon nigrc 1 nuclear ribonucleoprotein G OS=Anoplopoma fimbria GN=RUXG PE=4 SV=1

rdrolipoyl dehydrogenase $0 \mathrm{~S}=$ Danio rerio $\mathrm{GN}=\mathrm{d} 1 \mathrm{dh} \mathrm{PE}=2 \mathrm{SV}=1$

repsin B OS=Paralichthys olivaceus $\mathrm{PE}=2 \mathrm{SV}=1$

bonucleoside 5'-monophosphate $\mathrm{N}$-glycosidase $0 \mathrm{~S}=$ Tetraodon nigroviridis $\mathrm{GN}=\mathrm{rcl} \mathrm{PE}=3$ :yuridine 5-triphosphate nucleotidohydrolase, mitochondrial OS=Anoplopoma fimbria -ribosylation factor-like protein 1 OS=0smerus mordax GN=ARL1 PE=2 SV=1

tive uncharacterized protein $0 \mathrm{~S}=$ Gallus gallus GN=RCJMB04_19p6 PE=2 SV=1

-like protein 1 OS=Anoplopoma fimbria GN=0RML1 PE=2 SV=1

;easome subunit beta type $0 \mathrm{~S}=$ Danio rerio $\mathrm{GN}=\mathrm{psmb} 2 \mathrm{PE}=2 \mathrm{SV}=1$

eronin subunit 7 (Fragment) $0 \mathrm{~S}=$ Pagrus major $\mathrm{PE}=2 \mathrm{SV}=1$

mosome 12 SCAF14999, whole genome shotgun sequence OS=Tetraodon nigroviridis GN=C. 
luitin carrier protein (Fragment) OS=Danio rerio GN=ube2131 PE=2 SV=1

Igation factor 1-de1ta $0 \mathrm{~S}=$ Anoplopoma fimbria $\mathrm{GN}=\mathrm{EF} 1 \mathrm{D}$ PE=2 SV=1

ribosomal protein $\mathrm{S} 15$ OS=Salmo salar GN=RS15 $\mathrm{PE}=2 \mathrm{SV}=1$

16-binding protein $1 \mathrm{~A} 0 \mathrm{~S}=$ Anoplopoma fimbria $\mathrm{GN}=\mathrm{FKB} 1 \mathrm{~A} \quad \mathrm{PE}=3 \mathrm{SV}=1$

-directed RNA polymerase OS=Salmo salar GN=RPB2 PE=2 SV=1

mosome 21 SCAF13835, whole genome shotgun sequence. (Fragment) OS=Tetraodon nigrc lal recognition particle $9 \mathrm{kDa}$ protein $\mathrm{OS}=$ Danio rerio $\mathrm{GN}=\operatorname{srp} 9 \mathrm{PE}=2 \mathrm{SV}=1$

ictin subunit 6 OS=Salmo salar GN=DCTN6 PE=2 SV=1

-type zinc finger protein 1 OS=Esox lucius GN=ZFAN1 PE=2 SV=1

mosome 7 SCAF14536, whole genome shotgun sequence $0 \mathrm{~S}=$ Tetraodon nigroviridis GN=G؟ ribosomal protein L52, mitochondrial OS=Salmo salar GN=RM52 PE=2 SV=1

somal protein L19 0S=Solea senegalensis GN=RPL19-1ike2 PE=2 SV=1

-52 homolog 0S=Salmo salar GN=LIN52 PE=4 SV=1

mosome 19 SCAF15045, whole genome shotgun sequence. (Fragment) OS=Tetraodon nigrc mosome undetermined SCAF14653, whole genome shotgun sequence. (Fragment) 0S=Tetra mosome 21 SCAF15022, whole genome shotgun sequence. (Fragment) OS=Tetraodon nigrc

lal sequence receptor beta $0 \mathrm{~S}=\mathrm{Salmo}$ salar $\mathrm{GN}=\mathrm{Ssr} 2 \mathrm{PE}=2 \mathrm{SV}=1$

:hrome c oxidase subunit 4 isoform 1 , mitochondrial $0 S=$ Thunnus obesus $\mathrm{PE}=2 \mathrm{SV}=1$

irf24 homolog OS=0smerus mordax GN=CT024 PE=2 SV=1

incer of rudimentary homolog $0 \mathrm{~S}=$ Xenopus laevis $\mathrm{GN}=\mathrm{erh} \mathrm{PE}=3 \mathrm{SV}=1$

18 0S=Esox lucius GN=NEDD8 PE=4 SV=1

1-dihydrouridine synthase 4-like PP35 OS=Anoplopoma fimbria GN=DUS4L PE=2 SV=1

isport protein Sec61 subunit gamma $0 \mathrm{~S}=\mathrm{Salmo}$ salar $\mathrm{GN}=\mathrm{SC6} 6 \mathrm{G} \mathrm{PE}=4 \mathrm{SV}=1$

atrophy 3 protein homolog $0 \mathrm{~S}=$ Danio rerio $\mathrm{GN}=\mathrm{opa} 3 \mathrm{PE}=2 \mathrm{SV}=1$

ıctivator polypeptide $0 \mathrm{~S}=\mathrm{Salmo}$ salar $\mathrm{GN}=\mathrm{SAP}$ PE=2 SV=1

iitoyl-protein thioesterase 1 OS=Anoplopoma fimbria GN=PPT1 PE=2 SV=1

:1opmentally-regulated GTP-binding protein 2 OS=0smerus mordax GN=DRG2 PE=2 SV=1

:ficking protein particle complex subunit 4 OS=Anoplopoma fimbria GN=TPPC4 PE=2 SV mosome 6 SCAF14768, whole genome shotgun sequence. (Fragment) 0S=Tetraodon nigrov

mosome undetermined SCAF13628, whole genome shotgun sequence. (Fragment) OS=Tetra

396 OS=Rattus norvegicus GN=Rbbp4 PE=2 SV=1

mosome 4 SCAF14533, whole genome shotgun sequence OS=Tetraodon nigroviridis GN=GS

Iryotic translation initiation factor 3 subunit M OS=Anoplopoma fimbria GN=EIF3M $F$ ra kinase $\mathrm{B}$ OS=0ryzias latipes $\mathrm{GN}=$ aurkb $\mathrm{PE}=2 \mathrm{SV}=1$

-structural maintenance of chromosomes element 1 homolog OS=Anoplopoma fimbria GN= n related protein $2 / 3$ complex, subunit $40 \mathrm{~S}=$ Danio rerio $\mathrm{GN}=\operatorname{arpc} 41 \mathrm{PE}=2 \mathrm{SV}=1$

somal protein L4 (Fragment) $0 \mathrm{~S}=$ Pagrus major $\mathrm{PE}=2 \mathrm{SV}=1$

matin modifying protein $4 \mathrm{C}$ OS=Danio rerio $\mathrm{GN}=\mathrm{chmp} 4 \mathrm{c} \mathrm{PE}=2 \mathrm{SV}=1$

mosome 17 SCAF15006, whole genome shotgun sequence. (Fragment) OS=Tetraodon nigrc eophosmin $0 \mathrm{~S}=\mathrm{Salmo}$ salar $\mathrm{GN}=\mathrm{NPM} \mathrm{PE}=2 \mathrm{SV}=1$ 
mosome 21 SCAF15029, whole genome shotgun sequence. (Fragment) OS=Tetraodon nigrc

mosome undetermined SCAF14764, whole genome shotgun sequence. (Fragment) OS=Tetra ' dipeptidase 2 (Metallopeptidase M20 family) OS=Danio rerio $\mathrm{GN}=\mathrm{cndp} 2 \mathrm{PE}=2 \mathrm{SV}=1$

licted protein (Fragment) $0 \mathrm{~S}=$ Nematostella vectensis $\mathrm{GN}=\mathrm{v} 1 \mathrm{~g} 134024 \mathrm{PE}=4 \mathrm{SV}=1$ ear protein $\mathrm{Hcc}^{-1} \mathrm{OS}=$ Anoplopoma fimbria $\mathrm{GN}=\mathrm{HCC} 1 \mathrm{PE}=2 \mathrm{SV}=1$

c17c08 protein 0S=Danio rerio GN=wu: $f \mathrm{c} 17 \mathrm{c} 08 \mathrm{PE}=2 \mathrm{SV}=1$ Im paraoxonase/arylesterase 2 OS=Salmo salar GN=PON2 PE=2 SV=1 incer of yellow 2 transcription factor homolog OS=Esox lucius GN=ENY2 PE=4 SV=1 lenosylmethionine synthase OS=Salmo salar GN=METK2 PE=2 SV=1 mosome undetermined SCAF14764, whole genome shotgun sequence. (Fragment) OS=Tetra mosome undetermined SCAF13844, whole genome shotgun sequence OS=Tetraodon nigrovi isynthetase complex auxiliary component p43 OS=Anoplopoma fimbria GN=MCA1 PE=2 SV mosome undetermined SCAF15107, whole genome shotgun sequence. (Fragment) 0S=Tetra mosome 16 SCAF14974, whole genome shotgun sequence OS=Tetraodon nigroviridis GN=C mosome 15 SCAF14556, whole genome shotgun sequence $0 \mathrm{~S}=$ Tetraodon nigroviridis $\mathrm{GN}=\mathrm{C}$ iscription elongation factor B polypeptide 2 OS=Anoplopoma fimbria GN=ELOB PE=4 SV artite motif-containing protein 25 OS=Esox lucius GN=TRI25 PE=2 SV=1

inRNA-associated Sm-1ike protein LSm5 0S=Anoplopoma fimbria GN=LSM5 PE=4 SV=1 เa3-fucosyltransferase $0 \mathrm{~S}=$ Takifugu rubripes $\mathrm{GN}=$ fut9b $\mathrm{PE}=2 \mathrm{SV}=1$

i finger protein 170 OS=Salmo salar GN=RN170 PE=2 SV=1

osomal glutathione S-transferase 3 OS=Anoplopoma fimbria GN=MGST3 PE=2 SV=1

I signalosome complex subunit 6 OS=Anoplopoma fimbria GN=CSN6 PE=2 SV=1

bosomal protein $\mathrm{SA} 0 \mathrm{~S}=$ Sparus aurata $\mathrm{GN}=$ rpsa $\mathrm{PE}=2 \mathrm{SV}=2$

mosome 3 SCAF14679, whole genome shotgun sequence OS=Tetraodon nigroviridis GN=GS

mosome undetermined SCAF11646, whole genome shotgun sequence. (Fragment) 0S=Tetra ${ }^{-}$-sulfur assembly protein IscA-like 2, mitochondrial OS=0smerus mordax GN=ISAM2 PE

mosome 16 SCAF15002, whole genome shotgun sequence OS=Tetraodon nigroviridis GN=C. 1414 transmembrane protein C20orf30 homolog OS=Anoplopoma fimbria GN=CT030 PE=2 SV mocysteine tRNA activating factor $0 \mathrm{~S}=$ Takifugu rubripes $\mathrm{PE}=2 \mathrm{SV}=1$

antigen cytoplasmic tail-binding protein 2 OS=Anoplopoma fimbria GN=CD2B2 $\mathrm{PE}=2 \mathrm{SV}$ 1293 protein YOR006C 0S=0smerus mordax GN=Y0006 PE=2 SV=1

iscription initiation factor IIE subunit beta $0 \mathrm{~S}=0$ smerus mordax $\mathrm{GN}=\mathrm{T} 2 \mathrm{~EB}$ PE=2 SV=1

ator of RNA polymerase II transcription subunit 10 OS=Anoplopoma fimbria GN=MED1C :eine-rich PDZ-binding protein OS=Anoplopoma fimbria GN=CRIPT PE=4 SV=1 
ridis $\mathrm{GN}=\mathrm{GSTENG} 00038513001 \mathrm{PE}=4 \mathrm{SV}=1$

${ }^{I}=1$

;TENG00022568001 PE=4 SV=1

' $\mathrm{PE}=3 \mathrm{SV}=1$

$\therefore \mathrm{SV}=1$

viridis $\mathrm{GN}=\mathrm{GSTENG} 00029438001 \mathrm{PE}=4 \mathrm{SV}=1$

viridis GN=GSTENG00013131001 PE=4 SV=1

, $\mathrm{GN}=\operatorname{gnav} 1 \mathrm{PE}=2 \mathrm{SV}=1$

riridis GN=GSTENG00023873001 PE=4 SV=1

${ }^{\prime} \mathrm{E}=2 \quad \mathrm{SV}=1$ 


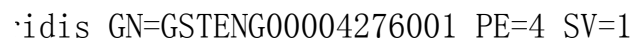

5.g $1 \quad \mathrm{PE}=3 \quad \mathrm{SV}=1$

riridis GN=GSTENG00017018001 PE=3 SV=1

lanio rerio $\mathrm{GN}=\mathrm{usp} 39 \mathrm{PE}=4 \mathrm{SV}=1$

เodon nigroviridis GN=GSTENG00009546001 PE=4 SV=1

don nigroviridis GN=GSTENG00004090001 PE=4 SV=1

iSTENG00015941001 PE=4 SV=1

: $\mathrm{SV}=1$

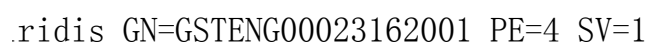

J=SLIRP $\quad \mathrm{PE}=4 \quad \mathrm{SV}=1$ 
don nigroviridis GN=GSTENG00004757001 PE=4 SV=1

ridis $\mathrm{GN}=\mathrm{GSTENG} 00034503001 \mathrm{PE}=3 \mathrm{SV}=1$

iN=NDUB5 $\quad$ PE=2 SV=1

GN=NDUA9 PE=2 SV=1

riridis GN=GSTENG00033388001 $\mathrm{PE}=3 \mathrm{SV}=1$ viridis $\mathrm{GN}=\mathrm{GSTENG} 00028388001 \mathrm{PE}=4 \mathrm{SV}=1$

riridis GN=GSTENG00027932001 $\mathrm{PE}=4 \mathrm{SV}=1$

;TENG00023858001 PE=4 SV=1

viridis GN=GSTENG00020229001 PE $=4$ SV=1

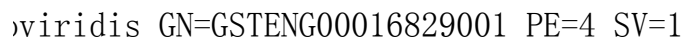

;TENG00025517001 PE=4 SV=1

riridis GN=GSTENG00027102001 $\mathrm{PE}=4 \mathrm{SV}=1$

เodon nigroviridis GN=GSTENG00021806001 PE=4 SV=1

;TENG00031888001 PE=4 SV=1 
$\mathrm{PE}=3 \quad \mathrm{SV}=1$

riridis GN=GSTENG00035051001 $\mathrm{PE}=4 \mathrm{SV}=1$

:1h14. $7 \mathrm{PE}=4 \mathrm{SV}=1$

'E $=2 \quad S V=1$

;TENG00033425001 PE=4 SV=1

;TENG00034700001 PE=4 SV=1

$i=2 \mathrm{SV}=1$

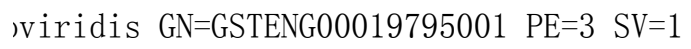

iSTENG00033341001 PE $=4 \quad \mathrm{SV}=1$

$=1$

riridis GN=GSTENG00020129001 $\mathrm{PE}=4 \mathrm{SV}=1$

riridis GN=GSTENG00017779001 $\mathrm{PE}=4 \mathrm{SV}=1$

$\mathrm{PE}=2 \quad \mathrm{SV}=1$

Iodon nigroviridis GN=GSTENG00009171001 PE=4 SV=1

ridis $\mathrm{GN}=\mathrm{GSTENG00031736001} \mathrm{PE}=4 \mathrm{SV}=1$ 
$\mathrm{PE}=2 \quad \mathrm{SV}=1$

; $\mathrm{PE}=4 \mathrm{SV}=1$

;TENG00023668001 PE=4 SV=1

riridis GN=GSTENG00023614001 $\mathrm{PE}=4 \mathrm{SV}=1$

${ }^{I}=1$

ridis $\mathrm{GN}=\mathrm{GSTENG} 00011891001 \mathrm{PE}=4 \mathrm{SV}=1$

riridis GN=GSTENG00017122001 $\mathrm{PE}=4 \mathrm{SV}=1$

; $=1$

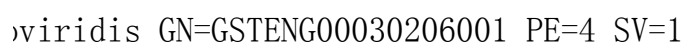

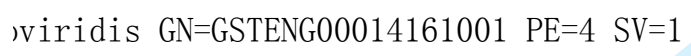

riridis GN=GSTENG00031216001 PE=4 SV=1

riridis GN=GSTENG00017123001 PE $=4 \quad \mathrm{SV}=1$

iSTENG00034902001 PE=4 SV=1

iSTENG00022692001 PE=4 SV=1

ridis $\mathrm{GN}=\mathrm{GSTENG00020488001} \mathrm{PE}=4 \mathrm{SV}=1$

riridis GN=GSTENG00024220001 PE=4 SV=1

, salar $\mathrm{GN}=\mathrm{STT} 3 \mathrm{~A} \mathrm{PE}=2 \mathrm{SV}=1$

PAB5 $\mathrm{PE}=3 \mathrm{SV}=1$ 


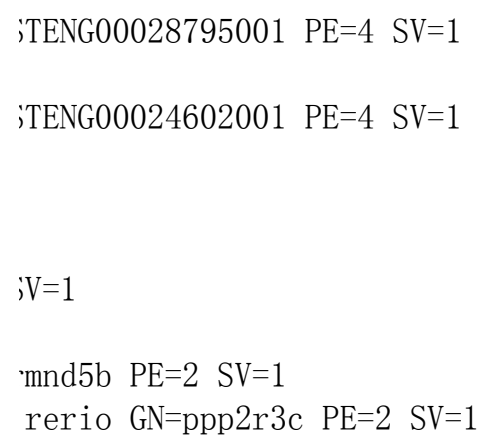

iN=NDUB8 PE=2 SV=1 
i111001 PE=3 SV=1

1) $\mathrm{PE}=4 \mathrm{SV}=1$

;TENG00024782001 PE=4 SV=1

;TENG00014630001 PE=4 SV=1

$\mathrm{I}=\mathrm{dnmt}-1 \quad \mathrm{PE}=2 \quad \mathrm{SV}=1$

·idis GN=GSTENG00003719001 PE=4 SV=1

viridis GN=GSTENG00029800001 PE=4 SV=1

;TENG00033260001 PE=4 SV=1

viridis GN=GSTENG00029514001 PE $=4$ SV=1

viridis GN=GSTENG00027582001 PE=3 SV=1

$i=2 \quad \mathrm{SV}=1$

https://mc06.manuscriptcentral.com/genome-pubs 
; $=1$

$101 \quad \mathrm{PE}=3 \quad \mathrm{SV}=1$

:atp5h PE=2 SV=1

$=2 \mathrm{SV}=1$

เodon nigroviridis GN=GSTENG00009758001 PE=4 SV=1 ;TENG00003506001 PE=4 SV=1

ETFA PE $=2 \quad \mathrm{SV}=1$

ridis $\mathrm{GN}=\mathrm{GSTENG} 00020465001 \mathrm{PE}=4 \mathrm{SV}=1$

;TENG00028899001 PE=4 SV=1

riridis GN=GSTENG00022244001 $\mathrm{PE}=4 \mathrm{SV}=1$ viridis GN=GSTENG00016036001 PE=4 SV=1

$=1$

riridis GN=GSTENG00022178001 PE $=4 \quad \mathrm{SV}=1$

(3) $\mathrm{PE}=2 \mathrm{SV}=1$ 


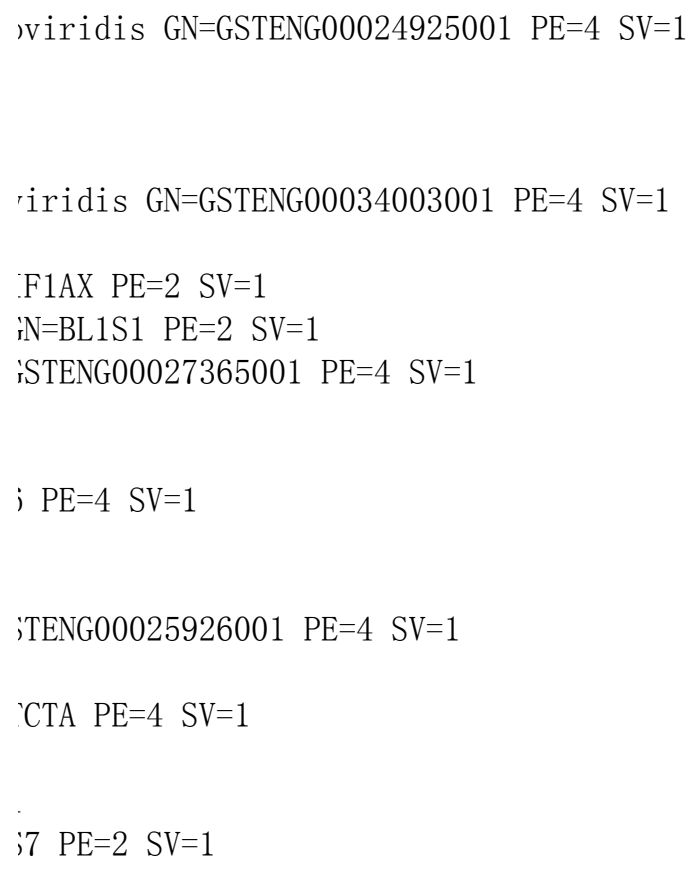


,viridis GN=GSTENG00012247001 PE=3 SV=1

;TENG00014695001 PE=4 SV=1

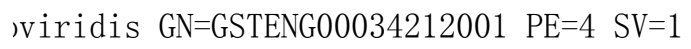

ıdon nigroviridis GN=GSTENG00020486001 PE $=4$ SV=1

viridis GN=GSTENG00032595001 PE $=4$ SV=1

$I=1$

riridis $\mathrm{GN}=\mathrm{GSTENG} 00025511001 \mathrm{PE}=4 \mathrm{SV}=1$

เodon nigroviridis GN=GSTENG00011542001 PE=4 SV=1

;TENG00014386001 PE=4 SV=1

'E $=2 \quad S V=1$

:NSE1 PE=2 SV=1

viridis GN=GSTENG00030881001 PE=4 SV=1 
viridis GN=GSTENG00033020001 PE=4 SV=1

เodon nigroviridis GN=GSTENG00025158001 PE=3 SV=1

เodon nigroviridis GN=GSTENG00025358001 PE $=4$ SV=1

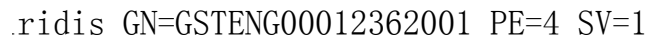

$r=1$ Iodon nigroviridis GN=GSTENG00035068001 PE $=4$ SV=1 iSTENG00026727001 PE=3 SV=1 iSTENG00016149001 PE=4 SV=1 ${ }^{I}=1$

;TENG00021231001 PE=4 SV=1

Iodon nigroviridis GN=GSTENG00009864001 PE $=4$ SV=1 $i=2 \mathrm{SV}=1$

iSTENG00030207001 PE=4 SV=1

${ }^{I}=1$

${ }^{I}=1$

1 $\mathrm{PE}=2 \mathrm{SV}=1$ 


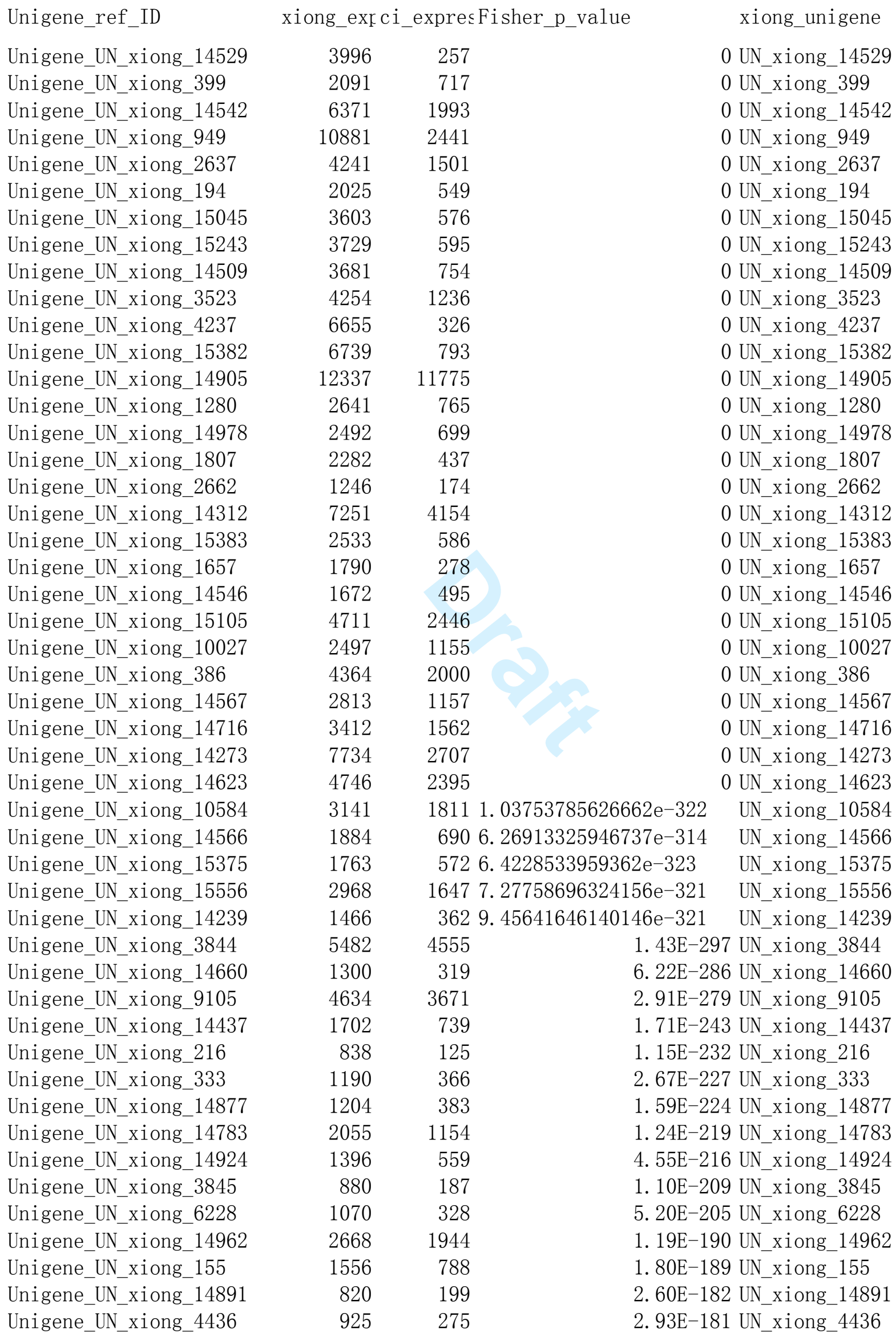




\begin{tabular}{|c|c|c|c|}
\hline Unigene_UN_xiong_14385 & 1219 & 515 & 6.99E-180 UN_xiong_14385 \\
\hline Unigene_UN_xiong_14665 & 1757 & 1058 & 2. 49E-171 UN_xiong_14665 \\
\hline Unigene_UN_xiong_1438 & 854 & 271 & 4. 67E-160 UN_xiong_1438 \\
\hline Unigene_UN_xiong_14648 & 896 & 304 & 9.91E-160 UN_xiong_14648 \\
\hline Unigene_UN_xiong_256 & 1100 & 476 & 1. 14E-158 UN_xiong_256 \\
\hline Unigene_UN_xiong_14285 & 2489 & 1939 & 1.13E-156 UN_xiong_14285 \\
\hline Unigene_UN_xiong_3390 & 3494 & 3184 & 4. $64 \mathrm{E}-153$ UN_xiong_3390 \\
\hline Unigene_UN_xiong_2317 & 2928 & 2498 & 3. 04E-151 UN_xiong_2317 \\
\hline Unigene_UN_xiong_8530 & 1935 & 1396 & 1.79E-141 UN_xiong_8530 \\
\hline Unigene_UN_xiong_3904 & 1200 & 635 & 3.79E-139 UN_xiong_3904 \\
\hline Unigene_UN_xiong_1478 & 1338 & 775 & 4. 45E-138 UN_xiong_1478 \\
\hline Unigene_UN_xiong_2555 & 1189 & 634 & 1.20E-136 UN_xiong_2555 \\
\hline Unigene_UN_xiong_13350 & 1252 & 709 & 2. 83E-133 UN_xiong_13350 \\
\hline Unigene_UN_xiong_3637 & 629 & 168 & 1.61E-132 UN_xiong_3637 \\
\hline Unigene_UN_xiong_14727 & 1181 & 649 & 7. 45E-131 UN_xiong_14727 \\
\hline Unigene_UN_xiong_6909 & 759 & 271 & 2. 05E-130 UN_xiong_6909 \\
\hline Unigene_UN_xiong_14274 & 616 & 164 & 3. 10E-130 UN_xiong_14274 \\
\hline Unigene_UN_xiong_14453 & 1023 & 514 & 2. 11E-126 UN_xiong_14453 \\
\hline Unigene_UN_xiong_14263 & 814 & 341 & 8.96E-122 UN_xiong_14263 \\
\hline Unigene_UN_xiong_14616 & 554 & 154 & 4. 06E-114 UN_xiong_14616 \\
\hline Unigene_UN_xiong_5777 & 814 & 366 & 1. 53E-113 UN_xiong_5777 \\
\hline Unigene_UN_xiong_15374 & 794 & 349 & 2. 50E-113 UN_xiong_15374 \\
\hline Unigene_UN_xiong_14859 & 645 & 226 & 8. 11E-113 UN_xiong_14859 \\
\hline Unigene_UN_xiong_3047 & 594 & 187 & 1.23E-112 UN_xiong_3047 \\
\hline Unigene_UN_xiong_2181 & 1021 & 564 & 1.55E-112 UN_xiong_2181 \\
\hline Unigene_UN_xiong_14937 & 583 & 180 & 4. 68E-112 UN_xiong_14937 \\
\hline Unigene_UN_xiong_14674 & 614 & 207 & 1. $12 \mathrm{E}-110$ UN_xiong_14674 \\
\hline Unigene_UN_xiong_5020 & 1176 & 741 & 3.99E-108 UN_xiong_5020 \\
\hline Unigene_UN_xiong_4401 & 602 & 204 & 4. 25E-108 UN_xiong_4401 \\
\hline Unigene_UN_xiong_14689 & 537 & 168 & 1.86E-102 UN_xiong_14689 \\
\hline Unigene_UN_xiong_751 & 779 & 376 & 3.43E-101 UN_xiong_751 \\
\hline Unigene_UN_xiong_14569 & 569 & 197 & 1. 11E-100 UN_xiong_14569 \\
\hline Unigene_UN_xiong_11645 & 524 & 165 & 1.46E-99 UN_xiong_11645 \\
\hline Unigene_UN_xiong_14286 & 799 & 401 & 2.40E-99 UN_xiong_14286 \\
\hline Unigene_UN_xiong_14334 & 1216 & 828 & 6. 22E-99 UN_xiong_14334 \\
\hline Unigene_UN_xiong_7854 & 661 & 278 & 1.02E-98 UN_xiong_7854 \\
\hline Unigene_UN_xiong_988 & 1029 & 631 & 1. 30E-98 UN_xiong_988 \\
\hline Unigene_UN_xiong_2611 & 1976 & 1719 & 3. 16E-98 UN_xiong_2611 \\
\hline Unigene_UN_xiong_14873 & 571 & 207 & 1.92E-97 UN_xiong_14873 \\
\hline Unigene_UN_xiong_5912 & 518 & 169 & 6.64E-96 UN_xiong_5912 \\
\hline Unigene_UN_xiong_145 & 459 & 135 & 1.94E-91 UN_xiong_145 \\
\hline Unigene_UN_xiong_790 & 821 & 461 & 3.89E-89 UN_xiong_790 \\
\hline Unigene_UN_xiong_7465 & 426 & 121 & 4. 06E-87 UN_xiong_7465 \\
\hline Unigene_UN_xiong_4109 & 884 & 535 & 1.33E-86 UN_xiong_4109 \\
\hline Unigene_UN_xiong_9546 & 1221 & 908 & 3.46E-85 UN_xiong_9546 \\
\hline Unigene_UN_xiong_1685 & 741 & 399 & 6. 15E-85 UN_xiong_1685 \\
\hline Unigene_UN_xiong_15543 & 781 & 439 & 9.24E-85 UN_xiong_15543 \\
\hline Unigene_UN_xiong_13667 & 1185 & 881 & 8.96E-83 UN_xiong_13667 \\
\hline Unigene_UN_xiong_1628 & 557 & 240 & 9.23E-82 UN_xiong_1628 \\
\hline Unigene_UN_xiong_7527 & 637 & 312 & 1. 18E-81 UN_xiong_7527 \\
\hline
\end{tabular}




\begin{tabular}{|c|c|c|c|}
\hline Unigene_UN_xiong_5941 & 535 & 225 & 2. 50E-80 UN_xiong_5941 \\
\hline Unigene_UN_xiong_8850 & 951 & 655 & 2. 63E-76 UN_xiong_8850 \\
\hline Unigene_UN_xiong_10847 & 697 & 398 & 3. 56E-74 UN_xiong_10847 \\
\hline Unigene_UN_xiong_14812 & 1115 & 852 & 4. 45E-74 UN_xiong_14812 \\
\hline Unigene_UN_xiong_6978 & 972 & 700 & 2. 74E-72 UN_xiong_6978 \\
\hline Unigene_UN_xiong_14438 & 2105 & 2102 & 3. 91E-72 UN_xiong_14438 \\
\hline Unigene_UN_xiong_14383 & 447 & 175 & 7. 86E-72 UN_xiong_14383 \\
\hline Unigene_UN_xiong_12363 & 420 & 157 & 2. 06E-70 UN_xiong_12363 \\
\hline Unigene_UN_xiong_14440 & 1775 & 1689 & 2. 51E-70 UN_xiong_14440 \\
\hline Unigene_UN_xiong_3675 & 494 & 221 & 4. 04E-70 UN_xiong_3675 \\
\hline Unigene_UN_xiong_8920 & 541 & 265 & 1. 03E-69 UN_xiong_8920 \\
\hline Unigene_UN_xiong_14490 & 689 & 418 & 9. 83E-68 UN_xiong_14490 \\
\hline Unigene_UN_xiong_2168 & 430 & 173 & 1. 41E-67 UN_xiong_2168 \\
\hline Unigene_UN_xiong_6384 & 675 & 413 & 1. 88E-65 UN_xiong_6384 \\
\hline Unigene_UN_xiong_2119 & 455 & 202 & 3. 86E-65 UN_xiong_2119 \\
\hline Unigene_UN_xiong_12342 & 371 & 138 & 1. 17E-62 UN_xiong_12342 \\
\hline Unigene_UN_xiong_9316 & 679 & 433 & 3. 66E-62 UN_xiong_9316 \\
\hline Unigene_UN_xiong_4494 & 692 & 447 & 4. 66E-62 UN_xiong_4494 \\
\hline Unigene_UN_xiong_14801 & 681 & 437 & 1. 27E-61 UN_xiong_14801 \\
\hline Unigene_UN_xiong_8994 & 592 & 346 & 1. 57E-61 UN_xiong_8994 \\
\hline Unigene_UN_xiong_2689 & 364 & 137 & 8.65E-61 UN_xiong_2689 \\
\hline Unigene_UN_xiong_6304 & 584 & 341 & 1. 16E-60 UN_xiong_6304 \\
\hline Unigene_UN_xiong_825 & 355 & 131 & 2.93E-60 UN_xiong_825 \\
\hline Unigene_UN_xiong_10733 & 917 & 707 & 3. 43E-60 UN_xiong_10733 \\
\hline Unigene_UN_xiong_3878 & 744 & 517 & 3. 15E-59 UN_xiong_3878 \\
\hline Unigene_UN_xiong_15520 & 921 & 719 & 5. 15E-59 UN_xiong_15520 \\
\hline Unigene_UN_xiong_12413 & 312 & 102 & 2. 59E-58 UN_xiong_12413 \\
\hline Unigene_UN_xiong_876 & 509 & 277 & 3. 31E-58 UN_xiong_876 \\
\hline Unigene_UN_xiong_9163 & 619 & 392 & 2. 58E-57 UN_xiong_9163 \\
\hline Unigene_UN_xiong_14497 & 568 & 341 & 5. 47E-57 UN_xiong_14497 \\
\hline Unigene_UN_xiong_3436 & 467 & 243 & 1. 77E-56 UN_xiong_3436 \\
\hline Unigene_UN_xiong_8136 & 407 & 188 & 4. 03E-56 UN_xiong_8136 \\
\hline Unigene_UN_xiong_4975 & 426 & 208 & 2. 46E-55 UN_xiong_4975 \\
\hline Unigene_UN_xiong_1201 & 520 & 303 & 2. 47E-54 UN_xiong_1201 \\
\hline Unigene_UN_xiong_14327 & 969 & 814 & 2. $68 \mathrm{E}-53$ UN_xiong_14327 \\
\hline Unigene_UN_xiong_5113 & 529 & 319 & 7.69E-53 UN_xiong_5113 \\
\hline Unigene_UN_xiong_2520 & 1151 & 1044 & 2.07E-52 UN_xiong_2520 \\
\hline Unigene_UN_xiong_142 & 607 & 404 & 2.93E-52 UN_xiong_142 \\
\hline Unigene_UN_xiong_4059 & 327 & 136 & 2. 52E-50 UN_xiong_4059 \\
\hline Unigene_UN_xiong_6694 & 713 & 538 & 3. 26E-49 UN_xiong_6694 \\
\hline Unigene_UN_xiong_10563 & 598 & 410 & 4. $72 \mathrm{E}-49$ UN_xiong_10563 \\
\hline Unigene_UN_xiong_1586 & 927 & 807 & 4.62E-47 UN_xiong_1586 \\
\hline Unigene_UN_xiong_14563 & 1317 & 1304 & 5. 05E-47 UN_xiong_14563 \\
\hline Unigene_UN_xiong_12491 & 366 & 189 & 4. 79E-45 UN_xiong_12491 \\
\hline Unigene_UN_xiong_15449 & 350 & 176 & 2. 09E-44 UN_xiong_15449 \\
\hline Unigene_UN_xiong_14271 & 852 & 737 & 4. 83E-44 UN_xiong_14271 \\
\hline Unigene_UN_xiong_15692 & 387 & 215 & 1. 74E-43 UN_xiong_15692 \\
\hline Unigene_UN_xiong_13462 & 273 & 111 & 3. 37E-43 UN_xiong_13462 \\
\hline Unigene_UN_xiong_12050 & 561 & 405 & 2. 32E-42 UN_xiong_12050 \\
\hline Unigene_UN_xiong_1436 & 681 & 545 & 5.98E-42 UN_xiong_1436 \\
\hline
\end{tabular}




\begin{tabular}{|c|c|c|c|}
\hline Unigene_UN_xiong_14884 & 242 & 89 & 7.50E-42 UN_xiong_14884 \\
\hline Unigene_UN_xiong_4179 & 778 & 662 & 7.97E-42 UN_xiong_4179 \\
\hline Unigene_UN_xiong_6936 & 290 & 130 & 1.02E-41 UN_xiong_6936 \\
\hline Unigene_UN_xiong_2808 & 570 & 423 & 5.69E-41 UN_xiong_2808 \\
\hline Unigene_UN_xiong_15372 & 649 & 516 & 1. 40E-40 UN_xiong_15372 \\
\hline Unigene_UN_xiong_3384 & 606 & 475 & 4.11E-39 UN_xiong_3384 \\
\hline Unigene_UN_xiong_14525 & 1055 & 1044 & 3. 86E-38 UN_xiong_14525 \\
\hline Unigene_UN_xiong_2973 & 823 & 746 & 4. 38E-38 UN_xiong_2973 \\
\hline Unigene_UN_xiong_557 & 393 & 246 & 1. 06E-37 UN_xiong_557 \\
\hline Unigene_UN_xiong_12975 & 369 & 221 & 1. 30E-37 UN_xiong_12975 \\
\hline Unigene_UN_xiong_14541 & 397 & 251 & 1.99E-37 UN_xiong_14541 \\
\hline Unigene_UN_xiong_14313 & 831 & 762 & 2. 31E-37 UN_xiong_14313 \\
\hline Unigene_UN_xiong_13097 & 898 & 848 & 3. 34E-37 UN_xiong_13097 \\
\hline Unigene_UN_xiong_6672 & 448 & 308 & 4.86E-37 UN_xiong_6672 \\
\hline Unigene_UN_xiong_4304 & 285 & 142 & 9.85E-37 UN_xiong_4304 \\
\hline Unigene_UN_xiong_14344 & 594 & 480 & 3. 46E-36 UN_xiong_14344 \\
\hline Unigene_UN_xiong_14299 & 305 & 166 & 1.62E-35 UN_xiong_14299 \\
\hline Unigene_UN_xiong_12671 & 848 & 810 & 3. 20E-34 UN_xiong_12671 \\
\hline Unigene_UN_xiong_14883 & 304 & 172 & 1.00E-33 UN_xiong_14883 \\
\hline Unigene_UN_xiong_2239 & 291 & 160 & 1.92E-33 UN_xiong_2239 \\
\hline Unigene_UN_xiong_4351 & 295 & 166 & 6. 11E-33 UN_xiong_4351 \\
\hline Unigene_UN_xiong_15034 & 395 & 274 & 2.58E-32 UN_xiong_15034 \\
\hline Unigene_UN_xiong_9964 & 239 & 116 & 4.80E-32 UN_xiong_9964 \\
\hline Unigene_UN_xiong_14637 & 300 & 176 & 8.09E-32 UN_xiong_14637 \\
\hline Unigene_UN_xiong_14354 & 529 & 435 & 3. 07E-31 UN_xiong_14354 \\
\hline Unigene_UN_xiong_15039 & 373 & 256 & 3. 39E-31 UN_xiong_15039 \\
\hline Unigene_UN_xiong_14970 & 305 & 184 & 3. $60 \mathrm{E}-31$ UN_xiong_14970 \\
\hline Unigene_UN_xiong_9243 & 787 & 764 & 2.02E-30 UN_xiong_9243 \\
\hline Unigene_UN_xiong_4995 & 355 & 241 & 3. 29E-30 UN_xiong_4995 \\
\hline Unigene_UN_xiong_14717 & 477 & 381 & 4. 36E-30 UN_xiong_14717 \\
\hline Unigene_UN_xiong_3793 & 240 & 125 & 7.99E-30 UN_xiong_3793 \\
\hline Unigene_UN_xiong_2413 & 302 & 190 & 3. 56E-29 UN_xiong_2413 \\
\hline Unigene_UN_xiong_14516 & 412 & 314 & 1. 51E-28 UN_xiong_14516 \\
\hline Unigene_UN_xiong_3968 & 433 & 344 & 9. 32E-28 UN_xiong_3968 \\
\hline Unigene_UN_xiong_3503 & 343 & 241 & 1.07E-27 UN_xiong_3503 \\
\hline Unigene_UN_xiong_3181 & 232 & 126 & 1.68E-27 UN_xiong_3181 \\
\hline Unigene_UN_xiong_1200 & 335 & 234 & 2.46E-27 UN_xiong_1200 \\
\hline Unigene_UN_xiong_6740 & 310 & 211 & 1. 37E-26 UN_xiong_6740 \\
\hline Unigene_UN_xiong_13283 & 255 & 154 & 3. 26E-26 UN_xiong_13283 \\
\hline Unigene_UN_xiong_12762 & 368 & 280 & 9.79E-26 UN_xiong_12762 \\
\hline Unigene_UN_xiong_14527 & 273 & 176 & 1.83E-25 UN_xiong_14527 \\
\hline Unigene_UN_xiong_662 & 455 & 386 & 2. 39E-25 UN_xiong_662 \\
\hline Unigene_UN_xiong_14683 & 315 & 223 & 2.55E-25 UN_xiong_14683 \\
\hline Unigene_UN_xiong_15786 & 398 & 324 & 2. $29 \mathrm{E}-24$ UN_xiong_15786 \\
\hline Unigene_UN_xiong_14398 & 328 & 245 & 5. 58E-24 UN_xiong_14398 \\
\hline Unigene_UN_xiong_3085 & 342 & 261 & 5. 75E-24 UN_xiong_3085 \\
\hline Unigene_UN_xiong_2677 & 215 & 124 & 1. 07E-23 UN_xiong_2677 \\
\hline Unigene_UN_xiong_15049 & 283 & 198 & 2.69E-23 UN_xiong_15049 \\
\hline Unigene_UN_xiong_14106 & 234 & 149 & 1.49E-22 UN_xiong_14106 \\
\hline Unigene_UN_xiong_7138 & 441 & 398 & 2. 29E-21 UN_xiong_7138 \\
\hline
\end{tabular}




\begin{tabular}{|c|c|c|c|}
\hline Unigene_UN_xiong_13092 & 274 & 202 & 9. 41E-21 UN_xiong_13092 \\
\hline Unigene_UN_xiong_14224 & 409 & 365 & 1. 70E-20 UN_xiong_14224 \\
\hline Unigene_UN_xiong_4865 & 259 & 188 & 3.60E-20 UN_xiong_4865 \\
\hline Unigene_UN_xiong_8591 & 543 & 542 & 5.88E-20 UN_xiong_8591 \\
\hline Unigene_UN_xiong_10069 & 399 & 357 & 7. 32E-20 UN_xiong_10069 \\
\hline Unigene_UN_xiong_607 & 458 & 436 & 2.17E-19 UN_xiong_607 \\
\hline Unigene_UN_xiong_3481 & 286 & 224 & 2. 19E-19 UN_xiong_3481 \\
\hline Unigene_UN_xiong_3495 & 426 & 397 & 3. 55E-19 UN_xiong_3495 \\
\hline Unigene_UN_xiong_15078 & 340 & 291 & 5. 22E-19 UN_xiong_15078 \\
\hline Unigene_UN_xiong_6002 & 340 & 292 & 7.97E-19 UN_xiong_6002 \\
\hline Unigene_UN_xiong_11708 & 273 & 213 & 9. $80 \mathrm{E}-19$ UN_xiong_11708 \\
\hline Unigene_UN_xiong_6878 & 343 & 300 & 3.40E-18 UN_xiong_6878 \\
\hline Unigene_UN_xiong_1920 & 316 & 270 & 8. 34E-18 UN_xiong_1920 \\
\hline Unigene_UN_xiong_10414 & 369 & 336 & 1. $01 \mathrm{E}-17$ UN_xiong_10414 \\
\hline Unigene_UN_xiong_14761 & 396 & 371 & 1. 20E-17 UN_xiong_14761 \\
\hline Unigene_UN_xiong_15624 & 462 & 460 & 3. 07E-17 UN_xiong_15624 \\
\hline Unigene_UN_xiong_1043 & 409 & 396 & 1. 01E-16 UN_xiong_1043 \\
\hline Unigene_UN_xiong_16 & 313 & 275 & 1. 34E-16 UN_xiong_16 \\
\hline Unigene_UN_xiong_8203 & 321 & 286 & 2.00E-16 UN_xiong_8203 \\
\hline Unigene_UN_xiong_14899 & 242 & 192 & 3.90E-16 UN_xiong_14899 \\
\hline Unigene_UN_xiong_11580 & 330 & 303 & 8.97E-16 UN_xiong_11580 \\
\hline Unigene_UN_xiong_6171 & 267 & 232 & 8. 94E-15 UN_xiong_6171 \\
\hline Unigene_UN_xiong_889 & 220 & 177 & 1.83E-14 UN_xiong_889 \\
\hline Unigene_UN_xiong_14731 & 266 & 234 & 2.83E-14 UN_xiong_14731 \\
\hline Unigene_UN_xiong_15790 & 332 & 318 & 2.99E-14 UN_xiong_15790 \\
\hline Unigene_UN_xiong_2288 & 244 & 208 & 3.95E-14 UN_xiong_2288 \\
\hline Unigene_UN_xiong_8411 & 310 & 291 & 3. $96 \mathrm{E}-14$ UN_xiong_8411 \\
\hline Unigene_UN_xiong_4628 & 272 & 243 & 4.79E-14 UN_xiong_4628 \\
\hline Unigene_UN_xiong_9969 & 122 & 74 & 2.89E-13 UN_xiong_9969 \\
\hline Unigene_UN_xiong_9738 & 296 & 281 & 3. 39E-13 UN_xiong_9738 \\
\hline Unigene_UN_xiong_14990 & 174 & 134 & 9. $14 \mathrm{E}-13$ UN_xiong_14990 \\
\hline Unigene_UN_xiong_2226 & 216 & 186 & 2.20E-12 UN_xiong_2226 \\
\hline Unigene_UN_xiong_14362 & 266 & 251 & 3. 55E-12 UN_xiong_14362 \\
\hline Unigene_UN_xiong_14684 & 271 & 258 & 4. 47E-12 UN_xiong_14684 \\
\hline Unigene_UN_xiong_8985 & 137 & 100 & 2. 84E-11 UN_xiong_8985 \\
\hline Unigene_UN_xiong_13205 & 197 & 175 & 9. 63E-11 UN_xiong_13205 \\
\hline Unigene_UN_xiong_4642 & 265 & 264 & 1.46E-10 UN_xiong_4642 \\
\hline Unigene_UN_xiong_10903 & 142 & 111 & 2. $11 \mathrm{E}-10$ UN_xiong_10903 \\
\hline Unigene_UN_xiong_4348 & 183 & 161 & 2. 45E-10 UN_xiong_4348 \\
\hline Unigene_UN_xiong_14352 & 242 & 236 & 2. 65E-10 UN_xiong_14352 \\
\hline Unigene_UN_xiong_14975 & 194 & 175 & 3. 39E-10 UN_xiong_14975 \\
\hline Unigene_UN_xiong_13896 & 191 & 172 & 3. 57E-10 UN_xiong_13896 \\
\hline Unigene_UN_xiong_3763 & 169 & 148 & 1.25E-09 UN_xiong_3763 \\
\hline Unigene_UN_xiong_14666 & 185 & 169 & 1. 31E-09 UN_xiong_14666 \\
\hline Unigene_UN_xiong_5310 & 206 & 196 & 1.42E-09 UN_xiong_5310 \\
\hline Unigene_UN_xiong_838 & 194 & 187 & 9.51E-09 UN_xiong_838 \\
\hline Unigene_UN_xiong_14997 & 182 & 181 & 1. 02E-07 UN_xiong_14997 \\
\hline Unigene_UN_xiong_891 & 152 & 147 & 3.80E-07 UN_xiong_891 \\
\hline Unigene_UN_xiong_10111 & 155 & 154 & 1.06E-06 UN_xiong_10111 \\
\hline
\end{tabular}




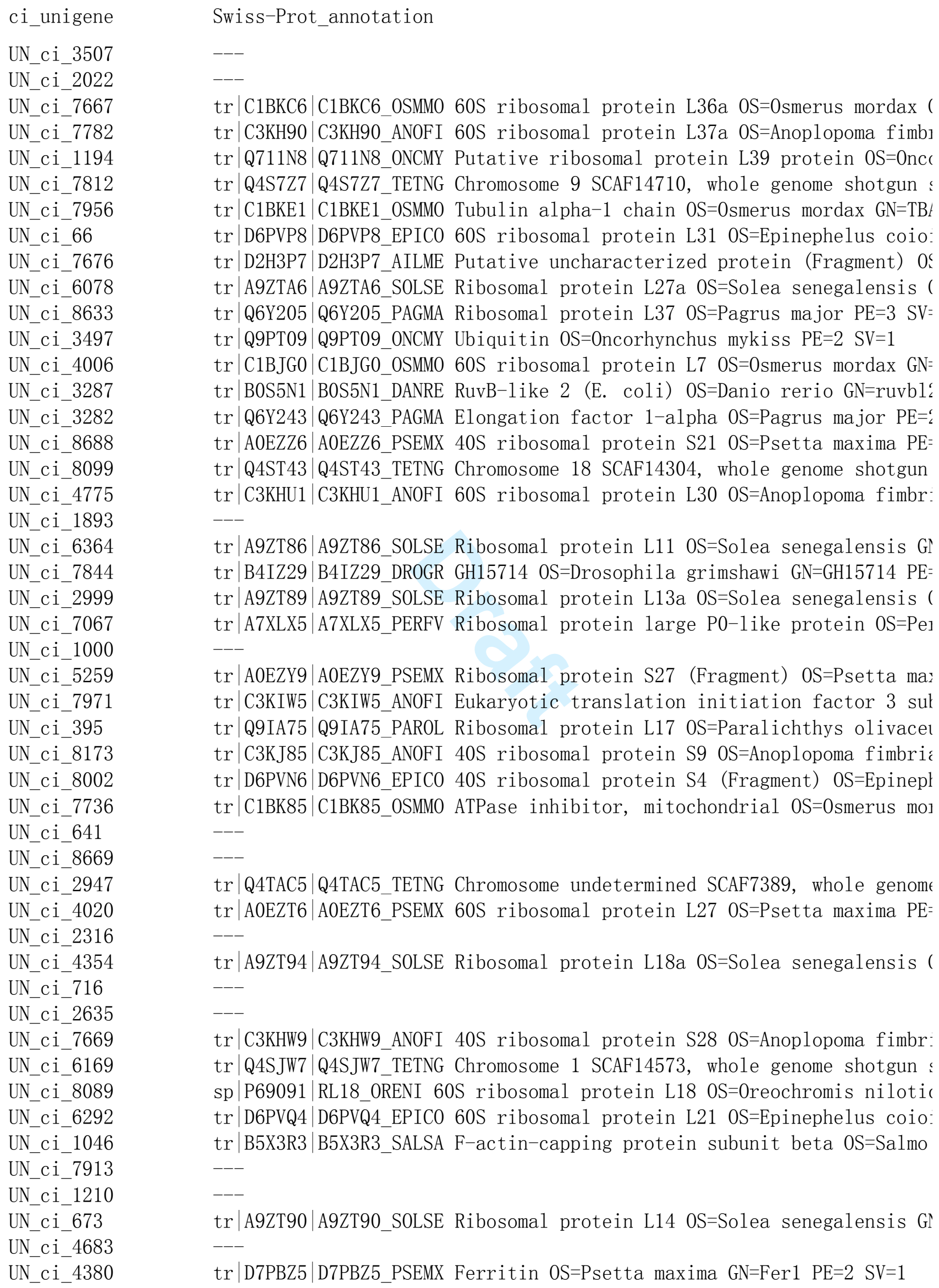

ci_unigene Swiss-Prot_annotation

tr $\mid$ C1BKC6 $\mid$ C1BKC6_0SMM0 60S ribosomal protein L36a 0S=0smerus mordax ( tr $\mid$ C3KH90|C3KH90_ANOFI 60S ribosomal protein L37a OS=Anoplopoma fimb] tr $\mid$ Q711N8|Q711N8_ONCMY Putative ribosomal protein L39 protein 0S=0nce tr $\mid$ Q4S7Z7|Q4S7Z7_TETNG Chromosome 9 SCAF14710, whole genome shotgun ؛ $\operatorname{tr} \mid$ C1BKE1 $\mid$ C1BKE1_0SMM0 Tubulin alpha-1 chain 0S=0smerus mordax GN=TB/ tr|D6PVP8|D6PVP8_EPIC0 60S ribosomal protein L31 OS=Epinephelus coio: tr $\mid$ D2H3P7 $\mid$ D2H3P7_AILME Putative uncharacterized protein (Fragment) 0s tr|A9ZTA6|A9ZTA6_SOLSE Ribosomal protein L27a 0S=Solea senegalensis ( tr $\mid$ Q6Y205|Q6Y205_PAGMA Ribosomal protein L37 OS=Pagrus major PE=3 SV= $\operatorname{tr} \mid$ Q9PT09 $\mid$ Q9PT09_0NCMY Ubiquitin OS=0ncorhynchus mykiss PE=2 SV=1 $\operatorname{tr} \mid$ C1BJG0|C1BJG0_0SMM0 60S ribosomal protein L7 0S=0smerus mordax GN= tr $\mid$ B0S5N1|B0S5N1_DANRE RuvB-1ike 2 (E. coli) OS=Danio rerio GN=ruvb1؛ $\operatorname{tr} \mid$ Q6Y243|Q6Y243_PAGMA Elongation factor 1-alpha OS=Pagrus major $\mathrm{PE=}$ ‘ tr $\mid$ A0EZZ6|A0EZZ6_PSEMX 40S ribosomal protein S21 OS=Psetta maxima PE= $\operatorname{tr} \mid$ Q4ST43|Q4ST43_TETNG Chromosome 18 SCAF14304, whole genome shotgun $\operatorname{tr} \mid$ C3KHU1 $\mid$ C3KHU1_ANOFI 60S ribosomal protein L30 0S=Anoplopoma fimbr:

tr $\mid$ A9ZT86 $\mid$ A9ZT86_SOLSE Ribosomal protein L11 OS=Solea senegalensis GN tr|B4IZ29|B4IZ29_DR0GR GH15714 OS=Drosophila grimshawi GN=GH15714 PE= tr $\mid$ A9ZT89|A9ZT89_SOLSE Ribosomal protein L13a OS=Solea senegalensis ( tr $\mid$ A7XLX5|A7XLX5_PERFV Ribosomal protein large P0-1ike protein 0S=Pe]

tr $\mid$ A0EZY9 $\mid$ A0EZY9_PSEMX Ribosomal protein S27 (Fragment) OS=Psetta ma: tr|C3KIW5|C3KIW5_ANOFI Eukaryotic translation initiation factor 3 sul tr $\mid$ Q9IA75 $\mid$ Q9IA75_PAR0L Ribosomal protein L17 0S=Paralichthys olivace tr|C3KJ85|C3KJ85_ANOFI 40S ribosomal protein S9 0S=Anoplopoma fimbric tr|D6PVN6|D6PVN6_EPIC0 40S ribosomal protein S4 (Fragment) 0S=Epinepl tr $\mid$ C1BK85 $\mid$ C1BK85_OSMM0 ATPase inhibitor, mitochondrial OS=0smerus mo]

tr $\mid$ Q4TAC5 $\mid$ Q4TAC5_TETNG Chromosome undetermined SCAF7389, whole genom tr|A0EZT6|A0EZT6_PSEMX 60S ribosomal protein L27 OS=Psetta maxima PE=

$\operatorname{tr} \mid$ A9ZT94 $\mid$ A9ZT94_SOLSE Ribosomal protein L18a OS=Solea senegalensis (

tr $\mid$ C3KHW9|C3KHW9_ANOFI 40S ribosomal protein S28 0S=Anoplopoma fimbr: tr $\mid$ Q4SJW7|Q4SJW7_TETNG Chromosome 1 SCAF14573, whole genome shotgun : sp $\mid$ P69091|RL18_ORENI 60S ribosomal protein L18 0S=0reochromis nilotic tr|D6PVQ4|D6PVQ4_EPIC0 60S ribosomal protein L21 OS=Epinephelus coio: tr $\mid$ B5X3R3|B5X3R3_SALSA F-actin-capping protein subunit beta 0S=Salmo

$\operatorname{tr} \mid$ A9ZT90|A9ZT90_SOLSE Ribosomal protein L14 0S=Solea senegalensis G! tr $\mid$ D7PBZ5|D7PBZ5_PSEMX Ferritin OS=Psetta maxima GN=Fer1 PE=2 SV=1 
UN_ci_7874

UN_ci_5561

UN_ci_1510

UN_ci_2832

UN_ci_4646

UN_ci_8021

UN_ci_8003

UN_ci_2644

UN_ci_3645

UN_ci_3151

UN_ci_1383

UN_ci_8499

UN_ci_7235

UN_ci_1908

UN_ci_4122

UN_ci_2980

UN_ci_8763

UN_ci_1711

UN_ci_2483

UN_ci 6411

UN_ci_5560

UN_ci_8285

UN_ci_7979

UN_ci_5511

UN_ci_7754

UN_ci_7922

UN_ci_8086

UN_ci_5262

UN_ci_4128

UN_ci 867

UN_ci_4792

UN_ci_3632

UN_ci_6200

UN_ci_4885

UN_ci_8322

UN_ci_3967

UN_ci_7375

UN_ci_3837

UN_ci_8106

UN_ci_1122

UN_ci_644

UN_ci 8330

UN_ci_6070

UN_ci_1594

UN_ci_1201

UN_ci_4379

UN_ci_8769

UN_ci_8108

UN_ci_6040

UN_ci_1567
tr|Q5DVI0|Q5DVI0_PLAFE 60S ribosomal protein L28 0S=Platichthys fles $\operatorname{tr} \mid$ Q6Y1Z9|Q6Y1Z9_PAGMA 40S ribosomal protein S24 OS=Pagrus major $\mathrm{PE=}$ ‘

$---$

$\operatorname{tr} \mid$ A2Q0R6 $\mid$ A2Q0R6_SOLSE Ribosomal protein S2 OS=Solea senegalensis GN= $\operatorname{tr} \mid$ A2Q0U0 $\mid$ A2Q0U0_SOLSE Ribosomal protein S25 OS=Solea senegalensis GI

tr $\mid$ Q7ZU62 $\mid$ Q7ZU62_DANRE Zgc:66168 protein (Fragment) OS=Danio rerio G! $\operatorname{tr} \mid$ C3KJ53 $\mid$ C3KJ53_ANOFI Elongation factor 1-delta OS=Anoplopoma fimbr: $\operatorname{tr} \mid$ A9ZTA4 $\mid$ A9ZTA4_SOLSE Ribosomal protein L26 OS=Solea senegalensis GI

$\operatorname{tr} \mid$ A2Q0S7 $\mid$ A2Q0S7_SOLSE 40S ribosomal protein S12 0S=Solea senegalens: tr $\mid$ Q4TCC0 $\mid$ Q4TCC0_TETNG Acylphosphatase (Fragment) OS=Tetraodon nigror tr $\mid$ Q4TAQ9 $\mid$ Q4TAQ9_TETNG Chromosome undetermined SCAF7267, whole genom tr $\mid$ C1BL06 $\mid$ C1BL06_OSMM0 TGF-beta-inducible nuclear protein 1 OS=0smer $\operatorname{tr} \mid$ Q7SZX9 $\mid$ Q7SZX9_DANRE FLJ10613-1ike OS=Danio rerio GN=gn131 PE=2 SV= tr $\mid$ Q4SM43|Q4SM43_TETNG Chromosome 13 SCAF14555, whole genome shotgun

$---$

$\operatorname{tr} \mid$ B5XBW1 $\mid$ B5XBW1_SALSA BCAS2 protein homolog 0S=Salmo salar GN=BCAS2 tr $\mid$ C1BIM0 $\mid$ C1BIM0_0SMM0 Growth hormone-inducible transmembrane proteir

$---$

tr $\mid$ Q7T2C6 $\mid$ Q7T2C6_DANRE RAB7, member RAS oncogene family OS=Danio rer: $\operatorname{tr} \mid$ C3KGT2 $\mid$ C3KGT2_ANOFI Thioredoxin domain-containing protein 9 OS=Anc tr $\mid$ Q4RH57 |Q4RH57_TETNG Chromosome undetermined SCAF15069, whole genor tr $\mid$ Q3ZLG0 $\mid$ Q3ZLG0_OREMO XNop56 (Fragment) OS=0reochromis mossambicus I tr $\mid$ C3KK43|C3KK43_ANOFI Myosin light polypeptide 6 OS=Anoplopoma fimb] sp|Q202I6|TCTP_PSEMX Translationally-controlled tumor protein homoloई

tr $\mid$ A0EZU4 $\mid$ A0EZU4_PSEMX 40S ribosomal protein S11 OS=Psetta maxima PE= $\operatorname{tr} \mid$ C3KI82|C3KI82_ANOFI Transmembrane protein 50A 0S=Anoplopoma fimbr: sp $\mid$ Q1L8Y6 $\mid$ BBP10_DANRE BBSome-interacting protein of $10 \mathrm{kDa}$ OS=Danio ] $\operatorname{tr} \mid$ B9USM4|B9USM4_9PERC 2-Cys peroxiredoxin 4 OS=Seriola lalandi GN=PI tr $\mid$ C3KHU8|C3KHU8_ANOFI NADH dehydrogenase 1 alpha subcomplex subunit tr $\mid$ Q4RJL0|Q4RJL0_TETNG Chromosome 3 SCAF15037, whole genome shotgun : $\operatorname{tr} \mid$ Q4RVD2 $\mid$ Q4RVD2_TETNG Chromosome 15 SCAF14992, whole genome shotgun $\operatorname{tr} \mid$ COHAQ6 $\mid$ COHAQ6_SALSA Elongation factor 2 OS=Salmo salar GN=EF2 $\mathrm{PE}=$ ‘ tr $\mid$ A4L7I8 $\mid$ A4L7I8_9SCOM Glutathione peroxidase 0S=Thunnus maccoyii PE= tr|Q4RWC3|Q4RWC3_TETNG Chromosome 2 SCAF14990, whole genome shotgun : tr $\mid$ Q4S578|Q4S578_TETNG Chromosome 6 SCAF14737, whole genome shotgun :

tr $\mid$ Q4SK90 $\mid$ Q4SK90_TETNG Chromosome 13 SCAF14566, whole genome shotgun 


\begin{tabular}{|c|c|}
\hline ci_749 & -- \\
\hline NN_ci_2189 & $\operatorname{tr} \mid$ C3KIZ9|C3KIZ9_ANOFI Ribosomal protein L19 0S=Anoplopoma fimbria GI \\
\hline JN_ci_7392 & r $\mid$ Q4RY82 $\mid$ Q4RY82_TETNG Chromosome 3 SCAF14978, whole genome shotgun ؛ \\
\hline N_ci_4626 & r $\mid$ C1BIV9 $\mid$ C1BIV9_0SMM0 FAM32A-1ike 0S=0smerus mordax GN=FA32A PE=4 SI \\
\hline N_ci_8164 & r|Q5DVH2|Q5DVH2_PLAFE 60S ribosomal protein L9 OS=Platichthys flesus \\
\hline N_ci_7851 & --- \\
\hline N_ci_7423 & --- \\
\hline N_ci_8 & $\operatorname{tr} \mid$ C1BL73|C1BL73_0SMM0 Charged multivesicular body protein 3 0S=0sme \\
\hline N_ci_7905 & $\operatorname{tr} \mid$ Q9IA74|Q9IA74_PAR0L 40S ribosomal protein S15A 0S=Paralichthys o1: \\
\hline N_ci_3657 & $\operatorname{tr} \mid$ Q6DEG5 |Q6DEG5_DANRE Polymerase (RNA) II (DNA directed) polypeptidt \\
\hline JN_ci_4072 & --- \\
\hline NN_ci_2946 & tr|C1BMA3|C1BMA3_0SMM0 Gamma-aminobutyric acid receptor-associated p] \\
\hline N_ci_t_7108 & $\operatorname{tr} \mid$ Q4RG23|Q4RG23_TETNG Chromosome 2 SCAF15106, whole genome shotgun ؛ \\
\hline N_ci_6452 & $\operatorname{tr} \mid$ C3KJY4|C3KJY4_ANOFI Coatomer subunit zeta-1 OS=Anoplopoma fimbria \\
\hline JN_ci_1844 & $\operatorname{tr} \mid$ C1BG03 $\mid$ C1BG03_ONCMY LYR motif-containing protein 2 OS=0ncorhynchus \\
\hline N_ci_- & $\operatorname{tr} \mid$ COPTZ1|COPTZ1_SALSA Transmembrane emp24 domain-containing protein \\
\hline N_ci_5545 & --- \\
\hline JN_ci_8338 & $\operatorname{tr} \mid$ A7UP40 $\mid$ A7UP40_PSEMX Heat shock protein 90 0S=Ps \\
\hline N_c & $\operatorname{tr} \mid$ Q5XGA8|Q5XGA8_XENTR Ribosomal protein S16 0S=Xenopus tropicalis GN \\
\hline JN_ci_5716 & --- \\
\hline N_c & $\operatorname{tr} \mid$ B6YZ85 $\mid$ B6YZ85_THUT0 Vasa OS=Thunnus thynnus orientalis $\mathrm{PE}=2 \mathrm{SV}=1$ \\
\hline 748 & --- \\
\hline N_ci_2310 & sp $\mid$ A8Y5U1|CE051_DANRE UPF0600 protein C5o1 \\
\hline N_c & $\operatorname{tr} \mid$ B5X4J9|B5X4J9_SALSA Ribosomal protein L7-1ike 1 OS=Salmo salar GN= \\
\hline JN_ci_8607 & tr $\mid$ A0EZU3|A0EZU3_PSEMX 40S ribosomal protein S14 (Fragment) 0S=Psetti \\
\hline UN_c & $\operatorname{tr} \mid$ B9V305|B9V305_EPIC0 40S ribosomal protein S19 (Fragment) 0S=Epiner \\
\hline UN_ci_2212 & $\operatorname{tr} \mid$ Q4RGQ9|Q4RGQ9_TETNG Chromosome 4 SCAF15093, whole genome shotgun : \\
\hline 3860 & --- \\
\hline 68 & $\operatorname{tr} \mid$ A5H1I3|A5H1I3_PAR0L Chaperonin contai \\
\hline 1441 & $\operatorname{tr} \mid$ Q4SDY7|Q4SDY7_TETNG Chromosome 13 SCAF14627, whole genome shotgun \\
\hline JN_ci_1100 & --- \\
\hline NN_ci_2297 & $\operatorname{tr} \mid$ B5DG90|B5DG90_SALSA RAB2A, member RAS oncogene family OS=Salmo sa. \\
\hline N_ci_1_1054 & tr|Q4RJP3|Q4RJP3_TETNG Chromosome 13 SCAF15035, whole genome shotgun \\
\hline JN_c & $\operatorname{tr} \mid$ Q4SDA6 $\mid$ Q4SDA6_TETNG Chromosome 1 SCAF14640, whole genome shotgun ؛ \\
\hline JN_ci_6832 & tr $\mid$ Q5RKM2|Q5RKM2_DANRE NADH-ubiquinone oxidoreductase 75 kDa subunit \\
\hline JN_c & tr|Q4SI99|Q4SI99_TETNG Chromosome 5 SCAF14581, whole genome shotgun ؛ \\
\hline UN_c & tr $\mid$ C3KK25 $\mid$ C3KK25_ANOFI Methyltransferase WBSCR22 OS=Anoplopoma fimbr: \\
\hline GPGRCDD02H2 & $\operatorname{tr} \mid$ C1BHB0 $\mid$ C1BHB0_0NCMY Eukaryotic translation initiation factor 3 sul \\
\hline UN_ci_7334 & --- \\
\hline UN_ci_6019 & $\operatorname{tr} \mid$ C3KJK1|C3KJK1_ANOFI 39S ribosomal protein L16, mitochondrial 0S=Ar \\
\hline UN_c & --- \\
\hline UN_c & $\operatorname{tr} \mid$ A2Q0T3 $\mid$ A2Q0T3_SOLSE Ribosomal protein S17 OS=Solea senegalensis GN \\
\hline UN_ci_362 & $\operatorname{tr} \mid$ Q6VEJ7 $\mid$ Q6VEJ7_OREM0 Receptor for activated protein kinase C OS=0rt \\
\hline UN_ci_5280 & $\operatorname{tr} \mid$ A6H913 $\mid$ A6H913_TAKRU TATA box binding protein 0S=Takifugu rubripes \\
\hline UN_ci_1239 & --- \\
\hline $\mathrm{UN}_{-}$ & $\operatorname{tr} \mid$ Q6Y249|Q6Y249_PAGMA BTF \\
\hline UN_ci_6939 & $\operatorname{tr} \mid$ Q5EB36|Q5EB36_XENTR MGC97656 protein 0S=Xenopus tropicalis GN=cop؛ \\
\hline UN_ci_5426 & $\operatorname{tr} \mid$ C3KIE3|C3KIE3_ANOFI Transcription factor A, mitochondrial OS=Anop- \\
\hline & --- \\
\hline Ji 448 & $A N B$ \\
\hline
\end{tabular}


UN_ci_3043

UN_ci_7855

UN_ci_2259

GP8I43W03GIU7K_ci

UN_ci_854

UN_ci_7788

UN_ci_4956

UN_ci_3621

UN_ci_4425

UN_ci_433

UN_ci_1287

UN_ci_8334

UN_ci_8275

UN_ci_4253

UN_ci_5589

UN_ci_1669

UN_ci_4248

UN_ci_7397

UN_ci_3196

UN_ci_855

UN_ci_5631

UN_ci_7798

UN_ci_122

UN_ci_1564

UN_ci_8068

UN_ci_4024

UN_ci_8794

UN_ci_8092

UN_ci_6179

UN_ci_7875

UN_ci_7329

UN_ci_3605

UN_ci_5578

UN_ci_7542

UN_ci_6513

UN_ci_7588

UN_ci_2516

UN_ci_71

UN_ci_1520

UN_ci_8067

UN_ci_3597

UN_ci_1590

UN_ci_8665

UN_ci_4091

UN_ci_6652

UN_ci_4584

UN_ci_8259

UN_ci_4919

UN_ci_8272

UN_ci_5775 tr $\mid$ Q7ZUZ4 $\mid$ Q7ZUZ4_DANRE Programmed cell death 5 OS=Danio rerio GN=pdec tr $\mid$ A1Y189|A1Y189_PAR0L Voltage-dependent anion channel OS=Paralichth! tr $\mid$ Q4S5R8|Q4S5R8_TETNG Chromosome 9 SCAF14729, whole genome shotgun : tr $\mid$ C3KH20|C3KH20_ANOFI NADH dehydrogenase 1 beta subcomplex subunit $t$ tr $\mid$ Q4SVP1|Q4SVP1_TETNG Chromosome undetermined SCAF13749, whole genor tr $\mid$ Q7ZX50|Q7ZX50_XENLA LOC444845 protein (Fragment) OS=Xenopus laevis tr $\mid$ C3KI17 $\mid$ C3KI17_ANOFI Proteasome subunit alpha type OS=Anoplopoma $\mathrm{f}$ : tr|Q4SQY6|Q4SQY6_TETNG Chromosome 11 SCAF14528, whole genome shotgun $\operatorname{tr} \mid$ C1BKU7 $\mid$ C1BKU7_0SMM0 Cellular nucleic acid-binding protein 0S=0sme] tr|Q4RP92|Q4RP92_TETNG Chromosome 1 SCAF15008, whole genome shotgun ؛

sp|Q2KKX7|CS042_SINCH UPF0608 protein C19orf42 homolog OS=Siniperca ( tr|B5DH25|B5DH25_SALSA 26S proteasome non-ATPase regulatory subunit :

tr $\mid$ Q71SZ0 $\mid$ Q71SZ0_9SC0M Cytochrome c oxidase subunit VIb OS=Scombridat tr |Q4SJP0|Q4SJP0_TETNG Chromosome 1 SCAF14573, whole genome shotgun : tr|Q4RG99|Q4RG99_TETNG Chromosome 12 SCAF15104, whole genome shotgun tr $\mid$ B5X4Q0|B5X4Q0_SALSA Tumor suppressor candidate 4 OS=Salmo salar GN tr $\mid$ Q4SCH5 $\mid$ Q4SCH5_TETNG Chromosome undetermined SCAF14653, whole genor $\operatorname{tr} \mid$ Q32PU7 $\mid$ Q32PU7_DANRE Zgc:123194 OS=Danio rerio GN=Zgc:123194 PE=2 ؛

$\operatorname{tr} \mid$ Q803B9 $\mid$ Q803B9_DANRE DCP2 decapping enzyme homolog (S. cerevisiae)

tr $\mid$ B3DLM7 |B3DLM7_XENTR LOC100170503 protein (Fragment) OS=Xenopus trc $\operatorname{tr} \mid$ C3KJD7 $\mid$ C3KJD7_ANOFI FAM92A1 OS=Anoplopoma fimbria GN=F92A1 PE=2 SI $\operatorname{tr} \mid$ B5X1I8|B5X1I8_SALSA Dolichyl-diphosphooligosaccharide--protein g1! tr $\mid$ C3KJM2 |C3KJM2_ANOFI DNA-directed RNA polymerases I, II, and III si

tr|B5X449|B5X449_SALSA Proteasome assembly chaperone 1 0S=Salmo sala] tr|Q4RUH3|Q4RUH3_TETNG Chromosome 1 SCAF14995, whole genome shotgun : tr $\mid$ C3KJB4|C3KJB4_ANOFI Leydig cell tumor $10 \mathrm{kDa}$ protein homolog 0S=Ar tr $\mid$ Q4S3L1 |Q4S3L1_TETNG Chromosome 1 SCAF14749, whole genome shotgun : tr $\mid$ B5X193|B5X193_SALSA Proliferation-associated protein 2G4 0S=Salmo $\operatorname{tr} \mid$ C3KHF2 $\mid$ C3KHF2_ANOFI Ubiquitin-1ike protein 4A OS=Anoplopoma fimbr: tr|D3TJL7|D3TJL7_EPICO Glutathione S-transferase OS=Epinephelus coio: tr $\mid$ C3KI11|C3KI11_ANOFI NADH dehydrogenase 1 alpha subcomplex subunit $\operatorname{tr} \mid$ Q6PBS0 $\mid$ Q6PBS0_DANRE Required for meiotic nuclear division 5 homolc sp $\mid$ Q803V3|P2R3C_DANRE Serine/threonine-protein phosphatase 2A regula1

tr|D1KY93|D1KY93_9CICH Oncogene DJ-1 OS=Maylandia sp. 'Kompakt Mbambc $\operatorname{tr} \mid$ C3KIS7|C3KIS7_ANOFI Mitochondrial fission 1 protein OS=Anoplopoma

tr $\mid$ Q4RHZ9|Q4RHZ9_TETNG Chromosome 8 SCAF15044, whole genome shotgun ؛ $\operatorname{tr} \mid$ Q6DBQ6 | Q6DBQ6_DANRE Zgc:92043 OS=Danio rerio GN=caprin1a PE=1 SV= $\operatorname{tr} \mid$ Q6PFS4 $\mid$ Q6PFS4_DANRE Inner membrane protein, mitochondrial (Mitofi: $\operatorname{tr} \mid$ B5DG05 |B5DG05_SALSA Serine/threonine-protein phosphatase 0S=Salmo tr $\mid$ Q9W6E6 $\mid$ Q9W6E6_9TELE Glutamine synthetase 0S=0psanus beta GN=GSase 
UN_ci_6531

UN_ci_5646

UN_ci_8157

UN_ci_5261

UN_ci_3141

UN_ci_1397

UN_ci_5872

UN_ci_8187

UN_ci_6636

UN_ci_4669

UN_ci_8614

UN_ci_2565

UN_ci_8541

UN_ci_7616

UN_ci_10

UN_ci_7163

UN_ci_7697

UN_ci_369

UN_ci_7472

UN_ci_294

UN_ci_307

UN_ci_1057

UN_ci_4771

UN_ci_2333

UN_ci_8319

UN_ci_1425

UN_ci_6171

UN_ci_8006

UN_ci_4751

UN_ci_4081

UN_ci_2854

UN_ci_8050

UN_ci_7283

UN_ci_5829

UN_ci_3585

UN_ci_48

UN_ci_5546

UN_ci_2932

UN_ci_4260

UN_ci_7837

UN_ci_2560

UN_ci_4738

UN_ci_1047

UN_ci_1717

UN_ci_7040

UN_ci_4105

UN_ci_7527

UN_ci_7571

UN_ci_4926 tr $\mid$ C3KK02 $\mid$ C3KK02_ANOFI UPF0414 transmembrane protein C20orf30 homolo

$\operatorname{tr} \mid$ COHBN2 $\mid$ COHBN2_SALSA Pre-mRNA-processing factor 19 OS=Salmo salar ( tr $\mid$ C1BKP9|C1BKP9_0SMM0 60S ribosomal protein L8 0S=0smerus mordax GN= tr $\mid$ Q4SLY5|Q4SLY5_TETNG Chromosome 13 SCAF14555, whole genome shotgun tr $\mid$ Q4SIZ7 $\mid$ Q4SIZ7_TETNG Chromosome 21 SCAF14577, whole genome shotgun tr $\mid$ A0EZW3 $\mid$ A0EZW3_PSEMX Receptor for activated protein kinase C (Fragr

tr $\mid$ A0EZYO $\mid$ A0EZYO_PSEMX Adenine phosphoribosyl transferase (Fragment) $\operatorname{tr} \mid$ Q8AYL1 $\mid$ Q8AYL1_ORYLA ZPC2 (Fragment) OS=0ryzias latipes PE=4 SV=1

tr $\mid$ Q4SBZ6 $\mid$ Q4SBZ6_TETNG Chromosome 14 SCAF14660, whole genome shotgun tr $\mid$ C1BKN5|C1BKN5_0SMM0 B-cel1 receptor-associated protein 29 0S=0sme] sp |Q803M5 $\mid$ SAAL1_DANRE Protein saal1 OS=Danio rerio GN=saal1 $\mathrm{PE}=2 \mathrm{SV}=\_$ tr $\mid$ Q4S6V3 $\mid$ Q4S6V3_TETNG T-complex protein 1 subunit alpha (Fragment) (

$\operatorname{tr} \mid$ Q6TEL5 $\mid$ Q6TEL5_DANRE Cullin 3 OS=Danio rerio GN=cul3a PE=2 SV=1

tr $\mid$ Q4S358|Q4S358_TETNG Chromosome 4 SCAF14752, whole genome shotgun : tr $\mid$ Q1PS66 $\mid$ Q1PS66_LATCA Deleted in azoospermia-like protein 0S=Lates ( tr $\mid$ Q4VBR8 $\mid$ Q4VBR8_DANRE Proteasome subunit alpha type OS=Danio rerio ( $\operatorname{tr} \mid$ C1J0J0 $\mid$ C1J0J0_GILSE ATP synthase subunit beta (Fragment) OS=Gi11ic

sp |Q2YDS5 |SCNM1_DANRE Sodium channe1 modifier 1 OS=Danio rerio GN=scr tr $\mid$ A5D6V4 $\mid$ A5D6V4_DANRE Zgc: 163141 protein OS=Danio rerio GN=zgc:1631 tr $\mid$ C1BM02 $\mid$ C1BM02_0SMM0 60S ribosomal protein L5 OS=0smerus mordax GN=

$\operatorname{tr} \mid$ Q08AY4 $\mid$ Q08AY4_XENLA LOC100158386 protein OS=Xenopus laevis GN=sfrs sp $\mid$ Q7ZWW7 $\mid$ TS31B_XENLA Tetraspanin-31-B OS=Xenopus laevis GN=tspan31-l tr|C3KK45|C3KK45_ANOFI Ribosome maturation protein SBDS 0S=Anoplopom

$\operatorname{tr} \mid$ Q6DHE6 |Q6DHE6_DANRE Lysy1-tRNA synthetase 0S=Danio rerio GN=kars I $\operatorname{tr} \mid$ C1BW85 $\mid$ C1BW85_ES0LU Lin-37 homolog OS=Esox lucius GN=LIN37 PE=2 SI tr|Q4RS99|Q4RS99_TETNG Chromosome 13 SCAF15000, whole genome shotgun tr|Q4RSW9|Q4RSW9_TETNG Chromosome 12 SCAF14999, whole genome shotgun

$\operatorname{tr} \mid$ Q67EQ7 $\mid$ Q67EQ7_ORYLA Zinc finger protein 183 OS=0ryzias latipes PE= $\operatorname{tr} \mid$ C3KHM3 $\mid$ C3KHM3_ANOFI 39S ribosomal protein L30, mitochondrial OS=Ar $\operatorname{tr} \mid$ B5X6W2 $\mid$ B5X6W2_SALSA Cystatin-B OS=Salmo salar GN=CYTB PE=4 SV=1 $\operatorname{tr} \mid$ A3QJW5 $\mid$ A3QJW5_DANRE CTP synthase OS=Danio rerio GN=ctps $\mathrm{PE}=3 \mathrm{SV}=1$ $\operatorname{tr} \mid$ Q6DC52 $\mid$ Q6DC52_DANRE Zgc:101107 OS=Danio rerio GN=Zgc:101107 PE=2 ؛ $\operatorname{tr} \mid$ C0H961|COH961_SALSA Vacuolar protein sorting-associated protein 3: tr|B5X958|B5X958_SALSA 0steoclast-stimulating factor 1 OS=Salmo sala] sp|Q7T2A5|EIF3L_DANRE Eukaryotic translation initiation factor 3 subi tr $\mid$ C3KH72 $\mid$ C3KH72_ANOFI Probable tRNAHis guanylyltransferase 0S=Anoplc tr $\mid$ Q4TC18|Q4TC18_TETNG Chromosome 21 SCAF7061, whole genome shotgun ؛

tr $\mid$ Q4RU94 |Q4RU94_TETNG Chromosome 1 SCAF14995, whole genome shotgun ؛ 
IN=RL36A PE=3 SV=1

ria $\mathrm{GN}=\mathrm{RL} 37 \mathrm{~A} \quad \mathrm{PE}=4 \mathrm{SV}=1$

orhynchus mykiss $\mathrm{PE}=4 \mathrm{SV}=1$

sequence $0 \mathrm{~S}=$ Tetraodon nigroviridis GN=GSTENG00022568001 PE=4 SV=1

$41 \quad \mathrm{PE}=2 \mathrm{SV}=1$

ides $\mathrm{PE}=2 \mathrm{SV}=1$

j=Ailuropoda melanoleuca GN=PANDA_004337 PE=3 SV=1

IN=RPL27a PE=2 SV=1

$=1$

$=\mathrm{RL} 7 \quad \mathrm{PE}=2 \quad \mathrm{SV}=1$

$2 \mathrm{PE}=4 \mathrm{SV}=1$

$2 \mathrm{SV}=1$

$=4 \mathrm{SV}=1$

sequence. (Fragment) OS=Tetraodon nigroviridis GN=GSTENG00013131001 PE=4 SV=1

ia $\mathrm{GN}=\mathrm{RL} 30 \mathrm{PE}=4 \mathrm{SV}=1$

$\mathrm{V}=\mathrm{RPL} 11 \quad \mathrm{PE}=2 \quad \mathrm{SV}=1$

$=4 \mathrm{SV}=1$

उN=RPL13a-1ike1 PE=2 SV=1

rca flavescens $\mathrm{PE}=2 \mathrm{SV}=1$

xima $\mathrm{PE}=2 \mathrm{SV}=1$

sunit E OS=Anoplopoma fimbria GN=EIF3E PE=2 SV=1

is $\mathrm{PE}=2 \mathrm{SV}=1$

э $\mathrm{GN}=\mathrm{RS} 9 \mathrm{PE}=2 \mathrm{SV}=1$

relus coioides $\mathrm{PE}=2 \mathrm{SV}=1$

rdax GN=ATIF1 PE=4 SV=1

2 shotgun sequence $0 \mathrm{~S}=$ Tetraodon nigroviridis GN=GSTENG00004276001 PE=4 SV=1

$=2 \mathrm{SV}=1$

IN $=$ RPL18a $\quad P E=2 \quad S V=1$

ia $\mathrm{GN}=\mathrm{RS} 28 \mathrm{PE}=4 \mathrm{SV}=1$

sequence. (Fragment) OS=Tetraodon nigroviridis GN=GSTENG00017018001 PE=3 SV=1

zus $\mathrm{GN}=\operatorname{rp} 118 \mathrm{PE}=2 \mathrm{SV}=2$

ides $\mathrm{PE}=2 \mathrm{SV}=1$

salar $\mathrm{GN}=\mathrm{CAPZB} \mathrm{PE}=2 \mathrm{SV}=1$

$\mathrm{V}=\mathrm{RPL} 14 \quad \mathrm{PE}=2 \quad \mathrm{SV}=1$ 
is $\mathrm{GN}=\mathrm{rp} 128 \mathrm{PE}=2 \mathrm{SV}=1$

$2 \mathrm{SV}=1$

$=\operatorname{rpS} 2 \mathrm{PE}=2 \mathrm{SV}=1$

$\mathrm{V}=\mathrm{rpS} 25 \quad \mathrm{PE}=2 \quad \mathrm{SV}=1$

$\mathrm{V}=\mathrm{rps} 27 \mathrm{a} \quad \mathrm{PE}=2 \mathrm{SV}=1$

ia $\mathrm{GN}=\mathrm{EF} 1 \mathrm{D} \quad \mathrm{PE}=2 \mathrm{SV}=1$

$\mathrm{V}=\mathrm{RPL} 26 \quad \mathrm{PE}=2 \mathrm{SV}=1$

is $\mathrm{GN}=\mathrm{rpS} 12 \mathrm{PE}=2 \mathrm{SV}=1$

viridis GN=GSTENG00003377001 PE=3 SV=1

? shotgun sequence. (Fragment) OS=Tetraodon nigroviridis GN=GSTENG00004090001 PE=4 SV=1

is mordax GN=TIP1 PE=2 SV=1

$=1$

sequence OS=Tetraodon nigroviridis GN=GSTENG00015941001 PE=4 SV=1

$\mathrm{PE}=2 \mathrm{SV}=1$

. $\mathrm{OS}=0$ smerus mordax $\mathrm{GN}=\mathrm{GHITM} \mathrm{PE}=2 \mathrm{SV}=1$

io $\mathrm{GN}=\mathrm{rab} 7 \mathrm{PE}=2 \mathrm{SV}=1$

splopoma fimbria GN=TXND9 PE=2 SV=1

ne shotgun sequence OS=Tetraodon nigroviridis GN=GSTENG00034503001 PE=3 SV=1

?E $=2 \quad \mathrm{SV}=1$

ria GN=MYL6 $\mathrm{PE}=2 \mathrm{SV}=1$

x $0 \mathrm{~S}=$ Psetta maxima $\mathrm{GN}=$ tpt $1 \quad \mathrm{PE}=2 \quad \mathrm{SV}=1$

$=2 \mathrm{SV}=1$

ia GN=TM50A PE=2 SV=1

rerio GN=bbip10 $\mathrm{PE}=2 \mathrm{SV}=1$

?DX PE=2 SV=1

9, mitochondrial 0S=Anoplopoma fimbria GN=NDUA9 PE=2 SV=1

sequence. (Fragment) OS=Tetraodon nigroviridis GN=GSTENG00033388001 PE=3 SV=1

sequence. (Fragment) OS=Tetraodon nigroviridis GN=GSTENG00028388001 PE=4 SV=1

$2 \mathrm{SV}=1$

$=2 \mathrm{SV}=2$

sequence. (Fragment) OS=Tetraodon nigroviridis GN=GSTENG00027932001 PE=4 SV=1

sequence OS=Tetraodon nigroviridis GN=GSTENG00023858001 PE=4 SV=1

sequence. (Fragment) OS=Tetraodon nigroviridis GN=GSTENG00016829001 PE=4 SV=1 
$\mathrm{V}=\mathrm{RL} 19$ PE=2 SV=1

sequence. (Fragment) OS=Tetraodon nigroviridis GN=GSTENG00027102001 PE=4 SV=1

$\mathrm{i}=1$

s $\mathrm{GN}=\mathrm{rp} 19 \mathrm{PE}=2 \mathrm{SV}=1$

rus mordax $\mathrm{GN}=\mathrm{CHMP} 3 \mathrm{PE}=2 \mathrm{SV}=1$

ivaceus $\mathrm{PE}=2 \mathrm{SV}=1$

ว $\mathrm{E}$ OS=Danio rerio $\mathrm{GN}=$ polr2eb $\mathrm{PE}=2 \mathrm{SV}=1$

rotein-1ike 1 OS=0smerus mordax GN=GRL1 PE=3 SV=1

sequence. (Fragment) OS=Tetraodon nigroviridis GN=GSTENG00035051001 PE=4 SV=1

$\mathrm{GN}=\mathrm{COPZ} 1 \quad \mathrm{PE}=2 \mathrm{SV}=1$

s mykiss GN=LYRM2 $\mathrm{PE}=4 \mathrm{SV}=1$

10 (Fragment) $0 \mathrm{~S}=$ Salmo salar GN=TMEDA $\mathrm{PE}=2 \mathrm{SV}=1$

$30 \quad \mathrm{PE}=2 \mathrm{SV}=1$

$\mathrm{V}=\operatorname{rps} 16 \quad \mathrm{PE}=2 \mathrm{SV}=1$

$\mathrm{GN}=\mathrm{si}: \mathrm{dkey}-46 \mathrm{a} 10.3 \mathrm{PE}=2 \mathrm{SV}=1$

$=\mathrm{RL} 7 \mathrm{~L} \quad \mathrm{PE}=2 \mathrm{SV}=1$

ə maxima $\mathrm{PE}=2 \mathrm{SV}=1$

shelus coioides $\mathrm{PE}=2 \mathrm{SV}=1$

sequence OS=Tetraodon nigroviridis GN=GSTENG00034700001 PE=4 SV=1

at) $0 \mathrm{~S}=$ Paralichthys olivaceus $\mathrm{GN}=\mathrm{Cctz} \mathrm{PE}=2 \mathrm{SV}=1$

sequence. (Fragment) OS=Tetraodon nigroviridis GN=GSTENG00019795001 PE=3 SV=1

lar $\mathrm{GN}=\mathrm{rab2a} \mathrm{PE}=2 \mathrm{SV}=1$

sequence OS=Tetraodon nigroviridis GN=GSTENG00033341001 PE=4 SV=1

sequence. (Fragment) OS=Tetraodon nigroviridis GN=GSTENG00020129001 PE=4 SV=1

OS=Danio rerio GN=ndufs $1 \mathrm{PE}=2 \mathrm{SV}=1$

sequence. (Fragment) OS=Tetraodon nigroviridis GN=GSTENG00017779001 PE=4 SV=1

ia $\mathrm{GN}=W B S 22 \mathrm{PE}=2 \mathrm{SV}=1$

Junit 12 OS=0ncorhynchus mykiss GN=IF3C PE=2 SV=1

.oplopoma fimbria GN=RM16 PE=2 SV=1

$\mathrm{V}=\mathrm{rpS} 17 \quad \mathrm{PE}=2 \quad \mathrm{SV}=1$

əochromis mossambicus $\mathrm{PE}=2 \mathrm{SV}=1$

$\mathrm{PE}=2 \mathrm{SV}=1$

s2 $\mathrm{PE}=2 \mathrm{SV}=1$

lopoma fimbria $\mathrm{GN}=\mathrm{TFAM} \mathrm{PE}=2 \mathrm{SV}=1$

unit I OS=Danio rerio $\mathrm{GN}=$ eif3i $\mathrm{PE}=2 \mathrm{SV}=1$ 
$15 \quad \mathrm{PE}=2 \mathrm{SV}=1$

ys olivaceus GN=VDAC $\mathrm{PE}=2 \mathrm{SV}=1$

sequence. (Fragment) OS=Tetraodon nigroviridis GN=GSTENG00023614001 PE=4 SV=1

j OS=Anoplopoma fimbria GN=NDUB6 PE=2 SV=1

ne shotgun sequence OS=Tetraodon nigroviridis GN=GSTENG00011891001 PE=4 SV=1

s $\mathrm{GN}=\mathrm{L} 0 \mathrm{C} 444845 \mathrm{PE}=2 \mathrm{SV}=1$

imbria $\mathrm{GN}=\mathrm{PSA} 3 \mathrm{PE}=2 \mathrm{SV}=1$

sequence. (Fragment) OS=Tetraodon nigroviridis GN=GSTENG00014161001 PE=4 SV=1

rus mordax $\mathrm{GN}=\mathrm{CNBP} \mathrm{PE}=2 \mathrm{SV}=1$

sequence. (Fragment) OS=Tetraodon nigroviridis GN=GSTENG00031216001 PE=4 SV=1

shuatsi $\mathrm{PE}=3 \mathrm{SV}=1$

7 like 2 OS=Salmo salar $\mathrm{PE}=2$ SV=1

ə gen. sp. $\mathrm{PE}=4 \mathrm{SV}=1$

sequence. (Fragment) OS=Tetraodon nigroviridis GN=GSTENG00017123001 PE=4 SV=1

sequence OS=Tetraodon nigroviridis GN=GSTENG00034902001 PE=4 SV=1

v=TUSC 4 PE=2 SV=1

ne shotgun sequence OS=Tetraodon nigroviridis GN=GSTENG00020488001 PE=4 SV=1

$\mathrm{jV}=1$

OS=Danio rerio $\mathrm{GN}=\mathrm{dcp} 2 \mathrm{PE}=2 \mathrm{SV}=1$

spicalis GN=LOC100170503 PE=2 SV=1

$\mathrm{i}=1$

ycosyltransferase subunit STT3A OS=Salmo salar GN=STT3A PE=2 SV=1

dbunit RPABC5 0S=Anoplopoma fimbria GN=RPAB5 PE=3 SV=1

$r$ GN=PSMG1 $\mathrm{PE}=2 \mathrm{SV}=1$

sequence OS=Tetraodon nigroviridis GN=GSTENG00028795001 PE=4 SV=1

.oplopoma fimbria GN=L10K PE=4 SV=1

sequence OS=Tetraodon nigroviridis GN=GSTENG00024602001 PE=4 SV=1

salar GN=PA2G4 $\mathrm{PE}=2 \mathrm{SV}=1$

ia $\mathrm{GN}=\mathrm{UBL} 4 \mathrm{~A} \quad \mathrm{PE}=2 \mathrm{SV}=1$

ides $\mathrm{PE}=2 \mathrm{SV}=1$

1 OS=Anoplopoma fimbria GN=NDUA1 PE=4 SV=1

sg B (S. cerevisiae) $0 \mathrm{~S}=$ Danio rerio $\mathrm{GN}=\mathrm{rmnd5b} \mathrm{PE}=2 \mathrm{SV}=1$

tory subunit B' subunit gamma OS=Danio rerio GN=ppp2r3c PE=2 SV=1

ə Bay' GN=Dj1 PE=2 SV=1

fimbria $\mathrm{GN}=\mathrm{FIS} 1 \mathrm{PE}=2 \mathrm{SV}=1$

sequence. (Fragment) OS=Tetraodon nigroviridis GN=GSTENG00034101001 PE=4 SV=1

1

lin) $0 \mathrm{~S}=$ Danio rerio $\mathrm{GN}=$ immt $\mathrm{PE}=2 \mathrm{SV}=1$

salar $\mathrm{GN}=\mathrm{ppp} 1 \mathrm{cc} \mathrm{PE}=2 \mathrm{SV}=1$

$\mathrm{PE}=2 \mathrm{SV}=1$ 
ร. OS=Anoplopoma fimbria GN=CT030 PE=4 SV=1

3N=PRP19 PE=2 SV=1

$=$ RL8 PE $=2 \quad \mathrm{SV}=1$ sequence. (Fragment) OS=Tetraodon nigroviridis GN=GSTENG00016015001 PE=4 SV=1 sequence OS=Tetraodon nigroviridis GN=GSTENG00017418001 PE=4 SV=1 nent) $0 \mathrm{~S}=$ Psetta maxima $\mathrm{PE}=2 \mathrm{SV}=1$

OS=Psetta maxima PE=2 SV=1

sequence OS=Tetraodon nigroviridis GN=GSTENG00020739001 PE=3 SV=1 rus mordax GN=BAP29 $\mathrm{PE}=2 \mathrm{SV}=1$

2

JS=Tetraodon nigroviridis GN=GSTENG00023111001 PE=3 SV=1

sequence OS=Tetraodon nigroviridis GN=GSTENG00024782001 PE=4 SV=1

zalcarifer $\mathrm{GN}=$ dazl $\mathrm{PE}=2 \mathrm{SV}=1$

$3 \mathrm{~N}=\mathrm{psma} 2 \mathrm{PE}=2 \mathrm{SV}=1$

shthys seta $\mathrm{PE}=2 \mathrm{SV}=1$

.m1 $\mathrm{PE}=2 \quad \mathrm{SV}=1$

$41 \quad \mathrm{PE}=2 \mathrm{SV}=1$

=RL5 PE=2 SV=1

s12ip1 PE=2 SV=1

ว $\mathrm{PE}=2 \mathrm{SV}=1$

ə fimbria GN=SBDS PE=2 SV=1

E $=2 \quad \mathrm{SV}=1$

$\mathrm{V}=1$

sequence. (Fragment) OS=Tetraodon nigroviridis GN=GSTENG00029800001 PE=4 SV=1 sequence. (Fragment) OS=Tetraodon nigroviridis GN=GSTENG00029514001 PE=4 SV=1

$=2 \quad \mathrm{SV}=1$

.oplopoma fimbria GN=RM30 PE=2 SV=1

jV $=1$

3A OS=Salmo salar GN=VP33A PE=2 SV=1

$r$ GN=0STF1 PE=2 SV=1

unit $\mathrm{L}$ OS=Danio rerio $\mathrm{GN}=$ eif31 $\mathrm{PE}=2 \mathrm{SV}=1$

spoma fimbria GN=THG1 PE=2 SV=1

sequence 0S=Tetraodon nigroviridis GN=GSTENG00003506001 PE=4 SV=1

sequence OS=Tetraodon nigroviridis GN=GSTENG00028899001 PE=4 SV=1 


\begin{tabular}{|c|c|c|c|}
\hline Unigene_ref_ID & xiong_expression & ci_expression & Fisher_p_value xiong_unigene \\
\hline Unigene_UN_xiong_14070 & 165 & 7554 & 0 UN_xiong_14070 \\
\hline Unigene_UN_xiong_13607 & 360 & 5649 & 0 UN_xiong_13607 \\
\hline Unigene_UN_xiong_11196 & 273 & 19628 & 0 UN_xiong_11196 \\
\hline Unigene_UN_xiong_7164 & 203 & 10946 & 0 UN_xiong_7164 \\
\hline Unigene_UN_xiong_1987 & 283 & 5118 & 0 UN_xiong_1987 \\
\hline Unigene_UN_xiong_5287 & 108 & 5966 & 0 UN_xiong_5287 \\
\hline Unigene_UN_xiong_11113 & 218 & 7116 & 0 UN_xiong_11113 \\
\hline Unigene_UN_xiong_4091 & 1332 & 68944 & 0 UN_xiong_4091 \\
\hline Unigene_UN_xiong_14868 & 155 & 3621 & 0 UN_xiong_14868 \\
\hline Unigene_UN_xiong_14272 & 233 & 4341 & 0 UN_xiong_14272 \\
\hline Unigene_UN_xiong_14842 & 502 & 4128 & 0 UN_xiong_14842 \\
\hline Unigene_UN_xiong_11680 & 139 & 5026 & 0 UN_xiong_11680 \\
\hline Unigene_UN_xiong_12139 & 244 & 4019 & 0 UN_xiong_12139 \\
\hline Unigene_UN_xiong_5130 & 202 & 10038 & 0 UN_xiong_5130 \\
\hline Unigene_UN_xiong_13569 & 4533 & 53675 & 0 UN_xiong_13569 \\
\hline Unigene_UN_xiong_13925 & 1128 & 12535 & 0 UN_xiong_13925 \\
\hline Unigene_UN_xiong_9552 & 233 & 3108 & 0 UN_xiong_9552 \\
\hline Unigene_UN_xiong_14377 & 1386 & 46636 & 0 UN_xiong_14377 \\
\hline Unigene_UN_xiong_15032 & 466 & 4948 & 0 UN_xiong_15032 \\
\hline Unigene_UN_xiong_14718 & 246 & 12389 & 0 UN_xiong_14718 \\
\hline Unigene_UN_xiong_8517 & 687 & 4441 & 9. 73E-299 UN_xiong_8517 \\
\hline Unigene_UN_xiong_4795 & 253 & 2864 & 1.80E-294 UN_xiong_4795 \\
\hline Unigene_UN_xiong_15664 & 209 & 2414 & 2. 01E-251 UN_xiong_15664 \\
\hline Unigene_UN_xiong_2880 & 590 & 3689 & 2. 82E-240 UN_xiong_2880 \\
\hline Unigene_UN_xiong_14677 & 496 & 3268 & 1. 16E-224 UN_xiong_14677 \\
\hline Unigene_UN_xiong_4430 & 143 & 1933 & 2. 36E-218 UN_xiong_4430 \\
\hline Unigene_UN_xiong_3079 & 256 & 2269 & 2.22E-200 UN_xiong_3079 \\
\hline Unigene_UN_xiong_3128 & 320 & 2409 & 1.97E-187 UN_xiong_3128 \\
\hline Unigene_UN_xiong_335 & 839 & 3951 & 2.17E-181 UN_xiong_335 \\
\hline Unigene_UN_xiong_5549 & 262 & 2153 & 6. 11E-180 UN_xiong_5549 \\
\hline Unigene_UN_xiong_10799 & 155 & 1701 & 9. 17E-173 UN_xiong_10799 \\
\hline Unigene_UN_xiong_15130 & 1065 & 4408 & 3. 01E-164 UN_xiong_15130 \\
\hline Unigene_UN_xiong_14747 & 500 & 2772 & 1. 10E-158 UN_xiong_14747 \\
\hline Unigene_UN_xiong_4712 & 632 & 2997 & 1.33E-139 UN_xiong_4712 \\
\hline Unigene_UN_xiong_3787 & 195 & 1564 & 8.66E-129 UN_xiong_3787 \\
\hline Unigene_UN_xiong_122 & 661 & 2915 & 1.22E-121 UN_xiong_122 \\
\hline Unigene_UN_xiong_13451 & 119 & 1203 & 6. 88E-117 UN_xiong_13451 \\
\hline Unigene_UN_xiong_8419 & 240 & 1634 & 6.92E-117 UN_xiong_8419 \\
\hline Unigene_UN_xiong_15660 & 553 & 2562 & 1. 51E-115 UN_xiong_15660 \\
\hline Unigene_UN_xiong_6273 & 299 & 1811 & 3.33E-115 UN_xiong_6273 \\
\hline Unigene_UN_xiong_14414 & 2462 & 7151 & 2. 43E-112 UN_xiong_14414 \\
\hline Unigene_UN_xiong_2958 & 266 & 1616 & 2.69E-103 UN_xiong_2958 \\
\hline Unigene_UN_xiong_14270 & 220 & 1442 & 8. 25E-100 UN_xiong_14270 \\
\hline Unigene_UN_xiong_15687 & 309 & 1692 & 3. 04E-96 UN_xiong_15687 \\
\hline Unigene_UN_xiong_3634 & 293 & 1630 & 1. 88E-94 UN_xiong_3634 \\
\hline Unigene_UN_xiong_1060 & 279 & 1544 & 5. 09E-89 UN_xiong_1060 \\
\hline Unigene_UN_xiong_14618 & 1356 & 4284 & 4. 92E-88 UN_xiong_14618 \\
\hline Unigene_UN_xiong_15785 & 542 & 2215 & 9.73E-82 UN_xiong_15785 \\
\hline
\end{tabular}


Unigene_UN_xiong_8790

Unigene_UN_xiong_12115

Unigene_UN_xiong_2248

Unigene_UN_xiong_4643

Unigene_UN_xiong_7498

Unigene_UN_xiong_13515

Unigene_UN_xiong_3105

Unigene_UN_xiong_5545

Unigene_UN_xiong_7707

Unigene_UN_xiong_2218

Unigene_UN_xiong_1570

Unigene_UN_xiong_1588

Unigene_UN_xiong_5453

Unigene_UN_xiong_14347

Unigene_UN_xiong_4932

Unigene_UN_xiong_5737

Unigene_UN_xiong_11251

Unigene_UN_xiong_15308

Unigene_UN_xiong_14307

Unigene_UN_xiong_4359

Unigene_UN_xiong_14308

Unigene_UN_xiong_12070

Unigene_UN_xiong_9359

Unigene_UN_xiong_307

Unigene_UN_xiong_15554

Unigene_UN_xiong_14840

Unigene_UN_xiong_14658

Unigene_UN_xiong_15533

Unigene_UN_xiong_9570

Unigene_UN_xiong_4039

Unigene_UN_xiong_2199

Unigene_UN_xiong_4453

Unigene_UN_xiong_15219

Unigene_UN_xiong_1428

Unigene_UN_xiong_7852

Unigene_UN_xiong_14795

Unigene_UN_xiong_3203

Unigene_UN_xiong_3600

Unigene_UN_xiong_14835

Unigene_UN_xiong_3265

Unigene_UN_xiong_14710

Unigene_UN_xiong_14441

Unigene_UN_xiong_15587

Unigene_UN_xiong_5589

Unigene_UN_xiong_1812

Unigene_UN_xiong_14805

Unigene_UN_xiong_14932

Unigene_UN_xiong_14593

Unigene_UN_xiong_8117

Unigene_UN_xiong_14444

\begin{tabular}{|c|c|c|}
\hline 149 & 1048 & 7.50E-78 UN_xiong_8790 \\
\hline 243 & 1345 & 9. 99E-78 UN_xiong_12115 \\
\hline 463 & 1949 & 5. 62E-76 UN_xiong_2248 \\
\hline 279 & 1417 & 1. $44 \mathrm{E}-73$ UN_xiong_4643 \\
\hline 248 & 1303 & 1. 01E-70 UN_xiong_7498 \\
\hline 619 & 2300 & 1. 14E-70 UN_xiong_13515 \\
\hline 984 & 3163 & 1. 21E-68 UN_xiong_3105 \\
\hline 451 & 1844 & 2.44E-68 UN_xiong_5545 \\
\hline 338 & 1511 & 2.40E-65 UN_xiong_7707 \\
\hline 213 & 1139 & 1.55E-63 UN_xiong_2218 \\
\hline 777 & 2591 & 3. 77E-62 UN_xiong_1570 \\
\hline 397 & 1639 & 5.61E-62 UN_xiong_1588 \\
\hline 122 & 841 & 1.03E-61 UN_xiong_5453 \\
\hline 5749 & 12920 & 6. 20E-58 UN_xiong_14347 \\
\hline 681 & 2295 & 9. 76E-57 UN_xiong_4932 \\
\hline 336 & 1417 & 7.28E-56 UN_xiong_5737 \\
\hline 198 & 1028 & 1. 84E-55 UN_xiong_11251 \\
\hline 342 & 1419 & 2. 20E-54 UN_xiong_15308 \\
\hline 839 & 2621 & 6. 78E-53 UN_xiong_14307 \\
\hline 1567 & 1585 & 3. 70E-52 UN_xiong_4359 \\
\hline 2896 & 3434 & 7.06E-52 UN_xiong_14308 \\
\hline 204 & 996 & 1. 52E-49 UN_xiong_12070 \\
\hline 433 & 1569 & 2. 72E-46 UN_xiong_9359 \\
\hline 238 & 1059 & 6. 11E-46 UN_xiong_307 \\
\hline 1435 & 1495 & 1. 50E-43 UN_xiong_ 15554 \\
\hline 162 & 823 & 1. 93E-43 UN_xiong_14840 \\
\hline 392 & 1433 & 3. 20E-43 UN_xiong_14658 \\
\hline 152 & 792 & 3. 26E-43 UN_xiong_15533 \\
\hline 1245 & 1254 & 2.57E-42 UN_xiong_9570 \\
\hline 192 & 886 & 6. 19E-41 UN_xiong_4039 \\
\hline 165 & 807 & 1. 72E-40 UN_xiong_2199 \\
\hline 166 & 808 & 2. 22E-40 UN_xiong_4453 \\
\hline 1857 & 2117 & 3. 37E-40 UN_xiong_15219 \\
\hline 226 & 954 & 2.98E-38 UN_xiong_1428 \\
\hline 234 & 950 & 1. 12E-35 UN_xiong_7852 \\
\hline 585 & 1784 & 3. 74E-34 UN_xiong_14795 \\
\hline 1131 & 1187 & 6. 41E-34 UN_xiong_3203 \\
\hline 176 & 778 & 8.87E-34 UN_xiong_3600 \\
\hline 328 & 1170 & 8. 94E-34 UN_xiong_14835 \\
\hline 318 & 1130 & 2. 12E-32 UN_xiong_3265 \\
\hline 283 & 1040 & 3. 86E-32 UN_xiong_14710 \\
\hline 325 & 1144 & 4. 32E-32 UN_xiong_14441 \\
\hline 554 & 1672 & 3. 53E-31 UN_xiong_15587 \\
\hline 164 & 682 & 4. 31E-27 UN_xiong_5589 \\
\hline 529 & 1527 & 5.65E-25 UN_xiong_1812 \\
\hline 716 & 725 & 1. 15E-24 UN_xiong_14805 \\
\hline 1037 & 1185 & 5. 33E-23 UN_xiong_14932 \\
\hline 686 & 707 & 1. 83E-22 UN_xiong_14593 \\
\hline 315 & 1002 & 2. 63E-22 UN_xiong_8117 \\
\hline 1570 & 3640 & 1. $68 \mathrm{E}-21$ UN_xiong_14444 \\
\hline
\end{tabular}




\begin{tabular}{|c|c|c|c|}
\hline Unigene_UN_xiong_14995 & 338 & 1040 & 3.95E-21 UN_xiong_14995 \\
\hline Unigene_UN_xiong_14907 & 2105 & 2812 & 3.98E-20 UN_xiong_14907 \\
\hline Unigene_UN_xiong_2251 & 299 & 933 & 7.78E-20 UN_xiong_2251 \\
\hline Unigene_UN_xiong_13033 & 748 & 822 & 1.22E-19 UN_xiong_13033 \\
\hline Unigene_UN_xiong_13924 & 865 & 988 & 1.89E-19 UN_xiong_13924 \\
\hline Unigene_UN_xiong_14283 & 178 & 640 & 1.89E-19 UN_xiong_14283 \\
\hline Unigene_UN_xiong_1931 & 588 & 606 & 1.90E-19 UN_xiong_1931 \\
\hline Unigene_UN_xiong_2209 & 518 & 525 & 2.99E-18 UN_xiong_2209 \\
\hline Unigene_UN_xiong_1494 & 1096 & 1338 & 3.71E-18 UN_xiong_1494 \\
\hline Unigene_UN_xiong_14885 & 855 & 992 & 4. 42E-18 UN_xiong_14885 \\
\hline Unigene_UN_xiong_4084 & 319 & 955 & 4. 54E-18 UN_xiong_4084 \\
\hline Unigene_UN_xiong_15557 & 512 & 519 & 4. 57E-18 UN_xiong_15557 \\
\hline Unigene_UN_xiong_983 & 232 & 748 & 1. 20E-17 UN_xiong_983 \\
\hline Unigene_UN_xiong_3521 & 859 & 2102 & 2.09E-17 UN_xiong_3521 \\
\hline Unigene_UN_xiong_9694 & 89 & 389 & 2.32E-17 UN_xiong_9694 \\
\hline Unigene_UN_xiong_8569 & 1036 & 2445 & 1.36E-16 UN_xiong_8569 \\
\hline Unigene_UN_xiong_2099 & 665 & 751 & 5. 53E-16 UN_xiong_2099 \\
\hline Unigene_UN_xiong_4533 & 470 & 1243 & 3. 74E-15 UN_xiong_4533 \\
\hline Unigene_UN_xiong_1942 & 282 & 826 & 7.29E-15 UN_xiong_1942 \\
\hline Unigene_UN_xiong_14251 & 865 & 1054 & 8. 57E-15 UN_xiong_14251 \\
\hline Unigene_UN_xiong_13480 & 5608 & 8578 & 2. 75E-14 UN_xiong_13480 \\
\hline Unigene_UN_xiong_14486 & 560 & 629 & 6. 03E-14 UN_xiong_14486 \\
\hline Unigene_UN_xiong_12764 & 197 & 620 & 6. 38E-14 UN_xiong_12764 \\
\hline Unigene_UN_xiong_14409 & 766 & 929 & 1.43E-13 UN_xiong_14409 \\
\hline Unigene_UN_xiong_7832 & 158 & 522 & 2. 05E-13 UN_xiong_7832 \\
\hline Unigene_UN_xiong_10741 & 202 & 614 & 1.28E-12 UN_xiong_10741 \\
\hline Unigene_UN_xiong_14968 & 323 & 323 & 1.99E-12 UN_xiong_14968 \\
\hline Unigene_UN_xiong_8120 & 169 & 535 & 2. 29E-12 UN_xiong_8120 \\
\hline Unigene_UN_xiong_14784 & 551 & 642 & 5.75E-12 UN_xiong_14784 \\
\hline Unigene_UN_xiong_14325 & 537 & 627 & 1. 17E-11 UN_xiong_14325 \\
\hline Unigene_UN_xiong_11308 & 370 & 969 & 1. 18E-11 UN_xiong_11308 \\
\hline Unigene_UN_xiong_2360 & 412 & 452 & 1.49E-11 UN_xiong_2360 \\
\hline Unigene_UN_xiong_14260 & 124 & 417 & 1.99E-11 UN_xiong_14260 \\
\hline Unigene_UN_xiong_6441 & 176 & 536 & 2. 82E-11 UN_xiong_6441 \\
\hline Unigene_UN_xiong_14258 & 682 & 844 & 3. $69 \mathrm{E}-11$ UN_xiong_14258 \\
\hline Unigene_UN_xiong_9761 & 781 & 990 & 3. 82E-11 UN_xiong_9761 \\
\hline Unigene_UN_xiong_3864 & 288 & 293 & 1.04E-10 UN_xiong_3864 \\
\hline Unigene_UN_xiong_4247 & 156 & 481 & 1.39E-10 UN_xiong_4247 \\
\hline Unigene_UN_xiong_14780 & 354 & 384 & 1. 75E-10 UN_xiong_14780 \\
\hline Unigene_UN_xiong_14608 & 533 & 638 & 1.93E-10 UN_xiong_14608 \\
\hline Unigene_UN_xiong_8140 & 441 & 507 & 2. 35E-10 UN_xiong_8140 \\
\hline Unigene_UN_xiong_6452 & 449 & 519 & 2.36E-10 UN_xiong_6452 \\
\hline Unigene_UN_xiong_13318 & 232 & 648 & 2.45E-10 UN_xiong_13318 \\
\hline Unigene_UN_xiong_4599 & 198 & 571 & 3.26E-10 UN_xiong_4599 \\
\hline Unigene_UN_xiong_11376 & 286 & 297 & 4. 71E-10 UN_xiong_11376 \\
\hline Unigene_UN_xiong_14736 & 501 & 1210 & 4.97E-10 UN_xiong_14736 \\
\hline Unigene_UN_xiong_11967 & 671 & 856 & 1.70E-09 UN_xiong_11967 \\
\hline Unigene_UN_xiong_15503 & 1081 & 2346 & 2. 26E-09 UN_xiong_15503 \\
\hline Unigene_UN_xiong_15191 & 510 & 623 & 3. 34E-09 UN_xiong_15191 \\
\hline Unigene_UN_xiong_14002 & 259 & 271 & 4.89E-09 UN_xiong_14002 \\
\hline
\end{tabular}




\begin{tabular}{|c|c|c|c|}
\hline Unigene_UN_xiong_13661 & 147 & 437 & 7. 14E-09 UN_xiong_13661 \\
\hline Unigene_UN_xiong_3420 & 490 & 599 & 7. 48E-09 UN_xiong_3420 \\
\hline Unigene_UN_xiong_1167 & 331 & 373 & 9.43E-09 UN_xiong_1167 \\
\hline Unigene_UN_xiong_4244 & 236 & 245 & 1.87E-08 UN_xiong_4244 \\
\hline Unigene_UN_xiong_14687 & 385 & 454 & 1.98E-08 UN_xiong_14687 \\
\hline Unigene_UN_xiong_10360 & 220 & 592 & 2. $15 \mathrm{E}-08$ UN_xiong_10360 \\
\hline Unigene_UN_xiong_2788 & 248 & 262 & 2. 17E-08 UN_xiong_2788 \\
\hline Unigene_UN_xiong_15787 & 559 & 1291 & 2.23E-08 UN_xiong_15787 \\
\hline Unigene_UN_xiong_9146 & 226 & 234 & 2.80E-08 UN_xiong_9146 \\
\hline Unigene_UN_xiong_3902 & 147 & 428 & 3. 25E-08 UN_xiong_3902 \\
\hline Unigene_UN_xiong_13970 & 379 & 452 & 5. 87E-08 UN_xiong_13970 \\
\hline Unigene_UN_xiong_9643 & 266 & 681 & 6.08E-08 UN_xiong_9643 \\
\hline Unigene_UN_xiong_14326 & 950 & 1315 & 6. 56E-08 UN_xiong_14326 \\
\hline Unigene_UN_xiong_13845 & 417 & 508 & 7. 45E-08 UN_xiong_13845 \\
\hline Unigene_UN_xiong_12519 & 187 & 187 & 8. 77E-08 UN_xiong_12519 \\
\hline Unigene_UN_xiong_7476 & 194 & 525 & 9. 89E-08 UN_xiong_7476 \\
\hline Unigene_UN_xiong_1596 & 289 & 328 & 1.22E-07 UN_xiong_1596 \\
\hline Unigene_UN_xiong_8683 & 244 & 268 & 2. 38E-07 UN_xiong_8683 \\
\hline Unigene_UN_xiong_14548 & 199 & 208 & 2. $96 \mathrm{E}-07$ UN_xiong_14548 \\
\hline Unigene_UN_xiong_1247 & 898 & 1921 & 4. 21E-07 UN_xiong_1247 \\
\hline Unigene_UN_xiong_14663 & 260 & 648 & 7. 71E-07 UN_xiong_14663 \\
\hline Unigene_UN_xiong_29 & 251 & 629 & 8. 78E-07 UN_xiong_29 \\
\hline Unigene_UN_xiong_4038 & 344 & 818 & 1.03E-06 UN_xiong_4038 \\
\hline Unigene_UN_xiong_14374 & 198 & 516 & 1.08E-06 UN_xiong_14374 \\
\hline Unigene_UN_xiong_11502 & 179 & 187 & 1. 18E-06 UN_xiong_11502 \\
\hline Unigene_UN_xiong_14681 & 209 & 229 & 1.34E-06 UN_xiong_14681 \\
\hline Unigene_UN_xiong_7586 & 212 & 543 & 1.46E-06 UN_xiong_7586 \\
\hline Unigene_UN_xiong_273 & 248 & 284 & 1.64E-06 UN_xiong_273 \\
\hline Unigene_UN_xiong_14856 & 207 & 228 & 2.01E-06 UN_xiong_14856 \\
\hline Unigene_UN_xiong_1364 & 713 & 983 & 2.02E-06 UN_xiong_1364 \\
\hline Unigene_UN_xiong_14906 & 169 & 177 & 2. 43E-06 UN_xiong_14906 \\
\hline Unigene_UN_xiong_553 & 121 & 343 & 2.62E-06 UN_xiong_553 \\
\hline Unigene_UN_xiong_15519 & 193 & 210 & 2. 86E-06 UN_xiong_15519 \\
\hline Unigene_UN_xiong_5132 & 260 & 305 & 3. 33E-06 UN_xiong_5132 \\
\hline Unigene_UN_xiong_4081 & 176 & 458 & 4.36E-06 UN_xiong_4081 \\
\hline Unigene_UN_xiong_4172 & 420 & 955 & 5.41E-06 UN_xiong_4172 \\
\hline Unigene_UN_xiong_7949 & 149 & 399 & 5. 55E-06 UN_xiong_7949 \\
\hline Unigene_UN_xiong_2313 & 222 & 254 & 5. 59E-06 UN_xiong_2313 \\
\hline Unigene_UN_xiong_6173 & 210 & 237 & 5.63E-06 UN_xiong_6173 \\
\hline Unigene_UN_xiong_1756 & 303 & 371 & 5.78E-06 UN_xiong_1756 \\
\hline Unigene_UN_xiong_2622 & 351 & 442 & 6. 40E-06 UN_xiong_2622 \\
\hline Unigene_UN_xiong_4067 & 143 & 147 & 7.62E-06 UN_xiong_4067 \\
\hline Unigene_UN_xiong_8071 & 255 & 618 & 7.62E-06 UN_xiong_8071 \\
\hline Unigene_UN_xiong_139 & 292 & 357 & 8. 27E-06 UN_xiong_139 \\
\hline Unigene_UN_xiong_10103 & 296 & 364 & 9. 85E-06 UN_xiong_10103 \\
\hline Unigene_UN_xiong_12679 & 420 & 946 & 1. 12E-05 UN_xiong_12679 \\
\hline Unigene_UN_xiong_14846 & 121 & 332 & 1.30E-05 UN_xiong_14846 \\
\hline Unigene_UN_xiong_4693 & 66 & 209 & 1.37E-05 UN_xiong_4693 \\
\hline Unigene_UN_xiong_1005 & 156 & 404 & 1.96E-05 UN_xiong_1005 \\
\hline Unigene_UN_xiong_3935 & 264 & 322 & 2.03E-05 UN_xiong_3935 \\
\hline
\end{tabular}




\begin{tabular}{|c|c|c|c|}
\hline Unigene_UN_xiong_2996 & 274 & 337 & 2.08E-05 UN_xiong_2996 \\
\hline Unigene_UN_xiong_10941 & 405 & 533 & 2. 16E-05 UN_xiong_10941 \\
\hline Unigene_UN_xiong_15055 & 120 & 324 & 3.24E-05 UN_xiong_15055 \\
\hline Unigene_UN_xiong_1512 & 278 & 349 & 4. 63E-05 UN_xiong_1512 \\
\hline Unigene_UN_xiong_14208 & 1351 & 2697 & 5. 68E-05 UN_xiong_14208 \\
\hline Unigene_UN_xiong_14470 & 380 & 846 & 6. 85E-05 UN_xiong_14470 \\
\hline Unigene_UN_xiong_8647 & 122 & 322 & 7.61E-05 UN_xiong_8647 \\
\hline Unigene_UN_xiong_4291 & 340 & 445 & 8. 19E-05 UN_xiong_4291 \\
\hline Unigene_UN_xiong_9232 & 192 & 465 & 0.000113842 UN_xiong_9232 \\
\hline Unigene_UN_xiong_14791 & 606 & 1279 & 0.000113925 UN_xiong_14791 \\
\hline Unigene_UN_xiong_14619 & 2290 & 4415 & 0.000115013 UN_xiong_14619 \\
\hline Unigene_UN_xiong_11417 & 110 & 114 & 0. 000123248 UN_xiong_11417 \\
\hline Unigene_UN_xiong_14042 & 202 & 482 & 0.000156752 UN_xiong_14042 \\
\hline Unigene_UN_xiong_5707 & 142 & 358 & 0. 000162563 UN_xiong_5707 \\
\hline Unigene_UN_xiong_5437 & 205 & 486 & 0. 000198153 UN_xiong_5437 \\
\hline Unigene_UN_xiong_14685 & 287 & 375 & 0. 000271018 UN_xiong_14685 \\
\hline Unigene_UN_xiong_8684 & 260 & 336 & 0.000340404 UN_xiong_8684 \\
\hline Unigene_UN_xiong_15755 & 962 & 1446 & 0. 000367605 UN_xiong_15755 \\
\hline Unigene_UN_xiong_13712 & 138 & 343 & 0.000371784 UN_xiong_13712 \\
\hline Unigene_UN_xiong_15511 & 719 & 1473 & 0. 000410546 UN_xiong_15511 \\
\hline Unigene_UN_xiong_14848 & 493 & 1041 & 0.000455935 UN_xiong_14848 \\
\hline Unigene_UN_xiong_13016 & 535 & 766 & 0. 000483569 UN_xiong_13016 \\
\hline Unigene_UN_xiong_9698 & 287 & 381 & 0.000534225 UN_xiong_9698 \\
\hline Unigene_UN_xiong_14737 & 367 & 504 & 0.000549823 UN_xiong_14737 \\
\hline Unigene_UN_xiong_1392 & 352 & 766 & 0. 000555159 UN_xiong_1392 \\
\hline Unigene_UN_xiong_55 & 236 & 313 & 0.001615934 UN_xiong_55 \\
\hline Unigene_UN_xiong_11332 & 269 & 366 & 0.002245386 UN_xiong_11332 \\
\hline Unigene_UN_xiong_14228 & 673 & 1356 & 0.002301629 UN_xiong_14228 \\
\hline Unigene_UN_xiong_6419 & 251 & 339 & 0.002354303 UN_xiong_6419 \\
\hline Unigene_UN_xiong_7955 & 98 & 113 & 0.003254974 UN_xiong_7955 \\
\hline Unigene_UN_xiong_4182 & 127 & 301 & 0.003544136 UN_xiong_4182 \\
\hline Unigene_UN_xiong_11475 & 226 & 496 & 0.004181513 UN_xiong_11475 \\
\hline Unigene_UN_xiong_12390 & 637 & 1276 & 0.004651094 UN_xiong_12390 \\
\hline Unigene_UN_xiong_6263 & 105 & 125 & 0.004844714 UN_xiong_6263 \\
\hline Unigene_UN_xiong_4289 & 280 & 392 & 0. 004998584 UN_xiong_4289 \\
\hline Unigene_UN_xiong_1206 & 158 & 204 & 0.005264702 UN_xiong_1206 \\
\hline Unigene_UN_xiong_1241 & 255 & 549 & 0.005331294 UN_xiong_1241 \\
\hline Unigene_UN_xiong_14951 & 96 & 234 & 0. 005945418 UN_xiong_14951 \\
\hline Unigene_UN_xiong_11755 & 277 & 589 & 0.006520803 UN_xiong_11755 \\
\hline Unigene_UN_xiong_14454 & 289 & 410 & 0. 007478477 UN_xiong_14454 \\
\hline Unigene_UN_xiong_5406 & 131 & 301 & 0. 008042897 UN_xiong_5406 \\
\hline Unigene_UN_xiong_2475 & 219 & 301 & 0.008141499 UN_xiong_2475 \\
\hline Unigene_UN_xiong_3161 & 187 & 255 & 0.011613861 UN_xiong_3161 \\
\hline Unigene_UN_xiong_13344 & 188 & 257 & 0. 011913152 UN_xiong_13344 \\
\hline Unigene_UN_xiong_3504 & 150 & 199 & 0. 012219281 UN_xiong_3504 \\
\hline Unigene_UN_xiong_2521 & 326 & 474 & 0. 012405025 UN_xiong_2521 \\
\hline Unigene_UN_xiong_13725 & 137 & 181 & 0. 014376075 UN_xiong_13725 \\
\hline Unigene_UN_xiong_14964 & 256 & 537 & 0. 016371574 UN_xiong_14964 \\
\hline Unigene_UN_xiong_12227 & 123 & 278 & 0. 016921315 UN_xiong_12227 \\
\hline Unigene UN xiong 15334 & 419 & 629 & $0.017471221 \mathrm{UN}$ xiong 1533 \\
\hline
\end{tabular}


Unigene_UN_xiong_5387
Unigene_UN_xiong_14520
Unigene_UN_xiong_4481
Unigene_UN_xiong_4168
Unigene_UN_xiong_4619
Unigene_UN_xiong_4204
Unigene_UN_xiong_5161
Unigene_UN_xiong_2186
Unigene_UN_xiong_12936
Unigene_UN_xiong_14512
Unigene_UN_xiong_15667
Unigene_UN_xiong_14590
Unigene_UN_xiong_7151
Unigene_UN_xiong_10090
Unigene_UN_xiong_7386

361

779

174

1238

239

247

205

418

168

964

200

529

194

247

179
536

1509

377

2348

502

515

289

838

233

1537

285

820

406

506

375
0.018248326 UN_xiong_5387

0. 018838957 UN_xiong_14520

0. 018875139 UN_xiong_4481

0. 018959565 UN_xiong_4168

0. 019779951 UN_xiong_4619

0. 021615442 UN_xiong_4204

0. 021844835 UN_xiong_5161

0. 022087708 UN_xiong_2186

0.025519663 UN_xiong_12936

0. 028951514 UN_xiong_14512

0.029863689 UN_xiong_15667

0. 033762136 UN_xiong_14590

0. 037636371 UN_xiong_7151

0. 040760726 UN_xiong_10090

0. 046839504 UN_xiong_7386 


\begin{tabular}{|c|c|}
\hline unigene & Swiss \\
\hline UN_ci_8324 & $\operatorname{tr} \mid$ Q4RBH1 $\mid$ Q4RBH1_TETNG Chromosome undetermined SCAF21483, whole genome \\
\hline UN_ci_8251 & tr|C3KGV9|C3KGV9_ANOFI Transcription elongation factor B polypeptide 10 \\
\hline UN_ci_4204 & $\operatorname{tr} \mid$ C3KJL5 $\mid$ C3KJL5_ANOFI Securin OS=Anoplopoma fimbria GN=PTTG1 PE=2 SV=1 \\
\hline UN_ci_8103 & $\operatorname{tr} \mid$ C3KIM6|C3KIM6_ANOFI Dynein 1ight chain 2, cytoplasmic 0S=Anoplopoma $f$ \\
\hline UN_ci_8019 & $\operatorname{tr} \mid$ C3KH05|C3KH05_ANOFI BUD31 homolog OS=Anoplopoma fimbria GN=BUD31 PE=2 \\
\hline UN_ci_8098 & $\operatorname{tr} \mid$ Q709D5 $\mid$ Q709D5_ORYLA Geminin OS=0ryzias latipes $\mathrm{PE}=2$ SV=1 \\
\hline UN_ci_7696 & $\operatorname{tr} \mid$ B7X8Y8|B7X8Y8_PAGMA Elongation factor 1 -alpha 0S=Pagrus major GN=42Sp \\
\hline UN_ci_6537 & $\operatorname{tr} \mid$ Q3ZLG2 $\mid$ Q3ZLG2_OREM0 Quinone reductase (Fragment) OS=0reochromis mossa \\
\hline UN_ci_6910 & $\operatorname{tr} \mid$ C1BIA8|C1BIA8_0NCMY Transcription initiation factor TFIID subunit 10 \\
\hline UN_ci_3721 & tr|C3KIM5|C3KIM5_ANOFI Epididymal secretory protein E1 OS=Anoplopoma fim \\
\hline UN_ci_7339 & $\operatorname{tr} \mid$ D6PVQ6|D6PVQ6_EPIC0 Mitochondrial ATP synthase lipid-binding protein \\
\hline UN_ci_8477 & \\
\hline UN_ci_7894 & 32|Q4RT32_TETNG Chromosome 12 SCAF14999, whole genome shotgun seq \\
\hline UN_ci_8317 & --- \\
\hline UN_ci_3186 & KK19|C3KK19_ANOFI Stathmin OS=Anoplopoma fimbria GN=STMN1 PE=2 SV=1 \\
\hline UN_ci_77720 & $\operatorname{tr} \mid$ C3UVG8 $\mid$ C3UVG8_9TELE Zona pellucida (Fragment) OS=Hypomesus transpacif \\
\hline UN_ci_8299 & $\operatorname{tr|B0V3V7|B0V3V7\_ DANRE~Guanine~nucleotide~binding~protein~alpha~subunit~}$ \\
\hline UN_ci_8490 & $\operatorname{tr} \mid$ Q27R93|Q27R93_FUNHE NAD (P)H dehydrogenase quinone 1 OS=Fundulus heter \\
\hline UN_ci_3128 & $\operatorname{tr} \mid$ Q2HYN5 $\mid$ Q2HYN5_ICTPU Translation factor sui1-1ike OS=Ictalurus punctat \\
\hline UN_ci_1523 & $\operatorname{tr} \mid$ Q4S564|Q4S564_TETNG Chromosome 6 SCAF14737, whole genome shotgun sequ \\
\hline UN_ci_7587 & $\operatorname{tr} \mid$ Q6DGD9|Q6DGD9_DANRE Trafficking protein particle complex 1 OS=Danio $r$ \\
\hline UN_ci_7945 & sp |Q7T3T9|ZAR1_TAKRU Zygote arrest protein 1 OS=Takifugu rubripes GN=zar \\
\hline UN_ci_8816 & $\operatorname{tr} \mid$ Q1KKY5|Q1KKY5_TAKRU Mitochondrial ATP synthase F0 complex subunit c i \\
\hline $\begin{array}{l}\text { GPGRCDD02H7PEA_ci } \\
\text { UN ci } 818\end{array}$ & tr|C1BJN5 $\mid$ C1BJN5_0SMM0 Cathepsin Z OS=0smerus mordax GN=CATZ PE=2 SV=1 \\
\hline UN_ci_8313 & LLS4|B9ELS4_SALSA Transmembrane protein 188 0S=Salmo sala \\
\hline UN_ci_8744 & protein OS=Danio rerio $\mathrm{GN}=\mathrm{L} 0 \mathrm{C} 51123 \mathrm{PE}=2 \mathrm{~S}$ \\
\hline UN_ci_6304 & $\operatorname{tr} \mid$ A0EZX2 $\mid$ A0EZX2_PSEMX Nucleoside diphosphate kinase (Fragment) OS=Psett \\
\hline UN_ci_8264 & $\operatorname{tr} \mid$ C3KI01|C3KI01_ANOFI Histone deacetylase complex subunit SAP18 0S=Anop \\
\hline UN_ci_1521 & $\operatorname{tr} \mid$ A3KQ59|A3KQ59_DANRE Nove1 protein similar to human and mouse ubiquiti \\
\hline UN_ci_8175 & $\operatorname{tr} \mid$ B5XE73|B5XE73_SALSA M-phase phosphoprotein 6 OS=Salmo salar GN=MPH6 P \\
\hline UN_ci_925 & $\operatorname{tr} \mid$ Q4T029|Q4T029_TETNG Chromosome undetermined SCAF11373, whole genome s \\
\hline UN_ci_2574 & $\operatorname{tr} \mid$ A0JPF1 $\mid$ A0JPF1_DANRE Zgc:153686 OS=Danio rerio GN=ubc PE=2 SV=1 \\
\hline & --- \\
\hline UN_ci_4781 & 1N7|B5T1N7_EPIC0 Mitochondrial cytochrome C oxidase subunit Vb (Fr \\
\hline UN_ci_8295 & $\operatorname{tr} \mid$ C3KIJ8|C3KIJ8_ANOFI Small nuclear ribonucleoprotein Sm D2 OS=Anoplopo \\
\hline UN_ci_5483 & $\operatorname{tr} \mid$ C3KIW9|C3KIW9_ANOFI Nuclear nucleic acid-binding protein C1D OS=Anop1 \\
\hline UN_ci_5288 & $\operatorname{tr} \mid$ Q4S6R2 $\mid$ Q4S6R2_TETNG Chromosome undetermined SCAF14724, whole genome s \\
\hline UN_ci_3921 & $\operatorname{tr} \mid$ C3KJ13|C3KJ13_ANOFI SRA stem-1oop-interacting RNA-binding protein, mi \\
\hline UN_ci_5805 & tr|Q4T9G5|Q4T9G5_TETNG Chromosome undetermined SCAF7571, whole genome sh \\
\hline UN_ci_239 & $\operatorname{tr} \mid$ C3KINO $\mid$ C3KINO_ANOFI GTP-binding nuclear protein Ran OS=Anoplopoma fim \\
\hline & --- \\
\hline UN_ci_7983 & $\operatorname{tr} \mid$ C3KIV4 $\mid$ C3KIV4_ANOFI FAM60A OS=Anoplopoma fiml \\
\hline UN_ci_3682 & $\operatorname{tr} \mid$ C3KJT2|C3KJT2_ANOFI NADH dehydrogenase 1 beta subcomplex subunit 5 , \\
\hline UN_ci_7946 & $\operatorname{tr} \mid$ C3KGU8|C3KGU8_ANOFI 40S ribosomal protein S8 0S=Anoplopoma fimbria GN \\
\hline UN_ci_7455 & $\operatorname{tr} \mid$ C3KJQ1|C3KJQ1_ANOFI Tumor suppressor candidate 2 OS=Anoplopoma fimbri \\
\hline UN_ci_7724 & $\operatorname{tr} \mid$ B9V3X3|B9V3X3_EPIC0 Muscle-specific beta 1 integrin binding protein 2 \\
\hline UN_ci_2481 & tr|Q4SD23|Q4SD23_TETNG Chromosome 14 SCAF14645, whole genome shotgun seq \\
\hline
\end{tabular}


UN_ci_8638

UN_ci_ 8221

UN_ci_8519

UN_ci_267

UN_ci_8256

UN_ci_8788

UN_ci_3655

UN_ci_8084

UN_ci_2057

UN_ci_2012

UN_ci_8493

UN_ci_7870

UN_ci_3905

UN_ci_8171

UN_ci_670

UN_ci_1772

UN_ci_8022

UN_ci_6115

UN_ci_7701

UN_ci 6001

UN_ci_7908

UN_ci_4671

UN_ci_7972

UN_ci_8506

UN_ci_7892

UN_ci_1277

UN_ci_6552

UN_ci_8250

UN_ci_3163

UN_ci_3692

UN_ci_5291

UN_ci_4439

UN_ci_257

UN_ci_8218

UN_ci_8468

UN_ci_8058

UN_ci_2242

UN_ci_8400

UN_ci_7356

UN_ci_4443

UN_ci_3927

UN_ci_8232

UN_ci_8163

UN_ci_7992

UN_ci_1380

UN_ci_8328

UN_ci_7661

UN_ci_8193

UN_ci_6119

UN_ci_8309 $\operatorname{tr} \mid$ Q4S1J7|Q4S1J7_TETNG Chromosome 6 SCAF14768, whole genome shotgun sequ tr $\mid$ D2CFU7 |D2CFU7_9PERC Superoxide dismutase [Cu-Zn] OS=Rachycentron cana tr $\mid$ Q4S9N2 $\mid$ Q4S9N2_TETNG Chromosome undetermined SCAF14696, whole genome s tr $\mid$ Q4RMT6 |Q4RMT6_TETNG Chromosome 3 SCAF15018, whole genome shotgun sequ $\operatorname{tr} \mid$ C3S171 $\mid$ C3S171_DANRE Glutathione peroxidase OS=Danio rerio GN=gpx4b PE tr $\mid$ C3KIH6|C3KIH6_ANOFI Chromobox protein homolog 3 OS=Anoplopoma fimbria tr $\mid$ D7PIW6 $\mid$ D7PIW6 PSEMX Ferritin OS=Psetta maxima GN=FerM PE=2 SV=1

$\operatorname{tr} \mid$ Q5RGQ1 |Q5RGQ1_DANRE Novel protein similar to vertebrate CD151 antigen tr $\mid$ Q4RJH9|Q4RJH9_TETNG Chromosome 3 SCAF15037, whole genome shotgun sequ

\section{$---$}

$\operatorname{tr} \mid$ Q800Z2|Q800Z2_PAROL Splicing factor arginine/serine-rich 3 OS=Paralic $\operatorname{tr} \mid$ A9ZT79|A9ZT79_SOLSE Ribosomal protein L6 OS=Solea senegalensis GN=RPL $\operatorname{tr} \mid$ Q90ZQ6 $\mid$ Q90ZQ6_ONCMY Polyubiquitin OS=0ncorhynchus mykiss PE=2 SV=1

$\operatorname{tr} \mid$ C3KH31 $\mid$ C3KH31_ANOFI EMI5 homolog, mitochondrial OS=Anoplopoma fimbria sp $\mid$ Q802F2 $\mid$ SELTA_DANRE Selenoprotein T1a OS=Danio rerio GN=selt1a PE=2 SV $\operatorname{tr} \mid$ Q3UBL7 $\mid$ Q3UBL7_MOUSE Putative uncharacterized protein (Fragment) OS=Mu $\operatorname{tr} \mid$ C1BXJ2|C1BXJ2_ESOLU 39S ribosomal protein L47, mitochondrial OS=Esox tr $\mid$ Q4T0S8|Q4T0S8_TETNG Chromosome undetermined SCAF10875, whole genome s $\operatorname{tr} \mid$ C1BJH9|C1BJH9_OSMMO ADP/ATP translocase 2 OS=0smerus mordax GN=ADT2 P $\operatorname{tr} \mid$ Q4V8Q4 $\mid$ Q4V8Q4_DANRE Zgc:114204 OS=Danio rerio GN=golt1b PE=2 SV=1 tr $\mid$ Q4RN49|Q4RN49_TETNG Chromosome undetermined SCAF15016, whole genome s

$\operatorname{tr} \mid$ C3KIQ6 $\mid$ C3KIQ6_ANOFI Myosin regulatory light chain 2, smooth muscle is

tr $\mid$ D2H9F9 $\mid$ D2H9F9_AILME Putative uncharacterized protein (Fragment) OS=Ai $\operatorname{tr} \mid$ Q4S5M1 $\mid$ Q4S5M1_TETNG Chromosome 9 SCAF14729, whole genome shotgun sequ $\operatorname{tr} \mid$ C3KIP5 $\mid$ C3KIP5_ANOFI Proteasome subunit beta type OS=Anoplopoma fimbri $\operatorname{tr} \mid$ C1BJ19|C1BJ19_0SMM0 Proteasome subunit beta type OS=0smerus mordax GN $\operatorname{tr} \mid$ Q4SJP1|Q4SJP1_TETNG Chromosome 1 SCAF14573, whole genome shotgun sequ tr|B5X8M2|B5X8M2_SALSA Mediator of RNA polymerase II transcription subun $\operatorname{tr} \mid$ Q8UVG1 |Q8UVG1_EPIC0 Ribosomal protein L32 0S=Epinephelus coioides PE= tr $\mid$ Q4RRE1|Q4RRE1_TETNG Chromosome 16 SCAF15002, whole genome shotgun seq tr $\mid$ C3KIU5 $\mid$ C3KIU5_ANOFI Transcription elongation factor SPT4 0S=Anoplopom $\operatorname{tr} \mid$ C3KJT1|C3KJT1_ANOFI High mobility group protein B2 OS=Anoplopoma fimb

tr $\mid$ Q4S7P2 $\mid$ Q4S7P2_TETNG Chromosome 18 SCAF14712, whole genome shotgun seq $\operatorname{tr} \mid$ B9EQ18|B9EQ18_SALSA CWC15 homolog 0S=Salmo salar GN=CWC15 PE=2 SV=1 tr $\mid$ B9EP79|B9EP79_SALSA Proteasome activator complex subunit 3 OS=Salmo s

tr $\mid$ COPUM3 $\mid$ COPUM3_SALSA ATP-dependent RNA helicase DDX39 (Fragment) OS=Sa tr|Q4S4D9|Q4S4D9_TETNG Chromosome 2 SCAF14738, whole genome shotgun sequ $\operatorname{tr} \mid$ C3KJDO $\mid$ C3KJD0_ANOFI RWD domain-containing protein 1 OS=Anoplopoma fim

$\operatorname{tr} \mid$ C1BL72 $\mid$ C1BL72_OSMM0 Elongation factor 1-gamma OS=0smerus mordax GN=EF $\operatorname{tr} \mid$ C3KJ11|C3KJ11_ANOFI Dolichol phosphate-mannose biosynthesis regulator $\operatorname{tr} \mid$ C1BEP1|C1BEP1_0NCMY Prefoldin subunit 6 OS=0ncorhynchus mykiss GN=PFD $\operatorname{tr} \mid$ C3KJV1 $\mid$ C3KJV1_ANOFI 39S ribosomal protein L51, mitochondrial OS=Anop1 $\operatorname{tr} \mid$ C3KHN8 $\mid$ C3KHN8_ANOFI Cofilin-2 OS=Anoplopoma fimbria GN=COF2 PE=2 SV=1 
UN_ci_8595

UN_ci_8420

UN_ci_160

UN_ci_3753

UN_ci_2798

UN_ci_6027

UN_ci_4049

UN_ci_539

UN_ci_4613

UN_ci_7761

UN_ci_2696

UN_ci_5849

UN_ci_647

UN_ci_3157

UN_ci_8153

UN_ci_7834

UN_ci_8670

UN_ci_7862

UN_ci_7952

UN_ci_7333

UN_ci_8343

UN_ci_3850

UN_ci_5250

UN_ci_8041

UN_ci_5796

UN_ci_8061

UN_ci_4275

UN_ci_4858

UN_ci_6572

UN_ci_3241

UN_ci_2416

UN_ci_6439

UN_ci_6184

UN_ci_8692

UN_ci_1011

UN_ci_7694

UN_ci_662

UN_ci_8076

UN_ci_231

UN_ci_2880

UN_ci_7662

UN_ci_597

UN_ci_5041

UN_ci_5248

UN_ci_8802

UN_ci_7311

UN_ci_8768

UN_ci_8708

UN_ci_5192

UN_ci_8764 $\operatorname{tr} \mid$ B5X3U0 $\mid$ B5X3U0_SALSA Ubiquitin carrier protein OS=Salmo salar GN=UBC9 $\operatorname{tr} \mid$ C8CH42 $\mid$ C8CH42_SCIOC QM-1ike protein OS=Sciaenops ocellatus GN=QM PE=2 $\operatorname{tr} \mid$ C3KHMO $\mid$ C3KHMO_ANOFI ATP synthase subunit g, mitochondrial OS=Anoplopo

tr $\mid$ Q4RT21 |Q4RT21_TETNG S-adenosylmethionine synthase OS=Tetraodon nigrov $\operatorname{tr} \mid$ C1BYE7 $\mid$ C1BYE7_ESOLU UPF0527 membrane protein OS=Esox lucius GN=U527 P

tr $\mid$ Q4SN52 $\mid$ Q4SN52_TETNG Chromosome 8 SCAF14543, whole genome shotgun sequ tr $\mid$ C1BL65|C1BL65_0SMM0 Dolichyl-diphosphooligosaccharide--protein glycos $\operatorname{tr} \mid$ COPTYO $\mid$ COPTYO_SALSA Nucleophosmin (Fragment) OS=Salmo salar GN=NPM PE $\operatorname{tr} \mid$ Q9DFT6 $\mid$ Q9DFT6_9PERC Beta tubulin OS=Notothenia coriiceps PE=2 SV=1

tr $\mid$ C3KIN7|C3KIN7_ANOFI NADH dehydrogenase 1 beta subcomplex subunit $8, \mathrm{~m}$ $\operatorname{tr} \mid$ D8U3C1 $\mid$ D8U3C1_VOLCA Putative uncharacterized protein OS=Volvox carter tr|B5X9E6|B5X9E6_SALSA Elongation factor 1-beta 0S=Salmo salar GN=EF1B P $\operatorname{tr} \mid$ B9EPJ4|B9EPJ4_SALSA Thioredoxin, mitochondrial OS=Salmo salar GN=THI0 tr $\mid$ COPTY4|COPTY4_SALSA Proliferation-associated protein 2G4 (Fragment) 0 tr $\mid$ Q4SPX5|Q4SPX5_TETNG Chromosome 7 SCAF14536, whole genome shotgun sequ tr $\mid$ C1BYB1|C1BYB1_ES0LU Transmembrane emp24 domain-containing protein 20 $\operatorname{tr} \mid$ C1BKW4 $\mid$ C1BKW4_0SMM0 KIAA0179 OS=0smerus mordax GN=K0179 PE=2 SV=1 $\operatorname{tr} \mid$ A9ZT78|A9ZT78_SOLSE Ribosomal protein L5 OS=Solea senegalensis GN=RPL $\operatorname{tr} \mid$ A9ZT85 $\mid$ A9ZT85_SOLSE Ribosomal protein OS=Solea senegalensis GN=RPL10a

tr $\mid$ Q9I8X6 |Q9I8X6_9SMEG Cytosine-specific methyltransferase OS=Xiphophoru $\operatorname{tr} \mid$ C3KH80|C3KH80_ANOFI Ran-specific GTPase-activating protein 0S=Anoplop $\operatorname{tr} \mid$ B5X8Y2|B5X8Y2_SALSA Methyltransferase-1ike protein 5 OS=Salmo salar G $\operatorname{tr} \mid$ Q8QFS3 $\mid$ Q8QFS3_TETNG H01CJC protein OS=Tetraodon nigroviridis GN=H01CJ

tr $\mid$ Q4TBK6 $\mid$ Q4TBK6_TETNG Chromosome undetermined SCAF7120, whole genome sh $\operatorname{tr} \mid$ C3KGT8 $\mid$ C3KGT8_ANOFI Coatomer subunit epsilon OS=Anoplopoma fimbria GN $\operatorname{tr} \mid$ C1BG20|C1BG20_0NCMY RING-box protein 2 OS=0ncorhynchus mykiss GN=RBX2 $\operatorname{tr} \mid$ C3KHA5|C3KHA5_ANOFI TRM112-like protein OS=Anoplopoma fimbria GN=TR11 tr $\mid$ Q4RJV4|Q4RJV4_TETNG Chromosome 9 SCAF15033, whole genome shotgun sequ tr|B5XB55|B5XB55_SALSA Signal peptidase complex subunit 3 0S=Salmo salar $\operatorname{tr} \mid$ Q1KLP9|Q1KLP9_PSEMX Natural killer enhancing factor OS=Psetta maxima

$\operatorname{tr} \mid$ C3KHK4 $\mid$ C3KHK4_ANOFI Prefoldin subunit 4 OS=Anoplopoma fimbria GN=PFD4 tr $\mid$ Q4RX35|Q4RX35_TETNG Chromosome 11 SCAF14979, whole genome shotgun seq

tr|C3KIW7|C3KIW7_ANOFI Survival of motor neuron-related-splicing factor $\operatorname{tr} \mid$ B5FY04|B5FY04_TAEGU Putative RBX1 protein 0S=Taeniopygia guttata $\mathrm{PE}=4$ $\operatorname{tr} \mid$ C3KIF4|C3KIF4_ANOFI 26S proteasome non-ATPase regulatory subunit 130 tr|B9EMJ6|B9EMJ6_SALSA DNA-directed RNA polymerase III subunit RPC6 OS=S $\operatorname{tr} \mid$ C1BKB6 $\mid$ C1BKB6_0SMM0 Adenosine kinase 0S=0smerus mordax GN=ADK PE=2 SV tr $\mid$ Q4RVH3 $\mid$ Q4RVH3_TETNG Proteasome subunit alpha type (Fragment) OS=Tetra tr|B3DFW5|B3DFW5_DANRE Si:dkey-13i19.8 OS=Danio rerio GN=si:dkey-13i19. 8 $\operatorname{tr} \mid$ Q6PC77|Q6PC77_DANRE ATP synthase, $\mathrm{H}+$ transporting, mitochondrial F0 c tr|C3KI25|C3KI25_ANOFI Small ubiquitin-related modifier 2 OS=Anoplopoma tr|B9ELR8|B9ELR8_SALSA Transmembrane protein 147 OS=Salmo salar GN=TM147 
UN_ci_1704

UN_ci_7784

UN_ci_3726

UN_ci_1737

UN_ci_8348

UN_ci_7095

UN_ci_4168

UN_ci_7884

UN_ci_4266

UN_ci_5104

UN_ci_7742

UN_ci_3600

UN_ci_8339

UN_ci_8596

UN_ci_3586

UN_ci_6015

UN_ci_3150

UN_ci_8151

UN_ci_3355

UN_ci_1165

UN_ci_8185

UN_ci_6583

UN_ci_7689

UN_ci_3785

UN_ci_542

UN_ci_47

UN_ci_4947

UN_ci_4159

UN_ci_6692

UN_ci_1151

UN_ci_6118

UN_ci_2779

UN_ci_6589

UN_ci_3409

UN_ci_7171

UN_ci_4353

UN_ci_7777

UN_ci_2373

UN_ci_8569

UN_ci_4993

UN_ci_5196

UN_ci_2937

UN_ci_8245

UN_ci_1294

UN_ci_4487

UN_ci_5521

UN_ci_7791

UN_ci_2008

UN_ci_1330

UN_ci_8216 tr $\mid$ C1BLM1|C1BLM1_0SMM0 Transmembrane BAX inhibitor motif-containing prot

tr $\mid$ Q4SZN8 |Q4SZN8_TETNG Chromosome undetermined SCAF11566, whole genome s

$\operatorname{tr} \mid$ C3KI81|C3KI81_ANOFI Electron transfer flavoprotein subunit alpha, mit $\operatorname{tr} \mid$ C3KIQ8|C3KIQ8_ANOFI 39S ribosomal protein L14, mitochondrial OS=Anop1 tr|C3KIZ6|C3KIZ6_ANOFI Cell division control protein 42 homolog 0S=Anop1 $\operatorname{tr} \mid$ A2Q0S1 $\mid$ A2Q0S1_SOLSE Ribosomal protein S6 OS=Solea senegalensis GN=rpS

tr|C1BKD0|C1BKD0_0SMM0 Transcription initiation factor TFIID subunit 11 $\operatorname{tr} \mid$ D8X0S5|D8X0S5_PSEMX Peroxiredoxin 6 OS=Psetta maxima GN=Prx6 PE=2 SV= tr $\mid$ B5XDC9|B5XDC9_SALSA Peptidy1-prolyl cis-trans isomerase 0S=Salmo sala $\operatorname{tr} \mid$ COH747|COH747_SALSA 40S ribosomal protein S17 0S=Salmo salar GN=RS17 tr $\mid$ A8JJG4 $\mid$ A8JJG4_CHLRE Predicted protein OS=Chlamydomonas reinhardtii GN

$\operatorname{tr} \mid$ C3KJ18 $\mid$ C3KJ18_ANOFI Probable protein BRICK1 OS=Anoplopoma fimbria GN= tr $\mid$ Q4SCJ6 $\mid$ Q4SCJ6_TETNG Chromosome undetermined SCAF14653, whole genome s tr $\mid$ Q5DW64 $\mid$ Q5DW64_ONCMY Glucose-regulated protein 78kDa (Fragment) OS=0nc $\operatorname{tr} \mid$ Q45KR0|Q45KR0_LARCR Heat shock protein 60 OS=Larimichthys crocea GN=H tr $\mid$ COH9Z2 $\mid$ COH9Z2_SALSA Cell division cycle 5-1ike protein OS=Salmo salar tr $\mid$ C3KK62 $\mid$ C3KK62_ANOFI Small EDRK-rich factor 2 OS=Anoplopoma fimbria GN $\operatorname{tr} \mid$ Q7ZV25 $\mid$ Q7ZV25_DANRE Tubulin cofactor a OS=Danio rerio GN=tbca PE=2 SV tr $\mid$ Q803D9 $\mid$ Q803D9_DANRE Prmt1 protein (Fragment) OS=Danio rerio GN=prmt1 $\operatorname{tr} \mid$ Q5HZ93 $\mid$ Q5HZ93_XENLA MGC85128 protein OS=Xenopus laevis GN=txn14a PE=2 $\operatorname{tr} \mid$ COH917 $\mid$ COH917_SALSA Heterogeneous nuclear ribonucleoprotein A/B OS=Sa

$\operatorname{tr} \mid$ Q6DRK2 $\mid$ Q6DRK2_DANRE Sp1icesome-associated factor 61 OS=Danio rerio GN tr $\mid$ Q4S8P8|Q4S8P8_TETNG Chromosome 7 SCAF14703, whole genome shotgun sequ tr $\mid$ Q4SLW7 |Q4SLW7_TETNG Chromosome 13 SCAF14555, whole genome shotgun seq $\operatorname{tr} \mid$ C1BJA0|C1BJA0_0SMM0 Surfeit locus protein 5 OS=0smerus mordax GN=SURF $\operatorname{tr} \mid$ C3KIG2 $\mid$ C3KIG2_ANOFI C-Myc-binding protein OS=Anoplopoma fimbria GN=MY $\operatorname{tr} \mid$ C3KJZ2 $\mid$ C3KJZ2_ANOFI ATP synthase-coupling factor 6, mitochondrial OS=

tr $\mid$ Q4S8V0|Q4S8V0_TETNG Chromosome 7 SCAF14703, whole genome shotgun sequ $\operatorname{tr} \mid$ C3KJR7|C3KJR7_ANOFI Thioredoxin-dependent peroxide reductase, mitocho $\operatorname{tr} \mid$ C3KHZ2 $\mid$ C3KHZ2_ANOFI Mitochondrial 28S ribosomal protein S21 0S=Anoplo tr $\mid$ Q4S2U7 |Q4S2U7_TETNG Chromosome 17 SCAF14760, whole genome shotgun seq $\operatorname{tr} \mid$ B5X2G7|B5X2G7_SALSA 28S ribosomal protein S31, mitochondrial 0S=Salmo $\operatorname{tr} \mid$ C3KIH2 $\mid$ C3KIH2_ANOFI Tumor suppressor candidate 2 OS=Anoplopoma fimbri tr $\mid$ C3KHX1|C3KHX1_ANOFI 26S protease regulatory subunit 8 OS=Anoplopoma $\mathrm{f}$ $\operatorname{tr} \mid$ Q4RI74|Q4RI74_TETNG Chromosome 8 SCAF15044, whole genome shotgun sequ sp|Q566X6|PTCD2_DANRE Pentatricopeptide repeat-containing protein 2 OS=D tr $\mid$ C3KHK1|C3KHK1_ANOFI Eukaryotic translation initiation factor $1 \mathrm{~A}, \mathrm{X}-\mathrm{ch}$ tr|C3KJU5|C3KJU5_ANOFI Biogenesis of 1ysosome-related organelles complex tr $\mid$ Q4RXL8|Q4RXL8_TETNG Chromosome 11 SCAF14979, whole genome shotgun seq $\operatorname{tr} \mid$ B9ENS2 $\mid$ B9ENS2_SALSA Thioredoxin OS=Salmo salar GN=THI0 PE=4 SV=1

tr|C3KJX5|C3KJX5_ANOFI Probable signal peptidase complex subunit 2 OS=An $\operatorname{tr} \mid$ D2H728|D2H728_AILME Putative uncharacterized protein (Fragment) OS=Ai tr|C3KIU9|C3KIU9_ANOFI Replication protein A $32 \mathrm{kDa}$ subunit OS=Anoplopom tr $\mid$ D2CFQ6 |D2CFQ6_LARCR Transcription factor Dp-1 OS=Larimichthys crocea 
UN_ci_7967

UN_ci 8268

UN_ci_5492

UN_ci_2749

UN_ci_2655

UN_ci_4718

UN_ci_6413

UN_ci_221

UN_ci_8634

UN_ci_8072

UN_ci_7652

UN_ci_7596

UN_ci_8070

UN_ci_4023

UN_ci_5388

UN_ci_8270

UN_ci_4164

UN_ci_4712

UN_ci_7252

UN_ci_198

UN_ci_863

UN_ci_8321

UN_ci_3516

UN_ci_6484

UN_ci_5475

UN_ci_1428

UN_ci_3697

UN_ci_3370

UN_ci_4293

UN_ci_3238

UN_ci_6714

UN_ci_5685

UN_ci_7693

UN_ci_8167

UN_ci_1617

UN_ci_8190

UN_ci_8128

UN_ci_4078

UN_ci_2833

UN_ci_8213

UN_ci_5538

UN_ci_3486

UN_ci_3942

UN_ci_6445

UN_ci_8739

UN_ci_7809

UN_ci_5649

UN_ci_7937

UN_ci_6606

UN_ci_7063 tr $\mid$ Q4S0Q0 $\mid$ Q4S0Q0_TETNG Chromosome 2 SCAF14781, whole genome shotgun sequ tr $\mid$ Q4T4L7 $\mid$ Q4T4L7_TETNG Profilin OS=Tetraodon nigroviridis GN=GSTENG00007 tr $\mid$ C3KK67|C3KK67_ANOFI T-cell leukemia translocation-altered gene protei

tr $\mid$ C3KJ47|C3KJ47_ANOFI Histidine triad nucleotide-binding protein 1 OS=A $\operatorname{tr} \mid$ C3KID9|C3KID9_ANOFI NADH dehydrogenase iron-sulfur protein 7, mitocho tr|B5XGK6|B5XGK6_SALSA Crystallin J1A 0S=Salmo salar GN=CRJ1A PE=2 SV=1

tr $\mid$ B9EL49|B9EL49_SALSA Ubiquitin carrier protein OS=Salmo salar GN=UBE2B $\operatorname{tr} \mid$ C3KHR9 $\mid$ C3KHR9_ANOFI Thioredoxin OS=Anoplopoma fimbria GN=THI0 PE=4 SV $\operatorname{tr} \mid$ A2Q0S2 $\mid$ A2Q0S2_SOLSE Ribosomal protein S7 OS=Solea senegalensis GN=rpS tr $\mid$ Q4SL28 $\mid$ Q4SL28_TETNG Chromosome 17 SCAF14563, whole genome shotgun seq tr $\mid$ C1BIP5|C1BIP5 0SMM0 U3 small nucleolar RNA-interacting protein 2 OS=0 $\operatorname{tr} \mid$ C3KHL7 $\mid$ C3KHL7_ANOFI Ubiquitin carrier protein OS=Anoplopoma fimbria G

$\operatorname{tr} \mid$ Q6PGV9|Q6PGV9_DANRE Peptidylproly1 isomerase (Cyclophilin)-like 4 0S= sp|Q6TH15|EIF3D_DANRE Eukaryotic translation initiation factor 3 subunit tr $\mid$ Q4S7N9|Q4S7N9_TETNG Chromosome 18 SCAF14712, whole genome shotgun seq tr $\mid$ C3KI46|C3KI46_ANOFI Probable palmitoyltransferase ZDHHC4 OS=Anoplopom tr $\mid$ Q27HW5 $\mid$ Q27HW5_PSEMX Ce11 death-regulatory protein OS=Psetta maxima PE tr $\mid$ Q4SS97|Q4SS97_TETNG Chromosome 11 SCAF14479, whole genome shotgun seq tr $\mid$ Q4RRB6|Q4RRB6_TETNG Chromosome 14 SCAF15003, whole genome shotgun seq $\operatorname{tr} \mid$ Q6P959|Q6P959_DANRE ATP synthase gamma chain 0S=Danio rerio GN=atp5c1 $\operatorname{tr} \mid$ A0F005 $\mid$ A0F005_PSEMX RNA polymerase B transcription factor 3 (Fragment $\operatorname{tr} \mid$ B5X218|B5X218_SALSA Nucleolar protein 5 OS=Salmo salar GN=NOL5 PE=2 S tr $\mid$ Q4RKM2 |Q4RKM2_TETNG Chromosome 18 SCAF15027, whole genome shotgun seq $\operatorname{tr} \mid$ Q4SLR0|Q4SLR0_TETNG Chromosome 15 SCAF14556, whole genome shotgun seq $\operatorname{tr} \mid$ C3KJH2 $\mid$ C3KJH2_ANOFI Smal1 nuclear ribonucleoprotein G OS=Anoplopoma $\mathrm{f}$

$\operatorname{tr} \mid$ Q6TNU6 $\mid$ Q6TNU6_DANRE Dihydrolipoyl dehydrogenase OS=Danio rerio GN=dld $\operatorname{tr} \mid$ Q68J69|Q68J69_PAR0L Cathepsin B OS=Paralichthys olivaceus PE=2 SV=1 sp $\mid$ Q4S2L4|RCL_TETNG Deoxyribonucleoside 5'-monophosphate N-glycosidase 0 tr|C3KK54|C3KK54_ANOFI Deoxyuridine 5-triphosphate nucleotidohydrolase, $\operatorname{tr} \mid$ C1BKV1 $\mid$ C1BKV1_OSMM0 ADP-ribosylation factor-like protein 1 OS=0smerus $\operatorname{tr} \mid$ Q5F3E1 $\mid$ Q5F3E1_CHICK Putative uncharacterized protein OS=Gallus gallus $\operatorname{tr} \mid$ C3KJ43 $\mid$ C3KJ43_ANOFI ORM1-1ike protein 1 OS=Anoplopoma fimbria GN=0RML tr $\mid$ Q6DHI9 $\mid$ Q6DHI9_DANRE Proteasome subunit beta type OS=Danio rerio GN=ps $\operatorname{tr} \mid$ Q6Y248 $\mid$ Q6Y248_PAGMA Chaperonin subunit 7 (Fragment) OS=Pagrus major P tr $\mid$ Q4RSM1 |Q4RSM1_TETNG Chromosome 12 SCAF14999, whole genome shotgun seq $\operatorname{tr} \mid$ Q6PH42 $\mid$ Q6PH42_DANRE Ubiquitin carrier protein (Fragment) OS=Danio rer $\operatorname{tr} \mid$ C3KI18 $\mid$ C3KI18_ANOFI Elongation factor 1-delta OS=Anoplopoma fimbria G tr $\mid$ COH7I9|COH7I9_SALSA 40S ribosomal protein S15 OS=Salmo salar GN=RS15 $\operatorname{tr} \mid$ C3KH14 $\mid$ C3KH14_ANOFI FK506-binding protein 1A OS=Anoplopoma fimbria GN tr $\mid$ COHA74 $\mid$ COHA74_SALSA DNA-directed RNA polymerase OS=Salmo salar GN=RPB tr $\mid$ Q4SUY7|Q4SUY7_TETNG Chromosome 21 SCAF13835, whole genome shotgun seq tr $\mid$ Q7ZW94|Q7ZW94_DANRE Signal recognition particle $9 \mathrm{kDa}$ protein OS=Dani $\operatorname{tr} \mid$ B5XFF1|B5XFF1_SALSA Dynactin subunit 6 OS=Salmo salar GN=DCTN6 PE=2 S $\operatorname{tr} \mid$ C1BYA1 $\mid$ C1BYA1_ES0LU AN1-type zinc finger protein 1 OS=Esox lucius GN=

$\operatorname{tr} \mid$ Q4SPS2 $\mid$ Q4SPS2_TETNG Chromosome 7 SCAF14536, whole genome shotgun sequ 
UN_ci_769

UN_ci_7865

UN_ci_5114

UN_ci_560

UN_ci_8109

UN_ci_8137

UN_ci_2879

UN_ci_3805

UN_ci_8520

UN_ci_7803

UN_ci_6336

UN_ci_8004

UN_ci_2975

UN_ci_8260

UN_ci_7921 $\operatorname{tr} \mid$ B5XA79|B5XA79_SALSA 39S ribosomal protein L52, mitochondrial 0S=Salmo $\operatorname{tr} \mid$ A9ZT96 $\mid$ A9ZT96_SOLSE Ribosomal protein L19 OS=Solea senegalensis GN=RP

$\operatorname{tr} \mid$ B5X7F1|B5X7F1_SALSA Lin-52 homolog OS=Salmo salar GN=LIN52 PE=4 SV=1 tr $\mid$ Q4RHR2|Q4RHR2_TETNG Chromosome 19 SCAF15045, whole genome shotgun seq tr $\mid$ Q4SCH7 |Q4SCH7_TETNG Chromosome undetermined SCAF14653, whole genome s tr|Q4RL89|Q4RL89_TETNG Chromosome 21 SCAF15022, whole genome shotgun seq

tr $\mid$ B5DGF1|B5DGF1_SALSA Signal sequence receptor beta OS=Salmo salar GN=S sp $\mid$ Q9I8U0|COX41_THU0B Cytochrome c oxidase subunit 4 isoform 1, mitochon $\operatorname{tr} \mid$ C1BLD2 $\mid$ C1BLD2_OSMM0 C20orf24 homolog 0S=0smerus mordax GN=CT024 PE=2 $\operatorname{tr} \mid$ Q71U69|Q71U69_XENLA Enhancer of rudimentary homolog OS=Xenopus laevis 
hotgun sequence OS=Tetraodon nigroviridis GN=GSTENG00038513001 PE=4 SV=1

IS=Anoplopoma fimbria GN=ELOC PE=4 SV=1

'imbria GN=DYL2 PE=4 SV=1

$\mathrm{SV}=1$

$50 \quad \mathrm{PE}=2 \mathrm{SV}=1$

mbicus $\mathrm{PE}=2 \mathrm{SV}=1$

OS=0ncorhynchus mykiss GN=TAF10 PE=2 SV=1

bria GN=NPC2 PE=2 SV=1

OS=Epinephelus coioides $\mathrm{PE}=2 \mathrm{SV}=1$

uence. (Fragment) OS=Tetraodon nigroviridis GN=GSTENG00029438001 PE=4 SV=1

icus $\mathrm{PE}=2 \mathrm{SV}=1$

v1 subtype (Fragment) OS=Danio rerio GN=gnav1 PE=2 SV=1

oclitus $\mathrm{PE}=2 \mathrm{SV}=1$

us $\mathrm{PE}=4 \mathrm{SV}=1$

ence. (Fragment) OS=Tetraodon nigroviridis GN=GSTENG00023873001 PE=4 SV=1

erio $\mathrm{GN}=$ trappc1 $\mathrm{PE}=2 \mathrm{SV}=1$

$1 \mathrm{PE}=2 \mathrm{SV}=1$

soform 1 OS=Takifugu rubripes GN=Atp5g1 PE=3 SV=1

$\mathrm{PE}=2 \mathrm{SV}=1$

$\mathrm{V}=1$

a maxima $\mathrm{PE}=2 \mathrm{SV}=1$

lopoma fimbria GN=SAP18 PE=2 SV=1

$\mathrm{n}$ specific peptidase 39 (USP39) OS=Danio rerio GN=usp39 $\mathrm{PE}=4 \mathrm{SV}=1$

'E=2 SV=1

hotgun sequence. (Fragment) OS=Tetraodon nigroviridis GN=GSTENG00009546001 PE=4 SV=1

agment) OS=Epinephelus coioides $\mathrm{PE}=2 \mathrm{SV}=1$

ma fimbria GN=SMD2 $\mathrm{PE}=4 \mathrm{SV}=1$

opoma fimbria GN=C1D $\mathrm{PE}=2 \mathrm{SV}=1$

hotgun sequence OS=Tetraodon nigroviridis GN=GSTENG00023162001 PE=4 SV=1

tochondrial OS=Anoplopoma fimbria GN=SLIRP PE=4 SV=1

otgun sequence. (Fragment) OS=Tetraodon nigroviridis GN=GSTENG00004757001 PE=4 SV=1

bria GN=RAN PE=2 SV=1

itochondrial 0S=Anoplopoma fimbria GN=NDUB5 PE=2 SV=1

$=\mathrm{RS} 8 \mathrm{PE}=2 \mathrm{SV}=1$

a $\mathrm{GN}=\mathrm{TUSC} 2 \mathrm{PE}=4 \mathrm{SV}=1$

OS=Epinephelus coioides $\mathrm{PE}=2 \mathrm{SV}=1$

uence. (Fragment) OS=Tetraodon nigroviridis GN=GSTENG00020229001 PE=4 SV=1 
ence OS=Tetraodon nigroviridis GN=GSTENG00025517001 PE=4 SV=1

dum $\mathrm{GN}=\mathrm{S} 0 \mathrm{D} 1 \mathrm{PE}=2 \mathrm{SV}=1$

hotgun sequence. (Fragment) OS=Tetraodon nigroviridis GN=GSTENG00021806001 PE=4 SV=1

ence OS=Tetraodon nigroviridis GN=GSTENG00031888001 PE=4 SV=1

$:=2 \mathrm{SV}=1$

GN=CBX3 $\quad \mathrm{PE}=2 \quad \mathrm{SV}=1$

(CD151) OS=Danio rerio GN=si:dkey-21h14. 7 PE=4 SV=1

ence OS=Tetraodon nigroviridis GN=GSTENG00033425001 PE=4 SV=1

hthys olivaceus $\mathrm{PE}=2 \mathrm{SV}=1$

$6 \mathrm{PE}=2 \mathrm{SV}=1$

GN=EMI5 PE=2 SV=1

$=3$

s musculus $\mathrm{GN}=2410042 \mathrm{D} 21 \mathrm{Rik} \mathrm{PE}=2 \mathrm{SV}=1$

lucius GN=RM47 PE=2 SV=1

hotgun sequence. (Fragment) OS=Tetraodon nigroviridis GN=GSTENG00009171001 PE=4 SV=1

'E=2 SV=1

hotgun sequence OS=Tetraodon nigroviridis GN=GSTENG00031736001 PE=4 SV=1

oform OS=Anoplopoma fimbria GN=MLRN PE=2 SV=1

luropoda melanoleuca GN=PANDA_006953 PE=4 SV=1

ence OS=Tetraodon nigroviridis GN=GSTENG00023668001 PE $=4 \quad \mathrm{SV}=1$

a $\mathrm{GN}=\mathrm{PSB} 1 \mathrm{~A} \quad \mathrm{PE}=2 \mathrm{SV}=1$

$=\mathrm{PSB} 3 \mathrm{PE}=2 \mathrm{SV}=1$

ence. (Fragment) OS=Tetraodon nigroviridis GN=GSTENG00017122001 PE=4 SV=1

it 20 OS=Salmo salar GN=MED20 $\mathrm{PE}=2 \mathrm{SV}=1$

$2 \mathrm{SV}=1$

uence. (Fragment) OS=Tetraodon nigroviridis GN=GSTENG00030206001 PE=4 SV=1

la fimbria GN=SPT4H $\mathrm{PE}=4 \mathrm{SV}=1$

ria GN=HMGB2 $\mathrm{PE}=2 \mathrm{SV}=1$

uence OS=Tetraodon nigroviridis GN=GSTENG00022692001 PE=4 SV=1

alar GN=PSME3 PE=2 SV=1

.1mo salar GN=DDX39 PE=2 SV=1

ence. (Fragment) 0S=Tetraodon nigroviridis GN=GSTENG00024220001 PE=4 SV=1

bria GN=RWDD1 PE=2 SV=1

'1G PE=2 SV=1

$\mathrm{y}$ protein $\mathrm{OS}=$ Anoplopoma fimbria $\mathrm{GN}=\mathrm{DPM} 2 \mathrm{PE}=4 \mathrm{SV}=1$

$16 \mathrm{PE}=2 \mathrm{SV}=1$

opoma fimbria GN=RM51 PE=2 SV=1 
$\mathrm{PE}=2 \mathrm{SV}=1$

$\mathrm{SV}=1$

ma fimbria GN=ATP5L PE=4 SV=1

iridis GN=GSTENG00029452001 PE=3 SV=1

'E=2 SV=1

ence. (Fragment) OS=Tetraodon nigroviridis GN=GSTENG00015434001 PE=4 SV=1

yltransferase subunit DAD1 OS=0smerus mordax GN=DAD1 PE=4 SV=1

$:=2 \mathrm{SV}=1$

it tochondrial 0S=Anoplopoma fimbria GN=NDUB8 PE=2 SV=1

i f. nagariensis GN=VOLCADRAFT_93889 PE $=4$ SV=1

'E=2 SV=1

IM PE=2 SV=1

IS=Salmo salar GN=PA2G4 PE=2 SV=1

ence OS=Tetraodon nigroviridis GN=GSTENG00014630001 PE=4 SV=1

IS=Esox lucius GN=TMED2 PE=2 SV=1

$5 \mathrm{PE}=2 \mathrm{SV}=1$

$\mathrm{PE}=2 \mathrm{SV}=1$

s maculatus x Xiphophorus helleri GN=dnmt $-1 \quad \mathrm{PE}=2 \mathrm{SV}=1$

,oma fimbria GN=RANG PE=2 SV=1

N=METL5 PE $=2 \quad \mathrm{SV}=1$

C $\mathrm{PE}=2 \mathrm{SV}=1$

otgun sequence OS=Tetraodon nigroviridis GN=GSTENG00003719001 PE=4 SV=1

$=\mathrm{COPE} \quad \mathrm{PE}=2 \quad \mathrm{SV}=1$

$\mathrm{PE}=4 \mathrm{SV}=1$

$2 \mathrm{PE}=2 \mathrm{SV}=1$

ence OS=Tetraodon nigroviridis GN=GSTENG00033260001 PE=4 SV=1

- $\mathrm{GN}=\mathrm{SPCS} 3 \mathrm{PE}=2 \mathrm{SV}=1$

$\mathrm{PE}=2 \quad \mathrm{SV}=1$

$\mathrm{PE}=2 \quad \mathrm{SV}=1$

uence. (Fragment) OS=Tetraodon nigroviridis GN=GSTENG00027582001 PE=3 SV=1

30 OS=Anoplopoma fimbria GN=SPF30 PE=2 SV=1

$\mathrm{SV}=1$

IS=Anoplopoma fimbria GN=PSD13 PE=2 SV=1

almo salar GN=RPC6 $\mathrm{PE}=2 \mathrm{SV}=1$

$=1$

odon nigroviridis GN=GSTENG00028335001 PE=3 SV=1

: $\mathrm{PE}=4 \mathrm{SV}=1$

omplex, subunit d OS=Danio rerio $\mathrm{GN}=$ atp5h $\mathrm{PE}=2 \mathrm{SV}=1$

fimbria $\mathrm{GN}=\mathrm{SUM} 02 \mathrm{PE}=4 \mathrm{SV}=1$

$\mathrm{PE}=2 \mathrm{SV}=1$ 
ein 4 OS=0smerus mordax GN=TMBI4 PE=2 SV=1

hotgun sequence. (Fragment) OS=Tetraodon nigroviridis GN=GSTENG00009758001 PE=4 SV=1

ochondrial OS=Anoplopoma fimbria GN=ETFA PE=2 SV=1

opoma fimbria GN=RM14 PE=2 SV=1

opoma fimbria GN=CDC42 $\mathrm{PE}=2 \mathrm{SV}=1$

$6 \mathrm{PE}=2 \mathrm{SV}=1$

OS=0smerus mordax GN=TAF11 PE=2 SV=1

$: 1$

.r $\mathrm{GN}=\mathrm{PPIL} 3 \mathrm{PE}=2 \mathrm{SV}=1$

$\mathrm{PE}=2 \mathrm{SV}=1$

=CHLREDRAFT_155068 PE=4 SV=1

BRK1 PE=4 SV=1

hotgun sequence OS=Tetraodon nigroviridis GN=GSTENG00020465001 PE=4 SV=1

orhynchus mykiss GN=GRP78 PE=2 SV=1

Isp60 $\mathrm{PE}=4 \mathrm{SV}=1$

- $\mathrm{GN}=\mathrm{CDC} 5 \mathrm{~L} \quad \mathrm{PE}=2 \mathrm{SV}=1$

$=\mathrm{SERF} 2 \mathrm{PE}=4 \mathrm{SV}=1$

$=1$

$\mathrm{PE}=2 \mathrm{SV}=1$

$\mathrm{SV}=1$

.1mo salar $\mathrm{GN}=\mathrm{ROAA} \quad \mathrm{PE}=2 \mathrm{SV}=1$

$=$ sf $3 a 3 \quad$ PE $=2 \quad \mathrm{SV}=1$

ence. (Fragment) OS=Tetraodon nigroviridis GN=GSTENG00022244001 PE=4 SV=1

uence. (Fragment) OS=Tetraodon nigroviridis GN=GSTENG00016036001 PE=4 SV=1

$5 \mathrm{PE}=2 \mathrm{SV}=1$

CBP $\mathrm{PE}=4 \mathrm{SV}=1$

Anoplopoma fimbria GN=ATP5J PE=3 SV=1

ence. (Fragment) OS=Tetraodon nigroviridis GN=GSTENG00022178001 PE=4 SV=1

ndrial OS=Anoplopoma fimbria GN=PRDX3 PE=2 SV=1

poma fimbria GN=RT21 PE=4 SV=1

uence. (Fragment) OS=Tetraodon nigroviridis GN=GSTENG00024925001 PE=4 SV=1

, salar GN=RT31 PE=2 SV=1

a $\mathrm{GN}=\mathrm{TUSC} 2 \mathrm{PE}=4 \mathrm{SV}=1$

imbria GN=PRS8 PE=2 SV=1

ence. (Fragment) 0S=Tetraodon nigroviridis GN=GSTENG00034003001 PE=4 SV=1

anio rerio GN=ptcd2 $\mathrm{PE}=2 \mathrm{SV}=1$

romosomal OS=Anoplopoma fimbria GN=IF1AX PE=2 SV=1

-1 subunit 1 OS=Anoplopoma fimbria GN=BL1S1 PE=2 SV=1

uence OS=Tetraodon nigroviridis GN=GSTENG00027365001 PE=4 SV=1

oplopoma fimbria GN=SPCS2 PE=2 SV=1

luropoda melanoleuca GN=PANDA_005916 PE=4 SV=1

la fimbria $\mathrm{GN}=\mathrm{RFA} 2 \mathrm{PE}=2 \mathrm{SV}=1$

$\mathrm{PE}=2 \mathrm{SV}=1$ 
ence OS=Tetraodon nigroviridis GN=GSTENG00025926001 PE=4 SV=1

$244001 \mathrm{PE}=3 \mathrm{SV}=1$

n homolog OS=Anoplopoma fimbria GN=TCTA PE=4 SV=1

noplopoma fimbria GN=HINT1 $\mathrm{PE}=2 \mathrm{SV}=1$

ndrial 0S=Anoplopoma fimbria GN=NDUS7 PE=2 SV=1

: $\mathrm{PE}=2 \mathrm{SV}=1$

$=1$

$7 \mathrm{PE}=2 \mathrm{SV}=1$

uence. (Fragment) OS=Tetraodon nigroviridis GN=GSTENG00016445001 PE=4 SV=1

ismerus mordax $\mathrm{GN}=\mathrm{U} 3 \mathrm{IP} 2 \mathrm{PE}=2 \mathrm{SV}=1$

$\mathrm{N}=\mathrm{UBE} 2 \mathrm{~A} \quad \mathrm{PE}=2 \mathrm{SV}=1$

Danio rerio $\mathrm{GN}=$ ppil $14 \mathrm{PE}=2 \mathrm{SV}=1$

D OS=Danio rerio $\mathrm{GN}=$ eif3d $\mathrm{PE}=2 \mathrm{SV}=1$

uence. (Fragment) OS=Tetraodon nigroviridis GN=GSTENG00022695001 PE=4 SV=1

la fimbria GN=ZDHC4 $\mathrm{PE}=2 \mathrm{SV}=1$

$:=2 \quad \mathrm{SV}=2$

uence. (Fragment) OS=Tetraodon nigroviridis GN=GSTENG00013537001 PE=4 SV=1

uence. (Fragment) OS=Tetraodon nigroviridis GN=GSTENG00030239001 PE=4 SV=1 $\mathrm{PE}=2 \mathrm{SV}=1$

) $0 \mathrm{~S}=\mathrm{Psetta}$ maxima $\mathrm{PE}=2 \mathrm{SV}=1$

$\mathrm{V}=1$

uence OS=Tetraodon nigroviridis GN=GSTENG00032873001 PE=4 SV=1

uence. (Fragment) OS=Tetraodon nigroviridis GN=GSTENG00016123001 PE=4 SV=1

imbria GN=RUXG PE=4 SV=1

h $\mathrm{PE}=2 \quad \mathrm{SV}=1$

IS=Tetraodon nigroviridis GN=rc1 PE=3 SV=2

mitochondrial OS=Anoplopoma fimbria GN=DUT PE=2 SV=1

mordax GN=ARL1 PE=2 SV=1

GN=RCJMB04_19p6 PE=2 SV=1

1 $\mathrm{PE}=2 \mathrm{SV}=1$

$\mathrm{mb} 2 \mathrm{PE}=2 \mathrm{SV}=1$

'E=2 $\mathrm{SV}=1$

uence OS=Tetraodon nigroviridis GN=GSTENG00029628001 PE=4 SV=1

io $\mathrm{GN}=u b e 2131 \mathrm{PE}=2 \mathrm{SV}=1$

$\mathrm{N}=\mathrm{EF} 1 \mathrm{D} \quad \mathrm{PE}=2 \quad \mathrm{SV}=1$

$\mathrm{PE}=2 \quad \mathrm{SV}=1$

$=$ FKB1A PE $=3 \quad \mathrm{SV}=1$

$2 \mathrm{PE}=2 \mathrm{SV}=1$

uence. (Fragment) OS=Tetraodon nigroviridis GN=GSTENG00012247001 PE=3 SV=1

o rerio $\mathrm{GN}=\mathrm{srp} 9 \mathrm{PE}=2 \mathrm{SV}=1$

$\mathrm{V}=1$

ZFAN1 PE=2 SV=1

ence OS=Tetraodon nigroviridis GN=GSTENG00014695001 PE=4 SV=1 
. salar GN=RM52 $\mathrm{PE}=2 \mathrm{SV}=1$

'L19-1ike2 PE=2 SV=1

uence. (Fragment) OS=Tetraodon nigroviridis GN=GSTENG00034212001 PE=4 SV=1

hotgun sequence. (Fragment) OS=Tetraodon nigroviridis GN=GSTENG00020486001 PE=4 SV=1

uence. (Fragment) OS=Tetraodon nigroviridis GN=GSTENG00032595001 PE=4 SV=1

sr2 $\mathrm{PE}=2 \mathrm{SV}=1$

drial OS=Thunnus obesus $\mathrm{PE}=2 \mathrm{SV}=1$

$\mathrm{SV}=1$

$\mathrm{GN}=$ erh $\quad \mathrm{PE}=3 \quad \mathrm{SV}=1$ 


\begin{tabular}{|c|c|c|}
\hline $\begin{array}{l}\text { Contig_Name } \\
\text { UN_xiong_3 }\end{array}$ & $\begin{array}{c}\text { Offset_On_Contig } \\
340\end{array}$ & $\begin{array}{c}\text { ALLELE } \\
\text { A/G }\end{array}$ \\
\hline UN_xiong_11 & 525 & $\mathrm{~T} / \mathrm{C}$ \\
\hline UN_xiong_11 & 626 & $\mathrm{~A} / \mathrm{T}$ \\
\hline UN_xiong_11 & 847 & $\mathrm{~T} / \mathrm{C}$ \\
\hline UN_xiong_11 & 903 & $\mathrm{~T} / \mathrm{C}$ \\
\hline UN_xiong_12 & 257 & $\mathrm{G} / \mathrm{A}$ \\
\hline UN_xiong_12 & 362 & $A / G$ \\
\hline UN_xiong_12 & 387 & $\mathrm{G} / \mathrm{A}$ \\
\hline UN_xiong_16 & 152 & $\mathrm{~T} / \mathrm{C}$ \\
\hline UN_xiong_16 & 376 & $A / G$ \\
\hline UN_xiong_29 & 634 & $\mathrm{~A} / \mathrm{C}$ \\
\hline UN_xiong_51 & 22 & $A / G$ \\
\hline UN_xiong_55 & 93 & $\mathrm{~T} / \mathrm{C}$ \\
\hline UN_xiong_55 & 117 & $\mathrm{C} / \mathrm{T}$ \\
\hline UN_xiong_59 & 161 & $\mathrm{G} / \mathrm{A}$ \\
\hline UN_xiong_66 & 335 & $\mathrm{G} / \mathrm{A}$ \\
\hline UN_xiong_66 & 420 & $\mathrm{G} / \mathrm{A}$ \\
\hline UN_xiong_68 & 138 & $\mathrm{C} / \mathrm{T}$ \\
\hline UN_xiong_73 & 94 & $\mathrm{~T} / \mathrm{C}$ \\
\hline UN_xiong_73 & 157 & $\mathrm{G} / \mathrm{A}$ \\
\hline UN_xiong_81 & 102 & $A / G$ \\
\hline UN_xiong_81 & 148 & $\mathrm{G} / \mathrm{A}$ \\
\hline UN_xiong_90 & 119 & $C / A$ \\
\hline UN_xiong_97 & 293 & $\mathrm{G} / \mathrm{A}$ \\
\hline UN_xiong_98 & 119 & $T / A$ \\
\hline UN_xiong_98 & 595 & $C / G$ \\
\hline UN_xiong_98 & 634 & $A / G$ \\
\hline UN_xiong_98 & 654 & $\mathrm{C} / \mathrm{T}$ \\
\hline UN_xiong_98 & 694 & $\mathrm{~T} / \mathrm{C}$ \\
\hline UN_xiong_98 & 758 & $A / G$ \\
\hline UN_xiong_98 & 805 & $\mathrm{G} / \mathrm{A}$ \\
\hline UN_xiong_98 & 887 & $A / G$ \\
\hline UN_xiong_101 & 446 & $A / G$ \\
\hline UN_xiong_101 & 468 & $A / G$ \\
\hline UN_xiong_101 & 569 & $\mathrm{G} / \mathrm{C}$ \\
\hline UN_xiong_108 & 206 & $\mathrm{~T} / \mathrm{C}$ \\
\hline UN_xiong_108 & 262 & $\mathrm{~T} / \mathrm{C}$ \\
\hline UN_xiong_111 & 127 & $\mathrm{C} / \mathrm{T}$ \\
\hline UN_xiong_122 & 302 & $\mathrm{G} / \mathrm{A}$ \\
\hline UN_xiong_122 & 382 & $\mathrm{G} / \mathrm{T}$ \\
\hline UN_xiong_122 & 406 & $\mathrm{~T} / \mathrm{C}$ \\
\hline UN_xiong_122 & 509 & $\mathrm{~T} / \mathrm{C}$ \\
\hline UN_xiong_122 & 642 & $C / A$ \\
\hline UN_xiong_142 & 127 & $A / G$ \\
\hline UN_xiong_145 & 502 & $\mathrm{C} / \mathrm{T}$ \\
\hline UN_xiong_145 & 585 & $\mathrm{~T} / \mathrm{C}$ \\
\hline
\end{tabular}




\begin{tabular}{|c|c|c|}
\hline UN_xiong_145 & 619 & $\mathrm{C} / \mathrm{T}$ \\
\hline UN_xiong_145 & 867 & $\mathrm{~T} / \mathrm{C}$ \\
\hline UN_xiong_146 & 8 & $\mathrm{~A} / \mathrm{T}$ \\
\hline UN_xiong_146 & 53 & $\mathrm{~A} / \mathrm{G}$ \\
\hline UN_xiong_146 & 134 & $\mathrm{C} / \mathrm{T}$ \\
\hline UN_xiong_146 & 384 & $\mathrm{G} / \mathrm{A}$ \\
\hline UN_xiong_146 & 430 & $\mathrm{~T} / \mathrm{C}$ \\
\hline UN_xiong_146 & 836 & $\mathrm{~T} / \mathrm{C}$ \\
\hline UN_xiong_148 & 159 & $\mathrm{~T} / \mathrm{C}$ \\
\hline UN_xiong_149 & 108 & $\mathrm{~T} / \mathrm{C}$ \\
\hline UN_xiong_153 & 171 & $\mathrm{~A} / \mathrm{T}$ \\
\hline UN_xiong_155 & 15 & $A / G$ \\
\hline UN_xiong_165 & 449 & $\mathrm{~T} / \mathrm{C}$ \\
\hline UN_xiong_169 & 139 & $\mathrm{~A} / \mathrm{G}$ \\
\hline UN_xiong_169 & 209 & $\mathrm{C} / \mathrm{T}$ \\
\hline UN_xiong_169 & 277 & $A / G$ \\
\hline UN_xiong_175 & 205 & $\mathrm{C} / \mathrm{A}$ \\
\hline UN_xiong_178 & 64 & $\mathrm{~T} / \mathrm{C}$ \\
\hline UN_xiong_178 & 200 & $\mathrm{~T} / \mathrm{C}$ \\
\hline UN_xiong_187 & 77 & $\mathrm{G} / \mathrm{A}$ \\
\hline UN_xiong_192 & 61 & $\mathrm{C} / \mathrm{G}$ \\
\hline UN_xiong_194 & 61 & $\mathrm{~T} / \mathrm{C}$ \\
\hline UN_xiong_194 & 147 & $\mathrm{~A} / \mathrm{G}$ \\
\hline UN_xiong_194 & 205 & $\mathrm{~T} / \mathrm{C}$ \\
\hline UN_xiong_194 & 251 & $\mathrm{C} / \mathrm{T}$ \\
\hline UN_xiong_208 & 75 & $\mathrm{~T} / \mathrm{C}$ \\
\hline UN_xiong_208 & 102 & $A / G$ \\
\hline UN_xiong_213 & 206 & $\mathrm{~A} / \mathrm{G}$ \\
\hline UN_xiong_213 & 445 & $\mathrm{~A} / \mathrm{G}$ \\
\hline UN_xiong_216 & 197 & $\mathrm{C} / \mathrm{A}$ \\
\hline UN_xiong_216 & 315 & $\mathrm{~T} / \mathrm{G}$ \\
\hline UN_xiong_216 & 406 & $\mathrm{~T} / \mathrm{C}$ \\
\hline UN_xiong_216 & 446 & $\mathrm{~A} / \mathrm{T}$ \\
\hline UN_xiong_216 & 661 & $\mathrm{G} / \mathrm{A}$ \\
\hline UN_xiong_216 & 886 & $\mathrm{C} / \mathrm{T}$ \\
\hline UN_xiong_216 & 914 & $\mathrm{~T} / \mathrm{G}$ \\
\hline UN_xiong_216 & 960 & $\mathrm{~T} / \mathrm{C}$ \\
\hline UN_xiong_216 & 1267 & $\mathrm{~A} / \mathrm{T}$ \\
\hline UN_xiong_228 & 79 & $\mathrm{~A} / \mathrm{G}$ \\
\hline UN_xiong_228 & 99 & $\mathrm{G} / \mathrm{A}$ \\
\hline UN_xiong_228 & 127 & $A / G$ \\
\hline UN_xiong_228 & 190 & $\mathrm{~A} / \mathrm{G}$ \\
\hline UN_xiong_228 & 309 & $\mathrm{G} / \mathrm{A}$ \\
\hline UN_xiong_235 & 154 & $\mathrm{~A} / \mathrm{G}$ \\
\hline UN_xiong_246 & 200 & $\mathrm{C} / \mathrm{T}$ \\
\hline UN_xiong_251 & 253 & $\mathrm{G} / \mathrm{A}$ \\
\hline UN_xiong_252 & 93 & $A / G$ \\
\hline
\end{tabular}




\begin{tabular}{|c|c|c|}
\hline UN_xiong_256 & 267 & $\mathrm{~T} / \mathrm{A}$ \\
\hline UN_xiong_257 & 523 & $A / G$ \\
\hline UN_xiong_257 & 879 & $\mathrm{G} / \mathrm{C}$ \\
\hline UN_xiong_260 & 56 & $\mathrm{C} / \mathrm{T}$ \\
\hline UN_xiong_261 & 241 & $A / G$ \\
\hline UN_xiong_263 & 121 & $\mathrm{~T} / \mathrm{C}$ \\
\hline UN_xiong_269 & 425 & $\mathrm{~T} / \mathrm{C}$ \\
\hline UN_xiong_269 & 548 & $A / G$ \\
\hline UN_xiong_269 & 588 & $\mathrm{C} / \mathrm{T}$ \\
\hline UN_xiong_274 & 66 & $\mathrm{G} / \mathrm{A}$ \\
\hline UN_xiong_278 & 145 & $\mathrm{G} / \mathrm{A}$ \\
\hline UN_xiong_283 & 234 & $\mathrm{G} / \mathrm{A}$ \\
\hline UN_xiong_290 & 43 & $\mathrm{~A} / \mathrm{T}$ \\
\hline UN_xiong_298 & 259 & $\mathrm{G} / \mathrm{A}$ \\
\hline UN_xiong_298 & 291 & $A / G$ \\
\hline UN_xiong_306 & 33 & $\mathrm{G} / \mathrm{C}$ \\
\hline UN_xiong_307 & 52 & $\mathrm{C} / \mathrm{A}$ \\
\hline UN_xiong_307 & 111 & $\mathrm{~T} / \mathrm{A}$ \\
\hline UN_xiong_307 & 598 & $\mathrm{~T} / \mathrm{C}$ \\
\hline UN_xiong_310 & 685 & $A / C$ \\
\hline UN_xiong_310 & 740 & $\mathrm{~T} / \mathrm{C}$ \\
\hline UN_xiong_310 & 942 & $A / G$ \\
\hline UN_xiong_310 & 1071 & $\mathrm{~T} / \mathrm{A}$ \\
\hline UN_xiong_318 & 224 & $\mathrm{C} / \mathrm{T}$ \\
\hline UN_xiong_335 & 131 & $A / G$ \\
\hline UN_xiong_335 & 161 & $\mathrm{G} / \mathrm{T}$ \\
\hline UN_xiong_335 & 233 & $\mathrm{C} / \mathrm{T}$ \\
\hline UN_xiong_335 & 316 & $\mathrm{~T} / \mathrm{C}$ \\
\hline UN_xiong_335 & 351 & $A / G$ \\
\hline UN_xiong_335 & 515 & $\mathrm{G} / \mathrm{A}$ \\
\hline UN_xiong_335 & 548 & $\mathrm{G} / \mathrm{A}$ \\
\hline UN_xiong_340 & 226 & $\mathrm{G} / \mathrm{A}$ \\
\hline UN_xiong_340 & 490 & $\mathrm{~T} / \mathrm{C}$ \\
\hline UN_xiong_342 & 145 & $A / G$ \\
\hline UN_xiong_342 & 516 & $\mathrm{~T} / \mathrm{C}$ \\
\hline UN_xiong_342 & 589 & $\mathrm{~T} / \mathrm{C}$ \\
\hline UN_xiong_342 & 622 & $\mathrm{G} / \mathrm{A}$ \\
\hline UN_xiong_342 & 659 & $\mathrm{C} / \mathrm{T}$ \\
\hline UN_xiong_342 & 790 & $A / G$ \\
\hline UN_xiong_353 & 203 & $\mathrm{C} / \mathrm{T}$ \\
\hline UN_xiong_354 & 72 & $\mathrm{~T} / \mathrm{A}$ \\
\hline UN_xiong_357 & 15 & $\mathrm{~T} / \mathrm{C}$ \\
\hline UN_xiong_357 & 179 & $\mathrm{~T} / \mathrm{A}$ \\
\hline UN_xiong_361 & 335 & $\mathrm{C} / \mathrm{T}$ \\
\hline UN_xiong_369 & 461 & $T / G$ \\
\hline UN_xiong_371 & 29 & $\mathrm{G} / \mathrm{T}$ \\
\hline UN_xiong_373 & 125 & $A / T$ \\
\hline
\end{tabular}




\begin{tabular}{|c|c|c|}
\hline UN_xiong_373 & 162 & $\mathrm{C} / \mathrm{A}$ \\
\hline UN_xiong_376 & 85 & $\mathrm{~T} / \mathrm{C}$ \\
\hline UN_xiong_379 & 316 & $\mathrm{~T} / \mathrm{C}$ \\
\hline UN_xiong_379 & 489 & $\mathrm{C} / \mathrm{T}$ \\
\hline UN_xiong_380 & 270 & $\mathrm{G} / \mathrm{A}$ \\
\hline UN_xiong_385 & 304 & $\mathrm{~T} / \mathrm{C}$ \\
\hline UN_xiong_386 & 25 & $C / G$ \\
\hline UN_xiong_389 & 196 & $A / G$ \\
\hline UN_xiong_389 & 343 & $\mathrm{~A} / \mathrm{T}$ \\
\hline UN_xiong_419 & 239 & $\mathrm{C} / \mathrm{A}$ \\
\hline UN_xiong_432 & 176 & $\mathrm{G} / \mathrm{A}$ \\
\hline UN_xiong_432 & 249 & $\mathrm{~T} / \mathrm{A}$ \\
\hline UN_xiong_432 & 703 & $A / G$ \\
\hline UN_xiong_444 & 190 & $\mathrm{C} / \mathrm{T}$ \\
\hline UN_xiong_444 & 253 & $\mathrm{C} / \mathrm{T}$ \\
\hline UN_xiong_444 & 436 & $A / G$ \\
\hline UN_xiong_444 & 573 & $\mathrm{~T} / \mathrm{C}$ \\
\hline UN_xiong_460 & 187 & $\mathrm{G} / \mathrm{A}$ \\
\hline UN_xiong_460 & 309 & $\mathrm{G} / \mathrm{C}$ \\
\hline UN_xiong_465 & 55 & $\mathrm{C} / \mathrm{T}$ \\
\hline UN_xiong_465 & 99 & $\mathrm{~T} / \mathrm{C}$ \\
\hline UN_xiong_465 & 153 & $\mathrm{G} / \mathrm{C}$ \\
\hline UN_xiong_465 & 322 & $\mathrm{~A} / \mathrm{C}$ \\
\hline UN_xiong_469 & 220 & $A / G$ \\
\hline UN_xiong_473 & 29 & $\mathrm{G} / \mathrm{C}$ \\
\hline UN_xiong_473 & 169 & $C / G$ \\
\hline UN_xiong_473 & 228 & $\mathrm{G} / \mathrm{A}$ \\
\hline UN_xiong_473 & 316 & $\mathrm{G} / \mathrm{A}$ \\
\hline UN_xiong_473 & 350 & $\mathrm{~T} / \mathrm{A}$ \\
\hline UN_xiong_483 & 23 & $\mathrm{~T} / \mathrm{C}$ \\
\hline UN_xiong_483 & 121 & $A / G$ \\
\hline UN_xiong_483 & 217 & $A / G$ \\
\hline UN_xiong_483 & 501 & $A / G$ \\
\hline UN_xiong_483 & 590 & $\mathrm{C} / \mathrm{T}$ \\
\hline UN_xiong_485 & 384 & $\mathrm{G} / \mathrm{A}$ \\
\hline UN_xiong_488 & 438 & $\mathrm{G} / \mathrm{A}$ \\
\hline UN_xiong_490 & 328 & $\mathrm{~T} / \mathrm{A}$ \\
\hline UN_xiong_499 & 361 & $T / G$ \\
\hline UN_xiong_534 & 330 & $\mathrm{G} / \mathrm{T}$ \\
\hline UN_xiong_549 & 294 & $\mathrm{~T} / \mathrm{C}$ \\
\hline UN_xiong_553 & 341 & $\mathrm{~T} / \mathrm{C}$ \\
\hline UN_xiong_557 & 294 & $\mathrm{~T} / \mathrm{C}$ \\
\hline UN_xiong_569 & 207 & $\mathrm{G} / \mathrm{A}$ \\
\hline UN_xiong_569 & 244 & $A / G$ \\
\hline UN_xiong_579 & 662 & $\mathrm{~T} / \mathrm{C}$ \\
\hline UN_xiong_582 & 161 & $\mathrm{G} / \mathrm{A}$ \\
\hline UN_xiong_582 & 186 & $\mathrm{C} / \mathrm{A}$ \\
\hline
\end{tabular}




\begin{tabular}{|c|c|c|}
\hline UN_xiong_582 & 245 & $\mathrm{~T} / \mathrm{C}$ \\
\hline UN_xiong_582 & 295 & $\mathrm{~T} / \mathrm{C}$ \\
\hline UN_xiong_582 & 322 & $\mathrm{~T} / \mathrm{C}$ \\
\hline UN_xiong_582 & 404 & $\mathrm{C} / \mathrm{T}$ \\
\hline UN_xiong_582 & 454 & $\mathrm{~A} / \mathrm{G}$ \\
\hline UN_xiong_582 & 504 & $\mathrm{~T} / \mathrm{C}$ \\
\hline UN_xiong_582 & 526 & $\mathrm{~T} / \mathrm{C}$ \\
\hline UN_xiong_582 & 555 & $\mathrm{~T} / \mathrm{C}$ \\
\hline UN_xiong_582 & 671 & $\mathrm{~T} / \mathrm{C}$ \\
\hline UN_xiong_586 & 228 & $\mathrm{C} / \mathrm{T}$ \\
\hline UN_xiong_588 & 43 & $\mathrm{G} / \mathrm{T}$ \\
\hline UN_xiong_588 & 233 & $\mathrm{C} / \mathrm{T}$ \\
\hline UN_xiong_588 & 356 & $\mathrm{~T} / \mathrm{C}$ \\
\hline UN_xiong_588 & 386 & $\mathrm{~A} / \mathrm{G}$ \\
\hline UN_xiong_589 & 11 & $\mathrm{G} / \mathrm{T}$ \\
\hline UN_xiong_589 & 53 & $\mathrm{~A} / \mathrm{G}$ \\
\hline UN_xiong_595 & 121 & $\mathrm{~T} / \mathrm{A}$ \\
\hline UN_xiong_597 & 56 & $\mathrm{~T} / \mathrm{C}$ \\
\hline UN_xiong_607 & 390 & $\mathrm{~A} / \mathrm{G}$ \\
\hline UN_xiong_618 & 42 & $\mathrm{~T} / \mathrm{C}$ \\
\hline UN_xiong_621 & 196 & $\mathrm{~A} / \mathrm{G}$ \\
\hline UN_xiong_621 & 237 & $\mathrm{~T} / \mathrm{A}$ \\
\hline UN_xiong_621 & 260 & $\mathrm{C} / \mathrm{A}$ \\
\hline UN_xiong_621 & 285 & $\mathrm{~T} / \mathrm{A}$ \\
\hline UN_xiong_629 & 13 & $\mathrm{~T} / \mathrm{A}$ \\
\hline UN_xiong_629 & 64 & $\mathrm{C} / \mathrm{T}$ \\
\hline UN_xiong_629 & 105 & $\mathrm{~A} / \mathrm{G}$ \\
\hline UN_xiong_629 & 242 & $\mathrm{~T} / \mathrm{G}$ \\
\hline UN_xiong_637 & 138 & $\mathrm{C} / \mathrm{T}$ \\
\hline UN_xiong_641 & 316 & $\mathrm{C} / \mathrm{T}$ \\
\hline UN_xiong_644 & 121 & $\mathrm{C} / \mathrm{T}$ \\
\hline UN_xiong_659 & 486 & $\mathrm{C} / \mathrm{T}$ \\
\hline UN_xiong_662 & 602 & $\mathrm{~T} / \mathrm{C}$ \\
\hline UN_xiong_662 & 630 & $\mathrm{~T} / \mathrm{C}$ \\
\hline UN_xiong_662 & 779 & $\mathrm{~T} / \mathrm{C}$ \\
\hline UN_xiong_688 & 392 & $\mathrm{~T} / \mathrm{C}$ \\
\hline UN_xiong_708 & 442 & $\mathrm{G} / \mathrm{A}$ \\
\hline UN_xiong_710 & 9 & $\mathrm{C} / \mathrm{G}$ \\
\hline UN_xiong_710 & 42 & $\mathrm{~T} / \mathrm{C}$ \\
\hline UN_xiong_710 & 63 & $\mathrm{G} / \mathrm{A}$ \\
\hline UN_xiong_710 & 98 & $\mathrm{~A} / \mathrm{G}$ \\
\hline UN_xiong_710 & 121 & $\mathrm{~A} / \mathrm{G}$ \\
\hline UN_xiong_710 & 155 & $\mathrm{~T} / \mathrm{C}$ \\
\hline UN_xiong_710 & 196 & $\mathrm{~T} / \mathrm{A}$ \\
\hline UN_xiong_710 & 221 & $\mathrm{~A} / \mathrm{G}$ \\
\hline UN_xiong_710 & 244 & $\mathrm{~A} / \mathrm{T}$ \\
\hline UN_xiong_710 & 271 & $\mathrm{~T} / \mathrm{C}$ \\
\hline
\end{tabular}




\begin{tabular}{|c|c|c|}
\hline UN_xiong_710 & 292 & $\mathrm{~T} / \mathrm{G}$ \\
\hline UN_xiong_710 & 314 & $\mathrm{~T} / \mathrm{C}$ \\
\hline UN_xiong_710 & 338 & $\mathrm{~T} / \mathrm{C}$ \\
\hline UN_xiong_710 & 376 & $\mathrm{~T} / \mathrm{C}$ \\
\hline UN_xiong_710 & 400 & $\mathrm{~T} / \mathrm{C}$ \\
\hline UN_xiong_710 & 423 & $A / T$ \\
\hline UN_xiong_710 & 449 & $A / G$ \\
\hline UN_xiong_710 & 476 & $\mathrm{G} / \mathrm{A}$ \\
\hline UN_xiong_710 & 505 & $\mathrm{G} / \mathrm{T}$ \\
\hline UN_xiong_726 & 79 & $A / G$ \\
\hline UN_xiong_734 & 258 & $T / G$ \\
\hline UN_xiong_736 & 165 & $A / G$ \\
\hline UN_xiong_743 & 16 & $A / G$ \\
\hline UN_xiong_743 & 51 & $A / G$ \\
\hline UN_xiong_743 & 240 & $\mathrm{G} / \mathrm{A}$ \\
\hline UN_xiong_743 & 333 & $\mathrm{C} / \mathrm{A}$ \\
\hline UN_xiong_743 & 394 & $A / C$ \\
\hline UN_xiong_743 & 416 & $\mathrm{~A} / \mathrm{C}$ \\
\hline UN_xiong_743 & 476 & $\mathrm{G} / \mathrm{A}$ \\
\hline UN_xiong_743 & 600 & $\mathrm{G} / \mathrm{A}$ \\
\hline UN_xiong_743 & 631 & $\mathrm{G} / \mathrm{A}$ \\
\hline UN_xiong_749 & 283 & $A / C$ \\
\hline UN_xiong_751 & 121 & $\mathrm{~T} / \mathrm{C}$ \\
\hline UN_xiong_751 & 258 & $A / G$ \\
\hline UN_xiong_751 & 292 & $\mathrm{~T} / \mathrm{C}$ \\
\hline UN_xiong_751 & 484 & $A / G$ \\
\hline UN_xiong_751 & 631 & $\mathrm{G} / \mathrm{A}$ \\
\hline UN_xiong_752 & 136 & $\mathrm{G} / \mathrm{T}$ \\
\hline UN_xiong_759 & 349 & $\mathrm{C} / \mathrm{T}$ \\
\hline UN_xiong_765 & 74 & $\mathrm{~T} / \mathrm{C}$ \\
\hline UN_xiong_765 & 168 & $\mathrm{C} / \mathrm{T}$ \\
\hline UN_xiong_766 & 15 & $\mathrm{G} / \mathrm{A}$ \\
\hline UN_xiong_766 & 450 & $\mathrm{G} / \mathrm{A}$ \\
\hline UN_xiong_766 & 500 & $\mathrm{~T} / \mathrm{C}$ \\
\hline UN_xiong_766 & 622 & $\mathrm{~T} / \mathrm{C}$ \\
\hline UN_xiong_767 & 54 & $\mathrm{G} / \mathrm{A}$ \\
\hline UN_xiong_772 & 68 & $\mathrm{G} / \mathrm{A}$ \\
\hline UN_xiong_785 & 149 & $A / G$ \\
\hline UN_xiong_785 & 193 & $\mathrm{~T} / \mathrm{C}$ \\
\hline UN_xiong_790 & 160 & $A / G$ \\
\hline UN_xiong_790 & 319 & $\mathrm{~T} / \mathrm{C}$ \\
\hline UN_xiong_790 & 397 & $\mathrm{~T} / \mathrm{C}$ \\
\hline UN_xiong_790 & 421 & $A / C$ \\
\hline UN_xiong_790 & 621 & $\mathrm{~T} / \mathrm{C}$ \\
\hline UN_xiong_790 & 1013 & $\mathrm{~T} / \mathrm{C}$ \\
\hline UN_xiong_793 & 31 & $\mathrm{C} / \mathrm{T}$ \\
\hline UN_xiong_804 & 348 & $\mathrm{~T} / \mathrm{A}$ \\
\hline
\end{tabular}




\begin{tabular}{|c|c|c|}
\hline UN_xiong_804 & 457 & $\mathrm{G} / \mathrm{A}$ \\
\hline UN_xiong_805 & 351 & $A / G$ \\
\hline UN_xiong_823 & 95 & $A / G$ \\
\hline UN_xiong_829 & 247 & $\mathrm{G} / \mathrm{A}$ \\
\hline UN_xiong_830 & 254 & $\mathrm{C} / \mathrm{T}$ \\
\hline UN_xiong_838 & 455 & $\mathrm{~A} / \mathrm{G}$ \\
\hline UN_xiong_840 & 60 & $\mathrm{G} / \mathrm{A}$ \\
\hline UN_xiong_840 & 263 & $\mathrm{G} / \mathrm{A}$ \\
\hline UN_xiong_840 & 419 & $\mathrm{~A} / \mathrm{C}$ \\
\hline UN_xiong_840 & 832 & $\mathrm{C} / \mathrm{T}$ \\
\hline UN_xiong_840 & 2183 & $A / G$ \\
\hline UN_xiong_841 & 84 & $\mathrm{~T} / \mathrm{A}$ \\
\hline UN_xiong_841 & 142 & $\mathrm{G} / \mathrm{A}$ \\
\hline UN_xiong_851 & 236 & $\mathrm{~A} / \mathrm{G}$ \\
\hline UN_xiong_851 & 314 & $\mathrm{G} / \mathrm{A}$ \\
\hline UN_xiong_856 & 54 & $\mathrm{C} / \mathrm{T}$ \\
\hline UN_xiong_861 & 169 & $\mathrm{C} / \mathrm{T}$ \\
\hline UN_xiong_864 & 471 & $\mathrm{~T} / \mathrm{C}$ \\
\hline UN_xiong_864 & 525 & $A / G$ \\
\hline UN_xiong_864 & 586 & $\mathrm{~A} / \mathrm{G}$ \\
\hline UN_xiong_864 & 650 & $\mathrm{C} / \mathrm{A}$ \\
\hline UN_xiong_864 & 753 & $\mathrm{~T} / \mathrm{G}$ \\
\hline UN_xiong_864 & 914 & $\mathrm{~T} / \mathrm{C}$ \\
\hline UN_xiong_864 & 953 & $C / G$ \\
\hline UN_xiong_865 & 388 & $\mathrm{~T} / \mathrm{C}$ \\
\hline UN_xiong_866 & 428 & $A / G$ \\
\hline UN_xiong_866 & 585 & $A / G$ \\
\hline UN_xiong_867 & 139 & $\mathrm{G} / \mathrm{A}$ \\
\hline UN_xiong_867 & 289 & $A / G$ \\
\hline UN_xiong_867 & 599 & $\mathrm{~A} / \mathrm{T}$ \\
\hline UN_xiong_868 & 135 & $\mathrm{~T} / \mathrm{C}$ \\
\hline UN_xiong_868 & 192 & $T / G$ \\
\hline UN_xiong_871 & 629 & $\mathrm{~T} / \mathrm{A}$ \\
\hline UN_xiong_871 & 666 & $\mathrm{~T} / \mathrm{C}$ \\
\hline UN_xiong_871 & 761 & $\mathrm{~A} / \mathrm{G}$ \\
\hline UN_xiong_871 & 781 & $\mathrm{G} / \mathrm{A}$ \\
\hline UN_xiong_871 & 801 & $\mathrm{~A} / \mathrm{T}$ \\
\hline UN_xiong_871 & 822 & $\mathrm{~T} / \mathrm{A}$ \\
\hline UN_xiong_871 & 845 & $\mathrm{~T} / \mathrm{C}$ \\
\hline UN_xiong_871 & 865 & $\mathrm{~A} / \mathrm{G}$ \\
\hline UN_xiong_871 & 889 & $\mathrm{~A} / \mathrm{T}$ \\
\hline UN_xiong_871 & 920 & $\mathrm{~T} / \mathrm{C}$ \\
\hline UN_xiong_871 & 945 & $\mathrm{~T} / \mathrm{C}$ \\
\hline UN_xiong_871 & 965 & $\mathrm{~T} / \mathrm{C}$ \\
\hline UN_xiong_871 & 985 & $\mathrm{~A} / \mathrm{T}$ \\
\hline UN_xiong_871 & 1006 & $\mathrm{~A} / \mathrm{G}$ \\
\hline UN_xiong_871 & 1036 & $\mathrm{~A} / \mathrm{G}$ \\
\hline
\end{tabular}




\begin{tabular}{|c|c|c|}
\hline UN_xiong_871 & 1057 & $\mathrm{~T} / \mathrm{C}$ \\
\hline UN_xiong_871 & 1077 & $\mathrm{C} / \mathrm{A}$ \\
\hline UN_xiong_876 & 228 & $\mathrm{~A} / \mathrm{T}$ \\
\hline UN_xiong_876 & 268 & $\mathrm{~T} / \mathrm{C}$ \\
\hline UN_xiong_876 & 299 & $\mathrm{G} / \mathrm{A}$ \\
\hline UN_xiong_889 & 42 & $\mathrm{~A} / \mathrm{C}$ \\
\hline UN_xiong_889 & 154 & $\mathrm{G} / \mathrm{A}$ \\
\hline UN_xiong_894 & 245 & $\mathrm{~T} / \mathrm{G}$ \\
\hline UN_xiong_897 & 404 & $\mathrm{~T} / \mathrm{C}$ \\
\hline UN_xiong_897 & 598 & $\mathrm{~A} / \mathrm{G}$ \\
\hline UN_xiong_901 & 159 & $\mathrm{G} / \mathrm{T}$ \\
\hline UN_xiong_901 & 620 & $\mathrm{G} / \mathrm{A}$ \\
\hline UN_xiong_901 & 1000 & $A / G$ \\
\hline UN_xiong_901 & 1171 & $\mathrm{~T} / \mathrm{C}$ \\
\hline UN_xiong_901 & 1556 & $A / G$ \\
\hline UN_xiong_912 & 187 & $\mathrm{G} / \mathrm{A}$ \\
\hline UN_xiong_912 & 367 & $\mathrm{~A} / \mathrm{T}$ \\
\hline UN_xiong_924 & 263 & $\mathrm{G} / \mathrm{C}$ \\
\hline UN_xiong_924 & 459 & $\mathrm{~A} / \mathrm{G}$ \\
\hline UN_xiong_924 & 563 & $\mathrm{C} / \mathrm{T}$ \\
\hline UN_xiong_924 & 651 & $\mathrm{C} / \mathrm{T}$ \\
\hline UN_xiong_924 & 719 & $A / G$ \\
\hline UN_xiong_932 & 26 & $\mathrm{~A} / \mathrm{G}$ \\
\hline UN_xiong_937 & 685 & $A / G$ \\
\hline UN_xiong_942 & 251 & $A / G$ \\
\hline UN_xiong_942 & 272 & $\mathrm{~T} / \mathrm{C}$ \\
\hline UN_xiong_942 & 324 & $A / G$ \\
\hline UN_xiong_946 & 766 & $\mathrm{G} / \mathrm{A}$ \\
\hline UN_xiong_948 & 126 & $\mathrm{~T} / \mathrm{C}$ \\
\hline UN_xiong_948 & 307 & $\mathrm{C} / \mathrm{T}$ \\
\hline UN_xiong_948 & 368 & $\mathrm{~A} / \mathrm{G}$ \\
\hline UN_xiong_949 & 53 & $C / A$ \\
\hline UN_xiong_949 & 75 & $\mathrm{C} / \mathrm{T}$ \\
\hline UN_xiong_949 & 96 & $\mathrm{~T} / \mathrm{C}$ \\
\hline UN_xiong_949 & 122 & $\mathrm{~A} / \mathrm{G}$ \\
\hline UN_xiong_949 & 147 & $\mathrm{~A} / \mathrm{G}$ \\
\hline UN_xiong_949 & 167 & $\mathrm{~A} / \mathrm{G}$ \\
\hline UN_xiong_949 & 188 & $\mathrm{~T} / \mathrm{C}$ \\
\hline UN_xiong_949 & 212 & $\mathrm{~T} / \mathrm{C}$ \\
\hline UN_xiong_949 & 233 & $\mathrm{~A} / \mathrm{G}$ \\
\hline UN_xiong_949 & 256 & $\mathrm{C} / \mathrm{T}$ \\
\hline UN_xiong_949 & 276 & $\mathrm{~A} / \mathrm{G}$ \\
\hline UN_xiong_949 & 296 & $\mathrm{G} / \mathrm{A}$ \\
\hline UN_xiong_949 & 318 & $C / G$ \\
\hline UN_xiong_949 & 338 & $\mathrm{G} / \mathrm{A}$ \\
\hline UN_xiong_949 & 362 & $\mathrm{~A} / \mathrm{G}$ \\
\hline UN_xiong_949 & 384 & $\mathrm{~T} / \mathrm{C}$ \\
\hline
\end{tabular}




\begin{tabular}{|c|c|c|}
\hline UN_xiong_949 & 409 & $A / G$ \\
\hline UN_xiong_949 & 442 & $\mathrm{~T} / \mathrm{A}$ \\
\hline UN_xiong_949 & 471 & $C / A$ \\
\hline UN_xiong_957 & 14 & $\mathrm{C} / \mathrm{T}$ \\
\hline UN_xiong_959 & 129 & $\mathrm{~A} / \mathrm{T}$ \\
\hline UN_xiong_965 & 103 & $\mathrm{~A} / \mathrm{T}$ \\
\hline UN_xiong_965 & 261 & $\mathrm{~T} / \mathrm{C}$ \\
\hline UN_xiong_965 & 310 & $\mathrm{~T} / \mathrm{C}$ \\
\hline UN_xiong_965 & 376 & $\mathrm{G} / \mathrm{A}$ \\
\hline UN_xiong_965 & 397 & $A / G$ \\
\hline UN_xiong_965 & 433 & $A / G$ \\
\hline UN_xiong_967 & 154 & $A / G$ \\
\hline UN_xiong_967 & 230 & $\mathrm{~T} / \mathrm{C}$ \\
\hline UN_xiong_967 & 373 & $\mathrm{~A} / \mathrm{G}$ \\
\hline UN_xiong_972 & 8 & $\mathrm{C} / \mathrm{T}$ \\
\hline UN_xiong_972 & 48 & $\mathrm{G} / \mathrm{A}$ \\
\hline UN_xiong_972 & 463 & $A / G$ \\
\hline UN_xiong_972 & 569 & $\mathrm{~T} / \mathrm{G}$ \\
\hline UN_xiong_972 & 627 & $\mathrm{~T} / \mathrm{C}$ \\
\hline UN_xiong_972 & 685 & $A / G$ \\
\hline UN_xiong_974 & 45 & $\mathrm{G} / \mathrm{A}$ \\
\hline UN_xiong_980 & 511 & $\mathrm{C} / \mathrm{T}$ \\
\hline UN_xiong_983 & 491 & $\mathrm{~T} / \mathrm{C}$ \\
\hline UN_xiong_983 & 719 & $\mathrm{~T} / \mathrm{C}$ \\
\hline UN_xiong_988 & 309 & $\mathrm{C} / \mathrm{T}$ \\
\hline UN_xiong_988 & 348 & $\mathrm{C} / \mathrm{T}$ \\
\hline UN_xiong_988 & 381 & $\mathrm{G} / \mathrm{T}$ \\
\hline UN_xiong_989 & 129 & $\mathrm{C} / \mathrm{T}$ \\
\hline UN_xiong_990 & 144 & $\mathrm{C} / \mathrm{T}$ \\
\hline UN_xiong_997 & 54 & $\mathrm{~T} / \mathrm{C}$ \\
\hline UN_xiong_999 & 216 & $A / G$ \\
\hline UN_xiong_1001 & 97 & $\mathrm{G} / \mathrm{A}$ \\
\hline UN_xiong_1003 & 215 & $A / G$ \\
\hline UN_xiong_1003 & 442 & $C / G$ \\
\hline UN_xiong_1005 & 244 & $\mathrm{~T} / \mathrm{C}$ \\
\hline UN_xiong_1005 & 430 & $\mathrm{C} / \mathrm{T}$ \\
\hline UN_xiong_1005 & 609 & $A / G$ \\
\hline UN_xiong_1005 & 1124 & $\mathrm{~T} / \mathrm{C}$ \\
\hline UN_xiong_1016 & 168 & $\mathrm{~A} / \mathrm{G}$ \\
\hline UN_xiong_1027 & 100 & $\mathrm{G} / \mathrm{A}$ \\
\hline UN_xiong_1038 & 470 & $\mathrm{~T} / \mathrm{C}$ \\
\hline UN_xiong_1043 & 156 & $A / G$ \\
\hline UN_xiong_1043 & 354 & $\mathrm{~T} / \mathrm{G}$ \\
\hline UN_xiong_1049 & 134 & $\mathrm{~A} / \mathrm{T}$ \\
\hline UN_xiong_1049 & 326 & $A / G$ \\
\hline UN_xiong_1052 & 192 & $\mathrm{G} / \mathrm{T}$ \\
\hline UN_xiong_1057 & 164 & $\mathrm{G} / \mathrm{A}$ \\
\hline
\end{tabular}




\begin{tabular}{|c|c|c|}
\hline UN_xiong_1060 & 202 & $\mathrm{~T} / \mathrm{C}$ \\
\hline UN_xiong_1078 & 137 & $\mathrm{G} / \mathrm{A}$ \\
\hline UN_xiong_1085 & 226 & $C / G$ \\
\hline UN_xiong_1091 & 214 & $\mathrm{C} / \mathrm{T}$ \\
\hline UN_xiong_1091 & 456 & $\mathrm{~A} / \mathrm{C}$ \\
\hline UN_xiong_1092 & 203 & $\mathrm{~A} / \mathrm{C}$ \\
\hline UN_xiong_1102 & 247 & $\mathrm{~T} / \mathrm{C}$ \\
\hline UN_xiong_1105 & 30 & $\mathrm{G} / \mathrm{A}$ \\
\hline UN_xiong_1105 & 74 & $A / T$ \\
\hline UN_xiong_1105 & 196 & $\mathrm{G} / \mathrm{A}$ \\
\hline UN_xiong_1113 & 498 & $\mathrm{G} / \mathrm{A}$ \\
\hline UN_xiong_1113 & 542 & $\mathrm{~T} / \mathrm{C}$ \\
\hline UN_xiong_1113 & 781 & $\mathrm{C} / \mathrm{T}$ \\
\hline UN_xiong_1113 & 880 & $\mathrm{C} / \mathrm{T}$ \\
\hline UN_xiong_1129 & 169 & $\mathrm{~T} / \mathrm{C}$ \\
\hline UN_xiong_1129 & 248 & $\mathrm{C} / \mathrm{T}$ \\
\hline UN_xiong_1141 & 220 & $\mathrm{~T} / \mathrm{C}$ \\
\hline UN_xiong_1162 & 84 & $\mathrm{G} / \mathrm{C}$ \\
\hline UN_xiong_1164 & 207 & $\mathrm{~T} / \mathrm{C}$ \\
\hline UN_xiong_1167 & 101 & $A / G$ \\
\hline UN_xiong_1167 & 647 & $\mathrm{C} / \mathrm{T}$ \\
\hline UN_xiong_1167 & 761 & $\mathrm{~T} / \mathrm{C}$ \\
\hline UN_xiong_1167 & 786 & $\mathrm{~T} / \mathrm{C}$ \\
\hline UN_xiong_1170 & 91 & $A / G$ \\
\hline UN_xiong_1174 & 89 & $A / G$ \\
\hline UN_xiong_1174 & 152 & $A / G$ \\
\hline UN_xiong_1174 & 258 & $C / G$ \\
\hline UN_xiong_1174 & 279 & $A / G$ \\
\hline UN_xiong_1174 & 314 & $\mathrm{~T} / \mathrm{C}$ \\
\hline UN_xiong_1176 & 72 & $\mathrm{~A} / \mathrm{C}$ \\
\hline UN_xiong_1179 & 295 & $\mathrm{~A} / \mathrm{T}$ \\
\hline UN_xiong_1191 & 51 & $\mathrm{~T} / \mathrm{A}$ \\
\hline UN_xiong_1191 & 158 & $A / G$ \\
\hline UN_xiong_1196 & 365 & $\mathrm{G} / \mathrm{A}$ \\
\hline UN_xiong_1197 & 42 & $A / G$ \\
\hline UN_xiong_1199 & 201 & $\mathrm{C} / \mathrm{T}$ \\
\hline UN_xiong_1201 & 550 & $\mathrm{G} / \mathrm{A}$ \\
\hline UN_xiong_1201 & 573 & $\mathrm{~T} / \mathrm{C}$ \\
\hline UN_xiong_1201 & 834 & $\mathrm{~T} / \mathrm{C}$ \\
\hline UN_xiong_1201 & 1069 & $A / G$ \\
\hline UN_xiong_1201 & 1269 & $\mathrm{G} / \mathrm{A}$ \\
\hline UN_xiong_1201 & 1452 & $\mathrm{C} / \mathrm{T}$ \\
\hline UN_xiong_1201 & 1555 & $\mathrm{~A} / \mathrm{C}$ \\
\hline UN_xiong_1225 & 15 & $\mathrm{G} / \mathrm{A}$ \\
\hline UN_xiong_1226 & 456 & $\mathrm{~T} / \mathrm{G}$ \\
\hline UN_xiong_1226 & 556 & $A / G$ \\
\hline UN_xiong_1226 & 616 & $A / G$ \\
\hline
\end{tabular}




\begin{tabular}{|c|c|c|}
\hline UN_xiong_1226 & 681 & $\mathrm{~T} / \mathrm{A}$ \\
\hline UN_xiong_1226 & 818 & $\mathrm{~T} / \mathrm{C}$ \\
\hline UN_xiong_1239 & 51 & $A / G$ \\
\hline UN_xiong_1241 & 721 & $\mathrm{~T} / \mathrm{C}$ \\
\hline UN_xiong_1246 & 263 & $C / A$ \\
\hline UN_xiong_1246 & 358 & $\mathrm{~T} / \mathrm{C}$ \\
\hline UN_xiong_1246 & 430 & $\mathrm{~T} / \mathrm{C}$ \\
\hline UN_xiong_1247 & 188 & $\mathrm{~T} / \mathrm{C}$ \\
\hline UN_xiong_1247 & 348 & $A / G$ \\
\hline UN_xiong_1247 & 417 & $\mathrm{C} / \mathrm{T}$ \\
\hline UN_xiong_1247 & 449 & $\mathrm{~A} / \mathrm{T}$ \\
\hline UN_xiong_1247 & 533 & $\mathrm{G} / \mathrm{A}$ \\
\hline UN_xiong_1247 & 611 & $\mathrm{G} / \mathrm{A}$ \\
\hline UN_xiong_1263 & 335 & $\mathrm{~T} / \mathrm{C}$ \\
\hline UN_xiong_1264 & 66 & $\mathrm{C} / \mathrm{T}$ \\
\hline UN_xiong_1264 & 130 & $\mathrm{~T} / \mathrm{C}$ \\
\hline UN_xiong_1267 & 510 & $A / G$ \\
\hline UN_xiong_1267 & 559 & $A / G$ \\
\hline UN_xiong_1267 & 916 & $\mathrm{~T} / \mathrm{C}$ \\
\hline UN_xiong_1270 & 300 & $\mathrm{~T} / \mathrm{C}$ \\
\hline UN_xiong_1271 & 396 & $\mathrm{~T} / \mathrm{A}$ \\
\hline UN_xiong_1271 & 418 & $\mathrm{~T} / \mathrm{C}$ \\
\hline UN_xiong_1271 & 450 & $\mathrm{~T} / \mathrm{C}$ \\
\hline UN_xiong_1272 & 371 & $\mathrm{~T} / \mathrm{C}$ \\
\hline UN_xiong_1279 & 72 & $\mathrm{C} / \mathrm{T}$ \\
\hline UN_xiong_1279 & 444 & $\mathrm{~T} / \mathrm{C}$ \\
\hline UN_xiong_1280 & 251 & $\mathrm{G} / \mathrm{T}$ \\
\hline UN_xiong_1280 & 425 & $C / G$ \\
\hline UN_xiong_1280 & 804 & $A / G$ \\
\hline UN_xiong_1280 & 926 & $\mathrm{G} / \mathrm{T}$ \\
\hline UN_xiong_1280 & 950 & $\mathrm{~A} / \mathrm{C}$ \\
\hline UN_xiong_1280 & 1151 & $\mathrm{G} / \mathrm{T}$ \\
\hline UN_xiong_1281 & 877 & $\mathrm{~T} / \mathrm{C}$ \\
\hline UN_xiong_1287 & 74 & $\mathrm{G} / \mathrm{A}$ \\
\hline UN_xiong_1287 & 278 & $\mathrm{C} / \mathrm{T}$ \\
\hline UN_xiong_1291 & 163 & $\mathrm{C} / \mathrm{T}$ \\
\hline UN_xiong_1293 & 235 & $\mathrm{~T} / \mathrm{C}$ \\
\hline UN_xiong_1293 & 270 & $\mathrm{G} / \mathrm{A}$ \\
\hline UN_xiong_1293 & 359 & $\mathrm{G} / \mathrm{A}$ \\
\hline UN_xiong_1293 & 485 & $\mathrm{~A} / \mathrm{T}$ \\
\hline UN_xiong_1293 & 591 & $\mathrm{G} / \mathrm{A}$ \\
\hline UN_xiong_1293 & 616 & $A / G$ \\
\hline UN_xiong_1293 & 702 & $\mathrm{C} / \mathrm{T}$ \\
\hline UN_xiong_1298 & 41 & $\mathrm{G} / \mathrm{C}$ \\
\hline UN_xiong_1298 & 139 & $\mathrm{~T} / \mathrm{G}$ \\
\hline UN_xiong_1300 & 257 & $\mathrm{G} / \mathrm{A}$ \\
\hline UN_xiong_1300 & 336 & $\mathrm{~T} / \mathrm{C}$ \\
\hline
\end{tabular}




\begin{tabular}{|c|c|c|}
\hline UN_xiong_1306 & 147 & $A / G$ \\
\hline UN_xiong_1308 & 412 & $\mathrm{~A} / \mathrm{G}$ \\
\hline UN_xiong_1314 & 73 & $\mathrm{G} / \mathrm{C}$ \\
\hline UN_xiong_1325 & 23 & $\mathrm{~T} / \mathrm{C}$ \\
\hline UN_xiong_1325 & 163 & $\mathrm{G} / \mathrm{T}$ \\
\hline UN_xiong_1333 & 53 & $\mathrm{~A} / \mathrm{G}$ \\
\hline UN_xiong_1333 & 119 & $\mathrm{~A} / \mathrm{C}$ \\
\hline UN_xiong_1333 & 181 & $A / G$ \\
\hline UN_xiong_1333 & 445 & $\mathrm{C} / \mathrm{A}$ \\
\hline UN_xiong_1341 & 12 & $\mathrm{~A} / \mathrm{G}$ \\
\hline UN_xiong_1341 & 128 & $\mathrm{G} / \mathrm{A}$ \\
\hline UN_xiong_1342 & 109 & $\mathrm{C} / \mathrm{T}$ \\
\hline UN_xiong_1364 & 218 & $\mathrm{G} / \mathrm{A}$ \\
\hline UN_xiong_1364 & 300 & $\mathrm{G} / \mathrm{A}$ \\
\hline UN_xiong_1364 & 560 & $\mathrm{~T} / \mathrm{C}$ \\
\hline UN_xiong_1364 & 587 & $\mathrm{~A} / \mathrm{T}$ \\
\hline UN_xiong_1364 & 670 & $\mathrm{C} / \mathrm{T}$ \\
\hline UN_xiong_1373 & 37 & $\mathrm{~T} / \mathrm{A}$ \\
\hline UN_xiong_1376 & 122 & $\mathrm{~A} / \mathrm{G}$ \\
\hline UN_xiong_1376 & 212 & $\mathrm{C} / \mathrm{T}$ \\
\hline UN_xiong_1381 & 379 & $\mathrm{~T} / \mathrm{C}$ \\
\hline UN_xiong_1381 & 469 & $\mathrm{G} / \mathrm{T}$ \\
\hline UN_xiong_1381 & 599 & $\mathrm{~T} / \mathrm{C}$ \\
\hline UN_xiong_1381 & 631 & $\mathrm{C} / \mathrm{T}$ \\
\hline UN_xiong_1386 & 162 & $\mathrm{~T} / \mathrm{G}$ \\
\hline UN_xiong_1387 & 554 & $\mathrm{C} / \mathrm{T}$ \\
\hline UN_xiong_1392 & 283 & $\mathrm{~T} / \mathrm{A}$ \\
\hline UN_xiong_1392 & 656 & $\mathrm{G} / \mathrm{A}$ \\
\hline UN_xiong_1392 & 773 & $\mathrm{~A} / \mathrm{T}$ \\
\hline UN_xiong_1392 & 1016 & $\mathrm{~T} / \mathrm{C}$ \\
\hline UN_xiong_1392 & 1188 & $\mathrm{~T} / \mathrm{C}$ \\
\hline UN_xiong_1392 & 1228 & $A / G$ \\
\hline UN_xiong_1392 & 1320 & $\mathrm{~T} / \mathrm{C}$ \\
\hline UN_xiong_1392 & 1357 & $\mathrm{G} / \mathrm{A}$ \\
\hline UN_xiong_1397 & 169 & $\mathrm{G} / \mathrm{T}$ \\
\hline UN_xiong_1397 & 296 & $A / G$ \\
\hline UN_xiong_1397 & 339 & $A / G$ \\
\hline UN_xiong_1397 & 368 & $\mathrm{~A} / \mathrm{G}$ \\
\hline UN_xiong_1397 & 421 & $\mathrm{C} / \mathrm{T}$ \\
\hline UN_xiong_1397 & 444 & $A / G$ \\
\hline UN_xiong_1397 & 468 & $\mathrm{~T} / \mathrm{C}$ \\
\hline UN_xiong_1397 & 488 & $A / G$ \\
\hline UN_xiong_1397 & 510 & $\mathrm{~T} / \mathrm{C}$ \\
\hline UN_xiong_1397 & 532 & $A / G$ \\
\hline UN_xiong_1397 & 561 & $A / G$ \\
\hline UN_xiong_1397 & 584 & $\mathrm{G} / \mathrm{A}$ \\
\hline UN_xiong_1397 & 610 & $\mathrm{~T} / \mathrm{C}$ \\
\hline
\end{tabular}




\begin{tabular}{|c|c|c|}
\hline UN_xiong_1397 & 646 & $A / G$ \\
\hline UN_xiong_1397 & 671 & $\mathrm{~T} / \mathrm{A}$ \\
\hline UN_xiong_1397 & 783 & $C / A$ \\
\hline UN_xiong_1397 & 878 & $\mathrm{~A} / \mathrm{T}$ \\
\hline UN_xiong_1397 & 1021 & $\mathrm{C} / \mathrm{A}$ \\
\hline UN_xiong_1410 & 64 & $A / G$ \\
\hline UN_xiong_1410 & 154 & $\mathrm{~A} / \mathrm{T}$ \\
\hline UN_xiong_1411 & 335 & $A / G$ \\
\hline UN_xiong_1428 & 185 & $A / G$ \\
\hline UN_xiong_1436 & 64 & $A / G$ \\
\hline UN_xiong_1436 & 436 & $A / G$ \\
\hline UN_xiong_1438 & 35 & $\mathrm{~T} / \mathrm{C}$ \\
\hline UN_xiong_1438 & 101 & $C / A$ \\
\hline UN_xiong_1438 & 265 & $\mathrm{G} / \mathrm{C}$ \\
\hline UN_xiong_1438 & 288 & $\mathrm{C} / \mathrm{T}$ \\
\hline UN_xiong_1438 & 311 & $\mathrm{~T} / \mathrm{C}$ \\
\hline UN_xiong_1438 & 354 & $\mathrm{G} / \mathrm{C}$ \\
\hline UN_xiong_1438 & 420 & $\mathrm{~T} / \mathrm{C}$ \\
\hline UN_xiong_1441 & 343 & $\mathrm{~T} / \mathrm{C}$ \\
\hline UN_xiong_1445 & 61 & $\mathrm{G} / \mathrm{A}$ \\
\hline UN_xiong_1445 & 336 & $\mathrm{G} / \mathrm{T}$ \\
\hline UN_xiong_1445 & 699 & $\mathrm{~T} / \mathrm{G}$ \\
\hline UN_xiong_1452 & 329 & $A / G$ \\
\hline UN_xiong_1460 & 279 & $\mathrm{G} / \mathrm{T}$ \\
\hline UN_xiong_1463 & 591 & $A / G$ \\
\hline UN_xiong_1469 & 392 & $\mathrm{~T} / \mathrm{C}$ \\
\hline UN_xiong_1478 & 46 & $\mathrm{~T} / \mathrm{C}$ \\
\hline UN_xiong_1483 & 443 & $\mathrm{G} / \mathrm{A}$ \\
\hline UN_xiong_1492 & 197 & $\mathrm{~A} / \mathrm{T}$ \\
\hline UN_xiong_1492 & 253 & $\mathrm{~A} / \mathrm{T}$ \\
\hline UN_xiong_1492 & 303 & $\mathrm{G} / \mathrm{T}$ \\
\hline UN_xiong_1494 & 339 & $\mathrm{G} / \mathrm{A}$ \\
\hline UN_xiong_1494 & 387 & $\mathrm{~T} / \mathrm{A}$ \\
\hline UN_xiong_1494 & 564 & $\mathrm{~T} / \mathrm{C}$ \\
\hline UN_xiong_1496 & 283 & $\mathrm{G} / \mathrm{A}$ \\
\hline UN_xiong_1496 & 365 & $\mathrm{~T} / \mathrm{C}$ \\
\hline UN_xiong_1498 & 84 & $A / G$ \\
\hline UN_xiong_1498 & 188 & $A / G$ \\
\hline UN_xiong_1498 & 328 & $\mathrm{G} / \mathrm{A}$ \\
\hline UN_xiong_1498 & 351 & $\mathrm{C} / \mathrm{G}$ \\
\hline UN_xiong_1510 & 34 & $\mathrm{G} / \mathrm{T}$ \\
\hline UN_xiong_1512 & 288 & $\mathrm{~A} / \mathrm{T}$ \\
\hline UN_xiong_1513 & 706 & $C / G$ \\
\hline UN_xiong_1517 & 617 & $\mathrm{G} / \mathrm{A}$ \\
\hline UN_xiong_1525 & 86 & $\mathrm{G} / \mathrm{A}$ \\
\hline UN_xiong_1525 & 300 & $A / G$ \\
\hline UN_xiong_1525 & 539 & $\mathrm{~A} / \mathrm{T}$ \\
\hline
\end{tabular}




\begin{tabular}{|c|c|c|}
\hline UN_xiong_1528 & 537 & $\mathrm{~T} / \mathrm{C}$ \\
\hline UN_xiong_1528 & 642 & $\mathrm{~T} / \mathrm{C}$ \\
\hline UN_xiong_1548 & 147 & $C / G$ \\
\hline UN_xiong_1551 & 492 & $A / G$ \\
\hline UN_xiong_1551 & 522 & $\mathrm{~T} / \mathrm{C}$ \\
\hline UN_xiong_1551 & 667 & $\mathrm{~T} / \mathrm{C}$ \\
\hline UN_xiong_1551 & 693 & $\mathrm{G} / \mathrm{T}$ \\
\hline UN_xiong_1551 & 780 & $A / G$ \\
\hline UN_xiong_1551 & 822 & $A / G$ \\
\hline UN_xiong_1551 & 891 & $\mathrm{~T} / \mathrm{A}$ \\
\hline UN_xiong_1551 & 1174 & $\mathrm{C} / \mathrm{T}$ \\
\hline UN_xiong_1557 & 208 & $\mathrm{C} / \mathrm{G}$ \\
\hline UN_xiong_1557 & 366 & $\mathrm{C} / \mathrm{T}$ \\
\hline UN_xiong_1570 & 696 & $A / G$ \\
\hline UN_xiong_1570 & 717 & $C / A$ \\
\hline UN_xiong_1573 & 15 & $\mathrm{~T} / \mathrm{C}$ \\
\hline UN_xiong_1579 & 58 & $\mathrm{C} / \mathrm{T}$ \\
\hline UN_xiong_1580 & 181 & $\mathrm{~A} / \mathrm{C}$ \\
\hline UN_xiong_1586 & 100 & $A / G$ \\
\hline UN_xiong_1586 & 181 & $A / G$ \\
\hline UN_xiong_1586 & 251 & $A / G$ \\
\hline UN_xiong_1586 & 287 & $A / G$ \\
\hline UN_xiong_1586 & 481 & $\mathrm{G} / \mathrm{T}$ \\
\hline UN_xiong_1587 & 85 & $\mathrm{G} / \mathrm{A}$ \\
\hline UN_xiong_1587 & 184 & $\mathrm{C} / \mathrm{T}$ \\
\hline UN_xiong_1593 & 211 & $\mathrm{C} / \mathrm{T}$ \\
\hline UN_xiong_1593 & 246 & $A / G$ \\
\hline UN_xiong_1593 & 375 & $G / A$ \\
\hline UN_xiong_1593 & 431 & $A / G$ \\
\hline UN_xiong_1593 & 511 & $A / G$ \\
\hline UN_xiong_1593 & 627 & $\mathrm{G} / \mathrm{A}$ \\
\hline UN_xiong_1593 & 721 & $A / G$ \\
\hline UN_xiong_1593 & 797 & $\mathrm{G} / \mathrm{C}$ \\
\hline UN_xiong_1596 & 447 & $\mathrm{~T} / \mathrm{A}$ \\
\hline UN_xiong_1599 & 31 & $A / G$ \\
\hline UN_xiong_1599 & 51 & $\mathrm{C} / \mathrm{T}$ \\
\hline UN_xiong_1599 & 103 & $A / G$ \\
\hline UN_xiong_1599 & 150 & $A / G$ \\
\hline UN_xiong_1599 & 201 & $A / G$ \\
\hline UN_xiong_1599 & 235 & $\mathrm{~A} / \mathrm{T}$ \\
\hline UN_xiong_1599 & 310 & $A / T$ \\
\hline UN_xiong_1599 & 404 & $G / A$ \\
\hline UN_xiong_1599 & 446 & $A / G$ \\
\hline UN_xiong_1599 & 505 & $A / G$ \\
\hline UN_xiong_1599 & 550 & $\mathrm{~T} / \mathrm{C}$ \\
\hline UN_xiong_1599 & 599 & $\mathrm{G} / \mathrm{A}$ \\
\hline UN_xiong_1599 & 707 & $C / A$ \\
\hline
\end{tabular}




\begin{tabular}{|c|c|c|}
\hline UN_xiong_1602 & 457 & $\mathrm{~T} / \mathrm{A}$ \\
\hline UN_xiong_1602 & 874 & $\mathrm{~T} / \mathrm{C}$ \\
\hline UN_xiong_1603 & 79 & $C / T$ \\
\hline UN_xiong_1603 & 145 & $A / G$ \\
\hline UN_xiong_1603 & 222 & $\mathrm{G} / \mathrm{T}$ \\
\hline UN_xiong_1603 & 281 & $\mathrm{G} / \mathrm{T}$ \\
\hline UN_xiong_1603 & 341 & $A / G$ \\
\hline UN_xiong_1603 & 411 & $\mathrm{~T} / \mathrm{C}$ \\
\hline UN_xiong_1607 & 311 & $\mathrm{C} / \mathrm{T}$ \\
\hline UN_xiong_1608 & 77 & $\mathrm{~A} / \mathrm{T}$ \\
\hline UN_xiong_1611 & 299 & $\mathrm{G} / \mathrm{A}$ \\
\hline UN_xiong_1619 & 337 & $A / C$ \\
\hline UN_xiong_1619 & 393 & $\mathrm{~T} / \mathrm{A}$ \\
\hline UN_xiong_1619 & 419 & $C / A$ \\
\hline UN_xiong_1619 & 448 & $\mathrm{G} / \mathrm{T}$ \\
\hline UN_xiong_1620 & 264 & $A / G$ \\
\hline UN_xiong_1620 & 306 & $A / G$ \\
\hline UN_xiong_1623 & 71 & $\mathrm{C} / \mathrm{T}$ \\
\hline UN_xiong_1628 & 154 & $\mathrm{~T} / \mathrm{C}$ \\
\hline UN_xiong_1628 & 512 & $G / A$ \\
\hline UN_xiong_1637 & 131 & $\mathrm{~T} / \mathrm{C}$ \\
\hline UN_xiong_1637 & 217 & $\mathrm{~T} / \mathrm{A}$ \\
\hline UN_xiong_1637 & 337 & $\mathrm{C} / \mathrm{T}$ \\
\hline UN_xiong_1639 & 403 & $A / G$ \\
\hline UN_xiong_1641 & 102 & $G / A$ \\
\hline UN_xiong_1641 & 124 & $\mathrm{~T} / \mathrm{C}$ \\
\hline UN_xiong_1642 & 195 & $A / G$ \\
\hline UN_xiong_1646 & 632 & $A / G$ \\
\hline UN_xiong_1654 & 313 & $\mathrm{~T} / \mathrm{A}$ \\
\hline UN_xiong_1654 & 405 & $C / A$ \\
\hline UN_xiong_1657 & 522 & $A / G$ \\
\hline UN_xiong_1657 & 588 & $\mathrm{C} / \mathrm{T}$ \\
\hline UN_xiong_1657 & 1457 & $\mathrm{~T} / \mathrm{C}$ \\
\hline UN_xiong_1657 & 1484 & $A / G$ \\
\hline UN_xiong_1660 & 150 & $A / G$ \\
\hline UN_xiong_1676 & 17 & $A / G$ \\
\hline UN_xiong_1676 & 141 & $\mathrm{C} / \mathrm{T}$ \\
\hline UN_xiong_1682 & 349 & $A / G$ \\
\hline UN_xiong_1683 & 152 & $\mathrm{~T} / \mathrm{A}$ \\
\hline UN_xiong_1683 & 173 & $A / G$ \\
\hline UN_xiong_1685 & 345 & $\mathrm{~T} / \mathrm{C}$ \\
\hline UN_xiong_1685 & 608 & $\mathrm{C} / \mathrm{T}$ \\
\hline UN_xiong_1685 & 705 & $A / G$ \\
\hline UN_xiong_1686 & 187 & $\mathrm{C} / \mathrm{T}$ \\
\hline UN_xiong_1690 & 317 & $G / A$ \\
\hline UN_xiong_1692 & 83 & $G / A$ \\
\hline UN_xiong_1704 & 16 & $\mathrm{~T} / \mathrm{C}$ \\
\hline
\end{tabular}




\begin{tabular}{|c|c|c|}
\hline UN_xiong_1704 & 162 & $\mathrm{~A} / \mathrm{T}$ \\
\hline UN_xiong_1705 & 94 & $\mathrm{G} / \mathrm{C}$ \\
\hline UN_xiong_1714 & 288 & $\mathrm{~A} / \mathrm{G}$ \\
\hline UN_xiong_1726 & 98 & $\mathrm{~T} / \mathrm{C}$ \\
\hline UN_xiong_1726 & 123 & $\mathrm{~T} / \mathrm{G}$ \\
\hline UN_xiong_1726 & 157 & $\mathrm{G} / \mathrm{A}$ \\
\hline UN_xiong_1741 & 283 & $\mathrm{C} / \mathrm{T}$ \\
\hline UN_xiong_1750 & 289 & $\mathrm{~T} / \mathrm{C}$ \\
\hline UN_xiong_1750 & 707 & $A / G$ \\
\hline UN_xiong_1752 & 46 & $A / G$ \\
\hline UN_xiong_1756 & 345 & $\mathrm{C} / \mathrm{T}$ \\
\hline UN_xiong_1761 & 604 & $\mathrm{~A} / \mathrm{T}$ \\
\hline UN_xiong_1775 & 122 & $\mathrm{~T} / \mathrm{C}$ \\
\hline UN_xiong_1775 & 233 & $A / G$ \\
\hline UN_xiong_1785 & 167 & $\mathrm{G} / \mathrm{A}$ \\
\hline UN_xiong_1785 & 230 & $\mathrm{~T} / \mathrm{C}$ \\
\hline UN_xiong_1807 & 115 & $\mathrm{C} / \mathrm{T}$ \\
\hline UN_xiong_1807 & 162 & $\mathrm{C} / \mathrm{T}$ \\
\hline UN_xiong_1807 & 203 & $\mathrm{~T} / \mathrm{C}$ \\
\hline UN_xiong_1807 & 226 & $\mathrm{~T} / \mathrm{C}$ \\
\hline UN_xiong_1807 & 360 & $\mathrm{~A} / \mathrm{G}$ \\
\hline UN_xiong_1807 & 412 & $\mathrm{~T} / \mathrm{A}$ \\
\hline UN_xiong_1812 & 15 & $\mathrm{C} / \mathrm{T}$ \\
\hline UN_xiong_1814 & 6 & $\mathrm{C} / \mathrm{T}$ \\
\hline UN_xiong_1814 & 33 & $\mathrm{~T} / \mathrm{C}$ \\
\hline UN_xiong_1814 & 103 & $\mathrm{~A} / \mathrm{G}$ \\
\hline UN_xiong_1814 & 220 & $\mathrm{~T} / \mathrm{C}$ \\
\hline UN_xiong_1814 & 871 & $\mathrm{~T} / \mathrm{C}$ \\
\hline UN_xiong_1814 & 999 & $\mathrm{~A} / \mathrm{C}$ \\
\hline UN_xiong_1814 & 1151 & $\mathrm{G} / \mathrm{A}$ \\
\hline UN_xiong_1819 & 433 & $\mathrm{C} / \mathrm{G}$ \\
\hline UN_xiong_1820 & 42 & $\mathrm{~A} / \mathrm{C}$ \\
\hline UN_xiong_1826 & 51 & $\mathrm{~A} / \mathrm{G}$ \\
\hline UN_xiong_1831 & 92 & $\mathrm{G} / \mathrm{T}$ \\
\hline UN_xiong_1831 & 446 & $\mathrm{C} / \mathrm{T}$ \\
\hline UN_xiong_1841 & 27 & $\mathrm{~T} / \mathrm{C}$ \\
\hline UN_xiong_1841 & 338 & $\mathrm{G} / \mathrm{C}$ \\
\hline UN_xiong_1841 & 454 & $\mathrm{~A} / \mathrm{C}$ \\
\hline UN_xiong_1842 & 777 & $\mathrm{G} / \mathrm{A}$ \\
\hline UN_xiong_1842 & 841 & $\mathrm{C} / \mathrm{T}$ \\
\hline UN_xiong_1842 & 886 & $\mathrm{~T} / \mathrm{C}$ \\
\hline UN_xiong_1850 & 88 & $\mathrm{~A} / \mathrm{G}$ \\
\hline UN_xiong_1850 & 249 & $\mathrm{~T} / \mathrm{C}$ \\
\hline UN_xiong_1850 & 282 & $\mathrm{G} / \mathrm{A}$ \\
\hline UN_xiong_1850 & 326 & $\mathrm{~A} / \mathrm{G}$ \\
\hline UN_xiong_1850 & 530 & $A / G$ \\
\hline UN_xiong_1869 & 340 & $\mathrm{G} / \mathrm{A}$ \\
\hline
\end{tabular}




\begin{tabular}{|c|c|c|}
\hline UN_xiong_1875 & 15 & $\mathrm{C} / \mathrm{T}$ \\
\hline UN_xiong_1875 & 207 & $\mathrm{~T} / \mathrm{C}$ \\
\hline UN_xiong_1875 & 563 & $\mathrm{~A} / \mathrm{G}$ \\
\hline UN_xiong_1875 & 640 & $\mathrm{C} / \mathrm{T}$ \\
\hline UN_xiong_1875 & 696 & $A / G$ \\
\hline UN_xiong_1875 & 721 & $\mathrm{G} / \mathrm{A}$ \\
\hline UN_xiong_1880 & 265 & $\mathrm{~A} / \mathrm{G}$ \\
\hline UN_xiong_1881 & 358 & $\mathrm{G} / \mathrm{A}$ \\
\hline UN_xiong_1902 & 290 & $\mathrm{C} / \mathrm{T}$ \\
\hline UN_xiong_1904 & 14 & $\mathrm{C} / \mathrm{T}$ \\
\hline UN_xiong_1904 & 78 & $\mathrm{~T} / \mathrm{C}$ \\
\hline UN_xiong_1904 & 167 & $\mathrm{G} / \mathrm{A}$ \\
\hline UN_xiong_1904 & 202 & $\mathrm{~T} / \mathrm{G}$ \\
\hline UN_xiong_1904 & 235 & $\mathrm{~T} / \mathrm{C}$ \\
\hline UN_xiong_1904 & 602 & $\mathrm{G} / \mathrm{A}$ \\
\hline UN_xiong_1913 & 216 & $\mathrm{G} / \mathrm{T}$ \\
\hline UN_xiong_1913 & 378 & $\mathrm{C} / \mathrm{T}$ \\
\hline UN_xiong_1913 & 668 & $\mathrm{~A} / \mathrm{T}$ \\
\hline UN_xiong_1913 & 696 & $\mathrm{~A} / \mathrm{G}$ \\
\hline UN_xiong_1913 & 734 & $\mathrm{~T} / \mathrm{C}$ \\
\hline UN_xiong_1913 & 793 & $\mathrm{C} / \mathrm{T}$ \\
\hline UN_xiong_1913 & 929 & $\mathrm{~A} / \mathrm{G}$ \\
\hline UN_xiong_1913 & 969 & $\mathrm{G} / \mathrm{A}$ \\
\hline UN_xiong_1913 & 1042 & $\mathrm{~A} / \mathrm{G}$ \\
\hline UN_xiong_1913 & 1153 & $\mathrm{G} / \mathrm{A}$ \\
\hline UN_xiong_1913 & 1303 & $\mathrm{~T} / \mathrm{C}$ \\
\hline UN_xiong_1920 & 700 & $\mathrm{~T} / \mathrm{C}$ \\
\hline UN_xiong_1939 & 295 & $A / G$ \\
\hline UN_xiong_1942 & 219 & $\mathrm{G} / \mathrm{A}$ \\
\hline UN_xiong_1942 & 1312 & $\mathrm{C} / \mathrm{T}$ \\
\hline UN_xiong_1942 & 1523 & $\mathrm{G} / \mathrm{A}$ \\
\hline UN_xiong_1952 & 101 & $\mathrm{G} / \mathrm{T}$ \\
\hline UN_xiong_1952 & 279 & $\mathrm{G} / \mathrm{A}$ \\
\hline UN_xiong_1958 & 101 & $\mathrm{~T} / \mathrm{C}$ \\
\hline UN_xiong_1958 & 168 & $\mathrm{G} / \mathrm{A}$ \\
\hline UN_xiong_1958 & 241 & $\mathrm{C} / \mathrm{T}$ \\
\hline UN_xiong_1959 & 259 & $\mathrm{~T} / \mathrm{A}$ \\
\hline UN_xiong_1959 & 340 & $\mathrm{C} / \mathrm{T}$ \\
\hline UN_xiong_1978 & 222 & $\mathrm{G} / \mathrm{A}$ \\
\hline UN_xiong_1982 & 126 & $\mathrm{C} / \mathrm{T}$ \\
\hline UN_xiong_1982 & 325 & $\mathrm{G} / \mathrm{A}$ \\
\hline UN_xiong_1987 & 467 & $\mathrm{C} / \mathrm{T}$ \\
\hline UN_xiong_1987 & 852 & $\mathrm{~T} / \mathrm{C}$ \\
\hline UN_xiong_1987 & 874 & $A / G$ \\
\hline UN_xiong_1987 & 1020 & $\mathrm{C} / \mathrm{T}$ \\
\hline UN_xiong_1990 & 756 & $\mathrm{C} / \mathrm{T}$ \\
\hline UN_xiong_1990 & 838 & $\mathrm{C} / \mathrm{T}$ \\
\hline
\end{tabular}




\begin{tabular}{|c|c|c|}
\hline UN_xiong_1990 & 942 & $\mathrm{~T} / \mathrm{C}$ \\
\hline UN_xiong_1990 & 965 & $\mathrm{G} / \mathrm{A}$ \\
\hline UN_xiong_1990 & 1198 & $A / G$ \\
\hline UN_xiong_1990 & 1320 & $\mathrm{~T} / \mathrm{C}$ \\
\hline UN_xiong_1990 & 1382 & $\mathrm{~T} / \mathrm{C}$ \\
\hline UN_xiong_1990 & 1506 & $\mathrm{G} / \mathrm{A}$ \\
\hline UN_xiong_1990 & 1646 & $\mathrm{G} / \mathrm{T}$ \\
\hline UN_xiong_1994 & 171 & $A / G$ \\
\hline UN_xiong_2000 & 211 & $A / G$ \\
\hline UN_xiong_2000 & 349 & $\mathrm{~T} / \mathrm{C}$ \\
\hline UN_xiong_2000 & 712 & $\mathrm{~T} / \mathrm{C}$ \\
\hline UN_xiong_2000 & 937 & $\mathrm{~T} / \mathrm{C}$ \\
\hline UN_xiong_2000 & 1002 & $A / G$ \\
\hline UN_xiong_2000 & 1056 & $A / G$ \\
\hline UN_xiong_2016 & 300 & $C / A$ \\
\hline UN_xiong_2016 & 473 & $A / G$ \\
\hline UN_xiong_2016 & 509 & $A / G$ \\
\hline UN_xiong_2016 & 687 & $\mathrm{~T} / \mathrm{C}$ \\
\hline UN_xiong_2024 & 208 & $\mathrm{C} / \mathrm{T}$ \\
\hline UN_xiong_2024 & 287 & $\mathrm{~T} / \mathrm{C}$ \\
\hline UN_xiong_2024 & 337 & $\mathrm{~T} / \mathrm{C}$ \\
\hline UN_xiong_2024 & 394 & $\mathrm{C} / \mathrm{T}$ \\
\hline UN_xiong_2024 & 585 & $C / A$ \\
\hline UN_xiong_2024 & 697 & $A / G$ \\
\hline UN_xiong_2024 & 765 & $\mathrm{~T} / \mathrm{C}$ \\
\hline UN_xiong_2026 & 91 & $\mathrm{C} / \mathrm{T}$ \\
\hline UN_xiong_2032 & 511 & $\mathrm{~A} / \mathrm{T}$ \\
\hline UN_xiong_2035 & 125 & $A / G$ \\
\hline UN_xiong_2035 & 253 & $A / G$ \\
\hline UN_xiong_2037 & 440 & $\mathrm{~T} / \mathrm{C}$ \\
\hline UN_xiong_2037 & 610 & $T / G$ \\
\hline UN_xiong_2037 & 635 & $\mathrm{~T} / \mathrm{C}$ \\
\hline UN_xiong_2052 & 428 & $T / G$ \\
\hline UN_xiong_2054 & 95 & $\mathrm{G} / \mathrm{T}$ \\
\hline UN_xiong_2062 & 12 & $\mathrm{~A} / \mathrm{T}$ \\
\hline UN_xiong_2063 & 322 & $\mathrm{C} / \mathrm{T}$ \\
\hline UN_xiong_2077 & 253 & $\mathrm{G} / \mathrm{T}$ \\
\hline UN_xiong_2077 & 339 & $G / A$ \\
\hline UN_xiong_2086 & 193 & $\mathrm{G} / \mathrm{A}$ \\
\hline UN_xiong_2086 & 583 & $\mathrm{G} / \mathrm{A}$ \\
\hline UN_xiong_2086 & 1121 & $\mathrm{G} / \mathrm{T}$ \\
\hline UN_xiong_2088 & 122 & $\mathrm{~T} / \mathrm{C}$ \\
\hline UN_xiong_2091 & 196 & $\mathrm{C} / \mathrm{T}$ \\
\hline UN_xiong_2098 & 77 & $\mathrm{C} / \mathrm{T}$ \\
\hline UN_xiong_2098 & 117 & $\mathrm{G} / \mathrm{T}$ \\
\hline UN_xiong_2098 & 215 & $G / A$ \\
\hline UN_xiong_2098 & 293 & $\mathrm{~T} / \mathrm{C}$ \\
\hline
\end{tabular}




\begin{tabular}{|c|c|c|}
\hline UN_xiong_2098 & 522 & $\mathrm{C} / \mathrm{G}$ \\
\hline UN_xiong_2098 & 624 & $\mathrm{~T} / \mathrm{G}$ \\
\hline UN_xiong_2098 & 644 & $\mathrm{~A} / \mathrm{C}$ \\
\hline UN_xiong_2099 & 45 & $\mathrm{~A} / \mathrm{G}$ \\
\hline UN_xiong_2099 & 118 & $\mathrm{~T} / \mathrm{C}$ \\
\hline UN_xiong_2099 & 398 & $\mathrm{~A} / \mathrm{C}$ \\
\hline UN_xiong_2100 & 91 & $\mathrm{G} / \mathrm{A}$ \\
\hline UN_xiong_2103 & 40 & $\mathrm{~A} / \mathrm{G}$ \\
\hline UN_xiong_2106 & 224 & $\mathrm{G} / \mathrm{T}$ \\
\hline UN_xiong_2109 & 116 & $\mathrm{~A} / \mathrm{G}$ \\
\hline UN_xiong_2119 & 303 & $C / T$ \\
\hline UN_xiong_2127 & 83 & $\mathrm{C} / \mathrm{T}$ \\
\hline UN_xiong_2129 & 200 & $A / G$ \\
\hline UN_xiong_2138 & 62 & $\mathrm{~T} / \mathrm{G}$ \\
\hline UN_xiong_2138 & 114 & $A / G$ \\
\hline UN_xiong_2138 & 134 & $\mathrm{~T} / \mathrm{C}$ \\
\hline UN_xiong_2154 & 28 & $\mathrm{C} / \mathrm{T}$ \\
\hline UN_xiong_2154 & 90 & $\mathrm{C} / \mathrm{T}$ \\
\hline UN_xiong_2157 & 430 & $\mathrm{C} / \mathrm{T}$ \\
\hline UN_xiong_2168 & 155 & $\mathrm{~T} / \mathrm{C}$ \\
\hline UN_xiong_2181 & 221 & $\mathrm{~T} / \mathrm{C}$ \\
\hline UN_xiong_2181 & 365 & $\mathrm{~T} / \mathrm{C}$ \\
\hline UN_xiong_2181 & 472 & $A / G$ \\
\hline UN_xiong_2182 & 282 & $\mathrm{G} / \mathrm{A}$ \\
\hline UN_xiong_2186 & 231 & $\mathrm{G} / \mathrm{C}$ \\
\hline UN_xiong_2186 & 480 & $A / G$ \\
\hline UN_xiong_2198 & 381 & $\mathrm{~T} / \mathrm{C}$ \\
\hline UN_xiong_2198 & 408 & $\mathrm{C} / \mathrm{T}$ \\
\hline UN_xiong_2198 & 435 & $\mathrm{~T} / \mathrm{C}$ \\
\hline UN_xiong_2198 & 460 & $A / G$ \\
\hline UN_xiong_2198 & 480 & $\mathrm{G} / \mathrm{A}$ \\
\hline UN_xiong_2203 & 294 & $A / G$ \\
\hline UN_xiong_2209 & 111 & $\mathrm{~A} / \mathrm{T}$ \\
\hline UN_xiong_2209 & 160 & $A / G$ \\
\hline UN_xiong_2213 & 210 & $\mathrm{G} / \mathrm{A}$ \\
\hline UN_xiong_2213 & 233 & $\mathrm{~A} / \mathrm{C}$ \\
\hline UN_xiong_2213 & 362 & $\mathrm{~T} / \mathrm{C}$ \\
\hline UN_xiong_2216 & 168 & $\mathrm{~T} / \mathrm{A}$ \\
\hline UN_xiong_2218 & 646 & $\mathrm{C} / \mathrm{T}$ \\
\hline UN_xiong_2218 & 780 & $\mathrm{~T} / \mathrm{C}$ \\
\hline UN_xiong_2237 & 53 & $\mathrm{~A} / \mathrm{G}$ \\
\hline UN_xiong_2237 & 73 & $A / G$ \\
\hline UN_xiong_2237 & 957 & $\mathrm{G} / \mathrm{A}$ \\
\hline UN_xiong_2239 & 337 & $\mathrm{G} / \mathrm{A}$ \\
\hline UN_xiong_2241 & 160 & $\mathrm{~T} / \mathrm{C}$ \\
\hline UN_xiong_2248 & 623 & $A / G$ \\
\hline UN_xiong_2251 & 449 & $\mathrm{~A} / \mathrm{G}$ \\
\hline
\end{tabular}




\begin{tabular}{|c|c|c|}
\hline UN_xiong_2265 & 501 & $A / G$ \\
\hline UN_xiong_2265 & 805 & $\mathrm{G} / \mathrm{A}$ \\
\hline UN_xiong_2265 & 989 & $A / G$ \\
\hline UN_xiong_2265 & 1232 & $\mathrm{C} / \mathrm{T}$ \\
\hline UN_xiong_2266 & 362 & $\mathrm{C} / \mathrm{A}$ \\
\hline UN_xiong_2269 & 129 & $\mathrm{~T} / \mathrm{C}$ \\
\hline UN_xiong_2277 & 571 & $\mathrm{C} / \mathrm{T}$ \\
\hline UN_xiong_2283 & 300 & $\mathrm{~T} / \mathrm{A}$ \\
\hline UN_xiong_2284 & 285 & $A / G$ \\
\hline UN_xiong_2284 & 313 & $A / G$ \\
\hline UN_xiong_2288 & 304 & $\mathrm{C} / \mathrm{T}$ \\
\hline UN_xiong_2307 & 179 & $\mathrm{C} / \mathrm{T}$ \\
\hline UN_xiong_2307 & 402 & $A / G$ \\
\hline UN_xiong_2313 & 137 & $\mathrm{~T} / \mathrm{C}$ \\
\hline UN_xiong_2313 & 176 & $\mathrm{~T} / \mathrm{C}$ \\
\hline UN_xiong_2317 & 105 & $\mathrm{~A} / \mathrm{C}$ \\
\hline UN_xiong_2317 & 291 & $A / G$ \\
\hline UN_xiong_2317 & 314 & $A / G$ \\
\hline UN_xiong_2317 & 408 & $\mathrm{~T} / \mathrm{C}$ \\
\hline UN_xiong_2317 & 479 & $\mathrm{~T} / \mathrm{G}$ \\
\hline UN_xiong_2317 & 506 & $\mathrm{~T} / \mathrm{C}$ \\
\hline UN_xiong_2317 & 561 & $\mathrm{~A} / \mathrm{C}$ \\
\hline UN_xiong_2320 & 61 & $\mathrm{G} / \mathrm{A}$ \\
\hline UN_xiong_2320 & 299 & $\mathrm{C} / \mathrm{T}$ \\
\hline UN_xiong_2324 & 772 & $\mathrm{C} / \mathrm{T}$ \\
\hline UN_xiong_2325 & 99 & $\mathrm{C} / \mathrm{T}$ \\
\hline UN_xiong_2325 & 125 & $\mathrm{G} / \mathrm{A}$ \\
\hline UN_xiong_2338 & 735 & $\mathrm{~T} / \mathrm{C}$ \\
\hline UN_xiong_2340 & 265 & $A / G$ \\
\hline UN_xiong_2340 & 749 & $\mathrm{~T} / \mathrm{C}$ \\
\hline UN_xiong_2346 & 382 & $\mathrm{G} / \mathrm{A}$ \\
\hline UN_xiong_2353 & 376 & $\mathrm{G} / \mathrm{A}$ \\
\hline UN_xiong_2360 & 252 & $A / G$ \\
\hline UN_xiong_2360 & 331 & $A / G$ \\
\hline UN_xiong_2368 & 67 & $\mathrm{G} / \mathrm{T}$ \\
\hline UN_xiong_2368 & 126 & $A / G$ \\
\hline UN_xiong_2368 & 260 & $\mathrm{C} / \mathrm{T}$ \\
\hline UN_xiong_2368 & 652 & $\mathrm{~T} / \mathrm{C}$ \\
\hline UN_xiong_2369 & 452 & $A / G$ \\
\hline UN_xiong_2374 & 302 & $\mathrm{~A} / \mathrm{C}$ \\
\hline UN_xiong_2382 & 693 & $\mathrm{~T} / \mathrm{G}$ \\
\hline UN_xiong_2382 & 947 & $\mathrm{~T} / \mathrm{C}$ \\
\hline UN_xiong_2382 & 1247 & $A / G$ \\
\hline UN_xiong_2399 & 22 & $\mathrm{G} / \mathrm{C}$ \\
\hline UN_xiong_2399 & 132 & $\mathrm{~T} / \mathrm{G}$ \\
\hline UN_xiong_2404 & 473 & $A / G$ \\
\hline UN_xiong_2408 & 265 & $\mathrm{~T} / \mathrm{C}$ \\
\hline
\end{tabular}




\begin{tabular}{|c|c|c|}
\hline UN_xiong_2408 & 298 & $\mathrm{~T} / \mathrm{C}$ \\
\hline UN_xiong_2413 & 478 & $A / G$ \\
\hline UN_xiong_2416 & 452 & $G / A$ \\
\hline UN_xiong_2417 & 486 & $\mathrm{~T} / \mathrm{C}$ \\
\hline UN_xiong_2427 & 562 & $\mathrm{G} / \mathrm{C}$ \\
\hline UN_xiong_2429 & 221 & $C / A$ \\
\hline UN_xiong_2429 & 267 & $\mathrm{~T} / \mathrm{A}$ \\
\hline UN_xiong_2435 & 770 & $\mathrm{C} / \mathrm{T}$ \\
\hline UN_xiong_2435 & 822 & $A / G$ \\
\hline UN_xiong_2438 & 265 & $A / G$ \\
\hline UN_xiong_2442 & 208 & $A / G$ \\
\hline UN_xiong_2446 & 133 & $\mathrm{G} / \mathrm{C}$ \\
\hline UN_xiong_2454 & 248 & $\mathrm{~T} / \mathrm{C}$ \\
\hline UN_xiong_2454 & 298 & $\mathrm{C} / \mathrm{T}$ \\
\hline UN_xiong_2460 & 187 & $\mathrm{G} / \mathrm{C}$ \\
\hline UN_xiong_2463 & 133 & $\mathrm{G} / \mathrm{A}$ \\
\hline UN_xiong_2467 & 347 & $\mathrm{G} / \mathrm{T}$ \\
\hline UN_xiong_2470 & 335 & $\mathrm{C} / \mathrm{T}$ \\
\hline UN_xiong_2472 & 70 & $\mathrm{C} / \mathrm{T}$ \\
\hline UN_xiong_2480 & 136 & $A / G$ \\
\hline UN_xiong_2481 & 286 & $A / G$ \\
\hline UN_xiong_2483 & 321 & $A / G$ \\
\hline UN_xiong_2493 & 173 & $\mathrm{C} / \mathrm{T}$ \\
\hline UN_xiong_2509 & 237 & $\mathrm{~A} / \mathrm{T}$ \\
\hline UN_xiong_2510 & 285 & $A / G$ \\
\hline UN_xiong_2515 & 418 & $A / G$ \\
\hline UN_xiong_2519 & 176 & $\mathrm{G} / \mathrm{A}$ \\
\hline UN_xiong_2519 & 205 & $\mathrm{C} / \mathrm{T}$ \\
\hline UN_xiong_2519 & 393 & $\mathrm{~T} / \mathrm{C}$ \\
\hline UN_xiong_2519 & 463 & $\mathrm{C} / \mathrm{T}$ \\
\hline UN_xiong_2519 & 487 & $\mathrm{~T} / \mathrm{C}$ \\
\hline UN_xiong_2519 & 618 & $A / C$ \\
\hline UN_xiong_2519 & 686 & $A / C$ \\
\hline UN_xiong_2519 & 753 & $G / A$ \\
\hline UN_xiong_2519 & 927 & $\mathrm{G} / \mathrm{C}$ \\
\hline UN_xiong_2519 & 956 & $\mathrm{~T} / \mathrm{A}$ \\
\hline UN_xiong_2519 & 982 & $A / G$ \\
\hline UN_xiong_2519 & 1003 & $\mathrm{~T} / \mathrm{A}$ \\
\hline UN_xiong_2520 & 40 & $\mathrm{C} / \mathrm{T}$ \\
\hline UN_xiong_2521 & 551 & $\mathrm{~T} / \mathrm{C}$ \\
\hline UN_xiong_2521 & 619 & $\mathrm{G} / \mathrm{A}$ \\
\hline UN_xiong_2521 & 770 & $A / G$ \\
\hline UN_xiong_2521 & 934 & $\mathrm{~A} / \mathrm{C}$ \\
\hline UN_xiong_2522 & 112 & $A / G$ \\
\hline UN_xiong_2522 & 279 & $C / A$ \\
\hline UN_xiong_2523 & 61 & $A / G$ \\
\hline UN_xiong_2524 & 49 & $\mathrm{~T} / \mathrm{A}$ \\
\hline
\end{tabular}




\begin{tabular}{|c|c|c|}
\hline UN_xiong_2530 & 728 & $\mathrm{C} / \mathrm{T}$ \\
\hline UN_xiong_2530 & 894 & $\mathrm{G} / \mathrm{A}$ \\
\hline UN_xiong_2534 & 372 & $\mathrm{~T} / \mathrm{C}$ \\
\hline UN_xiong_2540 & 32 & $\mathrm{~A} / \mathrm{G}$ \\
\hline UN_xiong_2555 & 67 & $\mathrm{G} / \mathrm{C}$ \\
\hline UN_xiong_2555 & 155 & $\mathrm{~T} / \mathrm{G}$ \\
\hline UN_xiong_2555 & 184 & $\mathrm{~T} / \mathrm{C}$ \\
\hline UN_xiong_2555 & 313 & $\mathrm{~A} / \mathrm{G}$ \\
\hline UN_xiong_2555 & 383 & $\mathrm{G} / \mathrm{A}$ \\
\hline UN_xiong_2558 & 183 & $\mathrm{~T} / \mathrm{A}$ \\
\hline UN_xiong_2558 & 374 & $\mathrm{~T} / \mathrm{C}$ \\
\hline UN_xiong_2562 & 463 & $\mathrm{~A} / \mathrm{G}$ \\
\hline UN_xiong_2569 & 329 & $\mathrm{~T} / \mathrm{C}$ \\
\hline UN_xiong_2575 & 155 & $\mathrm{G} / \mathrm{A}$ \\
\hline UN_xiong_2577 & 385 & $\mathrm{~A} / \mathrm{T}$ \\
\hline UN_xiong_2580 & 97 & $\mathrm{~T} / \mathrm{A}$ \\
\hline UN_xiong_2580 & 185 & $\mathrm{~T} / \mathrm{C}$ \\
\hline UN_xiong_2593 & 421 & $C / G$ \\
\hline UN_xiong_2593 & 442 & $\mathrm{~A} / \mathrm{T}$ \\
\hline UN_xiong_2604 & 56 & $\mathrm{~A} / \mathrm{G}$ \\
\hline UN_xiong_2605 & 260 & $\mathrm{~A} / \mathrm{T}$ \\
\hline UN_xiong_2606 & 138 & $\mathrm{~A} / \mathrm{G}$ \\
\hline UN_xiong_2606 & 231 & $\mathrm{G} / \mathrm{A}$ \\
\hline UN_xiong_2608 & 444 & $\mathrm{C} / \mathrm{T}$ \\
\hline UN_xiong_2611 & 116 & $\mathrm{~A} / \mathrm{G}$ \\
\hline UN_xiong_2611 & 207 & $\mathrm{~T} / \mathrm{C}$ \\
\hline UN_xiong_2611 & 315 & $\mathrm{C} / \mathrm{T}$ \\
\hline UN_xiong_2611 & 383 & $\mathrm{~T} / \mathrm{A}$ \\
\hline UN_xiong_2611 & 473 & $\mathrm{~T} / \mathrm{C}$ \\
\hline UN_xiong_2611 & 574 & $\mathrm{C} / \mathrm{A}$ \\
\hline UN_xiong_2622 & 48 & $\mathrm{~T} / \mathrm{C}$ \\
\hline UN_xiong_2622 & 342 & $\mathrm{~A} / \mathrm{G}$ \\
\hline UN_xiong_2623 & 262 & $\mathrm{C} / \mathrm{A}$ \\
\hline UN_xiong_2636 & 23 & $\mathrm{G} / \mathrm{C}$ \\
\hline UN_xiong_2636 & 53 & $\mathrm{~T} / \mathrm{A}$ \\
\hline UN_xiong_2636 & 90 & $\mathrm{G} / \mathrm{A}$ \\
\hline UN_xiong_2637 & 13 & $\mathrm{G} / \mathrm{T}$ \\
\hline UN_xiong_2637 & 38 & $\mathrm{G} / \mathrm{T}$ \\
\hline UN_xiong_2637 & 105 & $\mathrm{~A} / \mathrm{G}$ \\
\hline UN_xiong_2637 & 148 & $\mathrm{~T} / \mathrm{A}$ \\
\hline UN_xiong_2637 & 186 & $\mathrm{G} / \mathrm{A}$ \\
\hline UN_xiong_2637 & 251 & $\mathrm{~A} / \mathrm{G}$ \\
\hline UN_xiong_2637 & 296 & $\mathrm{G} / \mathrm{A}$ \\
\hline UN_xiong_2638 & 248 & $\mathrm{G} / \mathrm{A}$ \\
\hline UN_xiong_2639 & 116 & $\mathrm{G} / \mathrm{T}$ \\
\hline UN_xiong_2649 & 405 & $\mathrm{C} / \mathrm{T}$ \\
\hline UN_xiong_2649 & 452 & $\mathrm{~A} / \mathrm{G}$ \\
\hline
\end{tabular}




\begin{tabular}{|c|c|c|}
\hline UN_xiong_2649 & 515 & $\mathrm{C} / \mathrm{T}$ \\
\hline UN_xiong_2649 & 636 & $\mathrm{~T} / \mathrm{C}$ \\
\hline UN_xiong_2652 & 55 & $\mathrm{G} / \mathrm{A}$ \\
\hline UN_xiong_2656 & 65 & $\mathrm{~T} / \mathrm{G}$ \\
\hline UN_xiong_2656 & 104 & $\mathrm{C} / \mathrm{T}$ \\
\hline UN_xiong_2656 & 163 & $A / G$ \\
\hline UN_xiong_2656 & 203 & $\mathrm{G} / \mathrm{A}$ \\
\hline UN_xiong_2656 & 260 & $A / G$ \\
\hline UN_xiong_2656 & 283 & $A / G$ \\
\hline UN_xiong_2656 & 310 & $\mathrm{~T} / \mathrm{C}$ \\
\hline UN_xiong_2656 & 343 & $A / G$ \\
\hline UN_xiong_2656 & 369 & $\mathrm{C} / \mathrm{T}$ \\
\hline UN_xiong_2656 & 392 & $\mathrm{~A} / \mathrm{T}$ \\
\hline UN_xiong_2656 & 417 & $\mathrm{~T} / \mathrm{C}$ \\
\hline UN_xiong_2662 & 265 & $\mathrm{C} / \mathrm{T}$ \\
\hline UN_xiong_2662 & 409 & $A / G$ \\
\hline UN_xiong_2662 & 519 & $\mathrm{~T} / \mathrm{C}$ \\
\hline UN_xiong_2662 & 566 & $A / G$ \\
\hline UN_xiong_2662 & 591 & $C / A$ \\
\hline UN_xiong_2663 & 59 & $\mathrm{G} / \mathrm{T}$ \\
\hline UN_xiong_2663 & 93 & $\mathrm{~A} / \mathrm{T}$ \\
\hline UN_xiong_2663 & 300 & $A / G$ \\
\hline UN_xiong_2677 & 237 & $\mathrm{C} / \mathrm{A}$ \\
\hline UN_xiong_2677 & 375 & $\mathrm{~T} / \mathrm{A}$ \\
\hline UN_xiong_2677 & 701 & $\mathrm{C} / \mathrm{T}$ \\
\hline UN_xiong_2689 & 600 & $\mathrm{G} / \mathrm{A}$ \\
\hline UN_xiong_2689 & 1176 & $\mathrm{G} / \mathrm{T}$ \\
\hline UN_xiong_2706 & 367 & $\mathrm{G} / \mathrm{A}$ \\
\hline UN_xiong_2708 & 296 & $\mathrm{G} / \mathrm{A}$ \\
\hline UN_xiong_2710 & 45 & $\mathrm{~T} / \mathrm{C}$ \\
\hline UN_xiong_2714 & 101 & $\mathrm{C} / \mathrm{T}$ \\
\hline UN_xiong_2714 & 218 & $\mathrm{~A} / \mathrm{T}$ \\
\hline UN_xiong_2714 & 320 & $\mathrm{C} / \mathrm{T}$ \\
\hline UN_xiong_2714 & 489 & $A / G$ \\
\hline UN_xiong_2714 & 579 & $A / G$ \\
\hline UN_xiong_2724 & 61 & $C / A$ \\
\hline UN_xiong_2724 & 175 & $\mathrm{G} / \mathrm{T}$ \\
\hline UN_xiong_2725 & 251 & $\mathrm{C} / \mathrm{T}$ \\
\hline UN_xiong_2729 & 400 & $\mathrm{C} / \mathrm{T}$ \\
\hline UN_xiong_2729 & 585 & $\mathrm{~T} / \mathrm{A}$ \\
\hline UN_xiong_2732 & 69 & $\mathrm{G} / \mathrm{C}$ \\
\hline UN_xiong_2732 & 187 & $\mathrm{C} / \mathrm{T}$ \\
\hline UN_xiong_2737 & 313 & $\mathrm{C} / \mathrm{T}$ \\
\hline UN_xiong_2742 & 32 & $\mathrm{C} / \mathrm{T}$ \\
\hline UN_xiong_2742 & 54 & $A / G$ \\
\hline UN_xiong_2742 & 224 & $\mathrm{~T} / \mathrm{C}$ \\
\hline UN_xiong_2742 & 553 & $A / G$ \\
\hline
\end{tabular}




\begin{tabular}{|c|c|c|}
\hline UN_xiong_2742 & 625 & $A / G$ \\
\hline UN_xiong_2752 & 468 & $\mathrm{~A} / \mathrm{C}$ \\
\hline UN_xiong_2764 & 91 & $\mathrm{~A} / \mathrm{G}$ \\
\hline UN_xiong_2771 & 120 & $A / G$ \\
\hline UN_xiong_2773 & 214 & $A / G$ \\
\hline UN_xiong_2773 & 266 & $\mathrm{~T} / \mathrm{C}$ \\
\hline UN_xiong_2773 & 329 & $\mathrm{~T} / \mathrm{C}$ \\
\hline UN_xiong_2777 & 97 & $\mathrm{~A} / \mathrm{T}$ \\
\hline UN_xiong_2783 & 122 & $\mathrm{~A} / \mathrm{T}$ \\
\hline UN_xiong_2791 & 334 & $\mathrm{C} / \mathrm{A}$ \\
\hline UN_xiong_2792 & 115 & $A / G$ \\
\hline UN_xiong_2793 & 437 & $\mathrm{~T} / \mathrm{A}$ \\
\hline UN_xiong_2796 & 624 & $\mathrm{G} / \mathrm{C}$ \\
\hline UN_xiong_2800 & 247 & $\mathrm{C} / \mathrm{A}$ \\
\hline UN_xiong_2807 & 195 & $\mathrm{C} / \mathrm{A}$ \\
\hline UN_xiong_2808 & 30 & $\mathrm{G} / \mathrm{A}$ \\
\hline UN_xiong_2815 & 314 & $\mathrm{~A} / \mathrm{T}$ \\
\hline UN_xiong_2815 & 341 & $A / G$ \\
\hline UN_xiong_2818 & 874 & $\mathrm{~T} / \mathrm{G}$ \\
\hline UN_xiong_2825 & 150 & $\mathrm{G} / \mathrm{T}$ \\
\hline UN_xiong_2825 & 310 & $\mathrm{C} / \mathrm{T}$ \\
\hline UN_xiong_2825 & 370 & $\mathrm{G} / \mathrm{T}$ \\
\hline UN_xiong_2826 & 101 & $A / G$ \\
\hline UN_xiong_2826 & 241 & $\mathrm{~A} / \mathrm{T}$ \\
\hline UN_xiong_2826 & 270 & $\mathrm{~T} / \mathrm{C}$ \\
\hline UN_xiong_2826 & 298 & $\mathrm{~T} / \mathrm{C}$ \\
\hline UN_xiong_2826 & 428 & $\mathrm{~T} / \mathrm{C}$ \\
\hline UN_xiong_2848 & 55 & $\mathrm{G} / \mathrm{A}$ \\
\hline UN_xiong_2848 & 78 & $\mathrm{~T} / \mathrm{A}$ \\
\hline UN_xiong_2848 & 144 & $\mathrm{G} / \mathrm{A}$ \\
\hline UN_xiong_2848 & 241 & $\mathrm{G} / \mathrm{A}$ \\
\hline UN_xiong_2848 & 275 & $A / G$ \\
\hline UN_xiong_2848 & 308 & $\mathrm{~A} / \mathrm{C}$ \\
\hline UN_xiong_2848 & 352 & $\mathrm{C} / \mathrm{T}$ \\
\hline UN_xiong_2848 & 453 & $\mathrm{~T} / \mathrm{C}$ \\
\hline UN_xiong_2848 & 477 & $\mathrm{~A} / \mathrm{G}$ \\
\hline UN_xiong_2848 & 540 & $A / G$ \\
\hline UN_xiong_2848 & 581 & $\mathrm{~A} / \mathrm{T}$ \\
\hline UN_xiong_2848 & 602 & $A / G$ \\
\hline UN_xiong_2848 & 656 & $\mathrm{~T} / \mathrm{C}$ \\
\hline UN_xiong_2848 & 683 & $\mathrm{~T} / \mathrm{C}$ \\
\hline UN_xiong_2849 & 556 & $A / G$ \\
\hline UN_xiong_2849 & 693 & $\mathrm{~T} / \mathrm{C}$ \\
\hline UN_xiong_2849 & 799 & $A / G$ \\
\hline UN_xiong_2849 & 833 & $C / A$ \\
\hline UN_xiong_2850 & 214 & $\mathrm{~T} / \mathrm{G}$ \\
\hline UN_xiong_2850 & 1281 & $\mathrm{~T} / \mathrm{C}$ \\
\hline
\end{tabular}




\begin{tabular}{|c|c|c|}
\hline UN_xiong_2857 & 278 & $\mathrm{C} / \mathrm{T}$ \\
\hline UN_xiong_2875 & 143 & $A / G$ \\
\hline UN_xiong_2877 & 521 & $\mathrm{~T} / \mathrm{C}$ \\
\hline UN_xiong_2877 & 688 & $A / G$ \\
\hline UN_xiong_2877 & 732 & $A / G$ \\
\hline UN_xiong_2880 & 191 & $\mathrm{C} / \mathrm{T}$ \\
\hline UN_xiong_2882 & 557 & $\mathrm{C} / \mathrm{T}$ \\
\hline UN_xiong_2887 & 208 & $A / G$ \\
\hline UN_xiong_2887 & 375 & $G / A$ \\
\hline UN_xiong_2887 & 567 & $\mathrm{~T} / \mathrm{C}$ \\
\hline UN_xiong_2889 & 271 & $\mathrm{~T} / \mathrm{C}$ \\
\hline UN_xiong_2896 & 89 & $\mathrm{G} / \mathrm{A}$ \\
\hline UN_xiong_2918 & 335 & $\mathrm{G} / \mathrm{C}$ \\
\hline UN_xiong_2918 & 407 & $\mathrm{G} / \mathrm{C}$ \\
\hline UN_xiong_2918 & 444 & $C / G$ \\
\hline UN_xiong_2941 & 197 & $A / G$ \\
\hline UN_xiong_2941 & 224 & $\mathrm{~T} / \mathrm{C}$ \\
\hline UN_xiong_2958 & 214 & $A / G$ \\
\hline UN_xiong_2962 & 149 & $A / T$ \\
\hline UN_xiong_2964 & 36 & $\mathrm{~T} / \mathrm{C}$ \\
\hline UN_xiong_2965 & 48 & $A / G$ \\
\hline UN_xiong_2973 & 18 & $\mathrm{G} / \mathrm{C}$ \\
\hline UN_xiong_2973 & 110 & $\mathrm{~T} / \mathrm{A}$ \\
\hline UN_xiong_2973 & 294 & $\mathrm{C} / \mathrm{T}$ \\
\hline UN_xiong_2983 & 300 & $A / G$ \\
\hline UN_xiong_2996 & 276 & $G / A$ \\
\hline UN_xiong_2996 & 468 & $A / G$ \\
\hline UN_xiong_3002 & 333 & $\mathrm{~T} / \mathrm{C}$ \\
\hline UN_xiong_3008 & 538 & $\mathrm{~T} / \mathrm{C}$ \\
\hline UN_xiong_3015 & 556 & $\mathrm{G} / \mathrm{C}$ \\
\hline UN_xiong_3021 & 360 & $\mathrm{G} / \mathrm{A}$ \\
\hline UN_xiong_3029 & 540 & $\mathrm{G} / \mathrm{A}$ \\
\hline UN_xiong_3032 & 141 & $\mathrm{~T} / \mathrm{C}$ \\
\hline UN_xiong_3032 & 245 & $G / A$ \\
\hline UN_xiong_3043 & 83 & $\mathrm{~A} / \mathrm{C}$ \\
\hline UN_xiong_3047 & 138 & $\mathrm{G} / \mathrm{A}$ \\
\hline UN_xiong_3047 & 309 & $A / G$ \\
\hline UN_xiong_3047 & 336 & $A / G$ \\
\hline UN_xiong_3047 & 510 & $G / A$ \\
\hline UN_xiong_3047 & 684 & $\mathrm{C} / \mathrm{T}$ \\
\hline UN_xiong_3047 & 741 & $A / G$ \\
\hline UN_xiong_3068 & 326 & $\mathrm{~T} / \mathrm{C}$ \\
\hline UN_xiong_3076 & 247 & $\mathrm{G} / \mathrm{T}$ \\
\hline UN_xiong_3079 & 123 & $\mathrm{G} / \mathrm{T}$ \\
\hline UN_xiong_3079 & 170 & $\mathrm{G} / \mathrm{C}$ \\
\hline UN_xiong_3079 & 251 & $\mathrm{~T} / \mathrm{C}$ \\
\hline UN_xiong_3084 & 111 & $A / G$ \\
\hline
\end{tabular}




\begin{tabular}{|c|c|c|}
\hline UN_xiong_3099 & 187 & $\mathrm{C} / \mathrm{T}$ \\
\hline UN_xiong_3105 & 194 & $\mathrm{~A} / \mathrm{T}$ \\
\hline UN_xiong_3105 & 420 & $\mathrm{C} / \mathrm{T}$ \\
\hline UN_xiong_3105 & 488 & $\mathrm{G} / \mathrm{A}$ \\
\hline UN_xiong_3105 & 591 & $A / G$ \\
\hline UN_xiong_3105 & 756 & $A / G$ \\
\hline UN_xiong_3105 & 923 & $G / A$ \\
\hline UN_xiong_3105 & 964 & $A / G$ \\
\hline UN_xiong_3107 & 55 & $A / G$ \\
\hline UN_xiong_3109 & 125 & $A / G$ \\
\hline UN_xiong_3114 & 116 & $\mathrm{~T} / \mathrm{C}$ \\
\hline UN_xiong_3114 & 182 & $A / G$ \\
\hline UN_xiong_3117 & 346 & $\mathrm{~T} / \mathrm{C}$ \\
\hline UN_xiong_3117 & 385 & $\mathrm{C} / \mathrm{T}$ \\
\hline UN_xiong_3118 & 182 & $\mathrm{~T} / \mathrm{C}$ \\
\hline UN_xiong_3124 & 100 & $\mathrm{C} / \mathrm{T}$ \\
\hline UN_xiong_3126 & 186 & $\mathrm{~T} / \mathrm{C}$ \\
\hline UN_xiong_3126 & 242 & $\mathrm{~T} / \mathrm{C}$ \\
\hline UN_xiong_3126 & 307 & $\mathrm{G} / \mathrm{C}$ \\
\hline UN_xiong_3126 & 362 & $\mathrm{C} / \mathrm{T}$ \\
\hline UN_xiong_3126 & 383 & $\mathrm{C} / \mathrm{T}$ \\
\hline UN_xiong_3126 & 460 & $\mathrm{~T} / \mathrm{C}$ \\
\hline UN_xiong_3126 & 514 & $A / G$ \\
\hline UN_xiong_3126 & 593 & $\mathrm{G} / \mathrm{A}$ \\
\hline UN_xiong_3126 & 620 & $\mathrm{C} / \mathrm{T}$ \\
\hline UN_xiong_3126 & 650 & $\mathrm{C} / \mathrm{G}$ \\
\hline UN_xiong_3126 & 957 & $\mathrm{~T} / \mathrm{C}$ \\
\hline UN_xiong_3126 & 1098 & $\mathrm{C} / \mathrm{T}$ \\
\hline UN_xiong_3128 & 643 & $\mathrm{~T} / \mathrm{C}$ \\
\hline UN_xiong_3140 & 15 & $A / G$ \\
\hline UN_xiong_3140 & 66 & $\mathrm{C} / \mathrm{T}$ \\
\hline UN_xiong_3140 & 125 & $\mathrm{G} / \mathrm{T}$ \\
\hline UN_xiong_3140 & 558 & $\mathrm{~T} / \mathrm{A}$ \\
\hline UN_xiong_3143 & 33 & $C / A$ \\
\hline UN_xiong_3143 & 82 & $G / A$ \\
\hline UN_xiong_3143 & 388 & $A / G$ \\
\hline UN_xiong_3157 & 167 & $A / G$ \\
\hline UN_xiong_3161 & 539 & $\mathrm{~T} / \mathrm{A}$ \\
\hline UN_xiong_3180 & 306 & $G / A$ \\
\hline UN_xiong_3181 & 723 & $G / A$ \\
\hline UN_xiong_3181 & 773 & $\mathrm{~T} / \mathrm{C}$ \\
\hline UN_xiong_3184 & 176 & $T / G$ \\
\hline UN_xiong_3193 & 31 & $\mathrm{~T} / \mathrm{C}$ \\
\hline UN_xiong_3200 & 328 & $\mathrm{G} / \mathrm{T}$ \\
\hline UN_xiong_3201 & 267 & $\mathrm{~T} / \mathrm{G}$ \\
\hline UN_xiong_3201 & 418 & $\mathrm{C} / \mathrm{T}$ \\
\hline UN_xiong_3203 & 201 & $T / G$ \\
\hline
\end{tabular}




\begin{tabular}{|c|c|c|}
\hline UN_xiong_3203 & 288 & $\mathrm{C} / \mathrm{T}$ \\
\hline UN_xiong_3210 & 26 & $\mathrm{~T} / \mathrm{A}$ \\
\hline UN_xiong_3212 & 369 & $\mathrm{C} / \mathrm{T}$ \\
\hline UN_xiong_3231 & 392 & $\mathrm{~T} / \mathrm{A}$ \\
\hline UN_xiong_3231 & 434 & $\mathrm{C} / \mathrm{T}$ \\
\hline UN_xiong_3244 & 159 & $A / G$ \\
\hline UN_xiong_3245 & 535 & $A / G$ \\
\hline UN_xiong_3245 & 583 & $A / G$ \\
\hline UN_xiong_3252 & 155 & $\mathrm{G} / \mathrm{A}$ \\
\hline UN_xiong_3253 & 194 & $\mathrm{C} / \mathrm{T}$ \\
\hline UN_xiong_3265 & 308 & $\mathrm{~T} / \mathrm{C}$ \\
\hline UN_xiong_3265 & 384 & $\mathrm{C} / \mathrm{T}$ \\
\hline UN_xiong_3265 & 540 & $\mathrm{C} / \mathrm{T}$ \\
\hline UN_xiong_3266 & 165 & $T / G$ \\
\hline UN_xiong_3271 & 268 & $\mathrm{~T} / \mathrm{C}$ \\
\hline UN_xiong_3271 & 308 & $\mathrm{~T} / \mathrm{C}$ \\
\hline UN_xiong_3271 & 508 & $\mathrm{G} / \mathrm{T}$ \\
\hline UN_xiong_3272 & 65 & $\mathrm{~T} / \mathrm{C}$ \\
\hline UN_xiong_3272 & 813 & $C / A$ \\
\hline UN_xiong_3282 & 282 & $\mathrm{~T} / \mathrm{A}$ \\
\hline UN_xiong_3293 & 275 & $\mathrm{G} / \mathrm{A}$ \\
\hline UN_xiong_3293 & 489 & $A / G$ \\
\hline UN_xiong_3293 & 519 & $\mathrm{~T} / \mathrm{C}$ \\
\hline UN_xiong_3299 & 317 & $\mathrm{C} / \mathrm{T}$ \\
\hline UN_xiong_3320 & 593 & $A / G$ \\
\hline UN_xiong_3320 & 1041 & $\mathrm{C} / \mathrm{T}$ \\
\hline UN_xiong_3320 & 1166 & $\mathrm{G} / \mathrm{A}$ \\
\hline UN_xiong_3320 & 1282 & $\mathrm{~T} / \mathrm{C}$ \\
\hline UN_xiong_3321 & 198 & $A / C$ \\
\hline UN_xiong_3321 & 352 & $C / A$ \\
\hline UN_xiong_3323 & 312 & $\mathrm{G} / \mathrm{A}$ \\
\hline UN_xiong_3326 & 673 & $\mathrm{~T} / \mathrm{A}$ \\
\hline UN_xiong_3327 & 116 & $\mathrm{G} / \mathrm{A}$ \\
\hline UN_xiong_3340 & 110 & $\mathrm{C} / \mathrm{T}$ \\
\hline UN_xiong_3351 & 169 & $A / G$ \\
\hline UN_xiong_3351 & 202 & $A / G$ \\
\hline UN_xiong_3369 & 455 & $A / T$ \\
\hline UN_xiong_3369 & 476 & $A / G$ \\
\hline UN_xiong_3369 & 765 & $\mathrm{G} / \mathrm{T}$ \\
\hline UN_xiong_3369 & 942 & $\mathrm{C} / \mathrm{T}$ \\
\hline UN_xiong_3369 & 1080 & $\mathrm{~T} / \mathrm{C}$ \\
\hline UN_xiong_3369 & 1136 & $\mathrm{~T} / \mathrm{C}$ \\
\hline UN_xiong_3378 & 29 & $\mathrm{~T} / \mathrm{C}$ \\
\hline UN_xiong_3378 & 175 & $A / G$ \\
\hline UN_xiong_3378 & 312 & $\mathrm{C} / \mathrm{T}$ \\
\hline UN_xiong_3384 & 393 & $A / T$ \\
\hline UN_xiong_3384 & 485 & $\mathrm{~T} / \mathrm{A}$ \\
\hline
\end{tabular}




\begin{tabular}{|c|c|c|}
\hline UN_xiong_3384 & 653 & $\mathrm{G} / \mathrm{C}$ \\
\hline UN_xiong_3384 & 679 & $\mathrm{G} / \mathrm{C}$ \\
\hline UN_xiong_3384 & 845 & $\mathrm{G} / \mathrm{T}$ \\
\hline UN_xiong_3384 & 894 & $\mathrm{~T} / \mathrm{A}$ \\
\hline UN_xiong_3384 & 952 & $\mathrm{~T} / \mathrm{C}$ \\
\hline UN_xiong_3385 & 88 & $\mathrm{~T} / \mathrm{C}$ \\
\hline UN_xiong_3390 & 196 & $\mathrm{~A} / \mathrm{G}$ \\
\hline UN_xiong_3390 & 249 & $\mathrm{C} / \mathrm{T}$ \\
\hline UN_xiong_3390 & 284 & $\mathrm{~T} / \mathrm{C}$ \\
\hline UN_xiong_3390 & 319 & $\mathrm{~T} / \mathrm{C}$ \\
\hline UN_xiong_3390 & 347 & $\mathrm{~T} / \mathrm{G}$ \\
\hline UN_xiong_3390 & 388 & $\mathrm{C} / \mathrm{T}$ \\
\hline UN_xiong_3390 & 500 & $\mathrm{~T} / \mathrm{C}$ \\
\hline UN_xiong_3401 & 147 & $\mathrm{~A} / \mathrm{T}$ \\
\hline UN_xiong_3402 & 149 & $\mathrm{~T} / \mathrm{C}$ \\
\hline UN_xiong_3408 & 183 & $\mathrm{C} / \mathrm{T}$ \\
\hline UN_xiong_3410 & 96 & $\mathrm{G} / \mathrm{C}$ \\
\hline UN_xiong_3410 & 387 & $\mathrm{~A} / \mathrm{G}$ \\
\hline UN_xiong_3410 & 962 & $\mathrm{~A} / \mathrm{G}$ \\
\hline UN_xiong_3416 & 337 & $\mathrm{~T} / \mathrm{A}$ \\
\hline UN_xiong_3416 & 360 & $\mathrm{~T} / \mathrm{C}$ \\
\hline UN_xiong_3416 & 452 & $\mathrm{~T} / \mathrm{C}$ \\
\hline UN_xiong_3421 & 739 & $\mathrm{C} / \mathrm{T}$ \\
\hline UN_xiong_3445 & 33 & $\mathrm{G} / \mathrm{C}$ \\
\hline UN_xiong_3445 & 62 & $\mathrm{G} / \mathrm{A}$ \\
\hline UN_xiong_3445 & 87 & $\mathrm{G} / \mathrm{C}$ \\
\hline UN_xiong_3445 & 123 & $\mathrm{G} / \mathrm{C}$ \\
\hline UN_xiong_3445 & 159 & $\mathrm{G} / \mathrm{C}$ \\
\hline UN_xiong_3445 & 189 & $\mathrm{~T} / \mathrm{G}$ \\
\hline UN_xiong_3445 & 211 & $\mathrm{C} / \mathrm{G}$ \\
\hline UN_xiong_3445 & 240 & $\mathrm{G} / \mathrm{T}$ \\
\hline UN_xiong_3445 & 265 & $\mathrm{G} / \mathrm{C}$ \\
\hline UN_xiong_3445 & 295 & $\mathrm{~T} / \mathrm{G}$ \\
\hline UN_xiong_3445 & 317 & $\mathrm{C} / \mathrm{G}$ \\
\hline UN_xiong_3459 & 541 & $\mathrm{~T} / \mathrm{A}$ \\
\hline UN_xiong_3462 & 193 & $A / G$ \\
\hline UN_xiong_3495 & 105 & $A / T$ \\
\hline UN_xiong_3498 & 244 & $\mathrm{~A} / \mathrm{C}$ \\
\hline UN_xiong_3499 & 295 & $\mathrm{~A} / \mathrm{C}$ \\
\hline UN_xiong_3499 & 348 & $\mathrm{G} / \mathrm{A}$ \\
\hline UN_xiong_3499 & 617 & $A / T$ \\
\hline UN_xiong_3500 & 810 & $\mathrm{C} / \mathrm{T}$ \\
\hline UN_xiong_3500 & 1102 & $\mathrm{~A} / \mathrm{C}$ \\
\hline UN_xiong_3500 & 1305 & $A / C$ \\
\hline UN_xiong_3503 & 287 & $A / C$ \\
\hline UN_xiong_3503 & 448 & $A / C$ \\
\hline UN_xiong_3503 & 989 & $A / C$ \\
\hline
\end{tabular}




\begin{tabular}{|c|c|c|}
\hline UN_xiong_3504 & 604 & $\mathrm{G} / \mathrm{A}$ \\
\hline UN_xiong_3504 & 903 & $A / G$ \\
\hline UN_xiong_3518 & 18 & $\mathrm{~T} / \mathrm{G}$ \\
\hline UN_xiong_3518 & 189 & $\mathrm{~T} / \mathrm{C}$ \\
\hline UN_xiong_3518 & 210 & $\mathrm{C} / \mathrm{T}$ \\
\hline UN_xiong_3518 & 464 & $A / G$ \\
\hline UN_xiong_3518 & 702 & $\mathrm{~T} / \mathrm{C}$ \\
\hline UN_xiong_3521 & 253 & $\mathrm{~T} / \mathrm{C}$ \\
\hline UN_xiong_3521 & 310 & $A / G$ \\
\hline UN_xiong_3521 & 367 & $A / G$ \\
\hline UN_xiong_3521 & 390 & $\mathrm{~T} / \mathrm{C}$ \\
\hline UN_xiong_3523 & 25 & $\mathrm{G} / \mathrm{T}$ \\
\hline UN_xiong_3523 & 47 & $\mathrm{G} / \mathrm{A}$ \\
\hline UN_xiong_3523 & 126 & $A / G$ \\
\hline UN_xiong_3523 & 160 & $\mathrm{~T} / \mathrm{C}$ \\
\hline UN_xiong_3523 & 190 & $A / G$ \\
\hline UN_xiong_3523 & 220 & $\mathrm{~T} / \mathrm{C}$ \\
\hline UN_xiong_3523 & 254 & $\mathrm{C} / \mathrm{T}$ \\
\hline UN_xiong_3523 & 294 & $\mathrm{~A} / \mathrm{C}$ \\
\hline UN_xiong_3523 & 317 & $\mathrm{~T} / \mathrm{C}$ \\
\hline UN_xiong_3523 & 339 & $\mathrm{G} / \mathrm{C}$ \\
\hline UN_xiong_3523 & 418 & $\mathrm{~T} / \mathrm{C}$ \\
\hline UN_xiong_3523 & 441 & $\mathrm{C} / \mathrm{T}$ \\
\hline UN_xiong_3523 & 461 & $\mathrm{G} / \mathrm{A}$ \\
\hline UN_xiong_3523 & 536 & $A / G$ \\
\hline UN_xiong_3535 & 23 & $\mathrm{~T} / \mathrm{C}$ \\
\hline UN_xiong_3537 & 711 & $\mathrm{C} / \mathrm{T}$ \\
\hline UN_xiong_3543 & 16 & $\mathrm{C} / \mathrm{T}$ \\
\hline UN_xiong_3544 & 29 & $\mathrm{G} / \mathrm{A}$ \\
\hline UN_xiong_3544 & 76 & $A / G$ \\
\hline UN_xiong_3544 & 164 & $A / G$ \\
\hline UN_xiong_3544 & 293 & $\mathrm{~T} / \mathrm{C}$ \\
\hline UN_xiong_3544 & 321 & $A / G$ \\
\hline UN_xiong_3544 & 391 & $A / G$ \\
\hline UN_xiong_3544 & 464 & $\mathrm{~T} / \mathrm{C}$ \\
\hline UN_xiong_3544 & 534 & $A / G$ \\
\hline UN_xiong_3544 & 781 & $\mathrm{C} / \mathrm{T}$ \\
\hline UN_xiong_3544 & 887 & $\mathrm{~T} / \mathrm{A}$ \\
\hline UN_xiong_3553 & 179 & $\mathrm{C} / \mathrm{T}$ \\
\hline UN_xiong_3561 & 291 & $\mathrm{C} / \mathrm{T}$ \\
\hline UN_xiong_3565 & 65 & $\mathrm{C} / \mathrm{T}$ \\
\hline UN_xiong_3565 & 95 & $\mathrm{~T} / \mathrm{C}$ \\
\hline UN_xiong_3565 & 184 & $A / G$ \\
\hline UN_xiong_3576 & 235 & $\mathrm{~T} / \mathrm{C}$ \\
\hline UN_xiong_3582 & 271 & $\mathrm{G} / \mathrm{A}$ \\
\hline UN_xiong_3582 & 537 & $\mathrm{G} / \mathrm{A}$ \\
\hline UN_xiong_3585 & 158 & $C / A$ \\
\hline
\end{tabular}




\begin{tabular}{|c|c|c|}
\hline UN_xiong_3588 & 382 & $\mathrm{~A} / \mathrm{C}$ \\
\hline UN_xiong_3588 & 650 & $\mathrm{~T} / \mathrm{C}$ \\
\hline UN_xiong_3590 & 191 & $\mathrm{~T} / \mathrm{C}$ \\
\hline UN_xiong_3595 & 192 & $\mathrm{~T} / \mathrm{C}$ \\
\hline UN_xiong_3595 & 409 & $\mathrm{~T} / \mathrm{C}$ \\
\hline UN_xiong_3597 & 246 & $\mathrm{G} / \mathrm{A}$ \\
\hline UN_xiong_3600 & 421 & $\mathrm{G} / \mathrm{A}$ \\
\hline UN_xiong_3600 & 726 & $\mathrm{~A} / \mathrm{G}$ \\
\hline UN_xiong_3605 & 283 & $\mathrm{C} / \mathrm{T}$ \\
\hline UN_xiong_3606 & 231 & $\mathrm{~A} / \mathrm{T}$ \\
\hline UN_xiong_3611 & 490 & $A / G$ \\
\hline UN_xiong_3634 & 209 & $\mathrm{~A} / \mathrm{G}$ \\
\hline UN_xiong_3634 & 428 & $\mathrm{~T} / \mathrm{C}$ \\
\hline UN_xiong_3637 & 630 & $\mathrm{~A} / \mathrm{G}$ \\
\hline UN_xiong_3649 & 202 & $\mathrm{C} / \mathrm{T}$ \\
\hline UN_xiong_3655 & 254 & $\mathrm{C} / \mathrm{T}$ \\
\hline UN_xiong_3655 & 384 & $\mathrm{~T} / \mathrm{C}$ \\
\hline UN_xiong_3655 & 409 & $\mathrm{G} / \mathrm{T}$ \\
\hline UN_xiong_3659 & 259 & $\mathrm{~A} / \mathrm{T}$ \\
\hline UN_xiong_3662 & 179 & $\mathrm{G} / \mathrm{T}$ \\
\hline UN_xiong_3662 & 358 & $\mathrm{G} / \mathrm{A}$ \\
\hline UN_xiong_3669 & 377 & $\mathrm{C} / \mathrm{T}$ \\
\hline UN_xiong_3669 & 876 & $A / G$ \\
\hline UN_xiong_3669 & 952 & $\mathrm{C} / \mathrm{T}$ \\
\hline UN_xiong_3669 & 1051 & $\mathrm{~A} / \mathrm{T}$ \\
\hline UN_xiong_3669 & 1536 & $\mathrm{~A} / \mathrm{T}$ \\
\hline UN_xiong_3675 & 303 & $A / G$ \\
\hline UN_xiong_3680 & 87 & $\mathrm{C} / \mathrm{T}$ \\
\hline UN_xiong_3688 & 83 & $\mathrm{~A} / \mathrm{T}$ \\
\hline UN_xiong_3688 & 329 & $\mathrm{G} / \mathrm{T}$ \\
\hline UN_xiong_3699 & 139 & $\mathrm{~T} / \mathrm{A}$ \\
\hline UN_xiong_3721 & 147 & $\mathrm{~T} / \mathrm{A}$ \\
\hline UN_xiong_3727 & 130 & $\mathrm{~T} / \mathrm{C}$ \\
\hline UN_xiong_3728 & 257 & $\mathrm{~A} / \mathrm{T}$ \\
\hline UN_xiong_3728 & 429 & $\mathrm{C} / \mathrm{G}$ \\
\hline UN_xiong_3730 & 171 & $\mathrm{~A} / \mathrm{C}$ \\
\hline UN_xiong_3731 & 70 & $\mathrm{~A} / \mathrm{G}$ \\
\hline UN_xiong_3733 & 44 & $\mathrm{G} / \mathrm{A}$ \\
\hline UN_xiong_3733 & 298 & $\mathrm{~T} / \mathrm{C}$ \\
\hline UN_xiong_3733 & 511 & $\mathrm{~A} / \mathrm{T}$ \\
\hline UN_xiong_3733 & 711 & $\mathrm{~A} / \mathrm{G}$ \\
\hline UN_xiong_3733 & 1371 & $\mathrm{G} / \mathrm{A}$ \\
\hline UN_xiong_3733 & 1403 & $\mathrm{G} / \mathrm{A}$ \\
\hline UN_xiong_3733 & 1437 & $\mathrm{G} / \mathrm{A}$ \\
\hline UN_xiong_3733 & 1844 & $A / G$ \\
\hline UN_xiong_3733 & 2061 & $\mathrm{~T} / \mathrm{G}$ \\
\hline UN_xiong_3733 & 2131 & $\mathrm{C} / \mathrm{T}$ \\
\hline
\end{tabular}




\begin{tabular}{|c|c|c|}
\hline UN_xiong_3740 & 541 & $A / G$ \\
\hline UN_xiong_3740 & 754 & $\mathrm{C} / \mathrm{A}$ \\
\hline UN_xiong_3741 & 145 & $A / G$ \\
\hline UN_xiong_3741 & 173 & $\mathrm{~T} / \mathrm{G}$ \\
\hline UN_xiong_3741 & 226 & $A / G$ \\
\hline UN_xiong_3741 & 370 & $\mathrm{~T} / \mathrm{G}$ \\
\hline UN_xiong_3741 & 414 & $\mathrm{~A} / \mathrm{T}$ \\
\hline UN_xiong_3741 & 464 & $\mathrm{G} / \mathrm{A}$ \\
\hline UN_xiong_3741 & 529 & $\mathrm{~T} / \mathrm{C}$ \\
\hline UN_xiong_3741 & 561 & $\mathrm{G} / \mathrm{A}$ \\
\hline UN_xiong_3741 & 708 & $A / G$ \\
\hline UN_xiong_3744 & 336 & $\mathrm{~A} / \mathrm{C}$ \\
\hline UN_xiong_3776 & 135 & $\mathrm{G} / \mathrm{A}$ \\
\hline UN_xiong_3787 & 158 & $\mathrm{C} / \mathrm{A}$ \\
\hline UN_xiong_3787 & 188 & $\mathrm{G} / \mathrm{A}$ \\
\hline UN_xiong_3793 & 270 & $A / G$ \\
\hline UN_xiong_3793 & 308 & $\mathrm{C} / \mathrm{T}$ \\
\hline UN_xiong_3793 & 341 & $\mathrm{G} / \mathrm{T}$ \\
\hline UN_xiong_3793 & 466 & $A / G$ \\
\hline UN_xiong_3799 & 462 & $\mathrm{~A} / \mathrm{T}$ \\
\hline UN_xiong_3805 & 274 & $\mathrm{G} / \mathrm{T}$ \\
\hline UN_xiong_3805 & 452 & $\mathrm{G} / \mathrm{A}$ \\
\hline UN_xiong_3805 & 476 & $\mathrm{C} / \mathrm{T}$ \\
\hline UN_xiong_3805 & 505 & $\mathrm{G} / \mathrm{T}$ \\
\hline UN_xiong_3813 & 170 & $\mathrm{C} / \mathrm{T}$ \\
\hline UN_xiong_3813 & 290 & $\mathrm{G} / \mathrm{A}$ \\
\hline UN_xiong_3824 & 157 & $\mathrm{~A} / \mathrm{T}$ \\
\hline UN_xiong_3827 & 371 & $\mathrm{~T} / \mathrm{C}$ \\
\hline UN_xiong_3832 & 180 & $\mathrm{~T} / \mathrm{G}$ \\
\hline UN_xiong_3832 & 213 & $\mathrm{C} / \mathrm{G}$ \\
\hline UN_xiong_3832 & 267 & $\mathrm{~T} / \mathrm{A}$ \\
\hline UN_xiong_3836 & 10 & $\mathrm{G} / \mathrm{A}$ \\
\hline UN_xiong_3836 & 271 & $\mathrm{C} / \mathrm{T}$ \\
\hline UN_xiong_3836 & 304 & $\mathrm{~T} / \mathrm{C}$ \\
\hline UN_xiong_3836 & 532 & $\mathrm{~A} / \mathrm{T}$ \\
\hline UN_xiong_3838 & 219 & $A / G$ \\
\hline UN_xiong_3839 & 321 & $\mathrm{G} / \mathrm{T}$ \\
\hline UN_xiong_3839 & 520 & $\mathrm{~T} / \mathrm{C}$ \\
\hline UN_xiong_3840 & 513 & $\mathrm{G} / \mathrm{C}$ \\
\hline UN_xiong_3844 & 167 & $\mathrm{C} / \mathrm{T}$ \\
\hline UN_xiong_3844 & 395 & $C / A$ \\
\hline UN_xiong_3844 & 429 & $A / G$ \\
\hline UN_xiong_3844 & 501 & $\mathrm{~T} / \mathrm{A}$ \\
\hline UN_xiong_3845 & 226 & $\mathrm{G} / \mathrm{A}$ \\
\hline UN_xiong_3845 & 265 & $\mathrm{G} / \mathrm{A}$ \\
\hline UN_xiong_3845 & 419 & $\mathrm{~T} / \mathrm{C}$ \\
\hline UN_xiong_3845 & 475 & $\mathrm{~T} / \mathrm{C}$ \\
\hline
\end{tabular}




\begin{tabular}{|c|c|c|}
\hline UN_xiong_3854 & 309 & $\mathrm{C} / \mathrm{T}$ \\
\hline UN_xiong_3855 & 441 & $\mathrm{~T} / \mathrm{C}$ \\
\hline UN_xiong_3861 & 228 & $\mathrm{C} / \mathrm{T}$ \\
\hline UN_xiong_3863 & 185 & $A / G$ \\
\hline UN_xiong_3863 & 233 & $\mathrm{~T} / \mathrm{C}$ \\
\hline UN_xiong_3864 & 502 & $\mathrm{~T} / \mathrm{A}$ \\
\hline UN_xiong_3864 & 533 & $\mathrm{~T} / \mathrm{C}$ \\
\hline UN_xiong_3866 & 313 & $\mathrm{G} / \mathrm{A}$ \\
\hline UN_xiong_3870 & 346 & $\mathrm{C} / \mathrm{T}$ \\
\hline UN_xiong_3870 & 575 & $\mathrm{~T} / \mathrm{C}$ \\
\hline UN_xiong_3878 & 125 & $A / G$ \\
\hline UN_xiong_3878 & 196 & $\mathrm{G} / \mathrm{C}$ \\
\hline UN_xiong_3878 & 263 & $\mathrm{~T} / \mathrm{C}$ \\
\hline UN_xiong_3878 & 340 & $\mathrm{G} / \mathrm{C}$ \\
\hline UN_xiong_3885 & 413 & $A / G$ \\
\hline UN_xiong_3885 & 512 & $G / A$ \\
\hline UN_xiong_3890 & 161 & $A / G$ \\
\hline UN_xiong_3890 & 403 & $\mathrm{~T} / \mathrm{C}$ \\
\hline UN_xiong_3900 & 269 & $A / G$ \\
\hline UN_xiong_3909 & 136 & $A / G$ \\
\hline UN_xiong_3910 & 287 & $A / G$ \\
\hline UN_xiong_3910 & 317 & $A / G$ \\
\hline UN_xiong_3914 & 394 & $\mathrm{G} / \mathrm{C}$ \\
\hline UN_xiong_3914 & 581 & $\mathrm{~T} / \mathrm{C}$ \\
\hline UN_xiong_3914 & 903 & $C / A$ \\
\hline UN_xiong_3922 & 154 & $\mathrm{~T} / \mathrm{C}$ \\
\hline UN_xiong_3922 & 399 & $A / G$ \\
\hline UN_xiong_3927 & 20 & $\mathrm{~A} / \mathrm{T}$ \\
\hline UN_xiong_3927 & 45 & $A / G$ \\
\hline UN_xiong_3927 & 118 & $\mathrm{~T} / \mathrm{C}$ \\
\hline UN_xiong_3927 & 220 & $\mathrm{C} / \mathrm{T}$ \\
\hline UN_xiong_3927 & 254 & $G / A$ \\
\hline UN_xiong_3927 & 316 & $A / G$ \\
\hline UN_xiong_3927 & 349 & $\mathrm{G} / \mathrm{A}$ \\
\hline UN_xiong_3932 & 146 & $\mathrm{G} / \mathrm{A}$ \\
\hline UN_xiong_3932 & 282 & $\mathrm{~T} / \mathrm{C}$ \\
\hline UN_xiong_3932 & 302 & $\mathrm{C} / \mathrm{T}$ \\
\hline UN_xiong_3935 & 688 & $\mathrm{G} / \mathrm{T}$ \\
\hline UN_xiong_3959 & 384 & $A / G$ \\
\hline UN_xiong_3968 & 547 & $\mathrm{~T} / \mathrm{C}$ \\
\hline UN_xiong_3968 & 767 & $\mathrm{~T} / \mathrm{C}$ \\
\hline UN_xiong_3976 & 245 & $G / A$ \\
\hline UN_xiong_3976 & 291 & $\mathrm{C} / \mathrm{T}$ \\
\hline UN_xiong_3976 & 378 & $\mathrm{~A} / \mathrm{T}$ \\
\hline UN_xiong_3976 & 468 & $\mathrm{~T} / \mathrm{C}$ \\
\hline UN_xiong_3976 & 501 & $A / G$ \\
\hline UN_xiong_3976 & 555 & $\mathrm{~A} / \mathrm{T}$ \\
\hline
\end{tabular}




\begin{tabular}{|c|c|c|}
\hline UN_xiong_3976 & 576 & $\mathrm{~A} / \mathrm{C}$ \\
\hline UN_xiong_3985 & 36 & $\mathrm{~A} / \mathrm{T}$ \\
\hline UN_xiong_3991 & 244 & $\mathrm{G} / \mathrm{A}$ \\
\hline UN_xiong_3996 & 186 & $\mathrm{~T} / \mathrm{C}$ \\
\hline UN_xiong_3996 & 284 & $\mathrm{~A} / \mathrm{C}$ \\
\hline UN_xiong_3997 & 396 & $\mathrm{~T} / \mathrm{C}$ \\
\hline UN_xiong_3999 & 222 & $\mathrm{~T} / \mathrm{C}$ \\
\hline UN_xiong_4011 & 28 & $\mathrm{~T} / \mathrm{C}$ \\
\hline UN_xiong_4011 & 118 & $\mathrm{C} / \mathrm{T}$ \\
\hline UN_xiong_4011 & 317 & $\mathrm{G} / \mathrm{A}$ \\
\hline UN_xiong_4013 & 453 & $\mathrm{C} / \mathrm{T}$ \\
\hline UN_xiong_4028 & 131 & $\mathrm{~T} / \mathrm{C}$ \\
\hline UN_xiong_4030 & 170 & $\mathrm{~T} / \mathrm{C}$ \\
\hline UN_xiong_4030 & 315 & $\mathrm{~T} / \mathrm{C}$ \\
\hline UN_xiong_4032 & 76 & $\mathrm{C} / \mathrm{T}$ \\
\hline UN_xiong_4038 & 904 & $\mathrm{~T} / \mathrm{C}$ \\
\hline UN_xiong_4039 & 524 & $\mathrm{~T} / \mathrm{C}$ \\
\hline UN_xiong_4055 & 208 & $\mathrm{C} / \mathrm{T}$ \\
\hline UN_xiong_4059 & 339 & $A / G$ \\
\hline UN_xiong_4059 & 404 & $\mathrm{C} / \mathrm{T}$ \\
\hline UN_xiong_4059 & 525 & $\mathrm{~T} / \mathrm{G}$ \\
\hline UN_xiong_4059 & 573 & $\mathrm{~T} / \mathrm{C}$ \\
\hline UN_xiong_4059 & 662 & $\mathrm{~T} / \mathrm{C}$ \\
\hline UN_xiong_4059 & 724 & $A / G$ \\
\hline UN_xiong_4059 & 777 & $\mathrm{G} / \mathrm{A}$ \\
\hline UN_xiong_4067 & 450 & $A / G$ \\
\hline UN_xiong_4083 & 103 & $\mathrm{C} / \mathrm{T}$ \\
\hline UN_xiong_4084 & 401 & $\mathrm{C} / \mathrm{T}$ \\
\hline UN_xiong_4084 & 476 & $\mathrm{~T} / \mathrm{C}$ \\
\hline UN_xiong_4084 & 732 & $\mathrm{~T} / \mathrm{C}$ \\
\hline UN_xiong_4085 & 58 & $\mathrm{C} / \mathrm{T}$ \\
\hline UN_xiong_4085 & 142 & $A / G$ \\
\hline UN_xiong_4085 & 354 & $\mathrm{~T} / \mathrm{A}$ \\
\hline UN_xiong_4085 & 389 & $\mathrm{~A} / \mathrm{T}$ \\
\hline UN_xiong_4091 & 34 & $\mathrm{G} / \mathrm{T}$ \\
\hline UN_xiong_4091 & 60 & $C / A$ \\
\hline UN_xiong_4091 & 98 & $\mathrm{C} / \mathrm{T}$ \\
\hline UN_xiong_4091 & 135 & $\mathrm{~T} / \mathrm{C}$ \\
\hline UN_xiong_4091 & 182 & $\mathrm{~T} / \mathrm{C}$ \\
\hline UN_xiong_4091 & 207 & $A / G$ \\
\hline UN_xiong_4091 & 268 & $A / G$ \\
\hline UN_xiong_4091 & 312 & $\mathrm{~T} / \mathrm{C}$ \\
\hline UN_xiong_4091 & 461 & $\mathrm{~A} / \mathrm{C}$ \\
\hline UN_xiong_4091 & 591 & $\mathrm{C} / \mathrm{T}$ \\
\hline UN_xiong_4091 & 707 & $C / A$ \\
\hline UN_xiong_4091 & 774 & $\mathrm{C} / \mathrm{T}$ \\
\hline UN_xiong_4091 & 840 & $C / A$ \\
\hline
\end{tabular}




\begin{tabular}{|c|c|c|}
\hline UN_xiong_4091 & 860 & $\mathrm{~A} / \mathrm{C}$ \\
\hline UN_xiong_4091 & 937 & $\mathrm{~T} / \mathrm{G}$ \\
\hline UN_xiong_4109 & 10 & $\mathrm{~T} / \mathrm{C}$ \\
\hline UN_xiong_4109 & 75 & $A / G$ \\
\hline UN_xiong_4109 & 184 & $\mathrm{~A} / \mathrm{G}$ \\
\hline UN_xiong_4109 & 363 & $\mathrm{~T} / \mathrm{A}$ \\
\hline UN_xiong_4109 & 440 & $\mathrm{C} / \mathrm{G}$ \\
\hline UN_xiong_4109 & 490 & $\mathrm{~A} / \mathrm{G}$ \\
\hline UN_xiong_4109 & 574 & $\mathrm{G} / \mathrm{A}$ \\
\hline UN_xiong_4109 & 792 & $\mathrm{C} / \mathrm{G}$ \\
\hline UN_xiong_4109 & 1220 & $A / G$ \\
\hline UN_xiong_4109 & 1329 & $\mathrm{~T} / \mathrm{C}$ \\
\hline UN_xiong_4109 & 1352 & $\mathrm{~T} / \mathrm{C}$ \\
\hline UN_xiong_4109 & 1374 & $\mathrm{~T} / \mathrm{C}$ \\
\hline UN_xiong_4109 & 1400 & $\mathrm{C} / \mathrm{A}$ \\
\hline UN_xiong_4109 & 1452 & $\mathrm{~T} / \mathrm{C}$ \\
\hline UN_xiong_4109 & 1479 & $\mathrm{G} / \mathrm{A}$ \\
\hline UN_xiong_4109 & 1508 & $\mathrm{~T} / \mathrm{C}$ \\
\hline UN_xiong_4109 & 1529 & $\mathrm{~T} / \mathrm{G}$ \\
\hline UN_xiong_4115 & 69 & $\mathrm{~T} / \mathrm{C}$ \\
\hline UN_xiong_4117 & 347 & $\mathrm{~A} / \mathrm{T}$ \\
\hline UN_xiong_4128 & 147 & $\mathrm{~A} / \mathrm{T}$ \\
\hline UN_xiong_4154 & 96 & $\mathrm{~A} / \mathrm{G}$ \\
\hline UN_xiong_4157 & 101 & $\mathrm{~A} / \mathrm{G}$ \\
\hline UN_xiong_4160 & 257 & $\mathrm{C} / \mathrm{T}$ \\
\hline UN_xiong_4161 & 139 & $\mathrm{~T} / \mathrm{C}$ \\
\hline UN_xiong_4161 & 229 & $\mathrm{~T} / \mathrm{C}$ \\
\hline UN_xiong_4161 & 454 & $\mathrm{~T} / \mathrm{C}$ \\
\hline UN_xiong_4168 & 75 & $\mathrm{G} / \mathrm{C}$ \\
\hline UN_xiong_4168 & 196 & $\mathrm{G} / \mathrm{A}$ \\
\hline UN_xiong_4172 & 52 & $\mathrm{C} / \mathrm{T}$ \\
\hline UN_xiong_4172 & 76 & $\mathrm{~T} / \mathrm{A}$ \\
\hline UN_xiong_4178 & 40 & $\mathrm{~A} / \mathrm{T}$ \\
\hline UN_xiong_4178 & 114 & $\mathrm{~A} / \mathrm{T}$ \\
\hline UN_xiong_4179 & 157 & $\mathrm{~T} / \mathrm{C}$ \\
\hline UN_xiong_4179 & 275 & $\mathrm{C} / \mathrm{G}$ \\
\hline UN_xiong_4179 & 358 & $\mathrm{~T} / \mathrm{C}$ \\
\hline UN_xiong_4179 & 392 & $\mathrm{~T} / \mathrm{G}$ \\
\hline UN_xiong_4179 & 516 & $\mathrm{~A} / \mathrm{G}$ \\
\hline UN_xiong_4179 & 536 & $\mathrm{~T} / \mathrm{G}$ \\
\hline UN_xiong_4179 & 614 & $\mathrm{~T} / \mathrm{A}$ \\
\hline UN_xiong_4179 & 850 & $\mathrm{C} / \mathrm{T}$ \\
\hline UN_xiong_4179 & 1150 & $\mathrm{~A} / \mathrm{C}$ \\
\hline UN_xiong_4186 & 186 & $\mathrm{C} / \mathrm{T}$ \\
\hline UN_xiong_4186 & 283 & $\mathrm{G} / \mathrm{C}$ \\
\hline UN_xiong_4186 & 323 & $\mathrm{~T} / \mathrm{A}$ \\
\hline UN_xiong_4198 & 169 & $\mathrm{~A} / \mathrm{G}$ \\
\hline
\end{tabular}




\begin{tabular}{|c|c|c|}
\hline UN_xiong_4204 & 43 & $C / A$ \\
\hline UN_xiong_4204 & 353 & $\mathrm{C} / \mathrm{T}$ \\
\hline UN_xiong_4205 & 534 & $\mathrm{~T} / \mathrm{C}$ \\
\hline UN_xiong_4206 & 190 & $\mathrm{~T} / \mathrm{A}$ \\
\hline UN_xiong_4206 & 238 & $\mathrm{~T} / \mathrm{G}$ \\
\hline UN_xiong_4206 & 433 & $\mathrm{C} / \mathrm{T}$ \\
\hline UN_xiong_4219 & 262 & $\mathrm{G} / \mathrm{A}$ \\
\hline UN_xiong_4219 & 319 & $T / G$ \\
\hline UN_xiong_4219 & 528 & $A / G$ \\
\hline UN_xiong_4233 & 7 & $A / G$ \\
\hline UN_xiong_4233 & 515 & $A / G$ \\
\hline UN_xiong_4237 & 159 & $\mathrm{~A} / \mathrm{T}$ \\
\hline UN_xiong_4237 & 187 & $\mathrm{C} / \mathrm{T}$ \\
\hline UN_xiong_4237 & 217 & $\mathrm{~T} / \mathrm{G}$ \\
\hline UN_xiong_4237 & 237 & $\mathrm{C} / \mathrm{T}$ \\
\hline UN_xiong_4237 & 283 & $A / G$ \\
\hline UN_xiong_4237 & 319 & $A / G$ \\
\hline UN_xiong_4244 & 117 & $\mathrm{G} / \mathrm{A}$ \\
\hline UN_xiong_4244 & 267 & $\mathrm{~T} / \mathrm{C}$ \\
\hline UN_xiong_4244 & 292 & $A / G$ \\
\hline UN_xiong_4244 & 334 & $T / G$ \\
\hline UN_xiong_4252 & 490 & $A / G$ \\
\hline UN_xiong_4263 & 22 & $\mathrm{C} / \mathrm{T}$ \\
\hline UN_xiong_4263 & 227 & $\mathrm{~T} / \mathrm{C}$ \\
\hline UN_xiong_4263 & 359 & $\mathrm{C} / \mathrm{T}$ \\
\hline UN_xiong_4268 & 60 & $A / G$ \\
\hline UN_xiong_4268 & 115 & $\mathrm{G} / \mathrm{C}$ \\
\hline UN_xiong_4268 & 143 & $\mathrm{G} / \mathrm{C}$ \\
\hline UN_xiong_4268 & 183 & $T / G$ \\
\hline UN_xiong_4268 & 211 & $T / G$ \\
\hline UN_xiong_4268 & 239 & $T / G$ \\
\hline UN_xiong_4268 & 267 & $T / G$ \\
\hline UN_xiong_4268 & 295 & $\mathrm{G} / \mathrm{T}$ \\
\hline UN_xiong_4268 & 323 & $\mathrm{G} / \mathrm{C}$ \\
\hline UN_xiong_4268 & 351 & $\mathrm{G} / \mathrm{T}$ \\
\hline UN_xiong_4270 & 452 & $C / A$ \\
\hline UN_xiong_4270 & 671 & $\mathrm{G} / \mathrm{A}$ \\
\hline UN_xiong_4279 & 216 & $A / G$ \\
\hline UN_xiong_4281 & 239 & $T / G$ \\
\hline UN_xiong_4286 & 218 & $T / G$ \\
\hline UN_xiong_4288 & 198 & $C / G$ \\
\hline UN_xiong_4288 & 382 & $G / A$ \\
\hline UN_xiong_4289 & 224 & $\mathrm{~T} / \mathrm{C}$ \\
\hline UN_xiong_4291 & 318 & $\mathrm{~T} / \mathrm{C}$ \\
\hline UN_xiong_4291 & 410 & $\mathrm{~A} / \mathrm{T}$ \\
\hline UN_xiong_4292 & 296 & $\mathrm{G} / \mathrm{A}$ \\
\hline UN_xiong_4298 & 596 & $\mathrm{G} / \mathrm{T}$ \\
\hline
\end{tabular}




\begin{tabular}{|c|c|c|}
\hline UN_xiong_4298 & 695 & $\mathrm{C} / \mathrm{T}$ \\
\hline UN_xiong_4300 & 458 & $\mathrm{~T} / \mathrm{C}$ \\
\hline UN_xiong_4302 & 155 & $\mathrm{~A} / \mathrm{T}$ \\
\hline UN_xiong_4302 & 341 & $\mathrm{C} / \mathrm{T}$ \\
\hline UN_xiong_4302 & 463 & $\mathrm{G} / \mathrm{A}$ \\
\hline UN_xiong_4329 & 189 & $\mathrm{~T} / \mathrm{C}$ \\
\hline UN_xiong_4331 & 34 & $A / G$ \\
\hline UN_xiong_4331 & 68 & $\mathrm{~A} / \mathrm{G}$ \\
\hline UN_xiong_4344 & 315 & $\mathrm{~T} / \mathrm{C}$ \\
\hline UN_xiong_4346 & 337 & $\mathrm{~A} / \mathrm{G}$ \\
\hline UN_xiong_4348 & 93 & $\mathrm{G} / \mathrm{T}$ \\
\hline UN_xiong_4348 & 516 & $\mathrm{~T} / \mathrm{C}$ \\
\hline UN_xiong_4349 & 483 & $\mathrm{~A} / \mathrm{G}$ \\
\hline UN_xiong_4349 & 521 & $\mathrm{~A} / \mathrm{G}$ \\
\hline UN_xiong_4349 & 562 & $\mathrm{C} / \mathrm{T}$ \\
\hline UN_xiong_4349 & 582 & $\mathrm{G} / \mathrm{A}$ \\
\hline UN_xiong_4349 & 605 & $\mathrm{C} / \mathrm{A}$ \\
\hline UN_xiong_4349 & 659 & $\mathrm{~A} / \mathrm{G}$ \\
\hline UN_xiong_4351 & 78 & $\mathrm{G} / \mathrm{A}$ \\
\hline UN_xiong_4351 & 138 & $\mathrm{G} / \mathrm{A}$ \\
\hline UN_xiong_4351 & 261 & $\mathrm{~T} / \mathrm{C}$ \\
\hline UN_xiong_4351 & 294 & $\mathrm{~A} / \mathrm{G}$ \\
\hline UN_xiong_4351 & 426 & $A / G$ \\
\hline UN_xiong_4359 & 84 & $\mathrm{~T} / \mathrm{C}$ \\
\hline UN_xiong_4359 & 154 & $A / G$ \\
\hline UN_xiong_4376 & 170 & $A / G$ \\
\hline UN_xiong_4380 & 109 & $\mathrm{G} / \mathrm{A}$ \\
\hline UN_xiong_4380 & 214 & $\mathrm{C} / \mathrm{T}$ \\
\hline UN_xiong_4380 & 239 & $\mathrm{G} / \mathrm{C}$ \\
\hline UN_xiong_4380 & 355 & $\mathrm{G} / \mathrm{T}$ \\
\hline UN_xiong_4380 & 378 & $\mathrm{G} / \mathrm{A}$ \\
\hline UN_xiong_4380 & 400 & $\mathrm{C} / \mathrm{T}$ \\
\hline UN_xiong_4380 & 437 & $\mathrm{G} / \mathrm{A}$ \\
\hline UN_xiong_4380 & 500 & $\mathrm{G} / \mathrm{A}$ \\
\hline UN_xiong_4380 & 532 & $\mathrm{~T} / \mathrm{C}$ \\
\hline UN_xiong_4380 & 556 & $\mathrm{C} / \mathrm{G}$ \\
\hline UN_xiong_4380 & 582 & $\mathrm{~T} / \mathrm{C}$ \\
\hline UN_xiong_4380 & 605 & $\mathrm{G} / \mathrm{A}$ \\
\hline UN_xiong_4380 & 637 & $\mathrm{C} / \mathrm{T}$ \\
\hline UN_xiong_4380 & 696 & $\mathrm{C} / \mathrm{T}$ \\
\hline UN_xiong_4380 & 732 & $\mathrm{C} / \mathrm{T}$ \\
\hline UN_xiong_4380 & 759 & $\mathrm{~A} / \mathrm{C}$ \\
\hline UN_xiong_4380 & 817 & $\mathrm{G} / \mathrm{C}$ \\
\hline UN_xiong_4380 & 867 & $\mathrm{~T} / \mathrm{C}$ \\
\hline UN_xiong_4380 & 888 & $A / G$ \\
\hline UN_xiong_4380 & 1179 & $A / G$ \\
\hline UN_xiong_4381 & 446 & $\mathrm{~A} / \mathrm{C}$ \\
\hline
\end{tabular}




\begin{tabular}{|c|c|c|}
\hline UN_xiong_4393 & 312 & $\mathrm{C} / \mathrm{T}$ \\
\hline UN_xiong_4401 & 323 & $\mathrm{~T} / \mathrm{C}$ \\
\hline UN_xiong_4403 & 109 & $A / G$ \\
\hline UN_xiong_4404 & 377 & $\mathrm{~T} / \mathrm{C}$ \\
\hline UN_xiong_4404 & 454 & $G / A$ \\
\hline UN_xiong_4404 & 493 & $A / G$ \\
\hline UN_xiong_4404 & 522 & $A / G$ \\
\hline UN_xiong_4404 & 600 & $C / A$ \\
\hline UN_xiong_4406 & 51 & $\mathrm{G} / \mathrm{T}$ \\
\hline UN_xiong_4406 & 118 & $\mathrm{C} / \mathrm{T}$ \\
\hline UN_xiong_4406 & 143 & $A / G$ \\
\hline UN_xiong_4406 & 284 & $\mathrm{G} / \mathrm{A}$ \\
\hline UN_xiong_4406 & 418 & $\mathrm{G} / \mathrm{A}$ \\
\hline UN_xiong_4406 & 667 & $\mathrm{~A} / \mathrm{T}$ \\
\hline UN_xiong_4410 & 6 & $\mathrm{C} / \mathrm{T}$ \\
\hline UN_xiong_4414 & 378 & $\mathrm{~T} / \mathrm{C}$ \\
\hline UN_xiong_4428 & 240 & $\mathrm{G} / \mathrm{C}$ \\
\hline UN_xiong_4428 & 340 & $\mathrm{~T} / \mathrm{C}$ \\
\hline UN_xiong_4430 & 302 & $\mathrm{~T} / \mathrm{C}$ \\
\hline UN_xiong_4434 & 129 & $\mathrm{C} / \mathrm{T}$ \\
\hline UN_xiong_4435 & 120 & $A / G$ \\
\hline UN_xiong_4436 & 46 & $\mathrm{G} / \mathrm{A}$ \\
\hline UN_xiong_4436 & 259 & $A / G$ \\
\hline UN_xiong_4436 & 308 & $A / G$ \\
\hline UN_xiong_4436 & 399 & $A / G$ \\
\hline UN_xiong_4436 & 622 & $G / A$ \\
\hline UN_xiong_4436 & 934 & $\mathrm{~A} / \mathrm{C}$ \\
\hline UN_xiong_4437 & 576 & $A / G$ \\
\hline UN_xiong_4437 & 596 & $\mathrm{G} / \mathrm{A}$ \\
\hline UN_xiong_4453 & 8 & $\mathrm{C} / \mathrm{T}$ \\
\hline UN_xiong_4463 & 117 & $\mathrm{G} / \mathrm{A}$ \\
\hline UN_xiong_4463 & 226 & $\mathrm{~T} / \mathrm{C}$ \\
\hline UN_xiong_4463 & 363 & $C / A$ \\
\hline UN_xiong_4473 & 46 & $\mathrm{~T} / \mathrm{C}$ \\
\hline UN_xiong_4474 & 115 & $\mathrm{~T} / \mathrm{C}$ \\
\hline UN_xiong_4481 & 366 & $\mathrm{~T} / \mathrm{C}$ \\
\hline UN_xiong_4481 & 645 & $A / G$ \\
\hline UN_xiong_4485 & 212 & $\mathrm{~T} / \mathrm{A}$ \\
\hline UN_xiong_4486 & 241 & $\mathrm{G} / \mathrm{A}$ \\
\hline UN_xiong_4489 & 39 & $A / G$ \\
\hline UN_xiong_4489 & 73 & $\mathrm{C} / \mathrm{T}$ \\
\hline UN_xiong_4489 & 104 & $G / A$ \\
\hline UN_xiong_4489 & 136 & $\mathrm{~A} / \mathrm{T}$ \\
\hline UN_xiong_4489 & 156 & $\mathrm{G} / \mathrm{A}$ \\
\hline UN_xiong_4492 & 350 & $\mathrm{C} / \mathrm{T}$ \\
\hline UN_xiong_4494 & 423 & $\mathrm{~T} / \mathrm{C}$ \\
\hline UN_xiong_4494 & 446 & $A / G$ \\
\hline
\end{tabular}




\begin{tabular}{|c|c|c|}
\hline UN_xiong_4494 & 527 & $\mathrm{C} / \mathrm{T}$ \\
\hline UN_xiong_4494 & 552 & $A / G$ \\
\hline UN_xiong_4494 & 587 & $\mathrm{G} / \mathrm{A}$ \\
\hline UN_xiong_4494 & 623 & $\mathrm{G} / \mathrm{A}$ \\
\hline UN_xiong_4495 & 133 & $\mathrm{~T} / \mathrm{C}$ \\
\hline UN_xiong_4495 & 167 & $\mathrm{~T} / \mathrm{C}$ \\
\hline UN_xiong_4495 & 362 & $\mathrm{~T} / \mathrm{A}$ \\
\hline UN_xiong_4496 & 201 & $\mathrm{~T} / \mathrm{C}$ \\
\hline UN_xiong_4496 & 852 & $A / G$ \\
\hline UN_xiong_4518 & 60 & $\mathrm{~A} / \mathrm{G}$ \\
\hline UN_xiong_4518 & 370 & $\mathrm{~A} / \mathrm{G}$ \\
\hline UN_xiong_4518 & 412 & $\mathrm{~A} / \mathrm{T}$ \\
\hline UN_xiong_4518 & 507 & $\mathrm{~T} / \mathrm{A}$ \\
\hline UN_xiong_4518 & 531 & $\mathrm{C} / \mathrm{T}$ \\
\hline UN_xiong_4533 & 199 & $\mathrm{~A} / \mathrm{C}$ \\
\hline UN_xiong_4533 & 221 & $\mathrm{C} / \mathrm{T}$ \\
\hline UN_xiong_4533 & 425 & $\mathrm{~A} / \mathrm{G}$ \\
\hline UN_xiong_4533 & 556 & $\mathrm{~T} / \mathrm{C}$ \\
\hline UN_xiong_4539 & 165 & $\mathrm{~T} / \mathrm{G}$ \\
\hline UN_xiong_4542 & 139 & $\mathrm{C} / \mathrm{T}$ \\
\hline UN_xiong_4544 & 125 & $\mathrm{C} / \mathrm{T}$ \\
\hline UN_xiong_4544 & 159 & $\mathrm{G} / \mathrm{A}$ \\
\hline UN_xiong_4544 & 243 & $\mathrm{~T} / \mathrm{C}$ \\
\hline UN_xiong_4544 & 598 & $\mathrm{C} / \mathrm{A}$ \\
\hline UN_xiong_4551 & 128 & $\mathrm{~T} / \mathrm{G}$ \\
\hline UN_xiong_4561 & 147 & $\mathrm{G} / \mathrm{A}$ \\
\hline UN_xiong_4572 & 117 & $\mathrm{~T} / \mathrm{C}$ \\
\hline UN_xiong_4572 & 380 & $\mathrm{G} / \mathrm{A}$ \\
\hline UN_xiong_4572 & 448 & $\mathrm{~A} / \mathrm{C}$ \\
\hline UN_xiong_4572 & 528 & $\mathrm{G} / \mathrm{A}$ \\
\hline UN_xiong_4574 & 216 & $\mathrm{~A} / \mathrm{G}$ \\
\hline UN_xiong_4574 & 242 & $\mathrm{G} / \mathrm{T}$ \\
\hline UN_xiong_4574 & 478 & $\mathrm{~T} / \mathrm{G}$ \\
\hline UN_xiong_4578 & 128 & $\mathrm{~T} / \mathrm{C}$ \\
\hline UN_xiong_4578 & 198 & $\mathrm{~T} / \mathrm{A}$ \\
\hline UN_xiong_4579 & 256 & $\mathrm{~T} / \mathrm{A}$ \\
\hline UN_xiong_4579 & 353 & $\mathrm{~T} / \mathrm{G}$ \\
\hline UN_xiong_4580 & 431 & $\mathrm{~A} / \mathrm{G}$ \\
\hline UN_xiong_4584 & 794 & $\mathrm{~T} / \mathrm{C}$ \\
\hline UN_xiong_4585 & 594 & $\mathrm{~A} / \mathrm{G}$ \\
\hline UN_xiong_4592 & 1140 & $\mathrm{~T} / \mathrm{C}$ \\
\hline UN_xiong_4592 & 1247 & $A / G$ \\
\hline UN_xiong_4614 & 61 & $\mathrm{~A} / \mathrm{G}$ \\
\hline UN_xiong_4614 & 94 & $\mathrm{~T} / \mathrm{C}$ \\
\hline UN_xiong_4616 & 449 & $\mathrm{~A} / \mathrm{C}$ \\
\hline UN_xiong_4616 & 529 & $\mathrm{~T} / \mathrm{A}$ \\
\hline UN_xiong_4619 & 427 & $\mathrm{G} / \mathrm{A}$ \\
\hline
\end{tabular}




\begin{tabular}{|c|c|c|}
\hline UN_xiong_4619 & 534 & $C / A$ \\
\hline UN_xiong_4628 & 276 & $C / A$ \\
\hline UN_xiong_4628 & 628 & $\mathrm{C} / \mathrm{T}$ \\
\hline UN_xiong_4629 & 206 & $\mathrm{~A} / \mathrm{T}$ \\
\hline UN_xiong_4629 & 294 & $C / G$ \\
\hline UN_xiong_4637 & 205 & $\mathrm{~T} / \mathrm{C}$ \\
\hline UN_xiong_4638 & 360 & $\mathrm{~T} / \mathrm{A}$ \\
\hline UN_xiong_4642 & 251 & $\mathrm{C} / \mathrm{T}$ \\
\hline UN_xiong_4643 & 12 & $\mathrm{~T} / \mathrm{A}$ \\
\hline UN_xiong_4643 & 62 & $A / G$ \\
\hline UN_xiong_4643 & 168 & $\mathrm{C} / \mathrm{T}$ \\
\hline UN_xiong_4650 & 241 & $C / A$ \\
\hline UN_xiong_4659 & 47 & $\mathrm{G} / \mathrm{A}$ \\
\hline UN_xiong_4659 & 68 & $\mathrm{C} / \mathrm{T}$ \\
\hline UN_xiong_4659 & 90 & $\mathrm{C} / \mathrm{T}$ \\
\hline UN_xiong_4664 & 895 & $A / G$ \\
\hline UN_xiong_4664 & 1155 & $\mathrm{C} / \mathrm{T}$ \\
\hline UN_xiong_4664 & 1643 & $\mathrm{~T} / \mathrm{C}$ \\
\hline UN_xiong_4664 & 1816 & $\mathrm{~T} / \mathrm{C}$ \\
\hline UN_xiong_4664 & 1951 & $\mathrm{~T} / \mathrm{C}$ \\
\hline UN_xiong_4664 & 1986 & $\mathrm{C} / \mathrm{T}$ \\
\hline UN_xiong_4664 & 2070 & $\mathrm{~T} / \mathrm{C}$ \\
\hline UN_xiong_4664 & 2124 & $\mathrm{C} / \mathrm{T}$ \\
\hline UN_xiong_4673 & 192 & $A / G$ \\
\hline UN_xiong_4686 & 100 & $\mathrm{~T} / \mathrm{C}$ \\
\hline UN_xiong_4687 & 171 & $A / G$ \\
\hline UN_xiong_4687 & 209 & $\mathrm{~T} / \mathrm{C}$ \\
\hline UN_xiong_4687 & 393 & $A / G$ \\
\hline UN_xiong_4701 & 650 & $\mathrm{G} / \mathrm{A}$ \\
\hline UN_xiong_4703 & 234 & $A / T$ \\
\hline UN_xiong_4703 & 254 & $\mathrm{~A} / \mathrm{T}$ \\
\hline UN_xiong_4707 & 122 & $A / C$ \\
\hline UN_xiong_4711 & 228 & $\mathrm{C} / \mathrm{T}$ \\
\hline UN_xiong_4711 & 302 & $G / A$ \\
\hline UN_xiong_4715 & 80 & $\mathrm{C} / \mathrm{T}$ \\
\hline UN_xiong_4722 & 263 & $C / G$ \\
\hline UN_xiong_4726 & 48 & $\mathrm{~T} / \mathrm{C}$ \\
\hline UN_xiong_4744 & 71 & $\mathrm{C} / \mathrm{T}$ \\
\hline UN_xiong_4757 & 92 & $\mathrm{~T} / \mathrm{C}$ \\
\hline UN_xiong_4772 & 160 & $G / A$ \\
\hline UN_xiong_4772 & 224 & $C / A$ \\
\hline UN_xiong_4772 & 572 & $\mathrm{C} / \mathrm{T}$ \\
\hline UN_xiong_4780 & 93 & $C / A$ \\
\hline UN_xiong_4782 & 642 & $A / G$ \\
\hline UN_xiong_4782 & 710 & $\mathrm{G} / \mathrm{C}$ \\
\hline UN_xiong_4782 & 810 & $\mathrm{C} / \mathrm{T}$ \\
\hline UN_xiong_4783 & 116 & $\mathrm{~T} / \mathrm{C}$ \\
\hline
\end{tabular}




\begin{tabular}{|c|c|c|}
\hline UN_xiong_4783 & 144 & $\mathrm{G} / \mathrm{T}$ \\
\hline UN_xiong_4796 & 431 & $\mathrm{~T} / \mathrm{G}$ \\
\hline UN_xiong_4796 & 895 & $\mathrm{~T} / \mathrm{C}$ \\
\hline UN_xiong_4796 & 1305 & $\mathrm{~T} / \mathrm{C}$ \\
\hline UN_xiong_4796 & 1390 & $A / G$ \\
\hline UN_xiong_4803 & 143 & $\mathrm{G} / \mathrm{T}$ \\
\hline UN_xiong_4803 & 217 & $\mathrm{~A} / \mathrm{T}$ \\
\hline UN_xiong_4803 & 466 & $\mathrm{G} / \mathrm{T}$ \\
\hline UN_xiong_4811 & 135 & $T / G$ \\
\hline UN_xiong_4811 & 158 & $\mathrm{G} / \mathrm{T}$ \\
\hline UN_xiong_4811 & 266 & $A / G$ \\
\hline UN_xiong_4811 & 415 & $\mathrm{~T} / \mathrm{C}$ \\
\hline UN_xiong_4833 & 154 & $G / A$ \\
\hline UN_xiong_4834 & 373 & $C / A$ \\
\hline UN_xiong_4834 & 426 & $A / G$ \\
\hline UN_xiong_4834 & 665 & $\mathrm{~T} / \mathrm{C}$ \\
\hline UN_xiong_4834 & 692 & $\mathrm{G} / \mathrm{A}$ \\
\hline UN_xiong_4843 & 609 & $A / G$ \\
\hline UN_xiong_4850 & 172 & $\mathrm{G} / \mathrm{A}$ \\
\hline UN_xiong_4865 & 348 & $\mathrm{~T} / \mathrm{C}$ \\
\hline UN_xiong_4865 & 1521 & $A / G$ \\
\hline UN_xiong_4865 & 1711 & $\mathrm{~T} / \mathrm{C}$ \\
\hline UN_xiong_4865 & 1906 & $\mathrm{~A} / \mathrm{T}$ \\
\hline UN_xiong_4872 & 154 & $\mathrm{G} / \mathrm{A}$ \\
\hline UN_xiong_4875 & 21 & $A / G$ \\
\hline UN_xiong_4878 & 33 & $\mathrm{C} / \mathrm{T}$ \\
\hline UN_xiong_4878 & 334 & $\mathrm{~A} / \mathrm{C}$ \\
\hline UN_xiong_4887 & 197 & $\mathrm{G} / \mathrm{C}$ \\
\hline UN_xiong_4913 & 127 & $\mathrm{~T} / \mathrm{C}$ \\
\hline UN_xiong_4914 & 212 & $\mathrm{~T} / \mathrm{C}$ \\
\hline UN_xiong_4914 & 331 & $\mathrm{~T} / \mathrm{C}$ \\
\hline UN_xiong_4914 & 412 & $A / G$ \\
\hline UN_xiong_4922 & 201 & $\mathrm{C} / \mathrm{T}$ \\
\hline UN_xiong_4932 & 267 & $\mathrm{C} / \mathrm{T}$ \\
\hline UN_xiong_4932 & 334 & $\mathrm{C} / \mathrm{T}$ \\
\hline UN_xiong_4932 & 396 & $\mathrm{G} / \mathrm{A}$ \\
\hline UN_xiong_4932 & 475 & $\mathrm{C} / \mathrm{T}$ \\
\hline UN_xiong_4932 & 693 & $\mathrm{G} / \mathrm{A}$ \\
\hline UN_xiong_4932 & 788 & $A / G$ \\
\hline UN_xiong_4932 & 836 & $\mathrm{~A} / \mathrm{T}$ \\
\hline UN_xiong_4948 & 39 & $\mathrm{C} / \mathrm{T}$ \\
\hline UN_xiong_4951 & 252 & $\mathrm{C} / \mathrm{T}$ \\
\hline UN_xiong_4951 & 350 & $\mathrm{~T} / \mathrm{A}$ \\
\hline UN_xiong_4951 & 752 & $A / G$ \\
\hline UN_xiong_4952 & 137 & $A / G$ \\
\hline UN_xiong_4956 & 248 & $\mathrm{~T} / \mathrm{C}$ \\
\hline UN_xiong_4956 & 277 & $A / G$ \\
\hline
\end{tabular}




\begin{tabular}{|c|c|c|}
\hline UN_xiong_4956 & 566 & $\mathrm{C} / \mathrm{T}$ \\
\hline UN_xiong_4960 & 32 & $\mathrm{C} / \mathrm{T}$ \\
\hline UN_xiong_4960 & 68 & $A / G$ \\
\hline UN_xiong_4963 & 19 & $\mathrm{C} / \mathrm{T}$ \\
\hline UN_xiong_4963 & 132 & $\mathrm{C} / \mathrm{T}$ \\
\hline UN_xiong_4988 & 103 & $\mathrm{~T} / \mathrm{C}$ \\
\hline UN_xiong_4988 & 522 & $A / G$ \\
\hline UN_xiong_4988 & 601 & $\mathrm{G} / \mathrm{A}$ \\
\hline UN_xiong_4988 & 750 & $\mathrm{~T} / \mathrm{C}$ \\
\hline UN_xiong_4988 & 881 & $\mathrm{~A} / \mathrm{T}$ \\
\hline UN_xiong_4990 & 392 & $\mathrm{G} / \mathrm{A}$ \\
\hline UN_xiong_4995 & 19 & $\mathrm{~T} / \mathrm{C}$ \\
\hline UN_xiong_4995 & 55 & $\mathrm{~A} / \mathrm{T}$ \\
\hline UN_xiong_4995 & 121 & $\mathrm{~A} / \mathrm{T}$ \\
\hline UN_xiong_4995 & 201 & $\mathrm{~A} / \mathrm{C}$ \\
\hline UN_xiong_4995 & 232 & $\mathrm{~T} / \mathrm{G}$ \\
\hline UN_xiong_4995 & 320 & $\mathrm{~T} / \mathrm{C}$ \\
\hline UN_xiong_4995 & 561 & $\mathrm{C} / \mathrm{T}$ \\
\hline UN_xiong_4999 & 284 & $\mathrm{G} / \mathrm{A}$ \\
\hline UN_xiong_4999 & 329 & $\mathrm{C} / \mathrm{A}$ \\
\hline UN_xiong_5001 & 29 & $\mathrm{C} / \mathrm{T}$ \\
\hline UN_xiong_5001 & 103 & $\mathrm{G} / \mathrm{A}$ \\
\hline UN_xiong_5001 & 137 & $\mathrm{G} / \mathrm{A}$ \\
\hline UN_xiong_5001 & 279 & $\mathrm{G} / \mathrm{A}$ \\
\hline UN_xiong_5010 & 266 & $A / G$ \\
\hline UN_xiong_5011 & 185 & $\mathrm{~A} / \mathrm{T}$ \\
\hline UN_xiong_5011 & 335 & $A / G$ \\
\hline UN_xiong_5011 & 601 & $\mathrm{C} / \mathrm{A}$ \\
\hline UN_xiong_5013 & 25 & $\mathrm{~T} / \mathrm{C}$ \\
\hline UN_xiong_5013 & 212 & $A / G$ \\
\hline UN_xiong_5013 & 240 & $\mathrm{~A} / \mathrm{T}$ \\
\hline UN_xiong_5013 & 443 & $\mathrm{~T} / \mathrm{C}$ \\
\hline UN_xiong_5020 & 34 & $C / A$ \\
\hline UN_xiong_5020 & 311 & $A / G$ \\
\hline UN_xiong_5020 & 440 & $\mathrm{G} / \mathrm{A}$ \\
\hline UN_xiong_5026 & 146 & $\mathrm{~T} / \mathrm{A}$ \\
\hline UN_xiong_5026 & 579 & $\mathrm{~T} / \mathrm{C}$ \\
\hline UN_xiong_5026 & 735 & $\mathrm{~T} / \mathrm{C}$ \\
\hline UN_xiong_5026 & 762 & $\mathrm{G} / \mathrm{T}$ \\
\hline UN_xiong_5026 & 795 & $\mathrm{~T} / \mathrm{A}$ \\
\hline UN_xiong_5027 & 121 & $\mathrm{~T} / \mathrm{C}$ \\
\hline UN_xiong_5036 & 386 & $\mathrm{G} / \mathrm{A}$ \\
\hline UN_xiong_5067 & 439 & $\mathrm{~T} / \mathrm{C}$ \\
\hline UN_xiong_5078 & 14 & $A / G$ \\
\hline UN_xiong_5081 & 152 & $\mathrm{G} / \mathrm{T}$ \\
\hline UN_xiong_5090 & 437 & $\mathrm{G} / \mathrm{A}$ \\
\hline UN_xiong_5098 & 69 & $\mathrm{G} / \mathrm{T}$ \\
\hline
\end{tabular}




\begin{tabular}{|c|c|c|}
\hline UN_xiong_5098 & 187 & $\mathrm{~A} / \mathrm{C}$ \\
\hline UN_xiong_5098 & 301 & $\mathrm{~T} / \mathrm{A}$ \\
\hline UN_xiong_5113 & 144 & $A / G$ \\
\hline UN_xiong_5113 & 256 & $\mathrm{C} / \mathrm{T}$ \\
\hline UN_xiong_5113 & 295 & $A / G$ \\
\hline UN_xiong_5113 & 348 & $\mathrm{C} / \mathrm{T}$ \\
\hline UN_xiong_5113 & 375 & $\mathrm{C} / \mathrm{T}$ \\
\hline UN_xiong_5113 & 738 & $\mathrm{~T} / \mathrm{C}$ \\
\hline UN_xiong_5115 & 162 & $\mathrm{C} / \mathrm{T}$ \\
\hline UN_xiong_5117 & 20 & $\mathrm{G} / \mathrm{A}$ \\
\hline UN_xiong_5117 & 166 & $A / G$ \\
\hline UN_xiong_5117 & 188 & $\mathrm{~T} / \mathrm{A}$ \\
\hline UN_xiong_5119 & 70 & $\mathrm{G} / \mathrm{C}$ \\
\hline UN_xiong_5119 & 97 & $\mathrm{C} / \mathrm{T}$ \\
\hline UN_xiong_5119 & 242 & $\mathrm{~A} / \mathrm{C}$ \\
\hline UN_xiong_5119 & 509 & $\mathrm{~T} / \mathrm{C}$ \\
\hline UN_xiong_5119 & 588 & $A / G$ \\
\hline UN_xiong_5119 & 726 & $\mathrm{C} / \mathrm{T}$ \\
\hline UN_xiong_5119 & 821 & $\mathrm{~T} / \mathrm{C}$ \\
\hline UN_xiong_5125 & 30 & $A / G$ \\
\hline UN_xiong_5125 & 90 & $\mathrm{G} / \mathrm{A}$ \\
\hline UN_xiong_5125 & 165 & $A / G$ \\
\hline UN_xiong_5125 & 267 & $\mathrm{G} / \mathrm{A}$ \\
\hline UN_xiong_5130 & 142 & $A / G$ \\
\hline UN_xiong_5130 & 185 & $\mathrm{C} / \mathrm{T}$ \\
\hline UN_xiong_5130 & 282 & $\mathrm{G} / \mathrm{A}$ \\
\hline UN_xiong_5130 & 452 & $\mathrm{~T} / \mathrm{A}$ \\
\hline UN_xiong_5131 & 167 & $A / G$ \\
\hline UN_xiong_5135 & 42 & $\mathrm{G} / \mathrm{A}$ \\
\hline UN_xiong_5135 & 228 & $\mathrm{C} / \mathrm{T}$ \\
\hline UN_xiong_5147 & 146 & $A / G$ \\
\hline UN_xiong_5147 & 226 & $\mathrm{~T} / \mathrm{C}$ \\
\hline UN_xiong_5165 & 474 & $A / G$ \\
\hline UN_xiong_5165 & 578 & $\mathrm{~T} / \mathrm{C}$ \\
\hline UN_xiong_5165 & 669 & $A / G$ \\
\hline UN_xiong_5167 & 245 & $A / G$ \\
\hline UN_xiong_5186 & 289 & $\mathrm{~T} / \mathrm{C}$ \\
\hline UN_xiong_5196 & 319 & $\mathrm{~T} / \mathrm{A}$ \\
\hline UN_xiong_5211 & 90 & $\mathrm{~T} / \mathrm{C}$ \\
\hline UN_xiong_5212 & 257 & $\mathrm{~T} / \mathrm{C}$ \\
\hline UN_xiong_5227 & 153 & $A / G$ \\
\hline UN_xiong_5227 & 243 & $\mathrm{C} / \mathrm{T}$ \\
\hline UN_xiong_5233 & 490 & $A / G$ \\
\hline UN_xiong_5233 & 646 & $\mathrm{~A} / \mathrm{C}$ \\
\hline UN_xiong_5233 & 718 & $\mathrm{~T} / \mathrm{A}$ \\
\hline UN_xiong_5233 & 753 & $\mathrm{G} / \mathrm{A}$ \\
\hline UN_xiong_5233 & 798 & $\mathrm{C} / \mathrm{T}$ \\
\hline
\end{tabular}




\begin{tabular}{|c|c|c|}
\hline UN_xiong_5233 & 1064 & $\mathrm{~T} / \mathrm{A}$ \\
\hline UN_xiong_5248 & 393 & $\mathrm{G} / \mathrm{T}$ \\
\hline UN_xiong_5267 & 437 & $\mathrm{~A} / \mathrm{C}$ \\
\hline UN_xiong_5268 & 106 & $A / C$ \\
\hline UN_xiong_5268 & 226 & $\mathrm{C} / \mathrm{T}$ \\
\hline UN_xiong_5273 & 209 & $\mathrm{~T} / \mathrm{C}$ \\
\hline UN_xiong_5273 & 287 & $\mathrm{C} / \mathrm{T}$ \\
\hline UN_xiong_5280 & 143 & $\mathrm{~T} / \mathrm{C}$ \\
\hline UN_xiong_5290 & 439 & $\mathrm{G} / \mathrm{T}$ \\
\hline UN_xiong_5290 & 520 & $A / G$ \\
\hline UN_xiong_5294 & 80 & $\mathrm{C} / \mathrm{T}$ \\
\hline UN_xiong_5294 & 252 & $A / C$ \\
\hline UN_xiong_5299 & 76 & $A / G$ \\
\hline UN_xiong_5299 & 108 & $\mathrm{~A} / \mathrm{T}$ \\
\hline UN_xiong_5301 & 399 & $\mathrm{~T} / \mathrm{A}$ \\
\hline UN_xiong_5301 & 448 & $A / T$ \\
\hline UN_xiong_5301 & 546 & $G / A$ \\
\hline UN_xiong_5301 & 589 & $\mathrm{G} / \mathrm{A}$ \\
\hline UN_xiong_5325 & 28 & $\mathrm{G} / \mathrm{T}$ \\
\hline UN_xiong_5325 & 332 & $\mathrm{G} / \mathrm{A}$ \\
\hline UN_xiong_5329 & 154 & $\mathrm{~T} / \mathrm{C}$ \\
\hline UN_xiong_5329 & 200 & $\mathrm{G} / \mathrm{C}$ \\
\hline UN_xiong_5333 & 370 & $\mathrm{C} / \mathrm{T}$ \\
\hline UN_xiong_5341 & 236 & $\mathrm{C} / \mathrm{T}$ \\
\hline UN_xiong_5350 & 531 & $\mathrm{~A} / \mathrm{T}$ \\
\hline UN_xiong_5355 & 214 & $\mathrm{~T} / \mathrm{A}$ \\
\hline UN_xiong_5356 & 6 & $\mathrm{C} / \mathrm{T}$ \\
\hline UN_xiong_5369 & 86 & $\mathrm{G} / \mathrm{A}$ \\
\hline UN_xiong_5370 & 498 & $\mathrm{~T} / \mathrm{C}$ \\
\hline UN_xiong_5372 & 264 & $\mathrm{~T} / \mathrm{C}$ \\
\hline UN_xiong_5372 & 348 & $A / G$ \\
\hline UN_xiong_5372 & 423 & $\mathrm{~T} / \mathrm{C}$ \\
\hline UN_xiong_5372 & 458 & $A / G$ \\
\hline UN_xiong_5372 & 635 & $G / A$ \\
\hline UN_xiong_5385 & 102 & $A / G$ \\
\hline UN_xiong_5387 & 165 & $A / G$ \\
\hline UN_xiong_5398 & 348 & $\mathrm{~T} / \mathrm{C}$ \\
\hline UN_xiong_5398 & 407 & $A / T$ \\
\hline UN_xiong_5400 & 354 & $C / A$ \\
\hline UN_xiong_5410 & 166 & $A / G$ \\
\hline UN_xiong_5424 & 173 & $\mathrm{G} / \mathrm{A}$ \\
\hline UN_xiong_5424 & 428 & $\mathrm{~T} / \mathrm{C}$ \\
\hline UN_xiong_5424 & 715 & $C / A$ \\
\hline UN_xiong_5424 & 916 & $\mathrm{G} / \mathrm{A}$ \\
\hline UN_xiong_5425 & 357 & $\mathrm{G} / \mathrm{A}$ \\
\hline UN_xiong_5434 & 115 & $C / A$ \\
\hline UN_xiong_5441 & 183 & $\mathrm{G} / \mathrm{A}$ \\
\hline
\end{tabular}




\begin{tabular}{|c|c|c|}
\hline UN_xiong_5441 & 258 & $\mathrm{C} / \mathrm{T}$ \\
\hline UN_xiong_5443 & 387 & $\mathrm{~T} / \mathrm{A}$ \\
\hline UN_xiong_5454 & 173 & $\mathrm{C} / \mathrm{T}$ \\
\hline UN_xiong_5457 & 270 & $\mathrm{~T} / \mathrm{A}$ \\
\hline UN_xiong_5459 & 241 & $\mathrm{~A} / \mathrm{C}$ \\
\hline UN_xiong_5460 & 312 & $\mathrm{~T} / \mathrm{C}$ \\
\hline UN_xiong_5466 & 20 & $A / G$ \\
\hline UN_xiong_5466 & 149 & $A / G$ \\
\hline UN_xiong_5466 & 224 & $A / G$ \\
\hline UN_xiong_5466 & 329 & $A / G$ \\
\hline UN_xiong_5466 & 522 & $\mathrm{~T} / \mathrm{C}$ \\
\hline UN_xiong_5473 & 338 & $\mathrm{G} / \mathrm{A}$ \\
\hline UN_xiong_5480 & 67 & $\mathrm{~A} / \mathrm{C}$ \\
\hline UN_xiong_5480 & 116 & $\mathrm{~A} / \mathrm{C}$ \\
\hline UN_xiong_5480 & 208 & $A / G$ \\
\hline UN_xiong_5480 & 306 & $G / A$ \\
\hline UN_xiong_5485 & 1222 & $A / G$ \\
\hline UN_xiong_5485 & 1442 & $\mathrm{~T} / \mathrm{C}$ \\
\hline UN_xiong_5487 & 204 & $\mathrm{C} / \mathrm{T}$ \\
\hline UN_xiong_5491 & 69 & $T / G$ \\
\hline UN_xiong_5491 & 99 & $\mathrm{~T} / \mathrm{C}$ \\
\hline UN_xiong_5491 & 141 & $A / G$ \\
\hline UN_xiong_5491 & 346 & $\mathrm{G} / \mathrm{A}$ \\
\hline UN_xiong_5507 & 57 & $C / A$ \\
\hline UN_xiong_5518 & 87 & $\mathrm{C} / \mathrm{T}$ \\
\hline UN_xiong_5518 & 172 & $\mathrm{C} / \mathrm{T}$ \\
\hline UN_xiong_5518 & 261 & $A / G$ \\
\hline UN_xiong_5518 & 283 & $\mathrm{~A} / \mathrm{T}$ \\
\hline UN_xiong_5527 & 178 & $C / A$ \\
\hline UN_xiong_5527 & 274 & $\mathrm{C} / \mathrm{T}$ \\
\hline UN_xiong_5527 & 305 & $\mathrm{C} / \mathrm{T}$ \\
\hline UN_xiong_5527 & 368 & $\mathrm{~T} / \mathrm{C}$ \\
\hline UN_xiong_5527 & 406 & $\mathrm{C} / \mathrm{T}$ \\
\hline UN_xiong_5527 & 555 & $\mathrm{~T} / \mathrm{C}$ \\
\hline UN_xiong_5527 & 611 & $\mathrm{~A} / \mathrm{C}$ \\
\hline UN_xiong_5534 & 132 & $\mathrm{G} / \mathrm{A}$ \\
\hline UN_xiong_5534 & 197 & $\mathrm{~T} / \mathrm{A}$ \\
\hline UN_xiong_5540 & 67 & $\mathrm{~T} / \mathrm{C}$ \\
\hline UN_xiong_5540 & 176 & $\mathrm{~T} / \mathrm{C}$ \\
\hline UN_xiong_5545 & 15 & $G / A$ \\
\hline UN_xiong_5545 & 398 & $A / T$ \\
\hline UN_xiong_5545 & 423 & $\mathrm{C} / \mathrm{G}$ \\
\hline UN_xiong_5545 & 460 & $\mathrm{C} / \mathrm{T}$ \\
\hline UN_xiong_5545 & 505 & $\mathrm{G} / \mathrm{A}$ \\
\hline UN_xiong_5567 & 301 & $\mathrm{G} / \mathrm{A}$ \\
\hline UN_xiong_5575 & 833 & $A / G$ \\
\hline UN_xiong_5575 & 955 & $\mathrm{G} / \mathrm{A}$ \\
\hline
\end{tabular}




\begin{tabular}{|c|c|c|}
\hline UN_xiong_5576 & 751 & $\mathrm{C} / \mathrm{T}$ \\
\hline UN_xiong_5589 & 359 & $\mathrm{~T} / \mathrm{C}$ \\
\hline UN_xiong_5591 & 116 & $\mathrm{C} / \mathrm{A}$ \\
\hline UN_xiong_5591 & 246 & $\mathrm{~T} / \mathrm{C}$ \\
\hline UN_xiong_5592 & 547 & $\mathrm{C} / \mathrm{T}$ \\
\hline UN_xiong_5592 & 657 & $\mathrm{~T} / \mathrm{C}$ \\
\hline UN_xiong_5593 & 40 & $\mathrm{~T} / \mathrm{C}$ \\
\hline UN_xiong_5593 & 153 & $\mathrm{~A} / \mathrm{T}$ \\
\hline UN_xiong_5611 & 52 & $\mathrm{~T} / \mathrm{C}$ \\
\hline UN_xiong_5614 & 49 & $\mathrm{G} / \mathrm{A}$ \\
\hline UN_xiong_5614 & 350 & $\mathrm{~T} / \mathrm{C}$ \\
\hline UN_xiong_5633 & 113 & $\mathrm{G} / \mathrm{A}$ \\
\hline UN_xiong_5637 & 118 & $\mathrm{~T} / \mathrm{C}$ \\
\hline UN_xiong_5637 & 347 & $\mathrm{~T} / \mathrm{A}$ \\
\hline UN_xiong_5637 & 465 & $\mathrm{C} / \mathrm{G}$ \\
\hline UN_xiong_5637 & 902 & $\mathrm{~T} / \mathrm{C}$ \\
\hline UN_xiong_5646 & 147 & $\mathrm{~T} / \mathrm{C}$ \\
\hline UN_xiong_5650 & 197 & $\mathrm{~T} / \mathrm{C}$ \\
\hline UN_xiong_5651 & 490 & $\mathrm{G} / \mathrm{A}$ \\
\hline UN_xiong_5662 & 71 & $\mathrm{~T} / \mathrm{G}$ \\
\hline UN_xiong_5662 & 349 & $A / G$ \\
\hline UN_xiong_5662 & 492 & $\mathrm{G} / \mathrm{A}$ \\
\hline UN_xiong_5662 & 513 & $\mathrm{G} / \mathrm{A}$ \\
\hline UN_xiong_5664 & 304 & $\mathrm{~T} / \mathrm{C}$ \\
\hline UN_xiong_5668 & 303 & $\mathrm{~A} / \mathrm{G}$ \\
\hline UN_xiong_5668 & 659 & $\mathrm{G} / \mathrm{A}$ \\
\hline UN_xiong_5670 & 34 & $\mathrm{G} / \mathrm{T}$ \\
\hline UN_xiong_5670 & 267 & $\mathrm{~T} / \mathrm{A}$ \\
\hline UN_xiong_5680 & 928 & $\mathrm{~T} / \mathrm{C}$ \\
\hline UN_xiong_5683 & 123 & $\mathrm{~T} / \mathrm{C}$ \\
\hline UN_xiong_5683 & 280 & $\mathrm{~A} / \mathrm{G}$ \\
\hline UN_xiong_5683 & 333 & $A / G$ \\
\hline UN_xiong_5683 & 446 & $\mathrm{C} / \mathrm{T}$ \\
\hline UN_xiong_5688 & 461 & $\mathrm{G} / \mathrm{C}$ \\
\hline UN_xiong_5693 & 21 & $\mathrm{C} / \mathrm{T}$ \\
\hline UN_xiong_5698 & 98 & $\mathrm{G} / \mathrm{A}$ \\
\hline UN_xiong_5698 & 148 & $\mathrm{C} / \mathrm{T}$ \\
\hline UN_xiong_5698 & 203 & $\mathrm{~T} / \mathrm{C}$ \\
\hline UN_xiong_5698 & 247 & $\mathrm{C} / \mathrm{T}$ \\
\hline UN_xiong_5700 & 197 & $\mathrm{G} / \mathrm{C}$ \\
\hline UN_xiong_5705 & 170 & $\mathrm{~A} / \mathrm{G}$ \\
\hline UN_xiong_5706 & 580 & $\mathrm{G} / \mathrm{A}$ \\
\hline UN_xiong_5714 & 376 & $\mathrm{C} / \mathrm{T}$ \\
\hline UN_xiong_5718 & 43 & $\mathrm{~T} / \mathrm{C}$ \\
\hline UN_xiong_5723 & 23 & $\mathrm{G} / \mathrm{T}$ \\
\hline UN_xiong_5724 & 314 & $\mathrm{C} / \mathrm{T}$ \\
\hline UN_xiong_5724 & 448 & $\mathrm{~A} / \mathrm{G}$ \\
\hline
\end{tabular}




\begin{tabular}{|c|c|c|}
\hline UN_xiong_5730 & 81 & $\mathrm{C} / \mathrm{T}$ \\
\hline UN_xiong_5732 & 445 & $\mathrm{~T} / \mathrm{A}$ \\
\hline UN_xiong_5737 & 7 & $\mathrm{~A} / \mathrm{T}$ \\
\hline UN_xiong_5737 & 311 & $A / G$ \\
\hline UN_xiong_5737 & 362 & $G / A$ \\
\hline UN_xiong_5737 & 421 & $\mathrm{~T} / \mathrm{C}$ \\
\hline UN_xiong_5737 & 994 & $C / A$ \\
\hline UN_xiong_5741 & 335 & $\mathrm{~T} / \mathrm{C}$ \\
\hline UN_xiong_5742 & 223 & $T / G$ \\
\hline UN_xiong_5742 & 463 & $\mathrm{C} / \mathrm{T}$ \\
\hline UN_xiong_5747 & 74 & $\mathrm{~A} / \mathrm{C}$ \\
\hline UN_xiong_5749 & 15 & $\mathrm{~T} / \mathrm{A}$ \\
\hline UN_xiong_5753 & 516 & $A / G$ \\
\hline UN_xiong_5761 & 380 & $\mathrm{G} / \mathrm{A}$ \\
\hline UN_xiong_5761 & 601 & $\mathrm{~T} / \mathrm{C}$ \\
\hline UN_xiong_5773 & 25 & $\mathrm{~A} / \mathrm{T}$ \\
\hline UN_xiong_5773 & 369 & $A / G$ \\
\hline UN_xiong_5777 & 102 & $A / G$ \\
\hline UN_xiong_5777 & 201 & $\mathrm{~T} / \mathrm{C}$ \\
\hline UN_xiong_5777 & 224 & $C / A$ \\
\hline UN_xiong_5777 & 495 & $\mathrm{~A} / \mathrm{T}$ \\
\hline UN_xiong_5777 & 622 & $\mathrm{~T} / \mathrm{C}$ \\
\hline UN_xiong_5777 & 695 & $A / G$ \\
\hline UN_xiong_5789 & 368 & $A / G$ \\
\hline UN_xiong_5794 & 258 & $C / A$ \\
\hline UN_xiong_5794 & 400 & $A / G$ \\
\hline UN_xiong_5794 & 461 & $G / A$ \\
\hline UN_xiong_5794 & 518 & $A / G$ \\
\hline UN_xiong_5802 & 125 & $\mathrm{C} / \mathrm{T}$ \\
\hline UN_xiong_5809 & 200 & $A / C$ \\
\hline UN_xiong_5809 & 342 & $\mathrm{~T} / \mathrm{C}$ \\
\hline UN_xiong_5815 & 449 & $\mathrm{G} / \mathrm{A}$ \\
\hline UN_xiong_5827 & 56 & $A / G$ \\
\hline UN_xiong_5827 & 146 & $A / C$ \\
\hline UN_xiong_5827 & 447 & $T / G$ \\
\hline UN_xiong_5853 & 13 & $\mathrm{C} / \mathrm{T}$ \\
\hline UN_xiong_5853 & 393 & $\mathrm{~T} / \mathrm{C}$ \\
\hline UN_xiong_5856 & 417 & $\mathrm{C} / \mathrm{T}$ \\
\hline UN_xiong_5856 & 446 & $\mathrm{C} / \mathrm{T}$ \\
\hline UN_xiong_5856 & 524 & $A / G$ \\
\hline UN_xiong_5872 & 231 & $\mathrm{C} / \mathrm{T}$ \\
\hline UN_xiong_5880 & 114 & $\mathrm{G} / \mathrm{T}$ \\
\hline UN_xiong_5880 & 184 & $C / A$ \\
\hline UN_xiong_5880 & 246 & $\mathrm{G} / \mathrm{A}$ \\
\hline UN_xiong_5881 & 339 & $\mathrm{C} / \mathrm{T}$ \\
\hline UN_xiong_5881 & 364 & $A / G$ \\
\hline UN_xiong_5883 & 122 & $\mathrm{C} / \mathrm{T}$ \\
\hline
\end{tabular}




\begin{tabular}{|c|c|c|}
\hline UN_xiong_5883 & 164 & $A / G$ \\
\hline UN_xiong_5883 & 443 & $\mathrm{G} / \mathrm{A}$ \\
\hline UN_xiong_5885 & 372 & $\mathrm{~A} / \mathrm{T}$ \\
\hline UN_xiong_5885 & 476 & $\mathrm{~T} / \mathrm{A}$ \\
\hline UN_xiong_5903 & 124 & $\mathrm{C} / \mathrm{T}$ \\
\hline UN_xiong_5903 & 273 & $\mathrm{C} / \mathrm{T}$ \\
\hline UN_xiong_5912 & 84 & $A / G$ \\
\hline UN_xiong_5912 & 323 & $\mathrm{G} / \mathrm{A}$ \\
\hline UN_xiong_5920 & 138 & $A / G$ \\
\hline UN_xiong_5920 & 222 & $\mathrm{~A} / \mathrm{C}$ \\
\hline UN_xiong_5920 & 322 & $A / G$ \\
\hline UN_xiong_5935 & 7 & $\mathrm{C} / \mathrm{T}$ \\
\hline UN_xiong_5938 & 242 & $\mathrm{G} / \mathrm{A}$ \\
\hline UN_xiong_5941 & 132 & $C / A$ \\
\hline UN_xiong_5941 & 249 & $A / G$ \\
\hline UN_xiong_5941 & 386 & $\mathrm{G} / \mathrm{A}$ \\
\hline UN_xiong_5941 & 554 & $\mathrm{C} / \mathrm{T}$ \\
\hline UN_xiong_5941 & 589 & $\mathrm{C} / \mathrm{T}$ \\
\hline UN_xiong_5960 & 230 & $\mathrm{~A} / \mathrm{G}$ \\
\hline UN_xiong_5962 & 122 & $\mathrm{C} / \mathrm{T}$ \\
\hline UN_xiong_5980 & 256 & $\mathrm{C} / \mathrm{T}$ \\
\hline UN_xiong_5981 & 395 & $C / A$ \\
\hline UN_xiong_5991 & 92 & $\mathrm{G} / \mathrm{A}$ \\
\hline UN_xiong_5991 & 172 & $\mathrm{~A} / \mathrm{G}$ \\
\hline UN_xiong_5991 & 235 & $A / G$ \\
\hline UN_xiong_5991 & 640 & $\mathrm{~T} / \mathrm{C}$ \\
\hline UN_xiong_6002 & 20 & $A / G$ \\
\hline UN_xiong_6002 & 304 & $A / G$ \\
\hline UN_xiong_6002 & 349 & $\mathrm{G} / \mathrm{A}$ \\
\hline UN_xiong_6010 & 369 & $\mathrm{~T} / \mathrm{A}$ \\
\hline UN_xiong_6012 & 312 & $\mathrm{~A} / \mathrm{G}$ \\
\hline UN_xiong_6021 & 31 & $\mathrm{~T} / \mathrm{C}$ \\
\hline UN_xiong_6021 & 201 & $\mathrm{~T} / \mathrm{C}$ \\
\hline UN_xiong_6041 & 111 & $\mathrm{C} / \mathrm{T}$ \\
\hline UN_xiong_6079 & 491 & $\mathrm{G} / \mathrm{T}$ \\
\hline UN_xiong_6083 & 456 & $\mathrm{C} / \mathrm{T}$ \\
\hline UN_xiong_6083 & 689 & $\mathrm{C} / \mathrm{T}$ \\
\hline UN_xiong_6084 & 58 & $\mathrm{C} / \mathrm{G}$ \\
\hline UN_xiong_6084 & 596 & $\mathrm{C} / \mathrm{T}$ \\
\hline UN_xiong_6088 & 567 & $\mathrm{G} / \mathrm{A}$ \\
\hline UN_xiong_6101 & 272 & $\mathrm{C} / \mathrm{G}$ \\
\hline UN_xiong_6115 & 911 & $\mathrm{G} / \mathrm{A}$ \\
\hline UN_xiong_6131 & 236 & $\mathrm{~T} / \mathrm{C}$ \\
\hline UN_xiong_6152 & 33 & $\mathrm{C} / \mathrm{T}$ \\
\hline UN_xiong_6154 & 98 & $\mathrm{~T} / \mathrm{C}$ \\
\hline UN_xiong_6161 & 173 & $\mathrm{C} / \mathrm{T}$ \\
\hline UN_xiong_6173 & 362 & $\mathrm{~T} / \mathrm{C}$ \\
\hline
\end{tabular}




\begin{tabular}{|c|c|c|}
\hline UN_xiong_6174 & 439 & $\mathrm{G} / \mathrm{A}$ \\
\hline UN_xiong_6176 & 507 & $\mathrm{~T} / \mathrm{G}$ \\
\hline UN_xiong_6176 & 532 & $\mathrm{~A} / \mathrm{G}$ \\
\hline UN_xiong_6201 & 306 & $\mathrm{~T} / \mathrm{C}$ \\
\hline UN_xiong_6202 & 70 & $\mathrm{C} / \mathrm{T}$ \\
\hline UN_xiong_6202 & 329 & $\mathrm{~T} / \mathrm{A}$ \\
\hline UN_xiong_6210 & 83 & $\mathrm{~A} / \mathrm{C}$ \\
\hline UN_xiong_6210 & 444 & $\mathrm{G} / \mathrm{A}$ \\
\hline UN_xiong_6212 & 164 & $\mathrm{~A} / \mathrm{G}$ \\
\hline UN_xiong_6221 & 553 & $\mathrm{~T} / \mathrm{C}$ \\
\hline UN_xiong_6222 & 406 & $\mathrm{G} / \mathrm{T}$ \\
\hline UN_xiong_6222 & 724 & $\mathrm{C} / \mathrm{T}$ \\
\hline UN_xiong_6224 & 440 & $\mathrm{~T} / \mathrm{A}$ \\
\hline UN_xiong_6228 & 16 & $\mathrm{C} / \mathrm{A}$ \\
\hline UN_xiong_6228 & 64 & $\mathrm{C} / \mathrm{A}$ \\
\hline UN_xiong_6228 & 251 & $\mathrm{~T} / \mathrm{C}$ \\
\hline UN_xiong_6228 & 349 & $\mathrm{~T} / \mathrm{C}$ \\
\hline UN_xiong_6228 & 376 & $\mathrm{G} / \mathrm{A}$ \\
\hline UN_xiong_6228 & 405 & $\mathrm{~T} / \mathrm{C}$ \\
\hline UN_xiong_6228 & 431 & $\mathrm{G} / \mathrm{C}$ \\
\hline UN_xiong_6231 & 109 & $\mathrm{C} / \mathrm{G}$ \\
\hline UN_xiong_6251 & 325 & $\mathrm{~A} / \mathrm{G}$ \\
\hline UN_xiong_6251 & 426 & $\mathrm{C} / \mathrm{A}$ \\
\hline UN_xiong_6256 & 367 & $\mathrm{~A} / \mathrm{C}$ \\
\hline UN_xiong_6256 & 392 & $\mathrm{~T} / \mathrm{C}$ \\
\hline UN_xiong_6263 & 378 & $\mathrm{C} / \mathrm{A}$ \\
\hline UN_xiong_6263 & 630 & $\mathrm{~A} / \mathrm{T}$ \\
\hline UN_xiong_6267 & 254 & $\mathrm{C} / \mathrm{T}$ \\
\hline UN_xiong_6270 & 561 & $\mathrm{~T} / \mathrm{C}$ \\
\hline UN_xiong_6271 & 146 & $\mathrm{C} / \mathrm{T}$ \\
\hline UN_xiong_6271 & 176 & $\mathrm{~A} / \mathrm{G}$ \\
\hline UN_xiong_6271 & 414 & $\mathrm{~A} / \mathrm{T}$ \\
\hline UN_xiong_6271 & 461 & $\mathrm{G} / \mathrm{A}$ \\
\hline UN_xiong_6274 & 479 & $\mathrm{~A} / \mathrm{G}$ \\
\hline UN_xiong_6274 & 499 & $\mathrm{~T} / \mathrm{C}$ \\
\hline UN_xiong_6278 & 387 & $\mathrm{~T} / \mathrm{C}$ \\
\hline UN_xiong_6281 & 368 & $\mathrm{~T} / \mathrm{C}$ \\
\hline UN_xiong_6286 & 122 & $\mathrm{~T} / \mathrm{C}$ \\
\hline UN_xiong_6309 & 330 & $\mathrm{~T} / \mathrm{C}$ \\
\hline UN_xiong_6309 & 401 & $\mathrm{G} / \mathrm{A}$ \\
\hline UN_xiong_6309 & 557 & $\mathrm{G} / \mathrm{A}$ \\
\hline UN_xiong_6309 & 722 & $\mathrm{C} / \mathrm{T}$ \\
\hline UN_xiong_6309 & 776 & $\mathrm{C} / \mathrm{T}$ \\
\hline UN_xiong_6312 & 105 & $\mathrm{C} / \mathrm{A}$ \\
\hline UN_xiong_6321 & 202 & $\mathrm{~A} / \mathrm{G}$ \\
\hline UN_xiong_6334 & 208 & $\mathrm{~A} / \mathrm{T}$ \\
\hline UN_xiong_6334 & 261 & $\mathrm{C} / \mathrm{A}$ \\
\hline
\end{tabular}




\begin{tabular}{|c|c|c|}
\hline UN_xiong_6353 & 417 & $T / G$ \\
\hline UN_xiong_6384 & 69 & $\mathrm{G} / \mathrm{T}$ \\
\hline UN_xiong_6384 & 689 & $T / G$ \\
\hline UN_xiong_6401 & 93 & $G / A$ \\
\hline UN_xiong_6402 & 780 & $A / G$ \\
\hline UN_xiong_6402 & 830 & $C / A$ \\
\hline UN_xiong_6402 & 940 & $\mathrm{~T} / \mathrm{A}$ \\
\hline UN_xiong_6413 & 153 & $A / G$ \\
\hline UN_xiong_6413 & 348 & $\mathrm{~T} / \mathrm{C}$ \\
\hline UN_xiong_6414 & 187 & $\mathrm{~T} / \mathrm{C}$ \\
\hline UN_xiong_6418 & 301 & $\mathrm{C} / \mathrm{T}$ \\
\hline UN_xiong_6418 & 481 & $G / A$ \\
\hline UN_xiong_6419 & 212 & $\mathrm{~A} / \mathrm{T}$ \\
\hline UN_xiong_6422 & 28 & $\mathrm{C} / \mathrm{T}$ \\
\hline UN_xiong_6422 & 336 & $\mathrm{~T} / \mathrm{C}$ \\
\hline UN_xiong_6423 & 52 & $\mathrm{C} / \mathrm{T}$ \\
\hline UN_xiong_6423 & 340 & $A / G$ \\
\hline UN_xiong_6432 & 180 & $\mathrm{~A} / \mathrm{C}$ \\
\hline UN_xiong_6436 & 941 & $\mathrm{~T} / \mathrm{C}$ \\
\hline UN_xiong_6436 & 1017 & $\mathrm{~T} / \mathrm{C}$ \\
\hline UN_xiong_6444 & 247 & $C / T$ \\
\hline UN_xiong_6445 & 181 & $A / G$ \\
\hline UN_xiong_6452 & 406 & $\mathrm{~T} / \mathrm{C}$ \\
\hline UN_xiong_6452 & 506 & $A / G$ \\
\hline UN_xiong_6470 & 277 & $A / G$ \\
\hline UN_xiong_6475 & 131 & $A / C$ \\
\hline UN_xiong_6475 & 157 & $\mathrm{~A} / \mathrm{T}$ \\
\hline UN_xiong_6475 & 192 & $\mathrm{~T} / \mathrm{C}$ \\
\hline UN_xiong_6475 & 268 & $A / G$ \\
\hline UN_xiong_6477 & 62 & $\mathrm{~T} / \mathrm{C}$ \\
\hline UN_xiong_6477 & 100 & $\mathrm{~T} / \mathrm{C}$ \\
\hline UN_xiong_6477 & 185 & $T / G$ \\
\hline UN_xiong_6482 & 385 & $\mathrm{~T} / \mathrm{C}$ \\
\hline UN_xiong_6489 & 324 & $A / G$ \\
\hline UN_xiong_6493 & 13 & $A / G$ \\
\hline UN_xiong_6493 & 89 & $\mathrm{~T} / \mathrm{C}$ \\
\hline UN_xiong_6493 & 128 & $A / G$ \\
\hline UN_xiong_6497 & 364 & $\mathrm{~T} / \mathrm{C}$ \\
\hline UN_xiong_6498 & 202 & $\mathrm{G} / \mathrm{T}$ \\
\hline UN_xiong_6502 & 241 & $\mathrm{~T} / \mathrm{C}$ \\
\hline UN_xiong_6510 & 38 & $\mathrm{G} / \mathrm{T}$ \\
\hline UN_xiong_6510 & 167 & $A / G$ \\
\hline UN_xiong_6510 & 215 & $A / G$ \\
\hline UN_xiong_6515 & 340 & $G / A$ \\
\hline UN_xiong_6516 & 392 & $\mathrm{~T} / \mathrm{C}$ \\
\hline UN_xiong_6516 & 458 & $A / G$ \\
\hline UN_xiong_6516 & 538 & $A / C$ \\
\hline
\end{tabular}




\begin{tabular}{|c|c|c|}
\hline UN_xiong_6516 & 569 & $\mathrm{~T} / \mathrm{C}$ \\
\hline UN_xiong_6525 & 273 & $\mathrm{G} / \mathrm{A}$ \\
\hline UN_xiong_6540 & 47 & $\mathrm{~T} / \mathrm{C}$ \\
\hline UN_xiong_6540 & 175 & $A / G$ \\
\hline UN_xiong_6540 & 311 & $\mathrm{~A} / \mathrm{T}$ \\
\hline UN_xiong_6540 & 453 & $A / G$ \\
\hline UN_xiong_6548 & 62 & $\mathrm{~T} / \mathrm{C}$ \\
\hline UN_xiong_6551 & 288 & $\mathrm{G} / \mathrm{T}$ \\
\hline UN_xiong_6554 & 251 & $A / G$ \\
\hline UN_xiong_6554 & 304 & $\mathrm{G} / \mathrm{A}$ \\
\hline UN_xiong_6556 & 542 & $\mathrm{~T} / \mathrm{A}$ \\
\hline UN_xiong_6576 & 16 & $\mathrm{G} / \mathrm{C}$ \\
\hline UN_xiong_6584 & 171 & $\mathrm{~T} / \mathrm{C}$ \\
\hline UN_xiong_6584 & 303 & $\mathrm{~T} / \mathrm{C}$ \\
\hline UN_xiong_6589 & 558 & $\mathrm{G} / \mathrm{T}$ \\
\hline UN_xiong_6593 & 63 & $\mathrm{~T} / \mathrm{C}$ \\
\hline UN_xiong_6593 & 177 & $A / G$ \\
\hline UN_xiong_6596 & 319 & $A / G$ \\
\hline UN_xiong_6608 & 309 & $\mathrm{C} / \mathrm{G}$ \\
\hline UN_xiong_6616 & 41 & $\mathrm{C} / \mathrm{T}$ \\
\hline UN_xiong_6630 & 440 & $\mathrm{~T} / \mathrm{C}$ \\
\hline UN_xiong_6637 & 104 & $A / G$ \\
\hline UN_xiong_6657 & 227 & $\mathrm{G} / \mathrm{T}$ \\
\hline UN_xiong_6659 & 12 & $\mathrm{~T} / \mathrm{C}$ \\
\hline UN_xiong_6659 & 44 & $A / T$ \\
\hline UN_xiong_6659 & 170 & $G / A$ \\
\hline UN_xiong_6659 & 613 & $\mathrm{~T} / \mathrm{C}$ \\
\hline UN_xiong_6663 & 568 & $\mathrm{~T} / \mathrm{G}$ \\
\hline UN_xiong_6663 & 722 & $\mathrm{~T} / \mathrm{C}$ \\
\hline UN_xiong_6663 & 820 & $\mathrm{~T} / \mathrm{C}$ \\
\hline UN_xiong_6663 & 991 & $\mathrm{~T} / \mathrm{C}$ \\
\hline UN_xiong_6663 & 1056 & $\mathrm{~T} / \mathrm{C}$ \\
\hline UN_xiong_6663 & 1110 & $\mathrm{G} / \mathrm{A}$ \\
\hline UN_xiong_6668 & 94 & $\mathrm{C} / \mathrm{T}$ \\
\hline UN_xiong_6674 & 376 & $\mathrm{C} / \mathrm{T}$ \\
\hline UN_xiong_6674 & 689 & $\mathrm{C} / \mathrm{T}$ \\
\hline UN_xiong_6694 & 91 & $\mathrm{~T} / \mathrm{C}$ \\
\hline UN_xiong_6694 & 404 & $G / A$ \\
\hline UN_xiong_6694 & 451 & $\mathrm{~T} / \mathrm{A}$ \\
\hline UN_xiong_6722 & 358 & $A / G$ \\
\hline UN_xiong_6730 & 37 & $\mathrm{~T} / \mathrm{C}$ \\
\hline UN_xiong_6739 & 347 & $A / G$ \\
\hline UN_xiong_6740 & 513 & $A / G$ \\
\hline UN_xiong_6740 & 636 & $A / G$ \\
\hline UN_xiong_6748 & 264 & $A / G$ \\
\hline UN_xiong_6750 & 235 & $\mathrm{~T} / \mathrm{C}$ \\
\hline UN_xiong_6761 & 295 & $\mathrm{G} / \mathrm{A}$ \\
\hline
\end{tabular}




\begin{tabular}{|c|c|c|}
\hline UN_xiong_6761 & 492 & $\mathrm{~T} / \mathrm{G}$ \\
\hline UN_xiong_6769 & 173 & $A / G$ \\
\hline UN_xiong_6770 & 44 & $\mathrm{~T} / \mathrm{C}$ \\
\hline UN_xiong_6772 & 291 & $A / G$ \\
\hline UN_xiong_6778 & 460 & $A / G$ \\
\hline UN_xiong_6780 & 99 & $\mathrm{~T} / \mathrm{C}$ \\
\hline UN_xiong_6782 & 714 & $\mathrm{G} / \mathrm{C}$ \\
\hline UN_xiong_6787 & 172 & $\mathrm{G} / \mathrm{T}$ \\
\hline UN_xiong_6787 & 297 & $\mathrm{C} / \mathrm{T}$ \\
\hline UN_xiong_6791 & 103 & $A / G$ \\
\hline UN_xiong_6794 & 526 & $\mathrm{~T} / \mathrm{A}$ \\
\hline UN_xiong_6801 & 489 & $\mathrm{~A} / \mathrm{T}$ \\
\hline UN_xiong_6801 & 769 & $A / G$ \\
\hline UN_xiong_6801 & 795 & $A / G$ \\
\hline UN_xiong_6809 & 307 & $\mathrm{C} / \mathrm{T}$ \\
\hline UN_xiong_6820 & 237 & $G / A$ \\
\hline UN_xiong_6830 & 137 & $\mathrm{G} / \mathrm{T}$ \\
\hline UN_xiong_6830 & 212 & $A / G$ \\
\hline UN_xiong_6837 & 357 & $\mathrm{~A} / \mathrm{T}$ \\
\hline UN_xiong_6840 & 31 & $A / T$ \\
\hline UN_xiong_6849 & 479 & $\mathrm{C} / \mathrm{T}$ \\
\hline UN_xiong_6849 & 656 & $\mathrm{C} / \mathrm{T}$ \\
\hline UN_xiong_6849 & 682 & $\mathrm{C} / \mathrm{T}$ \\
\hline UN_xiong_6850 & 136 & $A / G$ \\
\hline UN_xiong_6860 & 210 & $C / A$ \\
\hline UN_xiong_6867 & 142 & $G / A$ \\
\hline UN_xiong_6871 & 6 & $A / G$ \\
\hline UN_xiong_6877 & 110 & $\mathrm{~T} / \mathrm{C}$ \\
\hline UN_xiong_6885 & 864 & $\mathrm{~T} / \mathrm{C}$ \\
\hline UN_xiong_6887 & 438 & $A / G$ \\
\hline UN_xiong_6891 & 216 & $A / G$ \\
\hline UN_xiong_6904 & 258 & $A / G$ \\
\hline UN_xiong_6907 & 12 & $A / G$ \\
\hline UN_xiong_6909 & 8 & $A / G$ \\
\hline UN_xiong_6909 & 179 & $\mathrm{~A} / \mathrm{C}$ \\
\hline UN_xiong_6909 & 339 & $A / G$ \\
\hline UN_xiong_6909 & 470 & $\mathrm{G} / \mathrm{A}$ \\
\hline UN_xiong_6909 & 500 & $\mathrm{G} / \mathrm{C}$ \\
\hline UN_xiong_6909 & 673 & $\mathrm{~A} / \mathrm{T}$ \\
\hline UN_xiong_6918 & 235 & $\mathrm{C} / \mathrm{T}$ \\
\hline UN_xiong_6918 & 286 & $\mathrm{~T} / \mathrm{C}$ \\
\hline UN_xiong_6918 & 311 & $\mathrm{~T} / \mathrm{C}$ \\
\hline UN_xiong_6920 & 6 & $\mathrm{G} / \mathrm{T}$ \\
\hline UN_xiong_6920 & 260 & $\mathrm{G} / \mathrm{T}$ \\
\hline UN_xiong_6920 & 494 & $\mathrm{~T} / \mathrm{C}$ \\
\hline UN_xiong_6920 & 729 & $\mathrm{~T} / \mathrm{C}$ \\
\hline UN_xiong_6920 & 769 & $\mathrm{~T} / \mathrm{C}$ \\
\hline
\end{tabular}




\begin{tabular}{|c|c|c|}
\hline UN_xiong_6936 & 14 & $\mathrm{~A} / \mathrm{G}$ \\
\hline UN_xiong_6938 & 34 & $\mathrm{C} / \mathrm{T}$ \\
\hline UN_xiong_6938 & 153 & $\mathrm{G} / \mathrm{A}$ \\
\hline UN_xiong_6938 & 245 & $\mathrm{~A} / \mathrm{C}$ \\
\hline UN_xiong_6938 & 739 & $A / G$ \\
\hline UN_xiong_6941 & 111 & $\mathrm{G} / \mathrm{A}$ \\
\hline UN_xiong_6946 & 413 & $\mathrm{C} / \mathrm{G}$ \\
\hline UN_xiong_6955 & 314 & $\mathrm{G} / \mathrm{A}$ \\
\hline UN_xiong_6964 & 89 & $\mathrm{~T} / \mathrm{C}$ \\
\hline UN_xiong_6964 & 231 & $\mathrm{~T} / \mathrm{C}$ \\
\hline UN_xiong_6964 & 344 & $\mathrm{~T} / \mathrm{C}$ \\
\hline UN_xiong_6964 & 452 & $\mathrm{~A} / \mathrm{G}$ \\
\hline UN_xiong_6964 & 502 & $A / G$ \\
\hline UN_xiong_6971 & 8 & $\mathrm{~T} / \mathrm{C}$ \\
\hline UN_xiong_6978 & 6 & $\mathrm{G} / \mathrm{A}$ \\
\hline UN_xiong_6978 & 115 & $A / G$ \\
\hline UN_xiong_6978 & 141 & $\mathrm{G} / \mathrm{T}$ \\
\hline UN_xiong_6978 & 254 & $\mathrm{~T} / \mathrm{C}$ \\
\hline UN_xiong_6978 & 566 & $\mathrm{C} / \mathrm{T}$ \\
\hline UN_xiong_7002 & 262 & $A / G$ \\
\hline UN_xiong_7002 & 494 & $G / A$ \\
\hline UN_xiong_7004 & 214 & $\mathrm{G} / \mathrm{A}$ \\
\hline UN_xiong_7004 & 414 & $\mathrm{~T} / \mathrm{C}$ \\
\hline UN_xiong_7011 & 207 & $\mathrm{C} / \mathrm{T}$ \\
\hline UN_xiong_7021 & 323 & $A / G$ \\
\hline UN_xiong_7030 & 288 & $\mathrm{G} / \mathrm{A}$ \\
\hline UN_xiong_7042 & 195 & $G / A$ \\
\hline UN_xiong_7043 & 27 & $\mathrm{G} / \mathrm{T}$ \\
\hline UN_xiong_7043 & 86 & $G / A$ \\
\hline UN_xiong_7043 & 113 & $\mathrm{C} / \mathrm{T}$ \\
\hline UN_xiong_7043 & 203 & $\mathrm{G} / \mathrm{A}$ \\
\hline UN_xiong_7051 & 439 & $\mathrm{C} / \mathrm{T}$ \\
\hline UN_xiong_7069 & 154 & $\mathrm{G} / \mathrm{A}$ \\
\hline UN_xiong_7083 & 113 & $\mathrm{G} / \mathrm{A}$ \\
\hline UN_xiong_7095 & 351 & $\mathrm{G} / \mathrm{T}$ \\
\hline UN_xiong_7097 & 12 & $\mathrm{C} / \mathrm{T}$ \\
\hline UN_xiong_7097 & 257 & $\mathrm{G} / \mathrm{T}$ \\
\hline UN_xiong_7097 & 320 & $\mathrm{C} / \mathrm{T}$ \\
\hline UN_xiong_7097 & 379 & $\mathrm{C} / \mathrm{T}$ \\
\hline UN_xiong_7097 & 474 & $\mathrm{~T} / \mathrm{C}$ \\
\hline UN_xiong_7099 & 234 & $\mathrm{G} / \mathrm{T}$ \\
\hline UN_xiong_7113 & 48 & $\mathrm{C} / \mathrm{T}$ \\
\hline UN_xiong_7113 & 208 & $\mathrm{~A} / \mathrm{C}$ \\
\hline UN_xiong_7113 & 713 & $\mathrm{~A} / \mathrm{G}$ \\
\hline UN_xiong_7124 & 86 & $\mathrm{G} / \mathrm{A}$ \\
\hline UN_xiong_7124 & 135 & $\mathrm{C} / \mathrm{T}$ \\
\hline UN_xiong_7124 & 444 & $\mathrm{~A} / \mathrm{G}$ \\
\hline
\end{tabular}




\begin{tabular}{|c|c|c|}
\hline UN_xiong_7133 & 310 & $\mathrm{~A} / \mathrm{C}$ \\
\hline UN_xiong_7133 & 879 & $\mathrm{~T} / \mathrm{C}$ \\
\hline UN_xiong_7133 & 913 & $\mathrm{~A} / \mathrm{T}$ \\
\hline UN_xiong_7135 & 464 & $\mathrm{G} / \mathrm{A}$ \\
\hline UN_xiong_7135 & 561 & $\mathrm{~T} / \mathrm{C}$ \\
\hline UN_xiong_7138 & 139 & $\mathrm{C} / \mathrm{T}$ \\
\hline UN_xiong_7138 & 408 & $\mathrm{~T} / \mathrm{G}$ \\
\hline UN_xiong_7150 & 385 & $\mathrm{~T} / \mathrm{C}$ \\
\hline UN_xiong_7163 & 195 & $A / G$ \\
\hline UN_xiong_7164 & 317 & $\mathrm{~A} / \mathrm{G}$ \\
\hline UN_xiong_7164 & 727 & $\mathrm{C} / \mathrm{A}$ \\
\hline UN_xiong_7164 & 967 & $\mathrm{~T} / \mathrm{C}$ \\
\hline UN_xiong_7183 & 43 & $\mathrm{G} / \mathrm{T}$ \\
\hline UN_xiong_7183 & 309 & $\mathrm{C} / \mathrm{T}$ \\
\hline UN_xiong_7183 & 629 & $\mathrm{~T} / \mathrm{C}$ \\
\hline UN_xiong_7183 & 1030 & $A / G$ \\
\hline UN_xiong_7202 & 309 & $\mathrm{~A} / \mathrm{C}$ \\
\hline UN_xiong_7203 & 837 & $\mathrm{~A} / \mathrm{G}$ \\
\hline UN_xiong_7203 & 876 & $\mathrm{~A} / \mathrm{G}$ \\
\hline UN_xiong_7203 & 899 & $\mathrm{~A} / \mathrm{T}$ \\
\hline UN_xiong_7203 & 923 & $\mathrm{C} / \mathrm{A}$ \\
\hline UN_xiong_7203 & 943 & $\mathrm{~T} / \mathrm{C}$ \\
\hline UN_xiong_7206 & 428 & $\mathrm{C} / \mathrm{T}$ \\
\hline UN_xiong_7228 & 569 & $\mathrm{~A} / \mathrm{C}$ \\
\hline UN_xiong_7228 & 686 & $\mathrm{G} / \mathrm{A}$ \\
\hline UN_xiong_7231 & 467 & $A / G$ \\
\hline UN_xiong_7232 & 7 & $\mathrm{G} / \mathrm{A}$ \\
\hline UN_xiong_7232 & 132 & $\mathrm{G} / \mathrm{A}$ \\
\hline UN_xiong_7234 & 277 & $\mathrm{G} / \mathrm{A}$ \\
\hline UN_xiong_7234 & 298 & $\mathrm{~T} / \mathrm{C}$ \\
\hline UN_xiong_7234 & 408 & $\mathrm{G} / \mathrm{T}$ \\
\hline UN_xiong_7234 & 504 & $\mathrm{G} / \mathrm{A}$ \\
\hline UN_xiong_7236 & 194 & $\mathrm{~T} / \mathrm{C}$ \\
\hline UN_xiong_7251 & 103 & $\mathrm{~T} / \mathrm{C}$ \\
\hline UN_xiong_7252 & 441 & $\mathrm{~T} / \mathrm{C}$ \\
\hline UN_xiong_7252 & 490 & $\mathrm{~A} / \mathrm{C}$ \\
\hline UN_xiong_7263 & 712 & $\mathrm{~A} / \mathrm{C}$ \\
\hline UN_xiong_7280 & 423 & $\mathrm{~A} / \mathrm{G}$ \\
\hline UN_xiong_7284 & 404 & $\mathrm{~A} / \mathrm{G}$ \\
\hline UN_xiong_7291 & 156 & $\mathrm{~T} / \mathrm{C}$ \\
\hline UN_xiong_7292 & 253 & $\mathrm{~A} / \mathrm{G}$ \\
\hline UN_xiong_7299 & 134 & $\mathrm{G} / \mathrm{A}$ \\
\hline UN_xiong_7300 & 236 & $\mathrm{~A} / \mathrm{G}$ \\
\hline UN_xiong_7301 & 31 & $\mathrm{C} / \mathrm{T}$ \\
\hline UN_xiong_7303 & 183 & $A / G$ \\
\hline UN_xiong_7304 & 381 & $A / G$ \\
\hline UN_xiong_7326 & 143 & $\mathrm{G} / \mathrm{A}$ \\
\hline
\end{tabular}




\begin{tabular}{|c|c|c|}
\hline UN_xiong_7326 & 243 & $\mathrm{G} / \mathrm{A}$ \\
\hline UN_xiong_7327 & 250 & $\mathrm{C} / \mathrm{A}$ \\
\hline UN_xiong_7328 & 328 & $\mathrm{~T} / \mathrm{C}$ \\
\hline UN_xiong_7330 & 162 & $\mathrm{G} / \mathrm{A}$ \\
\hline UN_xiong_7332 & 104 & $\mathrm{~A} / \mathrm{C}$ \\
\hline UN_xiong_7358 & 24 & $\mathrm{C} / \mathrm{G}$ \\
\hline UN_xiong_7358 & 254 & $\mathrm{~T} / \mathrm{C}$ \\
\hline UN_xiong_7373 & 431 & $\mathrm{~A} / \mathrm{C}$ \\
\hline UN_xiong_7378 & 150 & $\mathrm{C} / \mathrm{A}$ \\
\hline UN_xiong_7378 & 236 & $\mathrm{~T} / \mathrm{C}$ \\
\hline UN_xiong_7378 & 290 & $\mathrm{C} / \mathrm{T}$ \\
\hline UN_xiong_7378 & 522 & $\mathrm{G} / \mathrm{A}$ \\
\hline UN_xiong_7378 & 699 & $\mathrm{G} / \mathrm{A}$ \\
\hline UN_xiong_7384 & 340 & $\mathrm{~T} / \mathrm{C}$ \\
\hline UN_xiong_7386 & 760 & $\mathrm{~A} / \mathrm{C}$ \\
\hline UN_xiong_7392 & 234 & $A / G$ \\
\hline UN_xiong_7392 & 264 & $\mathrm{~T} / \mathrm{G}$ \\
\hline UN_xiong_7398 & 560 & $\mathrm{C} / \mathrm{T}$ \\
\hline UN_xiong_7413 & 907 & $\mathrm{G} / \mathrm{A}$ \\
\hline UN_xiong_7442 & 69 & $\mathrm{G} / \mathrm{A}$ \\
\hline UN_xiong_7447 & 265 & $\mathrm{~T} / \mathrm{A}$ \\
\hline UN_xiong_7464 & 377 & $\mathrm{~T} / \mathrm{G}$ \\
\hline UN_xiong_7465 & 137 & $\mathrm{~T} / \mathrm{A}$ \\
\hline UN_xiong_7465 & 309 & $\mathrm{~T} / \mathrm{G}$ \\
\hline UN_xiong_7465 & 688 & $\mathrm{G} / \mathrm{A}$ \\
\hline UN_xiong_7471 & 336 & $\mathrm{~T} / \mathrm{A}$ \\
\hline UN_xiong_7472 & 434 & $A / G$ \\
\hline UN_xiong_7474 & 186 & $\mathrm{G} / \mathrm{A}$ \\
\hline UN_xiong_7476 & 7 & $\mathrm{~T} / \mathrm{C}$ \\
\hline UN_xiong_7491 & 321 & $A / G$ \\
\hline UN_xiong_7495 & 33 & $\mathrm{G} / \mathrm{A}$ \\
\hline UN_xiong_7495 & 194 & $A / G$ \\
\hline UN_xiong_7498 & 372 & $\mathrm{~T} / \mathrm{C}$ \\
\hline UN_xiong_7498 & 750 & $\mathrm{~A} / \mathrm{C}$ \\
\hline UN_xiong_7498 & 777 & $\mathrm{G} / \mathrm{C}$ \\
\hline UN_xiong_7505 & 71 & $A / G$ \\
\hline UN_xiong_7505 & 117 & $A / G$ \\
\hline UN_xiong_7511 & 103 & $\mathrm{~A} / \mathrm{G}$ \\
\hline UN_xiong_7515 & 556 & $A / G$ \\
\hline UN_xiong_7515 & 646 & $\mathrm{~T} / \mathrm{C}$ \\
\hline UN_xiong_7517 & 163 & $\mathrm{G} / \mathrm{A}$ \\
\hline UN_xiong_7517 & 255 & $A / G$ \\
\hline UN_xiong_7527 & 80 & $\mathrm{C} / \mathrm{A}$ \\
\hline UN_xiong_7527 & 207 & $\mathrm{~T} / \mathrm{C}$ \\
\hline UN_xiong_7527 & 715 & $\mathrm{~A} / \mathrm{C}$ \\
\hline UN_xiong_7527 & 771 & $\mathrm{~A} / \mathrm{G}$ \\
\hline UN_xiong_7527 & 1229 & $\mathrm{~A} / \mathrm{T}$ \\
\hline
\end{tabular}




\begin{tabular}{|c|c|c|}
\hline UN_xiong_7529 & 311 & $\mathrm{G} / \mathrm{A}$ \\
\hline UN_xiong_7529 & 409 & $\mathrm{~T} / \mathrm{C}$ \\
\hline UN_xiong_7529 & 479 & $\mathrm{~T} / \mathrm{C}$ \\
\hline UN_xiong_7529 & 756 & $\mathrm{~T} / \mathrm{C}$ \\
\hline UN_xiong_7529 & 956 & $\mathrm{~T} / \mathrm{C}$ \\
\hline UN_xiong_7529 & 987 & $\mathrm{G} / \mathrm{T}$ \\
\hline UN_xiong_7529 & 1055 & $\mathrm{~T} / \mathrm{C}$ \\
\hline UN_xiong_7529 & 1125 & $\mathrm{~A} / \mathrm{G}$ \\
\hline UN_xiong_7529 & 1250 & $\mathrm{~T} / \mathrm{C}$ \\
\hline UN_xiong_7552 & 279 & $\mathrm{C} / \mathrm{G}$ \\
\hline UN_xiong_7559 & 642 & $\mathrm{C} / \mathrm{T}$ \\
\hline UN_xiong_7560 & 268 & $\mathrm{C} / \mathrm{T}$ \\
\hline UN_xiong_7561 & 306 & $A / G$ \\
\hline UN_xiong_7565 & 9 & $\mathrm{~A} / \mathrm{C}$ \\
\hline UN_xiong_7565 & 114 & $\mathrm{G} / \mathrm{A}$ \\
\hline UN_xiong_7565 & 168 & $\mathrm{~T} / \mathrm{C}$ \\
\hline UN_xiong_7565 & 226 & $\mathrm{G} / \mathrm{T}$ \\
\hline UN_xiong_7566 & 106 & $\mathrm{C} / \mathrm{T}$ \\
\hline UN_xiong_7596 & 284 & $\mathrm{G} / \mathrm{A}$ \\
\hline UN_xiong_7596 & 591 & $A / G$ \\
\hline UN_xiong_7596 & 615 & $\mathrm{~T} / \mathrm{A}$ \\
\hline UN_xiong_7599 & 84 & $\mathrm{G} / \mathrm{A}$ \\
\hline UN_xiong_7619 & 222 & $\mathrm{~T} / \mathrm{C}$ \\
\hline UN_xiong_7627 & 171 & $\mathrm{~A} / \mathrm{G}$ \\
\hline UN_xiong_7659 & 136 & $\mathrm{G} / \mathrm{A}$ \\
\hline UN_xiong_7659 & 223 & $\mathrm{~A} / \mathrm{C}$ \\
\hline UN_xiong_7665 & 362 & $\mathrm{G} / \mathrm{A}$ \\
\hline UN_xiong_7665 & 524 & $\mathrm{G} / \mathrm{C}$ \\
\hline UN_xiong_7665 & 718 & $\mathrm{G} / \mathrm{A}$ \\
\hline UN_xiong_7665 & 960 & $\mathrm{~T} / \mathrm{G}$ \\
\hline UN_xiong_7665 & 1004 & $\mathrm{G} / \mathrm{A}$ \\
\hline UN_xiong_7670 & 43 & $\mathrm{C} / \mathrm{T}$ \\
\hline UN_xiong_7677 & 267 & $\mathrm{~A} / \mathrm{C}$ \\
\hline UN_xiong_7682 & 530 & $\mathrm{~T} / \mathrm{C}$ \\
\hline UN_xiong_7682 & 561 & $\mathrm{~T} / \mathrm{G}$ \\
\hline UN_xiong_7685 & 536 & $\mathrm{C} / \mathrm{T}$ \\
\hline UN_xiong_7694 & 103 & $\mathrm{~A} / \mathrm{T}$ \\
\hline UN_xiong_7694 & 301 & $\mathrm{G} / \mathrm{A}$ \\
\hline UN_xiong_7698 & 223 & $\mathrm{~T} / \mathrm{C}$ \\
\hline UN_xiong_7701 & 266 & $\mathrm{G} / \mathrm{A}$ \\
\hline UN_xiong_7717 & 245 & $\mathrm{G} / \mathrm{A}$ \\
\hline UN_xiong_7743 & 321 & $\mathrm{G} / \mathrm{A}$ \\
\hline UN_xiong_7746 & 170 & $\mathrm{C} / \mathrm{T}$ \\
\hline UN_xiong_7770 & 173 & $\mathrm{C} / \mathrm{T}$ \\
\hline UN_xiong_7784 & 266 & $\mathrm{C} / \mathrm{T}$ \\
\hline UN_xiong_7784 & 324 & $\mathrm{~T} / \mathrm{A}$ \\
\hline UN_xiong_7786 & 71 & $\mathrm{~T} / \mathrm{G}$ \\
\hline
\end{tabular}




\begin{tabular}{|c|c|c|}
\hline UN_xiong_7791 & 232 & $\mathrm{G} / \mathrm{A}$ \\
\hline UN_xiong_7794 & 266 & $\mathrm{~T} / \mathrm{C}$ \\
\hline UN_xiong_7794 & 316 & $\mathrm{~T} / \mathrm{C}$ \\
\hline UN_xiong_7794 & 360 & $\mathrm{~T} / \mathrm{C}$ \\
\hline UN_xiong_7799 & 326 & $\mathrm{~T} / \mathrm{C}$ \\
\hline UN_xiong_7799 & 404 & $\mathrm{C} / \mathrm{T}$ \\
\hline UN_xiong_7799 & 541 & $\mathrm{~T} / \mathrm{C}$ \\
\hline UN_xiong_7802 & 109 & $C / A$ \\
\hline UN_xiong_7823 & 69 & $\mathrm{C} / \mathrm{T}$ \\
\hline UN_xiong_7832 & 412 & $A / G$ \\
\hline UN_xiong_7844 & 259 & $\mathrm{C} / \mathrm{T}$ \\
\hline UN_xiong_7851 & 275 & $\mathrm{C} / \mathrm{T}$ \\
\hline UN_xiong_7854 & 312 & $\mathrm{G} / \mathrm{T}$ \\
\hline UN_xiong_7854 & 333 & $\mathrm{G} / \mathrm{A}$ \\
\hline UN_xiong_7854 & 360 & $A / G$ \\
\hline UN_xiong_7872 & 103 & $\mathrm{C} / \mathrm{T}$ \\
\hline UN_xiong_7875 & 386 & $\mathrm{~A} / \mathrm{T}$ \\
\hline UN_xiong_7899 & 1467 & $C / A$ \\
\hline UN_xiong_7902 & 236 & $\mathrm{C} / \mathrm{G}$ \\
\hline UN_xiong_7922 & 151 & $\mathrm{G} / \mathrm{A}$ \\
\hline UN_xiong_7922 & 422 & $\mathrm{G} / \mathrm{A}$ \\
\hline UN_xiong_7936 & 374 & $\mathrm{G} / \mathrm{A}$ \\
\hline UN_xiong_7949 & 165 & $\mathrm{~T} / \mathrm{C}$ \\
\hline UN_xiong_7950 & 188 & $A / G$ \\
\hline UN_xiong_7953 & 76 & $\mathrm{G} / \mathrm{T}$ \\
\hline UN_xiong_7974 & 60 & $\mathrm{~T} / \mathrm{G}$ \\
\hline UN_xiong_7988 & 920 & $A / G$ \\
\hline UN_xiong_7994 & 563 & $\mathrm{~T} / \mathrm{C}$ \\
\hline UN_xiong_8025 & 89 & $C / G$ \\
\hline UN_xiong_8036 & 377 & $\mathrm{G} / \mathrm{A}$ \\
\hline UN_xiong_8036 & 538 & $\mathrm{C} / \mathrm{T}$ \\
\hline UN_xiong_8037 & 196 & $A / G$ \\
\hline UN_xiong_8049 & 446 & $\mathrm{~T} / \mathrm{C}$ \\
\hline UN_xiong_8049 & 571 & $A / G$ \\
\hline UN_xiong_8049 & 977 & $\mathrm{G} / \mathrm{A}$ \\
\hline UN_xiong_8049 & 1015 & $\mathrm{G} / \mathrm{T}$ \\
\hline UN_xiong_8062 & 53 & $\mathrm{C} / \mathrm{T}$ \\
\hline UN_xiong_8065 & 154 & $\mathrm{C} / \mathrm{T}$ \\
\hline UN_xiong_8065 & 207 & $\mathrm{C} / \mathrm{T}$ \\
\hline UN_xiong_8065 & 537 & $\mathrm{C} / \mathrm{T}$ \\
\hline UN_xiong_8065 & 609 & $\mathrm{~T} / \mathrm{C}$ \\
\hline UN_xiong_8067 & 283 & $A / G$ \\
\hline UN_xiong_8067 & 333 & $C / A$ \\
\hline UN_xiong_8071 & 32 & $\mathrm{~T} / \mathrm{C}$ \\
\hline UN_xiong_8071 & 131 & $A / G$ \\
\hline UN_xiong_8071 & 923 & $\mathrm{C} / \mathrm{T}$ \\
\hline UN_xiong_8074 & 219 & $\mathrm{C} / \mathrm{T}$ \\
\hline
\end{tabular}




\begin{tabular}{|c|c|c|}
\hline UN_xiong_8081 & 290 & $\mathrm{~A} / \mathrm{T}$ \\
\hline UN_xiong_8082 & 255 & $\mathrm{G} / \mathrm{A}$ \\
\hline UN_xiong_8087 & 56 & $\mathrm{~T} / \mathrm{C}$ \\
\hline UN_xiong_8096 & 333 & $\mathrm{~T} / \mathrm{G}$ \\
\hline UN_xiong_8096 & 406 & $A / G$ \\
\hline UN_xiong_8100 & 241 & $\mathrm{~A} / \mathrm{G}$ \\
\hline UN_xiong_8100 & 384 & $\mathrm{G} / \mathrm{A}$ \\
\hline UN_xiong_8113 & 337 & $\mathrm{G} / \mathrm{A}$ \\
\hline UN_xiong_8115 & 134 & $\mathrm{C} / \mathrm{T}$ \\
\hline UN_xiong_8117 & 128 & $\mathrm{G} / \mathrm{A}$ \\
\hline UN_xiong_8117 & 695 & $\mathrm{~A} / \mathrm{G}$ \\
\hline UN_xiong_8117 & 777 & $\mathrm{C} / \mathrm{T}$ \\
\hline UN_xiong_8119 & 253 & $\mathrm{C} / \mathrm{T}$ \\
\hline UN_xiong_8119 & 275 & $C / A$ \\
\hline UN_xiong_8120 & 48 & $\mathrm{G} / \mathrm{A}$ \\
\hline UN_xiong_8120 & 190 & $\mathrm{G} / \mathrm{T}$ \\
\hline UN_xiong_8122 & 241 & $\mathrm{~A} / \mathrm{T}$ \\
\hline UN_xiong_8122 & 415 & $\mathrm{~A} / \mathrm{T}$ \\
\hline UN_xiong_8122 & 754 & $\mathrm{~T} / \mathrm{C}$ \\
\hline UN_xiong_8122 & 775 & $A / G$ \\
\hline UN_xiong_8122 & 811 & $\mathrm{G} / \mathrm{A}$ \\
\hline UN_xiong_8122 & 1347 & $\mathrm{~A} / \mathrm{G}$ \\
\hline UN_xiong_8125 & 15 & $\mathrm{G} / \mathrm{T}$ \\
\hline UN_xiong_8129 & 279 & $\mathrm{G} / \mathrm{A}$ \\
\hline UN_xiong_8130 & 431 & $\mathrm{G} / \mathrm{A}$ \\
\hline UN_xiong_8136 & 482 & $\mathrm{C} / \mathrm{T}$ \\
\hline UN_xiong_8140 & 349 & $C / A$ \\
\hline UN_xiong_8143 & 305 & $\mathrm{C} / \mathrm{T}$ \\
\hline UN_xiong_8153 & 177 & $\mathrm{C} / \mathrm{T}$ \\
\hline UN_xiong_8153 & 246 & $\mathrm{G} / \mathrm{A}$ \\
\hline UN_xiong_8154 & 126 & $\mathrm{~T} / \mathrm{C}$ \\
\hline UN_xiong_8154 & 148 & $\mathrm{~T} / \mathrm{C}$ \\
\hline UN_xiong_8158 & 199 & $\mathrm{G} / \mathrm{T}$ \\
\hline UN_xiong_8159 & 879 & $\mathrm{~T} / \mathrm{C}$ \\
\hline UN_xiong_8159 & 954 & $\mathrm{C} / \mathrm{T}$ \\
\hline UN_xiong_8168 & 166 & $A / G$ \\
\hline UN_xiong_8168 & 222 & $\mathrm{~T} / \mathrm{C}$ \\
\hline UN_xiong_8172 & 257 & $\mathrm{G} / \mathrm{A}$ \\
\hline UN_xiong_8182 & 144 & $\mathrm{C} / \mathrm{T}$ \\
\hline UN_xiong_8182 & 217 & $\mathrm{G} / \mathrm{T}$ \\
\hline UN_xiong_8184 & 458 & $\mathrm{C} / \mathrm{A}$ \\
\hline UN_xiong_8184 & 502 & $\mathrm{~T} / \mathrm{C}$ \\
\hline UN_xiong_8199 & 130 & $\mathrm{~T} / \mathrm{A}$ \\
\hline UN_xiong_8203 & 607 & $A / G$ \\
\hline UN_xiong_8215 & 170 & $\mathrm{C} / \mathrm{G}$ \\
\hline UN_xiong_8223 & 258 & $A / G$ \\
\hline UN_xiong_8233 & 62 & $\mathrm{~T} / \mathrm{C}$ \\
\hline
\end{tabular}




\begin{tabular}{|c|c|c|}
\hline UN_xiong_8241 & 41 & $\mathrm{~T} / \mathrm{C}$ \\
\hline UN_xiong_8252 & 228 & $\mathrm{~T} / \mathrm{C}$ \\
\hline UN_xiong_8253 & 52 & $\mathrm{G} / \mathrm{A}$ \\
\hline UN_xiong_8253 & 207 & $\mathrm{~T} / \mathrm{C}$ \\
\hline UN_xiong_8253 & 445 & $\mathrm{C} / \mathrm{T}$ \\
\hline UN_xiong_8253 & 469 & $\mathrm{~T} / \mathrm{G}$ \\
\hline UN_xiong_8256 & 68 & $\mathrm{C} / \mathrm{T}$ \\
\hline UN_xiong_8256 & 144 & $\mathrm{~T} / \mathrm{C}$ \\
\hline UN_xiong_8256 & 271 & $\mathrm{~T} / \mathrm{C}$ \\
\hline UN_xiong_8256 & 630 & $\mathrm{G} / \mathrm{C}$ \\
\hline UN_xiong_8256 & 707 & $\mathrm{~T} / \mathrm{C}$ \\
\hline UN_xiong_8256 & 807 & $\mathrm{~T} / \mathrm{C}$ \\
\hline UN_xiong_8271 & 165 & $\mathrm{~A} / \mathrm{T}$ \\
\hline UN_xiong_8271 & 269 & $\mathrm{~T} / \mathrm{C}$ \\
\hline UN_xiong_8286 & 39 & $\mathrm{C} / \mathrm{T}$ \\
\hline UN_xiong_8286 & 278 & $A / G$ \\
\hline UN_xiong_8287 & 72 & $\mathrm{G} / \mathrm{A}$ \\
\hline UN_xiong_8291 & 14 & $\mathrm{~T} / \mathrm{C}$ \\
\hline UN_xiong_8291 & 158 & $\mathrm{G} / \mathrm{A}$ \\
\hline UN_xiong_8291 & 211 & $A / G$ \\
\hline UN_xiong_8295 & 132 & $\mathrm{C} / \mathrm{T}$ \\
\hline UN_xiong_8295 & 268 & $A / G$ \\
\hline UN_xiong_8296 & 43 & $\mathrm{~A} / \mathrm{T}$ \\
\hline UN_xiong_8296 & 785 & $\mathrm{G} / \mathrm{T}$ \\
\hline UN_xiong_8299 & 291 & $\mathrm{~T} / \mathrm{C}$ \\
\hline UN_xiong_8299 & 331 & $\mathrm{~T} / \mathrm{C}$ \\
\hline UN_xiong_8307 & 179 & $A / G$ \\
\hline UN_xiong_8307 & 347 & $\mathrm{G} / \mathrm{A}$ \\
\hline UN_xiong_8315 & 136 & $\mathrm{G} / \mathrm{T}$ \\
\hline UN_xiong_8329 & 347 & $C / A$ \\
\hline UN_xiong_8333 & 243 & $A / G$ \\
\hline UN_xiong_8339 & 193 & $\mathrm{G} / \mathrm{A}$ \\
\hline UN_xiong_8340 & 241 & $\mathrm{C} / \mathrm{T}$ \\
\hline UN_xiong_8344 & 77 & $\mathrm{~A} / \mathrm{G}$ \\
\hline UN_xiong_8345 & 107 & $\mathrm{G} / \mathrm{T}$ \\
\hline UN_xiong_8345 & 133 & $A / G$ \\
\hline UN_xiong_8348 & 70 & $\mathrm{C} / \mathrm{T}$ \\
\hline UN_xiong_8348 & 90 & $C / G$ \\
\hline UN_xiong_8348 & 150 & $\mathrm{~T} / \mathrm{G}$ \\
\hline UN_xiong_8348 & 189 & $\mathrm{C} / \mathrm{T}$ \\
\hline UN_xiong_8348 & 322 & $\mathrm{~A} / \mathrm{C}$ \\
\hline UN_xiong_8348 & 360 & $A / G$ \\
\hline UN_xiong_8348 & 497 & $\mathrm{~A} / \mathrm{T}$ \\
\hline UN_xiong_8358 & 404 & $\mathrm{~A} / \mathrm{G}$ \\
\hline UN_xiong_8358 & 513 & $\mathrm{~T} / \mathrm{C}$ \\
\hline UN_xiong_8358 & 693 & $\mathrm{~A} / \mathrm{T}$ \\
\hline UN_xiong_8375 & 85 & $\mathrm{C} / \mathrm{T}$ \\
\hline
\end{tabular}




\begin{tabular}{|c|c|c|}
\hline UN_xiong_8375 & 211 & $\mathrm{~T} / \mathrm{C}$ \\
\hline UN_xiong_8376 & 205 & $A / G$ \\
\hline UN_xiong_8389 & 104 & $A / G$ \\
\hline UN_xiong_8390 & 232 & $A / G$ \\
\hline UN_xiong_8390 & 276 & $G / A$ \\
\hline UN_xiong_8390 & 312 & $\mathrm{G} / \mathrm{T}$ \\
\hline UN_xiong_8405 & 260 & $\mathrm{~T} / \mathrm{C}$ \\
\hline UN_xiong_8411 & 203 & $\mathrm{G} / \mathrm{C}$ \\
\hline UN_xiong_8411 & 247 & $\mathrm{~T} / \mathrm{A}$ \\
\hline UN_xiong_8411 & 347 & $\mathrm{G} / \mathrm{A}$ \\
\hline UN_xiong_8413 & 261 & $\mathrm{C} / \mathrm{T}$ \\
\hline UN_xiong_8417 & 314 & $\mathrm{C} / \mathrm{T}$ \\
\hline UN_xiong_8419 & 493 & $\mathrm{~T} / \mathrm{C}$ \\
\hline UN_xiong_8452 & 94 & $\mathrm{C} / \mathrm{T}$ \\
\hline UN_xiong_8452 & 624 & $A / G$ \\
\hline UN_xiong_8452 & 736 & $\mathrm{~T} / \mathrm{C}$ \\
\hline UN_xiong_8452 & 807 & $G / A$ \\
\hline UN_xiong_8452 & 846 & $\mathrm{G} / \mathrm{A}$ \\
\hline UN_xiong_8462 & 589 & $\mathrm{C} / \mathrm{T}$ \\
\hline UN_xiong_8466 & 257 & $T / G$ \\
\hline UN_xiong_8469 & 281 & $\mathrm{~T} / \mathrm{C}$ \\
\hline UN_xiong_8479 & 120 & $\mathrm{G} / \mathrm{C}$ \\
\hline UN_xiong_8479 & 168 & $C / G$ \\
\hline UN_xiong_8480 & 340 & $\mathrm{~T} / \mathrm{C}$ \\
\hline UN_xiong_8480 & 481 & $\mathrm{~T} / \mathrm{C}$ \\
\hline UN_xiong_8480 & 543 & $A / G$ \\
\hline UN_xiong_8480 & 579 & $A / G$ \\
\hline UN_xiong_8483 & 300 & $\mathrm{~A} / \mathrm{T}$ \\
\hline UN_xiong_8493 & 51 & $\mathrm{~T} / \mathrm{C}$ \\
\hline UN_xiong_8519 & 284 & $A / G$ \\
\hline UN_xiong_8519 & 329 & $\mathrm{G} / \mathrm{C}$ \\
\hline UN_xiong_8519 & 382 & $A / G$ \\
\hline UN_xiong_8520 & 221 & $A / G$ \\
\hline UN_xiong_8520 & 284 & $A / G$ \\
\hline UN_xiong_8520 & 407 & $\mathrm{G} / \mathrm{A}$ \\
\hline UN_xiong_8521 & 38 & $C / A$ \\
\hline UN_xiong_8521 & 100 & $\mathrm{~A} / \mathrm{T}$ \\
\hline UN_xiong_8523 & 291 & $\mathrm{~T} / \mathrm{A}$ \\
\hline UN_xiong_8530 & 288 & $\mathrm{~T} / \mathrm{C}$ \\
\hline UN_xiong_8530 & 325 & $\mathrm{~T} / \mathrm{C}$ \\
\hline UN_xiong_8530 & 465 & $A / G$ \\
\hline UN_xiong_8530 & 588 & $A / G$ \\
\hline UN_xiong_8530 & 727 & $\mathrm{C} / \mathrm{T}$ \\
\hline UN_xiong_8530 & 826 & $\mathrm{~T} / \mathrm{C}$ \\
\hline UN_xiong_8530 & 861 & $\mathrm{G} / \mathrm{A}$ \\
\hline UN_xiong_8536 & 173 & $\mathrm{~T} / \mathrm{C}$ \\
\hline UN_xiong_8536 & 556 & $A / T$ \\
\hline
\end{tabular}




\begin{tabular}{|c|c|c|}
\hline UN_xiong_8543 & 36 & $\mathrm{G} / \mathrm{T}$ \\
\hline UN_xiong_8543 & 602 & $A / T$ \\
\hline UN_xiong_8549 & 327 & $\mathrm{C} / \mathrm{T}$ \\
\hline UN_xiong_8555 & 48 & $\mathrm{~A} / \mathrm{C}$ \\
\hline UN_xiong_8555 & 69 & $\mathrm{G} / \mathrm{C}$ \\
\hline UN_xiong_8555 & 113 & $\mathrm{~T} / \mathrm{C}$ \\
\hline UN_xiong_8555 & 143 & $\mathrm{G} / \mathrm{A}$ \\
\hline UN_xiong_8555 & 164 & $\mathrm{~T} / \mathrm{C}$ \\
\hline UN_xiong_8555 & 187 & $\mathrm{C} / \mathrm{T}$ \\
\hline UN_xiong_8555 & 216 & $A / G$ \\
\hline UN_xiong_8555 & 239 & $C / G$ \\
\hline UN_xiong_8555 & 345 & $C / G$ \\
\hline UN_xiong_8555 & 384 & $\mathrm{G} / \mathrm{C}$ \\
\hline UN_xiong_8559 & 635 & $\mathrm{~T} / \mathrm{G}$ \\
\hline UN_xiong_8560 & 176 & $A / G$ \\
\hline UN_xiong_8568 & 367 & $\mathrm{~A} / \mathrm{T}$ \\
\hline UN_xiong_8568 & 426 & $T / G$ \\
\hline UN_xiong_8568 & 580 & $\mathrm{G} / \mathrm{C}$ \\
\hline UN_xiong_8569 & 382 & $\mathrm{~T} / \mathrm{C}$ \\
\hline UN_xiong_8569 & 647 & $\mathrm{~T} / \mathrm{C}$ \\
\hline UN_xiong_8569 & 796 & $\mathrm{C} / \mathrm{G}$ \\
\hline UN_xiong_8569 & 1214 & $A / T$ \\
\hline UN_xiong_8573 & 403 & $\mathrm{~T} / \mathrm{A}$ \\
\hline UN_xiong_8573 & 683 & $\mathrm{G} / \mathrm{A}$ \\
\hline UN_xiong_8575 & 45 & $\mathrm{~T} / \mathrm{C}$ \\
\hline UN_xiong_8583 & 133 & $C / G$ \\
\hline UN_xiong_8583 & 297 & $\mathrm{~A} / \mathrm{C}$ \\
\hline UN_xiong_8586 & 102 & $\mathrm{G} / \mathrm{A}$ \\
\hline UN_xiong_8591 & 302 & $\mathrm{~T} / \mathrm{C}$ \\
\hline UN_xiong_8591 & 731 & $\mathrm{C} / \mathrm{T}$ \\
\hline UN_xiong_8591 & 761 & $A / G$ \\
\hline UN_xiong_8591 & 787 & $\mathrm{~T} / \mathrm{C}$ \\
\hline UN_xiong_8593 & 49 & $\mathrm{G} / \mathrm{C}$ \\
\hline UN_xiong_8600 & 97 & $\mathrm{~T} / \mathrm{C}$ \\
\hline UN_xiong_8622 & 44 & $\mathrm{G} / \mathrm{T}$ \\
\hline UN_xiong_8622 & 325 & $\mathrm{~T} / \mathrm{C}$ \\
\hline UN_xiong_8628 & 309 & $\mathrm{~T} / \mathrm{C}$ \\
\hline UN_xiong_8628 & 487 & $\mathrm{C} / \mathrm{T}$ \\
\hline UN_xiong_8634 & 589 & $\mathrm{~A} / \mathrm{T}$ \\
\hline UN_xiong_8647 & 456 & $A / G$ \\
\hline UN_xiong_8651 & 80 & $A / G$ \\
\hline UN_xiong_8651 & 302 & $\mathrm{C} / \mathrm{T}$ \\
\hline UN_xiong_8668 & 13 & $A / G$ \\
\hline UN_xiong_8668 & 397 & $\mathrm{~A} / \mathrm{C}$ \\
\hline UN_xiong_8670 & 270 & $C / A$ \\
\hline UN_xiong_8683 & 919 & $\mathrm{C} / \mathrm{T}$ \\
\hline UN_xiong_8684 & 545 & $A / G$ \\
\hline
\end{tabular}




\begin{tabular}{|c|c|c|}
\hline UN_xiong_8684 & 611 & $\mathrm{G} / \mathrm{A}$ \\
\hline UN_xiong_8690 & 373 & $\mathrm{~T} / \mathrm{C}$ \\
\hline UN_xiong_8690 & 442 & $\mathrm{~A} / \mathrm{T}$ \\
\hline UN_xiong_8702 & 54 & $A / G$ \\
\hline UN_xiong_8710 & 498 & $C / G$ \\
\hline UN_xiong_8722 & 179 & $\mathrm{G} / \mathrm{A}$ \\
\hline UN_xiong_8724 & 285 & $A / G$ \\
\hline UN_xiong_8732 & 182 & $\mathrm{G} / \mathrm{A}$ \\
\hline UN_xiong_8732 & 235 & $\mathrm{~A} / \mathrm{T}$ \\
\hline UN_xiong_8732 & 296 & $\mathrm{~T} / \mathrm{C}$ \\
\hline UN_xiong_8733 & 550 & $\mathrm{~A} / \mathrm{C}$ \\
\hline UN_xiong_8733 & 575 & $\mathrm{~T} / \mathrm{C}$ \\
\hline UN_xiong_8744 & 106 & $\mathrm{C} / \mathrm{T}$ \\
\hline UN_xiong_8765 & 471 & $\mathrm{C} / \mathrm{A}$ \\
\hline UN_xiong_8773 & 806 & $\mathrm{~T} / \mathrm{A}$ \\
\hline UN_xiong_8776 & 348 & $\mathrm{G} / \mathrm{A}$ \\
\hline UN_xiong_8786 & 26 & $\mathrm{~T} / \mathrm{A}$ \\
\hline UN_xiong_8790 & 297 & $\mathrm{G} / \mathrm{A}$ \\
\hline UN_xiong_8797 & 231 & $\mathrm{~T} / \mathrm{A}$ \\
\hline UN_xiong_8803 & 189 & $\mathrm{~T} / \mathrm{C}$ \\
\hline UN_xiong_8811 & 750 & $\mathrm{~T} / \mathrm{C}$ \\
\hline UN_xiong_8812 & 197 & $\mathrm{~A} / \mathrm{T}$ \\
\hline UN_xiong_8812 & 258 & $\mathrm{~T} / \mathrm{A}$ \\
\hline UN_xiong_8812 & 301 & $\mathrm{~T} / \mathrm{A}$ \\
\hline UN_xiong_8812 & 409 & $\mathrm{G} / \mathrm{A}$ \\
\hline UN_xiong_8820 & 309 & $A / G$ \\
\hline UN_xiong_8822 & 183 & $\mathrm{C} / \mathrm{T}$ \\
\hline UN_xiong_8831 & 230 & $\mathrm{~T} / \mathrm{C}$ \\
\hline UN_xiong_8831 & 268 & $\mathrm{G} / \mathrm{A}$ \\
\hline UN_xiong_8840 & 388 & $\mathrm{C} / \mathrm{T}$ \\
\hline UN_xiong_8843 & 214 & $\mathrm{~A} / \mathrm{T}$ \\
\hline UN_xiong_8850 & 85 & $A / G$ \\
\hline UN_xiong_8850 & 377 & $A / G$ \\
\hline UN_xiong_8850 & 513 & $\mathrm{C} / \mathrm{T}$ \\
\hline UN_xiong_8850 & 559 & $\mathrm{G} / \mathrm{A}$ \\
\hline UN_xiong_8850 & 599 & $\mathrm{~A} / \mathrm{G}$ \\
\hline UN_xiong_8853 & 309 & $A / G$ \\
\hline UN_xiong_8853 & 1105 & $\mathrm{C} / \mathrm{T}$ \\
\hline UN_xiong_8861 & 129 & $\mathrm{C} / \mathrm{T}$ \\
\hline UN_xiong_8862 & 252 & $C / G$ \\
\hline UN_xiong_8862 & 290 & $\mathrm{C} / \mathrm{T}$ \\
\hline UN_xiong_8862 & 318 & $\mathrm{G} / \mathrm{T}$ \\
\hline UN_xiong_8862 & 440 & $\mathrm{C} / \mathrm{T}$ \\
\hline UN_xiong_8862 & 471 & $A / G$ \\
\hline UN_xiong_8862 & 595 & $\mathrm{G} / \mathrm{A}$ \\
\hline UN_xiong_8862 & 676 & $A / G$ \\
\hline UN_xiong_8862 & 712 & $\mathrm{C} / \mathrm{G}$ \\
\hline
\end{tabular}




\begin{tabular}{|c|c|c|}
\hline UN_xiong_8868 & 485 & $\mathrm{~T} / \mathrm{G}$ \\
\hline UN_xiong_8868 & 512 & $A / G$ \\
\hline UN_xiong_8875 & 31 & $T / G$ \\
\hline UN_xiong_8884 & 256 & $\mathrm{~T} / \mathrm{C}$ \\
\hline UN_xiong_8884 & 291 & $\mathrm{~T} / \mathrm{C}$ \\
\hline UN_xiong_8892 & 188 & $\mathrm{G} / \mathrm{A}$ \\
\hline UN_xiong_8892 & 272 & $A / G$ \\
\hline UN_xiong_8897 & 944 & $C / A$ \\
\hline UN_xiong_8906 & 353 & $\mathrm{~T} / \mathrm{C}$ \\
\hline UN_xiong_8908 & 125 & $\mathrm{~T} / \mathrm{C}$ \\
\hline UN_xiong_8908 & 265 & $\mathrm{G} / \mathrm{A}$ \\
\hline UN_xiong_8912 & 276 & $\mathrm{G} / \mathrm{A}$ \\
\hline UN_xiong_8920 & 562 & $A / G$ \\
\hline UN_xiong_8922 & 257 & $\mathrm{G} / \mathrm{C}$ \\
\hline UN_xiong_8922 & 310 & $\mathrm{G} / \mathrm{A}$ \\
\hline UN_xiong_8922 & 449 & $A / G$ \\
\hline UN_xiong_8922 & 794 & $\mathrm{~T} / \mathrm{C}$ \\
\hline UN_xiong_8922 & 940 & $\mathrm{~T} / \mathrm{C}$ \\
\hline UN_xiong_8933 & 364 & $\mathrm{~A} / \mathrm{T}$ \\
\hline UN_xiong_8934 & 100 & $A / G$ \\
\hline UN_xiong_8934 & 207 & $\mathrm{~A} / \mathrm{C}$ \\
\hline UN_xiong_8937 & 151 & $\mathrm{C} / \mathrm{T}$ \\
\hline UN_xiong_8937 & 937 & $\mathrm{C} / \mathrm{T}$ \\
\hline UN_xiong_8937 & 1143 & $\mathrm{~T} / \mathrm{C}$ \\
\hline UN_xiong_8944 & 109 & $T / G$ \\
\hline UN_xiong_8944 & 231 & $C / A$ \\
\hline UN_xiong_8944 & 304 & $\mathrm{~T} / \mathrm{C}$ \\
\hline UN_xiong_8956 & 839 & $A / G$ \\
\hline UN_xiong_8963 & 187 & $G / A$ \\
\hline UN_xiong_8963 & 252 & $\mathrm{G} / \mathrm{A}$ \\
\hline UN_xiong_8977 & 278 & $A / G$ \\
\hline UN_xiong_8979 & 408 & $C / G$ \\
\hline UN_xiong_8985 & 90 & $G / A$ \\
\hline UN_xiong_8985 & 201 & $\mathrm{G} / \mathrm{A}$ \\
\hline UN_xiong_8985 & 337 & $\mathrm{~T} / \mathrm{C}$ \\
\hline UN_xiong_8985 & 430 & $C / A$ \\
\hline UN_xiong_8994 & 301 & $\mathrm{G} / \mathrm{A}$ \\
\hline UN_xiong_8994 & 431 & $T / G$ \\
\hline UN_xiong_8994 & 588 & $\mathrm{~T} / \mathrm{C}$ \\
\hline UN_xiong_8996 & 330 & $C / A$ \\
\hline UN_xiong_8996 & 652 & $\mathrm{~T} / \mathrm{C}$ \\
\hline UN_xiong_8996 & 678 & $A / G$ \\
\hline UN_xiong_8999 & 757 & $\mathrm{C} / \mathrm{T}$ \\
\hline UN_xiong_9004 & 232 & $\mathrm{G} / \mathrm{A}$ \\
\hline UN_xiong_9004 & 267 & $\mathrm{G} / \mathrm{T}$ \\
\hline UN_xiong_9004 & 306 & $A / G$ \\
\hline UN_xiong_9004 & 404 & $\mathrm{G} / \mathrm{T}$ \\
\hline
\end{tabular}




\begin{tabular}{|c|c|c|}
\hline UN_xiong_9004 & 450 & $A / G$ \\
\hline UN_xiong_9004 & 502 & $\mathrm{C} / \mathrm{G}$ \\
\hline UN_xiong_9004 & 557 & $\mathrm{G} / \mathrm{T}$ \\
\hline UN_xiong_9004 & 654 & $\mathrm{~A} / \mathrm{G}$ \\
\hline UN_xiong_9004 & 683 & $\mathrm{G} / \mathrm{A}$ \\
\hline UN_xiong_9017 & 322 & $\mathrm{~T} / \mathrm{A}$ \\
\hline UN_xiong_9035 & 6 & $\mathrm{~A} / \mathrm{T}$ \\
\hline UN_xiong_9035 & 44 & $\mathrm{C} / \mathrm{T}$ \\
\hline UN_xiong_9035 & 160 & $A / G$ \\
\hline UN_xiong_9035 & 214 & $\mathrm{G} / \mathrm{A}$ \\
\hline UN_xiong_9035 & 471 & $\mathrm{~T} / \mathrm{C}$ \\
\hline UN_xiong_9035 & 844 & $\mathrm{C} / \mathrm{T}$ \\
\hline UN_xiong_9035 & 938 & $\mathrm{~A} / \mathrm{C}$ \\
\hline UN_xiong_9035 & 978 & $\mathrm{~T} / \mathrm{G}$ \\
\hline UN_xiong_9035 & 1000 & $\mathrm{~T} / \mathrm{C}$ \\
\hline UN_xiong_9035 & 1020 & $\mathrm{G} / \mathrm{T}$ \\
\hline UN_xiong_9035 & 1041 & $\mathrm{~T} / \mathrm{A}$ \\
\hline UN_xiong_9035 & 1090 & $\mathrm{~A} / \mathrm{C}$ \\
\hline UN_xiong_9038 & 372 & $\mathrm{~T} / \mathrm{C}$ \\
\hline UN_xiong_9039 & 112 & $A / G$ \\
\hline UN_xiong_9047 & 100 & $\mathrm{C} / \mathrm{A}$ \\
\hline UN_xiong_9066 & 70 & $\mathrm{G} / \mathrm{A}$ \\
\hline UN_xiong_9066 & 132 & $\mathrm{~T} / \mathrm{C}$ \\
\hline UN_xiong_9066 & 204 & $\mathrm{C} / \mathrm{T}$ \\
\hline UN_xiong_9066 & 314 & $A / G$ \\
\hline UN_xiong_9066 & 500 & $G / A$ \\
\hline UN_xiong_9066 & 658 & $C / G$ \\
\hline UN_xiong_9066 & 739 & $\mathrm{G} / \mathrm{A}$ \\
\hline UN_xiong_9083 & 53 & $\mathrm{~T} / \mathrm{C}$ \\
\hline UN_xiong_9083 & 319 & $\mathrm{~T} / \mathrm{C}$ \\
\hline UN_xiong_9083 & 342 & $\mathrm{~A} / \mathrm{G}$ \\
\hline UN_xiong_9083 & 585 & $\mathrm{~T} / \mathrm{A}$ \\
\hline UN_xiong_9083 & 606 & $\mathrm{~A} / \mathrm{G}$ \\
\hline UN_xiong_9083 & 638 & $\mathrm{G} / \mathrm{C}$ \\
\hline UN_xiong_9083 & 700 & $\mathrm{~A} / \mathrm{G}$ \\
\hline UN_xiong_9083 & 853 & $\mathrm{~A} / \mathrm{C}$ \\
\hline UN_xiong_9097 & 340 & $\mathrm{G} / \mathrm{A}$ \\
\hline UN_xiong_9097 & 677 & $\mathrm{G} / \mathrm{A}$ \\
\hline UN_xiong_9105 & 33 & $\mathrm{G} / \mathrm{A}$ \\
\hline UN_xiong_9105 & 77 & $\mathrm{C} / \mathrm{T}$ \\
\hline UN_xiong_9105 & 117 & $\mathrm{~A} / \mathrm{G}$ \\
\hline UN_xiong_9105 & 140 & $\mathrm{C} / \mathrm{A}$ \\
\hline UN_xiong_9105 & 200 & $C / A$ \\
\hline UN_xiong_9105 & 238 & $A / G$ \\
\hline UN_xiong_9105 & 272 & $\mathrm{C} / \mathrm{T}$ \\
\hline UN_xiong_9105 & 300 & $A / G$ \\
\hline UN_xiong_9105 & 339 & $\mathrm{C} / \mathrm{T}$ \\
\hline
\end{tabular}




\begin{tabular}{|c|c|c|}
\hline UN_xiong_9105 & 398 & $\mathrm{C} / \mathrm{T}$ \\
\hline UN_xiong_9105 & 418 & $\mathrm{~T} / \mathrm{C}$ \\
\hline UN_xiong_9105 & 472 & $\mathrm{~T} / \mathrm{C}$ \\
\hline UN_xiong_9105 & 630 & $\mathrm{C} / \mathrm{T}$ \\
\hline UN_xiong_9105 & 663 & $C / A$ \\
\hline UN_xiong_9107 & 192 & $A / G$ \\
\hline UN_xiong_9133 & 282 & $\mathrm{~T} / \mathrm{C}$ \\
\hline UN_xiong_9136 & 98 & $\mathrm{~T} / \mathrm{C}$ \\
\hline UN_xiong_9146 & 60 & $\mathrm{C} / \mathrm{T}$ \\
\hline UN_xiong_9158 & 88 & $\mathrm{C} / \mathrm{T}$ \\
\hline UN_xiong_9158 & 143 & $\mathrm{~T} / \mathrm{C}$ \\
\hline UN_xiong_9163 & 231 & $A / G$ \\
\hline UN_xiong_9163 & 583 & $A / G$ \\
\hline UN_xiong_9163 & 652 & $A / G$ \\
\hline UN_xiong_9163 & 727 & $\mathrm{~A} / \mathrm{T}$ \\
\hline UN_xiong_9165 & 225 & $\mathrm{~T} / \mathrm{C}$ \\
\hline UN_xiong_9166 & 363 & $\mathrm{~T} / \mathrm{C}$ \\
\hline UN_xiong_9166 & 383 & $\mathrm{C} / \mathrm{T}$ \\
\hline UN_xiong_9167 & 274 & $\mathrm{~T} / \mathrm{C}$ \\
\hline UN_xiong_9170 & 506 & $A / G$ \\
\hline UN_xiong_9185 & 31 & $\mathrm{~A} / \mathrm{T}$ \\
\hline UN_xiong_9185 & 242 & $\mathrm{G} / \mathrm{T}$ \\
\hline UN_xiong_9185 & 528 & $\mathrm{G} / \mathrm{T}$ \\
\hline UN_xiong_9185 & 709 & $\mathrm{~T} / \mathrm{C}$ \\
\hline UN_xiong_9185 & 729 & $\mathrm{C} / \mathrm{T}$ \\
\hline UN_xiong_9190 & 232 & $A / G$ \\
\hline UN_xiong_9200 & 188 & $\mathrm{~T} / \mathrm{C}$ \\
\hline UN_xiong_9203 & 192 & $A / G$ \\
\hline UN_xiong_9203 & 374 & $A / G$ \\
\hline UN_xiong_9203 & 434 & $\mathrm{~T} / \mathrm{A}$ \\
\hline UN_xiong_9219 & 136 & $\mathrm{~T} / \mathrm{C}$ \\
\hline UN_xiong_9219 & 323 & $C / A$ \\
\hline UN_xiong_9221 & 232 & $\mathrm{C} / \mathrm{T}$ \\
\hline UN_xiong_9224 & 145 & $A / G$ \\
\hline UN_xiong_9227 & 319 & $\mathrm{C} / \mathrm{T}$ \\
\hline UN_xiong_9227 & 340 & $\mathrm{G} / \mathrm{T}$ \\
\hline UN_xiong_9227 & 528 & $\mathrm{G} / \mathrm{C}$ \\
\hline UN_xiong_9230 & 118 & $A / G$ \\
\hline UN_xiong_9230 & 328 & $\mathrm{~T} / \mathrm{C}$ \\
\hline UN_xiong_9230 & 567 & $\mathrm{~A} / \mathrm{T}$ \\
\hline UN_xiong_9230 & 618 & $\mathrm{~T} / \mathrm{G}$ \\
\hline UN_xiong_9230 & 798 & $\mathrm{G} / \mathrm{T}$ \\
\hline UN_xiong_9230 & 855 & $\mathrm{~T} / \mathrm{A}$ \\
\hline UN_xiong_9230 & 931 & $A / G$ \\
\hline UN_xiong_9230 & 970 & $\mathrm{~T} / \mathrm{C}$ \\
\hline UN_xiong_9230 & 1116 & $\mathrm{C} / \mathrm{T}$ \\
\hline UN_xiong_9230 & 1178 & $A / G$ \\
\hline
\end{tabular}




\begin{tabular}{|c|c|c|}
\hline UN_xiong_9230 & 1230 & $\mathrm{C} / \mathrm{A}$ \\
\hline UN_xiong_9230 & 1268 & $\mathrm{C} / \mathrm{T}$ \\
\hline UN_xiong_9243 & 73 & $\mathrm{~A} / \mathrm{G}$ \\
\hline UN_xiong_9243 & 221 & $\mathrm{G} / \mathrm{A}$ \\
\hline UN_xiong_9243 & 256 & $\mathrm{~T} / \mathrm{G}$ \\
\hline UN_xiong_9267 & 647 & $\mathrm{G} / \mathrm{A}$ \\
\hline UN_xiong_9267 & 682 & $\mathrm{~T} / \mathrm{C}$ \\
\hline UN_xiong_9267 & 848 & $\mathrm{~A} / \mathrm{C}$ \\
\hline UN_xiong_9267 & 1057 & $A / G$ \\
\hline UN_xiong_9267 & 1104 & $\mathrm{~A} / \mathrm{G}$ \\
\hline UN_xiong_9283 & 168 & $\mathrm{~T} / \mathrm{C}$ \\
\hline UN_xiong_9283 & 270 & $\mathrm{~A} / \mathrm{T}$ \\
\hline UN_xiong_9283 & 293 & $\mathrm{G} / \mathrm{A}$ \\
\hline UN_xiong_9283 & 417 & $\mathrm{C} / \mathrm{G}$ \\
\hline UN_xiong_9287 & 74 & $\mathrm{~T} / \mathrm{C}$ \\
\hline UN_xiong_9287 & 105 & $\mathrm{G} / \mathrm{A}$ \\
\hline UN_xiong_9300 & 48 & $\mathrm{~A} / \mathrm{T}$ \\
\hline UN_xiong_9303 & 242 & $\mathrm{C} / \mathrm{T}$ \\
\hline UN_xiong_9311 & 74 & $\mathrm{~T} / \mathrm{C}$ \\
\hline UN_xiong_9322 & 157 & $\mathrm{~T} / \mathrm{C}$ \\
\hline UN_xiong_9322 & 210 & $\mathrm{C} / \mathrm{T}$ \\
\hline UN_xiong_9322 & 320 & $\mathrm{~T} / \mathrm{G}$ \\
\hline UN_xiong_9324 & 55 & $\mathrm{C} / \mathrm{G}$ \\
\hline UN_xiong_9335 & 329 & $\mathrm{G} / \mathrm{A}$ \\
\hline UN_xiong_9343 & 390 & $\mathrm{C} / \mathrm{T}$ \\
\hline UN_xiong_9343 & 777 & $\mathrm{~T} / \mathrm{C}$ \\
\hline UN_xiong_9343 & 834 & $\mathrm{~T} / \mathrm{G}$ \\
\hline UN_xiong_9343 & 1045 & $\mathrm{~A} / \mathrm{T}$ \\
\hline UN_xiong_9359 & 1082 & $\mathrm{~T} / \mathrm{A}$ \\
\hline UN_xiong_9365 & 86 & $\mathrm{G} / \mathrm{A}$ \\
\hline UN_xiong_9382 & 536 & $\mathrm{~T} / \mathrm{C}$ \\
\hline UN_xiong_9382 & 614 & $\mathrm{G} / \mathrm{A}$ \\
\hline UN_xiong_9403 & 164 & $\mathrm{C} / \mathrm{A}$ \\
\hline UN_xiong_9403 & 284 & $\mathrm{~A} / \mathrm{C}$ \\
\hline UN_xiong_9405 & 335 & $\mathrm{C} / \mathrm{T}$ \\
\hline UN_xiong_9415 & 40 & $\mathrm{C} / \mathrm{G}$ \\
\hline UN_xiong_9415 & 73 & $\mathrm{G} / \mathrm{T}$ \\
\hline UN_xiong_9424 & 144 & $\mathrm{C} / \mathrm{T}$ \\
\hline UN_xiong_9424 & 195 & $\mathrm{~T} / \mathrm{C}$ \\
\hline UN_xiong_9424 & 239 & $\mathrm{~A} / \mathrm{T}$ \\
\hline UN_xiong_9424 & 262 & $\mathrm{G} / \mathrm{T}$ \\
\hline UN_xiong_9424 & 312 & $\mathrm{G} / \mathrm{T}$ \\
\hline UN_xiong_9424 & 344 & $\mathrm{G} / \mathrm{A}$ \\
\hline UN_xiong_9424 & 387 & $\mathrm{C} / \mathrm{T}$ \\
\hline UN_xiong_9424 & 491 & $\mathrm{~T} / \mathrm{G}$ \\
\hline UN_xiong_9426 & 150 & $\mathrm{C} / \mathrm{T}$ \\
\hline UN_xiong_9426 & 318 & $\mathrm{~T} / \mathrm{C}$ \\
\hline
\end{tabular}




\begin{tabular}{|c|c|c|}
\hline UN_xiong_9426 & 380 & $A / G$ \\
\hline UN_xiong_9426 & 434 & $\mathrm{C} / \mathrm{T}$ \\
\hline UN_xiong_9427 & 57 & $\mathrm{G} / \mathrm{C}$ \\
\hline UN_xiong_9429 & 96 & $T / G$ \\
\hline UN_xiong_9456 & 246 & $\mathrm{C} / \mathrm{T}$ \\
\hline UN_xiong_9456 & 281 & $\mathrm{C} / \mathrm{T}$ \\
\hline UN_xiong_9482 & 45 & $\mathrm{G} / \mathrm{A}$ \\
\hline UN_xiong_9482 & 166 & $\mathrm{C} / \mathrm{T}$ \\
\hline UN_xiong_9488 & 199 & $C / A$ \\
\hline UN_xiong_9488 & 266 & $\mathrm{~T} / \mathrm{C}$ \\
\hline UN_xiong_9503 & 677 & $\mathrm{~T} / \mathrm{C}$ \\
\hline UN_xiong_9510 & 150 & $\mathrm{G} / \mathrm{T}$ \\
\hline UN_xiong_9513 & 120 & $T / G$ \\
\hline UN_xiong_9513 & 160 & $\mathrm{C} / \mathrm{T}$ \\
\hline UN_xiong_9538 & 379 & $A / G$ \\
\hline UN_xiong_9538 & 474 & $\mathrm{~T} / \mathrm{C}$ \\
\hline UN_xiong_9546 & 12 & $\mathrm{~T} / \mathrm{C}$ \\
\hline UN_xiong_9546 & 32 & $\mathrm{~T} / \mathrm{C}$ \\
\hline UN_xiong_9546 & 240 & $\mathrm{~T} / \mathrm{C}$ \\
\hline UN_xiong_9546 & 346 & $\mathrm{G} / \mathrm{T}$ \\
\hline UN_xiong_9546 & 370 & $A / G$ \\
\hline UN_xiong_9546 & 453 & $A / G$ \\
\hline UN_xiong_9546 & 473 & $G / A$ \\
\hline UN_xiong_9546 & 547 & $\mathrm{~T} / \mathrm{C}$ \\
\hline UN_xiong_9546 & 607 & $A / G$ \\
\hline UN_xiong_9546 & 670 & $\mathrm{~A} / \mathrm{T}$ \\
\hline UN_xiong_9546 & 691 & $G / A$ \\
\hline UN_xiong_9546 & 711 & $A / G$ \\
\hline UN_xiong_9546 & 925 & $A / G$ \\
\hline UN_xiong_9546 & 1471 & $\mathrm{G} / \mathrm{T}$ \\
\hline UN_xiong_9546 & 1621 & $\mathrm{G} / \mathrm{A}$ \\
\hline UN_xiong_9546 & 1864 & $A / G$ \\
\hline UN_xiong_9546 & 1979 & $A / G$ \\
\hline UN_xiong_9546 & 2036 & $\mathrm{~T} / \mathrm{C}$ \\
\hline UN_xiong_9546 & 2283 & $A / G$ \\
\hline UN_xiong_9548 & 158 & $A / G$ \\
\hline UN_xiong_9552 & 90 & $C / A$ \\
\hline UN_xiong_9553 & 111 & $\mathrm{~T} / \mathrm{C}$ \\
\hline UN_xiong_9555 & 75 & $A / G$ \\
\hline UN_xiong_9555 & 146 & $T / G$ \\
\hline UN_xiong_9570 & 617 & $\mathrm{C} / \mathrm{T}$ \\
\hline UN_xiong_9570 & 702 & $\mathrm{~T} / \mathrm{C}$ \\
\hline UN_xiong_9570 & 760 & $A / G$ \\
\hline UN_xiong_9570 & 785 & $\mathrm{G} / \mathrm{T}$ \\
\hline UN_xiong_9570 & 815 & $\mathrm{C} / \mathrm{T}$ \\
\hline UN_xiong_9570 & 890 & $\mathrm{G} / \mathrm{T}$ \\
\hline UN_xiong_9570 & 952 & $A / G$ \\
\hline
\end{tabular}




\begin{tabular}{|c|c|c|}
\hline UN_xiong_9570 & 1064 & $\mathrm{~A} / \mathrm{G}$ \\
\hline UN_xiong_9570 & 1138 & $A / G$ \\
\hline UN_xiong_9571 & 127 & $\mathrm{~T} / \mathrm{A}$ \\
\hline UN_xiong_9585 & 430 & $A / G$ \\
\hline UN_xiong_9585 & 470 & $\mathrm{~T} / \mathrm{A}$ \\
\hline UN_xiong_9585 & 735 & $\mathrm{C} / \mathrm{T}$ \\
\hline UN_xiong_9585 & 783 & $\mathrm{~T} / \mathrm{C}$ \\
\hline UN_xiong_9585 & 1011 & $\mathrm{G} / \mathrm{A}$ \\
\hline UN_xiong_9585 & 1087 & $\mathrm{~T} / \mathrm{C}$ \\
\hline UN_xiong_9586 & 528 & $A / G$ \\
\hline UN_xiong_9586 & 678 & $\mathrm{C} / \mathrm{T}$ \\
\hline UN_xiong_9588 & 71 & $\mathrm{G} / \mathrm{A}$ \\
\hline UN_xiong_9588 & 102 & $\mathrm{~A} / \mathrm{G}$ \\
\hline UN_xiong_9588 & 135 & $\mathrm{G} / \mathrm{A}$ \\
\hline UN_xiong_9589 & 318 & $\mathrm{G} / \mathrm{T}$ \\
\hline UN_xiong_9596 & 16 & $\mathrm{~A} / \mathrm{G}$ \\
\hline UN_xiong_9600 & 77 & $\mathrm{~T} / \mathrm{C}$ \\
\hline UN_xiong_9600 & 114 & $\mathrm{G} / \mathrm{A}$ \\
\hline UN_xiong_9603 & 77 & $\mathrm{C} / \mathrm{T}$ \\
\hline UN_xiong_9610 & 699 & $\mathrm{~A} / \mathrm{T}$ \\
\hline UN_xiong_9610 & 854 & $\mathrm{G} / \mathrm{T}$ \\
\hline UN_xiong_9617 & 172 & $\mathrm{C} / \mathrm{T}$ \\
\hline UN_xiong_9623 & 294 & $\mathrm{~A} / \mathrm{G}$ \\
\hline UN_xiong_9629 & 350 & $\mathrm{~A} / \mathrm{G}$ \\
\hline UN_xiong_9636 & 409 & $\mathrm{G} / \mathrm{A}$ \\
\hline UN_xiong_9636 & 565 & $A / G$ \\
\hline UN_xiong_9652 & 465 & $\mathrm{~T} / \mathrm{C}$ \\
\hline UN_xiong_9653 & 273 & $\mathrm{G} / \mathrm{A}$ \\
\hline UN_xiong_9653 & 600 & $\mathrm{C} / \mathrm{T}$ \\
\hline UN_xiong_9655 & 21 & $\mathrm{G} / \mathrm{A}$ \\
\hline UN_xiong_9655 & 448 & $\mathrm{~T} / \mathrm{C}$ \\
\hline UN_xiong_9655 & 480 & $\mathrm{~T} / \mathrm{C}$ \\
\hline UN_xiong_9655 & 646 & $\mathrm{~T} / \mathrm{C}$ \\
\hline UN_xiong_9668 & 21 & $A / G$ \\
\hline UN_xiong_9668 & 207 & $\mathrm{C} / \mathrm{T}$ \\
\hline UN_xiong_9671 & 262 & $\mathrm{G} / \mathrm{A}$ \\
\hline UN_xiong_9676 & 171 & $\mathrm{G} / \mathrm{A}$ \\
\hline UN_xiong_9682 & 166 & $\mathrm{~T} / \mathrm{A}$ \\
\hline UN_xiong_9683 & 17 & $A / G$ \\
\hline UN_xiong_9691 & 97 & $\mathrm{~A} / \mathrm{G}$ \\
\hline UN_xiong_9691 & 166 & $\mathrm{G} / \mathrm{T}$ \\
\hline UN_xiong_9693 & 710 & $\mathrm{~A} / \mathrm{T}$ \\
\hline UN_xiong_9698 & 155 & $\mathrm{G} / \mathrm{A}$ \\
\hline UN_xiong_9698 & 210 & $\mathrm{G} / \mathrm{C}$ \\
\hline UN_xiong_9698 & 241 & $A / G$ \\
\hline UN_xiong_9698 & 301 & $\mathrm{~T} / \mathrm{C}$ \\
\hline UN_xiong_9701 & 341 & $A / G$ \\
\hline
\end{tabular}




\begin{tabular}{|c|c|c|}
\hline UN_xiong_9703 & 107 & $A / G$ \\
\hline UN_xiong_9715 & 388 & $A / G$ \\
\hline UN_xiong_9715 & 540 & $\mathrm{C} / \mathrm{T}$ \\
\hline UN_xiong_9720 & 185 & $\mathrm{~A} / \mathrm{T}$ \\
\hline UN_xiong_9726 & 456 & $\mathrm{G} / \mathrm{A}$ \\
\hline UN_xiong_9726 & 544 & $C / G$ \\
\hline UN_xiong_9727 & 260 & $\mathrm{C} / \mathrm{T}$ \\
\hline UN_xiong_9735 & 433 & $\mathrm{G} / \mathrm{A}$ \\
\hline UN_xiong_9735 & 618 & $\mathrm{~T} / \mathrm{C}$ \\
\hline UN_xiong_9737 & 80 & $\mathrm{C} / \mathrm{T}$ \\
\hline UN_xiong_9737 & 267 & $\mathrm{G} / \mathrm{A}$ \\
\hline UN_xiong_9738 & 15 & $\mathrm{~T} / \mathrm{A}$ \\
\hline UN_xiong_9738 & 205 & $\mathrm{~T} / \mathrm{C}$ \\
\hline UN_xiong_9738 & 237 & $\mathrm{C} / \mathrm{T}$ \\
\hline UN_xiong_9738 & 798 & $C / A$ \\
\hline UN_xiong_9739 & 47 & $\mathrm{G} / \mathrm{A}$ \\
\hline UN_xiong_9739 & 226 & $\mathrm{~T} / \mathrm{C}$ \\
\hline UN_xiong_9739 & 601 & $\mathrm{~T} / \mathrm{C}$ \\
\hline UN_xiong_9739 & 622 & $G / A$ \\
\hline UN_xiong_9743 & 39 & $\mathrm{G} / \mathrm{A}$ \\
\hline UN_xiong_9743 & 65 & $A / G$ \\
\hline UN_xiong_9743 & 290 & $A / C$ \\
\hline UN_xiong_9760 & 328 & $\mathrm{C} / \mathrm{T}$ \\
\hline UN_xiong_9760 & 348 & $\mathrm{G} / \mathrm{A}$ \\
\hline UN_xiong_9760 & 522 & $\mathrm{C} / \mathrm{T}$ \\
\hline UN_xiong_9760 & 639 & $\mathrm{G} / \mathrm{A}$ \\
\hline UN_xiong_9760 & 682 & $G / A$ \\
\hline UN_xiong_9761 & 71 & $A / G$ \\
\hline UN_xiong_9761 & 260 & $\mathrm{~A} / \mathrm{T}$ \\
\hline UN_xiong_9761 & 291 & $\mathrm{~T} / \mathrm{C}$ \\
\hline UN_xiong_9761 & 502 & $A / G$ \\
\hline UN_xiong_9761 & 578 & $\mathrm{C} / \mathrm{T}$ \\
\hline UN_xiong_9767 & 345 & $\mathrm{~T} / \mathrm{A}$ \\
\hline UN_xiong_9779 & 79 & $\mathrm{C} / \mathrm{T}$ \\
\hline UN_xiong_9790 & 234 & $\mathrm{C} / \mathrm{T}$ \\
\hline UN_xiong_9796 & 43 & $A / G$ \\
\hline UN_xiong_9803 & 128 & $T / G$ \\
\hline UN_xiong_9803 & 340 & $C / A$ \\
\hline UN_xiong_9803 & 440 & $\mathrm{~T} / \mathrm{A}$ \\
\hline UN_xiong_9809 & 89 & $A / G$ \\
\hline UN_xiong_9817 & 137 & $A / G$ \\
\hline UN_xiong_9817 & 159 & $A / G$ \\
\hline UN_xiong_9817 & 245 & $T / G$ \\
\hline UN_xiong_9817 & 310 & $A / G$ \\
\hline UN_xiong_9833 & 514 & $G / A$ \\
\hline UN_xiong_9844 & 177 & $\mathrm{C} / \mathrm{T}$ \\
\hline UN_xiong_9845 & 140 & $\mathrm{~T} / \mathrm{A}$ \\
\hline
\end{tabular}




\begin{tabular}{|c|c|c|}
\hline UN_xiong_9845 & 531 & $A / G$ \\
\hline UN_xiong_9845 & 752 & $\mathrm{G} / \mathrm{A}$ \\
\hline UN_xiong_9845 & 827 & $\mathrm{~T} / \mathrm{C}$ \\
\hline UN_xiong_9845 & 916 & $A / G$ \\
\hline UN_xiong_9854 & 6 & $\mathrm{G} / \mathrm{A}$ \\
\hline UN_xiong_9854 & 124 & $\mathrm{~T} / \mathrm{C}$ \\
\hline UN_xiong_9854 & 174 & $\mathrm{~A} / \mathrm{T}$ \\
\hline UN_xiong_9868 & 253 & $\mathrm{C} / \mathrm{A}$ \\
\hline UN_xiong_9868 & 365 & $A / G$ \\
\hline UN_xiong_9868 & 524 & $\mathrm{~A} / \mathrm{T}$ \\
\hline UN_xiong_9876 & 321 & $A / G$ \\
\hline UN_xiong_9876 & 407 & $\mathrm{~T} / \mathrm{C}$ \\
\hline UN_xiong_9876 & 653 & $\mathrm{C} / \mathrm{G}$ \\
\hline UN_xiong_9876 & 968 & $\mathrm{G} / \mathrm{A}$ \\
\hline UN_xiong_9888 & 807 & $\mathrm{G} / \mathrm{A}$ \\
\hline UN_xiong_9896 & 325 & $\mathrm{~T} / \mathrm{C}$ \\
\hline UN_xiong_9902 & 716 & $\mathrm{~T} / \mathrm{C}$ \\
\hline UN_xiong_9911 & 416 & $\mathrm{C} / \mathrm{T}$ \\
\hline UN_xiong_9917 & 220 & $\mathrm{~T} / \mathrm{C}$ \\
\hline UN_xiong_9941 & 505 & $\mathrm{~T} / \mathrm{C}$ \\
\hline UN_xiong_9955 & 212 & $\mathrm{G} / \mathrm{A}$ \\
\hline UN_xiong_9969 & 313 & $\mathrm{C} / \mathrm{T}$ \\
\hline UN_xiong_9969 & 601 & $\mathrm{G} / \mathrm{C}$ \\
\hline UN_xiong_9969 & 622 & $\mathrm{C} / \mathrm{T}$ \\
\hline UN_xiong_9969 & 722 & $\mathrm{G} / \mathrm{A}$ \\
\hline UN_xiong_9969 & 848 & $A / G$ \\
\hline UN_xiong_9970 & 369 & $\mathrm{G} / \mathrm{A}$ \\
\hline UN_xiong_9985 & 138 & $\mathrm{G} / \mathrm{C}$ \\
\hline UN_xiong_9985 & 606 & $\mathrm{~T} / \mathrm{A}$ \\
\hline UN_xiong_9985 & 648 & $\mathrm{~T} / \mathrm{G}$ \\
\hline UN_xiong_9985 & 698 & $\mathrm{~T} / \mathrm{C}$ \\
\hline UN_xiong_9985 & 867 & $A / G$ \\
\hline UN_xiong_9988 & 81 & $\mathrm{C} / \mathrm{T}$ \\
\hline UN_xiong_9988 & 108 & $\mathrm{G} / \mathrm{A}$ \\
\hline UN_xiong_9988 & 170 & $\mathrm{G} / \mathrm{A}$ \\
\hline UN_xiong_9995 & 341 & $\mathrm{~A} / \mathrm{C}$ \\
\hline UN_xiong_9995 & 388 & $\mathrm{G} / \mathrm{T}$ \\
\hline UN_xiong_10005 & 170 & $\mathrm{G} / \mathrm{A}$ \\
\hline UN_xiong_10027 & 6 & $\mathrm{~A} / \mathrm{T}$ \\
\hline UN_xiong_10027 & 34 & $\mathrm{~T} / \mathrm{C}$ \\
\hline UN_xiong_10027 & 122 & $\mathrm{~T} / \mathrm{C}$ \\
\hline UN_xiong_10027 & 173 & $\mathrm{~T} / \mathrm{C}$ \\
\hline UN_xiong_10027 & 199 & $\mathrm{~A} / \mathrm{G}$ \\
\hline UN_xiong_10027 & 246 & $A / G$ \\
\hline UN_xiong_10027 & 270 & $A / G$ \\
\hline UN_xiong_10027 & 290 & $A / G$ \\
\hline UN_xiong_10027 & 460 & $\mathrm{~T} / \mathrm{C}$ \\
\hline
\end{tabular}




\begin{tabular}{|c|c|c|}
\hline UN_xiong_10027 & 514 & $\mathrm{~T} / \mathrm{C}$ \\
\hline UN_xiong_10027 & 550 & $\mathrm{C} / \mathrm{T}$ \\
\hline UN_xiong_10027 & 592 & $\mathrm{~T} / \mathrm{G}$ \\
\hline UN_xiong_10027 & 635 & $A / G$ \\
\hline UN_xiong_10027 & 702 & $A / G$ \\
\hline UN_xiong_10027 & 753 & $\mathrm{~A} / \mathrm{G}$ \\
\hline UN_xiong_10027 & 791 & $\mathrm{C} / \mathrm{T}$ \\
\hline UN_xiong_10035 & 82 & $\mathrm{G} / \mathrm{A}$ \\
\hline UN_xiong_10035 & 216 & $A / G$ \\
\hline UN_xiong_10058 & 316 & $\mathrm{~T} / \mathrm{C}$ \\
\hline UN_xiong_10060 & 48 & $\mathrm{G} / \mathrm{A}$ \\
\hline UN_xiong_10060 & 292 & $\mathrm{~T} / \mathrm{G}$ \\
\hline UN_xiong_10060 & 320 & $\mathrm{~A} / \mathrm{G}$ \\
\hline UN_xiong_10064 & 149 & $\mathrm{~A} / \mathrm{G}$ \\
\hline UN_xiong_10067 & 696 & $A / G$ \\
\hline UN_xiong_10067 & 727 & $\mathrm{G} / \mathrm{C}$ \\
\hline UN_xiong_10069 & 175 & $\mathrm{C} / \mathrm{G}$ \\
\hline UN_xiong_10069 & 275 & $\mathrm{~A} / \mathrm{G}$ \\
\hline UN_xiong_10083 & 19 & $\mathrm{C} / \mathrm{T}$ \\
\hline UN_xiong_10083 & 254 & $\mathrm{~T} / \mathrm{C}$ \\
\hline UN_xiong_10083 & 290 & $\mathrm{G} / \mathrm{T}$ \\
\hline UN_xiong_10083 & 339 & $\mathrm{C} / \mathrm{A}$ \\
\hline UN_xiong_10083 & 379 & $\mathrm{~A} / \mathrm{G}$ \\
\hline UN_xiong_10083 & 452 & $\mathrm{~T} / \mathrm{C}$ \\
\hline UN_xiong_10083 & 517 & $\mathrm{C} / \mathrm{A}$ \\
\hline UN_xiong_10083 & 640 & $\mathrm{G} / \mathrm{C}$ \\
\hline UN_xiong_10083 & 667 & $\mathrm{~T} / \mathrm{C}$ \\
\hline UN_xiong_10083 & 808 & $\mathrm{C} / \mathrm{T}$ \\
\hline UN_xiong_10088 & 151 & $A / G$ \\
\hline UN_xiong_10088 & 171 & $\mathrm{G} / \mathrm{A}$ \\
\hline UN_xiong_10088 & 232 & $\mathrm{G} / \mathrm{A}$ \\
\hline UN_xiong_10090 & 182 & $A / G$ \\
\hline UN_xiong_10090 & 469 & $\mathrm{~A} / \mathrm{T}$ \\
\hline UN_xiong_10096 & 95 & $\mathrm{~T} / \mathrm{C}$ \\
\hline UN_xiong_10096 & 391 & $\mathrm{G} / \mathrm{A}$ \\
\hline UN_xiong_10103 & 733 & $\mathrm{~T} / \mathrm{C}$ \\
\hline UN_xiong_10109 & 314 & $\mathrm{~A} / \mathrm{T}$ \\
\hline UN_xiong_10111 & 314 & $\mathrm{C} / \mathrm{T}$ \\
\hline UN_xiong_10111 & 420 & $\mathrm{C} / \mathrm{T}$ \\
\hline UN_xiong_10122 & 229 & $A / G$ \\
\hline UN_xiong_10127 & 371 & $\mathrm{~A} / \mathrm{T}$ \\
\hline UN_xiong_10127 & 492 & $\mathrm{G} / \mathrm{A}$ \\
\hline UN_xiong_10135 & 267 & $\mathrm{~A} / \mathrm{G}$ \\
\hline UN_xiong_10135 & 551 & $\mathrm{G} / \mathrm{A}$ \\
\hline UN_xiong_10142 & 270 & $\mathrm{G} / \mathrm{T}$ \\
\hline UN_xiong_10159 & 95 & $\mathrm{~T} / \mathrm{C}$ \\
\hline UN_xiong_10169 & 211 & $\mathrm{~A} / \mathrm{C}$ \\
\hline
\end{tabular}




\begin{tabular}{|c|c|c|}
\hline UN_xiong_10169 & 278 & $\mathrm{~T} / \mathrm{C}$ \\
\hline UN_xiong_10169 & 415 & $A / G$ \\
\hline UN_xiong_10179 & 138 & $C / G$ \\
\hline UN_xiong_10179 & 211 & $\mathrm{~T} / \mathrm{C}$ \\
\hline UN_xiong_10179 & 250 & $\mathrm{G} / \mathrm{A}$ \\
\hline UN_xiong_10179 & 286 & $\mathrm{C} / \mathrm{T}$ \\
\hline UN_xiong_10179 & 392 & $\mathrm{~A} / \mathrm{T}$ \\
\hline UN_xiong_10179 & 824 & $\mathrm{G} / \mathrm{A}$ \\
\hline UN_xiong_10185 & 316 & $\mathrm{C} / \mathrm{T}$ \\
\hline UN_xiong_10187 & 111 & $\mathrm{C} / \mathrm{T}$ \\
\hline UN_xiong_10187 & 313 & $\mathrm{C} / \mathrm{T}$ \\
\hline UN_xiong_10187 & 335 & $\mathrm{~A} / \mathrm{C}$ \\
\hline UN_xiong_10187 & 546 & $\mathrm{G} / \mathrm{C}$ \\
\hline UN_xiong_10187 & 591 & $\mathrm{~T} / \mathrm{C}$ \\
\hline UN_xiong_10187 & 633 & $\mathrm{~T} / \mathrm{A}$ \\
\hline UN_xiong_10187 & 715 & $\mathrm{C} / \mathrm{T}$ \\
\hline UN_xiong_10187 & 896 & $\mathrm{~T} / \mathrm{A}$ \\
\hline UN_xiong_10187 & 985 & $\mathrm{~A} / \mathrm{C}$ \\
\hline UN_xiong_10187 & 1030 & $\mathrm{C} / \mathrm{T}$ \\
\hline UN_xiong_10197 & 536 & $\mathrm{~T} / \mathrm{C}$ \\
\hline UN_xiong_10203 & 70 & $\mathrm{C} / \mathrm{T}$ \\
\hline UN_xiong_10203 & 254 & $\mathrm{C} / \mathrm{T}$ \\
\hline UN_xiong_10219 & 260 & $\mathrm{~T} / \mathrm{C}$ \\
\hline UN_xiong_10233 & 355 & $\mathrm{~T} / \mathrm{A}$ \\
\hline UN_xiong_10238 & 163 & $A / G$ \\
\hline UN_xiong_10253 & 1077 & $\mathrm{C} / \mathrm{T}$ \\
\hline UN_xiong_10262 & 682 & $\mathrm{~T} / \mathrm{C}$ \\
\hline UN_xiong_10303 & 41 & $\mathrm{C} / \mathrm{A}$ \\
\hline UN_xiong_10303 & 66 & $\mathrm{G} / \mathrm{C}$ \\
\hline UN_xiong_10303 & 144 & $\mathrm{~T} / \mathrm{C}$ \\
\hline UN_xiong_10303 & 165 & $A / G$ \\
\hline UN_xiong_10303 & 198 & $G / A$ \\
\hline UN_xiong_10303 & 305 & $\mathrm{~A} / \mathrm{T}$ \\
\hline UN_xiong_10303 & 346 & $\mathrm{C} / \mathrm{T}$ \\
\hline UN_xiong_10303 & 500 & $\mathrm{~A} / \mathrm{T}$ \\
\hline UN_xiong_10303 & 532 & $\mathrm{~T} / \mathrm{C}$ \\
\hline UN_xiong_10303 & 864 & $A / G$ \\
\hline UN_xiong_10303 & 978 & $\mathrm{~T} / \mathrm{C}$ \\
\hline UN_xiong_10303 & 1027 & $\mathrm{G} / \mathrm{T}$ \\
\hline UN_xiong_10303 & 1077 & $\mathrm{G} / \mathrm{T}$ \\
\hline UN_xiong_10303 & 1103 & $\mathrm{~T} / \mathrm{C}$ \\
\hline UN_xiong_10303 & 1307 & $\mathrm{G} / \mathrm{A}$ \\
\hline UN_xiong_10303 & 1359 & $C / A$ \\
\hline UN_xiong_10337 & 199 & $\mathrm{~T} / \mathrm{A}$ \\
\hline UN_xiong_10347 & 524 & $\mathrm{~T} / \mathrm{C}$ \\
\hline UN_xiong_10347 & 1185 & $\mathrm{~T} / \mathrm{C}$ \\
\hline UN_xiong_10354 & 143 & $\mathrm{~T} / \mathrm{C}$ \\
\hline
\end{tabular}




\begin{tabular}{|c|c|c|}
\hline UN_xiong_10367 & 348 & $\mathrm{G} / \mathrm{C}$ \\
\hline UN_xiong_10367 & 410 & $\mathrm{C} / \mathrm{T}$ \\
\hline UN_xiong_10370 & 52 & $\mathrm{~T} / \mathrm{C}$ \\
\hline UN_xiong_10370 & 197 & $\mathrm{G} / \mathrm{T}$ \\
\hline UN_xiong_10374 & 548 & $A / G$ \\
\hline UN_xiong_10400 & 309 & $\mathrm{C} / \mathrm{T}$ \\
\hline UN_xiong_10401 & 205 & $A / G$ \\
\hline UN_xiong_10414 & 203 & $G / A$ \\
\hline UN_xiong_10414 & 262 & $\mathrm{C} / \mathrm{T}$ \\
\hline UN_xiong_10422 & 86 & $A / G$ \\
\hline UN_xiong_10431 & 39 & $\mathrm{~A} / \mathrm{C}$ \\
\hline UN_xiong_10435 & 84 & $\mathrm{G} / \mathrm{A}$ \\
\hline UN_xiong_10435 & 220 & $\mathrm{C} / \mathrm{A}$ \\
\hline UN_xiong_10435 & 246 & $\mathrm{~T} / \mathrm{C}$ \\
\hline UN_xiong_10435 & 290 & $A / G$ \\
\hline UN_xiong_10435 & 369 & $A / G$ \\
\hline UN_xiong_10435 & 449 & $A / G$ \\
\hline UN_xiong_10435 & 705 & $\mathrm{~T} / \mathrm{C}$ \\
\hline UN_xiong_10435 & 728 & $\mathrm{G} / \mathrm{A}$ \\
\hline UN_xiong_10435 & 815 & $\mathrm{C} / \mathrm{T}$ \\
\hline UN_xiong_10438 & 113 & $G / A$ \\
\hline UN_xiong_10438 & 187 & $A / G$ \\
\hline UN_xiong_10438 & 244 & $\mathrm{G} / \mathrm{A}$ \\
\hline UN_xiong_10438 & 331 & $\mathrm{~T} / \mathrm{C}$ \\
\hline UN_xiong_10438 & 400 & $\mathrm{G} / \mathrm{T}$ \\
\hline UN_xiong_10443 & 180 & $A / G$ \\
\hline UN_xiong_10443 & 217 & $\mathrm{~T} / \mathrm{C}$ \\
\hline UN_xiong_10446 & 187 & $A / G$ \\
\hline UN_xiong_10446 & 888 & $A / G$ \\
\hline UN_xiong_10446 & 988 & $\mathrm{C} / \mathrm{T}$ \\
\hline UN_xiong_10449 & 114 & $\mathrm{C} / \mathrm{T}$ \\
\hline UN_xiong_10452 & 334 & $\mathrm{~T} / \mathrm{C}$ \\
\hline UN_xiong_10463 & 55 & $\mathrm{G} / \mathrm{A}$ \\
\hline UN_xiong_10473 & 364 & $\mathrm{~T} / \mathrm{C}$ \\
\hline UN_xiong_10473 & 392 & $\mathrm{~T} / \mathrm{C}$ \\
\hline UN_xiong_10474 & 250 & $\mathrm{C} / \mathrm{T}$ \\
\hline UN_xiong_10485 & 316 & $\mathrm{C} / \mathrm{T}$ \\
\hline UN_xiong_10485 & 414 & $\mathrm{~T} / \mathrm{C}$ \\
\hline UN_xiong_10489 & 233 & $\mathrm{~T} / \mathrm{C}$ \\
\hline UN_xiong_10489 & 289 & $A / G$ \\
\hline UN_xiong_10496 & 295 & $\mathrm{C} / \mathrm{T}$ \\
\hline UN_xiong_10497 & 35 & $\mathrm{~A} / \mathrm{T}$ \\
\hline UN_xiong_10497 & 107 & $A / G$ \\
\hline UN_xiong_10497 & 165 & $\mathrm{G} / \mathrm{A}$ \\
\hline UN_xiong_10497 & 242 & $\mathrm{~T} / \mathrm{C}$ \\
\hline UN_xiong_10499 & 83 & $\mathrm{~T} / \mathrm{G}$ \\
\hline UN_xiong_10516 & 159 & $\mathrm{~T} / \mathrm{C}$ \\
\hline
\end{tabular}




\begin{tabular}{|c|c|c|}
\hline UN_xiong_10524 & 132 & $\mathrm{~A} / \mathrm{G}$ \\
\hline UN_xiong_10533 & 108 & $\mathrm{~A} / \mathrm{T}$ \\
\hline UN_xiong_10533 & 345 & $\mathrm{~T} / \mathrm{G}$ \\
\hline UN_xiong_10540 & 369 & $\mathrm{C} / \mathrm{T}$ \\
\hline UN_xiong_10540 & 439 & $\mathrm{C} / \mathrm{T}$ \\
\hline UN_xiong_10540 & 976 & $\mathrm{G} / \mathrm{A}$ \\
\hline UN_xiong_10542 & 898 & $\mathrm{C} / \mathrm{T}$ \\
\hline UN_xiong_10548 & 101 & $\mathrm{C} / \mathrm{T}$ \\
\hline UN_xiong_10548 & 155 & $\mathrm{G} / \mathrm{T}$ \\
\hline UN_xiong_10551 & 263 & $\mathrm{~T} / \mathrm{C}$ \\
\hline UN_xiong_10563 & 86 & $A / G$ \\
\hline UN_xiong_10578 & 124 & $\mathrm{C} / \mathrm{T}$ \\
\hline UN_xiong_10584 & 242 & $G / A$ \\
\hline UN_xiong_10584 & 451 & $\mathrm{~T} / \mathrm{C}$ \\
\hline UN_xiong_10584 & 494 & $\mathrm{C} / \mathrm{T}$ \\
\hline UN_xiong_10584 & 618 & $\mathrm{~A} / \mathrm{G}$ \\
\hline UN_xiong_10584 & 653 & $\mathrm{~A} / \mathrm{G}$ \\
\hline UN_xiong_10584 & 694 & $\mathrm{~T} / \mathrm{C}$ \\
\hline UN_xiong_10587 & 44 & $A / G$ \\
\hline UN_xiong_10594 & 72 & $\mathrm{C} / \mathrm{T}$ \\
\hline UN_xiong_10599 & 138 & $\mathrm{~A} / \mathrm{T}$ \\
\hline UN_xiong_10603 & 415 & $\mathrm{~A} / \mathrm{G}$ \\
\hline UN_xiong_10610 & 353 & $\mathrm{~A} / \mathrm{G}$ \\
\hline UN_xiong_10614 & 32 & $\mathrm{G} / \mathrm{A}$ \\
\hline UN_xiong_10614 & 73 & $\mathrm{~T} / \mathrm{C}$ \\
\hline UN_xiong_10614 & 294 & $A / G$ \\
\hline UN_xiong_10614 & 515 & $\mathrm{C} / \mathrm{G}$ \\
\hline UN_xiong_10630 & 424 & $\mathrm{~A} / \mathrm{C}$ \\
\hline UN_xiong_10652 & 120 & $\mathrm{C} / \mathrm{T}$ \\
\hline UN_xiong_10652 & 213 & $\mathrm{C} / \mathrm{T}$ \\
\hline UN_xiong_10652 & 267 & $A / G$ \\
\hline UN_xiong_10652 & 318 & $\mathrm{~A} / \mathrm{G}$ \\
\hline UN_xiong_10652 & 360 & $\mathrm{~A} / \mathrm{G}$ \\
\hline UN_xiong_10652 & 417 & $\mathrm{C} / \mathrm{T}$ \\
\hline UN_xiong_10654 & 396 & $\mathrm{G} / \mathrm{A}$ \\
\hline UN_xiong_10655 & 634 & $\mathrm{C} / \mathrm{T}$ \\
\hline UN_xiong_10656 & 75 & $\mathrm{~A} / \mathrm{T}$ \\
\hline UN_xiong_10656 & 214 & $\mathrm{~T} / \mathrm{A}$ \\
\hline UN_xiong_10656 & 282 & $\mathrm{~T} / \mathrm{C}$ \\
\hline UN_xiong_10681 & 349 & $\mathrm{~A} / \mathrm{C}$ \\
\hline UN_xiong_10691 & 266 & $\mathrm{~A} / \mathrm{T}$ \\
\hline UN_xiong_10700 & 103 & $A / G$ \\
\hline UN_xiong_10706 & 568 & $\mathrm{~A} / \mathrm{C}$ \\
\hline UN_xiong_10706 & 747 & $\mathrm{~T} / \mathrm{G}$ \\
\hline UN_xiong_10707 & 314 & $\mathrm{C} / \mathrm{T}$ \\
\hline UN_xiong_10707 & 672 & $\mathrm{~T} / \mathrm{A}$ \\
\hline UN_xiong_10707 & 1835 & $T / G$ \\
\hline
\end{tabular}




\begin{tabular}{|c|c|c|}
\hline UN_xiong_10707 & 1913 & $\mathrm{~A} / \mathrm{T}$ \\
\hline UN_xiong_10715 & 17 & $\mathrm{C} / \mathrm{T}$ \\
\hline UN_xiong_10715 & 93 & $\mathrm{~A} / \mathrm{G}$ \\
\hline UN_xiong_10721 & 62 & $\mathrm{~A} / \mathrm{G}$ \\
\hline UN_xiong_10723 & 121 & $\mathrm{~A} / \mathrm{T}$ \\
\hline UN_xiong_10724 & 46 & $A / G$ \\
\hline UN_xiong_10726 & 272 & $\mathrm{~T} / \mathrm{C}$ \\
\hline UN_xiong_10726 & 514 & $\mathrm{G} / \mathrm{C}$ \\
\hline UN_xiong_10726 & 850 & $\mathrm{G} / \mathrm{A}$ \\
\hline UN_xiong_10733 & 102 & $\mathrm{~T} / \mathrm{C}$ \\
\hline UN_xiong_10733 & 171 & $\mathrm{~T} / \mathrm{C}$ \\
\hline UN_xiong_10733 & 477 & $\mathrm{~T} / \mathrm{C}$ \\
\hline UN_xiong_10733 & 500 & $\mathrm{~A} / \mathrm{G}$ \\
\hline UN_xiong_10733 & 626 & $\mathrm{G} / \mathrm{T}$ \\
\hline UN_xiong_10733 & 655 & $\mathrm{G} / \mathrm{A}$ \\
\hline UN_xiong_10733 & 742 & $\mathrm{~T} / \mathrm{C}$ \\
\hline UN_xiong_10733 & 780 & $\mathrm{~A} / \mathrm{C}$ \\
\hline UN_xiong_10741 & 848 & $\mathrm{C} / \mathrm{A}$ \\
\hline UN_xiong_10741 & 1122 & $\mathrm{~T} / \mathrm{C}$ \\
\hline UN_xiong_10741 & 1282 & $\mathrm{G} / \mathrm{A}$ \\
\hline UN_xiong_10742 & 330 & $\mathrm{C} / \mathrm{T}$ \\
\hline UN_xiong_10748 & 321 & $\mathrm{~T} / \mathrm{G}$ \\
\hline UN_xiong_10749 & 47 & $A / G$ \\
\hline UN_xiong_10749 & 348 & $\mathrm{G} / \mathrm{A}$ \\
\hline UN_xiong_10756 & 258 & $G / A$ \\
\hline UN_xiong_10756 & 309 & $\mathrm{~A} / \mathrm{G}$ \\
\hline UN_xiong_10763 & 561 & $\mathrm{C} / \mathrm{T}$ \\
\hline UN_xiong_10763 & 581 & $\mathrm{G} / \mathrm{A}$ \\
\hline UN_xiong_10764 & 66 & $\mathrm{~A} / \mathrm{C}$ \\
\hline UN_xiong_10767 & 253 & $\mathrm{G} / \mathrm{T}$ \\
\hline UN_xiong_10770 & 362 & $\mathrm{G} / \mathrm{A}$ \\
\hline UN_xiong_10776 & 274 & $\mathrm{~T} / \mathrm{G}$ \\
\hline UN_xiong_10799 & 436 & $\mathrm{~T} / \mathrm{C}$ \\
\hline UN_xiong_10802 & 147 & $\mathrm{G} / \mathrm{A}$ \\
\hline UN_xiong_10808 & 383 & $\mathrm{~T} / \mathrm{C}$ \\
\hline UN_xiong_10809 & 240 & $\mathrm{~A} / \mathrm{G}$ \\
\hline UN_xiong_10815 & 122 & $\mathrm{~T} / \mathrm{C}$ \\
\hline UN_xiong_10815 & 201 & $\mathrm{G} / \mathrm{A}$ \\
\hline UN_xiong_10835 & 201 & $\mathrm{C} / \mathrm{T}$ \\
\hline UN_xiong_10883 & 56 & $\mathrm{G} / \mathrm{A}$ \\
\hline UN_xiong_10884 & 89 & $\mathrm{G} / \mathrm{C}$ \\
\hline UN_xiong_10884 & 409 & $\mathrm{C} / \mathrm{T}$ \\
\hline UN_xiong_10894 & 183 & $A / G$ \\
\hline UN_xiong_10894 & 214 & $\mathrm{~A} / \mathrm{G}$ \\
\hline UN_xiong_10905 & 124 & $G / A$ \\
\hline UN_xiong_10906 & 192 & $\mathrm{~T} / \mathrm{G}$ \\
\hline UN_xiong_10911 & 35 & $\mathrm{C} / \mathrm{T}$ \\
\hline
\end{tabular}




\begin{tabular}{|c|c|c|}
\hline UN_xiong_10911 & 102 & $A / G$ \\
\hline UN_xiong_10911 & 133 & $\mathrm{C} / \mathrm{T}$ \\
\hline UN_xiong_10911 & 254 & $\mathrm{~T} / \mathrm{C}$ \\
\hline UN_xiong_10911 & 368 & $\mathrm{~T} / \mathrm{C}$ \\
\hline UN_xiong_10911 & 432 & $\mathrm{C} / \mathrm{A}$ \\
\hline UN_xiong_10911 & 466 & $\mathrm{~T} / \mathrm{C}$ \\
\hline UN_xiong_10911 & 761 & $\mathrm{G} / \mathrm{T}$ \\
\hline UN_xiong_10911 & 842 & $\mathrm{~A} / \mathrm{G}$ \\
\hline UN_xiong_10911 & 864 & $\mathrm{~T} / \mathrm{A}$ \\
\hline UN_xiong_10924 & 64 & $A / G$ \\
\hline UN_xiong_10924 & 212 & $\mathrm{C} / \mathrm{T}$ \\
\hline UN_xiong_10924 & 262 & $\mathrm{~A} / \mathrm{G}$ \\
\hline UN_xiong_10926 & 39 & $\mathrm{G} / \mathrm{A}$ \\
\hline UN_xiong_10928 & 125 & $\mathrm{~A} / \mathrm{G}$ \\
\hline UN_xiong_10941 & 417 & $\mathrm{~A} / \mathrm{G}$ \\
\hline UN_xiong_10941 & 653 & $\mathrm{~A} / \mathrm{G}$ \\
\hline UN_xiong_10941 & 748 & $\mathrm{~A} / \mathrm{T}$ \\
\hline UN_xiong_10941 & 906 & $A / G$ \\
\hline UN_xiong_10959 & 68 & $\mathrm{~A} / \mathrm{T}$ \\
\hline UN_xiong_10959 & 102 & $A / G$ \\
\hline UN_xiong_10959 & 139 & $\mathrm{C} / \mathrm{T}$ \\
\hline UN_xiong_10959 & 255 & $\mathrm{~A} / \mathrm{G}$ \\
\hline UN_xiong_10959 & 301 & $\mathrm{~A} / \mathrm{G}$ \\
\hline UN_xiong_10959 & 368 & $\mathrm{C} / \mathrm{T}$ \\
\hline UN_xiong_10959 & 501 & $A / G$ \\
\hline UN_xiong_10959 & 590 & $\mathrm{~T} / \mathrm{A}$ \\
\hline UN_xiong_10959 & 649 & $\mathrm{~T} / \mathrm{A}$ \\
\hline UN_xiong_10959 & 836 & $\mathrm{~T} / \mathrm{C}$ \\
\hline UN_xiong_10959 & 862 & $\mathrm{~T} / \mathrm{C}$ \\
\hline UN_xiong_10959 & 894 & $\mathrm{~T} / \mathrm{C}$ \\
\hline UN_xiong_10959 & 943 & $\mathrm{G} / \mathrm{A}$ \\
\hline UN_xiong_10959 & 997 & $\mathrm{~T} / \mathrm{C}$ \\
\hline UN_xiong_10959 & 1084 & $A / G$ \\
\hline UN_xiong_10971 & 309 & $\mathrm{G} / \mathrm{A}$ \\
\hline UN_xiong_10974 & 409 & $\mathrm{~T} / \mathrm{C}$ \\
\hline UN_xiong_10981 & 624 & $\mathrm{C} / \mathrm{T}$ \\
\hline UN_xiong_10999 & 230 & $A / G$ \\
\hline UN_xiong_11012 & 278 & $\mathrm{~T} / \mathrm{A}$ \\
\hline UN_xiong_11012 & 634 & $\mathrm{~T} / \mathrm{C}$ \\
\hline UN_xiong_11012 & 673 & $\mathrm{G} / \mathrm{A}$ \\
\hline UN_xiong_11012 & 1048 & $\mathrm{~T} / \mathrm{C}$ \\
\hline UN_xiong_11012 & 1080 & $\mathrm{G} / \mathrm{A}$ \\
\hline UN_xiong_11012 & 1142 & $\mathrm{~T} / \mathrm{C}$ \\
\hline UN_xiong_11012 & 1204 & $\mathrm{G} / \mathrm{A}$ \\
\hline UN_xiong_11012 & 1226 & $C / G$ \\
\hline UN_xiong_11012 & 1300 & $A / G$ \\
\hline UN_xiong_11012 & 1352 & $\mathrm{~T} / \mathrm{C}$ \\
\hline
\end{tabular}




\begin{tabular}{|c|c|c|}
\hline UN_xiong_11012 & 1468 & $\mathrm{~T} / \mathrm{C}$ \\
\hline UN_xiong_11012 & 1696 & $\mathrm{~A} / \mathrm{C}$ \\
\hline UN_xiong_11012 & 1775 & $\mathrm{~T} / \mathrm{C}$ \\
\hline UN_xiong_11021 & 304 & $\mathrm{C} / \mathrm{A}$ \\
\hline UN_xiong_11023 & 675 & $\mathrm{C} / \mathrm{T}$ \\
\hline UN_xiong_11026 & 220 & $\mathrm{~T} / \mathrm{C}$ \\
\hline UN_xiong_11026 & 265 & $\mathrm{G} / \mathrm{A}$ \\
\hline UN_xiong_11031 & 111 & $\mathrm{C} / \mathrm{T}$ \\
\hline UN_xiong_11031 & 134 & $A / G$ \\
\hline UN_xiong_11041 & 277 & $A / G$ \\
\hline UN_xiong_11054 & 298 & $\mathrm{G} / \mathrm{C}$ \\
\hline UN_xiong_11054 & 358 & $\mathrm{C} / \mathrm{T}$ \\
\hline UN_xiong_11054 & 393 & $A / G$ \\
\hline UN_xiong_11056 & 238 & $\mathrm{C} / \mathrm{T}$ \\
\hline UN_xiong_11064 & 50 & $\mathrm{G} / \mathrm{A}$ \\
\hline UN_xiong_11064 & 166 & $A / T$ \\
\hline UN_xiong_11064 & 278 & $\mathrm{~A} / \mathrm{C}$ \\
\hline UN_xiong_11064 & 331 & $\mathrm{~A} / \mathrm{C}$ \\
\hline UN_xiong_11086 & 201 & $\mathrm{C} / \mathrm{T}$ \\
\hline UN_xiong_11113 & 589 & $A / G$ \\
\hline UN_xiong_11125 & 245 & $A / G$ \\
\hline UN_xiong_11125 & 314 & $\mathrm{~A} / \mathrm{C}$ \\
\hline UN_xiong_11128 & 488 & $\mathrm{C} / \mathrm{A}$ \\
\hline UN_xiong_11142 & 114 & $\mathrm{C} / \mathrm{T}$ \\
\hline UN_xiong_11146 & 401 & $A / G$ \\
\hline UN_xiong_11146 & 552 & $A / G$ \\
\hline UN_xiong_11148 & 158 & $\mathrm{~T} / \mathrm{C}$ \\
\hline UN_xiong_11153 & 427 & $A / T$ \\
\hline UN_xiong_11153 & 491 & $A / G$ \\
\hline UN_xiong_11153 & 637 & $A / G$ \\
\hline UN_xiong_11167 & 677 & $\mathrm{G} / \mathrm{T}$ \\
\hline UN_xiong_11170 & 129 & $A / G$ \\
\hline UN_xiong_11170 & 195 & $\mathrm{~A} / \mathrm{C}$ \\
\hline UN_xiong_11181 & 668 & $\mathrm{~T} / \mathrm{G}$ \\
\hline UN_xiong_11195 & 352 & $\mathrm{G} / \mathrm{A}$ \\
\hline UN_xiong_11196 & 64 & $A / G$ \\
\hline UN_xiong_11196 & 633 & $\mathrm{G} / \mathrm{A}$ \\
\hline UN_xiong_11196 & 680 & $\mathrm{~T} / \mathrm{A}$ \\
\hline UN_xiong_11213 & 383 & $\mathrm{G} / \mathrm{A}$ \\
\hline UN_xiong_11213 & 461 & $\mathrm{C} / \mathrm{T}$ \\
\hline UN_xiong_11226 & 775 & $\mathrm{~T} / \mathrm{C}$ \\
\hline UN_xiong_11227 & 71 & $\mathrm{G} / \mathrm{A}$ \\
\hline UN_xiong_11238 & 260 & $\mathrm{G} / \mathrm{A}$ \\
\hline UN_xiong_11251 & 106 & $\mathrm{~T} / \mathrm{G}$ \\
\hline UN_xiong_11251 & 228 & $\mathrm{~T} / \mathrm{A}$ \\
\hline UN_xiong_11251 & 314 & $A / G$ \\
\hline UN_xiong_11251 & 502 & $\mathrm{G} / \mathrm{A}$ \\
\hline
\end{tabular}




\begin{tabular}{|c|c|c|}
\hline UN_xiong_11264 & 173 & $\mathrm{G} / \mathrm{C}$ \\
\hline UN_xiong_11274 & 164 & $\mathrm{~A} / \mathrm{G}$ \\
\hline UN_xiong_11274 & 236 & $A / G$ \\
\hline UN_xiong_11274 & 383 & $A / G$ \\
\hline UN_xiong_11275 & 105 & $\mathrm{G} / \mathrm{A}$ \\
\hline UN_xiong_11286 & 31 & $\mathrm{~A} / \mathrm{G}$ \\
\hline UN_xiong_11288 & 195 & $\mathrm{G} / \mathrm{A}$ \\
\hline UN_xiong_11295 & 182 & $\mathrm{~T} / \mathrm{A}$ \\
\hline UN_xiong_11302 & 198 & $\mathrm{~T} / \mathrm{C}$ \\
\hline UN_xiong_11302 & 314 & $\mathrm{~T} / \mathrm{A}$ \\
\hline UN_xiong_11302 & 334 & $\mathrm{C} / \mathrm{A}$ \\
\hline UN_xiong_11306 & 159 & $A / G$ \\
\hline UN_xiong_11308 & 33 & $\mathrm{C} / \mathrm{A}$ \\
\hline UN_xiong_11308 & 150 & $\mathrm{~T} / \mathrm{C}$ \\
\hline UN_xiong_11308 & 258 & $\mathrm{~T} / \mathrm{C}$ \\
\hline UN_xiong_11317 & 375 & $\mathrm{~T} / \mathrm{C}$ \\
\hline UN_xiong_11317 & 702 & $\mathrm{G} / \mathrm{A}$ \\
\hline UN_xiong_11324 & 124 & $\mathrm{C} / \mathrm{T}$ \\
\hline UN_xiong_11327 & 115 & $A / G$ \\
\hline UN_xiong_11327 & 153 & $\mathrm{G} / \mathrm{T}$ \\
\hline UN_xiong_11327 & 268 & $\mathrm{G} / \mathrm{T}$ \\
\hline UN_xiong_11327 & 302 & $\mathrm{~T} / \mathrm{C}$ \\
\hline UN_xiong_11332 & 76 & $\mathrm{~A} / \mathrm{G}$ \\
\hline UN_xiong_11336 & 187 & $\mathrm{C} / \mathrm{T}$ \\
\hline UN_xiong_11336 & 257 & $\mathrm{C} / \mathrm{T}$ \\
\hline UN_xiong_11336 & 297 & $\mathrm{~T} / \mathrm{C}$ \\
\hline UN_xiong_11338 & 168 & $\mathrm{G} / \mathrm{T}$ \\
\hline UN_xiong_11342 & 641 & $A / G$ \\
\hline UN_xiong_11342 & 1478 & $A / G$ \\
\hline UN_xiong_11342 & 1686 & $\mathrm{C} / \mathrm{T}$ \\
\hline UN_xiong_11351 & 93 & $\mathrm{G} / \mathrm{C}$ \\
\hline UN_xiong_11364 & 262 & $\mathrm{C} / \mathrm{T}$ \\
\hline UN_xiong_11364 & 282 & $\mathrm{C} / \mathrm{T}$ \\
\hline UN_xiong_11364 & 499 & $\mathrm{~A} / \mathrm{C}$ \\
\hline UN_xiong_11366 & 157 & $\mathrm{~A} / \mathrm{T}$ \\
\hline UN_xiong_11371 & 227 & $A / G$ \\
\hline UN_xiong_11374 & 8 & $\mathrm{G} / \mathrm{T}$ \\
\hline UN_xiong_11378 & 27 & $A / G$ \\
\hline UN_xiong_11378 & 116 & $\mathrm{~T} / \mathrm{A}$ \\
\hline UN_xiong_11403 & 156 & $\mathrm{C} / \mathrm{T}$ \\
\hline UN_xiong_11431 & 254 & $\mathrm{~T} / \mathrm{C}$ \\
\hline UN_xiong_11431 & 294 & $\mathrm{C} / \mathrm{T}$ \\
\hline UN_xiong_11443 & 77 & $\mathrm{C} / \mathrm{T}$ \\
\hline UN_xiong_11449 & 112 & $A / G$ \\
\hline UN_xiong_11464 & 683 & $\mathrm{G} / \mathrm{A}$ \\
\hline UN_xiong_11464 & 1223 & $\mathrm{G} / \mathrm{T}$ \\
\hline UN_xiong_11470 & 144 & $\mathrm{C} / \mathrm{A}$ \\
\hline
\end{tabular}




\begin{tabular}{|c|c|c|}
\hline UN_xiong_11470 & 338 & $\mathrm{C} / \mathrm{T}$ \\
\hline UN_xiong_11474 & 149 & $\mathrm{G} / \mathrm{T}$ \\
\hline UN_xiong_11475 & 353 & $\mathrm{~T} / \mathrm{C}$ \\
\hline UN_xiong_11484 & 483 & $G / A$ \\
\hline UN_xiong_11484 & 687 & $A / T$ \\
\hline UN_xiong_11495 & 216 & $A / G$ \\
\hline UN_xiong_11502 & 203 & $\mathrm{~T} / \mathrm{C}$ \\
\hline UN_xiong_11502 & 459 & $\mathrm{~T} / \mathrm{C}$ \\
\hline UN_xiong_11502 & 495 & $\mathrm{~T} / \mathrm{C}$ \\
\hline UN_xiong_11516 & 401 & $\mathrm{C} / \mathrm{T}$ \\
\hline UN_xiong_11521 & 143 & $A / G$ \\
\hline UN_xiong_11523 & 96 & $\mathrm{C} / \mathrm{T}$ \\
\hline UN_xiong_11528 & 10 & $\mathrm{~A} / \mathrm{T}$ \\
\hline UN_xiong_11531 & 158 & $A / G$ \\
\hline UN_xiong_11550 & 113 & $\mathrm{~A} / \mathrm{C}$ \\
\hline UN_xiong_11568 & 316 & $\mathrm{~T} / \mathrm{C}$ \\
\hline UN_xiong_11568 & 374 & $\mathrm{~T} / \mathrm{A}$ \\
\hline UN_xiong_11568 & 1154 & $\mathrm{~T} / \mathrm{C}$ \\
\hline UN_xiong_11569 & 84 & $\mathrm{~T} / \mathrm{C}$ \\
\hline UN_xiong_11569 & 119 & $\mathrm{~T} / \mathrm{C}$ \\
\hline UN_xiong_11569 & 267 & $\mathrm{~A} / \mathrm{T}$ \\
\hline UN_xiong_11580 & 103 & $\mathrm{G} / \mathrm{A}$ \\
\hline UN_xiong_11580 & 213 & $\mathrm{~A} / \mathrm{T}$ \\
\hline UN_xiong_11580 & 237 & $A / G$ \\
\hline UN_xiong_11580 & 304 & $G / A$ \\
\hline UN_xiong_11585 & 209 & $\mathrm{G} / \mathrm{A}$ \\
\hline UN_xiong_11585 & 267 & $\mathrm{G} / \mathrm{C}$ \\
\hline UN_xiong_11600 & 134 & $A / G$ \\
\hline UN_xiong_11600 & 169 & $G / A$ \\
\hline UN_xiong_11600 & 283 & $\mathrm{~T} / \mathrm{C}$ \\
\hline UN_xiong_11613 & 182 & $\mathrm{~T} / \mathrm{G}$ \\
\hline UN_xiong_11613 & 374 & $A / G$ \\
\hline UN_xiong_11615 & 169 & $A / G$ \\
\hline UN_xiong_11627 & 681 & $\mathrm{~T} / \mathrm{C}$ \\
\hline UN_xiong_11636 & 132 & $\mathrm{~T} / \mathrm{C}$ \\
\hline UN_xiong_11636 & 314 & $\mathrm{C} / \mathrm{T}$ \\
\hline UN_xiong_11645 & 437 & $A / G$ \\
\hline UN_xiong_11645 & 692 & $\mathrm{C} / \mathrm{T}$ \\
\hline UN_xiong_11645 & 786 & $\mathrm{~T} / \mathrm{G}$ \\
\hline UN_xiong_11650 & 71 & $A / G$ \\
\hline UN_xiong_11652 & 303 & $\mathrm{C} / \mathrm{T}$ \\
\hline UN_xiong_11652 & 394 & $\mathrm{G} / \mathrm{C}$ \\
\hline UN_xiong_11652 & 471 & $\mathrm{~T} / \mathrm{G}$ \\
\hline UN_xiong_11670 & 314 & $T / G$ \\
\hline UN_xiong_11680 & 24 & $\mathrm{~T} / \mathrm{C}$ \\
\hline UN_xiong_11685 & 263 & $A / G$ \\
\hline UN_xiong_11687 & 359 & $\mathrm{~T} / \mathrm{C}$ \\
\hline
\end{tabular}




\begin{tabular}{|c|c|c|}
\hline UN_xiong_11692 & 201 & $\mathrm{~T} / \mathrm{C}$ \\
\hline UN_xiong_11708 & 88 & $A / G$ \\
\hline UN_xiong_11708 & 168 & $\mathrm{C} / \mathrm{T}$ \\
\hline UN_xiong_11731 & 358 & $C / A$ \\
\hline UN_xiong_11755 & 73 & $C / A$ \\
\hline UN_xiong_11755 & 144 & $\mathrm{~T} / \mathrm{G}$ \\
\hline UN_xiong_11755 & 497 & $A / G$ \\
\hline UN_xiong_11755 & 569 & $\mathrm{G} / \mathrm{A}$ \\
\hline UN_xiong_11755 & 710 & $C / G$ \\
\hline UN_xiong_11761 & 310 & $\mathrm{C} / \mathrm{T}$ \\
\hline UN_xiong_11761 & 332 & $G / A$ \\
\hline UN_xiong_11761 & 601 & $\mathrm{G} / \mathrm{T}$ \\
\hline UN_xiong_11761 & 1116 & $A / G$ \\
\hline UN_xiong_11761 & 1224 & $\mathrm{~A} / \mathrm{C}$ \\
\hline UN_xiong_11761 & 1305 & $\mathrm{G} / \mathrm{A}$ \\
\hline UN_xiong_11761 & 1349 & $\mathrm{G} / \mathrm{A}$ \\
\hline UN_xiong_11761 & 1428 & $\mathrm{~T} / \mathrm{G}$ \\
\hline UN_xiong_11761 & 1528 & $\mathrm{~T} / \mathrm{C}$ \\
\hline UN_xiong_11783 & 28 & $G / A$ \\
\hline UN_xiong_11783 & 193 & $\mathrm{~A} / \mathrm{C}$ \\
\hline UN_xiong_11783 & 234 & $\mathrm{G} / \mathrm{C}$ \\
\hline UN_xiong_11795 & 332 & $\mathrm{C} / \mathrm{T}$ \\
\hline UN_xiong_11797 & 267 & $\mathrm{C} / \mathrm{T}$ \\
\hline UN_xiong_11805 & 351 & $A / G$ \\
\hline UN_xiong_11811 & 97 & $\mathrm{C} / \mathrm{T}$ \\
\hline UN_xiong_11827 & 479 & $\mathrm{C} / \mathrm{T}$ \\
\hline UN_xiong_11827 & 745 & $A / G$ \\
\hline UN_xiong_11855 & 552 & $\mathrm{C} / \mathrm{T}$ \\
\hline UN_xiong_11863 & 640 & $\mathrm{G} / \mathrm{A}$ \\
\hline UN_xiong_11870 & 396 & $\mathrm{C} / \mathrm{T}$ \\
\hline UN_xiong_11870 & 454 & $G / A$ \\
\hline UN_xiong_11880 & 557 & $\mathrm{C} / \mathrm{T}$ \\
\hline UN_xiong_11880 & 660 & $\mathrm{~T} / \mathrm{C}$ \\
\hline UN_xiong_11902 & 24 & $A / G$ \\
\hline UN_xiong_11902 & 50 & $\mathrm{~A} / \mathrm{C}$ \\
\hline UN_xiong_11907 & 80 & $A / G$ \\
\hline UN_xiong_11919 & 352 & $\mathrm{G} / \mathrm{T}$ \\
\hline UN_xiong_11935 & 180 & $\mathrm{~T} / \mathrm{C}$ \\
\hline UN_xiong_11935 & 452 & $\mathrm{~A} / \mathrm{C}$ \\
\hline UN_xiong_11942 & 633 & $A / G$ \\
\hline UN_xiong_11967 & 338 & $A / G$ \\
\hline UN_xiong_11967 & 494 & $\mathrm{~T} / \mathrm{C}$ \\
\hline UN_xiong_11967 & 563 & $\mathrm{G} / \mathrm{T}$ \\
\hline UN_xiong_11967 & 694 & $\mathrm{~T} / \mathrm{C}$ \\
\hline UN_xiong_11980 & 393 & $\mathrm{C} / \mathrm{T}$ \\
\hline UN_xiong_11980 & 1018 & $\mathrm{C} / \mathrm{T}$ \\
\hline UN_xiong_11986 & 178 & $\mathrm{~T} / \mathrm{C}$ \\
\hline
\end{tabular}




\begin{tabular}{|c|c|c|}
\hline UN_xiong_12004 & 12 & $C / G$ \\
\hline UN_xiong_12004 & 61 & $\mathrm{G} / \mathrm{A}$ \\
\hline UN_xiong_12004 & 190 & $\mathrm{~T} / \mathrm{C}$ \\
\hline UN_xiong_12007 & 7 & $A / G$ \\
\hline UN_xiong_12007 & 190 & $\mathrm{~T} / \mathrm{C}$ \\
\hline UN_xiong_12010 & 286 & $\mathrm{~T} / \mathrm{C}$ \\
\hline UN_xiong_12023 & 91 & $\mathrm{C} / \mathrm{T}$ \\
\hline UN_xiong_12024 & 60 & $\mathrm{C} / \mathrm{T}$ \\
\hline UN_xiong_12036 & 25 & $\mathrm{G} / \mathrm{A}$ \\
\hline UN_xiong_12036 & 907 & $A / G$ \\
\hline UN_xiong_12061 & 568 & $\mathrm{~A} / \mathrm{C}$ \\
\hline UN_xiong_12061 & 590 & $\mathrm{C} / \mathrm{T}$ \\
\hline UN_xiong_12061 & 716 & $\mathrm{~A} / \mathrm{C}$ \\
\hline UN_xiong_12061 & 766 & $\mathrm{G} / \mathrm{A}$ \\
\hline UN_xiong_12061 & 847 & $\mathrm{~T} / \mathrm{C}$ \\
\hline UN_xiong_12064 & 343 & $A / G$ \\
\hline UN_xiong_12066 & 158 & $\mathrm{C} / \mathrm{T}$ \\
\hline UN_xiong_12066 & 197 & $\mathrm{G} / \mathrm{A}$ \\
\hline UN_xiong_12067 & 188 & $\mathrm{C} / \mathrm{A}$ \\
\hline UN_xiong_12067 & 346 & $\mathrm{G} / \mathrm{A}$ \\
\hline UN_xiong_12070 & 116 & $\mathrm{~T} / \mathrm{A}$ \\
\hline UN_xiong_12078 & 143 & $\mathrm{~A} / \mathrm{C}$ \\
\hline UN_xiong_12078 & 165 & $\mathrm{~T} / \mathrm{C}$ \\
\hline UN_xiong_12078 & 1141 & $\mathrm{G} / \mathrm{T}$ \\
\hline UN_xiong_12088 & 835 & $\mathrm{C} / \mathrm{T}$ \\
\hline UN_xiong_12088 & 866 & $\mathrm{~A} / \mathrm{T}$ \\
\hline UN_xiong_12091 & 320 & $C / A$ \\
\hline UN_xiong_12093 & 57 & $\mathrm{~T} / \mathrm{A}$ \\
\hline UN_xiong_12093 & 206 & $C / G$ \\
\hline UN_xiong_12093 & 261 & $\mathrm{G} / \mathrm{A}$ \\
\hline UN_xiong_12093 & 307 & $A / G$ \\
\hline UN_xiong_12093 & 469 & $\mathrm{G} / \mathrm{A}$ \\
\hline UN_xiong_12101 & 74 & $\mathrm{C} / \mathrm{T}$ \\
\hline UN_xiong_12101 & 309 & $\mathrm{G} / \mathrm{A}$ \\
\hline UN_xiong_12115 & 131 & $\mathrm{G} / \mathrm{A}$ \\
\hline UN_xiong_12115 & 230 & $\mathrm{~A} / \mathrm{T}$ \\
\hline UN_xiong_12115 & 318 & $\mathrm{~T} / \mathrm{C}$ \\
\hline UN_xiong_12115 & 351 & $\mathrm{G} / \mathrm{A}$ \\
\hline UN_xiong_12115 & 640 & $\mathrm{~A} / \mathrm{T}$ \\
\hline UN_xiong_12115 & 686 & $C / G$ \\
\hline UN_xiong_12115 & 889 & $A / G$ \\
\hline UN_xiong_12130 & 83 & $\mathrm{~A} / \mathrm{T}$ \\
\hline UN_xiong_12130 & 139 & $A / G$ \\
\hline UN_xiong_12130 & 232 & $\mathrm{G} / \mathrm{T}$ \\
\hline UN_xiong_12137 & 616 & $C / G$ \\
\hline UN_xiong_12137 & 777 & $\mathrm{C} / \mathrm{T}$ \\
\hline UN_xiong_12137 & 1330 & $A / G$ \\
\hline
\end{tabular}




\begin{tabular}{|c|c|c|}
\hline UN_xiong_12137 & 1857 & $A / G$ \\
\hline UN_xiong_12137 & 2362 & $\mathrm{C} / \mathrm{A}$ \\
\hline UN_xiong_12137 & 2731 & $A / G$ \\
\hline UN_xiong_12139 & 246 & $\mathrm{G} / \mathrm{A}$ \\
\hline UN_xiong_12139 & 383 & $A / G$ \\
\hline UN_xiong_12139 & 426 & $\mathrm{G} / \mathrm{A}$ \\
\hline UN_xiong_12140 & 73 & $\mathrm{C} / \mathrm{G}$ \\
\hline UN_xiong_12140 & 249 & $A / G$ \\
\hline UN_xiong_12140 & 473 & $\mathrm{G} / \mathrm{A}$ \\
\hline UN_xiong_12149 & 134 & $\mathrm{~T} / \mathrm{C}$ \\
\hline UN_xiong_12149 & 189 & $\mathrm{~A} / \mathrm{T}$ \\
\hline UN_xiong_12149 & 317 & $\mathrm{G} / \mathrm{A}$ \\
\hline UN_xiong_12170 & 420 & $\mathrm{~A} / \mathrm{C}$ \\
\hline UN_xiong_12170 & 654 & $\mathrm{G} / \mathrm{A}$ \\
\hline UN_xiong_12177 & 292 & $\mathrm{~T} / \mathrm{C}$ \\
\hline UN_xiong_12177 & 426 & $\mathrm{~A} / \mathrm{T}$ \\
\hline UN_xiong_12177 & 466 & $A / G$ \\
\hline UN_xiong_12184 & 262 & $\mathrm{~A} / \mathrm{C}$ \\
\hline UN_xiong_12190 & 210 & $\mathrm{~T} / \mathrm{C}$ \\
\hline UN_xiong_12190 & 241 & $A / G$ \\
\hline UN_xiong_12200 & 294 & $\mathrm{~T} / \mathrm{G}$ \\
\hline UN_xiong_12205 & 134 & $\mathrm{~T} / \mathrm{G}$ \\
\hline UN_xiong_12214 & 232 & $\mathrm{C} / \mathrm{T}$ \\
\hline UN_xiong_12226 & 215 & $\mathrm{G} / \mathrm{T}$ \\
\hline UN_xiong_12227 & 157 & $\mathrm{C} / \mathrm{T}$ \\
\hline UN_xiong_12227 & 324 & $\mathrm{G} / \mathrm{A}$ \\
\hline UN_xiong_12237 & 293 & $\mathrm{C} / \mathrm{G}$ \\
\hline UN_xiong_12237 & 1581 & $\mathrm{~T} / \mathrm{C}$ \\
\hline UN_xiong_12237 & 1833 & $\mathrm{G} / \mathrm{C}$ \\
\hline UN_xiong_12264 & 329 & $\mathrm{~T} / \mathrm{C}$ \\
\hline UN_xiong_12278 & 64 & $\mathrm{~T} / \mathrm{A}$ \\
\hline UN_xiong_12278 & 131 & $\mathrm{~A} / \mathrm{T}$ \\
\hline UN_xiong_12278 & 160 & $A / G$ \\
\hline UN_xiong_12278 & 184 & $\mathrm{~A} / \mathrm{G}$ \\
\hline UN_xiong_12278 & 226 & $\mathrm{~A} / \mathrm{G}$ \\
\hline UN_xiong_12278 & 255 & $\mathrm{G} / \mathrm{A}$ \\
\hline UN_xiong_12278 & 280 & $\mathrm{~T} / \mathrm{G}$ \\
\hline UN_xiong_12278 & 339 & $\mathrm{G} / \mathrm{A}$ \\
\hline UN_xiong_12278 & 371 & $A / G$ \\
\hline UN_xiong_12278 & 434 & $\mathrm{~T} / \mathrm{C}$ \\
\hline UN_xiong_12278 & 491 & $\mathrm{G} / \mathrm{T}$ \\
\hline UN_xiong_12278 & 520 & $\mathrm{C} / \mathrm{T}$ \\
\hline UN_xiong_12278 & 556 & $\mathrm{~A} / \mathrm{G}$ \\
\hline UN_xiong_12278 & 591 & $\mathrm{G} / \mathrm{A}$ \\
\hline UN_xiong_12278 & 623 & $A / G$ \\
\hline UN_xiong_12278 & 696 & $\mathrm{C} / \mathrm{T}$ \\
\hline UN_xiong_12278 & 721 & $A / G$ \\
\hline
\end{tabular}




\begin{tabular}{|c|c|c|}
\hline UN_xiong_12278 & 747 & $\mathrm{~T} / \mathrm{C}$ \\
\hline UN_xiong_12278 & 780 & $\mathrm{C} / \mathrm{T}$ \\
\hline UN_xiong_12278 & 827 & $\mathrm{~A} / \mathrm{T}$ \\
\hline UN_xiong_12278 & 863 & $A / G$ \\
\hline UN_xiong_12278 & 897 & $A / G$ \\
\hline UN_xiong_12278 & 1133 & $\mathrm{~A} / \mathrm{G}$ \\
\hline UN_xiong_12288 & 32 & $\mathrm{~T} / \mathrm{C}$ \\
\hline UN_xiong_12296 & 548 & $\mathrm{~T} / \mathrm{C}$ \\
\hline UN_xiong_12299 & 329 & $A / G$ \\
\hline UN_xiong_12300 & 207 & $\mathrm{G} / \mathrm{A}$ \\
\hline UN_xiong_12313 & 28 & $\mathrm{C} / \mathrm{T}$ \\
\hline UN_xiong_12325 & 244 & $\mathrm{G} / \mathrm{A}$ \\
\hline UN_xiong_12327 & 154 & $\mathrm{~A} / \mathrm{G}$ \\
\hline UN_xiong_12334 & 259 & $\mathrm{G} / \mathrm{A}$ \\
\hline UN_xiong_12336 & 155 & $\mathrm{~T} / \mathrm{C}$ \\
\hline UN_xiong_12342 & 121 & $\mathrm{~A} / \mathrm{T}$ \\
\hline UN_xiong_12343 & 27 & $A / G$ \\
\hline UN_xiong_12343 & 104 & $\mathrm{G} / \mathrm{T}$ \\
\hline UN_xiong_12343 & 157 & $A / G$ \\
\hline UN_xiong_12355 & 202 & $\mathrm{G} / \mathrm{A}$ \\
\hline UN_xiong_12358 & 71 & $\mathrm{G} / \mathrm{C}$ \\
\hline UN_xiong_12363 & 121 & $\mathrm{~T} / \mathrm{C}$ \\
\hline UN_xiong_12363 & 626 & $\mathrm{~A} / \mathrm{G}$ \\
\hline UN_xiong_12363 & 791 & $\mathrm{~T} / \mathrm{C}$ \\
\hline UN_xiong_12363 & 1128 & $\mathrm{~T} / \mathrm{C}$ \\
\hline UN_xiong_12363 & 1178 & $\mathrm{G} / \mathrm{T}$ \\
\hline UN_xiong_12363 & 1249 & $\mathrm{~T} / \mathrm{A}$ \\
\hline UN_xiong_12363 & 1287 & $\mathrm{~T} / \mathrm{C}$ \\
\hline UN_xiong_12363 & 1331 & $\mathrm{~T} / \mathrm{C}$ \\
\hline UN_xiong_12363 & 1533 & $\mathrm{C} / \mathrm{T}$ \\
\hline UN_xiong_12374 & 211 & $A / G$ \\
\hline UN_xiong_12384 & 389 & $\mathrm{G} / \mathrm{A}$ \\
\hline UN_xiong_12386 & 356 & $A / G$ \\
\hline UN_xiong_12390 & 523 & $\mathrm{~A} / \mathrm{G}$ \\
\hline UN_xiong_12390 & 557 & $\mathrm{~T} / \mathrm{C}$ \\
\hline UN_xiong_12390 & 650 & $A / G$ \\
\hline UN_xiong_12396 & 33 & $\mathrm{~T} / \mathrm{C}$ \\
\hline UN_xiong_12396 & 159 & $\mathrm{C} / \mathrm{T}$ \\
\hline UN_xiong_12405 & 40 & $\mathrm{G} / \mathrm{A}$ \\
\hline UN_xiong_12405 & 384 & $\mathrm{~T} / \mathrm{C}$ \\
\hline UN_xiong_12413 & 35 & $\mathrm{G} / \mathrm{A}$ \\
\hline UN_xiong_12424 & 326 & $\mathrm{C} / \mathrm{A}$ \\
\hline UN_xiong_12424 & 445 & $\mathrm{G} / \mathrm{A}$ \\
\hline UN_xiong_12424 & 491 & $\mathrm{C} / \mathrm{T}$ \\
\hline UN_xiong_12426 & 165 & $\mathrm{G} / \mathrm{C}$ \\
\hline UN_xiong_12428 & 379 & $\mathrm{C} / \mathrm{A}$ \\
\hline UN_xiong_12442 & 89 & $\mathrm{C} / \mathrm{T}$ \\
\hline
\end{tabular}




\begin{tabular}{|c|c|c|}
\hline UN_xiong_12451 & 249 & $\mathrm{C} / \mathrm{T}$ \\
\hline UN_xiong_12458 & 64 & $\mathrm{~T} / \mathrm{C}$ \\
\hline UN_xiong_12466 & 62 & $\mathrm{~A} / \mathrm{G}$ \\
\hline UN_xiong_12466 & 287 & $\mathrm{~T} / \mathrm{C}$ \\
\hline UN_xiong_12466 & 307 & $\mathrm{C} / \mathrm{T}$ \\
\hline UN_xiong_12466 & 437 & $\mathrm{~A} / \mathrm{G}$ \\
\hline UN_xiong_12505 & 110 & $\mathrm{C} / \mathrm{T}$ \\
\hline UN_xiong_12518 & 223 & $\mathrm{G} / \mathrm{A}$ \\
\hline UN_xiong_12518 & 429 & $\mathrm{~A} / \mathrm{C}$ \\
\hline UN_xiong_12520 & 145 & $\mathrm{~T} / \mathrm{C}$ \\
\hline UN_xiong_12522 & 236 & $\mathrm{G} / \mathrm{A}$ \\
\hline UN_xiong_12564 & 519 & $\mathrm{G} / \mathrm{A}$ \\
\hline UN_xiong_12564 & 783 & $\mathrm{C} / \mathrm{A}$ \\
\hline UN_xiong_12579 & 469 & $\mathrm{G} / \mathrm{A}$ \\
\hline UN_xiong_12580 & 937 & $\mathrm{G} / \mathrm{A}$ \\
\hline UN_xiong_12580 & 1007 & $\mathrm{G} / \mathrm{A}$ \\
\hline UN_xiong_12580 & 2151 & $A / G$ \\
\hline UN_xiong_12580 & 2623 & $\mathrm{~T} / \mathrm{C}$ \\
\hline UN_xiong_12583 & 192 & $\mathrm{~T} / \mathrm{C}$ \\
\hline UN_xiong_12583 & 317 & $\mathrm{~T} / \mathrm{C}$ \\
\hline UN_xiong_12586 & 44 & $\mathrm{~T} / \mathrm{G}$ \\
\hline UN_xiong_12606 & 63 & $\mathrm{~A} / \mathrm{G}$ \\
\hline UN_xiong_12617 & 1093 & $\mathrm{C} / \mathrm{T}$ \\
\hline UN_xiong_12628 & 169 & $\mathrm{C} / \mathrm{T}$ \\
\hline UN_xiong_12628 & 235 & $\mathrm{C} / \mathrm{A}$ \\
\hline UN_xiong_12628 & 348 & $\mathrm{C} / \mathrm{T}$ \\
\hline UN_xiong_12628 & 396 & $A / G$ \\
\hline UN_xiong_12628 & 630 & $\mathrm{C} / \mathrm{T}$ \\
\hline UN_xiong_12628 & 693 & $\mathrm{~T} / \mathrm{C}$ \\
\hline UN_xiong_12628 & 842 & $\mathrm{~T} / \mathrm{A}$ \\
\hline UN_xiong_12628 & 896 & $A / G$ \\
\hline UN_xiong_12629 & 147 & $\mathrm{G} / \mathrm{C}$ \\
\hline UN_xiong_12641 & 383 & $\mathrm{G} / \mathrm{A}$ \\
\hline UN_xiong_12651 & 31 & $\mathrm{~T} / \mathrm{A}$ \\
\hline UN_xiong_12651 & 69 & $\mathrm{~A} / \mathrm{G}$ \\
\hline UN_xiong_12663 & 279 & $\mathrm{C} / \mathrm{T}$ \\
\hline UN_xiong_12665 & 661 & $A / G$ \\
\hline UN_xiong_12671 & 102 & $\mathrm{G} / \mathrm{A}$ \\
\hline UN_xiong_12671 & 308 & $\mathrm{C} / \mathrm{T}$ \\
\hline UN_xiong_12679 & 40 & $A / G$ \\
\hline UN_xiong_12679 & 269 & $\mathrm{G} / \mathrm{A}$ \\
\hline UN_xiong_12679 & 314 & $\mathrm{~T} / \mathrm{C}$ \\
\hline UN_xiong_12706 & 407 & $\mathrm{~T} / \mathrm{C}$ \\
\hline UN_xiong_12707 & 31 & $\mathrm{G} / \mathrm{A}$ \\
\hline UN_xiong_12729 & 327 & $\mathrm{C} / \mathrm{T}$ \\
\hline UN_xiong_12729 & 507 & $\mathrm{G} / \mathrm{A}$ \\
\hline UN_xiong_12757 & 295 & $\mathrm{C} / \mathrm{T}$ \\
\hline
\end{tabular}




\begin{tabular}{|c|c|c|}
\hline UN_xiong_12761 & 170 & $\mathrm{C} / \mathrm{A}$ \\
\hline UN_xiong_12761 & 262 & $\mathrm{~T} / \mathrm{A}$ \\
\hline UN_xiong_12761 & 329 & $\mathrm{C} / \mathrm{T}$ \\
\hline UN_xiong_12762 & 500 & $A / G$ \\
\hline UN_xiong_12762 & 637 & $\mathrm{~T} / \mathrm{C}$ \\
\hline UN_xiong_12762 & 718 & $\mathrm{C} / \mathrm{A}$ \\
\hline UN_xiong_12762 & 869 & $\mathrm{C} / \mathrm{T}$ \\
\hline UN_xiong_12789 & 314 & $\mathrm{~T} / \mathrm{C}$ \\
\hline UN_xiong_12797 & 162 & $\mathrm{~T} / \mathrm{C}$ \\
\hline UN_xiong_12803 & 154 & $\mathrm{~T} / \mathrm{C}$ \\
\hline UN_xiong_12804 & 269 & $\mathrm{G} / \mathrm{A}$ \\
\hline UN_xiong_12804 & 1072 & $\mathrm{~T} / \mathrm{C}$ \\
\hline UN_xiong_12804 & 1120 & $\mathrm{C} / \mathrm{T}$ \\
\hline UN_xiong_12804 & 1234 & $\mathrm{G} / \mathrm{C}$ \\
\hline UN_xiong_12816 & 302 & $\mathrm{C} / \mathrm{A}$ \\
\hline UN_xiong_12825 & 900 & $\mathrm{~A} / \mathrm{T}$ \\
\hline UN_xiong_12828 & 101 & $\mathrm{C} / \mathrm{A}$ \\
\hline UN_xiong_12830 & 125 & $\mathrm{C} / \mathrm{A}$ \\
\hline UN_xiong_12830 & 190 & $\mathrm{~T} / \mathrm{C}$ \\
\hline UN_xiong_12830 & 465 & $\mathrm{G} / \mathrm{A}$ \\
\hline UN_xiong_12830 & 732 & $\mathrm{C} / \mathrm{A}$ \\
\hline UN_xiong_12852 & 139 & $\mathrm{G} / \mathrm{A}$ \\
\hline UN_xiong_12859 & 659 & $\mathrm{~A} / \mathrm{C}$ \\
\hline UN_xiong_12859 & 687 & $\mathrm{G} / \mathrm{C}$ \\
\hline UN_xiong_12859 & 718 & $\mathrm{C} / \mathrm{T}$ \\
\hline UN_xiong_12859 & 757 & $\mathrm{~T} / \mathrm{C}$ \\
\hline UN_xiong_12868 & 183 & $\mathrm{~T} / \mathrm{A}$ \\
\hline UN_xiong_12868 & 354 & $\mathrm{G} / \mathrm{A}$ \\
\hline UN_xiong_12868 & 380 & $G / A$ \\
\hline UN_xiong_12868 & 433 & $\mathrm{C} / \mathrm{A}$ \\
\hline UN_xiong_12868 & 458 & $\mathrm{~T} / \mathrm{G}$ \\
\hline UN_xiong_12868 & 510 & $\mathrm{C} / \mathrm{T}$ \\
\hline UN_xiong_12868 & 641 & $\mathrm{C} / \mathrm{T}$ \\
\hline UN_xiong_12868 & 662 & $\mathrm{C} / \mathrm{T}$ \\
\hline UN_xiong_12875 & 211 & $\mathrm{C} / \mathrm{A}$ \\
\hline UN_xiong_12875 & 466 & $\mathrm{~A} / \mathrm{G}$ \\
\hline UN_xiong_12890 & 475 & $\mathrm{C} / \mathrm{T}$ \\
\hline UN_xiong_12890 & 691 & $\mathrm{G} / \mathrm{A}$ \\
\hline UN_xiong_12893 & 145 & $A / G$ \\
\hline UN_xiong_12894 & 153 & $A / G$ \\
\hline UN_xiong_12896 & 294 & $\mathrm{~T} / \mathrm{C}$ \\
\hline UN_xiong_12898 & 226 & $\mathrm{~T} / \mathrm{C}$ \\
\hline UN_xiong_12899 & 207 & $\mathrm{C} / \mathrm{T}$ \\
\hline UN_xiong_12900 & 43 & $\mathrm{~T} / \mathrm{C}$ \\
\hline UN_xiong_12932 & 138 & $\mathrm{C} / \mathrm{T}$ \\
\hline UN_xiong_12932 & 373 & $\mathrm{C} / \mathrm{T}$ \\
\hline UN_xiong_12936 & 964 & $\mathrm{~A} / \mathrm{G}$ \\
\hline
\end{tabular}




\begin{tabular}{|c|c|c|}
\hline UN_xiong_12936 & 1010 & $\mathrm{C} / \mathrm{T}$ \\
\hline UN_xiong_12963 & 622 & $\mathrm{C} / \mathrm{T}$ \\
\hline UN_xiong_12975 & 375 & $\mathrm{~A} / \mathrm{G}$ \\
\hline UN_xiong_12982 & 250 & $\mathrm{G} / \mathrm{A}$ \\
\hline UN_xiong_12985 & 192 & $\mathrm{C} / \mathrm{G}$ \\
\hline UN_xiong_12985 & 333 & $\mathrm{~A} / \mathrm{G}$ \\
\hline UN_xiong_12987 & 222 & $\mathrm{C} / \mathrm{A}$ \\
\hline UN_xiong_12988 & 84 & $\mathrm{~T} / \mathrm{C}$ \\
\hline UN_xiong_12988 & 206 & $\mathrm{~A} / \mathrm{C}$ \\
\hline UN_xiong_12988 & 275 & $\mathrm{~T} / \mathrm{C}$ \\
\hline UN_xiong_12998 & 189 & $\mathrm{~T} / \mathrm{C}$ \\
\hline UN_xiong_13004 & 511 & $\mathrm{~T} / \mathrm{C}$ \\
\hline UN_xiong_13013 & 227 & $\mathrm{~A} / \mathrm{G}$ \\
\hline UN_xiong_13013 & 248 & $\mathrm{G} / \mathrm{A}$ \\
\hline UN_xiong_13016 & 88 & $\mathrm{G} / \mathrm{A}$ \\
\hline UN_xiong_13016 & 191 & $A / G$ \\
\hline UN_xiong_13016 & 314 & $A / G$ \\
\hline UN_xiong_13016 & 401 & $\mathrm{~T} / \mathrm{C}$ \\
\hline UN_xiong_13016 & 580 & $\mathrm{C} / \mathrm{T}$ \\
\hline UN_xiong_13016 & 635 & $\mathrm{G} / \mathrm{T}$ \\
\hline UN_xiong_13019 & 434 & $\mathrm{G} / \mathrm{A}$ \\
\hline UN_xiong_13033 & 443 & $A / G$ \\
\hline UN_xiong_13033 & 497 & $\mathrm{~T} / \mathrm{A}$ \\
\hline UN_xiong_13033 & 524 & $\mathrm{~T} / \mathrm{A}$ \\
\hline UN_xiong_13033 & 621 & $\mathrm{~T} / \mathrm{C}$ \\
\hline UN_xiong_13033 & 652 & $A / G$ \\
\hline UN_xiong_13033 & 740 & $\mathrm{~T} / \mathrm{A}$ \\
\hline UN_xiong_13033 & 778 & $\mathrm{~T} / \mathrm{C}$ \\
\hline UN_xiong_13035 & 178 & $A / G$ \\
\hline UN_xiong_13036 & 467 & $\mathrm{G} / \mathrm{A}$ \\
\hline UN_xiong_13046 & 244 & $A / G$ \\
\hline UN_xiong_13049 & 418 & $\mathrm{C} / \mathrm{T}$ \\
\hline UN_xiong_13068 & 93 & $\mathrm{G} / \mathrm{A}$ \\
\hline UN_xiong_13068 & 175 & $A / G$ \\
\hline UN_xiong_13072 & 84 & $\mathrm{G} / \mathrm{C}$ \\
\hline UN_xiong_13072 & 119 & $\mathrm{~T} / \mathrm{C}$ \\
\hline UN_xiong_13084 & 668 & $\mathrm{G} / \mathrm{T}$ \\
\hline UN_xiong_13084 & 911 & $\mathrm{C} / \mathrm{T}$ \\
\hline UN_xiong_13084 & 1010 & $\mathrm{G} / \mathrm{A}$ \\
\hline UN_xiong_13084 & 1093 & $\mathrm{~T} / \mathrm{C}$ \\
\hline UN_xiong_13092 & 256 & $\mathrm{G} / \mathrm{C}$ \\
\hline UN_xiong_13092 & 281 & $\mathrm{~T} / \mathrm{C}$ \\
\hline UN_xiong_13093 & 163 & $\mathrm{C} / \mathrm{A}$ \\
\hline UN_xiong_13095 & 503 & $\mathrm{~T} / \mathrm{C}$ \\
\hline UN_xiong_13095 & 708 & $\mathrm{G} / \mathrm{A}$ \\
\hline UN_xiong_13097 & 315 & $A / G$ \\
\hline UN_xiong_13097 & 668 & $\mathrm{C} / \mathrm{G}$ \\
\hline
\end{tabular}




\begin{tabular}{|c|c|c|}
\hline UN_xiong_13097 & 691 & $\mathrm{C} / \mathrm{A}$ \\
\hline UN_xiong_13097 & 753 & $\mathrm{G} / \mathrm{A}$ \\
\hline UN_xiong_13097 & 828 & $\mathrm{~T} / \mathrm{C}$ \\
\hline UN_xiong_13102 & 715 & $\mathrm{~T} / \mathrm{A}$ \\
\hline UN_xiong_13122 & 136 & $\mathrm{G} / \mathrm{A}$ \\
\hline UN_xiong_13122 & 168 & $\mathrm{~T} / \mathrm{C}$ \\
\hline UN_xiong_13122 & 222 & $A / G$ \\
\hline UN_xiong_13122 & 320 & $\mathrm{G} / \mathrm{T}$ \\
\hline UN_xiong_13122 & 363 & $\mathrm{~A} / \mathrm{T}$ \\
\hline UN_xiong_13122 & 384 & $A / G$ \\
\hline UN_xiong_13122 & 450 & $A / G$ \\
\hline UN_xiong_13122 & 511 & $\mathrm{~A} / \mathrm{T}$ \\
\hline UN_xiong_13122 & 616 & $\mathrm{C} / \mathrm{T}$ \\
\hline UN_xiong_13123 & 143 & $\mathrm{~A} / \mathrm{T}$ \\
\hline UN_xiong_13127 & 213 & $\mathrm{~A} / \mathrm{C}$ \\
\hline UN_xiong_13142 & 68 & $\mathrm{~T} / \mathrm{A}$ \\
\hline UN_xiong_13142 & 363 & $\mathrm{C} / \mathrm{A}$ \\
\hline UN_xiong_13143 & 203 & $\mathrm{~T} / \mathrm{C}$ \\
\hline UN_xiong_13144 & 503 & $\mathrm{G} / \mathrm{A}$ \\
\hline UN_xiong_13144 & 1301 & $\mathrm{C} / \mathrm{T}$ \\
\hline UN_xiong_13144 & 1465 & $A / G$ \\
\hline UN_xiong_13153 & 659 & $C / G$ \\
\hline UN_xiong_13160 & 216 & $G / A$ \\
\hline UN_xiong_13166 & 293 & $G / A$ \\
\hline UN_xiong_13166 & 360 & $\mathrm{~T} / \mathrm{C}$ \\
\hline UN_xiong_13166 & 407 & $\mathrm{C} / \mathrm{T}$ \\
\hline UN_xiong_13172 & 139 & $A / G$ \\
\hline UN_xiong_13172 & 203 & $\mathrm{G} / \mathrm{T}$ \\
\hline UN_xiong_13174 & 899 & $A / G$ \\
\hline UN_xiong_13174 & 1408 & $\mathrm{~T} / \mathrm{C}$ \\
\hline UN_xiong_13191 & 82 & $A / G$ \\
\hline UN_xiong_13191 & 193 & $A / G$ \\
\hline UN_xiong_13191 & 259 & $\mathrm{G} / \mathrm{A}$ \\
\hline UN_xiong_13191 & 346 & $\mathrm{~A} / \mathrm{C}$ \\
\hline UN_xiong_13191 & 382 & $\mathrm{~T} / \mathrm{C}$ \\
\hline UN_xiong_13191 & 487 & $C / G$ \\
\hline UN_xiong_13191 & 608 & $\mathrm{~T} / \mathrm{A}$ \\
\hline UN_xiong_13198 & 865 & $\mathrm{~A} / \mathrm{T}$ \\
\hline UN_xiong_13200 & 194 & $\mathrm{C} / \mathrm{A}$ \\
\hline UN_xiong_13231 & 261 & $\mathrm{~A} / \mathrm{C}$ \\
\hline UN_xiong_13231 & 369 & $\mathrm{C} / \mathrm{T}$ \\
\hline UN_xiong_13260 & 211 & $\mathrm{~T} / \mathrm{C}$ \\
\hline UN_xiong_13260 & 1003 & $\mathrm{~T} / \mathrm{C}$ \\
\hline UN_xiong_13267 & 207 & $A / G$ \\
\hline UN_xiong_13270 & 97 & $\mathrm{G} / \mathrm{A}$ \\
\hline UN_xiong_13270 & 119 & $T / G$ \\
\hline UN_xiong_13270 & 162 & $\mathrm{C} / \mathrm{A}$ \\
\hline
\end{tabular}




\begin{tabular}{|c|c|c|}
\hline UN_xiong_13270 & 622 & $\mathrm{G} / \mathrm{A}$ \\
\hline UN_xiong_13278 & 371 & $\mathrm{G} / \mathrm{C}$ \\
\hline UN_xiong_13281 & 243 & $\mathrm{~T} / \mathrm{C}$ \\
\hline UN_xiong_13297 & 121 & $\mathrm{~T} / \mathrm{C}$ \\
\hline UN_xiong_13304 & 168 & $\mathrm{~T} / \mathrm{G}$ \\
\hline UN_xiong_13311 & 285 & $\mathrm{C} / \mathrm{A}$ \\
\hline UN_xiong_13312 & 59 & $\mathrm{~A} / \mathrm{C}$ \\
\hline UN_xiong_13313 & 344 & $\mathrm{C} / \mathrm{T}$ \\
\hline UN_xiong_13316 & 106 & $\mathrm{~T} / \mathrm{C}$ \\
\hline UN_xiong_13316 & 189 & $\mathrm{~T} / \mathrm{C}$ \\
\hline UN_xiong_13321 & 373 & $\mathrm{C} / \mathrm{T}$ \\
\hline UN_xiong_13321 & 448 & $\mathrm{C} / \mathrm{G}$ \\
\hline UN_xiong_13323 & 117 & $\mathrm{G} / \mathrm{A}$ \\
\hline UN_xiong_13327 & 106 & $\mathrm{G} / \mathrm{A}$ \\
\hline UN_xiong_13332 & 58 & $\mathrm{~A} / \mathrm{G}$ \\
\hline UN_xiong_13350 & 303 & $\mathrm{~T} / \mathrm{G}$ \\
\hline UN_xiong_13350 & 521 & $\mathrm{G} / \mathrm{A}$ \\
\hline UN_xiong_13352 & 175 & $\mathrm{G} / \mathrm{A}$ \\
\hline UN_xiong_13355 & 222 & $\mathrm{C} / \mathrm{T}$ \\
\hline UN_xiong_13355 & 271 & $\mathrm{~T} / \mathrm{C}$ \\
\hline UN_xiong_13357 & 145 & $A / G$ \\
\hline UN_xiong_13359 & 275 & $A / G$ \\
\hline UN_xiong_13369 & 356 & $A / G$ \\
\hline UN_xiong_13394 & 786 & $\mathrm{G} / \mathrm{T}$ \\
\hline UN_xiong_13413 & 245 & $\mathrm{G} / \mathrm{T}$ \\
\hline UN_xiong_13420 & 124 & $\mathrm{C} / \mathrm{T}$ \\
\hline UN_xiong_13420 & 489 & $\mathrm{G} / \mathrm{C}$ \\
\hline UN_xiong_13427 & 206 & $\mathrm{~T} / \mathrm{C}$ \\
\hline UN_xiong_13451 & 619 & $\mathrm{~T} / \mathrm{A}$ \\
\hline UN_xiong_13462 & 837 & $\mathrm{~T} / \mathrm{C}$ \\
\hline UN_xiong_13475 & 330 & $\mathrm{~T} / \mathrm{C}$ \\
\hline UN_xiong_13476 & 169 & $\mathrm{G} / \mathrm{A}$ \\
\hline UN_xiong_13476 & 495 & $\mathrm{G} / \mathrm{T}$ \\
\hline UN_xiong_13479 & 487 & $\mathrm{~T} / \mathrm{A}$ \\
\hline UN_xiong_13479 & 712 & $A / G$ \\
\hline UN_xiong_13479 & 757 & $\mathrm{C} / \mathrm{T}$ \\
\hline UN_xiong_13479 & 819 & $\mathrm{G} / \mathrm{A}$ \\
\hline UN_xiong_13479 & 932 & $\mathrm{~A} / \mathrm{G}$ \\
\hline UN_xiong_13479 & 981 & $\mathrm{~T} / \mathrm{A}$ \\
\hline UN_xiong_13479 & 1002 & $\mathrm{~T} / \mathrm{C}$ \\
\hline UN_xiong_13480 & 25 & $\mathrm{~T} / \mathrm{C}$ \\
\hline UN_xiong_13480 & 69 & $\mathrm{~A} / \mathrm{G}$ \\
\hline UN_xiong_13480 & 125 & $\mathrm{G} / \mathrm{A}$ \\
\hline UN_xiong_13480 & 176 & $\mathrm{~T} / \mathrm{C}$ \\
\hline UN_xiong_13480 & 200 & $\mathrm{C} / \mathrm{T}$ \\
\hline UN_xiong_13480 & 253 & $\mathrm{~T} / \mathrm{C}$ \\
\hline UN_xiong_13480 & 274 & $A / G$ \\
\hline
\end{tabular}




\begin{tabular}{|c|c|c|}
\hline UN_xiong_13480 & 313 & $\mathrm{~A} / \mathrm{G}$ \\
\hline UN_xiong_13480 & 375 & $\mathrm{G} / \mathrm{A}$ \\
\hline UN_xiong_13480 & 512 & $\mathrm{C} / \mathrm{T}$ \\
\hline UN_xiong_13480 & 535 & $\mathrm{~T} / \mathrm{C}$ \\
\hline UN_xiong_13480 & 562 & $\mathrm{~A} / \mathrm{G}$ \\
\hline UN_xiong_13480 & 585 & $\mathrm{C} / \mathrm{T}$ \\
\hline UN_xiong_13480 & 624 & $\mathrm{~T} / \mathrm{C}$ \\
\hline UN_xiong_13480 & 655 & $\mathrm{G} / \mathrm{A}$ \\
\hline UN_xiong_13480 & 686 & $\mathrm{~A} / \mathrm{T}$ \\
\hline UN_xiong_13480 & 724 & $\mathrm{~T} / \mathrm{A}$ \\
\hline UN_xiong_13480 & 744 & $\mathrm{C} / \mathrm{A}$ \\
\hline UN_xiong_13484 & 458 & $\mathrm{~A} / \mathrm{T}$ \\
\hline UN_xiong_13495 & 424 & $\mathrm{~T} / \mathrm{C}$ \\
\hline UN_xiong_13515 & 305 & $A / G$ \\
\hline UN_xiong_13530 & 56 & $\mathrm{~A} / \mathrm{G}$ \\
\hline UN_xiong_13530 & 241 & $\mathrm{C} / \mathrm{A}$ \\
\hline UN_xiong_13530 & 387 & $\mathrm{~A} / \mathrm{G}$ \\
\hline UN_xiong_13530 & 483 & $\mathrm{~T} / \mathrm{C}$ \\
\hline UN_xiong_13530 & 736 & $\mathrm{C} / \mathrm{T}$ \\
\hline UN_xiong_13534 & 146 & $\mathrm{G} / \mathrm{A}$ \\
\hline UN_xiong_13548 & 106 & $\mathrm{C} / \mathrm{T}$ \\
\hline UN_xiong_13551 & 302 & $\mathrm{~T} / \mathrm{C}$ \\
\hline UN_xiong_13552 & 307 & $\mathrm{C} / \mathrm{T}$ \\
\hline UN_xiong_13553 & 236 & $\mathrm{~A} / \mathrm{T}$ \\
\hline UN_xiong_13559 & 114 & $\mathrm{~T} / \mathrm{C}$ \\
\hline UN_xiong_13569 & 22 & $\mathrm{~A} / \mathrm{G}$ \\
\hline UN_xiong_13569 & 49 & $\mathrm{~A} / \mathrm{T}$ \\
\hline UN_xiong_13569 & 79 & $A / G$ \\
\hline UN_xiong_13569 & 129 & $G / A$ \\
\hline UN_xiong_13569 & 162 & $\mathrm{~T} / \mathrm{C}$ \\
\hline UN_xiong_13569 & 219 & $\mathrm{~T} / \mathrm{C}$ \\
\hline UN_xiong_13569 & 269 & $\mathrm{~T} / \mathrm{A}$ \\
\hline UN_xiong_13569 & 311 & $\mathrm{G} / \mathrm{A}$ \\
\hline UN_xiong_13569 & 400 & $\mathrm{~A} / \mathrm{G}$ \\
\hline UN_xiong_13569 & 435 & $\mathrm{~T} / \mathrm{C}$ \\
\hline UN_xiong_13569 & 460 & $\mathrm{~A} / \mathrm{G}$ \\
\hline UN_xiong_13569 & 588 & $A / G$ \\
\hline UN_xiong_13569 & 653 & $\mathrm{~T} / \mathrm{A}$ \\
\hline UN_xiong_13569 & 678 & $\mathrm{C} / \mathrm{T}$ \\
\hline UN_xiong_13569 & 734 & $\mathrm{~T} / \mathrm{C}$ \\
\hline UN_xiong_13569 & 754 & $\mathrm{~T} / \mathrm{C}$ \\
\hline UN_xiong_13569 & 775 & $\mathrm{~T} / \mathrm{C}$ \\
\hline UN_xiong_13569 & 831 & $A / G$ \\
\hline UN_xiong_13569 & 971 & $\mathrm{~A} / \mathrm{T}$ \\
\hline UN_xiong_13569 & 1013 & $A / G$ \\
\hline UN_xiong_13569 & 1081 & $\mathrm{G} / \mathrm{A}$ \\
\hline UN_xiong_13569 & 1109 & $\mathrm{G} / \mathrm{A}$ \\
\hline
\end{tabular}




\begin{tabular}{|c|c|c|}
\hline UN_xiong_13573 & 171 & $\mathrm{C} / \mathrm{T}$ \\
\hline UN_xiong_13578 & 684 & $\mathrm{~T} / \mathrm{C}$ \\
\hline UN_xiong_13578 & 935 & $\mathrm{~T} / \mathrm{C}$ \\
\hline UN_xiong_13578 & 1066 & $A / G$ \\
\hline UN_xiong_13578 & 1187 & $\mathrm{~A} / \mathrm{T}$ \\
\hline UN_xiong_13584 & 370 & $\mathrm{~A} / \mathrm{T}$ \\
\hline UN_xiong_13591 & 747 & $A / G$ \\
\hline UN_xiong_13593 & 185 & $A / G$ \\
\hline UN_xiong_13598 & 14 & $\mathrm{G} / \mathrm{T}$ \\
\hline UN_xiong_13598 & 67 & $\mathrm{G} / \mathrm{A}$ \\
\hline UN_xiong_13600 & 21 & $\mathrm{G} / \mathrm{T}$ \\
\hline UN_xiong_13600 & 199 & $\mathrm{C} / \mathrm{T}$ \\
\hline UN_xiong_13607 & 670 & $\mathrm{G} / \mathrm{A}$ \\
\hline UN_xiong_13629 & 1609 & $\mathrm{~A} / \mathrm{G}$ \\
\hline UN_xiong_13629 & 1762 & $\mathrm{~T} / \mathrm{C}$ \\
\hline UN_xiong_13632 & 159 & $\mathrm{~T} / \mathrm{A}$ \\
\hline UN_xiong_13644 & 31 & $\mathrm{~T} / \mathrm{A}$ \\
\hline UN_xiong_13644 & 180 & $\mathrm{C} / \mathrm{T}$ \\
\hline UN_xiong_13651 & 45 & $\mathrm{C} / \mathrm{T}$ \\
\hline UN_xiong_13651 & 127 & $\mathrm{~A} / \mathrm{C}$ \\
\hline UN_xiong_13651 & 166 & $\mathrm{C} / \mathrm{T}$ \\
\hline UN_xiong_13651 & 204 & $\mathrm{C} / \mathrm{T}$ \\
\hline UN_xiong_13651 & 243 & $\mathrm{C} / \mathrm{T}$ \\
\hline UN_xiong_13651 & 294 & $\mathrm{G} / \mathrm{A}$ \\
\hline UN_xiong_13651 & 342 & $\mathrm{~T} / \mathrm{G}$ \\
\hline UN_xiong_13651 & 363 & $\mathrm{C} / \mathrm{T}$ \\
\hline UN_xiong_13651 & 432 & $\mathrm{C} / \mathrm{T}$ \\
\hline UN_xiong_13651 & 479 & $\mathrm{~T} / \mathrm{A}$ \\
\hline UN_xiong_13661 & 36 & $\mathrm{G} / \mathrm{C}$ \\
\hline UN_xiong_13661 & 344 & $\mathrm{C} / \mathrm{T}$ \\
\hline UN_xiong_13661 & 435 & $\mathrm{C} / \mathrm{T}$ \\
\hline UN_xiong_13664 & 180 & $\mathrm{~T} / \mathrm{C}$ \\
\hline UN_xiong_13667 & 502 & $A / G$ \\
\hline UN_xiong_13667 & 538 & $\mathrm{~A} / \mathrm{G}$ \\
\hline UN_xiong_13667 & 610 & $\mathrm{~A} / \mathrm{G}$ \\
\hline UN_xiong_13667 & 703 & $\mathrm{~T} / \mathrm{C}$ \\
\hline UN_xiong_13667 & 731 & $\mathrm{C} / \mathrm{T}$ \\
\hline UN_xiong_13690 & 142 & $A / G$ \\
\hline UN_xiong_13690 & 320 & $\mathrm{~T} / \mathrm{G}$ \\
\hline UN_xiong_13690 & 377 & $\mathrm{~T} / \mathrm{C}$ \\
\hline UN_xiong_13690 & 478 & $\mathrm{~T} / \mathrm{C}$ \\
\hline UN_xiong_13690 & 644 & $\mathrm{C} / \mathrm{T}$ \\
\hline UN_xiong_13712 & 223 & $\mathrm{~A} / \mathrm{G}$ \\
\hline UN_xiong_13712 & 294 & $\mathrm{C} / \mathrm{T}$ \\
\hline UN_xiong_13713 & 432 & $\mathrm{~A} / \mathrm{C}$ \\
\hline UN_xiong_13713 & 468 & $\mathrm{~T} / \mathrm{C}$ \\
\hline UN_xiong_13713 & 639 & $\mathrm{G} / \mathrm{A}$ \\
\hline
\end{tabular}




\begin{tabular}{|c|c|c|}
\hline UN_xiong_13713 & 705 & $\mathrm{~T} / \mathrm{C}$ \\
\hline UN_xiong_13713 & 885 & $A / G$ \\
\hline UN_xiong_13716 & 296 & $\mathrm{G} / \mathrm{T}$ \\
\hline UN_xiong_13744 & 103 & $\mathrm{~A} / \mathrm{T}$ \\
\hline UN_xiong_13763 & 78 & $A / G$ \\
\hline UN_xiong_13763 & 131 & $\mathrm{~T} / \mathrm{C}$ \\
\hline UN_xiong_13767 & 64 & $T / G$ \\
\hline UN_xiong_13773 & 398 & $A / C$ \\
\hline UN_xiong_13785 & 94 & $A / G$ \\
\hline UN_xiong_13786 & 102 & $\mathrm{C} / \mathrm{T}$ \\
\hline UN_xiong_13786 & 145 & $\mathrm{C} / \mathrm{T}$ \\
\hline UN_xiong_13786 & 224 & $A / G$ \\
\hline UN_xiong_13810 & 155 & $A / G$ \\
\hline UN_xiong_13821 & 142 & $\mathrm{C} / \mathrm{A}$ \\
\hline UN_xiong_13821 & 220 & $\mathrm{G} / \mathrm{A}$ \\
\hline UN_xiong_13821 & 264 & $\mathrm{C} / \mathrm{T}$ \\
\hline UN_xiong_13821 & 368 & $\mathrm{C} / \mathrm{T}$ \\
\hline UN_xiong_13821 & 569 & $\mathrm{C} / \mathrm{T}$ \\
\hline UN_xiong_13842 & 825 & $\mathrm{G} / \mathrm{T}$ \\
\hline UN_xiong_13845 & 379 & $C / G$ \\
\hline UN_xiong_13855 & 854 & $\mathrm{~A} / \mathrm{C}$ \\
\hline UN_xiong_13855 & 913 & $\mathrm{C} / \mathrm{T}$ \\
\hline UN_xiong_13855 & 1056 & $\mathrm{~T} / \mathrm{C}$ \\
\hline UN_xiong_13855 & 1138 & $\mathrm{~A} / \mathrm{T}$ \\
\hline UN_xiong_13855 & 1192 & $\mathrm{G} / \mathrm{A}$ \\
\hline UN_xiong_13855 & 1229 & $\mathrm{~T} / \mathrm{C}$ \\
\hline UN_xiong_13883 & 40 & $\mathrm{~T} / \mathrm{C}$ \\
\hline UN_xiong_13883 & 100 & $A / G$ \\
\hline UN_xiong_13883 & 396 & $\mathrm{C} / \mathrm{T}$ \\
\hline UN_xiong_13883 & 750 & $\mathrm{G} / \mathrm{A}$ \\
\hline UN_xiong_13889 & 547 & $A / G$ \\
\hline UN_xiong_13924 & 124 & $\mathrm{~T} / \mathrm{C}$ \\
\hline UN_xiong_13924 & 474 & $\mathrm{~T} / \mathrm{C}$ \\
\hline UN_xiong_13924 & 662 & $A / G$ \\
\hline UN_xiong_13924 & 719 & $A / G$ \\
\hline UN_xiong_13925 & 241 & $\mathrm{C} / \mathrm{T}$ \\
\hline UN_xiong_13934 & 199 & $\mathrm{G} / \mathrm{A}$ \\
\hline UN_xiong_13944 & 138 & $\mathrm{~T} / \mathrm{C}$ \\
\hline UN_xiong_13944 & 207 & $\mathrm{~T} / \mathrm{C}$ \\
\hline UN_xiong_13970 & 578 & $A / G$ \\
\hline UN_xiong_13970 & 709 & $\mathrm{C} / \mathrm{T}$ \\
\hline UN_xiong_13970 & 804 & $\mathrm{C} / \mathrm{T}$ \\
\hline UN_xiong_13970 & 852 & $\mathrm{G} / \mathrm{A}$ \\
\hline UN_xiong_13970 & 966 & $\mathrm{~T} / \mathrm{C}$ \\
\hline UN_xiong_13980 & 221 & $\mathrm{~T} / \mathrm{C}$ \\
\hline UN_xiong_13988 & 612 & $\mathrm{G} / \mathrm{A}$ \\
\hline UN_xiong_13993 & 223 & $\mathrm{C} / \mathrm{T}$ \\
\hline
\end{tabular}




\begin{tabular}{|c|c|c|}
\hline UN_xiong_13993 & 363 & $\mathrm{C} / \mathrm{T}$ \\
\hline UN_xiong_13993 & 400 & $\mathrm{G} / \mathrm{A}$ \\
\hline UN_xiong_13993 & 547 & $\mathrm{C} / \mathrm{T}$ \\
\hline UN_xiong_13993 & 613 & $\mathrm{~A} / \mathrm{T}$ \\
\hline UN_xiong_13994 & 110 & $\mathrm{C} / \mathrm{T}$ \\
\hline UN_xiong_14024 & 191 & $\mathrm{G} / \mathrm{C}$ \\
\hline UN_xiong_14027 & 149 & $A / G$ \\
\hline UN_xiong_14038 & 495 & $A / G$ \\
\hline UN_xiong_14042 & 16 & $\mathrm{C} / \mathrm{T}$ \\
\hline UN_xiong_14042 & 131 & $\mathrm{~T} / \mathrm{C}$ \\
\hline UN_xiong_14044 & 189 & $\mathrm{C} / \mathrm{T}$ \\
\hline UN_xiong_14044 & 589 & $\mathrm{~T} / \mathrm{C}$ \\
\hline UN_xiong_14059 & 260 & $\mathrm{~A} / \mathrm{G}$ \\
\hline UN_xiong_14060 & 752 & $\mathrm{~T} / \mathrm{C}$ \\
\hline UN_xiong_14064 & 170 & $\mathrm{C} / \mathrm{T}$ \\
\hline UN_xiong_14070 & 48 & $\mathrm{~T} / \mathrm{G}$ \\
\hline UN_xiong_14070 & 174 & $\mathrm{~A} / \mathrm{C}$ \\
\hline UN_xiong_14075 & 90 & $\mathrm{G} / \mathrm{A}$ \\
\hline UN_xiong_14091 & 574 & $\mathrm{G} / \mathrm{A}$ \\
\hline UN_xiong_14091 & 642 & $\mathrm{C} / \mathrm{T}$ \\
\hline UN_xiong_14118 & 884 & $\mathrm{~T} / \mathrm{C}$ \\
\hline UN_xiong_14120 & 354 & $A / G$ \\
\hline UN_xiong_14145 & 238 & $\mathrm{C} / \mathrm{T}$ \\
\hline UN_xiong_14145 & 518 & $\mathrm{~T} / \mathrm{C}$ \\
\hline UN_xiong_14145 & 595 & $\mathrm{~A} / \mathrm{G}$ \\
\hline UN_xiong_14145 & 626 & $\mathrm{G} / \mathrm{A}$ \\
\hline UN_xiong_14145 & 744 & $\mathrm{~T} / \mathrm{C}$ \\
\hline UN_xiong_14150 & 148 & $A / G$ \\
\hline UN_xiong_14170 & 143 & $\mathrm{C} / \mathrm{T}$ \\
\hline UN_xiong_14171 & 209 & $\mathrm{C} / \mathrm{T}$ \\
\hline UN_xiong_14176 & 119 & $A / G$ \\
\hline UN_xiong_14176 & 281 & $\mathrm{~T} / \mathrm{C}$ \\
\hline UN_xiong_14196 & 394 & $\mathrm{C} / \mathrm{T}$ \\
\hline UN_xiong_14206 & 40 & $\mathrm{G} / \mathrm{A}$ \\
\hline UN_xiong_14206 & 67 & $\mathrm{~A} / \mathrm{T}$ \\
\hline UN_xiong_14207 & 451 & $\mathrm{C} / \mathrm{G}$ \\
\hline UN_xiong_14207 & 529 & $\mathrm{~T} / \mathrm{C}$ \\
\hline UN_xiong_14207 & 579 & $A / G$ \\
\hline UN_xiong_14207 & 605 & $\mathrm{~T} / \mathrm{C}$ \\
\hline UN_xiong_14207 & 626 & $A / G$ \\
\hline UN_xiong_14207 & 818 & $\mathrm{~A} / \mathrm{G}$ \\
\hline UN_xiong_14207 & 852 & $A / G$ \\
\hline UN_xiong_14207 & 1047 & $\mathrm{G} / \mathrm{A}$ \\
\hline UN_xiong_14207 & 1139 & $\mathrm{C} / \mathrm{T}$ \\
\hline UN_xiong_14208 & 127 & $\mathrm{C} / \mathrm{T}$ \\
\hline UN_xiong_14208 & 176 & $\mathrm{~T} / \mathrm{C}$ \\
\hline UN_xiong_14208 & 203 & $\mathrm{~A} / \mathrm{G}$ \\
\hline
\end{tabular}




\begin{tabular}{|c|c|c|}
\hline UN_xiong_14208 & 378 & $\mathrm{~T} / \mathrm{C}$ \\
\hline UN_xiong_14208 & 503 & $A / G$ \\
\hline UN_xiong_14208 & 562 & $A / G$ \\
\hline UN_xiong_14215 & 131 & $\mathrm{C} / \mathrm{A}$ \\
\hline UN_xiong_14216 & 20 & $\mathrm{G} / \mathrm{A}$ \\
\hline UN_xiong_14219 & 103 & $A / G$ \\
\hline UN_xiong_14219 & 260 & $\mathrm{~A} / \mathrm{C}$ \\
\hline UN_xiong_14220 & 33 & $\mathrm{G} / \mathrm{A}$ \\
\hline UN_xiong_14220 & 68 & $A / G$ \\
\hline UN_xiong_14220 & 148 & $\mathrm{~T} / \mathrm{G}$ \\
\hline UN_xiong_14224 & 633 & $\mathrm{~T} / \mathrm{C}$ \\
\hline UN_xiong_14225 & 484 & $\mathrm{~T} / \mathrm{G}$ \\
\hline UN_xiong_14225 & 708 & $A / G$ \\
\hline UN_xiong_14225 & 747 & $\mathrm{C} / \mathrm{T}$ \\
\hline UN_xiong_14227 & 127 & $A / G$ \\
\hline UN_xiong_14227 & 162 & $\mathrm{~T} / \mathrm{C}$ \\
\hline UN_xiong_14227 & 214 & $A / G$ \\
\hline UN_xiong_14227 & 351 & $A / G$ \\
\hline UN_xiong_14227 & 378 & $\mathrm{~T} / \mathrm{A}$ \\
\hline UN_xiong_14227 & 598 & $\mathrm{~T} / \mathrm{C}$ \\
\hline UN_xiong_14227 & 725 & $\mathrm{~A} / \mathrm{T}$ \\
\hline UN_xiong_14228 & 444 & $\mathrm{~T} / \mathrm{G}$ \\
\hline UN_xiong_14229 & 240 & $A / G$ \\
\hline UN_xiong_14232 & 133 & $\mathrm{C} / \mathrm{A}$ \\
\hline UN_xiong_14232 & 228 & $A / G$ \\
\hline UN_xiong_14232 & 315 & $\mathrm{G} / \mathrm{C}$ \\
\hline UN_xiong_14232 & 360 & $\mathrm{~T} / \mathrm{A}$ \\
\hline UN_xiong_14233 & 177 & $\mathrm{G} / \mathrm{A}$ \\
\hline UN_xiong_14233 & 203 & $\mathrm{C} / \mathrm{T}$ \\
\hline UN_xiong_14233 & 733 & $A / G$ \\
\hline UN_xiong_14238 & 157 & $\mathrm{C} / \mathrm{T}$ \\
\hline UN_xiong_14238 & 383 & $A / G$ \\
\hline UN_xiong_14239 & 124 & $\mathrm{C} / \mathrm{T}$ \\
\hline UN_xiong_14239 & 212 & $A / G$ \\
\hline UN_xiong_14239 & 386 & $A / G$ \\
\hline UN_xiong_14239 & 461 & $\mathrm{C} / \mathrm{T}$ \\
\hline UN_xiong_14239 & 514 & $\mathrm{~A} / \mathrm{T}$ \\
\hline UN_xiong_14239 & 534 & $\mathrm{C} / \mathrm{T}$ \\
\hline UN_xiong_14239 & 603 & $\mathrm{~A} / \mathrm{T}$ \\
\hline UN_xiong_14239 & 741 & $\mathrm{C} / \mathrm{T}$ \\
\hline UN_xiong_14240 & 56 & $\mathrm{C} / \mathrm{T}$ \\
\hline UN_xiong_14240 & 177 & $\mathrm{~A} / \mathrm{C}$ \\
\hline UN_xiong_14240 & 407 & $\mathrm{~T} / \mathrm{C}$ \\
\hline UN_xiong_14240 & 446 & $\mathrm{~T} / \mathrm{C}$ \\
\hline UN_xiong_14240 & 565 & $A / G$ \\
\hline UN_xiong_14240 & 592 & $\mathrm{G} / \mathrm{A}$ \\
\hline UN_xiong_14240 & 612 & $A / G$ \\
\hline
\end{tabular}




\begin{tabular}{|c|c|c|}
\hline UN_xiong_14240 & 640 & $\mathrm{~T} / \mathrm{C}$ \\
\hline UN_xiong_14240 & 701 & $\mathrm{~T} / \mathrm{C}$ \\
\hline UN_xiong_14240 & 743 & $\mathrm{G} / \mathrm{T}$ \\
\hline UN_xiong_14240 & 790 & $\mathrm{~A} / \mathrm{G}$ \\
\hline UN_xiong_14241 & 61 & $\mathrm{~T} / \mathrm{G}$ \\
\hline UN_xiong_14243 & 49 & $\mathrm{~T} / \mathrm{G}$ \\
\hline UN_xiong_14243 & 85 & $A / G$ \\
\hline UN_xiong_14243 & 251 & $\mathrm{~A} / \mathrm{G}$ \\
\hline UN_xiong_14244 & 153 & $\mathrm{~T} / \mathrm{C}$ \\
\hline UN_xiong_14244 & 341 & $\mathrm{G} / \mathrm{A}$ \\
\hline UN_xiong_14244 & 375 & $\mathrm{~T} / \mathrm{C}$ \\
\hline UN_xiong_14244 & 553 & $\mathrm{G} / \mathrm{T}$ \\
\hline UN_xiong_14246 & 22 & $\mathrm{G} / \mathrm{T}$ \\
\hline UN_xiong_14246 & 94 & $\mathrm{C} / \mathrm{T}$ \\
\hline UN_xiong_14246 & 131 & $\mathrm{~A} / \mathrm{C}$ \\
\hline UN_xiong_14246 & 309 & $\mathrm{C} / \mathrm{T}$ \\
\hline UN_xiong_14246 & 345 & $\mathrm{G} / \mathrm{A}$ \\
\hline UN_xiong_14247 & 183 & $\mathrm{~A} / \mathrm{T}$ \\
\hline UN_xiong_14247 & 282 & $\mathrm{G} / \mathrm{A}$ \\
\hline UN_xiong_14248 & 301 & $\mathrm{G} / \mathrm{A}$ \\
\hline UN_xiong_14250 & 466 & $\mathrm{~T} / \mathrm{C}$ \\
\hline UN_xiong_14250 & 606 & $\mathrm{~A} / \mathrm{G}$ \\
\hline UN_xiong_14250 & 639 & $\mathrm{~T} / \mathrm{C}$ \\
\hline UN_xiong_14250 & 736 & $\mathrm{C} / \mathrm{A}$ \\
\hline UN_xiong_14251 & 294 & $\mathrm{~T} / \mathrm{A}$ \\
\hline UN_xiong_14251 & 328 & $\mathrm{~T} / \mathrm{C}$ \\
\hline UN_xiong_14251 & 376 & $\mathrm{~T} / \mathrm{C}$ \\
\hline UN_xiong_14251 & 404 & $\mathrm{~T} / \mathrm{C}$ \\
\hline UN_xiong_14251 & 455 & $\mathrm{~A} / \mathrm{C}$ \\
\hline UN_xiong_14251 & 519 & $\mathrm{~A} / \mathrm{G}$ \\
\hline UN_xiong_14251 & 737 & $\mathrm{~A} / \mathrm{G}$ \\
\hline UN_xiong_14251 & 838 & $\mathrm{~T} / \mathrm{C}$ \\
\hline UN_xiong_14251 & 858 & $\mathrm{G} / \mathrm{A}$ \\
\hline UN_xiong_14257 & 14 & $\mathrm{~A} / \mathrm{C}$ \\
\hline UN_xiong_14257 & 175 & $\mathrm{~A} / \mathrm{G}$ \\
\hline UN_xiong_14257 & 243 & $A / G$ \\
\hline UN_xiong_14257 & 338 & $\mathrm{~T} / \mathrm{A}$ \\
\hline UN_xiong_14257 & 550 & $\mathrm{G} / \mathrm{T}$ \\
\hline UN_xiong_14257 & 571 & $A / C$ \\
\hline UN_xiong_14257 & 608 & $\mathrm{C} / \mathrm{T}$ \\
\hline UN_xiong_14257 & 656 & $\mathrm{C} / \mathrm{T}$ \\
\hline UN_xiong_14257 & 678 & $\mathrm{G} / \mathrm{A}$ \\
\hline UN_xiong_14257 & 773 & $A / C$ \\
\hline UN_xiong_14257 & 847 & $\mathrm{G} / \mathrm{A}$ \\
\hline UN_xiong_14257 & 871 & $\mathrm{G} / \mathrm{T}$ \\
\hline UN_xiong_14257 & 947 & $C / T$ \\
\hline UN_xiong_14257 & 1001 & $\mathrm{G} / \mathrm{C}$ \\
\hline
\end{tabular}




\begin{tabular}{|c|c|c|}
\hline UN_xiong_14257 & 1050 & $\mathrm{~T} / \mathrm{A}$ \\
\hline UN_xiong_14260 & 69 & $\mathrm{~A} / \mathrm{G}$ \\
\hline UN_xiong_14263 & 85 & $\mathrm{C} / \mathrm{G}$ \\
\hline UN_xiong_14266 & 339 & $\mathrm{G} / \mathrm{T}$ \\
\hline UN_xiong_14266 & 380 & $\mathrm{C} / \mathrm{T}$ \\
\hline UN_xiong_14266 & 434 & $\mathrm{~T} / \mathrm{C}$ \\
\hline UN_xiong_14266 & 485 & $A / G$ \\
\hline UN_xiong_14267 & 394 & $\mathrm{G} / \mathrm{A}$ \\
\hline UN_xiong_14267 & 420 & $\mathrm{~T} / \mathrm{C}$ \\
\hline UN_xiong_14267 & 480 & $A / G$ \\
\hline UN_xiong_14270 & 382 & $A / G$ \\
\hline UN_xiong_14270 & 545 & $\mathrm{G} / \mathrm{A}$ \\
\hline UN_xiong_14271 & 278 & $\mathrm{~T} / \mathrm{C}$ \\
\hline UN_xiong_14272 & 182 & $\mathrm{G} / \mathrm{T}$ \\
\hline UN_xiong_14273 & 524 & $\mathrm{~T} / \mathrm{C}$ \\
\hline UN_xiong_14273 & 544 & $\mathrm{~T} / \mathrm{G}$ \\
\hline UN_xiong_14273 & 575 & $\mathrm{~T} / \mathrm{C}$ \\
\hline UN_xiong_14273 & 598 & $\mathrm{~A} / \mathrm{G}$ \\
\hline UN_xiong_14273 & 619 & $\mathrm{C} / \mathrm{A}$ \\
\hline UN_xiong_14274 & 95 & $A / G$ \\
\hline UN_xiong_14274 & 315 & $\mathrm{~T} / \mathrm{C}$ \\
\hline UN_xiong_14274 & 342 & $\mathrm{~T} / \mathrm{C}$ \\
\hline UN_xiong_14274 & 617 & $C / A$ \\
\hline UN_xiong_14275 & 47 & $\mathrm{G} / \mathrm{C}$ \\
\hline UN_xiong_14275 & 152 & $\mathrm{~T} / \mathrm{G}$ \\
\hline UN_xiong_14275 & 174 & $\mathrm{~T} / \mathrm{C}$ \\
\hline UN_xiong_14275 & 207 & $\mathrm{~T} / \mathrm{C}$ \\
\hline UN_xiong_14275 & 245 & $\mathrm{~T} / \mathrm{C}$ \\
\hline UN_xiong_14275 & 270 & $\mathrm{~T} / \mathrm{A}$ \\
\hline UN_xiong_14275 & 298 & $\mathrm{~T} / \mathrm{C}$ \\
\hline UN_xiong_14275 & 331 & $\mathrm{~T} / \mathrm{C}$ \\
\hline UN_xiong_14275 & 367 & $\mathrm{C} / \mathrm{A}$ \\
\hline UN_xiong_14275 & 394 & $A / G$ \\
\hline UN_xiong_14275 & 417 & $\mathrm{C} / \mathrm{T}$ \\
\hline UN_xiong_14275 & 440 & $\mathrm{~T} / \mathrm{G}$ \\
\hline UN_xiong_14276 & 34 & $A / G$ \\
\hline UN_xiong_14276 & 85 & $\mathrm{~A} / \mathrm{G}$ \\
\hline UN_xiong_14276 & 321 & $\mathrm{~A} / \mathrm{T}$ \\
\hline UN_xiong_14276 & 443 & $\mathrm{C} / \mathrm{T}$ \\
\hline UN_xiong_14276 & 502 & $A / G$ \\
\hline UN_xiong_14279 & 146 & $\mathrm{C} / \mathrm{T}$ \\
\hline UN_xiong_14279 & 193 & $\mathrm{~T} / \mathrm{C}$ \\
\hline UN_xiong_14283 & 239 & $\mathrm{C} / \mathrm{T}$ \\
\hline UN_xiong_14283 & 1277 & $\mathrm{G} / \mathrm{T}$ \\
\hline UN_xiong_14285 & 368 & $\mathrm{G} / \mathrm{A}$ \\
\hline UN_xiong_14285 & 395 & $\mathrm{C} / \mathrm{T}$ \\
\hline UN_xiong_14285 & 436 & $\mathrm{~T} / \mathrm{C}$ \\
\hline
\end{tabular}




\begin{tabular}{|c|c|c|}
\hline UN_xiong_14285 & 464 & $\mathrm{~A} / \mathrm{G}$ \\
\hline UN_xiong_14286 & 82 & $\mathrm{C} / \mathrm{T}$ \\
\hline UN_xiong_14286 & 203 & $\mathrm{~T} / \mathrm{C}$ \\
\hline UN_xiong_14286 & 334 & $A / G$ \\
\hline UN_xiong_14286 & 383 & $\mathrm{~T} / \mathrm{C}$ \\
\hline UN_xiong_14286 & 584 & $\mathrm{~T} / \mathrm{C}$ \\
\hline UN_xiong_14288 & 258 & $\mathrm{C} / \mathrm{A}$ \\
\hline UN_xiong_14290 & 46 & $\mathrm{G} / \mathrm{T}$ \\
\hline UN_xiong_14292 & 333 & $\mathrm{G} / \mathrm{A}$ \\
\hline UN_xiong_14292 & 428 & $\mathrm{C} / \mathrm{A}$ \\
\hline UN_xiong_14292 & 1000 & $\mathrm{G} / \mathrm{T}$ \\
\hline UN_xiong_14292 & 1049 & $A / G$ \\
\hline UN_xiong_14292 & 1200 & $\mathrm{~A} / \mathrm{G}$ \\
\hline UN_xiong_14292 & 1220 & $\mathrm{C} / \mathrm{T}$ \\
\hline UN_xiong_14292 & 1292 & $\mathrm{C} / \mathrm{A}$ \\
\hline UN_xiong_14292 & 1325 & $A / G$ \\
\hline UN_xiong_14292 & 1418 & $\mathrm{~T} / \mathrm{A}$ \\
\hline UN_xiong_14292 & 1609 & $A / G$ \\
\hline UN_xiong_14292 & 1649 & $A / G$ \\
\hline UN_xiong_14292 & 1695 & $\mathrm{C} / \mathrm{T}$ \\
\hline UN_xiong_14292 & 1750 & $\mathrm{C} / \mathrm{T}$ \\
\hline UN_xiong_14292 & 1810 & $A / G$ \\
\hline UN_xiong_14292 & 1861 & $\mathrm{~T} / \mathrm{C}$ \\
\hline UN_xiong_14292 & 1932 & $\mathrm{~T} / \mathrm{C}$ \\
\hline UN_xiong_14292 & 2005 & $\mathrm{~T} / \mathrm{G}$ \\
\hline UN_xiong_14292 & 2050 & $\mathrm{~A} / \mathrm{G}$ \\
\hline UN_xiong_14292 & 2286 & $\mathrm{G} / \mathrm{A}$ \\
\hline UN_xiong_14292 & 2375 & $C / A$ \\
\hline UN_xiong_14294 & 12 & $\mathrm{G} / \mathrm{T}$ \\
\hline UN_xiong_14294 & 42 & $\mathrm{~T} / \mathrm{C}$ \\
\hline UN_xiong_14294 & 114 & $\mathrm{~T} / \mathrm{A}$ \\
\hline UN_xiong_14294 & 193 & $\mathrm{G} / \mathrm{A}$ \\
\hline UN_xiong_14295 & 190 & $\mathrm{G} / \mathrm{A}$ \\
\hline UN_xiong_14297 & 106 & $\mathrm{~A} / \mathrm{G}$ \\
\hline UN_xiong_14299 & 326 & $\mathrm{~A} / \mathrm{C}$ \\
\hline UN_xiong_14302 & 366 & $\mathrm{~A} / \mathrm{G}$ \\
\hline UN_xiong_14302 & 394 & $\mathrm{G} / \mathrm{A}$ \\
\hline UN_xiong_14302 & 416 & $\mathrm{G} / \mathrm{A}$ \\
\hline UN_xiong_14302 & 1024 & $\mathrm{G} / \mathrm{C}$ \\
\hline UN_xiong_14302 & 1257 & $\mathrm{~T} / \mathrm{A}$ \\
\hline UN_xiong_14302 & 1380 & $\mathrm{G} / \mathrm{A}$ \\
\hline UN_xiong_14302 & 1552 & $\mathrm{~T} / \mathrm{C}$ \\
\hline UN_xiong_14303 & 116 & $\mathrm{G} / \mathrm{T}$ \\
\hline UN_xiong_14303 & 204 & $\mathrm{~A} / \mathrm{G}$ \\
\hline UN_xiong_14303 & 240 & $A / G$ \\
\hline UN_xiong_14303 & 269 & $\mathrm{G} / \mathrm{A}$ \\
\hline UN_xiong_14303 & 365 & $A / G$ \\
\hline
\end{tabular}




\begin{tabular}{|c|c|c|}
\hline UN_xiong_14305 & 219 & $\mathrm{C} / \mathrm{T}$ \\
\hline UN_xiong_14307 & 145 & $\mathrm{~T} / \mathrm{G}$ \\
\hline UN_xiong_14307 & 188 & $\mathrm{~T} / \mathrm{A}$ \\
\hline UN_xiong_14307 & 271 & $A / G$ \\
\hline UN_xiong_14307 & 363 & $\mathrm{G} / \mathrm{A}$ \\
\hline UN_xiong_14307 & 404 & $A / G$ \\
\hline UN_xiong_14308 & 168 & $\mathrm{~T} / \mathrm{C}$ \\
\hline UN_xiong_14308 & 200 & $\mathrm{~T} / \mathrm{C}$ \\
\hline UN_xiong_14308 & 367 & $\mathrm{~A} / \mathrm{T}$ \\
\hline UN_xiong_14308 & 462 & $\mathrm{~T} / \mathrm{C}$ \\
\hline UN_xiong_14308 & 603 & $\mathrm{G} / \mathrm{A}$ \\
\hline UN_xiong_14309 & 140 & $\mathrm{G} / \mathrm{A}$ \\
\hline UN_xiong_14309 & 230 & $\mathrm{C} / \mathrm{A}$ \\
\hline UN_xiong_14309 & 271 & $\mathrm{G} / \mathrm{A}$ \\
\hline UN_xiong_14309 & 348 & $\mathrm{~A} / \mathrm{G}$ \\
\hline UN_xiong_14309 & 422 & $\mathrm{C} / \mathrm{A}$ \\
\hline UN_xiong_14312 & 92 & $\mathrm{~A} / \mathrm{G}$ \\
\hline UN_xiong_14312 & 159 & $\mathrm{~T} / \mathrm{G}$ \\
\hline UN_xiong_14312 & 183 & $\mathrm{~T} / \mathrm{C}$ \\
\hline UN_xiong_14312 & 210 & $\mathrm{~T} / \mathrm{C}$ \\
\hline UN_xiong_14312 & 233 & $G / A$ \\
\hline UN_xiong_14312 & 278 & $A / G$ \\
\hline UN_xiong_14312 & 316 & $\mathrm{~T} / \mathrm{C}$ \\
\hline UN_xiong_14312 & 343 & $G / A$ \\
\hline UN_xiong_14312 & 384 & $\mathrm{~T} / \mathrm{C}$ \\
\hline UN_xiong_14312 & 411 & $\mathrm{~A} / \mathrm{G}$ \\
\hline UN_xiong_14312 & 443 & $\mathrm{~T} / \mathrm{C}$ \\
\hline UN_xiong_14312 & 470 & $\mathrm{~T} / \mathrm{C}$ \\
\hline UN_xiong_14313 & 46 & $\mathrm{G} / \mathrm{T}$ \\
\hline UN_xiong_14313 & 184 & $\mathrm{~T} / \mathrm{A}$ \\
\hline UN_xiong_14313 & 365 & $\mathrm{G} / \mathrm{T}$ \\
\hline UN_xiong_14313 & 683 & $A / G$ \\
\hline UN_xiong_14313 & 717 & $\mathrm{~T} / \mathrm{C}$ \\
\hline UN_xiong_14313 & 762 & $\mathrm{~T} / \mathrm{C}$ \\
\hline UN_xiong_14313 & 834 & $\mathrm{C} / \mathrm{T}$ \\
\hline UN_xiong_14314 & 54 & $\mathrm{~T} / \mathrm{C}$ \\
\hline UN_xiong_14316 & 443 & $\mathrm{C} / \mathrm{T}$ \\
\hline UN_xiong_14320 & 202 & $\mathrm{~T} / \mathrm{A}$ \\
\hline UN_xiong_14320 & 228 & $\mathrm{~T} / \mathrm{C}$ \\
\hline UN_xiong_14322 & 51 & $\mathrm{G} / \mathrm{T}$ \\
\hline UN_xiong_14322 & 77 & $A / G$ \\
\hline UN_xiong_14322 & 127 & $\mathrm{C} / \mathrm{G}$ \\
\hline UN_xiong_14322 & 175 & $\mathrm{C} / \mathrm{G}$ \\
\hline UN_xiong_14325 & 319 & $\mathrm{~T} / \mathrm{C}$ \\
\hline UN_xiong_14325 & 468 & $\mathrm{~A} / \mathrm{T}$ \\
\hline UN_xiong_14326 & 205 & $\mathrm{~A} / \mathrm{G}$ \\
\hline UN_xiong_14326 & 303 & $\mathrm{G} / \mathrm{A}$ \\
\hline
\end{tabular}




\begin{tabular}{|c|c|c|}
\hline UN_xiong_14326 & 324 & $\mathrm{C} / \mathrm{T}$ \\
\hline UN_xiong_14326 & 509 & $A / G$ \\
\hline UN_xiong_14326 & 597 & $G / A$ \\
\hline UN_xiong_14326 & 1068 & $\mathrm{~A} / \mathrm{T}$ \\
\hline UN_xiong_14327 & 50 & $\mathrm{~A} / \mathrm{T}$ \\
\hline UN_xiong_14327 & 165 & $A / G$ \\
\hline UN_xiong_14327 & 283 & $\mathrm{G} / \mathrm{T}$ \\
\hline UN_xiong_14327 & 338 & $\mathrm{~T} / \mathrm{C}$ \\
\hline UN_xiong_14327 & 517 & $A / G$ \\
\hline UN_xiong_14327 & 606 & $A / G$ \\
\hline UN_xiong_14327 & 655 & $\mathrm{C} / \mathrm{A}$ \\
\hline UN_xiong_14327 & 787 & $A / G$ \\
\hline UN_xiong_14327 & 811 & $\mathrm{~T} / \mathrm{C}$ \\
\hline UN_xiong_14327 & 841 & $\mathrm{C} / \mathrm{A}$ \\
\hline UN_xiong_14330 & 221 & $\mathrm{C} / \mathrm{T}$ \\
\hline UN_xiong_14331 & 79 & $\mathrm{~T} / \mathrm{C}$ \\
\hline UN_xiong_14334 & 197 & $A / G$ \\
\hline UN_xiong_14334 & 238 & $T / G$ \\
\hline UN_xiong_14334 & 319 & $\mathrm{~A} / \mathrm{C}$ \\
\hline UN_xiong_14334 & 343 & $\mathrm{G} / \mathrm{A}$ \\
\hline UN_xiong_14334 & 520 & $\mathrm{G} / \mathrm{A}$ \\
\hline UN_xiong_14334 & 585 & $\mathrm{G} / \mathrm{A}$ \\
\hline UN_xiong_14334 & 631 & $\mathrm{~A} / \mathrm{T}$ \\
\hline UN_xiong_14334 & 710 & $\mathrm{~A} / \mathrm{T}$ \\
\hline UN_xiong_14334 & 896 & $\mathrm{~T} / \mathrm{C}$ \\
\hline UN_xiong_14334 & 927 & $\mathrm{~T} / \mathrm{C}$ \\
\hline UN_xiong_14334 & 1090 & $\mathrm{G} / \mathrm{A}$ \\
\hline UN_xiong_14337 & 412 & $A / C$ \\
\hline UN_xiong_14337 & 802 & $\mathrm{G} / \mathrm{T}$ \\
\hline UN_xiong_14341 & 2074 & $\mathrm{~T} / \mathrm{C}$ \\
\hline UN_xiong_14341 & 2132 & $\mathrm{C} / \mathrm{T}$ \\
\hline UN_xiong_14343 & 59 & $C / G$ \\
\hline UN_xiong_14343 & 118 & $A / G$ \\
\hline UN_xiong_14344 & 244 & $\mathrm{~T} / \mathrm{C}$ \\
\hline UN_xiong_14344 & 338 & $A / G$ \\
\hline UN_xiong_14346 & 266 & $\mathrm{~T} / \mathrm{A}$ \\
\hline UN_xiong_14346 & 2032 & $\mathrm{~T} / \mathrm{C}$ \\
\hline UN_xiong_14347 & 24 & $A / G$ \\
\hline UN_xiong_14347 & 60 & $\mathrm{G} / \mathrm{T}$ \\
\hline UN_xiong_14347 & 94 & $\mathrm{C} / \mathrm{T}$ \\
\hline UN_xiong_14347 & 129 & $\mathrm{~T} / \mathrm{C}$ \\
\hline UN_xiong_14347 & 149 & $A / G$ \\
\hline UN_xiong_14347 & 272 & $\mathrm{~T} / \mathrm{C}$ \\
\hline UN_xiong_14347 & 330 & $A / G$ \\
\hline UN_xiong_14347 & 359 & $A / C$ \\
\hline UN_xiong_14347 & 381 & $\mathrm{~T} / \mathrm{C}$ \\
\hline UN_xiong_14347 & 411 & $\mathrm{~T} / \mathrm{C}$ \\
\hline
\end{tabular}




\begin{tabular}{|c|c|c|}
\hline UN_xiong_14347 & 448 & $\mathrm{~T} / \mathrm{A}$ \\
\hline UN_xiong_14347 & 481 & $\mathrm{C} / \mathrm{T}$ \\
\hline UN_xiong_14347 & 515 & $\mathrm{~A} / \mathrm{C}$ \\
\hline UN_xiong_14347 & 542 & $A / G$ \\
\hline UN_xiong_14347 & 587 & $A / G$ \\
\hline UN_xiong_14347 & 623 & $\mathrm{~T} / \mathrm{C}$ \\
\hline UN_xiong_14347 & 659 & $\mathrm{C} / \mathrm{T}$ \\
\hline UN_xiong_14347 & 681 & $\mathrm{C} / \mathrm{T}$ \\
\hline UN_xiong_14347 & 708 & $\mathrm{~T} / \mathrm{C}$ \\
\hline UN_xiong_14347 & 756 & $\mathrm{~T} / \mathrm{G}$ \\
\hline UN_xiong_14347 & 785 & $\mathrm{~A} / \mathrm{C}$ \\
\hline UN_xiong_14347 & 828 & $\mathrm{C} / \mathrm{A}$ \\
\hline UN_xiong_14351 & 73 & $\mathrm{C} / \mathrm{T}$ \\
\hline UN_xiong_14351 & 142 & $\mathrm{~A} / \mathrm{C}$ \\
\hline UN_xiong_14352 & 249 & $\mathrm{C} / \mathrm{T}$ \\
\hline UN_xiong_14352 & 384 & $\mathrm{~T} / \mathrm{C}$ \\
\hline UN_xiong_14354 & 33 & $\mathrm{~T} / \mathrm{C}$ \\
\hline UN_xiong_14354 & 166 & $\mathrm{~T} / \mathrm{C}$ \\
\hline UN_xiong_14359 & 161 & $A / G$ \\
\hline UN_xiong_14359 & 211 & $\mathrm{G} / \mathrm{A}$ \\
\hline UN_xiong_14359 & 444 & $\mathrm{~T} / \mathrm{C}$ \\
\hline UN_xiong_14359 & 634 & $A / G$ \\
\hline UN_xiong_14359 & 656 & $\mathrm{~A} / \mathrm{C}$ \\
\hline UN_xiong_14361 & 342 & $\mathrm{~A} / \mathrm{C}$ \\
\hline UN_xiong_14361 & 409 & $\mathrm{~T} / \mathrm{C}$ \\
\hline UN_xiong_14362 & 310 & $A / C$ \\
\hline UN_xiong_14362 & 883 & $\mathrm{~A} / \mathrm{C}$ \\
\hline UN_xiong_14362 & 1031 & $C / G$ \\
\hline UN_xiong_14362 & 1060 & $\mathrm{C} / \mathrm{T}$ \\
\hline UN_xiong_14362 & 1128 & $\mathrm{C} / \mathrm{A}$ \\
\hline UN_xiong_14363 & 373 & $\mathrm{G} / \mathrm{T}$ \\
\hline UN_xiong_14364 & 750 & $T / G$ \\
\hline UN_xiong_14367 & 471 & $\mathrm{G} / \mathrm{A}$ \\
\hline UN_xiong_14368 & 61 & $\mathrm{~A} / \mathrm{C}$ \\
\hline UN_xiong_14370 & 49 & $\mathrm{C} / \mathrm{T}$ \\
\hline UN_xiong_14370 & 92 & $A / G$ \\
\hline UN_xiong_14373 & 337 & $A / G$ \\
\hline UN_xiong_14375 & 27 & $A / G$ \\
\hline UN_xiong_14375 & 484 & $A / G$ \\
\hline UN_xiong_14375 & 565 & $\mathrm{~T} / \mathrm{C}$ \\
\hline UN_xiong_14375 & 601 & $\mathrm{G} / \mathrm{A}$ \\
\hline UN_xiong_14375 & 626 & $\mathrm{~T} / \mathrm{C}$ \\
\hline UN_xiong_14376 & 56 & $\mathrm{~T} / \mathrm{C}$ \\
\hline UN_xiong_14377 & 133 & $\mathrm{~T} / \mathrm{A}$ \\
\hline UN_xiong_14377 & 196 & $\mathrm{~A} / \mathrm{C}$ \\
\hline UN_xiong_14377 & 288 & $\mathrm{~T} / \mathrm{C}$ \\
\hline UN_xiong_14377 & 313 & $A / G$ \\
\hline
\end{tabular}




\begin{tabular}{|c|c|c|}
\hline UN_xiong_14377 & 369 & $\mathrm{~A} / \mathrm{G}$ \\
\hline UN_xiong_14377 & 393 & $\mathrm{~A} / \mathrm{T}$ \\
\hline UN_xiong_14377 & 436 & $\mathrm{~T} / \mathrm{C}$ \\
\hline UN_xiong_14377 & 678 & $\mathrm{~A} / \mathrm{G}$ \\
\hline UN_xiong_14377 & 781 & $\mathrm{~A} / \mathrm{G}$ \\
\hline UN_xiong_14377 & 956 & $\mathrm{C} / \mathrm{T}$ \\
\hline UN_xiong_14381 & 108 & $A / G$ \\
\hline UN_xiong_14383 & 498 & $\mathrm{~A} / \mathrm{G}$ \\
\hline UN_xiong_14383 & 542 & $\mathrm{G} / \mathrm{A}$ \\
\hline UN_xiong_14383 & 581 & $\mathrm{C} / \mathrm{T}$ \\
\hline UN_xiong_14383 & 706 & $\mathrm{~T} / \mathrm{C}$ \\
\hline UN_xiong_14383 & 732 & $G / A$ \\
\hline UN_xiong_14383 & 806 & $\mathrm{~A} / \mathrm{G}$ \\
\hline UN_xiong_14383 & 986 & $\mathrm{C} / \mathrm{T}$ \\
\hline UN_xiong_14384 & 60 & $A / G$ \\
\hline UN_xiong_14385 & 162 & $\mathrm{~T} / \mathrm{C}$ \\
\hline UN_xiong_14385 & 400 & $\mathrm{~T} / \mathrm{C}$ \\
\hline UN_xiong_14386 & 383 & $\mathrm{G} / \mathrm{C}$ \\
\hline UN_xiong_14388 & 446 & $\mathrm{~T} / \mathrm{C}$ \\
\hline UN_xiong_14388 & 544 & $\mathrm{~A} / \mathrm{C}$ \\
\hline UN_xiong_14388 & 825 & $\mathrm{~T} / \mathrm{C}$ \\
\hline UN_xiong_14395 & 341 & $A / G$ \\
\hline UN_xiong_14395 & 629 & $\mathrm{C} / \mathrm{T}$ \\
\hline UN_xiong_14395 & 662 & $\mathrm{G} / \mathrm{T}$ \\
\hline UN_xiong_14395 & 685 & $\mathrm{C} / \mathrm{A}$ \\
\hline UN_xiong_14395 & 737 & $\mathrm{~T} / \mathrm{A}$ \\
\hline UN_xiong_14395 & 769 & $C / G$ \\
\hline UN_xiong_14395 & 823 & $C / G$ \\
\hline UN_xiong_14395 & 852 & $\mathrm{G} / \mathrm{T}$ \\
\hline UN_xiong_14395 & 934 & $\mathrm{C} / \mathrm{T}$ \\
\hline UN_xiong_14396 & 39 & $\mathrm{~T} / \mathrm{C}$ \\
\hline UN_xiong_14396 & 93 & $\mathrm{C} / \mathrm{T}$ \\
\hline UN_xiong_14396 & 115 & $\mathrm{~A} / \mathrm{G}$ \\
\hline UN_xiong_14396 & 235 & $\mathrm{~T} / \mathrm{A}$ \\
\hline UN_xiong_14396 & 295 & $\mathrm{C} / \mathrm{T}$ \\
\hline UN_xiong_14396 & 333 & $\mathrm{~A} / \mathrm{C}$ \\
\hline UN_xiong_14397 & 734 & $\mathrm{G} / \mathrm{T}$ \\
\hline UN_xiong_14397 & 838 & $\mathrm{~A} / \mathrm{C}$ \\
\hline UN_xiong_14397 & 893 & $\mathrm{~A} / \mathrm{C}$ \\
\hline UN_xiong_14397 & 1145 & $\mathrm{C} / \mathrm{T}$ \\
\hline UN_xiong_14397 & 1442 & $\mathrm{C} / \mathrm{T}$ \\
\hline UN_xiong_14397 & 1592 & $\mathrm{C} / \mathrm{T}$ \\
\hline UN_xiong_14398 & 859 & $\mathrm{~T} / \mathrm{C}$ \\
\hline UN_xiong_14398 & 917 & $A / G$ \\
\hline UN_xiong_14399 & 283 & $G / A$ \\
\hline UN_xiong_14400 & 165 & $\mathrm{~A} / \mathrm{T}$ \\
\hline UN_xiong_14400 & 392 & $\mathrm{~A} / \mathrm{C}$ \\
\hline
\end{tabular}




\begin{tabular}{|c|c|c|}
\hline UN_xiong_14400 & 856 & $\mathrm{~A} / \mathrm{T}$ \\
\hline UN_xiong_14403 & 405 & $\mathrm{~T} / \mathrm{C}$ \\
\hline UN_xiong_14403 & 851 & $\mathrm{G} / \mathrm{A}$ \\
\hline UN_xiong_14404 & 198 & $A / G$ \\
\hline UN_xiong_14404 & 375 & $\mathrm{C} / \mathrm{A}$ \\
\hline UN_xiong_14404 & 1101 & $A / G$ \\
\hline UN_xiong_14404 & 1547 & $\mathrm{~T} / \mathrm{G}$ \\
\hline UN_xiong_14404 & 1603 & $\mathrm{C} / \mathrm{T}$ \\
\hline UN_xiong_14404 & 1656 & $\mathrm{~T} / \mathrm{C}$ \\
\hline UN_xiong_14404 & 1678 & $\mathrm{~A} / \mathrm{C}$ \\
\hline UN_xiong_14406 & 301 & $\mathrm{G} / \mathrm{T}$ \\
\hline UN_xiong_14407 & 644 & $A / G$ \\
\hline UN_xiong_14407 & 797 & $A / G$ \\
\hline UN_xiong_14407 & 910 & $\mathrm{G} / \mathrm{A}$ \\
\hline UN_xiong_14407 & 1070 & $\mathrm{~T} / \mathrm{C}$ \\
\hline UN_xiong_14408 & 209 & $\mathrm{G} / \mathrm{A}$ \\
\hline UN_xiong_14408 & 313 & $\mathrm{~T} / \mathrm{C}$ \\
\hline UN_xiong_14409 & 61 & $\mathrm{C} / \mathrm{T}$ \\
\hline UN_xiong_14409 & 146 & $\mathrm{~T} / \mathrm{C}$ \\
\hline UN_xiong_14409 & 301 & $A / G$ \\
\hline UN_xiong_14409 & 447 & $\mathrm{C} / \mathrm{T}$ \\
\hline UN_xiong_14409 & 570 & $\mathrm{~A} / \mathrm{C}$ \\
\hline UN_xiong_14409 & 599 & $\mathrm{~T} / \mathrm{A}$ \\
\hline UN_xiong_14409 & 1128 & $\mathrm{~A} / \mathrm{T}$ \\
\hline UN_xiong_14409 & 1161 & $\mathrm{~A} / \mathrm{T}$ \\
\hline UN_xiong_14409 & 1220 & $\mathrm{G} / \mathrm{A}$ \\
\hline UN_xiong_14411 & 299 & $\mathrm{C} / \mathrm{A}$ \\
\hline UN_xiong_14411 & 465 & $\mathrm{C} / \mathrm{T}$ \\
\hline UN_xiong_14412 & 995 & $A / G$ \\
\hline UN_xiong_14414 & 137 & $A / G$ \\
\hline UN_xiong_14414 & 205 & $\mathrm{~A} / \mathrm{T}$ \\
\hline UN_xiong_14414 & 643 & $A / G$ \\
\hline UN_xiong_14414 & 792 & $\mathrm{~T} / \mathrm{C}$ \\
\hline UN_xiong_14414 & 839 & $\mathrm{~T} / \mathrm{C}$ \\
\hline UN_xiong_14414 & 870 & $\mathrm{~A} / \mathrm{T}$ \\
\hline UN_xiong_14418 & 65 & $\mathrm{G} / \mathrm{A}$ \\
\hline UN_xiong_14419 & 899 & $\mathrm{~T} / \mathrm{C}$ \\
\hline UN_xiong_14419 & 1055 & $T / G$ \\
\hline UN_xiong_14419 & 1206 & $\mathrm{C} / \mathrm{A}$ \\
\hline UN_xiong_14421 & 153 & $\mathrm{~A} / \mathrm{C}$ \\
\hline UN_xiong_14422 & 109 & $\mathrm{C} / \mathrm{T}$ \\
\hline UN_xiong_14422 & 193 & $\mathrm{~T} / \mathrm{C}$ \\
\hline UN_xiong_14423 & 71 & $\mathrm{G} / \mathrm{A}$ \\
\hline UN_xiong_14423 & 111 & $A / G$ \\
\hline UN_xiong_14434 & 222 & $\mathrm{C} / \mathrm{A}$ \\
\hline UN_xiong_14437 & 187 & $\mathrm{~T} / \mathrm{C}$ \\
\hline UN_xiong_14437 & 386 & $A / G$ \\
\hline
\end{tabular}




\begin{tabular}{|c|c|c|}
\hline UN_xiong_14437 & 624 & $\mathrm{~A} / \mathrm{G}$ \\
\hline UN_xiong_14437 & 832 & $\mathrm{~A} / \mathrm{T}$ \\
\hline UN_xiong_14437 & 875 & $\mathrm{~T} / \mathrm{C}$ \\
\hline UN_xiong_14437 & 970 & $\mathrm{~T} / \mathrm{A}$ \\
\hline UN_xiong_14438 & 173 & $\mathrm{~A} / \mathrm{G}$ \\
\hline UN_xiong_14440 & 121 & $\mathrm{G} / \mathrm{A}$ \\
\hline UN_xiong_14440 & 143 & $\mathrm{~A} / \mathrm{T}$ \\
\hline UN_xiong_14440 & 315 & $\mathrm{~A} / \mathrm{G}$ \\
\hline UN_xiong_14442 & 305 & $\mathrm{~A} / \mathrm{T}$ \\
\hline UN_xiong_14443 & 636 & $\mathrm{G} / \mathrm{T}$ \\
\hline UN_xiong_14444 & 52 & $\mathrm{C} / \mathrm{T}$ \\
\hline UN_xiong_14444 & 310 & $\mathrm{~A} / \mathrm{G}$ \\
\hline UN_xiong_14444 & 757 & $\mathrm{~A} / \mathrm{G}$ \\
\hline UN_xiong_14444 & 815 & $A / G$ \\
\hline UN_xiong_14444 & 843 & $A / G$ \\
\hline UN_xiong_14444 & 1006 & $\mathrm{~A} / \mathrm{G}$ \\
\hline UN_xiong_14444 & 1083 & $\mathrm{~A} / \mathrm{G}$ \\
\hline UN_xiong_14444 & 1315 & $\mathrm{~T} / \mathrm{C}$ \\
\hline UN_xiong_14444 & 1338 & $\mathrm{~T} / \mathrm{C}$ \\
\hline UN_xiong_14444 & 1363 & $\mathrm{~A} / \mathrm{G}$ \\
\hline UN_xiong_14444 & 1398 & $\mathrm{~A} / \mathrm{G}$ \\
\hline UN_xiong_14444 & 1478 & $\mathrm{C} / \mathrm{T}$ \\
\hline UN_xiong_14453 & 92 & $C / G$ \\
\hline UN_xiong_14453 & 453 & $\mathrm{~T} / \mathrm{C}$ \\
\hline UN_xiong_14454 & 831 & $G / A$ \\
\hline UN_xiong_14455 & 202 & $\mathrm{G} / \mathrm{A}$ \\
\hline UN_xiong_14456 & 70 & $A / G$ \\
\hline UN_xiong_14456 & 375 & $\mathrm{C} / \mathrm{T}$ \\
\hline UN_xiong_14456 & 501 & $\mathrm{~T} / \mathrm{C}$ \\
\hline UN_xiong_14458 & 408 & $\mathrm{~A} / \mathrm{T}$ \\
\hline UN_xiong_14459 & 152 & $\mathrm{~T} / \mathrm{C}$ \\
\hline UN_xiong_14459 & 366 & $\mathrm{C} / \mathrm{T}$ \\
\hline UN_xiong_14466 & 100 & $\mathrm{C} / \mathrm{T}$ \\
\hline UN_xiong_14466 & 416 & $\mathrm{~A} / \mathrm{G}$ \\
\hline UN_xiong_14466 & 447 & $\mathrm{C} / \mathrm{A}$ \\
\hline UN_xiong_14466 & 478 & $\mathrm{C} / \mathrm{T}$ \\
\hline UN_xiong_14466 & 795 & $\mathrm{~A} / \mathrm{G}$ \\
\hline UN_xiong_14469 & 175 & $\mathrm{~T} / \mathrm{C}$ \\
\hline UN_xiong_14469 & 260 & $A / G$ \\
\hline UN_xiong_14469 & 352 & $\mathrm{G} / \mathrm{A}$ \\
\hline UN_xiong_14469 & 418 & $\mathrm{~T} / \mathrm{C}$ \\
\hline UN_xiong_14469 & 512 & $\mathrm{G} / \mathrm{T}$ \\
\hline UN_xiong_14469 & 768 & $\mathrm{~T} / \mathrm{A}$ \\
\hline UN_xiong_14470 & 682 & $A / G$ \\
\hline UN_xiong_14470 & 742 & $\mathrm{~A} / \mathrm{G}$ \\
\hline UN_xiong_14471 & 129 & $\mathrm{~A} / \mathrm{T}$ \\
\hline UN_xiong_14475 & 182 & $\mathrm{~T} / \mathrm{G}$ \\
\hline
\end{tabular}




\begin{tabular}{|c|c|c|}
\hline UN_xiong_14475 & 221 & $\mathrm{~A} / \mathrm{G}$ \\
\hline UN_xiong_14475 & 396 & $\mathrm{~T} / \mathrm{G}$ \\
\hline UN_xiong_14477 & 623 & $\mathrm{~A} / \mathrm{T}$ \\
\hline UN_xiong_14477 & 648 & $\mathrm{~T} / \mathrm{C}$ \\
\hline UN_xiong_14477 & 822 & $\mathrm{C} / \mathrm{T}$ \\
\hline UN_xiong_14480 & 13 & $\mathrm{G} / \mathrm{T}$ \\
\hline UN_xiong_14480 & 59 & $A / G$ \\
\hline UN_xiong_14480 & 119 & $\mathrm{C} / \mathrm{T}$ \\
\hline UN_xiong_14480 & 194 & $A / G$ \\
\hline UN_xiong_14480 & 261 & $\mathrm{G} / \mathrm{C}$ \\
\hline UN_xiong_14480 & 295 & $\mathrm{C} / \mathrm{T}$ \\
\hline UN_xiong_14480 & 323 & $\mathrm{~T} / \mathrm{C}$ \\
\hline UN_xiong_14480 & 435 & $\mathrm{~T} / \mathrm{C}$ \\
\hline UN_xiong_14480 & 472 & $A / G$ \\
\hline UN_xiong_14480 & 602 & $\mathrm{~T} / \mathrm{C}$ \\
\hline UN_xiong_14480 & 696 & $\mathrm{C} / \mathrm{T}$ \\
\hline UN_xiong_14480 & 983 & $\mathrm{~T} / \mathrm{C}$ \\
\hline UN_xiong_14481 & 393 & $\mathrm{G} / \mathrm{A}$ \\
\hline UN_xiong_14481 & 849 & $\mathrm{~T} / \mathrm{C}$ \\
\hline UN_xiong_14482 & 742 & $\mathrm{G} / \mathrm{T}$ \\
\hline UN_xiong_14482 & 855 & $\mathrm{C} / \mathrm{T}$ \\
\hline UN_xiong_14482 & 897 & $\mathrm{~T} / \mathrm{C}$ \\
\hline UN_xiong_14482 & 937 & $\mathrm{~A} / \mathrm{C}$ \\
\hline UN_xiong_14482 & 999 & $\mathrm{~A} / \mathrm{T}$ \\
\hline UN_xiong_14482 & 1033 & $\mathrm{C} / \mathrm{T}$ \\
\hline UN_xiong_14482 & 1465 & $\mathrm{~T} / \mathrm{C}$ \\
\hline UN_xiong_14482 & 1485 & $\mathrm{~T} / \mathrm{C}$ \\
\hline UN_xiong_14482 & 1847 & $\mathrm{G} / \mathrm{A}$ \\
\hline UN_xiong_14482 & 1893 & $G / A$ \\
\hline UN_xiong_14482 & 2063 & $\mathrm{G} / \mathrm{C}$ \\
\hline UN_xiong_14484 & 36 & $\mathrm{~T} / \mathrm{A}$ \\
\hline UN_xiong_14484 & 235 & $\mathrm{~T} / \mathrm{C}$ \\
\hline UN_xiong_14484 & 326 & $\mathrm{~T} / \mathrm{C}$ \\
\hline UN_xiong_14484 & 358 & $\mathrm{~T} / \mathrm{C}$ \\
\hline UN_xiong_14484 & 441 & $\mathrm{~T} / \mathrm{A}$ \\
\hline UN_xiong_14484 & 463 & $\mathrm{~T} / \mathrm{C}$ \\
\hline UN_xiong_14484 & 805 & $A / G$ \\
\hline UN_xiong_14484 & 851 & $\mathrm{~T} / \mathrm{C}$ \\
\hline UN_xiong_14485 & 80 & $\mathrm{G} / \mathrm{C}$ \\
\hline UN_xiong_14485 & 538 & $\mathrm{C} / \mathrm{T}$ \\
\hline UN_xiong_14485 & 579 & $\mathrm{G} / \mathrm{C}$ \\
\hline UN_xiong_14485 & 619 & $\mathrm{C} / \mathrm{T}$ \\
\hline UN_xiong_14485 & 669 & $\mathrm{~T} / \mathrm{G}$ \\
\hline UN_xiong_14485 & 690 & $\mathrm{~A} / \mathrm{G}$ \\
\hline UN_xiong_14485 & 721 & $A / G$ \\
\hline UN_xiong_14485 & 743 & $\mathrm{~T} / \mathrm{C}$ \\
\hline UN_xiong_14485 & 774 & $\mathrm{~A} / \mathrm{G}$ \\
\hline
\end{tabular}




\begin{tabular}{|c|c|c|}
\hline UN_xiong_14486 & 75 & $\mathrm{G} / \mathrm{A}$ \\
\hline UN_xiong_14489 & 579 & $\mathrm{G} / \mathrm{A}$ \\
\hline UN_xiong_14489 & 655 & $\mathrm{~A} / \mathrm{G}$ \\
\hline UN_xiong_14489 & 718 & $\mathrm{~A} / \mathrm{T}$ \\
\hline UN_xiong_14489 & 745 & $\mathrm{~A} / \mathrm{G}$ \\
\hline UN_xiong_14489 & 778 & $\mathrm{C} / \mathrm{T}$ \\
\hline UN_xiong_14489 & 798 & $\mathrm{~T} / \mathrm{C}$ \\
\hline UN_xiong_14489 & 822 & $A / G$ \\
\hline UN_xiong_14489 & 843 & $\mathrm{~T} / \mathrm{C}$ \\
\hline UN_xiong_14489 & 869 & $\mathrm{C} / \mathrm{A}$ \\
\hline UN_xiong_14489 & 908 & $\mathrm{G} / \mathrm{A}$ \\
\hline UN_xiong_14489 & 1030 & $\mathrm{~T} / \mathrm{C}$ \\
\hline UN_xiong_14489 & 1105 & $\mathrm{~T} / \mathrm{C}$ \\
\hline UN_xiong_14489 & 1125 & $\mathrm{~T} / \mathrm{A}$ \\
\hline UN_xiong_14490 & 53 & $\mathrm{C} / \mathrm{A}$ \\
\hline UN_xiong_14490 & 224 & $\mathrm{~T} / \mathrm{C}$ \\
\hline UN_xiong_14490 & 525 & $\mathrm{~T} / \mathrm{C}$ \\
\hline UN_xiong_14491 & 97 & $\mathrm{C} / \mathrm{A}$ \\
\hline UN_xiong_14491 & 118 & $\mathrm{C} / \mathrm{T}$ \\
\hline UN_xiong_14491 & 158 & $\mathrm{C} / \mathrm{T}$ \\
\hline UN_xiong_14491 & 276 & $G / A$ \\
\hline UN_xiong_14493 & 436 & $A / G$ \\
\hline UN_xiong_14493 & 567 & $A / G$ \\
\hline UN_xiong_14493 & 908 & $A / G$ \\
\hline UN_xiong_14493 & 966 & $\mathrm{~T} / \mathrm{G}$ \\
\hline UN_xiong_14493 & 1054 & $\mathrm{C} / \mathrm{T}$ \\
\hline UN_xiong_14494 & 313 & $\mathrm{G} / \mathrm{T}$ \\
\hline UN_xiong_14494 & 371 & $\mathrm{C} / \mathrm{T}$ \\
\hline UN_xiong_14494 & 818 & $\mathrm{~A} / \mathrm{G}$ \\
\hline UN_xiong_14495 & 335 & $\mathrm{C} / \mathrm{T}$ \\
\hline UN_xiong_14496 & 98 & $\mathrm{C} / \mathrm{A}$ \\
\hline UN_xiong_14496 & 154 & $\mathrm{C} / \mathrm{A}$ \\
\hline UN_xiong_14497 & 65 & $\mathrm{~A} / \mathrm{C}$ \\
\hline UN_xiong_14497 & 257 & $\mathrm{C} / \mathrm{T}$ \\
\hline UN_xiong_14497 & 359 & $\mathrm{~A} / \mathrm{C}$ \\
\hline UN_xiong_14497 & 1022 & $\mathrm{G} / \mathrm{A}$ \\
\hline UN_xiong_14497 & 1085 & $\mathrm{~T} / \mathrm{C}$ \\
\hline UN_xiong_14497 & 1164 & $\mathrm{~A} / \mathrm{G}$ \\
\hline UN_xiong_14497 & 1242 & $A / G$ \\
\hline UN_xiong_14500 & 495 & $\mathrm{G} / \mathrm{A}$ \\
\hline UN_xiong_14500 & 548 & $\mathrm{~T} / \mathrm{G}$ \\
\hline UN_xiong_14500 & 684 & $\mathrm{C} / \mathrm{T}$ \\
\hline UN_xiong_14500 & 812 & $A / G$ \\
\hline UN_xiong_14500 & 839 & $\mathrm{~T} / \mathrm{A}$ \\
\hline UN_xiong_14503 & 293 & $\mathrm{C} / \mathrm{T}$ \\
\hline UN_xiong_14505 & 456 & $\mathrm{~T} / \mathrm{C}$ \\
\hline UN_xiong_14505 & 610 & $\mathrm{G} / \mathrm{A}$ \\
\hline
\end{tabular}




\begin{tabular}{|c|c|c|}
\hline UN_xiong_14508 & 442 & $\mathrm{~T} / \mathrm{C}$ \\
\hline UN_xiong_14508 & 465 & $\mathrm{~T} / \mathrm{C}$ \\
\hline UN_xiong_14509 & 245 & $\mathrm{~A} / \mathrm{G}$ \\
\hline UN_xiong_14509 & 292 & $\mathrm{~T} / \mathrm{G}$ \\
\hline UN_xiong_14509 & 364 & $\mathrm{G} / \mathrm{A}$ \\
\hline UN_xiong_14509 & 401 & $\mathrm{C} / \mathrm{T}$ \\
\hline UN_xiong_14509 & 448 & $A / G$ \\
\hline UN_xiong_14512 & 325 & $\mathrm{G} / \mathrm{A}$ \\
\hline UN_xiong_14512 & 506 & $\mathrm{G} / \mathrm{A}$ \\
\hline UN_xiong_14512 & 600 & $\mathrm{~A} / \mathrm{T}$ \\
\hline UN_xiong_14516 & 175 & $\mathrm{C} / \mathrm{G}$ \\
\hline UN_xiong_14518 & 240 & $\mathrm{G} / \mathrm{T}$ \\
\hline UN_xiong_14522 & 536 & $\mathrm{~T} / \mathrm{C}$ \\
\hline UN_xiong_14522 & 729 & $C / A$ \\
\hline UN_xiong_14522 & 848 & $\mathrm{C} / \mathrm{T}$ \\
\hline UN_xiong_14522 & 891 & $\mathrm{G} / \mathrm{C}$ \\
\hline UN_xiong_14522 & 967 & $A / G$ \\
\hline UN_xiong_14522 & 998 & $\mathrm{~T} / \mathrm{C}$ \\
\hline UN_xiong_14522 & 1057 & $\mathrm{~A} / \mathrm{T}$ \\
\hline UN_xiong_14522 & 1135 & $\mathrm{C} / \mathrm{T}$ \\
\hline UN_xiong_14522 & 1162 & $\mathrm{~T} / \mathrm{C}$ \\
\hline UN_xiong_14522 & 1295 & $A / G$ \\
\hline UN_xiong_14522 & 1349 & $\mathrm{~T} / \mathrm{G}$ \\
\hline UN_xiong_14522 & 1394 & $\mathrm{~A} / \mathrm{T}$ \\
\hline UN_xiong_14522 & 1459 & $G / A$ \\
\hline UN_xiong_14522 & 2025 & $\mathrm{~T} / \mathrm{C}$ \\
\hline UN_xiong_14522 & 2152 & $\mathrm{~A} / \mathrm{G}$ \\
\hline UN_xiong_14522 & 2772 & $A / G$ \\
\hline UN_xiong_14522 & 2826 & $A / G$ \\
\hline UN_xiong_14523 & 398 & $C / G$ \\
\hline UN_xiong_14523 & 459 & $\mathrm{~A} / \mathrm{T}$ \\
\hline UN_xiong_14523 & 834 & $\mathrm{~T} / \mathrm{G}$ \\
\hline UN_xiong_14524 & 323 & $\mathrm{~T} / \mathrm{C}$ \\
\hline UN_xiong_14525 & 67 & $\mathrm{~T} / \mathrm{G}$ \\
\hline UN_xiong_14525 & 335 & $\mathrm{~T} / \mathrm{C}$ \\
\hline UN_xiong_14525 & 373 & $\mathrm{~A} / \mathrm{T}$ \\
\hline UN_xiong_14525 & 623 & $\mathrm{~A} / \mathrm{G}$ \\
\hline UN_xiong_14525 & 826 & $A / G$ \\
\hline UN_xiong_14525 & 900 & $A / G$ \\
\hline UN_xiong_14527 & 258 & $A / G$ \\
\hline UN_xiong_14527 & 306 & $\mathrm{G} / \mathrm{A}$ \\
\hline UN_xiong_14527 & 445 & $\mathrm{C} / \mathrm{T}$ \\
\hline UN_xiong_14529 & 14 & $\mathrm{G} / \mathrm{C}$ \\
\hline UN_xiong_14529 & 34 & $\mathrm{~T} / \mathrm{C}$ \\
\hline UN_xiong_14529 & 57 & $\mathrm{G} / \mathrm{A}$ \\
\hline UN_xiong_14529 & 84 & $A / G$ \\
\hline UN_xiong_14529 & 105 & $\mathrm{~A} / \mathrm{C}$ \\
\hline
\end{tabular}




\begin{tabular}{|c|c|c|}
\hline UN_xiong_14529 & 127 & $A / G$ \\
\hline UN_xiong_14529 & 152 & $\mathrm{C} / \mathrm{T}$ \\
\hline UN_xiong_14529 & 180 & $\mathrm{C} / \mathrm{T}$ \\
\hline UN_xiong_14529 & 203 & $\mathrm{~A} / \mathrm{G}$ \\
\hline UN_xiong_14529 & 223 & $\mathrm{G} / \mathrm{T}$ \\
\hline UN_xiong_14529 & 244 & $A / G$ \\
\hline UN_xiong_14529 & 266 & $\mathrm{C} / \mathrm{T}$ \\
\hline UN_xiong_14529 & 289 & $C / A$ \\
\hline UN_xiong_14529 & 312 & $\mathrm{C} / \mathrm{T}$ \\
\hline UN_xiong_14529 & 338 & $\mathrm{C} / \mathrm{T}$ \\
\hline UN_xiong_14529 & 365 & $\mathrm{~A} / \mathrm{T}$ \\
\hline UN_xiong_14529 & 392 & $A / G$ \\
\hline UN_xiong_14530 & 266 & $\mathrm{C} / \mathrm{T}$ \\
\hline UN_xiong_14530 & 409 & $\mathrm{~T} / \mathrm{G}$ \\
\hline UN_xiong_14530 & 807 & $A / G$ \\
\hline UN_xiong_14531 & 131 & $\mathrm{C} / \mathrm{A}$ \\
\hline UN_xiong_14534 & 556 & $\mathrm{~A} / \mathrm{G}$ \\
\hline UN_xiong_14534 & 671 & $\mathrm{~T} / \mathrm{A}$ \\
\hline UN_xiong_14534 & 774 & $\mathrm{~A} / \mathrm{T}$ \\
\hline UN_xiong_14535 & 62 & $\mathrm{~T} / \mathrm{G}$ \\
\hline UN_xiong_14535 & 127 & $G / A$ \\
\hline UN_xiong_14535 & 244 & $\mathrm{G} / \mathrm{A}$ \\
\hline UN_xiong_14535 & 273 & $\mathrm{G} / \mathrm{C}$ \\
\hline UN_xiong_14535 & 360 & $\mathrm{C} / \mathrm{T}$ \\
\hline UN_xiong_14537 & 385 & $\mathrm{C} / \mathrm{T}$ \\
\hline UN_xiong_14538 & 66 & $G / A$ \\
\hline UN_xiong_14539 & 604 & $\mathrm{G} / \mathrm{A}$ \\
\hline UN_xiong_14539 & 750 & $\mathrm{G} / \mathrm{A}$ \\
\hline UN_xiong_14541 & 38 & $A / G$ \\
\hline UN_xiong_14541 & 139 & $\mathrm{G} / \mathrm{T}$ \\
\hline UN_xiong_14541 & 216 & $\mathrm{C} / \mathrm{G}$ \\
\hline UN_xiong_14541 & 602 & $\mathrm{~A} / \mathrm{G}$ \\
\hline UN_xiong_14542 & 126 & $\mathrm{~A} / \mathrm{G}$ \\
\hline UN_xiong_14542 & 230 & $\mathrm{~A} / \mathrm{G}$ \\
\hline UN_xiong_14542 & 266 & $A / G$ \\
\hline UN_xiong_14542 & 295 & $\mathrm{~A} / \mathrm{T}$ \\
\hline UN_xiong_14542 & 323 & $\mathrm{~A} / \mathrm{G}$ \\
\hline UN_xiong_14542 & 353 & $\mathrm{~T} / \mathrm{G}$ \\
\hline UN_xiong_14542 & 379 & $\mathrm{~T} / \mathrm{C}$ \\
\hline UN_xiong_14545 & 503 & $\mathrm{~T} / \mathrm{C}$ \\
\hline UN_xiong_14545 & 549 & $\mathrm{~A} / \mathrm{G}$ \\
\hline UN_xiong_14545 & 636 & $\mathrm{C} / \mathrm{T}$ \\
\hline UN_xiong_14545 & 717 & $A / G$ \\
\hline UN_xiong_14545 & 786 & $\mathrm{G} / \mathrm{T}$ \\
\hline UN_xiong_14545 & 885 & $\mathrm{~T} / \mathrm{C}$ \\
\hline UN_xiong_14545 & 921 & $\mathrm{C} / \mathrm{T}$ \\
\hline UN_xiong_14545 & 1028 & $\mathrm{C} / \mathrm{T}$ \\
\hline
\end{tabular}




\begin{tabular}{|c|c|c|}
\hline UN_xiong_14545 & 1168 & $\mathrm{~T} / \mathrm{C}$ \\
\hline UN_xiong_14545 & 1207 & $A / G$ \\
\hline UN_xiong_14545 & 1252 & $\mathrm{~A} / \mathrm{C}$ \\
\hline UN_xiong_14545 & 1298 & $A / G$ \\
\hline UN_xiong_14545 & 1330 & $\mathrm{~T} / \mathrm{C}$ \\
\hline UN_xiong_14545 & 1357 & $\mathrm{G} / \mathrm{T}$ \\
\hline UN_xiong_14545 & 1380 & $A / G$ \\
\hline UN_xiong_14545 & 1403 & $\mathrm{~T} / \mathrm{C}$ \\
\hline UN_xiong_14545 & 1515 & $\mathrm{~T} / \mathrm{C}$ \\
\hline UN_xiong_14545 & 1553 & $\mathrm{~T} / \mathrm{C}$ \\
\hline UN_xiong_14545 & 1673 & $\mathrm{~T} / \mathrm{C}$ \\
\hline UN_xiong_14545 & 1760 & $\mathrm{~T} / \mathrm{C}$ \\
\hline UN_xiong_14545 & 1781 & $\mathrm{~T} / \mathrm{C}$ \\
\hline UN_xiong_14545 & 1819 & $\mathrm{~T} / \mathrm{C}$ \\
\hline UN_xiong_14545 & 1865 & $A / G$ \\
\hline UN_xiong_14545 & 1914 & $\mathrm{C} / \mathrm{T}$ \\
\hline UN_xiong_14546 & 115 & $\mathrm{G} / \mathrm{T}$ \\
\hline UN_xiong_14546 & 136 & $\mathrm{C} / \mathrm{T}$ \\
\hline UN_xiong_14546 & 157 & $\mathrm{G} / \mathrm{A}$ \\
\hline UN_xiong_14546 & 205 & $\mathrm{C} / \mathrm{T}$ \\
\hline UN_xiong_14546 & 244 & $\mathrm{C} / \mathrm{T}$ \\
\hline UN_xiong_14550 & 345 & $\mathrm{C} / \mathrm{T}$ \\
\hline UN_xiong_14553 & 85 & $A / G$ \\
\hline UN_xiong_14553 & 180 & $\mathrm{~T} / \mathrm{C}$ \\
\hline UN_xiong_14553 & 240 & $\mathrm{~T} / \mathrm{C}$ \\
\hline UN_xiong_14553 & 447 & $A / G$ \\
\hline UN_xiong_14553 & 591 & $\mathrm{~T} / \mathrm{C}$ \\
\hline UN_xiong_14553 & 613 & $\mathrm{C} / \mathrm{T}$ \\
\hline UN_xiong_14553 & 724 & $A / G$ \\
\hline UN_xiong_14553 & 750 & $\mathrm{~T} / \mathrm{C}$ \\
\hline UN_xiong_14553 & 816 & $A / G$ \\
\hline UN_xiong_14553 & 846 & $A / G$ \\
\hline UN_xiong_14553 & 1039 & $\mathrm{~T} / \mathrm{C}$ \\
\hline UN_xiong_14553 & 1083 & $A / G$ \\
\hline UN_xiong_14553 & 1470 & $\mathrm{~T} / \mathrm{A}$ \\
\hline UN_xiong_14553 & 1491 & $\mathrm{~T} / \mathrm{G}$ \\
\hline UN_xiong_14555 & 13 & $\mathrm{G} / \mathrm{T}$ \\
\hline UN_xiong_14555 & 287 & $\mathrm{G} / \mathrm{T}$ \\
\hline UN_xiong_14555 & 313 & $\mathrm{~T} / \mathrm{C}$ \\
\hline UN_xiong_14555 & 338 & $\mathrm{G} / \mathrm{T}$ \\
\hline UN_xiong_14555 & 469 & $\mathrm{~A} / \mathrm{C}$ \\
\hline UN_xiong_14557 & 356 & $\mathrm{~A} / \mathrm{C}$ \\
\hline UN_xiong_14561 & 495 & $\mathrm{~T} / \mathrm{C}$ \\
\hline UN_xiong_14562 & 331 & $A / T$ \\
\hline UN_xiong_14562 & 425 & $\mathrm{C} / \mathrm{T}$ \\
\hline UN_xiong_14563 & 32 & $\mathrm{~T} / \mathrm{G}$ \\
\hline UN_xiong_14563 & 374 & $\mathrm{C} / \mathrm{A}$ \\
\hline
\end{tabular}




\begin{tabular}{|c|c|c|}
\hline UN_xiong_14563 & 895 & $\mathrm{~T} / \mathrm{C}$ \\
\hline UN_xiong_14563 & 1052 & $\mathrm{C} / \mathrm{A}$ \\
\hline UN_xiong_14565 & 1118 & $\mathrm{C} / \mathrm{T}$ \\
\hline UN_xiong_14565 & 1151 & $\mathrm{~T} / \mathrm{C}$ \\
\hline UN_xiong_14565 & 1205 & $A / G$ \\
\hline UN_xiong_14565 & 1227 & $\mathrm{G} / \mathrm{T}$ \\
\hline UN_xiong_14565 & 1271 & $\mathrm{C} / \mathrm{T}$ \\
\hline UN_xiong_14565 & 1358 & $A / G$ \\
\hline UN_xiong_14565 & 1380 & $\mathrm{G} / \mathrm{A}$ \\
\hline UN_xiong_14565 & 1412 & $\mathrm{G} / \mathrm{A}$ \\
\hline UN_xiong_14565 & 1454 & $\mathrm{G} / \mathrm{C}$ \\
\hline UN_xiong_14565 & 1499 & $\mathrm{C} / \mathrm{T}$ \\
\hline UN_xiong_14565 & 1520 & $\mathrm{G} / \mathrm{A}$ \\
\hline UN_xiong_14565 & 1564 & $\mathrm{G} / \mathrm{A}$ \\
\hline UN_xiong_14565 & 1608 & $A / G$ \\
\hline UN_xiong_14565 & 1640 & $A / G$ \\
\hline UN_xiong_14565 & 1732 & $\mathrm{~T} / \mathrm{C}$ \\
\hline UN_xiong_14565 & 1775 & $A / G$ \\
\hline UN_xiong_14566 & 187 & $\mathrm{~T} / \mathrm{C}$ \\
\hline UN_xiong_14566 & 222 & $\mathrm{G} / \mathrm{A}$ \\
\hline UN_xiong_14566 & 306 & $A / G$ \\
\hline UN_xiong_14566 & 360 & $\mathrm{~T} / \mathrm{A}$ \\
\hline UN_xiong_14567 & 164 & $\mathrm{~A} / \mathrm{C}$ \\
\hline UN_xiong_14567 & 221 & $\mathrm{C} / \mathrm{T}$ \\
\hline UN_xiong_14567 & 275 & $\mathrm{~T} / \mathrm{C}$ \\
\hline UN_xiong_14567 & 331 & $A / G$ \\
\hline UN_xiong_14567 & 354 & $\mathrm{~T} / \mathrm{C}$ \\
\hline UN_xiong_14567 & 397 & $\mathrm{C} / \mathrm{T}$ \\
\hline UN_xiong_14567 & 467 & $A / G$ \\
\hline UN_xiong_14567 & 496 & $\mathrm{~T} / \mathrm{C}$ \\
\hline UN_xiong_14567 & 536 & $\mathrm{~T} / \mathrm{A}$ \\
\hline UN_xiong_14568 & 287 & $\mathrm{~A} / \mathrm{T}$ \\
\hline UN_xiong_14568 & 321 & $\mathrm{~A} / \mathrm{C}$ \\
\hline UN_xiong_14568 & 360 & $\mathrm{~T} / \mathrm{A}$ \\
\hline UN_xiong_14568 & 395 & $\mathrm{~T} / \mathrm{C}$ \\
\hline UN_xiong_14568 & 449 & $\mathrm{C} / \mathrm{T}$ \\
\hline UN_xiong_14568 & 564 & $\mathrm{C} / \mathrm{T}$ \\
\hline UN_xiong_14568 & 642 & $\mathrm{~A} / \mathrm{T}$ \\
\hline UN_xiong_14568 & 692 & $\mathrm{C} / \mathrm{T}$ \\
\hline UN_xiong_14568 & 759 & $\mathrm{~T} / \mathrm{C}$ \\
\hline UN_xiong_14569 & 78 & $A / G$ \\
\hline UN_xiong_14569 & 324 & $\mathrm{~T} / \mathrm{C}$ \\
\hline UN_xiong_14569 & 542 & $\mathrm{G} / \mathrm{A}$ \\
\hline UN_xiong_14571 & 150 & $A / G$ \\
\hline UN_xiong_14574 & 267 & $A / G$ \\
\hline UN_xiong_14574 & 581 & $\mathrm{~T} / \mathrm{C}$ \\
\hline UN_xiong_14576 & 92 & $\mathrm{C} / \mathrm{T}$ \\
\hline
\end{tabular}




\begin{tabular}{|c|c|c|}
\hline UN_xiong_14577 & 252 & $\mathrm{G} / \mathrm{A}$ \\
\hline UN_xiong_14578 & 365 & $A / G$ \\
\hline UN_xiong_14578 & 557 & $\mathrm{~A} / \mathrm{G}$ \\
\hline UN_xiong_14578 & 627 & $\mathrm{G} / \mathrm{A}$ \\
\hline UN_xiong_14578 & 757 & $\mathrm{~A} / \mathrm{G}$ \\
\hline UN_xiong_14578 & 777 & $\mathrm{~T} / \mathrm{A}$ \\
\hline UN_xiong_14578 & 799 & $\mathrm{~T} / \mathrm{C}$ \\
\hline UN_xiong_14579 & 10 & $\mathrm{C} / \mathrm{G}$ \\
\hline UN_xiong_14579 & 113 & $\mathrm{~A} / \mathrm{G}$ \\
\hline UN_xiong_14579 & 174 & $\mathrm{C} / \mathrm{T}$ \\
\hline UN_xiong_14579 & 237 & $\mathrm{G} / \mathrm{A}$ \\
\hline UN_xiong_14580 & 209 & $A / G$ \\
\hline UN_xiong_14581 & 89 & $\mathrm{~A} / \mathrm{C}$ \\
\hline UN_xiong_14581 & 444 & $\mathrm{~A} / \mathrm{C}$ \\
\hline UN_xiong_14581 & 469 & $\mathrm{~A} / \mathrm{G}$ \\
\hline UN_xiong_14581 & 497 & $\mathrm{~T} / \mathrm{C}$ \\
\hline UN_xiong_14581 & 555 & $\mathrm{~A} / \mathrm{G}$ \\
\hline UN_xiong_14581 & 580 & $\mathrm{~A} / \mathrm{T}$ \\
\hline UN_xiong_14581 & 715 & $\mathrm{G} / \mathrm{T}$ \\
\hline UN_xiong_14581 & 994 & $\mathrm{G} / \mathrm{A}$ \\
\hline UN_xiong_14581 & 1068 & $\mathrm{~T} / \mathrm{C}$ \\
\hline UN_xiong_14582 & 397 & $\mathrm{~T} / \mathrm{C}$ \\
\hline UN_xiong_14584 & 305 & $\mathrm{C} / \mathrm{T}$ \\
\hline UN_xiong_14584 & 564 & $\mathrm{~A} / \mathrm{G}$ \\
\hline UN_xiong_14584 & 771 & $\mathrm{~T} / \mathrm{C}$ \\
\hline UN_xiong_14584 & 796 & $\mathrm{~A} / \mathrm{G}$ \\
\hline UN_xiong_14584 & 824 & $\mathrm{~T} / \mathrm{C}$ \\
\hline UN_xiong_14584 & 994 & $A / G$ \\
\hline UN_xiong_14584 & 1050 & $\mathrm{~T} / \mathrm{C}$ \\
\hline UN_xiong_14584 & 1104 & $\mathrm{~T} / \mathrm{C}$ \\
\hline UN_xiong_14584 & 1284 & $\mathrm{C} / \mathrm{G}$ \\
\hline UN_xiong_14584 & 1335 & $\mathrm{~A} / \mathrm{C}$ \\
\hline UN_xiong_14584 & 1434 & $\mathrm{~T} / \mathrm{C}$ \\
\hline UN_xiong_14584 & 1504 & $\mathrm{~T} / \mathrm{C}$ \\
\hline UN_xiong_14584 & 1689 & $\mathrm{~T} / \mathrm{A}$ \\
\hline UN_xiong_14584 & 1782 & $\mathrm{~T} / \mathrm{G}$ \\
\hline UN_xiong_14585 & 34 & $A / G$ \\
\hline UN_xiong_14585 & 72 & $\mathrm{~A} / \mathrm{T}$ \\
\hline UN_xiong_14585 & 104 & $\mathrm{C} / \mathrm{A}$ \\
\hline UN_xiong_14585 & 182 & $\mathrm{~T} / \mathrm{C}$ \\
\hline UN_xiong_14585 & 204 & $\mathrm{C} / \mathrm{T}$ \\
\hline UN_xiong_14585 & 227 & $\mathrm{~T} / \mathrm{G}$ \\
\hline UN_xiong_14585 & 259 & $A / G$ \\
\hline UN_xiong_14585 & 291 & $\mathrm{~T} / \mathrm{C}$ \\
\hline UN_xiong_14585 & 325 & $\mathrm{C} / \mathrm{T}$ \\
\hline UN_xiong_14585 & 376 & $\mathrm{C} / \mathrm{G}$ \\
\hline UN_xiong_14585 & 418 & $A / G$ \\
\hline
\end{tabular}




\begin{tabular}{|c|c|c|}
\hline UN_xiong_14590 & 635 & $\mathrm{~T} / \mathrm{A}$ \\
\hline UN_xiong_14590 & 716 & $\mathrm{~T} / \mathrm{C}$ \\
\hline UN_xiong_14591 & 181 & $\mathrm{C} / \mathrm{T}$ \\
\hline UN_xiong_14591 & 771 & $C / G$ \\
\hline UN_xiong_14591 & 918 & $\mathrm{~T} / \mathrm{C}$ \\
\hline UN_xiong_14591 & 954 & $C / G$ \\
\hline UN_xiong_14591 & 1080 & $\mathrm{G} / \mathrm{A}$ \\
\hline UN_xiong_14592 & 130 & $\mathrm{~A} / \mathrm{T}$ \\
\hline UN_xiong_14593 & 98 & $\mathrm{~T} / \mathrm{C}$ \\
\hline UN_xiong_14593 & 227 & $A / G$ \\
\hline UN_xiong_14593 & 279 & $\mathrm{G} / \mathrm{A}$ \\
\hline UN_xiong_14593 & 628 & $\mathrm{~T} / \mathrm{C}$ \\
\hline UN_xiong_14597 & 255 & $\mathrm{~A} / \mathrm{C}$ \\
\hline UN_xiong_14599 & 137 & $\mathrm{~T} / \mathrm{C}$ \\
\hline UN_xiong_14600 & 278 & $A / T$ \\
\hline UN_xiong_14600 & 476 & $A / G$ \\
\hline UN_xiong_14600 & 496 & $A / G$ \\
\hline UN_xiong_14600 & 533 & $A / G$ \\
\hline UN_xiong_14601 & 345 & $\mathrm{C} / \mathrm{T}$ \\
\hline UN_xiong_14603 & 163 & $\mathrm{~A} / \mathrm{C}$ \\
\hline UN_xiong_14605 & 427 & $\mathrm{~T} / \mathrm{C}$ \\
\hline UN_xiong_14605 & 490 & $\mathrm{G} / \mathrm{A}$ \\
\hline UN_xiong_14606 & 393 & $\mathrm{~T} / \mathrm{C}$ \\
\hline UN_xiong_14607 & 236 & $\mathrm{C} / \mathrm{T}$ \\
\hline UN_xiong_14608 & 298 & $\mathrm{~A} / \mathrm{T}$ \\
\hline UN_xiong_14612 & 55 & $\mathrm{C} / \mathrm{A}$ \\
\hline UN_xiong_14612 & 107 & $A / G$ \\
\hline UN_xiong_14612 & 204 & $\mathrm{G} / \mathrm{A}$ \\
\hline UN_xiong_14612 & 253 & $\mathrm{C} / \mathrm{T}$ \\
\hline UN_xiong_14612 & 350 & $C / G$ \\
\hline UN_xiong_14612 & 378 & $\mathrm{~T} / \mathrm{C}$ \\
\hline UN_xiong_14612 & 420 & $\mathrm{G} / \mathrm{C}$ \\
\hline UN_xiong_14612 & 487 & $\mathrm{~T} / \mathrm{C}$ \\
\hline UN_xiong_14612 & 630 & $\mathrm{~T} / \mathrm{C}$ \\
\hline UN_xiong_14612 & 693 & $\mathrm{G} / \mathrm{A}$ \\
\hline UN_xiong_14616 & 50 & $\mathrm{~T} / \mathrm{C}$ \\
\hline UN_xiong_14616 & 121 & $\mathrm{C} / \mathrm{T}$ \\
\hline UN_xiong_14616 & 206 & $A / G$ \\
\hline UN_xiong_14616 & 367 & $A / G$ \\
\hline UN_xiong_14617 & 67 & $\mathrm{G} / \mathrm{C}$ \\
\hline UN_xiong_14617 & 402 & $\mathrm{G} / \mathrm{T}$ \\
\hline UN_xiong_14617 & 440 & $A / G$ \\
\hline UN_xiong_14617 & 466 & $\mathrm{~A} / \mathrm{T}$ \\
\hline UN_xiong_14617 & 550 & $\mathrm{~T} / \mathrm{G}$ \\
\hline UN_xiong_14617 & 788 & $\mathrm{G} / \mathrm{A}$ \\
\hline UN_xiong_14617 & 915 & $A / G$ \\
\hline UN_xiong_14617 & 1051 & $\mathrm{C} / \mathrm{T}$ \\
\hline
\end{tabular}




\begin{tabular}{|c|c|c|}
\hline UN_xiong_14617 & 1159 & $\mathrm{~T} / \mathrm{C}$ \\
\hline UN_xiong_14617 & 1256 & $A / G$ \\
\hline UN_xiong_14617 & 1297 & $A / G$ \\
\hline UN_xiong_14617 & 1366 & $A / G$ \\
\hline UN_xiong_14617 & 1409 & $\mathrm{C} / \mathrm{T}$ \\
\hline UN_xiong_14617 & 1465 & $\mathrm{G} / \mathrm{T}$ \\
\hline UN_xiong_14617 & 1560 & $\mathrm{~T} / \mathrm{C}$ \\
\hline UN_xiong_14617 & 1626 & $\mathrm{~T} / \mathrm{C}$ \\
\hline UN_xiong_14617 & 1656 & $\mathrm{~T} / \mathrm{G}$ \\
\hline UN_xiong_14617 & 1695 & $\mathrm{~T} / \mathrm{G}$ \\
\hline UN_xiong_14617 & 1727 & $\mathrm{~T} / \mathrm{C}$ \\
\hline UN_xiong_14618 & 37 & $\mathrm{G} / \mathrm{A}$ \\
\hline UN_xiong_14618 & 139 & $\mathrm{C} / \mathrm{T}$ \\
\hline UN_xiong_14618 & 240 & $\mathrm{~A} / \mathrm{T}$ \\
\hline UN_xiong_14618 & 382 & $\mathrm{C} / \mathrm{T}$ \\
\hline UN_xiong_14618 & 477 & $\mathrm{~T} / \mathrm{C}$ \\
\hline UN_xiong_14618 & 548 & $\mathrm{~A} / \mathrm{C}$ \\
\hline UN_xiong_14619 & 141 & $A / G$ \\
\hline UN_xiong_14619 & 185 & $\mathrm{~A} / \mathrm{T}$ \\
\hline UN_xiong_14619 & 295 & $\mathrm{G} / \mathrm{A}$ \\
\hline UN_xiong_14619 & 356 & $A / G$ \\
\hline UN_xiong_14619 & 419 & $\mathrm{G} / \mathrm{A}$ \\
\hline UN_xiong_14619 & 549 & $A / G$ \\
\hline UN_xiong_14619 & 576 & $\mathrm{~T} / \mathrm{C}$ \\
\hline UN_xiong_14619 & 601 & $A / G$ \\
\hline UN_xiong_14619 & 666 & $\mathrm{~T} / \mathrm{C}$ \\
\hline UN_xiong_14620 & 35 & $\mathrm{G} / \mathrm{T}$ \\
\hline UN_xiong_14620 & 232 & $\mathrm{~T} / \mathrm{C}$ \\
\hline UN_xiong_14620 & 410 & $A / G$ \\
\hline UN_xiong_14620 & 704 & $A / G$ \\
\hline UN_xiong_14622 & 592 & $\mathrm{~T} / \mathrm{A}$ \\
\hline UN_xiong_14623 & 35 & $\mathrm{C} / \mathrm{T}$ \\
\hline UN_xiong_14623 & 466 & $\mathrm{~T} / \mathrm{C}$ \\
\hline UN_xiong_14623 & 490 & $\mathrm{C} / \mathrm{T}$ \\
\hline UN_xiong_14623 & 520 & $A / G$ \\
\hline UN_xiong_14623 & 553 & $\mathrm{C} / \mathrm{T}$ \\
\hline UN_xiong_14623 & 578 & $A / G$ \\
\hline UN_xiong_14623 & 600 & $\mathrm{G} / \mathrm{T}$ \\
\hline UN_xiong_14623 & 622 & $\mathrm{~T} / \mathrm{C}$ \\
\hline UN_xiong_14623 & 653 & $\mathrm{~A} / \mathrm{C}$ \\
\hline UN_xiong_14623 & 685 & $\mathrm{~A} / \mathrm{T}$ \\
\hline UN_xiong_14623 & 705 & $\mathrm{G} / \mathrm{A}$ \\
\hline UN_xiong_14625 & 581 & $\mathrm{~T} / \mathrm{C}$ \\
\hline UN_xiong_14625 & 689 & $A / G$ \\
\hline UN_xiong_14625 & 755 & $\mathrm{~T} / \mathrm{C}$ \\
\hline UN_xiong_14625 & 785 & $\mathrm{~T} / \mathrm{G}$ \\
\hline UN_xiong_14625 & 839 & $A / G$ \\
\hline
\end{tabular}




\begin{tabular}{|c|c|c|}
\hline UN_xiong_14625 & 860 & $\mathrm{C} / \mathrm{G}$ \\
\hline UN_xiong_14627 & 188 & $\mathrm{C} / \mathrm{T}$ \\
\hline UN_xiong_14627 & 348 & $\mathrm{~T} / \mathrm{C}$ \\
\hline UN_xiong_14629 & 551 & $C / G$ \\
\hline UN_xiong_14629 & 585 & $\mathrm{G} / \mathrm{A}$ \\
\hline UN_xiong_14629 & 613 & $\mathrm{~T} / \mathrm{C}$ \\
\hline UN_xiong_14631 & 726 & $\mathrm{~A} / \mathrm{T}$ \\
\hline UN_xiong_14631 & 789 & $A / G$ \\
\hline UN_xiong_14631 & 838 & $\mathrm{C} / \mathrm{T}$ \\
\hline UN_xiong_14631 & 947 & $\mathrm{C} / \mathrm{T}$ \\
\hline UN_xiong_14631 & 1194 & $\mathrm{G} / \mathrm{A}$ \\
\hline UN_xiong_14631 & 1837 & $A / G$ \\
\hline UN_xiong_14631 & 1989 & $\mathrm{~A} / \mathrm{T}$ \\
\hline UN_xiong_14631 & 2111 & $A / G$ \\
\hline UN_xiong_14631 & 2150 & $\mathrm{~T} / \mathrm{C}$ \\
\hline UN_xiong_14631 & 2177 & $\mathrm{G} / \mathrm{A}$ \\
\hline UN_xiong_14631 & 2198 & $\mathrm{G} / \mathrm{A}$ \\
\hline UN_xiong_14631 & 2274 & $T / G$ \\
\hline UN_xiong_14631 & 2326 & $\mathrm{~T} / \mathrm{C}$ \\
\hline UN_xiong_14631 & 2417 & $\mathrm{~A} / \mathrm{T}$ \\
\hline UN_xiong_14631 & 2479 & $A / G$ \\
\hline UN_xiong_14631 & 2535 & $\mathrm{~T} / \mathrm{C}$ \\
\hline UN_xiong_14631 & 2598 & $\mathrm{~T} / \mathrm{C}$ \\
\hline UN_xiong_14631 & 2652 & $A / G$ \\
\hline UN_xiong_14631 & 2686 & $\mathrm{~T} / \mathrm{C}$ \\
\hline UN_xiong_14631 & 2729 & $\mathrm{G} / \mathrm{T}$ \\
\hline UN_xiong_14631 & 2882 & $A / G$ \\
\hline UN_xiong_14631 & 3470 & $\mathrm{~T} / \mathrm{C}$ \\
\hline UN_xiong_14632 & 390 & $\mathrm{~A} / \mathrm{T}$ \\
\hline UN_xiong_14635 & 416 & $\mathrm{~T} / \mathrm{C}$ \\
\hline UN_xiong_14637 & 45 & $A / G$ \\
\hline UN_xiong_14637 & 251 & $\mathrm{C} / \mathrm{T}$ \\
\hline UN_xiong_14637 & 452 & $A / G$ \\
\hline UN_xiong_14638 & 1093 & $\mathrm{C} / \mathrm{G}$ \\
\hline UN_xiong_14640 & 218 & $A / G$ \\
\hline UN_xiong_14640 & 308 & $A / G$ \\
\hline UN_xiong_14640 & 328 & $\mathrm{~T} / \mathrm{C}$ \\
\hline UN_xiong_14640 & 372 & $\mathrm{~T} / \mathrm{C}$ \\
\hline UN_xiong_14640 & 415 & $\mathrm{~T} / \mathrm{A}$ \\
\hline UN_xiong_14640 & 440 & $\mathrm{~T} / \mathrm{C}$ \\
\hline UN_xiong_14640 & 540 & $\mathrm{~T} / \mathrm{C}$ \\
\hline UN_xiong_14640 & 576 & $\mathrm{G} / \mathrm{A}$ \\
\hline UN_xiong_14640 & 663 & $\mathrm{~T} / \mathrm{C}$ \\
\hline UN_xiong_14641 & 43 & $\mathrm{G} / \mathrm{T}$ \\
\hline UN_xiong_14641 & 85 & $\mathrm{~T} / \mathrm{C}$ \\
\hline UN_xiong_14641 & 244 & $A / G$ \\
\hline UN_xiong_14641 & 316 & $\mathrm{~A} / \mathrm{C}$ \\
\hline
\end{tabular}




\begin{tabular}{|c|c|c|}
\hline UN_xiong_14641 & 597 & $\mathrm{~T} / \mathrm{G}$ \\
\hline UN_xiong_14641 & 759 & $\mathrm{G} / \mathrm{T}$ \\
\hline UN_xiong_14641 & 812 & $\mathrm{~T} / \mathrm{C}$ \\
\hline UN_xiong_14641 & 869 & $\mathrm{G} / \mathrm{A}$ \\
\hline UN_xiong_14641 & 960 & $\mathrm{C} / \mathrm{T}$ \\
\hline UN_xiong_14641 & 1097 & $\mathrm{C} / \mathrm{T}$ \\
\hline UN_xiong_14641 & 1338 & $\mathrm{~T} / \mathrm{C}$ \\
\hline UN_xiong_14641 & 1476 & $A / G$ \\
\hline UN_xiong_14641 & 1516 & $\mathrm{~T} / \mathrm{C}$ \\
\hline UN_xiong_14641 & 1613 & $\mathrm{C} / \mathrm{T}$ \\
\hline UN_xiong_14641 & 1680 & $\mathrm{G} / \mathrm{A}$ \\
\hline UN_xiong_14641 & 1776 & $\mathrm{~T} / \mathrm{C}$ \\
\hline UN_xiong_14641 & 1803 & $\mathrm{~T} / \mathrm{C}$ \\
\hline UN_xiong_14641 & 1828 & $\mathrm{~T} / \mathrm{C}$ \\
\hline UN_xiong_14641 & 1983 & $\mathrm{~T} / \mathrm{C}$ \\
\hline UN_xiong_14643 & 48 & $\mathrm{~T} / \mathrm{A}$ \\
\hline UN_xiong_14644 & 772 & $\mathrm{~T} / \mathrm{C}$ \\
\hline UN_xiong_14645 & 415 & $\mathrm{C} / \mathrm{T}$ \\
\hline UN_xiong_14645 & 468 & $\mathrm{C} / \mathrm{T}$ \\
\hline UN_xiong_14645 & 569 & $A / G$ \\
\hline UN_xiong_14648 & 87 & $A / G$ \\
\hline UN_xiong_14648 & 121 & $\mathrm{~T} / \mathrm{C}$ \\
\hline UN_xiong_14648 & 340 & $\mathrm{~T} / \mathrm{C}$ \\
\hline UN_xiong_14652 & 60 & $\mathrm{~A} / \mathrm{G}$ \\
\hline UN_xiong_14652 & 345 & $\mathrm{G} / \mathrm{T}$ \\
\hline UN_xiong_14655 & 279 & $\mathrm{~T} / \mathrm{G}$ \\
\hline UN_xiong_14656 & 222 & $\mathrm{~T} / \mathrm{C}$ \\
\hline UN_xiong_14658 & 402 & $\mathrm{~A} / \mathrm{C}$ \\
\hline UN_xiong_14658 & 496 & $\mathrm{~T} / \mathrm{C}$ \\
\hline UN_xiong_14660 & 316 & $\mathrm{~T} / \mathrm{A}$ \\
\hline UN_xiong_14660 & 438 & $\mathrm{C} / \mathrm{A}$ \\
\hline UN_xiong_14660 & 527 & $\mathrm{~T} / \mathrm{C}$ \\
\hline UN_xiong_14660 & 560 & $A / G$ \\
\hline UN_xiong_14660 & 585 & $\mathrm{~A} / \mathrm{G}$ \\
\hline UN_xiong_14660 & 676 & $\mathrm{C} / \mathrm{T}$ \\
\hline UN_xiong_14660 & 698 & $\mathrm{~T} / \mathrm{C}$ \\
\hline UN_xiong_14660 & 730 & $\mathrm{C} / \mathrm{T}$ \\
\hline UN_xiong_14660 & 826 & $\mathrm{C} / \mathrm{T}$ \\
\hline UN_xiong_14660 & 870 & $\mathrm{~T} / \mathrm{C}$ \\
\hline UN_xiong_14660 & 1044 & $\mathrm{~T} / \mathrm{C}$ \\
\hline UN_xiong_14660 & 1186 & $\mathrm{~T} / \mathrm{C}$ \\
\hline UN_xiong_14660 & 1435 & $\mathrm{~T} / \mathrm{A}$ \\
\hline UN_xiong_14661 & 95 & $\mathrm{~A} / \mathrm{G}$ \\
\hline UN_xiong_14662 & 698 & $\mathrm{G} / \mathrm{T}$ \\
\hline UN_xiong_14663 & 557 & $\mathrm{C} / \mathrm{T}$ \\
\hline UN_xiong_14665 & 460 & $\mathrm{~T} / \mathrm{C}$ \\
\hline UN_xiong_14665 & 503 & $\mathrm{~A} / \mathrm{T}$ \\
\hline
\end{tabular}




\begin{tabular}{|c|c|c|}
\hline UN_xiong_14667 & 294 & $\mathrm{C} / \mathrm{T}$ \\
\hline UN_xiong_14670 & 78 & $\mathrm{~T} / \mathrm{A}$ \\
\hline UN_xiong_14670 & 373 & $\mathrm{~A} / \mathrm{C}$ \\
\hline UN_xiong_14670 & 397 & $\mathrm{~T} / \mathrm{C}$ \\
\hline UN_xiong_14670 & 528 & $A / G$ \\
\hline UN_xiong_14670 & 577 & $\mathrm{G} / \mathrm{A}$ \\
\hline UN_xiong_14670 & 1435 & $\mathrm{~T} / \mathrm{C}$ \\
\hline UN_xiong_14672 & 285 & $A / G$ \\
\hline UN_xiong_14672 & 564 & $A / G$ \\
\hline UN_xiong_14672 & 989 & $\mathrm{~T} / \mathrm{G}$ \\
\hline UN_xiong_14672 & 1039 & $\mathrm{G} / \mathrm{T}$ \\
\hline UN_xiong_14672 & 1197 & $\mathrm{C} / \mathrm{T}$ \\
\hline UN_xiong_14672 & 1367 & $\mathrm{C} / \mathrm{T}$ \\
\hline UN_xiong_14672 & 1493 & $A / G$ \\
\hline UN_xiong_14672 & 1592 & $\mathrm{~T} / \mathrm{C}$ \\
\hline UN_xiong_14672 & 1671 & $A / G$ \\
\hline UN_xiong_14672 & 1895 & $A / G$ \\
\hline UN_xiong_14672 & 1984 & $\mathrm{~A} / \mathrm{T}$ \\
\hline UN_xiong_14672 & 2014 & $\mathrm{C} / \mathrm{T}$ \\
\hline UN_xiong_14674 & 216 & $\mathrm{G} / \mathrm{C}$ \\
\hline UN_xiong_14674 & 369 & $\mathrm{C} / \mathrm{T}$ \\
\hline UN_xiong_14674 & 997 & $A / G$ \\
\hline UN_xiong_14675 & 80 & $A / G$ \\
\hline UN_xiong_14676 & 298 & $\mathrm{~T} / \mathrm{A}$ \\
\hline UN_xiong_14679 & 670 & $\mathrm{G} / \mathrm{T}$ \\
\hline UN_xiong_14681 & 438 & $\mathrm{~T} / \mathrm{C}$ \\
\hline UN_xiong_14683 & 90 & $\mathrm{~T} / \mathrm{C}$ \\
\hline UN_xiong_14683 & 191 & $\mathrm{~T} / \mathrm{G}$ \\
\hline UN_xiong_14683 & 448 & $\mathrm{~T} / \mathrm{C}$ \\
\hline UN_xiong_14684 & 217 & $\mathrm{G} / \mathrm{C}$ \\
\hline UN_xiong_14685 & 76 & $\mathrm{~T} / \mathrm{C}$ \\
\hline UN_xiong_14685 & 319 & $A / G$ \\
\hline UN_xiong_14685 & 1196 & $\mathrm{G} / \mathrm{A}$ \\
\hline UN_xiong_14687 & 90 & $A / G$ \\
\hline UN_xiong_14687 & 171 & $A / G$ \\
\hline UN_xiong_14687 & 662 & $\mathrm{~T} / \mathrm{C}$ \\
\hline UN_xiong_14687 & 1144 & $\mathrm{G} / \mathrm{T}$ \\
\hline UN_xiong_14687 & 1295 & $A / G$ \\
\hline UN_xiong_14687 & 1379 & $\mathrm{~T} / \mathrm{C}$ \\
\hline UN_xiong_14689 & 365 & $\mathrm{C} / \mathrm{T}$ \\
\hline UN_xiong_14689 & 790 & $\mathrm{G} / \mathrm{A}$ \\
\hline UN_xiong_14689 & 957 & $\mathrm{~T} / \mathrm{C}$ \\
\hline UN_xiong_14689 & 1053 & $\mathrm{G} / \mathrm{A}$ \\
\hline UN_xiong_14689 & 1096 & $A / G$ \\
\hline UN_xiong_14689 & 1322 & $\mathrm{~T} / \mathrm{C}$ \\
\hline UN_xiong_14691 & 545 & $\mathrm{C} / \mathrm{T}$ \\
\hline UN_xiong_14691 & 669 & $A / G$ \\
\hline
\end{tabular}




\begin{tabular}{|c|c|c|}
\hline UN_xiong_14692 & 613 & $\mathrm{C} / \mathrm{A}$ \\
\hline UN_xiong_14692 & 784 & $A / G$ \\
\hline UN_xiong_14692 & 1011 & $\mathrm{~T} / \mathrm{G}$ \\
\hline UN_xiong_14692 & 1038 & $\mathrm{~A} / \mathrm{T}$ \\
\hline UN_xiong_14692 & 1060 & $\mathrm{~A} / \mathrm{T}$ \\
\hline UN_xiong_14692 & 1090 & $\mathrm{~T} / \mathrm{C}$ \\
\hline UN_xiong_14692 & 1148 & $\mathrm{~T} / \mathrm{C}$ \\
\hline UN_xiong_14692 & 1201 & $\mathrm{~T} / \mathrm{C}$ \\
\hline UN_xiong_14692 & 1227 & $\mathrm{C} / \mathrm{T}$ \\
\hline UN_xiong_14692 & 1375 & $\mathrm{G} / \mathrm{A}$ \\
\hline UN_xiong_14692 & 1417 & $A / G$ \\
\hline UN_xiong_14696 & 536 & $A / G$ \\
\hline UN_xiong_14697 & 207 & $\mathrm{~T} / \mathrm{C}$ \\
\hline UN_xiong_14697 & 588 & $\mathrm{~T} / \mathrm{C}$ \\
\hline UN_xiong_14700 & 67 & $A / G$ \\
\hline UN_xiong_14700 & 153 & $\mathrm{~T} / \mathrm{A}$ \\
\hline UN_xiong_14702 & 360 & $\mathrm{G} / \mathrm{T}$ \\
\hline UN_xiong_14704 & 55 & $\mathrm{C} / \mathrm{T}$ \\
\hline UN_xiong_14705 & 157 & $\mathrm{G} / \mathrm{A}$ \\
\hline UN_xiong_14710 & 343 & $\mathrm{G} / \mathrm{A}$ \\
\hline UN_xiong_14710 & 373 & $\mathrm{G} / \mathrm{A}$ \\
\hline UN_xiong_14712 & 117 & $\mathrm{~T} / \mathrm{C}$ \\
\hline UN_xiong_14712 & 285 & $\mathrm{G} / \mathrm{A}$ \\
\hline UN_xiong_14716 & 18 & $C / G$ \\
\hline UN_xiong_14716 & 39 & $A / G$ \\
\hline UN_xiong_14716 & 80 & $\mathrm{C} / \mathrm{T}$ \\
\hline UN_xiong_14716 & 215 & $\mathrm{C} / \mathrm{T}$ \\
\hline UN_xiong_14716 & 242 & $A / G$ \\
\hline UN_xiong_14716 & 469 & $\mathrm{C} / \mathrm{T}$ \\
\hline UN_xiong_14716 & 860 & $\mathrm{~T} / \mathrm{C}$ \\
\hline UN_xiong_14716 & 935 & $\mathrm{~A} / \mathrm{T}$ \\
\hline UN_xiong_14716 & 1028 & $A / G$ \\
\hline UN_xiong_14716 & 1054 & $\mathrm{~T} / \mathrm{G}$ \\
\hline UN_xiong_14716 & 1087 & $\mathrm{C} / \mathrm{T}$ \\
\hline UN_xiong_14716 & 1130 & $\mathrm{G} / \mathrm{A}$ \\
\hline UN_xiong_14716 & 1284 & $\mathrm{~T} / \mathrm{C}$ \\
\hline UN_xiong_14716 & 1314 & $\mathrm{C} / \mathrm{A}$ \\
\hline UN_xiong_14716 & 1415 & $\mathrm{~T} / \mathrm{A}$ \\
\hline UN_xiong_14716 & 1456 & $A / G$ \\
\hline UN_xiong_14716 & 1479 & $A / G$ \\
\hline UN_xiong_14716 & 1512 & $A / G$ \\
\hline UN_xiong_14716 & 1532 & $A / G$ \\
\hline UN_xiong_14716 & 1554 & $A / G$ \\
\hline UN_xiong_14716 & 1579 & $\mathrm{~T} / \mathrm{C}$ \\
\hline UN_xiong_14716 & 1605 & $\mathrm{~T} / \mathrm{G}$ \\
\hline UN_xiong_14716 & 1626 & $\mathrm{~T} / \mathrm{C}$ \\
\hline UN_xiong_14716 & 1647 & $\mathrm{G} / \mathrm{A}$ \\
\hline
\end{tabular}




\begin{tabular}{|c|c|c|}
\hline UN_xiong_14716 & 1688 & $\mathrm{C} / \mathrm{T}$ \\
\hline UN_xiong_14716 & 1708 & $A / G$ \\
\hline UN_xiong_14716 & 1794 & $\mathrm{G} / \mathrm{C}$ \\
\hline UN_xiong_14717 & 19 & $\mathrm{G} / \mathrm{A}$ \\
\hline UN_xiong_14717 & 372 & $A / G$ \\
\hline UN_xiong_14717 & 550 & $A / G$ \\
\hline UN_xiong_14717 & 588 & $\mathrm{~T} / \mathrm{C}$ \\
\hline UN_xiong_14717 & 703 & $A / G$ \\
\hline UN_xiong_14718 & 344 & $\mathrm{~T} / \mathrm{C}$ \\
\hline UN_xiong_14718 & 583 & $\mathrm{~A} / \mathrm{T}$ \\
\hline UN_xiong_14720 & 401 & $\mathrm{~T} / \mathrm{C}$ \\
\hline UN_xiong_14720 & 1476 & $\mathrm{G} / \mathrm{A}$ \\
\hline UN_xiong_14720 & 1499 & $\mathrm{~T} / \mathrm{C}$ \\
\hline UN_xiong_14721 & 427 & $\mathrm{~T} / \mathrm{C}$ \\
\hline UN_xiong_14721 & 501 & $\mathrm{G} / \mathrm{A}$ \\
\hline UN_xiong_14721 & 524 & $A / G$ \\
\hline UN_xiong_14721 & 1060 & $A / G$ \\
\hline UN_xiong_14722 & 327 & $A / G$ \\
\hline UN_xiong_14722 & 701 & $\mathrm{~T} / \mathrm{A}$ \\
\hline UN_xiong_14722 & 746 & $\mathrm{C} / \mathrm{T}$ \\
\hline UN_xiong_14724 & 410 & $\mathrm{C} / \mathrm{G}$ \\
\hline UN_xiong_14725 & 425 & $\mathrm{C} / \mathrm{T}$ \\
\hline UN_xiong_14727 & 805 & $\mathrm{G} / \mathrm{A}$ \\
\hline UN_xiong_14727 & 940 & $\mathrm{C} / \mathrm{A}$ \\
\hline UN_xiong_14727 & 1014 & $\mathrm{C} / \mathrm{T}$ \\
\hline UN_xiong_14727 & 1036 & $\mathrm{C} / \mathrm{T}$ \\
\hline UN_xiong_14727 & 1068 & $\mathrm{~T} / \mathrm{C}$ \\
\hline UN_xiong_14727 & 1088 & $\mathrm{C} / \mathrm{T}$ \\
\hline UN_xiong_14728 & 488 & $\mathrm{C} / \mathrm{T}$ \\
\hline UN_xiong_14729 & 1922 & $\mathrm{C} / \mathrm{T}$ \\
\hline UN_xiong_14729 & 1983 & $\mathrm{C} / \mathrm{A}$ \\
\hline UN_xiong_14730 & 107 & $\mathrm{~T} / \mathrm{C}$ \\
\hline UN_xiong_14731 & 265 & $\mathrm{C} / \mathrm{T}$ \\
\hline UN_xiong_14731 & 326 & $\mathrm{~T} / \mathrm{C}$ \\
\hline UN_xiong_14732 & 479 & $\mathrm{G} / \mathrm{C}$ \\
\hline UN_xiong_14736 & 72 & $\mathrm{C} / \mathrm{T}$ \\
\hline UN_xiong_14736 & 262 & $\mathrm{C} / \mathrm{A}$ \\
\hline UN_xiong_14736 & 516 & $\mathrm{~T} / \mathrm{C}$ \\
\hline UN_xiong_14736 & 812 & $A / G$ \\
\hline UN_xiong_14736 & 920 & $A / G$ \\
\hline UN_xiong_14736 & 979 & $\mathrm{~T} / \mathrm{C}$ \\
\hline UN_xiong_14736 & 1098 & $A / G$ \\
\hline UN_xiong_14736 & 1120 & $A / G$ \\
\hline UN_xiong_14737 & 193 & $\mathrm{~A} / \mathrm{C}$ \\
\hline UN_xiong_14738 & 50 & $\mathrm{~A} / \mathrm{C}$ \\
\hline UN_xiong_14740 & 487 & $\mathrm{G} / \mathrm{T}$ \\
\hline UN_xiong_14741 & 850 & $A / G$ \\
\hline
\end{tabular}




\begin{tabular}{|c|c|c|}
\hline UN_xiong_14742 & 357 & $\mathrm{~A} / \mathrm{T}$ \\
\hline UN_xiong_14743 & 713 & $\mathrm{G} / \mathrm{T}$ \\
\hline UN_xiong_14747 & 94 & $\mathrm{~A} / \mathrm{G}$ \\
\hline UN_xiong_14747 & 135 & $\mathrm{C} / \mathrm{T}$ \\
\hline UN_xiong_14747 & 168 & $\mathrm{~A} / \mathrm{G}$ \\
\hline UN_xiong_14749 & 229 & $\mathrm{~T} / \mathrm{C}$ \\
\hline UN_xiong_14749 & 254 & $\mathrm{~T} / \mathrm{A}$ \\
\hline UN_xiong_14749 & 312 & $\mathrm{C} / \mathrm{T}$ \\
\hline UN_xiong_14749 & 333 & $\mathrm{C} / \mathrm{T}$ \\
\hline UN_xiong_14749 & 448 & $\mathrm{~A} / \mathrm{C}$ \\
\hline UN_xiong_14749 & 576 & $\mathrm{~T} / \mathrm{C}$ \\
\hline UN_xiong_14749 & 684 & $\mathrm{G} / \mathrm{A}$ \\
\hline UN_xiong_14751 & 1696 & $\mathrm{C} / \mathrm{T}$ \\
\hline UN_xiong_14751 & 1819 & $\mathrm{~A} / \mathrm{C}$ \\
\hline UN_xiong_14752 & 832 & $\mathrm{~T} / \mathrm{C}$ \\
\hline UN_xiong_14752 & 862 & $\mathrm{G} / \mathrm{C}$ \\
\hline UN_xiong_14753 & 111 & $\mathrm{~A} / \mathrm{T}$ \\
\hline UN_xiong_14753 & 131 & $\mathrm{C} / \mathrm{A}$ \\
\hline UN_xiong_14755 & 58 & $A / G$ \\
\hline UN_xiong_14756 & 177 & $\mathrm{C} / \mathrm{A}$ \\
\hline UN_xiong_14756 & 718 & $\mathrm{C} / \mathrm{T}$ \\
\hline UN_xiong_14756 & 981 & $\mathrm{C} / \mathrm{T}$ \\
\hline UN_xiong_14761 & 485 & $\mathrm{~T} / \mathrm{C}$ \\
\hline UN_xiong_14762 & 125 & $A / G$ \\
\hline UN_xiong_14763 & 291 & $G / A$ \\
\hline UN_xiong_14763 & 756 & $\mathrm{~A} / \mathrm{G}$ \\
\hline UN_xiong_14763 & 1001 & $\mathrm{~T} / \mathrm{C}$ \\
\hline UN_xiong_14763 & 1027 & $\mathrm{C} / \mathrm{T}$ \\
\hline UN_xiong_14766 & 140 & $\mathrm{~A} / \mathrm{G}$ \\
\hline UN_xiong_14766 & 189 & $\mathrm{~T} / \mathrm{A}$ \\
\hline UN_xiong_14766 & 242 & $\mathrm{C} / \mathrm{T}$ \\
\hline UN_xiong_14766 & 281 & $\mathrm{G} / \mathrm{A}$ \\
\hline UN_xiong_14766 & 316 & $A / G$ \\
\hline UN_xiong_14766 & 376 & $\mathrm{~T} / \mathrm{C}$ \\
\hline UN_xiong_14766 & 461 & $\mathrm{G} / \mathrm{A}$ \\
\hline UN_xiong_14766 & 513 & $\mathrm{~T} / \mathrm{C}$ \\
\hline UN_xiong_14766 & 541 & $\mathrm{C} / \mathrm{T}$ \\
\hline UN_xiong_14766 & 707 & $\mathrm{C} / \mathrm{T}$ \\
\hline UN_xiong_14766 & 1684 & $\mathrm{~T} / \mathrm{C}$ \\
\hline UN_xiong_14766 & 1755 & $A / G$ \\
\hline UN_xiong_14766 & 1899 & $\mathrm{C} / \mathrm{G}$ \\
\hline UN_xiong_14768 & 630 & $\mathrm{~T} / \mathrm{C}$ \\
\hline UN_xiong_14768 & 697 & $\mathrm{~A} / \mathrm{T}$ \\
\hline UN_xiong_14772 & 104 & $\mathrm{~T} / \mathrm{G}$ \\
\hline UN_xiong_14772 & 209 & $A / G$ \\
\hline UN_xiong_14772 & 229 & $A / G$ \\
\hline UN_xiong_14772 & 341 & $\mathrm{~T} / \mathrm{C}$ \\
\hline
\end{tabular}




\begin{tabular}{|c|c|c|}
\hline UN_xiong_14772 & 370 & $\mathrm{~T} / \mathrm{G}$ \\
\hline UN_xiong_14772 & 684 & $\mathrm{~T} / \mathrm{C}$ \\
\hline UN_xiong_14772 & 1058 & $\mathrm{C} / \mathrm{T}$ \\
\hline UN_xiong_14772 & 1186 & $\mathrm{G} / \mathrm{A}$ \\
\hline UN_xiong_14772 & 1334 & $T / G$ \\
\hline UN_xiong_14772 & 1405 & $\mathrm{~T} / \mathrm{C}$ \\
\hline UN_xiong_14772 & 1481 & $\mathrm{~T} / \mathrm{A}$ \\
\hline UN_xiong_14772 & 1517 & $\mathrm{~T} / \mathrm{A}$ \\
\hline UN_xiong_14773 & 175 & $\mathrm{~T} / \mathrm{A}$ \\
\hline UN_xiong_14774 & 417 & $\mathrm{C} / \mathrm{T}$ \\
\hline UN_xiong_14775 & 153 & $G / A$ \\
\hline UN_xiong_14775 & 214 & $A / G$ \\
\hline UN_xiong_14778 & 393 & $A / G$ \\
\hline UN_xiong_14778 & 491 & $\mathrm{~T} / \mathrm{G}$ \\
\hline UN_xiong_14778 & 566 & $A / G$ \\
\hline UN_xiong_14780 & 253 & $\mathrm{~A} / \mathrm{T}$ \\
\hline UN_xiong_14781 & 764 & $\mathrm{G} / \mathrm{A}$ \\
\hline UN_xiong_14781 & 787 & $\mathrm{C} / \mathrm{T}$ \\
\hline UN_xiong_14781 & 853 & $A / G$ \\
\hline UN_xiong_14781 & 991 & $A / G$ \\
\hline UN_xiong_14783 & 64 & $\mathrm{~T} / \mathrm{C}$ \\
\hline UN_xiong_14783 & 123 & $A / G$ \\
\hline UN_xiong_14783 & 201 & $\mathrm{C} / \mathrm{T}$ \\
\hline UN_xiong_14783 & 227 & $\mathrm{C} / \mathrm{T}$ \\
\hline UN_xiong_14783 & 446 & $A / G$ \\
\hline UN_xiong_14783 & 505 & $A / G$ \\
\hline UN_xiong_14783 & 571 & $\mathrm{C} / \mathrm{T}$ \\
\hline UN_xiong_14783 & 633 & $\mathrm{~A} / \mathrm{T}$ \\
\hline UN_xiong_14784 & 45 & $A / G$ \\
\hline UN_xiong_14784 & 169 & $\mathrm{~T} / \mathrm{C}$ \\
\hline UN_xiong_14784 & 333 & $\mathrm{~T} / \mathrm{C}$ \\
\hline UN_xiong_14784 & 646 & $\mathrm{G} / \mathrm{A}$ \\
\hline UN_xiong_14784 & 670 & $\mathrm{~T} / \mathrm{A}$ \\
\hline UN_xiong_14786 & 373 & $\mathrm{~T} / \mathrm{C}$ \\
\hline UN_xiong_14786 & 755 & $A / G$ \\
\hline UN_xiong_14786 & 789 & $A / G$ \\
\hline UN_xiong_14786 & 901 & $\mathrm{~T} / \mathrm{C}$ \\
\hline UN_xiong_14786 & 926 & $\mathrm{~T} / \mathrm{C}$ \\
\hline UN_xiong_14786 & 966 & $A / G$ \\
\hline UN_xiong_14788 & 123 & $\mathrm{G} / \mathrm{A}$ \\
\hline UN_xiong_14788 & 159 & $\mathrm{C} / \mathrm{T}$ \\
\hline UN_xiong_14789 & 268 & $\mathrm{C} / \mathrm{T}$ \\
\hline UN_xiong_14789 & 303 & $A / G$ \\
\hline UN_xiong_14789 & 332 & $\mathrm{~A} / \mathrm{C}$ \\
\hline UN_xiong_14789 & 603 & $\mathrm{~T} / \mathrm{C}$ \\
\hline UN_xiong_14791 & 19 & $A / G$ \\
\hline UN_xiong_14791 & 283 & $A / G$ \\
\hline
\end{tabular}




\begin{tabular}{|c|c|c|}
\hline UN_xiong_14791 & 310 & $\mathrm{G} / \mathrm{A}$ \\
\hline UN_xiong_14791 & 352 & $\mathrm{C} / \mathrm{T}$ \\
\hline UN_xiong_14791 & 432 & $\mathrm{C} / \mathrm{T}$ \\
\hline UN_xiong_14791 & 598 & $\mathrm{~T} / \mathrm{C}$ \\
\hline UN_xiong_14791 & 667 & $\mathrm{G} / \mathrm{A}$ \\
\hline UN_xiong_14793 & 205 & $\mathrm{G} / \mathrm{C}$ \\
\hline UN_xiong_14793 & 229 & $\mathrm{~A} / \mathrm{T}$ \\
\hline UN_xiong_14793 & 446 & $G / A$ \\
\hline UN_xiong_14795 & 358 & $\mathrm{~T} / \mathrm{C}$ \\
\hline UN_xiong_14795 & 521 & $A / G$ \\
\hline UN_xiong_14795 & 738 & $\mathrm{~T} / \mathrm{C}$ \\
\hline UN_xiong_14796 & 125 & $\mathrm{~A} / \mathrm{T}$ \\
\hline UN_xiong_14800 & 83 & $\mathrm{~T} / \mathrm{C}$ \\
\hline UN_xiong_14800 & 498 & $\mathrm{G} / \mathrm{A}$ \\
\hline UN_xiong_14801 & 81 & $\mathrm{G} / \mathrm{T}$ \\
\hline UN_xiong_14801 & 157 & $\mathrm{G} / \mathrm{A}$ \\
\hline UN_xiong_14801 & 213 & $\mathrm{~A} / \mathrm{G}$ \\
\hline UN_xiong_14801 & 507 & $\mathrm{~T} / \mathrm{C}$ \\
\hline UN_xiong_14801 & 550 & $\mathrm{~T} / \mathrm{C}$ \\
\hline UN_xiong_14802 & 636 & $\mathrm{~T} / \mathrm{C}$ \\
\hline UN_xiong_14802 & 1025 & $\mathrm{~A} / \mathrm{T}$ \\
\hline UN_xiong_14803 & 899 & $\mathrm{G} / \mathrm{A}$ \\
\hline UN_xiong_14803 & 2251 & $\mathrm{~T} / \mathrm{C}$ \\
\hline UN_xiong_14804 & 97 & $\mathrm{~A} / \mathrm{G}$ \\
\hline UN_xiong_14804 & 164 & $\mathrm{C} / \mathrm{T}$ \\
\hline UN_xiong_14805 & 361 & $\mathrm{C} / \mathrm{T}$ \\
\hline UN_xiong_14805 & 1194 & $\mathrm{~T} / \mathrm{C}$ \\
\hline UN_xiong_14805 & 1238 & $\mathrm{~A} / \mathrm{T}$ \\
\hline UN_xiong_14805 & 1286 & $\mathrm{~A} / \mathrm{G}$ \\
\hline UN_xiong_14805 & 1490 & $\mathrm{C} / \mathrm{T}$ \\
\hline UN_xiong_14805 & 1585 & $\mathrm{~T} / \mathrm{C}$ \\
\hline UN_xiong_14805 & 1639 & $\mathrm{~A} / \mathrm{G}$ \\
\hline UN_xiong_14805 & 1804 & $A / G$ \\
\hline UN_xiong_14806 & 349 & $\mathrm{~A} / \mathrm{T}$ \\
\hline UN_xiong_14806 & 634 & $A / G$ \\
\hline UN_xiong_14806 & 658 & $\mathrm{C} / \mathrm{T}$ \\
\hline UN_xiong_14808 & 669 & $\mathrm{~T} / \mathrm{C}$ \\
\hline UN_xiong_14808 & 752 & $\mathrm{~T} / \mathrm{C}$ \\
\hline UN_xiong_14810 & 179 & $\mathrm{~T} / \mathrm{A}$ \\
\hline UN_xiong_14810 & 199 & $A / G$ \\
\hline UN_xiong_14811 & 304 & $\mathrm{G} / \mathrm{A}$ \\
\hline UN_xiong_14812 & 150 & $\mathrm{G} / \mathrm{T}$ \\
\hline UN_xiong_14815 & 220 & $\mathrm{C} / \mathrm{T}$ \\
\hline UN_xiong_14815 & 261 & $\mathrm{C} / \mathrm{T}$ \\
\hline UN_xiong_14816 & 340 & $\mathrm{C} / \mathrm{T}$ \\
\hline UN_xiong_14817 & 221 & $\mathrm{C} / \mathrm{T}$ \\
\hline UN_xiong_14817 & 288 & $\mathrm{~A} / \mathrm{T}$ \\
\hline
\end{tabular}




\begin{tabular}{|c|c|c|}
\hline UN_xiong_14818 & 316 & $A / G$ \\
\hline UN_xiong_14819 & 239 & $A / G$ \\
\hline UN_xiong_14819 & 308 & $\mathrm{~A} / \mathrm{T}$ \\
\hline UN_xiong_14819 & 347 & $\mathrm{~T} / \mathrm{C}$ \\
\hline UN_xiong_14820 & 91 & $\mathrm{C} / \mathrm{T}$ \\
\hline UN_xiong_14820 & 155 & $\mathrm{G} / \mathrm{A}$ \\
\hline UN_xiong_14820 & 182 & $\mathrm{~T} / \mathrm{A}$ \\
\hline UN_xiong_14820 & 250 & $\mathrm{~T} / \mathrm{G}$ \\
\hline UN_xiong_14820 & 271 & $A / G$ \\
\hline UN_xiong_14820 & 328 & $\mathrm{G} / \mathrm{T}$ \\
\hline UN_xiong_14823 & 333 & $A / G$ \\
\hline UN_xiong_14823 & 453 & $\mathrm{C} / \mathrm{T}$ \\
\hline UN_xiong_14823 & 663 & $\mathrm{~T} / \mathrm{C}$ \\
\hline UN_xiong_14826 & 289 & $A / G$ \\
\hline UN_xiong_14827 & 1012 & $\mathrm{C} / \mathrm{A}$ \\
\hline UN_xiong_14828 & 210 & $A / G$ \\
\hline UN_xiong_14828 & 406 & $\mathrm{~A} / \mathrm{C}$ \\
\hline UN_xiong_14829 & 333 & $A / T$ \\
\hline UN_xiong_14829 & 891 & $\mathrm{~T} / \mathrm{C}$ \\
\hline UN_xiong_14832 & 342 & $\mathrm{~T} / \mathrm{C}$ \\
\hline UN_xiong_14833 & 458 & $\mathrm{~T} / \mathrm{C}$ \\
\hline UN_xiong_14833 & 635 & $A / G$ \\
\hline UN_xiong_14833 & 662 & $\mathrm{C} / \mathrm{A}$ \\
\hline UN_xiong_14835 & 100 & $A / G$ \\
\hline UN_xiong_14835 & 332 & $\mathrm{~T} / \mathrm{C}$ \\
\hline UN_xiong_14835 & 679 & $\mathrm{~T} / \mathrm{C}$ \\
\hline UN_xiong_14836 & 166 & $\mathrm{~T} / \mathrm{C}$ \\
\hline UN_xiong_14836 & 344 & $A / G$ \\
\hline UN_xiong_14836 & 448 & $G / A$ \\
\hline UN_xiong_14836 & 628 & $A / G$ \\
\hline UN_xiong_14836 & 1043 & $\mathrm{~T} / \mathrm{C}$ \\
\hline UN_xiong_14842 & 19 & $\mathrm{G} / \mathrm{T}$ \\
\hline UN_xiong_14842 & 62 & $C / G$ \\
\hline UN_xiong_14842 & 227 & $\mathrm{~T} / \mathrm{C}$ \\
\hline UN_xiong_14842 & 462 & $\mathrm{~T} / \mathrm{C}$ \\
\hline UN_xiong_14846 & 1017 & $\mathrm{G} / \mathrm{A}$ \\
\hline UN_xiong_14846 & 1298 & $A / G$ \\
\hline UN_xiong_14852 & 1033 & $\mathrm{~T} / \mathrm{C}$ \\
\hline UN_xiong_14852 & 1054 & $\mathrm{~A} / \mathrm{T}$ \\
\hline UN_xiong_14852 & 1198 & $A / G$ \\
\hline UN_xiong_14852 & 1219 & $\mathrm{~T} / \mathrm{C}$ \\
\hline UN_xiong_14852 & 1241 & $\mathrm{~T} / \mathrm{G}$ \\
\hline UN_xiong_14852 & 1273 & $\mathrm{G} / \mathrm{A}$ \\
\hline UN_xiong_14852 & 1351 & $\mathrm{~A} / \mathrm{T}$ \\
\hline UN_xiong_14854 & 202 & $A / G$ \\
\hline UN_xiong_14854 & 381 & $\mathrm{C} / \mathrm{T}$ \\
\hline UN_xiong_14854 & 475 & $\mathrm{~T} / \mathrm{C}$ \\
\hline
\end{tabular}




\begin{tabular}{|c|c|c|}
\hline UN_xiong_14855 & 592 & $\mathrm{~T} / \mathrm{C}$ \\
\hline UN_xiong_14856 & 744 & $\mathrm{G} / \mathrm{C}$ \\
\hline UN_xiong_14858 & 140 & $\mathrm{G} / \mathrm{T}$ \\
\hline UN_xiong_14859 & 569 & $\mathrm{G} / \mathrm{A}$ \\
\hline UN_xiong_14859 & 647 & $A / G$ \\
\hline UN_xiong_14859 & 993 & $\mathrm{~T} / \mathrm{G}$ \\
\hline UN_xiong_14860 & 643 & $\mathrm{G} / \mathrm{A}$ \\
\hline UN_xiong_14862 & 76 & $\mathrm{~T} / \mathrm{G}$ \\
\hline UN_xiong_14864 & 341 & $C / G$ \\
\hline UN_xiong_14868 & 287 & $\mathrm{G} / \mathrm{A}$ \\
\hline UN_xiong_14868 & 405 & $A / G$ \\
\hline UN_xiong_14868 & 516 & $\mathrm{~A} / \mathrm{T}$ \\
\hline UN_xiong_14868 & 611 & $\mathrm{~T} / \mathrm{C}$ \\
\hline UN_xiong_14869 & 183 & $\mathrm{C} / \mathrm{T}$ \\
\hline UN_xiong_14869 & 240 & $\mathrm{G} / \mathrm{A}$ \\
\hline UN_xiong_14869 & 261 & $\mathrm{~A} / \mathrm{T}$ \\
\hline UN_xiong_14869 & 401 & $\mathrm{G} / \mathrm{C}$ \\
\hline UN_xiong_14869 & 1191 & $C / G$ \\
\hline UN_xiong_14869 & 1239 & $\mathrm{G} / \mathrm{A}$ \\
\hline UN_xiong_14869 & 1330 & $\mathrm{~T} / \mathrm{G}$ \\
\hline UN_xiong_14869 & 1443 & $\mathrm{G} / \mathrm{A}$ \\
\hline UN_xiong_14869 & 1498 & $\mathrm{G} / \mathrm{A}$ \\
\hline UN_xiong_14869 & 1518 & $\mathrm{G} / \mathrm{A}$ \\
\hline UN_xiong_14871 & 43 & $A / G$ \\
\hline UN_xiong_14871 & 67 & $G / A$ \\
\hline UN_xiong_14871 & 102 & $\mathrm{C} / \mathrm{A}$ \\
\hline UN_xiong_14871 & 130 & $\mathrm{~A} / \mathrm{G}$ \\
\hline UN_xiong_14871 & 329 & $\mathrm{~T} / \mathrm{C}$ \\
\hline UN_xiong_14873 & 420 & $\mathrm{G} / \mathrm{A}$ \\
\hline UN_xiong_14873 & 720 & $\mathrm{C} / \mathrm{T}$ \\
\hline UN_xiong_14873 & 831 & $\mathrm{~T} / \mathrm{C}$ \\
\hline UN_xiong_14873 & 976 & $\mathrm{~T} / \mathrm{C}$ \\
\hline UN_xiong_14873 & 1042 & $\mathrm{~A} / \mathrm{T}$ \\
\hline UN_xiong_14873 & 1078 & $A / G$ \\
\hline UN_xiong_14873 & 1173 & $\mathrm{~T} / \mathrm{C}$ \\
\hline UN_xiong_14874 & 262 & $\mathrm{~T} / \mathrm{C}$ \\
\hline UN_xiong_14874 & 284 & $\mathrm{~A} / \mathrm{T}$ \\
\hline UN_xiong_14874 & 382 & $A / G$ \\
\hline UN_xiong_14874 & 441 & $\mathrm{~T} / \mathrm{C}$ \\
\hline UN_xiong_14875 & 290 & $\mathrm{C} / \mathrm{T}$ \\
\hline UN_xiong_14876 & 50 & $\mathrm{~T} / \mathrm{C}$ \\
\hline UN_xiong_14876 & 94 & $\mathrm{~T} / \mathrm{C}$ \\
\hline UN_xiong_14876 & 159 & $\mathrm{C} / \mathrm{T}$ \\
\hline UN_xiong_14876 & 240 & $\mathrm{~T} / \mathrm{C}$ \\
\hline UN_xiong_14876 & 279 & $\mathrm{~T} / \mathrm{C}$ \\
\hline UN_xiong_14876 & 435 & $\mathrm{C} / \mathrm{T}$ \\
\hline UN_xiong_14876 & 458 & $\mathrm{~T} / \mathrm{G}$ \\
\hline
\end{tabular}




\begin{tabular}{|c|c|c|}
\hline UN_xiong_14876 & 489 & $\mathrm{C} / \mathrm{A}$ \\
\hline UN_xiong_14876 & 729 & $\mathrm{G} / \mathrm{A}$ \\
\hline UN_xiong_14876 & 886 & $A / G$ \\
\hline UN_xiong_14877 & 193 & $\mathrm{~T} / \mathrm{G}$ \\
\hline UN_xiong_14877 & 251 & $\mathrm{C} / \mathrm{T}$ \\
\hline UN_xiong_14877 & 301 & $A / G$ \\
\hline UN_xiong_14877 & 380 & $\mathrm{C} / \mathrm{A}$ \\
\hline UN_xiong_14877 & 718 & $\mathrm{G} / \mathrm{C}$ \\
\hline UN_xiong_14877 & 772 & $A / G$ \\
\hline UN_xiong_14877 & 844 & $A / G$ \\
\hline UN_xiong_14877 & 878 & $\mathrm{~A} / \mathrm{C}$ \\
\hline UN_xiong_14877 & 944 & $\mathrm{~T} / \mathrm{C}$ \\
\hline UN_xiong_14877 & 1375 & $\mathrm{~T} / \mathrm{C}$ \\
\hline UN_xiong_14877 & 1586 & $\mathrm{C} / \mathrm{A}$ \\
\hline UN_xiong_14877 & 1789 & $\mathrm{~A} / \mathrm{C}$ \\
\hline UN_xiong_14877 & 1831 & $A / G$ \\
\hline UN_xiong_14877 & 1853 & $\mathrm{G} / \mathrm{T}$ \\
\hline UN_xiong_14877 & 1926 & $A / G$ \\
\hline UN_xiong_14877 & 2052 & $\mathrm{G} / \mathrm{A}$ \\
\hline UN_xiong_14880 & 277 & $\mathrm{G} / \mathrm{A}$ \\
\hline UN_xiong_14881 & 198 & $\mathrm{C} / \mathrm{T}$ \\
\hline UN_xiong_14881 & 603 & $\mathrm{~T} / \mathrm{A}$ \\
\hline UN_xiong_14881 & 690 & $\mathrm{G} / \mathrm{A}$ \\
\hline UN_xiong_14881 & 909 & $\mathrm{C} / \mathrm{T}$ \\
\hline UN_xiong_14881 & 1004 & $\mathrm{~T} / \mathrm{C}$ \\
\hline UN_xiong_14884 & 88 & $\mathrm{C} / \mathrm{T}$ \\
\hline UN_xiong_14884 & 470 & $A / G$ \\
\hline UN_xiong_14884 & 510 & $\mathrm{~T} / \mathrm{A}$ \\
\hline UN_xiong_14884 & 873 & $G / A$ \\
\hline UN_xiong_14884 & 939 & $\mathrm{G} / \mathrm{A}$ \\
\hline UN_xiong_14885 & 81 & $\mathrm{C} / \mathrm{T}$ \\
\hline UN_xiong_14885 & 223 & $A / G$ \\
\hline UN_xiong_14885 & 286 & $A / G$ \\
\hline UN_xiong_14885 & 446 & $\mathrm{G} / \mathrm{A}$ \\
\hline UN_xiong_14885 & 518 & $\mathrm{G} / \mathrm{A}$ \\
\hline UN_xiong_14885 & 632 & $\mathrm{G} / \mathrm{A}$ \\
\hline UN_xiong_14887 & 432 & $\mathrm{C} / \mathrm{A}$ \\
\hline UN_xiong_14887 & 482 & $\mathrm{~T} / \mathrm{C}$ \\
\hline UN_xiong_14887 & 766 & $\mathrm{~T} / \mathrm{C}$ \\
\hline UN_xiong_14889 & 621 & $\mathrm{~A} / \mathrm{C}$ \\
\hline UN_xiong_14889 & 665 & $\mathrm{G} / \mathrm{A}$ \\
\hline UN_xiong_14889 & 797 & $A / G$ \\
\hline UN_xiong_14890 & 1098 & $\mathrm{C} / \mathrm{T}$ \\
\hline UN_xiong_14891 & 119 & $A / G$ \\
\hline UN_xiong_14891 & 511 & $\mathrm{G} / \mathrm{T}$ \\
\hline UN_xiong_14893 & 40 & $\mathrm{G} / \mathrm{T}$ \\
\hline UN_xiong_14893 & 63 & $A / G$ \\
\hline
\end{tabular}




\begin{tabular}{|c|c|c|}
\hline UN_xiong_14893 & 92 & $\mathrm{~A} / \mathrm{G}$ \\
\hline UN_xiong_14894 & 68 & $\mathrm{C} / \mathrm{A}$ \\
\hline UN_xiong_14894 & 239 & $\mathrm{~A} / \mathrm{C}$ \\
\hline UN_xiong_14894 & 283 & $\mathrm{C} / \mathrm{T}$ \\
\hline UN_xiong_14895 & 11 & $\mathrm{G} / \mathrm{C}$ \\
\hline UN_xiong_14895 & 392 & $\mathrm{G} / \mathrm{T}$ \\
\hline UN_xiong_14895 & 698 & $\mathrm{C} / \mathrm{T}$ \\
\hline UN_xiong_14896 & 93 & $\mathrm{~T} / \mathrm{C}$ \\
\hline UN_xiong_14896 & 228 & $\mathrm{~T} / \mathrm{C}$ \\
\hline UN_xiong_14896 & 427 & $\mathrm{G} / \mathrm{A}$ \\
\hline UN_xiong_14896 & 597 & $\mathrm{G} / \mathrm{T}$ \\
\hline UN_xiong_14896 & 773 & $\mathrm{C} / \mathrm{T}$ \\
\hline UN_xiong_14897 & 286 & $\mathrm{~T} / \mathrm{A}$ \\
\hline UN_xiong_14897 & 315 & $\mathrm{C} / \mathrm{T}$ \\
\hline UN_xiong_14897 & 338 & $\mathrm{G} / \mathrm{T}$ \\
\hline UN_xiong_14898 & 81 & $\mathrm{~A} / \mathrm{T}$ \\
\hline UN_xiong_14899 & 401 & $\mathrm{C} / \mathrm{T}$ \\
\hline UN_xiong_14899 & 828 & $\mathrm{~A} / \mathrm{T}$ \\
\hline UN_xiong_14900 & 833 & $\mathrm{~T} / \mathrm{A}$ \\
\hline UN_xiong_14902 & 80 & $A / G$ \\
\hline UN_xiong_14903 & 24 & $\mathrm{~T} / \mathrm{C}$ \\
\hline UN_xiong_14904 & 367 & $\mathrm{G} / \mathrm{A}$ \\
\hline UN_xiong_14905 & 256 & $\mathrm{~T} / \mathrm{C}$ \\
\hline UN_xiong_14905 & 276 & $\mathrm{~A} / \mathrm{G}$ \\
\hline UN_xiong_14905 & 298 & $G / A$ \\
\hline UN_xiong_14905 & 332 & $\mathrm{~T} / \mathrm{A}$ \\
\hline UN_xiong_14905 & 371 & $\mathrm{~A} / \mathrm{T}$ \\
\hline UN_xiong_14905 & 395 & $A / G$ \\
\hline UN_xiong_14905 & 415 & $\mathrm{C} / \mathrm{T}$ \\
\hline UN_xiong_14905 & 460 & $\mathrm{~T} / \mathrm{C}$ \\
\hline UN_xiong_14905 & 485 & $A / G$ \\
\hline UN_xiong_14905 & 508 & $\mathrm{~A} / \mathrm{G}$ \\
\hline UN_xiong_14905 & 530 & $A / G$ \\
\hline UN_xiong_14905 & 563 & $\mathrm{~T} / \mathrm{C}$ \\
\hline UN_xiong_14905 & 589 & $\mathrm{G} / \mathrm{T}$ \\
\hline UN_xiong_14905 & 609 & $\mathrm{~A} / \mathrm{G}$ \\
\hline UN_xiong_14905 & 639 & $\mathrm{~A} / \mathrm{C}$ \\
\hline UN_xiong_14905 & 663 & $\mathrm{~T} / \mathrm{C}$ \\
\hline UN_xiong_14905 & 683 & $\mathrm{~T} / \mathrm{C}$ \\
\hline UN_xiong_14905 & 707 & $\mathrm{~T} / \mathrm{C}$ \\
\hline UN_xiong_14905 & 738 & $\mathrm{G} / \mathrm{A}$ \\
\hline UN_xiong_14905 & 762 & $\mathrm{~T} / \mathrm{C}$ \\
\hline UN_xiong_14905 & 791 & $\mathrm{~T} / \mathrm{C}$ \\
\hline UN_xiong_14905 & 817 & $\mathrm{~T} / \mathrm{C}$ \\
\hline UN_xiong_14905 & 840 & $A / G$ \\
\hline UN_xiong_14905 & 863 & $A / G$ \\
\hline UN_xiong_14905 & 884 & $\mathrm{~T} / \mathrm{C}$ \\
\hline
\end{tabular}




\begin{tabular}{|c|c|c|}
\hline UN_xiong_14905 & 908 & $\mathrm{~A} / \mathrm{T}$ \\
\hline UN_xiong_14905 & 940 & $\mathrm{~A} / \mathrm{G}$ \\
\hline UN_xiong_14905 & 961 & $\mathrm{C} / \mathrm{T}$ \\
\hline UN_xiong_14905 & 986 & $\mathrm{~T} / \mathrm{C}$ \\
\hline UN_xiong_14905 & 1007 & $\mathrm{~T} / \mathrm{C}$ \\
\hline UN_xiong_14905 & 1027 & $\mathrm{~T} / \mathrm{C}$ \\
\hline UN_xiong_14905 & 1060 & $\mathrm{~A} / \mathrm{G}$ \\
\hline UN_xiong_14907 & 126 & $\mathrm{~T} / \mathrm{G}$ \\
\hline UN_xiong_14907 & 169 & $\mathrm{~T} / \mathrm{C}$ \\
\hline UN_xiong_14907 & 359 & $\mathrm{G} / \mathrm{A}$ \\
\hline UN_xiong_14907 & 401 & $\mathrm{~T} / \mathrm{C}$ \\
\hline UN_xiong_14907 & 554 & $\mathrm{~T} / \mathrm{C}$ \\
\hline UN_xiong_14907 & 616 & $\mathrm{~T} / \mathrm{G}$ \\
\hline UN_xiong_14907 & 697 & $\mathrm{~A} / \mathrm{G}$ \\
\hline UN_xiong_14907 & 762 & $\mathrm{~T} / \mathrm{C}$ \\
\hline UN_xiong_14907 & 833 & $\mathrm{~A} / \mathrm{T}$ \\
\hline UN_xiong_14908 & 318 & $\mathrm{C} / \mathrm{T}$ \\
\hline UN_xiong_14908 & 568 & $\mathrm{~A} / \mathrm{C}$ \\
\hline UN_xiong_14908 & 716 & $A / G$ \\
\hline UN_xiong_14908 & 896 & $\mathrm{~A} / \mathrm{G}$ \\
\hline UN_xiong_14910 & 224 & $\mathrm{C} / \mathrm{T}$ \\
\hline UN_xiong_14910 & 313 & $A / G$ \\
\hline UN_xiong_14910 & 488 & $\mathrm{C} / \mathrm{A}$ \\
\hline UN_xiong_14912 & 413 & $\mathrm{~A} / \mathrm{G}$ \\
\hline UN_xiong_14917 & 333 & $\mathrm{C} / \mathrm{T}$ \\
\hline UN_xiong_14917 & 525 & $\mathrm{G} / \mathrm{T}$ \\
\hline UN_xiong_14917 & 577 & $\mathrm{C} / \mathrm{T}$ \\
\hline UN_xiong_14917 & 627 & $\mathrm{~T} / \mathrm{C}$ \\
\hline UN_xiong_14917 & 703 & $\mathrm{C} / \mathrm{A}$ \\
\hline UN_xiong_14917 & 741 & $\mathrm{~T} / \mathrm{C}$ \\
\hline UN_xiong_14918 & 390 & $\mathrm{~A} / \mathrm{T}$ \\
\hline UN_xiong_14918 & 468 & $\mathrm{C} / \mathrm{T}$ \\
\hline UN_xiong_14918 & 596 & $\mathrm{C} / \mathrm{T}$ \\
\hline UN_xiong_14918 & 648 & $\mathrm{~T} / \mathrm{C}$ \\
\hline UN_xiong_14918 & 726 & $A / G$ \\
\hline UN_xiong_14918 & 818 & $\mathrm{G} / \mathrm{A}$ \\
\hline UN_xiong_14919 & 657 & $A / G$ \\
\hline UN_xiong_14920 & 370 & $\mathrm{G} / \mathrm{A}$ \\
\hline UN_xiong_14920 & 422 & $A / G$ \\
\hline UN_xiong_14920 & 503 & $\mathrm{~T} / \mathrm{C}$ \\
\hline UN_xiong_14921 & 408 & $\mathrm{~A} / \mathrm{T}$ \\
\hline UN_xiong_14924 & 132 & $\mathrm{G} / \mathrm{C}$ \\
\hline UN_xiong_14924 & 203 & $\mathrm{~T} / \mathrm{C}$ \\
\hline UN_xiong_14924 & 301 & $\mathrm{~A} / \mathrm{G}$ \\
\hline UN_xiong_14924 & 367 & $\mathrm{~A} / \mathrm{G}$ \\
\hline UN_xiong_14924 & 410 & $\mathrm{~T} / \mathrm{C}$ \\
\hline UN_xiong_14924 & 578 & $\mathrm{C} / \mathrm{T}$ \\
\hline
\end{tabular}




\begin{tabular}{|c|c|c|}
\hline UN_xiong_14925 & 21 & $\mathrm{G} / \mathrm{T}$ \\
\hline UN_xiong_14925 & 482 & $\mathrm{G} / \mathrm{A}$ \\
\hline UN_xiong_14925 & 566 & $\mathrm{~A} / \mathrm{G}$ \\
\hline UN_xiong_14925 & 676 & $\mathrm{~T} / \mathrm{C}$ \\
\hline UN_xiong_14925 & 713 & $\mathrm{~T} / \mathrm{C}$ \\
\hline UN_xiong_14925 & 1056 & $\mathrm{C} / \mathrm{T}$ \\
\hline UN_xiong_14925 & 1354 & $\mathrm{~T} / \mathrm{C}$ \\
\hline UN_xiong_14926 & 240 & $\mathrm{~T} / \mathrm{A}$ \\
\hline UN_xiong_14926 & 284 & $\mathrm{~A} / \mathrm{C}$ \\
\hline UN_xiong_14926 & 545 & $A / G$ \\
\hline UN_xiong_14927 & 186 & $A / G$ \\
\hline UN_xiong_14928 & 80 & $\mathrm{~T} / \mathrm{A}$ \\
\hline UN_xiong_14928 & 180 & $\mathrm{G} / \mathrm{A}$ \\
\hline UN_xiong_14929 & 189 & $\mathrm{~A} / \mathrm{T}$ \\
\hline UN_xiong_14930 & 155 & $\mathrm{C} / \mathrm{T}$ \\
\hline UN_xiong_14930 & 376 & $\mathrm{~T} / \mathrm{A}$ \\
\hline UN_xiong_14930 & 462 & $\mathrm{~T} / \mathrm{C}$ \\
\hline UN_xiong_14930 & 493 & $\mathrm{~T} / \mathrm{C}$ \\
\hline UN_xiong_14930 & 801 & $\mathrm{~A} / \mathrm{T}$ \\
\hline UN_xiong_14930 & 875 & $\mathrm{~T} / \mathrm{C}$ \\
\hline UN_xiong_14932 & 45 & $\mathrm{G} / \mathrm{T}$ \\
\hline UN_xiong_14932 & 602 & $\mathrm{G} / \mathrm{A}$ \\
\hline UN_xiong_14932 & 671 & $\mathrm{C} / \mathrm{T}$ \\
\hline UN_xiong_14932 & 708 & $\mathrm{~T} / \mathrm{C}$ \\
\hline UN_xiong_14933 & 412 & $\mathrm{~A} / \mathrm{G}$ \\
\hline UN_xiong_14933 & 1152 & $\mathrm{G} / \mathrm{A}$ \\
\hline UN_xiong_14936 & 409 & $\mathrm{~T} / \mathrm{C}$ \\
\hline UN_xiong_14937 & 139 & $\mathrm{~T} / \mathrm{C}$ \\
\hline UN_xiong_14937 & 311 & $\mathrm{C} / \mathrm{T}$ \\
\hline UN_xiong_14937 & 491 & $A / G$ \\
\hline UN_xiong_14937 & 529 & $\mathrm{~T} / \mathrm{C}$ \\
\hline UN_xiong_14937 & 817 & $\mathrm{C} / \mathrm{T}$ \\
\hline UN_xiong_14937 & 900 & $\mathrm{~A} / \mathrm{G}$ \\
\hline UN_xiong_14937 & 988 & $\mathrm{~T} / \mathrm{C}$ \\
\hline UN_xiong_14940 & 56 & $\mathrm{G} / \mathrm{A}$ \\
\hline UN_xiong_14941 & 268 & $\mathrm{C} / \mathrm{T}$ \\
\hline UN_xiong_14943 & 345 & $\mathrm{~T} / \mathrm{G}$ \\
\hline UN_xiong_14943 & 396 & $A / G$ \\
\hline UN_xiong_14943 & 442 & $\mathrm{~T} / \mathrm{C}$ \\
\hline UN_xiong_14943 & 507 & $\mathrm{~A} / \mathrm{G}$ \\
\hline UN_xiong_14945 & 155 & $\mathrm{G} / \mathrm{C}$ \\
\hline UN_xiong_14947 & 308 & $\mathrm{C} / \mathrm{A}$ \\
\hline UN_xiong_14947 & 668 & $\mathrm{C} / \mathrm{T}$ \\
\hline UN_xiong_14952 & 461 & $A / G$ \\
\hline UN_xiong_14952 & 513 & $\mathrm{~A} / \mathrm{G}$ \\
\hline UN_xiong_14954 & 495 & $\mathrm{G} / \mathrm{T}$ \\
\hline UN_xiong_14954 & 526 & $\mathrm{~T} / \mathrm{C}$ \\
\hline
\end{tabular}




\begin{tabular}{|c|c|c|}
\hline UN_xiong_14956 & 628 & $\mathrm{~A} / \mathrm{T}$ \\
\hline UN_xiong_14956 & 1210 & $\mathrm{C} / \mathrm{G}$ \\
\hline UN_xiong_14957 & 263 & $\mathrm{G} / \mathrm{T}$ \\
\hline UN_xiong_14958 & 57 & $\mathrm{C} / \mathrm{G}$ \\
\hline UN_xiong_14958 & 428 & $\mathrm{C} / \mathrm{T}$ \\
\hline UN_xiong_14958 & 542 & $\mathrm{G} / \mathrm{A}$ \\
\hline UN_xiong_14958 & 656 & $\mathrm{~T} / \mathrm{C}$ \\
\hline UN_xiong_14958 & 690 & $\mathrm{~T} / \mathrm{G}$ \\
\hline UN_xiong_14959 & 23 & $\mathrm{G} / \mathrm{T}$ \\
\hline UN_xiong_14959 & 370 & $\mathrm{~T} / \mathrm{G}$ \\
\hline UN_xiong_14959 & 684 & $A / G$ \\
\hline UN_xiong_14959 & 875 & $A / G$ \\
\hline UN_xiong_14959 & 896 & $\mathrm{G} / \mathrm{C}$ \\
\hline UN_xiong_14962 & 17 & $\mathrm{~A} / \mathrm{G}$ \\
\hline UN_xiong_14962 & 140 & $\mathrm{~T} / \mathrm{G}$ \\
\hline UN_xiong_14962 & 185 & $\mathrm{C} / \mathrm{T}$ \\
\hline UN_xiong_14962 & 239 & $\mathrm{C} / \mathrm{T}$ \\
\hline UN_xiong_14962 & 298 & $\mathrm{G} / \mathrm{A}$ \\
\hline UN_xiong_14962 & 349 & $\mathrm{~T} / \mathrm{G}$ \\
\hline UN_xiong_14962 & 401 & $\mathrm{~T} / \mathrm{C}$ \\
\hline UN_xiong_14964 & 22 & $\mathrm{~T} / \mathrm{C}$ \\
\hline UN_xiong_14964 & 130 & $\mathrm{~T} / \mathrm{C}$ \\
\hline UN_xiong_14964 & 291 & $\mathrm{G} / \mathrm{A}$ \\
\hline UN_xiong_14966 & 537 & $\mathrm{~A} / \mathrm{G}$ \\
\hline UN_xiong_14966 & 637 & $A / G$ \\
\hline UN_xiong_14968 & 308 & $A / G$ \\
\hline UN_xiong_14968 & 359 & $\mathrm{~T} / \mathrm{C}$ \\
\hline UN_xiong_14968 & 661 & $\mathrm{~A} / \mathrm{T}$ \\
\hline UN_xiong_14968 & 837 & $\mathrm{~T} / \mathrm{G}$ \\
\hline UN_xiong_14970 & 625 & $\mathrm{~A} / \mathrm{G}$ \\
\hline UN_xiong_14970 & 857 & $\mathrm{~T} / \mathrm{C}$ \\
\hline UN_xiong_14970 & 1249 & $\mathrm{~A} / \mathrm{G}$ \\
\hline UN_xiong_14972 & 88 & $\mathrm{G} / \mathrm{A}$ \\
\hline UN_xiong_14972 & 197 & $\mathrm{~T} / \mathrm{C}$ \\
\hline UN_xiong_14972 & 735 & $\mathrm{~T} / \mathrm{C}$ \\
\hline UN_xiong_14973 & 379 & $\mathrm{C} / \mathrm{T}$ \\
\hline UN_xiong_14974 & 192 & $\mathrm{G} / \mathrm{A}$ \\
\hline UN_xiong_14974 & 246 & $\mathrm{C} / \mathrm{A}$ \\
\hline UN_xiong_14975 & 91 & $\mathrm{C} / \mathrm{T}$ \\
\hline UN_xiong_14975 & 551 & $\mathrm{C} / \mathrm{T}$ \\
\hline UN_xiong_14976 & 253 & $\mathrm{C} / \mathrm{G}$ \\
\hline UN_xiong_14976 & 288 & $A / G$ \\
\hline UN_xiong_14976 & 495 & $\mathrm{~T} / \mathrm{G}$ \\
\hline UN_xiong_14976 & 550 & $\mathrm{~A} / \mathrm{T}$ \\
\hline UN_xiong_14976 & 595 & $\mathrm{~T} / \mathrm{C}$ \\
\hline UN_xiong_14976 & 654 & $A / G$ \\
\hline UN_xiong_14976 & 678 & $\mathrm{~A} / \mathrm{T}$ \\
\hline
\end{tabular}




\begin{tabular}{|c|c|c|}
\hline UN_xiong_14976 & 701 & $\mathrm{~T} / \mathrm{G}$ \\
\hline UN_xiong_14976 & 963 & $\mathrm{G} / \mathrm{C}$ \\
\hline UN_xiong_14976 & 1021 & $\mathrm{~A} / \mathrm{G}$ \\
\hline UN_xiong_14976 & 1075 & $\mathrm{C} / \mathrm{A}$ \\
\hline UN_xiong_14976 & 1154 & $\mathrm{C} / \mathrm{G}$ \\
\hline UN_xiong_14976 & 1227 & $\mathrm{G} / \mathrm{A}$ \\
\hline UN_xiong_14976 & 1284 & $\mathrm{C} / \mathrm{T}$ \\
\hline UN_xiong_14976 & 1318 & $\mathrm{C} / \mathrm{G}$ \\
\hline UN_xiong_14976 & 1346 & $\mathrm{~T} / \mathrm{C}$ \\
\hline UN_xiong_14976 & 1613 & $A / G$ \\
\hline UN_xiong_14976 & 1715 & $\mathrm{~A} / \mathrm{T}$ \\
\hline UN_xiong_14976 & 1754 & $\mathrm{~A} / \mathrm{C}$ \\
\hline UN_xiong_14976 & 1846 & $\mathrm{~T} / \mathrm{C}$ \\
\hline UN_xiong_14976 & 1930 & $\mathrm{C} / \mathrm{A}$ \\
\hline UN_xiong_14976 & 1967 & $\mathrm{~A} / \mathrm{T}$ \\
\hline UN_xiong_14977 & 191 & $\mathrm{G} / \mathrm{T}$ \\
\hline UN_xiong_14978 & 52 & $\mathrm{C} / \mathrm{G}$ \\
\hline UN_xiong_14978 & 140 & $\mathrm{C} / \mathrm{T}$ \\
\hline UN_xiong_14978 & 224 & $\mathrm{C} / \mathrm{T}$ \\
\hline UN_xiong_14978 & 256 & $A / G$ \\
\hline UN_xiong_14978 & 340 & $\mathrm{C} / \mathrm{T}$ \\
\hline UN_xiong_14978 & 368 & $\mathrm{~T} / \mathrm{C}$ \\
\hline UN_xiong_14978 & 401 & $\mathrm{C} / \mathrm{T}$ \\
\hline UN_xiong_14978 & 451 & $\mathrm{~A} / \mathrm{G}$ \\
\hline UN_xiong_14978 & 491 & $\mathrm{~T} / \mathrm{C}$ \\
\hline UN_xiong_14978 & 544 & $\mathrm{~T} / \mathrm{C}$ \\
\hline UN_xiong_14978 & 597 & $\mathrm{G} / \mathrm{A}$ \\
\hline UN_xiong_14978 & 671 & $\mathrm{C} / \mathrm{T}$ \\
\hline UN_xiong_14978 & 740 & $\mathrm{~A} / \mathrm{T}$ \\
\hline UN_xiong_14978 & 989 & $\mathrm{G} / \mathrm{A}$ \\
\hline UN_xiong_14978 & 1096 & $\mathrm{~T} / \mathrm{C}$ \\
\hline UN_xiong_14978 & 1130 & $\mathrm{C} / \mathrm{T}$ \\
\hline UN_xiong_14978 & 1169 & $\mathrm{C} / \mathrm{T}$ \\
\hline UN_xiong_14978 & 1236 & $\mathrm{~T} / \mathrm{C}$ \\
\hline UN_xiong_14978 & 1263 & $\mathrm{C} / \mathrm{T}$ \\
\hline UN_xiong_14978 & 1285 & $A / G$ \\
\hline UN_xiong_14978 & 1324 & $\mathrm{~T} / \mathrm{C}$ \\
\hline UN_xiong_14978 & 1410 & $A / G$ \\
\hline UN_xiong_14978 & 1442 & $\mathrm{~T} / \mathrm{C}$ \\
\hline UN_xiong_14978 & 1491 & $\mathrm{G} / \mathrm{A}$ \\
\hline UN_xiong_14978 & 1575 & $\mathrm{~T} / \mathrm{C}$ \\
\hline UN_xiong_14978 & 1631 & $\mathrm{G} / \mathrm{A}$ \\
\hline UN_xiong_14978 & 1711 & $\mathrm{~T} / \mathrm{C}$ \\
\hline UN_xiong_14978 & 1788 & $\mathrm{~T} / \mathrm{C}$ \\
\hline UN_xiong_14978 & 1829 & $\mathrm{~T} / \mathrm{A}$ \\
\hline UN_xiong_14985 & 91 & $\mathrm{G} / \mathrm{A}$ \\
\hline UN_xiong_14985 & 426 & $\mathrm{C} / \mathrm{A}$ \\
\hline
\end{tabular}




\begin{tabular}{|c|c|c|}
\hline UN_xiong_14985 & 465 & $\mathrm{C} / \mathrm{T}$ \\
\hline UN_xiong_14985 & 587 & $A / G$ \\
\hline UN_xiong_14985 & 771 & $A / G$ \\
\hline UN_xiong_14985 & 872 & $\mathrm{~T} / \mathrm{G}$ \\
\hline UN_xiong_14985 & 1059 & $\mathrm{~A} / \mathrm{T}$ \\
\hline UN_xiong_14986 & 260 & $\mathrm{C} / \mathrm{T}$ \\
\hline UN_xiong_14987 & 164 & $\mathrm{~T} / \mathrm{C}$ \\
\hline UN_xiong_14987 & 309 & $\mathrm{G} / \mathrm{A}$ \\
\hline UN_xiong_14987 & 764 & $A / G$ \\
\hline UN_xiong_14988 & 203 & $\mathrm{~T} / \mathrm{A}$ \\
\hline UN_xiong_14989 & 74 & $\mathrm{~A} / \mathrm{T}$ \\
\hline UN_xiong_14990 & 422 & $\mathrm{~A} / \mathrm{T}$ \\
\hline UN_xiong_14992 & 701 & $\mathrm{C} / \mathrm{T}$ \\
\hline UN_xiong_14994 & 102 & $\mathrm{~T} / \mathrm{A}$ \\
\hline UN_xiong_14996 & 297 & $A / C$ \\
\hline UN_xiong_14996 & 402 & $\mathrm{~T} / \mathrm{A}$ \\
\hline UN_xiong_14996 & 1053 & $\mathrm{C} / \mathrm{T}$ \\
\hline UN_xiong_14996 & 1206 & $\mathrm{~A} / \mathrm{T}$ \\
\hline UN_xiong_14997 & 593 & $\mathrm{G} / \mathrm{A}$ \\
\hline UN_xiong_14997 & 645 & $\mathrm{~T} / \mathrm{C}$ \\
\hline UN_xiong_14998 & 71 & $A / G$ \\
\hline UN_xiong_14998 & 167 & $\mathrm{~T} / \mathrm{C}$ \\
\hline UN_xiong_14998 & 525 & $A / G$ \\
\hline UN_xiong_14998 & 582 & $A / G$ \\
\hline UN_xiong_14998 & 603 & $\mathrm{~T} / \mathrm{C}$ \\
\hline UN_xiong_14998 & 762 & $\mathrm{~T} / \mathrm{C}$ \\
\hline UN_xiong_14998 & 913 & $\mathrm{~T} / \mathrm{C}$ \\
\hline UN_xiong_14998 & 989 & $\mathrm{~T} / \mathrm{C}$ \\
\hline UN_xiong_14998 & 1678 & $\mathrm{G} / \mathrm{A}$ \\
\hline UN_xiong_15000 & 589 & $\mathrm{G} / \mathrm{A}$ \\
\hline UN_xiong_15000 & 927 & $\mathrm{~T} / \mathrm{A}$ \\
\hline UN_xiong_15000 & 1007 & $A / G$ \\
\hline UN_xiong_15000 & 1058 & $A / G$ \\
\hline UN_xiong_15000 & 1718 & $\mathrm{~T} / \mathrm{A}$ \\
\hline UN_xiong_15000 & 2015 & $\mathrm{C} / \mathrm{T}$ \\
\hline UN_xiong_15001 & 185 & $\mathrm{G} / \mathrm{A}$ \\
\hline UN_xiong_15001 & 313 & $\mathrm{~T} / \mathrm{G}$ \\
\hline UN_xiong_15001 & 514 & $\mathrm{~A} / \mathrm{C}$ \\
\hline UN_xiong_15001 & 1010 & $\mathrm{G} / \mathrm{A}$ \\
\hline UN_xiong_15005 & 386 & $\mathrm{G} / \mathrm{A}$ \\
\hline UN_xiong_15005 & 611 & $\mathrm{~T} / \mathrm{A}$ \\
\hline UN_xiong_15005 & 658 & $\mathrm{G} / \mathrm{A}$ \\
\hline UN_xiong_15006 & 174 & $\mathrm{~A} / \mathrm{C}$ \\
\hline UN_xiong_15006 & 332 & $\mathrm{~T} / \mathrm{C}$ \\
\hline UN_xiong_15006 & 536 & $\mathrm{C} / \mathrm{T}$ \\
\hline UN_xiong_15008 & 151 & $A / G$ \\
\hline UN_xiong_15011 & 407 & $\mathrm{G} / \mathrm{A}$ \\
\hline
\end{tabular}




\begin{tabular}{|c|c|c|}
\hline UN_xiong_15011 & 655 & $\mathrm{C} / \mathrm{A}$ \\
\hline UN_xiong_15013 & 81 & $\mathrm{~T} / \mathrm{G}$ \\
\hline UN_xiong_15013 & 477 & $\mathrm{C} / \mathrm{T}$ \\
\hline UN_xiong_15015 & 375 & $\mathrm{G} / \mathrm{A}$ \\
\hline UN_xiong_15015 & 574 & $\mathrm{~T} / \mathrm{C}$ \\
\hline UN_xiong_15015 & 684 & $A / G$ \\
\hline UN_xiong_15015 & 709 & $A / G$ \\
\hline UN_xiong_15015 & 731 & $\mathrm{~A} / \mathrm{C}$ \\
\hline UN_xiong_15015 & 761 & $\mathrm{G} / \mathrm{A}$ \\
\hline UN_xiong_15015 & 825 & $\mathrm{G} / \mathrm{A}$ \\
\hline UN_xiong_15015 & 845 & $\mathrm{~T} / \mathrm{A}$ \\
\hline UN_xiong_15015 & 911 & $\mathrm{~A} / \mathrm{T}$ \\
\hline UN_xiong_15015 & 933 & $\mathrm{~T} / \mathrm{C}$ \\
\hline UN_xiong_15015 & 1090 & $A / G$ \\
\hline UN_xiong_15015 & 1202 & $\mathrm{~T} / \mathrm{C}$ \\
\hline UN_xiong_15015 & 1239 & $A / G$ \\
\hline UN_xiong_15015 & 1263 & $\mathrm{~T} / \mathrm{G}$ \\
\hline UN_xiong_15015 & 1497 & $A / G$ \\
\hline UN_xiong_15015 & 1692 & $\mathrm{G} / \mathrm{A}$ \\
\hline UN_xiong_15015 & 1805 & $A / G$ \\
\hline UN_xiong_15015 & 1870 & $\mathrm{~A} / \mathrm{G}$ \\
\hline UN_xiong_15016 & 273 & $\mathrm{C} / \mathrm{T}$ \\
\hline UN_xiong_15016 & 607 & $\mathrm{G} / \mathrm{A}$ \\
\hline UN_xiong_15019 & 98 & $\mathrm{~A} / \mathrm{G}$ \\
\hline UN_xiong_15020 & 456 & $\mathrm{C} / \mathrm{A}$ \\
\hline UN_xiong_15020 & 592 & $\mathrm{~T} / \mathrm{C}$ \\
\hline UN_xiong_15020 & 646 & $\mathrm{C} / \mathrm{T}$ \\
\hline UN_xiong_15020 & 820 & $\mathrm{C} / \mathrm{A}$ \\
\hline UN_xiong_15021 & 58 & $\mathrm{C} / \mathrm{A}$ \\
\hline UN_xiong_15022 & 78 & $\mathrm{~T} / \mathrm{C}$ \\
\hline UN_xiong_15022 & 117 & $C / G$ \\
\hline UN_xiong_15023 & 71 & $\mathrm{~T} / \mathrm{C}$ \\
\hline UN_xiong_15023 & 177 & $A / G$ \\
\hline UN_xiong_15027 & 56 & $A / G$ \\
\hline UN_xiong_15027 & 552 & $\mathrm{~T} / \mathrm{C}$ \\
\hline UN_xiong_15027 & 1000 & $\mathrm{G} / \mathrm{A}$ \\
\hline UN_xiong_15027 & 1170 & $\mathrm{~T} / \mathrm{C}$ \\
\hline UN_xiong_15032 & 278 & $A / G$ \\
\hline UN_xiong_15032 & 459 & $A / G$ \\
\hline UN_xiong_15032 & 651 & $\mathrm{G} / \mathrm{A}$ \\
\hline UN_xiong_15032 & 690 & $\mathrm{C} / \mathrm{T}$ \\
\hline UN_xiong_15032 & 775 & $A / G$ \\
\hline UN_xiong_15032 & 910 & $\mathrm{G} / \mathrm{A}$ \\
\hline UN_xiong_15034 & 11 & $\mathrm{G} / \mathrm{T}$ \\
\hline UN_xiong_15034 & 431 & $A / G$ \\
\hline UN_xiong_15034 & 550 & $\mathrm{~T} / \mathrm{C}$ \\
\hline UN_xiong_15035 & 191 & $\mathrm{G} / \mathrm{A}$ \\
\hline
\end{tabular}




\begin{tabular}{|c|c|c|}
\hline UN_xiong_15035 & 613 & $\mathrm{C} / \mathrm{T}$ \\
\hline UN_xiong_15036 & 269 & $\mathrm{~T} / \mathrm{C}$ \\
\hline UN_xiong_15036 & 684 & $\mathrm{G} / \mathrm{C}$ \\
\hline UN_xiong_15039 & 88 & $\mathrm{~T} / \mathrm{C}$ \\
\hline UN_xiong_15039 & 170 & $\mathrm{~A} / \mathrm{C}$ \\
\hline UN_xiong_15039 & 290 & $\mathrm{~T} / \mathrm{C}$ \\
\hline UN_xiong_15039 & 335 & $C / G$ \\
\hline UN_xiong_15039 & 410 & $\mathrm{~T} / \mathrm{C}$ \\
\hline UN_xiong_15040 & 86 & $\mathrm{C} / \mathrm{T}$ \\
\hline UN_xiong_15041 & 122 & $\mathrm{C} / \mathrm{T}$ \\
\hline UN_xiong_15041 & 148 & $\mathrm{G} / \mathrm{A}$ \\
\hline UN_xiong_15043 & 512 & $\mathrm{G} / \mathrm{A}$ \\
\hline UN_xiong_15043 & 977 & $A / G$ \\
\hline UN_xiong_15043 & 1102 & $\mathrm{~A} / \mathrm{G}$ \\
\hline UN_xiong_15045 & 11 & $\mathrm{G} / \mathrm{T}$ \\
\hline UN_xiong_15045 & 40 & $\mathrm{~T} / \mathrm{C}$ \\
\hline UN_xiong_15045 & 62 & $\mathrm{~T} / \mathrm{C}$ \\
\hline UN_xiong_15045 & 90 & $\mathrm{~T} / \mathrm{C}$ \\
\hline UN_xiong_15045 & 134 & $\mathrm{~A} / \mathrm{C}$ \\
\hline UN_xiong_15045 & 172 & $\mathrm{~T} / \mathrm{C}$ \\
\hline UN_xiong_15045 & 201 & $\mathrm{G} / \mathrm{A}$ \\
\hline UN_xiong_15045 & 226 & $A / G$ \\
\hline UN_xiong_15045 & 249 & $A / G$ \\
\hline UN_xiong_15045 & 269 & $A / G$ \\
\hline UN_xiong_15045 & 290 & $\mathrm{~A} / \mathrm{T}$ \\
\hline UN_xiong_15045 & 313 & $\mathrm{G} / \mathrm{A}$ \\
\hline UN_xiong_15045 & 477 & $\mathrm{~T} / \mathrm{C}$ \\
\hline UN_xiong_15045 & 618 & $\mathrm{~A} / \mathrm{T}$ \\
\hline UN_xiong_15045 & 726 & $\mathrm{G} / \mathrm{A}$ \\
\hline UN_xiong_15045 & 781 & $\mathrm{~T} / \mathrm{C}$ \\
\hline UN_xiong_15045 & 851 & $\mathrm{~T} / \mathrm{C}$ \\
\hline UN_xiong_15045 & 879 & $\mathrm{~T} / \mathrm{C}$ \\
\hline UN_xiong_15045 & 918 & $\mathrm{~T} / \mathrm{C}$ \\
\hline UN_xiong_15045 & 1023 & $\mathrm{G} / \mathrm{A}$ \\
\hline UN_xiong_15045 & 1044 & $\mathrm{C} / \mathrm{A}$ \\
\hline UN_xiong_15045 & 1068 & $\mathrm{~T} / \mathrm{G}$ \\
\hline UN_xiong_15045 & 1121 & $\mathrm{~T} / \mathrm{A}$ \\
\hline UN_xiong_15045 & 1158 & $A / G$ \\
\hline UN_xiong_15045 & 1247 & $\mathrm{C} / \mathrm{T}$ \\
\hline UN_xiong_15045 & 1299 & $\mathrm{C} / \mathrm{T}$ \\
\hline UN_xiong_15045 & 1344 & $\mathrm{G} / \mathrm{T}$ \\
\hline UN_xiong_15045 & 1398 & $\mathrm{~T} / \mathrm{A}$ \\
\hline UN_xiong_15045 & 1433 & $\mathrm{G} / \mathrm{A}$ \\
\hline UN_xiong_15045 & 1503 & $\mathrm{~T} / \mathrm{C}$ \\
\hline UN_xiong_15045 & 1525 & $\mathrm{~T} / \mathrm{G}$ \\
\hline UN_xiong_15045 & 1553 & $\mathrm{G} / \mathrm{A}$ \\
\hline UN_xiong_15045 & 1650 & $\mathrm{G} / \mathrm{A}$ \\
\hline
\end{tabular}




\begin{tabular}{|c|c|c|}
\hline UN_xiong_15045 & 1705 & $\mathrm{~T} / \mathrm{C}$ \\
\hline UN_xiong_15045 & 1743 & $\mathrm{C} / \mathrm{T}$ \\
\hline UN_xiong_15045 & 1842 & $\mathrm{~T} / \mathrm{C}$ \\
\hline UN_xiong_15045 & 1876 & $\mathrm{~T} / \mathrm{C}$ \\
\hline UN_xiong_15047 & 580 & $\mathrm{~T} / \mathrm{A}$ \\
\hline UN_xiong_15048 & 14 & $A / G$ \\
\hline UN_xiong_15048 & 171 & $A / G$ \\
\hline UN_xiong_15048 & 211 & $A / G$ \\
\hline UN_xiong_15048 & 321 & $\mathrm{G} / \mathrm{A}$ \\
\hline UN_xiong_15048 & 391 & $\mathrm{G} / \mathrm{T}$ \\
\hline UN_xiong_15048 & 711 & $\mathrm{C} / \mathrm{T}$ \\
\hline UN_xiong_15049 & 177 & $\mathrm{G} / \mathrm{A}$ \\
\hline UN_xiong_15049 & 231 & $\mathrm{~T} / \mathrm{C}$ \\
\hline UN_xiong_15049 & 690 & $A / G$ \\
\hline UN_xiong_15049 & 860 & $A / G$ \\
\hline UN_xiong_15049 & 882 & $\mathrm{~T} / \mathrm{G}$ \\
\hline UN_xiong_15051 & 249 & $\mathrm{G} / \mathrm{A}$ \\
\hline UN_xiong_15056 & 250 & $\mathrm{C} / \mathrm{T}$ \\
\hline UN_xiong_15059 & 74 & $\mathrm{G} / \mathrm{T}$ \\
\hline UN_xiong_15059 & 752 & $A / G$ \\
\hline UN_xiong_15059 & 806 & $\mathrm{~T} / \mathrm{C}$ \\
\hline UN_xiong_15060 & 128 & $\mathrm{C} / \mathrm{T}$ \\
\hline UN_xiong_15060 & 380 & $A / G$ \\
\hline UN_xiong_15060 & 534 & $A / G$ \\
\hline UN_xiong_15060 & 580 & $A / G$ \\
\hline UN_xiong_15060 & 615 & $\mathrm{G} / \mathrm{C}$ \\
\hline UN_xiong_15060 & 721 & $C / G$ \\
\hline UN_xiong_15060 & 802 & $A / G$ \\
\hline UN_xiong_15060 & 964 & $\mathrm{~A} / \mathrm{T}$ \\
\hline UN_xiong_15060 & 1001 & $\mathrm{C} / \mathrm{T}$ \\
\hline UN_xiong_15060 & 1067 & $A / G$ \\
\hline UN_xiong_15060 & 1169 & $\mathrm{G} / \mathrm{T}$ \\
\hline UN_xiong_15061 & 228 & $\mathrm{~T} / \mathrm{G}$ \\
\hline UN_xiong_15062 & 224 & $\mathrm{~A} / \mathrm{T}$ \\
\hline UN_xiong_15063 & 311 & $\mathrm{~A} / \mathrm{T}$ \\
\hline UN_xiong_15063 & 381 & $A / G$ \\
\hline UN_xiong_15063 & 506 & $\mathrm{~T} / \mathrm{C}$ \\
\hline UN_xiong_15065 & 7 & $\mathrm{~T} / \mathrm{C}$ \\
\hline UN_xiong_15065 & 71 & $A / C$ \\
\hline UN_xiong_15065 & 133 & $\mathrm{~T} / \mathrm{C}$ \\
\hline UN_xiong_15065 & 219 & $\mathrm{G} / \mathrm{A}$ \\
\hline UN_xiong_15065 & 362 & $\mathrm{C} / \mathrm{T}$ \\
\hline UN_xiong_15066 & 438 & $A / G$ \\
\hline UN_xiong_15066 & 869 & $A / G$ \\
\hline UN_xiong_15066 & 908 & $\mathrm{~T} / \mathrm{G}$ \\
\hline UN_xiong_15066 & 1109 & $\mathrm{G} / \mathrm{A}$ \\
\hline UN_xiong_15071 & 257 & $\mathrm{~T} / \mathrm{C}$ \\
\hline
\end{tabular}




\begin{tabular}{|c|c|c|}
\hline UN_xiong_15071 & 360 & $\mathrm{C} / \mathrm{T}$ \\
\hline UN_xiong_15079 & 300 & $\mathrm{C} / \mathrm{T}$ \\
\hline UN_xiong_15081 & 673 & $\mathrm{C} / \mathrm{G}$ \\
\hline UN_xiong_15081 & 750 & $\mathrm{~T} / \mathrm{A}$ \\
\hline UN_xiong_15081 & 1049 & $\mathrm{G} / \mathrm{T}$ \\
\hline UN_xiong_15081 & 1254 & $\mathrm{G} / \mathrm{A}$ \\
\hline UN_xiong_15081 & 1344 & $A / G$ \\
\hline UN_xiong_15081 & 1428 & $\mathrm{~T} / \mathrm{C}$ \\
\hline UN_xiong_15081 & 1515 & $\mathrm{C} / \mathrm{T}$ \\
\hline UN_xiong_15081 & 1657 & $\mathrm{~T} / \mathrm{C}$ \\
\hline UN_xiong_15088 & 366 & $\mathrm{C} / \mathrm{T}$ \\
\hline UN_xiong_15088 & 494 & $\mathrm{~A} / \mathrm{G}$ \\
\hline UN_xiong_15095 & 188 & $\mathrm{G} / \mathrm{A}$ \\
\hline UN_xiong_15105 & 516 & $\mathrm{C} / \mathrm{T}$ \\
\hline UN_xiong_15105 & 656 & $A / G$ \\
\hline UN_xiong_15105 & 766 & $\mathrm{G} / \mathrm{A}$ \\
\hline UN_xiong_15114 & 24 & $\mathrm{~T} / \mathrm{C}$ \\
\hline UN_xiong_15114 & 63 & $\mathrm{G} / \mathrm{C}$ \\
\hline UN_xiong_15114 & 142 & $A / G$ \\
\hline UN_xiong_15120 & 383 & $\mathrm{C} / \mathrm{T}$ \\
\hline UN_xiong_15124 & 68 & $\mathrm{C} / \mathrm{T}$ \\
\hline UN_xiong_15124 & 268 & $\mathrm{G} / \mathrm{A}$ \\
\hline UN_xiong_15124 & 376 & $\mathrm{~T} / \mathrm{A}$ \\
\hline UN_xiong_15124 & 452 & $\mathrm{G} / \mathrm{A}$ \\
\hline UN_xiong_15130 & 110 & $\mathrm{~T} / \mathrm{C}$ \\
\hline UN_xiong_15130 & 154 & $\mathrm{C} / \mathrm{T}$ \\
\hline UN_xiong_15130 & 184 & $\mathrm{C} / \mathrm{A}$ \\
\hline UN_xiong_15130 & 231 & $\mathrm{~T} / \mathrm{C}$ \\
\hline UN_xiong_15130 & 358 & $\mathrm{~T} / \mathrm{C}$ \\
\hline UN_xiong_15130 & 508 & $\mathrm{~T} / \mathrm{C}$ \\
\hline UN_xiong_15130 & 631 & $\mathrm{~A} / \mathrm{C}$ \\
\hline UN_xiong_15130 & 651 & $\mathrm{~T} / \mathrm{C}$ \\
\hline UN_xiong_15133 & 385 & $\mathrm{G} / \mathrm{A}$ \\
\hline UN_xiong_15139 & 58 & $\mathrm{~A} / \mathrm{T}$ \\
\hline UN_xiong_15152 & 305 & $\mathrm{~T} / \mathrm{C}$ \\
\hline UN_xiong_15160 & 691 & $\mathrm{C} / \mathrm{A}$ \\
\hline UN_xiong_15163 & 246 & $\mathrm{G} / \mathrm{A}$ \\
\hline UN_xiong_15163 & 278 & $A / G$ \\
\hline UN_xiong_15169 & 47 & $\mathrm{~A} / \mathrm{G}$ \\
\hline UN_xiong_15169 & 158 & $\mathrm{~T} / \mathrm{G}$ \\
\hline UN_xiong_15169 & 275 & $\mathrm{~A} / \mathrm{G}$ \\
\hline UN_xiong_15169 & 388 & $A / G$ \\
\hline UN_xiong_15171 & 73 & $\mathrm{C} / \mathrm{G}$ \\
\hline UN_xiong_15171 & 105 & $A / G$ \\
\hline UN_xiong_15171 & 162 & $\mathrm{C} / \mathrm{A}$ \\
\hline UN_xiong_15171 & 213 & $\mathrm{C} / \mathrm{T}$ \\
\hline UN_xiong_15171 & 244 & $\mathrm{C} / \mathrm{T}$ \\
\hline
\end{tabular}




\begin{tabular}{|c|c|c|}
\hline UN_xiong_15171 & 336 & $\mathrm{~T} / \mathrm{C}$ \\
\hline UN_xiong_15181 & 193 & $\mathrm{C} / \mathrm{G}$ \\
\hline UN_xiong_15181 & 277 & $\mathrm{C} / \mathrm{G}$ \\
\hline UN_xiong_15181 & 313 & $\mathrm{C} / \mathrm{G}$ \\
\hline UN_xiong_15191 & 114 & $\mathrm{C} / \mathrm{G}$ \\
\hline UN_xiong_15193 & 22 & $\mathrm{G} / \mathrm{T}$ \\
\hline UN_xiong_15198 & 132 & $C / A$ \\
\hline UN_xiong_15200 & 351 & $A / G$ \\
\hline UN_xiong_15200 & 431 & $\mathrm{G} / \mathrm{A}$ \\
\hline UN_xiong_15207 & 51 & $\mathrm{G} / \mathrm{A}$ \\
\hline UN_xiong_15219 & 22 & $\mathrm{~A} / \mathrm{T}$ \\
\hline UN_xiong_15219 & 101 & $\mathrm{~T} / \mathrm{C}$ \\
\hline UN_xiong_15219 & 408 & $\mathrm{~A} / \mathrm{G}$ \\
\hline UN_xiong_15219 & 444 & $\mathrm{~T} / \mathrm{C}$ \\
\hline UN_xiong_15219 & 465 & $\mathrm{G} / \mathrm{A}$ \\
\hline UN_xiong_15237 & 168 & $\mathrm{~A} / \mathrm{T}$ \\
\hline UN_xiong_15240 & 422 & $\mathrm{~T} / \mathrm{C}$ \\
\hline UN_xiong_15243 & 240 & $\mathrm{C} / \mathrm{T}$ \\
\hline UN_xiong_15243 & 314 & $\mathrm{~A} / \mathrm{T}$ \\
\hline UN_xiong_15243 & 377 & $\mathrm{C} / \mathrm{T}$ \\
\hline UN_xiong_15253 & 346 & $\mathrm{G} / \mathrm{C}$ \\
\hline UN_xiong_15253 & 380 & $\mathrm{~T} / \mathrm{G}$ \\
\hline UN_xiong_15254 & 66 & $\mathrm{~A} / \mathrm{T}$ \\
\hline UN_xiong_15254 & 154 & $\mathrm{C} / \mathrm{T}$ \\
\hline UN_xiong_15259 & 132 & $A / G$ \\
\hline UN_xiong_15259 & 379 & $\mathrm{C} / \mathrm{T}$ \\
\hline UN_xiong_15259 & 521 & $\mathrm{~T} / \mathrm{C}$ \\
\hline UN_xiong_15259 & 709 & $\mathrm{G} / \mathrm{A}$ \\
\hline UN_xiong_15259 & 1069 & $\mathrm{~T} / \mathrm{C}$ \\
\hline UN_xiong_15259 & 1255 & $A / G$ \\
\hline UN_xiong_15260 & 124 & $\mathrm{~T} / \mathrm{A}$ \\
\hline UN_xiong_15267 & 81 & $\mathrm{C} / \mathrm{T}$ \\
\hline UN_xiong_15272 & 800 & $\mathrm{~T} / \mathrm{C}$ \\
\hline UN_xiong_15273 & 411 & $\mathrm{C} / \mathrm{A}$ \\
\hline UN_xiong_15274 & 77 & $\mathrm{~T} / \mathrm{C}$ \\
\hline UN_xiong_15275 & 716 & $\mathrm{C} / \mathrm{A}$ \\
\hline UN_xiong_15280 & 625 & $\mathrm{G} / \mathrm{A}$ \\
\hline UN_xiong_15280 & 648 & $\mathrm{~T} / \mathrm{C}$ \\
\hline UN_xiong_15286 & 140 & $\mathrm{C} / \mathrm{T}$ \\
\hline UN_xiong_15286 & 220 & $\mathrm{~T} / \mathrm{C}$ \\
\hline UN_xiong_15286 & 270 & $\mathrm{~A} / \mathrm{G}$ \\
\hline UN_xiong_15286 & 318 & $\mathrm{G} / \mathrm{A}$ \\
\hline UN_xiong_15293 & 161 & $\mathrm{C} / \mathrm{T}$ \\
\hline UN_xiong_15293 & 193 & $A / G$ \\
\hline UN_xiong_15293 & 265 & $\mathrm{~T} / \mathrm{G}$ \\
\hline UN_xiong_15293 & 364 & $\mathrm{C} / \mathrm{T}$ \\
\hline UN_xiong_15304 & 194 & $\mathrm{G} / \mathrm{C}$ \\
\hline
\end{tabular}




\begin{tabular}{|c|c|c|}
\hline UN_xiong_15304 & 256 & $\mathrm{G} / \mathrm{T}$ \\
\hline UN_xiong_15308 & 225 & $\mathrm{G} / \mathrm{A}$ \\
\hline UN_xiong_15308 & 263 & $\mathrm{~T} / \mathrm{C}$ \\
\hline UN_xiong_15308 & 499 & $A / G$ \\
\hline UN_xiong_15312 & 727 & $\mathrm{~T} / \mathrm{C}$ \\
\hline UN_xiong_15323 & 164 & $\mathrm{~T} / \mathrm{A}$ \\
\hline UN_xiong_15323 & 236 & $\mathrm{C} / \mathrm{T}$ \\
\hline UN_xiong_15326 & 152 & $\mathrm{G} / \mathrm{T}$ \\
\hline UN_xiong_15326 & 214 & $\mathrm{~T} / \mathrm{C}$ \\
\hline UN_xiong_15328 & 337 & $A / G$ \\
\hline UN_xiong_15328 & 527 & $\mathrm{C} / \mathrm{A}$ \\
\hline UN_xiong_15328 & 620 & $\mathrm{~T} / \mathrm{C}$ \\
\hline UN_xiong_15328 & 734 & $\mathrm{G} / \mathrm{A}$ \\
\hline UN_xiong_15328 & 807 & $\mathrm{~A} / \mathrm{T}$ \\
\hline UN_xiong_15328 & 1005 & $\mathrm{~A} / \mathrm{G}$ \\
\hline UN_xiong_15328 & 1040 & $\mathrm{G} / \mathrm{A}$ \\
\hline UN_xiong_15328 & 1083 & $\mathrm{~A} / \mathrm{C}$ \\
\hline UN_xiong_15328 & 1108 & $A / G$ \\
\hline UN_xiong_15328 & 1128 & $A / G$ \\
\hline UN_xiong_15328 & 1174 & $A / G$ \\
\hline UN_xiong_15328 & 1218 & $\mathrm{G} / \mathrm{A}$ \\
\hline UN_xiong_15334 & 463 & $\mathrm{~T} / \mathrm{C}$ \\
\hline UN_xiong_15337 & 174 & $\mathrm{~A} / \mathrm{G}$ \\
\hline UN_xiong_15340 & 39 & $\mathrm{G} / \mathrm{A}$ \\
\hline UN_xiong_15340 & 152 & $\mathrm{C} / \mathrm{G}$ \\
\hline UN_xiong_15340 & 278 & $\mathrm{~A} / \mathrm{T}$ \\
\hline UN_xiong_15340 & 524 & $\mathrm{~A} / \mathrm{G}$ \\
\hline UN_xiong_15341 & 287 & $\mathrm{G} / \mathrm{T}$ \\
\hline UN_xiong_15341 & 311 & $\mathrm{C} / \mathrm{T}$ \\
\hline UN_xiong_15345 & 238 & $\mathrm{C} / \mathrm{A}$ \\
\hline UN_xiong_15345 & 666 & $C / G$ \\
\hline UN_xiong_15345 & 1192 & $\mathrm{~T} / \mathrm{C}$ \\
\hline UN_xiong_15345 & 1226 & $\mathrm{~T} / \mathrm{G}$ \\
\hline UN_xiong_15347 & 227 & $\mathrm{~A} / \mathrm{T}$ \\
\hline UN_xiong_15348 & 743 & $\mathrm{C} / \mathrm{T}$ \\
\hline UN_xiong_15350 & 291 & $\mathrm{G} / \mathrm{A}$ \\
\hline UN_xiong_15350 & 331 & $\mathrm{~T} / \mathrm{C}$ \\
\hline UN_xiong_15350 & 421 & $\mathrm{G} / \mathrm{T}$ \\
\hline UN_xiong_15372 & 213 & $\mathrm{G} / \mathrm{A}$ \\
\hline UN_xiong_15372 & 250 & $A / G$ \\
\hline UN_xiong_15372 & 406 & $\mathrm{~T} / \mathrm{G}$ \\
\hline UN_xiong_15374 & 79 & $\mathrm{G} / \mathrm{C}$ \\
\hline UN_xiong_15374 & 202 & $\mathrm{C} / \mathrm{T}$ \\
\hline UN_xiong_15374 & 242 & $A / G$ \\
\hline UN_xiong_15374 & 670 & $\mathrm{~A} / \mathrm{T}$ \\
\hline UN_xiong_15375 & 95 & $\mathrm{G} / \mathrm{A}$ \\
\hline UN_xiong_15375 & 119 & $\mathrm{~T} / \mathrm{A}$ \\
\hline
\end{tabular}




\begin{tabular}{|c|c|c|}
\hline UN_xiong_15375 & 201 & $\mathrm{~A} / \mathrm{C}$ \\
\hline UN_xiong_15375 & 312 & $\mathrm{C} / \mathrm{A}$ \\
\hline UN_xiong_15382 & 53 & $\mathrm{~T} / \mathrm{C}$ \\
\hline UN_xiong_15382 & 89 & $A / G$ \\
\hline UN_xiong_15382 & 132 & $\mathrm{G} / \mathrm{A}$ \\
\hline UN_xiong_15382 & 157 & $\mathrm{C} / \mathrm{T}$ \\
\hline UN_xiong_15382 & 185 & $A / G$ \\
\hline UN_xiong_15382 & 214 & $\mathrm{C} / \mathrm{T}$ \\
\hline UN_xiong_15382 & 258 & $\mathrm{~A} / \mathrm{T}$ \\
\hline UN_xiong_15382 & 367 & $\mathrm{C} / \mathrm{T}$ \\
\hline UN_xiong_15382 & 402 & $\mathrm{~T} / \mathrm{C}$ \\
\hline UN_xiong_15386 & 360 & $A / G$ \\
\hline UN_xiong_15401 & 259 & $\mathrm{~A} / \mathrm{G}$ \\
\hline UN_xiong_15401 & 715 & $\mathrm{G} / \mathrm{A}$ \\
\hline UN_xiong_15401 & 761 & $\mathrm{~T} / \mathrm{G}$ \\
\hline UN_xiong_15440 & 495 & $\mathrm{C} / \mathrm{T}$ \\
\hline UN_xiong_15449 & 511 & $\mathrm{~T} / \mathrm{G}$ \\
\hline UN_xiong_15449 & 625 & $\mathrm{G} / \mathrm{C}$ \\
\hline UN_xiong_15449 & 934 & $\mathrm{~A} / \mathrm{C}$ \\
\hline UN_xiong_15451 & 225 & $\mathrm{~T} / \mathrm{C}$ \\
\hline UN_xiong_15451 & 287 & $\mathrm{C} / \mathrm{T}$ \\
\hline UN_xiong_15451 & 336 & $\mathrm{~T} / \mathrm{C}$ \\
\hline UN_xiong_15451 & 361 & $\mathrm{~T} / \mathrm{C}$ \\
\hline UN_xiong_15453 & 186 & $\mathrm{C} / \mathrm{T}$ \\
\hline UN_xiong_15453 & 243 & $\mathrm{~A} / \mathrm{G}$ \\
\hline UN_xiong_15460 & 158 & $\mathrm{~T} / \mathrm{C}$ \\
\hline UN_xiong_15470 & 87 & $\mathrm{G} / \mathrm{T}$ \\
\hline UN_xiong_15470 & 115 & $\mathrm{C} / \mathrm{T}$ \\
\hline UN_xiong_15486 & 391 & $\mathrm{~T} / \mathrm{C}$ \\
\hline UN_xiong_15486 & 442 & $\mathrm{~T} / \mathrm{C}$ \\
\hline UN_xiong_15486 & 707 & $\mathrm{~T} / \mathrm{C}$ \\
\hline UN_xiong_15486 & 1594 & $\mathrm{~T} / \mathrm{A}$ \\
\hline UN_xiong_15486 & 1842 & $\mathrm{G} / \mathrm{A}$ \\
\hline UN_xiong_15487 & 503 & $\mathrm{G} / \mathrm{A}$ \\
\hline UN_xiong_15487 & 598 & $\mathrm{G} / \mathrm{A}$ \\
\hline UN_xiong_15487 & 630 & $\mathrm{C} / \mathrm{T}$ \\
\hline UN_xiong_15488 & 785 & $A / G$ \\
\hline UN_xiong_15496 & 202 & $\mathrm{~A} / \mathrm{G}$ \\
\hline UN_xiong_15503 & 378 & $\mathrm{~A} / \mathrm{C}$ \\
\hline UN_xiong_15503 & 614 & $\mathrm{C} / \mathrm{T}$ \\
\hline UN_xiong_15511 & 254 & $A / G$ \\
\hline UN_xiong_15511 & 312 & $\mathrm{~A} / \mathrm{G}$ \\
\hline UN_xiong_15511 & 450 & $\mathrm{G} / \mathrm{A}$ \\
\hline UN_xiong_15511 & 506 & $\mathrm{C} / \mathrm{A}$ \\
\hline UN_xiong_15511 & 621 & $\mathrm{C} / \mathrm{T}$ \\
\hline UN_xiong_15519 & 115 & $\mathrm{G} / \mathrm{A}$ \\
\hline UN_xiong_15529 & 276 & $\mathrm{~T} / \mathrm{C}$ \\
\hline
\end{tabular}




\begin{tabular}{|c|c|c|}
\hline UN_xiong_15529 & 343 & $\mathrm{~T} / \mathrm{C}$ \\
\hline UN_xiong_15529 & 368 & $\mathrm{C} / \mathrm{T}$ \\
\hline UN_xiong_15529 & 393 & $\mathrm{~A} / \mathrm{T}$ \\
\hline UN_xiong_15529 & 592 & $\mathrm{G} / \mathrm{A}$ \\
\hline UN_xiong_15532 & 409 & $\mathrm{~A} / \mathrm{T}$ \\
\hline UN_xiong_15533 & 141 & $\mathrm{C} / \mathrm{T}$ \\
\hline UN_xiong_15533 & 805 & $\mathrm{~A} / \mathrm{T}$ \\
\hline UN_xiong_15536 & 124 & $\mathrm{C} / \mathrm{T}$ \\
\hline UN_xiong_15536 & 199 & $A / G$ \\
\hline UN_xiong_15536 & 544 & $\mathrm{~T} / \mathrm{C}$ \\
\hline UN_xiong_15536 & 571 & $\mathrm{~T} / \mathrm{A}$ \\
\hline UN_xiong_15536 & 643 & $\mathrm{~A} / \mathrm{C}$ \\
\hline UN_xiong_15541 & 634 & $\mathrm{~T} / \mathrm{G}$ \\
\hline UN_xiong_15543 & 395 & $A / G$ \\
\hline UN_xiong_15543 & 472 & $A / G$ \\
\hline UN_xiong_15543 & 658 & $\mathrm{~T} / \mathrm{A}$ \\
\hline UN_xiong_15554 & 379 & $A / G$ \\
\hline UN_xiong_15554 & 455 & $\mathrm{~T} / \mathrm{C}$ \\
\hline UN_xiong_15554 & 489 & $\mathrm{G} / \mathrm{T}$ \\
\hline UN_xiong_15554 & 587 & $A / G$ \\
\hline UN_xiong_15554 & 609 & $A / G$ \\
\hline UN_xiong_15554 & 639 & $\mathrm{~T} / \mathrm{C}$ \\
\hline UN_xiong_15554 & 674 & $\mathrm{C} / \mathrm{A}$ \\
\hline UN_xiong_15554 & 705 & $A / G$ \\
\hline UN_xiong_15554 & 802 & $A / G$ \\
\hline UN_xiong_15554 & 867 & $\mathrm{~T} / \mathrm{C}$ \\
\hline UN_xiong_15554 & 894 & $A / G$ \\
\hline UN_xiong_15554 & 988 & C/A \\
\hline UN_xiong_15554 & 1302 & $A / G$ \\
\hline UN_xiong_15556 & 60 & $A / G$ \\
\hline UN_xiong_15556 & 83 & $\mathrm{~T} / \mathrm{A}$ \\
\hline UN_xiong_15556 & 179 & $A / G$ \\
\hline UN_xiong_15556 & 235 & $\mathrm{C} / \mathrm{T}$ \\
\hline UN_xiong_15556 & 287 & $A / G$ \\
\hline UN_xiong_15556 & 357 & $\mathrm{G} / \mathrm{A}$ \\
\hline UN_xiong_15556 & 378 & $\mathrm{G} / \mathrm{C}$ \\
\hline UN_xiong_15556 & 442 & $\mathrm{~A} / \mathrm{T}$ \\
\hline UN_xiong_15556 & 463 & $\mathrm{G} / \mathrm{A}$ \\
\hline UN_xiong_15556 & 633 & $A / G$ \\
\hline UN_xiong_15556 & 743 & $\mathrm{C} / \mathrm{T}$ \\
\hline UN_xiong_15557 & 254 & $A / G$ \\
\hline UN_xiong_15557 & 344 & $A / G$ \\
\hline UN_xiong_15557 & 446 & $\mathrm{~T} / \mathrm{C}$ \\
\hline UN_xiong_15557 & 472 & $\mathrm{G} / \mathrm{C}$ \\
\hline UN_xiong_15557 & 562 & $\mathrm{G} / \mathrm{A}$ \\
\hline UN_xiong_15562 & 34 & $\mathrm{~T} / \mathrm{G}$ \\
\hline UN_xiong_15562 & 452 & $\mathrm{G} / \mathrm{A}$ \\
\hline
\end{tabular}




\begin{tabular}{|c|c|c|}
\hline UN_xiong_15563 & 21 & $\mathrm{G} / \mathrm{A}$ \\
\hline UN_xiong_15563 & 322 & $A / G$ \\
\hline UN_xiong_15566 & 609 & $\mathrm{~A} / \mathrm{T}$ \\
\hline UN_xiong_15574 & 230 & $\mathrm{G} / \mathrm{A}$ \\
\hline UN_xiong_15574 & 604 & $\mathrm{C} / \mathrm{T}$ \\
\hline UN_xiong_15574 & 722 & $\mathrm{~T} / \mathrm{C}$ \\
\hline UN_xiong_15575 & 252 & $\mathrm{G} / \mathrm{C}$ \\
\hline UN_xiong_15587 & 519 & $\mathrm{C} / \mathrm{T}$ \\
\hline UN_xiong_15587 & 632 & $\mathrm{G} / \mathrm{A}$ \\
\hline UN_xiong_15587 & 696 & $\mathrm{~T} / \mathrm{C}$ \\
\hline UN_xiong_15587 & 1160 & $\mathrm{G} / \mathrm{A}$ \\
\hline UN_xiong_15587 & 1184 & $A / G$ \\
\hline UN_xiong_15587 & 1333 & $\mathrm{~T} / \mathrm{C}$ \\
\hline UN_xiong_15587 & 1365 & $\mathrm{~A} / \mathrm{T}$ \\
\hline UN_xiong_15592 & 106 & $\mathrm{~T} / \mathrm{C}$ \\
\hline UN_xiong_15611 & 313 & $\mathrm{G} / \mathrm{A}$ \\
\hline UN_xiong_15615 & 126 & $\mathrm{~A} / \mathrm{T}$ \\
\hline UN_xiong_15624 & 340 & $\mathrm{G} / \mathrm{A}$ \\
\hline UN_xiong_15624 & 1143 & $\mathrm{~A} / \mathrm{T}$ \\
\hline UN_xiong_15628 & 247 & $\mathrm{C} / \mathrm{T}$ \\
\hline UN_xiong_15631 & 35 & $\mathrm{~T} / \mathrm{G}$ \\
\hline UN_xiong_15631 & 79 & $\mathrm{~T} / \mathrm{C}$ \\
\hline UN_xiong_15631 & 221 & $\mathrm{~T} / \mathrm{C}$ \\
\hline UN_xiong_15631 & 260 & $\mathrm{C} / \mathrm{T}$ \\
\hline UN_xiong_15631 & 344 & $\mathrm{~T} / \mathrm{C}$ \\
\hline UN_xiong_15631 & 374 & $\mathrm{G} / \mathrm{C}$ \\
\hline UN_xiong_15631 & 411 & $\mathrm{~A} / \mathrm{G}$ \\
\hline UN_xiong_15631 & 468 & $\mathrm{G} / \mathrm{A}$ \\
\hline UN_xiong_15631 & 909 & $\mathrm{C} / \mathrm{A}$ \\
\hline UN_xiong_15633 & 485 & $A / G$ \\
\hline UN_xiong_15633 & 567 & $\mathrm{C} / \mathrm{T}$ \\
\hline UN_xiong_15633 & 1299 & $\mathrm{G} / \mathrm{C}$ \\
\hline UN_xiong_15633 & 1329 & $A / G$ \\
\hline UN_xiong_15633 & 1411 & $\mathrm{~A} / \mathrm{G}$ \\
\hline UN_xiong_15633 & 1436 & $\mathrm{G} / \mathrm{T}$ \\
\hline UN_xiong_15633 & 1621 & $\mathrm{G} / \mathrm{A}$ \\
\hline UN_xiong_15638 & 145 & $A / G$ \\
\hline UN_xiong_15641 & 475 & $\mathrm{C} / \mathrm{T}$ \\
\hline UN_xiong_15644 & 32 & $A / G$ \\
\hline UN_xiong_15644 & 65 & $\mathrm{~A} / \mathrm{T}$ \\
\hline UN_xiong_15644 & 164 & $\mathrm{~T} / \mathrm{C}$ \\
\hline UN_xiong_15644 & 237 & $\mathrm{C} / \mathrm{T}$ \\
\hline UN_xiong_15644 & 302 & $\mathrm{~T} / \mathrm{G}$ \\
\hline UN_xiong_15644 & 326 & $\mathrm{~A} / \mathrm{T}$ \\
\hline UN_xiong_15644 & 368 & $\mathrm{C} / \mathrm{G}$ \\
\hline UN_xiong_15660 & 259 & $\mathrm{G} / \mathrm{A}$ \\
\hline UN_xiong_15664 & 207 & $\mathrm{~A} / \mathrm{C}$ \\
\hline
\end{tabular}




\begin{tabular}{|c|c|c|}
\hline UN_xiong_15667 & 565 & $\mathrm{~A} / \mathrm{G}$ \\
\hline UN_xiong_15672 & 228 & $\mathrm{C} / \mathrm{T}$ \\
\hline UN_xiong_15672 & 468 & $\mathrm{~T} / \mathrm{C}$ \\
\hline UN_xiong_15676 & 228 & $C / A$ \\
\hline UN_xiong_15676 & 320 & $\mathrm{~A} / \mathrm{T}$ \\
\hline UN_xiong_15676 & 525 & $\mathrm{G} / \mathrm{A}$ \\
\hline UN_xiong_15681 & 212 & $\mathrm{~T} / \mathrm{C}$ \\
\hline UN_xiong_15684 & 476 & $C / A$ \\
\hline UN_xiong_15687 & 69 & $\mathrm{~T} / \mathrm{C}$ \\
\hline UN_xiong_15689 & 178 & $\mathrm{C} / \mathrm{T}$ \\
\hline UN_xiong_15689 & 718 & $\mathrm{~T} / \mathrm{C}$ \\
\hline UN_xiong_15691 & 269 & $\mathrm{C} / \mathrm{T}$ \\
\hline UN_xiong_15691 & 361 & $\mathrm{~T} / \mathrm{C}$ \\
\hline UN_xiong_15692 & 143 & $\mathrm{G} / \mathrm{C}$ \\
\hline UN_xiong_15692 & 615 & $\mathrm{~T} / \mathrm{C}$ \\
\hline UN_xiong_15692 & 850 & $\mathrm{~T} / \mathrm{C}$ \\
\hline UN_xiong_15697 & 175 & $\mathrm{~A} / \mathrm{G}$ \\
\hline UN_xiong_15697 & 271 & $\mathrm{~A} / \mathrm{C}$ \\
\hline UN_xiong_15697 & 291 & $\mathrm{C} / \mathrm{T}$ \\
\hline UN_xiong_15699 & 956 & $\mathrm{G} / \mathrm{A}$ \\
\hline UN_xiong_15705 & 705 & $\mathrm{C} / \mathrm{G}$ \\
\hline UN_xiong_15707 & 659 & $A / G$ \\
\hline UN_xiong_15708 & 30 & $\mathrm{~T} / \mathrm{C}$ \\
\hline UN_xiong_15708 & 521 & $\mathrm{~T} / \mathrm{G}$ \\
\hline UN_xiong_15708 & 588 & $G / A$ \\
\hline UN_xiong_15708 & 835 & $\mathrm{~T} / \mathrm{C}$ \\
\hline UN_xiong_15708 & 866 & $\mathrm{~A} / \mathrm{G}$ \\
\hline UN_xiong_15708 & 1127 & $\mathrm{C} / \mathrm{G}$ \\
\hline UN_xiong_15708 & 1190 & $\mathrm{G} / \mathrm{T}$ \\
\hline UN_xiong_15708 & 1247 & $\mathrm{~T} / \mathrm{C}$ \\
\hline UN_xiong_15712 & 385 & $\mathrm{~T} / \mathrm{A}$ \\
\hline UN_xiong_15712 & 572 & $A / G$ \\
\hline UN_xiong_15712 & 667 & $\mathrm{G} / \mathrm{A}$ \\
\hline UN_xiong_15712 & 688 & $\mathrm{C} / \mathrm{T}$ \\
\hline UN_xiong_15715 & 664 & $A / G$ \\
\hline UN_xiong_15715 & 782 & $\mathrm{~A} / \mathrm{G}$ \\
\hline UN_xiong_15715 & 829 & $\mathrm{~T} / \mathrm{C}$ \\
\hline UN_xiong_15725 & 216 & $A / G$ \\
\hline UN_xiong_15733 & 301 & $\mathrm{~A} / \mathrm{C}$ \\
\hline UN_xiong_15736 & 384 & $\mathrm{~A} / \mathrm{C}$ \\
\hline UN_xiong_15736 & 572 & $\mathrm{~T} / \mathrm{C}$ \\
\hline UN_xiong_15736 & 1014 & $\mathrm{G} / \mathrm{A}$ \\
\hline UN_xiong_15736 & 1551 & $\mathrm{~T} / \mathrm{C}$ \\
\hline UN_xiong_15736 & 1580 & $\mathrm{~A} / \mathrm{G}$ \\
\hline UN_xiong_15742 & 46 & $A / G$ \\
\hline UN_xiong_15742 & 100 & $\mathrm{~T} / \mathrm{C}$ \\
\hline UN_xiong_15742 & 257 & $\mathrm{~T} / \mathrm{C}$ \\
\hline
\end{tabular}




\begin{tabular}{|c|c|c|}
\hline UN_xiong_15742 & 303 & $\mathrm{G} / \mathrm{A}$ \\
\hline UN_xiong_15742 & 370 & $\mathrm{C} / \mathrm{T}$ \\
\hline UN_xiong_15742 & 694 & $\mathrm{~T} / \mathrm{C}$ \\
\hline UN_xiong_15747 & 246 & $\mathrm{G} / \mathrm{T}$ \\
\hline UN_xiong_15747 & 323 & $\mathrm{G} / \mathrm{A}$ \\
\hline UN_xiong_15747 & 623 & $\mathrm{~T} / \mathrm{C}$ \\
\hline UN_xiong_15750 & 200 & $\mathrm{~A} / \mathrm{C}$ \\
\hline UN_xiong_15753 & 398 & $\mathrm{~T} / \mathrm{A}$ \\
\hline UN_xiong_15754 & 1114 & $\mathrm{C} / \mathrm{T}$ \\
\hline UN_xiong_15754 & 1236 & $A / G$ \\
\hline UN_xiong_15755 & 64 & $\mathrm{G} / \mathrm{A}$ \\
\hline UN_xiong_15755 & 427 & $\mathrm{~A} / \mathrm{T}$ \\
\hline UN_xiong_15755 & 676 & $\mathrm{C} / \mathrm{T}$ \\
\hline UN_xiong_15758 & 323 & $A / G$ \\
\hline UN_xiong_15758 & 383 & $\mathrm{G} / \mathrm{T}$ \\
\hline UN_xiong_15765 & 50 & $\mathrm{C} / \mathrm{T}$ \\
\hline UN_xiong_15770 & 159 & $\mathrm{~A} / \mathrm{G}$ \\
\hline UN_xiong_15771 & 139 & $\mathrm{~T} / \mathrm{C}$ \\
\hline UN_xiong_15774 & 273 & $A / G$ \\
\hline UN_xiong_15779 & 111 & $\mathrm{~A} / \mathrm{C}$ \\
\hline UN_xiong_15784 & 175 & $\mathrm{G} / \mathrm{T}$ \\
\hline UN_xiong_15784 & 207 & $\mathrm{~T} / \mathrm{C}$ \\
\hline UN_xiong_15784 & 428 & $\mathrm{~T} / \mathrm{C}$ \\
\hline UN_xiong_15784 & 538 & $\mathrm{C} / \mathrm{T}$ \\
\hline UN_xiong_15784 & 609 & $\mathrm{~T} / \mathrm{C}$ \\
\hline UN_xiong_15784 & 634 & $\mathrm{~T} / \mathrm{C}$ \\
\hline UN_xiong_15784 & 683 & $\mathrm{~A} / \mathrm{G}$ \\
\hline UN_xiong_15784 & 845 & $\mathrm{C} / \mathrm{T}$ \\
\hline UN_xiong_15785 & 330 & $\mathrm{~A} / \mathrm{G}$ \\
\hline UN_xiong_15785 & 410 & $\mathrm{C} / \mathrm{T}$ \\
\hline UN_xiong_15786 & 18 & $A / G$ \\
\hline UN_xiong_15786 & 308 & $A / G$ \\
\hline UN_xiong_15786 & 645 & $\mathrm{G} / \mathrm{A}$ \\
\hline UN_xiong_15787 & 473 & $A / G$ \\
\hline UN_xiong_15787 & 515 & $\mathrm{~A} / \mathrm{C}$ \\
\hline UN_xiong_15787 & 605 & $C / A$ \\
\hline UN_xiong_15789 & 449 & $\mathrm{G} / \mathrm{A}$ \\
\hline UN_xiong_15789 & 758 & $\mathrm{~T} / \mathrm{C}$ \\
\hline UN_xiong_15789 & 781 & $\mathrm{~T} / \mathrm{C}$ \\
\hline UN_xiong_15790 & 177 & $\mathrm{~T} / \mathrm{A}$ \\
\hline UN_xiong_15790 & 731 & $\mathrm{C} / \mathrm{T}$ \\
\hline UN_xiong_15791 & 504 & $\mathrm{~A} / \mathrm{G}$ \\
\hline UN_xiong_15791 & 591 & $\mathrm{C} / \mathrm{T}$ \\
\hline UN_xiong_15791 & 823 & $\mathrm{~T} / \mathrm{C}$ \\
\hline UN_xiong_15791 & 915 & $A / G$ \\
\hline UN_xiong_15794 & 77 & $\mathrm{C} / \mathrm{G}$ \\
\hline
\end{tabular}




\begin{tabular}{|c|c|}
\hline Contig_Name & Offset_On_Contig ALLE \\
\hline UN_ci_2 & $422 \mathrm{G} / \mathrm{A}$ \\
\hline UN_ci_10 & $488 \mathrm{C} / \mathrm{A}$ \\
\hline UN_ci_10 & $955 \mathrm{G} / \mathrm{A}$ \\
\hline UN_ci_10 & $1045 \mathrm{~T} / \mathrm{C}$ \\
\hline UN_ci_16 & $272 \mathrm{C} / \mathrm{T}$ \\
\hline UN_ci_17 & $242 \mathrm{~T} / \mathrm{C}$ \\
\hline UN_ci_23 & $225 \mathrm{~T} / \mathrm{C}$ \\
\hline UN_ci_33 & $282 \mathrm{G} / \mathrm{A}$ \\
\hline UN_ci_43 & $328 \mathrm{G} / \mathrm{C}$ \\
\hline UN_ci_47 & $140 \mathrm{~T} / \mathrm{C}$ \\
\hline UN_ci_47 & $405 \mathrm{~T} / \mathrm{C}$ \\
\hline UN_ci_48 & $97 \mathrm{C} / \mathrm{T}$ \\
\hline UN_ci_48 & $214 \mathrm{~A} / \mathrm{G}$ \\
\hline UN_ci_48 & $252 \mathrm{~A} / \mathrm{G}$ \\
\hline UN_ci_51 & $322 \mathrm{~A} / \mathrm{T}$ \\
\hline UN_ci_53 & $57 \mathrm{~T} / \mathrm{C}$ \\
\hline UN_ci_60 & $41 \mathrm{C} / \mathrm{T}$ \\
\hline UN_ci_60 & $499 \mathrm{G} / \mathrm{T}$ \\
\hline UN_ci_60 & $727 \mathrm{G} / \mathrm{C}$ \\
\hline UN_ci_73 & $276 \mathrm{~T} / \mathrm{C}$ \\
\hline UN_ci_73 & $318 \mathrm{~T} / \mathrm{C}$ \\
\hline UN_ci_73 & $371 \mathrm{~T} / \mathrm{A}$ \\
\hline UN_ci_73 & $417 \mathrm{~A} / \mathrm{G}$ \\
\hline UN_ci_74 & $51 \mathrm{G} / \mathrm{A}$ \\
\hline UN_ci_74 & $356 \mathrm{G} / \mathrm{A}$ \\
\hline UN_ci_74 & $389 \mathrm{G} / \mathrm{A}$ \\
\hline UN_ci_79 & $980 \mathrm{~A} / \mathrm{G}$ \\
\hline UN_ci_85 & $9 \mathrm{~T} / \mathrm{G}$ \\
\hline UN_ci_99 & $24 \mathrm{~A} / \mathrm{G}$ \\
\hline UN_ci_99 & $47 \mathrm{C} / \mathrm{T}$ \\
\hline UN_ci_99 & $211 \mathrm{C} / \mathrm{A}$ \\
\hline UN_ci_99 & $314 \mathrm{G} / \mathrm{A}$ \\
\hline UN_ci_103 & $311 \mathrm{G} / \mathrm{A}$ \\
\hline UN_ci_109 & $301 \mathrm{~A} / \mathrm{G}$ \\
\hline UN_ci_115 & $115 \mathrm{~A} / \mathrm{G}$ \\
\hline UN_ci_115 & $168 \mathrm{~A} / \mathrm{G}$ \\
\hline UN_ci_121 & $265 \mathrm{C} / \mathrm{T}$ \\
\hline UN_ci_121 & $1049 \mathrm{~T} / \mathrm{C}$ \\
\hline UN_ci_121 & $1150 \mathrm{~A} / \mathrm{G}$ \\
\hline UN_ci_121 & $1383 \mathrm{~T} / \mathrm{A}$ \\
\hline UN_ci_142 & $575 \mathrm{~T} / \mathrm{G}$ \\
\hline UN_ci_142 & $637 \mathrm{C} / \mathrm{T}$ \\
\hline UN_ci_142 & $777 \mathrm{~A} / \mathrm{G}$ \\
\hline UN_ci_143 & $418 \mathrm{~T} / \mathrm{C}$ \\
\hline UN_ci_143 & $471 \mathrm{~A} / \mathrm{C}$ \\
\hline UN_ci_150 & $197 \mathrm{~A} / \mathrm{G}$ \\
\hline UN_ci_158 & $76 \mathrm{C} / \mathrm{A}$ \\
\hline UN_ci_158 & $163 \mathrm{C} / \mathrm{T}$ \\
\hline UN_ci_158 & $243 \mathrm{~T} / \mathrm{C}$ \\
\hline
\end{tabular}




\begin{tabular}{|c|c|}
\hline UN_ci_158 & $358 \mathrm{~A} / \mathrm{G}$ \\
\hline UN_ci_160 & $121 \mathrm{~T} / \mathrm{C}$ \\
\hline UN_ci_169 & $304 \mathrm{~A} / \mathrm{G}$ \\
\hline UN_ci_178 & $263 \mathrm{~A} / \mathrm{T}$ \\
\hline UN_ci_183 & $330 \mathrm{~A} / \mathrm{G}$ \\
\hline UN_ci_183 & $371 \mathrm{~T} / \mathrm{A}$ \\
\hline UN_ci_198 & $75 \mathrm{G} / \mathrm{T}$ \\
\hline UN_ci_198 & $146 \mathrm{~T} / \mathrm{A}$ \\
\hline UN_ci_198 & $241 \mathrm{~T} / \mathrm{G}$ \\
\hline UN_ci_198 & $325 \mathrm{~T} / \mathrm{C}$ \\
\hline UN_ci_198 & $369 \mathrm{~T} / \mathrm{G}$ \\
\hline UN_ci_198 & $671 \mathrm{C} / \mathrm{A}$ \\
\hline UN_ci_208 & $56 \mathrm{C} / \mathrm{A}$ \\
\hline UN_ci_210 & $185 \mathrm{~T} / \mathrm{C}$ \\
\hline UN_ci_214 & $38 \mathrm{G} / \mathrm{A}$ \\
\hline UN_ci_221 & 202 T/G \\
\hline UN_ci_222 & $80 \mathrm{~A} / \mathrm{C}$ \\
\hline UN_ci_231 & $182 \mathrm{C} / \mathrm{T}$ \\
\hline UN_ci_231 & $256 \mathrm{~A} / \mathrm{G}$ \\
\hline UN_ci_234 & $40 \mathrm{~A} / \mathrm{G}$ \\
\hline UN_ci_234 & $163 \mathrm{~A} / \mathrm{T}$ \\
\hline UN_ci_234 & $203 \mathrm{~T} / \mathrm{C}$ \\
\hline UN_ci_239 & $135 \mathrm{~A} / \mathrm{G}$ \\
\hline UN_ci_239 & $311 \mathrm{~A} / \mathrm{G}$ \\
\hline UN_ci_239 & $363 \mathrm{G} / \mathrm{A}$ \\
\hline UN_ci_239 & $394 \mathrm{C} / \mathrm{G}$ \\
\hline UN_ci_239 & $420 \mathrm{~T} / \mathrm{C}$ \\
\hline UN_ci_239 & $527 \mathrm{~A} / \mathrm{G}$ \\
\hline UN_ci_239 & $606 \mathrm{~A} / \mathrm{G}$ \\
\hline UN_ci_239 & $651 \mathrm{~A} / \mathrm{G}$ \\
\hline UN_ci_239 & $717 \mathrm{~A} / \mathrm{G}$ \\
\hline UN_ci_239 & $779 \mathrm{~T} / \mathrm{C}$ \\
\hline UN_ci_239 & $820 \mathrm{G} / \mathrm{T}$ \\
\hline UN_ci_239 & $845 \mathrm{~T} / \mathrm{C}$ \\
\hline UN_ci_239 & $865 \mathrm{~T} / \mathrm{C}$ \\
\hline UN_ci_239 & $888 \mathrm{~T} / \mathrm{C}$ \\
\hline UN_ci_239 & $953 \mathrm{~A} / \mathrm{G}$ \\
\hline UN_ci_239 & $1051 \mathrm{C} / \mathrm{G}$ \\
\hline UN_ci_243 & $56 \mathrm{G} / \mathrm{A}$ \\
\hline UN_ci_245 & $325 \mathrm{G} / \mathrm{A}$ \\
\hline UN_ci_246 & $226 \mathrm{~T} / \mathrm{G}$ \\
\hline UN_ci_257 & $69 \mathrm{C} / \mathrm{T}$ \\
\hline UN_ci_257 & $242 \mathrm{C} / \mathrm{T}$ \\
\hline UN_ci_257 & $300 \mathrm{~A} / \mathrm{G}$ \\
\hline UN_ci_257 & $401 \mathrm{~A} / \mathrm{G}$ \\
\hline UN_ci_261 & $23 \mathrm{G} / \mathrm{T}$ \\
\hline UN_ci_262 & $126 \mathrm{G} / \mathrm{A}$ \\
\hline UN_ci_262 & $155 \mathrm{G} / \mathrm{A}$ \\
\hline UN_ci_267 & $38 \mathrm{G} / \mathrm{A}$ \\
\hline UN_ci_267 & $300 \mathrm{~A} / \mathrm{G}$ \\
\hline
\end{tabular}




\begin{tabular}{|c|c|}
\hline UN_ci_267 & $401 \mathrm{~T} / \mathrm{C}$ \\
\hline UN_ci_267 & $495 \mathrm{~A} / \mathrm{G}$ \\
\hline UN_ci_267 & $556 \mathrm{~A} / \mathrm{G}$ \\
\hline UN_ci_267 & $640 \mathrm{G} / \mathrm{A}$ \\
\hline UN_ci_267 & $683 \mathrm{C} / \mathrm{T}$ \\
\hline UN_ci_267 & $791 \mathrm{~T} / \mathrm{C}$ \\
\hline UN_ci_268 & $102 \mathrm{C} / \mathrm{T}$ \\
\hline UN_ci_268 & $122 \mathrm{C} / \mathrm{T}$ \\
\hline UN_ci_268 & $175 \mathrm{~T} / \mathrm{C}$ \\
\hline UN_ci_269 & $70 \mathrm{~T} / \mathrm{A}$ \\
\hline UN_ci_280 & $382 \mathrm{~T} / \mathrm{A}$ \\
\hline UN_ci_283 & $372 \mathrm{~A} / \mathrm{G}$ \\
\hline UN_ci_297 & $168 \mathrm{C} / \mathrm{T}$ \\
\hline UN_ci_297 & $504 \mathrm{~T} / \mathrm{A}$ \\
\hline UN_ci_297 & $627 \mathrm{G} / \mathrm{A}$ \\
\hline UN_ci_304 & $241 \mathrm{C} / \mathrm{A}$ \\
\hline UN_ci_316 & $415 \mathrm{~T} / \mathrm{C}$ \\
\hline UN_ci_317 & $37 \mathrm{~A} / \mathrm{T}$ \\
\hline UN_ci_317 & $255 \mathrm{~T} / \mathrm{A}$ \\
\hline UN_ci_323 & $110 \mathrm{G} / \mathrm{A}$ \\
\hline UN_ci_333 & $801 \mathrm{~A} / \mathrm{G}$ \\
\hline UN_ci_333 & $1140 \mathrm{~T} / \mathrm{C}$ \\
\hline UN_ci_343 & $114 \mathrm{~T} / \mathrm{C}$ \\
\hline UN_ci_344 & $168 \mathrm{G} / \mathrm{A}$ \\
\hline UN_ci_344 & $198 \mathrm{~A} / \mathrm{G}$ \\
\hline UN_ci_356 & $250 \mathrm{~A} / \mathrm{T}$ \\
\hline UN_ci_362 & $40 \mathrm{G} / \mathrm{A}$ \\
\hline UN_ci_362 & $176 \mathrm{~A} / \mathrm{G}$ \\
\hline UN_ci_362 & $947 \mathrm{G} / \mathrm{A}$ \\
\hline UN_ci_362 & $971 \mathrm{~A} / \mathrm{G}$ \\
\hline UN_ci_362 & $1005 \mathrm{~T} / \mathrm{C}$ \\
\hline UN_ci_362 & $1030 \mathrm{~A} / \mathrm{G}$ \\
\hline UN_ci_362 & $1188 \mathrm{~T} / \mathrm{C}$ \\
\hline UN_ci_362 & $1240 \mathrm{~A} / \mathrm{C}$ \\
\hline UN_ci_362 & $1279 \mathrm{~T} / \mathrm{A}$ \\
\hline UN_ci_362 & $1309 \mathrm{~A} / \mathrm{C}$ \\
\hline UN_ci_368 & $123 \mathrm{~T} / \mathrm{C}$ \\
\hline UN_ci_373 & $127 \mathrm{~T} / \mathrm{C}$ \\
\hline UN_ci_373 & $727 \mathrm{C} / \mathrm{T}$ \\
\hline UN_ci_373 & $838 \mathrm{~T} / \mathrm{G}$ \\
\hline UN_ci_379 & $205 \mathrm{~A} / \mathrm{G}$ \\
\hline UN_ci_379 & $372 \mathrm{G} / \mathrm{A}$ \\
\hline UN_ci_389 & $134 \mathrm{G} / \mathrm{A}$ \\
\hline UN_ci_392 & $139 \mathrm{~T} / \mathrm{C}$ \\
\hline UN_ci_395 & $556 \mathrm{G} / \mathrm{A}$ \\
\hline UN_ci_395 & $590 \mathrm{G} / \mathrm{A}$ \\
\hline UN_ci_395 & $619 \mathrm{C} / \mathrm{T}$ \\
\hline UN_ci_395 & $652 \mathrm{~T} / \mathrm{G}$ \\
\hline UN_ci_395 & 946 G/T \\
\hline UN_ci_398 & $35 \mathrm{~T} / \mathrm{C}$ \\
\hline
\end{tabular}




\begin{tabular}{|c|c|}
\hline UN_ci_398 & $63 \mathrm{C} / \mathrm{G}$ \\
\hline UN_ci_398 & $84 \mathrm{~A} / \mathrm{T}$ \\
\hline UN_ci_409 & $237 \mathrm{~A} / \mathrm{T}$ \\
\hline UN_ci_420 & $53 \mathrm{~A} / \mathrm{G}$ \\
\hline UN_ci_420 & $84 \mathrm{~T} / \mathrm{C}$ \\
\hline UN_ci_420 & $562 \mathrm{C} / \mathrm{T}$ \\
\hline UN_ci_420 & $655 \mathrm{C} / \mathrm{T}$ \\
\hline UN_ci_435 & $136 \mathrm{C} / \mathrm{T}$ \\
\hline UN_ci_440 & $910 \mathrm{C} / \mathrm{T}$ \\
\hline UN_ci_447 & $35 \mathrm{~A} / \mathrm{G}$ \\
\hline UN_ci_447 & $93 \mathrm{C} / \mathrm{T}$ \\
\hline UN_ci_447 & $296 \mathrm{C} / \mathrm{T}$ \\
\hline UN_ci_448 & $415 \mathrm{~T} / \mathrm{C}$ \\
\hline UN_ci_448 & $593 \mathrm{~T} / \mathrm{C}$ \\
\hline UN_ci_448 & $805 \mathrm{~T} / \mathrm{G}$ \\
\hline UN_ci_460 & $332 \mathrm{C} / \mathrm{T}$ \\
\hline UN_ci_474 & $131 \mathrm{C} / \mathrm{T}$ \\
\hline UN_ci_474 & $166 \mathrm{~A} / \mathrm{G}$ \\
\hline UN_ci_483 & $260 \mathrm{~A} / \mathrm{T}$ \\
\hline UN_ci_490 & $69 \mathrm{~A} / \mathrm{G}$ \\
\hline UN_ci_490 & $99 \mathrm{~A} / \mathrm{G}$ \\
\hline UN_ci_490 & $353 \mathrm{C} / \mathrm{T}$ \\
\hline UN_ci_493 & $351 \mathrm{~T} / \mathrm{C}$ \\
\hline UN_ci_495 & $122 \mathrm{~A} / \mathrm{G}$ \\
\hline UN_ci_498 & $33 \mathrm{~A} / \mathrm{T}$ \\
\hline UN_ci_506 & $125 \mathrm{G} / \mathrm{A}$ \\
\hline UN_ci_521 & $238 \mathrm{~A} / \mathrm{G}$ \\
\hline UN_ci_525 & $683 \mathrm{G} / \mathrm{C}$ \\
\hline UN_ci_539 & 109 T/G \\
\hline UN_ci_540 & $64 \mathrm{~T} / \mathrm{C}$ \\
\hline UN_ci_540 & $536 \mathrm{C} / \mathrm{G}$ \\
\hline UN_ci_541 & $468 \mathrm{~T} / \mathrm{C}$ \\
\hline UN_ci_551 & 327 T/C \\
\hline UN_ci_560 & $9 \mathrm{C} / \mathrm{G}$ \\
\hline UN_ci_560 & $120 \mathrm{~A} / \mathrm{G}$ \\
\hline UN_ci_563 & $252 \mathrm{~T} / \mathrm{C}$ \\
\hline UN_ci_564 & $54 \mathrm{C} / \mathrm{T}$ \\
\hline UN_ci_577 & $353 \mathrm{~A} / \mathrm{C}$ \\
\hline UN_ci_578 & $472 \mathrm{~A} / \mathrm{G}$ \\
\hline UN_ci_584 & $44 \mathrm{~T} / \mathrm{C}$ \\
\hline UN_ci_587 & $32 \mathrm{C} / \mathrm{G}$ \\
\hline UN_ci_589 & $11 \mathrm{G} / \mathrm{T}$ \\
\hline UN_ci_589 & $143 \mathrm{C} / \mathrm{T}$ \\
\hline UN_ci_594 & $21 \mathrm{~A} / \mathrm{C}$ \\
\hline UN_ci_594 & $51 \mathrm{~A} / \mathrm{G}$ \\
\hline UN_ci_594 & $92 \mathrm{C} / \mathrm{T}$ \\
\hline UN_ci_594 & $186 \mathrm{~A} / \mathrm{G}$ \\
\hline UN_ci_594 & $257 \mathrm{~A} / \mathrm{T}$ \\
\hline UN_ci_594 & $288 \mathrm{~T} / \mathrm{C}$ \\
\hline UN_ci_594 & $348 \mathrm{~T} / \mathrm{C}$ \\
\hline
\end{tabular}




\begin{tabular}{|c|c|}
\hline UN_ci_597 & $624 \mathrm{~T} / \mathrm{C}$ \\
\hline UN_ci_600 & $25 \mathrm{~T} / \mathrm{C}$ \\
\hline UN_ci_601 & $453 \mathrm{~T} / \mathrm{C}$ \\
\hline UN_ci_610 & $370 \mathrm{C} / \mathrm{T}$ \\
\hline UN_ci_617 & $164 \mathrm{~A} / \mathrm{G}$ \\
\hline UN_ci_620 & $491 \mathrm{C} / \mathrm{T}$ \\
\hline UN_ci_620 & $534 \mathrm{~T} / \mathrm{C}$ \\
\hline UN_ci_620 & $789 \mathrm{~T} / \mathrm{A}$ \\
\hline UN_ci_620 & $856 \mathrm{G} / \mathrm{T}$ \\
\hline UN_ci_620 & $910 \mathrm{G} / \mathrm{A}$ \\
\hline UN_ci_641 & $51 \mathrm{~T} / \mathrm{C}$ \\
\hline UN_ci_641 & $74 \mathrm{G} / \mathrm{A}$ \\
\hline UN_ci_641 & $98 \mathrm{~T} / \mathrm{A}$ \\
\hline UN_ci_641 & $180 \mathrm{~A} / \mathrm{C}$ \\
\hline UN_ci_641 & $207 \mathrm{~A} / \mathrm{G}$ \\
\hline UN_ci_641 & $259 \mathrm{~T} / \mathrm{C}$ \\
\hline UN_ci_641 & $284 \mathrm{~A} / \mathrm{T}$ \\
\hline UN_ci_647 & $329 \mathrm{C} / \mathrm{T}$ \\
\hline UN_ci_647 & $371 \mathrm{~T} / \mathrm{C}$ \\
\hline UN_ci_647 & $498 \mathrm{~T} / \mathrm{C}$ \\
\hline UN_ci_647 & $588 \mathrm{C} / \mathrm{A}$ \\
\hline UN_ci_650 & $140 \mathrm{~A} / \mathrm{G}$ \\
\hline UN_ci_662 & $158 \mathrm{~A} / \mathrm{G}$ \\
\hline UN_ci_670 & $26 \mathrm{C} / \mathrm{G}$ \\
\hline UN_ci_670 & $184 \mathrm{~T} / \mathrm{A}$ \\
\hline UN_ci_670 & $212 \mathrm{G} / \mathrm{A}$ \\
\hline UN_ci_670 & $241 \mathrm{~A} / \mathrm{T}$ \\
\hline UN_ci_670 & $265 \mathrm{~A} / \mathrm{G}$ \\
\hline UN_ci_670 & 419 G/T \\
\hline UN_ci_670 & $442 \mathrm{C} / \mathrm{A}$ \\
\hline UN_ci_670 & $545 \mathrm{~A} / \mathrm{G}$ \\
\hline UN_ci_670 & $609 \mathrm{~A} / \mathrm{G}$ \\
\hline UN_ci_670 & $686 \mathrm{~A} / \mathrm{G}$ \\
\hline UN_ci_670 & 708 G/A \\
\hline UN_ci_670 & 735 G/C \\
\hline UN_ci_670 & $762 \mathrm{~T} / \mathrm{C}$ \\
\hline UN_ci_670 & $783 \mathrm{G} / \mathrm{A}$ \\
\hline UN_ci_670 & $807 \mathrm{C} / \mathrm{T}$ \\
\hline UN_ci_670 & $852 \mathrm{C} / \mathrm{T}$ \\
\hline UN_ci_670 & $879 \mathrm{~T} / \mathrm{G}$ \\
\hline UN_ci_670 & $903 \mathrm{~A} / \mathrm{G}$ \\
\hline UN_ci_670 & $969 \mathrm{G} / \mathrm{C}$ \\
\hline UN_ci_670 & 1011 G/A \\
\hline UN_ci_670 & $1131 \mathrm{G} / \mathrm{A}$ \\
\hline UN_ci_673 & $470 \mathrm{~T} / \mathrm{G}$ \\
\hline UN_ci_675 & $94 \mathrm{G} / \mathrm{T}$ \\
\hline UN_ci_675 & $274 \mathrm{~T} / \mathrm{C}$ \\
\hline UN_ci_675 & $642 \mathrm{~T} / \mathrm{C}$ \\
\hline UN_ci_679 & $774 \mathrm{~A} / \mathrm{G}$ \\
\hline UN_ci_680 & $47 \mathrm{G} / \mathrm{A}$ \\
\hline
\end{tabular}




\begin{tabular}{|c|c|}
\hline UN_ci_680 & $932 \mathrm{G} / \mathrm{A}$ \\
\hline UN_ci_680 & $1154 \mathrm{C} / \mathrm{T}$ \\
\hline UN_ci_681 & $177 \mathrm{~T} / \mathrm{C}$ \\
\hline UN_ci_682 & $276 \mathrm{~T} / \mathrm{G}$ \\
\hline UN_ci_683 & $443 \mathrm{~T} / \mathrm{C}$ \\
\hline UN_ci_685 & $490 \mathrm{C} / \mathrm{T}$ \\
\hline UN_ci_697 & $215 \mathrm{G} / \mathrm{A}$ \\
\hline UN_ci_697 & $255 \mathrm{~T} / \mathrm{C}$ \\
\hline UN_ci_697 & $421 \mathrm{C} / \mathrm{T}$ \\
\hline UN_ci_697 & $509 \mathrm{C} / \mathrm{T}$ \\
\hline UN_ci_697 & $529 \mathrm{~T} / \mathrm{C}$ \\
\hline UN_ci_697 & $565 \mathrm{~A} / \mathrm{T}$ \\
\hline UN_ci_697 & $595 \mathrm{C} / \mathrm{T}$ \\
\hline UN_ci_697 & $616 \mathrm{G} / \mathrm{A}$ \\
\hline UN_ci_697 & $667 \mathrm{~A} / \mathrm{G}$ \\
\hline UN_ci_697 & $690 \mathrm{~A} / \mathrm{G}$ \\
\hline UN_ci_699 & $228 \mathrm{C} / \mathrm{G}$ \\
\hline UN_ci_716 & 137 T/C \\
\hline UN_ci_716 & $551 \mathrm{~T} / \mathrm{C}$ \\
\hline UN_ci_716 & $843 \mathrm{C} / \mathrm{T}$ \\
\hline UN_ci_727 & $720 \mathrm{G} / \mathrm{A}$ \\
\hline UN_ci_727 & $742 \mathrm{C} / \mathrm{T}$ \\
\hline UN_ci_727 & $813 \mathrm{~T} / \mathrm{C}$ \\
\hline UN_ci_727 & $1016 \mathrm{C} / \mathrm{T}$ \\
\hline UN_ci_739 & $53 \mathrm{G} / \mathrm{C}$ \\
\hline UN_ci_739 & $93 \mathrm{~A} / \mathrm{C}$ \\
\hline UN_ci_739 & $123 \mathrm{~A} / \mathrm{G}$ \\
\hline UN_ci_739 & $164 \mathrm{~A} / \mathrm{G}$ \\
\hline UN_ci_739 & $223 \mathrm{~A} / \mathrm{G}$ \\
\hline UN_ci_739 & $267 \mathrm{~A} / \mathrm{T}$ \\
\hline UN_ci_739 & $314 \mathrm{~A} / \mathrm{C}$ \\
\hline UN_ci_739 & $358 \mathrm{~T} / \mathrm{A}$ \\
\hline UN_ci_739 & $384 \mathrm{~A} / \mathrm{C}$ \\
\hline UN_ci_745 & $15 \mathrm{~T} / \mathrm{C}$ \\
\hline UN_ci_749 & $310 \mathrm{G} / \mathrm{A}$ \\
\hline UN_ci_752 & $234 \mathrm{~A} / \mathrm{G}$ \\
\hline UN_ci_756 & 79 A/G \\
\hline UN_ci_756 & 397 A/T \\
\hline UN_ci_756 & $482 \mathrm{C} / \mathrm{A}$ \\
\hline UN_ci_765 & $125 \mathrm{~T} / \mathrm{C}$ \\
\hline UN_ci_769 & $227 \mathrm{~T} / \mathrm{C}$ \\
\hline UN_ci_769 & $282 \mathrm{G} / \mathrm{C}$ \\
\hline UN_ci_772 & 7 T/G \\
\hline UN_ci_772 & $239 \mathrm{C} / \mathrm{A}$ \\
\hline UN_ci_780 & $569 \mathrm{C} / \mathrm{T}$ \\
\hline UN_ci_784 & $71 \mathrm{G} / \mathrm{C}$ \\
\hline UN_ci_784 & $104 \mathrm{~A} / \mathrm{G}$ \\
\hline UN_ci_784 & $154 \mathrm{C} / \mathrm{G}$ \\
\hline UN_ci_784 & $288 \mathrm{C} / \mathrm{T}$ \\
\hline UN_ci_784 & $426 \mathrm{~A} / \mathrm{G}$ \\
\hline
\end{tabular}




\begin{tabular}{|c|c|}
\hline UN_ci_785 & $103 \mathrm{~A} / \mathrm{G}$ \\
\hline UN_ci_796 & $263 \mathrm{~T} / \mathrm{C}$ \\
\hline UN_ci_801 & $1214 \mathrm{~A} / \mathrm{C}$ \\
\hline UN_ci_818 & $58 \mathrm{~T} / \mathrm{A}$ \\
\hline UN_ci_818 & $130 \mathrm{C} / \mathrm{T}$ \\
\hline UN_ci_818 & $159 \mathrm{~A} / \mathrm{G}$ \\
\hline UN_ci_818 & $181 \mathrm{~A} / \mathrm{T}$ \\
\hline UN_ci_818 & $205 \mathrm{~T} / \mathrm{C}$ \\
\hline UN_ci_818 & $262 \mathrm{~A} / \mathrm{G}$ \\
\hline UN_ci_818 & $390 \mathrm{~A} / \mathrm{G}$ \\
\hline UN_ci_832 & $326 \mathrm{~T} / \mathrm{C}$ \\
\hline UN_ci_840 & $106 \mathrm{C} / \mathrm{T}$ \\
\hline UN_ci_842 & $57 \mathrm{~A} / \mathrm{T}$ \\
\hline UN_ci_843 & $602 \mathrm{C} / \mathrm{A}$ \\
\hline UN_ci_843 & $2745 \mathrm{~A} / \mathrm{G}$ \\
\hline UN_ci_843 & $2813 \mathrm{~A} / \mathrm{G}$ \\
\hline UN_ci_844 & 96 A/G \\
\hline UN_ci_844 & $219 \mathrm{G} / \mathrm{T}$ \\
\hline UN_ci_846 & $103 \mathrm{~A} / \mathrm{G}$ \\
\hline UN_ci_846 & $318 \mathrm{~T} / \mathrm{C}$ \\
\hline UN_ci_853 & $80 \mathrm{~T} / \mathrm{C}$ \\
\hline UN_ci_855 & $330 \mathrm{~T} / \mathrm{C}$ \\
\hline UN_ci_863 & $23 \mathrm{G} / \mathrm{T}$ \\
\hline UN_ci_863 & $60 \mathrm{~A} / \mathrm{G}$ \\
\hline UN_ci_863 & $266 \mathrm{~T} / \mathrm{C}$ \\
\hline UN_ci_863 & $366 \mathrm{~A} / \mathrm{T}$ \\
\hline UN_ci_863 & $569 \mathrm{~A} / \mathrm{C}$ \\
\hline UN_ci_867 & $125 \mathrm{~A} / \mathrm{C}$ \\
\hline UN_ci_867 & $209 \mathrm{~T} / \mathrm{C}$ \\
\hline UN_ci_867 & $599 \mathrm{G} / \mathrm{C}$ \\
\hline UN_ci_877 & $176 \mathrm{~A} / \mathrm{G}$ \\
\hline UN_ci_879 & $215 \mathrm{G} / \mathrm{A}$ \\
\hline UN_ci_881 & $289 \mathrm{C} / \mathrm{T}$ \\
\hline UN_ci_889 & $51 \mathrm{C} / \mathrm{T}$ \\
\hline UN_ci_896 & $132 \mathrm{~T} / \mathrm{G}$ \\
\hline UN_ci_901 & $67 \mathrm{~T} / \mathrm{A}$ \\
\hline UN_ci_903 & $392 \mathrm{~A} / \mathrm{C}$ \\
\hline UN_ci_906 & $79 \mathrm{~T} / \mathrm{C}$ \\
\hline UN_ci_906 & $119 \mathrm{~A} / \mathrm{G}$ \\
\hline UN_ci_906 & $222 \mathrm{~T} / \mathrm{C}$ \\
\hline UN_ci_906 & $581 \mathrm{~T} / \mathrm{C}$ \\
\hline UN_ci_908 & $661 \mathrm{C} / \mathrm{T}$ \\
\hline UN_ci_916 & $126 \mathrm{~T} / \mathrm{A}$ \\
\hline UN_ci_918 & $275 \mathrm{~T} / \mathrm{G}$ \\
\hline UN_ci_918 & $322 \mathrm{~T} / \mathrm{A}$ \\
\hline UN_ci_925 & $369 \mathrm{C} / \mathrm{T}$ \\
\hline UN_ci_925 & $412 \mathrm{~T} / \mathrm{C}$ \\
\hline UN_ci_925 & $439 \mathrm{G} / \mathrm{A}$ \\
\hline UN_ci_925 & $484 \mathrm{~A} / \mathrm{G}$ \\
\hline UN_ci_925 & $507 \mathrm{~T} / \mathrm{G}$ \\
\hline
\end{tabular}




\begin{tabular}{|c|c|}
\hline UN_ci_925 & $551 \mathrm{~A} / \mathrm{C}$ \\
\hline UN_ci_925 & $587 \mathrm{~A} / \mathrm{G}$ \\
\hline UN_ci_925 & 609 A/G \\
\hline UN_ci_925 & $636 \mathrm{C} / \mathrm{T}$ \\
\hline UN_ci_925 & 692 A/G \\
\hline UN_ci_925 & $733 \mathrm{C} / \mathrm{T}$ \\
\hline UN_ci_925 & 757 T/C \\
\hline UN_ci_925 & 832 T/C \\
\hline UN_ci_925 & $921 \mathrm{~T} / \mathrm{C}$ \\
\hline UN_ci_925 & 949 T/G \\
\hline UN_ci_925 & $969 \mathrm{G} / \mathrm{A}$ \\
\hline UN_ci_925 & 996 T/C \\
\hline UN_ci_925 & $1025 \mathrm{~T} / \mathrm{C}$ \\
\hline UN_ci_925 & $1065 \mathrm{~T} / \mathrm{C}$ \\
\hline UN_ci_942 & $158 \mathrm{C} / \mathrm{T}$ \\
\hline UN_ci_942 & 197 T/G \\
\hline UN_ci_942 & $227 \mathrm{~A} / \mathrm{G}$ \\
\hline UN_ci_942 & $270 \mathrm{C} / \mathrm{A}$ \\
\hline UN_ci_945 & $45 \mathrm{G} / \mathrm{A}$ \\
\hline UN_ci_945 & $165 \mathrm{G} / \mathrm{T}$ \\
\hline UN_ci_993 & $187 \mathrm{C} / \mathrm{A}$ \\
\hline UN_ci_1005 & $110 \mathrm{~A} / \mathrm{G}$ \\
\hline UN_ci_1005 & 199 T/G \\
\hline UN_ci_1005 & $265 \mathrm{~A} / \mathrm{C}$ \\
\hline UN_ci_1005 & $286 \mathrm{~A} / \mathrm{G}$ \\
\hline UN_ci_1007 & $578 \mathrm{~A} / \mathrm{G}$ \\
\hline UN_ci_1011 & $45 \mathrm{G} / \mathrm{T}$ \\
\hline UN_ci_1030 & $131 \mathrm{~A} / \mathrm{G}$ \\
\hline UN_ci_1038 & $987 \mathrm{C} / \mathrm{T}$ \\
\hline UN_ci_1040 & $11 \mathrm{C} / \mathrm{G}$ \\
\hline UN_ci_1040 & $39 \mathrm{C} / \mathrm{T}$ \\
\hline UN_ci_1046 & $191 \mathrm{~T} / \mathrm{C}$ \\
\hline UN_ci_1046 & 379 A/G \\
\hline UN_ci_1047 & $94 \mathrm{G} / \mathrm{A}$ \\
\hline UN_ci_1047 & $258 \mathrm{~A} / \mathrm{G}$ \\
\hline UN_ci_1054 & $88 \mathrm{~A} / \mathrm{T}$ \\
\hline UN_ci_1054 & $112 \mathrm{C} / \mathrm{T}$ \\
\hline UN_ci_1054 & $493 \mathrm{~T} / \mathrm{G}$ \\
\hline UN_ci_1054 & $835 \mathrm{~T} / \mathrm{C}$ \\
\hline UN_ci_1054 & $1090 \mathrm{G} / \mathrm{A}$ \\
\hline UN_ci_1063 & $317 \mathrm{C} / \mathrm{T}$ \\
\hline UN_ci_1066 & $73 \mathrm{G} / \mathrm{A}$ \\
\hline UN_ci_1067 & $397 \mathrm{~T} / \mathrm{C}$ \\
\hline UN_ci_1067 & $490 \mathrm{G} / \mathrm{A}$ \\
\hline UN_ci_1075 & 309 G/A \\
\hline UN_ci_1075 & 366 A/G \\
\hline UN_ci_1081 & $125 \mathrm{C} / \mathrm{T}$ \\
\hline UN_ci_1088 & $33 \mathrm{~T} / \mathrm{C}$ \\
\hline UN_ci_1090 & $82 \mathrm{G} / \mathrm{A}$ \\
\hline UN_ci_1090 & $430 \mathrm{~A} / \mathrm{G}$ \\
\hline
\end{tabular}




\begin{tabular}{|c|c|}
\hline UN_ci_1093 & $112 \mathrm{C} / \mathrm{T}$ \\
\hline UN_ci_1095 & $207 \mathrm{~T} / \mathrm{A}$ \\
\hline UN_ci_1098 & $140 \mathrm{C} / \mathrm{T}$ \\
\hline UN_ci_1109 & $187 \mathrm{G} / \mathrm{T}$ \\
\hline UN_ci_1109 & $231 \mathrm{G} / \mathrm{T}$ \\
\hline UN_ci_1109 & $263 \mathrm{G} / \mathrm{A}$ \\
\hline UN_ci_1113 & $432 \mathrm{C} / \mathrm{T}$ \\
\hline UN_ci_1114 & $64 \mathrm{C} / \mathrm{T}$ \\
\hline UN_ci_1115 & $29 \mathrm{G} / \mathrm{T}$ \\
\hline UN_ci_1115 & $223 \mathrm{~T} / \mathrm{C}$ \\
\hline UN_ci_1115 & $428 \mathrm{~A} / \mathrm{C}$ \\
\hline UN_ci_1115 & $511 \mathrm{~T} / \mathrm{C}$ \\
\hline UN_ci_1115 & $551 \mathrm{~A} / \mathrm{G}$ \\
\hline UN_ci_1117 & $176 \mathrm{~A} / \mathrm{G}$ \\
\hline UN_ci_1117 & $446 \mathrm{G} / \mathrm{A}$ \\
\hline UN_ci_1120 & $222 \mathrm{~A} / \mathrm{G}$ \\
\hline UN_ci_1122 & $643 \mathrm{C} / \mathrm{T}$ \\
\hline UN_ci_1130 & $281 \mathrm{C} / \mathrm{T}$ \\
\hline UN_ci_1134 & $110 \mathrm{C} / \mathrm{A}$ \\
\hline UN_ci_1134 & $267 \mathrm{C} / \mathrm{T}$ \\
\hline UN_ci_1140 & $112 \mathrm{C} / \mathrm{A}$ \\
\hline UN_ci_1148 & $214 \mathrm{C} / \mathrm{T}$ \\
\hline UN_ci_1148 & $310 \mathrm{~A} / \mathrm{T}$ \\
\hline UN_ci_1151 & $251 \mathrm{G} / \mathrm{A}$ \\
\hline UN_ci_1151 & $508 \mathrm{~A} / \mathrm{G}$ \\
\hline UN_ci_1151 & $675 \mathrm{G} / \mathrm{A}$ \\
\hline UN_ci_1152 & $251 \mathrm{G} / \mathrm{T}$ \\
\hline UN_ci_1165 & $372 \mathrm{~A} / \mathrm{G}$ \\
\hline UN_ci_1165 & $421 \mathrm{~A} / \mathrm{G}$ \\
\hline UN_ci_1165 & $636 \mathrm{C} / \mathrm{T}$ \\
\hline UN_ci_1165 & $671 \mathrm{~T} / \mathrm{A}$ \\
\hline UN_ci_1165 & $755 \mathrm{~A} / \mathrm{G}$ \\
\hline UN_ci_1165 & $832 \mathrm{C} / \mathrm{T}$ \\
\hline UN_ci_1168 & $266 \mathrm{~A} / \mathrm{T}$ \\
\hline UN_ci_1172 & $430 \mathrm{~A} / \mathrm{G}$ \\
\hline UN_ci_1172 & $467 \mathrm{~T} / \mathrm{A}$ \\
\hline UN_ci_1172 & $577 \mathrm{G} / \mathrm{A}$ \\
\hline UN_ci_1172 & $667 \mathrm{~A} / \mathrm{G}$ \\
\hline UN_ci_1172 & $715 \mathrm{~A} / \mathrm{T}$ \\
\hline UN_ci_1172 & $744 \mathrm{G} / \mathrm{A}$ \\
\hline UN_ci_1172 & $783 \mathrm{C} / \mathrm{T}$ \\
\hline UN_ci_1176 & $55 \mathrm{~T} / \mathrm{C}$ \\
\hline UN_ci_1176 & $77 \mathrm{~T} / \mathrm{C}$ \\
\hline UN_ci_1176 & $622 \mathrm{~A} / \mathrm{C}$ \\
\hline UN_ci_1180 & $228 \mathrm{~A} / \mathrm{G}$ \\
\hline UN_ci_1180 & 393 T/C \\
\hline UN_ci_1180 & $426 \mathrm{~A} / \mathrm{T}$ \\
\hline UN_ci_1191 & $378 \mathrm{G} / \mathrm{A}$ \\
\hline UN_ci_1191 & $401 \mathrm{~T} / \mathrm{C}$ \\
\hline UN_ci_1191 & $427 \mathrm{~T} / \mathrm{C}$ \\
\hline
\end{tabular}




\begin{tabular}{|c|c|}
\hline UN_ci_1191 & $460 \mathrm{G} / \mathrm{A}$ \\
\hline UN_ci_1191 & $514 \mathrm{~A} / \mathrm{G}$ \\
\hline UN_ci_1191 & $548 \mathrm{~A} / \mathrm{G}$ \\
\hline UN_ci_1194 & $123 \mathrm{G} / \mathrm{A}$ \\
\hline UN_ci_1194 & $282 \mathrm{~A} / \mathrm{G}$ \\
\hline UN_ci_1194 & $372 \mathrm{~A} / \mathrm{G}$ \\
\hline UN_ci_1199 & $234 \mathrm{C} / \mathrm{T}$ \\
\hline UN_ci_1201 & $343 \mathrm{~A} / \mathrm{G}$ \\
\hline UN_ci_1201 & $1682 \mathrm{C} / \mathrm{G}$ \\
\hline UN_ci_1201 & 1727 T/C \\
\hline UN_ci_1201 & $1769 \mathrm{~T} / \mathrm{C}$ \\
\hline UN_ci_1201 & $1805 \mathrm{~T} / \mathrm{C}$ \\
\hline UN_ci_1201 & $1908 \mathrm{C} / \mathrm{T}$ \\
\hline UN_ci_1210 & $130 \mathrm{~T} / \mathrm{G}$ \\
\hline UN_ci_1210 & 259 T/C \\
\hline UN_ci_1210 & $279 \mathrm{G} / \mathrm{T}$ \\
\hline UN_ci_1212 & $19 \mathrm{G} / \mathrm{T}$ \\
\hline UN_ci_1212 & $186 \mathrm{C} / \mathrm{A}$ \\
\hline UN_ci_1212 & $215 \mathrm{~A} / \mathrm{G}$ \\
\hline UN_ci_1212 & $293 \mathrm{G} / \mathrm{A}$ \\
\hline UN_ci_1212 & $427 \mathrm{~T} / \mathrm{C}$ \\
\hline UN_ci_1212 & $499 \mathrm{C} / \mathrm{T}$ \\
\hline UN_ci_1212 & 859 T/C \\
\hline UN_ci_1212 & $959 \mathrm{G} / \mathrm{T}$ \\
\hline UN_ci_1212 & 1039 T/A \\
\hline UN_ci_1212 & $1059 \mathrm{G} / \mathrm{T}$ \\
\hline UN_ci_1212 & $1133 \mathrm{~T} / \mathrm{C}$ \\
\hline UN_ci_1225 & $177 \mathrm{~A} / \mathrm{T}$ \\
\hline UN_ci_1225 & $224 \mathrm{C} / \mathrm{G}$ \\
\hline UN_ci_1231 & $582 \mathrm{G} / \mathrm{A}$ \\
\hline UN_ci_1236 & $179 \mathrm{G} / \mathrm{A}$ \\
\hline UN_ci_1237 & $12 \mathrm{G} / \mathrm{T}$ \\
\hline UN_ci_1239 & $150 \mathrm{G} / \mathrm{A}$ \\
\hline UN_ci_1239 & $266 \mathrm{C} / \mathrm{T}$ \\
\hline UN_ci_1239 & 386 G/C \\
\hline UN_ci_1240 & $207 \mathrm{~A} / \mathrm{G}$ \\
\hline UN_ci_1250 & $255 \mathrm{~T} / \mathrm{C}$ \\
\hline UN_ci_1251 & $360 \mathrm{~T} / \mathrm{C}$ \\
\hline UN_ci_1255 & $110 \mathrm{C} / \mathrm{T}$ \\
\hline UN_ci_1257 & $549 \mathrm{C} / \mathrm{A}$ \\
\hline UN_ci_1266 & $603 \mathrm{G} / \mathrm{A}$ \\
\hline UN_ci_1273 & $598 \mathrm{~T} / \mathrm{C}$ \\
\hline UN_ci_1273 & $646 \mathrm{~T} / \mathrm{G}$ \\
\hline UN_ci_1277 & $259 \mathrm{~A} / \mathrm{G}$ \\
\hline UN_ci_1277 & $459 \mathrm{G} / \mathrm{C}$ \\
\hline UN_ci_1277 & $714 \mathrm{~A} / \mathrm{G}$ \\
\hline UN_ci_1277 & $844 \mathrm{G} / \mathrm{C}$ \\
\hline UN_ci_1277 & 1083 T/C \\
\hline UN_ci_1277 & $1105 \mathrm{~T} / \mathrm{C}$ \\
\hline UN_ci_1278 & $184 \mathrm{~A} / \mathrm{C}$ \\
\hline
\end{tabular}




\begin{tabular}{|c|c|}
\hline UN_ci_1278 & $411 \mathrm{C} / \mathrm{A}$ \\
\hline UN_ci_1283 & 69 A/G \\
\hline UN_ci_1283 & $277 \mathrm{~T} / \mathrm{G}$ \\
\hline UN_ci_1283 & $323 \mathrm{~A} / \mathrm{G}$ \\
\hline UN_ci_1283 & $390 \mathrm{~T} / \mathrm{C}$ \\
\hline UN_ci_1286 & $102 \mathrm{G} / \mathrm{A}$ \\
\hline UN_ci_1287 & $620 \mathrm{G} / \mathrm{A}$ \\
\hline UN_ci_1287 & $712 \mathrm{C} / \mathrm{T}$ \\
\hline UN_ci_1295 & $168 \mathrm{~T} / \mathrm{A}$ \\
\hline UN_ci_1310 & $132 \mathrm{~T} / \mathrm{C}$ \\
\hline UN_ci_1310 & $186 \mathrm{~A} / \mathrm{T}$ \\
\hline UN_ci_1310 & $234 \mathrm{G} / \mathrm{A}$ \\
\hline UN_ci_1310 & $312 \mathrm{G} / \mathrm{A}$ \\
\hline UN_ci_1310 & $352 \mathrm{G} / \mathrm{A}$ \\
\hline UN_ci_1310 & $409 \mathrm{C} / \mathrm{A}$ \\
\hline UN_ci_1310 & $432 \mathrm{~A} / \mathrm{G}$ \\
\hline UN_ci_1310 & $470 \mathrm{~A} / \mathrm{G}$ \\
\hline UN_ci_1310 & $549 \mathrm{~A} / \mathrm{G}$ \\
\hline UN_ci_1310 & $583 \mathrm{G} / \mathrm{T}$ \\
\hline UN_ci_1315 & $284 \mathrm{~A} / \mathrm{G}$ \\
\hline UN_ci_1315 & $722 \mathrm{~A} / \mathrm{G}$ \\
\hline UN_ci_1315 & $929 \mathrm{C} / \mathrm{T}$ \\
\hline UN_ci_1316 & $291 \mathrm{C} / \mathrm{A}$ \\
\hline UN_ci_1330 & $241 \mathrm{C} / \mathrm{T}$ \\
\hline UN_ci_1330 & $361 \mathrm{~A} / \mathrm{C}$ \\
\hline UN_ci_1330 & $398 \mathrm{~T} / \mathrm{C}$ \\
\hline UN_ci_1330 & $664 \mathrm{G} / \mathrm{T}$ \\
\hline UN_ci_1337 & $221 \mathrm{~A} / \mathrm{G}$ \\
\hline UN_ci_1340 & $320 \mathrm{~A} / \mathrm{G}$ \\
\hline UN_ci_1340 & $577 \mathrm{G} / \mathrm{A}$ \\
\hline UN_ci_1340 & $679 \mathrm{~A} / \mathrm{C}$ \\
\hline UN_ci_1340 & $1100 \mathrm{C} / \mathrm{T}$ \\
\hline UN_ci_1340 & $1168 \mathrm{G} / \mathrm{A}$ \\
\hline UN_ci_1340 & $1453 \mathrm{~T} / \mathrm{A}$ \\
\hline UN_ci_1340 & $1552 \mathrm{~T} / \mathrm{G}$ \\
\hline UN_ci_1342 & $281 \mathrm{~A} / \mathrm{G}$ \\
\hline UN_ci_1342 & $332 \mathrm{~T} / \mathrm{C}$ \\
\hline UN_ci_1342 & $403 \mathrm{~T} / \mathrm{G}$ \\
\hline UN_ci_1342 & $473 \mathrm{G} / \mathrm{T}$ \\
\hline UN_ci_1342 & $570 \mathrm{~A} / \mathrm{G}$ \\
\hline UN_ci_1342 & $694 \mathrm{~A} / \mathrm{G}$ \\
\hline UN_ci_1342 & 919 A/G \\
\hline UN_ci_1342 & $1640 \mathrm{C} / \mathrm{T}$ \\
\hline UN_ci_1342 & $1754 \mathrm{~T} / \mathrm{C}$ \\
\hline UN_ci_1348 & $248 \mathrm{C} / \mathrm{G}$ \\
\hline UN_ci_1348 & $814 \mathrm{G} / \mathrm{A}$ \\
\hline UN_ci_1352 & $225 \mathrm{~T} / \mathrm{C}$ \\
\hline UN_ci_1360 & $234 \mathrm{~A} / \mathrm{G}$ \\
\hline UN_ci_1360 & $284 \mathrm{~A} / \mathrm{G}$ \\
\hline UN_ci_1366 & $324 \mathrm{~A} / \mathrm{G}$ \\
\hline
\end{tabular}




\begin{tabular}{|c|c|}
\hline UN_ci_1366 & $418 \mathrm{~A} / \mathrm{G}$ \\
\hline UN_ci_1366 & $667 \mathrm{G} / \mathrm{A}$ \\
\hline UN_ci_1366 & $714 \mathrm{~T} / \mathrm{A}$ \\
\hline UN_ci_1366 & $1034 \mathrm{~T} / \mathrm{C}$ \\
\hline UN_ci_1369 & $606 \mathrm{~A} / \mathrm{T}$ \\
\hline UN_ci_1372 & $595 \mathrm{~A} / \mathrm{T}$ \\
\hline UN_ci_1372 & $618 \mathrm{~T} / \mathrm{C}$ \\
\hline UN_ci_1378 & $401 \mathrm{G} / \mathrm{C}$ \\
\hline UN_ci_1380 & $16 \mathrm{C} / \mathrm{T}$ \\
\hline UN_ci_1380 & $104 \mathrm{~T} / \mathrm{C}$ \\
\hline UN_ci_1380 & $136 \mathrm{G} / \mathrm{A}$ \\
\hline UN_ci_1380 & 189 A/G \\
\hline UN_ci_1383 & $138 \mathrm{~A} / \mathrm{C}$ \\
\hline UN_ci_1384 & $397 \mathrm{C} / \mathrm{T}$ \\
\hline UN_ci_1397 & $429 \mathrm{C} / \mathrm{T}$ \\
\hline UN_ci_1407 & $85 \mathrm{G} / \mathrm{T}$ \\
\hline UN_ci_1407 & $112 \mathrm{G} / \mathrm{A}$ \\
\hline UN_ci_1407 & $265 \mathrm{~T} / \mathrm{C}$ \\
\hline UN_ci_1407 & $446 \mathrm{~T} / \mathrm{C}$ \\
\hline UN_ci_1407 & $1183 \mathrm{~A} / \mathrm{T}$ \\
\hline UN_ci_1407 & $1215 \mathrm{G} / \mathrm{A}$ \\
\hline UN_ci_1407 & $1466 \mathrm{C} / \mathrm{A}$ \\
\hline UN_ci_1407 & $1513 \mathrm{~A} / \mathrm{C}$ \\
\hline UN_ci_1407 & 1788 A/G \\
\hline UN_ci_1407 & $1809 \mathrm{G} / \mathrm{A}$ \\
\hline UN_ci_1407 & $1913 \mathrm{~A} / \mathrm{G}$ \\
\hline UN_ci_1407 & 2028 G/A \\
\hline UN_ci_1407 & $2170 \mathrm{~A} / \mathrm{G}$ \\
\hline UN_ci_1407 & $2322 \mathrm{C} / \mathrm{T}$ \\
\hline UN_ci_1407 & $2575 \mathrm{~T} / \mathrm{C}$ \\
\hline UN_ci_1409 & $100 \mathrm{~A} / \mathrm{G}$ \\
\hline UN_ci_1409 & $155 \mathrm{~T} / \mathrm{G}$ \\
\hline UN_ci_1418 & $466 \mathrm{~A} / \mathrm{G}$ \\
\hline UN_ci_1418 & $637 \mathrm{~A} / \mathrm{G}$ \\
\hline UN_ci_1418 & $1000 \mathrm{C} / \mathrm{T}$ \\
\hline UN_ci_1418 & $1054 \mathrm{~A} / \mathrm{G}$ \\
\hline UN_ci_1418 & 1495 T/G \\
\hline UN_ci_1419 & $32 \mathrm{C} / \mathrm{T}$ \\
\hline UN_ci_1419 & $157 \mathrm{C} / \mathrm{T}$ \\
\hline UN_ci_1419 & $234 \mathrm{C} / \mathrm{T}$ \\
\hline UN_ci_1419 & $292 \mathrm{~A} / \mathrm{G}$ \\
\hline UN_ci_1419 & $582 \mathrm{C} / \mathrm{T}$ \\
\hline UN_ci_1425 & $263 \mathrm{C} / \mathrm{T}$ \\
\hline UN_ci_1428 & $414 \mathrm{G} / \mathrm{A}$ \\
\hline UN_ci_1435 & $402 \mathrm{G} / \mathrm{A}$ \\
\hline UN_ci_1436 & $49 \mathrm{G} / \mathrm{T}$ \\
\hline UN_ci_1436 & $142 \mathrm{~A} / \mathrm{G}$ \\
\hline UN_ci_1436 & $166 \mathrm{G} / \mathrm{A}$ \\
\hline UN_ci_1436 & $252 \mathrm{C} / \mathrm{G}$ \\
\hline UN_ci_1436 & $280 \mathrm{~A} / \mathrm{G}$ \\
\hline
\end{tabular}




$\begin{array}{lr}\text { UN_ci_1436 } & 354 \mathrm{~T} / \mathrm{C} \\ \text { UN_ci_1436 } & 453 \mathrm{~A} / \mathrm{T} \\ \text { UN_ci_1436 } & 578 \mathrm{~A} / \mathrm{G} \\ \text { UN_ci_1436 } & 661 \mathrm{~T} / \mathrm{C} \\ \text { UN_ci_1437 } & 347 \mathrm{C} / \mathrm{T} \\ \text { UN_ci_1437 } & 395 \mathrm{G} / \mathrm{T} \\ \text { UN_ci_1440 } & 196 \mathrm{G} / \mathrm{A} \\ \text { UN_ci_1441 } & 1114 \mathrm{~T} / \mathrm{C} \\ \text { UN_ci_1441 } & 1195 \mathrm{G} / \mathrm{T} \\ \text { UN_ci_1441 } & 1346 \mathrm{C} / \mathrm{T} \\ \text { UN_ci_1455 } & 189 \mathrm{C} / \mathrm{A} \\ \text { UN_ci_1461 } & 155 \mathrm{~T} / \mathrm{A} \\ \text { UN_ci_1475 } & 193 \mathrm{C} / \mathrm{T} \\ \text { UN_ci_1475 } & 448 \mathrm{G} / \mathrm{T} \\ \text { UN_ci_1480 } & 74 \mathrm{~A} / \mathrm{C} \\ \text { UN_ci_1480 } & 170 \mathrm{~A} / \mathrm{G} \\ \text { UN_ci_1481 } & 55 \mathrm{~A} / \mathrm{T} \\ \text { UN_ci_1498 } & 170 \mathrm{~T} / \mathrm{C} \\ \text { UN_ci_1501 } & 65 \mathrm{G} / \mathrm{A} \\ \text { UN_ci_1506 } & 148 \mathrm{G} / \mathrm{A} \\ \text { UN_ci_1506 } & 987 \mathrm{~A} / \mathrm{G} \\ \text { UN_ci_1507 } & 915 \mathrm{~T} / \mathrm{C} \\ \text { UN_ci_1507 } & \end{array}$




\begin{tabular}{|c|c|}
\hline UN_ci_1523 & 1037 A/G \\
\hline UN_ci_1523 & $1068 \mathrm{~A} / \mathrm{T}$ \\
\hline UN_ci_1523 & $1092 \mathrm{~T} / \mathrm{C}$ \\
\hline UN_ci_1523 & $1147 \mathrm{C} / \mathrm{T}$ \\
\hline UN_ci_1523 & 1189 T/A \\
\hline UN_ci_1526 & $185 \mathrm{C} / \mathrm{T}$ \\
\hline UN_ci_1531 & $920 \mathrm{C} / \mathrm{A}$ \\
\hline UN_ci_1531 & 962 A/G \\
\hline UN_ci_1531 & 1109 A/G \\
\hline UN_ci_1531 & $1130 \mathrm{C} / \mathrm{T}$ \\
\hline UN_ci_1531 & 1186 G/C \\
\hline UN_ci_1531 & 1206 A/G \\
\hline UN_ci_1531 & $1253 \mathrm{C} / \mathrm{T}$ \\
\hline UN_ci_1531 & $1452 \mathrm{G} / \mathrm{C}$ \\
\hline UN_ci_1532 & $52 \mathrm{C} / \mathrm{T}$ \\
\hline UN_ci_1532 & $257 \mathrm{~T} / \mathrm{G}$ \\
\hline UN_ci_1533 & $777 \mathrm{C} / \mathrm{T}$ \\
\hline UN_ci_1547 & 219 A/G \\
\hline UN_ci_1547 & $361 \mathrm{~A} / \mathrm{C}$ \\
\hline UN_ci_1558 & $180 \mathrm{~T} / \mathrm{A}$ \\
\hline UN_ci_1564 & $249 \mathrm{~A} / \mathrm{T}$ \\
\hline UN_ci_1567 & $44 \mathrm{C} / \mathrm{A}$ \\
\hline UN_ci_1576 & $52 \mathrm{~T} / \mathrm{A}$ \\
\hline UN_ci_1576 & $170 \mathrm{~T} / \mathrm{C}$ \\
\hline UN_ci_1576 & 203 T/C \\
\hline UN_ci_1576 & $294 \mathrm{~A} / \mathrm{C}$ \\
\hline UN_ci_1580 & $22 \mathrm{G} / \mathrm{A}$ \\
\hline UN_ci_1580 & $88 \mathrm{~T} / \mathrm{C}$ \\
\hline UN_ci_1580 & $238 \mathrm{G} / \mathrm{C}$ \\
\hline UN_ci_1580 & $603 \mathrm{~T} / \mathrm{C}$ \\
\hline UN_ci_1580 & $873 \mathrm{~T} / \mathrm{C}$ \\
\hline UN_ci_1580 & $949 \mathrm{G} / \mathrm{T}$ \\
\hline UN_ci_1580 & $1104 \mathrm{C} / \mathrm{T}$ \\
\hline UN_ci_1580 & $1135 \mathrm{~T} / \mathrm{C}$ \\
\hline UN_ci_1582 & $154 \mathrm{~T} / \mathrm{C}$ \\
\hline UN_ci_1589 & $283 \mathrm{~T} / \mathrm{G}$ \\
\hline UN_ci_1590 & $702 \mathrm{~A} / \mathrm{G}$ \\
\hline UN_ci_1590 & $745 \mathrm{C} / \mathrm{T}$ \\
\hline UN_ci_1594 & $76 \mathrm{C} / \mathrm{T}$ \\
\hline UN_ci_1594 & $155 \mathrm{G} / \mathrm{T}$ \\
\hline UN_ci_1594 & $203 \mathrm{G} / \mathrm{A}$ \\
\hline UN_ci_1594 & $763 \mathrm{G} / \mathrm{C}$ \\
\hline UN_ci_1594 & $981 \mathrm{C} / \mathrm{T}$ \\
\hline UN_ci_1594 & $1546 \mathrm{G} / \mathrm{C}$ \\
\hline UN_ci_1599 & $106 \mathrm{~T} / \mathrm{C}$ \\
\hline UN_ci_1599 & $134 \mathrm{~A} / \mathrm{G}$ \\
\hline UN_ci_1608 & $340 \mathrm{G} / \mathrm{T}$ \\
\hline UN_ci_1617 & $162 \mathrm{~T} / \mathrm{A}$ \\
\hline UN_ci_1624 & $18 \mathrm{~T} / \mathrm{A}$ \\
\hline UN_ci_1624 & $320 \mathrm{~A} / \mathrm{C}$ \\
\hline
\end{tabular}




\begin{tabular}{|c|c|}
\hline UN_ci_1626 & $422 \mathrm{~A} / \mathrm{G}$ \\
\hline UN_ci_1641 & $76 \mathrm{C} / \mathrm{T}$ \\
\hline UN_ci_1652 & $138 \mathrm{~T} / \mathrm{C}$ \\
\hline UN_ci_1652 & $234 \mathrm{C} / \mathrm{T}$ \\
\hline UN_ci_1652 & $958 \mathrm{~A} / \mathrm{G}$ \\
\hline UN_ci_1652 & $1378 \mathrm{G} / \mathrm{T}$ \\
\hline UN_ci_1661 & $150 \mathrm{C} / \mathrm{T}$ \\
\hline UN_ci_1666 & $13 \mathrm{~A} / \mathrm{C}$ \\
\hline UN_ci_1666 & $569 \mathrm{~T} / \mathrm{C}$ \\
\hline UN_ci_1666 & $795 \mathrm{~A} / \mathrm{T}$ \\
\hline UN_ci_1668 & $469 \mathrm{C} / \mathrm{T}$ \\
\hline UN_ci_1668 & $637 \mathrm{C} / \mathrm{G}$ \\
\hline UN_ci_1668 & $735 \mathrm{C} / \mathrm{T}$ \\
\hline UN_ci_1669 & $608 \mathrm{C} / \mathrm{T}$ \\
\hline UN_ci_1672 & $246 \mathrm{~A} / \mathrm{G}$ \\
\hline UN_ci_1673 & $204 \mathrm{~T} / \mathrm{G}$ \\
\hline UN_ci_1686 & 75 T/G \\
\hline UN_ci_1687 & $87 \mathrm{G} / \mathrm{A}$ \\
\hline UN_ci_1691 & $130 \mathrm{~A} / \mathrm{T}$ \\
\hline UN_ci_1702 & $45 \mathrm{G} / \mathrm{A}$ \\
\hline UN_ci_1702 & $119 \mathrm{G} / \mathrm{A}$ \\
\hline UN_ci_1704 & $189 \mathrm{G} / \mathrm{A}$ \\
\hline UN_ci_1704 & 895 C/G \\
\hline UN_ci_1707 & $234 \mathrm{C} / \mathrm{T}$ \\
\hline UN_ci_1710 & $81 \mathrm{G} / \mathrm{A}$ \\
\hline UN_ci_1710 & $658 \mathrm{~A} / \mathrm{T}$ \\
\hline UN_ci_1710 & $880 \mathrm{~T} / \mathrm{C}$ \\
\hline UN_ci_1711 & $11 \mathrm{G} / \mathrm{C}$ \\
\hline UN_ci_1711 & $389 \mathrm{C} / \mathrm{G}$ \\
\hline UN_ci_1713 & $213 \mathrm{~A} / \mathrm{G}$ \\
\hline UN_ci_1726 & 1076 T/C \\
\hline UN_ci_1733 & $502 \mathrm{C} / \mathrm{G}$ \\
\hline UN_ci_1733 & $551 \mathrm{~A} / \mathrm{T}$ \\
\hline UN_ci_1737 & $349 \mathrm{~T} / \mathrm{G}$ \\
\hline UN_ci_1744 & $32 \mathrm{C} / \mathrm{T}$ \\
\hline UN_ci_1744 & $635 \mathrm{~T} / \mathrm{C}$ \\
\hline UN_ci_1749 & $107 \mathrm{~T} / \mathrm{A}$ \\
\hline UN_ci_1753 & $616 \mathrm{~T} / \mathrm{G}$ \\
\hline UN_ci_1767 & $224 \mathrm{C} / \mathrm{T}$ \\
\hline UN_ci_1767 & $303 \mathrm{~A} / \mathrm{G}$ \\
\hline UN_ci_1767 & $326 \mathrm{G} / \mathrm{A}$ \\
\hline UN_ci_1767 & $410 \mathrm{~A} / \mathrm{C}$ \\
\hline UN_ci_1767 & $508 \mathrm{~A} / \mathrm{T}$ \\
\hline UN_ci_1768 & $384 \mathrm{C} / \mathrm{T}$ \\
\hline UN_ci_1768 & $1467 \mathrm{C} / \mathrm{T}$ \\
\hline UN_ci_1772 & $83 \mathrm{G} / \mathrm{T}$ \\
\hline UN_ci_1772 & $183 \mathrm{~A} / \mathrm{G}$ \\
\hline UN_ci_1772 & 296 G/A \\
\hline UN_ci_1772 & $534 \mathrm{~T} / \mathrm{C}$ \\
\hline UN_ci_1772 & $1005 \mathrm{G} / \mathrm{A}$ \\
\hline
\end{tabular}




\begin{tabular}{|c|c|}
\hline UN_ci_1772 & $1057 \mathrm{G} / \mathrm{A}$ \\
\hline UN_ci_1774 & $294 \mathrm{~A} / \mathrm{T}$ \\
\hline UN_ci_1774 & 351 T/A \\
\hline UN_ci_1776 & $507 \mathrm{G} / \mathrm{T}$ \\
\hline UN_ci_1785 & $175 \mathrm{~T} / \mathrm{C}$ \\
\hline UN_ci_1785 & $211 \mathrm{C} / \mathrm{T}$ \\
\hline UN_ci_1785 & $315 \mathrm{~A} / \mathrm{G}$ \\
\hline UN_ci_1796 & $400 \mathrm{~A} / \mathrm{G}$ \\
\hline UN_ci_1796 & $425 \mathrm{~T} / \mathrm{C}$ \\
\hline UN_ci_1798 & $317 \mathrm{G} / \mathrm{T}$ \\
\hline UN_ci_1805 & $54 \mathrm{~T} / \mathrm{C}$ \\
\hline UN_ci_1805 & $98 \mathrm{~T} / \mathrm{C}$ \\
\hline UN_ci_1805 & $141 \mathrm{~A} / \mathrm{G}$ \\
\hline UN_ci_1805 & $210 \mathrm{~T} / \mathrm{C}$ \\
\hline UN_ci_1811 & $233 \mathrm{~A} / \mathrm{G}$ \\
\hline UN_ci_1811 & $272 \mathrm{G} / \mathrm{A}$ \\
\hline UN_ci_1811 & 304 G/C \\
\hline UN_ci_1811 & $374 \mathrm{~A} / \mathrm{T}$ \\
\hline UN_ci_1811 & $574 \mathrm{C} / \mathrm{T}$ \\
\hline UN_ci_1811 & $871 \mathrm{~A} / \mathrm{C}$ \\
\hline UN_ci_1811 & $893 \mathrm{~A} / \mathrm{T}$ \\
\hline UN_ci_1819 & $80 \mathrm{~A} / \mathrm{G}$ \\
\hline UN_ci_1827 & $36 \mathrm{~T} / \mathrm{G}$ \\
\hline UN_ci_1827 & $170 \mathrm{~T} / \mathrm{G}$ \\
\hline UN_ci_1828 & $21 \mathrm{G} / \mathrm{C}$ \\
\hline UN_ci_1828 & $142 \mathrm{~T} / \mathrm{C}$ \\
\hline UN_ci_1837 & $229 \mathrm{~T} / \mathrm{C}$ \\
\hline UN_ci_1840 & $51 \mathrm{~T} / \mathrm{A}$ \\
\hline UN_ci_1842 & 199 T/C \\
\hline UN_ci_1868 & $159 \mathrm{C} / \mathrm{T}$ \\
\hline UN_ci_1890 & $176 \mathrm{G} / \mathrm{A}$ \\
\hline UN_ci_1893 & $217 \mathrm{C} / \mathrm{A}$ \\
\hline UN_ci_1911 & $125 \mathrm{C} / \mathrm{T}$ \\
\hline UN_ci_1918 & $55 \mathrm{~T} / \mathrm{C}$ \\
\hline UN_ci_1927 & $397 \mathrm{~A} / \mathrm{G}$ \\
\hline UN_ci_1927 & $418 \mathrm{~T} / \mathrm{G}$ \\
\hline UN_ci_1929 & $83 \mathrm{G} / \mathrm{A}$ \\
\hline UN_ci_1958 & $37 \mathrm{~A} / \mathrm{G}$ \\
\hline UN_ci_1974 & $150 \mathrm{G} / \mathrm{A}$ \\
\hline UN_ci_1988 & $204 \mathrm{~A} / \mathrm{G}$ \\
\hline UN_ci_1991 & $176 \mathrm{G} / \mathrm{A}$ \\
\hline UN_ci_1991 & $686 \mathrm{~A} / \mathrm{C}$ \\
\hline UN_ci_1994 & $10 \mathrm{G} / \mathrm{A}$ \\
\hline UN_ci_1994 & $38 \mathrm{~A} / \mathrm{G}$ \\
\hline UN_ci_1994 & $91 \mathrm{~T} / \mathrm{C}$ \\
\hline UN_ci_1994 & $250 \mathrm{~A} / \mathrm{G}$ \\
\hline UN_ci_2008 & $314 \mathrm{~T} / \mathrm{C}$ \\
\hline UN_ci_2008 & $1326 \mathrm{C} / \mathrm{T}$ \\
\hline UN_ci_2012 & $57 \mathrm{G} / \mathrm{C}$ \\
\hline UN_ci_2012 & $104 \mathrm{C} / \mathrm{A}$ \\
\hline
\end{tabular}




\begin{tabular}{|c|c|}
\hline UN_ci_2012 & $206 \mathrm{~A} / \mathrm{G}$ \\
\hline UN_ci_2012 & $606 \mathrm{G} / \mathrm{A}$ \\
\hline UN_ci_2012 & $664 \mathrm{~T} / \mathrm{C}$ \\
\hline UN_ci_2012 & $936 \mathrm{G} / \mathrm{C}$ \\
\hline UN_ci_2012 & $1030 \mathrm{C} / \mathrm{T}$ \\
\hline UN_ci_2012 & 1146 T/C \\
\hline UN_ci_2012 & $1263 \mathrm{~T} / \mathrm{A}$ \\
\hline UN_ci_2021 & $982 \mathrm{G} / \mathrm{A}$ \\
\hline UN_ci_2022 & $37 \mathrm{G} / \mathrm{A}$ \\
\hline UN_ci_2022 & $80 \mathrm{~T} / \mathrm{C}$ \\
\hline UN_ci_2035 & $17 \mathrm{~A} / \mathrm{T}$ \\
\hline UN_ci_2040 & $229 \mathrm{C} / \mathrm{T}$ \\
\hline UN_ci_2040 & $270 \mathrm{C} / \mathrm{T}$ \\
\hline UN_ci_2041 & $293 \mathrm{~A} / \mathrm{G}$ \\
\hline UN_ci_2048 & $306 \mathrm{~A} / \mathrm{C}$ \\
\hline UN_ci_2053 & $218 \mathrm{G} / \mathrm{A}$ \\
\hline UN_ci_2057 & $52 \mathrm{~A} / \mathrm{G}$ \\
\hline UN_ci_2057 & $118 \mathrm{C} / \mathrm{A}$ \\
\hline UN_ci_2057 & $390 \mathrm{~A} / \mathrm{G}$ \\
\hline UN_ci_2065 & $234 \mathrm{C} / \mathrm{T}$ \\
\hline UN_ci_2065 & $486 \mathrm{G} / \mathrm{A}$ \\
\hline UN_ci_2066 & $740 \mathrm{G} / \mathrm{A}$ \\
\hline UN_ci_2073 & $242 \mathrm{~T} / \mathrm{C}$ \\
\hline UN_ci_2074 & $154 \mathrm{~T} / \mathrm{C}$ \\
\hline UN_ci_2074 & 199 T/C \\
\hline UN_ci_2074 & $234 \mathrm{~T} / \mathrm{C}$ \\
\hline UN_ci_2075 & $314 \mathrm{~A} / \mathrm{G}$ \\
\hline UN_ci_2083 & $131 \mathrm{~A} / \mathrm{C}$ \\
\hline UN_ci_2083 & $164 \mathrm{G} / \mathrm{T}$ \\
\hline UN_ci_2108 & $123 \mathrm{~A} / \mathrm{G}$ \\
\hline UN_ci_2108 & $378 \mathrm{~A} / \mathrm{T}$ \\
\hline UN_ci_2109 & $96 \mathrm{~T} / \mathrm{C}$ \\
\hline UN_ci_2110 & $48 \mathrm{~T} / \mathrm{C}$ \\
\hline UN_ci_2110 & $153 \mathrm{G} / \mathrm{A}$ \\
\hline UN_ci_2115 & $180 \mathrm{~A} / \mathrm{T}$ \\
\hline UN_ci_2116 & $238 \mathrm{~A} / \mathrm{G}$ \\
\hline UN_ci_2137 & $160 \mathrm{~T} / \mathrm{C}$ \\
\hline UN_ci_2145 & $512 \mathrm{~A} / \mathrm{T}$ \\
\hline UN_ci_2145 & $629 \mathrm{C} / \mathrm{A}$ \\
\hline UN_ci_2145 & $884 \mathrm{~T} / \mathrm{G}$ \\
\hline UN_ci_2145 & $935 \mathrm{G} / \mathrm{A}$ \\
\hline UN_ci_2145 & $1002 \mathrm{G} / \mathrm{T}$ \\
\hline UN_ci_2145 & $1073 \mathrm{G} / \mathrm{A}$ \\
\hline UN_ci_2147 & $573 \mathrm{~A} / \mathrm{T}$ \\
\hline UN_ci_2152 & $104 \mathrm{~A} / \mathrm{G}$ \\
\hline UN_ci_2152 & $239 \mathrm{~T} / \mathrm{C}$ \\
\hline UN_ci_2152 & $266 \mathrm{~A} / \mathrm{G}$ \\
\hline UN_ci_2152 & $308 \mathrm{C} / \mathrm{A}$ \\
\hline UN_ci_2158 & $180 \mathrm{~T} / \mathrm{A}$ \\
\hline UN_ci_2158 & $216 \mathrm{G} / \mathrm{A}$ \\
\hline
\end{tabular}




\begin{tabular}{|c|c|}
\hline UN_ci_2161 & $278 \mathrm{~T} / \mathrm{C}$ \\
\hline UN_ci_2162 & $72 \mathrm{G} / \mathrm{T}$ \\
\hline UN_ci_2166 & $40 \mathrm{C} / \mathrm{T}$ \\
\hline UN_ci_2169 & $313 \mathrm{G} / \mathrm{A}$ \\
\hline UN_ci_2171 & $85 \mathrm{~T} / \mathrm{A}$ \\
\hline UN_ci_2171 & $122 \mathrm{~A} / \mathrm{G}$ \\
\hline UN_ci_2171 & $293 \mathrm{~T} / \mathrm{C}$ \\
\hline UN_ci_2171 & $325 \mathrm{~T} / \mathrm{C}$ \\
\hline UN_ci_2171 & $557 \mathrm{C} / \mathrm{T}$ \\
\hline UN_ci_2171 & $1655 \mathrm{~A} / \mathrm{T}$ \\
\hline UN_ci_2171 & $1705 \mathrm{~T} / \mathrm{G}$ \\
\hline UN_ci_2179 & $109 \mathrm{~A} / \mathrm{T}$ \\
\hline UN_ci_2179 & $173 \mathrm{G} / \mathrm{A}$ \\
\hline UN_ci_2189 & $129 \mathrm{G} / \mathrm{T}$ \\
\hline UN_ci_2189 & $252 \mathrm{~A} / \mathrm{G}$ \\
\hline UN_ci_2189 & $582 \mathrm{C} / \mathrm{T}$ \\
\hline UN_ci_2189 & $736 \mathrm{C} / \mathrm{T}$ \\
\hline UN_ci_2189 & 766 G/T \\
\hline UN_ci_2193 & $79 \mathrm{C} / \mathrm{T}$ \\
\hline UN_ci_2193 & $109 \mathrm{~T} / \mathrm{C}$ \\
\hline UN_ci_2193 & $186 \mathrm{~A} / \mathrm{G}$ \\
\hline UN_ci_2209 & 209 G/A \\
\hline UN_ci_2209 & $482 \mathrm{~A} / \mathrm{G}$ \\
\hline UN_ci_2233 & $278 \mathrm{C} / \mathrm{T}$ \\
\hline UN_ci_2233 & 386 A/G \\
\hline UN_ci_2235 & $242 \mathrm{C} / \mathrm{T}$ \\
\hline UN_ci_2239 & $39 \mathrm{C} / \mathrm{T}$ \\
\hline UN_ci_2242 & $153 \mathrm{C} / \mathrm{T}$ \\
\hline UN_ci_2245 & 190 T/C \\
\hline UN_ci_2246 & $80 \mathrm{~T} / \mathrm{A}$ \\
\hline UN_ci_2254 & $136 \mathrm{~A} / \mathrm{G}$ \\
\hline UN_ci_2254 & $158 \mathrm{~A} / \mathrm{G}$ \\
\hline UN_ci_2254 & $233 \mathrm{~A} / \mathrm{T}$ \\
\hline UN_ci_2259 & $1401 \mathrm{G} / \mathrm{A}$ \\
\hline UN_ci_2261 & $341 \mathrm{C} / \mathrm{T}$ \\
\hline UN_ci_2264 & $166 \mathrm{G} / \mathrm{A}$ \\
\hline UN_ci_2269 & $200 \mathrm{C} / \mathrm{A}$ \\
\hline UN_ci_2274 & $377 \mathrm{G} / \mathrm{A}$ \\
\hline UN_ci_2287 & $117 \mathrm{C} / \mathrm{A}$ \\
\hline UN_ci_2293 & $144 \mathrm{~A} / \mathrm{T}$ \\
\hline UN_ci_2293 & $215 \mathrm{C} / \mathrm{T}$ \\
\hline UN_ci_2305 & $135 \mathrm{~T} / \mathrm{A}$ \\
\hline UN_ci_2308 & $278 \mathrm{~A} / \mathrm{G}$ \\
\hline UN_ci_2308 & $347 \mathrm{C} / \mathrm{G}$ \\
\hline UN_ci_2309 & $298 \mathrm{~A} / \mathrm{G}$ \\
\hline UN_ci_2315 & $114 \mathrm{~T} / \mathrm{C}$ \\
\hline UN_ci_2315 & $274 \mathrm{~A} / \mathrm{G}$ \\
\hline UN_ci_2316 & $174 \mathrm{C} / \mathrm{T}$ \\
\hline UN_ci_2316 & $526 \mathrm{~T} / \mathrm{A}$ \\
\hline UN_ci_2316 & $883 \mathrm{~T} / \mathrm{C}$ \\
\hline
\end{tabular}




\begin{tabular}{|c|c|}
\hline UN_ci_2325 & $952 \mathrm{~T} / \mathrm{C}$ \\
\hline UN_ci_2333 & $655 \mathrm{C} / \mathrm{T}$ \\
\hline UN_ci_2339 & $28 \mathrm{~A} / \mathrm{G}$ \\
\hline UN_ci_2339 & $99 \mathrm{~A} / \mathrm{T}$ \\
\hline UN_ci_2340 & $51 \mathrm{~A} / \mathrm{T}$ \\
\hline UN_ci_2340 & $152 \mathrm{~T} / \mathrm{C}$ \\
\hline UN_ci_2342 & $56 \mathrm{~T} / \mathrm{C}$ \\
\hline UN_ci_2356 & $227 \mathrm{G} / \mathrm{A}$ \\
\hline UN_ci_2356 & $425 \mathrm{~T} / \mathrm{C}$ \\
\hline UN_ci_2359 & $36 \mathrm{G} / \mathrm{A}$ \\
\hline UN_ci_2359 & $92 \mathrm{C} / \mathrm{A}$ \\
\hline UN_ci_2359 & $122 \mathrm{C} / \mathrm{T}$ \\
\hline UN_ci_2366 & $167 \mathrm{~T} / \mathrm{G}$ \\
\hline UN_ci_2366 & $283 \mathrm{C} / \mathrm{T}$ \\
\hline UN_ci_2373 & $983 \mathrm{G} / \mathrm{C}$ \\
\hline UN_ci_2374 & $523 \mathrm{C} / \mathrm{T}$ \\
\hline UN_ci_2374 & $853 \mathrm{~A} / \mathrm{G}$ \\
\hline UN_ci_2374 & $1024 \mathrm{G} / \mathrm{C}$ \\
\hline UN_ci_2375 & $240 \mathrm{~A} / \mathrm{G}$ \\
\hline UN_ci_2375 & $263 \mathrm{C} / \mathrm{T}$ \\
\hline UN_ci_2375 & $296 \mathrm{C} / \mathrm{T}$ \\
\hline UN_ci_2375 & $333 \mathrm{~A} / \mathrm{C}$ \\
\hline UN_ci_2375 & $363 \mathrm{~A} / \mathrm{T}$ \\
\hline UN_ci_2375 & $410 \mathrm{~A} / \mathrm{G}$ \\
\hline UN_ci_2375 & $769 \mathrm{C} / \mathrm{T}$ \\
\hline UN_ci_2375 & $860 \mathrm{~A} / \mathrm{C}$ \\
\hline UN_ci_2375 & $892 \mathrm{~T} / \mathrm{C}$ \\
\hline UN_ci_2383 & $421 \mathrm{~T} / \mathrm{C}$ \\
\hline UN_ci_2389 & $411 \mathrm{C} / \mathrm{T}$ \\
\hline UN_ci_2389 & $453 \mathrm{~A} / \mathrm{T}$ \\
\hline UN_ci_2400 & $638 \mathrm{~A} / \mathrm{C}$ \\
\hline UN_ci_2400 & $752 \mathrm{~A} / \mathrm{G}$ \\
\hline UN_ci_2409 & $92 \mathrm{G} / \mathrm{T}$ \\
\hline UN_ci_2415 & $162 \mathrm{G} / \mathrm{A}$ \\
\hline UN_ci_2415 & $258 \mathrm{C} / \mathrm{T}$ \\
\hline UN_ci_2415 & $282 \mathrm{C} / \mathrm{T}$ \\
\hline UN_ci_2416 & $736 \mathrm{~T} / \mathrm{C}$ \\
\hline UN_ci_2416 & $788 \mathrm{~A} / \mathrm{C}$ \\
\hline UN_ci_2441 & $217 \mathrm{C} / \mathrm{G}$ \\
\hline UN_ci_2441 & $383 \mathrm{~A} / \mathrm{G}$ \\
\hline UN_ci_2441 & $404 \mathrm{~A} / \mathrm{G}$ \\
\hline UN_ci_2444 & $386 \mathrm{~T} / \mathrm{C}$ \\
\hline UN_ci_2449 & $284 \mathrm{~A} / \mathrm{G}$ \\
\hline UN_ci_2457 & $238 \mathrm{C} / \mathrm{T}$ \\
\hline UN_ci_2461 & $234 \mathrm{G} / \mathrm{C}$ \\
\hline UN_ci_2461 & $649 \mathrm{~A} / \mathrm{C}$ \\
\hline UN_ci_2461 & $758 \mathrm{~A} / \mathrm{G}$ \\
\hline UN_ci_2468 & 299 T/A \\
\hline UN_ci_2481 & $233 \mathrm{~A} / \mathrm{G}$ \\
\hline UN_ci_2481 & $349 \mathrm{C} / \mathrm{T}$ \\
\hline
\end{tabular}




\begin{tabular}{|c|c|}
\hline UN_ci_2481 & $441 \mathrm{~A} / \mathrm{G}$ \\
\hline UN_ci_2481 & $512 \mathrm{~T} / \mathrm{C}$ \\
\hline UN_ci_2481 & $538 \mathrm{C} / \mathrm{T}$ \\
\hline UN_ci_2483 & $170 \mathrm{C} / \mathrm{T}$ \\
\hline UN_ci_2492 & $176 \mathrm{~A} / \mathrm{G}$ \\
\hline UN_ci_2492 & $254 \mathrm{~T} / \mathrm{C}$ \\
\hline UN_ci_2492 & $313 \mathrm{C} / \mathrm{T}$ \\
\hline UN_ci_2496 & $63 \mathrm{~A} / \mathrm{T}$ \\
\hline UN_ci_2498 & $44 \mathrm{G} / \mathrm{C}$ \\
\hline UN_ci_2498 & $632 \mathrm{~T} / \mathrm{G}$ \\
\hline UN_ci_2498 & $788 \mathrm{C} / \mathrm{T}$ \\
\hline UN_ci_2506 & $157 \mathrm{~A} / \mathrm{C}$ \\
\hline UN_ci_2506 & $232 \mathrm{~A} / \mathrm{G}$ \\
\hline UN_ci_2506 & $253 \mathrm{~A} / \mathrm{G}$ \\
\hline UN_ci_2506 & $827 \mathrm{~A} / \mathrm{G}$ \\
\hline UN_ci_2517 & $505 \mathrm{~T} / \mathrm{C}$ \\
\hline UN_ci_2525 & $805 \mathrm{~A} / \mathrm{G}$ \\
\hline UN_ci_2527 & $83 \mathrm{~A} / \mathrm{G}$ \\
\hline UN_ci_2532 & $362 \mathrm{~T} / \mathrm{C}$ \\
\hline UN_ci_2532 & $453 \mathrm{C} / \mathrm{T}$ \\
\hline UN_ci_2532 & $487 \mathrm{~A} / \mathrm{G}$ \\
\hline UN_ci_2532 & $554 \mathrm{~T} / \mathrm{C}$ \\
\hline UN_ci_2533 & $471 \mathrm{~A} / \mathrm{G}$ \\
\hline UN_ci_2533 & $1120 \mathrm{~A} / \mathrm{G}$ \\
\hline UN_ci_2533 & $1328 \mathrm{C} / \mathrm{T}$ \\
\hline UN_ci_2533 & $1732 \mathrm{~A} / \mathrm{G}$ \\
\hline UN_ci_2533 & $1784 \mathrm{~T} / \mathrm{C}$ \\
\hline UN_ci_2543 & $201 \mathrm{C} / \mathrm{T}$ \\
\hline UN_ci_2543 & $277 \mathrm{G} / \mathrm{A}$ \\
\hline UN_ci_2553 & $218 \mathrm{~T} / \mathrm{C}$ \\
\hline UN_ci_2553 & $283 \mathrm{G} / \mathrm{C}$ \\
\hline UN_ci_2563 & $434 \mathrm{C} / \mathrm{T}$ \\
\hline UN_ci_2563 & $665 \mathrm{C} / \mathrm{T}$ \\
\hline UN_ci_2565 & $187 \mathrm{C} / \mathrm{A}$ \\
\hline UN_ci_2570 & 769 A/G \\
\hline UN_ci_2570 & $842 \mathrm{~A} / \mathrm{G}$ \\
\hline UN_ci_2570 & $874 \mathrm{~A} / \mathrm{G}$ \\
\hline UN_ci_2574 & $24 \mathrm{~A} / \mathrm{G}$ \\
\hline UN_ci_2574 & $123 \mathrm{~T} / \mathrm{C}$ \\
\hline UN_ci_2574 & $162 \mathrm{G} / \mathrm{A}$ \\
\hline UN_ci_2597 & $24 \mathrm{~A} / \mathrm{T}$ \\
\hline UN_ci_2597 & $55 \mathrm{~T} / \mathrm{C}$ \\
\hline UN_ci_2597 & $289 \mathrm{~A} / \mathrm{T}$ \\
\hline UN_ci_2613 & $827 \mathrm{~A} / \mathrm{C}$ \\
\hline UN_ci_2613 & 879 T/C \\
\hline UN_ci_2616 & $342 \mathrm{G} / \mathrm{C}$ \\
\hline UN_ci_2618 & $12 \mathrm{G} / \mathrm{A}$ \\
\hline UN_ci_2618 & $52 \mathrm{~T} / \mathrm{C}$ \\
\hline UN_ci_2618 & $181 \mathrm{~T} / \mathrm{C}$ \\
\hline UN_ci_2618 & $224 \mathrm{~T} / \mathrm{C}$ \\
\hline
\end{tabular}




$\begin{array}{lr}\text { UN_ci_2618 } & 346 \mathrm{~A} / \mathrm{G} \\ \text { UN_ci_2618 } & 445 \mathrm{~A} / \mathrm{G} \\ \text { UN_ci_2618 } & 544 \mathrm{G} / \mathrm{A} \\ \text { UN_ci_2622 } & 162 \mathrm{C} / \mathrm{T} \\ \text { UN_ci_2622 } & 195 \mathrm{~T} / \mathrm{C} \\ \text { UN_ci_2623 } & 193 \mathrm{G} / \mathrm{A} \\ \text { UN_ci_2625 } & 169 \mathrm{C} / \mathrm{G} \\ \text { UN_ci_2625 } & 267 \mathrm{G} / \mathrm{A} \\ \text { UN_ci_2629 } & 49 \mathrm{~T} / \mathrm{C} \\ \text { UN_ci_2631 } & 95 \mathrm{~A} / \mathrm{T} \\ \text { UN_ci_2631 } & 171 \mathrm{~T} / \mathrm{C} \\ \text { UN_ci_2631 } & 217 \mathrm{~A} / \mathrm{G} \\ \text { UN_ci_2631 } & 300 \mathrm{~T} / \mathrm{C} \\ \text { UN_ci_2635 } & 806 \mathrm{~T} / \mathrm{C} \\ \text { UN_ci_2635 } & 834 \mathrm{G} / \mathrm{T} \\ \text { UN_ci_2640 } & 187 \mathrm{~A} / \mathrm{G} \\ \text { UN_ci_2640 } & 299 \mathrm{C} / \mathrm{G} \\ \text { UN_ci_2640 } & 398 \mathrm{C} / \mathrm{T} \\ \text { UN_ci_2640 } & 528 \mathrm{C} / \mathrm{G} \\ \text { UN_ci_2642 } & 131 \mathrm{~A} / \mathrm{G} \\ \text { UN_ci_2644 } & 40 \mathrm{~T} / \mathrm{C} \\ \text { UN_ci_2644 } & 68 \mathrm{C} / \mathrm{A} \\ \text { UN_ci_2644 } & 121 \mathrm{~T} / \mathrm{C} \\ \text { UN_ci_2644 } & 168 \mathrm{G} / \mathrm{A} \\ \text { UN_ci_2644 } & \end{array}$




\begin{tabular}{|c|c|}
\hline UN_ci_2686 & $188 \mathrm{~A} / \mathrm{G}$ \\
\hline UN_ci_2691 & $117 \mathrm{C} / \mathrm{T}$ \\
\hline UN_ci_2691 & $275 \mathrm{G} / \mathrm{A}$ \\
\hline UN_ci_2696 & $340 \mathrm{G} / \mathrm{T}$ \\
\hline UN_ci_2696 & $460 \mathrm{~T} / \mathrm{C}$ \\
\hline UN_ci_2696 & $592 \mathrm{C} / \mathrm{T}$ \\
\hline UN_ci_2696 & $625 \mathrm{~A} / \mathrm{G}$ \\
\hline UN_ci_2696 & $744 \mathrm{~T} / \mathrm{C}$ \\
\hline UN_ci_2696 & $784 \mathrm{~T} / \mathrm{G}$ \\
\hline UN_ci_2696 & $805 \mathrm{C} / \mathrm{T}$ \\
\hline UN_ci_2696 & $903 \mathrm{~T} / \mathrm{C}$ \\
\hline UN_ci_2696 & 1219 T/C \\
\hline UN_ci_2696 & $1255 \mathrm{~T} / \mathrm{C}$ \\
\hline UN_ci_2696 & $1342 \mathrm{C} / \mathrm{A}$ \\
\hline UN_ci_2696 & $1372 \mathrm{C} / \mathrm{G}$ \\
\hline UN_ci_2696 & $1466 \mathrm{~A} / \mathrm{T}$ \\
\hline UN_ci_2699 & $34 \mathrm{G} / \mathrm{A}$ \\
\hline UN_ci_2699 & 205 T/C \\
\hline UN_ci_2718 & $191 \mathrm{C} / \mathrm{T}$ \\
\hline UN_ci_2718 & $244 \mathrm{~T} / \mathrm{C}$ \\
\hline UN_ci_2718 & $344 \mathrm{C} / \mathrm{A}$ \\
\hline UN_ci_2718 & $447 \mathrm{~A} / \mathrm{C}$ \\
\hline UN_ci_2718 & $831 \mathrm{~A} / \mathrm{G}$ \\
\hline UN_ci_2720 & 193 A/G \\
\hline UN_ci_2722 & $118 \mathrm{G} / \mathrm{C}$ \\
\hline UN_ci_2722 & $164 \mathrm{~A} / \mathrm{G}$ \\
\hline UN_ci_2726 & $149 \mathrm{G} / \mathrm{T}$ \\
\hline UN_ci_2726 & $347 \mathrm{C} / \mathrm{T}$ \\
\hline UN_ci_2727 & $35 \mathrm{~A} / \mathrm{G}$ \\
\hline UN_ci_2727 & $184 \mathrm{~A} / \mathrm{G}$ \\
\hline UN_ci_2727 & $240 \mathrm{C} / \mathrm{G}$ \\
\hline UN_ci_2727 & $261 \mathrm{~T} / \mathrm{C}$ \\
\hline UN_ci_2727 & 292 A/G \\
\hline UN_ci_2727 & $323 \mathrm{~T} / \mathrm{C}$ \\
\hline UN_ci_2727 & $352 \mathrm{~A} / \mathrm{G}$ \\
\hline UN_ci_2727 & $407 \mathrm{~A} / \mathrm{G}$ \\
\hline UN_ci_2727 & $459 \mathrm{~A} / \mathrm{T}$ \\
\hline UN_ci_2733 & $211 \mathrm{G} / \mathrm{A}$ \\
\hline UN_ci_2733 & $249 \mathrm{G} / \mathrm{A}$ \\
\hline UN_ci_2733 & $271 \mathrm{C} / \mathrm{T}$ \\
\hline UN_ci_2739 & $65 \mathrm{~T} / \mathrm{C}$ \\
\hline UN_ci_2739 & $152 \mathrm{~T} / \mathrm{A}$ \\
\hline UN_ci_2741 & $54 \mathrm{~T} / \mathrm{C}$ \\
\hline UN_ci_2741 & $425 \mathrm{G} / \mathrm{A}$ \\
\hline UN_ci_2745 & $130 \mathrm{~A} / \mathrm{G}$ \\
\hline UN_ci_2750 & $232 \mathrm{G} / \mathrm{A}$ \\
\hline UN_ci_2752 & $378 \mathrm{C} / \mathrm{T}$ \\
\hline UN_ci_2753 & $177 \mathrm{G} / \mathrm{A}$ \\
\hline UN_ci_2756 & $358 \mathrm{~T} / \mathrm{C}$ \\
\hline UN_ci_2757 & $308 \mathrm{~A} / \mathrm{G}$ \\
\hline
\end{tabular}




\begin{tabular}{|c|c|}
\hline UN_ci_2767 & $225 \mathrm{~A} / \mathrm{G}$ \\
\hline UN_ci_2771 & $431 \mathrm{~A} / \mathrm{G}$ \\
\hline UN_ci_2771 & $490 \mathrm{~A} / \mathrm{T}$ \\
\hline UN_ci_2771 & $555 \mathrm{~A} / \mathrm{T}$ \\
\hline UN_ci_2773 & $215 \mathrm{G} / \mathrm{T}$ \\
\hline UN_ci_2776 & $52 \mathrm{~A} / \mathrm{C}$ \\
\hline UN_ci_2776 & $147 \mathrm{~A} / \mathrm{T}$ \\
\hline UN_ci_2776 & $290 \mathrm{C} / \mathrm{A}$ \\
\hline UN_ci_2777 & 199 A/G \\
\hline UN_ci_2779 & $465 \mathrm{~A} / \mathrm{G}$ \\
\hline UN_ci_2789 & $71 \mathrm{C} / \mathrm{A}$ \\
\hline UN_ci_2789 & 207 T/C \\
\hline UN_ci_2789 & $244 \mathrm{~A} / \mathrm{G}$ \\
\hline UN_ci_2789 & $352 \mathrm{G} / \mathrm{A}$ \\
\hline UN_ci_2798 & $465 \mathrm{~A} / \mathrm{G}$ \\
\hline UN_ci_2798 & $634 \mathrm{~A} / \mathrm{T}$ \\
\hline UN_ci_2798 & 782 T/G \\
\hline UN_ci_2803 & $60 \mathrm{G} / \mathrm{A}$ \\
\hline UN_ci_2817 & $288 \mathrm{~A} / \mathrm{T}$ \\
\hline UN_ci_2819 & $211 \mathrm{C} / \mathrm{T}$ \\
\hline UN_ci_2820 & $83 \mathrm{~T} / \mathrm{C}$ \\
\hline UN_ci_2832 & $346 \mathrm{~T} / \mathrm{G}$ \\
\hline UN_ci_2832 & $382 \mathrm{G} / \mathrm{A}$ \\
\hline UN_ci_2833 & $10 \mathrm{G} / \mathrm{T}$ \\
\hline UN_ci_2833 & $386 \mathrm{C} / \mathrm{T}$ \\
\hline UN_ci_2835 & $182 \mathrm{C} / \mathrm{A}$ \\
\hline UN_ci_2835 & $224 \mathrm{~T} / \mathrm{C}$ \\
\hline UN_ci_2835 & $393 \mathrm{C} / \mathrm{G}$ \\
\hline UN_ci_2835 & $419 \mathrm{G} / \mathrm{C}$ \\
\hline UN_ci_2835 & $444 \mathrm{C} / \mathrm{A}$ \\
\hline UN_ci_2835 & $498 \mathrm{~A} / \mathrm{G}$ \\
\hline UN_ci_2838 & $715 \mathrm{G} / \mathrm{T}$ \\
\hline UN_ci_2841 & $170 \mathrm{C} / \mathrm{T}$ \\
\hline UN_ci_2847 & 119 T/C \\
\hline UN_ci_2847 & $157 \mathrm{~A} / \mathrm{C}$ \\
\hline UN_ci_2847 & $243 \mathrm{~T} / \mathrm{C}$ \\
\hline UN_ci_2847 & $289 \mathrm{~T} / \mathrm{C}$ \\
\hline UN_ci_2847 & $530 \mathrm{~A} / \mathrm{G}$ \\
\hline UN_ci_2847 & $568 \mathrm{G} / \mathrm{T}$ \\
\hline UN_ci_2847 & 695 T/C \\
\hline UN_ci_2847 & $760 \mathrm{~T} / \mathrm{C}$ \\
\hline UN_ci_2848 & $621 \mathrm{C} / \mathrm{T}$ \\
\hline UN_ci_2849 & $385 \mathrm{C} / \mathrm{A}$ \\
\hline UN_ci_2858 & $217 \mathrm{~T} / \mathrm{C}$ \\
\hline UN_ci_2858 & $237 \mathrm{G} / \mathrm{A}$ \\
\hline UN_ci_2875 & $146 \mathrm{C} / \mathrm{T}$ \\
\hline UN_ci_2879 & $142 \mathrm{G} / \mathrm{A}$ \\
\hline UN_ci_2880 & $251 \mathrm{~A} / \mathrm{T}$ \\
\hline UN_ci_2882 & $300 \mathrm{C} / \mathrm{T}$ \\
\hline UN_ci_2882 & $360 \mathrm{G} / \mathrm{A}$ \\
\hline
\end{tabular}




\begin{tabular}{|c|c|}
\hline UN_ci_2887 & $284 \mathrm{~T} / \mathrm{C}$ \\
\hline UN_ci_2887 & $534 \mathrm{~A} / \mathrm{G}$ \\
\hline UN_ci_2889 & $347 \mathrm{G} / \mathrm{A}$ \\
\hline UN_ci_2892 & $176 \mathrm{~A} / \mathrm{T}$ \\
\hline UN_ci_2895 & $107 \mathrm{~T} / \mathrm{A}$ \\
\hline UN_ci_2901 & $105 \mathrm{G} / \mathrm{A}$ \\
\hline UN_ci_2901 & $486 \mathrm{~A} / \mathrm{G}$ \\
\hline UN_ci_2904 & $61 \mathrm{~T} / \mathrm{C}$ \\
\hline UN_ci_2922 & $290 \mathrm{C} / \mathrm{G}$ \\
\hline UN_ci_2922 & 339 C/T \\
\hline UN_ci_2922 & $402 \mathrm{~T} / \mathrm{C}$ \\
\hline UN_ci_2922 & $422 \mathrm{~A} / \mathrm{C}$ \\
\hline UN_ci_2922 & $495 \mathrm{G} / \mathrm{A}$ \\
\hline UN_ci_2922 & $542 \mathrm{~T} / \mathrm{C}$ \\
\hline UN_ci_2922 & $743 \mathrm{~A} / \mathrm{G}$ \\
\hline UN_ci_2922 & $799 \mathrm{~T} / \mathrm{C}$ \\
\hline UN_ci_2922 & $881 \mathrm{~T} / \mathrm{C}$ \\
\hline UN_ci_2928 & $186 \mathrm{~A} / \mathrm{G}$ \\
\hline UN_ci_2930 & $122 \mathrm{C} / \mathrm{T}$ \\
\hline UN_ci_2930 & $259 \mathrm{~A} / \mathrm{G}$ \\
\hline UN_ci_2930 & $327 \mathrm{G} / \mathrm{A}$ \\
\hline UN_ci_2930 & 379 T/C \\
\hline UN_ci_2930 & $481 \mathrm{~A} / \mathrm{G}$ \\
\hline UN_ci_2930 & $694 \mathrm{G} / \mathrm{A}$ \\
\hline UN_ci_2947 & $520 \mathrm{G} / \mathrm{A}$ \\
\hline UN_ci_2959 & $338 \mathrm{~A} / \mathrm{G}$ \\
\hline UN_ci_2965 & $325 \mathrm{~T} / \mathrm{C}$ \\
\hline UN_ci_2971 & $44 \mathrm{G} / \mathrm{T}$ \\
\hline UN_ci_2973 & $254 \mathrm{G} / \mathrm{A}$ \\
\hline UN_ci_2973 & $470 \mathrm{~A} / \mathrm{G}$ \\
\hline UN_ci_2978 & $343 \mathrm{G} / \mathrm{A}$ \\
\hline UN_ci_2979 & $45 \mathrm{C} / \mathrm{T}$ \\
\hline UN_ci_2979 & $126 \mathrm{~T} / \mathrm{A}$ \\
\hline UN_ci_2979 & $186 \mathrm{C} / \mathrm{T}$ \\
\hline UN_ci_2980 & $588 \mathrm{~A} / \mathrm{G}$ \\
\hline UN_ci_2980 & 997 A/G \\
\hline UN_ci_2986 & $545 \mathrm{C} / \mathrm{T}$ \\
\hline UN_ci_2987 & $157 \mathrm{G} / \mathrm{A}$ \\
\hline UN_ci_2987 & $711 \mathrm{C} / \mathrm{T}$ \\
\hline UN_ci_2990 & $175 \mathrm{C} / \mathrm{T}$ \\
\hline UN_ci_2992 & 304 T/C \\
\hline UN_ci_2995 & $276 \mathrm{C} / \mathrm{T}$ \\
\hline UN_ci_2996 & $149 \mathrm{C} / \mathrm{T}$ \\
\hline UN_ci_2998 & $156 \mathrm{~A} / \mathrm{T}$ \\
\hline UN_ci_2998 & $374 \mathrm{C} / \mathrm{T}$ \\
\hline UN_ci_2998 & $738 \mathrm{C} / \mathrm{G}$ \\
\hline UN_ci_2999 & $118 \mathrm{~T} / \mathrm{C}$ \\
\hline UN_ci_2999 & $148 \mathrm{~T} / \mathrm{C}$ \\
\hline UN_ci_2999 & $168 \mathrm{~A} / \mathrm{C}$ \\
\hline UN_ci_2999 & $300 \mathrm{C} / \mathrm{T}$ \\
\hline
\end{tabular}




\begin{tabular}{|c|c|}
\hline UN_ci_2999 & $373 \mathrm{~A} / \mathrm{G}$ \\
\hline UN_ci_2999 & $421 \mathrm{~A} / \mathrm{G}$ \\
\hline UN_ci_2999 & $467 \mathrm{~A} / \mathrm{G}$ \\
\hline UN_ci_2999 & $533 \mathrm{~A} / \mathrm{G}$ \\
\hline UN_ci_2999 & $697 \mathrm{~T} / \mathrm{C}$ \\
\hline UN_ci_2999 & $742 \mathrm{~A} / \mathrm{G}$ \\
\hline UN_ci_3001 & $396 \mathrm{~T} / \mathrm{C}$ \\
\hline UN_ci_3006 & $245 \mathrm{~T} / \mathrm{C}$ \\
\hline UN_ci_3008 & $13 \mathrm{~T} / \mathrm{A}$ \\
\hline UN_ci_3016 & $167 \mathrm{~A} / \mathrm{G}$ \\
\hline UN_ci_3016 & $280 \mathrm{~T} / \mathrm{C}$ \\
\hline UN_ci_3016 & $390 \mathrm{G} / \mathrm{A}$ \\
\hline UN_ci_3033 & $373 \mathrm{G} / \mathrm{A}$ \\
\hline UN_ci_3044 & $266 \mathrm{~A} / \mathrm{G}$ \\
\hline UN_ci_3044 & $379 \mathrm{G} / \mathrm{A}$ \\
\hline UN_ci_3047 & $188 \mathrm{~T} / \mathrm{G}$ \\
\hline UN_ci_3047 & $257 \mathrm{G} / \mathrm{C}$ \\
\hline UN_ci_3047 & $514 \mathrm{~A} / \mathrm{G}$ \\
\hline UN_ci_3047 & $603 \mathrm{G} / \mathrm{A}$ \\
\hline UN_ci_3047 & 999 T/C \\
\hline UN_ci_3047 & $1203 \mathrm{~T} / \mathrm{C}$ \\
\hline UN_ci_3047 & $1240 \mathrm{C} / \mathrm{A}$ \\
\hline UN_ci_3047 & $1262 \mathrm{~T} / \mathrm{A}$ \\
\hline UN_ci_3047 & $1286 \mathrm{G} / \mathrm{A}$ \\
\hline UN_ci_3053 & $459 \mathrm{C} / \mathrm{T}$ \\
\hline UN_ci_3055 & $85 \mathrm{~T} / \mathrm{A}$ \\
\hline UN_ci_3060 & $165 \mathrm{C} / \mathrm{A}$ \\
\hline UN_ci_3060 & $456 \mathrm{~T} / \mathrm{C}$ \\
\hline UN_ci_3066 & $43 \mathrm{G} / \mathrm{A}$ \\
\hline UN_ci_3077 & $372 \mathrm{G} / \mathrm{A}$ \\
\hline UN_ci_3079 & $177 \mathrm{C} / \mathrm{T}$ \\
\hline UN_ci_3079 & $198 \mathrm{C} / \mathrm{T}$ \\
\hline UN_ci_3083 & $147 \mathrm{~T} / \mathrm{C}$ \\
\hline UN_ci_3085 & $21 \mathrm{~A} / \mathrm{T}$ \\
\hline UN_ci_3094 & $310 \mathrm{G} / \mathrm{T}$ \\
\hline UN_ci_3101 & $685 \mathrm{~A} / \mathrm{G}$ \\
\hline UN_ci_3102 & $343 \mathrm{G} / \mathrm{C}$ \\
\hline UN_ci_3104 & $169 \mathrm{G} / \mathrm{A}$ \\
\hline UN_ci_3104 & $317 \mathrm{~A} / \mathrm{G}$ \\
\hline UN_ci_3104 & $338 \mathrm{~T} / \mathrm{C}$ \\
\hline UN_ci_3104 & $492 \mathrm{G} / \mathrm{T}$ \\
\hline UN_ci_3106 & $184 \mathrm{C} / \mathrm{T}$ \\
\hline UN_ci_3108 & $243 \mathrm{~T} / \mathrm{C}$ \\
\hline UN_ci_3108 & $325 \mathrm{G} / \mathrm{A}$ \\
\hline UN_ci_3108 & $435 \mathrm{G} / \mathrm{T}$ \\
\hline UN_ci_3108 & $479 \mathrm{C} / \mathrm{T}$ \\
\hline UN_ci_3110 & $193 \mathrm{~T} / \mathrm{C}$ \\
\hline UN_ci_3110 & $233 \mathrm{C} / \mathrm{T}$ \\
\hline UN_ci_3110 & $421 \mathrm{~A} / \mathrm{G}$ \\
\hline UN_ci_3115 & $169 \mathrm{~A} / \mathrm{C}$ \\
\hline
\end{tabular}




$\begin{array}{lr}\text { UN_ci_3128 } & 197 \mathrm{C} / \mathrm{T} \\ \text { UN_ci_3128 } & 245 \mathrm{C} / \mathrm{T} \\ \text { UN_ci_3128 } & 290 \mathrm{C} / \mathrm{T} \\ \text { UN_ci_3128 } & 346 \mathrm{~A} / \mathrm{G} \\ \text { UN_ci_3128 } & 402 \mathrm{~T} / \mathrm{C} \\ \text { UN_ci_3128 } & 435 \mathrm{~A} / \mathrm{G} \\ \text { UN_ci_3128 } & 476 \mathrm{~T} / \mathrm{C} \\ \text { UN_ci_3128 } & 547 \mathrm{~A} / \mathrm{G} \\ \text { UN_ci_3128 } & 571 \mathrm{~A} / \mathrm{G} \\ \text { UN_ci_3128 } & 608 \mathrm{~T} / \mathrm{C} \\ \text { UN_ci_3131 } & 77 \mathrm{~A} / \mathrm{T} \\ \text { UN_ci_3131 } & 204 \mathrm{G} / \mathrm{A} \\ \text { UN_ci_3131 } & 290 \mathrm{~T} / \mathrm{C} \\ \text { UN_ci_3131 } & 411 \mathrm{~A} / \mathrm{C} \\ \text { UN_ci_3131 } & 431 \mathrm{G} / \mathrm{A} \\ \text { UN_ci_3132 } & 187 \mathrm{~A} / \mathrm{G} \\ \text { UN_ci_3137 } & 489 \mathrm{C} / \mathrm{A} \\ \text { UN_ci_3137 } & 525 \mathrm{~T} / \mathrm{G} \\ \text { UN_ci_3137 } & 684 \mathrm{~A} / \mathrm{C} \\ \text { UN_ci_3141 } & 30 \mathrm{~A} / \mathrm{T} \\ \text { UN_ci_3141 } & 62 \mathrm{~T} / \mathrm{C} \\ \text { UN_ci_3150 } & 117 \mathrm{~A} / \mathrm{T} \\ \text { UN_ci_3150 } & 137 \mathrm{~A} / \mathrm{G} \\ \text { UN_ci_3157 } & 157 \mathrm{~T} / \mathrm{C} \\ \text { UN_ci_3157 } & 178 \mathrm{C} / \mathrm{T} \\ \text { UN_ci_3157 } & \end{array}$




\begin{tabular}{|c|c|}
\hline UN_ci_3186 & $200 \mathrm{C} / \mathrm{T}$ \\
\hline UN_ci_3186 & $221 \mathrm{~A} / \mathrm{G}$ \\
\hline UN_ci_3186 & $241 \mathrm{C} / \mathrm{T}$ \\
\hline UN_ci_3186 & $261 \mathrm{~A} / \mathrm{G}$ \\
\hline UN_ci_3186 & $282 \mathrm{~A} / \mathrm{G}$ \\
\hline UN_ci_3186 & $302 \mathrm{~T} / \mathrm{C}$ \\
\hline UN_ci_3186 & $323 \mathrm{C} / \mathrm{T}$ \\
\hline UN_ci_3186 & $344 \mathrm{G} / \mathrm{T}$ \\
\hline UN_ci_3186 & $364 \mathrm{~A} / \mathrm{G}$ \\
\hline UN_ci_3186 & $384 \mathrm{C} / \mathrm{T}$ \\
\hline UN_ci_3186 & $406 \mathrm{~A} / \mathrm{G}$ \\
\hline UN_ci_3186 & $427 \mathrm{~A} / \mathrm{G}$ \\
\hline UN_ci_3186 & $447 \mathrm{~A} / \mathrm{G}$ \\
\hline UN_ci_3186 & $469 \mathrm{G} / \mathrm{A}$ \\
\hline UN_ci_3186 & $489 \mathrm{~A} / \mathrm{G}$ \\
\hline UN_ci_3186 & $510 \mathrm{~A} / \mathrm{G}$ \\
\hline UN_ci_3186 & $530 \mathrm{G} / \mathrm{A}$ \\
\hline UN_ci_3186 & $554 \mathrm{~A} / \mathrm{C}$ \\
\hline UN_ci_3186 & $575 \mathrm{C} / \mathrm{T}$ \\
\hline UN_ci_3186 & $598 \mathrm{C} / \mathrm{T}$ \\
\hline UN_ci_3186 & 629 A/G \\
\hline UN_ci_3186 & $649 \mathrm{~T} / \mathrm{C}$ \\
\hline UN_ci_3186 & $669 \mathrm{~T} / \mathrm{C}$ \\
\hline UN_ci_3186 & $690 \mathrm{~T} / \mathrm{A}$ \\
\hline UN_ci_3186 & $714 \mathrm{~T} / \mathrm{C}$ \\
\hline UN_ci_3186 & $735 \mathrm{C} / \mathrm{T}$ \\
\hline UN_ci_3186 & $756 \mathrm{~T} / \mathrm{C}$ \\
\hline UN_ci_3186 & $780 \mathrm{~T} / \mathrm{C}$ \\
\hline UN_ci_3186 & $802 \mathrm{~T} / \mathrm{A}$ \\
\hline UN_ci_3186 & $823 \mathrm{~T} / \mathrm{C}$ \\
\hline UN_ci_3186 & $843 \mathrm{~A} / \mathrm{C}$ \\
\hline UN_ci_3186 & $873 \mathrm{~T} / \mathrm{C}$ \\
\hline UN_ci_3186 & $910 \mathrm{~A} / \mathrm{G}$ \\
\hline UN_ci_3186 & $930 \mathrm{~T} / \mathrm{A}$ \\
\hline UN_ci_3186 & $953 \mathrm{~A} / \mathrm{G}$ \\
\hline UN_ci_3186 & $973 \mathrm{G} / \mathrm{A}$ \\
\hline UN_ci_3186 & $1002 \mathrm{~T} / \mathrm{G}$ \\
\hline UN_ci_3186 & $1029 \mathrm{~T} / \mathrm{C}$ \\
\hline UN_ci_3186 & $1050 \mathrm{~T} / \mathrm{G}$ \\
\hline UN_ci_3186 & $1072 \mathrm{~A} / \mathrm{G}$ \\
\hline UN_ci_3186 & 1093 T/C \\
\hline UN_ci_3186 & $1123 \mathrm{~A} / \mathrm{C}$ \\
\hline UN_ci_3186 & $1164 \mathrm{~T} / \mathrm{C}$ \\
\hline UN_ci_3186 & $1185 \mathrm{C} / \mathrm{T}$ \\
\hline UN_ci_3186 & $1226 \mathrm{C} / \mathrm{A}$ \\
\hline UN_ci_3186 & $1251 \mathrm{~T} / \mathrm{C}$ \\
\hline UN_ci_3186 & $1271 \mathrm{~T} / \mathrm{C}$ \\
\hline UN_ci_3186 & $1313 \mathrm{~A} / \mathrm{G}$ \\
\hline UN_ci_3195 & $49 \mathrm{~A} / \mathrm{G}$ \\
\hline UN_ci_3199 & $923 \mathrm{~T} / \mathrm{C}$ \\
\hline
\end{tabular}




\begin{tabular}{|c|c|}
\hline UN_ci_3199 & $988 \mathrm{C} / \mathrm{T}$ \\
\hline UN_ci_3216 & $1549 \mathrm{~A} / \mathrm{G}$ \\
\hline UN_ci_3216 & $1834 \mathrm{~A} / \mathrm{T}$ \\
\hline UN_ci_3216 & $2542 \mathrm{C} / \mathrm{T}$ \\
\hline UN_ci_3220 & $441 \mathrm{~T} / \mathrm{C}$ \\
\hline UN_ci_3222 & $15 \mathrm{C} / \mathrm{T}$ \\
\hline UN_ci_3222 & $53 \mathrm{G} / \mathrm{C}$ \\
\hline UN_ci_3222 & $120 \mathrm{C} / \mathrm{T}$ \\
\hline UN_ci_3222 & $171 \mathrm{G} / \mathrm{A}$ \\
\hline UN_ci_3222 & $249 \mathrm{~T} / \mathrm{C}$ \\
\hline UN_ci_3222 & $328 \mathrm{~A} / \mathrm{G}$ \\
\hline UN_ci_3222 & $402 \mathrm{C} / \mathrm{T}$ \\
\hline UN_ci_3222 & $445 \mathrm{C} / \mathrm{T}$ \\
\hline UN_ci_3222 & $470 \mathrm{~A} / \mathrm{G}$ \\
\hline UN_ci_3222 & $512 \mathrm{~A} / \mathrm{G}$ \\
\hline UN_ci_3222 & $649 \mathrm{~A} / \mathrm{C}$ \\
\hline UN_ci_3222 & $678 \mathrm{~A} / \mathrm{G}$ \\
\hline UN_ci_3222 & 706 A/G \\
\hline UN_ci_3222 & $738 \mathrm{~T} / \mathrm{C}$ \\
\hline UN_ci_3222 & $764 \mathrm{C} / \mathrm{T}$ \\
\hline UN_ci_3226 & $43 \mathrm{~A} / \mathrm{T}$ \\
\hline UN_ci_3226 & $288 \mathrm{~T} / \mathrm{C}$ \\
\hline UN_ci_3226 & $409 \mathrm{~A} / \mathrm{C}$ \\
\hline UN_ci_3226 & $750 \mathrm{C} / \mathrm{A}$ \\
\hline UN_ci_3235 & $431 \mathrm{G} / \mathrm{A}$ \\
\hline UN_ci_3235 & $642 \mathrm{~T} / \mathrm{C}$ \\
\hline UN_ci_3235 & $736 \mathrm{C} / \mathrm{T}$ \\
\hline UN_ci_3238 & $1140 \mathrm{~A} / \mathrm{G}$ \\
\hline UN_ci_3241 & $362 \mathrm{~T} / \mathrm{C}$ \\
\hline UN_ci_3241 & $433 \mathrm{~A} / \mathrm{G}$ \\
\hline UN_ci_3241 & $684 \mathrm{C} / \mathrm{A}$ \\
\hline UN_ci_3245 & $316 \mathrm{G} / \mathrm{A}$ \\
\hline UN_ci_3245 & $781 \mathrm{~A} / \mathrm{C}$ \\
\hline UN_ci_3245 & 837 T/C \\
\hline UN_ci_3245 & $1023 \mathrm{G} / \mathrm{A}$ \\
\hline UN_ci_3245 & $1148 \mathrm{~A} / \mathrm{G}$ \\
\hline UN_ci_3247 & $455 \mathrm{G} / \mathrm{A}$ \\
\hline UN_ci_3249 & $230 \mathrm{C} / \mathrm{T}$ \\
\hline UN_ci_3249 & $262 \mathrm{G} / \mathrm{C}$ \\
\hline UN_ci_3268 & $50 \mathrm{~A} / \mathrm{G}$ \\
\hline UN_ci_3278 & $362 \mathrm{~A} / \mathrm{C}$ \\
\hline UN_ci_3280 & 202 T/A \\
\hline UN_ci_3280 & $266 \mathrm{G} / \mathrm{A}$ \\
\hline UN_ci_3282 & $195 \mathrm{C} / \mathrm{T}$ \\
\hline UN_ci_3282 & $339 \mathrm{~T} / \mathrm{C}$ \\
\hline UN_ci_3282 & $441 \mathrm{C} / \mathrm{T}$ \\
\hline UN_ci_3282 & $777 \mathrm{G} / \mathrm{A}$ \\
\hline UN_ci_3282 & $938 \mathrm{C} / \mathrm{T}$ \\
\hline UN_ci_3282 & $1296 \mathrm{C} / \mathrm{T}$ \\
\hline UN_ci_3282 & $1511 \mathrm{~T} / \mathrm{C}$ \\
\hline
\end{tabular}




\begin{tabular}{|c|c|}
\hline UN_ci_3282 & $1546 \mathrm{~T} / \mathrm{C}$ \\
\hline UN_ci_3282 & $1600 \mathrm{~T} / \mathrm{C}$ \\
\hline UN_ci_3282 & $1770 \mathrm{G} / \mathrm{A}$ \\
\hline UN_ci_3282 & $1813 \mathrm{~A} / \mathrm{T}$ \\
\hline UN_ci_3287 & $366 \mathrm{~T} / \mathrm{C}$ \\
\hline UN_ci_3287 & 396 G/A \\
\hline UN_ci_3287 & $560 \mathrm{G} / \mathrm{C}$ \\
\hline UN_ci_3287 & $629 \mathrm{G} / \mathrm{A}$ \\
\hline UN_ci_3287 & $731 \mathrm{G} / \mathrm{T}$ \\
\hline UN_ci_3313 & $226 \mathrm{C} / \mathrm{A}$ \\
\hline UN_ci_3314 & $85 \mathrm{~T} / \mathrm{C}$ \\
\hline UN_ci_3317 & $122 \mathrm{G} / \mathrm{C}$ \\
\hline UN_ci_3325 & $245 \mathrm{C} / \mathrm{T}$ \\
\hline UN_ci_3326 & $6 \mathrm{G} / \mathrm{A}$ \\
\hline UN_ci_3338 & $492 \mathrm{~T} / \mathrm{C}$ \\
\hline UN_ci_3338 & $822 \mathrm{C} / \mathrm{T}$ \\
\hline UN_ci_3340 & $159 \mathrm{~A} / \mathrm{T}$ \\
\hline UN_ci_3354 & $279 \mathrm{C} / \mathrm{T}$ \\
\hline UN_ci_3355 & $1056 \mathrm{~A} / \mathrm{G}$ \\
\hline UN_ci_3355 & $1322 \mathrm{~T} / \mathrm{C}$ \\
\hline UN_ci_3356 & $484 \mathrm{~T} / \mathrm{A}$ \\
\hline UN_ci_3356 & $590 \mathrm{~T} / \mathrm{G}$ \\
\hline UN_ci_3356 & $665 \mathrm{G} / \mathrm{A}$ \\
\hline UN_ci_3361 & 326 G/A \\
\hline UN_ci_3370 & $86 \mathrm{~A} / \mathrm{G}$ \\
\hline UN_ci_3370 & $196 \mathrm{C} / \mathrm{G}$ \\
\hline UN_ci_3370 & 439 A/G \\
\hline UN_ci_3370 & $466 \mathrm{G} / \mathrm{T}$ \\
\hline UN_ci_3370 & $529 \mathrm{~A} / \mathrm{T}$ \\
\hline UN_ci_3390 & $177 \mathrm{~A} / \mathrm{G}$ \\
\hline UN_ci_3390 & 229 G/A \\
\hline UN_ci_3391 & $153 \mathrm{~T} / \mathrm{C}$ \\
\hline UN_ci_3391 & $361 \mathrm{~A} / \mathrm{C}$ \\
\hline UN_ci_3396 & $346 \mathrm{G} / \mathrm{A}$ \\
\hline UN_ci_3401 & $89 \mathrm{C} / \mathrm{T}$ \\
\hline UN_ci_3403 & $201 \mathrm{G} / \mathrm{A}$ \\
\hline UN_ci_3403 & $380 \mathrm{~A} / \mathrm{G}$ \\
\hline UN_ci_3403 & $407 \mathrm{G} / \mathrm{A}$ \\
\hline UN_ci_3407 & $7 \mathrm{C} / \mathrm{T}$ \\
\hline UN_ci_3407 & $43 \mathrm{~T} / \mathrm{C}$ \\
\hline UN_ci_3407 & $75 \mathrm{~A} / \mathrm{T}$ \\
\hline UN_ci_3407 & $145 \mathrm{C} / \mathrm{T}$ \\
\hline UN_ci_3407 & $169 \mathrm{C} / \mathrm{G}$ \\
\hline UN_ci_3407 & $218 \mathrm{C} / \mathrm{A}$ \\
\hline UN_ci_3407 & $240 \mathrm{C} / \mathrm{G}$ \\
\hline UN_ci_3407 & $282 \mathrm{~A} / \mathrm{T}$ \\
\hline UN_ci_3407 & $376 \mathrm{~T} / \mathrm{A}$ \\
\hline UN_ci_3409 & $291 \mathrm{~T} / \mathrm{G}$ \\
\hline UN_ci_3412 & $752 \mathrm{G} / \mathrm{A}$ \\
\hline UN_ci_3414 & $845 \mathrm{~A} / \mathrm{C}$ \\
\hline
\end{tabular}




\begin{tabular}{|c|c|}
\hline UN_ci_3414 & 994 T/G \\
\hline UN_ci_3417 & $105 \mathrm{~A} / \mathrm{C}$ \\
\hline UN_ci_3417 & $584 \mathrm{C} / \mathrm{T}$ \\
\hline UN_ci_3417 & $842 \mathrm{G} / \mathrm{C}$ \\
\hline UN_ci_3417 & $897 \mathrm{~A} / \mathrm{G}$ \\
\hline UN_ci_3418 & $13 \mathrm{G} / \mathrm{T}$ \\
\hline UN_ci_3418 & $148 \mathrm{C} / \mathrm{T}$ \\
\hline UN_ci_3418 & $342 \mathrm{~T} / \mathrm{C}$ \\
\hline UN_ci_3418 & $391 \mathrm{~A} / \mathrm{G}$ \\
\hline UN_ci_3418 & $569 \mathrm{G} / \mathrm{A}$ \\
\hline UN_ci_3418 & $590 \mathrm{~A} / \mathrm{G}$ \\
\hline UN_ci_3426 & $15 \mathrm{~T} / \mathrm{A}$ \\
\hline UN_ci_3434 & $229 \mathrm{C} / \mathrm{T}$ \\
\hline UN_ci_3434 & $270 \mathrm{~T} / \mathrm{C}$ \\
\hline UN_ci_3434 & $619 \mathrm{G} / \mathrm{A}$ \\
\hline UN_ci_3434 & $694 \mathrm{~A} / \mathrm{G}$ \\
\hline UN_ci_3439 & $108 \mathrm{~T} / \mathrm{C}$ \\
\hline UN_ci_3440 & $436 \mathrm{C} / \mathrm{A}$ \\
\hline UN_ci_3449 & $242 \mathrm{G} / \mathrm{T}$ \\
\hline UN_ci_3452 & $173 \mathrm{~A} / \mathrm{G}$ \\
\hline UN_ci_3453 & $8 \mathrm{~A} / \mathrm{G}$ \\
\hline UN_ci_3453 & $62 \mathrm{G} / \mathrm{A}$ \\
\hline UN_ci_3453 & $83 \mathrm{C} / \mathrm{T}$ \\
\hline UN_ci_3453 & $636 \mathrm{~T} / \mathrm{C}$ \\
\hline UN_ci_3469 & $345 \mathrm{~T} / \mathrm{C}$ \\
\hline UN_ci_3469 & $490 \mathrm{~T} / \mathrm{C}$ \\
\hline UN_ci_3478 & $291 \mathrm{~A} / \mathrm{G}$ \\
\hline UN_ci_3481 & $24 \mathrm{C} / \mathrm{T}$ \\
\hline UN_ci_3485 & $301 \mathrm{G} / \mathrm{A}$ \\
\hline UN_ci_3486 & $139 \mathrm{C} / \mathrm{T}$ \\
\hline UN_ci_3488 & $749 \mathrm{G} / \mathrm{A}$ \\
\hline UN_ci_3488 & $1445 \mathrm{C} / \mathrm{G}$ \\
\hline UN_ci_3495 & $167 \mathrm{~T} / \mathrm{C}$ \\
\hline UN_ci_3495 & $272 \mathrm{~T} / \mathrm{A}$ \\
\hline UN_ci_3497 & $216 \mathrm{~A} / \mathrm{T}$ \\
\hline UN_ci_3497 & $373 \mathrm{C} / \mathrm{T}$ \\
\hline UN_ci_3497 & $508 \mathrm{~A} / \mathrm{C}$ \\
\hline UN_ci_3497 & $538 \mathrm{C} / \mathrm{T}$ \\
\hline UN_ci_3498 & $392 \mathrm{~T} / \mathrm{C}$ \\
\hline UN_ci_3503 & $952 \mathrm{G} / \mathrm{A}$ \\
\hline UN_ci_3505 & $404 \mathrm{~A} / \mathrm{C}$ \\
\hline UN_ci_3505 & $524 \mathrm{~A} / \mathrm{G}$ \\
\hline UN_ci_3505 & $646 \mathrm{~T} / \mathrm{C}$ \\
\hline UN_ci_3505 & $846 \mathrm{~A} / \mathrm{G}$ \\
\hline UN_ci_3507 & $444 \mathrm{~A} / \mathrm{G}$ \\
\hline UN_ci_3510 & $601 \mathrm{~A} / \mathrm{G}$ \\
\hline UN_ci_3516 & $155 \mathrm{G} / \mathrm{A}$ \\
\hline UN_ci_3516 & $210 \mathrm{G} / \mathrm{C}$ \\
\hline UN_ci_3520 & $402 \mathrm{~T} / \mathrm{G}$ \\
\hline UN_ci_3531 & $386 \mathrm{C} / \mathrm{A}$ \\
\hline
\end{tabular}




\begin{tabular}{|c|c|}
\hline UN_ci_3531 & $430 \mathrm{G} / \mathrm{A}$ \\
\hline UN_ci_3534 & $172 \mathrm{G} / \mathrm{C}$ \\
\hline UN_ci_3536 & $364 \mathrm{C} / \mathrm{A}$ \\
\hline UN_ci_3536 & $405 \mathrm{~T} / \mathrm{C}$ \\
\hline UN_ci_3557 & $418 \mathrm{G} / \mathrm{A}$ \\
\hline UN_ci_3559 & $108 \mathrm{~A} / \mathrm{G}$ \\
\hline UN_ci_3559 & $250 \mathrm{G} / \mathrm{A}$ \\
\hline UN_ci_3568 & $239 \mathrm{C} / \mathrm{T}$ \\
\hline UN_ci_3574 & $1166 \mathrm{C} / \mathrm{T}$ \\
\hline UN_ci_3576 & $1192 \mathrm{C} / \mathrm{T}$ \\
\hline UN_ci_3578 & 519 A/G \\
\hline UN_ci_3578 & $576 \mathrm{~T} / \mathrm{C}$ \\
\hline UN_ci_3578 & $610 \mathrm{G} / \mathrm{A}$ \\
\hline UN_ci_3578 & $684 \mathrm{~A} / \mathrm{G}$ \\
\hline UN_ci_3586 & $385 \mathrm{~T} / \mathrm{C}$ \\
\hline UN_ci_3592 & $400 \mathrm{C} / \mathrm{T}$ \\
\hline UN_ci_3593 & $326 \mathrm{G} / \mathrm{A}$ \\
\hline UN_ci_3593 & $478 \mathrm{~T} / \mathrm{G}$ \\
\hline UN_ci_3594 & $288 \mathrm{~T} / \mathrm{G}$ \\
\hline UN_ci_3594 & $408 \mathrm{~A} / \mathrm{G}$ \\
\hline UN_ci_3594 & $433 \mathrm{~T} / \mathrm{C}$ \\
\hline UN_ci_3596 & $416 \mathrm{G} / \mathrm{A}$ \\
\hline UN_ci_3597 & $314 \mathrm{G} / \mathrm{A}$ \\
\hline UN_ci_3600 & $255 \mathrm{~T} / \mathrm{C}$ \\
\hline UN_ci_3600 & 599 A/G \\
\hline UN_ci_3600 & $870 \mathrm{G} / \mathrm{T}$ \\
\hline UN_ci_3600 & $998 \mathrm{~T} / \mathrm{C}$ \\
\hline UN_ci_3601 & $172 \mathrm{~A} / \mathrm{T}$ \\
\hline UN_ci_3601 & $195 \mathrm{C} / \mathrm{T}$ \\
\hline UN_ci_3601 & $225 \mathrm{G} / \mathrm{A}$ \\
\hline UN_ci_3601 & $256 \mathrm{~T} / \mathrm{C}$ \\
\hline UN_ci_3601 & $283 \mathrm{~A} / \mathrm{G}$ \\
\hline UN_ci_3601 & $312 \mathrm{C} / \mathrm{T}$ \\
\hline UN_ci_3601 & $337 \mathrm{~T} / \mathrm{C}$ \\
\hline UN_ci_3601 & $380 \mathrm{~A} / \mathrm{G}$ \\
\hline UN_ci_3601 & $404 \mathrm{C} / \mathrm{G}$ \\
\hline UN_ci_3601 & $441 \mathrm{~A} / \mathrm{G}$ \\
\hline UN_ci_3601 & $469 \mathrm{~A} / \mathrm{G}$ \\
\hline UN_ci_3601 & $489 \mathrm{~A} / \mathrm{G}$ \\
\hline UN_ci_3601 & $521 \mathrm{~T} / \mathrm{C}$ \\
\hline UN_ci_3601 & $563 \mathrm{G} / \mathrm{C}$ \\
\hline UN_ci_3605 & $119 \mathrm{~A} / \mathrm{C}$ \\
\hline UN_ci_3605 & $219 \mathrm{G} / \mathrm{A}$ \\
\hline UN_ci_3605 & $262 \mathrm{C} / \mathrm{T}$ \\
\hline UN_ci_3605 & $344 \mathrm{G} / \mathrm{A}$ \\
\hline UN_ci_3610 & $151 \mathrm{~T} / \mathrm{G}$ \\
\hline UN_ci_3610 & $399 \mathrm{G} / \mathrm{A}$ \\
\hline UN_ci_3611 & $45 \mathrm{~T} / \mathrm{G}$ \\
\hline UN_ci_3611 & $192 \mathrm{~T} / \mathrm{C}$ \\
\hline UN_ci_3611 & $521 \mathrm{C} / \mathrm{T}$ \\
\hline
\end{tabular}




\begin{tabular}{|c|c|}
\hline UN_ci_3611 & $584 \mathrm{C} / \mathrm{A}$ \\
\hline UN_ci_3611 & $712 \mathrm{~A} / \mathrm{T}$ \\
\hline UN_ci_3612 & $195 \mathrm{C} / \mathrm{T}$ \\
\hline UN_ci_3621 & $192 \mathrm{~A} / \mathrm{G}$ \\
\hline UN_ci_3621 & $239 \mathrm{~A} / \mathrm{G}$ \\
\hline UN_ci_3628 & $468 \mathrm{G} / \mathrm{A}$ \\
\hline UN_ci_3628 & $681 \mathrm{~T} / \mathrm{C}$ \\
\hline UN_ci_3632 & $240 \mathrm{~A} / \mathrm{G}$ \\
\hline UN_ci_3632 & $271 \mathrm{C} / \mathrm{T}$ \\
\hline UN_ci_3638 & $97 \mathrm{~T} / \mathrm{A}$ \\
\hline UN_ci_3642 & $315 \mathrm{G} / \mathrm{T}$ \\
\hline UN_ci_3645 & $365 \mathrm{C} / \mathrm{T}$ \\
\hline UN_ci_3645 & $500 \mathrm{~A} / \mathrm{G}$ \\
\hline UN_ci_3645 & $722 \mathrm{~T} / \mathrm{A}$ \\
\hline UN_ci_3645 & $872 \mathrm{G} / \mathrm{A}$ \\
\hline UN_ci_3645 & $906 \mathrm{~A} / \mathrm{T}$ \\
\hline UN_ci_3652 & $1076 \mathrm{~A} / \mathrm{T}$ \\
\hline UN_ci_3652 & $1119 \mathrm{C} / \mathrm{T}$ \\
\hline UN_ci_3655 & $13 \mathrm{G} / \mathrm{A}$ \\
\hline UN_ci_3655 & $211 \mathrm{~T} / \mathrm{C}$ \\
\hline UN_ci_3655 & $252 \mathrm{C} / \mathrm{T}$ \\
\hline UN_ci_3655 & $373 \mathrm{~T} / \mathrm{A}$ \\
\hline UN_ci_3655 & $419 \mathrm{~T} / \mathrm{C}$ \\
\hline UN_ci_3655 & $580 \mathrm{C} / \mathrm{A}$ \\
\hline UN_ci_3655 & $626 \mathrm{C} / \mathrm{T}$ \\
\hline UN_ci_3655 & $687 \mathrm{C} / \mathrm{T}$ \\
\hline UN_ci_3655 & $727 \mathrm{~A} / \mathrm{G}$ \\
\hline UN_ci_3655 & $793 \mathrm{~T} / \mathrm{A}$ \\
\hline UN_ci_3655 & $918 \mathrm{~T} / \mathrm{C}$ \\
\hline UN_ci_3655 & $981 \mathrm{~T} / \mathrm{A}$ \\
\hline UN_ci_3655 & $1023 \mathrm{G} / \mathrm{A}$ \\
\hline UN_ci_3655 & $1047 \mathrm{C} / \mathrm{A}$ \\
\hline UN_ci_3655 & $1135 \mathrm{C} / \mathrm{A}$ \\
\hline UN_ci_3657 & $373 \mathrm{G} / \mathrm{A}$ \\
\hline UN_ci_3657 & $464 \mathrm{C} / \mathrm{G}$ \\
\hline UN_ci_3658 & $201 \mathrm{~A} / \mathrm{T}$ \\
\hline UN_ci_3658 & $402 \mathrm{~A} / \mathrm{G}$ \\
\hline UN_ci_3660 & $170 \mathrm{G} / \mathrm{A}$ \\
\hline UN_ci_3661 & $395 \mathrm{C} / \mathrm{T}$ \\
\hline UN_ci_3666 & 89 A/G \\
\hline UN_ci_3666 & $224 \mathrm{C} / \mathrm{G}$ \\
\hline UN_ci_3666 & $451 \mathrm{~A} / \mathrm{G}$ \\
\hline UN_ci_3669 & $166 \mathrm{~T} / \mathrm{C}$ \\
\hline UN_ci_3669 & $272 \mathrm{~T} / \mathrm{C}$ \\
\hline UN_ci_3669 & $469 \mathrm{G} / \mathrm{A}$ \\
\hline UN_ci_3680 & $13 \mathrm{C} / \mathrm{T}$ \\
\hline UN_ci_3680 & $401 \mathrm{C} / \mathrm{G}$ \\
\hline UN_ci_3680 & $438 \mathrm{G} / \mathrm{A}$ \\
\hline UN_ci_3680 & $484 \mathrm{~T} / \mathrm{C}$ \\
\hline UN_ci_3680 & $551 \mathrm{~A} / \mathrm{G}$ \\
\hline
\end{tabular}




\begin{tabular}{|c|c|}
\hline UN_ci_3680 & $571 \mathrm{C} / \mathrm{T}$ \\
\hline UN_ci_3680 & $655 \mathrm{~A} / \mathrm{G}$ \\
\hline UN_ci_3680 & $720 \mathrm{~T} / \mathrm{A}$ \\
\hline UN_ci_3680 & $782 \mathrm{~T} / \mathrm{C}$ \\
\hline UN_ci_3680 & $891 \mathrm{~T} / \mathrm{G}$ \\
\hline UN_ci_3680 & $940 \mathrm{~T} / \mathrm{A}$ \\
\hline UN_ci_3682 & $31 \mathrm{G} / \mathrm{C}$ \\
\hline UN_ci_3682 & $103 \mathrm{C} / \mathrm{T}$ \\
\hline UN_ci_3682 & $129 \mathrm{~A} / \mathrm{G}$ \\
\hline UN_ci_3682 & $268 \mathrm{~A} / \mathrm{T}$ \\
\hline UN_ci_3682 & $339 \mathrm{~A} / \mathrm{G}$ \\
\hline UN_ci_3682 & $621 \mathrm{~A} / \mathrm{G}$ \\
\hline UN_ci_3682 & $710 \mathrm{C} / \mathrm{T}$ \\
\hline UN_ci_3690 & $7 \mathrm{~A} / \mathrm{G}$ \\
\hline UN_ci_3690 & $50 \mathrm{~T} / \mathrm{C}$ \\
\hline UN_ci_3690 & $116 \mathrm{G} / \mathrm{A}$ \\
\hline UN_ci_3690 & $152 \mathrm{~T} / \mathrm{C}$ \\
\hline UN_ci_3690 & $261 \mathrm{~T} / \mathrm{C}$ \\
\hline UN_ci_3690 & $366 \mathrm{G} / \mathrm{A}$ \\
\hline UN_ci_3690 & $455 \mathrm{C} / \mathrm{T}$ \\
\hline UN_ci_3692 & $81 \mathrm{~A} / \mathrm{G}$ \\
\hline UN_ci_3696 & $495 \mathrm{~A} / \mathrm{C}$ \\
\hline UN_ci_3698 & $300 \mathrm{G} / \mathrm{A}$ \\
\hline UN_ci_3698 & $607 \mathrm{~A} / \mathrm{C}$ \\
\hline UN_ci_3709 & $66 \mathrm{G} / \mathrm{T}$ \\
\hline UN_ci_3709 & $93 \mathrm{~T} / \mathrm{C}$ \\
\hline UN_ci_3709 & $114 \mathrm{~A} / \mathrm{G}$ \\
\hline UN_ci_3709 & $164 \mathrm{~A} / \mathrm{G}$ \\
\hline UN_ci_3709 & $207 \mathrm{G} / \mathrm{A}$ \\
\hline UN_ci_3709 & $238 \mathrm{~A} / \mathrm{G}$ \\
\hline UN_ci_3709 & $294 \mathrm{~A} / \mathrm{C}$ \\
\hline UN_ci_3709 & $315 \mathrm{G} / \mathrm{A}$ \\
\hline UN_ci_3709 & $347 \mathrm{~T} / \mathrm{C}$ \\
\hline UN_ci_3709 & $376 \mathrm{~T} / \mathrm{C}$ \\
\hline UN_ci_3709 & $417 \mathrm{~T} / \mathrm{C}$ \\
\hline UN_ci_3709 & $437 \mathrm{~T} / \mathrm{G}$ \\
\hline UN_ci_3714 & $229 \mathrm{G} / \mathrm{T}$ \\
\hline UN_ci_3719 & $218 \mathrm{~A} / \mathrm{C}$ \\
\hline UN_ci_3721 & $70 \mathrm{~T} / \mathrm{G}$ \\
\hline UN_ci_3721 & $103 \mathrm{~T} / \mathrm{C}$ \\
\hline UN_ci_3721 & $126 \mathrm{~T} / \mathrm{C}$ \\
\hline UN_ci_3721 & $161 \mathrm{~A} / \mathrm{G}$ \\
\hline UN_ci_3721 & $200 \mathrm{~A} / \mathrm{T}$ \\
\hline UN_ci_3721 & $332 \mathrm{~T} / \mathrm{C}$ \\
\hline UN_ci_3721 & $658 \mathrm{~A} / \mathrm{C}$ \\
\hline UN_ci_3721 & $744 \mathrm{~T} / \mathrm{C}$ \\
\hline UN_ci_3721 & $807 \mathrm{G} / \mathrm{T}$ \\
\hline UN_ci_3721 & $892 \mathrm{~T} / \mathrm{C}$ \\
\hline UN_ci_3721 & $918 \mathrm{C} / \mathrm{A}$ \\
\hline UN_ci_3721 & $967 \mathrm{G} / \mathrm{A}$ \\
\hline
\end{tabular}




\begin{tabular}{|c|c|}
\hline UN_ci_3721 & $989 \mathrm{~T} / \mathrm{C}$ \\
\hline UN_ci_3721 & $1044 \mathrm{C} / \mathrm{A}$ \\
\hline UN_ci_3726 & $602 \mathrm{G} / \mathrm{A}$ \\
\hline UN_ci_3726 & $964 \mathrm{C} / \mathrm{A}$ \\
\hline UN_ci_3730 & $300 \mathrm{C} / \mathrm{A}$ \\
\hline UN_ci_3730 & $366 \mathrm{~A} / \mathrm{G}$ \\
\hline UN_ci_3735 & $43 \mathrm{G} / \mathrm{A}$ \\
\hline UN_ci_3736 & $298 \mathrm{~A} / \mathrm{G}$ \\
\hline UN_ci_3753 & $80 \mathrm{~A} / \mathrm{G}$ \\
\hline UN_ci_3753 & $124 \mathrm{~A} / \mathrm{G}$ \\
\hline UN_ci_3753 & $155 \mathrm{~T} / \mathrm{A}$ \\
\hline UN_ci_3753 & $193 \mathrm{~A} / \mathrm{T}$ \\
\hline UN_ci_3753 & $245 \mathrm{~A} / \mathrm{G}$ \\
\hline UN_ci_3753 & $288 \mathrm{~A} / \mathrm{G}$ \\
\hline UN_ci_3753 & $339 \mathrm{~A} / \mathrm{T}$ \\
\hline UN_ci_3753 & $479 \mathrm{~A} / \mathrm{G}$ \\
\hline UN_ci_3753 & $500 \mathrm{~T} / \mathrm{C}$ \\
\hline UN_ci_3753 & $641 \mathrm{~T} / \mathrm{C}$ \\
\hline UN_ci_3755 & $433 \mathrm{~A} / \mathrm{G}$ \\
\hline UN_ci_3755 & $511 \mathrm{G} / \mathrm{A}$ \\
\hline UN_ci_3756 & $263 \mathrm{G} / \mathrm{A}$ \\
\hline UN_ci_3756 & $469 \mathrm{C} / \mathrm{T}$ \\
\hline UN_ci_3765 & $223 \mathrm{~T} / \mathrm{A}$ \\
\hline UN_ci_3768 & 59 A/G \\
\hline UN_ci_3771 & $228 \mathrm{G} / \mathrm{A}$ \\
\hline UN_ci_3775 & $71 \mathrm{~T} / \mathrm{G}$ \\
\hline UN_ci_3775 & $91 \mathrm{G} / \mathrm{C}$ \\
\hline UN_ci_3785 & $264 \mathrm{G} / \mathrm{A}$ \\
\hline UN_ci_3785 & $310 \mathrm{~A} / \mathrm{G}$ \\
\hline UN_ci_3797 & $407 \mathrm{G} / \mathrm{C}$ \\
\hline UN_ci_3800 & $129 \mathrm{C} / \mathrm{A}$ \\
\hline UN_ci_3805 & $6 \mathrm{C} / \mathrm{G}$ \\
\hline UN_ci_3805 & $127 \mathrm{~A} / \mathrm{C}$ \\
\hline UN_ci_3805 & $534 \mathrm{C} / \mathrm{G}$ \\
\hline UN_ci_3805 & $587 \mathrm{C} / \mathrm{T}$ \\
\hline UN_ci_3806 & $40 \mathrm{~A} / \mathrm{G}$ \\
\hline UN_ci_3806 & $245 \mathrm{~A} / \mathrm{G}$ \\
\hline UN_ci_3806 & 292 T/C \\
\hline UN_ci_3806 & $410 \mathrm{~A} / \mathrm{G}$ \\
\hline UN_ci_3806 & $463 \mathrm{C} / \mathrm{T}$ \\
\hline UN_ci_3806 & $496 \mathrm{G} / \mathrm{A}$ \\
\hline UN_ci_3806 & $543 \mathrm{~A} / \mathrm{G}$ \\
\hline UN_ci_3806 & $713 \mathrm{G} / \mathrm{A}$ \\
\hline UN_ci_3806 & $745 \mathrm{G} / \mathrm{A}$ \\
\hline UN_ci_3808 & $229 \mathrm{~A} / \mathrm{G}$ \\
\hline UN_ci_3808 & $337 \mathrm{~T} / \mathrm{C}$ \\
\hline UN_ci_3818 & $638 \mathrm{C} / \mathrm{T}$ \\
\hline UN_ci_3821 & $37 \mathrm{~A} / \mathrm{C}$ \\
\hline UN_ci_3821 & $85 \mathrm{G} / \mathrm{A}$ \\
\hline UN_ci_3822 & $334 \mathrm{~T} / \mathrm{G}$ \\
\hline
\end{tabular}




$\begin{array}{lr}\text { UN_ci_3822 } & 376 \mathrm{~T} / \mathrm{C} \\ \text { UN_ci_3822 } & 472 \mathrm{~A} / \mathrm{G} \\ \text { UN_ci_3822 } & 500 \mathrm{~A} / \mathrm{G} \\ \text { UN_ci_3822 } & 668 \mathrm{C} / \mathrm{T} \\ \text { UN_ci_3826 } & 258 \mathrm{~A} / \mathrm{G} \\ \text { UN_ci_3826 } & 427 \mathrm{G} / \mathrm{A} \\ \text { UN_ci_3826 } & 495 \mathrm{G} / \mathrm{A} \\ \text { UN_ci_3826 } & 688 \mathrm{G} / \mathrm{T} \\ \text { UN_ci_3834 } & 274 \mathrm{G} / \mathrm{T} \\ \text { UN_ci_3834 } & 391 \mathrm{~T} / \mathrm{C} \\ \text { UN_ci_3836 } & 575 \mathrm{~A} / \mathrm{G} \\ \text { UN_ci_3837 } & 181 \mathrm{C} / \mathrm{A} \\ \text { UN_ci_3837 } & 229 \mathrm{~A} / \mathrm{G} \\ \text { UN_ci_3837 } & 376 \mathrm{~T} / \mathrm{C} \\ \text { UN_ci_3837 } & 446 \mathrm{~A} / \mathrm{G} \\ \text { UN_ci_3837 } & 481 \mathrm{~A} / \mathrm{G} \\ \text { UN_ci_3837 } & 622 \mathrm{~T} / \mathrm{A} \\ \text { UN_ci_3844 } & 7 \mathrm{C} / \mathrm{G} \\ \text { UN_ci_3848 } & 123 \mathrm{C} / \mathrm{A} \\ \text { UN_ci_3850 } & 18 \mathrm{C} / \mathrm{G} \\ \text { UN_ci_3850 } & 247 \mathrm{~T} / \mathrm{C} \\ \text { UN_ci_3850 } & 311 \mathrm{~A} / \mathrm{G} \\ \text { UN_ci_3850 } & 276 \mathrm{C} / \mathrm{A} \\ \text { UN_ci_3850 } & 724 \mathrm{C} / \mathrm{T} \\ \text { UN_ci_3850 } & \end{array}$




\begin{tabular}{|c|c|}
\hline UN_ci_3927 & $246 \mathrm{~A} / \mathrm{G}$ \\
\hline UN_ci_3927 & $360 \mathrm{G} / \mathrm{A}$ \\
\hline UN_ci_3927 & $456 \mathrm{~T} / \mathrm{C}$ \\
\hline UN_ci_3942 & $549 \mathrm{C} / \mathrm{A}$ \\
\hline UN_ci_3942 & $1031 \mathrm{~T} / \mathrm{C}$ \\
\hline UN_ci_3942 & $1080 \mathrm{~T} / \mathrm{C}$ \\
\hline UN_ci_3942 & $1330 \mathrm{~T} / \mathrm{C}$ \\
\hline UN_ci_3949 & $140 \mathrm{G} / \mathrm{A}$ \\
\hline UN_ci_3949 & $200 \mathrm{~A} / \mathrm{T}$ \\
\hline UN_ci_3950 & $207 \mathrm{~A} / \mathrm{C}$ \\
\hline UN_ci_3964 & $170 \mathrm{G} / \mathrm{A}$ \\
\hline UN_ci_3968 & $667 \mathrm{G} / \mathrm{A}$ \\
\hline UN_ci_3968 & $985 \mathrm{~T} / \mathrm{C}$ \\
\hline UN_ci_3973 & $389 \mathrm{G} / \mathrm{A}$ \\
\hline UN_ci_3976 & $228 \mathrm{~A} / \mathrm{G}$ \\
\hline UN_ci_3998 & $219 \mathrm{G} / \mathrm{T}$ \\
\hline UN_ci_4006 & $112 \mathrm{C} / \mathrm{A}$ \\
\hline UN_ci_4006 & $148 \mathrm{G} / \mathrm{A}$ \\
\hline UN_ci_4006 & 199 T/C \\
\hline UN_ci_4006 & $225 \mathrm{C} / \mathrm{T}$ \\
\hline UN_ci_4006 & $269 \mathrm{~A} / \mathrm{G}$ \\
\hline UN_ci_4006 & 319 T/C \\
\hline UN_ci_4006 & $365 \mathrm{~A} / \mathrm{C}$ \\
\hline UN_ci_4006 & 390 A/G \\
\hline UN_ci_4006 & $447 \mathrm{~A} / \mathrm{G}$ \\
\hline UN_ci_4006 & $467 \mathrm{~T} / \mathrm{C}$ \\
\hline UN_ci_4006 & $512 \mathrm{C} / \mathrm{T}$ \\
\hline UN_ci_4006 & $534 \mathrm{~T} / \mathrm{C}$ \\
\hline UN_ci_4006 & $554 \mathrm{G} / \mathrm{A}$ \\
\hline UN_ci_4006 & $577 \mathrm{C} / \mathrm{T}$ \\
\hline UN_ci_4006 & $597 \mathrm{~T} / \mathrm{G}$ \\
\hline UN_ci_4006 & $626 \mathrm{~T} / \mathrm{C}$ \\
\hline UN_ci_4006 & $651 \mathrm{G} / \mathrm{T}$ \\
\hline UN_ci_4006 & $675 \mathrm{C} / \mathrm{T}$ \\
\hline UN_ci_4006 & $701 \mathrm{~A} / \mathrm{G}$ \\
\hline UN_ci_4006 & 739 A/G \\
\hline UN_ci_4006 & $803 \mathrm{~A} / \mathrm{G}$ \\
\hline UN_ci_4006 & $868 \mathrm{~A} / \mathrm{G}$ \\
\hline UN_ci_4006 & 915 G/C \\
\hline UN_ci_4010 & $227 \mathrm{G} / \mathrm{T}$ \\
\hline UN_ci_4020 & $168 \mathrm{C} / \mathrm{T}$ \\
\hline UN_ci_4020 & $350 \mathrm{~T} / \mathrm{C}$ \\
\hline UN_ci_4020 & 396 A/G \\
\hline UN_ci_4020 & $431 \mathrm{~A} / \mathrm{T}$ \\
\hline UN_ci_4020 & $452 \mathrm{~T} / \mathrm{C}$ \\
\hline UN_ci_4020 & $475 \mathrm{~A} / \mathrm{T}$ \\
\hline UN_ci_4020 & $496 \mathrm{~A} / \mathrm{G}$ \\
\hline UN_ci_4022 & $46 \mathrm{~T} / \mathrm{C}$ \\
\hline UN_ci_4023 & $565 \mathrm{C} / \mathrm{T}$ \\
\hline UN_ci_4023 & $648 \mathrm{~T} / \mathrm{C}$ \\
\hline
\end{tabular}




$\begin{array}{lr}\text { UN_ci_4024 } & 810 \mathrm{G} / \mathrm{A} \\ \text { UN_ci_4040 } & 386 \mathrm{G} / \mathrm{A} \\ \text { UN_ci_4040 } & 470 \mathrm{~T} / \mathrm{A} \\ \text { UN_ci_4048 } & 472 \mathrm{~A} / \mathrm{G} \\ \text { UN_ci_4049 } & 103 \mathrm{~A} / \mathrm{G} \\ \text { UN_ci_4050 } & 76 \mathrm{~A} / \mathrm{G} \\ \text { UN_ci_4057 } & 371 \mathrm{C} / \mathrm{T} \\ \text { UN_ci_4059 } & 300 \mathrm{G} / \mathrm{A} \\ \text { UN_ci_4060 } & 162 \mathrm{G} / \mathrm{A} \\ \text { UN_ci_4060 } & 329 \mathrm{C} / \mathrm{T} \\ \text { UN_ci_4060 } & 362 \mathrm{G} / \mathrm{A} \\ \text { UN_ci_4064 } & 141 \mathrm{C} / \mathrm{T} \\ \text { UN_ci_4064 } & 339 \mathrm{~A} / \mathrm{T} \\ \text { UN_ci_4064 } & 627 \mathrm{G} / \mathrm{T} \\ \text { UN_ci_4069 } & 472 \mathrm{C} / \mathrm{T} \\ \text { UN_ci_4069 } & 1035 \mathrm{~A} / \mathrm{G} \\ \text { UN_ci_4072 } & 480 \mathrm{C} / \mathrm{T} \\ \text { UN_ci_4073 } & 8 \mathrm{G} / \mathrm{A} \\ \text { UN_ci_4073 } & 263 \mathrm{~T} / \mathrm{C} \\ \text { UN_ci_4073 } & 407 \mathrm{~A} / \mathrm{G} \\ \text { UN_ci_4079 } & 178 \mathrm{~A} / \mathrm{G} \\ \text { UN_ci_4079 } & 39 \mathrm{G} / \mathrm{A} \\ \text { UN_ci_4085 } & 39 \mathrm{G} / \mathrm{A} / \mathrm{A} \\ \text { UN_ci_4086 } & 619 \mathrm{G} / \mathrm{C} \\ \text { UN_ci_4089 } & 423 \mathrm{G} / \mathrm{T} \\ \text { UN_ci_4091 } & \end{array}$




$\begin{array}{lr}\text { UN_ci_4160 } & 657 \mathrm{~T} / \mathrm{C} \\ \text { UN_ci_4160 } & 1034 \mathrm{~A} / \mathrm{G} \\ \text { UN_ci_4160 } & 1099 \mathrm{~T} / \mathrm{C} \\ \text { UN_ci_4161 } & 525 \mathrm{C} / \mathrm{A} \\ \text { UN_ci_4164 } & 545 \mathrm{G} / \mathrm{A} \\ \text { UN_ci_4165 } & 567 \mathrm{~A} / \mathrm{T} \\ \text { UN_ci_4168 } & 203 \mathrm{C} / \mathrm{T} \\ \text { UN_ci_4169 } & 324 \mathrm{~A} / \mathrm{C} \\ \text { UN_ci_4170 } & 146 \mathrm{~A} / \mathrm{G} \\ \text { UN_ci_4172 } & 871 \mathrm{C} / \mathrm{A} \\ \text { UN_ci_4182 } & 179 \mathrm{~A} / \mathrm{G} \\ \text { UN_ci_4186 } & 188 \mathrm{G} / \mathrm{A} \\ \text { UN_ci_4191 } & 543 \mathrm{~T} / \mathrm{G} \\ \text { UN_ci_4193 } & 241 \mathrm{G} / \mathrm{T} \\ \text { UN_ci_4193 } & 287 \mathrm{G} / \mathrm{A} \\ \text { UN_ci_4193 } & 317 \mathrm{G} / \mathrm{A} \\ \text { UN_ci_4193 } & 410 \mathrm{~T} / \mathrm{A} \\ \text { UN_ci_4193 } & 602 \mathrm{C} / \mathrm{T} \\ \text { UN_ci_4193 } & 629 \mathrm{C} / \mathrm{T} \\ \text { UN_ci_4193 } & 683 \mathrm{G} / \mathrm{A} \\ \text { UN_ci_4193 } & 1070 \mathrm{C} / \mathrm{A} \\ \text { UN_ci_4193 } & 745 \mathrm{C} / \mathrm{A} \\ \text { UN_ci_4193 } & \end{array}$




$\begin{array}{lr}\text { UN_ci_4217 } & 475 \mathrm{~T} / \mathrm{C} \\ \text { UN_ci_4217 } & 497 \mathrm{C} / \mathrm{T} \\ \text { UN_ci_4224 } & 175 \mathrm{~T} / \mathrm{C} \\ \text { UN_ci_4224 } & 352 \mathrm{G} / \mathrm{A} \\ \text { UN_ci_4226 } & 276 \mathrm{~T} / \mathrm{C} \\ \text { UN_ci_4227 } & 312 \mathrm{C} / \mathrm{T} \\ \text { UN_ci_4227 } & 344 \mathrm{~T} / \mathrm{A} \\ \text { UN_ci_4227 } & 408 \mathrm{~T} / \mathrm{C} \\ \text { UN_ci_4230 } & 279 \mathrm{G} / \mathrm{A} \\ \text { UN_ci_4230 } & 791 \mathrm{C} / \mathrm{A} \\ \text { UN_ci_4230 } & 823 \mathrm{G} / \mathrm{A} \\ \text { UN_ci_4230 } & 905 \mathrm{~T} / \mathrm{A} \\ \text { UN_ci_4250 } & 173 \mathrm{~A} / \mathrm{G} \\ \text { UN_ci_4252 } & 94 \mathrm{~T} / \mathrm{C} \\ \text { UN_ci_4252 } & 354 \mathrm{~A} / \mathrm{T} \\ \text { UN_ci_4256 } & 311 \mathrm{~A} / \mathrm{G} \\ \text { UN_ci_4256 } & 495 \mathrm{~A} / \mathrm{G} \\ \text { UN_ci_4256 } & 610 \mathrm{C} / \mathrm{A} \\ \text { UN_ci_4256 } & 654 \mathrm{~A} / \mathrm{G} \\ \text { UN_ci_4256 } & 801 \mathrm{~T} / \mathrm{C} \\ \text { UN_ci_4256 } & 832 \mathrm{C} / \mathrm{T} \\ \text { UN_ci_4256 } & 870 \mathrm{~T} / \mathrm{C} \\ \text { UN_ci_4256 } & 398 \mathrm{C} / \mathrm{T} \\ \text { UN_ci_4259 } & \end{array}$




\begin{tabular}{|c|c|}
\hline UN_ci_4316 & $248 \mathrm{~T} / \mathrm{C}$ \\
\hline UN_ci_4329 & $448 \mathrm{~T} / \mathrm{C}$ \\
\hline UN_ci_4329 & $583 \mathrm{~A} / \mathrm{T}$ \\
\hline UN_ci_4338 & $163 \mathrm{G} / \mathrm{T}$ \\
\hline UN_ci_4338 & 398 C/T \\
\hline UN_ci_4339 & $23 \mathrm{~T} / \mathrm{C}$ \\
\hline UN_ci_4339 & $171 \mathrm{~A} / \mathrm{C}$ \\
\hline UN_ci_4339 & $237 \mathrm{~T} / \mathrm{C}$ \\
\hline UN_ci_4339 & $258 \mathrm{G} / \mathrm{T}$ \\
\hline UN_ci_4342 & $725 \mathrm{C} / \mathrm{A}$ \\
\hline UN_ci_4343 & $6 \mathrm{~A} / \mathrm{G}$ \\
\hline UN_ci_4343 & $105 \mathrm{G} / \mathrm{A}$ \\
\hline UN_ci_4343 & $133 \mathrm{C} / \mathrm{A}$ \\
\hline UN_ci_4343 & $216 \mathrm{~T} / \mathrm{C}$ \\
\hline UN_ci_4343 & $247 \mathrm{G} / \mathrm{A}$ \\
\hline UN_ci_4343 & $299 \mathrm{C} / \mathrm{T}$ \\
\hline UN_ci_4343 & $321 \mathrm{G} / \mathrm{T}$ \\
\hline UN_ci_4343 & 356 A/G \\
\hline UN_ci_4343 & $377 \mathrm{G} / \mathrm{A}$ \\
\hline UN_ci_4343 & 397 A/G \\
\hline UN_ci_4343 & $423 \mathrm{~A} / \mathrm{G}$ \\
\hline UN_ci_4343 & $461 \mathrm{G} / \mathrm{A}$ \\
\hline UN_ci_4343 & $538 \mathrm{~T} / \mathrm{C}$ \\
\hline UN_ci_4343 & $564 \mathrm{~A} / \mathrm{G}$ \\
\hline UN_ci_4343 & $584 \mathrm{~A} / \mathrm{G}$ \\
\hline UN_ci_4343 & $609 \mathrm{~T} / \mathrm{C}$ \\
\hline UN_ci_4343 & $634 \mathrm{~A} / \mathrm{G}$ \\
\hline UN_ci_4343 & $671 \mathrm{~A} / \mathrm{C}$ \\
\hline UN_ci_4343 & $735 \mathrm{~T} / \mathrm{C}$ \\
\hline UN_ci_4343 & $758 \mathrm{~T} / \mathrm{C}$ \\
\hline UN_ci_4343 & $836 \mathrm{C} / \mathrm{T}$ \\
\hline UN_ci_4343 & $1394 \mathrm{C} / \mathrm{G}$ \\
\hline UN_ci_4343 & $1552 \mathrm{~T} / \mathrm{A}$ \\
\hline UN_ci_4348 & $317 \mathrm{~A} / \mathrm{G}$ \\
\hline UN_ci_4348 & $405 \mathrm{G} / \mathrm{A}$ \\
\hline UN_ci_4350 & $381 \mathrm{G} / \mathrm{A}$ \\
\hline UN_ci_4350 & $467 \mathrm{~A} / \mathrm{C}$ \\
\hline UN_ci_4350 & $856 \mathrm{G} / \mathrm{T}$ \\
\hline UN_ci_4352 & $476 \mathrm{~T} / \mathrm{C}$ \\
\hline UN_ci_4352 & $953 \mathrm{~A} / \mathrm{G}$ \\
\hline UN_ci_4352 & $1140 \mathrm{C} / \mathrm{A}$ \\
\hline UN_ci_4353 & $105 \mathrm{~A} / \mathrm{G}$ \\
\hline UN_ci_4353 & $272 \mathrm{~A} / \mathrm{T}$ \\
\hline UN_ci_4353 & $408 \mathrm{G} / \mathrm{A}$ \\
\hline UN_ci_4354 & $26 \mathrm{G} / \mathrm{T}$ \\
\hline UN_ci_4354 & $89 \mathrm{~A} / \mathrm{G}$ \\
\hline UN_ci_4354 & $161 \mathrm{C} / \mathrm{A}$ \\
\hline UN_ci_4354 & $189 \mathrm{~A} / \mathrm{G}$ \\
\hline UN_ci_4354 & $221 \mathrm{C} / \mathrm{A}$ \\
\hline UN_ci_4354 & $325 \mathrm{~T} / \mathrm{C}$ \\
\hline
\end{tabular}




\begin{tabular}{|c|c|}
\hline UN_ci_4354 & 365 T/C \\
\hline UN_ci_4354 & $393 \mathrm{~A} / \mathrm{G}$ \\
\hline UN_ci_4354 & $420 \mathrm{~A} / \mathrm{G}$ \\
\hline UN_ci_4354 & $443 \mathrm{~A} / \mathrm{G}$ \\
\hline UN_ci_4354 & $500 \mathrm{~T} / \mathrm{C}$ \\
\hline UN_ci_4354 & $593 \mathrm{~A} / \mathrm{G}$ \\
\hline UN_ci_4354 & $637 \mathrm{C} / \mathrm{T}$ \\
\hline UN_ci_4354 & $678 \mathrm{~A} / \mathrm{G}$ \\
\hline UN_ci_4358 & $232 \mathrm{C} / \mathrm{T}$ \\
\hline UN_ci_4358 & $397 \mathrm{C} / \mathrm{T}$ \\
\hline UN_ci_4363 & $70 \mathrm{C} / \mathrm{T}$ \\
\hline UN_ci_4363 & $259 \mathrm{C} / \mathrm{T}$ \\
\hline UN_ci_4363 & $311 \mathrm{G} / \mathrm{A}$ \\
\hline UN_ci_4363 & $356 \mathrm{~T} / \mathrm{A}$ \\
\hline UN_ci_4363 & $482 \mathrm{C} / \mathrm{T}$ \\
\hline UN_ci_4363 & 594 A/G \\
\hline UN_ci_4363 & $741 \mathrm{~T} / \mathrm{C}$ \\
\hline UN_ci_4363 & 776 T/C \\
\hline UN_ci_4366 & $134 \mathrm{G} / \mathrm{T}$ \\
\hline UN_ci_4378 & $358 \mathrm{~A} / \mathrm{C}$ \\
\hline UN_ci_4416 & $276 \mathrm{C} / \mathrm{A}$ \\
\hline UN_ci_4425 & 659 A/G \\
\hline UN_ci_4429 & 249 A/G \\
\hline UN_ci_4429 & $500 \mathrm{~A} / \mathrm{T}$ \\
\hline UN_ci_4429 & $557 \mathrm{G} / \mathrm{A}$ \\
\hline UN_ci_4429 & $662 \mathrm{C} / \mathrm{T}$ \\
\hline UN_ci_4429 & 735 A/G \\
\hline UN_ci_4433 & $117 \mathrm{~A} / \mathrm{C}$ \\
\hline UN_ci_4434 & $60 \mathrm{~A} / \mathrm{T}$ \\
\hline UN_ci_4434 & $297 \mathrm{G} / \mathrm{A}$ \\
\hline UN_ci_4439 & $158 \mathrm{C} / \mathrm{T}$ \\
\hline UN_ci_4439 & $185 \mathrm{~A} / \mathrm{G}$ \\
\hline UN_ci_4439 & $347 \mathrm{~A} / \mathrm{C}$ \\
\hline UN_ci_4439 & $698 \mathrm{~A} / \mathrm{T}$ \\
\hline UN_ci_4439 & $864 \mathrm{~A} / \mathrm{C}$ \\
\hline UN_ci_4443 & 198 T/C \\
\hline UN_ci_4443 & $296 \mathrm{~A} / \mathrm{G}$ \\
\hline UN_ci_4443 & $347 \mathrm{~T} / \mathrm{A}$ \\
\hline UN_ci_4443 & $497 \mathrm{G} / \mathrm{T}$ \\
\hline UN_ci_4445 & $287 \mathrm{G} / \mathrm{T}$ \\
\hline UN_ci_4461 & $49 \mathrm{C} / \mathrm{G}$ \\
\hline UN_ci_4472 & $210 \mathrm{~T} / \mathrm{C}$ \\
\hline UN_ci_4480 & $241 \mathrm{~A} / \mathrm{T}$ \\
\hline UN_ci_4485 & $238 \mathrm{C} / \mathrm{T}$ \\
\hline UN_ci_4485 & $290 \mathrm{~A} / \mathrm{C}$ \\
\hline UN_ci_4486 & $264 \mathrm{G} / \mathrm{A}$ \\
\hline UN_ci_4487 & $673 \mathrm{~T} / \mathrm{C}$ \\
\hline UN_ci_4490 & $196 \mathrm{C} / \mathrm{T}$ \\
\hline UN_ci_4515 & 902 T/C \\
\hline UN_ci_4516 & $453 \mathrm{~T} / \mathrm{A}$ \\
\hline
\end{tabular}




\begin{tabular}{|c|c|}
\hline UN_ci_4522 & $205 \mathrm{~A} / \mathrm{G}$ \\
\hline UN_ci_4552 & $740 \mathrm{~A} / \mathrm{T}$ \\
\hline UN_ci_4554 & $243 \mathrm{~T} / \mathrm{C}$ \\
\hline UN_ci_4558 & $483 \mathrm{~A} / \mathrm{T}$ \\
\hline UN_ci_4563 & $86 \mathrm{~T} / \mathrm{C}$ \\
\hline UN_ci_4563 & $168 \mathrm{G} / \mathrm{A}$ \\
\hline UN_ci_4564 & $502 \mathrm{~T} / \mathrm{C}$ \\
\hline UN_ci_4569 & $36 \mathrm{G} / \mathrm{A}$ \\
\hline UN_ci_4569 & 389 C/T \\
\hline UN_ci_4571 & $185 \mathrm{G} / \mathrm{A}$ \\
\hline UN_ci_4577 & $452 \mathrm{~T} / \mathrm{C}$ \\
\hline UN_ci_4583 & $346 \mathrm{G} / \mathrm{A}$ \\
\hline UN_ci_4584 & $254 \mathrm{~A} / \mathrm{G}$ \\
\hline UN_ci_4599 & $130 \mathrm{G} / \mathrm{A}$ \\
\hline UN_ci_4599 & $163 \mathrm{G} / \mathrm{A}$ \\
\hline UN_ci_4599 & $851 \mathrm{~A} / \mathrm{G}$ \\
\hline UN_ci_4603 & $84 \mathrm{~T} / \mathrm{C}$ \\
\hline UN_ci_4609 & $174 \mathrm{C} / \mathrm{T}$ \\
\hline UN_ci_4611 & 393 T/A \\
\hline UN_ci_4611 & 449 T/A \\
\hline UN_ci_4611 & $499 \mathrm{G} / \mathrm{T}$ \\
\hline UN_ci_4613 & $233 \mathrm{~T} / \mathrm{C}$ \\
\hline UN_ci_4613 & $285 \mathrm{C} / \mathrm{T}$ \\
\hline UN_ci_4613 & $570 \mathrm{C} / \mathrm{T}$ \\
\hline UN_ci_4625 & $466 \mathrm{~A} / \mathrm{G}$ \\
\hline UN_ci_4626 & $592 \mathrm{~T} / \mathrm{A}$ \\
\hline UN_ci_4630 & $482 \mathrm{C} / \mathrm{T}$ \\
\hline UN_ci_4635 & $19 \mathrm{~A} / \mathrm{G}$ \\
\hline UN_ci_4635 & $174 \mathrm{C} / \mathrm{T}$ \\
\hline UN_ci_4635 & $243 \mathrm{G} / \mathrm{A}$ \\
\hline UN_ci_4635 & $275 \mathrm{G} / \mathrm{A}$ \\
\hline UN_ci_4635 & $394 \mathrm{G} / \mathrm{T}$ \\
\hline UN_ci_4635 & $466 \mathrm{~T} / \mathrm{C}$ \\
\hline UN_ci_4635 & 629 T/C \\
\hline UN_ci_4635 & $690 \mathrm{G} / \mathrm{C}$ \\
\hline UN_ci_4638 & $169 \mathrm{G} / \mathrm{A}$ \\
\hline UN_ci_4641 & $126 \mathrm{G} / \mathrm{A}$ \\
\hline UN_ci_4646 & $282 \mathrm{~A} / \mathrm{G}$ \\
\hline UN_ci_4646 & $334 \mathrm{C} / \mathrm{T}$ \\
\hline UN_ci_4646 & $486 \mathrm{~T} / \mathrm{C}$ \\
\hline UN_ci_4646 & $531 \mathrm{G} / \mathrm{T}$ \\
\hline UN_ci_4646 & $658 \mathrm{~T} / \mathrm{C}$ \\
\hline UN_ci_4653 & $137 \mathrm{~T} / \mathrm{C}$ \\
\hline UN_ci_4653 & $176 \mathrm{G} / \mathrm{A}$ \\
\hline UN_ci_4653 & $241 \mathrm{~T} / \mathrm{C}$ \\
\hline UN_ci_4653 & $636 \mathrm{~A} / \mathrm{G}$ \\
\hline UN_ci_4653 & $1152 \mathrm{~T} / \mathrm{C}$ \\
\hline UN_ci_4653 & $1391 \mathrm{~A} / \mathrm{G}$ \\
\hline UN_ci_4655 & $241 \mathrm{G} / \mathrm{A}$ \\
\hline UN_ci_4655 & $420 \mathrm{~A} / \mathrm{C}$ \\
\hline
\end{tabular}




\begin{tabular}{|c|c|}
\hline UN_ci_4662 & $647 \mathrm{G} / \mathrm{C}$ \\
\hline UN_ci_4662 & $944 \mathrm{G} / \mathrm{A}$ \\
\hline UN_ci_4669 & $291 \mathrm{C} / \mathrm{T}$ \\
\hline UN_ci_4671 & $175 \mathrm{~A} / \mathrm{G}$ \\
\hline UN_ci_4671 & $687 \mathrm{C} / \mathrm{T}$ \\
\hline UN_ci_4676 & $269 \mathrm{G} / \mathrm{A}$ \\
\hline UN_ci_4679 & $80 \mathrm{G} / \mathrm{A}$ \\
\hline UN_ci_4679 & $120 \mathrm{~A} / \mathrm{C}$ \\
\hline UN_ci_4679 & $198 \mathrm{C} / \mathrm{T}$ \\
\hline UN_ci_4679 & $603 \mathrm{~A} / \mathrm{G}$ \\
\hline UN_ci_4679 & $628 \mathrm{~A} / \mathrm{G}$ \\
\hline UN_ci_4680 & $297 \mathrm{C} / \mathrm{T}$ \\
\hline UN_ci_4692 & $135 \mathrm{~A} / \mathrm{G}$ \\
\hline UN_ci_4692 & $172 \mathrm{C} / \mathrm{G}$ \\
\hline UN_ci_4696 & $724 \mathrm{C} / \mathrm{T}$ \\
\hline UN_ci_4696 & $778 \mathrm{G} / \mathrm{A}$ \\
\hline UN_ci_4696 & 895 G/T \\
\hline UN_ci_4699 & $154 \mathrm{G} / \mathrm{A}$ \\
\hline UN_ci_4699 & $307 \mathrm{G} / \mathrm{A}$ \\
\hline UN_ci_4699 & $341 \mathrm{~A} / \mathrm{G}$ \\
\hline UN_ci_4699 & $407 \mathrm{~A} / \mathrm{G}$ \\
\hline UN_ci_4699 & $539 \mathrm{~A} / \mathrm{T}$ \\
\hline UN_ci_4704 & $185 \mathrm{~A} / \mathrm{G}$ \\
\hline UN_ci_4712 & $486 \mathrm{~T} / \mathrm{C}$ \\
\hline UN_ci_4715 & $507 \mathrm{~A} / \mathrm{G}$ \\
\hline UN_ci_4715 & $536 \mathrm{~T} / \mathrm{A}$ \\
\hline UN_ci_4717 & $466 \mathrm{~A} / \mathrm{G}$ \\
\hline UN_ci_4718 & $135 \mathrm{~A} / \mathrm{C}$ \\
\hline UN_ci_4718 & $291 \mathrm{C} / \mathrm{T}$ \\
\hline UN_ci_4718 & $447 \mathrm{~T} / \mathrm{C}$ \\
\hline UN_ci_4719 & $680 \mathrm{~T} / \mathrm{C}$ \\
\hline UN_ci_4719 & $701 \mathrm{~A} / \mathrm{C}$ \\
\hline UN_ci_4728 & $309 \mathrm{C} / \mathrm{T}$ \\
\hline UN_ci_4728 & $444 \mathrm{~T} / \mathrm{A}$ \\
\hline UN_ci_4733 & $239 \mathrm{~A} / \mathrm{C}$ \\
\hline UN_ci_4738 & $883 \mathrm{~A} / \mathrm{G}$ \\
\hline UN_ci_4739 & $569 \mathrm{~A} / \mathrm{T}$ \\
\hline UN_ci_4739 & $758 \mathrm{~T} / \mathrm{A}$ \\
\hline UN_ci_4745 & $27 \mathrm{G} / \mathrm{T}$ \\
\hline UN_ci_4745 & $148 \mathrm{G} / \mathrm{A}$ \\
\hline UN_ci_4745 & $243 \mathrm{C} / \mathrm{G}$ \\
\hline UN_ci_4745 & $361 \mathrm{~A} / \mathrm{G}$ \\
\hline UN_ci_4745 & $428 \mathrm{C} / \mathrm{A}$ \\
\hline UN_ci_4745 & $468 \mathrm{C} / \mathrm{T}$ \\
\hline UN_ci_4746 & $37 \mathrm{C} / \mathrm{G}$ \\
\hline UN_ci_4757 & 394 A/G \\
\hline UN_ci_4761 & $834 \mathrm{~A} / \mathrm{G}$ \\
\hline UN_ci_4761 & $1000 \mathrm{~T} / \mathrm{C}$ \\
\hline UN_ci_4764 & $300 \mathrm{~T} / \mathrm{C}$ \\
\hline UN_ci_4767 & $580 \mathrm{C} / \mathrm{G}$ \\
\hline
\end{tabular}




\begin{tabular}{|c|c|}
\hline UN_ci_4779 & $17 \mathrm{~T} / \mathrm{G}$ \\
\hline UN_ci_4779 & $147 \mathrm{C} / \mathrm{T}$ \\
\hline UN_ci_4779 & $216 \mathrm{~T} / \mathrm{C}$ \\
\hline UN_ci_4779 & $503 \mathrm{~T} / \mathrm{C}$ \\
\hline UN_ci_4779 & $539 \mathrm{C} / \mathrm{A}$ \\
\hline UN_ci_4779 & $624 \mathrm{~A} / \mathrm{G}$ \\
\hline UN_ci_4781 & $106 \mathrm{~A} / \mathrm{G}$ \\
\hline UN_ci_4781 & $165 \mathrm{~A} / \mathrm{G}$ \\
\hline UN_ci_4781 & $212 \mathrm{G} / \mathrm{A}$ \\
\hline UN_ci_4781 & $261 \mathrm{C} / \mathrm{T}$ \\
\hline UN_ci_4781 & $408 \mathrm{G} / \mathrm{A}$ \\
\hline UN_ci_4781 & $642 \mathrm{~T} / \mathrm{C}$ \\
\hline UN_ci_4781 & $699 \mathrm{G} / \mathrm{A}$ \\
\hline UN_ci_4784 & $14 \mathrm{~T} / \mathrm{C}$ \\
\hline UN_ci_4784 & $180 \mathrm{~A} / \mathrm{T}$ \\
\hline UN_ci_4784 & $552 \mathrm{~A} / \mathrm{G}$ \\
\hline UN_ci_4784 & $673 \mathrm{G} / \mathrm{A}$ \\
\hline UN_ci_4784 & $846 \mathrm{~A} / \mathrm{T}$ \\
\hline UN_ci_4789 & $523 \mathrm{~T} / \mathrm{C}$ \\
\hline UN_ci_4794 & 229 A/G \\
\hline UN_ci_4808 & $123 \mathrm{G} / \mathrm{A}$ \\
\hline UN_ci_4808 & $327 \mathrm{C} / \mathrm{T}$ \\
\hline UN_ci_4825 & $717 \mathrm{G} / \mathrm{A}$ \\
\hline UN_ci_4828 & $72 \mathrm{G} / \mathrm{A}$ \\
\hline UN_ci_4828 & $297 \mathrm{C} / \mathrm{T}$ \\
\hline UN_ci_4830 & $688 \mathrm{~A} / \mathrm{T}$ \\
\hline UN_ci_4833 & $424 \mathrm{~A} / \mathrm{G}$ \\
\hline UN_ci_4835 & $126 \mathrm{C} / \mathrm{A}$ \\
\hline UN_ci_4835 & $147 \mathrm{G} / \mathrm{A}$ \\
\hline UN_ci_4837 & $104 \mathrm{C} / \mathrm{A}$ \\
\hline UN_ci_4837 & $305 \mathrm{C} / \mathrm{T}$ \\
\hline UN_ci_4853 & $25 \mathrm{~T} / \mathrm{C}$ \\
\hline UN_ci_4858 & $46 \mathrm{G} / \mathrm{A}$ \\
\hline UN_ci_4858 & $226 \mathrm{G} / \mathrm{A}$ \\
\hline UN_ci_4858 & $390 \mathrm{G} / \mathrm{T}$ \\
\hline UN_ci_4858 & $469 \mathrm{C} / \mathrm{T}$ \\
\hline UN_ci_4858 & $714 \mathrm{~T} / \mathrm{C}$ \\
\hline UN_ci_4873 & $101 \mathrm{~T} / \mathrm{C}$ \\
\hline UN_ci_4884 & $62 \mathrm{C} / \mathrm{T}$ \\
\hline UN_ci_4884 & $525 \mathrm{~T} / \mathrm{G}$ \\
\hline UN_ci_4885 & $44 \mathrm{~T} / \mathrm{C}$ \\
\hline UN_ci_4885 & $95 \mathrm{~T} / \mathrm{C}$ \\
\hline UN_ci_4899 & $1016 \mathrm{C} / \mathrm{T}$ \\
\hline UN_ci_4899 & $1208 \mathrm{C} / \mathrm{G}$ \\
\hline UN_ci_4913 & $313 \mathrm{G} / \mathrm{T}$ \\
\hline UN_ci_4917 & $88 \mathrm{~A} / \mathrm{G}$ \\
\hline UN_ci_4924 & $286 \mathrm{~T} / \mathrm{A}$ \\
\hline UN_ci_4931 & $406 \mathrm{~A} / \mathrm{G}$ \\
\hline UN_ci_4931 & $485 \mathrm{~A} / \mathrm{G}$ \\
\hline UN_ci_4933 & $325 \mathrm{C} / \mathrm{T}$ \\
\hline
\end{tabular}




\begin{tabular}{|c|c|}
\hline UN_ci_4937 & $431 \mathrm{~A} / \mathrm{G}$ \\
\hline UN_ci_4947 & $129 \mathrm{~A} / \mathrm{G}$ \\
\hline UN_ci_4950 & $258 \mathrm{G} / \mathrm{C}$ \\
\hline UN_ci_4954 & $12 \mathrm{~A} / \mathrm{G}$ \\
\hline UN_ci_4954 & $181 \mathrm{G} / \mathrm{A}$ \\
\hline UN_ci_4954 & $322 \mathrm{~A} / \mathrm{G}$ \\
\hline UN_ci_4956 & $481 \mathrm{~T} / \mathrm{C}$ \\
\hline UN_ci_4956 & $572 \mathrm{C} / \mathrm{T}$ \\
\hline UN_ci_4956 & $605 \mathrm{~T} / \mathrm{A}$ \\
\hline UN_ci_4956 & $911 \mathrm{C} / \mathrm{A}$ \\
\hline UN_ci_4962 & $126 \mathrm{C} / \mathrm{T}$ \\
\hline UN_ci_4964 & $541 \mathrm{~T} / \mathrm{C}$ \\
\hline UN_ci_4969 & $343 \mathrm{G} / \mathrm{A}$ \\
\hline UN_ci_4975 & $278 \mathrm{G} / \mathrm{A}$ \\
\hline UN_ci_4979 & $238 \mathrm{G} / \mathrm{A}$ \\
\hline UN_ci_4979 & $405 \mathrm{~A} / \mathrm{T}$ \\
\hline UN_ci_4981 & $228 \mathrm{~T} / \mathrm{C}$ \\
\hline UN_ci_4993 & $271 \mathrm{~A} / \mathrm{G}$ \\
\hline UN_ci_4993 & $663 \mathrm{C} / \mathrm{T}$ \\
\hline UN_ci_4993 & $880 \mathrm{~A} / \mathrm{G}$ \\
\hline UN_ci_4993 & $970 \mathrm{~A} / \mathrm{G}$ \\
\hline UN_ci_4997 & $80 \mathrm{~T} / \mathrm{C}$ \\
\hline UN_ci_4997 & $116 \mathrm{~T} / \mathrm{C}$ \\
\hline UN_ci_4997 & $160 \mathrm{C} / \mathrm{A}$ \\
\hline UN_ci_4997 & $256 \mathrm{~T} / \mathrm{C}$ \\
\hline UN_ci_4997 & $288 \mathrm{~T} / \mathrm{C}$ \\
\hline UN_ci_4997 & $322 \mathrm{G} / \mathrm{T}$ \\
\hline UN_ci_4997 & $391 \mathrm{~A} / \mathrm{G}$ \\
\hline UN_ci_4997 & $482 \mathrm{C} / \mathrm{T}$ \\
\hline UN_ci_4997 & $587 \mathrm{G} / \mathrm{A}$ \\
\hline UN_ci_4997 & $785 \mathrm{~T} / \mathrm{G}$ \\
\hline UN_ci_4997 & $838 \mathrm{G} / \mathrm{T}$ \\
\hline UN_ci_4997 & $871 \mathrm{~T} / \mathrm{G}$ \\
\hline UN_ci_4997 & $1091 \mathrm{~T} / \mathrm{C}$ \\
\hline UN_ci_4997 & $1156 \mathrm{~A} / \mathrm{T}$ \\
\hline UN_ci_4997 & $1207 \mathrm{G} / \mathrm{A}$ \\
\hline UN_ci_4997 & $1245 \mathrm{C} / \mathrm{A}$ \\
\hline UN_ci_4997 & $1290 \mathrm{C} / \mathrm{T}$ \\
\hline UN_ci_4997 & $1322 \mathrm{~T} / \mathrm{C}$ \\
\hline UN_ci_4997 & $1364 \mathrm{~A} / \mathrm{G}$ \\
\hline UN_ci_4997 & $1428 \mathrm{~T} / \mathrm{C}$ \\
\hline UN_ci_4997 & $1494 \mathrm{C} / \mathrm{T}$ \\
\hline UN_ci_4997 & $1606 \mathrm{G} / \mathrm{C}$ \\
\hline UN_ci_4997 & $1841 \mathrm{G} / \mathrm{A}$ \\
\hline UN_ci_4998 & $135 \mathrm{~T} / \mathrm{A}$ \\
\hline UN_ci_5009 & $50 \mathrm{~T} / \mathrm{C}$ \\
\hline UN_ci_5009 & $95 \mathrm{C} / \mathrm{T}$ \\
\hline UN_ci_5009 & $244 \mathrm{~T} / \mathrm{C}$ \\
\hline UN_ci_5009 & $265 \mathrm{C} / \mathrm{A}$ \\
\hline UN_ci_5009 & $289 \mathrm{~T} / \mathrm{C}$ \\
\hline
\end{tabular}




\begin{tabular}{|c|c|}
\hline UN_ci_5009 & $342 \mathrm{C} / \mathrm{G}$ \\
\hline UN_ci_5009 & $415 \mathrm{~T} / \mathrm{A}$ \\
\hline UN_ci_5009 & $529 \mathrm{~A} / \mathrm{T}$ \\
\hline UN_ci_5009 & $677 \mathrm{~A} / \mathrm{G}$ \\
\hline UN_ci_5009 & $754 \mathrm{~A} / \mathrm{T}$ \\
\hline UN_ci_5009 & 797 C/G \\
\hline UN_ci_5009 & $841 \mathrm{~A} / \mathrm{G}$ \\
\hline UN_ci_5009 & $870 \mathrm{~T} / \mathrm{C}$ \\
\hline UN_ci_5009 & $906 \mathrm{C} / \mathrm{T}$ \\
\hline UN_ci_5009 & $956 \mathrm{~A} / \mathrm{G}$ \\
\hline UN_ci_5009 & $1005 \mathrm{~T} / \mathrm{C}$ \\
\hline UN_ci_5009 & $1044 \mathrm{~T} / \mathrm{C}$ \\
\hline UN_ci_5009 & $1093 \mathrm{~A} / \mathrm{G}$ \\
\hline UN_ci_5009 & $1154 \mathrm{~A} / \mathrm{G}$ \\
\hline UN_ci_5009 & $1246 \mathrm{~A} / \mathrm{G}$ \\
\hline UN_ci_5009 & $1399 \mathrm{~T} / \mathrm{C}$ \\
\hline UN_ci_5009 & $1440 \mathrm{C} / \mathrm{G}$ \\
\hline UN_ci_5009 & $1462 \mathrm{~T} / \mathrm{C}$ \\
\hline UN_ci_5016 & $587 \mathrm{~A} / \mathrm{G}$ \\
\hline UN_ci_5026 & $142 \mathrm{~T} / \mathrm{A}$ \\
\hline UN_ci_5026 & $353 \mathrm{C} / \mathrm{T}$ \\
\hline UN_ci_5026 & $577 \mathrm{~A} / \mathrm{C}$ \\
\hline UN_ci_5026 & $812 \mathrm{~A} / \mathrm{C}$ \\
\hline UN_ci_5026 & $865 \mathrm{G} / \mathrm{A}$ \\
\hline UN_ci_5026 & $1081 \mathrm{C} / \mathrm{T}$ \\
\hline UN_ci_5029 & $385 \mathrm{~T} / \mathrm{C}$ \\
\hline UN_ci_5036 & $367 \mathrm{~T} / \mathrm{C}$ \\
\hline UN_ci_5041 & $266 \mathrm{~T} / \mathrm{A}$ \\
\hline UN_ci_5041 & $482 \mathrm{G} / \mathrm{A}$ \\
\hline UN_ci_5041 & $511 \mathrm{~T} / \mathrm{A}$ \\
\hline UN_ci_5041 & 576 A/G \\
\hline UN_ci_5041 & 729 T/C \\
\hline UN_ci_5041 & $858 \mathrm{~A} / \mathrm{G}$ \\
\hline UN_ci_5049 & $7 \mathrm{~A} / \mathrm{G}$ \\
\hline UN_ci_5049 & $50 \mathrm{~A} / \mathrm{T}$ \\
\hline UN_ci_5049 & $129 \mathrm{G} / \mathrm{T}$ \\
\hline UN_ci_5053 & 318 T/C \\
\hline UN_ci_5059 & $348 \mathrm{C} / \mathrm{T}$ \\
\hline UN_ci_5059 & $526 \mathrm{G} / \mathrm{C}$ \\
\hline UN_ci_5059 & $614 \mathrm{C} / \mathrm{T}$ \\
\hline UN_ci_5072 & $354 \mathrm{~T} / \mathrm{C}$ \\
\hline UN_ci_5072 & $468 \mathrm{~T} / \mathrm{C}$ \\
\hline UN_ci_5073 & $32 \mathrm{~T} / \mathrm{C}$ \\
\hline UN_ci_5076 & $243 \mathrm{~T} / \mathrm{G}$ \\
\hline UN_ci_5080 & $322 \mathrm{C} / \mathrm{T}$ \\
\hline UN_ci_5085 & $361 \mathrm{~T} / \mathrm{C}$ \\
\hline UN_ci_5097 & $25 \mathrm{~T} / \mathrm{G}$ \\
\hline UN_ci_5097 & $178 \mathrm{~T} / \mathrm{C}$ \\
\hline UN_ci_5097 & $200 \mathrm{~A} / \mathrm{G}$ \\
\hline UN_ci_5097 & $236 \mathrm{~T} / \mathrm{A}$ \\
\hline
\end{tabular}




\begin{tabular}{|c|c|}
\hline UN_ci_5097 & $289 \mathrm{~A} / \mathrm{T}$ \\
\hline UN_ci_5098 & $232 \mathrm{G} / \mathrm{A}$ \\
\hline UN_ci_5098 & $954 \mathrm{C} / \mathrm{T}$ \\
\hline UN_ci_5104 & $118 \mathrm{G} / \mathrm{A}$ \\
\hline UN_ci_5104 & $409 \mathrm{~A} / \mathrm{T}$ \\
\hline UN_ci_5104 & $698 \mathrm{C} / \mathrm{T}$ \\
\hline UN_ci_5104 & $862 \mathrm{G} / \mathrm{A}$ \\
\hline UN_ci_5104 & $976 \mathrm{~T} / \mathrm{A}$ \\
\hline UN_ci_5104 & $1006 \mathrm{~T} / \mathrm{C}$ \\
\hline UN_ci_5104 & $1074 \mathrm{G} / \mathrm{T}$ \\
\hline UN_ci_5104 & 1239 A/G \\
\hline UN_ci_5104 & $1264 \mathrm{G} / \mathrm{A}$ \\
\hline UN_ci_5104 & $1286 \mathrm{~A} / \mathrm{C}$ \\
\hline UN_ci_5111 & $233 \mathrm{~A} / \mathrm{G}$ \\
\hline UN_ci_5114 & $21 \mathrm{G} / \mathrm{C}$ \\
\hline UN_ci_5114 & $285 \mathrm{~A} / \mathrm{G}$ \\
\hline UN_ci_5114 & $575 \mathrm{~A} / \mathrm{G}$ \\
\hline UN_ci_5120 & $361 \mathrm{G} / \mathrm{T}$ \\
\hline UN_ci_5121 & $84 \mathrm{~A} / \mathrm{G}$ \\
\hline UN_ci_5121 & $308 \mathrm{~T} / \mathrm{C}$ \\
\hline UN_ci_5124 & $241 \mathrm{G} / \mathrm{A}$ \\
\hline UN_ci_5124 & 302 G/A \\
\hline UN_ci_5124 & $363 \mathrm{~T} / \mathrm{G}$ \\
\hline UN_ci_5124 & $601 \mathrm{C} / \mathrm{T}$ \\
\hline UN_ci_5124 & $718 \mathrm{~T} / \mathrm{C}$ \\
\hline UN_ci_5124 & $784 \mathrm{~T} / \mathrm{C}$ \\
\hline UN_ci_5129 & $354 \mathrm{G} / \mathrm{A}$ \\
\hline UN_ci_5134 & $361 \mathrm{~A} / \mathrm{G}$ \\
\hline UN_ci_5134 & $426 \mathrm{C} / \mathrm{T}$ \\
\hline UN_ci_5149 & $172 \mathrm{~T} / \mathrm{G}$ \\
\hline UN_ci_5149 & $377 \mathrm{C} / \mathrm{T}$ \\
\hline UN_ci_5154 & $321 \mathrm{G} / \mathrm{T}$ \\
\hline UN_ci_5166 & $177 \mathrm{~A} / \mathrm{G}$ \\
\hline UN_ci_5176 & $490 \mathrm{C} / \mathrm{T}$ \\
\hline UN_ci_5184 & $863 \mathrm{C} / \mathrm{G}$ \\
\hline UN_ci_5185 & $350 \mathrm{G} / \mathrm{A}$ \\
\hline UN_ci_5192 & $36 \mathrm{~T} / \mathrm{A}$ \\
\hline UN_ci_5192 & $106 \mathrm{~A} / \mathrm{G}$ \\
\hline UN_ci_5192 & $497 \mathrm{C} / \mathrm{A}$ \\
\hline UN_ci_5192 & $525 \mathrm{~A} / \mathrm{G}$ \\
\hline UN_ci_5196 & $61 \mathrm{G} / \mathrm{C}$ \\
\hline UN_ci_5196 & $182 \mathrm{G} / \mathrm{A}$ \\
\hline UN_ci_5196 & $459 \mathrm{C} / \mathrm{A}$ \\
\hline UN_ci_5200 & 69 A/G \\
\hline UN_ci_5200 & $229 \mathrm{~T} / \mathrm{C}$ \\
\hline UN_ci_5200 & $278 \mathrm{C} / \mathrm{T}$ \\
\hline UN_ci_5200 & $308 \mathrm{C} / \mathrm{A}$ \\
\hline UN_ci_5200 & $582 \mathrm{~T} / \mathrm{C}$ \\
\hline UN_ci_5200 & $731 \mathrm{C} / \mathrm{A}$ \\
\hline UN_ci_5201 & $574 \mathrm{~A} / \mathrm{C}$ \\
\hline
\end{tabular}




\begin{tabular}{|c|c|}
\hline UN_ci_5201 & $620 \mathrm{C} / \mathrm{T}$ \\
\hline UN_ci_5211 & $67 \mathrm{~T} / \mathrm{C}$ \\
\hline UN_ci_5211 & $316 \mathrm{G} / \mathrm{A}$ \\
\hline UN_ci_5212 & $249 \mathrm{C} / \mathrm{G}$ \\
\hline UN_ci_5219 & $86 \mathrm{~T} / \mathrm{C}$ \\
\hline UN_ci_5219 & $113 \mathrm{~A} / \mathrm{C}$ \\
\hline UN_ci_5219 & $242 \mathrm{G} / \mathrm{C}$ \\
\hline UN_ci_5219 & $368 \mathrm{~A} / \mathrm{G}$ \\
\hline UN_ci_5219 & $486 \mathrm{~A} / \mathrm{G}$ \\
\hline UN_ci_5219 & $587 \mathrm{G} / \mathrm{A}$ \\
\hline UN_ci_5219 & $688 \mathrm{~A} / \mathrm{G}$ \\
\hline UN_ci_5219 & $738 \mathrm{~T} / \mathrm{G}$ \\
\hline UN_ci_5219 & $802 \mathrm{C} / \mathrm{T}$ \\
\hline UN_ci_5219 & $822 \mathrm{~A} / \mathrm{T}$ \\
\hline UN_ci_5219 & $874 \mathrm{~A} / \mathrm{G}$ \\
\hline UN_ci_5219 & $947 \mathrm{~A} / \mathrm{C}$ \\
\hline UN_ci_5219 & $969 \mathrm{C} / \mathrm{T}$ \\
\hline UN_ci_5219 & $1060 \mathrm{G} / \mathrm{A}$ \\
\hline UN_ci_5219 & $1347 \mathrm{G} / \mathrm{A}$ \\
\hline UN_ci_5219 & $1378 \mathrm{~A} / \mathrm{G}$ \\
\hline UN_ci_5219 & $1399 \mathrm{C} / \mathrm{T}$ \\
\hline UN_ci_5219 & $1521 \mathrm{~T} / \mathrm{C}$ \\
\hline UN_ci_5219 & $1622 \mathrm{~A} / \mathrm{G}$ \\
\hline UN_ci_5219 & $1643 \mathrm{~A} / \mathrm{G}$ \\
\hline UN_ci_5220 & $690 \mathrm{G} / \mathrm{A}$ \\
\hline UN_ci_5228 & $381 \mathrm{~T} / \mathrm{C}$ \\
\hline UN_ci_5228 & $461 \mathrm{~A} / \mathrm{T}$ \\
\hline UN_ci_5228 & $518 \mathrm{C} / \mathrm{T}$ \\
\hline UN_ci_5228 & $642 \mathrm{G} / \mathrm{A}$ \\
\hline UN_ci_5229 & $288 \mathrm{C} / \mathrm{T}$ \\
\hline UN_ci_5239 & $185 \mathrm{~T} / \mathrm{C}$ \\
\hline UN_ci_5241 & $391 \mathrm{~T} / \mathrm{G}$ \\
\hline UN_ci_5248 & $220 \mathrm{G} / \mathrm{A}$ \\
\hline UN_ci_5248 & $465 \mathrm{~T} / \mathrm{C}$ \\
\hline UN_ci_5250 & $716 \mathrm{C} / \mathrm{T}$ \\
\hline UN_ci_5251 & $376 \mathrm{~T} / \mathrm{C}$ \\
\hline UN_ci_5251 & $464 \mathrm{~T} / \mathrm{C}$ \\
\hline UN_ci_5251 & $529 \mathrm{~T} / \mathrm{C}$ \\
\hline UN_ci_5251 & $572 \mathrm{C} / \mathrm{T}$ \\
\hline UN_ci_5254 & $520 \mathrm{G} / \mathrm{A}$ \\
\hline UN_ci_5258 & $661 \mathrm{~A} / \mathrm{G}$ \\
\hline UN_ci_5259 & $84 \mathrm{G} / \mathrm{C}$ \\
\hline UN_ci_5259 & $241 \mathrm{C} / \mathrm{T}$ \\
\hline UN_ci_5259 & $277 \mathrm{C} / \mathrm{T}$ \\
\hline UN_ci_5259 & $566 \mathrm{~T} / \mathrm{C}$ \\
\hline UN_ci_5259 & $618 \mathrm{~A} / \mathrm{G}$ \\
\hline UN_ci_5261 & $46 \mathrm{G} / \mathrm{C}$ \\
\hline UN_ci_5261 & $215 \mathrm{~A} / \mathrm{G}$ \\
\hline UN_ci_5261 & $881 \mathrm{C} / \mathrm{T}$ \\
\hline UN_ci_5262 & $42 \mathrm{G} / \mathrm{C}$ \\
\hline
\end{tabular}




\begin{tabular}{|c|c|}
\hline UN_ci_5262 & $72 \mathrm{C} / \mathrm{A}$ \\
\hline UN_ci_5262 & $454 \mathrm{~T} / \mathrm{C}$ \\
\hline UN_ci_5262 & $478 \mathrm{G} / \mathrm{A}$ \\
\hline UN_ci_5270 & $246 \mathrm{~A} / \mathrm{T}$ \\
\hline UN_ci_5280 & $1388 \mathrm{G} / \mathrm{A}$ \\
\hline UN_ci_5288 & $90 \mathrm{G} / \mathrm{C}$ \\
\hline UN_ci_5288 & $171 \mathrm{~T} / \mathrm{C}$ \\
\hline UN_ci_5288 & $356 \mathrm{C} / \mathrm{T}$ \\
\hline UN_ci_5291 & $140 \mathrm{~A} / \mathrm{G}$ \\
\hline UN_ci_5291 & $480 \mathrm{~A} / \mathrm{G}$ \\
\hline UN_ci_5291 & 794 C/G \\
\hline UN_ci_5294 & $486 \mathrm{~T} / \mathrm{C}$ \\
\hline UN_ci_5314 & $230 \mathrm{C} / \mathrm{G}$ \\
\hline UN_ci_5314 & $288 \mathrm{C} / \mathrm{T}$ \\
\hline UN_ci_5314 & $481 \mathrm{~A} / \mathrm{G}$ \\
\hline UN_ci_5314 & 629 A/G \\
\hline UN_ci_5322 & $391 \mathrm{~T} / \mathrm{A}$ \\
\hline UN_ci_5324 & $203 \mathrm{~A} / \mathrm{T}$ \\
\hline UN_ci_5326 & $494 \mathrm{C} / \mathrm{T}$ \\
\hline UN_ci_5329 & $563 \mathrm{C} / \mathrm{T}$ \\
\hline UN_ci_5330 & $53 \mathrm{~A} / \mathrm{C}$ \\
\hline UN_ci_5343 & $139 \mathrm{~A} / \mathrm{G}$ \\
\hline UN_ci_5343 & $236 \mathrm{~T} / \mathrm{A}$ \\
\hline UN_ci_5343 & $287 \mathrm{~A} / \mathrm{G}$ \\
\hline UN_ci_5343 & $403 \mathrm{C} / \mathrm{A}$ \\
\hline UN_ci_5346 & $328 \mathrm{~A} / \mathrm{T}$ \\
\hline UN_ci_5346 & $391 \mathrm{C} / \mathrm{T}$ \\
\hline UN_ci_5348 & $150 \mathrm{C} / \mathrm{T}$ \\
\hline UN_ci_5348 & $727 \mathrm{G} / \mathrm{T}$ \\
\hline UN_ci_5351 & $74 \mathrm{C} / \mathrm{T}$ \\
\hline UN_ci_5351 & $234 \mathrm{C} / \mathrm{T}$ \\
\hline UN_ci_5354 & $202 \mathrm{C} / \mathrm{T}$ \\
\hline UN_ci_5379 & $520 \mathrm{~T} / \mathrm{G}$ \\
\hline UN_ci_5379 & $648 \mathrm{~A} / \mathrm{T}$ \\
\hline UN_ci_5379 & $735 \mathrm{G} / \mathrm{A}$ \\
\hline UN_ci_5384 & $32 \mathrm{G} / \mathrm{T}$ \\
\hline UN_ci_5384 & $194 \mathrm{C} / \mathrm{A}$ \\
\hline UN_ci_5388 & $392 \mathrm{~T} / \mathrm{C}$ \\
\hline UN_ci_5388 & $606 \mathrm{G} / \mathrm{T}$ \\
\hline UN_ci_5388 & $971 \mathrm{~T} / \mathrm{A}$ \\
\hline UN_ci_5388 & $1094 \mathrm{~A} / \mathrm{G}$ \\
\hline UN_ci_5389 & 75 T/G \\
\hline UN_ci_5394 & $782 \mathrm{~T} / \mathrm{A}$ \\
\hline UN_ci_5411 & $262 \mathrm{G} / \mathrm{A}$ \\
\hline UN_ci_5455 & $335 \mathrm{~T} / \mathrm{G}$ \\
\hline UN_ci_5455 & 528 A/G \\
\hline UN_ci_5455 & $702 \mathrm{~A} / \mathrm{C}$ \\
\hline UN_ci_5458 & $115 \mathrm{G} / \mathrm{C}$ \\
\hline UN_ci_5458 & $206 \mathrm{~A} / \mathrm{T}$ \\
\hline UN_ci_5461 & $101 \mathrm{~A} / \mathrm{G}$ \\
\hline
\end{tabular}




\begin{tabular}{|c|c|}
\hline UN_ci_5468 & $123 \mathrm{G} / \mathrm{A}$ \\
\hline UN_ci_5468 & 289 A/G \\
\hline UN_ci_5470 & $175 \mathrm{~A} / \mathrm{T}$ \\
\hline UN_ci_5472 & $324 \mathrm{~A} / \mathrm{G}$ \\
\hline UN_ci_5475 & $68 \mathrm{~A} / \mathrm{C}$ \\
\hline UN_ci_5475 & $120 \mathrm{G} / \mathrm{A}$ \\
\hline UN_ci_5475 & $377 \mathrm{G} / \mathrm{C}$ \\
\hline UN_ci_5475 & $596 \mathrm{~T} / \mathrm{A}$ \\
\hline UN_ci_5475 & $943 \mathrm{~T} / \mathrm{C}$ \\
\hline UN_ci_5475 & $1250 \mathrm{~A} / \mathrm{G}$ \\
\hline UN_ci_5475 & $1710 \mathrm{~A} / \mathrm{G}$ \\
\hline UN_ci_5483 & $8 \mathrm{~T} / \mathrm{A}$ \\
\hline UN_ci_5483 & $95 \mathrm{~A} / \mathrm{G}$ \\
\hline UN_ci_5483 & $397 \mathrm{G} / \mathrm{A}$ \\
\hline UN_ci_5483 & $418 \mathrm{C} / \mathrm{T}$ \\
\hline UN_ci_5483 & $505 \mathrm{G} / \mathrm{A}$ \\
\hline UN_ci_5483 & $564 \mathrm{G} / \mathrm{A}$ \\
\hline UN_ci_5483 & $615 \mathrm{G} / \mathrm{A}$ \\
\hline UN_ci_5483 & $851 \mathrm{~A} / \mathrm{G}$ \\
\hline UN_ci_5483 & 937 T/C \\
\hline UN_ci_5488 & $104 \mathrm{~T} / \mathrm{G}$ \\
\hline UN_ci_5492 & $295 \mathrm{C} / \mathrm{T}$ \\
\hline UN_ci_5492 & $505 \mathrm{~A} / \mathrm{G}$ \\
\hline UN_ci_5500 & $510 \mathrm{~A} / \mathrm{G}$ \\
\hline UN_ci_5511 & $69 \mathrm{~A} / \mathrm{G}$ \\
\hline UN_ci_5511 & $170 \mathrm{C} / \mathrm{T}$ \\
\hline UN_ci_5511 & $240 \mathrm{~A} / \mathrm{G}$ \\
\hline UN_ci_5513 & $178 \mathrm{~T} / \mathrm{C}$ \\
\hline UN_ci_5513 & $294 \mathrm{~T} / \mathrm{C}$ \\
\hline UN_ci_5515 & $107 \mathrm{~A} / \mathrm{G}$ \\
\hline UN_ci_5517 & $399 \mathrm{C} / \mathrm{T}$ \\
\hline UN_ci_5517 & $601 \mathrm{~T} / \mathrm{A}$ \\
\hline UN_ci_5521 & $25 \mathrm{G} / \mathrm{A}$ \\
\hline UN_ci_5521 & $634 \mathrm{~T} / \mathrm{A}$ \\
\hline UN_ci_5521 & $712 \mathrm{C} / \mathrm{T}$ \\
\hline UN_ci_5521 & $744 \mathrm{~A} / \mathrm{G}$ \\
\hline UN_ci_5521 & $766 \mathrm{C} / \mathrm{A}$ \\
\hline UN_ci_5525 & $349 \mathrm{G} / \mathrm{T}$ \\
\hline UN_ci_5538 & $43 \mathrm{G} / \mathrm{A}$ \\
\hline UN_ci_5538 & $270 \mathrm{G} / \mathrm{A}$ \\
\hline UN_ci_5538 & $326 \mathrm{~T} / \mathrm{C}$ \\
\hline UN_ci_5538 & 489 A/G \\
\hline UN_ci_5538 & $603 \mathrm{~A} / \mathrm{C}$ \\
\hline UN_ci_5538 & $654 \mathrm{~A} / \mathrm{G}$ \\
\hline UN_ci_5538 & $801 \mathrm{~T} / \mathrm{C}$ \\
\hline UN_ci_5539 & $92 \mathrm{C} / \mathrm{T}$ \\
\hline UN_ci_5543 & $368 \mathrm{G} / \mathrm{A}$ \\
\hline UN_ci_5558 & $486 \mathrm{~T} / \mathrm{C}$ \\
\hline UN_ci_5560 & $225 \mathrm{~A} / \mathrm{C}$ \\
\hline UN_ci_5560 & $346 \mathrm{~T} / \mathrm{C}$ \\
\hline
\end{tabular}




\begin{tabular}{|c|c|}
\hline UN_ci_5560 & $484 \mathrm{G} / \mathrm{A}$ \\
\hline UN_ci_5561 & $190 \mathrm{~A} / \mathrm{G}$ \\
\hline UN_ci_5573 & $116 \mathrm{G} / \mathrm{A}$ \\
\hline UN_ci_5574 & $384 \mathrm{~T} / \mathrm{C}$ \\
\hline UN_ci_5574 & 429 A/G \\
\hline UN_ci_5574 & $523 \mathrm{~A} / \mathrm{G}$ \\
\hline UN_ci_5574 & $610 \mathrm{~A} / \mathrm{G}$ \\
\hline UN_ci_5574 & $773 \mathrm{G} / \mathrm{C}$ \\
\hline UN_ci_5578 & $421 \mathrm{G} / \mathrm{A}$ \\
\hline UN_ci_5583 & $94 \mathrm{G} / \mathrm{C}$ \\
\hline UN_ci_5583 & $305 \mathrm{C} / \mathrm{T}$ \\
\hline UN_ci_5583 & $687 \mathrm{~T} / \mathrm{C}$ \\
\hline UN_ci_5583 & $835 \mathrm{~T} / \mathrm{C}$ \\
\hline UN_ci_5594 & $482 \mathrm{~T} / \mathrm{C}$ \\
\hline UN_ci_5619 & $303 \mathrm{~A} / \mathrm{G}$ \\
\hline UN_ci_5619 & $385 \mathrm{G} / \mathrm{T}$ \\
\hline UN_ci_5631 & 767 A/G \\
\hline UN_ci_5633 & $130 \mathrm{~A} / \mathrm{G}$ \\
\hline UN_ci_5633 & $376 \mathrm{G} / \mathrm{A}$ \\
\hline UN_ci_5638 & $356 \mathrm{C} / \mathrm{G}$ \\
\hline UN_ci_5649 & $57 \mathrm{G} / \mathrm{A}$ \\
\hline UN_ci_5649 & $93 \mathrm{~A} / \mathrm{T}$ \\
\hline UN_ci_5649 & 378 A/G \\
\hline UN_ci_5661 & $67 \mathrm{C} / \mathrm{A}$ \\
\hline UN_ci_5661 & $89 \mathrm{~T} / \mathrm{G}$ \\
\hline UN_ci_5661 & 205 T/G \\
\hline UN_ci_5661 & $355 \mathrm{~T} / \mathrm{C}$ \\
\hline UN_ci_5661 & $425 \mathrm{C} / \mathrm{T}$ \\
\hline UN_ci_5662 & $26 \mathrm{~T} / \mathrm{G}$ \\
\hline UN_ci_5667 & $111 \mathrm{C} / \mathrm{T}$ \\
\hline UN_ci_5667 & $251 \mathrm{C} / \mathrm{T}$ \\
\hline UN_ci_5679 & $139 \mathrm{~A} / \mathrm{T}$ \\
\hline UN_ci_5685 & $11 \mathrm{~A} / \mathrm{G}$ \\
\hline UN_ci_5696 & $105 \mathrm{~A} / \mathrm{G}$ \\
\hline UN_ci_5713 & $203 \mathrm{~A} / \mathrm{T}$ \\
\hline UN_ci_5713 & $256 \mathrm{~T} / \mathrm{C}$ \\
\hline UN_ci_5718 & $400 \mathrm{~A} / \mathrm{C}$ \\
\hline UN_ci_5721 & $340 \mathrm{~T} / \mathrm{C}$ \\
\hline UN_ci_5721 & $412 \mathrm{G} / \mathrm{T}$ \\
\hline UN_ci_5721 & $475 \mathrm{C} / \mathrm{T}$ \\
\hline UN_ci_5724 & $218 \mathrm{~A} / \mathrm{G}$ \\
\hline UN_ci_5735 & $283 \mathrm{G} / \mathrm{A}$ \\
\hline UN_ci_5746 & $241 \mathrm{C} / \mathrm{G}$ \\
\hline UN_ci_5747 & $272 \mathrm{~A} / \mathrm{G}$ \\
\hline UN_ci_5747 & $383 \mathrm{~A} / \mathrm{G}$ \\
\hline UN_ci_5747 & $428 \mathrm{~A} / \mathrm{G}$ \\
\hline UN_ci_5747 & $453 \mathrm{C} / \mathrm{T}$ \\
\hline UN_ci_5747 & $490 \mathrm{~T} / \mathrm{C}$ \\
\hline UN_ci_5748 & $199 \mathrm{G} / \mathrm{A}$ \\
\hline UN_ci_5751 & $10 \mathrm{G} / \mathrm{A}$ \\
\hline
\end{tabular}




\begin{tabular}{|c|c|}
\hline UN_ci_5752 & $121 \mathrm{G} / \mathrm{C}$ \\
\hline UN_ci_5757 & $23 \mathrm{G} / \mathrm{T}$ \\
\hline UN_ci_5760 & $234 \mathrm{~A} / \mathrm{T}$ \\
\hline UN_ci_5764 & $259 \mathrm{C} / \mathrm{G}$ \\
\hline UN_ci_5774 & $376 \mathrm{G} / \mathrm{A}$ \\
\hline UN_ci_5775 & $408 \mathrm{~T} / \mathrm{G}$ \\
\hline UN_ci_5799 & $504 \mathrm{C} / \mathrm{T}$ \\
\hline UN_ci_5805 & $106 \mathrm{G} / \mathrm{T}$ \\
\hline UN_ci_5805 & $302 \mathrm{G} / \mathrm{A}$ \\
\hline UN_ci_5805 & $421 \mathrm{~T} / \mathrm{C}$ \\
\hline UN_ci_5805 & $558 \mathrm{~A} / \mathrm{G}$ \\
\hline UN_ci_5805 & $594 \mathrm{~T} / \mathrm{C}$ \\
\hline UN_ci_5805 & $771 \mathrm{~T} / \mathrm{C}$ \\
\hline UN_ci_5805 & $800 \mathrm{C} / \mathrm{A}$ \\
\hline UN_ci_5816 & $102 \mathrm{~T} / \mathrm{C}$ \\
\hline UN_ci_5819 & $249 \mathrm{~A} / \mathrm{C}$ \\
\hline UN_ci_5819 & $1215 \mathrm{~A} / \mathrm{G}$ \\
\hline UN_ci_5824 & $609 \mathrm{~T} / \mathrm{C}$ \\
\hline UN_ci_5829 & $183 \mathrm{~T} / \mathrm{A}$ \\
\hline UN_ci_5829 & 306 A/G \\
\hline UN_ci_5829 & $356 \mathrm{G} / \mathrm{A}$ \\
\hline UN_ci_5839 & $885 \mathrm{~A} / \mathrm{G}$ \\
\hline UN_ci_5839 & 1019 A/G \\
\hline UN_ci_5839 & 1197 T/G \\
\hline UN_ci_5839 & $1282 \mathrm{G} / \mathrm{A}$ \\
\hline UN_ci_5845 & $130 \mathrm{~T} / \mathrm{C}$ \\
\hline UN_ci_5849 & $85 \mathrm{~A} / \mathrm{G}$ \\
\hline UN_ci_5849 & $173 \mathrm{~T} / \mathrm{C}$ \\
\hline UN_ci_5849 & $443 \mathrm{G} / \mathrm{C}$ \\
\hline UN_ci_5857 & $107 \mathrm{G} / \mathrm{T}$ \\
\hline UN_ci_5870 & $10 \mathrm{G} / \mathrm{C}$ \\
\hline UN_ci_5870 & $759 \mathrm{~A} / \mathrm{T}$ \\
\hline UN_ci_5872 & $149 \mathrm{C} / \mathrm{T}$ \\
\hline UN_ci_5872 & 347 T/C \\
\hline UN_ci_5874 & $378 \mathrm{G} / \mathrm{A}$ \\
\hline UN_ci_5876 & 393 A/G \\
\hline UN_ci_5877 & $950 \mathrm{~A} / \mathrm{G}$ \\
\hline UN_ci_5884 & $549 \mathrm{~A} / \mathrm{C}$ \\
\hline UN_ci_5885 & $66 \mathrm{~A} / \mathrm{G}$ \\
\hline UN_ci_5890 & $19 \mathrm{G} / \mathrm{C}$ \\
\hline UN_ci_5890 & $42 \mathrm{~A} / \mathrm{G}$ \\
\hline UN_ci_5890 & $65 \mathrm{C} / \mathrm{T}$ \\
\hline UN_ci_5890 & $111 \mathrm{~A} / \mathrm{G}$ \\
\hline UN_ci_5890 & $200 \mathrm{~A} / \mathrm{G}$ \\
\hline UN_ci_5890 & $647 \mathrm{C} / \mathrm{T}$ \\
\hline UN_ci_5890 & $695 \mathrm{~T} / \mathrm{C}$ \\
\hline UN_ci_5892 & $178 \mathrm{~A} / \mathrm{C}$ \\
\hline UN_ci_5892 & 1279 T/C \\
\hline UN_ci_5892 & $1300 \mathrm{~A} / \mathrm{T}$ \\
\hline UN_ci_5908 & $387 \mathrm{~T} / \mathrm{G}$ \\
\hline
\end{tabular}




\begin{tabular}{|c|c|}
\hline UN_ci_5911 & $658 \mathrm{~A} / \mathrm{G}$ \\
\hline UN_ci_5911 & $734 \mathrm{~A} / \mathrm{G}$ \\
\hline UN_ci_5911 & $757 \mathrm{~A} / \mathrm{T}$ \\
\hline UN_ci_5911 & $955 \mathrm{~A} / \mathrm{G}$ \\
\hline UN_ci_5911 & $1062 \mathrm{~A} / \mathrm{G}$ \\
\hline UN_ci_5911 & $1154 \mathrm{G} / \mathrm{A}$ \\
\hline UN_ci_5911 & $1240 \mathrm{~T} / \mathrm{C}$ \\
\hline UN_ci_5911 & $1418 \mathrm{~A} / \mathrm{T}$ \\
\hline UN_ci_5916 & $171 \mathrm{~T} / \mathrm{A}$ \\
\hline UN_ci_5916 & $237 \mathrm{C} / \mathrm{A}$ \\
\hline UN_ci_5916 & $435 \mathrm{G} / \mathrm{A}$ \\
\hline UN_ci_5916 & $572 \mathrm{~T} / \mathrm{C}$ \\
\hline UN_ci_5918 & $120 \mathrm{C} / \mathrm{T}$ \\
\hline UN_ci_5918 & 292 T/A \\
\hline UN_ci_5925 & $380 \mathrm{~T} / \mathrm{C}$ \\
\hline UN_ci_5925 & $563 \mathrm{~T} / \mathrm{C}$ \\
\hline UN_ci_5925 & $932 \mathrm{~T} / \mathrm{G}$ \\
\hline UN_ci_5925 & $1157 \mathrm{~A} / \mathrm{G}$ \\
\hline UN_ci_5935 & $94 \mathrm{~A} / \mathrm{G}$ \\
\hline UN_ci_5939 & $142 \mathrm{~A} / \mathrm{G}$ \\
\hline UN_ci_5939 & $172 \mathrm{~A} / \mathrm{G}$ \\
\hline UN_ci_5939 & $220 \mathrm{C} / \mathrm{T}$ \\
\hline UN_ci_5939 & $334 \mathrm{C} / \mathrm{T}$ \\
\hline UN_ci_5939 & $355 \mathrm{~T} / \mathrm{C}$ \\
\hline UN_ci_5939 & $476 \mathrm{C} / \mathrm{G}$ \\
\hline UN_ci_5942 & $468 \mathrm{~A} / \mathrm{G}$ \\
\hline UN_ci_5942 & $547 \mathrm{C} / \mathrm{T}$ \\
\hline UN_ci_5943 & $289 \mathrm{~T} / \mathrm{A}$ \\
\hline UN_ci_5947 & $53 \mathrm{~A} / \mathrm{G}$ \\
\hline UN_ci_5960 & $213 \mathrm{~A} / \mathrm{G}$ \\
\hline UN_ci_5960 & $779 \mathrm{~A} / \mathrm{T}$ \\
\hline UN_ci_5962 & $155 \mathrm{~A} / \mathrm{T}$ \\
\hline UN_ci_5962 & $780 \mathrm{G} / \mathrm{A}$ \\
\hline UN_ci_5962 & $824 \mathrm{~A} / \mathrm{T}$ \\
\hline UN_ci_5962 & $946 \mathrm{G} / \mathrm{A}$ \\
\hline UN_ci_5965 & $174 \mathrm{~T} / \mathrm{C}$ \\
\hline UN_ci_5967 & $366 \mathrm{~A} / \mathrm{G}$ \\
\hline UN_ci_5976 & $542 \mathrm{G} / \mathrm{C}$ \\
\hline UN_ci_5978 & 297 G/A \\
\hline UN_ci_5979 & $302 \mathrm{~T} / \mathrm{C}$ \\
\hline UN_ci_5979 & $406 \mathrm{C} / \mathrm{T}$ \\
\hline UN_ci_5979 & $859 \mathrm{G} / \mathrm{A}$ \\
\hline UN_ci_5983 & $271 \mathrm{~A} / \mathrm{G}$ \\
\hline UN_ci_5983 & $526 \mathrm{~A} / \mathrm{G}$ \\
\hline UN_ci_5983 & $699 \mathrm{~T} / \mathrm{C}$ \\
\hline UN_ci_5983 & 819 G/A \\
\hline UN_ci_5983 & 915 G/T \\
\hline UN_ci_6001 & $27 \mathrm{~A} / \mathrm{G}$ \\
\hline UN_ci_6001 & 207 G/A \\
\hline UN_ci_6001 & $257 \mathrm{~A} / \mathrm{G}$ \\
\hline
\end{tabular}




\begin{tabular}{|c|c|}
\hline UN_ci_6001 & $646 \mathrm{~A} / \mathrm{G}$ \\
\hline UN_ci_6004 & $437 \mathrm{~A} / \mathrm{C}$ \\
\hline UN_ci_6004 & $691 \mathrm{C} / \mathrm{G}$ \\
\hline UN_ci_6010 & $382 \mathrm{~A} / \mathrm{C}$ \\
\hline UN_ci_6010 & 577 T/A \\
\hline UN_ci_6010 & $670 \mathrm{~A} / \mathrm{G}$ \\
\hline UN_ci_6012 & $94 \mathrm{C} / \mathrm{T}$ \\
\hline UN_ci_6012 & $583 \mathrm{C} / \mathrm{A}$ \\
\hline UN_ci_6015 & $152 \mathrm{~T} / \mathrm{C}$ \\
\hline UN_ci_6015 & $185 \mathrm{~T} / \mathrm{C}$ \\
\hline UN_ci_6015 & $428 \mathrm{~A} / \mathrm{G}$ \\
\hline UN_ci_6019 & 409 A/G \\
\hline UN_ci_6023 & $138 \mathrm{C} / \mathrm{T}$ \\
\hline UN_ci_6023 & $703 \mathrm{C} / \mathrm{T}$ \\
\hline UN_ci_6027 & $42 \mathrm{G} / \mathrm{C}$ \\
\hline UN_ci_6027 & $378 \mathrm{C} / \mathrm{T}$ \\
\hline UN_ci_6027 & $1112 \mathrm{G} / \mathrm{T}$ \\
\hline UN_ci_6037 & $139 \mathrm{C} / \mathrm{T}$ \\
\hline UN_ci_6059 & $127 \mathrm{G} / \mathrm{A}$ \\
\hline UN_ci_6059 & $184 \mathrm{C} / \mathrm{A}$ \\
\hline UN_ci_6059 & $325 \mathrm{~A} / \mathrm{G}$ \\
\hline UN_ci_6070 & 322 T/A \\
\hline UN_ci_6070 & $494 \mathrm{~T} / \mathrm{G}$ \\
\hline UN_ci_6075 & $34 \mathrm{~T} / \mathrm{C}$ \\
\hline UN_ci_6075 & 369 A/G \\
\hline UN_ci_6075 & $469 \mathrm{~T} / \mathrm{C}$ \\
\hline UN_ci_6075 & $535 \mathrm{C} / \mathrm{T}$ \\
\hline UN_ci_6075 & $782 \mathrm{C} / \mathrm{A}$ \\
\hline UN_ci_6078 & $286 \mathrm{~A} / \mathrm{G}$ \\
\hline UN_ci_6078 & $387 \mathrm{~A} / \mathrm{T}$ \\
\hline UN_ci_6078 & $458 \mathrm{C} / \mathrm{T}$ \\
\hline UN_ci_6078 & 489 A/G \\
\hline UN_ci_6078 & $598 \mathrm{G} / \mathrm{A}$ \\
\hline UN_ci_6081 & $285 \mathrm{~A} / \mathrm{C}$ \\
\hline UN_ci_6084 & $30 \mathrm{~T} / \mathrm{C}$ \\
\hline UN_ci_6096 & $883 \mathrm{C} / \mathrm{T}$ \\
\hline UN_ci_6112 & $56 \mathrm{~A} / \mathrm{T}$ \\
\hline UN_ci_6112 & $102 \mathrm{G} / \mathrm{A}$ \\
\hline UN_ci_6112 & $167 \mathrm{C} / \mathrm{T}$ \\
\hline UN_ci_6112 & $333 \mathrm{C} / \mathrm{T}$ \\
\hline UN_ci_6115 & 179 A/G \\
\hline UN_ci_6115 & $262 \mathrm{G} / \mathrm{A}$ \\
\hline UN_ci_6115 & 315 T/C \\
\hline UN_ci_6115 & $352 \mathrm{~A} / \mathrm{G}$ \\
\hline UN_ci_6115 & 395 T/C \\
\hline UN_ci_6115 & $534 \mathrm{C} / \mathrm{T}$ \\
\hline UN_ci_6115 & $615 \mathrm{~T} / \mathrm{C}$ \\
\hline UN_ci_6118 & $142 \mathrm{~A} / \mathrm{G}$ \\
\hline UN_ci_6118 & $208 \mathrm{C} / \mathrm{A}$ \\
\hline UN_ci_6119 & $68 \mathrm{~A} / \mathrm{C}$ \\
\hline
\end{tabular}




\begin{tabular}{|c|c|}
\hline UN_ci_6119 & $252 \mathrm{G} / \mathrm{T}$ \\
\hline UN_ci_6119 & $611 \mathrm{~T} / \mathrm{C}$ \\
\hline UN_ci_6119 & $650 \mathrm{~T} / \mathrm{C}$ \\
\hline UN_ci_6119 & $694 \mathrm{~T} / \mathrm{C}$ \\
\hline UN_ci_6119 & $726 \mathrm{~T} / \mathrm{C}$ \\
\hline UN_ci_6122 & $126 \mathrm{G} / \mathrm{A}$ \\
\hline UN_ci_6122 & $832 \mathrm{~A} / \mathrm{G}$ \\
\hline UN_ci_6124 & $24 \mathrm{G} / \mathrm{T}$ \\
\hline UN_ci_6124 & $159 \mathrm{G} / \mathrm{A}$ \\
\hline UN_ci_6124 & $424 \mathrm{~A} / \mathrm{G}$ \\
\hline UN_ci_6124 & $702 \mathrm{~A} / \mathrm{C}$ \\
\hline UN_ci_6129 & $265 \mathrm{~T} / \mathrm{C}$ \\
\hline UN_ci_6131 & $139 \mathrm{G} / \mathrm{A}$ \\
\hline UN_ci_6132 & $50 \mathrm{G} / \mathrm{A}$ \\
\hline UN_ci_6132 & $714 \mathrm{G} / \mathrm{A}$ \\
\hline UN_ci_6141 & $422 \mathrm{~A} / \mathrm{G}$ \\
\hline UN_ci_6142 & $1035 \mathrm{~T} / \mathrm{A}$ \\
\hline UN_ci_6142 & $1251 \mathrm{~A} / \mathrm{G}$ \\
\hline UN_ci_6148 & $37 \mathrm{~A} / \mathrm{T}$ \\
\hline UN_ci_6148 & $106 \mathrm{C} / \mathrm{T}$ \\
\hline UN_ci_6148 & $436 \mathrm{~T} / \mathrm{A}$ \\
\hline UN_ci_6148 & $522 \mathrm{~A} / \mathrm{C}$ \\
\hline UN_ci_6148 & $598 \mathrm{~T} / \mathrm{G}$ \\
\hline UN_ci_6148 & $636 \mathrm{C} / \mathrm{T}$ \\
\hline UN_ci_6148 & $660 \mathrm{~A} / \mathrm{G}$ \\
\hline UN_ci_6148 & $752 \mathrm{G} / \mathrm{A}$ \\
\hline UN_ci_6148 & $807 \mathrm{C} / \mathrm{T}$ \\
\hline UN_ci_6152 & $652 \mathrm{G} / \mathrm{A}$ \\
\hline UN_ci_6154 & $107 \mathrm{~A} / \mathrm{G}$ \\
\hline UN_ci_6154 & $165 \mathrm{G} / \mathrm{A}$ \\
\hline UN_ci_6154 & $190 \mathrm{G} / \mathrm{A}$ \\
\hline UN_ci_6155 & 379 A/G \\
\hline UN_ci_6156 & $36 \mathrm{C} / \mathrm{T}$ \\
\hline UN_ci_6156 & $81 \mathrm{G} / \mathrm{T}$ \\
\hline UN_ci_6156 & $130 \mathrm{C} / \mathrm{T}$ \\
\hline UN_ci_6156 & $177 \mathrm{G} / \mathrm{A}$ \\
\hline UN_ci_6156 & $285 \mathrm{~T} / \mathrm{C}$ \\
\hline UN_ci_6156 & $355 \mathrm{G} / \mathrm{A}$ \\
\hline UN_ci_6156 & $380 \mathrm{C} / \mathrm{G}$ \\
\hline UN_ci_6156 & $538 \mathrm{~A} / \mathrm{G}$ \\
\hline UN_ci_6157 & $250 \mathrm{~T} / \mathrm{G}$ \\
\hline UN_ci_6158 & $131 \mathrm{~T} / \mathrm{C}$ \\
\hline UN_ci_6160 & $52 \mathrm{~A} / \mathrm{G}$ \\
\hline UN_ci_6160 & $479 \mathrm{~A} / \mathrm{G}$ \\
\hline UN_ci_6163 & $385 \mathrm{~A} / \mathrm{C}$ \\
\hline UN_ci_6163 & $453 \mathrm{~A} / \mathrm{G}$ \\
\hline UN_ci_6163 & $486 \mathrm{~T} / \mathrm{C}$ \\
\hline UN_ci_6163 & $529 \mathrm{C} / \mathrm{T}$ \\
\hline UN_ci_6163 & $646 \mathrm{C} / \mathrm{T}$ \\
\hline UN_ci_6164 & $698 \mathrm{~T} / \mathrm{C}$ \\
\hline
\end{tabular}




\begin{tabular}{|c|c|}
\hline UN_ci_6164 & $739 \mathrm{G} / \mathrm{T}$ \\
\hline UN_ci_6164 & $850 \mathrm{C} / \mathrm{A}$ \\
\hline UN_ci_6164 & $886 \mathrm{~A} / \mathrm{G}$ \\
\hline UN_ci_6169 & $878 \mathrm{~A} / \mathrm{C}$ \\
\hline UN_ci_6179 & $187 \mathrm{C} / \mathrm{T}$ \\
\hline UN_ci_6179 & 219 A/G \\
\hline UN_ci_6183 & $83 \mathrm{~A} / \mathrm{G}$ \\
\hline UN_ci_6183 & $378 \mathrm{C} / \mathrm{A}$ \\
\hline UN_ci_6184 & $334 \mathrm{~A} / \mathrm{G}$ \\
\hline UN_ci_6184 & $509 \mathrm{~A} / \mathrm{C}$ \\
\hline UN_ci_6185 & $536 \mathrm{~A} / \mathrm{G}$ \\
\hline UN_ci_6190 & $178 \mathrm{~A} / \mathrm{G}$ \\
\hline UN_ci_6190 & $286 \mathrm{~A} / \mathrm{G}$ \\
\hline UN_ci_6202 & $838 \mathrm{~T} / \mathrm{C}$ \\
\hline UN_ci_6208 & $128 \mathrm{~T} / \mathrm{A}$ \\
\hline UN_ci_6215 & $396 \mathrm{~T} / \mathrm{C}$ \\
\hline UN_ci_6223 & $170 \mathrm{G} / \mathrm{C}$ \\
\hline UN_ci_6223 & $492 \mathrm{C} / \mathrm{T}$ \\
\hline UN_ci_6223 & $690 \mathrm{~T} / \mathrm{C}$ \\
\hline UN_ci_6226 & $304 \mathrm{C} / \mathrm{T}$ \\
\hline UN_ci_6226 & $640 \mathrm{~A} / \mathrm{T}$ \\
\hline UN_ci_6227 & $152 \mathrm{C} / \mathrm{A}$ \\
\hline UN_ci_6227 & $223 \mathrm{~A} / \mathrm{G}$ \\
\hline UN_ci_6250 & $185 \mathrm{~T} / \mathrm{A}$ \\
\hline UN_ci_6250 & $238 \mathrm{~T} / \mathrm{C}$ \\
\hline UN_ci_6250 & $277 \mathrm{G} / \mathrm{A}$ \\
\hline UN_ci_6251 & $176 \mathrm{G} / \mathrm{C}$ \\
\hline UN_ci_6251 & $224 \mathrm{G} / \mathrm{A}$ \\
\hline UN_ci_6251 & $520 \mathrm{~T} / \mathrm{C}$ \\
\hline UN_ci_6255 & $358 \mathrm{G} / \mathrm{C}$ \\
\hline UN_ci_6255 & $400 \mathrm{~T} / \mathrm{C}$ \\
\hline UN_ci_6258 & $330 \mathrm{~T} / \mathrm{C}$ \\
\hline UN_ci_6258 & $606 \mathrm{~T} / \mathrm{C}$ \\
\hline UN_ci_6268 & $56 \mathrm{~T} / \mathrm{G}$ \\
\hline UN_ci_6268 & $81 \mathrm{C} / \mathrm{T}$ \\
\hline UN_ci_6279 & $433 \mathrm{~A} / \mathrm{T}$ \\
\hline UN_ci_6279 & $458 \mathrm{~A} / \mathrm{T}$ \\
\hline UN_ci_6279 & $588 \mathrm{G} / \mathrm{A}$ \\
\hline UN_ci_6288 & $374 \mathrm{~A} / \mathrm{G}$ \\
\hline UN_ci_6289 & $169 \mathrm{C} / \mathrm{T}$ \\
\hline UN_ci_6290 & $132 \mathrm{~A} / \mathrm{T}$ \\
\hline UN_ci_6290 & $195 \mathrm{C} / \mathrm{T}$ \\
\hline UN_ci_6290 & $240 \mathrm{G} / \mathrm{T}$ \\
\hline UN_ci_6290 & $397 \mathrm{C} / \mathrm{T}$ \\
\hline UN_ci_6292 & $676 \mathrm{~T} / \mathrm{C}$ \\
\hline UN_ci_6295 & $38 \mathrm{G} / \mathrm{T}$ \\
\hline UN_ci_6295 & $695 \mathrm{~A} / \mathrm{C}$ \\
\hline UN_ci_6298 & $58 \mathrm{~T} / \mathrm{C}$ \\
\hline UN_ci_6298 & $325 \mathrm{C} / \mathrm{T}$ \\
\hline UN_ci_6304 & $148 \mathrm{~T} / \mathrm{C}$ \\
\hline
\end{tabular}




\begin{tabular}{|c|c|}
\hline UN_ci_6304 & $221 \mathrm{~A} / \mathrm{G}$ \\
\hline UN_ci_6304 & $373 \mathrm{~A} / \mathrm{G}$ \\
\hline UN_ci_6304 & $399 \mathrm{C} / \mathrm{T}$ \\
\hline UN_ci_6304 & $422 \mathrm{G} / \mathrm{C}$ \\
\hline UN_ci_6304 & $443 \mathrm{C} / \mathrm{T}$ \\
\hline UN_ci_6304 & $477 \mathrm{C} / \mathrm{T}$ \\
\hline UN_ci_6304 & 502 G/A \\
\hline UN_ci_6304 & $535 \mathrm{~T} / \mathrm{C}$ \\
\hline UN_ci_6304 & $557 \mathrm{C} / \mathrm{T}$ \\
\hline UN_ci_6304 & $612 \mathrm{G} / \mathrm{A}$ \\
\hline UN_ci_6304 & $646 \mathrm{~A} / \mathrm{C}$ \\
\hline UN_ci_6304 & $710 \mathrm{G} / \mathrm{A}$ \\
\hline UN_ci_6304 & $779 \mathrm{G} / \mathrm{A}$ \\
\hline UN_ci_6304 & $846 \mathrm{~T} / \mathrm{G}$ \\
\hline UN_ci_6305 & $38 \mathrm{~T} / \mathrm{A}$ \\
\hline UN_ci_6305 & $307 \mathrm{G} / \mathrm{C}$ \\
\hline UN_ci_6305 & $347 \mathrm{~A} / \mathrm{T}$ \\
\hline UN_ci_6305 & $613 \mathrm{C} / \mathrm{A}$ \\
\hline UN_ci_6311 & $868 \mathrm{G} / \mathrm{A}$ \\
\hline UN_ci_6315 & $47 \mathrm{G} / \mathrm{T}$ \\
\hline UN_ci_6315 & $185 \mathrm{~T} / \mathrm{A}$ \\
\hline UN_ci_6315 & $366 \mathrm{G} / \mathrm{T}$ \\
\hline UN_ci_6316 & $98 \mathrm{~A} / \mathrm{G}$ \\
\hline UN_ci_6316 & $236 \mathrm{~A} / \mathrm{G}$ \\
\hline UN_ci_6318 & $229 \mathrm{~T} / \mathrm{C}$ \\
\hline UN_ci_6318 & $277 \mathrm{G} / \mathrm{T}$ \\
\hline UN_ci_6318 & $302 \mathrm{~T} / \mathrm{C}$ \\
\hline UN_ci_6318 & $368 \mathrm{~A} / \mathrm{G}$ \\
\hline UN_ci_6324 & $160 \mathrm{~T} / \mathrm{C}$ \\
\hline UN_ci_6326 & $215 \mathrm{C} / \mathrm{T}$ \\
\hline UN_ci_6326 & $240 \mathrm{C} / \mathrm{A}$ \\
\hline UN_ci_6326 & $290 \mathrm{C} / \mathrm{G}$ \\
\hline UN_ci_6326 & $596 \mathrm{G} / \mathrm{T}$ \\
\hline UN_ci_6326 & $651 \mathrm{~T} / \mathrm{C}$ \\
\hline UN_ci_6327 & $185 \mathrm{C} / \mathrm{T}$ \\
\hline UN_ci_6327 & $425 \mathrm{~T} / \mathrm{A}$ \\
\hline UN_ci_6327 & $466 \mathrm{G} / \mathrm{A}$ \\
\hline UN_ci_6327 & $650 \mathrm{~A} / \mathrm{G}$ \\
\hline UN_ci_6327 & $761 \mathrm{G} / \mathrm{A}$ \\
\hline UN_ci_6329 & $382 \mathrm{G} / \mathrm{A}$ \\
\hline UN_ci_6329 & $541 \mathrm{~A} / \mathrm{T}$ \\
\hline UN_ci_6336 & $248 \mathrm{~T} / \mathrm{C}$ \\
\hline UN_ci_6339 & $94 \mathrm{G} / \mathrm{T}$ \\
\hline UN_ci_6339 & $162 \mathrm{~A} / \mathrm{G}$ \\
\hline UN_ci_6345 & $774 \mathrm{~A} / \mathrm{C}$ \\
\hline UN_ci_6351 & $70 \mathrm{C} / \mathrm{G}$ \\
\hline UN_ci_6351 & $182 \mathrm{~T} / \mathrm{C}$ \\
\hline UN_ci_6351 & $271 \mathrm{~A} / \mathrm{G}$ \\
\hline UN_ci_6351 & $452 \mathrm{G} / \mathrm{A}$ \\
\hline UN_ci_6351 & $496 \mathrm{C} / \mathrm{G}$ \\
\hline
\end{tabular}




\begin{tabular}{|c|c|}
\hline UN_ci_6364 & $833 \mathrm{~A} / \mathrm{G}$ \\
\hline UN_ci_6364 & $945 \mathrm{~A} / \mathrm{G}$ \\
\hline UN_ci_6364 & 1195 T/C \\
\hline UN_ci_6374 & $89 \mathrm{~T} / \mathrm{C}$ \\
\hline UN_ci_6374 & $180 \mathrm{~T} / \mathrm{G}$ \\
\hline UN_ci_6374 & $378 \mathrm{C} / \mathrm{T}$ \\
\hline UN_ci_6374 & $985 \mathrm{G} / \mathrm{A}$ \\
\hline UN_ci_6374 & $1080 \mathrm{~T} / \mathrm{C}$ \\
\hline UN_ci_6374 & $1126 \mathrm{G} / \mathrm{A}$ \\
\hline UN_ci_6375 & 767 T/G \\
\hline UN_ci_6379 & 1107 G/C \\
\hline UN_ci_6392 & $408 \mathrm{~T} / \mathrm{C}$ \\
\hline UN_ci_6392 & 865 G/C \\
\hline UN_ci_6394 & $651 \mathrm{~T} / \mathrm{C}$ \\
\hline UN_ci_6394 & 770 T/G \\
\hline UN_ci_6394 & $852 \mathrm{~T} / \mathrm{C}$ \\
\hline UN_ci_6401 & $159 \mathrm{~T} / \mathrm{C}$ \\
\hline UN_ci_6401 & $310 \mathrm{~A} / \mathrm{G}$ \\
\hline UN_ci_6407 & $88 \mathrm{C} / \mathrm{T}$ \\
\hline UN_ci_6413 & $596 \mathrm{C} / \mathrm{T}$ \\
\hline UN_ci_6413 & $759 \mathrm{G} / \mathrm{C}$ \\
\hline UN_ci_6413 & $1023 \mathrm{~A} / \mathrm{G}$ \\
\hline UN_ci_6417 & $263 \mathrm{~T} / \mathrm{G}$ \\
\hline UN_ci_6417 & $1064 \mathrm{G} / \mathrm{A}$ \\
\hline UN_ci_6418 & $371 \mathrm{G} / \mathrm{C}$ \\
\hline UN_ci_6420 & $208 \mathrm{~T} / \mathrm{C}$ \\
\hline UN_ci_6424 & $361 \mathrm{C} / \mathrm{T}$ \\
\hline UN_ci_6424 & $392 \mathrm{~A} / \mathrm{G}$ \\
\hline UN_ci_6433 & $128 \mathrm{~T} / \mathrm{C}$ \\
\hline UN_ci_6433 & $210 \mathrm{G} / \mathrm{A}$ \\
\hline UN_ci_6433 & $316 \mathrm{~T} / \mathrm{C}$ \\
\hline UN_ci_6439 & $567 \mathrm{G} / \mathrm{A}$ \\
\hline UN_ci_6439 & $598 \mathrm{~A} / \mathrm{G}$ \\
\hline UN_ci_6446 & $304 \mathrm{~A} / \mathrm{G}$ \\
\hline UN_ci_6446 & $820 \mathrm{C} / \mathrm{T}$ \\
\hline UN_ci_6450 & 349 T/C \\
\hline UN_ci_6452 & $136 \mathrm{C} / \mathrm{A}$ \\
\hline UN_ci_6452 & $264 \mathrm{~A} / \mathrm{T}$ \\
\hline UN_ci_6452 & $577 \mathrm{G} / \mathrm{A}$ \\
\hline UN_ci_6454 & $89 \mathrm{C} / \mathrm{T}$ \\
\hline UN_ci_6454 & $311 \mathrm{~A} / \mathrm{G}$ \\
\hline UN_ci_6463 & $114 \mathrm{C} / \mathrm{A}$ \\
\hline UN_ci_6469 & $251 \mathrm{C} / \mathrm{T}$ \\
\hline UN_ci_6474 & $346 \mathrm{~A} / \mathrm{G}$ \\
\hline UN_ci_6474 & $406 \mathrm{G} / \mathrm{C}$ \\
\hline UN_ci_6477 & $58 \mathrm{~T} / \mathrm{C}$ \\
\hline UN_ci_6477 & $127 \mathrm{C} / \mathrm{T}$ \\
\hline UN_ci_6477 & $420 \mathrm{~T} / \mathrm{C}$ \\
\hline UN_ci_6477 & $655 \mathrm{C} / \mathrm{T}$ \\
\hline UN_ci_6484 & $67 \mathrm{~A} / \mathrm{G}$ \\
\hline
\end{tabular}




\begin{tabular}{|c|c|}
\hline UN_ci_6484 & $188 \mathrm{~A} / \mathrm{C}$ \\
\hline UN_ci_6484 & $382 \mathrm{~T} / \mathrm{C}$ \\
\hline UN_ci_6484 & $888 \mathrm{C} / \mathrm{T}$ \\
\hline UN_ci_6495 & $236 \mathrm{G} / \mathrm{A}$ \\
\hline UN_ci_6495 & $375 \mathrm{~A} / \mathrm{C}$ \\
\hline UN_ci_6495 & $605 \mathrm{C} / \mathrm{T}$ \\
\hline UN_ci_6495 & $626 \mathrm{~A} / \mathrm{G}$ \\
\hline UN_ci_6495 & $724 \mathrm{C} / \mathrm{G}$ \\
\hline UN_ci_6498 & $33 \mathrm{~A} / \mathrm{G}$ \\
\hline UN_ci_6498 & $62 \mathrm{G} / \mathrm{A}$ \\
\hline UN_ci_6498 & $97 \mathrm{C} / \mathrm{T}$ \\
\hline UN_ci_6505 & $100 \mathrm{~A} / \mathrm{G}$ \\
\hline UN_ci_6505 & 295 T/C \\
\hline UN_ci_6505 & $390 \mathrm{~A} / \mathrm{C}$ \\
\hline UN_ci_6505 & $563 \mathrm{~A} / \mathrm{G}$ \\
\hline UN_ci_6513 & $238 \mathrm{G} / \mathrm{T}$ \\
\hline UN_ci_6513 & $483 \mathrm{~A} / \mathrm{G}$ \\
\hline UN_ci_6521 & $250 \mathrm{~A} / \mathrm{G}$ \\
\hline UN_ci_6530 & $300 \mathrm{~A} / \mathrm{C}$ \\
\hline UN_ci_6530 & $432 \mathrm{C} / \mathrm{T}$ \\
\hline UN_ci_6530 & $513 \mathrm{C} / \mathrm{T}$ \\
\hline UN_ci_6531 & $46 \mathrm{C} / \mathrm{T}$ \\
\hline UN_ci_6531 & 589 A/G \\
\hline UN_ci_6531 & $791 \mathrm{C} / \mathrm{T}$ \\
\hline UN_ci_6537 & $256 \mathrm{~T} / \mathrm{G}$ \\
\hline UN_ci_6537 & $276 \mathrm{C} / \mathrm{T}$ \\
\hline UN_ci_6537 & $296 \mathrm{~T} / \mathrm{A}$ \\
\hline UN_ci_6537 & $316 \mathrm{~A} / \mathrm{G}$ \\
\hline UN_ci_6537 & 336 A/G \\
\hline UN_ci_6537 & 356 A/G \\
\hline UN_ci_6537 & $376 \mathrm{C} / \mathrm{T}$ \\
\hline UN_ci_6537 & $396 \mathrm{C} / \mathrm{T}$ \\
\hline UN_ci_6537 & $416 \mathrm{~A} / \mathrm{G}$ \\
\hline UN_ci_6537 & $436 \mathrm{~A} / \mathrm{G}$ \\
\hline UN_ci_6537 & $456 \mathrm{G} / \mathrm{T}$ \\
\hline UN_ci_6537 & $476 \mathrm{C} / \mathrm{A}$ \\
\hline UN_ci_6537 & $496 \mathrm{C} / \mathrm{G}$ \\
\hline UN_ci_6537 & $516 \mathrm{~T} / \mathrm{C}$ \\
\hline UN_ci_6537 & $537 \mathrm{~A} / \mathrm{G}$ \\
\hline UN_ci_6537 & $557 \mathrm{~T} / \mathrm{C}$ \\
\hline UN_ci_6537 & $578 \mathrm{~T} / \mathrm{C}$ \\
\hline UN_ci_6537 & $599 \mathrm{C} / \mathrm{T}$ \\
\hline UN_ci_6537 & 619 T/C \\
\hline UN_ci_6537 & $642 \mathrm{~A} / \mathrm{G}$ \\
\hline UN_ci_6537 & $664 \mathrm{~A} / \mathrm{G}$ \\
\hline UN_ci_6537 & $685 \mathrm{~A} / \mathrm{G}$ \\
\hline UN_ci_6537 & $705 \mathrm{G} / \mathrm{A}$ \\
\hline UN_ci_6537 & $725 \mathrm{~T} / \mathrm{C}$ \\
\hline UN_ci_6537 & 746 T/C \\
\hline UN_ci_6537 & $766 \mathrm{~A} / \mathrm{G}$ \\
\hline
\end{tabular}




\begin{tabular}{|c|c|}
\hline UN_ci_6537 & $787 \mathrm{C} / \mathrm{A}$ \\
\hline UN_ci_6537 & $812 \mathrm{~T} / \mathrm{C}$ \\
\hline UN_ci_6537 & $832 \mathrm{C} / \mathrm{T}$ \\
\hline UN_ci_6537 & $852 \mathrm{G} / \mathrm{A}$ \\
\hline UN_ci_6537 & $872 \mathrm{~A} / \mathrm{G}$ \\
\hline UN_ci_6537 & $892 \mathrm{~A} / \mathrm{G}$ \\
\hline UN_ci_6537 & $915 \mathrm{~T} / \mathrm{A}$ \\
\hline UN_ci_6537 & $935 \mathrm{~A} / \mathrm{G}$ \\
\hline UN_ci_6537 & $955 \mathrm{C} / \mathrm{A}$ \\
\hline UN_ci_6537 & 976 A/G \\
\hline UN_ci_6537 & $1000 \mathrm{C} / \mathrm{T}$ \\
\hline UN_ci_6537 & $1022 \mathrm{C} / \mathrm{T}$ \\
\hline UN_ci_6537 & $1043 \mathrm{~T} / \mathrm{C}$ \\
\hline UN_ci_6537 & $1067 \mathrm{~A} / \mathrm{G}$ \\
\hline UN_ci_6537 & $1089 \mathrm{~A} / \mathrm{C}$ \\
\hline UN_ci_6537 & $1111 \mathrm{~T} / \mathrm{A}$ \\
\hline UN_ci_6537 & $1136 \mathrm{~T} / \mathrm{C}$ \\
\hline UN_ci_6537 & 1157 T/C \\
\hline UN_ci_6547 & 399 A/G \\
\hline UN_ci_6552 & $38 \mathrm{G} / \mathrm{T}$ \\
\hline UN_ci_6552 & $654 \mathrm{~T} / \mathrm{C}$ \\
\hline UN_ci_6552 & $712 \mathrm{~T} / \mathrm{C}$ \\
\hline UN_ci_6554 & 75 T/G \\
\hline UN_ci_6554 & $98 \mathrm{G} / \mathrm{T}$ \\
\hline UN_ci_6554 & $281 \mathrm{G} / \mathrm{C}$ \\
\hline UN_ci_6554 & 313 G/A \\
\hline UN_ci_6554 & $370 \mathrm{~A} / \mathrm{G}$ \\
\hline UN_ci_6554 & $462 \mathrm{~A} / \mathrm{G}$ \\
\hline UN_ci_6554 & $527 \mathrm{G} / \mathrm{C}$ \\
\hline UN_ci_6554 & 589 A/G \\
\hline UN_ci_6554 & $634 \mathrm{C} / \mathrm{G}$ \\
\hline UN_ci_6554 & $681 \mathrm{~A} / \mathrm{G}$ \\
\hline UN_ci_6572 & $779 \mathrm{C} / \mathrm{T}$ \\
\hline UN_ci_6577 & $184 \mathrm{C} / \mathrm{T}$ \\
\hline UN_ci_6583 & $13 \mathrm{~A} / \mathrm{G}$ \\
\hline UN_ci_6583 & $219 \mathrm{~A} / \mathrm{G}$ \\
\hline UN_ci_6583 & $311 \mathrm{~T} / \mathrm{C}$ \\
\hline UN_ci_6583 & $364 \mathrm{G} / \mathrm{A}$ \\
\hline UN_ci_6583 & $455 \mathrm{~A} / \mathrm{G}$ \\
\hline UN_ci_6589 & $519 \mathrm{~T} / \mathrm{C}$ \\
\hline UN_ci_6606 & $69 \mathrm{G} / \mathrm{C}$ \\
\hline UN_ci_6606 & $120 \mathrm{~A} / \mathrm{G}$ \\
\hline UN_ci_6606 & $157 \mathrm{~T} / \mathrm{C}$ \\
\hline UN_ci_6606 & $322 \mathrm{~A} / \mathrm{G}$ \\
\hline UN_ci_6606 & $370 \mathrm{C} / \mathrm{T}$ \\
\hline UN_ci_6606 & $448 \mathrm{~A} / \mathrm{C}$ \\
\hline UN_ci_6606 & $515 \mathrm{~A} / \mathrm{T}$ \\
\hline UN_ci_6606 & $602 \mathrm{C} / \mathrm{T}$ \\
\hline UN_ci_6619 & $493 \mathrm{G} / \mathrm{A}$ \\
\hline UN_ci_6619 & $717 \mathrm{G} / \mathrm{A}$ \\
\hline
\end{tabular}




\begin{tabular}{|c|c|}
\hline UN_ci_6620 & $140 \mathrm{~T} / \mathrm{C}$ \\
\hline UN_ci_6625 & $247 \mathrm{~A} / \mathrm{G}$ \\
\hline UN_ci_6630 & $682 \mathrm{C} / \mathrm{G}$ \\
\hline UN_ci_6630 & $787 \mathrm{G} / \mathrm{A}$ \\
\hline UN_ci_6630 & $1416 \mathrm{~T} / \mathrm{C}$ \\
\hline UN_ci_6630 & $1507 \mathrm{G} / \mathrm{T}$ \\
\hline UN_ci_6630 & $1564 \mathrm{~T} / \mathrm{G}$ \\
\hline UN_ci_6630 & $1702 \mathrm{G} / \mathrm{A}$ \\
\hline UN_ci_6630 & $1825 \mathrm{C} / \mathrm{A}$ \\
\hline UN_ci_6630 & 1987 T/C \\
\hline UN_ci_6630 & $2065 \mathrm{~A} / \mathrm{G}$ \\
\hline UN_ci_6630 & $2148 \mathrm{~A} / \mathrm{G}$ \\
\hline UN_ci_6630 & $2182 \mathrm{~T} / \mathrm{G}$ \\
\hline UN_ci_6636 & $39 \mathrm{~A} / \mathrm{C}$ \\
\hline UN_ci_6636 & $145 \mathrm{C} / \mathrm{T}$ \\
\hline UN_ci_6636 & $223 \mathrm{~A} / \mathrm{G}$ \\
\hline UN_ci_6637 & $253 \mathrm{~T} / \mathrm{A}$ \\
\hline UN_ci_6637 & $351 \mathrm{G} / \mathrm{A}$ \\
\hline UN_ci_6637 & $1148 \mathrm{~A} / \mathrm{G}$ \\
\hline UN_ci_6643 & $1063 \mathrm{G} / \mathrm{A}$ \\
\hline UN_ci_6646 & $425 \mathrm{~A} / \mathrm{G}$ \\
\hline UN_ci_6650 & $1265 \mathrm{~T} / \mathrm{C}$ \\
\hline UN_ci_6650 & $1302 \mathrm{~A} / \mathrm{T}$ \\
\hline UN_ci_6650 & $1648 \mathrm{~A} / \mathrm{G}$ \\
\hline UN_ci_6650 & $1856 \mathrm{C} / \mathrm{T}$ \\
\hline UN_ci_6652 & $82 \mathrm{~T} / \mathrm{C}$ \\
\hline UN_ci_6658 & $456 \mathrm{~A} / \mathrm{G}$ \\
\hline UN_ci_6674 & $936 \mathrm{C} / \mathrm{T}$ \\
\hline UN_ci_6674 & 1167 T/G \\
\hline UN_ci_6680 & $117 \mathrm{~A} / \mathrm{G}$ \\
\hline UN_ci_6680 & $856 \mathrm{~A} / \mathrm{C}$ \\
\hline UN_ci_6681 & $52 \mathrm{C} / \mathrm{T}$ \\
\hline UN_ci_6692 & $577 \mathrm{C} / \mathrm{T}$ \\
\hline UN_ci_6693 & $260 \mathrm{~A} / \mathrm{G}$ \\
\hline UN_ci_6696 & $88 \mathrm{C} / \mathrm{T}$ \\
\hline UN_ci_6700 & $199 \mathrm{G} / \mathrm{A}$ \\
\hline UN_ci_6705 & $116 \mathrm{C} / \mathrm{T}$ \\
\hline UN_ci_6705 & $161 \mathrm{~T} / \mathrm{C}$ \\
\hline UN_ci_6705 & $198 \mathrm{~A} / \mathrm{T}$ \\
\hline UN_ci_6711 & $368 \mathrm{G} / \mathrm{T}$ \\
\hline UN_ci_6725 & $209 \mathrm{~T} / \mathrm{C}$ \\
\hline UN_ci_6725 & $284 \mathrm{G} / \mathrm{A}$ \\
\hline UN_ci_6725 & $340 \mathrm{~A} / \mathrm{G}$ \\
\hline UN_ci_6726 & $125 \mathrm{C} / \mathrm{T}$ \\
\hline UN_ci_6726 & $248 \mathrm{~A} / \mathrm{G}$ \\
\hline UN_ci_6726 & $382 \mathrm{~T} / \mathrm{C}$ \\
\hline UN_ci_6728 & $174 \mathrm{~T} / \mathrm{C}$ \\
\hline UN_ci_6729 & $158 \mathrm{~A} / \mathrm{G}$ \\
\hline UN_ci_6729 & $184 \mathrm{~T} / \mathrm{C}$ \\
\hline UN_ci_6729 & $291 \mathrm{C} / \mathrm{A}$ \\
\hline
\end{tabular}




\begin{tabular}{|c|c|}
\hline UN_ci_6738 & $203 \mathrm{C} / \mathrm{T}$ \\
\hline UN_ci_6748 & $102 \mathrm{G} / \mathrm{C}$ \\
\hline UN_ci_6748 & $435 \mathrm{~A} / \mathrm{C}$ \\
\hline UN_ci_6751 & 249 A/G \\
\hline UN_ci_6751 & $384 \mathrm{C} / \mathrm{A}$ \\
\hline UN_ci_6756 & $369 \mathrm{C} / \mathrm{T}$ \\
\hline UN_ci_6756 & $610 \mathrm{~A} / \mathrm{T}$ \\
\hline UN_ci_6756 & $667 \mathrm{~A} / \mathrm{T}$ \\
\hline UN_ci_6776 & $704 \mathrm{C} / \mathrm{T}$ \\
\hline UN_ci_6791 & $238 \mathrm{~T} / \mathrm{C}$ \\
\hline UN_ci_6796 & $352 \mathrm{~T} / \mathrm{C}$ \\
\hline UN_ci_6798 & $143 \mathrm{~T} / \mathrm{A}$ \\
\hline UN_ci_6802 & $92 \mathrm{~A} / \mathrm{G}$ \\
\hline UN_ci_6802 & $212 \mathrm{~T} / \mathrm{C}$ \\
\hline UN_ci_6802 & $255 \mathrm{~A} / \mathrm{G}$ \\
\hline UN_ci_6804 & $7 \mathrm{G} / \mathrm{T}$ \\
\hline UN_ci_6804 & $54 \mathrm{~A} / \mathrm{C}$ \\
\hline UN_ci_6804 & $74 \mathrm{~A} / \mathrm{G}$ \\
\hline UN_ci_6804 & $255 \mathrm{C} / \mathrm{T}$ \\
\hline UN_ci_6804 & 357 T/C \\
\hline UN_ci_6804 & $708 \mathrm{~A} / \mathrm{G}$ \\
\hline UN_ci_6804 & 791 T/C \\
\hline UN_ci_6810 & $405 \mathrm{C} / \mathrm{A}$ \\
\hline UN_ci_6818 & 177 T/C \\
\hline UN_ci_6832 & $562 \mathrm{C} / \mathrm{T}$ \\
\hline UN_ci_6832 & $607 \mathrm{~A} / \mathrm{G}$ \\
\hline UN_ci_6832 & $627 \mathrm{C} / \mathrm{T}$ \\
\hline UN_ci_6832 & $680 \mathrm{~T} / \mathrm{G}$ \\
\hline UN_ci_6832 & $767 \mathrm{~A} / \mathrm{G}$ \\
\hline UN_ci_6832 & $824 \mathrm{~A} / \mathrm{G}$ \\
\hline UN_ci_6840 & $362 \mathrm{G} / \mathrm{A}$ \\
\hline UN_ci_6871 & $103 \mathrm{G} / \mathrm{A}$ \\
\hline UN_ci_6872 & $70 \mathrm{G} / \mathrm{A}$ \\
\hline UN_ci_6887 & $296 \mathrm{~T} / \mathrm{C}$ \\
\hline UN_ci_6891 & $671 \mathrm{C} / \mathrm{T}$ \\
\hline UN_ci_6900 & $328 \mathrm{~T} / \mathrm{A}$ \\
\hline UN_ci_6902 & 702 G/C \\
\hline UN_ci_6905 & $502 \mathrm{~A} / \mathrm{G}$ \\
\hline UN_ci_6907 & $707 \mathrm{~A} / \mathrm{G}$ \\
\hline UN_ci_6910 & $76 \mathrm{C} / \mathrm{A}$ \\
\hline UN_ci_6910 & $243 \mathrm{~A} / \mathrm{G}$ \\
\hline UN_ci_6910 & $338 \mathrm{~A} / \mathrm{G}$ \\
\hline UN_ci_6910 & $367 \mathrm{C} / \mathrm{T}$ \\
\hline UN_ci_6910 & $564 \mathrm{~T} / \mathrm{C}$ \\
\hline UN_ci_6910 & $617 \mathrm{C} / \mathrm{T}$ \\
\hline UN_ci_6910 & $686 \mathrm{C} / \mathrm{T}$ \\
\hline UN_ci_6910 & $824 \mathrm{~T} / \mathrm{C}$ \\
\hline UN_ci_6910 & $1068 \mathrm{C} / \mathrm{A}$ \\
\hline UN_ci_6918 & $371 \mathrm{~A} / \mathrm{T}$ \\
\hline UN_ci_6918 & $818 \mathrm{C} / \mathrm{T}$ \\
\hline
\end{tabular}




$\begin{array}{lr}\text { UN_ci_6929 } & 250 \mathrm{~A} / \mathrm{G} \\ \text { UN_ci_6929 } & 288 \mathrm{C} / \mathrm{G} \\ \text { UN_ci_6934 } & 68 \mathrm{G} / \mathrm{T} \\ \text { UN_ci_6934 } & 701 \mathrm{~A} / \mathrm{C} \\ \text { UN_ci_6942 } & 362 \mathrm{G} / \mathrm{C} \\ \text { UN_ci_6959 } & 948 \mathrm{G} / \mathrm{A} \\ \text { UN_ci_6964 } & 270 \mathrm{G} / \mathrm{A} \\ \text { UN_ci_6966 } & 1188 \mathrm{~T} / \mathrm{C} \\ \text { UN_ci_6967 } & 167 \mathrm{~A} / \mathrm{C} \\ \text { UN_ci_6967 } & 221 \mathrm{G} / \mathrm{A} \\ \text { UN_ci_6967 } & 345 \mathrm{~T} / \mathrm{A} \\ \text { UN_ci_6972 } & 582 \mathrm{~A} / \mathrm{G} \\ \text { UN_ci_6972 } & 604 \mathrm{C} / \mathrm{T} \\ \text { UN_ci_6972 } & 688 \mathrm{C} / \mathrm{T} \\ \text { UN_ci_6972 } & 808 \mathrm{~A} / \mathrm{C} \\ \text { UN_ci_6975 } & 343 \mathrm{~A} / \mathrm{T} \\ \text { UN_ci_6975 } & 456 \mathrm{C} / \mathrm{T} \\ \text { UN_ci_6977 } & 388 \mathrm{~A} / \mathrm{C} \\ \text { UN_ci_6977 } & 493 \mathrm{G} / \mathrm{A} \\ \text { UN_ci_6982 } & 37 \mathrm{C} / \mathrm{A} \\ \text { UN_ci_6985 } & 377 \mathrm{~A} / \mathrm{G} \\ \text { UN_ci_6993 } & 121 \mathrm{G} / \mathrm{A} \\ \text { UN_ci_6996 } & \end{array}$




\begin{tabular}{|c|c|}
\hline UN_ci_7063 & $261 \mathrm{G} / \mathrm{A}$ \\
\hline UN_ci_7063 & $414 \mathrm{~A} / \mathrm{G}$ \\
\hline UN_ci_7065 & $811 \mathrm{~A} / \mathrm{G}$ \\
\hline UN_ci_7065 & $985 \mathrm{~T} / \mathrm{C}$ \\
\hline UN_ci_7067 & $33 \mathrm{C} / \mathrm{T}$ \\
\hline UN_ci_7067 & $625 \mathrm{C} / \mathrm{T}$ \\
\hline UN_ci_7067 & $778 \mathrm{G} / \mathrm{A}$ \\
\hline UN_ci_7067 & $921 \mathrm{~A} / \mathrm{G}$ \\
\hline UN_ci_7067 & $946 \mathrm{G} / \mathrm{A}$ \\
\hline UN_ci_7067 & $989 \mathrm{G} / \mathrm{A}$ \\
\hline UN_ci_7067 & 1039 A/G \\
\hline UN_ci_7067 & $1110 \mathrm{~A} / \mathrm{C}$ \\
\hline UN_ci_7071 & $401 \mathrm{G} / \mathrm{A}$ \\
\hline UN_ci_7071 & $480 \mathrm{G} / \mathrm{A}$ \\
\hline UN_ci_7071 & $536 \mathrm{~A} / \mathrm{T}$ \\
\hline UN_ci_7071 & $627 \mathrm{~A} / \mathrm{G}$ \\
\hline UN_ci_7071 & $715 \mathrm{G} / \mathrm{A}$ \\
\hline UN_ci_7071 & $788 \mathrm{~T} / \mathrm{C}$ \\
\hline UN_ci_7071 & $814 \mathrm{~A} / \mathrm{G}$ \\
\hline UN_ci_7071 & $844 \mathrm{~T} / \mathrm{C}$ \\
\hline UN_ci_7071 & $888 \mathrm{G} / \mathrm{C}$ \\
\hline UN_ci_7071 & $915 \mathrm{~A} / \mathrm{G}$ \\
\hline UN_ci_7071 & 972 T/C \\
\hline UN_ci_7071 & 1002 T/C \\
\hline UN_ci_7071 & $1035 \mathrm{~A} / \mathrm{G}$ \\
\hline UN_ci_7071 & $1114 \mathrm{~T} / \mathrm{A}$ \\
\hline UN_ci_7071 & $1167 \mathrm{C} / \mathrm{T}$ \\
\hline UN_ci_7071 & $1231 \mathrm{~A} / \mathrm{T}$ \\
\hline UN_ci_7071 & $1252 \mathrm{~A} / \mathrm{C}$ \\
\hline UN_ci_7071 & $1288 \mathrm{~A} / \mathrm{C}$ \\
\hline UN_ci_7071 & $1308 \mathrm{C} / \mathrm{T}$ \\
\hline UN_ci_7071 & $1329 \mathrm{G} / \mathrm{T}$ \\
\hline UN_ci_7071 & $1351 \mathrm{G} / \mathrm{A}$ \\
\hline UN_ci_7071 & 1373 A/G \\
\hline UN_ci_7071 & 1398 A/G \\
\hline UN_ci_7071 & $1418 \mathrm{~T} / \mathrm{C}$ \\
\hline UN_ci_7071 & $1443 \mathrm{~T} / \mathrm{C}$ \\
\hline UN_ci_7071 & $1464 \mathrm{~A} / \mathrm{G}$ \\
\hline UN_ci_7071 & $1490 \mathrm{G} / \mathrm{A}$ \\
\hline UN_ci_7071 & $1510 \mathrm{~T} / \mathrm{A}$ \\
\hline UN_ci_7072 & $12 \mathrm{G} / \mathrm{T}$ \\
\hline UN_ci_7072 & $52 \mathrm{G} / \mathrm{T}$ \\
\hline UN_ci_7072 & 149 T/C \\
\hline UN_ci_7081 & 379 C/T \\
\hline UN_ci_7095 & $319 \mathrm{C} / \mathrm{T}$ \\
\hline UN_ci_7097 & 389 G/A \\
\hline UN_ci_7098 & $616 \mathrm{C} / \mathrm{G}$ \\
\hline UN_ci_7098 & $655 \mathrm{~A} / \mathrm{G}$ \\
\hline UN_ci_7098 & $699 \mathrm{C} / \mathrm{T}$ \\
\hline UN_ci_7098 & $721 \mathrm{~A} / \mathrm{C}$ \\
\hline
\end{tabular}




\begin{tabular}{|c|c|}
\hline UN_ci_7098 & $773 \mathrm{G} / \mathrm{A}$ \\
\hline UN_ci_7098 & $835 \mathrm{C} / \mathrm{T}$ \\
\hline UN_ci_7098 & $884 \mathrm{~A} / \mathrm{G}$ \\
\hline UN_ci_7098 & $941 \mathrm{~A} / \mathrm{G}$ \\
\hline UN_ci_7098 & $978 \mathrm{~T} / \mathrm{C}$ \\
\hline UN_ci_7100 & $12 \mathrm{G} / \mathrm{A}$ \\
\hline UN_ci_7101 & $259 \mathrm{G} / \mathrm{A}$ \\
\hline UN_ci_7101 & $520 \mathrm{C} / \mathrm{T}$ \\
\hline UN_ci_7108 & $402 \mathrm{C} / \mathrm{T}$ \\
\hline UN_ci_7111 & $692 \mathrm{~A} / \mathrm{G}$ \\
\hline UN_ci_7121 & $446 \mathrm{C} / \mathrm{T}$ \\
\hline UN_ci_7121 & $479 \mathrm{~T} / \mathrm{C}$ \\
\hline UN_ci_7121 & $542 \mathrm{~T} / \mathrm{C}$ \\
\hline UN_ci_7121 & $699 \mathrm{~T} / \mathrm{C}$ \\
\hline UN_ci_7121 & $750 \mathrm{~A} / \mathrm{G}$ \\
\hline UN_ci_7121 & $784 \mathrm{~A} / \mathrm{G}$ \\
\hline UN_ci_7121 & $826 \mathrm{~A} / \mathrm{G}$ \\
\hline UN_ci_7125 & $58 \mathrm{~A} / \mathrm{T}$ \\
\hline UN_ci_7125 & $159 \mathrm{~A} / \mathrm{G}$ \\
\hline UN_ci_7125 & $179 \mathrm{C} / \mathrm{T}$ \\
\hline UN_ci_7134 & $240 \mathrm{C} / \mathrm{A}$ \\
\hline UN_ci_7134 & $318 \mathrm{~T} / \mathrm{A}$ \\
\hline UN_ci_7143 & $8 \mathrm{~A} / \mathrm{T}$ \\
\hline UN_ci_7143 & $93 \mathrm{C} / \mathrm{A}$ \\
\hline UN_ci_7149 & $132 \mathrm{G} / \mathrm{A}$ \\
\hline UN_ci_7149 & $302 \mathrm{~T} / \mathrm{C}$ \\
\hline UN_ci_7149 & $351 \mathrm{G} / \mathrm{T}$ \\
\hline UN_ci_7149 & $824 \mathrm{~T} / \mathrm{G}$ \\
\hline UN_ci_7149 & 1059 A/G \\
\hline UN_ci_7154 & $84 \mathrm{C} / \mathrm{T}$ \\
\hline UN_ci_7163 & $644 \mathrm{C} / \mathrm{T}$ \\
\hline UN_ci_7163 & $722 \mathrm{~A} / \mathrm{G}$ \\
\hline UN_ci_7168 & $109 \mathrm{G} / \mathrm{A}$ \\
\hline UN_ci_7169 & $331 \mathrm{~T} / \mathrm{C}$ \\
\hline UN_ci_7171 & $167 \mathrm{~A} / \mathrm{T}$ \\
\hline UN_ci_7171 & $314 \mathrm{~A} / \mathrm{G}$ \\
\hline UN_ci_7171 & 489 A/G \\
\hline UN_ci_7171 & $734 \mathrm{C} / \mathrm{A}$ \\
\hline UN_ci_7171 & $882 \mathrm{G} / \mathrm{A}$ \\
\hline UN_ci_7175 & 229 G/A \\
\hline UN_ci_7175 & $340 \mathrm{~T} / \mathrm{C}$ \\
\hline UN_ci_7175 & $683 \mathrm{~A} / \mathrm{G}$ \\
\hline UN_ci_7183 & $72 \mathrm{G} / \mathrm{T}$ \\
\hline UN_ci_7184 & $171 \mathrm{G} / \mathrm{A}$ \\
\hline UN_ci_7185 & $347 \mathrm{~T} / \mathrm{C}$ \\
\hline UN_ci_7185 & $367 \mathrm{~T} / \mathrm{C}$ \\
\hline UN_ci_7185 & $647 \mathrm{~T} / \mathrm{C}$ \\
\hline UN_ci_7186 & $67 \mathrm{~A} / \mathrm{G}$ \\
\hline UN_ci_7186 & 386 G/C \\
\hline UN_ci_7186 & $413 \mathrm{G} / \mathrm{C}$ \\
\hline
\end{tabular}




$\begin{array}{lr}\text { UN_ci_7186 } & \\ \text { UN_ci_7187 } & 680 \mathrm{~A} / \mathrm{G} \\ \text { UN_ci_7188 } & 46 \mathrm{~T} / \mathrm{A} \\ \text { UN_ci_7188 } & 75 \mathrm{~T} / \mathrm{C} \\ \text { UN_ci_7195 } & 165 \mathrm{G} / \mathrm{A} \\ \text { UN_ci_7201 } & 721 \mathrm{G} / \mathrm{A} \\ \text { UN_ci_7201 } & 300 \mathrm{G} / \mathrm{A} \\ \text { UN_ci_7207 } & 404 \mathrm{~T} / \mathrm{C} \\ \text { UN_ci_7208 } & 682 \mathrm{G} / \mathrm{A} \\ \text { UN_ci_7213 } & 405 \mathrm{C} / \mathrm{T} \\ \text { UN_ci_7213 } & 54 \mathrm{G} / \mathrm{T} \\ \text { UN_ci_7213 } & 88 \mathrm{C} / \mathrm{A} \\ \text { UN_ci_7213 } & 145 \mathrm{C} / \mathrm{G} \\ \text { UN_ci_7213 } & 416 \mathrm{~A} / \mathrm{C} \\ \text { UN_ci_7213 } & 535 \mathrm{~T} / \mathrm{C} \\ \text { UN_ci_7228 } & 976 \mathrm{G} / \mathrm{A} \\ \text { UN_ci_7233 } & 272 \mathrm{~T} / \mathrm{C} \\ \text { UN_ci_7235 } & 31 \mathrm{C} / \mathrm{T} \\ \text { UN_ci_7235 } & 327 \mathrm{~A} / \mathrm{G} \\ \text { UN_ci_7235 } & 473 \mathrm{~A} / \mathrm{G} \\ \text { UN_ci_7238 } & 514 \mathrm{G} / \mathrm{A} \\ \text { UN_ci_7238 } & 229 \mathrm{G} / \mathrm{C} \\ \text { UN_ci_7239 } & 307 \mathrm{~T} / \mathrm{C} \\ \text { UN_ci_7246 } & 58 \mathrm{G} / \mathrm{C} \\ \text { UN_ci_7246 } & 328 \mathrm{G} / \mathrm{T} \\ \text { UN_ci_7246 } & 1057 \mathrm{~A} / \mathrm{C} \\ \text { UN_ci_7254 } & 53 \mathrm{C} / \mathrm{T} \\ \text { UN_ci_7254 } & \end{array}$




\begin{tabular}{|c|c|}
\hline UN_ci_7311 & $72 \mathrm{~T} / \mathrm{C}$ \\
\hline UN_ci_7311 & $187 \mathrm{G} / \mathrm{A}$ \\
\hline UN_ci_7311 & $258 \mathrm{C} / \mathrm{A}$ \\
\hline UN_ci_7311 & $353 \mathrm{~T} / \mathrm{C}$ \\
\hline UN_ci_7311 & $450 \mathrm{C} / \mathrm{T}$ \\
\hline UN_ci_7311 & $580 \mathrm{~A} / \mathrm{G}$ \\
\hline UN_ci_7311 & $624 \mathrm{C} / \mathrm{T}$ \\
\hline UN_ci_7311 & $957 \mathrm{~T} / \mathrm{C}$ \\
\hline UN_ci_7311 & $1031 \mathrm{~T} / \mathrm{C}$ \\
\hline UN_ci_7321 & $216 \mathrm{G} / \mathrm{A}$ \\
\hline UN_ci_7325 & $190 \mathrm{~A} / \mathrm{T}$ \\
\hline UN_ci_7325 & $478 \mathrm{~A} / \mathrm{G}$ \\
\hline UN_ci_7325 & $806 \mathrm{C} / \mathrm{T}$ \\
\hline UN_ci_7325 & $854 \mathrm{~T} / \mathrm{C}$ \\
\hline UN_ci_7325 & $893 \mathrm{C} / \mathrm{G}$ \\
\hline UN_ci_7329 & $360 \mathrm{C} / \mathrm{T}$ \\
\hline UN_ci_7329 & $422 \mathrm{~A} / \mathrm{G}$ \\
\hline UN_ci_7329 & $524 \mathrm{~T} / \mathrm{C}$ \\
\hline UN_ci_7329 & $557 \mathrm{~T} / \mathrm{G}$ \\
\hline UN_ci_7329 & $615 \mathrm{~T} / \mathrm{C}$ \\
\hline UN_ci_7333 & $469 \mathrm{C} / \mathrm{T}$ \\
\hline UN_ci_7333 & $599 \mathrm{C} / \mathrm{T}$ \\
\hline UN_ci_7333 & $808 \mathrm{~T} / \mathrm{C}$ \\
\hline UN_ci_7333 & $904 \mathrm{G} / \mathrm{T}$ \\
\hline UN_ci_7333 & $981 \mathrm{~A} / \mathrm{G}$ \\
\hline UN_ci_7333 & $1029 \mathrm{C} / \mathrm{A}$ \\
\hline UN_ci_7339 & $95 \mathrm{~T} / \mathrm{G}$ \\
\hline UN_ci_7339 & $116 \mathrm{~A} / \mathrm{C}$ \\
\hline UN_ci_7339 & $296 \mathrm{~A} / \mathrm{T}$ \\
\hline UN_ci_7339 & $402 \mathrm{C} / \mathrm{T}$ \\
\hline UN_ci_7339 & $790 \mathrm{~T} / \mathrm{C}$ \\
\hline UN_ci_7339 & $971 \mathrm{~T} / \mathrm{C}$ \\
\hline UN_ci_7339 & $1014 \mathrm{~A} / \mathrm{G}$ \\
\hline UN_ci_7342 & $200 \mathrm{G} / \mathrm{A}$ \\
\hline UN_ci_7342 & $224 \mathrm{~T} / \mathrm{A}$ \\
\hline UN_ci_7348 & $15 \mathrm{C} / \mathrm{G}$ \\
\hline UN_ci_7350 & $32 \mathrm{~T} / \mathrm{A}$ \\
\hline UN_ci_7350 & $187 \mathrm{~A} / \mathrm{T}$ \\
\hline UN_ci_7350 & $257 \mathrm{~A} / \mathrm{G}$ \\
\hline UN_ci_7350 & $326 \mathrm{~A} / \mathrm{C}$ \\
\hline UN_ci_7350 & $377 \mathrm{C} / \mathrm{T}$ \\
\hline UN_ci_7350 & $434 \mathrm{~A} / \mathrm{G}$ \\
\hline UN_ci_7350 & $463 \mathrm{G} / \mathrm{A}$ \\
\hline UN_ci_7350 & $848 \mathrm{~A} / \mathrm{G}$ \\
\hline UN_ci_7350 & $885 \mathrm{~A} / \mathrm{G}$ \\
\hline UN_ci_7352 & $340 \mathrm{G} / \mathrm{A}$ \\
\hline UN_ci_7354 & $53 \mathrm{G} / \mathrm{C}$ \\
\hline UN_ci_7354 & $121 \mathrm{C} / \mathrm{A}$ \\
\hline UN_ci_7354 & $763 \mathrm{C} / \mathrm{T}$ \\
\hline UN_ci_7356 & $110 \mathrm{~A} / \mathrm{G}$ \\
\hline
\end{tabular}




\begin{tabular}{|c|c|}
\hline UN_ci_7356 & $507 \mathrm{~A} / \mathrm{G}$ \\
\hline UN_ci_7356 & $759 \mathrm{C} / \mathrm{T}$ \\
\hline UN_ci_7356 & $1002 \mathrm{~T} / \mathrm{C}$ \\
\hline UN_ci_7362 & $355 \mathrm{C} / \mathrm{G}$ \\
\hline UN_ci_7362 & $412 \mathrm{G} / \mathrm{A}$ \\
\hline UN_ci_7368 & 1194 A/G \\
\hline UN_ci_7375 & $498 \mathrm{C} / \mathrm{T}$ \\
\hline UN_ci_7375 & $577 \mathrm{G} / \mathrm{A}$ \\
\hline UN_ci_7375 & $754 \mathrm{C} / \mathrm{T}$ \\
\hline UN_ci_7375 & $820 \mathrm{~A} / \mathrm{C}$ \\
\hline UN_ci_7379 & $313 \mathrm{G} / \mathrm{A}$ \\
\hline UN_ci_7397 & $100 \mathrm{G} / \mathrm{A}$ \\
\hline UN_ci_7397 & $135 \mathrm{C} / \mathrm{T}$ \\
\hline UN_ci_7397 & $306 \mathrm{C} / \mathrm{T}$ \\
\hline UN_ci_7397 & 376 T/C \\
\hline UN_ci_7397 & $738 \mathrm{~T} / \mathrm{G}$ \\
\hline UN_ci_7402 & $125 \mathrm{~T} / \mathrm{G}$ \\
\hline UN_ci_7403 & $118 \mathrm{G} / \mathrm{A}$ \\
\hline UN_ci_7403 & $584 \mathrm{G} / \mathrm{A}$ \\
\hline UN_ci_7405 & $686 \mathrm{G} / \mathrm{A}$ \\
\hline UN_ci_7405 & $750 \mathrm{G} / \mathrm{A}$ \\
\hline UN_ci_7405 & $968 \mathrm{~T} / \mathrm{C}$ \\
\hline UN_ci_7408 & $111 \mathrm{~A} / \mathrm{T}$ \\
\hline UN_ci_7408 & 202 T/C \\
\hline UN_ci_7408 & $225 \mathrm{~T} / \mathrm{C}$ \\
\hline UN_ci_7408 & $336 \mathrm{~T} / \mathrm{A}$ \\
\hline UN_ci_7408 & $475 \mathrm{G} / \mathrm{A}$ \\
\hline UN_ci_7410 & $101 \mathrm{~A} / \mathrm{G}$ \\
\hline UN_ci_7418 & $146 \mathrm{~A} / \mathrm{G}$ \\
\hline UN_ci_7421 & $613 \mathrm{~A} / \mathrm{T}$ \\
\hline UN_ci_7421 & 955 G/T \\
\hline UN_ci_7423 & $268 \mathrm{C} / \mathrm{T}$ \\
\hline UN_ci_7423 & 312 T/C \\
\hline UN_ci_7423 & $419 \mathrm{G} / \mathrm{A}$ \\
\hline UN_ci_7453 & $334 \mathrm{~A} / \mathrm{T}$ \\
\hline UN_ci_7455 & $152 \mathrm{G} / \mathrm{A}$ \\
\hline UN_ci_7455 & 797 C/G \\
\hline UN_ci_7457 & $65 \mathrm{C} / \mathrm{A}$ \\
\hline UN_ci_7469 & $902 \mathrm{~T} / \mathrm{A}$ \\
\hline UN_ci_7472 & $154 \mathrm{~T} / \mathrm{C}$ \\
\hline UN_ci_7472 & $266 \mathrm{C} / \mathrm{T}$ \\
\hline UN_ci_7486 & $62 \mathrm{~A} / \mathrm{G}$ \\
\hline UN_ci_7492 & $255 \mathrm{~T} / \mathrm{A}$ \\
\hline UN_ci_7515 & $592 \mathrm{C} / \mathrm{T}$ \\
\hline UN_ci_7515 & $868 \mathrm{G} / \mathrm{A}$ \\
\hline UN_ci_7527 & $594 \mathrm{~A} / \mathrm{G}$ \\
\hline UN_ci_7532 & $882 \mathrm{~A} / \mathrm{G}$ \\
\hline UN_ci_7542 & $735 \mathrm{~T} / \mathrm{C}$ \\
\hline UN_ci_7542 & $900 \mathrm{C} / \mathrm{T}$ \\
\hline UN_ci_7552 & $57 \mathrm{~T} / \mathrm{C}$ \\
\hline
\end{tabular}




\begin{tabular}{|c|c|}
\hline UN_ci_7552 & $188 \mathrm{C} / \mathrm{G}$ \\
\hline UN_ci_7552 & $223 \mathrm{G} / \mathrm{A}$ \\
\hline UN_ci_7552 & $268 \mathrm{G} / \mathrm{C}$ \\
\hline UN_ci_7558 & $237 \mathrm{~T} / \mathrm{C}$ \\
\hline UN_ci_7568 & $131 \mathrm{C} / \mathrm{T}$ \\
\hline UN_ci_7572 & $46 \mathrm{~T} / \mathrm{A}$ \\
\hline UN_ci_7575 & $561 \mathrm{~A} / \mathrm{C}$ \\
\hline UN_ci_7587 & $108 \mathrm{G} / \mathrm{T}$ \\
\hline UN_ci_7587 & $142 \mathrm{~A} / \mathrm{G}$ \\
\hline UN_ci_7587 & $328 \mathrm{G} / \mathrm{A}$ \\
\hline UN_ci_7587 & $373 \mathrm{~T} / \mathrm{C}$ \\
\hline UN_ci_7587 & $439 \mathrm{~T} / \mathrm{C}$ \\
\hline UN_ci_7587 & $568 \mathrm{~A} / \mathrm{G}$ \\
\hline UN_ci_7587 & $588 \mathrm{~A} / \mathrm{G}$ \\
\hline UN_ci_7587 & $612 \mathrm{~A} / \mathrm{G}$ \\
\hline UN_ci_7587 & $750 \mathrm{G} / \mathrm{C}$ \\
\hline UN_ci_7588 & $685 \mathrm{C} / \mathrm{T}$ \\
\hline UN_ci_7588 & $851 \mathrm{G} / \mathrm{A}$ \\
\hline UN_ci_7591 & $169 \mathrm{G} / \mathrm{T}$ \\
\hline UN_ci_7591 & $474 \mathrm{~A} / \mathrm{G}$ \\
\hline UN_ci_7596 & $332 \mathrm{G} / \mathrm{A}$ \\
\hline UN_ci_7605 & $287 \mathrm{~T} / \mathrm{A}$ \\
\hline UN_ci_7609 & $972 \mathrm{~T} / \mathrm{C}$ \\
\hline UN_ci_7609 & $1012 \mathrm{~A} / \mathrm{G}$ \\
\hline UN_ci_7610 & $300 \mathrm{C} / \mathrm{T}$ \\
\hline UN_ci_7616 & $103 \mathrm{G} / \mathrm{A}$ \\
\hline UN_ci_7616 & $514 \mathrm{~A} / \mathrm{C}$ \\
\hline UN_ci_7616 & $600 \mathrm{~A} / \mathrm{T}$ \\
\hline UN_ci_7618 & $28 \mathrm{G} / \mathrm{C}$ \\
\hline UN_ci_7618 & $105 \mathrm{C} / \mathrm{T}$ \\
\hline UN_ci_7618 & $145 \mathrm{C} / \mathrm{G}$ \\
\hline UN_ci_7618 & $216 \mathrm{G} / \mathrm{A}$ \\
\hline UN_ci_7618 & $815 \mathrm{C} / \mathrm{T}$ \\
\hline UN_ci_7618 & $1168 \mathrm{~T} / \mathrm{C}$ \\
\hline UN_ci_7618 & $1460 \mathrm{~A} / \mathrm{T}$ \\
\hline UN_ci_7623 & $116 \mathrm{G} / \mathrm{A}$ \\
\hline UN_ci_7627 & $605 \mathrm{G} / \mathrm{A}$ \\
\hline UN_ci_7628 & $223 \mathrm{~A} / \mathrm{C}$ \\
\hline UN_ci_7628 & $382 \mathrm{G} / \mathrm{T}$ \\
\hline UN_ci_7645 & $743 \mathrm{G} / \mathrm{C}$ \\
\hline UN_ci_7645 & $1193 \mathrm{C} / \mathrm{T}$ \\
\hline UN_ci_7645 & $1246 \mathrm{~T} / \mathrm{G}$ \\
\hline UN_ci_7650 & 394 C/G \\
\hline UN_ci_7650 & $492 \mathrm{~T} / \mathrm{A}$ \\
\hline UN_ci_7652 & $90 \mathrm{G} / \mathrm{A}$ \\
\hline UN_ci_7652 & $135 \mathrm{~T} / \mathrm{C}$ \\
\hline UN_ci_7652 & $298 \mathrm{C} / \mathrm{T}$ \\
\hline UN_ci_7652 & $413 \mathrm{~T} / \mathrm{C}$ \\
\hline UN_ci_7652 & $591 \mathrm{~T} / \mathrm{C}$ \\
\hline UN_ci_7652 & $650 \mathrm{~T} / \mathrm{C}$ \\
\hline
\end{tabular}




\begin{tabular}{|c|c|}
\hline UN_ci_7652 & $717 \mathrm{~A} / \mathrm{G}$ \\
\hline UN_ci_7652 & $749 \mathrm{~T} / \mathrm{A}$ \\
\hline UN_ci_7661 & $189 \mathrm{C} / \mathrm{T}$ \\
\hline UN_ci_7661 & $232 \mathrm{~A} / \mathrm{C}$ \\
\hline UN_ci_7661 & $389 \mathrm{C} / \mathrm{T}$ \\
\hline UN_ci_7661 & $598 \mathrm{G} / \mathrm{A}$ \\
\hline UN_ci_7662 & 579 T/C \\
\hline UN_ci_7662 & $648 \mathrm{C} / \mathrm{T}$ \\
\hline UN_ci_7662 & $885 \mathrm{C} / \mathrm{A}$ \\
\hline UN_ci_7662 & $984 \mathrm{~T} / \mathrm{A}$ \\
\hline UN_ci_7662 & $1238 \mathrm{~A} / \mathrm{G}$ \\
\hline UN_ci_7662 & $1265 \mathrm{~T} / \mathrm{G}$ \\
\hline UN_ci_7664 & $115 \mathrm{C} / \mathrm{T}$ \\
\hline UN_ci_7664 & $149 \mathrm{C} / \mathrm{T}$ \\
\hline UN_ci_7664 & $240 \mathrm{~T} / \mathrm{C}$ \\
\hline UN_ci_7664 & $432 \mathrm{~T} / \mathrm{G}$ \\
\hline UN_ci_7664 & $893 \mathrm{C} / \mathrm{G}$ \\
\hline UN_ci_7664 & $975 \mathrm{~A} / \mathrm{G}$ \\
\hline UN_ci_7664 & $1100 \mathrm{~T} / \mathrm{C}$ \\
\hline UN_ci_7664 & $1217 \mathrm{G} / \mathrm{A}$ \\
\hline UN_ci_7665 & $83 \mathrm{C} / \mathrm{T}$ \\
\hline UN_ci_7665 & $119 \mathrm{C} / \mathrm{T}$ \\
\hline UN_ci_7665 & $250 \mathrm{G} / \mathrm{T}$ \\
\hline UN_ci_7665 & $350 \mathrm{~T} / \mathrm{G}$ \\
\hline UN_ci_7665 & $486 \mathrm{~A} / \mathrm{G}$ \\
\hline UN_ci_7665 & $621 \mathrm{~A} / \mathrm{C}$ \\
\hline UN_ci_7665 & $673 \mathrm{~T} / \mathrm{C}$ \\
\hline UN_ci_7667 & $275 \mathrm{~A} / \mathrm{C}$ \\
\hline UN_ci_7667 & $322 \mathrm{G} / \mathrm{T}$ \\
\hline UN_ci_7667 & $422 \mathrm{G} / \mathrm{A}$ \\
\hline UN_ci_7668 & $611 \mathrm{C} / \mathrm{T}$ \\
\hline UN_ci_7670 & $133 \mathrm{~T} / \mathrm{C}$ \\
\hline UN_ci_7670 & $200 \mathrm{C} / \mathrm{T}$ \\
\hline UN_ci_7670 & $448 \mathrm{~A} / \mathrm{G}$ \\
\hline UN_ci_7670 & $661 \mathrm{C} / \mathrm{T}$ \\
\hline UN_ci_7670 & $776 \mathrm{~A} / \mathrm{T}$ \\
\hline UN_ci_7673 & $115 \mathrm{~A} / \mathrm{T}$ \\
\hline UN_ci_7673 & $143 \mathrm{~T} / \mathrm{G}$ \\
\hline UN_ci_7673 & $170 \mathrm{G} / \mathrm{T}$ \\
\hline UN_ci_7676 & $83 \mathrm{G} / \mathrm{T}$ \\
\hline UN_ci_7676 & $430 \mathrm{G} / \mathrm{A}$ \\
\hline UN_ci_7677 & $155 \mathrm{C} / \mathrm{T}$ \\
\hline UN_ci_7680 & $55 \mathrm{~A} / \mathrm{T}$ \\
\hline UN_ci_7681 & $268 \mathrm{~A} / \mathrm{G}$ \\
\hline UN_ci_7681 & $340 \mathrm{~A} / \mathrm{T}$ \\
\hline UN_ci_7681 & $693 \mathrm{G} / \mathrm{A}$ \\
\hline UN_ci_7681 & $1072 \mathrm{G} / \mathrm{A}$ \\
\hline UN_ci_7681 & $1123 \mathrm{~T} / \mathrm{C}$ \\
\hline UN_ci_7681 & $1162 \mathrm{~T} / \mathrm{A}$ \\
\hline UN_ci_7681 & $1271 \mathrm{~T} / \mathrm{G}$ \\
\hline
\end{tabular}




\begin{tabular}{|c|c|}
\hline UN_ci_7683 & $426 \mathrm{C} / \mathrm{T}$ \\
\hline UN_ci_7683 & $539 \mathrm{G} / \mathrm{A}$ \\
\hline UN_ci_7683 & $1165 \mathrm{~A} / \mathrm{G}$ \\
\hline UN_ci_7685 & $358 \mathrm{~A} / \mathrm{T}$ \\
\hline UN_ci_7685 & $402 \mathrm{~T} / \mathrm{C}$ \\
\hline UN_ci_7685 & $439 \mathrm{G} / \mathrm{T}$ \\
\hline UN_ci_7685 & $487 \mathrm{~T} / \mathrm{A}$ \\
\hline UN_ci_7687 & $85 \mathrm{~A} / \mathrm{G}$ \\
\hline UN_ci_7687 & $132 \mathrm{C} / \mathrm{G}$ \\
\hline UN_ci_7687 & $163 \mathrm{C} / \mathrm{G}$ \\
\hline UN_ci_7687 & $228 \mathrm{C} / \mathrm{G}$ \\
\hline UN_ci_7687 & $253 \mathrm{~A} / \mathrm{G}$ \\
\hline UN_ci_7687 & $415 \mathrm{~A} / \mathrm{T}$ \\
\hline UN_ci_7688 & $122 \mathrm{C} / \mathrm{A}$ \\
\hline UN_ci_7688 & $545 \mathrm{~T} / \mathrm{C}$ \\
\hline UN_ci_7689 & $9 \mathrm{G} / \mathrm{T}$ \\
\hline UN_ci_7689 & $82 \mathrm{G} / \mathrm{A}$ \\
\hline UN_ci_7689 & $145 \mathrm{G} / \mathrm{A}$ \\
\hline UN_ci_7689 & $177 \mathrm{C} / \mathrm{T}$ \\
\hline UN_ci_7689 & 299 G/A \\
\hline UN_ci_7689 & $351 \mathrm{~A} / \mathrm{G}$ \\
\hline UN_ci_7689 & $380 \mathrm{~A} / \mathrm{G}$ \\
\hline UN_ci_7689 & 779 A/G \\
\hline UN_ci_7689 & $965 \mathrm{~A} / \mathrm{G}$ \\
\hline UN_ci_7689 & $1024 \mathrm{~T} / \mathrm{C}$ \\
\hline UN_ci_7690 & $167 \mathrm{C} / \mathrm{T}$ \\
\hline UN_ci_7690 & $702 \mathrm{G} / \mathrm{A}$ \\
\hline UN_ci_7690 & $1063 \mathrm{~T} / \mathrm{C}$ \\
\hline UN_ci_7692 & $94 \mathrm{C} / \mathrm{A}$ \\
\hline UN_ci_7692 & $289 \mathrm{~T} / \mathrm{C}$ \\
\hline UN_ci_7692 & $342 \mathrm{C} / \mathrm{T}$ \\
\hline UN_ci_7692 & $435 \mathrm{C} / \mathrm{T}$ \\
\hline UN_ci_7692 & $493 \mathrm{C} / \mathrm{T}$ \\
\hline UN_ci_7692 & $908 \mathrm{C} / \mathrm{A}$ \\
\hline UN_ci_7692 & $1005 \mathrm{~A} / \mathrm{G}$ \\
\hline UN_ci_7694 & $290 \mathrm{~T} / \mathrm{C}$ \\
\hline UN_ci_7694 & $783 \mathrm{C} / \mathrm{T}$ \\
\hline UN_ci_7696 & $154 \mathrm{G} / \mathrm{T}$ \\
\hline UN_ci_7696 & $179 \mathrm{~A} / \mathrm{G}$ \\
\hline UN_ci_7696 & $205 \mathrm{G} / \mathrm{A}$ \\
\hline UN_ci_7696 & $290 \mathrm{~T} / \mathrm{C}$ \\
\hline UN_ci_7696 & $333 \mathrm{~A} / \mathrm{G}$ \\
\hline UN_ci_7696 & $371 \mathrm{~A} / \mathrm{G}$ \\
\hline UN_ci_7696 & $465 \mathrm{C} / \mathrm{T}$ \\
\hline UN_ci_7696 & $513 \mathrm{~T} / \mathrm{C}$ \\
\hline UN_ci_7696 & $546 \mathrm{C} / \mathrm{A}$ \\
\hline UN_ci_7696 & $570 \mathrm{~T} / \mathrm{C}$ \\
\hline UN_ci_7696 & $592 \mathrm{~T} / \mathrm{C}$ \\
\hline UN_ci_7696 & $631 \mathrm{~A} / \mathrm{G}$ \\
\hline UN_ci_7696 & $667 \mathrm{~A} / \mathrm{G}$ \\
\hline
\end{tabular}




\begin{tabular}{|c|c|}
\hline UN_ci_7696 & $693 \mathrm{C} / \mathrm{A}$ \\
\hline UN_ci_7696 & $731 \mathrm{G} / \mathrm{T}$ \\
\hline UN_ci_7696 & $752 \mathrm{~T} / \mathrm{C}$ \\
\hline UN_ci_7696 & $782 \mathrm{~A} / \mathrm{G}$ \\
\hline UN_ci_7696 & $804 \mathrm{~T} / \mathrm{C}$ \\
\hline UN_ci_7696 & 839 A/T \\
\hline UN_ci_7696 & $912 \mathrm{~A} / \mathrm{G}$ \\
\hline UN_ci_7696 & $939 \mathrm{G} / \mathrm{A}$ \\
\hline UN_ci_7696 & $967 \mathrm{~A} / \mathrm{G}$ \\
\hline UN_ci_7696 & $1025 \mathrm{C} / \mathrm{T}$ \\
\hline UN_ci_7696 & $1089 \mathrm{~T} / \mathrm{C}$ \\
\hline UN_ci_7696 & $1161 \mathrm{C} / \mathrm{T}$ \\
\hline UN_ci_7696 & $1221 \mathrm{~T} / \mathrm{C}$ \\
\hline UN_ci_7696 & $1251 \mathrm{~T} / \mathrm{C}$ \\
\hline UN_ci_7696 & $1293 \mathrm{~A} / \mathrm{G}$ \\
\hline UN_ci_7696 & $1333 \mathrm{C} / \mathrm{T}$ \\
\hline UN_ci_7696 & $1387 \mathrm{C} / \mathrm{T}$ \\
\hline UN_ci_7696 & 1426 A/G \\
\hline UN_ci_7696 & $1452 \mathrm{G} / \mathrm{A}$ \\
\hline UN_ci_7696 & $1507 \mathrm{G} / \mathrm{A}$ \\
\hline UN_ci_7696 & $1531 \mathrm{~A} / \mathrm{G}$ \\
\hline UN_ci_7696 & $1573 \mathrm{G} / \mathrm{A}$ \\
\hline UN_ci_7696 & 1755 A/G \\
\hline UN_ci_7696 & 1797 A/G \\
\hline UN_ci_7696 & $1818 \mathrm{~T} / \mathrm{C}$ \\
\hline UN_ci_7697 & $366 \mathrm{G} / \mathrm{T}$ \\
\hline UN_ci_7699 & $270 \mathrm{G} / \mathrm{A}$ \\
\hline UN_ci_7701 & $197 \mathrm{~A} / \mathrm{G}$ \\
\hline UN_ci_7701 & $248 \mathrm{G} / \mathrm{A}$ \\
\hline UN_ci_7701 & $355 \mathrm{~T} / \mathrm{G}$ \\
\hline UN_ci_7701 & $404 \mathrm{~T} / \mathrm{C}$ \\
\hline UN_ci_7701 & $450 \mathrm{G} / \mathrm{A}$ \\
\hline UN_ci_7701 & $546 \mathrm{~T} / \mathrm{C}$ \\
\hline UN_ci_7701 & $567 \mathrm{~A} / \mathrm{T}$ \\
\hline UN_ci_7701 & 599 A/G \\
\hline UN_ci_7701 & $644 \mathrm{~T} / \mathrm{C}$ \\
\hline UN_ci_7701 & $665 \mathrm{~A} / \mathrm{G}$ \\
\hline UN_ci_7701 & $717 \mathrm{G} / \mathrm{A}$ \\
\hline UN_ci_7701 & $766 \mathrm{G} / \mathrm{A}$ \\
\hline UN_ci_7701 & $840 \mathrm{~T} / \mathrm{C}$ \\
\hline UN_ci_7701 & 875 T/C \\
\hline UN_ci_7702 & $32 \mathrm{C} / \mathrm{T}$ \\
\hline UN_ci_7702 & $217 \mathrm{C} / \mathrm{A}$ \\
\hline UN_ci_7702 & $426 \mathrm{~A} / \mathrm{G}$ \\
\hline UN_ci_7702 & $914 \mathrm{C} / \mathrm{T}$ \\
\hline UN_ci_7703 & $276 \mathrm{G} / \mathrm{A}$ \\
\hline UN_ci_7704 & $234 \mathrm{~A} / \mathrm{G}$ \\
\hline UN_ci_7709 & $258 \mathrm{~A} / \mathrm{C}$ \\
\hline UN_ci_7713 & $62 \mathrm{C} / \mathrm{T}$ \\
\hline UN_ci_7713 & $201 \mathrm{C} / \mathrm{A}$ \\
\hline
\end{tabular}




\begin{tabular}{|c|c|}
\hline UN_ci_7713 & $229 \mathrm{~A} / \mathrm{C}$ \\
\hline UN_ci_7713 & $257 \mathrm{~A} / \mathrm{C}$ \\
\hline UN_ci_7713 & $285 \mathrm{~A} / \mathrm{C}$ \\
\hline UN_ci_7713 & $313 \mathrm{~A} / \mathrm{C}$ \\
\hline UN_ci_7713 & $338 \mathrm{~T} / \mathrm{G}$ \\
\hline UN_ci_7713 & $366 \mathrm{~T} / \mathrm{G}$ \\
\hline UN_ci_7713 & $396 \mathrm{C} / \mathrm{T}$ \\
\hline UN_ci_7713 & $426 \mathrm{~A} / \mathrm{C}$ \\
\hline UN_ci_7713 & $461 \mathrm{C} / \mathrm{T}$ \\
\hline UN_ci_7713 & $487 \mathrm{~A} / \mathrm{G}$ \\
\hline UN_ci_7713 & $527 \mathrm{~A} / \mathrm{G}$ \\
\hline UN_ci_7715 & $42 \mathrm{~T} / \mathrm{C}$ \\
\hline UN_ci_7720 & $103 \mathrm{~A} / \mathrm{G}$ \\
\hline UN_ci_7720 & $127 \mathrm{~A} / \mathrm{G}$ \\
\hline UN_ci_7720 & $154 \mathrm{~T} / \mathrm{C}$ \\
\hline UN_ci_7720 & $174 \mathrm{~T} / \mathrm{C}$ \\
\hline UN_ci_7720 & $204 \mathrm{~T} / \mathrm{A}$ \\
\hline UN_ci_7720 & $227 \mathrm{~T} / \mathrm{A}$ \\
\hline UN_ci_7720 & $254 \mathrm{~A} / \mathrm{G}$ \\
\hline UN_ci_7720 & $282 \mathrm{~A} / \mathrm{G}$ \\
\hline UN_ci_7720 & $303 \mathrm{C} / \mathrm{A}$ \\
\hline UN_ci_7720 & $332 \mathrm{~T} / \mathrm{A}$ \\
\hline UN_ci_7720 & $366 \mathrm{C} / \mathrm{A}$ \\
\hline UN_ci_7720 & $450 \mathrm{~A} / \mathrm{G}$ \\
\hline UN_ci_7722 & $59 \mathrm{G} / \mathrm{A}$ \\
\hline UN_ci_7722 & $106 \mathrm{~T} / \mathrm{A}$ \\
\hline UN_ci_7723 & $43 \mathrm{~A} / \mathrm{G}$ \\
\hline UN_ci_7723 & $169 \mathrm{~T} / \mathrm{C}$ \\
\hline UN_ci_7724 & $458 \mathrm{C} / \mathrm{A}$ \\
\hline UN_ci_7724 & $487 \mathrm{~A} / \mathrm{G}$ \\
\hline UN_ci_7724 & $721 \mathrm{~T} / \mathrm{C}$ \\
\hline UN_ci_7724 & $795 \mathrm{C} / \mathrm{T}$ \\
\hline UN_ci_7724 & 819 G/T \\
\hline UN_ci_7724 & $928 \mathrm{~A} / \mathrm{T}$ \\
\hline UN_ci_7724 & $1014 \mathrm{~A} / \mathrm{G}$ \\
\hline UN_ci_7730 & $32 \mathrm{G} / \mathrm{A}$ \\
\hline UN_ci_7730 & $160 \mathrm{~A} / \mathrm{C}$ \\
\hline UN_ci_7730 & $249 \mathrm{G} / \mathrm{C}$ \\
\hline UN_ci_7730 & 1002 G/A \\
\hline UN_ci_7732 & $76 \mathrm{G} / \mathrm{C}$ \\
\hline UN_ci_7732 & $106 \mathrm{~T} / \mathrm{C}$ \\
\hline UN_ci_7732 & $192 \mathrm{~A} / \mathrm{G}$ \\
\hline UN_ci_7732 & $226 \mathrm{~A} / \mathrm{C}$ \\
\hline UN_ci_7732 & $476 \mathrm{C} / \mathrm{T}$ \\
\hline UN_ci_7732 & $718 \mathrm{G} / \mathrm{A}$ \\
\hline UN_ci_7732 & $790 \mathrm{~T} / \mathrm{C}$ \\
\hline UN_ci_7732 & $1126 \mathrm{~T} / \mathrm{G}$ \\
\hline UN_ci_7732 & $1282 \mathrm{~T} / \mathrm{C}$ \\
\hline UN_ci_7733 & $12 \mathrm{G} / \mathrm{T}$ \\
\hline UN_ci_7733 & $70 \mathrm{C} / \mathrm{A}$ \\
\hline
\end{tabular}




\begin{tabular}{|c|c|}
\hline UN_ci_7733 & $165 \mathrm{~T} / \mathrm{C}$ \\
\hline UN_ci_7733 & $265 \mathrm{~A} / \mathrm{G}$ \\
\hline UN_ci_7733 & $344 \mathrm{~T} / \mathrm{G}$ \\
\hline UN_ci_7736 & $235 \mathrm{G} / \mathrm{A}$ \\
\hline UN_ci_7736 & $304 \mathrm{C} / \mathrm{T}$ \\
\hline UN_ci_7738 & $466 \mathrm{~T} / \mathrm{C}$ \\
\hline UN_ci_7738 & $578 \mathrm{G} / \mathrm{A}$ \\
\hline UN_ci_7738 & $612 \mathrm{~A} / \mathrm{G}$ \\
\hline UN_ci_7741 & $689 \mathrm{G} / \mathrm{A}$ \\
\hline UN_ci_7741 & 906 A/G \\
\hline UN_ci_7741 & $961 \mathrm{~A} / \mathrm{G}$ \\
\hline UN_ci_7742 & $264 \mathrm{~A} / \mathrm{G}$ \\
\hline UN_ci_7743 & $200 \mathrm{~A} / \mathrm{G}$ \\
\hline UN_ci_7743 & $815 \mathrm{~A} / \mathrm{G}$ \\
\hline UN_ci_7743 & $882 \mathrm{C} / \mathrm{T}$ \\
\hline UN_ci_7743 & $956 \mathrm{~A} / \mathrm{T}$ \\
\hline UN_ci_7743 & 1098 A/G \\
\hline UN_ci_7743 & $1367 \mathrm{C} / \mathrm{T}$ \\
\hline UN_ci_7744 & $89 \mathrm{C} / \mathrm{T}$ \\
\hline UN_ci_7744 & $135 \mathrm{~A} / \mathrm{G}$ \\
\hline UN_ci_7744 & $281 \mathrm{~A} / \mathrm{G}$ \\
\hline UN_ci_7744 & $301 \mathrm{C} / \mathrm{T}$ \\
\hline UN_ci_7744 & $330 \mathrm{~A} / \mathrm{C}$ \\
\hline UN_ci_7744 & $355 \mathrm{~T} / \mathrm{C}$ \\
\hline UN_ci_7744 & 397 T/C \\
\hline UN_ci_7744 & $430 \mathrm{~T} / \mathrm{C}$ \\
\hline UN_ci_7744 & $512 \mathrm{~A} / \mathrm{G}$ \\
\hline UN_ci_7744 & $532 \mathrm{~T} / \mathrm{G}$ \\
\hline UN_ci_7744 & $877 \mathrm{G} / \mathrm{A}$ \\
\hline UN_ci_7744 & $1281 \mathrm{~T} / \mathrm{C}$ \\
\hline UN_ci_7744 & $1359 \mathrm{C} / \mathrm{A}$ \\
\hline UN_ci_7744 & $1476 \mathrm{~A} / \mathrm{G}$ \\
\hline UN_ci_7744 & $1725 \mathrm{~A} / \mathrm{G}$ \\
\hline UN_ci_7744 & $1867 \mathrm{~T} / \mathrm{C}$ \\
\hline UN_ci_7745 & $37 \mathrm{~A} / \mathrm{G}$ \\
\hline UN_ci_7745 & $314 \mathrm{G} / \mathrm{T}$ \\
\hline UN_ci_7745 & $594 \mathrm{G} / \mathrm{A}$ \\
\hline UN_ci_7745 & $1081 \mathrm{G} / \mathrm{A}$ \\
\hline UN_ci_7748 & $126 \mathrm{~A} / \mathrm{C}$ \\
\hline UN_ci_7748 & $193 \mathrm{C} / \mathrm{G}$ \\
\hline UN_ci_7749 & $94 \mathrm{~T} / \mathrm{C}$ \\
\hline UN_ci_7750 & $775 \mathrm{~A} / \mathrm{T}$ \\
\hline UN_ci_7751 & $241 \mathrm{G} / \mathrm{T}$ \\
\hline UN_ci_7752 & $71 \mathrm{G} / \mathrm{A}$ \\
\hline UN_ci_7753 & $134 \mathrm{~A} / \mathrm{C}$ \\
\hline UN_ci_7753 & $370 \mathrm{~A} / \mathrm{G}$ \\
\hline UN_ci_7753 & $479 \mathrm{~A} / \mathrm{G}$ \\
\hline UN_ci_7753 & $541 \mathrm{G} / \mathrm{T}$ \\
\hline UN_ci_7753 & $567 \mathrm{~T} / \mathrm{C}$ \\
\hline UN_ci_7753 & $603 \mathrm{~A} / \mathrm{C}$ \\
\hline
\end{tabular}




\begin{tabular}{|c|c|}
\hline UN_ci_7753 & $719 \mathrm{C} / \mathrm{A}$ \\
\hline UN_ci_7753 & $769 \mathrm{~A} / \mathrm{G}$ \\
\hline UN_ci_7754 & $286 \mathrm{~A} / \mathrm{C}$ \\
\hline UN_ci_7754 & $465 \mathrm{~A} / \mathrm{G}$ \\
\hline UN_ci_7757 & $434 \mathrm{C} / \mathrm{T}$ \\
\hline UN_ci_7757 & $486 \mathrm{C} / \mathrm{T}$ \\
\hline UN_ci_7757 & $617 \mathrm{C} / \mathrm{A}$ \\
\hline UN_ci_7757 & $1023 \mathrm{C} / \mathrm{A}$ \\
\hline UN_ci_7761 & $116 \mathrm{~T} / \mathrm{C}$ \\
\hline UN_ci_7761 & $251 \mathrm{~A} / \mathrm{C}$ \\
\hline UN_ci_7761 & $324 \mathrm{~A} / \mathrm{G}$ \\
\hline UN_ci_7761 & $455 \mathrm{C} / \mathrm{T}$ \\
\hline UN_ci_7761 & $691 \mathrm{G} / \mathrm{A}$ \\
\hline UN_ci_7762 & $377 \mathrm{~A} / \mathrm{C}$ \\
\hline UN_ci_7765 & $96 \mathrm{~T} / \mathrm{C}$ \\
\hline UN_ci_7765 & $120 \mathrm{~T} / \mathrm{C}$ \\
\hline UN_ci_7766 & $403 \mathrm{G} / \mathrm{A}$ \\
\hline UN_ci_7772 & $435 \mathrm{~T} / \mathrm{A}$ \\
\hline UN_ci_7774 & $137 \mathrm{~A} / \mathrm{G}$ \\
\hline UN_ci_7774 & $561 \mathrm{~A} / \mathrm{C}$ \\
\hline UN_ci_7774 & $618 \mathrm{C} / \mathrm{T}$ \\
\hline UN_ci_7774 & $729 \mathrm{G} / \mathrm{T}$ \\
\hline UN_ci_7774 & $757 \mathrm{~A} / \mathrm{G}$ \\
\hline UN_ci_7774 & $852 \mathrm{G} / \mathrm{C}$ \\
\hline UN_ci_7774 & $910 \mathrm{~A} / \mathrm{T}$ \\
\hline UN_ci_7774 & $956 \mathrm{C} / \mathrm{T}$ \\
\hline UN_ci_7774 & $1101 \mathrm{G} / \mathrm{A}$ \\
\hline UN_ci_7774 & $1141 \mathrm{C} / \mathrm{T}$ \\
\hline UN_ci_7774 & $1368 \mathrm{G} / \mathrm{T}$ \\
\hline UN_ci_7774 & $1425 \mathrm{G} / \mathrm{T}$ \\
\hline UN_ci_7774 & $1575 \mathrm{~T} / \mathrm{A}$ \\
\hline UN_ci_7774 & $1789 \mathrm{~A} / \mathrm{T}$ \\
\hline UN_ci_7774 & $1887 \mathrm{C} / \mathrm{T}$ \\
\hline UN_ci_7774 & $1934 \mathrm{C} / \mathrm{A}$ \\
\hline UN_ci_7774 & $1960 \mathrm{G} / \mathrm{T}$ \\
\hline UN_ci_7774 & 1994 G/A \\
\hline UN_ci_7774 & 2063 A/G \\
\hline UN_ci_7774 & $2178 \mathrm{G} / \mathrm{C}$ \\
\hline UN_ci_7774 & $2370 \mathrm{C} / \mathrm{T}$ \\
\hline UN_ci_7775 & $271 \mathrm{G} / \mathrm{A}$ \\
\hline UN_ci_7775 & $363 \mathrm{~T} / \mathrm{A}$ \\
\hline UN_ci_7775 & $430 \mathrm{C} / \mathrm{T}$ \\
\hline UN_ci_7775 & $463 \mathrm{~T} / \mathrm{C}$ \\
\hline UN_ci_7775 & $514 \mathrm{C} / \mathrm{T}$ \\
\hline UN_ci_7775 & $542 \mathrm{~A} / \mathrm{G}$ \\
\hline UN_ci_7775 & $568 \mathrm{G} / \mathrm{A}$ \\
\hline UN_ci_7777 & $250 \mathrm{~A} / \mathrm{C}$ \\
\hline UN_ci_7777 & $564 \mathrm{~T} / \mathrm{C}$ \\
\hline UN_ci_7777 & $622 \mathrm{C} / \mathrm{T}$ \\
\hline UN_ci_7777 & $684 \mathrm{C} / \mathrm{T}$ \\
\hline
\end{tabular}




\begin{tabular}{|c|c|}
\hline UN_ci_7777 & $1011 \mathrm{C} / \mathrm{T}$ \\
\hline UN_ci_7779 & $186 \mathrm{G} / \mathrm{A}$ \\
\hline UN_ci_7782 & 52 G/C \\
\hline UN_ci_7782 & $138 \mathrm{C} / \mathrm{T}$ \\
\hline UN_ci_7782 & 202 T/C \\
\hline UN_ci_7782 & $236 \mathrm{~T} / \mathrm{C}$ \\
\hline UN_ci_7782 & $295 \mathrm{~A} / \mathrm{G}$ \\
\hline UN_ci_7782 & $373 \mathrm{~A} / \mathrm{G}$ \\
\hline UN_ci_7782 & 396 A/G \\
\hline UN_ci_7782 & $460 \mathrm{~A} / \mathrm{G}$ \\
\hline UN_ci_7785 & $272 \mathrm{C} / \mathrm{T}$ \\
\hline UN_ci_7788 & $249 \mathrm{~A} / \mathrm{T}$ \\
\hline UN_ci_7788 & 393 T/C \\
\hline UN_ci_7788 & $672 \mathrm{C} / \mathrm{A}$ \\
\hline UN_ci_7788 & $831 \mathrm{C} / \mathrm{T}$ \\
\hline UN_ci_7788 & $855 \mathrm{G} / \mathrm{T}$ \\
\hline UN_ci_7788 & $1032 \mathrm{~A} / \mathrm{T}$ \\
\hline UN_ci_7788 & $1072 \mathrm{~A} / \mathrm{T}$ \\
\hline UN_ci_7788 & $1286 \mathrm{~A} / \mathrm{G}$ \\
\hline UN_ci_7788 & $1680 \mathrm{C} / \mathrm{T}$ \\
\hline UN_ci_7788 & 2026 A/C \\
\hline UN_ci_7789 & $36 \mathrm{C} / \mathrm{G}$ \\
\hline UN_ci_7789 & $186 \mathrm{~T} / \mathrm{C}$ \\
\hline UN_ci_7789 & $299 \mathrm{~A} / \mathrm{G}$ \\
\hline UN_ci_7789 & $411 \mathrm{C} / \mathrm{T}$ \\
\hline UN_ci_7790 & $580 \mathrm{C} / \mathrm{T}$ \\
\hline UN_ci_7790 & $647 \mathrm{G} / \mathrm{A}$ \\
\hline UN_ci_7790 & $931 \mathrm{G} / \mathrm{A}$ \\
\hline UN_ci_7790 & $1130 \mathrm{~A} / \mathrm{G}$ \\
\hline UN_ci_7790 & $1166 \mathrm{G} / \mathrm{A}$ \\
\hline UN_ci_7791 & $457 \mathrm{~T} / \mathrm{C}$ \\
\hline UN_ci_7791 & $564 \mathrm{G} / \mathrm{A}$ \\
\hline UN_ci_7791 & $1243 \mathrm{~A} / \mathrm{T}$ \\
\hline UN_ci_7793 & $30 \mathrm{~A} / \mathrm{T}$ \\
\hline UN_ci_7793 & $105 \mathrm{~A} / \mathrm{G}$ \\
\hline UN_ci_7793 & $142 \mathrm{~A} / \mathrm{G}$ \\
\hline UN_ci_7793 & $182 \mathrm{~T} / \mathrm{C}$ \\
\hline UN_ci_7793 & 203 T/C \\
\hline UN_ci_7793 & $311 \mathrm{~A} / \mathrm{G}$ \\
\hline UN_ci_7793 & $388 \mathrm{G} / \mathrm{A}$ \\
\hline UN_ci_7793 & $423 \mathrm{G} / \mathrm{T}$ \\
\hline UN_ci_7793 & $477 \mathrm{~A} / \mathrm{C}$ \\
\hline UN_ci_7793 & $503 \mathrm{~A} / \mathrm{G}$ \\
\hline UN_ci_7793 & $531 \mathrm{G} / \mathrm{A}$ \\
\hline UN_ci_7793 & $566 \mathrm{C} / \mathrm{T}$ \\
\hline UN_ci_7793 & $647 \mathrm{~A} / \mathrm{G}$ \\
\hline UN_ci_7793 & $667 \mathrm{~A} / \mathrm{G}$ \\
\hline UN_ci_7793 & $723 \mathrm{G} / \mathrm{A}$ \\
\hline UN_ci_7793 & $761 \mathrm{~A} / \mathrm{G}$ \\
\hline UN_ci_7793 & $793 \mathrm{C} / \mathrm{T}$ \\
\hline
\end{tabular}




\begin{tabular}{|c|c|}
\hline UN_ci_7793 & $842 \mathrm{~T} / \mathrm{C}$ \\
\hline UN_ci_7793 & $888 \mathrm{C} / \mathrm{T}$ \\
\hline UN_ci_7793 & $940 \mathrm{G} / \mathrm{A}$ \\
\hline UN_ci_7793 & $974 \mathrm{~A} / \mathrm{T}$ \\
\hline UN_ci_7793 & $1036 \mathrm{~T} / \mathrm{C}$ \\
\hline UN_ci_7793 & 1192 T/C \\
\hline UN_ci_7793 & $1284 \mathrm{~T} / \mathrm{A}$ \\
\hline UN_ci_7793 & $1350 \mathrm{~A} / \mathrm{G}$ \\
\hline UN_ci_7797 & $161 \mathrm{~T} / \mathrm{C}$ \\
\hline UN_ci_7797 & $245 \mathrm{~A} / \mathrm{G}$ \\
\hline UN_ci_7797 & $265 \mathrm{G} / \mathrm{A}$ \\
\hline UN_ci_7797 & $292 \mathrm{~A} / \mathrm{G}$ \\
\hline UN_ci_7797 & $361 \mathrm{~T} / \mathrm{G}$ \\
\hline UN_ci_7797 & $390 \mathrm{~A} / \mathrm{G}$ \\
\hline UN_ci_7797 & $413 \mathrm{~T} / \mathrm{A}$ \\
\hline UN_ci_7798 & $968 \mathrm{C} / \mathrm{T}$ \\
\hline UN_ci_7799 & $254 \mathrm{C} / \mathrm{G}$ \\
\hline UN_ci_7799 & $467 \mathrm{~T} / \mathrm{C}$ \\
\hline UN_ci_7799 & $536 \mathrm{~A} / \mathrm{G}$ \\
\hline UN_ci_7799 & $594 \mathrm{~A} / \mathrm{G}$ \\
\hline UN_ci_7801 & $503 \mathrm{G} / \mathrm{A}$ \\
\hline UN_ci_7801 & $663 \mathrm{~A} / \mathrm{G}$ \\
\hline UN_ci_7801 & $702 \mathrm{~A} / \mathrm{G}$ \\
\hline UN_ci_7801 & $1068 \mathrm{~T} / \mathrm{C}$ \\
\hline UN_ci_7803 & $257 \mathrm{C} / \mathrm{T}$ \\
\hline UN_ci_7803 & $279 \mathrm{G} / \mathrm{A}$ \\
\hline UN_ci_7803 & $311 \mathrm{C} / \mathrm{T}$ \\
\hline UN_ci_7803 & $524 \mathrm{C} / \mathrm{T}$ \\
\hline UN_ci_7804 & $152 \mathrm{~A} / \mathrm{T}$ \\
\hline UN_ci_7806 & $341 \mathrm{G} / \mathrm{A}$ \\
\hline UN_ci_7806 & $412 \mathrm{C} / \mathrm{G}$ \\
\hline UN_ci_7806 & $475 \mathrm{G} / \mathrm{A}$ \\
\hline UN_ci_7806 & $536 \mathrm{G} / \mathrm{A}$ \\
\hline UN_ci_7806 & $578 \mathrm{C} / \mathrm{T}$ \\
\hline UN_ci_7809 & $795 \mathrm{~A} / \mathrm{G}$ \\
\hline UN_ci_7813 & $685 \mathrm{~T} / \mathrm{C}$ \\
\hline UN_ci_7813 & $1222 \mathrm{G} / \mathrm{A}$ \\
\hline UN_ci_7814 & $155 \mathrm{~T} / \mathrm{A}$ \\
\hline UN_ci_7814 & $205 \mathrm{G} / \mathrm{A}$ \\
\hline UN_ci_7814 & $245 \mathrm{~T} / \mathrm{C}$ \\
\hline UN_ci_7814 & $286 \mathrm{~T} / \mathrm{C}$ \\
\hline UN_ci_7814 & $327 \mathrm{~T} / \mathrm{C}$ \\
\hline UN_ci_7814 & $350 \mathrm{~T} / \mathrm{C}$ \\
\hline UN_ci_7814 & $379 \mathrm{C} / \mathrm{T}$ \\
\hline UN_ci_7814 & $399 \mathrm{G} / \mathrm{A}$ \\
\hline UN_ci_7814 & 419 A/G \\
\hline UN_ci_7814 & $443 \mathrm{~T} / \mathrm{C}$ \\
\hline UN_ci_7814 & $463 \mathrm{G} / \mathrm{A}$ \\
\hline UN_ci_7814 & $483 \mathrm{~T} / \mathrm{C}$ \\
\hline UN_ci_7814 & $503 \mathrm{~A} / \mathrm{G}$ \\
\hline
\end{tabular}




$\begin{array}{lr}\text { UN_ci_7814 } & 526 \mathrm{~A} / \mathrm{C} \\ \text { UN_ci_7814 } & 547 \mathrm{~T} / \mathrm{C} \\ \text { UN_ci_7814 } & 567 \mathrm{G} / \mathrm{A} \\ \text { UN_ci_7814 } & 588 \mathrm{G} / \mathrm{A} \\ \text { UN_ci_7814 } & 612 \mathrm{~A} / \mathrm{C} \\ \text { UN_ci_7814 } & 635 \mathrm{G} / \mathrm{T} \\ \text { UN_ci_7814 } & 658 \mathrm{G} / \mathrm{A} \\ \text { UN_ci_7814 } & 678 \mathrm{C} / \mathrm{T} \\ \text { UN_ci_7814 } & 711 \mathrm{~T} / \mathrm{G} \\ \text { UN_ci_7814 } & 749 \mathrm{~A} / \mathrm{G} \\ \text { UN_ci_7814 } & 774 \mathrm{~T} / \mathrm{C} \\ \text { UN_ci_7814 } & 799 \mathrm{C} / \mathrm{T} \\ \text { UN_ci_7814 } & 819 \mathrm{~T} / \mathrm{C} \\ \text { UN_ci_7814 } & 839 \mathrm{~A} / \mathrm{G} \\ \text { UN_ci_7814 } & 875 \mathrm{~T} / \mathrm{C} \\ \text { UN_ci_7814 } & 900 \mathrm{G} / \mathrm{T} \\ \text { UN_ci_7814 } & 920 \mathrm{~A} / \mathrm{G} \\ \text { UN_ci_7814 } & 946 \mathrm{C} / \mathrm{T} \\ \text { UN_ci_7814 } & 967 \mathrm{~A} / \mathrm{G} \\ \text { UN_ci_7814 } & 987 \mathrm{G} / \mathrm{A} \\ \text { UN_ci_7814 } & 1013 \mathrm{G} / \mathrm{A} \\ \text { UN_ci_7814 } & 793 \mathrm{~A} / \mathrm{G} \\ \text { UN_ci_7814 } & \end{array}$




$\begin{array}{lr}\text { UN_ci_7821 } & 933 \mathrm{~T} / \mathrm{C} \\ \text { UN_ci_7821 } & 1002 \mathrm{~A} / \mathrm{G} \\ \text { UN_ci_7821 } & 1027 \mathrm{G} / \mathrm{A} \\ \text { UN_ci_7821 } & 1107 \mathrm{C} / \mathrm{A} \\ \text { UN_ci_7822 } & 141 \mathrm{G} / \mathrm{A} \\ \text { UN_ci_7822 } & 194 \mathrm{~T} / \mathrm{C} \\ \text { UN_ci_7822 } & 326 \mathrm{C} / \mathrm{T} \\ \text { UN_ci_7822 } & 360 \mathrm{C} / \mathrm{T} \\ \text { UN_ci_7822 } & 852 \mathrm{G} / \mathrm{A} \\ \text { UN_ci_7825 } & 146 \mathrm{C} / \mathrm{T} \\ \text { UN_ci_7825 } & 1224 \mathrm{G} / \mathrm{A} \\ \text { UN_ci_7827 } & 502 \mathrm{C} / \mathrm{T} \\ \text { UN_ci_7827 } & 765 \mathrm{~T} / \mathrm{G} \\ \text { UN_ci_7827 } & 802 \mathrm{~T} / \mathrm{C} \\ \text { UN_ci_7827 } & 822 \mathrm{~T} / \mathrm{C} \\ \text { UN_ci_7827 } & 846 \mathrm{C} / \mathrm{T} \\ \text { UN_ci_7827 } & 893 \mathrm{C} / \mathrm{T} \\ \text { UN_ci_7827 } & 919 \mathrm{~T} / \mathrm{C} \\ \text { UN_ci_7827 } & 949 \mathrm{C} / \mathrm{A} \\ \text { UN_ci_7827 } & 978 \mathrm{~T} / \mathrm{C} \\ \text { UN_ci_7827 } & 1005 \mathrm{~T} / \mathrm{C} \\ \text { UN_ci_7827 } & 506 \mathrm{C} / \mathrm{A} \\ \text { UN_ci_7827 } & \end{array}$




\begin{tabular}{|c|c|}
\hline UN_ci_7839 & $855 \mathrm{C} / \mathrm{T}$ \\
\hline UN_ci_7840 & $405 \mathrm{C} / \mathrm{T}$ \\
\hline UN_ci_7840 & $1530 \mathrm{G} / \mathrm{C}$ \\
\hline UN_ci_7840 & $1691 \mathrm{~A} / \mathrm{C}$ \\
\hline UN_ci_7843 & $251 \mathrm{~T} / \mathrm{G}$ \\
\hline UN_ci_7843 & $371 \mathrm{C} / \mathrm{T}$ \\
\hline UN_ci_7844 & $77 \mathrm{C} / \mathrm{T}$ \\
\hline UN_ci_7844 & $135 \mathrm{~A} / \mathrm{G}$ \\
\hline UN_ci_7845 & $45 \mathrm{~T} / \mathrm{A}$ \\
\hline UN_ci_7846 & $586 \mathrm{G} / \mathrm{A}$ \\
\hline UN_ci_7846 & $631 \mathrm{C} / \mathrm{G}$ \\
\hline UN_ci_7846 & $658 \mathrm{C} / \mathrm{T}$ \\
\hline UN_ci_7846 & $688 \mathrm{C} / \mathrm{T}$ \\
\hline UN_ci_7846 & $742 \mathrm{~A} / \mathrm{G}$ \\
\hline UN_ci_7846 & $778 \mathrm{~A} / \mathrm{G}$ \\
\hline UN_ci_7846 & $826 \mathrm{~T} / \mathrm{G}$ \\
\hline UN_ci_7846 & $847 \mathrm{C} / \mathrm{T}$ \\
\hline UN_ci_7846 & 877 T/C \\
\hline UN_ci_7855 & $107 \mathrm{G} / \mathrm{C}$ \\
\hline UN_ci_7855 & 378 A/G \\
\hline UN_ci_7855 & $611 \mathrm{~T} / \mathrm{C}$ \\
\hline UN_ci_7855 & 869 A/G \\
\hline UN_ci_7855 & 1298 T/C \\
\hline UN_ci_7855 & 1469 C/G \\
\hline UN_ci_7855 & $1642 \mathrm{C} / \mathrm{G}$ \\
\hline UN_ci_7857 & $477 \mathrm{~A} / \mathrm{G}$ \\
\hline UN_ci_7859 & $121 \mathrm{~A} / \mathrm{G}$ \\
\hline UN_ci_7859 & $147 \mathrm{C} / \mathrm{T}$ \\
\hline UN_ci_7859 & $225 \mathrm{~T} / \mathrm{C}$ \\
\hline UN_ci_7860 & $8 \mathrm{C} / \mathrm{G}$ \\
\hline UN_ci_7860 & $448 \mathrm{~A} / \mathrm{G}$ \\
\hline UN_ci_7862 & $355 \mathrm{~T} / \mathrm{C}$ \\
\hline UN_ci_7862 & $441 \mathrm{~T} / \mathrm{C}$ \\
\hline UN_ci_7862 & $521 \mathrm{G} / \mathrm{T}$ \\
\hline UN_ci_7862 & $586 \mathrm{~T} / \mathrm{C}$ \\
\hline UN_ci_7862 & $635 \mathrm{G} / \mathrm{A}$ \\
\hline UN_ci_7865 & $251 \mathrm{~A} / \mathrm{T}$ \\
\hline UN_ci_7870 & $179 \mathrm{~A} / \mathrm{C}$ \\
\hline UN_ci_7870 & $277 \mathrm{~A} / \mathrm{G}$ \\
\hline UN_ci_7870 & 312 T/C \\
\hline UN_ci_7871 & $98 \mathrm{~A} / \mathrm{T}$ \\
\hline UN_ci_7871 & $338 \mathrm{C} / \mathrm{T}$ \\
\hline UN_ci_7871 & $602 \mathrm{C} / \mathrm{A}$ \\
\hline UN_ci_7871 & $747 \mathrm{~A} / \mathrm{G}$ \\
\hline UN_ci_7871 & $883 \mathrm{G} / \mathrm{A}$ \\
\hline UN_ci_7872 & $526 \mathrm{C} / \mathrm{A}$ \\
\hline UN_ci_7872 & $630 \mathrm{G} / \mathrm{A}$ \\
\hline UN_ci_7872 & $982 \mathrm{G} / \mathrm{A}$ \\
\hline UN_ci_7874 & $213 \mathrm{C} / \mathrm{T}$ \\
\hline UN_ci_7875 & $189 \mathrm{C} / \mathrm{T}$ \\
\hline
\end{tabular}




\begin{tabular}{|c|c|}
\hline UN_ci_7875 & $784 \mathrm{~T} / \mathrm{G}$ \\
\hline UN_ci_7877 & $39 \mathrm{~A} / \mathrm{T}$ \\
\hline UN_ci_7877 & $74 \mathrm{G} / \mathrm{A}$ \\
\hline UN_ci_7877 & $274 \mathrm{~T} / \mathrm{G}$ \\
\hline UN_ci_7877 & $348 \mathrm{~T} / \mathrm{G}$ \\
\hline UN_ci_7877 & $382 \mathrm{~T} / \mathrm{A}$ \\
\hline UN_ci_7877 & $429 \mathrm{C} / \mathrm{T}$ \\
\hline UN_ci_7877 & $463 \mathrm{G} / \mathrm{A}$ \\
\hline UN_ci_7877 & $495 \mathrm{~A} / \mathrm{G}$ \\
\hline UN_ci_7877 & $520 \mathrm{C} / \mathrm{T}$ \\
\hline UN_ci_7877 & $559 \mathrm{G} / \mathrm{T}$ \\
\hline UN_ci_7877 & $620 \mathrm{~A} / \mathrm{G}$ \\
\hline UN_ci_7877 & $672 \mathrm{~T} / \mathrm{C}$ \\
\hline UN_ci_7877 & $709 \mathrm{G} / \mathrm{T}$ \\
\hline UN_ci_7877 & $739 \mathrm{~A} / \mathrm{C}$ \\
\hline UN_ci_7877 & $762 \mathrm{~A} / \mathrm{T}$ \\
\hline UN_ci_7877 & 799 A/G \\
\hline UN_ci_7877 & $831 \mathrm{~A} / \mathrm{G}$ \\
\hline UN_ci_7877 & 919 A/G \\
\hline UN_ci_7877 & $940 \mathrm{C} / \mathrm{T}$ \\
\hline UN_ci_7877 & 1012 G/A \\
\hline UN_ci_7877 & 1039 T/A \\
\hline UN_ci_7877 & $1072 \mathrm{C} / \mathrm{T}$ \\
\hline UN_ci_7877 & 1102 T/A \\
\hline UN_ci_7877 & $1144 \mathrm{~T} / \mathrm{G}$ \\
\hline UN_ci_7877 & 1192 T/A \\
\hline UN_ci_7877 & $1222 \mathrm{G} / \mathrm{A}$ \\
\hline UN_ci_7877 & $1375 \mathrm{~A} / \mathrm{G}$ \\
\hline UN_ci_7877 & $1491 \mathrm{~T} / \mathrm{A}$ \\
\hline UN_ci_7881 & $235 \mathrm{C} / \mathrm{T}$ \\
\hline UN_ci_7881 & $264 \mathrm{~T} / \mathrm{C}$ \\
\hline UN_ci_7882 & $598 \mathrm{~A} / \mathrm{G}$ \\
\hline UN_ci_7882 & $725 \mathrm{~T} / \mathrm{C}$ \\
\hline UN_ci_7882 & $993 \mathrm{C} / \mathrm{T}$ \\
\hline UN_ci_7883 & $229 \mathrm{~A} / \mathrm{T}$ \\
\hline UN_ci_7884 & $201 \mathrm{G} / \mathrm{A}$ \\
\hline UN_ci_7884 & $276 \mathrm{C} / \mathrm{T}$ \\
\hline UN_ci_7884 & $423 \mathrm{~A} / \mathrm{C}$ \\
\hline UN_ci_7884 & $541 \mathrm{~A} / \mathrm{C}$ \\
\hline UN_ci_7884 & $586 \mathrm{~A} / \mathrm{G}$ \\
\hline UN_ci_7884 & $795 \mathrm{~A} / \mathrm{T}$ \\
\hline UN_ci_7884 & $845 \mathrm{~T} / \mathrm{C}$ \\
\hline UN_ci_7885 & $378 \mathrm{~T} / \mathrm{G}$ \\
\hline UN_ci_7888 & 199 G/C \\
\hline UN_ci_7889 & $287 \mathrm{~A} / \mathrm{T}$ \\
\hline UN_ci_7889 & $338 \mathrm{G} / \mathrm{C}$ \\
\hline UN_ci_7889 & $372 \mathrm{~A} / \mathrm{G}$ \\
\hline UN_ci_7889 & $466 \mathrm{~A} / \mathrm{T}$ \\
\hline UN_ci_7892 & $148 \mathrm{~A} / \mathrm{G}$ \\
\hline UN_ci_7892 & $419 \mathrm{C} / \mathrm{A}$ \\
\hline
\end{tabular}




\begin{tabular}{|c|c|}
\hline UN_ci_7892 & $554 \mathrm{~T} / \mathrm{G}$ \\
\hline UN_ci_7892 & $765 \mathrm{~A} / \mathrm{G}$ \\
\hline UN_ci_7892 & $834 \mathrm{G} / \mathrm{A}$ \\
\hline UN_ci_7894 & $102 \mathrm{G} / \mathrm{A}$ \\
\hline UN_ci_7894 & $146 \mathrm{~A} / \mathrm{G}$ \\
\hline UN_ci_7894 & $175 \mathrm{G} / \mathrm{A}$ \\
\hline UN_ci_7894 & $274 \mathrm{G} / \mathrm{A}$ \\
\hline UN_ci_7894 & $296 \mathrm{~T} / \mathrm{C}$ \\
\hline UN_ci_7894 & $363 \mathrm{C} / \mathrm{T}$ \\
\hline UN_ci_7894 & $411 \mathrm{G} / \mathrm{A}$ \\
\hline UN_ci_7894 & $440 \mathrm{~A} / \mathrm{G}$ \\
\hline UN_ci_7894 & 519 T/C \\
\hline UN_ci_7894 & $560 \mathrm{~A} / \mathrm{C}$ \\
\hline UN_ci_7894 & $740 \mathrm{~A} / \mathrm{G}$ \\
\hline UN_ci_7894 & 799 A/G \\
\hline UN_ci_7894 & $832 \mathrm{~A} / \mathrm{G}$ \\
\hline UN_ci_7894 & 1249 T/C \\
\hline UN_ci_7894 & $1270 \mathrm{~A} / \mathrm{G}$ \\
\hline UN_ci_7894 & $1493 \mathrm{C} / \mathrm{T}$ \\
\hline UN_ci_7894 & 1529 T/C \\
\hline UN_ci_7895 & $425 \mathrm{G} / \mathrm{A}$ \\
\hline UN_ci_7898 & 733 G/C \\
\hline UN_ci_7900 & $55 \mathrm{~A} / \mathrm{G}$ \\
\hline UN_ci_7900 & $160 \mathrm{~A} / \mathrm{G}$ \\
\hline UN_ci_7900 & $225 \mathrm{~A} / \mathrm{G}$ \\
\hline UN_ci_7900 & $470 \mathrm{~A} / \mathrm{C}$ \\
\hline UN_ci_7900 & $502 \mathrm{C} / \mathrm{G}$ \\
\hline UN_ci_7900 & $524 \mathrm{G} / \mathrm{A}$ \\
\hline UN_ci_7900 & $567 \mathrm{C} / \mathrm{G}$ \\
\hline UN_ci_7901 & $648 \mathrm{~T} / \mathrm{C}$ \\
\hline UN_ci_7901 & $675 \mathrm{~A} / \mathrm{G}$ \\
\hline UN_ci_7901 & $700 \mathrm{G} / \mathrm{T}$ \\
\hline UN_ci_7901 & $748 \mathrm{~A} / \mathrm{G}$ \\
\hline UN_ci_7901 & $864 \mathrm{~T} / \mathrm{C}$ \\
\hline UN_ci_7901 & $915 \mathrm{G} / \mathrm{T}$ \\
\hline UN_ci_7901 & $953 \mathrm{~T} / \mathrm{C}$ \\
\hline UN_ci_7901 & $973 \mathrm{G} / \mathrm{A}$ \\
\hline UN_ci_7901 & $1018 \mathrm{C} / \mathrm{T}$ \\
\hline UN_ci_7902 & $118 \mathrm{~A} / \mathrm{G}$ \\
\hline UN_ci_7902 & 367 T/C \\
\hline UN_ci_7902 & $387 \mathrm{~A} / \mathrm{G}$ \\
\hline UN_ci_7902 & $430 \mathrm{~A} / \mathrm{C}$ \\
\hline UN_ci_7902 & $516 \mathrm{~A} / \mathrm{G}$ \\
\hline UN_ci_7902 & $709 \mathrm{~T} / \mathrm{C}$ \\
\hline UN_ci_7902 & $731 \mathrm{~T} / \mathrm{C}$ \\
\hline UN_ci_7903 & $38 \mathrm{C} / \mathrm{T}$ \\
\hline UN_ci_7903 & $60 \mathrm{G} / \mathrm{T}$ \\
\hline UN_ci_7903 & $119 \mathrm{~A} / \mathrm{C}$ \\
\hline UN_ci_7903 & 374 T/C \\
\hline UN_ci_7903 & $454 \mathrm{~A} / \mathrm{T}$ \\
\hline
\end{tabular}




\begin{tabular}{|c|c|}
\hline UN_ci_7903 & $503 \mathrm{~A} / \mathrm{G}$ \\
\hline UN_ci_7903 & $556 \mathrm{G} / \mathrm{A}$ \\
\hline UN_ci_7903 & $578 \mathrm{~T} / \mathrm{A}$ \\
\hline UN_ci_7903 & $659 \mathrm{~T} / \mathrm{C}$ \\
\hline UN_ci_7903 & $762 \mathrm{~A} / \mathrm{T}$ \\
\hline UN_ci_7903 & $864 \mathrm{~T} / \mathrm{C}$ \\
\hline UN_ci_7903 & $930 \mathrm{~T} / \mathrm{G}$ \\
\hline UN_ci_7905 & $539 \mathrm{C} / \mathrm{T}$ \\
\hline UN_ci_7905 & $693 \mathrm{~A} / \mathrm{G}$ \\
\hline UN_ci_7905 & $780 \mathrm{~A} / \mathrm{G}$ \\
\hline UN_ci_7905 & $846 \mathrm{G} / \mathrm{A}$ \\
\hline UN_ci_7908 & $169 \mathrm{C} / \mathrm{G}$ \\
\hline UN_ci_7908 & 291 T/C \\
\hline UN_ci_7908 & $356 \mathrm{~T} / \mathrm{C}$ \\
\hline UN_ci_7908 & $549 \mathrm{G} / \mathrm{A}$ \\
\hline UN_ci_7908 & $698 \mathrm{~T} / \mathrm{C}$ \\
\hline UN_ci_7908 & $758 \mathrm{~T} / \mathrm{C}$ \\
\hline UN_ci_7908 & $788 \mathrm{~T} / \mathrm{C}$ \\
\hline UN_ci_7908 & $829 \mathrm{C} / \mathrm{T}$ \\
\hline UN_ci_7908 & $886 \mathrm{~T} / \mathrm{C}$ \\
\hline UN_ci_7908 & $918 \mathrm{~T} / \mathrm{C}$ \\
\hline UN_ci_7908 & $969 \mathrm{~A} / \mathrm{C}$ \\
\hline UN_ci_7908 & $1024 \mathrm{~T} / \mathrm{C}$ \\
\hline UN_ci_7908 & $1075 \mathrm{~T} / \mathrm{C}$ \\
\hline UN_ci_7908 & $1101 \mathrm{~A} / \mathrm{G}$ \\
\hline UN_ci_7908 & 1137 T/C \\
\hline UN_ci_7908 & $1158 \mathrm{~A} / \mathrm{G}$ \\
\hline UN_ci_7908 & 1299 T/C \\
\hline UN_ci_7908 & $1330 \mathrm{C} / \mathrm{T}$ \\
\hline UN_ci_7910 & $38 \mathrm{G} / \mathrm{A}$ \\
\hline UN_ci_7910 & $537 \mathrm{C} / \mathrm{T}$ \\
\hline UN_ci_7910 & $1324 \mathrm{G} / \mathrm{A}$ \\
\hline UN_ci_7910 & $1374 \mathrm{~A} / \mathrm{G}$ \\
\hline UN_ci_7913 & $351 \mathrm{G} / \mathrm{T}$ \\
\hline UN_ci_7914 & $55 \mathrm{G} / \mathrm{T}$ \\
\hline UN_ci_7914 & $78 \mathrm{G} / \mathrm{T}$ \\
\hline UN_ci_7918 & $116 \mathrm{~A} / \mathrm{G}$ \\
\hline UN_ci_7918 & $146 \mathrm{G} / \mathrm{A}$ \\
\hline UN_ci_7918 & $183 \mathrm{G} / \mathrm{A}$ \\
\hline UN_ci_7918 & $237 \mathrm{~A} / \mathrm{G}$ \\
\hline UN_ci_7918 & $263 \mathrm{G} / \mathrm{C}$ \\
\hline UN_ci_7918 & $318 \mathrm{~T} / \mathrm{C}$ \\
\hline UN_ci_7918 & 392 A/G \\
\hline UN_ci_7920 & $1254 \mathrm{~A} / \mathrm{G}$ \\
\hline UN_ci_7920 & $1276 \mathrm{~T} / \mathrm{G}$ \\
\hline UN_ci_7920 & $1609 \mathrm{~A} / \mathrm{G}$ \\
\hline UN_ci_7921 & $546 \mathrm{~A} / \mathrm{G}$ \\
\hline UN_ci_7921 & $925 \mathrm{~A} / \mathrm{C}$ \\
\hline UN_ci_7922 & $393 \mathrm{~T} / \mathrm{A}$ \\
\hline UN_ci_7922 & $435 \mathrm{C} / \mathrm{T}$ \\
\hline
\end{tabular}




\begin{tabular}{|c|c|}
\hline UN_ci_7922 & $1155 \mathrm{~T} / \mathrm{C}$ \\
\hline UN_ci_7922 & $1266 \mathrm{~A} / \mathrm{T}$ \\
\hline UN_ci_7925 & $501 \mathrm{~T} / \mathrm{A}$ \\
\hline UN_ci_7925 & $546 \mathrm{G} / \mathrm{A}$ \\
\hline UN_ci_7925 & 602 T/G \\
\hline UN_ci_7925 & $681 \mathrm{~A} / \mathrm{G}$ \\
\hline UN_ci_7925 & $720 \mathrm{G} / \mathrm{A}$ \\
\hline UN_ci_7925 & $819 \mathrm{C} / \mathrm{T}$ \\
\hline UN_ci_7925 & $874 \mathrm{~A} / \mathrm{T}$ \\
\hline UN_ci_7925 & $969 \mathrm{~A} / \mathrm{C}$ \\
\hline UN_ci_7925 & $1082 \mathrm{G} / \mathrm{A}$ \\
\hline UN_ci_7925 & $1426 \mathrm{~T} / \mathrm{C}$ \\
\hline UN_ci_7925 & $1499 \mathrm{C} / \mathrm{T}$ \\
\hline UN_ci_7925 & $1693 \mathrm{G} / \mathrm{C}$ \\
\hline UN_ci_7925 & $1773 \mathrm{~T} / \mathrm{C}$ \\
\hline UN_ci_7925 & $1951 \mathrm{~A} / \mathrm{G}$ \\
\hline UN_ci_7925 & 1972 T/C \\
\hline UN_ci_7925 & 2009 C/A \\
\hline UN_ci_7925 & $2140 \mathrm{C} / \mathrm{T}$ \\
\hline UN_ci_7925 & $2162 \mathrm{C} / \mathrm{T}$ \\
\hline UN_ci_7925 & $2220 \mathrm{C} / \mathrm{T}$ \\
\hline UN_ci_7925 & $2252 \mathrm{G} / \mathrm{A}$ \\
\hline UN_ci_7925 & $2282 \mathrm{~A} / \mathrm{G}$ \\
\hline UN_ci_7925 & 2317 T/C \\
\hline UN_ci_7925 & $2350 \mathrm{C} / \mathrm{T}$ \\
\hline UN_ci_7925 & 2375 A/G \\
\hline UN_ci_7925 & $2418 \mathrm{~T} / \mathrm{C}$ \\
\hline UN_ci_7925 & $2447 \mathrm{~T} / \mathrm{C}$ \\
\hline UN_ci_7925 & $2550 \mathrm{G} / \mathrm{C}$ \\
\hline UN_ci_7927 & $262 \mathrm{~A} / \mathrm{G}$ \\
\hline UN_ci_7927 & $398 \mathrm{G} / \mathrm{C}$ \\
\hline UN_ci_7928 & $7 \mathrm{~A} / \mathrm{T}$ \\
\hline UN_ci_7928 & $123 \mathrm{~A} / \mathrm{G}$ \\
\hline UN_ci_7928 & $248 \mathrm{~A} / \mathrm{T}$ \\
\hline UN_ci_7928 & $341 \mathrm{G} / \mathrm{A}$ \\
\hline UN_ci_7928 & $385 \mathrm{G} / \mathrm{A}$ \\
\hline UN_ci_7928 & $410 \mathrm{~T} / \mathrm{C}$ \\
\hline UN_ci_7928 & $449 \mathrm{~T} / \mathrm{C}$ \\
\hline UN_ci_7928 & $596 \mathrm{~T} / \mathrm{A}$ \\
\hline UN_ci_7928 & $938 \mathrm{~T} / \mathrm{C}$ \\
\hline UN_ci_7928 & $966 \mathrm{~A} / \mathrm{T}$ \\
\hline UN_ci_7928 & $1243 \mathrm{~T} / \mathrm{A}$ \\
\hline UN_ci_7929 & $164 \mathrm{C} / \mathrm{T}$ \\
\hline UN_ci_7929 & $205 \mathrm{~A} / \mathrm{G}$ \\
\hline UN_ci_7929 & $263 \mathrm{~T} / \mathrm{C}$ \\
\hline UN_ci_7929 & $409 \mathrm{C} / \mathrm{T}$ \\
\hline UN_ci_7929 & $456 \mathrm{~T} / \mathrm{C}$ \\
\hline UN_ci_7929 & $640 \mathrm{G} / \mathrm{A}$ \\
\hline UN_ci_7929 & $856 \mathrm{C} / \mathrm{T}$ \\
\hline UN_ci_7929 & $1055 \mathrm{~T} / \mathrm{C}$ \\
\hline
\end{tabular}




$\begin{array}{lr}\text { UN_ci_7929 } & 1381 \mathrm{~A} / \mathrm{C} \\ \text { UN_ci_7929 } & 1438 \mathrm{~A} / \mathrm{T} \\ \text { UN_ci_7929 } & 1458 \mathrm{~A} / \mathrm{G} \\ \text { UN_ci_7929 } & 1518 \mathrm{~A} / \mathrm{G} \\ \text { UN_ci_7929 } & 1542 \mathrm{~A} / \mathrm{T} \\ \text { UN_ci_7934 } & 37 \mathrm{C} / \mathrm{T} \\ \text { UN_ci_7934 } & 155 \mathrm{C} / \mathrm{T} \\ \text { UN_ci_7934 } & 187 \mathrm{C} / \mathrm{T} \\ \text { UN_ci_7934 } & 212 \mathrm{~A} / \mathrm{C} \\ \text { UN_ci_7934 } & 249 \mathrm{~A} / \mathrm{G} \\ \text { UN_ci_7934 } & 269 \mathrm{G} / \mathrm{T} \\ \text { UN_ci_7936 } & 182 \mathrm{~A} / \mathrm{C} \\ \text { UN_ci_7936 } & 713 \mathrm{G} / \mathrm{A} \\ \text { UN_ci_7936 } & 903 \mathrm{G} / \mathrm{A} \\ \text { UN_ci_7937 } & 12 \mathrm{~A} / \mathrm{C} \\ \text { UN_ci_7937 } & 310 \mathrm{C} / \mathrm{T} \\ \text { UN_ci_7938 } & 146 \mathrm{C} / \mathrm{T} \\ \text { UN_ci_7939 } & 422 \mathrm{~A} / \mathrm{G} \\ \text { UN_ci_7942 } & 27 \mathrm{~A} / \mathrm{G} \\ \text { UN_ci_7942 } & 70 \mathrm{~T} / \mathrm{C} \\ \text { UN_ci_7942 } & 324 \mathrm{G} / \mathrm{A} \\ \text { UN_ci_7942 } & 110 \mathrm{~A} / \mathrm{G} \\ \text { UN_ci_7945 } & 192 \mathrm{C} / \mathrm{T} \\ \text { UN_ci_7945 } & 244 \mathrm{G} / \mathrm{T} \\ \text { UN_ci_7945 } & 143 \mathrm{C} / \mathrm{T} \\ \text { UN_ci_7945 } & \end{array}$




\begin{tabular}{|c|c|}
\hline UN_ci_7952 & $302 \mathrm{~A} / \mathrm{G}$ \\
\hline UN_ci_7952 & 792 A/G \\
\hline UN_ci_7952 & $964 \mathrm{C} / \mathrm{T}$ \\
\hline UN_ci_7952 & $1060 \mathrm{G} / \mathrm{A}$ \\
\hline UN_ci_7953 & $182 \mathrm{~A} / \mathrm{G}$ \\
\hline UN_ci_7955 & $209 \mathrm{C} / \mathrm{A}$ \\
\hline UN_ci_7956 & $84 \mathrm{~A} / \mathrm{G}$ \\
\hline UN_ci_7956 & $110 \mathrm{G} / \mathrm{T}$ \\
\hline UN_ci_7956 & $403 \mathrm{~T} / \mathrm{C}$ \\
\hline UN_ci_7956 & $658 \mathrm{G} / \mathrm{A}$ \\
\hline UN_ci_7956 & $1174 \mathrm{C} / \mathrm{T}$ \\
\hline UN_ci_7956 & $1280 \mathrm{G} / \mathrm{A}$ \\
\hline UN_ci_7956 & 1335 G/T \\
\hline UN_ci_7956 & $1355 \mathrm{C} / \mathrm{A}$ \\
\hline UN_ci_7956 & $1376 \mathrm{~A} / \mathrm{G}$ \\
\hline UN_ci_7956 & $1396 \mathrm{~T} / \mathrm{A}$ \\
\hline UN_ci_7957 & $114 \mathrm{G} / \mathrm{A}$ \\
\hline UN_ci_7958 & $324 \mathrm{~A} / \mathrm{T}$ \\
\hline UN_ci_7961 & $670 \mathrm{~A} / \mathrm{G}$ \\
\hline UN_ci_7961 & $720 \mathrm{~A} / \mathrm{G}$ \\
\hline UN_ci_7961 & $771 \mathrm{~A} / \mathrm{G}$ \\
\hline UN_ci_7962 & $593 \mathrm{G} / \mathrm{A}$ \\
\hline UN_ci_7962 & $676 \mathrm{G} / \mathrm{A}$ \\
\hline UN_ci_7963 & $351 \mathrm{C} / \mathrm{T}$ \\
\hline UN_ci_7964 & $71 \mathrm{~A} / \mathrm{G}$ \\
\hline UN_ci_7964 & $204 \mathrm{C} / \mathrm{T}$ \\
\hline UN_ci_7964 & $233 \mathrm{~A} / \mathrm{G}$ \\
\hline UN_ci_7964 & $274 \mathrm{G} / \mathrm{T}$ \\
\hline UN_ci_7964 & 359 A/G \\
\hline UN_ci_7965 & $241 \mathrm{C} / \mathrm{T}$ \\
\hline UN_ci_7965 & $286 \mathrm{~T} / \mathrm{C}$ \\
\hline UN_ci_7965 & $331 \mathrm{~A} / \mathrm{G}$ \\
\hline UN_ci_7967 & $685 \mathrm{~T} / \mathrm{G}$ \\
\hline UN_ci_7967 & $746 \mathrm{~T} / \mathrm{C}$ \\
\hline UN_ci_7967 & $805 \mathrm{G} / \mathrm{A}$ \\
\hline UN_ci_7967 & 992 A/G \\
\hline UN_ci_7970 & $353 \mathrm{~A} / \mathrm{G}$ \\
\hline UN_ci_7971 & $132 \mathrm{~T} / \mathrm{C}$ \\
\hline UN_ci_7971 & $448 \mathrm{C} / \mathrm{A}$ \\
\hline UN_ci_7971 & $548 \mathrm{~A} / \mathrm{G}$ \\
\hline UN_ci_7971 & $631 \mathrm{~T} / \mathrm{C}$ \\
\hline UN_ci_7971 & $750 \mathrm{~T} / \mathrm{C}$ \\
\hline UN_ci_7971 & 959 C/G \\
\hline UN_ci_7971 & $1196 \mathrm{~T} / \mathrm{C}$ \\
\hline UN_ci_7971 & $1354 \mathrm{C} / \mathrm{T}$ \\
\hline UN_ci_7971 & $1376 \mathrm{C} / \mathrm{T}$ \\
\hline UN_ci_7971 & $1422 \mathrm{C} / \mathrm{T}$ \\
\hline UN_ci_7972 & $255 \mathrm{~T} / \mathrm{A}$ \\
\hline UN_ci_7972 & $291 \mathrm{~T} / \mathrm{C}$ \\
\hline UN_ci_7972 & $330 \mathrm{C} / \mathrm{T}$ \\
\hline
\end{tabular}




\begin{tabular}{|c|c|}
\hline UN_ci_7972 & $1023 \mathrm{G} / \mathrm{A}$ \\
\hline UN_ci_7972 & $1048 \mathrm{~T} / \mathrm{C}$ \\
\hline UN_ci_7972 & $1089 \mathrm{G} / \mathrm{A}$ \\
\hline UN_ci_7972 & $1148 \mathrm{C} / \mathrm{T}$ \\
\hline UN_ci_7972 & $1178 \mathrm{~T} / \mathrm{C}$ \\
\hline UN_ci_7972 & $1201 \mathrm{C} / \mathrm{T}$ \\
\hline UN_ci_7972 & $1303 \mathrm{~T} / \mathrm{A}$ \\
\hline UN_ci_7973 & $194 \mathrm{G} / \mathrm{T}$ \\
\hline UN_ci_7973 & 292 G/A \\
\hline UN_ci_7973 & $376 \mathrm{~T} / \mathrm{C}$ \\
\hline UN_ci_7973 & $458 \mathrm{C} / \mathrm{T}$ \\
\hline UN_ci_7973 & $520 \mathrm{~T} / \mathrm{G}$ \\
\hline UN_ci_7974 & $635 \mathrm{~T} / \mathrm{C}$ \\
\hline UN_ci_7976 & $340 \mathrm{G} / \mathrm{T}$ \\
\hline UN_ci_7976 & $900 \mathrm{C} / \mathrm{T}$ \\
\hline UN_ci_7976 & $1027 \mathrm{G} / \mathrm{A}$ \\
\hline UN_ci_7976 & $1348 \mathrm{~A} / \mathrm{G}$ \\
\hline UN_ci_7976 & $1518 \mathrm{G} / \mathrm{T}$ \\
\hline UN_ci_7979 & $278 \mathrm{~A} / \mathrm{G}$ \\
\hline UN_ci_7979 & 329 T/C \\
\hline UN_ci_7979 & $695 \mathrm{C} / \mathrm{T}$ \\
\hline UN_ci_7982 & $302 \mathrm{~A} / \mathrm{T}$ \\
\hline UN_ci_7982 & $362 \mathrm{C} / \mathrm{T}$ \\
\hline UN_ci_7982 & $426 \mathrm{~T} / \mathrm{C}$ \\
\hline UN_ci_7983 & $80 \mathrm{G} / \mathrm{A}$ \\
\hline UN_ci_7983 & $165 \mathrm{~A} / \mathrm{G}$ \\
\hline UN_ci_7983 & $204 \mathrm{~A} / \mathrm{T}$ \\
\hline UN_ci_7983 & $233 \mathrm{C} / \mathrm{T}$ \\
\hline UN_ci_7983 & $447 \mathrm{~A} / \mathrm{G}$ \\
\hline UN_ci_7983 & 579 A/G \\
\hline UN_ci_7984 & $44 \mathrm{~A} / \mathrm{T}$ \\
\hline UN_ci_7990 & $559 \mathrm{~A} / \mathrm{G}$ \\
\hline UN_ci_7990 & 906 G/A \\
\hline UN_ci_7990 & $1344 \mathrm{G} / \mathrm{T}$ \\
\hline UN_ci_7990 & $1759 \mathrm{~A} / \mathrm{T}$ \\
\hline UN_ci_7990 & 1797 T/C \\
\hline UN_ci_7990 & 1917 T/C \\
\hline UN_ci_7992 & $341 \mathrm{~A} / \mathrm{G}$ \\
\hline UN_ci_7992 & $594 \mathrm{~T} / \mathrm{G}$ \\
\hline UN_ci_7993 & $119 \mathrm{C} / \mathrm{G}$ \\
\hline UN_ci_7993 & $215 \mathrm{G} / \mathrm{A}$ \\
\hline UN_ci_7993 & $351 \mathrm{~T} / \mathrm{C}$ \\
\hline UN_ci_7994 & $184 \mathrm{G} / \mathrm{A}$ \\
\hline UN_ci_7994 & $296 \mathrm{~T} / \mathrm{C}$ \\
\hline UN_ci_7994 & $316 \mathrm{~T} / \mathrm{C}$ \\
\hline UN_ci_7994 & $352 \mathrm{~T} / \mathrm{G}$ \\
\hline UN_ci_7994 & $493 \mathrm{~T} / \mathrm{A}$ \\
\hline UN_ci_7994 & $639 \mathrm{C} / \mathrm{T}$ \\
\hline UN_ci_7994 & $716 \mathrm{~T} / \mathrm{C}$ \\
\hline UN_ci_7994 & $996 \mathrm{~T} / \mathrm{A}$ \\
\hline
\end{tabular}




\begin{tabular}{|c|c|}
\hline UN_ci_7994 & $1080 \mathrm{C} / \mathrm{T}$ \\
\hline UN_ci_7994 & $1247 \mathrm{G} / \mathrm{A}$ \\
\hline UN_ci_7997 & $222 \mathrm{G} / \mathrm{A}$ \\
\hline UN_ci_7998 & $40 \mathrm{~T} / \mathrm{G}$ \\
\hline UN_ci_7998 & $68 \mathrm{G} / \mathrm{A}$ \\
\hline UN_ci_7998 & $290 \mathrm{~T} / \mathrm{C}$ \\
\hline UN_ci_7998 & $410 \mathrm{~T} / \mathrm{C}$ \\
\hline UN_ci_7998 & $478 \mathrm{C} / \mathrm{T}$ \\
\hline UN_ci_7998 & $505 \mathrm{~A} / \mathrm{G}$ \\
\hline UN_ci_7998 & $605 \mathrm{~A} / \mathrm{G}$ \\
\hline UN_ci_7998 & $625 \mathrm{~A} / \mathrm{G}$ \\
\hline UN_ci_7998 & $951 \mathrm{~A} / \mathrm{G}$ \\
\hline UN_ci_8000 & $464 \mathrm{~A} / \mathrm{G}$ \\
\hline UN_ci_8000 & $660 \mathrm{~A} / \mathrm{C}$ \\
\hline UN_ci_8000 & $1075 \mathrm{~T} / \mathrm{A}$ \\
\hline UN_ci_8000 & $2142 \mathrm{~T} / \mathrm{C}$ \\
\hline UN_ci_8000 & $2181 \mathrm{~A} / \mathrm{C}$ \\
\hline UN_ci_8000 & $2319 \mathrm{C} / \mathrm{T}$ \\
\hline UN_ci_8002 & $446 \mathrm{~T} / \mathrm{C}$ \\
\hline UN_ci_8002 & $471 \mathrm{~A} / \mathrm{C}$ \\
\hline UN_ci_8002 & $638 \mathrm{~T} / \mathrm{C}$ \\
\hline UN_ci_8002 & $808 \mathrm{~A} / \mathrm{G}$ \\
\hline UN_ci_8002 & $863 \mathrm{G} / \mathrm{A}$ \\
\hline UN_ci_8003 & 289 G/T \\
\hline UN_ci_8003 & $342 \mathrm{~A} / \mathrm{G}$ \\
\hline UN_ci_8003 & $368 \mathrm{G} / \mathrm{A}$ \\
\hline UN_ci_8003 & $390 \mathrm{~A} / \mathrm{C}$ \\
\hline UN_ci_8003 & $439 \mathrm{G} / \mathrm{C}$ \\
\hline UN_ci_8003 & $513 \mathrm{~A} / \mathrm{G}$ \\
\hline UN_ci_8003 & $564 \mathrm{~A} / \mathrm{G}$ \\
\hline UN_ci_8003 & $588 \mathrm{G} / \mathrm{T}$ \\
\hline UN_ci_8003 & $610 \mathrm{~A} / \mathrm{G}$ \\
\hline UN_ci_8003 & $642 \mathrm{~A} / \mathrm{C}$ \\
\hline UN_ci_8003 & $662 \mathrm{~A} / \mathrm{G}$ \\
\hline UN_ci_8003 & $695 \mathrm{~T} / \mathrm{A}$ \\
\hline UN_ci_8004 & $343 \mathrm{G} / \mathrm{A}$ \\
\hline UN_ci_8004 & 395 T/C \\
\hline UN_ci_8004 & 617 T/C \\
\hline UN_ci_8005 & $457 \mathrm{~T} / \mathrm{C}$ \\
\hline UN_ci_8006 & $196 \mathrm{~A} / \mathrm{G}$ \\
\hline UN_ci_8006 & $254 \mathrm{C} / \mathrm{A}$ \\
\hline UN_ci_8006 & $285 \mathrm{~T} / \mathrm{G}$ \\
\hline UN_ci_8006 & $331 \mathrm{~T} / \mathrm{G}$ \\
\hline UN_ci_8006 & $371 \mathrm{~A} / \mathrm{G}$ \\
\hline UN_ci_8006 & $419 \mathrm{C} / \mathrm{A}$ \\
\hline UN_ci_8007 & $154 \mathrm{C} / \mathrm{T}$ \\
\hline UN_ci_8008 & 309 A/G \\
\hline UN_ci_8008 & $383 \mathrm{~A} / \mathrm{G}$ \\
\hline UN_ci_8008 & $615 \mathrm{~A} / \mathrm{T}$ \\
\hline UN_ci_8008 & $834 \mathrm{~T} / \mathrm{C}$ \\
\hline
\end{tabular}




\begin{tabular}{|c|c|}
\hline UN_ci_8010 & $57 \mathrm{G} / \mathrm{C}$ \\
\hline UN_ci_8010 & $83 \mathrm{~A} / \mathrm{G}$ \\
\hline UN_ci_8010 & $104 \mathrm{C} / \mathrm{T}$ \\
\hline UN_ci_8010 & $126 \mathrm{G} / \mathrm{T}$ \\
\hline UN_ci_8010 & $167 \mathrm{~A} / \mathrm{G}$ \\
\hline UN_ci_8010 & $198 \mathrm{G} / \mathrm{A}$ \\
\hline UN_ci_8010 & $233 \mathrm{~A} / \mathrm{G}$ \\
\hline UN_ci_8010 & $301 \mathrm{~A} / \mathrm{G}$ \\
\hline UN_ci_8010 & $355 \mathrm{~T} / \mathrm{C}$ \\
\hline UN_ci_8010 & $401 \mathrm{C} / \mathrm{A}$ \\
\hline UN_ci_8010 & $431 \mathrm{~A} / \mathrm{G}$ \\
\hline UN_ci_8010 & $545 \mathrm{~A} / \mathrm{G}$ \\
\hline UN_ci_8011 & $37 \mathrm{~T} / \mathrm{C}$ \\
\hline UN_ci_8011 & $92 \mathrm{~T} / \mathrm{G}$ \\
\hline UN_ci_8011 & $117 \mathrm{~T} / \mathrm{C}$ \\
\hline UN_ci_8011 & $140 \mathrm{~A} / \mathrm{G}$ \\
\hline UN_ci_8011 & $175 \mathrm{C} / \mathrm{T}$ \\
\hline UN_ci_8011 & $196 \mathrm{~A} / \mathrm{G}$ \\
\hline UN_ci_8011 & $217 \mathrm{~T} / \mathrm{C}$ \\
\hline UN_ci_8011 & $244 \mathrm{C} / \mathrm{T}$ \\
\hline UN_ci_8011 & $298 \mathrm{G} / \mathrm{A}$ \\
\hline UN_ci_8011 & $334 \mathrm{~T} / \mathrm{G}$ \\
\hline UN_ci_8013 & $401 \mathrm{G} / \mathrm{A}$ \\
\hline UN_ci_8013 & $468 \mathrm{G} / \mathrm{C}$ \\
\hline UN_ci_8013 & $626 \mathrm{~A} / \mathrm{T}$ \\
\hline UN_ci_8013 & $698 \mathrm{~A} / \mathrm{G}$ \\
\hline UN_ci_8013 & $842 \mathrm{~A} / \mathrm{T}$ \\
\hline UN_ci_8013 & $1018 \mathrm{~T} / \mathrm{C}$ \\
\hline UN_ci_8013 & $1093 \mathrm{G} / \mathrm{A}$ \\
\hline UN_ci_8017 & $365 \mathrm{~A} / \mathrm{G}$ \\
\hline UN_ci_8017 & $497 \mathrm{~A} / \mathrm{C}$ \\
\hline UN_ci_8018 & $314 \mathrm{~A} / \mathrm{G}$ \\
\hline UN_ci_8019 & $120 \mathrm{G} / \mathrm{C}$ \\
\hline UN_ci_8019 & $572 \mathrm{~A} / \mathrm{G}$ \\
\hline UN_ci_8019 & $615 \mathrm{~A} / \mathrm{G}$ \\
\hline UN_ci_8019 & $661 \mathrm{~T} / \mathrm{C}$ \\
\hline UN_ci_8019 & $748 \mathrm{~T} / \mathrm{C}$ \\
\hline UN_ci_8019 & $829 \mathrm{~T} / \mathrm{C}$ \\
\hline UN_ci_8019 & 919 T/C \\
\hline UN_ci_8019 & $976 \mathrm{C} / \mathrm{T}$ \\
\hline UN_ci_8019 & $1017 \mathrm{~A} / \mathrm{C}$ \\
\hline UN_ci_8019 & $1094 \mathrm{~T} / \mathrm{G}$ \\
\hline UN_ci_8019 & 1139 T/C \\
\hline UN_ci_8021 & $164 \mathrm{~T} / \mathrm{C}$ \\
\hline UN_ci_8021 & $197 \mathrm{C} / \mathrm{T}$ \\
\hline UN_ci_8021 & $403 \mathrm{~T} / \mathrm{C}$ \\
\hline UN_ci_8021 & $651 \mathrm{C} / \mathrm{G}$ \\
\hline UN_ci_8022 & $80 \mathrm{~T} / \mathrm{C}$ \\
\hline UN_ci_8022 & $528 \mathrm{~A} / \mathrm{G}$ \\
\hline UN_ci_8022 & $1014 \mathrm{~T} / \mathrm{C}$ \\
\hline
\end{tabular}




$\begin{array}{lr}\text { UN_ci_8022 } & \\ \text { UN_ci_8024 } & 1175 \mathrm{~T} / \mathrm{C} \\ \text { UN_ci_8024 } & 363 \mathrm{~A} / \mathrm{G} \\ \text { UN_ci_8026 } & 778 \mathrm{~A} / \mathrm{G} \\ \text { UN_ci_8026 } & 697 \mathrm{~A} / \mathrm{G} \\ \text { UN_ci_8026 } & 722 \mathrm{G} / \mathrm{A} \\ \text { UN_ci_8026 } & 758 \mathrm{~A} / \mathrm{G} \\ \text { UN_ci_8026 } & 968 \mathrm{G} / \mathrm{A} \\ \text { UN_ci_8026 } & 1127 \mathrm{~T} / \mathrm{A} \\ \text { UN_ci_8030 } & 1276 \mathrm{~T} / \mathrm{C} \\ \text { UN_ci_8030 } & 190 \mathrm{G} / \mathrm{C} \\ \text { UN_ci_8030 } & 284 \mathrm{~A} / \mathrm{G} \\ \text { UN_ci_8030 } & 360 \mathrm{~T} / \mathrm{C} \\ \text { UN_ci_8030 } & 603 \mathrm{C} / \mathrm{T} \\ \text { UN_ci_8030 } & 645 \mathrm{~A} / \mathrm{G} \\ \text { UN_ci_8030 } & 713 \mathrm{~T} / \mathrm{C} \\ \text { UN_ci_8030 } & 779 \mathrm{G} / \mathrm{A} \\ \text { UN_ci_8030 } & 855 \mathrm{~T} / \mathrm{C} \\ \text { UN_ci_8033 } & 1052 \mathrm{G} / \mathrm{A} \\ \text { UN_ci_8033 } & 373 \mathrm{~A} / \mathrm{G} \\ \text { UN_ci_8033 } & 572 \mathrm{G} / \mathrm{A} \\ \text { UN_ci_8033 } & 832 \mathrm{C} / \mathrm{T} \\ \text { UN_ci_8033 } & 872 \mathrm{C} / \mathrm{A} \\ \text { UN_ci_8033 } & 859 \mathrm{G} \text { C/T } \\ \text { UN_ci_8036 } & 505 \mathrm{~T} / \mathrm{C} \\ \text { UN_ci_8036 } & \end{array}$




$\begin{array}{lr}\text { UN_ci_8041 } & 1236 \mathrm{~A} / \mathrm{T} \\ \text { UN_ci_8041 } & 1527 \mathrm{~T} / \mathrm{C} \\ \text { UN_ci_8043 } & 85 \mathrm{~T} / \mathrm{A} \\ \text { UN_ci_8044 } & 726 \mathrm{C} / \mathrm{T} \\ \text { UN_ci_8044 } & 789 \mathrm{C} / \mathrm{T} \\ \text { UN_ci_8044 } & 901 \mathrm{C} / \mathrm{T} \\ \text { UN_ci_8045 } & 220 \mathrm{~T} / \mathrm{C} \\ \text { UN_ci_8045 } & 297 \mathrm{~A} / \mathrm{G} \\ \text { UN_ci_8045 } & 334 \mathrm{G} / \mathrm{A} \\ \text { UN_ci_8047 } & 71 \mathrm{G} / \mathrm{A} \\ \text { UN_ci_8049 } & 183 \mathrm{~A} / \mathrm{G} \\ \text { UN_ci_8049 } & 723 \mathrm{~T} / \mathrm{C} \\ \text { UN_ci_8052 } & 404 \mathrm{~A} / \mathrm{G} \\ \text { UN_ci_8054 } & 118 \mathrm{C} / \mathrm{T} \\ \text { UN_ci_8054 } & 153 \mathrm{G} / \mathrm{A} \\ \text { UN_ci_8054 } & 175 \mathrm{~A} / \mathrm{T} \\ \text { UN_ci_8054 } & 250 \mathrm{G} / \mathrm{A} \\ \text { UN_ci_8054 } & 279 \mathrm{C} / \mathrm{T} \\ \text { UN_ci_8054 } & 316 \mathrm{~T} / \mathrm{A} \\ \text { UN_ci_8054 } & 336 \mathrm{~A} / \mathrm{G} \\ \text { UN_ci_8054 } & 39 \mathrm{C} / \mathrm{T} \\ \text { UN_ci_8054 } & 573 \mathrm{G} / \mathrm{A} \\ \text { UN_ci_8054 } & 642 \mathrm{G} / \mathrm{A} \\ \text { UN_ci_8054 } & 705 \mathrm{G} / \mathrm{A} \\ \text { UN_ci_8054 } & \end{array}$




\begin{tabular}{|c|c|}
\hline UN_ci_8065 & $121 \mathrm{~T} / \mathrm{C}$ \\
\hline UN_ci_8065 & $306 \mathrm{~T} / \mathrm{C}$ \\
\hline UN_ci_8065 & 419 T/A \\
\hline UN_ci_8065 & $702 \mathrm{C} / \mathrm{T}$ \\
\hline UN_ci_8065 & 777 T/C \\
\hline UN_ci_8065 & $873 \mathrm{~T} / \mathrm{C}$ \\
\hline UN_ci_8065 & $903 \mathrm{~A} / \mathrm{C}$ \\
\hline UN_ci_8065 & $924 \mathrm{G} / \mathrm{T}$ \\
\hline UN_ci_8065 & $969 \mathrm{~T} / \mathrm{G}$ \\
\hline UN_ci_8065 & $1014 \mathrm{~T} / \mathrm{A}$ \\
\hline UN_ci_8065 & $1034 \mathrm{~A} / \mathrm{G}$ \\
\hline UN_ci_8067 & $210 \mathrm{G} / \mathrm{T}$ \\
\hline UN_ci_8067 & $290 \mathrm{~A} / \mathrm{G}$ \\
\hline UN_ci_8068 & $369 \mathrm{G} / \mathrm{A}$ \\
\hline UN_ci_8069 & $527 \mathrm{~A} / \mathrm{G}$ \\
\hline UN_ci_8069 & $578 \mathrm{C} / \mathrm{G}$ \\
\hline UN_ci_8069 & $692 \mathrm{~A} / \mathrm{C}$ \\
\hline UN_ci_8069 & $933 \mathrm{~A} / \mathrm{G}$ \\
\hline UN_ci_8069 & $987 \mathrm{~T} / \mathrm{G}$ \\
\hline UN_ci_8070 & $618 \mathrm{G} / \mathrm{A}$ \\
\hline UN_ci_8070 & $836 \mathrm{~T} / \mathrm{C}$ \\
\hline UN_ci_8070 & $930 \mathrm{~A} / \mathrm{G}$ \\
\hline UN_ci_8070 & 1076 A/G \\
\hline UN_ci_8071 & $933 \mathrm{~T} / \mathrm{C}$ \\
\hline UN_ci_8072 & $20 \mathrm{G} / \mathrm{A}$ \\
\hline UN_ci_8072 & $91 \mathrm{~A} / \mathrm{G}$ \\
\hline UN_ci_8072 & 209 T/C \\
\hline UN_ci_8072 & $283 \mathrm{G} / \mathrm{A}$ \\
\hline UN_ci_8072 & $310 \mathrm{G} / \mathrm{A}$ \\
\hline UN_ci_8072 & $355 \mathrm{C} / \mathrm{A}$ \\
\hline UN_ci_8072 & $432 \mathrm{~T} / \mathrm{C}$ \\
\hline UN_ci_8072 & $550 \mathrm{~A} / \mathrm{G}$ \\
\hline UN_ci_8073 & $74 \mathrm{G} / \mathrm{T}$ \\
\hline UN_ci_8073 & $156 \mathrm{C} / \mathrm{T}$ \\
\hline UN_ci_8073 & $191 \mathrm{C} / \mathrm{T}$ \\
\hline UN_ci_8073 & $227 \mathrm{C} / \mathrm{T}$ \\
\hline UN_ci_8073 & $251 \mathrm{~T} / \mathrm{C}$ \\
\hline UN_ci_8073 & $290 \mathrm{~A} / \mathrm{C}$ \\
\hline UN_ci_8073 & $362 \mathrm{~T} / \mathrm{C}$ \\
\hline UN_ci_8073 & $410 \mathrm{~T} / \mathrm{C}$ \\
\hline UN_ci_8073 & $441 \mathrm{~T} / \mathrm{C}$ \\
\hline UN_ci_8073 & $473 \mathrm{~T} / \mathrm{C}$ \\
\hline UN_ci_8073 & $678 \mathrm{C} / \mathrm{A}$ \\
\hline UN_ci_8073 & $715 \mathrm{C} / \mathrm{T}$ \\
\hline UN_ci_8073 & $737 \mathrm{~A} / \mathrm{T}$ \\
\hline UN_ci_8073 & $767 \mathrm{~T} / \mathrm{C}$ \\
\hline UN_ci_8073 & $814 \mathrm{~T} / \mathrm{C}$ \\
\hline UN_ci_8073 & $1098 \mathrm{~A} / \mathrm{G}$ \\
\hline UN_ci_8073 & $1142 \mathrm{G} / \mathrm{C}$ \\
\hline UN_ci_8076 & $228 \mathrm{C} / \mathrm{T}$ \\
\hline
\end{tabular}




\begin{tabular}{|c|c|}
\hline UN_ci_8076 & $535 \mathrm{~A} / \mathrm{G}$ \\
\hline UN_ci_8078 & $320 \mathrm{~T} / \mathrm{C}$ \\
\hline UN_ci_8078 & $453 \mathrm{~T} / \mathrm{G}$ \\
\hline UN_ci_8079 & $154 \mathrm{C} / \mathrm{G}$ \\
\hline UN_ci_8079 & $205 \mathrm{C} / \mathrm{T}$ \\
\hline UN_ci_8079 & $310 \mathrm{~T} / \mathrm{C}$ \\
\hline UN_ci_8079 & $374 \mathrm{G} / \mathrm{A}$ \\
\hline UN_ci_8080 & $22 \mathrm{G} / \mathrm{A}$ \\
\hline UN_ci_8080 & $151 \mathrm{G} / \mathrm{A}$ \\
\hline UN_ci_8080 & $712 \mathrm{G} / \mathrm{A}$ \\
\hline UN_ci_8080 & $798 \mathrm{G} / \mathrm{A}$ \\
\hline UN_ci_8080 & $861 \mathrm{G} / \mathrm{A}$ \\
\hline UN_ci_8081 & $107 \mathrm{G} / \mathrm{T}$ \\
\hline UN_ci_8084 & $12 \mathrm{~T} / \mathrm{C}$ \\
\hline UN_ci_8084 & $51 \mathrm{~A} / \mathrm{T}$ \\
\hline UN_ci_8084 & $387 \mathrm{G} / \mathrm{A}$ \\
\hline UN_ci_8084 & $551 \mathrm{G} / \mathrm{C}$ \\
\hline UN_ci_8084 & $675 \mathrm{~T} / \mathrm{C}$ \\
\hline UN_ci_8084 & 777 T/C \\
\hline UN_ci_8085 & $746 \mathrm{~A} / \mathrm{G}$ \\
\hline UN_ci_8086 & $769 \mathrm{C} / \mathrm{A}$ \\
\hline UN_ci_8086 & $885 \mathrm{~T} / \mathrm{G}$ \\
\hline UN_ci_8086 & $970 \mathrm{~A} / \mathrm{T}$ \\
\hline UN_ci_8088 & $58 \mathrm{C} / \mathrm{T}$ \\
\hline UN_ci_8089 & $448 \mathrm{~A} / \mathrm{G}$ \\
\hline UN_ci_8089 & $665 \mathrm{~A} / \mathrm{G}$ \\
\hline UN_ci_8092 & $485 \mathrm{C} / \mathrm{T}$ \\
\hline UN_ci_8093 & $663 \mathrm{~A} / \mathrm{C}$ \\
\hline UN_ci_8093 & $1184 \mathrm{~T} / \mathrm{C}$ \\
\hline UN_ci_8093 & $1582 \mathrm{~A} / \mathrm{G}$ \\
\hline UN_ci_8093 & $1683 \mathrm{C} / \mathrm{A}$ \\
\hline UN_ci_8093 & 1889 A/G \\
\hline UN_ci_8093 & $1992 \mathrm{~T} / \mathrm{C}$ \\
\hline UN_ci_8093 & $2118 \mathrm{~T} / \mathrm{C}$ \\
\hline UN_ci_8093 & $2285 \mathrm{C} / \mathrm{T}$ \\
\hline UN_ci_8094 & $525 \mathrm{G} / \mathrm{C}$ \\
\hline UN_ci_8094 & $622 \mathrm{G} / \mathrm{T}$ \\
\hline UN_ci_8095 & $219 \mathrm{C} / \mathrm{G}$ \\
\hline UN_ci_8095 & $342 \mathrm{G} / \mathrm{T}$ \\
\hline UN_ci_8095 & $445 \mathrm{~A} / \mathrm{G}$ \\
\hline UN_ci_8097 & $22 \mathrm{C} / \mathrm{T}$ \\
\hline UN_ci_8097 & $128 \mathrm{G} / \mathrm{A}$ \\
\hline UN_ci_8097 & $400 \mathrm{~T} / \mathrm{G}$ \\
\hline UN_ci_8097 & $420 \mathrm{~A} / \mathrm{G}$ \\
\hline UN_ci_8098 & $109 \mathrm{G} / \mathrm{A}$ \\
\hline UN_ci_8098 & $156 \mathrm{~A} / \mathrm{G}$ \\
\hline UN_ci_8098 & $197 \mathrm{~A} / \mathrm{G}$ \\
\hline UN_ci_8098 & $226 \mathrm{~A} / \mathrm{G}$ \\
\hline UN_ci_8098 & $246 \mathrm{~A} / \mathrm{G}$ \\
\hline UN_ci_8098 & $309 \mathrm{C} / \mathrm{T}$ \\
\hline
\end{tabular}




\begin{tabular}{|c|c|}
\hline UN_ci_8098 & $346 \mathrm{~A} / \mathrm{T}$ \\
\hline UN_ci_8098 & $374 \mathrm{~A} / \mathrm{G}$ \\
\hline UN_ci_8098 & $428 \mathrm{~A} / \mathrm{G}$ \\
\hline UN_ci_8098 & $474 \mathrm{~A} / \mathrm{G}$ \\
\hline UN_ci_8098 & $587 \mathrm{~A} / \mathrm{G}$ \\
\hline UN_ci_8098 & $607 \mathrm{~A} / \mathrm{T}$ \\
\hline UN_ci_8098 & $637 \mathrm{C} / \mathrm{T}$ \\
\hline UN_ci_8098 & $716 \mathrm{~A} / \mathrm{G}$ \\
\hline UN_ci_8098 & $747 \mathrm{~T} / \mathrm{C}$ \\
\hline UN_ci_8098 & $768 \mathrm{~A} / \mathrm{C}$ \\
\hline UN_ci_8098 & $816 \mathrm{~A} / \mathrm{G}$ \\
\hline UN_ci_8098 & 877 T/C \\
\hline UN_ci_8098 & $1040 \mathrm{~A} / \mathrm{G}$ \\
\hline UN_ci_8098 & $1078 \mathrm{~T} / \mathrm{C}$ \\
\hline UN_ci_8098 & $1098 \mathrm{G} / \mathrm{A}$ \\
\hline UN_ci_8098 & 1145 T/C \\
\hline UN_ci_8098 & $1241 \mathrm{G} / \mathrm{T}$ \\
\hline UN_ci_8098 & $1300 \mathrm{G} / \mathrm{A}$ \\
\hline UN_ci_8098 & $1361 \mathrm{G} / \mathrm{A}$ \\
\hline UN_ci_8098 & $1411 \mathrm{~A} / \mathrm{G}$ \\
\hline UN_ci_8099 & $52 \mathrm{C} / \mathrm{T}$ \\
\hline UN_ci_8099 & $724 \mathrm{~T} / \mathrm{G}$ \\
\hline UN_ci_8099 & 889 G/T \\
\hline UN_ci_8100 & $695 \mathrm{~A} / \mathrm{G}$ \\
\hline UN_ci_8101 & $365 \mathrm{C} / \mathrm{A}$ \\
\hline UN_ci_8101 & $393 \mathrm{~A} / \mathrm{G}$ \\
\hline UN_ci_8101 & $437 \mathrm{~T} / \mathrm{C}$ \\
\hline UN_ci_8102 & $609 \mathrm{~T} / \mathrm{C}$ \\
\hline UN_ci_8103 & $131 \mathrm{G} / \mathrm{T}$ \\
\hline UN_ci_8103 & $155 \mathrm{G} / \mathrm{A}$ \\
\hline UN_ci_8103 & $177 \mathrm{~A} / \mathrm{G}$ \\
\hline UN_ci_8103 & $200 \mathrm{~A} / \mathrm{G}$ \\
\hline UN_ci_8103 & $234 \mathrm{~T} / \mathrm{C}$ \\
\hline UN_ci_8103 & $262 \mathrm{~T} / \mathrm{C}$ \\
\hline UN_ci_8103 & $294 \mathrm{~T} / \mathrm{C}$ \\
\hline UN_ci_8103 & $314 \mathrm{~A} / \mathrm{T}$ \\
\hline UN_ci_8103 & $351 \mathrm{~A} / \mathrm{G}$ \\
\hline UN_ci_8103 & $374 \mathrm{~A} / \mathrm{G}$ \\
\hline UN_ci_8103 & $419 \mathrm{~T} / \mathrm{C}$ \\
\hline UN_ci_8103 & $448 \mathrm{~T} / \mathrm{C}$ \\
\hline UN_ci_8103 & $529 \mathrm{~A} / \mathrm{T}$ \\
\hline UN_ci_8103 & $553 \mathrm{C} / \mathrm{T}$ \\
\hline UN_ci_8103 & $573 \mathrm{~T} / \mathrm{C}$ \\
\hline UN_ci_8103 & 605 T/C \\
\hline UN_ci_8103 & $643 \mathrm{~A} / \mathrm{G}$ \\
\hline UN_ci_8103 & $665 \mathrm{~T} / \mathrm{C}$ \\
\hline UN_ci_8103 & $710 \mathrm{~A} / \mathrm{G}$ \\
\hline UN_ci_8103 & $736 \mathrm{~T} / \mathrm{C}$ \\
\hline UN_ci_8103 & $774 \mathrm{~T} / \mathrm{C}$ \\
\hline UN_ci_8103 & $794 \mathrm{~A} / \mathrm{G}$ \\
\hline
\end{tabular}




$\begin{array}{lr}\text { UN_ci_8103 } & 830 \mathrm{~A} / \mathrm{C} \\ \text { UN_ci_8103 } & 868 \mathrm{G} / \mathrm{A} \\ \text { UN_ci_8103 } & 938 \mathrm{G} / \mathrm{A} \\ \text { UN_ci_8103 } & 1002 \mathrm{~T} / \mathrm{C} \\ \text { UN_ci_8103 } & 1133 \mathrm{~T} / \mathrm{C} \\ \text { UN_ci_8104 } & 68 \mathrm{~A} / \mathrm{G} \\ \text { UN_ci_8104 } & 93 \mathrm{~T} / \mathrm{A} \\ \text { UN_ci_8104 } & 212 \mathrm{~T} / \mathrm{C} \\ \text { UN_ci_8104 } & 284 \mathrm{G} / \mathrm{A} \\ \text { UN_ci_8104 } & 405 \mathrm{C} / \mathrm{T} \\ \text { UN_ci_8104 } & 435 \mathrm{C} / \mathrm{T} \\ \text { UN_ci_8105 } & 8 \mathrm{~A} / \mathrm{G} \\ \text { UN_ci_8105 } & 103 \mathrm{C} / \mathrm{A} \\ \text { UN_ci_8105 } & 258 \mathrm{G} / \mathrm{A} \\ \text { UN_ci_8105 } & 489 \mathrm{~T} / \mathrm{C} \\ \text { UN_ci_8105 } & 627 \mathrm{C} / \mathrm{T} \\ \text { UN_ci_8105 } & 688 \mathrm{C} / \mathrm{A} \\ \text { UN_ci_8106 } & 580 \mathrm{C} / \mathrm{T} \\ \text { UN_ci_8106 } & 877 \mathrm{~T} / \mathrm{G} \\ \text { UN_ci_8108 } & 69 \mathrm{~T} / \mathrm{C} \\ \text { UN_ci_8108 } & 52 \mathrm{C} / \mathrm{G} \\ \text { UN_ci_8108 } & 158 \mathrm{G} / \mathrm{T} \\ \text { UN_ci_8108 } & 215 \mathrm{~T} / \mathrm{C} \\ \text { UN_ci_8108 } & 1255 \mathrm{~T} / \mathrm{C} \\ \text { UN_ci_8108 } & 1466 \mathrm{~T} / \mathrm{A} \\ \text { UN_ci_8109 } & \end{array}$




\begin{tabular}{|c|c|}
\hline UN_ci_8118 & $384 \mathrm{~A} / \mathrm{G}$ \\
\hline UN_ci_8118 & $477 \mathrm{C} / \mathrm{A}$ \\
\hline UN_ci_8118 & $662 \mathrm{C} / \mathrm{T}$ \\
\hline UN_ci_8118 & $781 \mathrm{~A} / \mathrm{T}$ \\
\hline UN_ci_8118 & $954 \mathrm{C} / \mathrm{T}$ \\
\hline UN_ci_8120 & $36 \mathrm{G} / \mathrm{C}$ \\
\hline UN_ci_8121 & $45 \mathrm{~A} / \mathrm{G}$ \\
\hline UN_ci_8121 & $92 \mathrm{C} / \mathrm{T}$ \\
\hline UN_ci_8121 & $255 \mathrm{C} / \mathrm{T}$ \\
\hline UN_ci_8121 & 289 A/G \\
\hline UN_ci_8121 & $396 \mathrm{~T} / \mathrm{C}$ \\
\hline UN_ci_8121 & $582 \mathrm{C} / \mathrm{T}$ \\
\hline UN_ci_8121 & $628 \mathrm{~T} / \mathrm{C}$ \\
\hline UN_ci_8121 & $708 \mathrm{C} / \mathrm{A}$ \\
\hline UN_ci_8123 & $283 \mathrm{C} / \mathrm{G}$ \\
\hline UN_ci_8123 & $320 \mathrm{~A} / \mathrm{G}$ \\
\hline UN_ci_8123 & $352 \mathrm{G} / \mathrm{A}$ \\
\hline UN_ci_8123 & $410 \mathrm{~A} / \mathrm{G}$ \\
\hline UN_ci_8123 & $457 \mathrm{~A} / \mathrm{G}$ \\
\hline UN_ci_8123 & $520 \mathrm{~A} / \mathrm{G}$ \\
\hline UN_ci_8123 & $558 \mathrm{G} / \mathrm{A}$ \\
\hline UN_ci_8123 & $583 \mathrm{~A} / \mathrm{G}$ \\
\hline UN_ci_8123 & $663 \mathrm{C} / \mathrm{T}$ \\
\hline UN_ci_8123 & $738 \mathrm{G} / \mathrm{C}$ \\
\hline UN_ci_8123 & $777 \mathrm{G} / \mathrm{A}$ \\
\hline UN_ci_8123 & $797 \mathrm{~A} / \mathrm{G}$ \\
\hline UN_ci_8123 & $855 \mathrm{~A} / \mathrm{C}$ \\
\hline UN_ci_8123 & $886 \mathrm{~A} / \mathrm{T}$ \\
\hline UN_ci_8123 & $915 \mathrm{~A} / \mathrm{G}$ \\
\hline UN_ci_8123 & $991 \mathrm{C} / \mathrm{A}$ \\
\hline UN_ci_8123 & $1099 \mathrm{~T} / \mathrm{C}$ \\
\hline UN_ci_8123 & $1141 \mathrm{~A} / \mathrm{G}$ \\
\hline UN_ci_8123 & $1244 \mathrm{~A} / \mathrm{G}$ \\
\hline UN_ci_8123 & $1313 \mathrm{~A} / \mathrm{G}$ \\
\hline UN_ci_8123 & $1350 \mathrm{~A} / \mathrm{G}$ \\
\hline UN_ci_8123 & 1437 T/C \\
\hline UN_ci_8123 & $1654 \mathrm{~T} / \mathrm{C}$ \\
\hline UN_ci_8123 & $1688 \mathrm{G} / \mathrm{A}$ \\
\hline UN_ci_8123 & $1721 \mathrm{G} / \mathrm{A}$ \\
\hline UN_ci_8123 & 1819 A/G \\
\hline UN_ci_8123 & $1854 \mathrm{~T} / \mathrm{G}$ \\
\hline UN_ci_8123 & 1928 T/C \\
\hline UN_ci_8124 & $162 \mathrm{~T} / \mathrm{C}$ \\
\hline UN_ci_8125 & $275 \mathrm{~T} / \mathrm{C}$ \\
\hline UN_ci_8125 & $692 \mathrm{~A} / \mathrm{G}$ \\
\hline UN_ci_8125 & $864 \mathrm{G} / \mathrm{T}$ \\
\hline UN_ci_8127 & $110 \mathrm{~T} / \mathrm{C}$ \\
\hline UN_ci_8127 & $211 \mathrm{~A} / \mathrm{G}$ \\
\hline UN_ci_8127 & $474 \mathrm{~A} / \mathrm{C}$ \\
\hline UN_ci_8127 & $505 \mathrm{~T} / \mathrm{G}$ \\
\hline
\end{tabular}




\begin{tabular}{|c|c|}
\hline UN_ci_8127 & $538 \mathrm{~A} / \mathrm{G}$ \\
\hline UN_ci_8127 & $696 \mathrm{G} / \mathrm{A}$ \\
\hline UN_ci_8127 & $762 \mathrm{G} / \mathrm{A}$ \\
\hline UN_ci_8128 & $153 \mathrm{~A} / \mathrm{G}$ \\
\hline UN_ci_8128 & $408 \mathrm{~A} / \mathrm{G}$ \\
\hline UN_ci_8128 & $757 \mathrm{~T} / \mathrm{C}$ \\
\hline UN_ci_8129 & $798 \mathrm{~A} / \mathrm{G}$ \\
\hline UN_ci_8129 & $860 \mathrm{~A} / \mathrm{G}$ \\
\hline UN_ci_8129 & $980 \mathrm{~A} / \mathrm{G}$ \\
\hline UN_ci_8129 & 1049 A/G \\
\hline UN_ci_8129 & $1154 \mathrm{~A} / \mathrm{G}$ \\
\hline UN_ci_8129 & 1202 G/A \\
\hline UN_ci_8129 & $1270 \mathrm{~T} / \mathrm{C}$ \\
\hline UN_ci_8129 & $1371 \mathrm{~A} / \mathrm{G}$ \\
\hline UN_ci_8129 & $1417 \mathrm{~T} / \mathrm{C}$ \\
\hline UN_ci_8129 & $1438 \mathrm{~A} / \mathrm{G}$ \\
\hline UN_ci_8129 & $1464 \mathrm{C} / \mathrm{T}$ \\
\hline UN_ci_8129 & $1486 \mathrm{~T} / \mathrm{C}$ \\
\hline UN_ci_8129 & $1508 \mathrm{~T} / \mathrm{C}$ \\
\hline UN_ci_8129 & $1544 \mathrm{~A} / \mathrm{G}$ \\
\hline UN_ci_8129 & $1566 \mathrm{~T} / \mathrm{G}$ \\
\hline UN_ci_8129 & $1602 \mathrm{~A} / \mathrm{G}$ \\
\hline UN_ci_8129 & $1627 \mathrm{~A} / \mathrm{G}$ \\
\hline UN_ci_8129 & $1666 \mathrm{~T} / \mathrm{C}$ \\
\hline UN_ci_8129 & $1695 \mathrm{~A} / \mathrm{C}$ \\
\hline UN_ci_8129 & $1722 \mathrm{C} / \mathrm{G}$ \\
\hline UN_ci_8129 & 1743 T/A \\
\hline UN_ci_8129 & $1764 \mathrm{~A} / \mathrm{G}$ \\
\hline UN_ci_8129 & $1788 \mathrm{~T} / \mathrm{C}$ \\
\hline UN_ci_8129 & $1808 \mathrm{~A} / \mathrm{G}$ \\
\hline UN_ci_8129 & $1831 \mathrm{~T} / \mathrm{C}$ \\
\hline UN_ci_8129 & $1860 \mathrm{~A} / \mathrm{G}$ \\
\hline UN_ci_8129 & $1887 \mathrm{~T} / \mathrm{C}$ \\
\hline UN_ci_8129 & $1911 \mathrm{~T} / \mathrm{C}$ \\
\hline UN_ci_8129 & $1933 \mathrm{C} / \mathrm{T}$ \\
\hline UN_ci_8129 & $1956 \mathrm{C} / \mathrm{T}$ \\
\hline UN_ci_8129 & $1978 \mathrm{~T} / \mathrm{C}$ \\
\hline UN_ci_8129 & $2034 \mathrm{~A} / \mathrm{G}$ \\
\hline UN_ci_8129 & $2059 \mathrm{C} / \mathrm{T}$ \\
\hline UN_ci_8129 & 2079 G/A \\
\hline UN_ci_8129 & 2099 T/C \\
\hline UN_ci_8129 & $2120 \mathrm{~T} / \mathrm{C}$ \\
\hline UN_ci_8130 & $178 \mathrm{~T} / \mathrm{G}$ \\
\hline UN_ci_8130 & $385 \mathrm{~A} / \mathrm{T}$ \\
\hline UN_ci_8130 & $747 \mathrm{G} / \mathrm{A}$ \\
\hline UN_ci_8132 & $6 \mathrm{~T} / \mathrm{G}$ \\
\hline UN_ci_8133 & $182 \mathrm{C} / \mathrm{T}$ \\
\hline UN_ci_8133 & $203 \mathrm{C} / \mathrm{T}$ \\
\hline UN_ci_8133 & $226 \mathrm{~A} / \mathrm{G}$ \\
\hline UN_ci_8134 & $34 \mathrm{~T} / \mathrm{G}$ \\
\hline
\end{tabular}




\begin{tabular}{|c|c|}
\hline UN_ci_8134 & $71 \mathrm{~A} / \mathrm{T}$ \\
\hline UN_ci_8134 & $112 \mathrm{~A} / \mathrm{G}$ \\
\hline UN_ci_8134 & $268 \mathrm{~T} / \mathrm{C}$ \\
\hline UN_ci_8134 & $309 \mathrm{~T} / \mathrm{C}$ \\
\hline UN_ci_8134 & $621 \mathrm{~T} / \mathrm{C}$ \\
\hline UN_ci_8134 & $652 \mathrm{C} / \mathrm{T}$ \\
\hline UN_ci_8134 & $696 \mathrm{~T} / \mathrm{G}$ \\
\hline UN_ci_8134 & $724 \mathrm{~T} / \mathrm{G}$ \\
\hline UN_ci_8136 & $495 \mathrm{C} / \mathrm{T}$ \\
\hline UN_ci_8136 & $580 \mathrm{C} / \mathrm{G}$ \\
\hline UN_ci_8138 & 369 A/G \\
\hline UN_ci_8139 & $145 \mathrm{~A} / \mathrm{T}$ \\
\hline UN_ci_8139 & $233 \mathrm{~T} / \mathrm{C}$ \\
\hline UN_ci_8139 & $310 \mathrm{~T} / \mathrm{A}$ \\
\hline UN_ci_8139 & $446 \mathrm{~T} / \mathrm{C}$ \\
\hline UN_ci_8139 & $470 \mathrm{G} / \mathrm{A}$ \\
\hline UN_ci_8139 & $648 \mathrm{~A} / \mathrm{C}$ \\
\hline UN_ci_8139 & $674 \mathrm{C} / \mathrm{T}$ \\
\hline UN_ci_8139 & $695 \mathrm{~A} / \mathrm{G}$ \\
\hline UN_ci_8139 & $718 \mathrm{G} / \mathrm{A}$ \\
\hline UN_ci_8139 & $741 \mathrm{G} / \mathrm{A}$ \\
\hline UN_ci_8139 & $767 \mathrm{~T} / \mathrm{A}$ \\
\hline UN_ci_8139 & $787 \mathrm{C} / \mathrm{T}$ \\
\hline UN_ci_8139 & $809 \mathrm{~T} / \mathrm{C}$ \\
\hline UN_ci_8139 & $831 \mathrm{C} / \mathrm{A}$ \\
\hline UN_ci_8139 & $854 \mathrm{~A} / \mathrm{G}$ \\
\hline UN_ci_8139 & $877 \mathrm{~T} / \mathrm{C}$ \\
\hline UN_ci_8139 & $898 \mathrm{G} / \mathrm{A}$ \\
\hline UN_ci_8139 & $920 \mathrm{G} / \mathrm{A}$ \\
\hline UN_ci_8139 & $940 \mathrm{C} / \mathrm{T}$ \\
\hline UN_ci_8139 & $965 \mathrm{~T} / \mathrm{C}$ \\
\hline UN_ci_8139 & $991 \mathrm{~T} / \mathrm{C}$ \\
\hline UN_ci_8139 & $1014 \mathrm{~T} / \mathrm{C}$ \\
\hline UN_ci_8139 & $1035 \mathrm{G} / \mathrm{C}$ \\
\hline UN_ci_8139 & $1095 \mathrm{G} / \mathrm{T}$ \\
\hline UN_ci_8139 & $1182 \mathrm{G} / \mathrm{A}$ \\
\hline UN_ci_8139 & $1217 \mathrm{~A} / \mathrm{G}$ \\
\hline UN_ci_8139 & $1249 \mathrm{C} / \mathrm{T}$ \\
\hline UN_ci_8139 & $1344 \mathrm{~T} / \mathrm{A}$ \\
\hline UN_ci_8139 & $1383 \mathrm{~A} / \mathrm{G}$ \\
\hline UN_ci_8139 & $1448 \mathrm{C} / \mathrm{A}$ \\
\hline UN_ci_8139 & $1528 \mathrm{~A} / \mathrm{G}$ \\
\hline UN_ci_8140 & $71 \mathrm{~A} / \mathrm{C}$ \\
\hline UN_ci_8140 & $141 \mathrm{~T} / \mathrm{C}$ \\
\hline UN_ci_8140 & $204 \mathrm{C} / \mathrm{T}$ \\
\hline UN_ci_8140 & $267 \mathrm{C} / \mathrm{A}$ \\
\hline UN_ci_8140 & $330 \mathrm{~T} / \mathrm{C}$ \\
\hline UN_ci_8140 & $443 \mathrm{C} / \mathrm{T}$ \\
\hline UN_ci_8140 & $502 \mathrm{C} / \mathrm{T}$ \\
\hline UN_ci_8140 & $604 \mathrm{~T} / \mathrm{C}$ \\
\hline
\end{tabular}




$\begin{array}{lr}\text { UN_ci_8140 } & 640 \mathrm{~A} / \mathrm{G} \\ \text { UN_ci_8140 } & 932 \mathrm{~A} / \mathrm{G} \\ \text { UN_ci_8140 } & 1026 \mathrm{C} / \mathrm{T} \\ \text { UN_ci_8141 } & 553 \mathrm{~T} / \mathrm{G} \\ \text { UN_ci_8141 } & 1223 \mathrm{G} / \mathrm{T} \\ \text { UN_ci_8142 } & 496 \mathrm{~A} / \mathrm{G} \\ \text { UN_ci_8142 } & 531 \mathrm{~A} / \mathrm{G} \\ \text { UN_ci_8142 } & 648 \mathrm{~A} / \mathrm{G} \\ \text { UN_ci_8142 } & 719 \mathrm{~A} / \mathrm{G} \\ \text { UN_ci_8143 } & 261 \mathrm{G} / \mathrm{A} \\ \text { UN_ci_8143 } & 404 \mathrm{~A} / \mathrm{G} \\ \text { UN_ci_8143 } & 680 \mathrm{~A} / \mathrm{G} \\ \text { UN_ci_8143 } & 1219 \mathrm{~T} / \mathrm{C} \\ \text { UN_ci_8145 } & 88 \mathrm{C} / \mathrm{T} \\ \text { UN_ci_8145 } & 110 \mathrm{~A} / \mathrm{T} \\ \text { UN_ci_8145 } & 287 \mathrm{~A} / \mathrm{C} \\ \text { UN_ci_8147 } & 233 \mathrm{~T} / \mathrm{C} \\ \text { UN_ci_8147 } & 362 \mathrm{~T} / \mathrm{C} \\ \text { UN_ci_8147 } & 1273 \mathrm{~T} / \mathrm{A} \\ \text { UN_ci_8147 } & 1347 \mathrm{~T} / \mathrm{G} \\ \text { UN_ci_8147 } & 511 \mathrm{~T} / \mathrm{C} \\ \text { UN_ci_8147 } & \end{array}$




\begin{tabular}{|c|c|}
\hline UN_ci_8162 & $962 \mathrm{~T} / \mathrm{C}$ \\
\hline UN_ci_8162 & $1105 \mathrm{~T} / \mathrm{C}$ \\
\hline UN_ci_8162 & 1196 C/G \\
\hline UN_ci_8163 & $64 \mathrm{G} / \mathrm{C}$ \\
\hline UN_ci_8163 & $199 \mathrm{G} / \mathrm{T}$ \\
\hline UN_ci_8163 & $307 \mathrm{~T} / \mathrm{C}$ \\
\hline UN_ci_8163 & $829 \mathrm{G} / \mathrm{A}$ \\
\hline UN_ci_8163 & $983 \mathrm{~A} / \mathrm{G}$ \\
\hline UN_ci_8163 & $1123 \mathrm{~A} / \mathrm{G}$ \\
\hline UN_ci_8163 & $1212 \mathrm{~A} / \mathrm{G}$ \\
\hline UN_ci_8163 & $1371 \mathrm{~T} / \mathrm{C}$ \\
\hline UN_ci_8163 & $1538 \mathrm{G} / \mathrm{A}$ \\
\hline UN_ci_8163 & $1603 \mathrm{G} / \mathrm{T}$ \\
\hline UN_ci_8163 & $1716 \mathrm{~A} / \mathrm{G}$ \\
\hline UN_ci_8164 & $76 \mathrm{~A} / \mathrm{G}$ \\
\hline UN_ci_8164 & $139 \mathrm{G} / \mathrm{T}$ \\
\hline UN_ci_8164 & $791 \mathrm{~A} / \mathrm{T}$ \\
\hline UN_ci_8165 & $288 \mathrm{C} / \mathrm{T}$ \\
\hline UN_ci_8165 & $550 \mathrm{~T} / \mathrm{A}$ \\
\hline UN_ci_8166 & $274 \mathrm{G} / \mathrm{C}$ \\
\hline UN_ci_8166 & $688 \mathrm{~A} / \mathrm{C}$ \\
\hline UN_ci_8167 & $148 \mathrm{~A} / \mathrm{G}$ \\
\hline UN_ci_8168 & $186 \mathrm{~A} / \mathrm{G}$ \\
\hline UN_ci_8168 & $234 \mathrm{C} / \mathrm{G}$ \\
\hline UN_ci_8168 & $1053 \mathrm{~A} / \mathrm{G}$ \\
\hline UN_ci_8168 & $1148 \mathrm{C} / \mathrm{T}$ \\
\hline UN_ci_8168 & 1219 T/C \\
\hline UN_ci_8168 & $1499 \mathrm{G} / \mathrm{A}$ \\
\hline UN_ci_8171 & $37 \mathrm{~A} / \mathrm{G}$ \\
\hline UN_ci_8171 & $71 \mathrm{~A} / \mathrm{G}$ \\
\hline UN_ci_8171 & $108 \mathrm{~T} / \mathrm{A}$ \\
\hline UN_ci_8171 & $195 \mathrm{C} / \mathrm{T}$ \\
\hline UN_ci_8171 & $225 \mathrm{~A} / \mathrm{G}$ \\
\hline UN_ci_8171 & $246 \mathrm{~T} / \mathrm{C}$ \\
\hline UN_ci_8171 & $278 \mathrm{~A} / \mathrm{G}$ \\
\hline UN_ci_8171 & $333 \mathrm{~T} / \mathrm{C}$ \\
\hline UN_ci_8171 & $364 \mathrm{~A} / \mathrm{C}$ \\
\hline UN_ci_8171 & 394 G/A \\
\hline UN_ci_8171 & $444 \mathrm{~T} / \mathrm{C}$ \\
\hline UN_ci_8171 & $473 \mathrm{~T} / \mathrm{C}$ \\
\hline UN_ci_8171 & $503 \mathrm{~A} / \mathrm{G}$ \\
\hline UN_ci_8171 & $526 \mathrm{~A} / \mathrm{G}$ \\
\hline UN_ci_8171 & $547 \mathrm{~A} / \mathrm{G}$ \\
\hline UN_ci_8171 & $594 \mathrm{G} / \mathrm{A}$ \\
\hline UN_ci_8171 & $618 \mathrm{~A} / \mathrm{G}$ \\
\hline UN_ci_8171 & $656 \mathrm{~T} / \mathrm{C}$ \\
\hline UN_ci_8171 & $710 \mathrm{~T} / \mathrm{C}$ \\
\hline UN_ci_8171 & $740 \mathrm{~T} / \mathrm{G}$ \\
\hline UN_ci_8171 & $765 \mathrm{~T} / \mathrm{C}$ \\
\hline UN_ci_8171 & $795 \mathrm{~T} / \mathrm{C}$ \\
\hline
\end{tabular}




\begin{tabular}{|c|c|}
\hline UN_ci_8171 & $829 \mathrm{C} / \mathrm{A}$ \\
\hline UN_ci_8173 & $506 \mathrm{~T} / \mathrm{C}$ \\
\hline UN_ci_8173 & $549 \mathrm{C} / \mathrm{T}$ \\
\hline UN_ci_8174 & $231 \mathrm{~T} / \mathrm{C}$ \\
\hline UN_ci_8174 & $260 \mathrm{~A} / \mathrm{G}$ \\
\hline UN_ci_8174 & 315 G/C \\
\hline UN_ci_8174 & $369 \mathrm{~A} / \mathrm{C}$ \\
\hline UN_ci_8174 & $513 \mathrm{~T} / \mathrm{C}$ \\
\hline UN_ci_8175 & $195 \mathrm{~A} / \mathrm{G}$ \\
\hline UN_ci_8175 & $267 \mathrm{G} / \mathrm{A}$ \\
\hline UN_ci_8175 & $317 \mathrm{C} / \mathrm{G}$ \\
\hline UN_ci_8175 & $405 \mathrm{~T} / \mathrm{C}$ \\
\hline UN_ci_8175 & $567 \mathrm{~A} / \mathrm{G}$ \\
\hline UN_ci_8175 & $654 \mathrm{~T} / \mathrm{C}$ \\
\hline UN_ci_8175 & $762 \mathrm{~A} / \mathrm{G}$ \\
\hline UN_ci_8176 & $504 \mathrm{~A} / \mathrm{T}$ \\
\hline UN_ci_8176 & $531 \mathrm{~T} / \mathrm{C}$ \\
\hline UN_ci_8176 & $1013 \mathrm{~A} / \mathrm{T}$ \\
\hline UN_ci_8178 & $127 \mathrm{~A} / \mathrm{G}$ \\
\hline UN_ci_8178 & $488 \mathrm{~A} / \mathrm{G}$ \\
\hline UN_ci_8181 & $213 \mathrm{G} / \mathrm{T}$ \\
\hline UN_ci_8181 & $237 \mathrm{~A} / \mathrm{G}$ \\
\hline UN_ci_8181 & $370 \mathrm{~A} / \mathrm{G}$ \\
\hline UN_ci_8181 & $396 \mathrm{~A} / \mathrm{C}$ \\
\hline UN_ci_8181 & $514 \mathrm{~T} / \mathrm{C}$ \\
\hline UN_ci_8181 & $583 \mathrm{C} / \mathrm{A}$ \\
\hline UN_ci_8181 & 798 T/G \\
\hline UN_ci_8181 & $1024 \mathrm{~A} / \mathrm{G}$ \\
\hline UN_ci_8182 & $979 \mathrm{C} / \mathrm{T}$ \\
\hline UN_ci_8184 & 766 G/T \\
\hline UN_ci_8185 & $233 \mathrm{G} / \mathrm{C}$ \\
\hline UN_ci_8185 & $342 \mathrm{~A} / \mathrm{G}$ \\
\hline UN_ci_8185 & $790 \mathrm{C} / \mathrm{T}$ \\
\hline UN_ci_8186 & $260 \mathrm{~A} / \mathrm{T}$ \\
\hline UN_ci_8187 & $181 \mathrm{~A} / \mathrm{T}$ \\
\hline UN_ci_8189 & $252 \mathrm{~T} / \mathrm{C}$ \\
\hline UN_ci_8189 & 299 C/A \\
\hline UN_ci_8189 & $420 \mathrm{~T} / \mathrm{G}$ \\
\hline UN_ci_8189 & $442 \mathrm{~T} / \mathrm{C}$ \\
\hline UN_ci_8189 & $552 \mathrm{C} / \mathrm{T}$ \\
\hline UN_ci_8189 & $575 \mathrm{~T} / \mathrm{C}$ \\
\hline UN_ci_8190 & $528 \mathrm{~A} / \mathrm{G}$ \\
\hline UN_ci_8191 & $189 \mathrm{C} / \mathrm{A}$ \\
\hline UN_ci_8191 & $343 \mathrm{G} / \mathrm{A}$ \\
\hline UN_ci_8193 & $193 \mathrm{~T} / \mathrm{C}$ \\
\hline UN_ci_8193 & $523 \mathrm{C} / \mathrm{G}$ \\
\hline UN_ci_8193 & $604 \mathrm{~A} / \mathrm{G}$ \\
\hline UN_ci_8197 & $545 \mathrm{C} / \mathrm{T}$ \\
\hline UN_ci_8198 & $167 \mathrm{~A} / \mathrm{C}$ \\
\hline UN_ci_8199 & $136 \mathrm{~T} / \mathrm{G}$ \\
\hline
\end{tabular}




\begin{tabular}{|c|c|}
\hline UN_ci_8200 & $265 \mathrm{~T} / \mathrm{C}$ \\
\hline UN_ci_8200 & $454 \mathrm{~A} / \mathrm{C}$ \\
\hline UN_ci_8200 & $654 \mathrm{G} / \mathrm{T}$ \\
\hline UN_ci_8200 & $777 \mathrm{~T} / \mathrm{C}$ \\
\hline UN_ci_8200 & $802 \mathrm{~T} / \mathrm{C}$ \\
\hline UN_ci_8201 & $623 \mathrm{~T} / \mathrm{C}$ \\
\hline UN_ci_8201 & $655 \mathrm{~A} / \mathrm{G}$ \\
\hline UN_ci_8201 & $681 \mathrm{G} / \mathrm{A}$ \\
\hline UN_ci_8201 & $702 \mathrm{G} / \mathrm{C}$ \\
\hline UN_ci_8201 & $724 \mathrm{~A} / \mathrm{G}$ \\
\hline UN_ci_8201 & $749 \mathrm{C} / \mathrm{T}$ \\
\hline UN_ci_8201 & $770 \mathrm{~A} / \mathrm{T}$ \\
\hline UN_ci_8201 & $790 \mathrm{~A} / \mathrm{G}$ \\
\hline UN_ci_8201 & $810 \mathrm{C} / \mathrm{G}$ \\
\hline UN_ci_8201 & $830 \mathrm{G} / \mathrm{A}$ \\
\hline UN_ci_8201 & $850 \mathrm{~A} / \mathrm{G}$ \\
\hline UN_ci_8201 & $878 \mathrm{G} / \mathrm{T}$ \\
\hline UN_ci_8201 & $923 \mathrm{~T} / \mathrm{C}$ \\
\hline UN_ci_8201 & $947 \mathrm{~A} / \mathrm{G}$ \\
\hline UN_ci_8201 & $975 \mathrm{~A} / \mathrm{G}$ \\
\hline UN_ci_8203 & $175 \mathrm{~T} / \mathrm{A}$ \\
\hline UN_ci_8203 & $224 \mathrm{C} / \mathrm{T}$ \\
\hline UN_ci_8203 & $311 \mathrm{~T} / \mathrm{A}$ \\
\hline UN_ci_8203 & $404 \mathrm{~T} / \mathrm{C}$ \\
\hline UN_ci_8204 & $95 \mathrm{G} / \mathrm{C}$ \\
\hline UN_ci_8205 & $539 \mathrm{~A} / \mathrm{G}$ \\
\hline UN_ci_8205 & $634 \mathrm{~T} / \mathrm{C}$ \\
\hline UN_ci_8205 & $673 \mathrm{C} / \mathrm{A}$ \\
\hline UN_ci_8205 & $794 \mathrm{G} / \mathrm{A}$ \\
\hline UN_ci_8205 & $1241 \mathrm{~A} / \mathrm{G}$ \\
\hline UN_ci_8205 & 1413 T/C \\
\hline UN_ci_8205 & $1637 \mathrm{G} / \mathrm{A}$ \\
\hline UN_ci_8205 & $1766 \mathrm{~T} / \mathrm{G}$ \\
\hline UN_ci_8206 & $262 \mathrm{G} / \mathrm{A}$ \\
\hline UN_ci_8206 & $359 \mathrm{G} / \mathrm{A}$ \\
\hline UN_ci_8206 & $457 \mathrm{G} / \mathrm{A}$ \\
\hline UN_ci_8206 & $557 \mathrm{~A} / \mathrm{G}$ \\
\hline UN_ci_8206 & $703 \mathrm{~T} / \mathrm{C}$ \\
\hline UN_ci_8206 & $760 \mathrm{~A} / \mathrm{C}$ \\
\hline UN_ci_8207 & $47 \mathrm{~A} / \mathrm{C}$ \\
\hline UN_ci_8207 & $272 \mathrm{~A} / \mathrm{G}$ \\
\hline UN_ci_8207 & $520 \mathrm{G} / \mathrm{A}$ \\
\hline UN_ci_8207 & $696 \mathrm{G} / \mathrm{A}$ \\
\hline UN_ci_8207 & $806 \mathrm{~T} / \mathrm{C}$ \\
\hline UN_ci_8212 & $613 \mathrm{~A} / \mathrm{G}$ \\
\hline UN_ci_8212 & $757 \mathrm{G} / \mathrm{T}$ \\
\hline UN_ci_8212 & $787 \mathrm{~T} / \mathrm{G}$ \\
\hline UN_ci_8212 & $1124 \mathrm{~T} / \mathrm{C}$ \\
\hline UN_ci_8212 & $1145 \mathrm{G} / \mathrm{A}$ \\
\hline UN_ci_8212 & $1168 \mathrm{~A} / \mathrm{G}$ \\
\hline
\end{tabular}




\begin{tabular}{|c|c|}
\hline UN_ci_8212 & $1189 \mathrm{~A} / \mathrm{G}$ \\
\hline UN_ci_8212 & $1210 \mathrm{G} / \mathrm{A}$ \\
\hline UN_ci_8212 & $1233 \mathrm{~T} / \mathrm{C}$ \\
\hline UN_ci_8212 & $1254 \mathrm{~T} / \mathrm{G}$ \\
\hline UN_ci_8212 & $1274 \mathrm{~A} / \mathrm{G}$ \\
\hline UN_ci_8212 & $1294 \mathrm{~A} / \mathrm{G}$ \\
\hline UN_ci_8212 & $1314 \mathrm{~A} / \mathrm{G}$ \\
\hline UN_ci_8212 & $1334 \mathrm{~T} / \mathrm{C}$ \\
\hline UN_ci_8212 & $1354 \mathrm{~T} / \mathrm{C}$ \\
\hline UN_ci_8212 & $1374 \mathrm{~A} / \mathrm{G}$ \\
\hline UN_ci_8212 & $1394 \mathrm{~A} / \mathrm{G}$ \\
\hline UN_ci_8212 & $1414 \mathrm{~A} / \mathrm{G}$ \\
\hline UN_ci_8212 & $1435 \mathrm{~T} / \mathrm{C}$ \\
\hline UN_ci_8212 & $1464 \mathrm{~A} / \mathrm{G}$ \\
\hline UN_ci_8212 & $1485 \mathrm{~A} / \mathrm{G}$ \\
\hline UN_ci_8212 & $1524 \mathrm{C} / \mathrm{A}$ \\
\hline UN_ci_8212 & $1554 \mathrm{C} / \mathrm{T}$ \\
\hline UN_ci_8212 & $1608 \mathrm{G} / \mathrm{A}$ \\
\hline UN_ci_8213 & $278 \mathrm{C} / \mathrm{T}$ \\
\hline UN_ci_8213 & $723 \mathrm{~A} / \mathrm{C}$ \\
\hline UN_ci_8216 & 209 C/G \\
\hline UN_ci_8216 & $1381 \mathrm{~T} / \mathrm{C}$ \\
\hline UN_ci_8216 & $1425 \mathrm{~A} / \mathrm{C}$ \\
\hline UN_ci_8217 & $331 \mathrm{C} / \mathrm{T}$ \\
\hline UN_ci_8218 & $106 \mathrm{~A} / \mathrm{G}$ \\
\hline UN_ci_8218 & $143 \mathrm{~A} / \mathrm{G}$ \\
\hline UN_ci_8218 & $327 \mathrm{G} / \mathrm{A}$ \\
\hline UN_ci_8218 & $348 \mathrm{C} / \mathrm{T}$ \\
\hline UN_ci_8218 & $580 \mathrm{G} / \mathrm{A}$ \\
\hline UN_ci_8220 & $274 \mathrm{~A} / \mathrm{T}$ \\
\hline UN_ci_8221 & $114 \mathrm{~T} / \mathrm{G}$ \\
\hline UN_ci_8221 & $194 \mathrm{~A} / \mathrm{G}$ \\
\hline UN_ci_8221 & $331 \mathrm{G} / \mathrm{A}$ \\
\hline UN_ci_8221 & $477 \mathrm{~T} / \mathrm{C}$ \\
\hline UN_ci_8221 & $500 \mathrm{G} / \mathrm{A}$ \\
\hline UN_ci_8221 & $620 \mathrm{~T} / \mathrm{A}$ \\
\hline UN_ci_8221 & 658 A/G \\
\hline UN_ci_8221 & 695 T/C \\
\hline UN_ci_8221 & $870 \mathrm{~A} / \mathrm{G}$ \\
\hline UN_ci_8221 & 1109 T/C \\
\hline UN_ci_8221 & $1233 \mathrm{~T} / \mathrm{C}$ \\
\hline UN_ci_8221 & $1257 \mathrm{C} / \mathrm{T}$ \\
\hline UN_ci_8225 & $385 \mathrm{C} / \mathrm{G}$ \\
\hline UN_ci_8225 & $980 \mathrm{C} / \mathrm{T}$ \\
\hline UN_ci_8225 & $1022 \mathrm{~A} / \mathrm{G}$ \\
\hline UN_ci_8225 & 1076 T/C \\
\hline UN_ci_8225 & $1114 \mathrm{C} / \mathrm{A}$ \\
\hline UN_ci_8225 & $1161 \mathrm{~A} / \mathrm{G}$ \\
\hline UN_ci_8225 & $1203 \mathrm{~A} / \mathrm{G}$ \\
\hline UN_ci_8225 & $1228 \mathrm{~A} / \mathrm{G}$ \\
\hline
\end{tabular}




$\begin{array}{lr}\text { UN_ci_8225 } & 1278 \mathrm{~A} / \mathrm{G} \\ \text { UN_ci_8225 } & 1344 \mathrm{G} / \mathrm{A} \\ \text { UN_ci_8225 } & 1441 \mathrm{~A} / \mathrm{G} \\ \text { UN_ci_8230 } & 711 \mathrm{~A} / \mathrm{G} \\ \text { UN_ci_8232 } & 201 \mathrm{~T} / \mathrm{C} \\ \text { UN_ci_8232 } & 580 \mathrm{G} / \mathrm{A} \\ \text { UN_ci_8233 } & 301 \mathrm{G} / \mathrm{C} \\ \text { UN_ci_8233 } & 322 \mathrm{C} / \mathrm{T} \\ \text { UN_ci_8233 } & 352 \mathrm{C} / \mathrm{T} \\ \text { UN_ci_8233 } & 528 \mathrm{C} / \mathrm{G} \\ \text { UN_ci_8233 } & 839 \mathrm{~A} / \mathrm{G} \\ \text { UN_ci_8233 } & 1093 \mathrm{C} / \mathrm{T} \\ \text { UN_ci_8233 } & 1420 \mathrm{~A} / \mathrm{G} \\ \text { UN_ci_8233 } & 1532 \mathrm{G} / \mathrm{A} \\ \text { UN_ci_8233 } & 1842 \mathrm{C} / \mathrm{G} \\ \text { UN_ci_8236 } & 28 \mathrm{~A} / \mathrm{C} \\ \text { UN_ci_8236 } & 94 \mathrm{~A} / \mathrm{G} \\ \text { UN_ci_8236 } & 122 \mathrm{C} / \mathrm{T} \\ \text { UN_ci_8236 } & 599 \mathrm{C} / \mathrm{T} \\ \text { UN_ci_8236 } & 755 \mathrm{C} / \mathrm{T} \\ \text { UN_ci_8236 } & 780 \mathrm{C} / \mathrm{T} \\ \text { UN_ci_8236 } & 55 \mathrm{C} / \mathrm{T} \\ \text { UN_ci_8236 } & 202 \mathrm{~A} / \mathrm{G} \\ \text { UN_ci_8236 } & 377 \mathrm{C} / \mathrm{T} \\ \text { UN_ci_8236 } & 407 \mathrm{C} / \mathrm{T} \\ \text { UN_ci_8237 } & 54 \mathrm{~A} / \mathrm{T} \\ \text { UN_ci_8237 } & \end{array}$




$\begin{array}{lr}\text { UN_ci_8250 } & 163 \mathrm{C} / \mathrm{T} \\ \text { UN_ci_8250 } & 313 \mathrm{~T} / \mathrm{A} \\ \text { UN_ci_8250 } & 786 \mathrm{~T} / \mathrm{G} \\ \text { UN_ci_8250 } & 1021 \mathrm{~T} / \mathrm{C} \\ \text { UN_ci_8250 } & 1327 \mathrm{C} / \mathrm{T} \\ \text { UN_ci_8251 } & 8 \mathrm{~A} / \mathrm{G} \\ \text { UN_ci_8251 } & 49 \mathrm{C} / \mathrm{T} \\ \text { UN_ci_8251 } & 148 \mathrm{C} / \mathrm{T} \\ \text { UN_ci_8251 } & 214 \mathrm{~T} / \mathrm{C} \\ \text { UN_ci_8251 } & 247 \mathrm{C} / \mathrm{A} \\ \text { UN_ci_8251 } & 435 \mathrm{~A} / \mathrm{T} \\ \text { UN_ci_8251 } & 614 \mathrm{~A} / \mathrm{G} \\ \text { UN_ci_8251 } & 636 \mathrm{~T} / \mathrm{C} \\ \text { UN_ci_8251 } & 691 \mathrm{~T} / \mathrm{C} \\ \text { UN_ci_8251 } & 758 \mathrm{C} / \mathrm{G} \\ \text { UN_ci_8254 } & 60 \mathrm{~T} / \mathrm{A} \\ \text { UN_ci_8255 } & 315 \mathrm{C} / \mathrm{T} \\ \text { UN_ci_8256 } & 332 \mathrm{C} / \mathrm{T} \\ \text { UN_ci_8256 } & 1081 \mathrm{~A} / \mathrm{G} \\ \text { UN_ci_8256 } & 1127 \mathrm{~A} / \mathrm{C} \\ \text { UN_ci_8256 } & 1177 \mathrm{C} / \mathrm{T} \\ \text { UN_ci_8256 } & 1125 \mathrm{C} / \mathrm{T} \\ \text { UN_ci_8256 } & \end{array}$




\begin{tabular}{|c|c|}
\hline UN_ci_8265 & 1182 G/C \\
\hline UN_ci_8266 & $402 \mathrm{~A} / \mathrm{G}$ \\
\hline UN_ci_8267 & $65 \mathrm{~T} / \mathrm{G}$ \\
\hline UN_ci_8267 & $141 \mathrm{G} / \mathrm{A}$ \\
\hline UN_ci_8267 & $257 \mathrm{~A} / \mathrm{G}$ \\
\hline UN_ci_8267 & 389 G/T \\
\hline UN_ci_8267 & $724 \mathrm{~A} / \mathrm{G}$ \\
\hline UN_ci_8267 & $801 \mathrm{~A} / \mathrm{T}$ \\
\hline UN_ci_8268 & $278 \mathrm{~T} / \mathrm{C}$ \\
\hline UN_ci_8268 & $360 \mathrm{~A} / \mathrm{T}$ \\
\hline UN_ci_8268 & $958 \mathrm{~A} / \mathrm{G}$ \\
\hline UN_ci_8269 & $549 \mathrm{~T} / \mathrm{C}$ \\
\hline UN_ci_8270 & $636 \mathrm{~T} / \mathrm{C}$ \\
\hline UN_ci_8270 & $962 \mathrm{~A} / \mathrm{G}$ \\
\hline UN_ci_8270 & $1521 \mathrm{~A} / \mathrm{G}$ \\
\hline UN_ci_8270 & $1692 \mathrm{G} / \mathrm{T}$ \\
\hline UN_ci_8272 & $1558 \mathrm{~A} / \mathrm{G}$ \\
\hline UN_ci_8273 & 292 T/C \\
\hline UN_ci_8274 & $695 \mathrm{G} / \mathrm{T}$ \\
\hline UN_ci_8274 & $826 \mathrm{~T} / \mathrm{C}$ \\
\hline UN_ci_8275 & $467 \mathrm{~A} / \mathrm{G}$ \\
\hline UN_ci_8275 & $951 \mathrm{G} / \mathrm{A}$ \\
\hline UN_ci_8275 & 974 G/C \\
\hline UN_ci_8275 & $1157 \mathrm{~T} / \mathrm{C}$ \\
\hline UN_ci_8275 & $1198 \mathrm{~T} / \mathrm{A}$ \\
\hline UN_ci_8275 & 1294 T/C \\
\hline UN_ci_8276 & 119 T/C \\
\hline UN_ci_8276 & $259 \mathrm{G} / \mathrm{C}$ \\
\hline UN_ci_8278 & $238 \mathrm{~T} / \mathrm{G}$ \\
\hline UN_ci_8279 & 869 A/G \\
\hline UN_ci_8279 & 929 A/G \\
\hline UN_ci_8279 & $984 \mathrm{G} / \mathrm{T}$ \\
\hline UN_ci_8279 & $1101 \mathrm{C} / \mathrm{T}$ \\
\hline UN_ci_8279 & $1188 \mathrm{~A} / \mathrm{G}$ \\
\hline UN_ci_8279 & $1228 \mathrm{~A} / \mathrm{T}$ \\
\hline UN_ci_8279 & 1315 T/C \\
\hline UN_ci_8279 & $1377 \mathrm{C} / \mathrm{T}$ \\
\hline UN_ci_8279 & $1581 \mathrm{~A} / \mathrm{G}$ \\
\hline UN_ci_8279 & $1651 \mathrm{~A} / \mathrm{G}$ \\
\hline UN_ci_8279 & $1722 \mathrm{C} / \mathrm{T}$ \\
\hline UN_ci_8281 & $121 \mathrm{G} / \mathrm{T}$ \\
\hline UN_ci_8281 & $153 \mathrm{~A} / \mathrm{C}$ \\
\hline UN_ci_8281 & $409 \mathrm{~A} / \mathrm{G}$ \\
\hline UN_ci_8282 & 1149 A/G \\
\hline UN_ci_8283 & 107 G/T \\
\hline UN_ci_8283 & $274 \mathrm{C} / \mathrm{T}$ \\
\hline UN_ci_8283 & $454 \mathrm{~T} / \mathrm{C}$ \\
\hline UN_ci_8283 & $618 \mathrm{G} / \mathrm{C}$ \\
\hline UN_ci_8283 & $747 \mathrm{~T} / \mathrm{C}$ \\
\hline UN_ci_8283 & $1047 \mathrm{C} / \mathrm{G}$ \\
\hline
\end{tabular}




$\begin{array}{lr}\text { UN_ci_8283 } & 1208 \mathrm{G} / \mathrm{A} \\ \text { UN_ci_8283 } & 1305 \mathrm{~T} / \mathrm{C} \\ \text { UN_ci_8283 } & 1429 \mathrm{G} / \mathrm{A} \\ \text { UN_ci_8283 } & 1465 \mathrm{C} / \mathrm{T} \\ \text { UN_ci_8284 } & 131 \mathrm{~A} / \mathrm{T} \\ \text { UN_ci_8284 } & 175 \mathrm{~A} / \mathrm{G} \\ \text { UN_ci_8286 } & 90 \mathrm{G} / \mathrm{A} \\ \text { UN_ci_8287 } & 477 \mathrm{~A} / \mathrm{T} \\ \text { UN_ci_8287 } & 989 \mathrm{~T} / \mathrm{A} \\ \text { UN_ci_8288 } & 212 \mathrm{G} / \mathrm{T} \\ \text { UN_ci_8288 } & 260 \mathrm{~T} / \mathrm{A} \\ \text { UN_ci_8288 } & 291 \mathrm{~T} / \mathrm{C} \\ \text { UN_ci_8288 } & 320 \mathrm{~A} / \mathrm{G} \\ \text { UN_ci_8288 } & 409 \mathrm{C} / \mathrm{T} \\ \text { UN_ci_8288 } & 437 \mathrm{~A} / \mathrm{G} \\ \text { UN_ci_8288 } & 457 \mathrm{G} / \mathrm{A} \\ \text { UN_ci_8288 } & 478 \mathrm{C} / \mathrm{T} \\ \text { UN_ci_8288 } & 498 \mathrm{~A} / \mathrm{G} \\ \text { UN_ci_8288 } & 521 \mathrm{~T} / \mathrm{C} \\ \text { UN_ci_8288 } & 566 \mathrm{~T} / \mathrm{C} \\ \text { UN_ci_8288 } & 587 \mathrm{~A} / \mathrm{G} \\ \text { UN_ci_8288 } & 7924 \mathrm{~A} / \mathrm{G} \\ \text { UN_ci_8288 } & 569 \mathrm{~T}\end{array}$




\begin{tabular}{|c|c|}
\hline UN_ci_8299 & $1071 \mathrm{G} / \mathrm{A}$ \\
\hline UN_ci_8299 & $1100 \mathrm{~T} / \mathrm{C}$ \\
\hline UN_ci_8299 & $1142 \mathrm{~T} / \mathrm{C}$ \\
\hline UN_ci_8299 & $1267 \mathrm{~T} / \mathrm{C}$ \\
\hline UN_ci_8299 & 1308 T/G \\
\hline UN_ci_8299 & $1344 \mathrm{~T} / \mathrm{C}$ \\
\hline UN_ci_8299 & $1367 \mathrm{G} / \mathrm{T}$ \\
\hline UN_ci_8301 & $118 \mathrm{~A} / \mathrm{T}$ \\
\hline UN_ci_8302 & $104 \mathrm{G} / \mathrm{A}$ \\
\hline UN_ci_8302 & $709 \mathrm{G} / \mathrm{A}$ \\
\hline UN_ci_8302 & 1162 T/C \\
\hline UN_ci_8302 & $1461 \mathrm{~A} / \mathrm{T}$ \\
\hline UN_ci_8302 & $1728 \mathrm{C} / \mathrm{G}$ \\
\hline UN_ci_8303 & $315 \mathrm{~A} / \mathrm{C}$ \\
\hline UN_ci_8303 & $510 \mathrm{C} / \mathrm{A}$ \\
\hline UN_ci_8303 & $586 \mathrm{~T} / \mathrm{C}$ \\
\hline UN_ci_8303 & 761 C/G \\
\hline UN_ci_8303 & 796 G/T \\
\hline UN_ci_8305 & $264 \mathrm{~T} / \mathrm{C}$ \\
\hline UN_ci_8308 & $105 \mathrm{C} / \mathrm{T}$ \\
\hline UN_ci_8308 & $342 \mathrm{C} / \mathrm{T}$ \\
\hline UN_ci_8308 & 379 C/T \\
\hline UN_ci_8308 & $550 \mathrm{G} / \mathrm{A}$ \\
\hline UN_ci_8309 & $26 \mathrm{G} / \mathrm{A}$ \\
\hline UN_ci_8309 & $58 \mathrm{~A} / \mathrm{G}$ \\
\hline UN_ci_8309 & $87 \mathrm{~T} / \mathrm{C}$ \\
\hline UN_ci_8309 & $488 \mathrm{~A} / \mathrm{G}$ \\
\hline UN_ci_8309 & $638 \mathrm{C} / \mathrm{T}$ \\
\hline UN_ci_8309 & $681 \mathrm{G} / \mathrm{C}$ \\
\hline UN_ci_8309 & $780 \mathrm{~A} / \mathrm{G}$ \\
\hline UN_ci_8309 & $852 \mathrm{~A} / \mathrm{C}$ \\
\hline UN_ci_8309 & $996 \mathrm{C} / \mathrm{T}$ \\
\hline UN_ci_8309 & $1054 \mathrm{~T} / \mathrm{C}$ \\
\hline UN_ci_8309 & $1187 \mathrm{~T} / \mathrm{C}$ \\
\hline UN_ci_8309 & $1242 \mathrm{~A} / \mathrm{C}$ \\
\hline UN_ci_8309 & $1269 \mathrm{C} / \mathrm{T}$ \\
\hline UN_ci_8309 & 1503 T/C \\
\hline UN_ci_8310 & $7 \mathrm{~A} / \mathrm{T}$ \\
\hline UN_ci_8310 & $42 \mathrm{G} / \mathrm{C}$ \\
\hline UN_ci_8310 & $84 \mathrm{C} / \mathrm{T}$ \\
\hline UN_ci_8310 & $163 \mathrm{C} / \mathrm{T}$ \\
\hline UN_ci_8310 & $356 \mathrm{~T} / \mathrm{A}$ \\
\hline UN_ci_8310 & $480 \mathrm{~A} / \mathrm{G}$ \\
\hline UN_ci_8313 & $8 \mathrm{C} / \mathrm{T}$ \\
\hline UN_ci_8313 & 197 T/C \\
\hline UN_ci_8313 & $294 \mathrm{C} / \mathrm{A}$ \\
\hline UN_ci_8313 & $468 \mathrm{G} / \mathrm{A}$ \\
\hline UN_ci_8313 & $606 \mathrm{C} / \mathrm{A}$ \\
\hline UN_ci_8314 & $615 \mathrm{~A} / \mathrm{T}$ \\
\hline UN_ci_8315 & $966 \mathrm{~A} / \mathrm{G}$ \\
\hline
\end{tabular}




\begin{tabular}{|c|c|}
\hline UN_ci_8316 & $1009 \mathrm{~A} / \mathrm{G}$ \\
\hline UN_ci_8316 & $1109 \mathrm{~T} / \mathrm{A}$ \\
\hline UN_ci_8316 & $1412 \mathrm{~A} / \mathrm{G}$ \\
\hline UN_ci_8316 & $1648 \mathrm{~A} / \mathrm{T}$ \\
\hline UN_ci_8316 & $1711 \mathrm{G} / \mathrm{A}$ \\
\hline UN_ci_8317 & $18 \mathrm{G} / \mathrm{A}$ \\
\hline UN_ci_8317 & $68 \mathrm{~A} / \mathrm{G}$ \\
\hline UN_ci_8317 & $149 \mathrm{~T} / \mathrm{C}$ \\
\hline UN_ci_8317 & $200 \mathrm{~A} / \mathrm{G}$ \\
\hline UN_ci_8317 & $221 \mathrm{~A} / \mathrm{G}$ \\
\hline UN_ci_8317 & $256 \mathrm{~A} / \mathrm{G}$ \\
\hline UN_ci_8317 & $283 \mathrm{~A} / \mathrm{G}$ \\
\hline UN_ci_8317 & 307 A/G \\
\hline UN_ci_8317 & $335 \mathrm{C} / \mathrm{T}$ \\
\hline UN_ci_8317 & $368 \mathrm{~A} / \mathrm{G}$ \\
\hline UN_ci_8317 & $397 \mathrm{C} / \mathrm{T}$ \\
\hline UN_ci_8317 & $424 \mathrm{~A} / \mathrm{G}$ \\
\hline UN_ci_8317 & $460 \mathrm{C} / \mathrm{T}$ \\
\hline UN_ci_8317 & $498 \mathrm{~A} / \mathrm{G}$ \\
\hline UN_ci_8317 & $554 \mathrm{G} / \mathrm{A}$ \\
\hline UN_ci_8317 & $584 \mathrm{~A} / \mathrm{G}$ \\
\hline UN_ci_8317 & $613 \mathrm{~T} / \mathrm{C}$ \\
\hline UN_ci_8317 & $638 \mathrm{C} / \mathrm{T}$ \\
\hline UN_ci_8317 & $668 \mathrm{G} / \mathrm{A}$ \\
\hline UN_ci_8317 & $736 \mathrm{G} / \mathrm{A}$ \\
\hline UN_ci_8317 & $815 \mathrm{~A} / \mathrm{C}$ \\
\hline UN_ci_8317 & $836 \mathrm{C} / \mathrm{T}$ \\
\hline UN_ci_8317 & $869 \mathrm{~T} / \mathrm{C}$ \\
\hline UN_ci_8317 & $910 \mathrm{~T} / \mathrm{C}$ \\
\hline UN_ci_8317 & $930 \mathrm{~A} / \mathrm{G}$ \\
\hline UN_ci_8317 & $951 \mathrm{~T} / \mathrm{C}$ \\
\hline UN_ci_8317 & $973 \mathrm{~A} / \mathrm{G}$ \\
\hline UN_ci_8317 & 998 G/C \\
\hline UN_ci_8317 & $1036 \mathrm{G} / \mathrm{A}$ \\
\hline UN_ci_8318 & $91 \mathrm{C} / \mathrm{T}$ \\
\hline UN_ci_8319 & $143 \mathrm{G} / \mathrm{A}$ \\
\hline UN_ci_8319 & $166 \mathrm{~A} / \mathrm{G}$ \\
\hline UN_ci_8319 & $720 \mathrm{~A} / \mathrm{T}$ \\
\hline UN_ci_8320 & $242 \mathrm{C} / \mathrm{T}$ \\
\hline UN_ci_8321 & $122 \mathrm{~A} / \mathrm{C}$ \\
\hline UN_ci_8321 & $226 \mathrm{~A} / \mathrm{C}$ \\
\hline UN_ci_8321 & $252 \mathrm{C} / \mathrm{T}$ \\
\hline UN_ci_8321 & 356 A/G \\
\hline UN_ci_8321 & $443 \mathrm{~T} / \mathrm{C}$ \\
\hline UN_ci_8321 & $835 \mathrm{~A} / \mathrm{C}$ \\
\hline UN_ci_8322 & $221 \mathrm{C} / \mathrm{A}$ \\
\hline UN_ci_8322 & $664 \mathrm{~T} / \mathrm{C}$ \\
\hline UN_ci_8322 & $972 \mathrm{G} / \mathrm{A}$ \\
\hline UN_ci_8323 & $129 \mathrm{~T} / \mathrm{C}$ \\
\hline UN_ci_8323 & $194 \mathrm{~T} / \mathrm{C}$ \\
\hline
\end{tabular}




\begin{tabular}{|c|c|}
\hline UN_ci_8323 & $325 \mathrm{C} / \mathrm{T}$ \\
\hline UN_ci_8323 & $463 \mathrm{C} / \mathrm{T}$ \\
\hline UN_ci_8324 & $109 \mathrm{~T} / \mathrm{C}$ \\
\hline UN_ci_8324 & $145 \mathrm{~A} / \mathrm{G}$ \\
\hline UN_ci_8324 & $180 \mathrm{~T} / \mathrm{C}$ \\
\hline UN_ci_8324 & $206 \mathrm{C} / \mathrm{T}$ \\
\hline UN_ci_8324 & $267 \mathrm{~T} / \mathrm{C}$ \\
\hline UN_ci_8324 & $287 \mathrm{C} / \mathrm{T}$ \\
\hline UN_ci_8324 & $345 \mathrm{~T} / \mathrm{C}$ \\
\hline UN_ci_8324 & $399 \mathrm{~T} / \mathrm{C}$ \\
\hline UN_ci_8324 & $455 \mathrm{~A} / \mathrm{C}$ \\
\hline UN_ci_8324 & $484 \mathrm{~T} / \mathrm{C}$ \\
\hline UN_ci_8324 & $627 \mathrm{~A} / \mathrm{G}$ \\
\hline UN_ci_8324 & $654 \mathrm{G} / \mathrm{T}$ \\
\hline UN_ci_8324 & $691 \mathrm{~A} / \mathrm{G}$ \\
\hline UN_ci_8324 & $790 \mathrm{~A} / \mathrm{C}$ \\
\hline UN_ci_8324 & $832 \mathrm{C} / \mathrm{T}$ \\
\hline UN_ci_8325 & $90 \mathrm{C} / \mathrm{T}$ \\
\hline UN_ci_8325 & $368 \mathrm{~A} / \mathrm{C}$ \\
\hline UN_ci_8327 & $484 \mathrm{~A} / \mathrm{G}$ \\
\hline UN_ci_8328 & $465 \mathrm{C} / \mathrm{G}$ \\
\hline UN_ci_8328 & $760 \mathrm{~T} / \mathrm{C}$ \\
\hline UN_ci_8328 & $844 \mathrm{~T} / \mathrm{A}$ \\
\hline UN_ci_8328 & $1074 \mathrm{G} / \mathrm{A}$ \\
\hline UN_ci_8328 & $1362 \mathrm{~A} / \mathrm{G}$ \\
\hline UN_ci_8328 & $1510 \mathrm{C} / \mathrm{T}$ \\
\hline UN_ci_8328 & $1571 \mathrm{C} / \mathrm{T}$ \\
\hline UN_ci_8328 & $1666 \mathrm{~T} / \mathrm{C}$ \\
\hline UN_ci_8329 & $192 \mathrm{~A} / \mathrm{G}$ \\
\hline UN_ci_8330 & $443 \mathrm{G} / \mathrm{A}$ \\
\hline UN_ci_8330 & $1030 \mathrm{~A} / \mathrm{G}$ \\
\hline UN_ci_8330 & $1234 \mathrm{C} / \mathrm{T}$ \\
\hline UN_ci_8332 & $474 \mathrm{C} / \mathrm{A}$ \\
\hline UN_ci_8332 & $536 \mathrm{~A} / \mathrm{G}$ \\
\hline UN_ci_8332 & $1168 \mathrm{~A} / \mathrm{G}$ \\
\hline UN_ci_8334 & $94 \mathrm{~A} / \mathrm{G}$ \\
\hline UN_ci_8334 & $121 \mathrm{~A} / \mathrm{G}$ \\
\hline UN_ci_8335 & $275 \mathrm{C} / \mathrm{A}$ \\
\hline UN_ci_8338 & $369 \mathrm{C} / \mathrm{T}$ \\
\hline UN_ci_8338 & $405 \mathrm{C} / \mathrm{T}$ \\
\hline UN_ci_8338 & $521 \mathrm{C} / \mathrm{T}$ \\
\hline UN_ci_8338 & $546 \mathrm{C} / \mathrm{T}$ \\
\hline UN_ci_8338 & $1057 \mathrm{~A} / \mathrm{T}$ \\
\hline UN_ci_8338 & $1100 \mathrm{~T} / \mathrm{A}$ \\
\hline UN_ci_8339 & $204 \mathrm{~A} / \mathrm{T}$ \\
\hline UN_ci_8339 & $533 \mathrm{~T} / \mathrm{C}$ \\
\hline UN_ci_8339 & 613 T/C \\
\hline UN_ci_8339 & 763 T/C \\
\hline UN_ci_8339 & $806 \mathrm{~A} / \mathrm{G}$ \\
\hline UN_ci_8340 & $211 \mathrm{~A} / \mathrm{T}$ \\
\hline
\end{tabular}




$\begin{array}{lr}\text { UN_ci_8343 } & 145 \mathrm{~T} / \mathrm{C} \\ \text { UN_ci_8343 } & 169 \mathrm{C} / \mathrm{T} \\ \text { UN_ci_8343 } & 252 \mathrm{~T} / \mathrm{C} \\ \text { UN_ci_8343 } & 295 \mathrm{~T} / \mathrm{C} \\ \text { UN_ci_8343 } & 375 \mathrm{~A} / \mathrm{G} \\ \text { UN_ci_8343 } & 423 \mathrm{~A} / \mathrm{G} \\ \text { UN_ci_8343 } & 458 \mathrm{~A} / \mathrm{C} \\ \text { UN_ci_8343 } & 490 \mathrm{~T} / \mathrm{C} \\ \text { UN_ci_8343 } & 540 \mathrm{C} / \mathrm{T} \\ \text { UN_ci_8343 } & 564 \mathrm{~A} / \mathrm{G} \\ \text { UN_ci_8343 } & 649 \mathrm{~A} / \mathrm{G} \\ \text { UN_ci_8343 } & 670 \mathrm{~T} / \mathrm{C} \\ \text { UN_ci_8343 } & 730 \mathrm{~A} / \mathrm{G} \\ \text { UN_ci_8343 } & 756 \mathrm{~A} / \mathrm{G} \\ \text { UN_ci_8343 } & 810 \mathrm{~A} / \mathrm{T} \\ \text { UN_ci_8343 } & 831 \mathrm{G} / \mathrm{A} \\ \text { UN_ci_8344 } & 26 \mathrm{G} / \mathrm{C} \\ \text { UN_ci_8344 } & 87 \mathrm{~T} / \mathrm{C} \\ \text { UN_ci_8344 } & 203 \mathrm{~A} / \mathrm{G} \\ \text { UN_ci_8344 } & 422 \mathrm{~T} / \mathrm{C} \\ \text { UN_ci_8344 } & 1024 \mathrm{~A} / \mathrm{G} \\ \text { UN_ci_8344 } & 1079 \mathrm{C} / \mathrm{T} \\ \text { UN_ci_8344 } & 1108 \mathrm{~A} / \mathrm{G} \\ \text { UN_ci_8344 } & 1140 \mathrm{~A} / \mathrm{T} \\ \text { UN_ci_8345 } & 1160 \mathrm{~A} / \mathrm{G} \\ \text { UN_ci_8345 } & 1183 \mathrm{~T} / \mathrm{A} \\ \text { UN_ci_8345 } & \end{array}$




\begin{tabular}{|c|c|}
\hline UN_ci_8346 & 1213 T/C \\
\hline UN_ci_8346 & $1233 \mathrm{C} / \mathrm{G}$ \\
\hline UN_ci_8346 & $1254 \mathrm{~T} / \mathrm{G}$ \\
\hline UN_ci_8346 & $1277 \mathrm{C} / \mathrm{T}$ \\
\hline UN_ci_8346 & $1299 \mathrm{G} / \mathrm{A}$ \\
\hline UN_ci_8346 & $1321 \mathrm{G} / \mathrm{A}$ \\
\hline UN_ci_8346 & $1349 \mathrm{C} / \mathrm{T}$ \\
\hline UN_ci_8346 & $1370 \mathrm{~A} / \mathrm{G}$ \\
\hline UN_ci_8346 & $1405 \mathrm{~A} / \mathrm{G}$ \\
\hline UN_ci_8346 & $1428 \mathrm{~T} / \mathrm{C}$ \\
\hline UN_ci_8346 & $1464 \mathrm{G} / \mathrm{A}$ \\
\hline UN_ci_8346 & $1495 \mathrm{C} / \mathrm{T}$ \\
\hline UN_ci_8346 & $1518 \mathrm{C} / \mathrm{A}$ \\
\hline UN_ci_8346 & $1538 \mathrm{~A} / \mathrm{G}$ \\
\hline UN_ci_8346 & $1567 \mathrm{~A} / \mathrm{G}$ \\
\hline UN_ci_8346 & 1587 T/C \\
\hline UN_ci_8346 & $1622 \mathrm{C} / \mathrm{T}$ \\
\hline UN_ci_8346 & $1646 \mathrm{~T} / \mathrm{C}$ \\
\hline UN_ci_8346 & $1670 \mathrm{~T} / \mathrm{C}$ \\
\hline UN_ci_8346 & 1717 T/C \\
\hline UN_ci_8346 & $1815 \mathrm{~T} / \mathrm{A}$ \\
\hline UN_ci_8346 & $1884 \mathrm{~A} / \mathrm{T}$ \\
\hline UN_ci_8346 & $1920 \mathrm{~A} / \mathrm{G}$ \\
\hline UN_ci_8346 & $1950 \mathrm{G} / \mathrm{T}$ \\
\hline UN_ci_8346 & 1995 T/C \\
\hline UN_ci_8346 & $2017 \mathrm{C} / \mathrm{T}$ \\
\hline UN_ci_8346 & 2095 A/G \\
\hline UN_ci_8346 & $2138 \mathrm{C} / \mathrm{G}$ \\
\hline UN_ci_8346 & $2242 \mathrm{G} / \mathrm{A}$ \\
\hline UN_ci_8346 & $2272 \mathrm{~A} / \mathrm{G}$ \\
\hline UN_ci_8346 & 2327 T/C \\
\hline UN_ci_8346 & $2390 \mathrm{~A} / \mathrm{G}$ \\
\hline UN_ci_8346 & $2429 \mathrm{G} / \mathrm{A}$ \\
\hline UN_ci_8346 & $2544 \mathrm{~T} / \mathrm{C}$ \\
\hline UN_ci_8346 & 2703 A/G \\
\hline UN_ci_8346 & $2755 \mathrm{G} / \mathrm{A}$ \\
\hline UN_ci_8346 & 2786 A/G \\
\hline UN_ci_8346 & $2817 \mathrm{~T} / \mathrm{C}$ \\
\hline UN_ci_8346 & 2879 A/G \\
\hline UN_ci_8346 & $2902 \mathrm{C} / \mathrm{T}$ \\
\hline UN_ci_8346 & 2922 A/G \\
\hline UN_ci_8346 & 2949 A/G \\
\hline UN_ci_8347 & $102 \mathrm{G} / \mathrm{T}$ \\
\hline UN_ci_8347 & $122 \mathrm{C} / \mathrm{T}$ \\
\hline UN_ci_8347 & $457 \mathrm{~T} / \mathrm{C}$ \\
\hline UN_ci_8347 & $789 \mathrm{G} / \mathrm{T}$ \\
\hline UN_ci_8348 & $359 \mathrm{C} / \mathrm{A}$ \\
\hline UN_ci_8348 & $1413 \mathrm{C} / \mathrm{T}$ \\
\hline UN_ci_8350 & $249 \mathrm{~T} / \mathrm{A}$ \\
\hline UN_ci_8353 & $165 \mathrm{C} / \mathrm{T}$ \\
\hline
\end{tabular}




\begin{tabular}{|c|c|}
\hline UN_ci_8353 & $289 \mathrm{~T} / \mathrm{C}$ \\
\hline UN_ci_8356 & $374 \mathrm{C} / \mathrm{T}$ \\
\hline UN_ci_8382 & $153 \mathrm{~T} / \mathrm{C}$ \\
\hline UN_ci_8385 & $430 \mathrm{C} / \mathrm{T}$ \\
\hline UN_ci_8391 & $91 \mathrm{C} / \mathrm{T}$ \\
\hline UN_ci_8391 & $131 \mathrm{C} / \mathrm{G}$ \\
\hline UN_ci_8393 & $315 \mathrm{~T} / \mathrm{A}$ \\
\hline UN_ci_8393 & $651 \mathrm{~A} / \mathrm{G}$ \\
\hline UN_ci_8400 & $828 \mathrm{~T} / \mathrm{C}$ \\
\hline UN_ci_8400 & 1189 T/C \\
\hline UN_ci_8400 & $1225 \mathrm{G} / \mathrm{A}$ \\
\hline UN_ci_8400 & 1343 T/A \\
\hline UN_ci_8406 & $469 \mathrm{~A} / \mathrm{G}$ \\
\hline UN_ci_8406 & $586 \mathrm{C} / \mathrm{T}$ \\
\hline UN_ci_8406 & $1414 \mathrm{G} / \mathrm{A}$ \\
\hline UN_ci_8409 & $311 \mathrm{G} / \mathrm{A}$ \\
\hline UN_ci_8420 & $450 \mathrm{~T} / \mathrm{G}$ \\
\hline UN_ci_8420 & $543 \mathrm{~T} / \mathrm{C}$ \\
\hline UN_ci_8420 & $689 \mathrm{~A} / \mathrm{C}$ \\
\hline UN_ci_8420 & $713 \mathrm{~A} / \mathrm{G}$ \\
\hline UN_ci_8420 & $748 \mathrm{~A} / \mathrm{C}$ \\
\hline UN_ci_8420 & $887 \mathrm{~T} / \mathrm{C}$ \\
\hline UN_ci_8420 & $935 \mathrm{~A} / \mathrm{G}$ \\
\hline UN_ci_8420 & 1040 T/C \\
\hline UN_ci_8420 & $1071 \mathrm{~A} / \mathrm{G}$ \\
\hline UN_ci_8425 & $583 \mathrm{~A} / \mathrm{G}$ \\
\hline UN_ci_8427 & $9 \mathrm{~T} / \mathrm{G}$ \\
\hline UN_ci_8427 & $455 \mathrm{~A} / \mathrm{G}$ \\
\hline UN_ci_8427 & $531 \mathrm{C} / \mathrm{T}$ \\
\hline UN_ci_8427 & $1562 \mathrm{C} / \mathrm{T}$ \\
\hline UN_ci_8434 & $93 \mathrm{~A} / \mathrm{G}$ \\
\hline UN_ci_8449 & $288 \mathrm{~T} / \mathrm{C}$ \\
\hline UN_ci_8449 & $342 \mathrm{~T} / \mathrm{A}$ \\
\hline UN_ci_8468 & $191 \mathrm{C} / \mathrm{T}$ \\
\hline UN_ci_8468 & $388 \mathrm{~T} / \mathrm{C}$ \\
\hline UN_ci_8468 & $526 \mathrm{~A} / \mathrm{G}$ \\
\hline UN_ci_8472 & $322 \mathrm{G} / \mathrm{A}$ \\
\hline UN_ci_8472 & $412 \mathrm{~T} / \mathrm{C}$ \\
\hline UN_ci_8472 & $438 \mathrm{~A} / \mathrm{G}$ \\
\hline UN_ci_8472 & $558 \mathrm{C} / \mathrm{A}$ \\
\hline UN_ci_8474 & $242 \mathrm{~A} / \mathrm{C}$ \\
\hline UN_ci_8474 & $388 \mathrm{~T} / \mathrm{C}$ \\
\hline UN_ci_8474 & $559 \mathrm{~T} / \mathrm{C}$ \\
\hline UN_ci_8474 & $692 \mathrm{~A} / \mathrm{G}$ \\
\hline UN_ci_8477 & $267 \mathrm{G} / \mathrm{A}$ \\
\hline UN_ci_8477 & $392 \mathrm{~A} / \mathrm{G}$ \\
\hline UN_ci_8477 & $414 \mathrm{~A} / \mathrm{C}$ \\
\hline UN_ci_8477 & $480 \mathrm{G} / \mathrm{C}$ \\
\hline UN_ci_8477 & $531 \mathrm{~A} / \mathrm{C}$ \\
\hline UN_ci_8477 & $566 \mathrm{C} / \mathrm{G}$ \\
\hline
\end{tabular}




\begin{tabular}{|c|c|}
\hline UN_ci_8477 & $633 \mathrm{~A} / \mathrm{G}$ \\
\hline UN_ci_8477 & $739 \mathrm{G} / \mathrm{A}$ \\
\hline UN_ci_8477 & $775 \mathrm{C} / \mathrm{T}$ \\
\hline UN_ci_8477 & $803 \mathrm{~T} / \mathrm{G}$ \\
\hline UN_ci_8477 & $858 \mathrm{~A} / \mathrm{G}$ \\
\hline UN_ci_8477 & $904 \mathrm{~T} / \mathrm{C}$ \\
\hline UN_ci_8477 & $932 \mathrm{~A} / \mathrm{G}$ \\
\hline UN_ci_8477 & $964 \mathrm{~A} / \mathrm{G}$ \\
\hline UN_ci_8477 & 997 A/G \\
\hline UN_ci_8477 & 1022 T/C \\
\hline UN_ci_8477 & 1091 T/C \\
\hline UN_ci_8478 & $208 \mathrm{G} / \mathrm{A}$ \\
\hline UN_ci_8478 & $338 \mathrm{G} / \mathrm{T}$ \\
\hline UN_ci_8479 & $307 \mathrm{G} / \mathrm{T}$ \\
\hline UN_ci_8479 & $385 \mathrm{G} / \mathrm{T}$ \\
\hline UN_ci_8479 & $710 \mathrm{~T} / \mathrm{G}$ \\
\hline UN_ci_8483 & $76 \mathrm{~A} / \mathrm{T}$ \\
\hline UN_ci_8488 & $437 \mathrm{C} / \mathrm{T}$ \\
\hline UN_ci_8488 & $525 \mathrm{~A} / \mathrm{T}$ \\
\hline UN_ci_8490 & $180 \mathrm{~A} / \mathrm{G}$ \\
\hline UN_ci_8490 & $208 \mathrm{~A} / \mathrm{G}$ \\
\hline UN_ci_8490 & $233 \mathrm{~A} / \mathrm{G}$ \\
\hline UN_ci_8490 & $254 \mathrm{~A} / \mathrm{G}$ \\
\hline UN_ci_8490 & $282 \mathrm{~T} / \mathrm{C}$ \\
\hline UN_ci_8490 & 306 A/G \\
\hline UN_ci_8490 & $326 \mathrm{C} / \mathrm{T}$ \\
\hline UN_ci_8490 & $351 \mathrm{~T} / \mathrm{C}$ \\
\hline UN_ci_8490 & $378 \mathrm{~T} / \mathrm{C}$ \\
\hline UN_ci_8490 & $400 \mathrm{C} / \mathrm{T}$ \\
\hline UN_ci_8490 & $420 \mathrm{~T} / \mathrm{C}$ \\
\hline UN_ci_8490 & $443 \mathrm{C} / \mathrm{T}$ \\
\hline UN_ci_8490 & $464 \mathrm{G} / \mathrm{A}$ \\
\hline UN_ci_8490 & $484 \mathrm{G} / \mathrm{C}$ \\
\hline UN_ci_8490 & $507 \mathrm{~A} / \mathrm{G}$ \\
\hline UN_ci_8490 & $527 \mathrm{C} / \mathrm{T}$ \\
\hline UN_ci_8490 & $547 \mathrm{~T} / \mathrm{C}$ \\
\hline UN_ci_8490 & $567 \mathrm{G} / \mathrm{A}$ \\
\hline UN_ci_8490 & $587 \mathrm{C} / \mathrm{T}$ \\
\hline UN_ci_8490 & 607 T/C \\
\hline UN_ci_8490 & $628 \mathrm{~A} / \mathrm{G}$ \\
\hline UN_ci_8490 & $650 \mathrm{~A} / \mathrm{G}$ \\
\hline UN_ci_8490 & $670 \mathrm{~T} / \mathrm{C}$ \\
\hline UN_ci_8490 & $693 \mathrm{C} / \mathrm{T}$ \\
\hline UN_ci_8490 & $713 \mathrm{~A} / \mathrm{G}$ \\
\hline UN_ci_8490 & $735 \mathrm{~T} / \mathrm{C}$ \\
\hline UN_ci_8490 & $758 \mathrm{~T} / \mathrm{C}$ \\
\hline UN_ci_8490 & $778 \mathrm{G} / \mathrm{A}$ \\
\hline UN_ci_8490 & 799 A/G \\
\hline UN_ci_8490 & $820 \mathrm{C} / \mathrm{T}$ \\
\hline UN_ci_8490 & $840 \mathrm{C} / \mathrm{T}$ \\
\hline
\end{tabular}




$\begin{array}{lr}\text { UN_ci_8490 } & 865 \mathrm{G} / \mathrm{A} \\ \text { UN_ci_8490 } & 891 \mathrm{C} / \mathrm{T} \\ \text { UN_ci_8490 } & 915 \mathrm{~A} / \mathrm{G} \\ \text { UN_ci_8490 } & 936 \mathrm{C} / \mathrm{A} \\ \text { UN_ci_8490 } & 956 \mathrm{G} / \mathrm{A} \\ \text { UN_ci_8490 } & 976 \mathrm{G} / \mathrm{C} \\ \text { UN_ci_8490 } & 996 \mathrm{G} / \mathrm{A} \\ \text { UN_ci_8490 } & 1016 \mathrm{~A} / \mathrm{G} \\ \text { UN_ci_8490 } & 1039 \mathrm{~A} / \mathrm{G} \\ \text { UN_ci_8490 } & 1067 \mathrm{~T} / \mathrm{C} \\ \text { UN_ci_8490 } & 1092 \mathrm{~T} / \mathrm{C} \\ \text { UN_ci_8493 } & 70 \mathrm{~T} / \mathrm{C} \\ \text { UN_ci_8493 } & 92 \mathrm{G} / \mathrm{A} \\ \text { UN_ci_8493 } & 129 \mathrm{~T} / \mathrm{A} \\ \text { UN_ci_8493 } & 171 \mathrm{C} / \mathrm{T} \\ \text { UN_ci_8493 } & 236 \mathrm{~T} / \mathrm{C} \\ \text { UN_ci_8493 } & 288 \mathrm{G} / \mathrm{A} \\ \text { UN_ci_8493 } & 366 \mathrm{G} / \mathrm{A} \\ \text { UN_ci_8493 } & 682 \mathrm{C} / \mathrm{A} \\ \text { UN_ci_8499 } & 235 \mathrm{~A} / \mathrm{G} \\ \text { UN_ci_8500 } & 915 \mathrm{~A} / \mathrm{G} \\ \text { UN_ci_8506 } & 1001 \mathrm{C} / \mathrm{T} \\ \text { UN_ci_8506 } & 1074 \mathrm{G} / \mathrm{T} \\ \text { UN_ci_8506 } & 1149 \mathrm{~T} / \mathrm{C} \\ \text { UN_ci_8506 } & 1278 \mathrm{~A} / \mathrm{G} \\ \text { UN_ci_8507 } & \end{array}$




\begin{tabular}{|c|c|}
\hline UN_ci_8522 & $387 \mathrm{~A} / \mathrm{G}$ \\
\hline UN_ci_8522 & $839 \mathrm{~T} / \mathrm{C}$ \\
\hline UN_ci_8526 & $220 \mathrm{~A} / \mathrm{T}$ \\
\hline UN_ci_8533 & 167 T/A \\
\hline UN_ci_8533 & 302 T/C \\
\hline UN_ci_8533 & $661 \mathrm{~A} / \mathrm{G}$ \\
\hline UN_ci_8537 & $1292 \mathrm{~A} / \mathrm{G}$ \\
\hline UN_ci_8537 & $1396 \mathrm{~A} / \mathrm{C}$ \\
\hline UN_ci_8537 & $1520 \mathrm{G} / \mathrm{A}$ \\
\hline UN_ci_8538 & $341 \mathrm{C} / \mathrm{A}$ \\
\hline UN_ci_8538 & $555 \mathrm{C} / \mathrm{T}$ \\
\hline UN_ci_8538 & $588 \mathrm{C} / \mathrm{T}$ \\
\hline UN_ci_8540 & $439 \mathrm{~T} / \mathrm{C}$ \\
\hline UN_ci_8544 & $278 \mathrm{G} / \mathrm{T}$ \\
\hline UN_ci_8544 & 359 T/G \\
\hline UN_ci_8544 & $382 \mathrm{~A} / \mathrm{G}$ \\
\hline UN_ci_8544 & $403 \mathrm{~T} / \mathrm{C}$ \\
\hline UN_ci_8559 & $495 \mathrm{C} / \mathrm{T}$ \\
\hline UN_ci_8559 & $615 \mathrm{~A} / \mathrm{G}$ \\
\hline UN_ci_8559 & 775 C/G \\
\hline UN_ci_8562 & $418 \mathrm{~T} / \mathrm{A}$ \\
\hline UN_ci_8569 & $431 \mathrm{~T} / \mathrm{C}$ \\
\hline UN_ci_8569 & $625 \mathrm{~A} / \mathrm{G}$ \\
\hline UN_ci_8569 & $656 \mathrm{C} / \mathrm{T}$ \\
\hline UN_ci_8580 & $151 \mathrm{G} / \mathrm{C}$ \\
\hline UN_ci_8582 & $218 \mathrm{~T} / \mathrm{G}$ \\
\hline UN_ci_8582 & $856 \mathrm{C} / \mathrm{A}$ \\
\hline UN_ci_8595 & $190 \mathrm{G} / \mathrm{T}$ \\
\hline UN_ci_8595 & $932 \mathrm{~A} / \mathrm{G}$ \\
\hline UN_ci_8595 & $1096 \mathrm{G} / \mathrm{C}$ \\
\hline UN_ci_8595 & $1200 \mathrm{~T} / \mathrm{C}$ \\
\hline UN_ci_8595 & $1544 \mathrm{~A} / \mathrm{T}$ \\
\hline UN_ci_8596 & $190 \mathrm{G} / \mathrm{C}$ \\
\hline UN_ci_8596 & $366 \mathrm{G} / \mathrm{T}$ \\
\hline UN_ci_8596 & $555 \mathrm{C} / \mathrm{T}$ \\
\hline UN_ci_8598 & $164 \mathrm{G} / \mathrm{T}$ \\
\hline UN_ci_8598 & $189 \mathrm{G} / \mathrm{T}$ \\
\hline UN_ci_8605 & $554 \mathrm{C} / \mathrm{T}$ \\
\hline UN_ci_8605 & $800 \mathrm{C} / \mathrm{G}$ \\
\hline UN_ci_8606 & $404 \mathrm{~T} / \mathrm{C}$ \\
\hline UN_ci_8606 & $497 \mathrm{~A} / \mathrm{G}$ \\
\hline UN_ci_8607 & $205 \mathrm{C} / \mathrm{T}$ \\
\hline UN_ci_8607 & $274 \mathrm{G} / \mathrm{C}$ \\
\hline UN_ci_8607 & $382 \mathrm{C} / \mathrm{T}$ \\
\hline UN_ci_8607 & $414 \mathrm{~A} / \mathrm{C}$ \\
\hline UN_ci_8607 & $503 \mathrm{~A} / \mathrm{G}$ \\
\hline UN_ci_8607 & $601 \mathrm{G} / \mathrm{A}$ \\
\hline UN_ci_8611 & $493 \mathrm{~A} / \mathrm{C}$ \\
\hline UN_ci_8611 & $562 \mathrm{G} / \mathrm{A}$ \\
\hline UN_ci_8611 & $609 \mathrm{G} / \mathrm{A}$ \\
\hline
\end{tabular}




\begin{tabular}{|c|c|}
\hline UN_ci_8611 & $630 \mathrm{G} / \mathrm{T}$ \\
\hline UN_ci_8611 & $863 \mathrm{G} / \mathrm{A}$ \\
\hline UN_ci_8612 & 359 A/G \\
\hline UN_ci_8612 & $380 \mathrm{~T} / \mathrm{A}$ \\
\hline UN_ci_8612 & $402 \mathrm{G} / \mathrm{A}$ \\
\hline UN_ci_8612 & $426 \mathrm{C} / \mathrm{T}$ \\
\hline UN_ci_8612 & $446 \mathrm{~T} / \mathrm{C}$ \\
\hline UN_ci_8612 & $471 \mathrm{~T} / \mathrm{C}$ \\
\hline UN_ci_8612 & $493 \mathrm{~A} / \mathrm{G}$ \\
\hline UN_ci_8612 & $515 \mathrm{C} / \mathrm{T}$ \\
\hline UN_ci_8612 & $542 \mathrm{~T} / \mathrm{C}$ \\
\hline UN_ci_8612 & $568 \mathrm{~A} / \mathrm{G}$ \\
\hline UN_ci_8612 & $590 \mathrm{~T} / \mathrm{A}$ \\
\hline UN_ci_8612 & $610 \mathrm{C} / \mathrm{T}$ \\
\hline UN_ci_8612 & $630 \mathrm{~T} / \mathrm{A}$ \\
\hline UN_ci_8612 & $654 \mathrm{~A} / \mathrm{G}$ \\
\hline UN_ci_8612 & $675 \mathrm{C} / \mathrm{T}$ \\
\hline UN_ci_8612 & $695 \mathrm{G} / \mathrm{A}$ \\
\hline UN_ci_8612 & $719 \mathrm{~T} / \mathrm{C}$ \\
\hline UN_ci_8612 & $748 \mathrm{C} / \mathrm{T}$ \\
\hline UN_ci_8612 & $770 \mathrm{~T} / \mathrm{C}$ \\
\hline UN_ci_8612 & $793 \mathrm{C} / \mathrm{T}$ \\
\hline UN_ci_8612 & $822 \mathrm{~A} / \mathrm{C}$ \\
\hline UN_ci_8612 & $846 \mathrm{~A} / \mathrm{G}$ \\
\hline UN_ci_8612 & $867 \mathrm{G} / \mathrm{A}$ \\
\hline UN_ci_8612 & $892 \mathrm{~A} / \mathrm{G}$ \\
\hline UN_ci_8612 & $912 \mathrm{~A} / \mathrm{G}$ \\
\hline UN_ci_8612 & $933 \mathrm{~A} / \mathrm{C}$ \\
\hline UN_ci_8612 & $961 \mathrm{G} / \mathrm{A}$ \\
\hline UN_ci_8612 & $984 \mathrm{~A} / \mathrm{G}$ \\
\hline UN_ci_8612 & 1019 A/G \\
\hline UN_ci_8612 & $1057 \mathrm{C} / \mathrm{G}$ \\
\hline UN_ci_8612 & $1135 \mathrm{C} / \mathrm{G}$ \\
\hline UN_ci_8612 & $1164 \mathrm{G} / \mathrm{T}$ \\
\hline UN_ci_8612 & $1396 \mathrm{~A} / \mathrm{T}$ \\
\hline UN_ci_8612 & $1425 \mathrm{G} / \mathrm{C}$ \\
\hline UN_ci_8612 & $1458 \mathrm{~T} / \mathrm{A}$ \\
\hline UN_ci_8612 & $1494 \mathrm{~T} / \mathrm{A}$ \\
\hline UN_ci_8612 & $1547 \mathrm{~A} / \mathrm{G}$ \\
\hline UN_ci_8614 & $73 \mathrm{G} / \mathrm{A}$ \\
\hline UN_ci_8614 & $538 \mathrm{G} / \mathrm{A}$ \\
\hline UN_ci_8618 & $348 \mathrm{C} / \mathrm{A}$ \\
\hline UN_ci_8618 & $490 \mathrm{C} / \mathrm{G}$ \\
\hline UN_ci_8618 & $707 \mathrm{~A} / \mathrm{G}$ \\
\hline UN_ci_8618 & $772 \mathrm{~A} / \mathrm{G}$ \\
\hline UN_ci_8618 & $871 \mathrm{~A} / \mathrm{T}$ \\
\hline UN_ci_8618 & $898 \mathrm{~A} / \mathrm{G}$ \\
\hline UN_ci_8618 & $1037 \mathrm{~A} / \mathrm{C}$ \\
\hline UN_ci_8618 & $1090 \mathrm{G} / \mathrm{A}$ \\
\hline UN_ci_8618 & $1151 \mathrm{G} / \mathrm{A}$ \\
\hline
\end{tabular}




\begin{tabular}{|c|c|}
\hline UN_ci_8618 & $1243 \mathrm{~A} / \mathrm{T}$ \\
\hline UN_ci_8618 & $1272 \mathrm{C} / \mathrm{A}$ \\
\hline UN_ci_8618 & $1561 \mathrm{G} / \mathrm{A}$ \\
\hline UN_ci_8618 & $1684 \mathrm{G} / \mathrm{A}$ \\
\hline UN_ci_8618 & 2055 G/A \\
\hline UN_ci_8618 & $2182 \mathrm{~T} / \mathrm{C}$ \\
\hline UN_ci_8618 & 2264 T/A \\
\hline UN_ci_8626 & $488 \mathrm{C} / \mathrm{T}$ \\
\hline UN_ci_8635 & $437 \mathrm{~A} / \mathrm{T}$ \\
\hline UN_ci_8635 & $480 \mathrm{~A} / \mathrm{G}$ \\
\hline UN_ci_8635 & $521 \mathrm{~T} / \mathrm{C}$ \\
\hline UN_ci_8635 & 643 A/G \\
\hline UN_ci_8635 & $692 \mathrm{~A} / \mathrm{T}$ \\
\hline UN_ci_8635 & $731 \mathrm{C} / \mathrm{A}$ \\
\hline UN_ci_8635 & $860 \mathrm{~T} / \mathrm{G}$ \\
\hline UN_ci_8636 & $41 \mathrm{~T} / \mathrm{A}$ \\
\hline UN_ci_8636 & $502 \mathrm{G} / \mathrm{T}$ \\
\hline UN_ci_8638 & $350 \mathrm{G} / \mathrm{A}$ \\
\hline UN_ci_8638 & $433 \mathrm{C} / \mathrm{T}$ \\
\hline UN_ci_8638 & 508 A/G \\
\hline UN_ci_8638 & $571 \mathrm{~T} / \mathrm{C}$ \\
\hline UN_ci_8638 & 648 A/G \\
\hline UN_ci_8643 & $9 \mathrm{~T} / \mathrm{G}$ \\
\hline UN_ci_8643 & $42 \mathrm{C} / \mathrm{G}$ \\
\hline UN_ci_8643 & 228 A/G \\
\hline UN_ci_8643 & 447 T/C \\
\hline UN_ci_8644 & $150 \mathrm{~A} / \mathrm{G}$ \\
\hline UN_ci_8644 & 219 A/T \\
\hline UN_ci_8646 & 299 A/G \\
\hline UN_ci_8646 & $591 \mathrm{~T} / \mathrm{A}$ \\
\hline UN_ci_8646 & $650 \mathrm{G} / \mathrm{T}$ \\
\hline UN_ci_8646 & $787 \mathrm{G} / \mathrm{A}$ \\
\hline UN_ci_8647 & $106 \mathrm{C} / \mathrm{T}$ \\
\hline UN_ci_8651 & $211 \mathrm{G} / \mathrm{A}$ \\
\hline UN_ci_8651 & 357 G/C \\
\hline UN_ci_8651 & 617 T/C \\
\hline UN_ci_8651 & $753 \mathrm{~T} / \mathrm{C}$ \\
\hline UN_ci_8652 & 109 A/T \\
\hline UN_ci_8652 & $228 \mathrm{C} / \mathrm{T}$ \\
\hline UN_ci_8654 & $40 \mathrm{~T} / \mathrm{G}$ \\
\hline UN_ci_8660 & $6 \mathrm{G} / \mathrm{A}$ \\
\hline UN_ci_8660 & $40 \mathrm{~T} / \mathrm{A}$ \\
\hline UN_ci_8661 & $717 \mathrm{G} / \mathrm{A}$ \\
\hline UN_ci_8668 & $117 \mathrm{C} / \mathrm{T}$ \\
\hline UN_ci_8668 & $371 \mathrm{~T} / \mathrm{C}$ \\
\hline UN_ci_8669 & $98 \mathrm{G} / \mathrm{A}$ \\
\hline UN_ci_8669 & 402 G/C \\
\hline UN_ci_8669 & $490 \mathrm{~A} / \mathrm{G}$ \\
\hline UN_ci_8669 & $713 \mathrm{~A} / \mathrm{T}$ \\
\hline UN_ci_8669 & $760 \mathrm{C} / \mathrm{G}$ \\
\hline
\end{tabular}




\begin{tabular}{|c|c|}
\hline UN_ci_8675 & $530 \mathrm{~A} / \mathrm{C}$ \\
\hline UN_ci_8676 & $356 \mathrm{C} / \mathrm{T}$ \\
\hline UN_ci_8679 & $161 \mathrm{~A} / \mathrm{C}$ \\
\hline UN_ci_8679 & $216 \mathrm{C} / \mathrm{T}$ \\
\hline UN_ci_8679 & $239 \mathrm{~T} / \mathrm{C}$ \\
\hline UN_ci_8679 & $277 \mathrm{~A} / \mathrm{G}$ \\
\hline UN_ci_8679 & $378 \mathrm{~T} / \mathrm{C}$ \\
\hline UN_ci_8679 & $398 \mathrm{~A} / \mathrm{G}$ \\
\hline UN_ci_8679 & $442 \mathrm{~T} / \mathrm{C}$ \\
\hline UN_ci_8679 & $519 \mathrm{~A} / \mathrm{G}$ \\
\hline UN_ci_8679 & $565 \mathrm{~T} / \mathrm{C}$ \\
\hline UN_ci_8685 & $533 \mathrm{~A} / \mathrm{G}$ \\
\hline UN_ci_8685 & $745 \mathrm{C} / \mathrm{T}$ \\
\hline UN_ci_8687 & $88 \mathrm{~A} / \mathrm{C}$ \\
\hline UN_ci_8687 & $316 \mathrm{~A} / \mathrm{G}$ \\
\hline UN_ci_8687 & $516 \mathrm{C} / \mathrm{T}$ \\
\hline UN_ci_8688 & $500 \mathrm{~T} / \mathrm{C}$ \\
\hline UN_ci_8688 & $551 \mathrm{~T} / \mathrm{C}$ \\
\hline UN_ci_8692 & $241 \mathrm{C} / \mathrm{G}$ \\
\hline UN_ci_8692 & $771 \mathrm{~A} / \mathrm{G}$ \\
\hline UN_ci_8699 & $430 \mathrm{~T} / \mathrm{C}$ \\
\hline UN_ci_8699 & $491 \mathrm{~T} / \mathrm{C}$ \\
\hline UN_ci_8699 & $574 \mathrm{~T} / \mathrm{G}$ \\
\hline UN_ci_8699 & $606 \mathrm{~A} / \mathrm{G}$ \\
\hline UN_ci_8699 & $665 \mathrm{C} / \mathrm{T}$ \\
\hline UN_ci_8704 & $134 \mathrm{~A} / \mathrm{C}$ \\
\hline UN_ci_8704 & $242 \mathrm{G} / \mathrm{T}$ \\
\hline UN_ci_8704 & $516 \mathrm{~T} / \mathrm{C}$ \\
\hline UN_ci_8705 & $29 \mathrm{C} / \mathrm{T}$ \\
\hline UN_ci_8705 & $373 \mathrm{G} / \mathrm{A}$ \\
\hline UN_ci_8708 & $413 \mathrm{~A} / \mathrm{G}$ \\
\hline UN_ci_8708 & $567 \mathrm{G} / \mathrm{A}$ \\
\hline UN_ci_8708 & $635 \mathrm{C} / \mathrm{T}$ \\
\hline UN_ci_8708 & $723 \mathrm{~A} / \mathrm{G}$ \\
\hline UN_ci_8708 & $816 \mathrm{~A} / \mathrm{G}$ \\
\hline UN_ci_8708 & $871 \mathrm{C} / \mathrm{T}$ \\
\hline UN_ci_8708 & $898 \mathrm{~T} / \mathrm{C}$ \\
\hline UN_ci_8711 & $127 \mathrm{C} / \mathrm{T}$ \\
\hline UN_ci_8717 & $211 \mathrm{G} / \mathrm{A}$ \\
\hline UN_ci_8717 & $234 \mathrm{~A} / \mathrm{G}$ \\
\hline UN_ci_8717 & $304 \mathrm{~A} / \mathrm{G}$ \\
\hline UN_ci_8717 & $349 \mathrm{G} / \mathrm{A}$ \\
\hline UN_ci_8718 & $482 \mathrm{~T} / \mathrm{C}$ \\
\hline UN_ci_8718 & $549 \mathrm{~A} / \mathrm{G}$ \\
\hline UN_ci_8725 & $391 \mathrm{~T} / \mathrm{C}$ \\
\hline UN_ci_8736 & $478 \mathrm{~A} / \mathrm{T}$ \\
\hline UN_ci_8736 & $571 \mathrm{~A} / \mathrm{C}$ \\
\hline UN_ci_8736 & $709 \mathrm{G} / \mathrm{T}$ \\
\hline UN_ci_8736 & $748 \mathrm{G} / \mathrm{A}$ \\
\hline UN_ci_8736 & $825 \mathrm{~T} / \mathrm{C}$ \\
\hline
\end{tabular}




\begin{tabular}{|c|c|}
\hline UN_ci_8736 & $863 \mathrm{~A} / \mathrm{C}$ \\
\hline UN_ci_8739 & $370 \mathrm{C} / \mathrm{T}$ \\
\hline UN_ci_8739 & $973 \mathrm{~T} / \mathrm{C}$ \\
\hline UN_ci_8740 & $532 \mathrm{C} / \mathrm{T}$ \\
\hline UN_ci_8744 & $485 \mathrm{~A} / \mathrm{G}$ \\
\hline UN_ci_8744 & $568 \mathrm{~T} / \mathrm{C}$ \\
\hline UN_ci_8744 & $595 \mathrm{~T} / \mathrm{C}$ \\
\hline UN_ci_8744 & $860 \mathrm{C} / \mathrm{T}$ \\
\hline UN_ci_8744 & $887 \mathrm{~A} / \mathrm{T}$ \\
\hline UN_ci_8744 & $910 \mathrm{~A} / \mathrm{G}$ \\
\hline UN_ci_8744 & $976 \mathrm{~T} / \mathrm{C}$ \\
\hline UN_ci_8744 & $1046 \mathrm{C} / \mathrm{T}$ \\
\hline UN_ci_8744 & $1102 \mathrm{~T} / \mathrm{C}$ \\
\hline UN_ci_8744 & $1266 \mathrm{~A} / \mathrm{G}$ \\
\hline UN_ci_8744 & $1327 \mathrm{G} / \mathrm{A}$ \\
\hline UN_ci_8752 & $658 \mathrm{C} / \mathrm{A}$ \\
\hline UN_ci_8757 & $338 \mathrm{G} / \mathrm{T}$ \\
\hline UN_ci_8757 & $775 \mathrm{~T} / \mathrm{A}$ \\
\hline UN_ci_8758 & $67 \mathrm{G} / \mathrm{T}$ \\
\hline UN_ci_8758 & $123 \mathrm{~A} / \mathrm{G}$ \\
\hline UN_ci_8764 & $796 \mathrm{C} / \mathrm{T}$ \\
\hline UN_ci_8765 & $395 \mathrm{~A} / \mathrm{C}$ \\
\hline UN_ci_8767 & $133 \mathrm{C} / \mathrm{T}$ \\
\hline UN_ci_8768 & $149 \mathrm{C} / \mathrm{A}$ \\
\hline UN_ci_8768 & $374 \mathrm{~T} / \mathrm{C}$ \\
\hline UN_ci_8768 & 593 T/C \\
\hline UN_ci_8771 & $332 \mathrm{G} / \mathrm{A}$ \\
\hline UN_ci_8771 & $459 \mathrm{~T} / \mathrm{C}$ \\
\hline UN_ci_8771 & $761 \mathrm{C} / \mathrm{A}$ \\
\hline UN_ci_8785 & 185 T/C \\
\hline UN_ci_8785 & $304 \mathrm{G} / \mathrm{A}$ \\
\hline UN_ci_8785 & $633 \mathrm{C} / \mathrm{A}$ \\
\hline UN_ci_8785 & $750 \mathrm{C} / \mathrm{T}$ \\
\hline UN_ci_8785 & $1235 \mathrm{C} / \mathrm{A}$ \\
\hline UN_ci_8786 & $125 \mathrm{G} / \mathrm{T}$ \\
\hline UN_ci_8788 & $52 \mathrm{~T} / \mathrm{C}$ \\
\hline UN_ci_8788 & $154 \mathrm{~A} / \mathrm{G}$ \\
\hline UN_ci_8788 & $260 \mathrm{~T} / \mathrm{C}$ \\
\hline UN_ci_8788 & 297 T/C \\
\hline UN_ci_8788 & $344 \mathrm{~T} / \mathrm{C}$ \\
\hline UN_ci_8788 & $460 \mathrm{~T} / \mathrm{G}$ \\
\hline UN_ci_8788 & $507 \mathrm{~T} / \mathrm{C}$ \\
\hline UN_ci_8788 & $564 \mathrm{~A} / \mathrm{G}$ \\
\hline UN_ci_8788 & $670 \mathrm{~T} / \mathrm{C}$ \\
\hline UN_ci_8788 & $748 \mathrm{C} / \mathrm{G}$ \\
\hline UN_ci_8789 & $237 \mathrm{~A} / \mathrm{C}$ \\
\hline UN_ci_8792 & $349 \mathrm{~T} / \mathrm{A}$ \\
\hline UN_ci_8792 & $400 \mathrm{G} / \mathrm{A}$ \\
\hline UN_ci_8792 & $440 \mathrm{~T} / \mathrm{A}$ \\
\hline UN_ci_8792 & $529 \mathrm{~A} / \mathrm{T}$ \\
\hline
\end{tabular}




$\begin{array}{lr}\text { UN_ci_8792 } & 564 \mathrm{C} / \mathrm{T} \\ \text { UN_ci_8792 } & 590 \mathrm{G} / \mathrm{A} \\ \text { UN_ci_8794 } & 904 \mathrm{~A} / \mathrm{C} \\ \text { UN_ci_8794 } & 1228 \mathrm{~T} / \mathrm{G} \\ \text { UN_ci_8799 } & 32 \mathrm{~A} / \mathrm{G} \\ \text { UN_ci_8799 } & 61 \mathrm{~T} / \mathrm{A} \\ \text { UN_ci_8799 } & 171 \mathrm{~T} / \mathrm{A} \\ \text { UN_ci_8806 } & 328 \mathrm{G} / \mathrm{A} \\ \text { UN_ci_8806 } & 490 \mathrm{~A} / \mathrm{G} \\ \text { UN_ci_8806 } & 522 \mathrm{C} / \mathrm{T} \\ \text { UN_ci_8806 } & 598 \mathrm{C} / \mathrm{A} \\ \text { UN_ci_8806 } & 661 \mathrm{~T} / \mathrm{C} \\ \text { UN_ci_8815 } & 9 \mathrm{~A} / \mathrm{G} \\ \text { UN_ci_8815 } & 76 \mathrm{~A} / \mathrm{C} \\ \text { UN_ci_8815 } & 283 \mathrm{G} / \mathrm{A} \\ \text { UN_ci_8815 } & 314 \mathrm{~A} / \mathrm{C} \\ \text { UN_ci_8815 } & 478 \mathrm{G} / \mathrm{A} \\ \text { UN_ci_8815 } & 557 \mathrm{C} / \mathrm{T} \\ \text { UN_ci_8815 } & 625 \mathrm{~T} / \mathrm{C} \\ \text { UN_ci_8815 } & 675 \mathrm{~A} / \mathrm{G} \\ \text { UN_ci_8815 } & 747 \mathrm{~T} / \mathrm{C} \\ \text { UN_ci_8815 } & 785 \mathrm{C} / \mathrm{A} \\ \text { UN_ci_8815 } & 889 \mathrm{~T} / \mathrm{A} \\ \text { UN_ci_8815 } & 977 \mathrm{G} / \mathrm{A} \\ \text { UN_ci_8815 } & 1032 \mathrm{C} / \mathrm{T} \\ \text { UN_ci_8815 } & 1134 \mathrm{~T} / \mathrm{C} \\ \text { UN_ci_8815 } & 1450 \mathrm{~A} / \mathrm{C} \\ \text { UN_ci_8815 } & 1636 \mathrm{C} / \mathrm{T} \\ \text { UN_ci_8815 } & 1731 \mathrm{~A} / \mathrm{G} \\ \text { UN_ci_8816 } & 171 \mathrm{G} / \mathrm{A} \\ \text { UN_ci_8816 } & 199 \mathrm{~A} / \mathrm{G} \\ \text { UN_ci_8816 } & 413 \mathrm{C} / \mathrm{T} \\ \text { UN_ci_8816 } & 631 \mathrm{G} / \mathrm{T} \\ \text { UN_ci_8816 } & 656 \mathrm{~A} / \mathrm{G} \\ \text { UN_ci_8816 } & 790 \mathrm{G} / \mathrm{T} \\ \text { UN_ci_8816 } & 829 \mathrm{~T} / \mathrm{C} \\ & \\ \text { Un } & \end{array}$




\section{Figure S1}

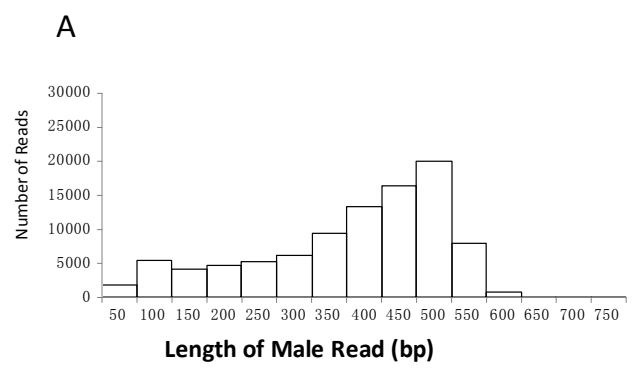

B

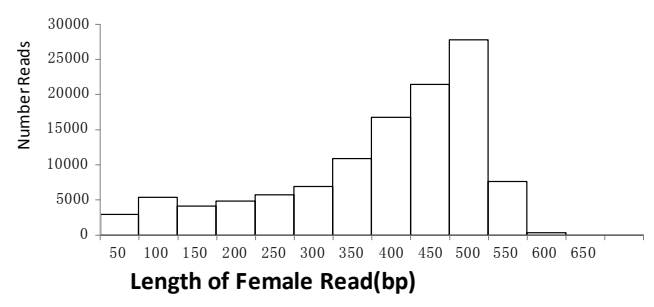

Figure S1 Overview of the raw reads obtained from the Scophthalmus maximus transcriptome. (A) Size distribution of the reads from male turbots; (B) Size distribution of the reads from female turbots. 